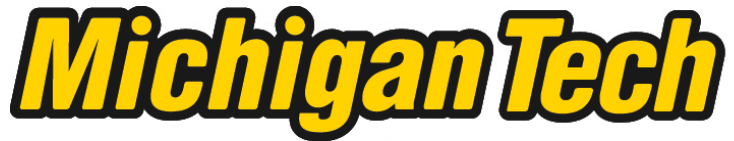 \\ Michigan Technological University Create the Future Digital Commons @ Michigan Tech
}

Planning and adaptation measures for urban slum communities in West Africa : stochastic rainfall modeling applied to domestic rainwater harvesting and climate change adaptation

Joshua R. Cowden

Michigan Technological University

Follow this and additional works at: https://digitalcommons.mtu.edu/etds

Part of the Civil and Environmental Engineering Commons

Copyright 2008 Joshua R. Cowden

Recommended Citation

Cowden, Joshua R., "Planning and adaptation measures for urban slum communities in West Africa : stochastic rainfall modeling applied to domestic rainwater harvesting and climate change adaptation", Dissertation, Michigan Technological University, 2008.

https://doi.org/10.37099/mtu.dc.etds/724

Follow this and additional works at: https://digitalcommons.mtu.edu/etds

Part of the Civil and Environmental Engineering Commons 


\title{
Planning and Adaptation Measures for Urban Slum Communities in West Africa: Stochastic Rainfall Modeling Applied to Domestic Rainwater Harvesting and Climate Change Adaptation
}

\author{
By \\ Joshua R Cowden \\ A DISSERTATION \\ Submitted in partial fulfillment of the requirements \\ for the degree of \\ DOCTOR OF PHILOSOPHY IN ENVIRONMENTAL ENGINEERING \\ MICHIGAN TECHNOLOGICAL UNIVERSITY \\ 2008
}

Copyright (C) Joshua R Cowden 2008 

This dissertation, "Planning and Adaptation Measures for Urban Slum Communities in West Africa: Stochastic Rainfall Modeling Applied to Domestic Rainwater Harvesting and Climate Change Adaptation," is hereby approved in partial fulfillment of the requirements for the degree of DOCTOR OF PHILOSOPHY IN ENVIRONMENTAL ENGINEERING.

DEPARTMENT:

Civil and Environmental Engineering

Signatures:

Dr. James R. Mihelcic, Dissertation Co-Advisor

Date

Dr. David W. Watkins Jr., Dissertation Co-Advisor

Date

Dr. Judith Perlinger, Non-Departmental Program Chair

Date 



\begin{abstract}
Over half of the world's population is living in urban settlements, and most urban growth is occurring in developing countries. These countries' economies are often unable to accommodate these rural-urban immigrations, resulting in millions of people settling in insecure communities known as urban slums. Current efforts to prevent urban slums include UN-HABITAT strategies to upgrade slums and stimulate urban/regional development. Urban metabolism analysis, which studies material and energy flows/stocks through urban processes, help decision-makers better understand their urban system. Nine lessons from past urban metabolism studies are presented to assist in finding answers on how to best solve urban slum challenges.
\end{abstract}

Worldwide, improved water access rates are lowest for Sub-Saharan Africa and these low rates have important implications on the health and economy of the region. Domestic rainwater harvesting (DRWH) is proposed as a potential mechanism for water supply enhancement, especially for the poor urban households in the region, which is essential for development planning and poverty alleviation initiatives. Several parsimonious stochastic rainfall models are developed and compared for application to DRWH assessment in West Africa. A first-order Markov occurrence model with a mixed exponential amount model is selected as the best option for unconditioned Markov models. However, there is no clear advantage in selecting Markov models over spelllength models for DRWH, with each model having distinct strengths and weaknesses. It is clear DRWH can be successfully used as a water enhancement mechanism in West Africa for significant portions of the year.

Climate model output is used to determine climate change impacts to DRWH and to assess the technology as an adaptation measure to climate change. Several statistical downscaling methods are used to downscale multiple climate models to the local level. Climate change is expected to have little impact on DRWH reliability in West Africa by the mid- $21^{\text {st }}$ century, with only slight temporal shifts in rainfall. Developing communities in this region can invest with confidence in DRWH systems for drinking water enhancement. Study results also suggest that community improvements toward implementing DRWH systems should be focused on increasing water storage, due to storm size frequency changes. 


\section{Acknowledgements}

The author gratefully acknowledges support from the Sustainable Futures IGERT project sponsored by the U.S. National Science Foundation (under Grant No. DGE 0333401), the Michigan Technological University Graduate School, and the Department of Civil and Environmental Engineering.

My many thanks also go to my advisors, Dr. James R. Mihelcic and Dr. David W. Watkins Jr. for their unending support and mentorship. I am also grateful to my committee members, Dr. Alex S. Mayer and Dr. Mary H. Durfee, as well as to the entire graduate faculty, for their instruction and insights.

Finally, my love and appreciation go to my wife and children, for their patience, love, and sacrifice. 


\section{Contents}

Chapter 1 Objectives, Introduction, and Organization............................................ 1

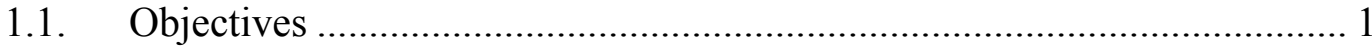

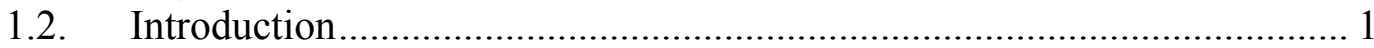

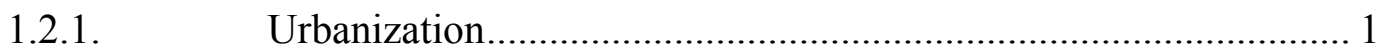

1.2.2. Climate

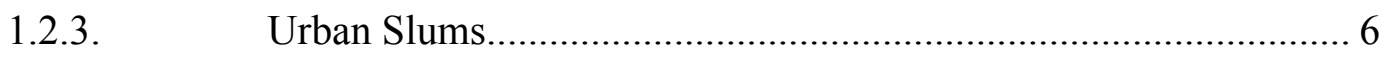

1.2.4. Domestic Rainwater Harvesting ................................................ 8

1.2.5. Stochastic Weather Generators .................................................... 8

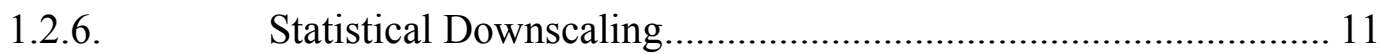

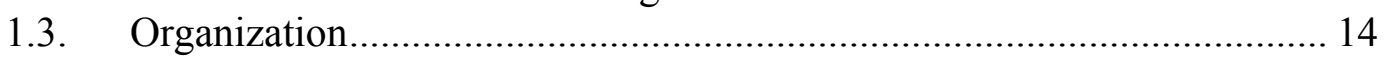

Chapter 2 Urban Metabolism Analysis: Nine Lessons for Urban Slums in the

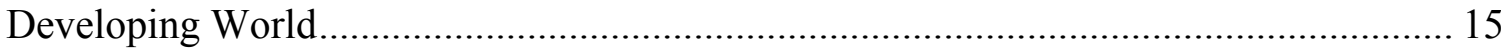

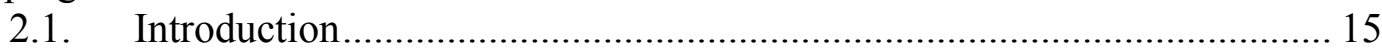

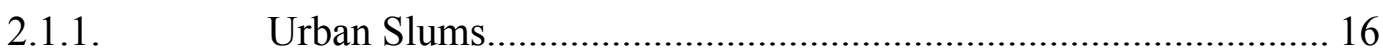

2.1.2. Urban Slums Programs and Policies........................................... 18

2.2. Urban Metabolism Lessons.................................................................. 20

2.2.1. Metabolism Lessons for Developing Countries............................. 22

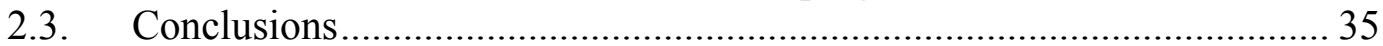

Chapter 3 Stochastic Rainfall Modeling in West Africa: Parsimonious Approaches for Domestic Rainwater Harvesting Assessment................................................................ 37

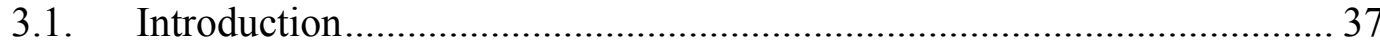

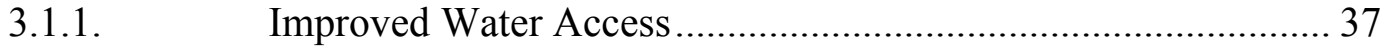

3.1.2. Stochastic Weather Generators ................................................. 40

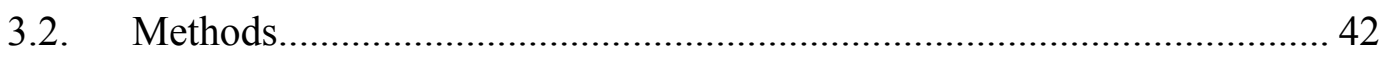

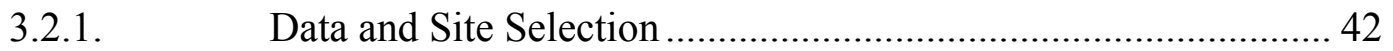

3.2.2. Stochastic Rainfall Modeling.................................................... 43

3.2.3. Model Comparison................................................................ 45

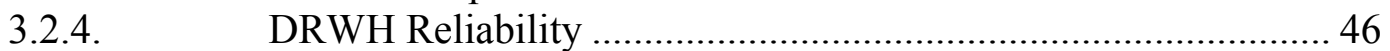

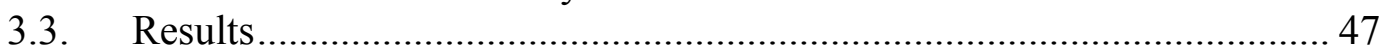

3.3.1. Markov Order..................................................................... 47

3.3.2. Occurrence Sensitivity to Missing Data .................................... 50

3.3.3. Amount Model ....................................................................... 52

3.3.4. Model Comparison................................................................... 53

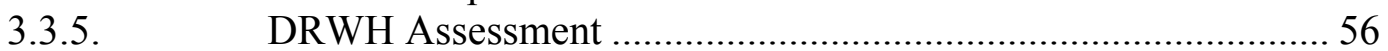

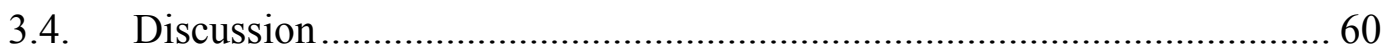

Chapter 4 Climate Change Impact Assessment on Domestic Rainwater Harvesting in

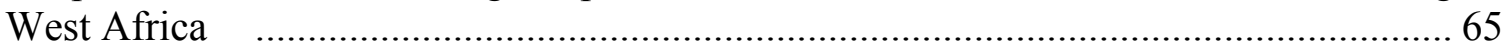

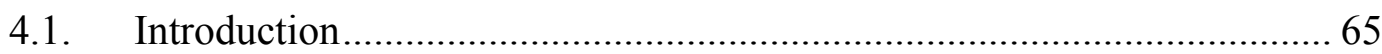

4.1.1. Regression Models................................................................. 71

4.1.2. Weather Classification ............................................................ 72

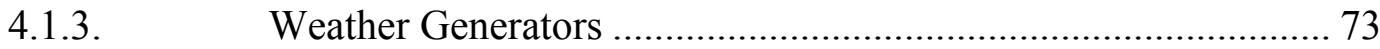

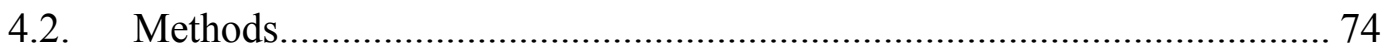

4.2.1. Markov Weather Generator Downscaling ..................................... 75

vii 
4.2.2. Spell-length Weather Generator Downscaling ..... .76

4.2.3. Climate Change Impact Analysis on Domestic Rainwater

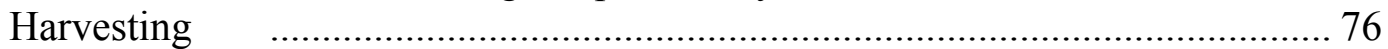

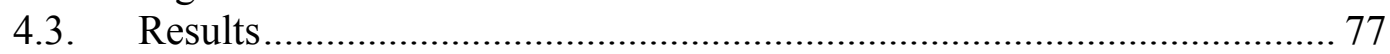

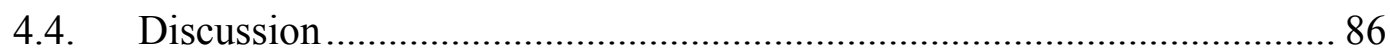

Chapter 5 Findings and Recommendations for Future Work.................................. 91

5.1. Urban Slums and Urban Metabolism..................................................... 91

5.2. Stochastic Weather Models and Domestic Rainwater Harvesting ........... 93

5.3. Climate Change Impact on Domestic Rainwater Harvesting ................... 94

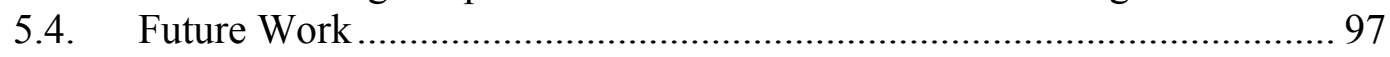

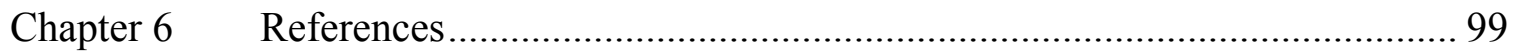

Appendix A: Map of Gauge Locations

Appendix B: Markov Model Transition Probabilities

Appendix C: LARS-WG Parameters

Appendix D: Conditioned 1st-Order Markov Transition Probabilities

Appendix E: Gamma Distribution Parameters for Wet Day Amounts

Appendix F: Mixed Exponential Distribution Parameters for Wet Day Amounts

Appendix G: Conditioned Mixed Exponential Distribution Parameters for Wet Day

Amounts

Appendix H: Domestic Rainwater Harvesting Reliabilities Using the $1^{\text {st }}$-Order Markov Model

Appendix I: Domestic Rainwater Harvesting Reliabilities Using the LARS-WG Spelllength Model

Appendix J: Domestic Rainwater Harvesting Reliabilities Using the Conditioned $1^{\text {st }}$ Order Markov Model

Appendix K: Statistical Downscaling Results 


\section{Chapter 1 Objectives, Introduction, and Organization}

\subsection{Objectives}

Human civilizations and the natural environment have always been subject to

change as they both respond and adapt to new threats and opportunities. Global change is rapidly advancing, and may soon overwhelm this ability to adapt. Global change comes in many forms, including land use, climate, and consumption, and with the human sphere being intertwined with the natural ecology of the planet, what impacts one can no longer be ignored by the other. This research has three objectives in an exploration of possible solutions to the global change issues facing West African urban populations, especially challenges related to improved water access:

- Build several lessons for slum communities and their host cities based on the experiences garnered from urban metabolism studies in the developed world.

- Assess the feasibility and reliability of domestic rainwater harvesting systems in West African slum households using stochastic weather generator models.

- Assess the potential impacts of climate change on domestic rainwater harvesting systems in slum communities by statistically downscaling global circulation model (GCM) predictions.

\subsection{Introduction}

\subsubsection{Urbanization}

The world's urban population in 2005 was 3.15 billion and by 2030 will increase to 4.91 billion, nearly $60 \%$ of the global population (UNESA, 2006). The next few years will see over half of the global population living in urban areas for the first time in 
recorded history. This urbanization, however, is not evenly distributed throughout the world. Developing nations are experiencing dramatic shifts from their large rural base to urban regions due to rural economic decline, wars, drought, etc., and are expected to account for and absorb most of the world's total population growth between 2000 and 2030 (UNESA, 2006). Sub-Saharan Africa is currently experiencing some of the planet's highest urbanization rates (UNESA, 2006). West African urban populations, already the highest in sub-Saharan Africa, will continue to grow in the coming decades, even under a declining urban growth rate (Figure 1-1 and Figure 1-2).

Rapid population growth strains urban water supplies and infrastructure, as well as the local government's capability of providing new infrastructure to what often are large, illegal settlements (Cowden et al., 2006; UN-HABITAT, 2003). At the same time, climate change has the potential to have a large impact on water availability because $20 \%$ of the one billion people estimated to experience water scarcity by the year 2025 are associated with direct effects of climate change (Vorosmarty et al. 2000). Figure 1-3 and Figure 1-4 illustrate the improved water access deficiencies and associated lack of sanitation for urban areas in West Africa, which leaves millions at risk to water-borne disease. The northern areas of West Africa below the Sahara do suffer from physical water scarcity; however, many of the coastal regions containing most of the population seem to have adequate hydrologic resources, pointing to limited economic and planning capacity as the culprit for poor drinking water access (Cowden et al, 2006). 


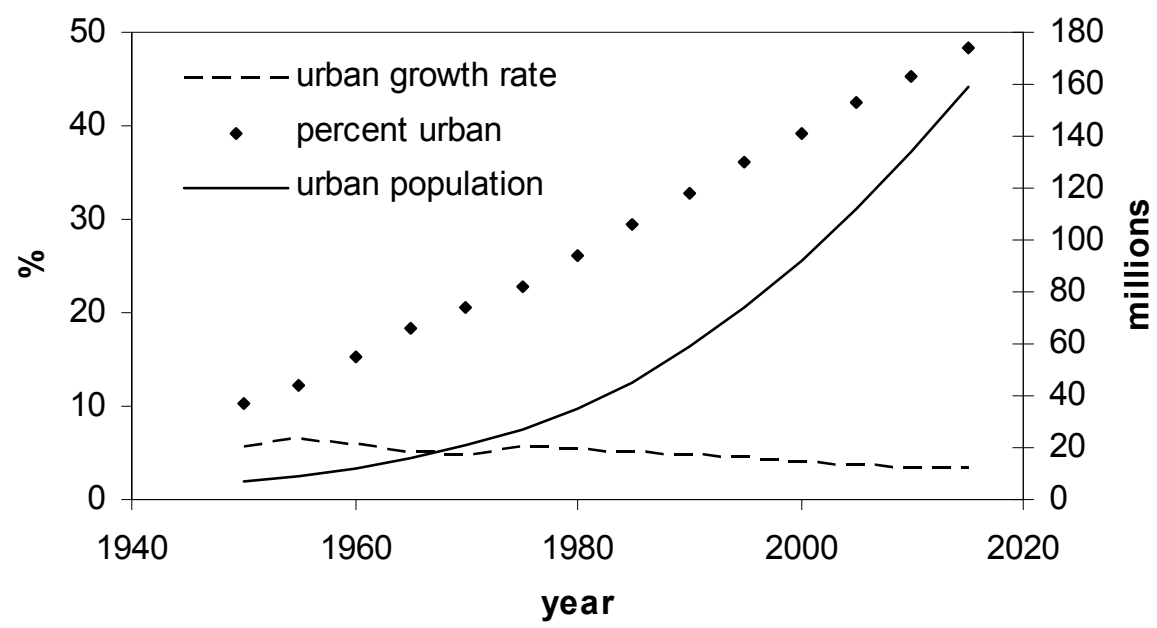

Figure 1-1: West Africa Urban Population and Growth Rates

(Figure created by author from data in UNESA, 2006)

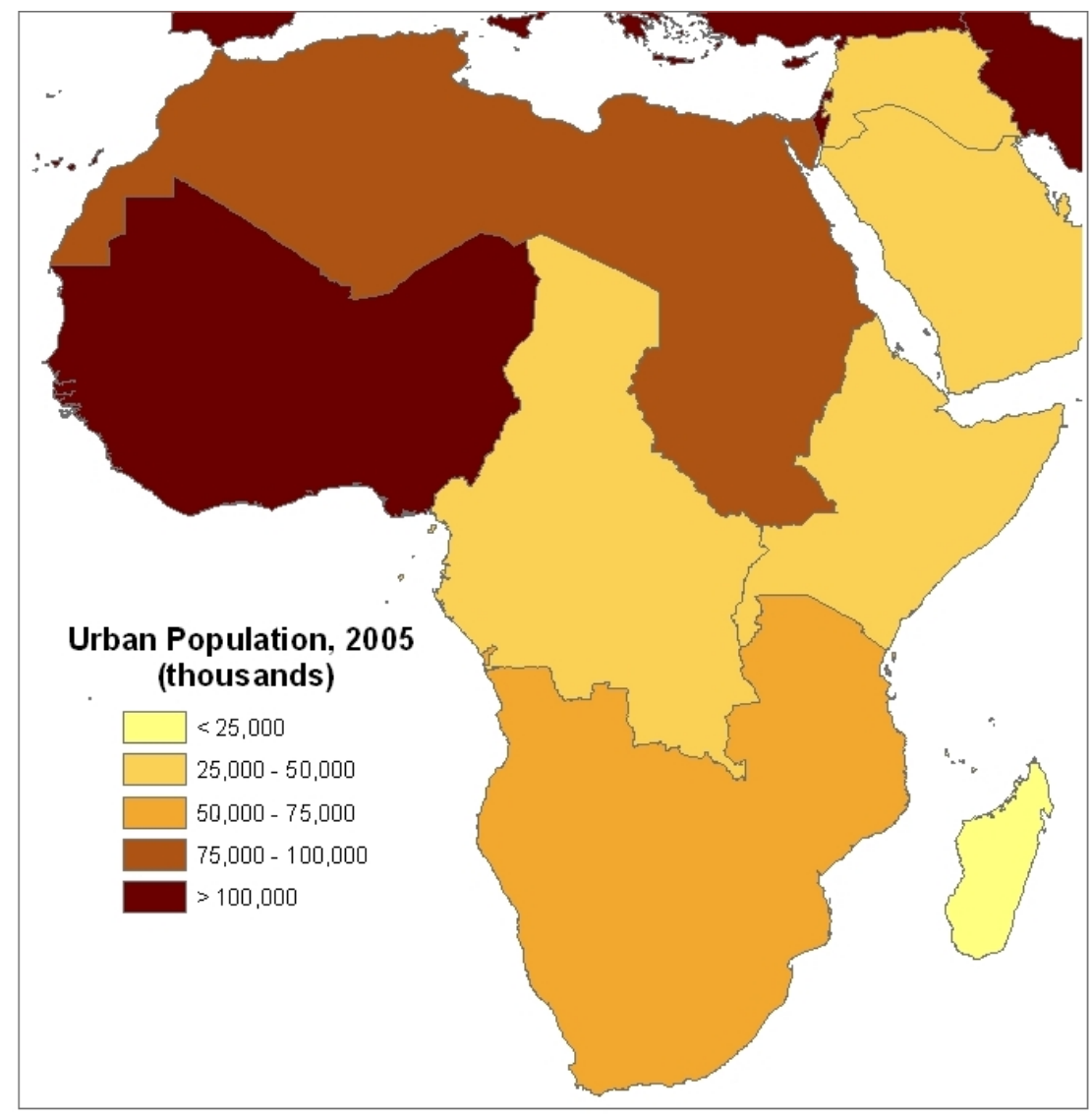

Figure 1-2: Urban Population Totals in Africa (Map created by author from data in UNEP, 2005) 


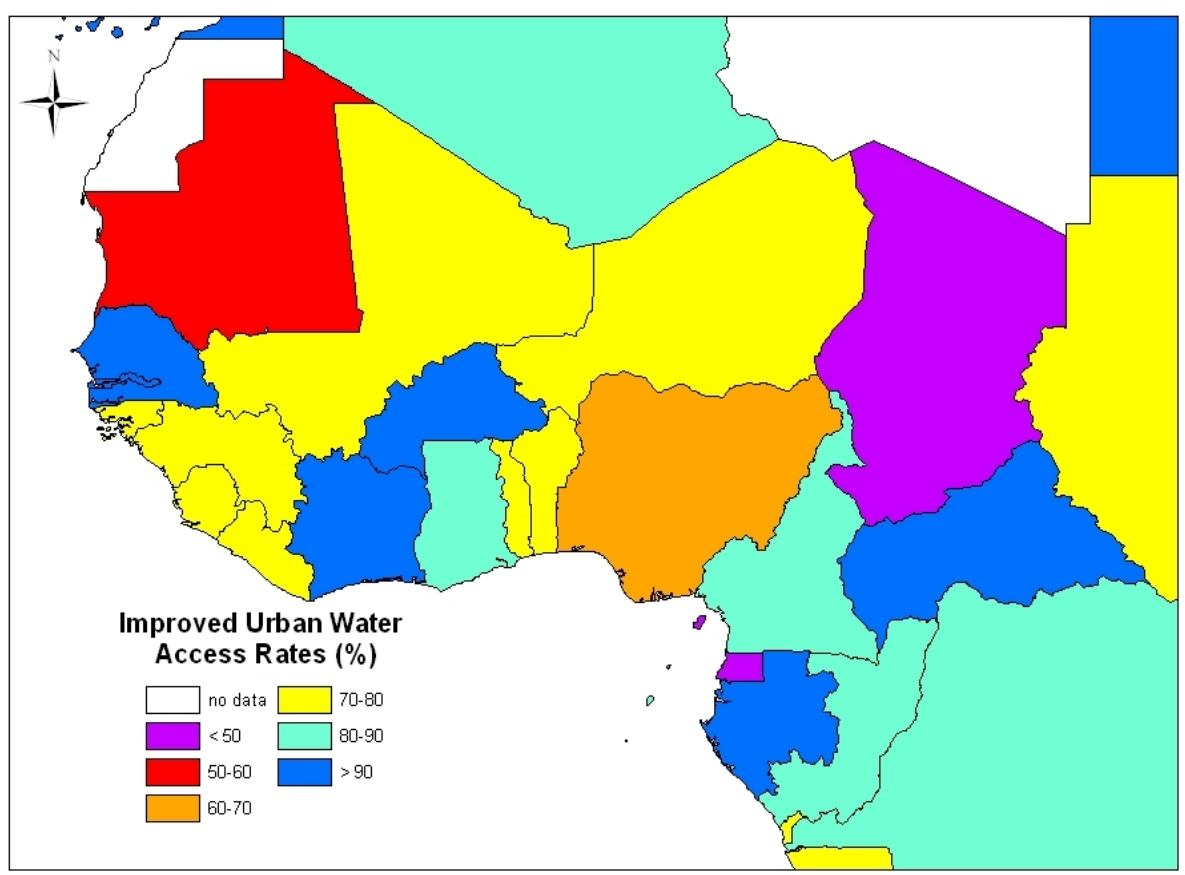

Figure 1-3: Improved Urban Water Access Rates in West Africa (Map created by author from data in UNEP, 2005)

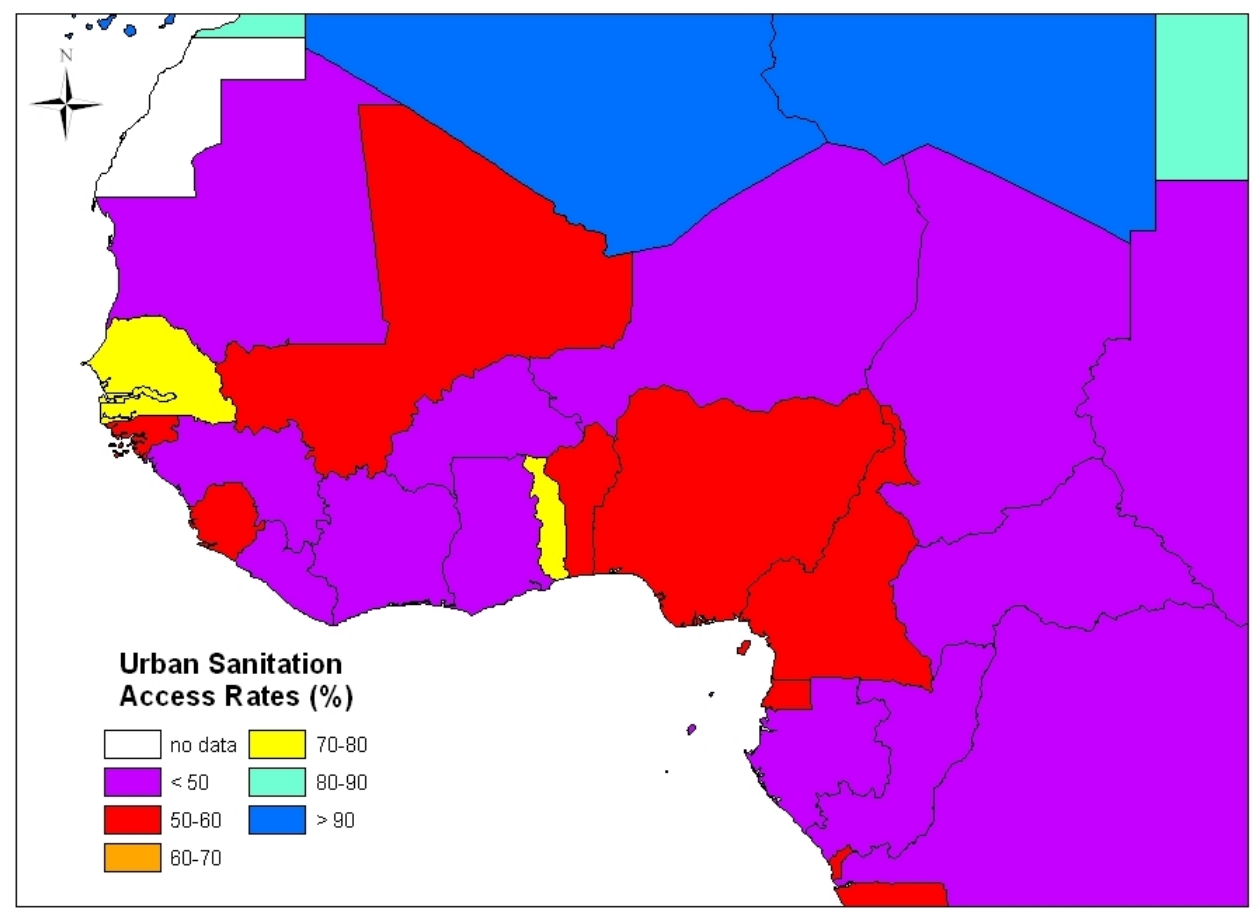

Figure 1-4: Urban Sanitation Access Rates in West Africa (Map created by author from data in UNEP, 2005) 


\subsubsection{Climate}

The climate of West Africa is variable both spatially and temporally (Figure 1-5). Rainfall is relatively abundant in the humid tropics along the southern coastal areas. The Sahelian region, the boundary between the Sahara desert and the more humid coastal and interior regions, is more arid and therefore more susceptible to unexpected rainfall. Much of the region experiences an annual wet and dry oscillation, associated with movement of the Inter-tropical Convergence Zone (ITCZ). The ITCZ is the boundary in which the trade winds of the Northern and Southern hemispheres meet, creating a band of low pressure and associated thunderstorms around the equator. The arrival of these systems creates monsoon conditions during the wet season for much of the tropics (Sultan and Janicot, 2003).

Interannual climate variability is also evident in the area, with a major drought occurring in the early 1970's and 1980's, and general drought conditions existing from the late 1960's into the 1990's (Le Barbe et al., 2002). Drought conditions in the area have been strongly correlated to sea surface temperatures. Temperature variations in the Gulf of Guinea and the tropical Atlantic, and the associated atmospheric responses, have been shown to explain historical changes in wet and dry precipitation patterns in both the Sahel and Guinea Coast (Balas et al., 2007; Giannini et al., 2003; Hoerling et al., 2006). Interannual variability can disrupt the stationarity of precipitation record of limited length. Rainfall records during the latter half of the $20^{\text {th }}$ century show slight nonstationarity due to a rising trend of precipitation as the aforementioned drought ended.

The impacts of climate change on the precipitation patterns of West Africa are uncertain at this time. Many of the climate models are incapable of producing current 
climate patterns associated with the West African monsoon (Cook and Vizy, 2006).

Several of the models do not agree on the sign change of precipitation impacts in the coming decades; however general conclusions indicate a drying in the sub-tropics due to minor increases in precipitation in the tropical regions (Christensen et al., 2007).

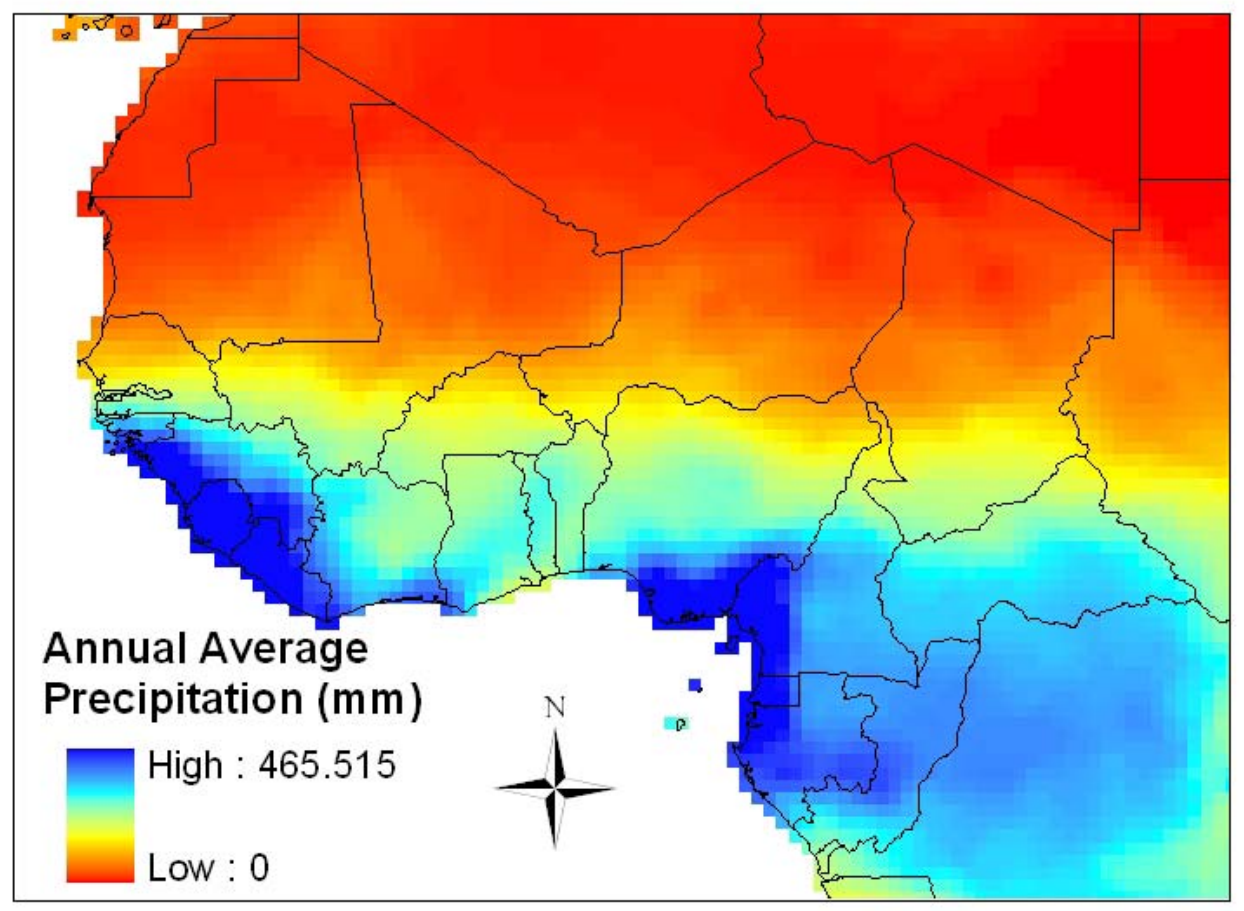

Figure 1-5. Annual Precipitation Patterns West Africa (Map created by author from data in VASClimO, 2005)

\subsubsection{Urban Slums}

Rapidly growing cities challenge efforts to improve quality of life and environmental conditions for their inhabitants, as well as cause environmental and economic change on a global scale due to high material consumption, fuel use (i.e. greenhouse gas emissions), waste production, and land use changes (e.g. deforestation). As urban populations increase in cities with inadequate services, so does the percentage of urban slum dwellers, defined as lacking any combination of adequate and safe drinking 
water, sanitation, durable housing, living space, and security of tenure (UN-HABITAT, 2005). Unfortunately, slum dwellers often do not have the political decision-making access to change these conditions on a community-wide scale (UN-HABITAT, 2005). In 2001, there were 924 million slum dwellers worldwide living in these conditions, a $28 \%$ increase from 1990 (UN-HABITAT, 2005). The slum dweller population in Sub-Saharan Africa represents $72 \%$ of the urban population.

The world community has resolved, through the United Nations Millennium Development Goals (MDG) Target 11, to achieve "significant improvement in the lives of at least 100 million slum dwellers" by 2020 (United Nations, 2005). MDG Target 10 aims to halve, by 2015, the proportion of people without sustainable access to safe drinking water and basic sanitation. Abundant, improved water is key to the health and quality of life of slum dwellers, but urban research indicates that clean water access rates are dismal in many slum areas, with many more cities forecasting an inability to maintain access to growing demand (UN-HABITAT, 2003).

The challenges to slum water provision brought on by increasing urbanization are also exacerbated by predicted climate change scenarios. Understanding the dynamics between slum communities, their associated governments, and their surrounding environment are essential in achieving and surpassing the MDG goals. The challenge is daunting, however, as the number of urban slum dwellers increases. If slum dweller proportions remain the same, West Africa could see an additional 34 million urban dwellers living in these conditions, possibly negating MDG Target 11 efforts. Chapter 2 discusses urban metabolism methodologies and how they can increase understanding between slums and their environment. 


\subsubsection{Domestic Rainwater Harvesting}

Domestic rainwater harvesting (DRWH) is an ancient technology still practiced formally (e.g. permanent storage systems) and informally (e.g. pots under roof edges) in many dry and wet climates alike. DRWH has been experiencing increasing popularity in providing water where supplies are low due to water scarcity or poverty. These systems may be very compatible to the conditions found in impoverished urban communities, as they save time, money, and are less complex then other centralized treatment technologies (Thomas, 1998).

This technology may also empower slum households in enhancing their own water supply, regardless of the availability and willingness of government infrastructure support and international economic aid (Cowden, 2006). DRWH can be a viable replacement for conventional services that are not being provided due to their illegal tenure condition. Large scale community planning, management, and operation issues are also negligible as this technology is primarily managed at the household level. Finally, once in place, the resource is provided at no monetary cost or travel time to the slum dweller. Granted, all these advantages are dependent on adequate rainfall supplies, and slum dwellers must be provided access to information that will assist them in decisions regarding rainwater harvesting. Figure 1-6 illustrates the area of Africa where DRWH is capable of enhancing domestic water supplies.

\subsubsection{Stochastic Weather Generators}

Extensive rainfall records are needed to adequately assess DRWH reliability. Complete or long daily rainfall records, however, are not available in many regions of sub-Saharan Africa. Stochastic weather generators offer a solution by creating multiple, 
continuous synthetic sequences of rainfall for use in hydrologic modeling and reliability assessment (Wilks and Wilby, 1999). Stochastic weather generators can also be used in climate change impact assessments via parameter adjustment by global climate model output (Semenov and Barrow, 1997; Wilby et al., 1998). Chapter 3 discusses the methodology and application of weather generators to DRWH systems in West Africa. 

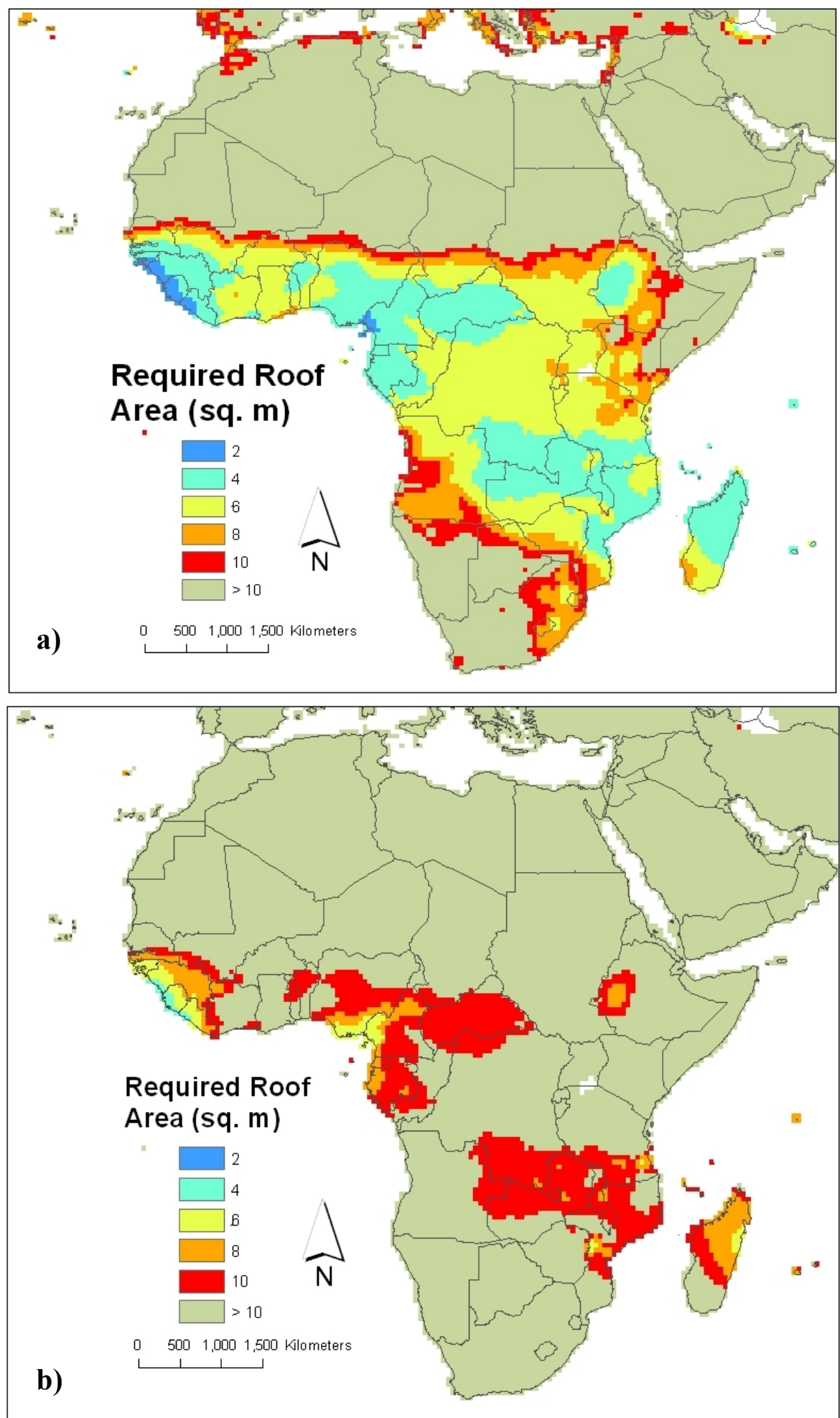

Figure 1-6. Required roof area ( $\mathrm{m}^{2} /$ capita) to supply at least three months of a) 20 $\mathrm{L} /$ day/capita, and b) $50 \mathrm{~L} /$ day/capita of rainwater (Cowden, 2006; With permission from ASCE) 


\subsubsection{Statistical Downscaling}

The following sections discuss the methods of using GCM data to determine climate change impacts. These methods are also summarized in Chapter 4, along with their use in determining climate change impacts on domestic rainwater harvesting.

\subsubsection{General Circulation Models}

The past twenty years have seen remarkable advances in our understanding of climate processes and the transfer of this knowledge into climate models. The most advanced and complex of these models are coupled atmosphere-ocean general circulation models (GCM), which are commonly used for climate projections based on anthropogenic forcings (e.g. greenhouse gas emissions) (IPCC, 2001). GCMs, however, are currently not capable of providing adequate weather variable outputs at the spatial or temporal scales required by most hydrological models, making direct GCM climate change impact studies vague at best. Using current GCM output for regional or local studies is inadvisable for two reasons: 1) the coarse spatial scale of GCM requires certain weather variables, such as precipitation, to be parameterized, and therefore more vulnerable to large-scale model error easily propagated down to local situations; and 2) sub-grid scale forcings, such as land-use, topography, and cloud cover, are often not accounted for in GCMs, even though these forcings are important in regional weather variability (Wilby and Wigley, 1997; IPCC, 2001).

Initial attempts to downscale GCM results to local impacts used the change factor method, where only the differences between controlled and perturbed GCM runs were applied to a local baseline climate. For example, if the GCM predicts a 2-degree temperature rise between simulated periods of 1990 and 2080, then 2 degrees would be 
added to the observed 1990 temperature records to simulate the predicted future.

Although there is no defined relationship between the coarse and finer scale variables, and no change in variable time structure, this method is useful for rapid climate change scenario analysis or where resources are not available for more complex methods (Wilby et al., 2004; Diaz-Nieto and Wilby, 2005). Recognizing the need for variable temporal structure in impact studies, several more complex methods have been developed, categorized as dynamical and statistical downscaling methods (Figure 1-7).

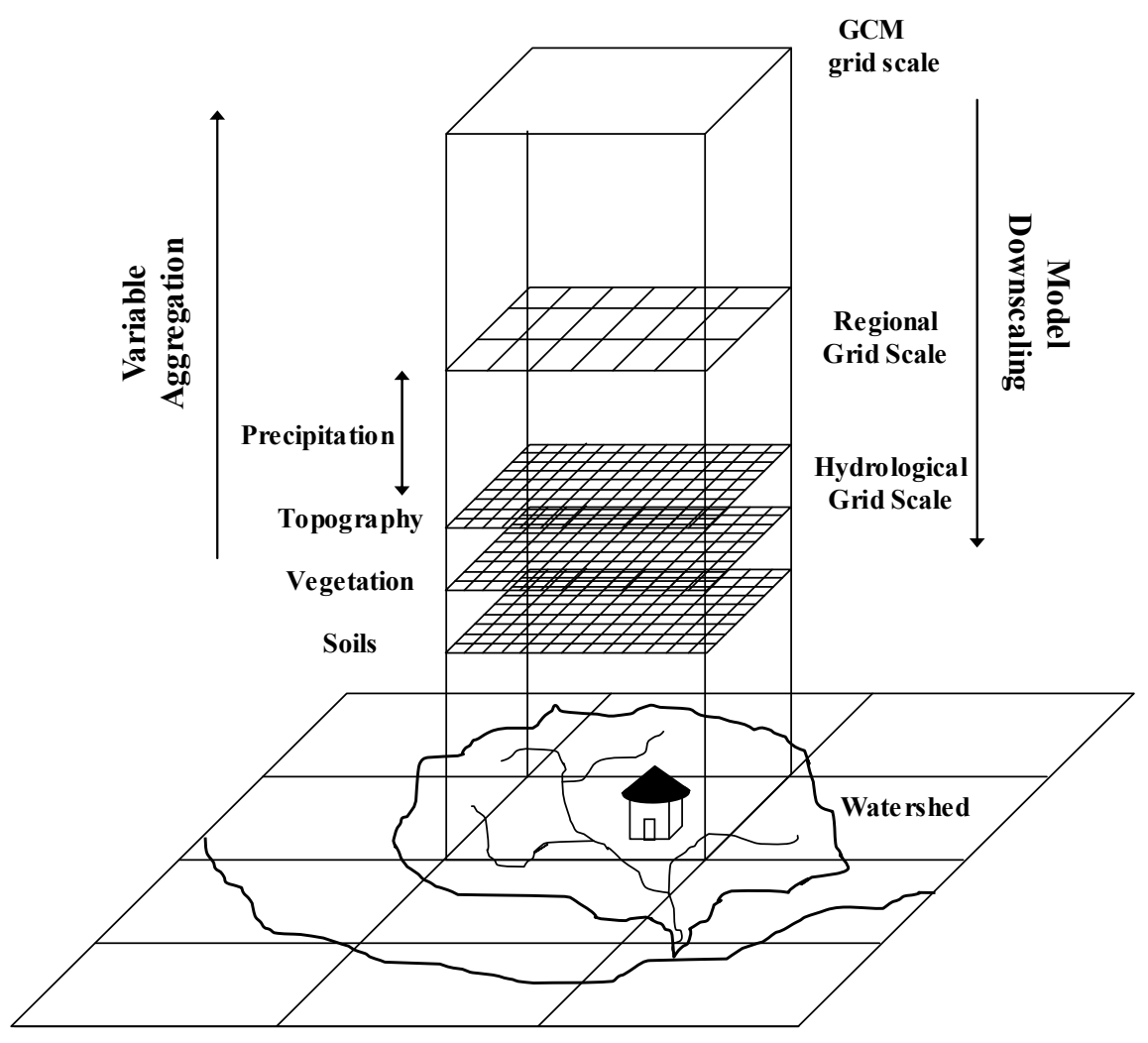

Figure 1-7: General Approach to GCM Downscaling

Variables essential to watershed modeling, such as precipitation and soils, are not modeled in GCMs at the necessary scale. Statistical downscaling GCM output ties largescale predictions to local variable response. (adapted from Wilby and Dawson, 2004) 


\subsubsection{Dynamical Methods}

Dynamical methods increase the horizontal (i.e. spatial) resolution of climate models. Impact studies using high and variable resolution Regional Climate Models (RCM) and GCMs are becoming more common, hopefully leading to performance analysis of such models (IPCC, 2001). Ultimately, physically-based climate models with fine enough resolution to account for all global and local forcings would best project the future climate and indeed, this is the goal of GCM research. Physical understanding and computational ability, however, currently prevent such models, especially in the developing world where computational and monetary resources are low.

\subsubsection{Statistical Methods}

Statistical downscaling (SD) methods define a quantitative relationship between large-scale atmospheric predictors and local surface variables or predictands (Wilby et al., 2004). The general form of this relationship is

$$
R_{t}=F\left(X_{T}\right) \quad \text { for } T \leq t
$$

where $R_{t}$ is the predictand at time $t, F$ is the method to quantify the relationship, and $X_{t}$ is the predictor set of current or past atmospheric variables (Charles et al., 2004). Possible predictors include pressure, geopotential height, wind speed and direction, precipitation, and humidity. Predictor sets can be derived from the GCM results, but can also come from RCMs, creating a hybrid dynamical-statistical downscaling approach. IPCC guidelines ultimately recommend predictor variables to be strongly correlated with the predictand, make physical sense, be realistically characterized by the GCM, able to represent interannual variability, and reflect the climate change signal (Wilby et. al, 2004). 


\subsubsection{Downscaling Models}

Statistical downscaling models can be grouped into three broad categories, namely regression models, weather classification schemes, and weather generators (IPCC, 2001). Regression models use linear or nonlinear regression transfer function to relate the predictors and predictand. Weather classification schemes or weather typing uses synoptic similarity to classify or group days into a finite number of discrete weather types or "states". Predictand values or probabilities are then assigned to each class by weighting the predictand(s) with the relative frequencies of the weather classes. Weather generators are stochastic models able to reproduce the statistical characteristics of a local predictand. The models are conditioned on large-scale predictors, weather states, or precipitation properties

\subsection{Organization}

This dissertation is organized into the following chapters.

- "Urban Metabolism Analysis: Nine Lessons for Urban Slums in the Developing World" (Chapter 2)

- "Stochastic Rainfall Modeling in West Africa: Parsimonious Approaches for Domestic Rainwater Harvesting Assessment” (Chapter 3)

- "Climate Change Impact Assessment on Domestic Rainwater Harvesting in West Africa" (Chapter 4) 


\section{Chapter 2 Urban Metabolism Analysis: Nine Lessons for Urban Slums in the Developing World}

\subsection{Introduction}

The world's rural population in 2003 was 3.26 billion and is predicted to decrease to 3.19 billion by 2030 . For the first time in human history, human urban population will exceed rural population in 2007 (Figure 2-1) and by 2030, 60.8\% of the global population is expected to be living in urban areas. During this period, the world's urban population growth rate is predicted to be $1.83 \%$, nearly double the world's total population growth rate (UNESA, 2004).

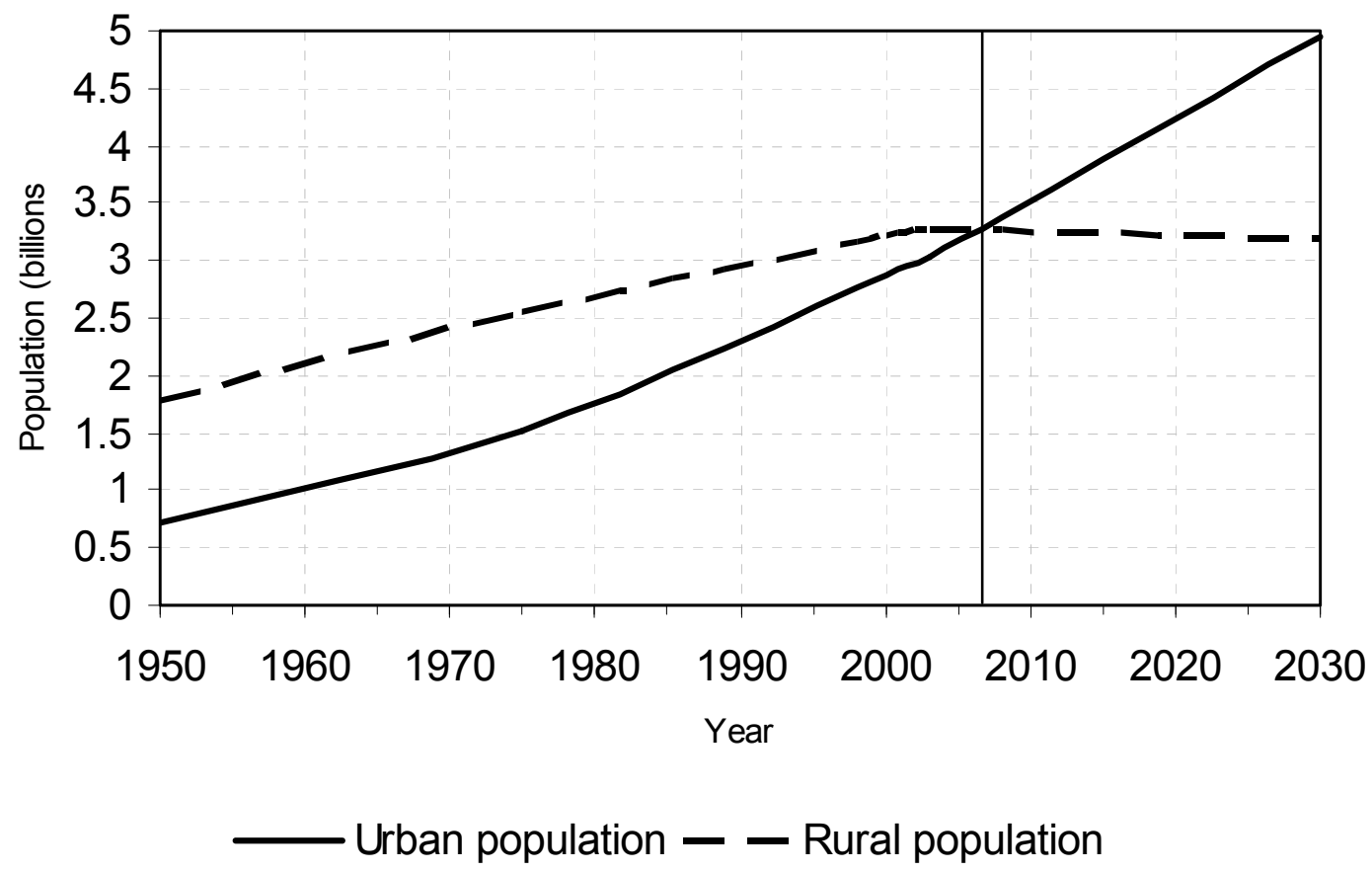

Figure 2-1: Urban and Rural Populations of the World: 1950-2030 (Figure created by author from data in UNESA, 2004) 
The large increase in urban population is not evenly distributed throughout the world, however. Most of the more developed countries are already highly urbanized, Europe being 73\% urbanized and North America being 80\% urbanized in 2003 . Although these percentages are expected to increase in the future, they are small compared to the rate of urbanization occurring in developing nations, which are currently experiencing dramatic shifts from their large rural base to urban regions. These urban regions of the developing world are thus expected to account for and absorb most of the world's total population growth between 2000 and 2030 (UNESA, 2004). These rapidly growing cities are and will continue to present unique and significant challenges in sustaining a quality of life and a clean environment for their inhabitants (Ezcurra \& Mazari-Hiriart, 1996).

\subsubsection{Urban Slums}

As the number and size of the world's cities increase, so do the absolute numbers of people living in urban poverty. These urban poor are often designated as slum dwellers, defined as lacking any combination of adequate and safe drinking water, sanitation, durable housing, living space, and security of tenure (UN-HABITAT, 2005). These inadequate conditions results in slum dwellers being more vulnerable to disease, natural disasters (e.g. flooding, land slides, earthquakes), crime, and inequality. Urban slums, especially in developing countries, are often settled by large masses of people fleeing rural economic decline, wars, drought, etc., and are considered illegal squatter settlements on public or private land. These people are given little to no political decision-making access and are usually denied basic municipal services (UN, 2005). As a result, urban growth in developing countries has occurred in a haphazard manner, with 
little planning regarding the welfare and sustainability of the community or the environment.

In 2001, there were 924 million slum dwellers worldwide living in these conditions, a 28\% increase from 1990 (UN, 2005). However, slum dwellers as a percentage of the total urban population have decreased in most developing regions of the world during the same time period (Table 2-1). Due to rising urbanization trends, though, the absolute number of slum dwellers is projected to grow to 1.4 billion people by 2020 if there is no significant improvement to urban slums. Even with decreasing trends in slum dweller proportions, some areas of the world continue to have high percentages of their urban population living in slum conditions (e.g. $72 \%$ of urban SubSaharan Africans are slum dwellers).

Table 2-1: Urban Slum Dweller Population and Percentage of Urban Population (UN, 2005)

\begin{tabular}{|lllll|}
\hline & \multicolumn{2}{l}{$\begin{array}{l}\text { Slum dwellers } \\
\text { (millions) }\end{array}$} & \multicolumn{2}{l|}{$\begin{array}{l}\text { Slum dwellers as } \\
\text { percentage of } \\
\text { urban population }\end{array}$} \\
& $\mathbf{1 9 9 0}$ & $\mathbf{2 0 0 1}$ & $\mathbf{1 9 9 0}$ & $\mathbf{2 0 0 1}$ \\
\hline World & 721.6 & 924.0 & 31.6 & 31.6 \\
Developed Regions & 41.8 & 45.2 & 6.0 & 6.0 \\
CIS, Europe & 9.2 & 8.9 & 6.0 & 6.0 \\
CIS, Asia & 9.7 & 9.8 & 30.3 & 29.4 \\
Developing regions & 660.9 & 860.1 & 47.0 & 43.3 \\
Northern Africa & 21.7 & 21.4 & 37.7 & 28.2 \\
Sub-Saharan Africa & 101.0 & 166.2 & 72.3 & 71.9 \\
Latin America and the Caribbean & 110.8 & 127.6 & 35.4 & 31.9 \\
Eastern Asia & 150.8 & 193.8 & 41.1 & 36.4 \\
Eastern Asia excluding China & 12.8 & 15.6 & 25.3 & 25.4 \\
Southern Asia & 198.7 & 253.1 & 63.7 & 59.0 \\
South-Eastern Asia & 49.0 & 56.8 & 36.8 & 28.0 \\
Western Asia & 28.6 & 40.8 & 34.4 & 35.3 \\
Oceania & 0.4 & 0.5 & 24.5 & 24.1 \\
\hline
\end{tabular}




\subsubsection{Urban Slums Programs and Policies}

One of the first major global policies that specifically targeted the often neglected urban slum dwellers was the United Nations Millennium Development Goals. This series of goals, targets, and indicators was developed to "uphold the principles of human dignity, equality and equity at the global level”, with specific goals aimed at, e.g., eliminating poverty, gender inequality and child mortality (UN, 2000). Goal 7 endeavors to ensure environmental sustainability, with Target 11 being to achieve "significant improvement in the lives of at least 100 million slum dwellers" by 2020 (UN, 2005). The United Nations Human Settlements Programme (UN-HABITAT) was delegated the responsibility of assisting member states of the UN to achieve Target 11 .

Urban slums often result from poor planning and lack of funds in both the government and the slum populations (Cohen, 2004). UN-HABITAT has developed several global, regional, and local programs to assist countries in improving slum dwellings and building cities around sustainable principles. Most of these programs, such as the Urban Management Programme and the Sustainable Cities Programme, have objectives in strengthening urban planning/management capacity and efficiency in order to improve resource use, environmental conditions, and economic growth. To reach these objectives, these programs often strive to what the Urban Management Programmes describes as "consolidate experiences and deepen knowledge and understanding on urban management" (UN-HABITAT, 2005). The aims and objectives of UN-HABITAT programs stem from a three-part strategy developed for tackling slum growth (Table 2-2). 
Table 2-2: UN-HABITAT Strategies to Improve Slums and Prevent Future Slum Growth (UN-HABITAT, 2005)

A) Slum Upgrading- accomplish physical, social, and governance upgrading of existing slums

B) Urban Development- improve job creation via effective management of land use, revenue base, infrastructure, amenities, security, community empowerment, etc.

C) Regional Development- maximize positive and reduce negative impacts of urbanization via regional investment, distribution of development services,

The three strategies of slum upgrading and urban/regional development have been put into practice to not only improve existing slums, but also to spur sustainable economic development in order to prevent new slum growth. These strategies and the programs that implement them have been successful in reducing proportions and absolute numbers of urban slum dwellers in several cities in China, Thailand, India, and Columbia. Their success seems to be founded on the same principles of building efficient networks, increasing understanding of the urban system, developing effective urban growth planning strategies, strong and wise infrastructure investment, and continuing commitment at all levels of government (UN, 2005). An emerging tool that fits within the UN-HABITAT slum strategies and can aid city planners and managers in understanding their urban systems and developing planning strategies for urban slums is urban metabolism analysis. 


\subsection{Urban Metabolism Lessons}

Although not a biological entity itself, a human city can be described metaphorically as having a metabolism, with various energy and material flows moving through the urban system. The urban metabolism concept was initially developed in the 1960's by Abel Wolman, and is defined as the sum of urban processes (technological and societal) that provide sustenance, maintenance, growth and innovation in cities, as measured through stocks and flows of energy and materials. These large fluxes, stocks, and processes of materials and energy are one of the most prominent features of a city and are used by large populations at high densities to sustain health, economy, and infrastructure (Wolman, 1965; Baccini and Brunner, 1991).

For example, the United States, which is currently highly urbanized ( $\sim 8 \%)$, went from consuming $\sim 200$ million metric tons of construction material in 1900 , to 2,800 million metric tons in 1995 (Matos and Wagner, 1998). This material flux is dwarfed by the amount of water and air that flow through urban systems each year. Cities also produce large waste outputs which have often been discarded back to the natural environment, causing ecological damage.

Several methodologies have been used to represent the metabolism of cities, including material flow analysis, embodied energy analysis, and ecological footprinting (Hendriks et al., 2000, Huang and Hsu, 2003; Wackernagel and Rees, 1995). Figure 2-2 illustrates a basic material flow analysis methodology common to many urban metabolism studies. This knowledge can assist decision makers to better manage resources and waste as well as develop other feedback loops between urban policy and 
the environment, thereby revealing the directions best suited for sustainable development (Kennedy et al., 2007; Baccini and Brunner, 1991).

To date, a handful of urban metabolism studies have been conducted on large cities in developed countries (e.g. Toronto, Vienna) (Sahely et al., 2003; Hendriks et al., 2000). However, none are known to be performed on rapidly expanding urban slum communities in the developing world. Understanding the material flows, storages, and processes and how they can be related back to policy making (i.e. feedback mechanisms) is vital if the world's developing cities and urban slum communities hope to improve material and energy efficiency while increasing quality of life.

The knowledge gained through existing metabolism studies should be considered by international, national, and regional decision makers as a potential pathway to upgrade current slums and improve regional and urban development in a sustainable manner. Accordingly, the objective of this paper is to assemble several lessons learned from previous urban metabolism studies for potential application to developing cities and slums, as well as offer guidance in conducting studies for these growing cities. These lessons can help illuminate the plans best suited for slum improvement and prevention, based on UN-HABITAT strategies. Table 2-3 and the ensuing text highlight nine lessons derived from urban metabolism studies, with the first five ordered to correspond to the procedures of a typical urban metabolism study. 


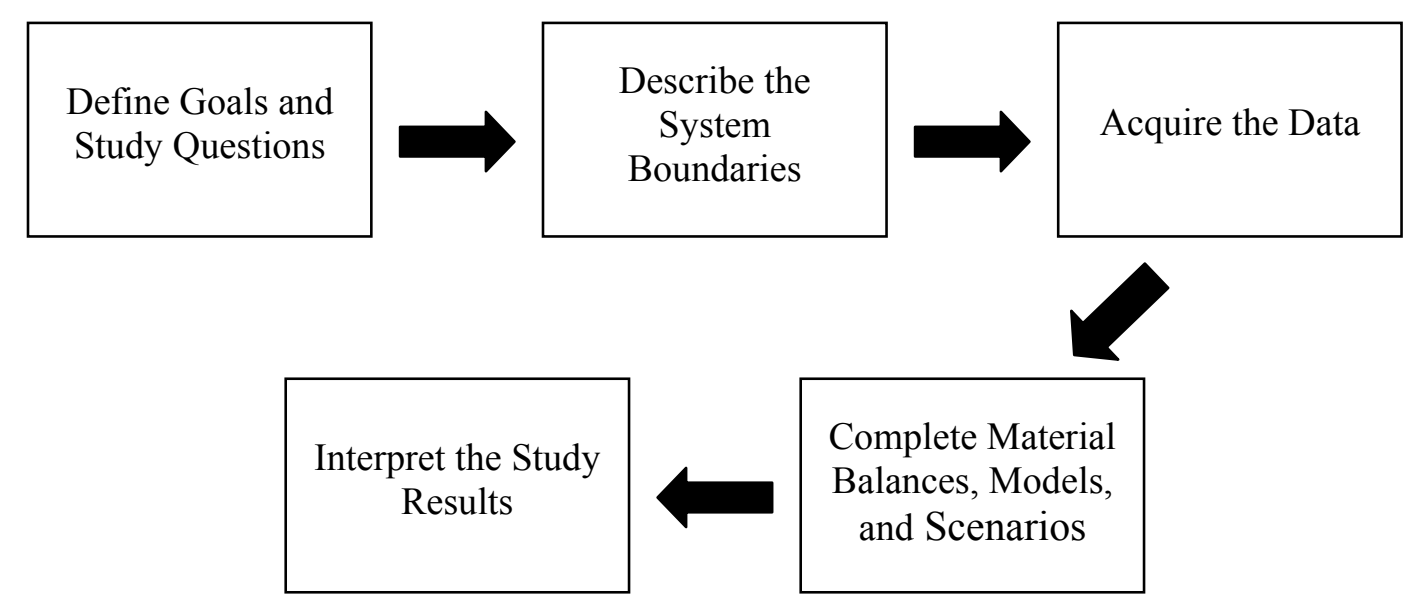

Figure 2-2: Material Flow Analysis Methodology for Use in Urban Metabolism Studies

\subsubsection{Metabolism Lessons for Developing Countries}

\subsubsection{Integrate Models}

Once urban metabolism methodology found a small niche in academia it was realized that for these models to truly be applicable to urban policy-making, social and economic issues must be connected in some manner to the metabolic pathways. Urban ecologists stress that to fully understand urban systems a framework must be employed that integrates environmental and socioeconomic processes (Pickett et al., 2001). Urban metabolism studies which do not take into account these socioeconomic drivers are limited in their ability to uncover root causes of environmental and social problems (Fung and Kennedy, 2005). This is especially true for urban slums where environmental conditions are often a result of socioeconomic factors and where socioeconomic conditions can be a result of environmental factors. 
Table 2-3: Urban Metabolism Lessons for Developing Countries

\begin{tabular}{|c|c|c|}
\hline Lesson & $\begin{array}{l}\text { Applicable UN- } \\
\text { HABITAT Slum } \\
\text { Strategy }\end{array}$ & Details \\
\hline Integrate Models & $\begin{array}{l}\text { Urban Development, } \\
\text { Regional } \\
\text { Development }\end{array}$ & $\begin{array}{l}\text { Use an integrated framework when studying } \\
\text { the metabolisms and socioeconomics of } \\
\text { urban systems. }\end{array}$ \\
\hline $\begin{array}{l}\text { Include the Whole } \\
\text { Urbanized Region in } \\
\text { Specifying } \\
\text { Metabolism } \\
\text { Boundaries }\end{array}$ & Urban Development & $\begin{array}{l}\text { Include smaller peri-urban systems in } \\
\text { metabolism analysis by using urban } \\
\text { conditions or indicators as guides for policy, } \\
\text { not just political boundaries. }\end{array}$ \\
\hline Define Efficiency & $\begin{array}{l}\text { Urban Development, } \\
\text { Regional } \\
\text { Development }\end{array}$ & $\begin{array}{l}\text { Establish comprehensive, accurate, and } \\
\text { efficient goals/indicators for use with } \\
\text { metabolic data. }\end{array}$ \\
\hline $\begin{array}{l}\text { Understand the } \\
\text { Mechanisms Within } \\
\text { "Black Box" } \\
\text { Processes }\end{array}$ & $\begin{array}{l}\text { Slum upgrading, } \\
\text { Urban Development }\end{array}$ & $\begin{array}{l}\text { Gather and analyze detailed process } \\
\text { information in order to improve resource } \\
\text { management and appropriate technology } \\
\text { implementation. }\end{array}$ \\
\hline $\begin{array}{l}\text { Conduct Metabolism } \\
\text { Checkups }\end{array}$ & Urban Development & $\begin{array}{l}\text { Continue to monitor a city's metabolism to } \\
\text { understand the long-term needs and impacts } \\
\text { of slums, allowing decision makers to find, } \\
\text { solve, and/or prevent problems. }\end{array}$ \\
\hline $\begin{array}{l}\text { Mitigate Solid and } \\
\text { Hazardous Waste } \\
\text { Outputs }\end{array}$ & $\begin{array}{l}\text { Slum Upgrading, } \\
\text { Urban Development, } \\
\text { Regional } \\
\text { Development }\end{array}$ & $\begin{array}{l}\text { Create and enforce sound policy in material } \\
\text { use reduction, recycling, treatment, and } \\
\text { disposal based on metabolism knowledge. }\end{array}$ \\
\hline $\begin{array}{l}\text { Manage Water and } \\
\text { Wastewater Flows }\end{array}$ & $\begin{array}{l}\text { Slum Upgrading, } \\
\text { Urban Development, } \\
\text { Regional } \\
\text { Development }\end{array}$ & $\begin{array}{l}\text { Understand and manage the water and } \\
\text { nutrient balances of both city and slum } \\
\text { dwellings without negating water/sanitation } \\
\text { access through damaging surrounding } \\
\text { ecosystems. }\end{array}$ \\
\hline $\begin{array}{l}\text { Extend Metabolism } \\
\text { Parameters to } \\
\text { Measure Quality of } \\
\text { Life }\end{array}$ & Slum Upgrading & $\begin{array}{l}\text { Find the right balance between livability } \\
\text { and metabolism indicators to improve the } \\
\text { lives of slum dwellers without necessarily } \\
\text { increasing consumption. }\end{array}$ \\
\hline $\begin{array}{l}\text { Pay Attention to } \\
\text { "Change } \\
\text { in Storage" }\end{array}$ & $\begin{array}{l}\text { Urban Development, } \\
\text { Slum Upgrading }\end{array}$ & $\begin{array}{l}\text { Understand imbalances and accumulations } \\
\text { of materials and waste within the urban } \\
\text { system to prevent future problems and to } \\
\text { utilize potential resources. }\end{array}$ \\
\hline
\end{tabular}


A recent metabolism study that attempts to combine multiple models is an integrated macroeconomic/metabolism model of the Greater Toronto Area (Fung and Kennedy, 2005). The econometric modeling technique was noted to be less complex than other economic modeling techniques, but still able to model long-term urban dynamics and maintain the option of connecting with more detailed microeconomic models. Multiple-Scale Integrated Assessment of Societal Metabolism (MSIASM) is another recent approach to integrated modeling and provides a framework for connecting biophysical and economic variables in an analysis of human development towards sustainability (Giampietro et al., 2001). Developing cities are urged to use an integrated framework when studying and/or attempting to improve the metabolism and socioeconomics of their urban system. If they do not integrate these analytical techniques they run the risk of shifting their problems, such as air pollution or hazardous waste, to other sectors, locations, and communities, including slum dwellings which have little defense against their city's shortsightedness (Giampietro et al., 2001).

\subsubsection{Include the Whole Urbanized Region in Specifying Metabolism Boundaries}

Many people think of large megalopolises, such as Mexico City and Tokyo, when they hear about increasing urbanization. Although these metropolises are expected to increase in number, most of the world's urban population currently resides in cities with less than 500,000 people and this trend is expected to continue in the future (UN, 2004). In developing countries these small urban settlements are often associated with one, large city, and herein lies the problem.

The definition of what is urban is often arbitrary and can change over time to best suit the statistical desires of the country (Cohen, 2004). City political boundaries in 
developing countries are often drawn so that many outlying areas (e.g. slum areas) are excluded, thereby perpetuating these areas' lack of infrastructure, service, tenure, and government. Ignoring these populations, however, can have detrimental consequences as many cities are dependent on their surrounding areas, whether urban or rural, for resource supply as well as residual disposal. Vienna discovered a strong material and waste sink dependence on its outlying areas (Hendriks et al., 2000), and if developing cities ignore their urbanizing outskirts, a false sense of metabolism improvement will be seen as unsustainable practices (e.g. solid waste dumping, toxic material use) are moved outward into ignored slum neighborhoods.

Developing cities must not disregard that the entire urban system, including the urban core, suburban areas, outlying cities, and even surrounding rural areas are ecologically and economically connected (Pickett et al., 2001). An urban metabolism study of Toronto recognized these connections and included several surrounding municipalities in what the study termed the city-region or Greater Toronto Area (Sahely et al., 2003). If and when developing countries use urban metabolism methodologies to become more sustainable, they must also include smaller outlying systems in the analysis. To improve urban slums, cities must use the entire urban system as a target for policy, and not just convenient political boundaries.

\subsubsection{Define Efficiency}

One of the benefits of conducting urban metabolism studies, especially multiple studies over time, is the ability to gauge the city's improvement in resource and energy use efficiency. A city's urban metabolism data are useful for comparative analysis, whether it is to the city's own past metabolism results or to those of another city (Sahely 
et al., 2003). These comparisons can help decision-makers see the sustainability or efficiency of management practices. The metabolism data can also be compared to previously set goals and/or indicators dealing with resource use and even the improvement of urban slum dwellers' lives.

The development of sustainable goals and/or indicators combined with metabolism data can help cities improve their decision making by highlighting potential environmental problems (e.g. surface water eutrophication) before they become a crisis (For more detail on indicators see Alberti, 1996; Foxon et. al., 1999; Azar et. al., 1996). One concern with goals and indicators is often there are problems in defining what is efficient and sustainable (Bohle, 1994). For instance metabolism results may indicate that a city is improving and reaching its goals regarding resource efficiency, as desired by UN-HABITAT programs, but it may be at the cost of providing adequate standards of living to urban slum dwellers, an often ignored component of the urban system. The problems in defining efficiency in past urban metabolism studies have often been caused by inattention to social factors, such as race and wealth, which are difficult to account for in metabolic flow analysis (Bohle, 1994). Unless accurate, efficient and equitable goals or indicators are specifically outlined for urban slums, and city resource efficiency definitions encompass slum areas, metabolism data will do little to improve their conditions.

\subsubsection{Understand the Mechanisms Within "Black Box" Processes}

There are several components within a city's metabolism which can effectively be categorized within the concepts of materials, processes, goods, and activities, with examples being surface water, treatment, potable water, and bathing, respectively 
(Baccini and Brunner, 1991). Processes create a change in materials and goods, whether in quality or location, thereby enabling these materials and goods to be used in designated activities. Past urban methodologies have often been concerned with conducting mass and energy balances in order to quantify material fluxes through the urban system, treating the processes themselves as "black boxes" (Baccini and Brunner, 1991). Often these "black boxes" are the result of limited data and resources in research, as is the case in many developing countries' intra-urban food distribution processes (Bohle, 1994).

Although the material balance is still an important tool in urban metabolism research, recent ideas in urban ecosystem analysis require a more detailed look at processes in order to accurately model and manage the material's interactions and changes within the processes (Pickett et al., 2001). Hong Kong's second urban metabolism study noted that management patterns of pollution treatment versus prevention inhibited environmental progress (Warren-Rhodes and Koenig, 2001). Understanding the waste processes more thoroughly might have helped reveal alternative residual management options.

Process knowledge is also important when integrating socioeconomic models into the metabolism models. Sustainable material management can only be achieved if the economic, social, and technological drivers are fully understood and integrated into the selection and operation of these processes. For example, urban slums are often in need of basic goods, such as drinking water. There are many treatment processes which could provide this good, but without adequate understanding of how the population's education, culture, and economics interact with the workings of the process, appropriate technologies which could be sustained by the local population might be overlooked. 
Therefore, as developing cities conduct material/energy flow analyses of their cities, including the slums, process information should also be gathered and analyzed to improve resource management.

\subsubsection{Conduct Metabolism Checkups}

A city's growth and development will determine and/or alter its urban form, defined as "the spatial configuration of fixed elements within [the] metropolitan region" (Anderson et al., 1996). Urban form can significantly direct or alter metabolic flows and stocks in a city (Anderson et al., 1996). The flow and stock changes are even more pronounced in the often transient slum dwellings. Due to this inevitable and sometimes rapid change in urban form, any attempt of a developing city to use urban metabolism data to improve water access, sanitation, durable housing, etc., would be meaningless without continual monitoring of the metabolic pathways.

It is apparent that urban slums will not go away in the near future. Long term understanding of the needs and impacts of these communities is therefore needed in order to avoid short-sighted quick fixes that do not lead communities toward sustainable resource use and quality living. Urban metabolism "checkups" also separate vital trends from temporary noise, allowing decision makers to find, solve, and/or prevent environmental problems using sustainable indicators as guides (Warren-Rhodes and Koenig, 2001). Follow-up studies are one of the largest weaknesses in past urban metabolism studies, but they are essential to any long term policy incorporating urban metabolism methodology. 


\subsubsection{Mitigate Solid and Hazardous Waste Outputs}

The industrial age ushered in the urbanization of the world's population, with concentrations of industry continuing to draw more people into ever growing and developing urban systems (Bai, 2002). This large concentration of industry with its associated growth of commercial and residential sectors creates a large amount of solid and hazardous waste and is a large and troublesome output of a city's metabolism. Taipei's urban metabolism study suggested that waste dumps were one of the worst environmental problems in the city due to large waste volumes, inadequate treatment facilities, and dwindling landfill sites (Huang and Hsu, 2003). Hong Kong's solid waste output grew $245 \%$ between a 1971 urban metabolism study and a follow-up study in 1997 (Warren-Rhodes and Koenig, 2001). Both Taipei and Hong Kong are running out of space for waste and should serve as a warning to any developing city with similar growth characteristics. One review of material and energy flow through several large cities noted that less than $10 \%$ of material inputs leaves a city as goods, suggesting that large quantities are exported as waste or are potential wastes to be exported later (Decker et. al., 2000).

An unfortunate side effect of this unplanned waste in developing countries is that often times it finds its way to slum areas. Waste dumps can even promote slum conditions, attested by numerous accounts of makeshift cities springing up around dumps in developing countries across the globe. Some cities have even deliberately exacerbated this scene through industrial relocation, in which polluting industries are exported to outlying communities with little thought as to the health and environmental impacts such a move might cause (Bai, 2002). In order for developing cities to eliminate these 
growing waste trends and improve slum conditions, they must have adequate knowledge about material flows so that proper preparation can be made for reducing material consumption, recycling or creating circular metabolic pathways; and for safe treatment or disposal. Urban metabolism analysis can assist in acquiring this knowledge, but it must be acted upon. Hong Kong's initial metabolism study in 1971 offered many suggestions to avoid ecological damage due to over consumption and solid waste generation, but these suggestions were largely ignored by decision makers, resulting in their current deluge of waste (Warren-Rhodes and Koenig, 2001).

\subsubsection{Manage Water and Wastewater Flows}

The importance of water inputs and outputs in a city can not be overstated. Past urban metabolism studies have shown water to be one of the largest, if not the largest, material inputs into an urban system (Decker et. al., 2000; Sahely et al., 2003). Great strides have been made in past decades to improve water supply and sanitation in urban centers across the globe. As of 2002, improved global urban water supply and sanitation coverage was at $95 \%$ and $81 \%$, respectively (UNEP, 2005). Regional coverage, however, indicates a continuing need for improvement. For example, most of SubSaharan Africa has urban water supply and sanitation coverage below $85 \%$ and $50 \%$ respectively (UNEP, 2005). Another concern relates back to a country's arbitrary definition of "urban". If these countries do not categorize illegal slum areas surrounding the city as urban, then these populations figure into the rural water and sanitation coverage rates, which are worse than urban rates, sometimes by more than $50 \%$.

In an urban system, water is involved with many processes and activities (e.g. transport, cleaning, consumption) and is considered both a material input and good (e.g. 
chemical industry, drinking water) (Baccini and Brunner, 1991). But mismanagement of this vital resource can threaten both human and environmental health. The initial Hong Kong study found water to be highly polluted, with the author cautioning the government to not exceed the water's ability to assimilate waste safely (Newcombe et al., 1978). The follow-up metabolism study found even worse conditions and stated that benefits gained in apparent efficient and sustainable metabolism pathways were outweighed by the environmental degradation caused by some metabolic outputs, such as untreated wastewater (Warren-Rhodes and Koenig, 2001).

Urban metabolism methodologies can assist in making improvements in urban water and nutrient/wastewater management if used effectively by urban planners and understood by decision makers. Urban metabolism shows that nutrients typically enter the urban system in concentrated form (i.e. food) and then are diluted in discharged wastewater, creating potential eutrophication problems in receiving water bodies. Vienna's metabolism study found that although the Danube River had a high dilution potential, nitrogen discharges were 800 times higher than natural loadings (Hendriks et. al., 2000). Metabolism studies may help to eliminate this linear nutrient pathway by highlighting potential needs and possibilities for wastewater reuse in the agricultural sector, thereby closing the nutrient loop.

Vienna's metabolism study also identified that effective urban wastewater or nutrient output management must include hinterland or outlying areas, which contribute significantly to nutrient levels in the river due to agricultural runoff. This emphasizes the need to include urban slums in any water quality management plan. If a city seeks to improve nutrient pollution without considering hinterland areas, particularly those 
inhabited by large slum populations, it is unlikely to develop sustainable nutrient management and wastewater reuse options.

Urban metabolism studies can also help cities map out drinking water flows and help to improve coverage rates. Metabolism data can draw attention to such problems as distribution leaks, rising water tables due to excessive and often unintentional recharge, and sewage/gray water infiltration with its associated health impacts. As cities improve water access to slum dwellings, urban metabolism methodology can again assist in managing the resulting nutrient loadings (Baccini and Brunner, 1991). The ultimate goal is to improve the sustainability and access of the urban slum water system without negating these benefits with rising sanitation problems.

\subsubsection{Extend Metabolism Parameters to Measure Quality of Life}

Material use is one measure often used in urban metabolism studies, with developed cities usually having higher per capita rates of material consumption (Decker et. al., 2000). To reach sustainable levels of material consumption, however, cities must grow without increasing their overall material intensity and in many cases without increasing overall consumption of certain materials, such as scarce non-renewable resources or materials with exhausted waste sinks. This can be difficult for material intensive cities that wish to improve the lives of slum dwellers. Often the first reaction is to build bigger and better housing. Such was the case in Hong Kong, where the government used large amounts of resources to construct resettlement housing, contributing to their large imports and stocks of construction materials. It was noted that this new housing was not effective in improving the lives of slum dwellers, leading to the 
conclusion that excessive material consumption does not necessarily lead to a higher quality of life (Newcombe et al., 1978).

The extended metabolism model can assist a city in improving the quality of life for their slum dwellers without increasing material use intensity. The extended metabolism model was refined for use in Australian State of the Environment Reporting and consists of basic material and energy flow analysis coupled with measuring livability indicators (Newman et al., 1996). Livability is defined as "the human requirement for social amenity, health and well being and includes both individual and community well being" (Newman, 1999). Some of the livability indicators include decreasing proportion of substandard housing, but also include reducing crime and infant mortality to increasing leisure and education opportunities. Obviously the world community wishes to eliminate the dangerous, overcrowded, and illegal squatter settlements, and through finding the right balance between livability indicators and metabolism indicators (e.g. reductions in air and water pollution and increases in green space and transportation services), a city would be able to improve the lives of slum dwellers without necessarily increasing material consumption. Follow-up extended metabolism studies on Australian cities may offer more understanding of this balance.

\subsubsection{Pay Attention to "Change in Storage"}

An urban metabolism analysis will show data to address concerns over the sheer magnitudes of resources flowing into cities, and wastes flowing out. The "change in storage", i.e. the difference between inflows and outflows may, however, be just as critical in the long term. 
The importance of managing storage processes may be demonstrated by considering urban groundwater systems in the urban metabolism. Many cities in arid regions suffer from an imbalance in water management whereby large quantities of water are pumped into the cities, but wastewater is not pumped out. Often the wastewater is disposed of by discharge into the subsurface below the city, which causes a rising of the water table. Such rising water tables have been reported for several cities in the Middle East: Riyadh, Jeddah, Damman, Kuwait, Al-Ajman, Beirut, Cairo and Karachi (AbuRizaiza 1999). As well as posing problems of groundwater contamination, this rising of water levels threatens urban infrastructure: basements, foundations, tunnels, pipes and cables. In the long run this imbalance in the water component of the urban metabolism may threaten the physical integrity of cities.

Understanding of other storage processes in the urban metabolism is also important. The urban fabric contains many potentially hazardous materials, e.g., mercury, asbestos, PCBs. Modern cities may contain larger volumes of hazardous materials than available space in hazardous materials landfills (Brunner and Rechberger, 2001). Nutrients such as phosphorus and nitrogen also tend to accumulate in urban soils or waste sites, potentially providing a future resource. On a daily time scale, the urban heat island effect, whereby cities maintain temperatures higher then their rural surroundings, can also be recognized as a storage process in the energy component of urban metabolism. Strategies to mitigate urban heat islands may become increasingly important with global climate change. Thus, in addition to quantifying inputs and outputs, urban studies should also aim to characterize storage processes. This is especially important for developing cities that are consuming large material inputs to either improve or assimilate slum communities. 


\subsection{Conclusions}

In $2030,60.8 \%$ of the global population will be living in urban areas, with urban regions of the developing world absorbing most of the world's total population growth between 2000 and 2030 (UNESA, 2004). The global urban slum dweller population is forecasted to swell to 1.4 billion by 2020 . UN-HABITAT's three strategies of slum upgrading, urban development, and regional development, along with their goals of creating a shared vision, developing effecting planning strategies, and preventing future human/environmental catastrophe in urban slums, can only be achieved with adequate information about the urban system and processes. UN-HABITAT efforts to achieve the UN's Millennium Development Goal's Target 11 often recognize the need to develop and/or adapt knowledge. These nine urban metabolism lessons have been assembled from previous urban metabolism studies to contribute to the knowledge in the global effort of improving and preventing urban slums. By increasing the knowledge of the urban system we hope to improve, humans will be more adequately prepared to address rising slum issues.

Better understanding of the urban ecosystem can also assist progress towards other Millennium Development Goals, especially as improvements are made to integrate urban metabolism methodology with socioeconomic models. An improvement to water and sanitation access is one such area of potential progress. The physical planning and management of cities and urban-rural interaction areas cannot be isolated from the environmental and socioeconomic feedbacks, especially as it relates to water (Niemczynowicz, 1996). Urban metabolism knowledge and lessons can help identify appropriate technologies that may assist in providing improved water and sanitation 
access, which in turn has ramifications on local economy and environment. Ongoing work will determine if rainwater harvesting is one such tool for alleviating slum conditions by improving the sustainability of water and wastewater flows.

Smarter and sustainable economic development and city management can only facilitate efforts to decrease poverty, disease, inequality, etc. As more and more studies are made, greater material and energy efficiency will be possible with appropriate policy implementation. Urban centers must have the opportunity of becoming extremely efficient and allowing for the "sustainable" development of the human race. Meeting the United Nations Millennium Development Goals by 2015 will greatly depend on how the world community manages the urban situation, which will require innovative thinking in areas of food/water supply, sanitation, education, equality, and environmental sustainability. New management tools will be needed to head off the current slum crisis and looming catastrophe that is sure to happen if these cities continue to haphazardly grow and operate like tumors on the land. 


\section{Chapter 3 Stochastic Rainfall Modeling in West Africa: Parsimonious Approaches for Domestic Rainwater Harvesting Assessment ${ }^{\mathrm{a}}$}

\subsection{Introduction}

\subsubsection{Improved Water Access}

Global commitment to universal improved water access has escalated over the last decade as policy makers and water professionals have sought to alleviate many of the public health and economic development problems associated with water scarcity. The United Nation's Millennium Development Goals (MDGs) related to water and sanitation access are on the agendas of many governments and NGOs, and the other MDGs related to poverty reduction, education, gender equality, health, and sustainable development all have direct ties to water-related indicators (WWAP, 2006). Half of the MDGs' 2015 target period is now past, and although some progress has been made, the world community needs increased mobilization toward these targets as well as toward future aims of water provision.

Several metrics have been proposed to define physical or economic water stress or scarcity. A freshwater per capita value of $1700 \mathrm{~m}^{3} /$ year of total available water supply, accounting for all uses, is a common delineation of water stress (Falkenmark et al., 1989). Health impacts can also be incorporated into these metrics, as with the World Health Organization's (WHO) recommendation of $20 \mathrm{~L} /$ day to meet basic consumption and hygiene needs (Howard and Bartram, 2003). MDG Target 10 is focused on increasing improved water access (defined in Table 3-1). Figure 3-1 shows the current improved water access rates in Africa, which are the lowest of all continents. This figure may be an a- Previously published in Journal of Hydrology (Cowden et al., 2008) 
underestimation of the problem as aggregation can mask local and regional issues not reflected in national surveys (Vorosmarty et al., 2005).

Table 3-1: Improved Water Definitions (WHO/UNICEF, 2000)

\begin{tabular}{|lll|}
\hline Improved $\quad$ Water & Supply & Not Improved Water Supply Technologies \\
Household connection & Unprotected well \\
Public standpipe & Unprotected spring \\
Borehole & Vendor-provided water \\
Protected dug well & Bottled water \\
Protected spring & Tanker truck-provided water \\
Rainwater collection & \\
\hline
\end{tabular}

a- Due to insufficient quality, quantity, or unreasonable price

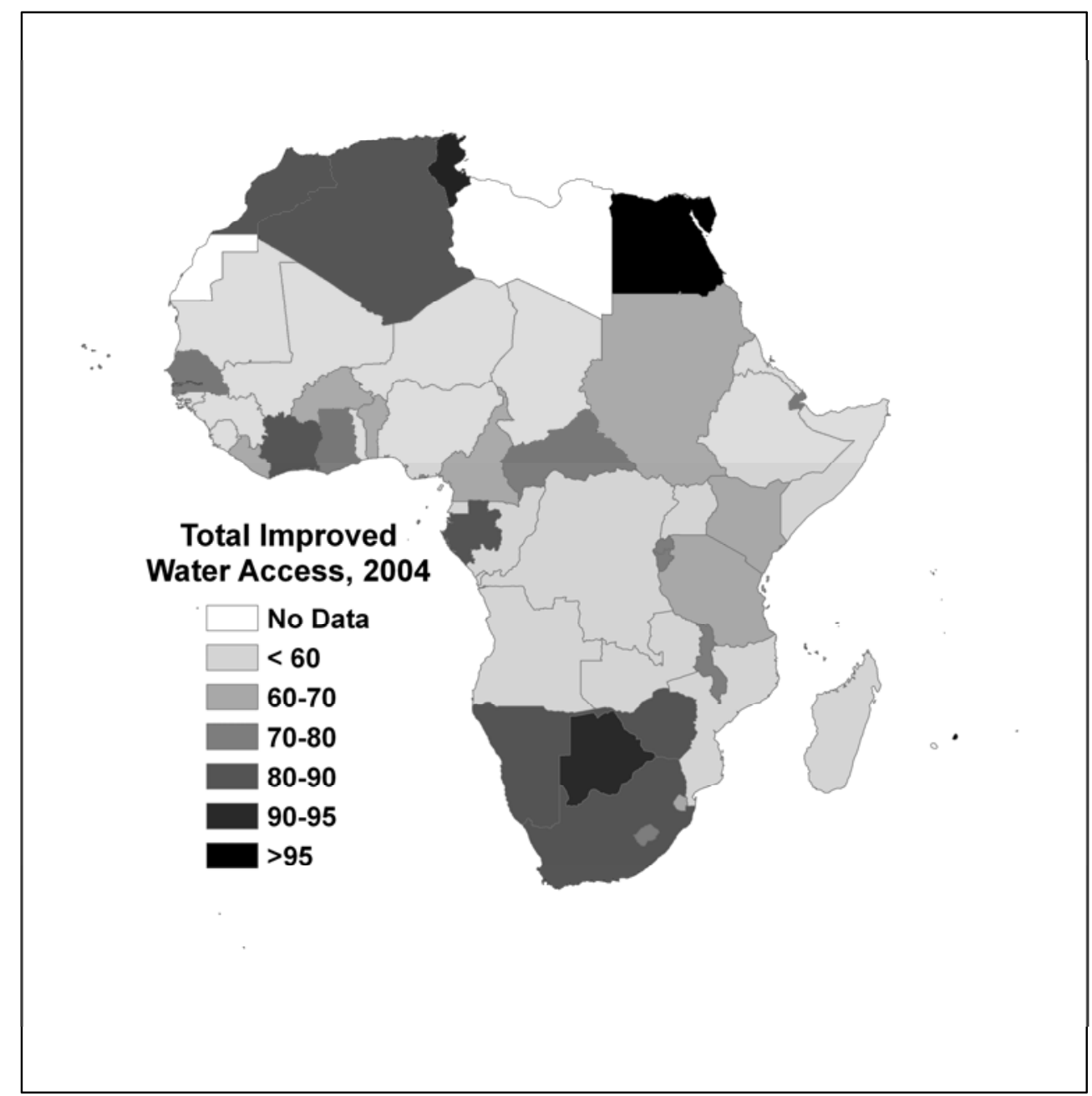

Figure 3-1: Improved Water Access Percent Coverage in Africa (Map created by author from data in UNEP, 2005) 
An exploration of alternative methods of water provision, in place of typical large, central engineering solutions, should be a part of any developing nation's water plan. Domestic Rainwater Harvesting (DRWH) is practiced formally (e.g. permanent collection/storage systems) and informally (e.g. pots under roof edges) in various parts of the world. This technology has seen increasing popularity in developing countries due to better reliability, simplicity, versatility, use of local materials, time savings, and lower costs than some centralized treatment systems. It is also compatible to the conditions found in impoverished urban communities (Mihelcic et al., 2007; Thomas, 1998). This technology can also empower households in enhancing their own water supply, regardless of the availability and willingness of government infrastructure support and international economic aid (Cowden et al., 2006). Such is the case within urban slums, for which lack of adequate improved water is a defining characteristic. There are 199 million slum dwellers in sub-Saharan Africa (UN-HABITAT, 2006), and DRWH could offer a viable enhancement of water supply and associated improved health.

Several areas in sub-Saharan Africa are now developing DRWH programs, and many case studies exist on the application of the technology, including implementation in urban areas of the continent (Ntale et al., 2005; Thomas, 2005; Handia et al., 2002; Gumbo, 1998). Many DRWH assessments, however, use aggregated monthly precipitation data in the determination of water availability. This method is sufficient for very approximate analysis, or if storage volumes are sufficient to capture most of the monthly precipitation, making day-to-day rainfall occurrence less of a concern. However, households with little storage capacity (e.g. urban slums) are dependent on the 
daily probability of precipitation if using a DRWH system. Daily precipitation data, therefore, are essential in assessing DRWH reliability for these systems.

\subsubsection{Stochastic Weather Generators}

Unfortunately, complete or long daily rainfall records are not readily available in many regions of sub-Saharan Africa. Many methods offer daily sequence construction from climate model-linked precipitation models or statistical disaggregation of monthly values, yet they are often too complicated (i.e. data intensive, high training requirements) or not readily available for use by water planners and NGOs in developing countries. "Off the shelf" stochastic weather generators, however, offer a solution by creating multiple, unbroken synthetic sequences of rainfall for use in hydrologic modeling and reliability assessment (Wilks and Wilby, 1999). The model parameters may also be interpolated to non-gauged sites, allowing for broad spatial assessment of DRWH systems (Semenov et al., 1998). Stochastic weather generators can also be used in climate change impact assessments via parameter adjustment by global climate model output (Semenov and Barrow, 1997; Wilby et al., 1998).

Two stochastic weather generator models, a $1^{\text {st }}$-order Markov model--used by WGEN (Richardson and Wright, 1984) and other freely available statistical software-and LARS-WG (Semenov and Barrow, 1997), are considered herein as appropriate, parsimonious options for water planners and non-governmental organizations (NGOs) in the developing world. The governing principles of both models have been developed over the past 30 to 40 years, and have been, along with their successors, used in studies and operations for various applications throughout the world (Lawless and Semenov, 2005; Neitsch et al., 2005). The $1^{\text {st }}$-order Markov model is a lag-one dependence model, 40 
and LARS-WG is spell-length model. These models offer distinct advantages over more sophisticated, highly parameterized, or physical research models, including Internet availability of modeling software, small input data requirements, fast computations, and ease of use.

One weakness of these methods, however, is the lack of extensive application to tropical to semi-arid equatorial climates such as West Africa, which differ from the midlatitude, temperate climates for which they were originally developed (Wilby et al., 2004). To date, only a handful of stochastic weather generators studies have been completed for West Africa, most using components of the WGEN model, with a few using the LARS-WG model (Schuol and Abbaspour, 2007; Jones and Thornton, 2000; Jackson, 1981; Jimoh and Webster, 1996, 1999; Garbutt et al., 1981). The models also have seen little if any application to DRWH, with no studies reported in rapidly urbanizing West Africa.

Accordingly, this paper will address the application of these stochastic weather generator models for several sites in West Africa. The models will then be used to assess the reliability of DRWH systems for meeting basic water needs in the region (i.e. ability to produce $\geq 20$ L per person per day) and to determine the impact this technology can have on water supply enhancement. The two stochastic weather generator models will be compared against each other as well as to a more complicated $2^{\text {nd }}$-order Markov model and a conditioned $1^{\text {st }}$-order Markov model to determine the suitability of these methods to DRWH assessment in West Africa. These comparisons will also allow investigation of benefits of using more complex research methods in developing water supply management systems that incorporate DRWH. 


\subsection{Methods}

\subsubsection{Data and Site Selection}

This research will focus on the tropical to semi-arid regions of West Africa. The rainfall data used to construct the stochastic rainfall models were obtained from the U.S. National Climatic Data Center's (NCDC) Global Summary of Day data (NCDC, 2007). These data have been subjected to extensive quality control by NCDC to remove random errors associated with the reported observations and daily derivations. Multiple gauges for the West African region were selected based on minimum record lengths of twenty years (Figure 3-2), with most records ranging between 1973 and 2005. Although there is no standard minimum on record lengths for stochastic rainfall models, this record length assures adequate stochastic parameter estimation (Richardson, 2000; Soltani and Hoogenboom, 2003). Mean monthly rainfalls have strong seasonality patterns and range from bimodal distributions along the Guinea coasts to unimodal distributions farther inland toward the Sahel. The gauge threshold (i.e. instrumentation limit) for this data set is 0.01 inches or $0.254 \mathrm{~mm}$, and this same threshold was used to distinguish a wet day from a dry day in the calculation of rainfall occurrence model parameters. Appendix A shows the location of gauges used in this study. 


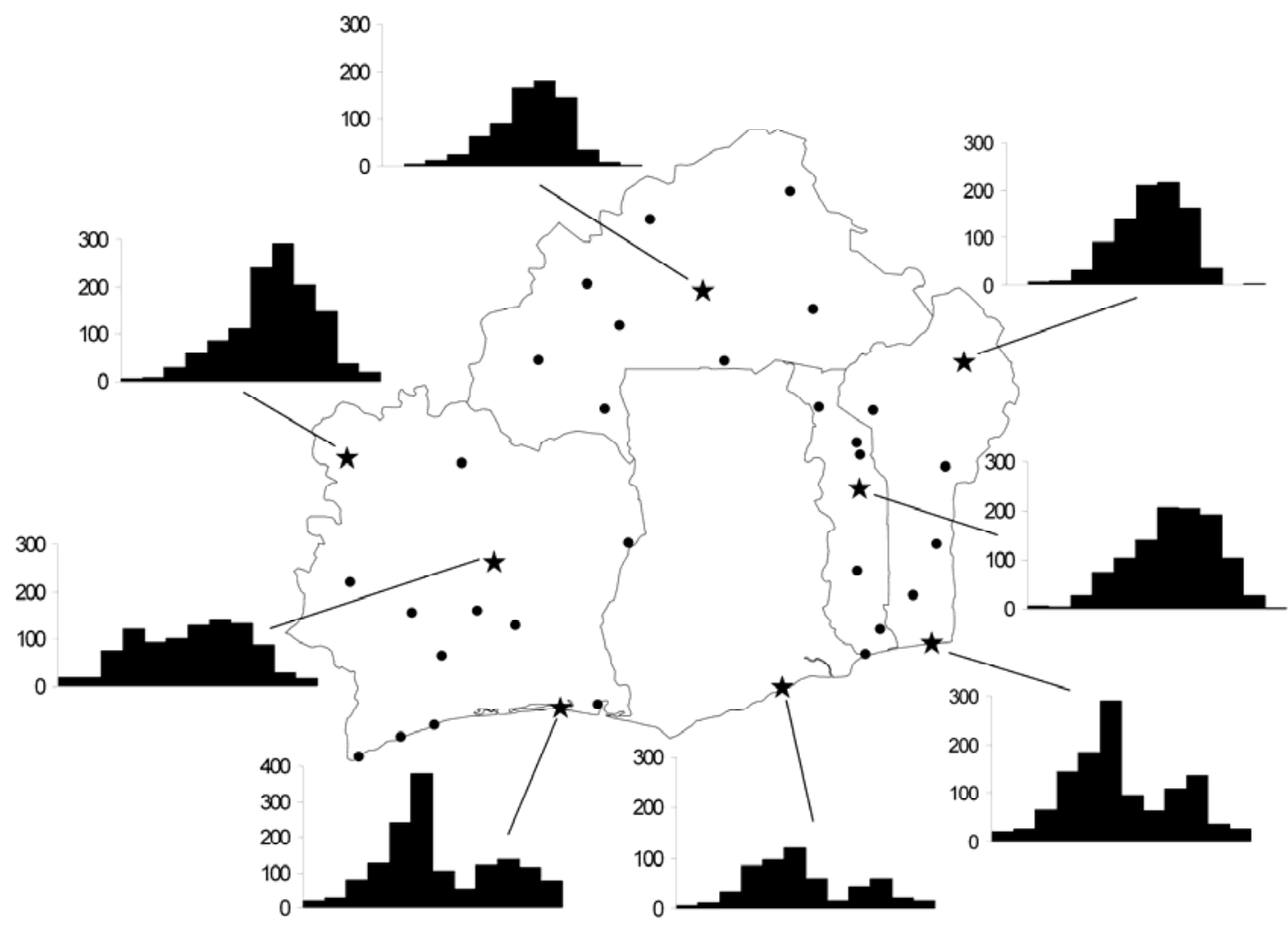

Figure 3-2: Precipitation gauges used in five countries of West Africa (Burkina Faso, Cote d'Ivoire, Ghana, Togo, Benin). Example rainfall distributions from selected gauges are shown as the monthly mean $(\mathrm{mm})$.

\subsubsection{Stochastic Rainfall Modeling}

\subsubsection{Markov Models}

WGEN and its successors use a $1^{\text {st }}$-order Markov chain, corresponding to lag-1 transition probability dependency. A zero-order Markov chain, or unconditional occurrence model, and a $2^{\text {nd }}$-order Markov chain, or lag- 2 model, were compared with the $1^{\text {st }}$-order model. Assuming a constant season length of one month, the maximum likelihood estimates of the transition probabilities were calculated from the observed proportional frequencies. State changes with missing days were ignored in this study. One hundred 30-year sequences of simulated precipitation occurrence were then 
produced using the transition probabilities and random number sequences. Assumptions were made regarding the first state(s) of the sequence, based on the unconditional probabilities of each gauge for the given month.

\subsubsection{Amount Model}

Two amount distributions were used in this study, the 2-parameter gamma distribution,

$$
f(x)=\frac{x^{\alpha-1} \exp (-x / \beta)}{\beta^{\alpha} \Gamma(\alpha)} \quad \text { for } x, \alpha, \beta>0
$$

and the mixed-exponential distribution (Wilks, 1999).

$$
f(x)=\left(\alpha / \beta_{1}\right) \exp \left(-x / \beta_{1}\right)+\left(\frac{1-\alpha}{\beta_{2}}\right) \exp \left(-x / \beta_{2}\right) \quad \text { for } x>0 ; 0 \leq \alpha \leq 1 ; 0<\beta_{1}<\beta_{2} \quad 3-2
$$

Maximum likelihood estimators were used for the shape $(\alpha)$ and scale $(\beta)$ parameters of the gamma distribution and the mixing $(\alpha)$ and mean $\left(\beta_{1}, \beta_{2}\right)$ parameters of the mixed exponential distribution. Random samples from these distributions produce the wet day amounts in the synthetic occurrence sequence.

\subsubsection{Spell-Length Model}

The spell-length model used in this study followed the precipitation component of the LARS-WG weather generator (Semenov and Barrow, 1997). This model samples from a semi-empirical distribution, or histogram, of the observed spell-lengths and precipitation values, rather than fitting a specified distribution to the data (Semenov et al., 1998). Alternating wet-dry sequences are produced by randomly selecting from the corresponding histogram's intervals, based on their relative frequencies, and then 
selecting a value from the uniform distribution within the interval. LARS-WG software was used for these steps in producing the 30 -year sequences.

\subsubsection{Conditioned Markov Model}

Several studies have shown the influence global sea-surface temperatures (SSTs) have on the rainfall variability of the West African region (Balas et al., 2007; Rowell, 2001). These SSTs may have direct impacts on rainfall processes, or indirect influences via teleconnections to mid-latitude Atlantic conditions, which then impact rainfall in the region. The $1^{\text {st }}$-order Markov model was conditioned on SSTs using the Oceanic Nino Index (ONI) (CPC, 2007). The rainfall gauge dataset was divided according to whether a month fell within and warm, neutral, or cold Pacific SST event, as defined by ONI. Transition probabilities and amount distributions were then calculated for each of the three states. Simulation proceeded by using the occurrence frequency of each ONI event to produce monthly sequences. The corresponding SWG parameters for each event were then used to complete the multiple 30-year sequences of daily precipitation.

\subsubsection{Model Comparison}

The Bayesian information criterion (BIC) was used in determining the optimum Markov chain order and amount model, operating on the premise of balancing the goodness of fit of a model with the model's complexity, as indicated by the degrees of freedom or number of independent parameters (Schwarz, 1978; Wilks, 1999). The BIC score is computed as

$$
B I C(k)=-2 L(\theta)+m \ln (n)
$$

where $m$ equals the degrees of freedom or number of independent parameters, $L$ equals the log-likelihood function, and $n$ is the sample size. 
All models were also compared using tests commonly found in the literature to examine several statistical properties of the time series, such as wet days per month, monthly amount totals, and wet/dry spell lengths. These tests include graphical comparisons, root mean square error (RMSE) (Mehrotra et al., 2006; Wilby et al., 1998), and the t-test and F-test for mean and variance analysis, respectively (Semenov et al., 1998). The latter two methods test the following null hypotheses, respectively:

$\left(\mathrm{H}_{0}: \mu_{\text {observed }}=\mu_{\text {model }} ; \mathrm{H}_{0}: \sigma_{\text {observed }}^{2}=\sigma_{\text {model }}^{2} ; \alpha=0.05\right)$

Overdispersion, which is the under-representation of the observed variability by the models, was also calculated as (Wilks, 1999)

Variance Overdispersion $=\left(\frac{\text { observed variance }}{\text { modeled variance }}-1\right) \times 100 \%$

\subsubsection{DRWH Reliability}

One hundred 30-year sequences of synthetic rainfall for each model were produced for use in the DRWH assessment. The daily per capita DRWH yield is the product of per capita roof area, runoff coefficient, and daily rainfall. For the initial statistical comparison of the models, an assumed roof area of $6 \mathrm{~m}^{2}$ per person is used. A runoff coefficient of 0.8 was used and is a common value in the DRWH literature (Preul,1994). Monthly reliability of the DRWH system was calculated as follows.

$$
\text { Reliability }=\frac{\# \text { of days yield } \geq 20 \mathrm{~L}}{\text { total } \# \text { of days }}
$$

A more thorough domestic rainwater harvesting assessment was then conducted in the study region using roof areas ranging from 2 to $10 \mathrm{~m}^{2}$ and storage volumes ranging from 0 to $1000 \mathrm{~L}$. The amount of water available for consumption was dependent on runoff 
and the previous day's storage. Consumption was assumed to be up to $20 \mathrm{~L} /$ day per capita. Runoff exceeding storage capacity was assumed to be discarded.

\subsection{Results}

\subsubsection{Markov Order}

Markov order selection was determined for comparison with the LARS-WG and conditioned Markov model. Appendices B, C, and D contain the estimates of transition probabilities and LARS-WG parameters examined in this study. Figure 3-3 shows the number of gauges selected for each model order, as determined by the lowest BIC score. The zero-order model is the preferred Markov order for the majority of gauges for 9 out of 12 months, with the $1^{\text {st }}$-order model being preferred slightly for the remaining 3

months. The $2^{\text {nd }}$-order model is rarely preferred for any of the gauges, receiving only a few low BIC scores during the latter part of the wet season in the region. These BIC scores indicate that the higher order Markov models do not contribute enough skill to the rainfall parameter estimation to justify the increase in complexity.

The dominance of the zero-order model is less clear, however, when the relative BIC scores are examined. When the $1^{\text {st }}$ - or $2^{\text {nd }}$-order model is not preferred by the BIC (i.e. the lowest score), the percent difference between that score and the minimum score is small. Median percent differences between the minimum BIC score and $1^{\text {st }}$-order BIC values are less than $3 \%$ for all months and gauges, and less than $4 \%$ for the $2^{\text {nd }}$-order model. 


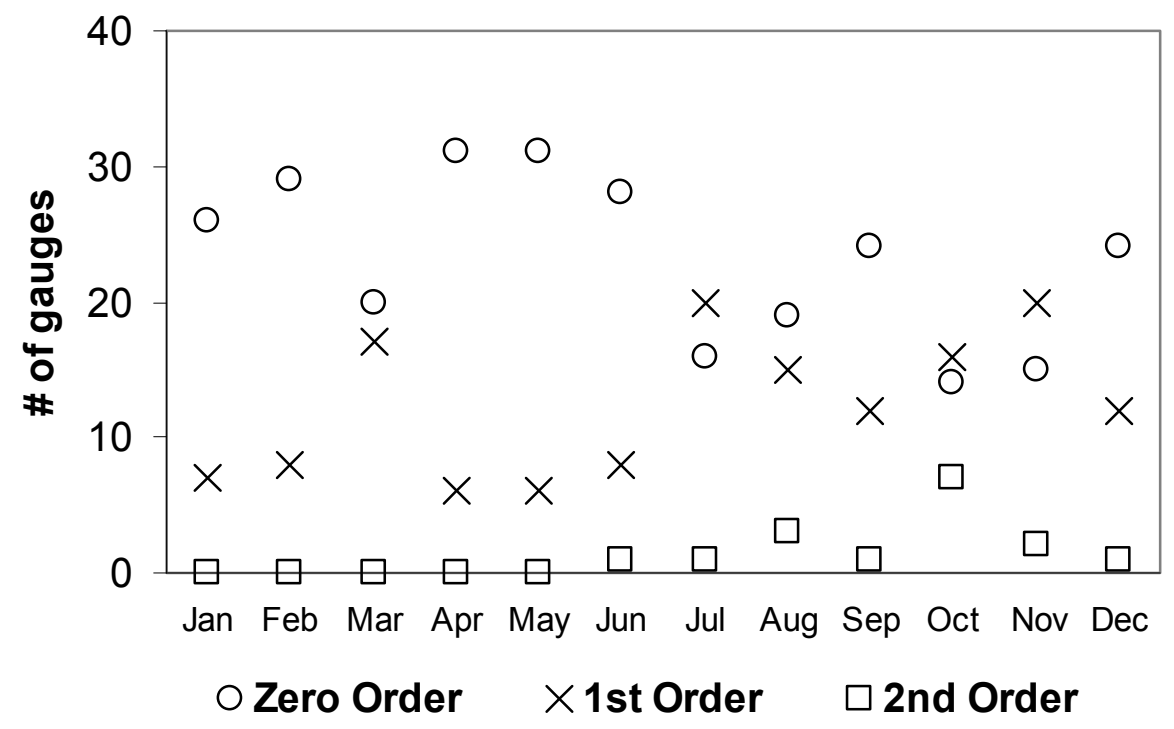

Figure 3-3: Bayesian Information Criterion (BIC) selection of Markov model order. The figure indicates, for each model order, the number of gauges with the lowest (preferred) BIC score.

Order selection is also determined by examining how well each of the Markov orders reproduces certain characteristics of the observed data. Figure 3-4 shows the standard deviation of the $1^{\text {st }}$-order Markov model plotted against the standard deviation of the observed data. The zero- and $2^{\text {nd }}$-order models show similar patterns. Overdispersion is evident for all three models, especially during the wetter months of Apr - Sep.

Overdispersion is expected here as it is a common weakness of Markov rainfall occurrence models. Table 3-2 contains the results of various statistical tests conducted on the results of Figure 3-4. The $1^{\text {st }}$-order Markov model has the lowest level of overdispersion and the lowest RMSE for all three rainfall characteristics in Figure 3-4. The 1st-order Markov model also has the least rejections of the null hypothesis $(\alpha=0.05)$ of the observed and model variance being equal. These tests favor the $1^{\text {st }}$-order model across all months, in contrast to the BIC scores. 

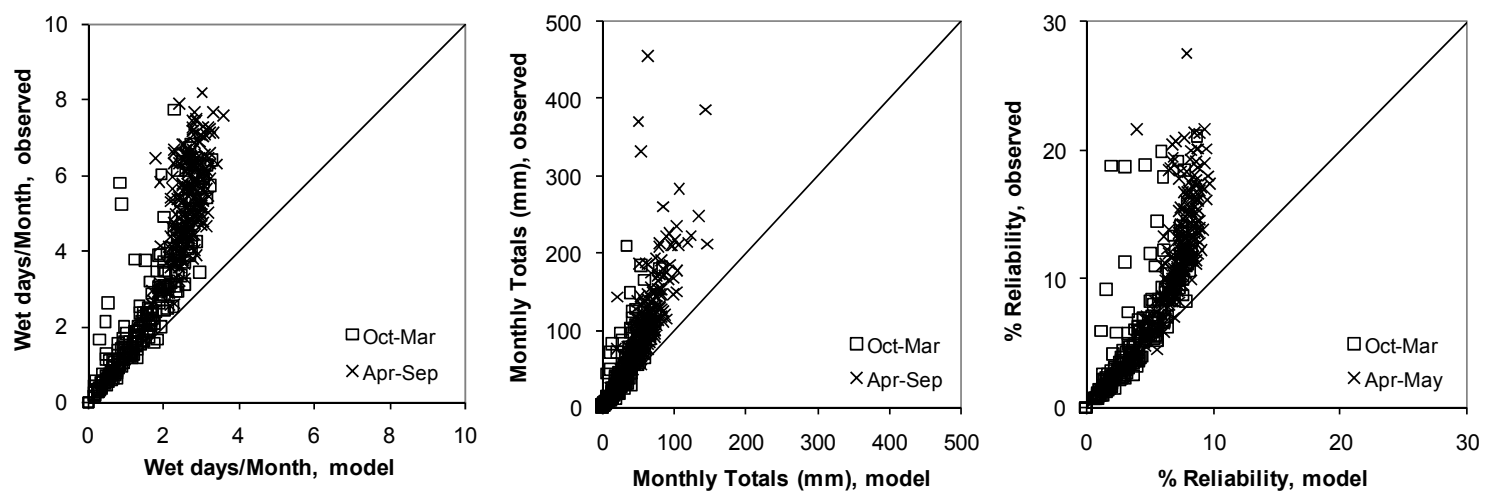

Figure 3-4: Standard Deviation of wet days per month, monthly totals (mm), and DRHW $\%$ Reliability ( $\geq 20 \mathrm{~L} /$ day per capita) for the $1^{\text {st }}$-order Markov model

Table 3-2: Rainfall characteristics statistical test results for the Markov models.

Dispersion and RMSE values are averaged across all gauges and months. F-test values indicate the number of months (across all gauges and months) where there is insufficient evidence to reject $\mathrm{HO}$.

\begin{tabular}{|c|c|c|c|c|c|}
\hline & Zero & $1 \mathrm{st}$ & 2nd & Gamma & Mixed \\
\hline \multicolumn{6}{|l|}{ Wet Days/Month } \\
\hline dispersion & 297 & 238 & 267 & - & - \\
\hline RMSE (std. dev.) & 1.88 & 1.74 & 1.75 & - & - \\
\hline F-test & 51 & 93 & 63 & - & - \\
\hline \multicolumn{6}{|l|}{ Monthly Amount } \\
\hline dispersion & 273 & 246 & 271 & 246 & 168 \\
\hline RMSE (std. dev.) & 36.8 & 35.9 & 36.4 & 35.9 & 32.8 \\
\hline F-test & 102 & 113 & 100 & 113 & 165 \\
\hline \multicolumn{6}{|l|}{ DRWH Reliability } \\
\hline dispersion & 211 & 182 & 209 & 182 & 210 \\
\hline RMSE (std. dev.) & 3.64 & 3.49 & 3.53 & 3.49 & 3.65 \\
\hline F-test & 124 & 139 & 128 & 139 & 123 \\
\hline \multicolumn{6}{|l|}{ Wet Spells } \\
\hline RMSE (mean) & 0.090 & 0.081 & 0.121 & - & - \\
\hline RMSE (std. dev.) & 0.155 & 0.085 & 0.132 & - & - \\
\hline \multicolumn{6}{|l|}{ Dry Spells } \\
\hline RMSE (mean) & 1.64 & 1.94 & 2.25 & - & - \\
\hline RMSE (std. dev.) & 5.77 & 6.50 & 7.01 & - & - \\
\hline \multicolumn{6}{|l|}{ Max Wet Spell } \\
\hline RMSE & 6.15 & 7.59 & 8.59 & - & - \\
\hline \multicolumn{6}{|l|}{ Max Dry Spell } \\
\hline RMSE & 59.0 & 62.7 & 66.9 & - & - \\
\hline
\end{tabular}


Figure 3-5 illustrates the mean and standard deviation of wet and dry spells within the observed and model rainfall sequences. All three models reasonably reproduce the wet spell characteristic, with the zero-, $1^{\text {st }}$-, and $2^{\text {nd }}$-order Markov models having the RMSE values of $0.09,0.08$, and 0.12 , respectively. None of the models are able to adequately reproduce the dry spell lengths or the maximum wet and dry spells, though the zero-order model has the lowest RMSE. All three Markov models overshoot these rainfall characteristics.
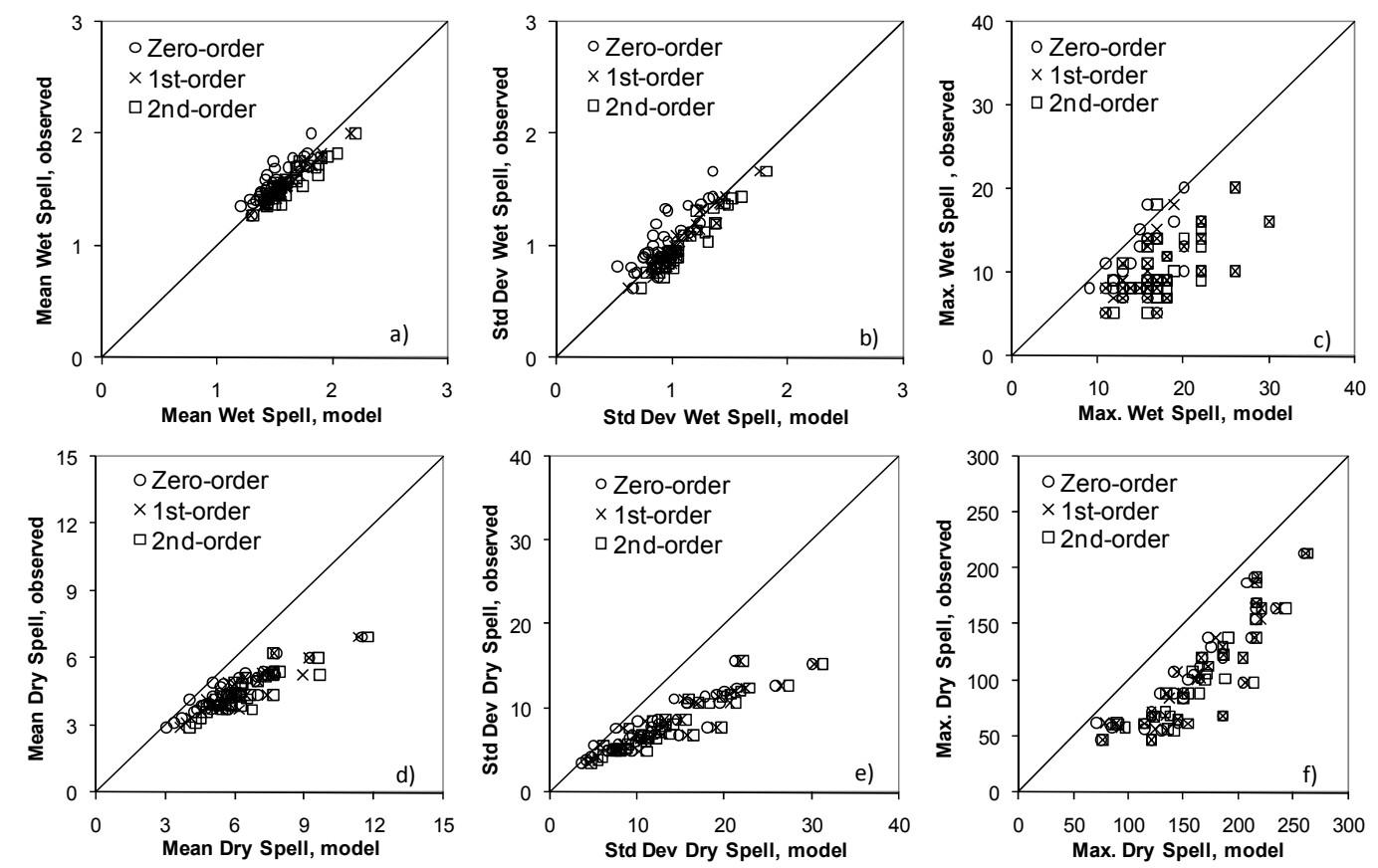

Figure 3-5: Mean, standard deviation, and maximum of a)-c) wet and d)-f) dry spells for zero-, $1^{\text {st }}$-, and $2^{\text {nd }}$-order Markov models.

\subsubsection{Occurrence Sensitivity to Missing Data}

Many meteorological records within the developing world suffer not only from sparse gauge networks and short record lengths, but also from incompleteness resulting from issues such as equipment downtime, funding problems, or operator absence/error. 
Figure 3-6 illustrates the sensitivity of Markov model order choice to missing data. Two assumptions were made regarding missing days: either they have the same probability of being rainy, $\mathrm{P}(\mathrm{w})$, as the rest of the record, or they were assumed to be dry, representing a conservative approach. BIC scores for the modified record filled in with wet or dry days behave similar to the original record, with a slightly higher preference for the zero-order model. Filling in the missing days with all dry days, however, significantly reduces the BIC preference for the zero-order model, especially during the wetter months. This is due to the mixing of more and longer dry spells with the multiple wet spells during this time. Models capable of representing this autocorrelation without large increases in parameters would be preferred by the BIC. The $\mathrm{P}(\mathrm{w})$-filled data set would have a more random scattering of wet and dry days and hence less autocorrelation.
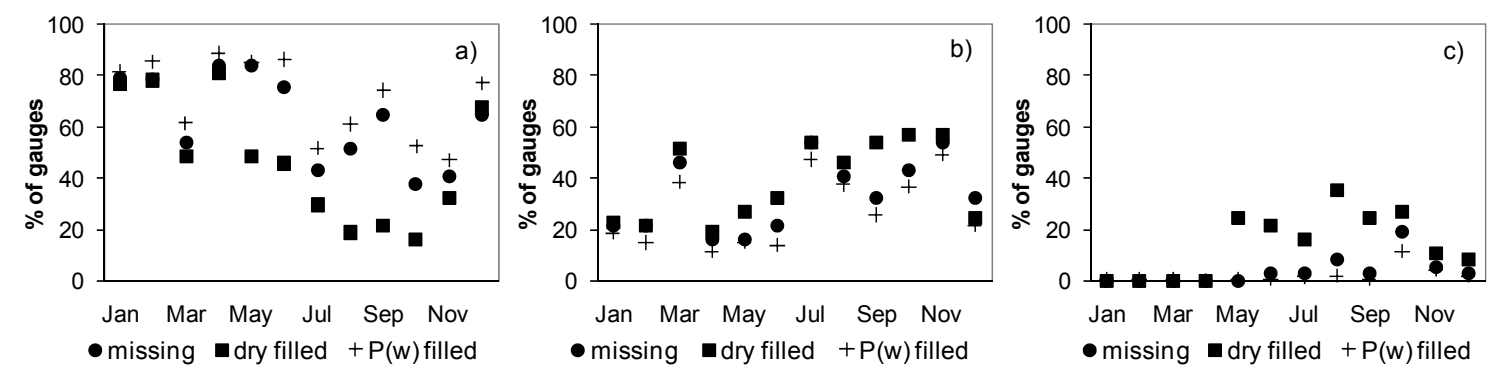

Figure 3-6: Sensitivity of BIC selection of Markov model order to missing days within the observations. The Figure indicates the $\%$ of gauges with the lowest (preferred) BIC score for a) a zero-order Markov model, b) a $1^{\text {st }}$-order Markov model, and c) a $2^{\text {nd }}$-order Markov model.

Rainfall characteristics can also be sensitive to missing data. Gauges with small percentages of missing data have small differences between the modified data sets, with an obvious but often slight drop in wet days/month in the dry-filled data set. Gauges with large amounts of missing data, of course, may have significant drops in wet days per month if missing days are filled with all dry days. Filling missing days using the wet day 
probabilities intuitively results in little change in wet days per month as the ratio of wet days to total days of data remains relatively the same.

\subsubsection{Amount Model}

BIC scores for the amount model overwhelmingly favor the three-parameter mixed exponential distribution over the two-parameter gamma distribution by 309 to 123 for all gauges and months. The reverse is true only during the drier months of the year, where few observations favor the gamma distribution, though not by much. Unlike the occurrence BIC results, the difference between the BIC scores for each model is often high. For comparison between rainfall statistics, the $1^{\text {st }}$-order Markov chain occurrence model was used to ensure that the variability associated with the occurrence process was equal for each amount model.

Both models reproduce the mean monthly totals but again underestimate the observed variability (Table 3-2). A large portion of this overdispersion is due to the occurrence model, but some of the variability is accounted for by the amount models. The mixed exponential distribution outperforms the gamma distribution for monthly totals, whereas the gamma distribution is able to better reproduce the observed variability of DRWH reliability (Table 3-2). The gamma distribution, however, overestimates the mean DRWH reliability by $19.6 \%$ (not shown), which might lead to a more favorable outlook of DRWH harvesting than is actually warranted by the historical record. Appendices E, F, and G contain the estimated parameters for the gamma and mixed exponential rainfall amount distributions. 


\subsubsection{Model Comparison}

Figure 3-7 shows the mean and standard deviation of the models plotted against the observed data for DRWH reliability. Similar patterns are seen in the monthly amount totals, both of which result from the underlying wet days per month behavior. Both Markov models adequately reproduce the means of the rainfall characteristics, with the $1^{\text {st }}$-order Markov model having the lowest averaged RMSE (mean). The LARS-WG overestimates the observed wet days per month, especially in the drier months of DecJan. This overestimation is carried through into monthly totals and DRWH reliability. Overdispersion is also evident for these three models, especially during the wetter months of Apr - Sep; however, the LARS-WG model exhibits underdispersion during the dry season, resulting in higher wet day variability than the historical record (see Figure 3-8). Figure 3-8 illustrates that during the wetter months, the conditioned Markov model has the least overdispersion, whereas the unconditioned $1^{\text {st }}$-order Markov model has the lowest RMSE of the mean. 

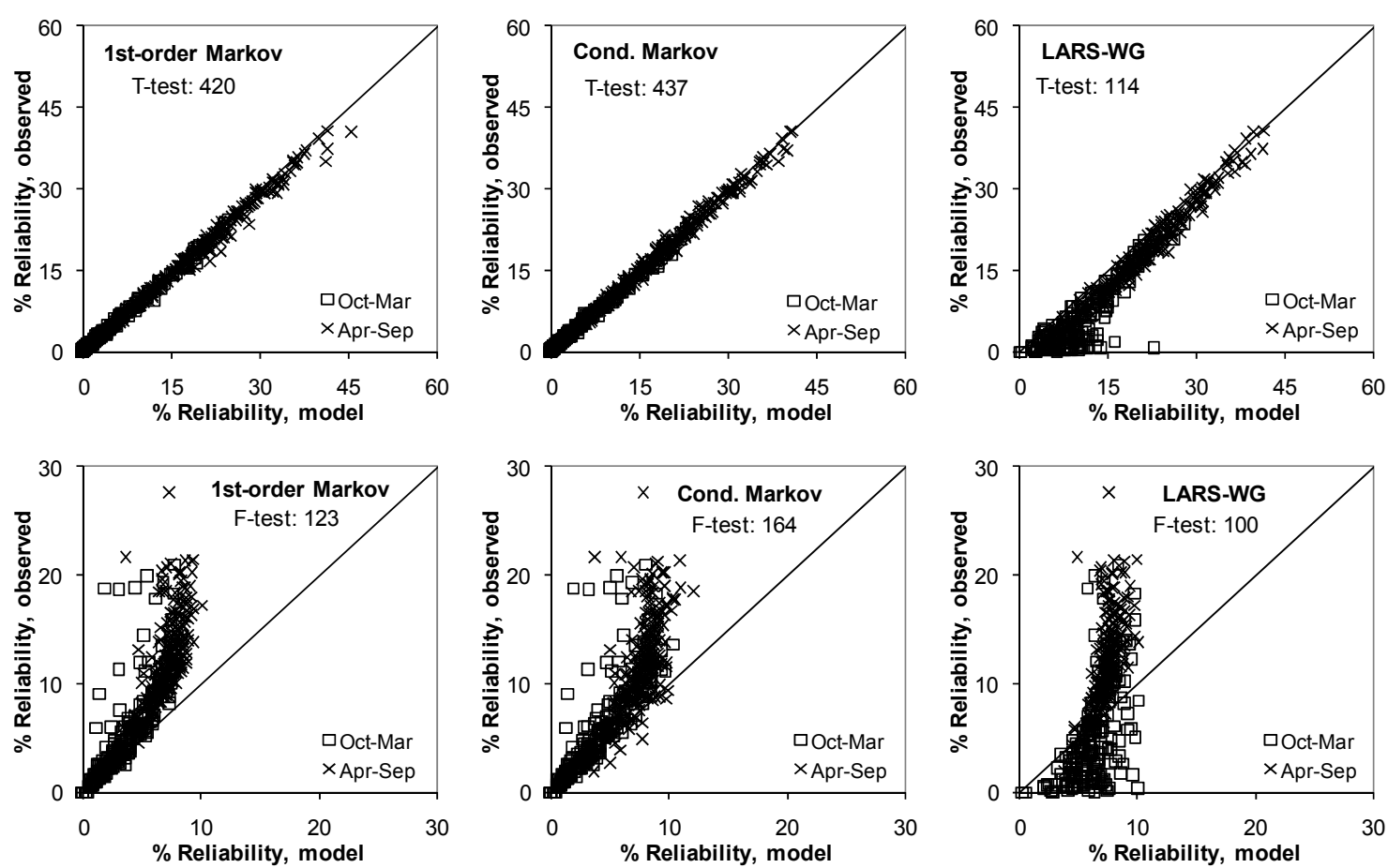

Figure 3-7: Mean and standard deviation of \% reliability. T-test and F-test values indicate the number of months (across all gauges and months) where there is insufficient evidence to reject $\mathrm{HO}$.

The RMSE of the standard deviation is relatively the same through the year for all three models, except during the dry season when LARS-WG overestimates the observations. The conditioned Markov has the lowest averaged RMSE (std. dev.) for all three rainfall characteristics. According to the $\mathrm{t}$ - and F-tests, the conditioned Markov model performs the best out of the three, with the unconditioned $1^{\text {st }}$-order Markov model outperforming the LARS-WG model in simulating the application characteristic of DRWH reliability (See Figure 3-7).

The reverse is true, however, when the spell lengths are considered (see Figure 39 and Table 3-3). Here the LARS-WG far outperforms the Markov models, able to reproduce both the means and standard deviations of wet and dry spells, whereas the Markov models were incapable of matching the dry spell characteristics. This is a 
strength of the LARS-WG model, as it samples directly from the observed spell lengths when constructing synthetic weather sequences.
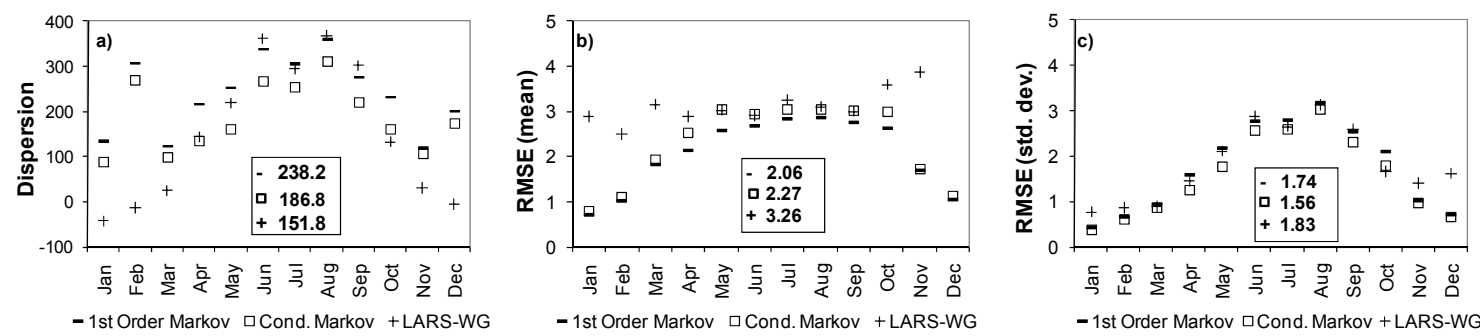

-1st Order Markov aCond. Markov + LARS-WG

- 1st Order Markov $\square$ Cond. Markov + LARS-WG
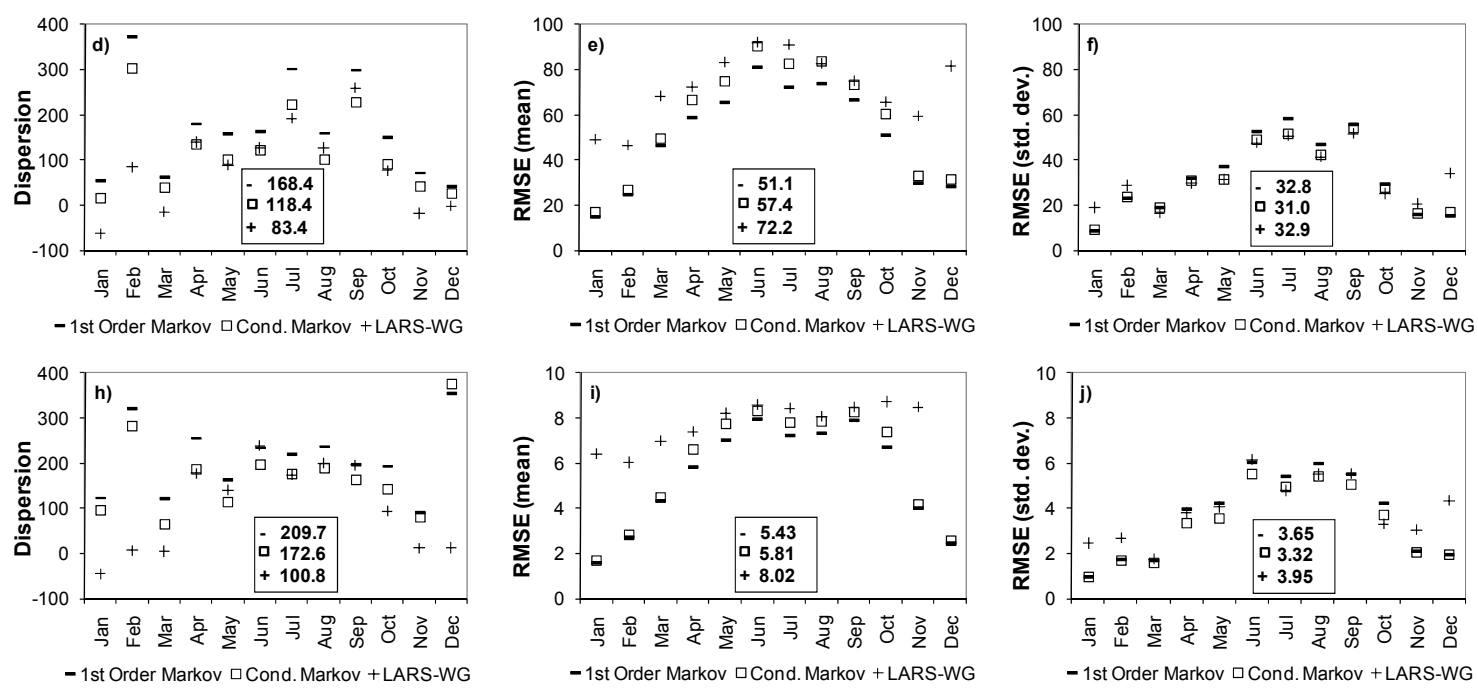

Figure 3-8: Monthly dispersion and RMSE values for a)-c) wet days/month, d)-f) month totals (mm), and h)-j) DRWH \% reliability. Boxed values are dispersion and RMSE values averaged across all gauges and months. 

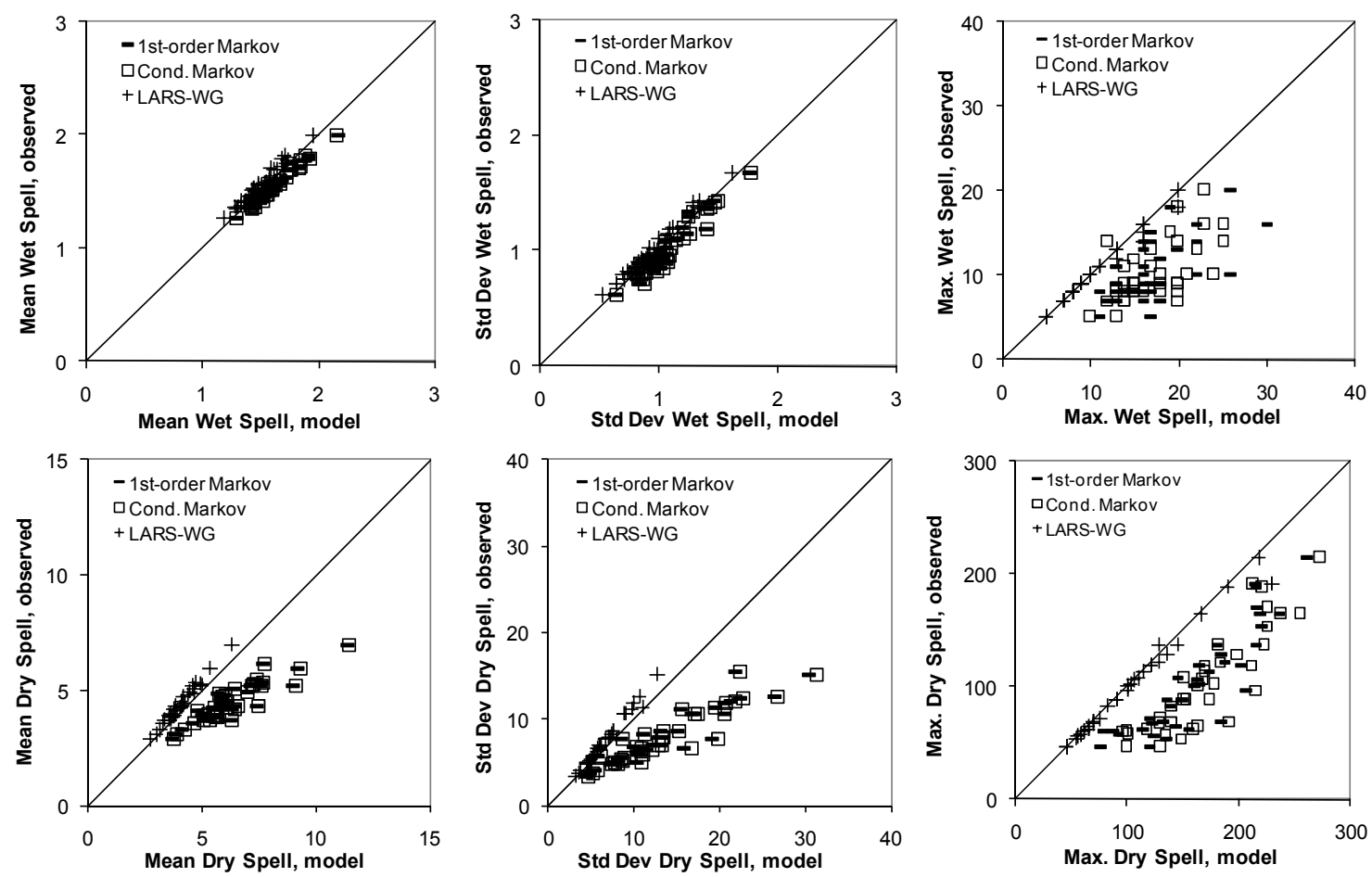

Figure 3-9: Mean, standard deviation, and maximum of wet and dry spells (days)

Table 3-3: Spell length characteristics statistical test results for the stochastic weather generators. RMSE values are averaged across all gauges and months.

\begin{tabular}{cccc}
\hline & 1st Order & Conditioned & LARS-WG \\
\hline Wet Spells & & & \\
RMSE (mean) & 0.081 & 0.080 & 0.056 \\
RMSE (std. dev.) & 0.085 & 0.103 & 0.056 \\
\hline Dry Spells & & & \\
RMSE (mean) & 1.94 & 2.02 & 0.293 \\
RMSE (std. dev.) & 6.50 & 6.85 & 0.955 \\
\hline Max Wet Spell & & & \\
RMSE & 7.59 & 7.84 & 0.615 \\
\hline Max Dry Spell & & & \\
RMSE & 62.7 & 71.0 & 7.15 \\
\hline
\end{tabular}

\subsubsection{DRWH Assessment}

DRWH systems are often designed with an optimum storage size and catchment area, and the same considerations are made for DRWH systems in urban West Africa. Design alternatives, however, are somewhat constrained by the space available in 
developing world cities, especially in slum communities. For common per capita area and storage values in urban slums, the marginal benefits of increasing storage volume are much higher than increasing roof area when initial storage volumes are low, regardless of the roof size. However, as storage volumes increase, this gap decreases to a point where roof area increases must be considered as an alternative to storage augmentation.

Figure 3-10 and Figure 3-11 show multi-model DRHW reliability ( $\geq 20 \mathrm{~L} /$ day/capita) assessments for two gauges in West Africa. Both figures show results for varying roof and storage values, with Figure 3-10 showing the results of the LARSWG model and $1^{\text {st }}$-order Markov model, and Figure 3-11 showing the results of the LARS-WG model and the Conditioned Markov model. Households with little per capita storage capacity or roof area can only expect DRWH to partially enhance improved water supply for certain months of the year. Households with larger storage volumes and roof areas can enjoy a significant water supply enhancement, but are still unable to rely solely upon DRWH systems to meet demands.

Finally, Figure 3-12 allows water planners in government or NGOs to determine an optimum storage volume for varying roof sizes. Storage sizes were incrementally increased (in steps of $40 \mathrm{~L}$ ) and DRWH reliability estimated. The reliability benefits eventually diminish with increasing storage, indicating the reliability is also limited by roof area or rainfall. For small per capita roof areas, one $200 \mathrm{~L}$ drum would be optimum for most regions, whereas 2-5 drums would be needed to take advantage of increasing per capita roof size up to $10 \mathrm{~m}^{2}$. Appendices $\mathrm{H}$, I, and $\mathrm{J}$ contain the reliability results for all models and gauges. 

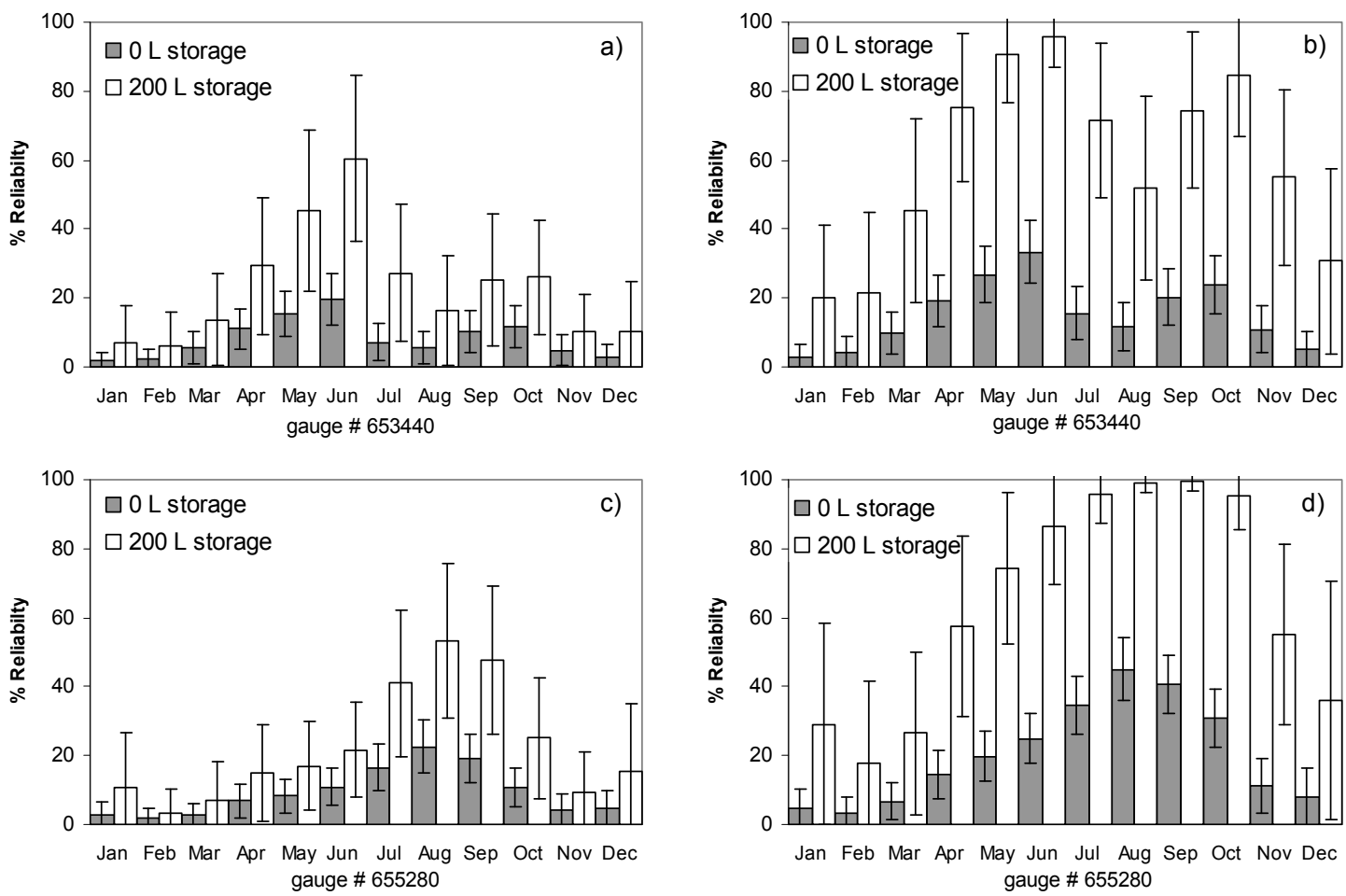

Figure 3-10: Multi-model DRWH reliability for a $2 \mathrm{~m}^{2}$ per capita roof area for gauges a) 653440 and c) 655280 , and for a $10 \mathrm{~m}^{2}$ per capita roof area for gauges b) 653440 and d) 655280. Models used are LARS-WG and $1^{\text {st }}$ order Markov. 

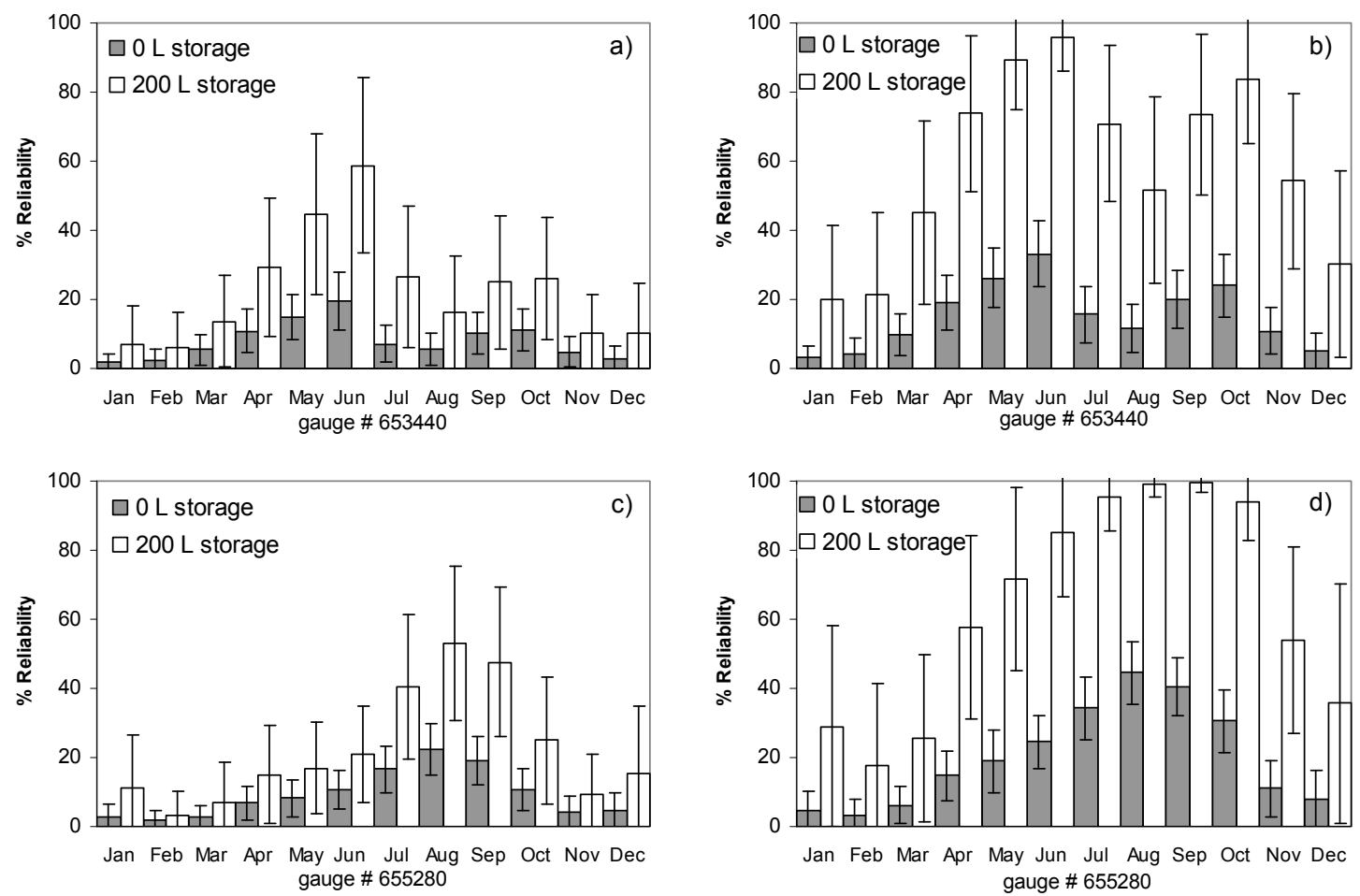

Figure 3-11: Multi-model DRWH reliability for a $2 \mathrm{~m}^{2}$ per capita roof area for gauges a) 653440 and c) 655280 , and for a $10 \mathrm{~m}^{2}$ per capita roof area for gauges b) 653440 and d) 655280. Models used are LARS-WG and Conditioned Markov. 


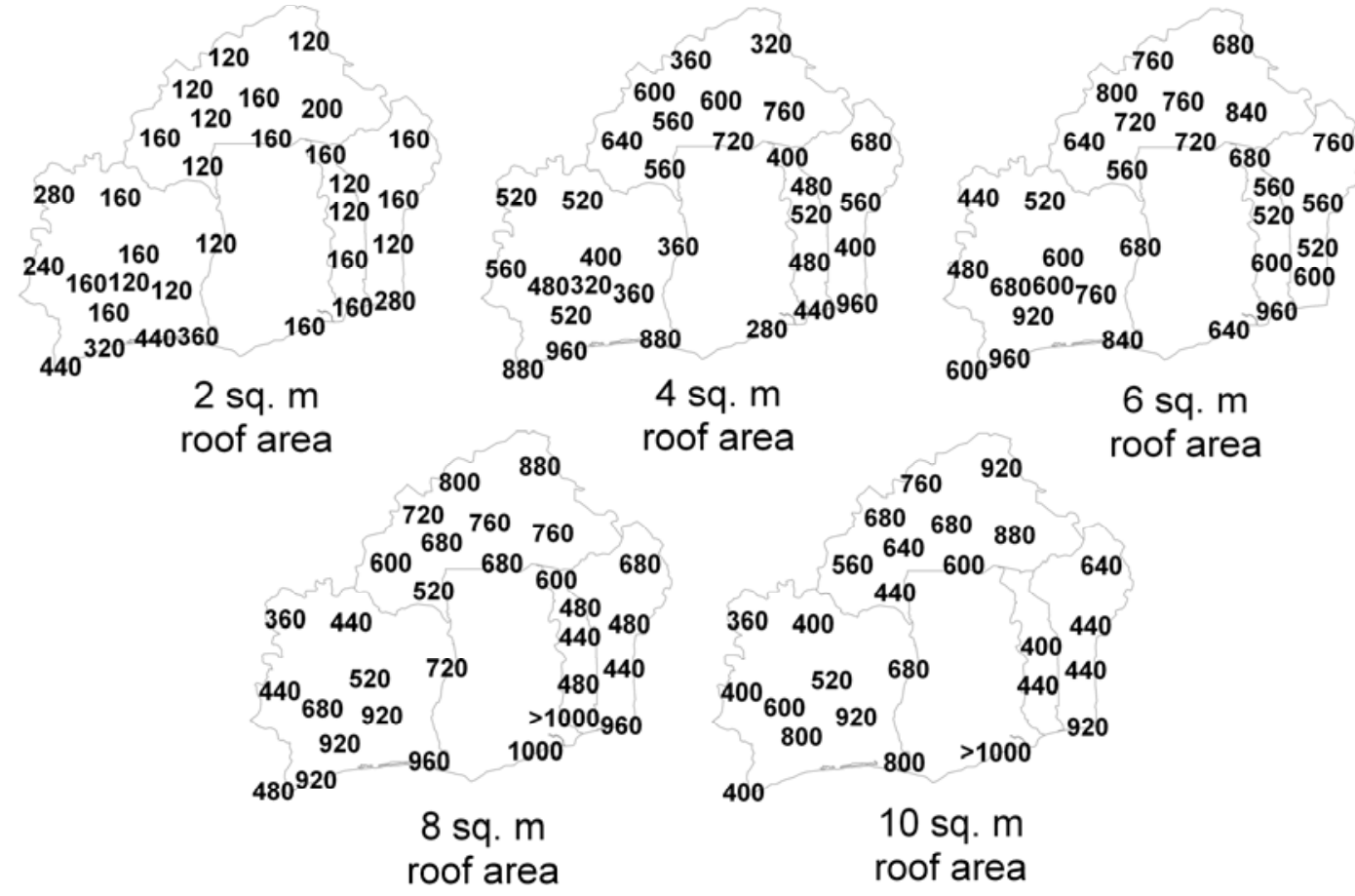

Figure 3-12: Optimum per capita storage size (L) for the wetter months of Apr-Oct.

Optimum storage size for each month is determined by either a) further increase in storage size (40 L increments) results in $<1 \%$ change in reliability, or b) the storage size results in $>95 \%$ reliability. The maximum of monthly values for each gauge is shown.

\subsection{Discussion}

The BIC scores indicate the zero-order Markov model to be the preferable model for this region. This is intuitive for the very dry months of Dec-Feb, as there are very few wet days recorded during this time and therefore little autocorrelation associated with the time series. Wet days are rare, and random events are easily represented by marginal wet-day probabilities. Selection for the remainder of the year, however, is less clear, as the zero-order Markov model has only a slight advantage over the $1^{\text {st }}$-order model.

Probability dependence on previous day conditions becomes more important as more wet days occur during the rise, peak, and fall of the uni- or bimodal rainy seasons. 
Sole reliance upon BIC scores for model determination, however, is not recommended. Several studies have noted the overdispersion tendencies of Markov models, and this known behavior is seen in this study (Katz and Parlange, 1998). Relying solely upon the BIC, especially when the score difference between orders is small, risks choosing a model that is less capable of capturing the variability of rainfall characteristics directly associated with the model application. Such is the case with DRWH reliability. The $1^{\text {st }}$-order model clearly outperforms the zero-order model in reproducing the variability of the rainfall characteristics, including DRWH reliability, examined in this study.

Some study locations have seen greater variability reproduced by the $2^{\text {nd }}$-order model than by the $1^{\text {st }}$-order model (Katz and Parlange, 1998); however this is not the case here. Perhaps the large number of missing days prevented the accurate estimation of $2^{\text {nd }}-$ order parameters. A few of the 2-lag transition probabilities for some of the gauges were very small or zero due to missing days, thereby affecting the accurate representation of the 2-lag autocorrelation. These missing observations are also responsible for the overestimation of spell length variability and maximums in the Markov models, as they interrupt spell lengths in the historical record.

The larger percentage of missing days in developing world records is yet another reason to be wary of BIC application as it is sensitive to the method of filling in these observations. Filling in missing days can also have detrimental effects on the model application. If a record's missing days are filled with dry days, it will give a conservative estimate of DRWH reliability. However, storage volume optimization will become 
highly uncertain, as larger storage volumes may be warranted than that recommended using dry-filled data.

The wet days per month are especially critical to small-storage DRWH systems dependent on frequent occurrence of rainfall. For this reason a $1^{\text {st }}$-order Markov model is recommended for DRHW assessment in this West African region. This conclusion is amenable to the availability of the "off the shelf" software that utilizes a $1^{\text {st }}$-order Markov chain. A mixture model could also be used, with a zero-order model for the dry season, in accordance to the BIC results; however, parsimony dictates a consistent method for DRWH assessment in developing countries.

Comparing the amount models, the mixed exponential distribution clearly outperforms the gamma distribution in BIC scores and in reproducing the mean and variance of the monthly totals. It also better matches the mean DRWH reliability but not the variance, though both distributions exhibit significant overdispersion. Although the gamma distribution matches this rainfall characteristic's variability better than the mixed exponential distribution, the mean reliability resulting from the gamma model would create a false sense of security in terms of DRWH system reliance as it overestimates the true mean reliability. Previous studies have also indicated the mixed exponential distribution's superior ability to reproduce the larger storm intensities, which the gamma model may miss entirely (Wilks, 1999). This is also evident in this study as the mixed exponential distribution better matches the observed monthly total variability. For DRWH studies involving large storage systems, capturing these large storms and accurately representing monthly amount totals is essential. The mixed exponential distribution is therefore recommended for this region and application. 
Neither the LARS-WG nor the unconditioned $1^{\text {st }}$-order Markov model seems to have a substantial advantage over the other during the wetter months of the year. Both can adequately reproduce rainfall characteristic means, and both suffer from the overdispersion common to stochastic weather generators. However, the LARS-WG cannot be recommended for drier months as it overestimates both the mean and the variance wet days during those months. This is due to sampling spell lengths interrupted by missing days. LARS-WG samples from the wet spell lengths after a dry spell, and does not insert missing days in the synthetic record. This results in the overestimation of wet days in the dry months. The conditioned Markov model behaves as expected and better matches the interannual variability of all the rainfall characteristics. This is, of course, due to its inclusion of a large-scale interannual model component.

A completely different conclusion is reached, however, if the DRWH application is fully thought out. In terms of this study, where little to no storage is considered, then the Markov models are of use as they adequately reproduce mean monthly values (e.g. when rainfall exceeds a threshold to allow for $>20 \mathrm{~L}$ collection). As storage volumes increase, however, and households in the developing world attempt to correctly size their tanks for perhaps larger consumptive demands, the realistic representation of spell lengths becomes more important. The storage volume available at the beginning of each day is dependent on the lengths of dry spells between storms. The LARS-WG model has a clear advantage in that it almost perfectly matches the observed spell length characteristics.

For these reasons a multi-model approach is used in assessing the capabilities of DRWH systems in providing improved water supply reliability. The variability of the 
assessments quantifies the reliability ranges produced by the models. It is clear that DRHW systems can provide improved water supplies through much of the year, although alternative sources must be available due to the aforementioned variability. Some months may see longer dry spells than others, and limited resources (space, funding) may preclude large enough storage and catchment areas to provide water through the dry season. Future work will include a more complete assessment over a range of catchment areas, as well as storage size optimization incorporating direct costs and health impacts. In accessing health impacts, additional consumption levels, such as 50/L and 100/L per day, will also be investigated. These same parsimonious models may also be used in general circulation downscaling studies to determine how DRWH system reliability could be impacted by various global climate change scenarios. Although only two gauges are represented here, these freely available models are easily applicable to gauges in other developing regions where cost, training, or data limitations may prohibit the use of more complicated methods. 


\section{Chapter 4 Climate Change Impact Assessment on Domestic Rainwater Harvesting in West Africa}

\subsection{Introduction}

Domestic rainwater harvesting (DRWH) consists of the conveyance of precipitation falling onto an impervious area (e.g. rooftop) into a storage container. This technology has been utilized for centuries to supply water at a household and community level for purposes such as drinking, cooking, washing, and laundry. DRWH continues to be practiced in several areas of the world, from wet regions such as Thailand, India, and parts of Africa, to arid regions such Australia, the southwestern U.S., and the Middle East. People in regions with limited water supplies (e.g. deserts, small islands) are especially adept at capturing infrequent, large storms and storing the water for extended periods of time.

Rainwater harvesting has been practiced in sub-Saharan Africa for centuries, especially by subsistence farmers seeking water for crops. Several studies have looked at the utility of DRWH in Africa, examining variables such as rainfall, roof area, and demand, and have found many regions of the continent suitable for the technology (Cowden et al., 2008; Thomas, 2005, 1998; Ntale et al., 2005). DRWH is now gaining popularity in several regions of the continent and has become a standard tool and program emphasis for several water resources non-governmental organizations (NGOs) and governmental agencies, especially in Eastern and Southern African countries.

Two intertwined environmental and social conditions exist in West Africa that make DRWH an appropriate technology for water provision. Water availability and water quality are not consistently high in several regions of West Africa, especially in the 
Sahelian region. Coupling this situation with institutional failings has resulted in low improved water and sanitation coverage, as was previously shown in Chapter 1 with regards to improved water and sanitation coverage in Africa (Figure 1-3 and Figure 1-4). Urbanization also strains local resources and institutional capacity. West African urbanization rates are the highest on the continent and among the highest in the world. In fact, by 2050, approximately 420 million people will be living in urban areas in West Africa, compared to roughly 130 million in 2008 (UNPD, 2008). This rapid urbanization creates large, informal settlements, or slums, that are typically not serviced by local water and sanitation agencies.

Future climate change has potential to exacerbate the water and urbanization problems of West Africa. Rising sea levels and salt water intrusion into heavily used aquifers could displace a high proportion of the population inland, creating an ecological refugee crisis. Changing precipitation patterns can also add uncertainty regarding the planning and use of surface and groundwater resources. The United Nations Millennium Development Goals (MDG) Target 10 seeks to halve, by 2015, the proportion of people without sustainable access to safe drinking water and basic sanitation. Climate change may hamper MDG efforts as populations and water resources availability shift both spatially and temporally.

DRWH, however, has been recognized as a means of achieving the MDG target in spite of climatic variability and uncertainty (UNEP, 2005). DRWH has also been an adaptation strategy for civilizations faced with abrupt climate fluctuations (Pandey et al., 2003). DRWH can, in effect, mitigate climate change impacts on displaced or slum communities faced with decreasing improved water access. 
The climate of West Africa ranges from the humid tropics in the South to the semi-arid Sahelian region to the North. Rainfall is relatively abundant in many areas, especially along the coasts (Figure 4-1). The Sahelian region, the boundary between the Sahara desert and the more humid coastal and interior regions, is more arid and therefore more susceptible to unexpected rainfall variability. This region experienced major drought periods in the early 1970's and 1980's, with general drought conditions existing from the late 1960's into the 1990's (Le Barbe et al., 2002).

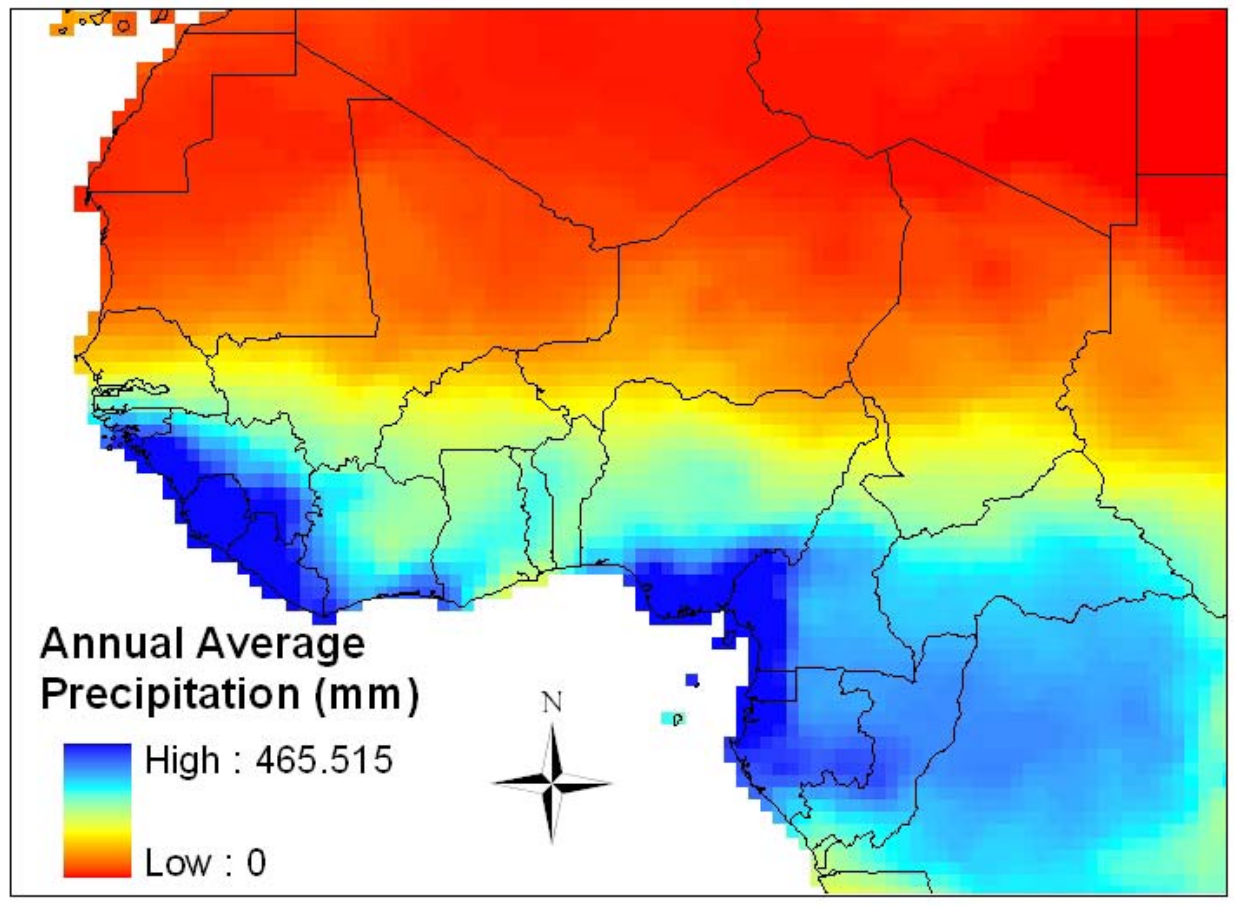

Figure 4-1. Annual Precipitation Patterns West Africa (Map created by author from data in VASClimO, 2005)

Much of the region experiences an annual wet and dry oscillation, associated with movement of the Inter-tropical Convergence Zone (ITCZ). The ITCZ is the boundary in which the trade winds of the Northern and Southern hemispheres meet, creating a band of low pressure and associated thunderstorms around the equator. The arrival of these 
systems creates monsoon conditions during the wet season for much of the tropics (Sultan and Janicot, 2003). Rainfall patterns in the West African region have been strongly correlated to sea surface temperatures. Temperature variations in the Gulf of Guinea and the tropical Atlantic, and the associated atmospheric responses, have been shown to explain historical changes in wet and dry precipitation patterns in both the Sahel and Guinea Coast (Balas et al., 2007; Giannini et al., 2003; Hoerling et al., 2006).

Ocean-atmospheric relationships can be captured in GCM simulations; however, the successful representation of West African precipitation has eluded many climate models, especially the dipolar rainfall variations and the annual monsoonal season (Christensen et al., 2007). The GCM failings in representing African rainfall stem from the inability to reproduce precipitation mechanisms, such as the hydrological cycle or orographic effect, or limitations in simulating teleconnections or feedback mechanisms (Boko et al., 2007). IPCC AR4 predictions for the West African region vary widely, with some predicting substantial drying and others progressive wetting (Boko et al., 2007). Cook and Vizy (2006) examined several GCM simulations over Africa and found the GFDL-CM2.0, MIROC3.2(medres), and MRI-CGCM2.3.2 models to reasonably simulate twentieth-century climate, though no single GCM had strengths in simulating all precipitation characteristics.

GCMs are also not currently able to provide adequate weather variable outputs at the spatial or temporal scales of many hydrological systems, including DRWH, making direct GCM climate change impact studies vague at best. Though computation advances enable finer spatial models, including regional climate models, the need to statistically downscale GCM output to local areas of concern still exists, especially in data and 68 
resource poor regions such as Africa. Statistical downscaling (SD) methods define a quantitative relationship between large-scale atmospheric predictors and local surface variables or predictands (Wilby et al., 2004). Many SD studies use precipitation for the predictand as this is the most important variable in agriculture and hydrologic studies. Commonly used predictors include sea level pressure fields and temperatures, geopotential heights and thickness, vorticity, and relative humidity (IPCC, 2001).

The general form of the predictor-predictand relationship is

$$
R_{t}=F\left(X_{T}\right) \quad \text { for } T \leq t
$$

where $R_{t}$ is the predictand at time t, F is the method to quantify the relationship, and $X_{t}$ is the predictor set of current or past atmospheric variables (Charles et al., 2004). The IPCC has identified three key assumptions of SD methods, each being fundamental to the validity of statistical downscaling (IPCC, 2001; Wilby et al, 2004, Charles et al., 2004). The first is that the predictors are relevant to the predictand and can be realistically modeled by the GCM. This balance between relevance and representation is an important skill of any SD study. The second assumption is one of stationarity, where the quantitative relationship or transfer function between the predictor and predictand does not change in future altered climates. This is impossible to verify because future observations do not exist, but it can be validated using a separate observation set with a different climate than the fitting record set (Charles et al., 2004). Calibration and validation using past and future regional climate model (RCM) data sets is also a possibility (IPCC, 2001). Along with this assumption is the caveat against using future predictor values outside the climatology used to calibrate the transfer function, as this will invalidate the model (Wilby et al., 2004). 
The third assumption is that the chosen predictors fully represent the climate change signal. Predictors that are not affected by model forcings and do not vary with forecasted climate change do little to translate this change to local predictands. On the other hand, if predictors that are very representative of climate change signals are left out of the model, unrealistic results may occur. Predictor selection methods must be chosen so as not to exclude these potentially important large-scale variables, and multiple predictor sets are encouraged in SD studies (Wilby et al., 2004).

The choice of predictor(s) is perhaps the most important step in statistical downscaling, as the validity of the aforementioned assumptions rest upon a sensible selection. This selection process can be difficult in that any one predictor may have low explanatory power, and this power may vary in time or space (Wilby et al., 2004). The emergence of climate reanalysis data sets has made a variety of predictor values available for selection, but these may be limited to the GCM data output that will be used in the climate change scenario assessment (Wilby et al., 2004).

Several correlation and selection methods have been used to determine an adequate set of predictors for downscaling models, including partial correlation analysis, step-wise regression, and information criteria (Charles, et al., 2004; IPCC, 2001). Many earlier studies have focused on atmospheric circulation and pressure variables, but recent investigations have revealed the importance of humidity predictors in rainfall downscaling. IPCC guidelines ultimately recommend predictor variables to be strongly correlated with the predictand, make physical sense, realistically characterized by the GCM, able to represent interannual variability, and reflective of the climate change signal 
(Wilby et. al, 2004). Whether or not any given predictor meets all these recommendations depends on the site and quality of data available.

Statistical downscaling models can be grouped into three broad categories, namely regression models, weather classification schemes, and weather generators.

\subsubsection{Regression Models}

After determining the correlation between predictors and predictand, a simple choice of relating them together would be identifying a transfer function:

$$
R_{t}=F_{Y}\left(X_{T} ; \theta\right) \quad \text { for } T \leq t
$$

where $\theta$ is the parameter set and $F_{Y}$ is the linear or nonlinear regression function (Charles et al., 2004). Several regression methods have been used, including simple and multivariate regression (Kidson and Thompson, 1998; Murphy, 1999; Sailor and Li, 1999; Wilby and Wigley, 2000;), singular value decomposition (von Storch and Zwiers, 1999), canonical correlation analysis (Karl et al., 1990; Wigley et al, 1990; von Storch et al., 1993; Busuioc et al., 2001), artificial neural networks (Hewitson and Crane, 1996; Crane and Hewitson, 1998; Trigo and Paluntikof, 1999; Schoof and Pryor, 2001; Tatli et al., 2004), and spatial interpolation methods (Brandsma and Buishand, 1997; Buishand and Brandsma, 1999; Biau et al., 1999; Wood et al., 2004). Hybrid regression models have also been developed that add a stochastic component to inflate certain desired predictand characteristics that may be underestimated by regression alone.

The strengths of the regression models include their ability to consider a wide range of predictor values, with many techniques using readily available software (Wilby et al, 2004). Weaknesses of the regression methods include the assumptions of linearity and normality in certain cases and an inability to reproduce observed variances in the 
predictand, especially for daily precipitation (von Storch, 1999; Wilby et al, 2004). This weakness in variance reproduction can be strengthened by bilinear linking of global circulation and predictand variable covariances (Burger, 1996) or by adding "noise" using stochastic processes (Charles et al., 1999; Wilby et al, 2003).

\subsubsection{Weather Classification}

Weather classification schemes or weather typing uses synoptic similarity to classify or group days into a finite number of discrete weather types or "states":

$$
\begin{aligned}
& R_{t}=F_{R}\left(S_{t}\right) \\
& S_{t}=F_{S}\left(X_{T}\right) \quad \text { for } T \leq t
\end{aligned}
$$

where $S_{t}$ is the weather state at time $t, F_{s}$ is the weather state definition method, and $F_{R}$ relates the weather state to the predictand (Charles et al., 2004). Both objective and subjective methods are used to define the weather states. Subjective methods were developed first and typically involved an "expert" classification of weather types for a specific region (Lamb, 1972; Bardossy and Caspary, 1990; Hay et al., 1991; Jones et al, 1993). The most common objective method is cluster analysis of atmospheric fields (Corte-Real et al., 1999; Huth, 2000; Kidson, 2000; Hewitson and Crane, 2002), with several other statistical methods also being used for state identification (Wilby and Wigly, 1997). Analog methods, which match current or predicted weather states to historical states, require long observation records to adequately capture the variance. Still, analog methods have compared favorably to more complex methods (Martin et al., 1997; Zorita and von Storch, 1999; Timbal and McAvaney, 2001).

Once the weather states are determined, $F_{R}$ must be determined. This involves assigning predictand values or probabilities to each class by weighting the predictand(s) 72 
with the relative frequencies of the weather classes (IPCC, 2001). Resampling, Monte Carlo analysis, regression, and GCM field output are methods of producing time sequences of the weather states. A stochastic version of the weather classification method uses a Markov chain process to determine the transition of the weather states followed by conditional or unconditional wet/dry probabilities assigned to each state. These hidden Markov models are found to adequately reproduce occurrence and persistence of wet/dry spells, as well as interannual variability (Hughes and Guttorp, 1994; Charles et al., 1999; Hughes et al., 1999; Robertson et al., 2004).

A strength of weather classification is the physically based link it provides between prevailing climate patterns and local predictand behavior. This strength, however, is also its greatest weakness in that it is heavily dependent on the main assumption that these physical links will not change in future climates. Another strength of this method is the ability to connect a wide assortment of predictands to weather classification schemes, ranging from snow (Martin et al., 1997) to landslides (Dehn, 1999). Weaknesses include insensitivity to climate change forcings and the additional work required in weather states identification (Wilby et al., 2004).

\subsubsection{Weather Generators}

Weather generators are stochastic models able to reproduce the statistical characteristics of a local predictand. For use in downscaling, these models are conditioned on large-scale predictors, weather states, or precipitation properties:

$$
R_{t}=F_{W}\left(\theta \mid X_{T}\right) \quad \text { for } T \leq t
$$

or 


$$
R_{t}=F_{W}\left(\theta \mid S_{t}\right)
$$

where $\theta$ is the parameter set of the weather generator $F_{w}$ (Charles et al., 2004). The basic methodology adjusts weather generator parameters based on temporal changes in GCM output or other criteria (Katz, 1996; Wilby et al., 1998; Wilks, 1999). Weather generator parameter adjustments that are also conditioned on large-scale predictors, however, can have better skill in reproducing rainfall characteristic statistics (Katz and Parlange, 1996; Wilks, 1999a; Qian et al., 2002). Adjustment of weather generator parameters can alter relationships between other climate variables (e.g. temperature) conditioned on precipitation occurrence (Wilks, 1992; Katz, 1996). The basic method of weather generator downscaling has two steps, extrapolation and downscaling (Wilby et al, 1998; Wilks, 1999b).

(IPCC, 2001). Two statistical downscaling methods will be employed in this study. Both use stochastic weather generators and involve the adjustment of the Markov and spell-length weather generator parameters to reflect climate change. The methods will be used to determine the impacts of climate change on DRWH the West Africa for the time period of 2045 to 2065 .

\subsection{Methods}

Due to recognized biases within individual GCM models, output data (i.e. precipitation fields) from several GCMs will be downscaled to the local scale by each downscaling method, as described below. These GCMs include the MIROC3.2 medres (MIROC), MRI-CGCM2.3.2 (MRI), and the CCCMA_CGCM3 (CGCM3) modes. The MIROC and MRI models were chosen due to adequate representation of sea-surface 
temperatures off the coast of West Africa, and the resulting rainfall patterns in the region (Cook and Vizy, 2006). The CGCM3 also adequately simulated some West Africa precipitation characteristics, though not to the degree of the other two GCMs. The CGCM3 model, however, is used in this study due to its common application in a downscaling method to be considered in future work.

\subsubsection{Markov Weather Generator Downscaling}

Markov weather generators are commonly used to generate long sequences of weather variable that are statistically similar to the historical record, and have been applied to rainwater harvesting reliability in West Africa (Cowden et al., 2008). Markov weather generator downscaling involves the adjustment of the model parameters of interest (e.g. precipitation transition probabilities) to reflect a changing climate, as predicted by each GCM. This adjustment entails two parts, extrapolation of GCM predictions to large-scale predictors, and downscaling of the altered large-scale predictors to local scale predictands. The methodology herein will follow the steps outlined in Wilby et al. (1998), using area-averaged precipitation parameters as the predictor.

The control and perturbed GCM daily rainfall statistics (i.e. unconditional wetday probability, $\pi$, and lag-1 autocorrelation, $r$ ) are determined. An area-average precipitation sequence, equal to the GCM spatial resolution, is then constructed using the local gauge observations. The same rainfall statistics are then calculated for the areaaverage sequence. All unconditional wet-day probabilities are linearly adjusted on a logodds scale, and a Fisher $Z$ transform procedure is completed for the lag-1 autocorrelations. The extrapolation step entails applying the GCM difference between the control and perturbed runs to the area-average values. The gamma distribution 
parameters, used for wet-day amounts, are extrapolated via a proportional adjustment. The downscaling method involves determining the relationship between the area-average precipitation and the local precipitation. These relationships are established using linear regression, and are then applied to the extrapolated area-average values to determine the downscaled local rainfall parameters.

\subsubsection{Spell-length Weather Generator Downscaling}

To produce climate scenario precipitation sequences, the LARS-WG software does not utilize the predictor/predictand extrapolation and downscaling steps. Instead, the software is set up to simply perturb the model parameters by entering the variable difference between control and perturbed GCM runs (Semenov et al., 1998). This is similar to change factor methods, but allows for differing time series on a day-to-day basis (Semenov, 2002). This convention will be followed in this study, with the control and perturbed GCM variables being the change in monthly mean rainfall and the change in wet/dry spell durations. The LARS-WG perturbed model will then be used to generate precipitation sequences for each of the GCMs.

\subsubsection{Climate Change Impact Analysis on Domestic Rainwater Harvesting}

The impact of climate change on the reliability of DRWH systems will be assessed at the gauged locations. System reliability will be the criterion in assessing DRWH systems in the study region and will be defined as

$$
\text { Reliability }=\frac{\# \text { of time periods, } \mathrm{t}, \text { where } X_{t} \geq X^{T}}{n}
$$

where $X_{t}(\mathrm{~mm})$ is the rainfall for day $t, X^{T}(\mathrm{~mm})$ is a defined rainfall threshold value, and $n$ is the total number of days (Loucks et al., 2005). $X^{T}$ is a function of water demand ( $S_{d}$, 
$\left.\mathrm{m}^{3}\right)$, roof area available for the DRWH system $\left(A, \mathrm{~m}^{2}\right)$, and a runoff coefficient $(C)$, and is defined as follows.

$$
X^{T}=\frac{1000 S_{d}}{C A}
$$

The water demand $\left(S_{d}\right)$ will be based on the World Health Organization's (WHO) recommendation of a minimum of $20 \mathrm{~L}$ of water per person per day and the average African daily water consumption of 50 L (Howard and Bartram, 2003; UN-HABITAT, 2003). The WHO recommendation of $20 \mathrm{~L}$ is the minimum quantity of water needed for basic drinking, cooking, sanitation, and hygiene. Average per capita roof areas for African slum households are not reported in the literature, so a range of values (between 2 and $10 \mathrm{~m}^{2}$ ) will be used, encompassing minimum recommendations taken from previous studies (Thomas, 1998). The runoff coefficient, 0.8 , accounts for rainfall lost due to splashing, leaking, and first-flush diversions. There is no carry-over storage for these systems in this analysis, as storage volumes in slum households are either limited or unknown.

\subsection{Results}

The average monthly DRWH reliability values of each downscaling method used on each GCM were averaged together to produce a multimodel value that was compared to the observed monthly DRWH reliabilities. Analysis based on ensembles of multiple GCMs is an established practice, and the ensemble means are expected to outperform individual model results (Meehl et al., 2007). The minimum and maximum average monthly DRWH reliability values were also determined in order to display a range of results, or as a representation of the uncertainties within the climate change impact 
assessment. These averages and ranges were determined for three IPCC Special Report on Emissions Scenarios (SRES). It must be recognized, however, that multimodel results cannot include all possible model configurations due to resource constraints (Meehl et al., 2007).

The SRES are the emission scenarios used by climate modeling centers to examine potential future climate change. The three SRES used in this study are the A1B, A2, and B1 scenarios. The A1B scenario assumes a rapid, global economic growth with a balance across fossil and non-fossil energy use. A2 assumes high population growth, but slow economic and technological development focused on the local scale. B1 is a similar globalization world like A1B, but with economic activity steered toward the service and information sectors. Figure 4-2 and Figure 4-3 illustrate the impacts these emission scenarios have on DRWH reliability at a 20 L/day per capita level.

Figure 4-2 represents the bimodal precipitation patterns prevalent in the coastal areas of the five-country region, and Figure 4-3 shows the unimodal pattern of gauges further inland. All four gauges show a climate change induced decline in DRWH reliability in the ascending limbs of the rainfall pattern (spring and/or early fall), and an increased DRWH reliability in the descending limbs (summer and/or fall). This signifies a slight temporal shift in the bimodal and unimodal precipitation patterns. The climate change impact on DRWH is minimal throughout most of the year, though 5 percent to 10 percent differences are evident during the peak wet times of the year. Large differences and uncertainties associated with the dry months (Nov - Feb) may be attributed to the inability of the weather generators to adequately represent sparse precipitation patterns in this region (Cowden et al., 2008). 
The differences in GCMs and downscaling methods are shown in Table 4-1 and Table 4-2. Table 4-1 shows the percentage, by GCM, of the maximum and minimum DRWH reliability values for all roof sizes, which in most cases exceeds or is less than the observed value (see Figure 4-2 and Figure 4-3). The MRI model predicts higher DRWH reliability overall, though the spell-length downscaling method shows wetter CGCM3 results. Both downscaling methods show that the MIROC model consistently predicts the lowest reliability. Table 4-2 shows that the spell-length downscaling model consistently results in higher reliability than the Markov method. 

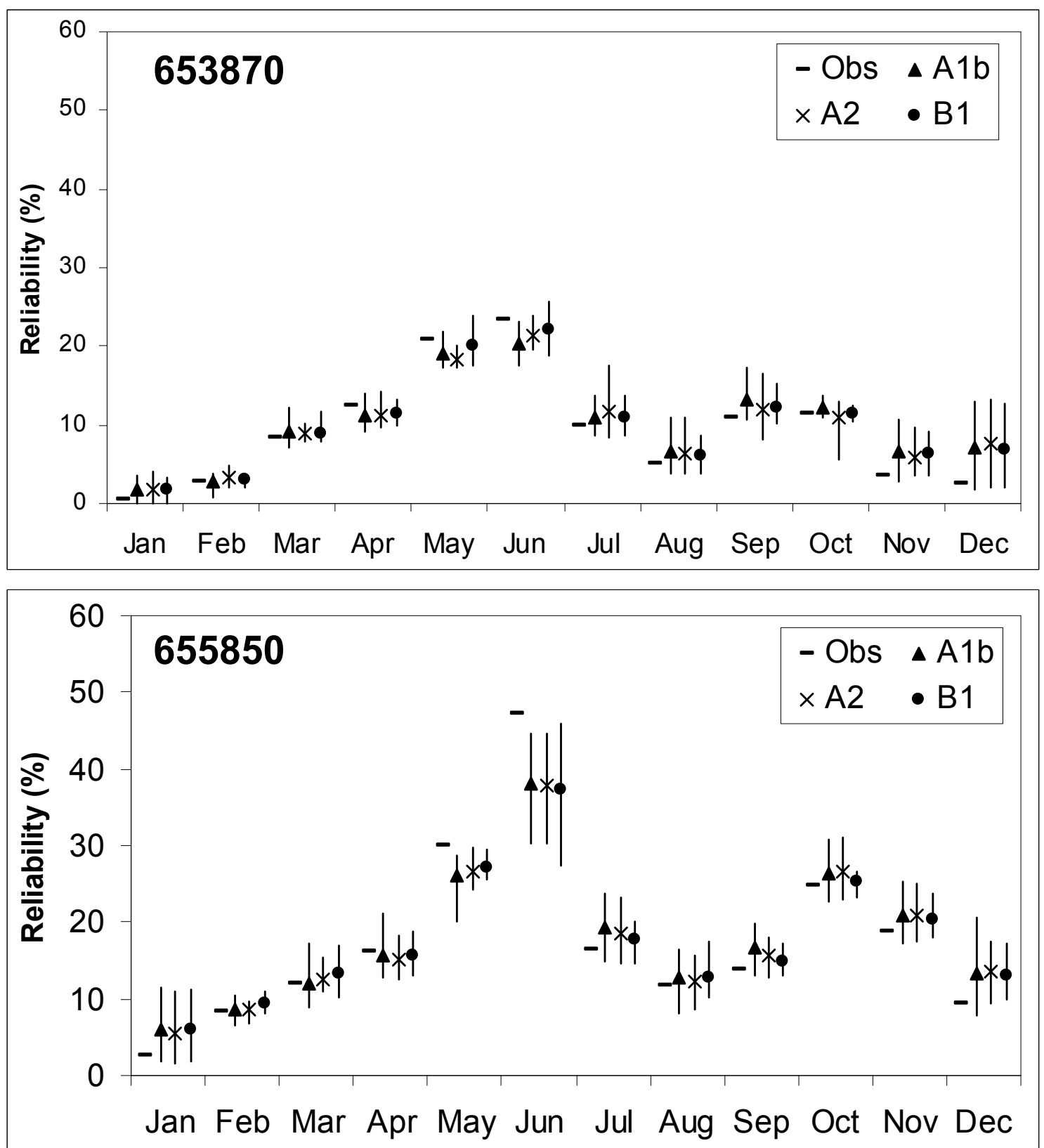

Figure 4-2: Climate Change Impact on 20 L/day Per Capita Reliability in the Coastal Region of West Africa. 

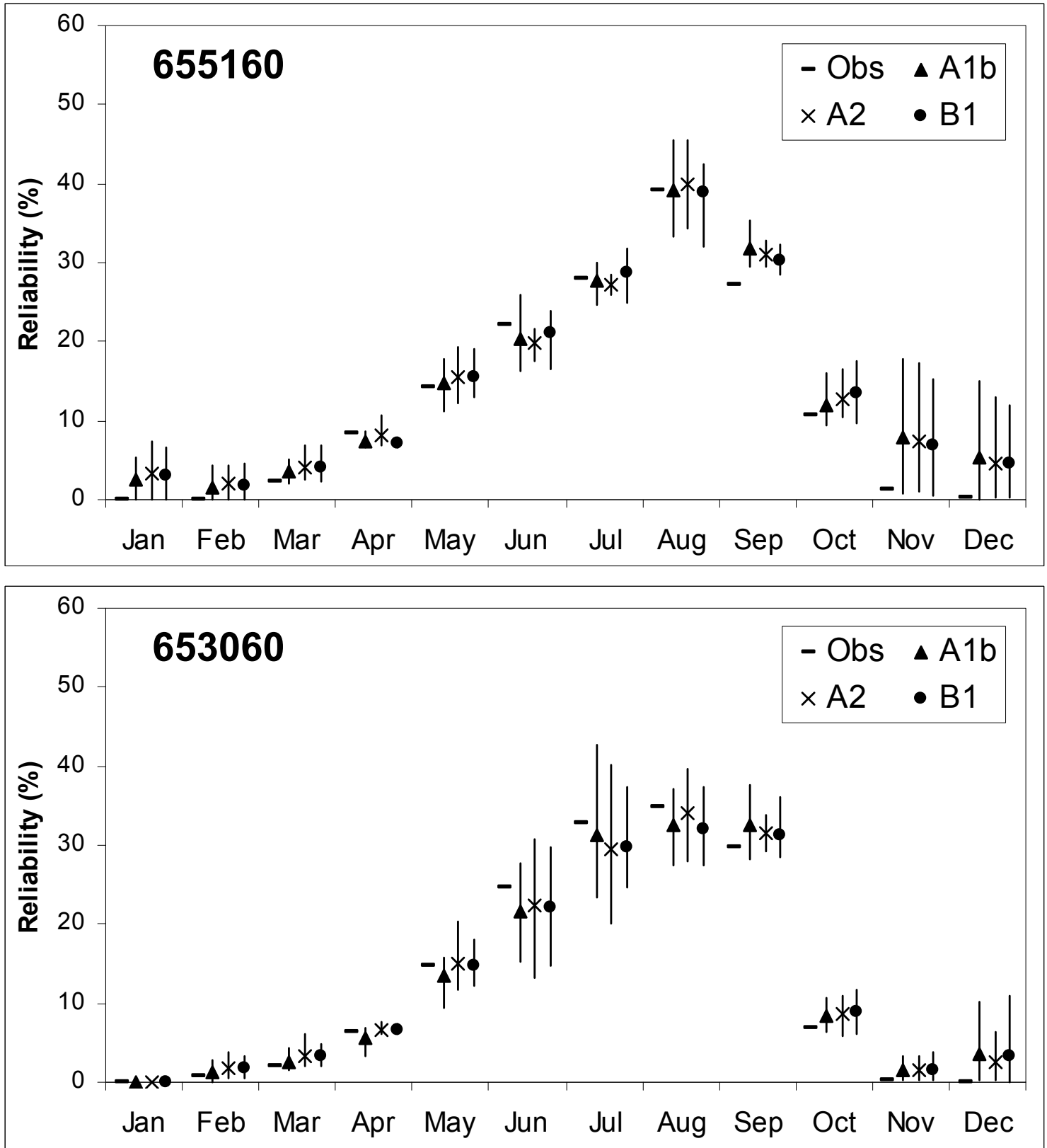

Figure 4-3: Climate Change Impact on 20 L/day Per Capita Reliability in the Inland (Sahel) Region of West Africa. 
The potential impact of climate change on DRWH associated with different roof surface areas can be seen in Figure 4-4 and Figure 4-5. Per capita roof areas are not large in most slum communities. A range of $2 \mathrm{~m}^{2}$ to $10 \mathrm{~m}^{2}$ was used in this study. In general, DWRH reliability improves with the smaller surface areas under the climate change scenarios, but worsens as surface area increases. These two figures show only the Alb scenario, but similar results are found for the A2 and B2 scenarios, though with slightly different change magnitudes. Again, multimodel results are shown with the shaded area representing the range associated with the differing GCMs and downscaling methods.

The results of each GCM and downscaling method are shown in Appendix K.

Table 4-1. The Percentage of Maximum and Minimum DRWH Reliability Values by General Circulation Model.

\begin{tabular}{|c|c|c|c|c|c|c|c|c|c|c|c|c|}
\hline \multicolumn{13}{|c|}{ Maximum Values } \\
\hline $\begin{array}{l}\text { Markov } \\
\text { Generator }\end{array}$ & $J$ & $F$ & $M$ & $A$ & $M$ & $J$ & $J$ & $A$ & $s$ & 0 & $N$ & $D$ \\
\hline CGCM3 & 20 & 34 & 37 & 19 & 33 & 46 & 35 & 16 & 29 & 19 & 10 & 54 \\
\hline MIROC & 38 & 10 & 38 & 4 & 20 & 6 & 8 & 33 & 10 & 52 & 72 & 22 \\
\hline MRI & 42 & 55 & 24 & 77 & 47 & 48 & 57 & 51 & 61 & 29 & 18 & 24 \\
\hline $\begin{array}{l}\text { Spell- } \\
\text { length } \\
\text { Generator }\end{array}$ & $J$ & $F$ & $M$ & $A$ & $M$ & $J$ & $J$ & $\boldsymbol{A}$ & $s$ & 0 & $N$ & $D$ \\
\hline CGCM3 & 38 & 67 & 70 & 49 & 63 & 39 & 51 & 27 & 28 & 20 & 22 & 54 \\
\hline MIROC & 50 & 12 & 8 & 2 & 10 & 26 & 8 & 20 & 12 & 40 & 48 & 38 \\
\hline MRI & 12 & 22 & 22 & 50 & 28 & 35 & 41 & 53 & 60 & 40 & 30 & 8 \\
\hline \multicolumn{13}{|c|}{ Minimum Values } \\
\hline $\begin{array}{l}\text { Markov } \\
\text { Generator }\end{array}$ & $J$ & $F$ & $M$ & $A$ & $M$ & $J$ & $J$ & $A$ & $s$ & 0 & $N$ & $D$ \\
\hline CGCM3 & 18 & 10 & 17 & 18 & 25 & 20 & 20 & 10 & 22 & 33 & 48 & 9 \\
\hline MIROC & 23 & 73 & 37 & 74 & 53 & 73 & 67 & 54 & 65 & 20 & 7 & 36 \\
\hline MRI & 58 & 17 & 46 & 8 & 22 & 7 & 13 & 36 & 13 & 47 & 45 & 55 \\
\hline $\begin{array}{l}\text { Spell- } \\
\text { length } \\
\text { Generator }\end{array}$ & $J$ & $F$ & $M$ & $A$ & $M$ & $J$ & $J$ & $A$ & $s$ & 0 & $N$ & $D$ \\
\hline CGCM3 & 14 & 7 & 2 & 3 & 10 & 28 & 17 & 28 & 25 & 42 & 46 & 19 \\
\hline MIROC & 14 & 73 & 74 & 91 & 66 & 51 & 67 & 61 & 59 & 28 & 22 & 17 \\
\hline MRI & 73 & 20 & 24 & 6 & 24 & 21 & 16 & 10 & 16 & 30 & 32 & 65 \\
\hline
\end{tabular}


Table 4-2. The Percentage of Maximum and Minimum DRWH Reliability Values by Downscaling Method and General Circulation Model.

\begin{tabular}{|c|c|c|c|c|c|c|c|c|c|c|c|c|}
\hline \multicolumn{13}{|c|}{ Maximum Values } \\
\hline CGCM3 & $J$ & $F$ & $M$ & $A$ & $M$ & $J$ & $J$ & $A$ & $S$ & 0 & $N$ & $D$ \\
\hline $\begin{array}{l}\text { Markov } \\
\text { Gener- } \\
\text { ator }\end{array}$ & 0 & 2 & 3 & 10 & 11 & 42 & 17 & 44 & 40 & 34 & 5 & 0 \\
\hline $\begin{array}{l}\text { Spell- } \\
\text { length } \\
\text { Gener- } \\
\text { ator }\end{array}$ & 100 & 98 & 97 & 90 & 89 & 58 & 83 & 56 & 60 & 66 & 95 & 100 \\
\hline MIROC & $J$ & $\boldsymbol{F}$ & $M$ & $A$ & $M$ & $J$ & $J$ & $A$ & $S$ & 0 & $N$ & $D$ \\
\hline $\begin{array}{l}\text { Markov } \\
\text { Gener- } \\
\text { ator }\end{array}$ & 0 & 3 & 21 & 38 & 38 & 45 & 37 & 46 & 41 & 30 & 3 & 0 \\
\hline $\begin{array}{l}\text { Spell- } \\
\text { length } \\
\text { Gener- } \\
\text { ator }\end{array}$ & 100 & 97 & 79 & 62 & 62 & 55 & 63 & 54 & 59 & 70 & 97 & 100 \\
\hline MRI & $J$ & $\boldsymbol{F}$ & $M$ & $A$ & $M$ & $J$ & $J$ & $A$ & $S$ & 0 & $\boldsymbol{N}$ & $D$ \\
\hline $\begin{array}{l}\text { Markov } \\
\text { Gener- } \\
\text { ator }\end{array}$ & 0 & 4 & 8 & 25 & 35 & 50 & 27 & 37 & 29 & 29 & 4 & 1 \\
\hline $\begin{array}{l}\text { Spell- } \\
\text { length } \\
\text { Gener- } \\
\text { ator }\end{array}$ & 100 & 96 & 92 & 75 & 65 & 50 & 73 & 63 & 71 & 71 & 96 & 99 \\
\hline
\end{tabular}




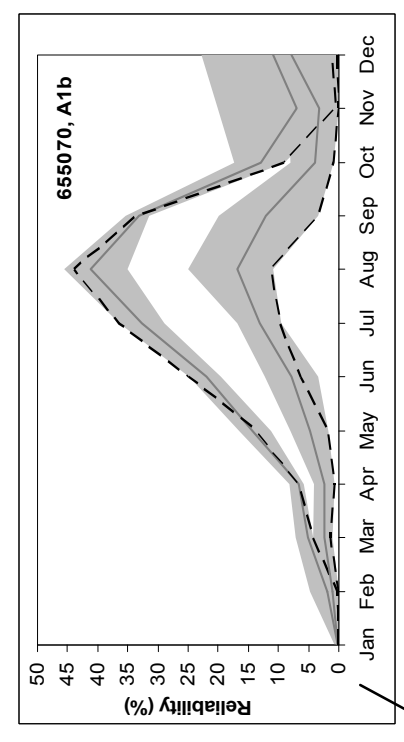
(\%) Kఛ!!!qu!|ә

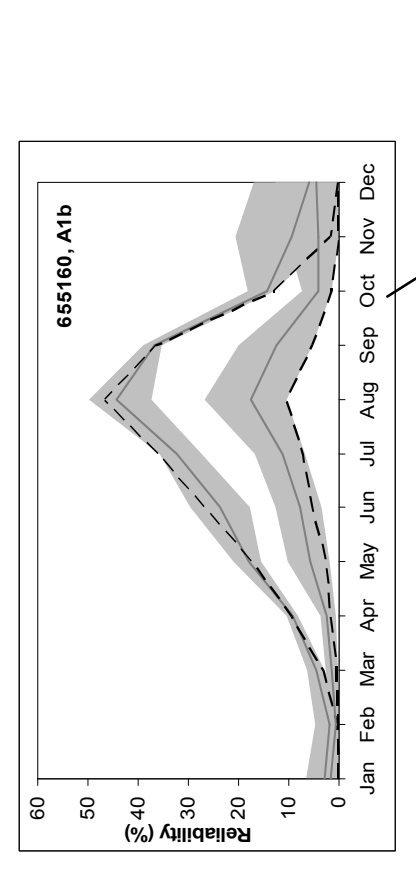

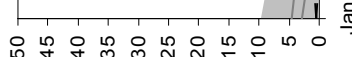

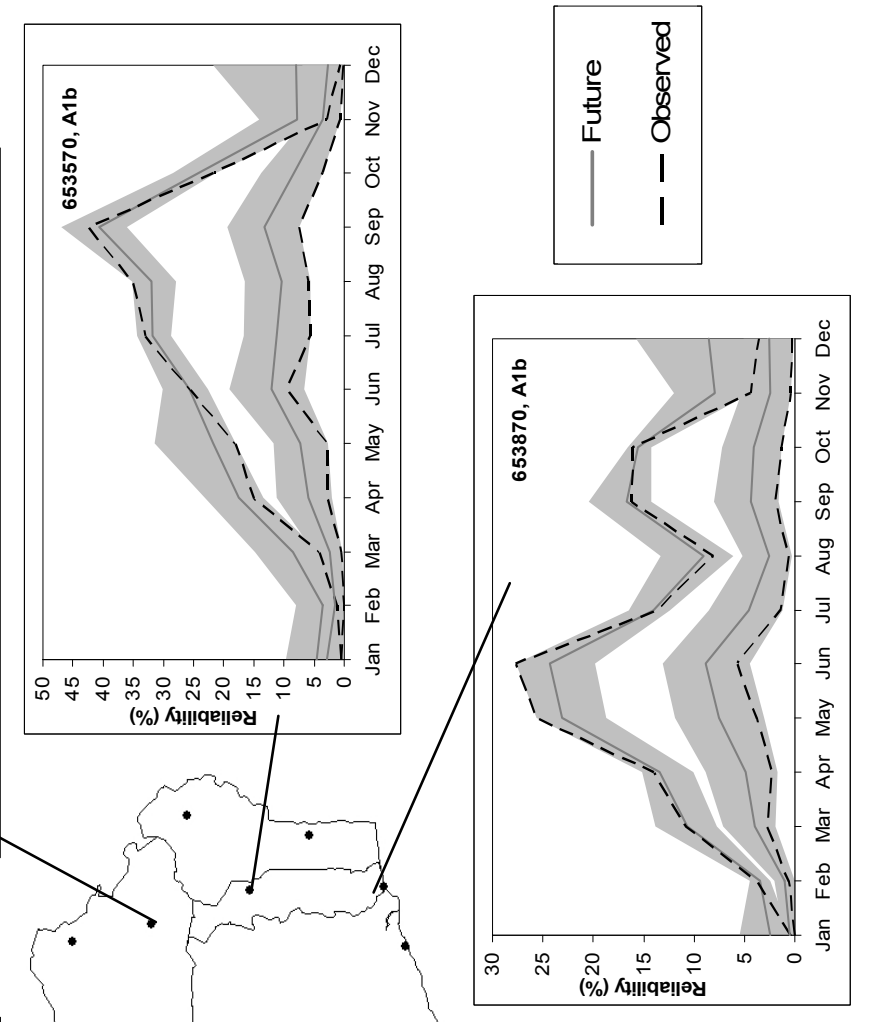

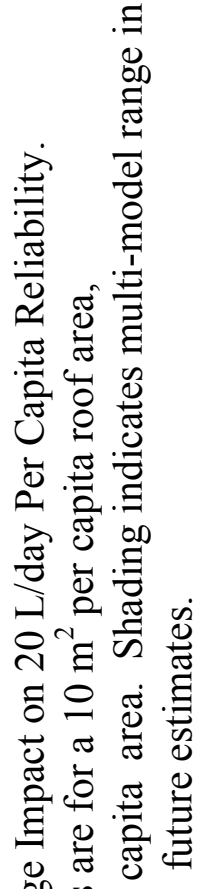

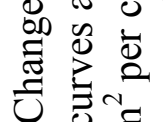

䒕 N
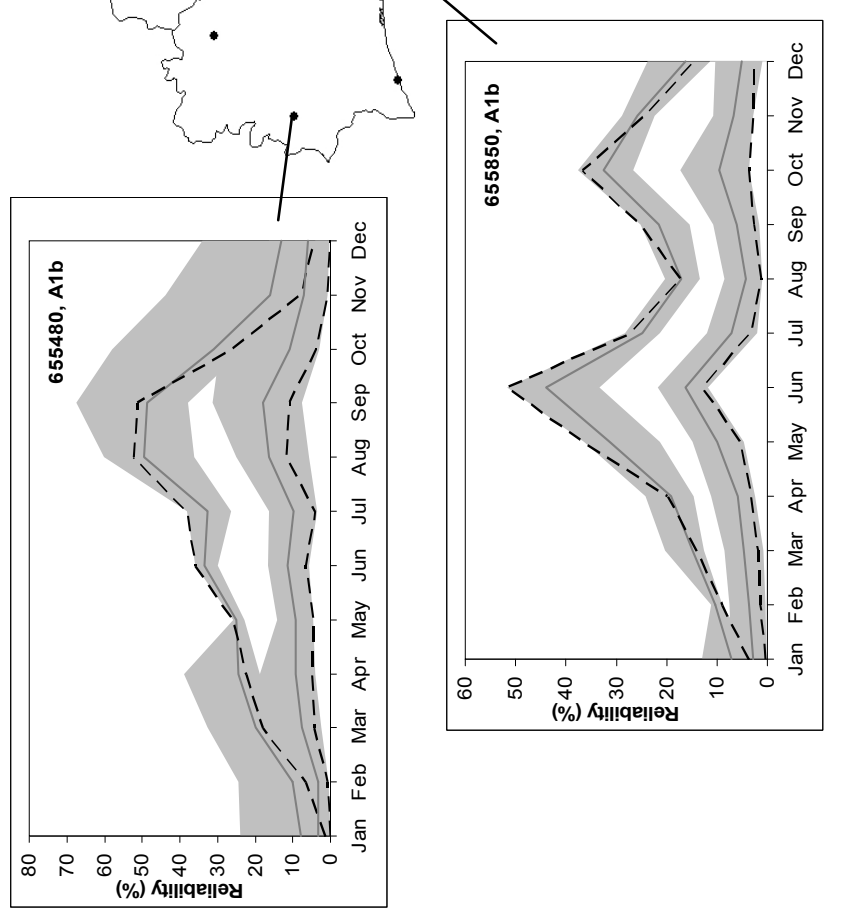

. 


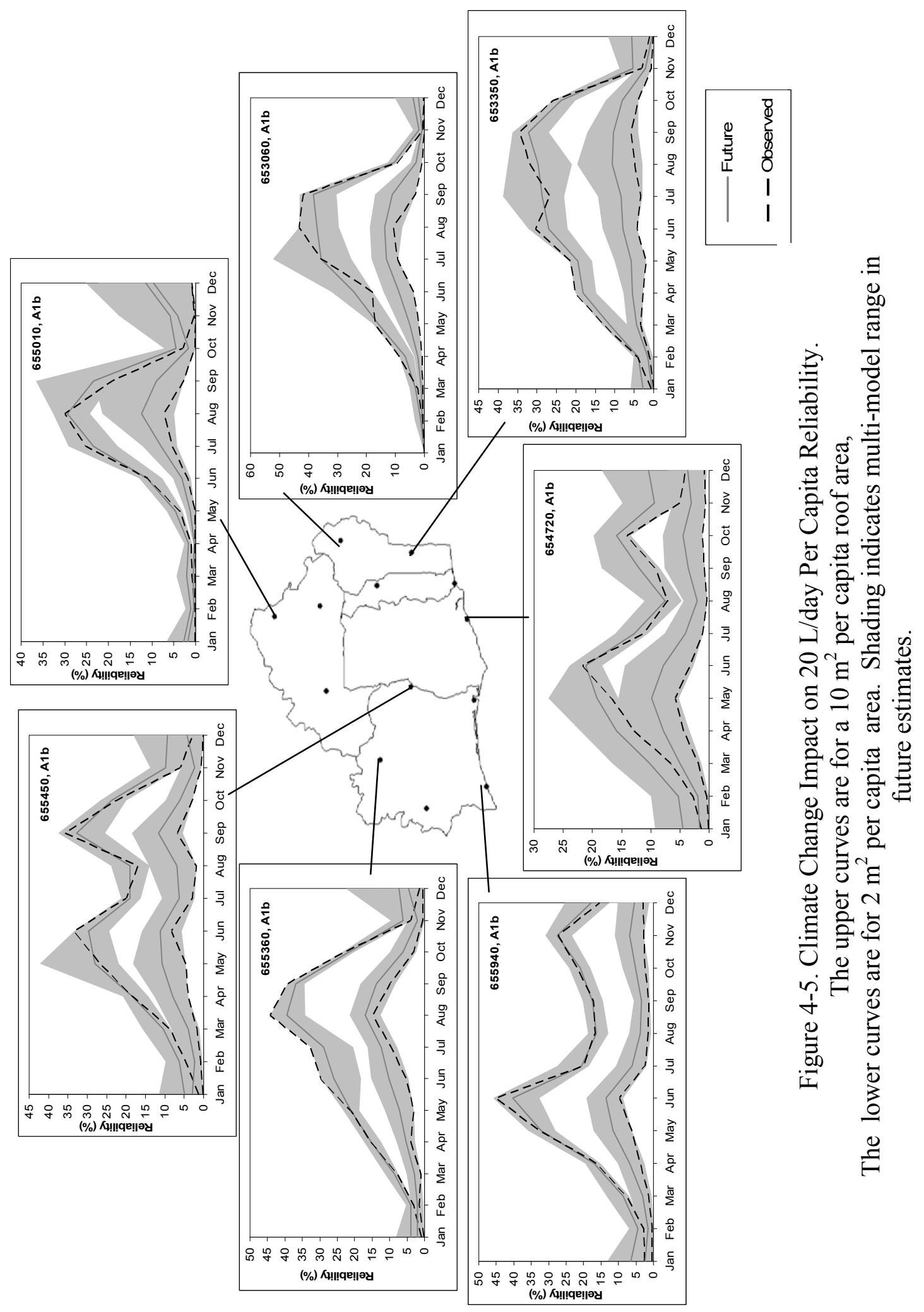




\subsection{Discussion}

Based on these results, climate change is expected to have little impact on domestic rainwater harvesting reliability in West Africa by the mid- $21^{\text {st }}$ century, with the slight temporal shift in rainfall patterns causing small increases or decreases in reliability at the larger per capita roof areas. The differences between the emission scenarios are also small. Both the averages and value ranges are similar across most months and gauges for the three SRES scenarios. The only substantial differences come in the extremely wet months, where climate change may drop DRWH reliability by 5 to 10 percent. This seems especially pronounced in the coastal areas with bimodal distributions of seasonal rainfall. These small changes in precipitation are consistent with IPCC observations, which found small changes in precipitation in the Guinean Coast Region (Christensen et al., 2007).

The differences between the GCMs found in this study are consistent with the Cook and Vizy (2006) results, which looked at GCM performance in the West African region. They found the MIROC model to produce strong warming in the Gulf of Guinea, which stopped the northward movement of the monsoon in the summer months. This large decrease in precipitation results in the minimum DRWH reliability values found here. The MRI model was found to have a more moderate sea surface temperature rise, which resulted in a wetter Guinea Coast in the later part of the $21^{\text {st }}$ century. The larger DRWH reliability values associated with MRI are consistent with these findings.

Climate change impacts on DRWH systems using the smaller $2 \mathrm{~m}^{2}$ per capita roof areas appear to me more beneficial than larger roof areas. Across most gauges and months there are improvements in reliability for these small roof areas. Using the 
DRWH equation mentioned previously, a $10 \mathrm{~m}^{2}$ roof needs a $2.5 \mathrm{~mm}$ storm to produce 20 L of water. A $2 \mathrm{~m}^{2}$ roof needs $12.5 \mathrm{~mm}$ of precipitation. The DRWH reliability results show how under the SRES climate scenarios, larger storms will increase in frequency in the region, whereas smaller storms will decrease in frequency. These results suggest that community improvements toward implementing DRWH system should be focused on increasing water storage. The larger storms need to be captured and stored for later use. Simply enlarging the catchment's surface area will not be adequate. Indeed, during several months of the year there is little difference in reliability between the two roof sizes.

The results of this study, though not showing any large increase in benefits under climate change, still offer up DRWH as a potential adaptation measure for other impacts. Sea level rises are expected to drive millions of West African inland, creating an ecological refugee crisis. Slum communities could emerge faster than infrastructure would be able to provide for them. The UN's Intergovernmental Panel on Climate Change has stated that,

"[A]daptation strategies [in developing countries] should be designed in the context of development, environment and health policies. Many of the options that can be used to reduce future vulnerability are of value in adapting to current climate and can be used to achieve other environmental and social objectives." (Bates et al., 2008)

Investments in DRWH technologies now could achieve these development, health, and social objectives, and buffer potential impacts due to population shifts in the future. Households can have assurance that their drinking water provided by these systems will not change drastically. This is not to say DRWH system can provide all water needs. The results of this study and Cowden et al. (2008) show that DRWH can only be used to 
enhance drinking supplies. This water enhancement, however, is expected to be stable in an otherwise uncertain future, based on GCM simulations.

Several sources of uncertainty exist within statistical downscaling methods, many of which originate in the GCM itself. One large source of uncertainty is the greenhouse gas forcings associated with future emission levels. Variables such as population growth, energy demand, and technology innovations inhibit a confident prediction of future greenhouse gas levels, resulting in the varied climate change scenarios in the current literature. The conversion of these future emissions to atmospheric concentrations and their potential radiative effects are also uncertain (IPCC, 2001).

The GCM's skill also contributes to uncertainty in downscaling results. With poor simulation of current climates and inadequate understanding or resolution of several feedback mechanisms and local forcings, including clouds and land-atmosphere interactions, GCM output will continue to create uncertainty in downscaled local climate variables. Another source of uncertainty in downscaling methods lies within the methods themselves. Using statistical methods and parameter estimation with several assumptions highlights the inadequate knowledge and representation of the physical precipitation processes. Numerical approximation techniques, as well as inadequate observational records due to measurement errors or gauge network sparseness, contribute to this uncertainty in the downscaling model (IPCC, 2001).

Ongoing and needed work in this field is plentiful. The most pressing tasks will be to update these results as more accurate climate and model data become available that lessens the aforementioned uncertainties. Although not within the scope of this work, improving data collection and modeling methods to better represent the precipitation 
mechanisms in this region will greatly improve these results. There is a great dearth of climate impact studies using downscaling or regional climate models on the African continent. The reasons and challenges behind this are well recognized, but they must be surmounted to enable planning and action in a region that is least able to adapt to potential global changes. 


\section{Chapter 5 Findings and Recommendations for Future Work}

\subsection{Urban Slums and Urban Metabolism}

A city's metabolism is defined as the sum of urban processes (technological and societal) that provide sustenance, maintenance, growth and innovation in cities, as measured through stocks and flows of energy and materials. A knowledge and manipulation of an urban metabolism can be instrumental in improving urban slum conditions and help developing cities avoid the chaotic and impoverished growth many now face. Table 5-1 outlines nine lessons obtained from urban metabolism studies in developed nations, and provides methods, tools, and conclusions applicable to developing countries. By increasing the knowledge of the urban system we hope to improve, humans will be more adequately prepared to address rising slum issues and progress towards other Millennium Development Goals. Smarter and sustainable economic development and city management can only improve efforts to decrease poverty, disease, inequality, etc. As more and more studies are made, greater material and energy efficiency will be possible with appropriate policy implementation. DRWH could contribute to this efficiency and should be a target of such policies. 
Table 5-1: Urban Metabolism Lessons for Developing Countries

\begin{tabular}{|c|c|c|}
\hline Lesson & $\begin{array}{l}\text { Applicable UN- } \\
\text { HABITAT Slum } \\
\text { Strategy }\end{array}$ & Details \\
\hline Integrate Models & $\begin{array}{l}\text { Urban Development, } \\
\text { Regional Development }\end{array}$ & $\begin{array}{l}\text { Use an integrated framework when } \\
\text { studying the metabolisms and } \\
\text { socioeconomics of urban systems. }\end{array}$ \\
\hline $\begin{array}{l}\text { Include the Whole } \\
\text { Urbanized Region in } \\
\text { Specifying } \\
\text { Metabolism } \\
\text { Boundaries }\end{array}$ & Urban Development & $\begin{array}{l}\text { Include smaller peri-urban systems in } \\
\text { metabolism analysis by using urban } \\
\text { conditions or indicators as guides for } \\
\text { policy, not just political boundaries. }\end{array}$ \\
\hline Define Efficiency & $\begin{array}{l}\text { Urban Development, } \\
\text { Regional Development }\end{array}$ & $\begin{array}{l}\text { Establish comprehensive, accurate, and } \\
\text { efficient goals/indicators for use with } \\
\text { metabolic data. }\end{array}$ \\
\hline $\begin{array}{l}\text { Understand the } \\
\text { Mechanisms Within } \\
\text { "Black Box" } \\
\text { Processes }\end{array}$ & $\begin{array}{l}\text { Slum upgrading, Urban } \\
\text { Development }\end{array}$ & $\begin{array}{l}\text { Gather and analyze detailed process } \\
\text { information in order to improve resource } \\
\text { management and appropriate technology } \\
\text { implementation. }\end{array}$ \\
\hline $\begin{array}{l}\text { Conduct Metabolism } \\
\text { Checkups }\end{array}$ & Urban Development & $\begin{array}{l}\text { Continue to monitor a city's metabolism } \\
\text { to understand the long-term needs and } \\
\text { impacts of slums, allowing decision } \\
\text { makers to find, solve, and/or prevent } \\
\text { problems. }\end{array}$ \\
\hline $\begin{array}{l}\text { Mitigate Solid and } \\
\text { Hazardous Waste } \\
\text { Outputs }\end{array}$ & $\begin{array}{l}\text { Slum Upgrading, Urban } \\
\text { Development, Regional } \\
\text { Development }\end{array}$ & $\begin{array}{l}\text { Create and enforce sound policy in } \\
\text { material use reduction, recycling, } \\
\text { treatment, and disposal based on } \\
\text { metabolism knowledge. }\end{array}$ \\
\hline $\begin{array}{l}\text { Manage Water and } \\
\text { Wastewater Flows }\end{array}$ & $\begin{array}{l}\text { Slum Upgrading, Urban } \\
\text { Development, Regional } \\
\text { Development }\end{array}$ & $\begin{array}{l}\text { Understand and manage the water and } \\
\text { nutrient balances of both city and slum } \\
\text { dwellings without negating } \\
\text { water/sanitation access through damaging } \\
\text { surrounding ecosystems. }\end{array}$ \\
\hline $\begin{array}{l}\text { Extend Metabolism } \\
\text { Parameters to } \\
\text { Measure Quality of } \\
\text { Life }\end{array}$ & Slum Upgrading & $\begin{array}{l}\text { Find the right balance between livability } \\
\text { and metabolism indicators to improve the } \\
\text { lives of slum dwellers without necessarily } \\
\text { increasing consumption. }\end{array}$ \\
\hline $\begin{array}{l}\text { Pay Attention to } \\
\text { "Change } \\
\text { in Storage" }\end{array}$ & $\begin{array}{l}\text { Urban Development, } \\
\text { Slum Upgrading }\end{array}$ & $\begin{array}{l}\text { Understand imbalances and } \\
\text { accumulations of materials and waste } \\
\text { within the urban system to prevent future } \\
\text { problems and to utilize potential } \\
\text { resources. }\end{array}$ \\
\hline
\end{tabular}




\subsection{Stochastic Weather Models and Domestic Rainwater Harvesting}

DRWH holds great promise for enhancing water supplies in urban slum areas in West Africa, and mitigating climate change impacts. However, DRWH policies need data for development, and sufficient and accurate data is a common problem in developing world meteorological records. To address this problem, parsimonious stochastic rainfall models are developed to produce long, continuous synthetic weather sequences that are statistically similar to the available observations.

The stochastic rainfall modeling outlined in Chapter 3 indicates that the $1^{\text {st }}$-order Markov model outperforms the zero-order model in reproducing the variability of the rainfall characteristics, including DRWH reliability, examined in this study. The wet days per month are especially critical to small-storage DRWH systems dependent on frequent occurrence of rainfall. For this reason a $1^{\text {st }}$-order Markov model is also recommended for DRHW assessment in the West African region. The mixed exponential distribution better matches the observed monthly total variability and is therefore recommended for rainfall amounts in stochastic rainfall models used in this region and for DRWH application.

To account for the uncertainty in these stochastic rainfall models, a multi-model approach was used in assessing the capabilities of DRWH systems in providing improved water supply reliability (Figure 5-1). DRHW systems were found to be able to provide improved water supplies through much of the year in West Africa, although alternative sources are needed due to extensive dry spells. If little to no storage is considered, the Markov stochastic rainfall models are of use as they adequately reproduce mean monthly values (e.g. when rainfall exceeds a threshold to allow for collection of $>20 \mathrm{~L}$ per capita 
per day). As storage volumes increase, however, and households in the developing world attempt to correctly size their tanks for perhaps larger consumptive demands, an accurate representation of spell lengths becomes more important. The LARS-WG stochastic model has a clear advantage in that it almost perfectly matches the observed spell length characteristics.
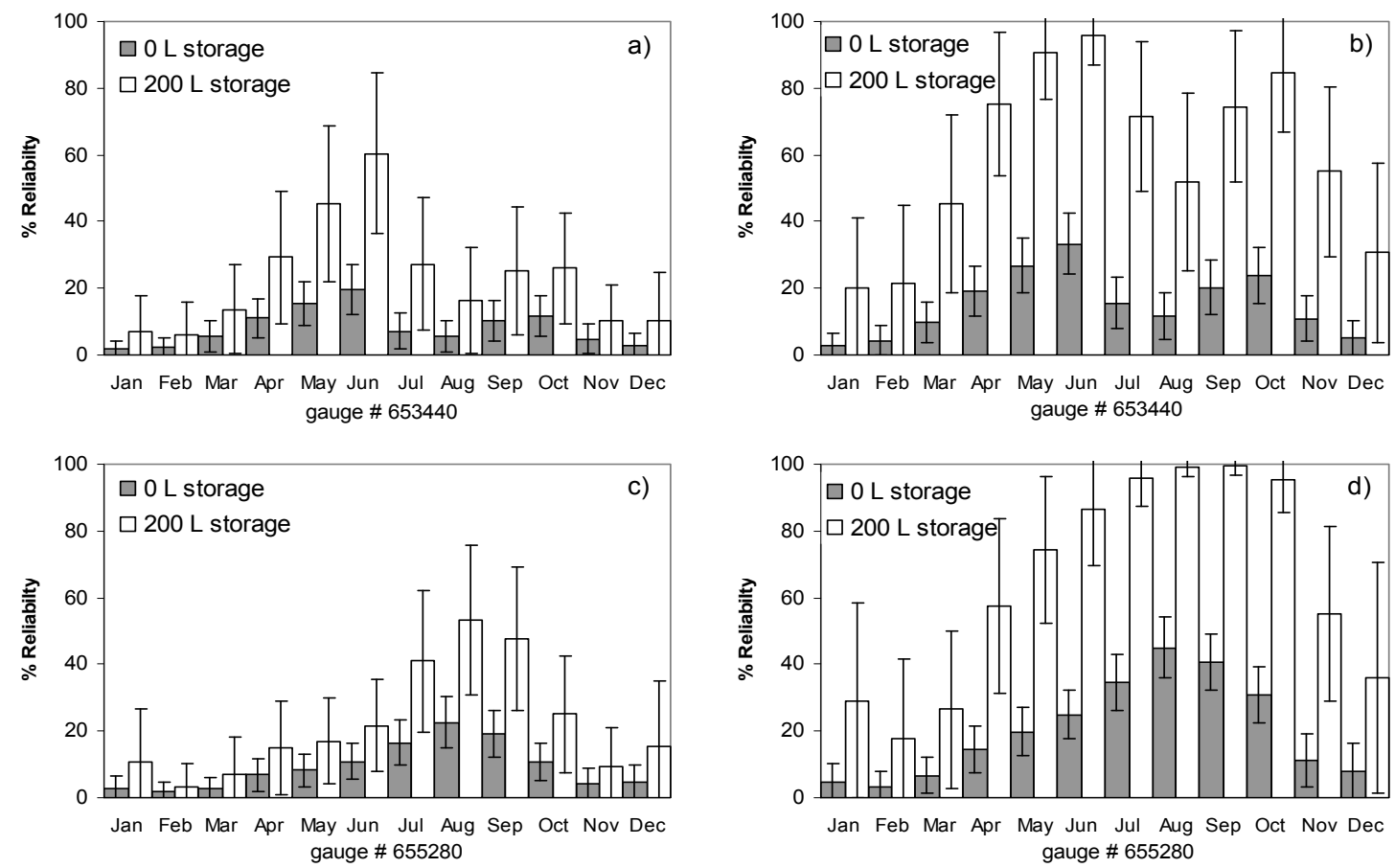

Figure 5-1. Multi-model DRWH reliability for a $2 \mathrm{~m}^{2}$ per capita roof area for gauges a) 653440 and c) 655280 , and for a $10 \mathrm{~m}^{2}$ per capita roof area for gauges b) 653440 and d) 655280. Models used are LARS-WG and $1^{\text {st }}$ order Markov.

\subsection{Climate Change Impact on Domestic Rainwater Harvesting}

Based on the output of three GCMs, climate change is expected to have little impact on DRWH reliability in West Africa by the mid- $21^{\text {st }}$ century, with the slight temporal shift in rainfall patterns causing small increases or decreases in reliability at the larger per capita roof areas (Figure 5-2). The differences between the SRES emission 
scenarios are also small. These small changes in precipitation are consistent with IPCC observations, which found small changes in precipitation in the Guinean Coast Region (Christensen et al., 2007). Developing communities in this region can invest with confidence in DRWH systems for drinking water enhancement.

Climate change impacts on DRWH systems using $2 \mathrm{~m}^{2}$ per capita roof areas appear to me more beneficial than larger roof areas (Figure 5-2). This indicates a potential change in precipitation frequency and amounts in the region due to climate change. The DRWH reliability results show how under the SRES climate scenarios, larger storms will increase in frequency in the region, whereas smaller storms will decrease in frequency. These results suggest that community improvements toward implementing DRWH systems should be focused on increasing water storage. The larger storms need to be captured and stored for later use. Simply enlarging the catchment's surface area will not be adequate. 


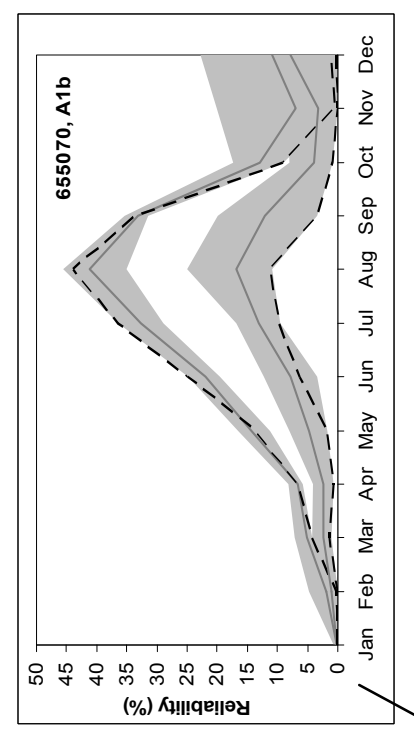

은 (\%) Ky!!!qu!|әy

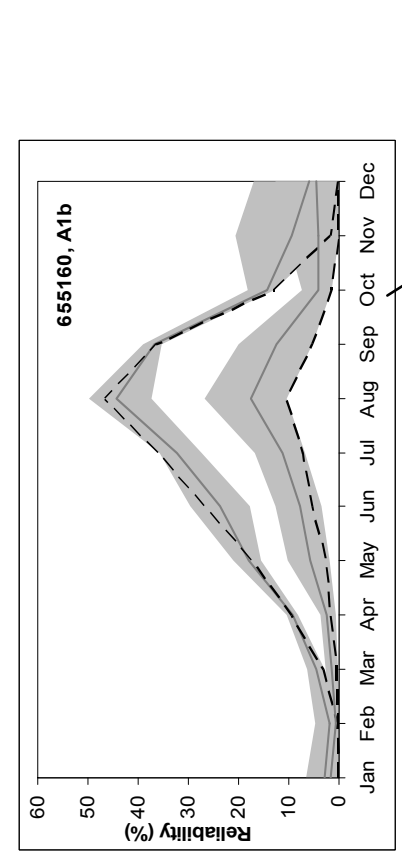




\subsection{Future Work}

Future work could include a more complete assessment of DRWH over a range of catchment areas, as well as storage size optimization incorporating direct costs and health impacts. In accessing health impacts, additional consumption levels, such as 50/L and 100/L per day, could also be investigated. These readily available stochastic rainfall models are easily applicable to gauges in other developing regions where cost, training, or data limitations may prohibit the use of more complicated methods.

Ongoing and needed work for the climate change impact assessment is plentiful. The IPCC has stated that climate change and water research is needed in developing nations, and research involving the socioeconomic dimension would be especially helpful (Bates, 2008). Ongoing work includes utilizing a third downscaling method, which is a hybrid regression-stochastic model. Regression downscaling requires identifying observed large-scale predictors that are well correlated with the local predictand and then fitting the regression equations to the relationship. The regression equations are then used to downscale predictand parameters using GCM predictor variables. The Statistical Downscaling Model (SDSM) software will be used for these steps. Future work also includes using a fourth GCM, the GFDL-CM2.0, to better represent GCM variability in precipitation performance.

Another pressing task regarding this study will be to update these results as more accurate climate and model data become available. Although not within the scope of this work, improving data collection and modeling methods to better represent the precipitation mechanisms in this region will greatly improve these results. There is a great dearth of climate impact studies on the African continent, both in downscaling and 
regional climate studies. The reasons and challenges behind this are well recognized, but they must be surmounted to enable planning and action in a region that is least able to adapt to potential global changes.

Significant uncertainties also continue to exist in relevant DRWH costs, water use, and health data. Data collection for estimation of these parameters would also greatly improve these results and would allow a robust quantification of the health impacts of improved water supplies. Further sensitivity analyses of cost and health data could also provide an indication of cost-effectiveness. This could be accomplished by connecting water enhancement via DRWH to health metrics, such as relative risk and disability adjusted life years (DALY). Finally, in addition to these potential areas of study, there is also a need to develop relatively simple and transparent assessment methods for informing policy makers. 


\section{Chapter 6 References}

Abu-Rizaiza, Q.S. 1999. Threats from ground water table rise in urban areas in developing countries. Water International 24(1): 46-52.

Alberti, M. 1996. Measuring urban sustainability. Environmental Impact Assessment Review 16: 381-424.

Anderson, W., Kanaroglou, P., \& Miller, E. 1996. Urban form, energy, and the environment: A review of issues, evidence and policy. Urban Studies 33(1):7-35.

Azar, C., Holmberg, J., and Lindgren, K. 1996. Socio-ecological Indicators for Sustainability. Ecological Economics 18:89-112.

Baccini, P. \& Brunner, P. 1991. Metabolism of the Anthroposphere. Springer-Verlag, New York.

Bai, X. 2002. Industrial relocation in Asia: A sound environmental management strategy? Environment 44(5):8-21.

Balas N., Nicholson, S.E., and Klotter, D., 2007. The relationship of rainfall variability in West Central Africa to sea-surface temperature fluctuations. International Journal of Climatology 27(10), 1335-1349.

Bates, B.C., Kundzewicz, Z.W., Wu, S. and Palutikof, J.P. (Eds.). 2008. Climate change and water. Technical Paper of the Intergovernmental Panel on Climate Change. IPCC Secretariat. Geneva, $210 \mathrm{pp}$.

Bohle, H.G. 1994. Metropolitan food systems in developing countries: The perspective of urban metabolism. GeoJournal 34(3):245-251. 
Boko, M., Niang, I., Nyong, A., Vogel, C., Githeko, A., Medany, M., Osman-Elasha, B., Tabo, R., and Yanda, P. 2007. Africa. In: Climate Change 2007: Impacts, Adaptation and Vulnerability. Contribution of Working Group II to the Fourth Assessment Report of the Intergovernmental Panel on Climate Change, M.L. Parry, O.F. Canziani, J.P. Palutikof, P.J. van der Linden and C.E. Hanson, Eds., Cambridge University Press, Cambridge UK, 433-467.

Brunner, P.H. and H. Rechberger. 2001. Anthropogenic metabolism and environmental legacies, in Encyclopedia of Global Environmental Change, Vol. 3 (ed Munn., T.) UK: John Willey \& Sons.

Christensen, J.H., Hewitson, B., Busuioc, A., Chen, A., Gao, X., Held, I., Jones, R., Kolli, R.K., Kwon, W.-T., Laprise, R., Magaña Rueda, V., Mearns, L., Menéndez, C.G., Räisänen, J., Rinke, A., Sarr A., and Whetton, P. 2007. Regional Climate Projections. In: Climate Change 2007: The Physical Science Basis. Contribution of Working Group I to the Fourth Assessment Report of the Intergovernmental Panel on Climate Change [Solomon, S., D. Qin, M. Manning, Z. Chen, M. Marquis, K.B. Averyt, M. Tignor and H.L. Miller (eds.)]. Cambridge University Press, Cambridge, United Kingdom and New York, NY, USA.

Climate Prediction Center (CPC). 2007. Oceanic Nino Index. Available at http://www.cpc.noaa.gov.

Cohen, B. 2004. Urban growth in developing countries: A review of current trends and a caution regarding existing forecasts. World Development 32(1):23:51. 
Cook, K.H. and Vizy, E.K. 2006. Coupled model simulations of the West African monsoon system: Twentieth- and twenty-first-century simulations. Journal of Climate 19, 3681-3702.

Cowden, J.R, Mihelcic, J.R., and Watkins, D.W. 2006. Domestic rainwater harvesting assessment to improve water supply and health in Africa's urban slums. Proc. 2006 World Environmental \& Water Resources Congress, Omaha, NE May 21$25,2006$.

Cowden, J.R, Watkins, D.W., and Mihelcic, J.R. 2008. Stochastic rainfall modeling in West Africa: Parsimonious approaches for domestic rainwater harvesting assessment. Journal of Hydrology 361(1-2), 64-77.

Decker, E. H., Elliott, S., Smith, F. A., Blake, D. R., and Rowland, F. S. 2000. Energy and material flow though the urban ecosystem. Annual Review of Energy Environment 25: 685-740.

Ezcurra, E. and Mazari-Hiriart, M. 1996. Are mega cities viable? A cautionary tale from Mexico City. Environment 38: 6-8.

Falkenmark, M., Lundqvist, J., and Widstrand, C. 1989. Macro-scale water scarcity requires micro-scale approaches. Natural Resources Forum 13, 258-267. Foxon, T., Leach, M., Butler, D., Dawess, J., Hutchinson, D., Pearson, P., and Rose, D. 1999. Useful indicators of urban sustainability: Some methodological issues. Local Environment 4(2):137-149.

Fung, M. and Kennedy, C.A. 2005. An integrated macroeconomic model for assessing urban sustainability. Environment and Planning B 32(5), 639-656. 
Garbutt, D.J., Stern, R.D., Dennett, M.D., and Elston, J. 1981. A comparison of the rainfall climate of eleven places in West Africa using a two-part model for daily rainfall. Archiv fur Meteorologie, Geophysik, und Bioclimatologie Ser. B 29, $137-155$.

Giampietro, M., Mayumi, K., \& Bukkens, S. 2001. Multiple-Scale integrated assessment of societal metabolism: An analytical tool to study development and sustainability. Environment, Development, and Sustainability 3:275-307.

Gumbo, B., 1998. Rain water harvesting in the urban environment: Options for water conservation and environmental protection in Harare. Proc. National Conference, Masvingo, Zimbabwe, Oct. 13-16, 1998.

Handia, L., Tembo, J.M., and Mwiindwa, C. 2002. Potential of rainwater harvesting in urban Zambia. Proc. 3rd WaterNet/Warfsa Symposium, Dar es Salaam, Tanzania, Oct. 30-31, 2002.

Hendriks, C., Obernosterer, R., Müller, D., Kytzia, S., Baccini, P., and Brunner, P. H. 2000. Material flow analysis: a tool to support environmental policy decision making. Case-studies on the city of Vienna and the Swiss lowlands. Local Environment 5(3): 311-328.

Howard, G. and Bartram, J. 2003. Domestic water quantity, service level, and health. World Health Organization, Geneva, Switzerland.

Huang, S. L. \& Hsu, W. L. 2003. Materials flow analysis and emergy evaluation of Taipei’s urban construction. Landscape and Urban Planning 63(2):61-74. 
IPCC. 2001. Third Assessment Report, Climate Change 2001. Cambridge University Press, London.

Jackson, I.J., 1981. Dependence of wet and dry days in the Tropics. Archiv fur Meteorologie, Geophysik, und Bioclimatologie Ser. B. 29, 167-179.

Jimoh, O.D. and Webster, P. 1996. Optimum order of Markov chain for daily rainfall in Nigeria. Journal of Hydrology 185, 45-69.

Jimoh, O.D. and Webster, P. 1999. Stochastic modelling daily rainfall in Nigeria, intraannual variation of model parameters. Journal of Hydrology 222, 1-17.

Jones, P.G. and Thornton, P.K. 2000. MarkSim: Software to generate daily weather data for Latin America and Africa. Agronomy Journal 92, 445-453.

Katz, R.W. and Parlange, M.B. 1998. Overdispersion phenomenon in stochastic modeling of precipitation. Journal of Climate 11, 591-601.

Kennedy, C.A., Cuddihy, J., and Engel Yan, J. 2007. The changing metabolism of cities. J. Industrial Ecology 11(2), 43-59.

Lawless, C. and Semenov, M.A. 2005. Assessing lead-time for predicting wheat growth using a crop simulation model. Agricultural and Forest Meteorology 135, 302313.

Le Barbe, L., Lebel, T., and Tapsoba, D. 2002. Rainfall variability in West Africa during the years 1950-90. Journal of Climate 15(2), 187-202.

Loucks, D.P., van Beek, E., Stedinger, J.R., Dijkman, J.P.M., and Villars, M.T. 2005. Water Resources Systems Planning and Management: An Introduction to Methods, Models and Applications. UNESCO, Paris. 
Matos, G. \& Wagner, L. 1998. Consumption of materials in the United States: 19001995. Annual Review of Energy and Environment 23:107-122.

Meehl, G.A., Stocker, T.F., Collins, W.D., Friedlingstein, P., Gaye, A.T., Gregory, J.M., Kitoh, A., Knutti, R., Murphy, J.M., Noda, A., Raper, S.C.B., Watterson, I.G., Weaver, A.J., and Zhao, Z.-C. 2007. Global Climate Projections. In: Climate Change 2007: The Physical Science Basis. Contribution of Working Group I to the Fourth Assessment Report of the Intergovernmental Panel on Climate Change [Solomon, S., D. Qin, M. Manning, Z. Chen, M. Marquis, K.B. Averyt, M. Tignor and H.L. Miller (eds.)]. Cambridge University Press, Cambridge, United Kingdom and New York, NY, USA.

Mehrotra, R., Srikanthan, R., and Sharma A. 2006. A comparison of three stochastic multi-site precipitation occurrence generators. Journal of Hydrology 331, 280292.

Mihelcic, J.R., Zimmerman, J.B., and Ramaswami, A. 2007. Integrating developed and developing world knowledge into global discussions and strategies for sustainability.1.Science and technology. Environmental Science \& Technology 41(10), 3415-3421.

U.S. National Climatic Data Center (NCDC). 2007. Global Surface Summary of Day. Available at: http://www.ncdc.noaa.gov/oa/ncdc.html.

National Oceanic \& Atmospheric Administration (NOAA). 2007. NCEP_Reanalysis 2 data, NOAA/OAR/ESRL PSD, Boulder, Colorado, USA. Acquired at http://www.cdc.noaa.gov/ 
Neitsch, S.L., Arnold, J.G., Kiniry, J.R., and Williams, J.R. 2005. Soil and Water Assessment Tool Theoretical Documentation: Version 2005. USDA-ARS, Temple, TX.

Newcombe, K. et al. 1978. The metabolism of a city: The case of Hong Kong. Ambio $7: 3-15$.

Newman, P.W.G. 1999. Sustainability and cities: extending the metabolism model. Landscape and Urban Planning 44: 219-226.

Newman, P.W.G. et. al. 1996. Human Settlements. In: Australian State of the Environment Report. Department of Environment, Sport, and Territories. Canberra, Australia.

Niemczynowicz, J. 1996. Megacities from a water perspective. Water International 21, 198-205.

Ntale, H.K., Naturinda, D.N., Rubarenzya, M.H., and Kyamugambi, K. 2005. The rainwater harvesting strategy for Uganda. Proc. 31st WEDC International Conference, Kampala, Uganda, 2005.

Pandey, D.N., Gupta, A.K., and Anderson, D.M. 2003. Rainwater harvesting as an adaptation to climate change. Current Science 85(1), 46-59.

Pickett, S.T.A., Cadenasso, M.L., Grove, J.M., Nilon, C.H., Pouyat, R.V., Zipperer, W.C., and Costanza, R. 2001. Urban ecological systems: Linking terrestrial ecological, physical, and socioeconomic components of metropolitan areas. Annual Review of Ecological Systems 32:127-157.

Preul, H. C.1994. Rainfall-runoff water harvesting prospects for Greater Ammanand Jordan. Water International 19(2), 82-85. 
Richardson, C.W. 2000. Data requirements for estimation of weather generation parameters. Trans. ASAE 43, 877-882.

Richardson, C.W. and Wright, D.A. 1984. WGEN: a model for generating daily weather variables. US Department of Agriculture, Agricultural Research Service, ARS-8. USDA, Washington DC.

Rowell, D.P., 2001. Teleconnections between the tropical Pacific and the Sahel. Quarterly Journal of the Royal Meteorological Society 127, 1683-1706.

Sahely, H.R., Dudding, S., and Kennedy, C.A.. 2003. Estimating the urban metabolism of Canadian cities: GTA case study. Canadian Journal for Civil Engineering 30: 468483.

Schuol, J. and Abbaspour, K.C. 2007. Using monthly weather statistics to generate daily data in SWAT model application to West Africa. Ecological Modelling 201, 301311.

Schwarz, G. 1978. Estimating the dimension of a model. The Annals of Statistics 6(2), 461-464.

Semenov, M.A. 2002. LARS-WG 3.0 User Manual. Last Access, Nov 30, 2006. $<$ http://www.rothamsted.bbsrc.ac.uk/mas-models/larswg.php>.

Semenov, M.A. and Barrow, E.M., 1997. Use of a stochastic weather generator in the development of climate change scenarios. Climatic Change 35, 397-414.

Semenov, M.A., Brooks, R.J., Barrow, E.M., and Richardson, C.W. 1998. Comparison of the WGEN and LARS-WG stochastic weather generators for diverse climates. Climate Research 10, 95-107. 
Soltani, A. and Hoogenboom, G. 2003. Minimum data requirements for parameter estimation of stochastic weather generators. Climate Research 25, 109-119.

Sultan, B. and Janicot, S. 2003. The West African monsoon dynamics. Part I: Documentation of intraseasonal variability. Journal of Climate 16(21), 33893406.

Thomas, T. 2005. The case for using roofwater harvesting in Uganda. Proc. IRCSA XII, New Delhi, November 2005.

Thomas, T. 2005. Mainstreaming roofwater harvesting: Ugandan experiences. Proc. IRCSA XII, New Delhi, November 2005.

Thomas, T. 1998. Domestic water supply using rainwater harvesting. Building Research \& Information 26(2), 94-101.

United Nations (UN). 2005. The Millennium Development Goals Report: 2005. UN Sale No. E.05.II6. UN Department of Public Information, New York.

United Nations (UN). 2000. United Nations Millennium Declaration. Accessed Aug. 2, 2005. <http://www.un.org/millenniumgoals/>.

United Nations Environmental Programme (UNEP). 2005. Global Environmental Outlook: GEO Data Portal. Accessed Sep. 8, 2005. $<$ http://geodata.grid.unep.ch/>

UNESA. 2004. World Urbanization Prospects: The 2003 Revision. UN Sale No. E.04.XIII.6. UNESA Population Division, New York.

UN-HABITAT. 2006. The State of the World's Cities Report 2006/7. Earthscan, London. UN-HABITAT. 2005. UN-HABITAT Annual Report 2005. ISBN: 92-1-131735. UNHABITAT, Nairobi, Kenya. 
UN-HABITAT. 2005. Operational Activities Report: 2005. ISBN No.: 92-1-131679-0.

United Nations Human Settlements Programme. Nairobi, Kenya.

UN-HABITAT. 2003. The challenge of slums: Global report on human settlement.

Earthscan Publications Ltd., London.

United Nations Population Division (UNPD). 2008. World Urbanization Prospects: The 2007 Revision, Population Division of the Department of Economic and Social Affairs of the United Nations Secretariat. Accessed on October 13, 2008. http://esa.un.org/unup.

VASClimO. 2005. Globally gridded monthly precipitation sums. German Climate Research Programme (DEKLIM). Accessed on October 16, 2005, http://www.dwd.de/en/FundE/Klima/KLIS/int/GPCC/Projects/VASCLimO.htm.

Vorosmarty, C.J., Douglas, E.M., Green, P.A., and Revenga, C. 2005. Geospatial indicators of emerging water stress: An application to Africa. Ambio 34(3), 230236.

Vorosmarty, C.J., Green, P., Salisbury, J., Lammers, R.B. 2000. Global water resources: Vulnerability from climate change and population growth. Science $289,284-288$

Wackernagel, R and Rees, W. 1995. Our Ecological Footprint: Reducing Human Impact on Earth. New Society Publishers, Gabrioea Island, BC.

Warren-Rhodes, K. and Koenig, A. 2001. Escalating trends in the u rban metabolism of Hong Kong: 1971-1997. Ambio 30: 429-438.

WHO/UNICEF. 2000. Global Water Supply and Sanitation Assessment, 2000 Report, Water Supply and Sanitation Collaborative Council, Geneva and New York. 
Wilby, R.L., Charles, S.P., Zorita, E., Timbal, B., Whetton, P., and Mearns, L.O. 2004. Guidelines for Use of Climate Scenarios Developed from Statistical Downscaling Methods. IPCC TGICA, Geneva, Switzerland.

Wilby, R.L. and Dawson, C.W. 2004. SDSM User Manual, Last Accessed Nov. 28, 2006, <http://unfccc.int/resource/cd_roms/na1/v_and_a/Resoursce_materials/ Climate/SDSM/SDSM.Manual.pdf $>$.

Wilby, R.L., Dawson, C.W. and Barrow, E.M. 2001. SDSM - a decision support tool for the assessment of regional climate change impacts. Environmental and Modelling Software 17, 145-157.

Wilby, R.L., Wigley, T.M.L., Conway, D., Jones, P.D., Hewitson, B.C., Main, J., and Wilks, D.S. 1998. Statistical downscaling of general circulation model output: A comparison of methods. Water Resources Research 34(11), 2995-3008.

Wilks, D.S. 1999. Interannual variability and extreme-value characteristics of several stochastic daily precipitation models. Agricultural and Forest Meteorology 93, 153-169.

Wilks, D.S. and Wilby, R.L. 1999. The weather generation game: A review of stochastic weather models. Progress in Physical Geography 23(3), 329-357.

Wolman, A. 1965. The metabolism of cities. Scientific American 213(3): 179-190.

World Water Assessment Program (WWAP). 2006. Water, a shared responsibility: The 2nd UN World Water Development Report. UNESCO, Paris and Berghahn Books, New York City. 
Appendix A: Map of Gauge Locations 


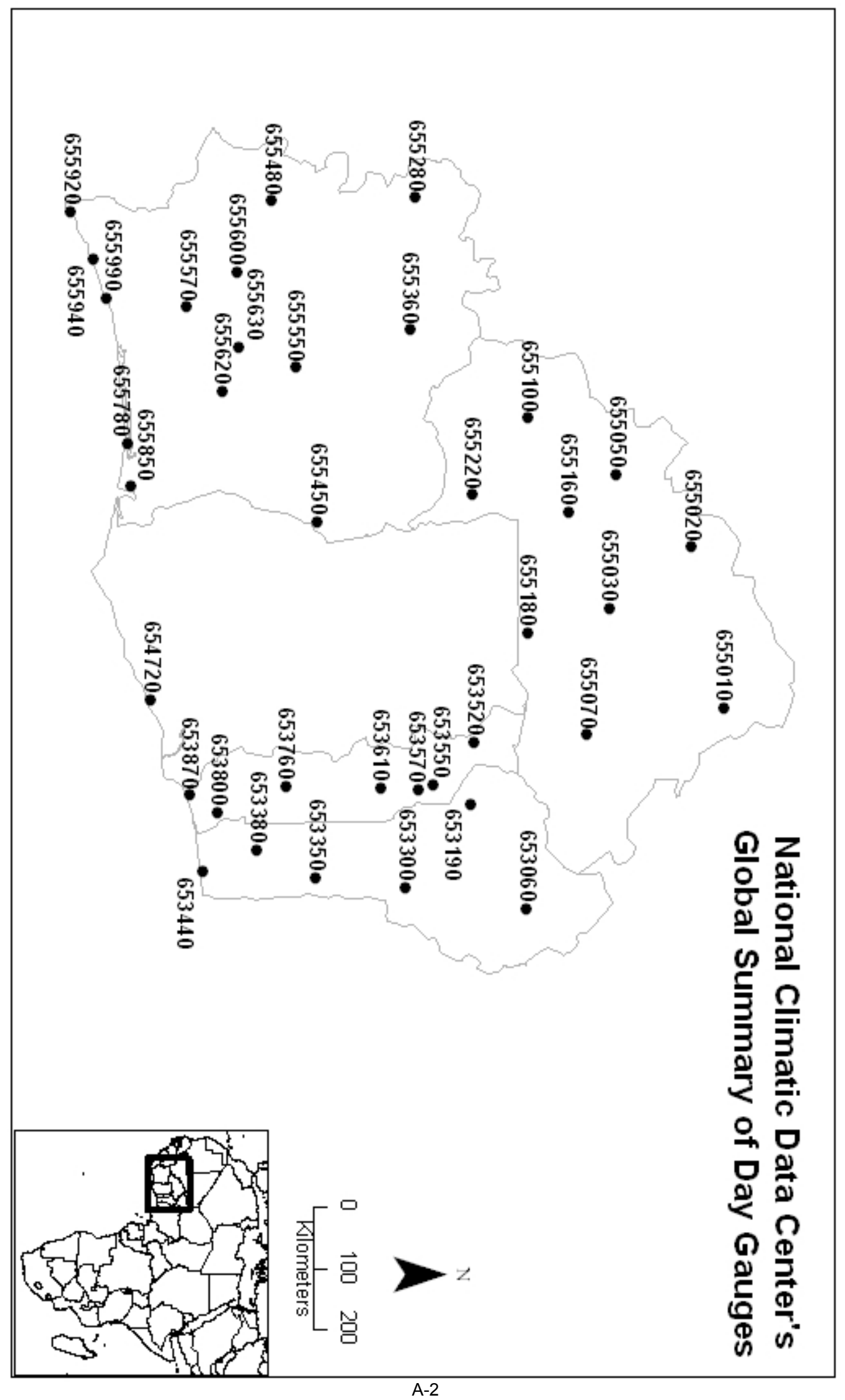


Appendix B: Markov Model Transition Probabilities 


\begin{tabular}{|c|c|c|c|c|c|c|c|c|}
\hline \multirow{3}{*}{ Gauge } & \multicolumn{8}{|c|}{ Zero-Order Markov Transition Probabilities } \\
\hline & Jan & & Feb & & Mar & & Apr & \\
\hline & $\mathbf{P}_{\mathrm{w}}$ & $\mathbf{P}_{\mathrm{d}}$ & $\mathbf{P}_{\mathrm{w}}$ & $\mathbf{P}_{\mathrm{d}}$ & $\mathbf{P}_{\mathrm{w}}$ & $\mathbf{P}_{\mathrm{d}}$ & $\mathbf{P}_{\mathrm{w}}$ & $\mathbf{P}_{\mathrm{d}}$ \\
\hline 653060 & 0.000 & 1.000 & 0.009 & 0.991 & 0.024 & 0.976 & 0.094 & 0.906 \\
\hline 653190 & 0.004 & 0.996 & 0.011 & 0.989 & 0.062 & 0.938 & 0.215 & 0.785 \\
\hline 653300 & 0.010 & 0.990 & 0.014 & 0.986 & 0.088 & 0.912 & 0.157 & 0.843 \\
\hline 653350 & 0.012 & 0.988 & 0.039 & 0.961 & 0.130 & 0.870 & 0.239 & 0.761 \\
\hline 653380 & 0.017 & 0.983 & 0.056 & 0.944 & 0.163 & 0.837 & 0.269 & 0.731 \\
\hline 653440 & 0.040 & 0.960 & 0.060 & 0.940 & 0.142 & 0.858 & 0.230 & 0.770 \\
\hline 653520 & 0.002 & 0.998 & 0.010 & 0.990 & 0.045 & 0.955 & 0.115 & 0.885 \\
\hline 653550 & 0.002 & 0.998 & 0.030 & 0.970 & 0.088 & 0.912 & 0.218 & 0.782 \\
\hline 653570 & 0.008 & 0.992 & 0.016 & 0.984 & 0.061 & 0.939 & 0.191 & 0.809 \\
\hline 653610 & 0.012 & 0.988 & 0.015 & 0.985 & 0.097 & 0.903 & 0.225 & 0.775 \\
\hline 653760 & 0.017 & 0.983 & 0.053 & 0.947 & 0.124 & 0.876 & 0.238 & 0.762 \\
\hline 653800 & 0.009 & 0.991 & 0.075 & 0.925 & 0.143 & 0.857 & 0.209 & 0.791 \\
\hline 653870 & 0.023 & 0.977 & 0.047 & 0.953 & 0.117 & 0.883 & 0.197 & 0.803 \\
\hline 654720 & 0.027 & 0.973 & 0.057 & 0.943 & 0.088 & 0.912 & 0.129 & 0.871 \\
\hline 655010 & 0.001 & 0.999 & 0.004 & 0.996 & 0.013 & 0.987 & 0.020 & 0.980 \\
\hline 655020 & 0.001 & 0.999 & 0.001 & 0.999 & 0.019 & 0.981 & 0.033 & 0.967 \\
\hline 655030 & 0.000 & 1.000 & 0.005 & 0.995 & 0.029 & 0.971 & 0.066 & 0.934 \\
\hline 655050 & 0.002 & 0.998 & 0.005 & 0.995 & 0.044 & 0.956 & 0.080 & 0.920 \\
\hline 655070 & 0.000 & 1.000 & 0.003 & 0.997 & 0.040 & 0.960 & 0.090 & 0.910 \\
\hline 655100 & 0.004 & 0.996 & 0.009 & 0.991 & 0.062 & 0.938 & 0.137 & 0.863 \\
\hline 655160 & 0.004 & 0.996 & 0.001 & 0.999 & 0.038 & 0.962 & 0.112 & 0.888 \\
\hline 655180 & 0.000 & 1.000 & 0.007 & 0.993 & 0.059 & 0.941 & 0.105 & 0.895 \\
\hline 655220 & 0.011 & 0.989 & 0.013 & 0.987 & 0.067 & 0.933 & 0.160 & 0.840 \\
\hline 655280 & 0.017 & 0.983 & 0.015 & 0.985 & 0.075 & 0.925 & 0.200 & 0.800 \\
\hline 655360 & 0.008 & 0.992 & 0.031 & 0.969 & 0.086 & 0.914 & 0.179 & 0.821 \\
\hline 655450 & 0.017 & 0.983 & 0.058 & 0.942 & 0.118 & 0.882 & 0.208 & 0.792 \\
\hline 655480 & 0.019 & 0.981 & 0.081 & 0.919 & 0.200 & 0.800 & 0.247 & 0.753 \\
\hline 655550 & 0.023 & 0.977 & 0.041 & 0.959 & 0.140 & 0.860 & 0.216 & 0.784 \\
\hline 655570 & 0.048 & 0.952 & 0.131 & 0.869 & 0.253 & 0.747 & 0.303 & 0.697 \\
\hline 655600 & 0.024 & 0.976 & 0.099 & 0.901 & 0.194 & 0.806 & 0.237 & 0.763 \\
\hline 655620 & 0.024 & 0.976 & 0.082 & 0.918 & 0.201 & 0.799 & 0.223 & 0.777 \\
\hline 655630 & 0.025 & 0.975 & 0.071 & 0.929 & 0.194 & 0.806 & 0.245 & 0.755 \\
\hline 655780 & 0.060 & 0.940 & 0.099 & 0.901 & 0.186 & 0.814 & 0.265 & 0.735 \\
\hline 655850 & 0.051 & 0.949 & 0.132 & 0.868 & 0.214 & 0.786 & 0.273 & 0.727 \\
\hline 655920 & 0.116 & 0.884 & 0.122 & 0.878 & 0.150 & 0.850 & 0.276 & 0.724 \\
\hline 655940 & 0.042 & 0.958 & 0.043 & 0.957 & 0.092 & 0.908 & 0.180 & 0.820 \\
\hline 655990 & 0.037 & 0.963 & 0.071 & 0.929 & 0.086 & 0.914 & 0.199 & 0.801 \\
\hline
\end{tabular}




\begin{tabular}{|c|c|c|c|c|c|c|c|c|}
\hline \multirow{3}{*}{ Gauge } & \multicolumn{8}{|c|}{ Zero-Order Markov Transition Probabilities } \\
\hline & May & & Jun & & Jul & & Aug & \\
\hline & $\mathbf{P}_{\mathrm{w}}$ & $\mathbf{P}_{\mathbf{d}}$ & $\mathbf{P}_{\mathrm{w}}$ & $\mathbf{P}_{\mathbf{d}}$ & $\mathbf{P}_{\mathrm{w}}$ & $\mathbf{P}_{\mathbf{d}}$ & $\mathbf{P}_{\mathrm{w}}$ & $\mathbf{P}_{\mathrm{d}}$ \\
\hline 653060 & 0.219 & 0.781 & 0.312 & 0.688 & 0.412 & 0.588 & 0.478 & 0.522 \\
\hline 653190 & 0.266 & 0.734 & 0.386 & 0.614 & 0.491 & 0.509 & 0.580 & 0.420 \\
\hline 653300 & 0.298 & 0.702 & 0.378 & 0.622 & 0.447 & 0.553 & 0.481 & 0.519 \\
\hline 653350 & 0.272 & 0.728 & 0.409 & 0.591 & 0.354 & 0.646 & 0.356 & 0.644 \\
\hline 653380 & 0.303 & 0.697 & 0.368 & 0.632 & 0.353 & 0.647 & 0.310 & 0.690 \\
\hline 653440 & 0.351 & 0.649 & 0.482 & 0.518 & 0.254 & 0.746 & 0.216 & 0.784 \\
\hline 653520 & 0.212 & 0.788 & 0.261 & 0.739 & 0.335 & 0.665 & 0.362 & 0.638 \\
\hline 653550 & 0.345 & 0.655 & 0.398 & 0.602 & 0.497 & 0.503 & 0.600 & 0.400 \\
\hline 653570 & 0.253 & 0.747 & 0.282 & 0.718 & 0.413 & 0.587 & 0.462 & 0.538 \\
\hline 653610 & 0.309 & 0.691 & 0.409 & 0.591 & 0.544 & 0.456 & 0.554 & 0.446 \\
\hline 653760 & 0.261 & 0.739 & 0.369 & 0.631 & 0.444 & 0.556 & 0.413 & 0.587 \\
\hline 653800 & 0.260 & 0.740 & 0.326 & 0.674 & 0.235 & 0.765 & 0.208 & 0.792 \\
\hline 653870 & 0.302 & 0.698 & 0.360 & 0.640 & 0.187 & 0.813 & 0.174 & 0.826 \\
\hline 654720 & 0.167 & 0.833 & 0.312 & 0.688 & 0.172 & 0.828 & 0.160 & 0.840 \\
\hline 655010 & 0.084 & 0.916 & 0.170 & 0.830 & 0.271 & 0.729 & 0.342 & 0.658 \\
\hline 655020 & 0.110 & 0.890 & 0.226 & 0.774 & 0.341 & 0.659 & 0.431 & 0.569 \\
\hline 655030 & 0.179 & 0.821 & 0.275 & 0.725 & 0.376 & 0.624 & 0.453 & 0.547 \\
\hline 655050 & 0.160 & 0.840 & 0.242 & 0.758 & 0.363 & 0.637 & 0.472 & 0.528 \\
\hline 655070 & 0.187 & 0.813 & 0.282 & 0.718 & 0.378 & 0.622 & 0.471 & 0.529 \\
\hline 655100 & 0.225 & 0.775 & 0.329 & 0.671 & 0.394 & 0.606 & 0.527 & 0.473 \\
\hline 655160 & 0.209 & 0.791 & 0.302 & 0.698 & 0.397 & 0.603 & 0.523 & 0.477 \\
\hline 655180 & 0.195 & 0.805 & 0.325 & 0.675 & 0.362 & 0.638 & 0.522 & 0.478 \\
\hline 655220 & 0.231 & 0.769 & 0.288 & 0.712 & 0.398 & 0.602 & 0.456 & 0.544 \\
\hline 655280 & 0.237 & 0.763 & 0.311 & 0.689 & 0.431 & 0.569 & 0.558 & 0.442 \\
\hline 655360 & 0.265 & 0.735 & 0.291 & 0.709 & 0.333 & 0.667 & 0.432 & 0.568 \\
\hline 655450 & 0.307 & 0.693 & 0.346 & 0.654 & 0.240 & 0.760 & 0.249 & 0.751 \\
\hline 655480 & 0.299 & 0.701 & 0.379 & 0.621 & 0.507 & 0.493 & 0.597 & 0.403 \\
\hline 655550 & 0.236 & 0.764 & 0.284 & 0.716 & 0.233 & 0.767 & 0.299 & 0.701 \\
\hline 655570 & 0.424 & 0.576 & 0.456 & 0.544 & 0.271 & 0.729 & 0.332 & 0.668 \\
\hline 655600 & 0.322 & 0.678 & 0.365 & 0.635 & 0.323 & 0.677 & 0.396 & 0.604 \\
\hline 655620 & 0.336 & 0.664 & 0.382 & 0.618 & 0.261 & 0.739 & 0.267 & 0.733 \\
\hline 655630 & 0.319 & 0.681 & 0.345 & 0.655 & 0.228 & 0.772 & 0.280 & 0.720 \\
\hline 655780 & 0.448 & 0.552 & 0.536 & 0.464 & 0.251 & 0.749 & 0.208 & 0.792 \\
\hline 655850 & 0.467 & 0.533 & 0.585 & 0.415 & 0.382 & 0.618 & 0.350 & 0.650 \\
\hline 655920 & 0.531 & 0.469 & 0.564 & 0.436 & 0.393 & 0.607 & 0.531 & 0.469 \\
\hline 655940 & 0.403 & 0.597 & 0.530 & 0.470 & 0.296 & 0.704 & 0.302 & 0.698 \\
\hline 655990 & 0.419 & 0.581 & 0.490 & 0.510 & 0.246 & 0.754 & 0.272 & 0.728 \\
\hline
\end{tabular}




\begin{tabular}{|c|c|c|c|c|c|c|c|c|}
\hline \multirow{3}{*}{ Gauge } & \multicolumn{8}{|c|}{ Zero-Order Markov Transition Probabilities } \\
\hline & Sep & & Oct & & Nov & & Dec & \\
\hline & $\mathbf{P}_{\mathrm{w}}$ & $\mathbf{P}_{\mathrm{d}}$ & $\mathbf{P}_{\mathrm{w}}$ & $\mathbf{P}_{\mathrm{d}}$ & $\mathbf{P}_{\mathrm{w}}$ & $\mathbf{P}_{\mathrm{d}}$ & $\mathbf{P}_{\mathrm{w}}$ & $\mathbf{P}_{\mathrm{d}}$ \\
\hline 653060 & 0.421 & 0.579 & 0.128 & 0.872 & 0.008 & 0.992 & 0.001 & 0.999 \\
\hline 653190 & 0.592 & 0.408 & 0.298 & 0.702 & 0.032 & 0.968 & 0.003 & 0.997 \\
\hline 653300 & 0.514 & 0.486 & 0.276 & 0.724 & 0.025 & 0.975 & 0.010 & 0.990 \\
\hline 653350 & 0.416 & 0.584 & 0.326 & 0.674 & 0.039 & 0.961 & 0.011 & 0.989 \\
\hline 653380 & 0.375 & 0.625 & 0.308 & 0.692 & 0.077 & 0.923 & 0.028 & 0.972 \\
\hline 653440 & 0.329 & 0.671 & 0.337 & 0.663 & 0.137 & 0.863 & 0.043 & 0.957 \\
\hline 653520 & 0.467 & 0.533 & 0.188 & 0.812 & 0.026 & 0.974 & 0.004 & 0.996 \\
\hline 653550 & 0.584 & 0.416 & 0.391 & 0.609 & 0.068 & 0.932 & 0.011 & 0.989 \\
\hline 653570 & 0.452 & 0.548 & 0.270 & 0.730 & 0.033 & 0.967 & 0.009 & 0.991 \\
\hline 653610 & 0.529 & 0.471 & 0.300 & 0.700 & 0.052 & 0.948 & 0.019 & 0.981 \\
\hline 653760 & 0.375 & 0.625 & 0.295 & 0.705 & 0.059 & 0.941 & 0.020 & 0.980 \\
\hline 653800 & 0.288 & 0.712 & 0.342 & 0.658 & 0.121 & 0.879 & 0.029 & 0.971 \\
\hline 653870 & 0.243 & 0.757 & 0.233 & 0.767 & 0.086 & 0.914 & 0.033 & 0.967 \\
\hline 654720 & 0.173 & 0.827 & 0.222 & 0.778 & 0.088 & 0.912 & 0.039 & 0.961 \\
\hline 655010 & 0.244 & 0.756 & 0.058 & 0.942 & 0.003 & 0.997 & 0.006 & 0.994 \\
\hline 655020 & 0.303 & 0.697 & 0.117 & 0.883 & 0.006 & 0.994 & 0.003 & 0.997 \\
\hline 655030 & 0.335 & 0.665 & 0.128 & 0.872 & 0.016 & 0.984 & 0.006 & 0.994 \\
\hline 655050 & 0.348 & 0.652 & 0.154 & 0.846 & 0.020 & 0.980 & 0.006 & 0.994 \\
\hline 655070 & 0.371 & 0.629 & 0.133 & 0.867 & 0.012 & 0.988 & 0.009 & 0.991 \\
\hline 655100 & 0.459 & 0.541 & 0.194 & 0.806 & 0.040 & 0.960 & 0.008 & 0.992 \\
\hline 655160 & 0.388 & 0.612 & 0.161 & 0.839 & 0.023 & 0.977 & 0.003 & 0.997 \\
\hline 655180 & 0.398 & 0.602 & 0.147 & 0.853 & 0.038 & 0.962 & 0.004 & 0.996 \\
\hline 655220 & 0.456 & 0.544 & 0.254 & 0.746 & 0.038 & 0.962 & 0.009 & 0.991 \\
\hline 655280 & 0.520 & 0.480 & 0.366 & 0.634 & 0.097 & 0.903 & 0.017 & 0.983 \\
\hline 655360 & 0.432 & 0.568 & 0.313 & 0.687 & 0.074 & 0.926 & 0.009 & 0.991 \\
\hline 655450 & 0.425 & 0.575 & 0.308 & 0.692 & 0.099 & 0.901 & 0.037 & 0.963 \\
\hline 655480 & 0.563 & 0.437 & 0.348 & 0.652 & 0.116 & 0.884 & 0.044 & 0.956 \\
\hline 655550 & 0.369 & 0.631 & 0.253 & 0.747 & 0.072 & 0.928 & 0.037 & 0.963 \\
\hline 655570 & 0.421 & 0.579 & 0.409 & 0.591 & 0.296 & 0.704 & 0.114 & 0.886 \\
\hline 655600 & 0.426 & 0.574 & 0.292 & 0.708 & 0.132 & 0.868 & 0.056 & 0.944 \\
\hline 655620 & 0.345 & 0.655 & 0.325 & 0.675 & 0.128 & 0.872 & 0.048 & 0.952 \\
\hline 655630 & 0.358 & 0.642 & 0.331 & 0.669 & 0.156 & 0.844 & 0.059 & 0.941 \\
\hline 655780 & 0.267 & 0.733 & 0.377 & 0.623 & 0.352 & 0.648 & 0.177 & 0.823 \\
\hline 655850 & 0.416 & 0.584 & 0.473 & 0.527 & 0.385 & 0.615 & 0.184 & 0.816 \\
\hline 655920 & 0.596 & 0.404 & 0.485 & 0.515 & 0.430 & 0.570 & 0.257 & 0.743 \\
\hline 655940 & 0.319 & 0.681 & 0.351 & 0.649 & 0.358 & 0.642 & 0.178 & 0.822 \\
\hline 655990 & 0.246 & 0.754 & 0.318 & 0.682 & 0.296 & 0.704 & 0.137 & 0.863 \\
\hline
\end{tabular}




\begin{tabular}{|c|c|c|c|c|c|c|c|c|}
\hline \multirow[t]{2}{*}{ Gauge } & \multicolumn{4}{|l|}{ Jan } & \multicolumn{4}{|l|}{ Feb } \\
\hline & $\mathbf{P}_{\mathrm{ww}}$ & $P_{w d}$ & $\mathbf{P}_{\mathrm{dw}}$ & $\mathbf{P}_{\mathrm{dd}}$ & $\mathbf{P}_{\mathrm{ww}}$ & $P_{\mathrm{wd}}$ & $\mathbf{P}_{\mathrm{dw}}$ & $\mathbf{P}_{\mathrm{dd}}$ \\
\hline 653060 & 0.000 & 1.000 & 0.000 & 1.000 & 0.000 & 1.000 & 0.009 & 0.991 \\
\hline 653190 & 0.000 & 1.000 & 0.004 & 0.996 & 0.333 & 0.667 & 0.008 & 0.992 \\
\hline 653300 & 0.286 & 0.714 & 0.008 & 0.992 & 0.167 & 0.833 & 0.012 & 0.988 \\
\hline 653350 & 0.100 & 0.900 & 0.011 & 0.989 & 0.207 & 0.793 & 0.032 & 0.968 \\
\hline 653380 & 0.000 & 1.000 & 0.018 & 0.982 & 0.132 & 0.868 & 0.051 & 0.949 \\
\hline 653440 & 0.121 & 0.879 & 0.037 & 0.963 & 0.140 & 0.860 & 0.055 & 0.945 \\
\hline 653520 & 0.000 & 1.000 & 0.002 & 0.998 & 0.000 & 1.000 & 0.010 & 0.990 \\
\hline 653550 & 0.000 & 0.000 & 0.002 & 0.998 & 0.100 & 0.900 & 0.028 & 0.972 \\
\hline 653570 & 0.333 & 0.667 & 0.006 & 0.994 & 0.167 & 0.833 & 0.013 & 0.987 \\
\hline 653610 & 0.000 & 1.000 & 0.012 & 0.988 & 0.000 & 1.000 & 0.016 & 0.984 \\
\hline 653760 & 0.000 & 1.000 & 0.017 & 0.983 & 0.120 & 0.880 & 0.050 & 0.950 \\
\hline 653800 & 0.000 & 1.000 & 0.009 & 0.991 & 0.214 & 0.786 & 0.064 & 0.936 \\
\hline 653870 & 0.150 & 0.850 & 0.020 & 0.980 & 0.094 & 0.906 & 0.045 & 0.955 \\
\hline 654720 & 0.111 & 0.889 & 0.025 & 0.975 & 0.063 & 0.938 & 0.056 & 0.944 \\
\hline 655010 & 0.000 & 1.000 & 0.001 & 0.999 & 0.000 & 1.000 & 0.004 & 0.996 \\
\hline 655020 & 0.000 & 1.000 & 0.001 & 0.999 & 0.000 & 1.000 & 0.001 & 0.999 \\
\hline 655030 & 0.000 & 1.000 & 0.000 & 1.000 & 0.000 & 1.000 & 0.005 & 0.995 \\
\hline 655050 & 0.000 & 1.000 & 0.002 & 0.998 & 0.000 & 1.000 & 0.005 & 0.995 \\
\hline 655070 & 0.000 & 0.000 & 0.000 & 1.000 & 0.000 & 1.000 & 0.003 & 0.997 \\
\hline 655100 & 0.000 & 1.000 & 0.004 & 0.996 & 0.143 & 0.857 & 0.008 & 0.992 \\
\hline 655160 & 0.000 & 1.000 & 0.004 & 0.996 & 0.000 & 1.000 & 0.001 & 0.999 \\
\hline 655180 & 0.000 & 0.000 & 0.000 & 1.000 & 0.000 & 1.000 & 0.007 & 0.993 \\
\hline 655220 & 0.000 & 1.000 & 0.011 & 0.989 & 0.000 & 1.000 & 0.013 & 0.987 \\
\hline 655280 & 0.154 & 0.846 & 0.014 & 0.986 & 0.000 & 1.000 & 0.015 & 0.985 \\
\hline 655360 & 0.125 & 0.875 & 0.007 & 0.993 & 0.071 & 0.929 & 0.030 & 0.970 \\
\hline 655450 & 0.167 & 0.833 & 0.016 & 0.984 & 0.242 & 0.758 & 0.047 & 0.953 \\
\hline 655480 & 0.200 & 0.800 & 0.016 & 0.984 & 0.245 & 0.755 & 0.067 & 0.933 \\
\hline 655550 & 0.167 & 0.833 & 0.020 & 0.980 & 0.130 & 0.870 & 0.037 & 0.963 \\
\hline 655570 & 0.207 & 0.793 & 0.040 & 0.960 & 0.211 & 0.789 & 0.119 & 0.881 \\
\hline 655600 & 0.000 & 1.000 & 0.025 & 0.975 & 0.161 & 0.839 & 0.092 & 0.908 \\
\hline 655620 & 0.000 & 1.000 & 0.025 & 0.975 & 0.106 & 0.894 & 0.080 & 0.920 \\
\hline 655630 & 0.111 & 0.889 & 0.023 & 0.977 & 0.083 & 0.917 & 0.070 & 0.930 \\
\hline 655780 & 0.229 & 0.771 & 0.050 & 0.950 & 0.203 & 0.797 & 0.088 & 0.912 \\
\hline 655850 & 0.154 & 0.846 & 0.046 & 0.954 & 0.179 & 0.821 & 0.125 & 0.875 \\
\hline 655920 & 0.250 & 0.750 & 0.096 & 0.904 & 0.218 & 0.782 & 0.109 & 0.891 \\
\hline 655940 & 0.188 & 0.813 & 0.036 & 0.964 & 0.038 & 0.962 & 0.044 & 0.956 \\
\hline 655990 & 0.250 & 0.750 & 0.027 & 0.973 & 0.108 & 0.892 & 0.069 & 0.931 \\
\hline
\end{tabular}




\begin{tabular}{|c|c|c|c|c|c|c|c|c|}
\hline \multirow[t]{2}{*}{ Gauge } & \multicolumn{4}{|l|}{ Mar } & \multicolumn{4}{|l|}{ Apr } \\
\hline & $\mathbf{P}_{\mathrm{ww}}$ & $\mathbf{P}_{\mathrm{wd}}$ & $\mathbf{P}_{\mathrm{dw}}$ & $\mathbf{P}_{\mathrm{dd}}$ & $\mathbf{P}_{\mathrm{ww}}$ & $\mathbf{P}_{\mathrm{wd}}$ & $\mathbf{P}_{\mathrm{dw}}$ & $\mathbf{P}_{\mathrm{dd}}$ \\
\hline 653060 & 0.158 & 0.842 & 0.020 & 0.980 & 0.186 & 0.814 & 0.085 & 0.915 \\
\hline 653190 & 0.245 & 0.755 & 0.049 & 0.951 & 0.290 & 0.710 & 0.196 & 0.804 \\
\hline 653300 & 0.185 & 0.815 & 0.080 & 0.920 & 0.205 & 0.795 & 0.148 & 0.852 \\
\hline 653350 & 0.239 & 0.761 & 0.115 & 0.885 & 0.279 & 0.721 & 0.226 & 0.774 \\
\hline 653380 & 0.160 & 0.840 & 0.164 & 0.836 & 0.285 & 0.715 & 0.263 & 0.737 \\
\hline 653440 & 0.222 & 0.778 & 0.128 & 0.872 & 0.261 & 0.739 & 0.222 & 0.778 \\
\hline 653520 & 0.217 & 0.783 & 0.036 & 0.964 & 0.109 & 0.891 & 0.116 & 0.884 \\
\hline 653550 & 0.081 & 0.919 & 0.089 & 0.911 & 0.233 & 0.767 & 0.215 & 0.785 \\
\hline 653570 & 0.174 & 0.826 & 0.054 & 0.946 & 0.239 & 0.761 & 0.180 & 0.820 \\
\hline 653610 & 0.119 & 0.881 & 0.095 & 0.905 & 0.217 & 0.783 & 0.227 & 0.773 \\
\hline 653760 & 0.186 & 0.814 & 0.114 & 0.886 & 0.274 & 0.726 & 0.226 & 0.774 \\
\hline 653800 & 0.193 & 0.807 & 0.135 & 0.865 & 0.123 & 0.877 & 0.229 & 0.771 \\
\hline 653870 & 0.225 & 0.775 & 0.103 & 0.897 & 0.295 & 0.705 & 0.176 & 0.824 \\
\hline 654720 & 0.132 & 0.868 & 0.083 & 0.917 & 0.170 & 0.830 & 0.122 & 0.878 \\
\hline 655010 & 0.200 & 0.800 & 0.011 & 0.989 & 0.000 & 1.000 & 0.020 & 0.980 \\
\hline 655020 & 0.077 & 0.923 & 0.018 & 0.982 & 0.045 & 0.955 & 0.033 & 0.967 \\
\hline 655030 & 0.208 & 0.792 & 0.024 & 0.976 & 0.140 & 0.860 & 0.061 & 0.939 \\
\hline 655050 & 0.300 & 0.700 & 0.033 & 0.967 & 0.265 & 0.735 & 0.065 & 0.935 \\
\hline 655070 & 0.250 & 0.750 & 0.031 & 0.969 & 0.176 & 0.824 & 0.082 & 0.918 \\
\hline 655100 & 0.229 & 0.771 & 0.052 & 0.948 & 0.255 & 0.745 & 0.119 & 0.881 \\
\hline 655160 & 0.233 & 0.767 & 0.030 & 0.970 & 0.197 & 0.803 & 0.101 & 0.899 \\
\hline 655180 & 0.346 & 0.654 & 0.042 & 0.958 & 0.238 & 0.762 & 0.089 & 0.911 \\
\hline 655220 & 0.261 & 0.739 & 0.054 & 0.946 & 0.200 & 0.800 & 0.152 & 0.848 \\
\hline 655280 & 0.125 & 0.875 & 0.071 & 0.929 & 0.245 & 0.755 & 0.189 & 0.811 \\
\hline 655360 & 0.163 & 0.837 & 0.079 & 0.921 & 0.208 & 0.792 & 0.173 & 0.827 \\
\hline 655450 & 0.230 & 0.770 & 0.102 & 0.898 & 0.218 & 0.782 & 0.206 & 0.794 \\
\hline 655480 & 0.239 & 0.761 & 0.190 & 0.810 & 0.234 & 0.766 & 0.252 & 0.748 \\
\hline 655550 & 0.171 & 0.829 & 0.135 & 0.865 & 0.248 & 0.752 & 0.207 & 0.793 \\
\hline 655570 & 0.365 & 0.635 & 0.212 & 0.788 & 0.285 & 0.715 & 0.312 & 0.688 \\
\hline 655600 & 0.178 & 0.822 & 0.198 & 0.802 & 0.212 & 0.788 & 0.244 & 0.756 \\
\hline 655620 & 0.311 & 0.689 & 0.174 & 0.826 & 0.250 & 0.750 & 0.215 & 0.785 \\
\hline 655630 & 0.270 & 0.730 & 0.177 & 0.823 & 0.231 & 0.769 & 0.250 & 0.750 \\
\hline 655780 & 0.258 & 0.742 & 0.170 & 0.830 & 0.307 & 0.693 & 0.250 & 0.750 \\
\hline 655850 & 0.265 & 0.735 & 0.199 & 0.801 & 0.291 & 0.709 & 0.267 & 0.733 \\
\hline 655920 & 0.216 & 0.784 & 0.138 & 0.862 & 0.378 & 0.622 & 0.239 & 0.761 \\
\hline 655940 & 0.077 & 0.923 & 0.093 & 0.907 & 0.258 & 0.742 & 0.163 & 0.837 \\
\hline 655990 & 0.125 & 0.875 & 0.083 & 0.917 & 0.291 & 0.709 & 0.175 & 0.825 \\
\hline
\end{tabular}




\begin{tabular}{|c|c|c|c|c|c|c|c|c|}
\hline \multirow[t]{2}{*}{ Gauge } & \multicolumn{4}{|l|}{ May } & \multicolumn{4}{|l|}{ Jun } \\
\hline & $\mathbf{P}_{\mathrm{ww}}$ & $\mathbf{P}_{\mathrm{wd}}$ & $\mathbf{P}_{\mathrm{dw}}$ & $\mathbf{P}_{\mathrm{dd}}$ & $\mathbf{P}_{\mathrm{ww}}$ & $\mathbf{P}_{\mathrm{wd}}$ & $\mathbf{P}_{\mathrm{dw}}$ & $\mathbf{P}_{\mathrm{dd}}$ \\
\hline 653060 & 0.241 & 0.759 & 0.213 & 0.787 & 0.292 & 0.708 & 0.321 & 0.679 \\
\hline 653190 & 0.325 & 0.675 & 0.244 & 0.756 & 0.417 & 0.583 & 0.366 & 0.634 \\
\hline 653300 & 0.255 & 0.745 & 0.318 & 0.682 & 0.391 & 0.609 & 0.369 & 0.631 \\
\hline 653350 & 0.274 & 0.726 & 0.271 & 0.729 & 0.450 & 0.550 & 0.381 & 0.619 \\
\hline 653380 & 0.352 & 0.648 & 0.282 & 0.718 & 0.364 & 0.636 & 0.371 & 0.629 \\
\hline 653440 & 0.349 & 0.651 & 0.352 & 0.648 & 0.538 & 0.462 & 0.428 & 0.572 \\
\hline 653520 & 0.284 & 0.716 & 0.194 & 0.806 & 0.276 & 0.724 & 0.256 & 0.744 \\
\hline 653550 & 0.400 & 0.600 & 0.316 & 0.684 & 0.455 & 0.545 & 0.357 & 0.643 \\
\hline 653570 & 0.327 & 0.673 & 0.227 & 0.773 & 0.277 & 0.723 & 0.283 & 0.717 \\
\hline 653610 & 0.302 & 0.698 & 0.312 & 0.688 & 0.403 & 0.597 & 0.413 & 0.587 \\
\hline 653760 & 0.306 & 0.694 & 0.245 & 0.755 & 0.404 & 0.596 & 0.349 & 0.651 \\
\hline 653800 & 0.294 & 0.706 & 0.248 & 0.752 & 0.357 & 0.643 & 0.311 & 0.689 \\
\hline 653870 & 0.322 & 0.678 & 0.293 & 0.707 & 0.417 & 0.583 & 0.328 & 0.672 \\
\hline 654720 & 0.277 & 0.723 & 0.145 & 0.855 & 0.439 & 0.561 & 0.259 & 0.741 \\
\hline 655010 & 0.197 & 0.803 & 0.073 & 0.927 & 0.157 & 0.843 & 0.173 & 0.827 \\
\hline 655020 & 0.192 & 0.808 & 0.100 & 0.900 & 0.199 & 0.801 & 0.234 & 0.766 \\
\hline 655030 & 0.201 & 0.799 & 0.175 & 0.825 & 0.217 & 0.783 & 0.297 & 0.703 \\
\hline 655050 & 0.262 & 0.738 & 0.142 & 0.858 & 0.200 & 0.800 & 0.256 & 0.744 \\
\hline 655070 & 0.211 & 0.789 & 0.182 & 0.818 & 0.295 & 0.705 & 0.277 & 0.723 \\
\hline 655100 & 0.208 & 0.792 & 0.230 & 0.770 & 0.319 & 0.681 & 0.335 & 0.665 \\
\hline 655160 & 0.264 & 0.736 & 0.195 & 0.805 & 0.269 & 0.731 & 0.317 & 0.683 \\
\hline 655180 & 0.228 & 0.772 & 0.188 & 0.812 & 0.257 & 0.743 & 0.356 & 0.644 \\
\hline 655220 & 0.257 & 0.743 & 0.224 & 0.776 & 0.250 & 0.750 & 0.306 & 0.694 \\
\hline 655280 & 0.206 & 0.794 & 0.247 & 0.753 & 0.226 & 0.774 & 0.348 & 0.652 \\
\hline 655360 & 0.257 & 0.743 & 0.268 & 0.732 & 0.259 & 0.741 & 0.304 & 0.696 \\
\hline 655450 & 0.332 & 0.668 & 0.296 & 0.704 & 0.337 & 0.663 & 0.351 & 0.649 \\
\hline 655480 & 0.304 & 0.696 & 0.297 & 0.703 & 0.411 & 0.589 & 0.359 & 0.641 \\
\hline 655550 & 0.262 & 0.738 & 0.228 & 0.772 & 0.290 & 0.710 & 0.282 & 0.718 \\
\hline 655570 & 0.476 & 0.524 & 0.387 & 0.613 & 0.520 & 0.480 & 0.401 & 0.599 \\
\hline 655600 & 0.303 & 0.697 & 0.330 & 0.670 & 0.415 & 0.585 & 0.336 & 0.664 \\
\hline 655620 & 0.354 & 0.646 & 0.327 & 0.673 & 0.415 & 0.585 & 0.360 & 0.640 \\
\hline 655630 & 0.309 & 0.691 & 0.324 & 0.676 & 0.446 & 0.554 & 0.291 & 0.709 \\
\hline 655780 & 0.504 & 0.496 & 0.402 & 0.598 & 0.589 & 0.411 & 0.469 & 0.531 \\
\hline 655850 & 0.487 & 0.513 & 0.450 & 0.550 & 0.660 & 0.340 & 0.472 & 0.528 \\
\hline 655920 & 0.620 & 0.380 & 0.430 & 0.570 & 0.624 & 0.376 & 0.486 & 0.514 \\
\hline 655940 & 0.498 & 0.502 & 0.345 & 0.655 & 0.574 & 0.426 & 0.478 & 0.522 \\
\hline 655990 & 0.534 & 0.466 & 0.340 & 0.660 & 0.556 & 0.444 & 0.424 & 0.576 \\
\hline
\end{tabular}




\begin{tabular}{|c|c|c|c|c|c|c|c|c|}
\hline \multirow[t]{2}{*}{ Gauge } & \multicolumn{8}{|l|}{ Jul } \\
\hline & $\mathbf{P}_{w w}$ & $P_{w d}$ & $\mathbf{P}_{\mathrm{dw}}$ & $\mathbf{P}_{\mathrm{dd}}$ & $\mathbf{P}_{\mathrm{ww}}$ & $P_{w d}$ & $\mathbf{P}_{\mathrm{dw}}$ & $\mathbf{P}_{\mathrm{dd}}$ \\
\hline 653060 & 0.408 & 0.592 & 0.414 & 0.586 & 0.482 & 0.518 & 0.474 & 0.526 \\
\hline 653190 & 0.502 & 0.498 & 0.482 & 0.518 & 0.578 & 0.422 & 0.583 & 0.417 \\
\hline 653300 & 0.512 & 0.488 & 0.394 & 0.606 & 0.535 & 0.465 & 0.427 & 0.573 \\
\hline 653350 & 0.467 & 0.533 & 0.292 & 0.708 & 0.466 & 0.534 & 0.296 & 0.704 \\
\hline 653380 & 0.409 & 0.591 & 0.325 & 0.675 & 0.449 & 0.551 & 0.242 & 0.758 \\
\hline 653440 & 0.430 & 0.570 & 0.189 & 0.811 & 0.378 & 0.622 & 0.172 & 0.828 \\
\hline 653520 & 0.320 & 0.680 & 0.343 & 0.657 & 0.345 & 0.655 & 0.372 & 0.628 \\
\hline 653550 & 0.506 & 0.494 & 0.488 & 0.512 & 0.601 & 0.399 & 0.599 & 0.401 \\
\hline 653570 & 0.382 & 0.618 & 0.435 & 0.565 & 0.503 & 0.497 & 0.426 & 0.574 \\
\hline 653610 & 0.569 & 0.431 & 0.515 & 0.485 & 0.608 & 0.392 & 0.485 & 0.515 \\
\hline 653760 & 0.542 & 0.458 & 0.371 & 0.629 & 0.445 & 0.555 & 0.389 & 0.611 \\
\hline 653800 & 0.340 & 0.660 & 0.202 & 0.798 & 0.404 & 0.596 & 0.151 & 0.849 \\
\hline 653870 & 0.347 & 0.653 & 0.149 & 0.851 & 0.279 & 0.721 & 0.151 & 0.849 \\
\hline 654720 & 0.400 & 0.600 & 0.121 & 0.879 & 0.242 & 0.758 & 0.144 & 0.856 \\
\hline 655010 & 0.161 & 0.839 & 0.312 & 0.688 & 0.316 & 0.684 & 0.355 & 0.645 \\
\hline 655020 & 0.332 & 0.668 & 0.345 & 0.655 & 0.399 & 0.601 & 0.454 & 0.546 \\
\hline 655030 & 0.314 & 0.686 & 0.415 & 0.585 & 0.442 & 0.558 & 0.463 & 0.537 \\
\hline 655050 & 0.317 & 0.683 & 0.388 & 0.612 & 0.444 & 0.556 & 0.495 & 0.505 \\
\hline 655070 & 0.346 & 0.654 & 0.396 & 0.604 & 0.440 & 0.560 & 0.498 & 0.502 \\
\hline 655100 & 0.372 & 0.628 & 0.407 & 0.593 & 0.522 & 0.478 & 0.532 & 0.468 \\
\hline 655160 & 0.363 & 0.637 & 0.419 & 0.581 & 0.526 & 0.474 & 0.519 & 0.481 \\
\hline 655180 & 0.347 & 0.653 & 0.370 & 0.630 & 0.530 & 0.470 & 0.514 & 0.486 \\
\hline 655220 & 0.384 & 0.616 & 0.408 & 0.592 & 0.454 & 0.546 & 0.457 & 0.543 \\
\hline 655280 & 0.469 & 0.531 & 0.401 & 0.599 & 0.567 & 0.433 & 0.547 & 0.453 \\
\hline 655360 & 0.333 & 0.667 & 0.333 & 0.667 & 0.457 & 0.543 & 0.414 & 0.586 \\
\hline 655450 & 0.333 & 0.667 & 0.211 & 0.789 & 0.349 & 0.651 & 0.218 & 0.782 \\
\hline 655480 & 0.580 & 0.420 & 0.425 & 0.575 & 0.608 & 0.392 & 0.580 & 0.420 \\
\hline 655550 & 0.358 & 0.642 & 0.194 & 0.806 & 0.411 & 0.589 & 0.253 & 0.747 \\
\hline 655570 & 0.429 & 0.571 & 0.211 & 0.789 & 0.447 & 0.553 & 0.277 & 0.723 \\
\hline 655600 & 0.466 & 0.534 & 0.255 & 0.745 & 0.460 & 0.540 & 0.353 & 0.647 \\
\hline 655620 & 0.406 & 0.594 & 0.210 & 0.790 & 0.400 & 0.600 & 0.222 & 0.778 \\
\hline 655630 & 0.320 & 0.680 & 0.200 & 0.800 & 0.393 & 0.607 & 0.236 & 0.764 \\
\hline 655780 & 0.465 & 0.535 & 0.172 & 0.828 & 0.389 & 0.611 & 0.159 & 0.841 \\
\hline 655850 & 0.528 & 0.472 & 0.291 & 0.709 & 0.495 & 0.505 & 0.273 & 0.727 \\
\hline 655920 & 0.560 & 0.440 & 0.272 & 0.728 & 0.613 & 0.387 & 0.441 & 0.559 \\
\hline 655940 & 0.464 & 0.536 & 0.221 & 0.779 & 0.456 & 0.544 & 0.234 & 0.766 \\
\hline 655990 & 0.451 & 0.549 & 0.179 & 0.821 & 0.437 & 0.563 & 0.212 & 0.788 \\
\hline
\end{tabular}




\section{1st-Order Markov Transition Probabilities}

\begin{tabular}{|c|c|c|c|c|c|c|c|c|}
\hline Gauge & Sep & & & & Oct & & & \\
\hline 653060 & $\begin{array}{l}\mathbf{P}_{\mathrm{ww}} \\
0.408\end{array}$ & $\begin{array}{l}\mathbf{P}_{\mathbf{w d}} \\
0.592\end{array}$ & $\begin{array}{l}\mathbf{P}_{\mathrm{dw}} \\
0.431\end{array}$ & $\begin{array}{l}\mathbf{P}_{\text {dd }} \\
0.569\end{array}$ & $\begin{array}{l}\mathbf{P}_{\mathbf{w w}} \\
0.255\end{array}$ & $\begin{array}{l}\mathbf{P}_{\mathbf{w d}} \\
0.745\end{array}$ & $\begin{array}{l}\mathbf{P}_{\mathrm{dw}} \\
0.108\end{array}$ & $\begin{array}{l}\mathbf{P}_{\text {dd }} \\
0.892\end{array}$ \\
\hline 653190 & 0.599 & 0.401 & 0.581 & 0.419 & 0.451 & 0.549 & 0.232 & 0.768 \\
\hline 653300 & 0.533 & 0.467 & 0.492 & 0.508 & 0.427 & 0.573 & 0.216 & 0.784 \\
\hline 653350 & 0.418 & 0.582 & 0.414 & 0.586 & 0.377 & 0.623 & 0.299 & 0.701 \\
\hline 653380 & 0.448 & 0.552 & 0.331 & 0.669 & 0.364 & 0.636 & 0.283 & 0.717 \\
\hline 653440 & 0.425 & 0.575 & 0.283 & 0.717 & 0.384 & 0.616 & 0.311 & 0.689 \\
\hline 653520 & 0.512 & 0.488 & 0.428 & 0.572 & 0.384 & 0.616 & 0.139 & 0.861 \\
\hline 653550 & 0.609 & 0.391 & 0.544 & 0.456 & 0.441 & & & 0.643 \\
\hline 653570 & 0.485 & 0.515 & 0.421 & 0.579 & 0.409 & 0.591 & 0.216 & 0.784 \\
\hline 653610 & 0.540 & 0.460 & 0.516 & 0.484 & 0.446 & 0.554 & 0.236 & 0.764 \\
\hline 653760 & 0.410 & 0.590 & 0.354 & 0.646 & 359 & .641 & 0.269 & 0.731 \\
\hline 653800 & 0.394 & 0.606 & 0.244 & 0.7 & 0.313 & 0.688 & 0.357 & 0.643 \\
\hline 653870 & 0.337 & 0.663 & 0.211 & 0.789 & 0.291 & 0.709 & 0.215 & 0.785 \\
\hline 654720 & 0.344 & 0.656 & 0.137 & 0.863 & 0.372 & 0.628 & 0.180 & 0.820 \\
\hline 655010 & 0.286 & 0.714 & 0.228 & 0.772 & 0.174 & 0.826 & 0.051 & 0.949 \\
\hline 655020 & 0.320 & 0.680 & 0.295 & 0.705 & 0.208 & 0.792 & 0.103 & 0.897 \\
\hline 655030 & 0.313 & 0.687 & 0.347 & 0.653 & 0.193 & 0.807 & 0.117 & 0.883 \\
\hline 655050 & 0.298 & 0.702 & 0.378 & 0.622 & 0.219 & 0.781 & 0.142 & 0.858 \\
\hline 655070 & 0.409 & 0.591 & 0.348 & 0.652 & 0.189 & 0.8 & & 0.876 \\
\hline 655100 & 0.455 & & 0.462 & 0.5 & 0.283 & 0.717 & & 0.829 \\
\hline 655160 & 0.438 & 62 & 0.354 & 0.6 & 0.236 & 0.764 & 0.145 & 0.855 \\
\hline 655180 & 0.376 & 0.624 & 0.414 & 0.586 & 0.283 & 0.717 & 0.122 & 0.878 \\
\hline 655220 & 0.433 & .567 & 0.475 & 0.525 & 0.294 & 0.706 & 0.240 & 0.760 \\
\hline 655280 & 0.491 & 0.509 & 0.554 & 0.446 & 0.403 & 0.597 & 0.343 & 0.657 \\
\hline 655360 & 0.410 & 0.590 & 0.450 & 0.550 & 0.429 & 0.571 & 0.257 & 0.743 \\
\hline 655450 & 0.469 & 0.531 & 0.394 & 0.606 & 0.408 & 0.592 & 0.262 & 0.738 \\
\hline 655480 & 0.571 & 0.429 & 0.551 & 0.449 & 0.474 & 0.526 & 0.280 & 0.720 \\
\hline 655550 & 0.437 & 0.563 & 0.328 & 0.672 & 0.321 & 0.679 & 0.230 & 0.770 \\
\hline 655570 & 0.506 & 0.494 & 0.358 & 0.642 & 0.388 & 0.612 & 0.424 & 0.576 \\
\hline 655600 & 0.497 & 0.503 & 0.369 & 0.631 & 0.325 & 0.675 & 0.277 & 0.723 \\
\hline 655620 & 0.449 & 0.551 & 0.286 & 0.714 & 0.374 & 0.626 & 0.301 & 0.699 \\
\hline 655630 & 0.423 & & & & & 0.625 & & 0.691 \\
\hline & 0.390 & & & 0.777 & & & 0.292 & 0.708 \\
\hline 655850 & 0.488 & 0.512 & 0.366 & 0.634 & 0.530 & 0.470 & 0.421 & 0.579 \\
\hline 655920 & 0.674 & 0.326 & 0.467 & 0.533 & 0.567 & 0.433 & 0.401 & 0.599 \\
\hline 655940 & 0.457 & 0.543 & 0.251 & 0.749 & 0.462 & 0.538 & 0.290 & 0.710 \\
\hline 655990 & 0.383 & 0.617 & 0.197 & 0.803 & 0.432 & 0.568 & 0.264 & 0.736 \\
\hline
\end{tabular}




\begin{tabular}{|c|c|c|c|c|c|c|c|c|}
\hline \multirow[t]{2}{*}{ Gauge } & \multicolumn{4}{|l|}{ Nov } & \multicolumn{4}{|l|}{ Dec } \\
\hline & $\mathbf{P}_{\mathrm{ww}}$ & $\mathbf{P}_{\mathrm{wd}}$ & $\mathbf{P}_{\mathrm{dw}}$ & $\mathbf{P}_{\mathrm{dd}}$ & $\mathbf{P}_{\mathrm{ww}}$ & $\mathbf{P}_{\mathrm{wd}}$ & $\mathbf{P}_{\mathrm{dw}}$ & $\mathbf{P}_{\mathrm{dd}}$ \\
\hline 653060 & 0.286 & 0.714 & 0.005 & 0.995 & 0.000 & 1.000 & 0.001 & 0.999 \\
\hline 653190 & 0.300 & 0.700 & 0.021 & 0.979 & 0.000 & 1.000 & 0.003 & 0.997 \\
\hline 653300 & 0.273 & 0.727 & 0.018 & 0.982 & 0.000 & 1.000 & 0.010 & 0.990 \\
\hline 653350 & 0.147 & 0.853 & 0.034 & 0.966 & 0.111 & 0.889 & 0.010 & 0.990 \\
\hline 653380 & 0.246 & 0.754 & 0.063 & 0.937 & 0.000 & 1.000 & 0.029 & 0.971 \\
\hline 653440 & 0.275 & 0.725 & 0.114 & 0.886 & 0.091 & 0.909 & 0.041 & 0.959 \\
\hline 653520 & 0.154 & 0.846 & 0.023 & 0.977 & 0.000 & 1.000 & 0.004 & 0.996 \\
\hline 653550 & 0.370 & 0.630 & 0.044 & 0.956 & 0.000 & 1.000 & 0.011 & 0.989 \\
\hline 653570 & 0.214 & 0.786 & 0.028 & 0.972 & 0.000 & 1.000 & 0.009 & 0.991 \\
\hline 653610 & 0.229 & 0.771 & 0.040 & 0.960 & 0.250 & 0.750 & 0.014 & 0.986 \\
\hline 653760 & 0.073 & 0.927 & 0.058 & 0.942 & 0.000 & 1.000 & 0.021 & 0.979 \\
\hline 653800 & 0.194 & 0.806 & 0.109 & 0.891 & 0.133 & 0.867 & 0.026 & 0.974 \\
\hline 653870 & 0.157 & 0.843 & 0.079 & 0.921 & 0.071 & 0.929 & 0.032 & 0.968 \\
\hline 654720 & 0.111 & 0.889 & 0.086 & 0.914 & 0.000 & 1.000 & 0.041 & 0.959 \\
\hline 655010 & 0.000 & 1.000 & 0.003 & 0.997 & 0.000 & 1.000 & 0.007 & 0.993 \\
\hline 655020 & 0.200 & 0.800 & 0.004 & 0.996 & 0.000 & 1.000 & 0.003 & 0.997 \\
\hline 655030 & 0.133 & 0.867 & 0.014 & 0.986 & 0.200 & 0.800 & 0.005 & 0.995 \\
\hline 655050 & 0.200 & 0.800 & 0.016 & 0.984 & 0.000 & 1.000 & 0.006 & 0.994 \\
\hline 655070 & 0.091 & 0.909 & 0.011 & 0.989 & 0.333 & 0.667 & 0.006 & 0.994 \\
\hline 655100 & 0.263 & 0.737 & 0.029 & 0.971 & 0.000 & 1.000 & 0.008 & 0.992 \\
\hline 655160 & 0.412 & 0.588 & 0.013 & 0.987 & 0.000 & 1.000 & 0.003 & 0.997 \\
\hline 655180 & 0.313 & 0.688 & 0.027 & 0.973 & 0.200 & 0.800 & 0.002 & 0.998 \\
\hline 655220 & 0.138 & 0.862 & 0.033 & 0.967 & 0.167 & 0.833 & 0.007 & 0.993 \\
\hline 655280 & 0.254 & 0.746 & 0.079 & 0.921 & 0.200 & 0.800 & 0.013 & 0.987 \\
\hline 655360 & 0.179 & 0.821 & 0.064 & 0.936 & 0.167 & 0.833 & 0.008 & 0.992 \\
\hline 655450 & 0.226 & 0.774 & 0.085 & 0.915 & 0.250 & 0.750 & 0.028 & 0.972 \\
\hline 655480 & 0.218 & 0.782 & 0.100 & 0.900 & 0.179 & 0.821 & 0.038 & 0.962 \\
\hline 655550 & 0.185 & 0.815 & 0.062 & 0.938 & 0.080 & 0.920 & 0.036 & 0.964 \\
\hline 655570 & 0.321 & 0.679 & 0.286 & 0.714 & 0.240 & 0.760 & 0.097 & 0.903 \\
\hline 655600 & 0.333 & 0.667 & 0.098 & 0.902 & 0.133 & 0.867 & 0.052 & 0.948 \\
\hline 655620 & 0.276 & 0.724 & 0.108 & 0.892 & 0.032 & 0.968 & 0.049 & 0.951 \\
\hline 655630 & 0.287 & 0.713 & 0.131 & 0.869 & 0.194 & 0.806 & 0.049 & 0.951 \\
\hline 655780 & 0.409 & 0.591 & 0.319 & 0.681 & 0.321 & 0.679 & 0.144 & 0.856 \\
\hline 655850 & 0.425 & 0.575 & 0.359 & 0.641 & 0.274 & 0.726 & 0.162 & 0.838 \\
\hline 655920 & 0.517 & 0.483 & 0.362 & 0.638 & 0.349 & 0.651 & 0.223 & 0.777 \\
\hline 655940 & 0.427 & 0.573 & 0.319 & 0.681 & 0.277 & 0.723 & 0.154 & 0.846 \\
\hline 655990 & 0.349 & 0.651 & 0.274 & 0.726 & 0.293 & 0.707 & 0.112 & 0.888 \\
\hline
\end{tabular}




\begin{tabular}{|c|c|c|c|c|c|c|c|c|}
\hline \multirow{2}{*}{ Gauge } & \multicolumn{8}{|c|}{ 2nd-Order Markov Transition Probabilities } \\
\hline & $\mathbf{P}_{\mathrm{www}}$ & $P_{w w d}$ & $\mathbf{P}_{\mathrm{wdw}}$ & $\mathbf{P}_{\text {wdd }}$ & $P_{d w w}$ & $\mathbf{P}_{\mathrm{dwd}}$ & $\mathbf{P}_{\mathrm{ddw}}$ & $\mathbf{P}_{\text {ddd }}$ \\
\hline 653060 & 0.000 & 0.000 & 0.000 & 1.000 & 0.000 & 1.000 & 0.000 & 1.000 \\
\hline 653190 & 0.000 & 0.000 & 0.000 & 1.000 & 0.000 & 1.000 & 0.004 & 0.996 \\
\hline 653300 & 0.000 & 1.000 & 0.000 & 1.000 & 0.333 & 0.667 & 0.008 & 0.992 \\
\hline 653350 & 0.000 & 1.000 & 0.000 & 1.000 & 0.111 & 0.889 & 0.011 & 0.989 \\
\hline 653380 & 0.000 & 0.000 & 0.000 & 1.000 & 0.000 & 1.000 & 0.018 & 0.982 \\
\hline 653440 & 0.000 & 1.000 & 0.059 & 0.941 & 0.138 & 0.862 & 0.036 & 0.964 \\
\hline 653520 & 0.000 & 0.000 & 0.000 & 1.000 & 0.000 & 1.000 & 0.002 & 0.998 \\
\hline 653550 & 0.000 & 0.000 & 0.000 & 0.000 & 0.000 & 0.000 & 0.002 & 0.998 \\
\hline 653570 & 0.000 & 1.000 & 0.000 & 1.000 & 0.500 & 0.500 & 0.006 & 0.994 \\
\hline 653610 & 0.000 & 0.000 & 0.167 & 0.833 & 0.000 & 1.000 & 0.011 & 0.989 \\
\hline 653760 & 0.000 & 1.000 & 0.000 & 1.000 & 0.000 & 1.000 & 0.018 & 0.982 \\
\hline 653800 & 0.000 & 0.000 & 0.143 & 0.857 & 0.000 & 1.000 & 0.007 & 0.993 \\
\hline 653870 & 0.000 & 1.000 & 0.059 & 0.941 & 0.176 & 0.824 & 0.019 & 0.981 \\
\hline 654720 & 0.000 & 1.000 & 0.000 & 1.000 & 0.125 & 0.875 & 0.025 & 0.975 \\
\hline 655010 & 0.000 & 0.000 & 0.000 & 1.000 & 0.000 & 1.000 & 0.001 & 0.999 \\
\hline 655020 & 0.000 & 0.000 & 0.000 & 1.000 & 0.000 & 1.000 & 0.001 & 0.999 \\
\hline 655030 & 0.000 & 0.000 & 0.000 & 1.000 & 0.000 & 1.000 & 0.000 & 1.000 \\
\hline 655050 & 0.000 & 0.000 & 0.000 & 1.000 & 0.000 & 1.000 & 0.002 & 0.998 \\
\hline 655070 & 0.000 & 0.000 & 0.000 & 0.000 & 0.000 & 0.000 & 0.000 & 1.000 \\
\hline 655100 & 0.000 & 0.000 & 0.000 & 1.000 & 0.000 & 1.000 & 0.004 & 0.996 \\
\hline 655160 & 0.000 & 0.000 & 0.000 & 1.000 & 0.000 & 1.000 & 0.004 & 0.996 \\
\hline 655180 & 0.000 & 0.000 & 0.000 & 0.000 & 0.000 & 0.000 & 0.000 & 1.000 \\
\hline 655220 & 0.000 & 1.000 & 0.000 & 1.000 & 0.000 & 1.000 & 0.011 & 0.989 \\
\hline 655280 & 0.250 & 0.750 & 0.000 & 1.000 & 0.111 & 0.889 & 0.014 & 0.986 \\
\hline 655360 & 0.000 & 1.000 & 0.000 & 1.000 & 0.143 & 0.857 & 0.007 & 0.993 \\
\hline 655450 & 0.000 & 1.000 & 0.000 & 1.000 & 0.200 & 0.800 & 0.016 & 0.984 \\
\hline 655480 & 0.000 & 1.000 & 0.000 & 1.000 & 0.222 & 0.778 & 0.016 & 0.984 \\
\hline 655550 & 0.000 & 1.000 & 0.111 & 0.889 & 0.222 & 0.778 & 0.018 & 0.982 \\
\hline 655570 & 0.286 & 0.714 & 0.087 & 0.913 & 0.182 & 0.818 & 0.038 & 0.962 \\
\hline 655600 & 0.000 & 1.000 & 0.056 & 0.944 & 0.000 & 1.000 & 0.024 & 0.976 \\
\hline 655620 & 0.000 & 1.000 & 0.167 & 0.833 & 0.000 & 1.000 & 0.021 & 0.979 \\
\hline 655630 & 0.000 & 1.000 & 0.000 & 1.000 & 0.125 & 0.875 & 0.023 & 0.977 \\
\hline 655780 & 0.000 & 1.000 & 0.098 & 0.902 & 0.282 & 0.718 & 0.048 & 0.952 \\
\hline 655850 & 0.250 & 0.750 & 0.179 & 0.821 & 0.136 & 0.864 & 0.039 & 0.961 \\
\hline 655920 & 0.227 & 0.773 & 0.155 & 0.845 & 0.259 & 0.741 & 0.089 & 0.911 \\
\hline 655940 & 0.250 & 0.750 & 0.071 & 0.929 & 0.167 & 0.833 & 0.035 & 0.965 \\
\hline 655990 & 0.167 & 0.833 & 0.000 & 1.000 & 0.278 & 0.722 & 0.028 & 0.972 \\
\hline
\end{tabular}




\begin{tabular}{|c|c|c|c|c|c|c|c|c|}
\hline \multirow{2}{*}{ Gauge } & \multicolumn{8}{|c|}{ 2nd-Order Markov Transition Probabilities } \\
\hline & $\mathbf{P}_{\text {www }}$ & $\mathbf{P}_{\text {wwd }}$ & $\mathbf{P}_{\mathrm{wdw}}$ & $\mathbf{P}_{\text {wdd }}$ & $\mathbf{P}_{\mathrm{dww}}$ & $\mathbf{P}_{\mathrm{dwd}}$ & $\mathbf{P}_{\mathrm{ddw}}$ & $\mathbf{P}_{\text {ddd }}$ \\
\hline 653060 & 0.000 & 0.000 & 0.000 & 1.000 & 0.000 & 1.000 & 0.009 & 0.991 \\
\hline 653190 & 0.000 & 1.000 & 0.250 & 0.750 & 0.400 & 0.600 & 0.007 & 0.993 \\
\hline 653300 & 0.000 & 1.000 & 0.000 & 1.000 & 0.200 & 0.800 & 0.012 & 0.988 \\
\hline 653350 & 0.167 & 0.833 & 0.000 & 1.000 & 0.217 & 0.783 & 0.033 & 0.967 \\
\hline 653380 & 0.000 & 1.000 & 0.121 & 0.879 & 0.147 & 0.853 & 0.048 & 0.952 \\
\hline 653440 & 0.000 & 1.000 & 0.081 & 0.919 & 0.159 & 0.841 & 0.054 & 0.946 \\
\hline 653520 & 0.000 & 0.000 & 0.333 & 0.667 & 0.000 & 1.000 & 0.008 & 0.992 \\
\hline 653550 & 0.000 & 1.000 & 0.143 & 0.857 & 0.111 & 0.889 & 0.026 & 0.974 \\
\hline 653570 & 0.000 & 1.000 & 0.250 & 0.750 & 0.200 & 0.800 & 0.011 & 0.989 \\
\hline 653610 & 0.000 & 0.000 & 0.000 & 1.000 & 0.000 & 1.000 & 0.016 & 0.984 \\
\hline 653760 & 0.000 & 1.000 & 0.250 & 0.750 & 0.130 & 0.870 & 0.042 & 0.958 \\
\hline 653800 & 0.167 & 0.833 & 0.273 & 0.727 & 0.227 & 0.773 & 0.050 & 0.950 \\
\hline 653870 & 0.000 & 1.000 & 0.074 & 0.926 & 0.103 & 0.897 & 0.044 & 0.956 \\
\hline 654720 & 0.000 & 0.000 & 0.125 & 0.875 & 0.063 & 0.938 & 0.052 & 0.948 \\
\hline 655010 & 0.000 & 0.000 & 0.000 & 1.000 & 0.000 & 1.000 & 0.004 & 0.996 \\
\hline 655020 & 0.000 & 0.000 & 0.000 & 1.000 & 0.000 & 1.000 & 0.001 & 0.999 \\
\hline 655030 & 0.000 & 0.000 & 0.333 & 0.667 & 0.000 & 1.000 & 0.004 & 0.996 \\
\hline 655050 & 0.000 & 0.000 & 0.000 & 1.000 & 0.000 & 1.000 & 0.005 & 0.995 \\
\hline 655070 & 0.000 & 0.000 & 0.000 & 1.000 & 0.000 & 1.000 & 0.003 & 0.997 \\
\hline 655100 & 0.000 & 1.000 & 0.000 & 1.000 & 0.167 & 0.833 & 0.008 & 0.992 \\
\hline 655160 & 0.000 & 0.000 & 0.000 & 1.000 & 0.000 & 1.000 & 0.001 & 0.999 \\
\hline 655180 & 0.000 & 1.000 & 0.000 & 1.000 & 0.000 & 1.000 & 0.007 & 0.993 \\
\hline 655220 & 0.000 & 1.000 & 0.286 & 0.714 & 0.000 & 1.000 & 0.010 & 0.990 \\
\hline 655280 & 0.000 & 1.000 & 0.000 & 1.000 & 0.000 & 1.000 & 0.016 & 0.984 \\
\hline 655360 & 0.000 & 1.000 & 0.071 & 0.929 & 0.077 & 0.923 & 0.029 & 0.971 \\
\hline 655450 & 0.250 & 0.750 & 0.174 & 0.826 & 0.240 & 0.760 & 0.041 & 0.959 \\
\hline 655480 & 0.083 & 0.917 & 0.135 & 0.865 & 0.297 & 0.703 & 0.062 & 0.938 \\
\hline 655550 & 0.000 & 1.000 & 0.000 & 1.000 & 0.150 & 0.850 & 0.039 & 0.961 \\
\hline 655570 & 0.125 & 0.875 & 0.228 & 0.772 & 0.233 & 0.767 & 0.105 & 0.895 \\
\hline 655600 & 0.111 & 0.889 & 0.111 & 0.889 & 0.170 & 0.830 & 0.090 & 0.910 \\
\hline 655620 & 0.000 & 1.000 & 0.125 & 0.875 & 0.116 & 0.884 & 0.076 & 0.924 \\
\hline 655630 & 0.000 & 1.000 & 0.133 & 0.867 & 0.091 & 0.909 & 0.066 & 0.934 \\
\hline 655780 & 0.385 & 0.615 & 0.167 & 0.833 & 0.164 & 0.836 & 0.082 & 0.918 \\
\hline 655850 & 0.000 & 1.000 & 0.264 & 0.736 & 0.207 & 0.793 & 0.108 & 0.892 \\
\hline 655920 & 0.211 & 0.789 & 0.121 & 0.879 & 0.220 & 0.780 & 0.107 & 0.893 \\
\hline 655940 & 0.000 & 1.000 & 0.042 & 0.958 & 0.040 & 0.960 & 0.044 & 0.956 \\
\hline 655990 & 0.000 & 1.000 & 0.069 & 0.931 & 0.121 & 0.879 & 0.069 & 0.931 \\
\hline
\end{tabular}




\begin{tabular}{|c|c|c|c|c|c|c|c|c|}
\hline \multirow[t]{2}{*}{ Gauge } & \multicolumn{8}{|c|}{$\begin{array}{l}\text { 2nd-Order Markov Transition Probabilities } \\
\text { Mar }\end{array}$} \\
\hline & $\mathbf{P}_{\text {www }}$ & $\mathbf{P}_{\mathrm{wwd}}$ & $\mathbf{P}_{\mathrm{wdw}}$ & $\mathbf{P}_{\text {wdd }}$ & $\mathbf{P}_{\mathrm{dww}}$ & $\mathbf{P}_{\mathrm{dwd}}$ & $\mathbf{P}_{\mathrm{ddw}}$ & $\mathbf{P}_{\text {ddd }}$ \\
\hline 653060 & 0.000 & 1.000 & 0.000 & 1.000 & 0.188 & 0.813 & 0.020 & 0.980 \\
\hline 653190 & 0.313 & 0.688 & 0.179 & 0.821 & 0.216 & 0.784 & 0.042 & 0.958 \\
\hline 653300 & 0.100 & 0.900 & 0.167 & 0.833 & 0.200 & 0.800 & 0.073 & 0.927 \\
\hline 653350 & 0.174 & 0.826 & 0.186 & 0.814 & 0.261 & 0.739 & 0.107 & 0.893 \\
\hline 653380 & 0.105 & 0.895 & 0.231 & 0.769 & 0.170 & 0.830 & 0.151 & 0.849 \\
\hline 653440 & 0.138 & 0.862 & 0.155 & 0.845 & 0.247 & 0.753 & 0.124 & 0.876 \\
\hline 653520 & 0.000 & 1.000 & 0.000 & 1.000 & 0.278 & 0.722 & 0.038 & 0.962 \\
\hline 653550 & 0.200 & 0.800 & 0.056 & 0.944 & 0.063 & 0.938 & 0.092 & 0.908 \\
\hline 653570 & 0.000 & 1.000 & 0.105 & 0.895 & 0.222 & 0.778 & 0.051 & 0.949 \\
\hline 653610 & 0.000 & 1.000 & 0.173 & 0.827 & 0.132 & 0.868 & 0.087 & 0.913 \\
\hline 653760 & 0.318 & 0.682 & 0.185 & 0.815 & 0.141 & 0.859 & 0.104 & 0.896 \\
\hline 653800 & 0.077 & 0.923 & 0.173 & 0.827 & 0.227 & 0.773 & 0.128 & 0.872 \\
\hline 653870 & 0.050 & 0.950 & 0.176 & 0.824 & 0.275 & 0.725 & 0.094 & 0.906 \\
\hline 654720 & 0.143 & 0.857 & 0.161 & 0.839 & 0.129 & 0.871 & 0.077 & 0.923 \\
\hline 655010 & 0.000 & 1.000 & 0.000 & 1.000 & 0.250 & 0.750 & 0.011 & 0.989 \\
\hline 655020 & 0.000 & 1.000 & 0.000 & 1.000 & 0.083 & 0.917 & 0.019 & 0.981 \\
\hline 655030 & 0.200 & 0.800 & 0.000 & 1.000 & 0.211 & 0.789 & 0.024 & 0.976 \\
\hline 655050 & 0.200 & 0.800 & 0.067 & 0.933 & 0.333 & 0.667 & 0.032 & 0.968 \\
\hline 655070 & 0.500 & 0.500 & 0.143 & 0.857 & 0.182 & 0.818 & 0.028 & 0.972 \\
\hline 655100 & 0.100 & 0.900 & 0.139 & 0.861 & 0.263 & 0.737 & 0.048 & 0.952 \\
\hline 655160 & 0.143 & 0.857 & 0.050 & 0.950 & 0.261 & 0.739 & 0.029 & 0.971 \\
\hline 655180 & 0.111 & 0.889 & 0.000 & 1.000 & 0.471 & 0.529 & 0.044 & 0.956 \\
\hline 655220 & 0.250 & 0.750 & 0.057 & 0.943 & 0.265 & 0.735 & 0.054 & 0.946 \\
\hline 655280 & 0.000 & 1.000 & 0.152 & 0.848 & 0.143 & 0.857 & 0.066 & 0.934 \\
\hline 655360 & 0.000 & 1.000 & 0.077 & 0.923 & 0.200 & 0.800 & 0.079 & 0.921 \\
\hline 655450 & 0.389 & 0.611 & 0.180 & 0.820 & 0.179 & 0.821 & 0.092 & 0.908 \\
\hline 655480 & 0.161 & 0.839 & 0.253 & 0.747 & 0.267 & 0.733 & 0.176 & 0.824 \\
\hline 655550 & 0.250 & 0.750 & 0.145 & 0.855 & 0.156 & 0.844 & 0.134 & 0.866 \\
\hline 655570 & 0.377 & 0.623 & 0.234 & 0.766 & 0.359 & 0.641 & 0.206 & 0.794 \\
\hline 655600 & 0.111 & 0.889 & 0.242 & 0.758 & 0.191 & 0.809 & 0.188 & 0.812 \\
\hline 655620 & 0.286 & 0.714 & 0.253 & 0.747 & 0.322 & 0.678 & 0.160 & 0.840 \\
\hline 655630 & 0.222 & 0.778 & 0.289 & 0.711 & 0.288 & 0.712 & 0.155 & 0.845 \\
\hline 655780 & 0.125 & 0.875 & 0.140 & 0.860 & 0.306 & 0.694 & 0.176 & 0.824 \\
\hline 655850 & 0.216 & 0.784 & 0.202 & 0.798 & 0.283 & 0.717 & 0.198 & 0.802 \\
\hline 655920 & 0.261 & 0.739 & 0.214 & 0.786 & 0.203 & 0.797 & 0.125 & 0.875 \\
\hline 655940 & 0.000 & 1.000 & 0.074 & 0.926 & 0.085 & 0.915 & 0.095 & 0.905 \\
\hline 655990 & 0.500 & 0.500 & 0.021 & 0.979 & 0.091 & 0.909 & 0.089 & 0.911 \\
\hline
\end{tabular}


2nd-Order Markov Transition Probabilities

\section{Gauge Apr}

\begin{tabular}{|c|c|c|c|c|c|c|c|c|}
\hline & $\mathbf{P}_{\mathrm{www}}$ & $P_{w w d}$ & $\mathbf{P}_{\mathrm{wdw}}$ & $\mathbf{P}_{\text {wdd }}$ & $\mathbf{P}_{\mathrm{dww}}$ & $\mathbf{P}_{\mathrm{dwd}}$ & $\mathbf{P}_{\mathrm{ddw}}$ & $\mathbf{P}_{\text {ddd }}$ \\
\hline 653060 & 0.111 & 0.889 & 0.114 & 0.886 & 0.200 & 0.800 & 0.083 & 0.917 \\
\hline 653190 & 0.257 & 0.743 & 0.287 & 0.713 & 0.301 & 0.699 & 0.175 & 0.825 \\
\hline 653300 & 0.053 & 0.947 & 0.172 & 0.828 & 0.237 & 0.763 & 0.143 & 0.857 \\
\hline 653350 & 0.200 & 0.800 & 0.229 & 0.771 & 0.307 & 0.693 & 0.226 & 0.774 \\
\hline 653380 & 0.275 & 0.725 & 0.242 & 0.758 & 0.289 & 0.711 & 0.270 & 0.730 \\
\hline 653440 & 0.227 & 0.773 & 0.238 & 0.762 & 0.273 & 0.727 & 0.217 & 0.783 \\
\hline 653520 & 0.000 & 1.000 & 0.205 & 0.795 & 0.122 & 0.878 & 0.106 & 0.894 \\
\hline 653550 & 0.357 & 0.643 & 0.281 & 0.719 & 0.203 & 0.797 & 0.198 & 0.802 \\
\hline 653570 & 0.375 & 0.625 & 0.250 & 0.750 & 0.196 & 0.804 & 0.167 & 0.833 \\
\hline 653610 & 0.240 & 0.760 & 0.200 & 0.800 & 0.211 & 0.789 & 0.234 & 0.766 \\
\hline 553760 & 0.244 & 0.756 & 0.237 & 0.763 & 0.287 & 0.713 & 0.223 & 0.777 \\
\hline 53800 & 0.100 & 0.900 & 0.213 & 0.787 & 0.127 & 0.873 & 0.233 & 0.767 \\
\hline 553870 & 0.222 & 0.778 & 0.258 & 0.742 & 0.323 & 0.677 & 0.161 & 0.839 \\
\hline 654720 & 0.200 & 0.800 & 0.125 & 0.875 & 0.163 & 0.837 & 0.122 & 0.878 \\
\hline 655010 & 0.000 & 0.000 & 0.067 & 0.933 & 0.000 & 1.000 & 0.019 & 0.981 \\
\hline 655020 & 0.000 & 1.000 & 0.091 & 0.909 & 0.048 & 0.952 & 0.031 & 0.969 \\
\hline 655030 & 0.000 & 1.000 & 0.063 & 0.938 & 0.163 & 0.837 & 0.060 & 0.940 \\
\hline 655050 & 0.111 & 0.889 & 0.115 & 0.885 & 0.320 & 0.680 & 0.061 & 0.939 \\
\hline 655070 & 0.250 & 0.750 & 0.116 & 0.884 & 0.163 & 0.837 & 0.080 & 0.920 \\
\hline 655100 & 0.240 & 0.760 & 0.148 & 0.852 & 0.259 & 0.7 & 0.1 & 0.885 \\
\hline 655160 & 0.308 & 0.692 & 0.127 & 0.873 & 0.175 & 0.825 & 0.098 & 0.902 \\
\hline 655180 & 0.222 & 0.778 & 0.061 & 0.939 & 0.242 & 0.758 & 0.091 & 0.909 \\
\hline 655220 & 0.095 & 0.905 & 0.172 & 0.828 & 0.226 & 0.774 & 0.148 & 0.852 \\
\hline 655280 & 0.385 & 0.615 & 0.210 & 0.790 & 0.200 & 0.800 & 0.184 & 0.816 \\
\hline 655360 & 0.136 & 0.864 & 0.178 & 0.822 & 0.226 & 0.774 & 0.171 & 0.829 \\
\hline 655450 & 0.074 & 0.926 & 0.200 & 0.800 & 0.258 & 0.742 & 0.208 & 0.792 \\
\hline 655480 & 0.194 & 0.806 & 0.220 & 0.780 & 0.245 & 0.755 & 0.262 & 0.738 \\
\hline 655550 & 0.194 & 0.806 & 0.220 & 0.780 & 0.267 & 0.733 & 0.203 & 0.797 \\
\hline 655570 & 0.254 & 0.746 & 0.333 & 0.667 & 0.300 & 0.700 & 0.302 & 0.698 \\
\hline 655600 & 0.182 & 0.818 & 0.320 & 0.680 & 0.222 & 0.778 & 0.220 & 0.780 \\
\hline 655620 & 0.279 & 0.721 & 0.226 & 0.774 & 0.239 & 0.761 & 0.211 & 0.789 \\
\hline 655630 & 0.313 & 0.688 & 0.267 & 0.733 & 0.206 & 0.794 & 0.244 & 0.756 \\
\hline 655780 & 0.359 & 0.641 & 0.313 & 0.687 & 0.283 & 0.717 & 0.227 & 0.773 \\
\hline 655850 & 0.293 & 0.707 & 0.288 & 0.712 & 0.290 & & 0.259 & 0.741 \\
\hline 655920 & 0.424 & 0.576 & 0.248 & 0.752 & 0.349 & & 0.236 & 0.764 \\
\hline 655940 & 0.318 & 0.682 & 0.167 & 0.833 & 0.239 & 0.761 & 0.162 & 0.838 \\
\hline 655990 & 0.405 & 0.595 & 0.239 & 0.761 & 0.244 & 0.756 & 0.161 & 0.839 \\
\hline
\end{tabular}




\begin{tabular}{|c|c|c|c|c|c|c|c|c|}
\hline \multirow[t]{2}{*}{ Gauge } & \multicolumn{8}{|c|}{ 2nd-Order Markov Transition Probabilities } \\
\hline & $\mathbf{P}_{\mathrm{www}}$ & $P_{\text {wwd }}$ & $\mathbf{P}_{\mathrm{wdw}}$ & $\mathbf{P}_{\text {wdd }}$ & $\mathbf{P}_{\mathrm{dww}}$ & $\mathbf{P}_{\mathrm{dwd}}$ & $\mathbf{P}_{\mathrm{ddw}}$ & $\mathbf{P}_{\text {ddd }}$ \\
\hline 653060 & 0.195 & 0.805 & 0.250 & 0.750 & 0.256 & 0.744 & 0.202 & 0.798 \\
\hline 653190 & 0.362 & 0.638 & 0.292 & 0.708 & 0.305 & 0.695 & 0.227 & 0.773 \\
\hline 653300 & 0.270 & 0.730 & 0.348 & 0.652 & 0.250 & 0.750 & 0.304 & 0.696 \\
\hline 653350 & 0.235 & 0.765 & 0.266 & 0.734 & 0.289 & 0.711 & 0.273 & 0.727 \\
\hline 653380 & 0.366 & 0.634 & 0.244 & 0.756 & 0.345 & 0.655 & 0.298 & 0.702 \\
\hline 653440 & 0.365 & 0.635 & 0.340 & 0.660 & 0.341 & 0.659 & 0.359 & 0.641 \\
\hline 653520 & 0.409 & 0.591 & 0.148 & 0.852 & 0.242 & 0.758 & 0.204 & 0.796 \\
\hline 653550 & 0.426 & 0.574 & 0.333 & 0.667 & 0.380 & 0.620 & 0.308 & 0.692 \\
\hline 653570 & 0.241 & 0.759 & 0.242 & 0.758 & 0.362 & 0.638 & 0.222 & 0.778 \\
\hline 653610 & 0.213 & 0.787 & 0.355 & 0.645 & 0.347 & 0.653 & 0.292 & 0.708 \\
\hline 653760 & 0.300 & 0.700 & 0.229 & 0.771 & 0.308 & 0.692 & 0.251 & 0.749 \\
\hline 653800 & 0.242 & 0.758 & 0.359 & 0.641 & 0.316 & 0.684 & 0.210 & 0.790 \\
\hline 653870 & 0.397 & 0.603 & 0.377 & 0.623 & 0.287 & 0.713 & 0.258 & 0.742 \\
\hline 654720 & 0.133 & 0.867 & 0.281 & 0.719 & 0.320 & 0.680 & 0.117 & 0.883 \\
\hline 655010 & 0.083 & 0.917 & 0.109 & 0.891 & 0.224 & 0.776 & 0.071 & 0.929 \\
\hline 655020 & 0.357 & 0.643 & 0.103 & 0.897 & 0.153 & 0.847 & 0.099 & 0.901 \\
\hline 655030 & 0.111 & 0.889 & 0.175 & 0.825 & 0.223 & 0.777 & 0.175 & 0.825 \\
\hline 655050 & 0.250 & 0.750 & 0.157 & 0.843 & 0.265 & 0.735 & 0.140 & 0.860 \\
\hline 655070 & 0.240 & 0.760 & 0.173 & 0.827 & 0.202 & 0.798 & 0.184 & 0.816 \\
\hline 655100 & 0.119 & 0.881 & 0.277 & 0.723 & 0.235 & 0.765 & 0.216 & 0.784 \\
\hline 655160 & 0.216 & 0.784 & 0.224 & 0.776 & 0.280 & 0.720 & 0.188 & 0.812 \\
\hline 655180 & 0.200 & 0.800 & 0.167 & 0.833 & 0.234 & 0.766 & 0.193 & 0.807 \\
\hline 655220 & 0.265 & 0.735 & 0.241 & 0.759 & 0.255 & 0.745 & 0.219 & 0.781 \\
\hline 655280 & 0.212 & 0.788 & 0.327 & 0.673 & 0.204 & 0.796 & 0.221 & 0.779 \\
\hline 655360 & 0.261 & 0.739 & 0.364 & 0.636 & 0.256 & 0.744 & 0.234 & 0.766 \\
\hline 655450 & 0.393 & 0.607 & 0.333 & 0.667 & 0.301 & 0.699 & 0.280 & 0.720 \\
\hline 655480 & 0.431 & 0.569 & 0.353 & 0.647 & 0.250 & 0.750 & 0.275 & 0.725 \\
\hline 655550 & 0.189 & 0.811 & 0.257 & 0.743 & 0.288 & 0.712 & 0.219 & 0.781 \\
\hline 655570 & 0.448 & 0.552 & 0.473 & 0.527 & 0.500 & 0.500 & 0.335 & 0.665 \\
\hline 655600 & 0.200 & 0.800 & 0.368 & 0.632 & 0.344 & 0.656 & 0.312 & 0.688 \\
\hline 655620 & 0.266 & 0.734 & 0.354 & 0.646 & 0.400 & 0.600 & 0.315 & 0.685 \\
\hline 655630 & 0.263 & 0.737 & 0.371 & 0.629 & 0.331 & 0.669 & 0.300 & 0.700 \\
\hline 655780 & 0.522 & 0.478 & 0.443 & 0.557 & 0.486 & 0.514 & 0.375 & 0.625 \\
\hline 655850 & 0.520 & 0.480 & 0.511 & 0.489 & 0.458 & 0.542 & 0.399 & 0.601 \\
\hline 655920 & 0.643 & 0.357 & 0.516 & 0.484 & 0.581 & 0.419 & 0.366 & 0.634 \\
\hline 655940 & 0.570 & 0.430 & 0.443 & 0.557 & 0.435 & 0.565 & 0.296 & 0.704 \\
\hline 655990 & 0.569 & 0.431 & 0.393 & 0.607 & 0.500 & 0.500 & 0.314 & 0.686 \\
\hline
\end{tabular}




\begin{tabular}{|c|c|c|c|c|c|c|c|c|}
\hline \multirow[t]{2}{*}{ Gauge } & \multicolumn{8}{|c|}{$\begin{array}{l}\text { 2nd-Order Markov Transition Probabilities } \\
\text { Jun }\end{array}$} \\
\hline & $\mathbf{P}_{\mathrm{www}}$ & $P_{w w d}$ & $\mathbf{P}_{\mathrm{wdw}}$ & $\mathbf{P}_{\text {wdd }}$ & $\mathbf{P}_{\mathrm{dww}}$ & $P_{d w d}$ & $\mathbf{P}_{\mathrm{ddw}}$ & $\mathbf{P}_{\text {ddd }}$ \\
\hline 653060 & 0.237 & 0.763 & 0.331 & 0.669 & 0.315 & 0.685 & 0.316 & 0.684 \\
\hline 653190 & 0.410 & 0.590 & 0.359 & 0.641 & 0.423 & 0.577 & 0.370 & 0.630 \\
\hline 653300 & 0.343 & 0.657 & 0.410 & 0.590 & 0.425 & 0.575 & 0.344 & 0.656 \\
\hline 653350 & 0.402 & 0.598 & 0.419 & 0.581 & 0.486 & 0.514 & 0.357 & 0.643 \\
\hline 653380 & 0.352 & 0.648 & 0.360 & 0.640 & 0.370 & 0.630 & 0.377 & 0.623 \\
\hline 653440 & 0.547 & 0.453 & 0.438 & 0.562 & 0.526 & 0.474 & 0.419 & 0.581 \\
\hline 653520 & 0.233 & 0.767 & 0.213 & 0.788 & 0.294 & 0.706 & 0.275 & 0.725 \\
\hline 653550 & 0.481 & 0.519 & 0.344 & 0.656 & 0.431 & 0.569 & 0.366 & 0.634 \\
\hline 653570 & 0.190 & 0.810 & 0.231 & 0.769 & 0.318 & 0.682 & 0.304 & 0.696 \\
\hline 653610 & 0.360 & 0.640 & 0.417 & 0.583 & 0.432 & 0.568 & 0.411 & 0.589 \\
\hline 653760 & 0.360 & 0.640 & 0.355 & 0.645 & 0.437 & 0.563 & 0.346 & 0.654 \\
\hline 653800 & 0.310 & 0.690 & 0.368 & 0.632 & 0.386 & 0.614 & 0.287 & 0.712 \\
\hline 653870 & 0.438 & 0.562 & 0.335 & 0.665 & 0.403 & 0.597 & 0.324 & 0.676 \\
\hline 654720 & 0.548 & 0.452 & 0.311 & 0.689 & 0.357 & 0.643 & 0.240 & 0.760 \\
\hline 655010 & 0.111 & 0.889 & 0.174 & 0.826 & 0.167 & 0.833 & 0.173 & 0.827 \\
\hline 655020 & 0.226 & 0.774 & 0.285 & 0.715 & 0.191 & 0.809 & 0.217 & 0.783 \\
\hline 655030 & 0.128 & 0.872 & 0.252 & 0.748 & 0.244 & 0.756 & 0.316 & 0.684 \\
\hline 655050 & 0.188 & 0.813 & 0.304 & 0.696 & 0.203 & 0.797 & 0.239 & 0.761 \\
\hline 655070 & 0.327 & 0.673 & 0.288 & 0.712 & 0.282 & 0.718 & 0.273 & 0.727 \\
\hline 655100 & 0.244 & 0.756 & 0.333 & 0.667 & 0.355 & 0.645 & 0.335 & 0.665 \\
\hline 655160 & 0.216 & 0.784 & 0.314 & 0.686 & 0.289 & 0.711 & 0.318 & 0.682 \\
\hline 655180 & 0.296 & 0.704 & 0.382 & 0.618 & 0.244 & 0.756 & 0.344 & 0.656 \\
\hline 655220 & 0.231 & 0.769 & 0.328 & 0.672 & 0.258 & 0.742 & 0.294 & 0.706 \\
\hline 655280 & 0.175 & 0.825 & 0.387 & 0.613 & 0.245 & 0.755 & 0.329 & 0.671 \\
\hline 655360 & 0.093 & 0.907 & 0.363 & 0.637 & 0.317 & 0.683 & 0.276 & 0.724 \\
\hline 655450 & 0.323 & 0.677 & 0.326 & 0.674 & 0.343 & 0.657 & 0.366 & 0.634 \\
\hline 655480 & 0.413 & 0.587 & 0.350 & 0.650 & 0.410 & 0.590 & 0.365 & 0.635 \\
\hline 655550 & 0.229 & 0.771 & 0.330 & 0.670 & 0.311 & 0.689 & 0.265 & 0.735 \\
\hline 655570 & 0.526 & 0.474 & 0.492 & 0.508 & 0.513 & 0.487 & 0.335 & 0.665 \\
\hline 655600 & 0.405 & 0.595 & 0.357 & 0.643 & 0.422 & 0.578 & 0.325 & 0.675 \\
\hline 655620 & 0.414 & 0.586 & 0.373 & 0.627 & 0.416 & 0.584 & 0.352 & 0.648 \\
\hline 655630 & 0.451 & 0.549 & 0.270 & 0.730 & 0.442 & 0.558 & 0.300 & 0.700 \\
\hline 655780 & 0.574 & 0.426 & 0.557 & 0.443 & 0.613 & 0.387 & 0.384 & 0.616 \\
\hline 655850 & 0.647 & 0.353 & 0.541 & 0.459 & 0.689 & 0.311 & 0.398 & 0.602 \\
\hline 655920 & 0.620 & 0.380 & 0.545 & 0.455 & 0.632 & 0.368 & 0.426 & 0.574 \\
\hline 655940 & 0.582 & 0.418 & 0.505 & 0.495 & 0.563 & 0.438 & 0.451 & 0.549 \\
\hline 655990 & 0.524 & 0.476 & 0.553 & 0.447 & 0.600 & 0.400 & 0.316 & 0.684 \\
\hline
\end{tabular}




\begin{tabular}{|c|c|c|c|c|c|c|c|c|}
\hline \multirow[t]{2}{*}{ Gauge } & \multicolumn{8}{|c|}{ 2nd-Order Markov Transition Probabilities } \\
\hline & $\mathbf{P}_{\text {www }}$ & $\mathbf{P}_{\mathrm{wwd}}$ & $\mathbf{P}_{\mathrm{wdw}}$ & $\mathbf{P}_{\text {wdd }}$ & $\mathbf{P}_{\mathrm{dww}}$ & $\mathbf{P}_{\mathrm{dwd}}$ & $\mathbf{P}_{\mathrm{ddw}}$ & $\mathbf{P}_{\text {ddd }}$ \\
\hline 653060 & 0.458 & 0.542 & 0.411 & 0.589 & 0.376 & 0.624 & 0.416 & 0.584 \\
\hline 653190 & 0.483 & 0.517 & 0.503 & 0.497 & 0.519 & 0.481 & 0.464 & 0.536 \\
\hline 653300 & 0.488 & 0.512 & 0.377 & 0.623 & 0.538 & 0.462 & 0.405 & 0.595 \\
\hline 653350 & 0.529 & 0.471 & 0.314 & 0.686 & 0.415 & 0.585 & 0.283 & 0.717 \\
\hline 653380 & 0.370 & 0.630 & 0.390 & 0.610 & 0.436 & 0.564 & 0.294 & 0.706 \\
\hline 653440 & 0.476 & 0.524 & 0.256 & 0.744 & 0.392 & 0.608 & 0.172 & 0.828 \\
\hline 653520 & 0.327 & 0.673 & 0.346 & 0.654 & 0.316 & 0.684 & 0.341 & 0.659 \\
\hline 653550 & 0.500 & 0.500 & 0.488 & 0.512 & 0.512 & 0.488 & 0.488 & 0.512 \\
\hline 653570 & 0.479 & 0.521 & 0.424 & 0.576 & 0.325 & 0.675 & 0.446 & 0.554 \\
\hline 653610 & 0.554 & 0.446 & 0.525 & 0.475 & 0.590 & 0.410 & 0.504 & 0.496 \\
\hline 653760 & 0.586 & 0.414 & 0.430 & 0.570 & 0.496 & 0.504 & 0.338 & 0.662 \\
\hline 653800 & 0.361 & 0.639 & 0.219 & 0.781 & 0.328 & 0.672 & 0.198 & 0.802 \\
\hline 653870 & 0.291 & 0.709 & 0.168 & 0.832 & 0.379 & 0.621 & 0.146 & 0.854 \\
\hline 654720 & 0.417 & 0.583 & 0.153 & 0.847 & 0.388 & 0.612 & 0.116 & 0.884 \\
\hline 655010 & 0.133 & 0.867 & 0.324 & 0.676 & 0.167 & 0.833 & 0.307 & 0.693 \\
\hline 655020 & 0.315 & 0.685 & 0.322 & 0.678 & 0.340 & 0.660 & 0.357 & 0.643 \\
\hline 655030 & 0.301 & 0.699 & 0.377 & 0.623 & 0.320 & 0.680 & 0.443 & 0.557 \\
\hline 655050 & 0.455 & 0.545 & 0.392 & 0.608 & 0.255 & 0.745 & 0.385 & 0.615 \\
\hline 655070 & 0.368 & 0.632 & 0.362 & 0.638 & 0.336 & 0.664 & 0.416 & 0.584 \\
\hline 655100 & 0.346 & 0.654 & 0.405 & 0.595 & 0.387 & 0.613 & 0.408 & 0.592 \\
\hline 655160 & 0.398 & 0.602 & 0.425 & 0.575 & 0.342 & 0.658 & 0.414 & 0.586 \\
\hline 655180 & 0.234 & 0.766 & 0.322 & 0.678 & 0.416 & 0.584 & 0.400 & 0.600 \\
\hline 655220 & 0.402 & 0.598 & 0.362 & 0.638 & 0.371 & 0.629 & 0.440 & 0.560 \\
\hline 655280 & 0.434 & 0.566 & 0.467 & 0.533 & 0.500 & 0.500 & 0.350 & 0.650 \\
\hline 655360 & 0.286 & 0.714 & 0.281 & 0.719 & 0.358 & 0.642 & 0.358 & 0.642 \\
\hline 655450 & 0.320 & 0.680 & 0.267 & 0.733 & 0.340 & 0.660 & 0.194 & 0.806 \\
\hline 655480 & 0.567 & 0.433 & 0.402 & 0.598 & 0.600 & 0.400 & 0.443 & 0.557 \\
\hline 655550 & 0.327 & 0.673 & 0.233 & 0.767 & 0.376 & 0.624 & 0.184 & 0.816 \\
\hline 655570 & 0.474 & 0.526 & 0.324 & 0.676 & 0.392 & 0.608 & 0.179 & 0.821 \\
\hline 655600 & 0.506 & 0.494 & 0.350 & 0.650 & 0.430 & 0.570 & 0.221 & 0.779 \\
\hline 655620 & 0.417 & 0.583 & 0.300 & 0.700 & 0.398 & 0.602 & 0.187 & 0.813 \\
\hline 655630 & 0.341 & 0.659 & 0.307 & 0.693 & 0.309 & 0.691 & 0.170 & 0.830 \\
\hline 655780 & 0.486 & 0.514 & 0.219 & 0.781 & 0.442 & 0.558 & 0.160 & 0.840 \\
\hline 655850 & 0.611 & 0.389 & 0.400 & 0.600 & 0.429 & 0.571 & 0.241 & 0.759 \\
\hline 655920 & 0.570 & 0.430 & 0.357 & 0.643 & 0.545 & 0.455 & 0.232 & 0.768 \\
\hline 655940 & 0.468 & 0.532 & 0.238 & 0.762 & 0.461 & 0.539 & 0.215 & 0.785 \\
\hline 655990 & 0.452 & 0.548 & 0.282 & 0.718 & 0.451 & 0.549 & 0.155 & 0.845 \\
\hline
\end{tabular}




\section{2nd-Order Markov Transition Probabilities}

\section{Gauge Aug}

\begin{tabular}{|c|c|c|c|c|c|c|c|c|}
\hline 653060 & $\begin{array}{l}\mathbf{P}_{\text {www }} \\
0.487\end{array}$ & $\begin{array}{l}\mathbf{P}_{\text {wwd }} \\
0.513\end{array}$ & $\begin{array}{l}\mathbf{P}_{\mathrm{wdw}} \\
0.509\end{array}$ & $\begin{array}{l}\mathbf{P}_{\text {wdd }} \\
0.491\end{array}$ & $\begin{array}{l}\mathbf{P}_{\text {dww }} \\
0.478\end{array}$ & $\begin{array}{l}\mathbf{P}_{\text {dwd }} \\
0.522\end{array}$ & $\begin{array}{l}\mathbf{P}_{\mathrm{ddw}} \\
0.442\end{array}$ & $\begin{array}{l}\mathbf{P}_{\text {ddd }} \\
0.558\end{array}$ \\
\hline 53190 & 0.616 & 0.384 & 0.611 & 0.389 & 0.532 & 0.468 & 0.547 & 0.453 \\
\hline 53300 & 0.528 & 0.472 & 0.485 & 0.515 & 0.544 & 0.456 & 0.375 & 0.625 \\
\hline 53350 & 0.510 & 0.490 & 0.313 & 0.687 & 0.422 & 0.578 & 0.288 & .712 \\
\hline 53380 & 0.455 & 0.545 & 0.327 & 0.673 & 0.443 & 0.557 & 0.212 & 0.788 \\
\hline 653440 & 0.470 & 0.530 & 0.231 & 0.769 & 0.321 & 0.679 & 0.159 & 0.841 \\
\hline 653520 & 0.269 & 0.731 & 0.479 & 0.521 & 0.391 & 0.609 & 0.301 & 0.699 \\
\hline 653550 & 0.647 & 0.353 & 0.658 & 0.342 & 0.532 & 0.468 & 0.517 & 0.483 \\
\hline 653570 & 0.614 & 0.386 & 0.425 & 0.575 & 0.385 & 0.615 & 0.427 & 0.573 \\
\hline 653610 & 0.602 & 0.398 & 0.525 & 0.475 & 0.617 & 0.383 & 0.443 & 0.557 \\
\hline 653760 & 0.403 & 0.597 & 0.421 & 0.579 & 0.487 & 0.513 & 0.367 & 0.633 \\
\hline 653800 & 0.256 & 0.744 & 0.291 & 0.709 & 0.509 & 0.491 & 0.122 & 0.878 \\
\hline 653870 & 0.341 & 0.659 & 0.215 & 0.785 & 0.253 & 0.747 & 0.139 & 0.861 \\
\hline 554720 & 0.313 & 0.688 & 0.304 & 0.696 & 0.220 & 0.780 & 0.121 & 0.879 \\
\hline 55010 & 0.387 & 0.613 & 0.375 & 0.625 & 0.288 & 0.712 & 0.343 & 0.657 \\
\hline 55020 & 0.515 & 0.485 & 0.476 & 0.524 & 0.325 & 0.675 & 0.435 & 0.565 \\
\hline 55030 & 0.454 & 0.546 & 0.430 & 0.570 & 0.432 & 0.568 & 0.493 & 0.507 \\
\hline 655050 & 0.434 & 0.566 & 0.467 & 0.533 & 0.452 & 0.548 & 0.525 & 0.475 \\
\hline 655070 & 0.514 & 0.486 & 0.466 & 0.534 & 0.387 & 0.613 & 0.530 & 0.470 \\
\hline 655100 & 0.536 & 0.464 & 0.511 & 0.489 & 0.506 & 0.494 & 0.555 & 0.445 \\
\hline 655160 & 0.476 & 0.524 & 0.541 & 0.459 & 0.579 & 0.421 & 0.497 & 0.503 \\
\hline 655180 & 0.538 & 0.462 & 0.427 & 0.573 & 0.523 & 0.477 & 0.593 & 0.407 \\
\hline 655220 & 0.459 & 0.541 & 0.441 & 0.559 & 0.449 & 0.551 & 0.470 & 0.530 \\
\hline 655280 & 0.607 & 0.393 & 0.574 & 0.426 & 0.512 & 0.488 & 0.515 & 0.485 \\
\hline 655360 & 0.423 & 0.577 & 0.409 & 0.591 & 0.485 & 0.515 & 0.418 & 0.582 \\
\hline 655450 & 0.341 & 0.659 & 0.247 & 0.753 & 0.353 & 0.647 & 0.210 & 0.790 \\
\hline 655480 & 0.596 & 0.404 & 0.553 & 0.447 & 0.627 & 0.373 & 0.622 & 0.378 \\
\hline 655550 & 0.367 & 0.633 & 0.272 & 0.728 & 0.439 & 0.561 & 0.247 & 0.753 \\
\hline 655570 & 0.464 & 0.536 & 0.345 & 0.655 & 0.434 & 0.566 & 0.252 & 0.748 \\
\hline 655600 & 0.454 & 0.546 & 0.500 & 0.500 & 0.465 & 0.535 & 0.270 & 0.730 \\
\hline 655620 & 0.407 & 0.593 & 0.290 & 0.710 & 0.396 & 0.604 & 0.202 & 0.798 \\
\hline 655630 & 0.472 & 0.528 & 0.310 & 0.690 & 0.341 & 0.659 & 0.212 & 0.788 \\
\hline 655780 & 0.386 & 0.614 & 0.207 & 0.793 & 0.390 & 0.610 & 0.149 & 0.851 \\
\hline 655850 & 0.477 & 0.523 & 0.366 & 0.634 & 0.510 & 0.490 & 0.239 & 0.761 \\
\hline 655920 & 0.622 & 0.378 & 0.532 & 0.468 & 0.598 & 0.402 & 0.366 & 0.634 \\
\hline 655940 & 0.451 & 0.549 & 0.337 & 0.663 & 0.462 & 0.538 & 0.199 & 0.801 \\
\hline 655990 & 0.586 & 0.414 & 0.289 & 0.711 & 0.318 & 0.682 & 0.191 & 0.809 \\
\hline
\end{tabular}




\section{2nd-Order Markov Transition Probabilities}

\section{Gauge Sep}

$653060 P_{\text {www }}$

$\mathbf{P}_{\text {wwd }}$

$653190 \quad 0.626$

$653300 \quad 0.519$

$653350 \quad 0.376$

$653380 \quad 0.450$

$\begin{array}{ll}653440 & 0.389\end{array}$

$\begin{array}{ll}653520 & 0.494\end{array}$

$\begin{array}{ll}653550 & 0.627\end{array}$

$\begin{array}{ll}653570 & 0.513\end{array}$

$\begin{array}{ll}653610 & 0.568\end{array}$

$\begin{array}{ll}653760 & 0.375\end{array}$

$\begin{array}{ll}653800 & 0.500\end{array}$

$\begin{array}{ll}653870 & 0.333\end{array}$

$\begin{array}{ll}654720 & 0.250\end{array}$

$\begin{array}{ll}655010 & 0.356\end{array}$

$\begin{array}{ll}655020 & 0.406\end{array}$

$\begin{array}{ll}655030 & 0.348\end{array}$

$\begin{array}{ll}655050 & 0.333\end{array}$

0.580

$\mathbf{P}_{\text {wdw }}$

0.374

0.477

$\mathbf{P}_{\text {wdd }}$

0.481

0.648

0.523

$\mathbf{P}_{\text {dww }}$

0.352

0.399

$\mathbf{P}_{\mathrm{dwd}}$

$\mathbf{P}_{\mathrm{ddw}}$

0.560

0.624

0.527

0.473

0.549

0.550

0.406

0.594

0.453

0.391

0.609

0.446

0.611

0.400

0.600

0.452

0.506

0.434

0.566

0.529

0.432

0.581

0.487

0.568

0.549

0.459

0.492

0.508

0.500

0.625

0.398

0.602

0.436

0.500

0.250

0.750

0.310

0.728

0.339

0.750

0.272

0.791

0.405

0.744

0.250

0.594

0.256

0.723

0.273

0.386

0.614

0.293

0.667

0.440

0.560

0.280

0.601

0.395

0.440

0.478

0.451

0.547

$0.454 \quad 0.546$

$\begin{array}{lll}0.547 & 0.420 & 0.580\end{array}$

$\begin{array}{lll}0.548 & 0.239 & 0.761\end{array}$

0.471

0.423

0.577

$\begin{array}{lll}0.419 & 0.510 & 0.490\end{array}$

0.541

0.396

0.604

$\begin{array}{lll}0.500 & 0.546 & 0.454\end{array}$

$\begin{array}{lll}0.564 & 0.330 & 0.670\end{array}$

0.690

0.242

0.758

0.661

0.192

0.808

0.595

0.125

0.875

0.750

0.218

0.782

0.727

0.304

0.696

$\begin{array}{lll}0.707 & 0.323 & 0.677\end{array}$

$\begin{array}{lll}0.720 & 0.335 & 0.665\end{array}$

0.578

0.381

0.619

0.398

0.602

0.329

0.671

$\begin{array}{ll}655100 & 0.396\end{array}$

0.604

0.449

0.551

0.503

0.497

0.475

0.525

$\begin{array}{ll}655180 & 0.345\end{array}$

0.565
0.655

0.387

0.633

0.441

0.559

0.345

0.655

$\begin{array}{ll}655220 & 0.514\end{array}$

0.486

0.486

0.514

0.400

0.600

0.359

0.641

$\begin{array}{lll}655280 & 0.547\end{array}$

0.453

0.554

0.446

0.369

0.631

0.465

0.535

$\begin{array}{lll}655360 & 0.412\end{array}$

0.588

0.436

0.564

0.431

0.569

0.554

0.446

$\begin{array}{ll}655450 & 0.505\end{array}$

0.495

0.391

0.609

0.438

0.592

0.464

0.536

$655480 \quad 0.591$

0.409

0.638

0.362

0.538

0.563

$0.395 \quad 0.605$

$\begin{array}{ll}655550 & 0.427\end{array}$

0.573

0.363

0.637

0.446

0.462

$0.427 \quad 0.573$

$\begin{array}{ll}655570 & 0.542\end{array}$

$\begin{array}{ll}655600 & 0.554\end{array}$

0.458

0.402

0.598

0.469

0.578

0.433

0.584

0.330

0.670

0.480

0.611

0.386

0.721

0.394

0.531

0.495

0.554

$0.311 \quad 0.689$

$\begin{array}{lll}0.531 & 0.332 & 0.668\end{array}$

$\begin{array}{lll}0.567 & 0.328 & 0.672\end{array}$

$\begin{array}{lll}0.520 & 0.267 & 0.733\end{array}$

$\begin{array}{ll}655780 & 0.386\end{array}$

0.614

0.279

0.614

$0.284 \quad 0.716$

$\begin{array}{lll}0.606 & 0.207 & 0.793\end{array}$

$\begin{array}{ll}655920 & 0.685\end{array}$

0.315

0.469

0.520

0.645

0.505

$0.302 \quad 0.698$

$0.507 \quad 0.256$

0.744

0.423

0.355

0.577

0.452

0.548

$655990 \quad 0.466$

0.534

0.253

0.747

0.325

0.675

0.180

0.751

0.820 


\begin{tabular}{|c|c|c|c|c|c|c|c|c|}
\hline \multirow{3}{*}{ Gauge } & \multicolumn{8}{|c|}{ 2nd-Order Markov Transition Probabilities } \\
\hline & \multicolumn{8}{|c|}{ Oct } \\
\hline & $\mathbf{P}_{\mathrm{www}}$ & $P_{w w d}$ & $\mathbf{P}_{\mathrm{wdw}}$ & $\mathbf{P}_{\text {wdd }}$ & $\mathbf{P}_{\mathrm{dww}}$ & $\mathbf{P}_{\mathrm{dwd}}$ & $\mathbf{P}_{\mathrm{ddw}}$ & $\mathbf{P}_{\text {ddd }}$ \\
\hline 653060 & 0.222 & 0.778 & 0.214 & 0.786 & 0.266 & 0.734 & 0.093 & 0.907 \\
\hline 653190 & 0.464 & 0.536 & 0.315 & 0.685 & 0.441 & 0.559 & 0.204 & 0.796 \\
\hline 653300 & 0.490 & 0.510 & 0.358 & 0.642 & 0.379 & 0.621 & 0.172 & 0.828 \\
\hline 653350 & 0.410 & 0.590 & 0.418 & 0.582 & 0.355 & 0.645 & 0.242 & 0.758 \\
\hline 653380 & 0.370 & 0.630 & 0.340 & 0.660 & 0.361 & 0.639 & 0.259 & 0.741 \\
\hline 653440 & 0.397 & 0.603 & 0.339 & 0.661 & 0.376 & 0.624 & 0.297 & 0.703 \\
\hline 653520 & 0.415 & 0.585 & 0.233 & 0.767 & 0.362 & 0.638 & 0.122 & 0.878 \\
\hline 653550 & 0.507 & 0.493 & 0.548 & 0.452 & 0.378 & 0.622 & 0.233 & 0.767 \\
\hline 653570 & 0.438 & 0.563 & 0.288 & 0.712 & 0.387 & 0.613 & 0.195 & 0.805 \\
\hline 653610 & 0.434 & 0.566 & 0.421 & 0.579 & 0.456 & 0.544 & 0.175 & 0.825 \\
\hline 653760 & 0.300 & 0.700 & 0.379 & 0.621 & 0.395 & 0.605 & 0.224 & 0.776 \\
\hline 653800 & 0.432 & 0.568 & 0.414 & 0.586 & 0.260 & 0.740 & 0.326 & 0.674 \\
\hline 653870 & 0.295 & 0.705 & 0.221 & 0.779 & 0.289 & 0.711 & 0.213 & 0.787 \\
\hline 654720 & 0.310 & 0.690 & 0.179 & 0.821 & 0.408 & 0.592 & 0.181 & 0.819 \\
\hline 655010 & 0.250 & 0.750 & 0.128 & 0.872 & 0.158 & 0.842 & 0.045 & 0.955 \\
\hline 655020 & 0.190 & 0.810 & 0.225 & 0.775 & 0.213 & 0.787 & 0.088 & 0.912 \\
\hline 655030 & 0.160 & 0.840 & 0.303 & 0.697 & 0.202 & 0.798 & 0.088 & 0.912 \\
\hline 655050 & 0.313 & 0.688 & 0.222 & 0.778 & 0.193 & 0.807 & 0.130 & 0.870 \\
\hline 655070 & 0.222 & 0.778 & 0.178 & 0.822 & 0.182 & 0.818 & 0.116 & 0.884 \\
\hline 655100 & 0.259 & 0.741 & 0.273 & 0.727 & 0.294 & 0.706 & 0.148 & 0.852 \\
\hline 655160 & 0.212 & 0.788 & 0.250 & 0.750 & 0.244 & 0.756 & 0.126 & 0.874 \\
\hline 655180 & 0.267 & 0.733 & 0.178 & 0.822 & 0.289 & 0.711 & 0.113 & 0.887 \\
\hline 655220 & 0.241 & 0.759 & 0.385 & 0.615 & 0.320 & 0.680 & 0.190 & 0.810 \\
\hline 655280 & 0.398 & 0.602 & 0.346 & 0.654 & 0.407 & 0.593 & 0.340 & 0.660 \\
\hline 655360 & 0.467 & 0.533 & 0.355 & 0.645 & 0.396 & 0.604 & 0.221 & 0.779 \\
\hline 655450 & 0.416 & 0.584 & 0.282 & 0.718 & 0.402 & 0.598 & 0.255 & 0.745 \\
\hline 655480 & 0.546 & 0.454 & 0.342 & 0.658 & 0.404 & 0.596 & 0.252 & 0.748 \\
\hline 655550 & 0.318 & 0.682 & 0.276 & 0.724 & 0.323 & 0.677 & 0.217 & 0.783 \\
\hline 655570 & 0.363 & 0.637 & 0.424 & 0.576 & 0.405 & 0.595 & 0.423 & 0.577 \\
\hline 655600 & 0.237 & 0.763 & 0.306 & 0.694 & 0.378 & 0.622 & 0.265 & 0.735 \\
\hline 655620 & 0.391 & 0.609 & 0.328 & 0.672 & 0.364 & 0.636 & 0.287 & 0.713 \\
\hline 655630 & 0.369 & 0.631 & 0.299 & 0.701 & 0.379 & 0.621 & 0.313 & 0.687 \\
\hline 655780 & 0.491 & 0.509 & 0.382 & 0.618 & 0.532 & 0.468 & 0.252 & 0.748 \\
\hline 655850 & 0.548 & 0.452 & 0.480 & 0.520 & 0.508 & 0.492 & 0.376 & 0.624 \\
\hline 655920 & 0.575 & 0.425 & 0.476 & 0.524 & 0.555 & 0.445 & 0.337 & 0.663 \\
\hline 655940 & 0.512 & 0.488 & 0.348 & 0.652 & 0.410 & 0.590 & 0.265 & 0.735 \\
\hline 655990 & 0.489 & 0.511 & 0.422 & 0.578 & 0.382 & 0.618 & 0.205 & 0.795 \\
\hline
\end{tabular}




\begin{tabular}{|c|c|c|c|c|c|c|c|c|}
\hline \multirow{2}{*}{ Gauge } & \multicolumn{8}{|c|}{ 2nd-Order Markov Transition Probabilities } \\
\hline & $\mathbf{P}_{\mathrm{www}}$ & $P_{w w d}$ & $\mathbf{P}_{\mathrm{wdw}}$ & $\mathbf{P}_{\text {wdd }}$ & $\mathbf{P}_{\mathrm{dww}}$ & $\mathbf{P}_{\mathrm{dwd}}$ & $\mathbf{P}_{\mathrm{ddw}}$ & $\mathbf{P}_{\text {ddd }}$ \\
\hline 653060 & 0.333 & 0.667 & 0.167 & 0.833 & 0.250 & 0.750 & 0.004 & 0.996 \\
\hline 653190 & 0.500 & 0.500 & 0.107 & 0.893 & 0.167 & 0.833 & 0.018 & 0.982 \\
\hline 653300 & 0.333 & 0.667 & 0.091 & 0.909 & 0.250 & 0.750 & 0.016 & 0.984 \\
\hline 653350 & 0.167 & 0.833 & 0.125 & 0.875 & 0.143 & 0.857 & 0.030 & 0.970 \\
\hline 653380 & 0.389 & 0.611 & 0.125 & 0.875 & 0.186 & 0.814 & 0.058 & 0.942 \\
\hline 653440 & 0.206 & 0.794 & 0.189 & 0.811 & 0.302 & 0.698 & 0.103 & 0.897 \\
\hline 653520 & 0.000 & 1.000 & 0.063 & 0.938 & 0.200 & 0.800 & 0.022 & 0.978 \\
\hline 653550 & 0.364 & 0.636 & 0.118 & 0.882 & 0.375 & 0.625 & 0.040 & 0.960 \\
\hline 653570 & 0.333 & 0.667 & 0.000 & 1.000 & 0.182 & 0.818 & 0.029 & 0.971 \\
\hline 653610 & 0.111 & 0.889 & 0.276 & 0.724 & 0.269 & 0.731 & 0.027 & 0.973 \\
\hline 653760 & 0.000 & 1.000 & 0.150 & 0.850 & 0.083 & 0.917 & 0.051 & 0.949 \\
\hline 653800 & 0.143 & 0.857 & 0.111 & 0.889 & 0.208 & 0.792 & 0.109 & 0.891 \\
\hline 653870 & 0.300 & 0.700 & 0.161 & 0.839 & 0.133 & 0.867 & 0.071 & 0.929 \\
\hline 654720 & 0.250 & 0.750 & 0.083 & 0.917 & 0.094 & 0.906 & 0.086 & 0.914 \\
\hline 655010 & 0.000 & 0.000 & 0.000 & 1.000 & 0.000 & 1.000 & 0.003 & 0.997 \\
\hline 655020 & 0.000 & 1.000 & 0.000 & 1.000 & 0.333 & 0.667 & 0.004 & 0.996 \\
\hline 655030 & 0.000 & 1.000 & 0.000 & 1.000 & 0.154 & 0.846 & 0.014 & 0.986 \\
\hline 655050 & 0.000 & 1.000 & 0.091 & 0.909 & 0.250 & 0.750 & 0.014 & 0.986 \\
\hline 655070 & 0.000 & 1.000 & 0.000 & 1.000 & 0.125 & 0.875 & 0.011 & 0.989 \\
\hline 655100 & 0.091 & 0.909 & 0.030 & 0.970 & 0.333 & 0.667 & 0.029 & 0.971 \\
\hline 655160 & 0.250 & 0.750 & 0.000 & 1.000 & 0.556 & 0.444 & 0.014 & 0.986 \\
\hline 655180 & 0.250 & 0.750 & 0.231 & 0.769 & 0.333 & 0.667 & 0.021 & 0.979 \\
\hline 655220 & 0.167 & 0.833 & 0.103 & 0.897 & 0.130 & 0.870 & 0.029 & 0.971 \\
\hline 655280 & 0.211 & 0.789 & 0.167 & 0.833 & 0.273 & 0.727 & 0.069 & 0.931 \\
\hline 655360 & 0.231 & 0.769 & 0.125 & 0.875 & 0.163 & 0.837 & 0.059 & 0.941 \\
\hline 655450 & 0.222 & 0.778 & 0.176 & 0.824 & 0.227 & 0.773 & 0.075 & 0.925 \\
\hline 655480 & 0.391 & 0.609 & 0.152 & 0.848 & 0.156 & 0.844 & 0.093 & 0.907 \\
\hline 655550 & 0.250 & 0.750 & 0.186 & 0.814 & 0.167 & 0.833 & 0.051 & 0.949 \\
\hline 655570 & 0.339 & 0.661 & 0.325 & 0.675 & 0.313 & 0.687 & 0.271 & 0.729 \\
\hline 655600 & 0.407 & 0.593 & 0.155 & 0.845 & 0.296 & 0.704 & 0.090 & 0.910 \\
\hline 655620 & 0.263 & 0.737 & 0.167 & 0.833 & 0.281 & 0.719 & 0.101 & 0.899 \\
\hline 655630 & 0.360 & 0.640 & 0.286 & 0.714 & 0.258 & 0.742 & 0.106 & 0.894 \\
\hline 655780 & 0.376 & 0.624 & 0.371 & 0.629 & 0.435 & 0.565 & 0.290 & 0.710 \\
\hline 655850 & 0.484 & 0.516 & 0.411 & 0.589 & 0.383 & 0.617 & 0.328 & 0.672 \\
\hline 655920 & 0.513 & 0.487 & 0.409 & 0.591 & 0.521 & 0.479 & 0.333 & 0.667 \\
\hline 655940 & 0.460 & 0.540 & 0.391 & 0.609 & 0.400 & 0.600 & 0.285 & 0.715 \\
\hline 655990 & 0.359 & 0.641 & 0.317 & 0.683 & 0.343 & 0.657 & 0.257 & 0.743 \\
\hline
\end{tabular}




\begin{tabular}{|c|c|c|c|c|c|c|c|c|}
\hline \multirow[t]{2}{*}{ Gauge } & \multicolumn{8}{|c|}{ 2nd-Order Markov Transition Probabilities } \\
\hline & $\mathbf{P}_{\text {www }}$ & $\mathbf{P}_{\text {wwd }}$ & $\mathbf{P}_{\mathrm{wdw}}$ & $\mathbf{P}_{\text {wdd }}$ & $\mathbf{P}_{\mathrm{dww}}$ & $\mathbf{P}_{\mathrm{dwd}}$ & $\mathbf{P}_{\mathrm{ddw}}$ & $\mathbf{P}_{\text {ddd }}$ \\
\hline 653060 & 0.000 & 0.000 & 0.000 & 1.000 & 0.000 & 1.000 & 0.001 & 0.999 \\
\hline 653190 & 0.000 & 0.000 & 0.000 & 1.000 & 0.000 & 1.000 & 0.003 & 0.997 \\
\hline 653300 & 0.000 & 0.000 & 0.000 & 1.000 & 0.000 & 1.000 & 0.010 & 0.990 \\
\hline 653350 & 0.000 & 1.000 & 0.000 & 1.000 & 0.125 & 0.875 & 0.010 & 0.990 \\
\hline 653380 & 0.000 & 0.000 & 0.111 & 0.889 & 0.000 & 1.000 & 0.025 & 0.975 \\
\hline 653440 & 0.000 & 1.000 & 0.053 & 0.947 & 0.103 & 0.897 & 0.040 & 0.960 \\
\hline 653520 & 0.000 & 0.000 & 0.000 & 1.000 & 0.000 & 1.000 & 0.004 & 0.996 \\
\hline 653550 & 0.000 & 0.000 & 0.333 & 0.667 & 0.000 & 1.000 & 0.008 & 0.992 \\
\hline 653570 & 0.000 & 0.000 & 0.167 & 0.833 & 0.000 & 1.000 & 0.007 & 0.993 \\
\hline 653610 & 0.333 & 0.667 & 0.000 & 1.000 & 0.222 & 0.778 & 0.014 & 0.986 \\
\hline 653760 & 0.000 & 1.000 & 0.063 & 0.938 & 0.000 & 1.000 & 0.019 & 0.981 \\
\hline 653800 & 0.500 & 0.500 & 0.143 & 0.857 & 0.077 & 0.923 & 0.022 & 0.978 \\
\hline 653870 & 0.000 & 1.000 & 0.074 & 0.926 & 0.080 & 0.920 & 0.030 & 0.970 \\
\hline 654720 & 0.000 & 0.000 & 0.158 & 0.842 & 0.000 & 1.000 & 0.035 & 0.965 \\
\hline 655010 & 0.000 & 0.000 & 0.000 & 1.000 & 0.000 & 1.000 & 0.007 & 0.993 \\
\hline 655020 & 0.000 & 0.000 & 0.000 & 1.000 & 0.000 & 1.000 & 0.003 & 0.997 \\
\hline 655030 & 0.000 & 1.000 & 0.000 & 1.000 & 0.250 & 0.750 & 0.005 & 0.995 \\
\hline 655050 & 0.000 & 0.000 & 0.000 & 1.000 & 0.000 & 1.000 & 0.006 & 0.994 \\
\hline 655070 & 0.000 & 1.000 & 0.000 & 1.000 & 0.500 & 0.500 & 0.006 & 0.994 \\
\hline 655100 & 0.000 & 0.000 & 0.000 & 1.000 & 0.000 & 1.000 & 0.008 & 0.992 \\
\hline 655160 & 0.000 & 0.000 & 0.000 & 1.000 & 0.000 & 1.000 & 0.003 & 0.997 \\
\hline 655180 & 0.000 & 1.000 & 0.000 & 1.000 & 0.333 & 0.667 & 0.002 & 0.998 \\
\hline 655220 & 0.000 & 1.000 & 0.000 & 1.000 & 0.200 & 0.800 & 0.007 & 0.993 \\
\hline 655280 & 0.000 & 0.000 & 0.000 & 1.000 & 0.200 & 0.800 & 0.014 & 0.986 \\
\hline 655360 & 0.000 & 1.000 & 0.000 & 1.000 & 0.200 & 0.800 & 0.008 & 0.992 \\
\hline 655450 & 0.000 & 1.000 & 0.105 & 0.895 & 0.333 & 0.667 & 0.025 & 0.975 \\
\hline 655480 & 0.000 & 1.000 & 0.120 & 0.880 & 0.208 & 0.792 & 0.034 & 0.966 \\
\hline 655550 & 0.000 & 1.000 & 0.043 & 0.957 & 0.087 & 0.913 & 0.035 & 0.965 \\
\hline 655570 & 0.286 & 0.714 & 0.183 & 0.817 & 0.222 & 0.778 & 0.087 & 0.913 \\
\hline 655600 & 0.400 & 0.600 & 0.120 & 0.880 & 0.080 & 0.920 & 0.048 & 0.952 \\
\hline 655620 & 0.000 & 1.000 & 0.219 & 0.781 & 0.034 & 0.966 & 0.040 & 0.960 \\
\hline 655630 & 0.000 & 1.000 & 0.067 & 0.933 & 0.269 & 0.731 & 0.048 & 0.952 \\
\hline 655780 & 0.179 & 0.821 & 0.233 & 0.767 & 0.393 & 0.607 & 0.127 & 0.873 \\
\hline 655850 & 0.314 & 0.686 & 0.284 & 0.716 & 0.256 & 0.744 & 0.137 & 0.863 \\
\hline 655920 & 0.333 & 0.667 & 0.291 & 0.709 & 0.360 & 0.640 & 0.199 & 0.801 \\
\hline 655940 & 0.273 & 0.727 & 0.253 & 0.747 & 0.279 & 0.721 & 0.132 & 0.868 \\
\hline 655990 & 0.333 & 0.667 & 0.246 & 0.754 & 0.273 & 0.727 & 0.090 & 0.910 \\
\hline
\end{tabular}




\section{Appendix C: LARS-WG Parameters}

These parameters are the wet spell length and wet day amount histograms that LARS-WG calculates from the observed data. These histograms are used in the model to create the synthetic rainfall sequences. 
[NAME]

C-2

653060WG

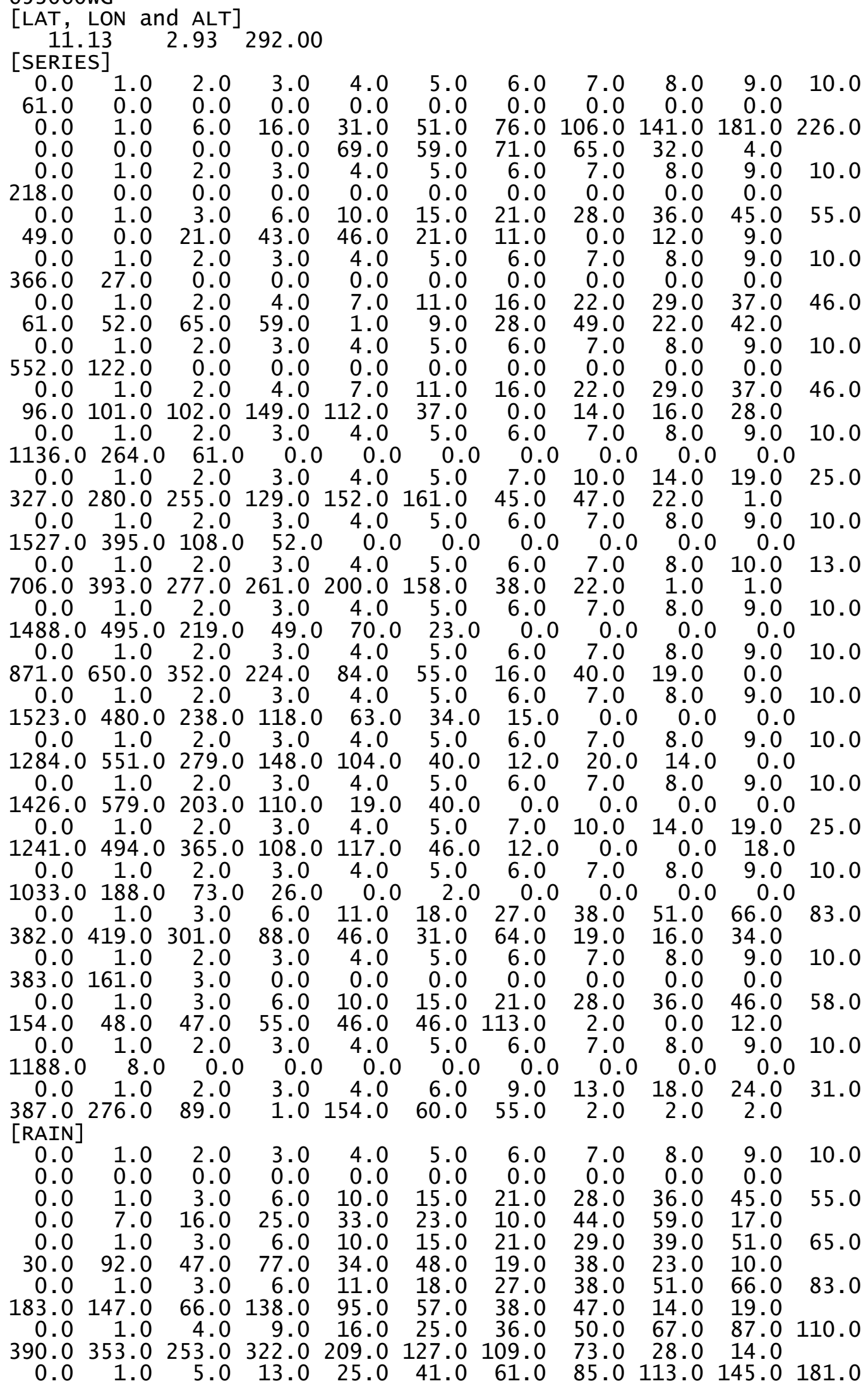




$\begin{array}{rrrrrrrrrrr}518.0 & 426.0 & 692.0 & 723.0 & 265.0 & 122.0 & 48.0 & 39.0 & 14.0 & 14.0 & \\ 0.0 & 1.0 & 5.0 & 13.0 & 25.0 & 41.0 & 61.0 & 85.0 & 113.0 & 145.0 & 181.0 \\ 607.0 & 552.0 & 952.0 & 876.0 & 519.0 & 183.0 & 26.0 & 53.0 & 0.0 & 12.0 & \\ 0.0 & 1.0 & 5.0 & 12.0 & 23.0 & 38.0 & 57.0 & 80.0 & 107.0 & 138.0 & 173.0 \\ 895.0 & 646.0 & 1003.0 & 696.0 & 684.0 & 285.0 & 34.0 & 48.0 & 4.0 & 15.0 & \\ 0.0 & 1.0 & 5.0 & 12.0 & 22.0 & 36.0 & 54.0 & 76.0 & 102.0 & 132.0 & 166.0 \\ 844.0 & 592.0 & 1093.0 & 602.0 & 494.0 & 242.0 & 38.0 & 39.0 & 10.0 & 18.0 & \\ 0.0 & 1.0 & 4.0 & 9.0 & 16.0 & 25.0 & 36.0 & 49.0 & 65.0 & 84.0 & 106.0 \\ 428.0 & 340.0 & 396.0 & 257.0 & 155.0 & 83.0 & 41.0 & 16.0 & 10.0 & 4.0 & \\ 0.0 & 1.0 & 2.0 & 3.0 & 5.0 & 8.0 & 12.0 & 17.0 & 23.0 & 30.0 & 38.0 \\ 279.0 & 102.0 & 41.0 & 54.0 & 56.0 & 49.0 & 55.0 & 29.0 & 27.0 & 5.0 & \\ 0.0 & 1.0 & 3.0 & 6.0 & 10.0 & 15.0 & 21.0 & 28.0 & 36.0 & 45.0 & 55.0 \\ 609.0 & 115.0 & 0.0 & 0.0 & 0.0 & 0.0 & 3.0 & 164.0 & 247.0 & 111.0 & \\ \text { [END] } & & & & & & & & & & \end{array}$


[NAME]

C-4

653190WG

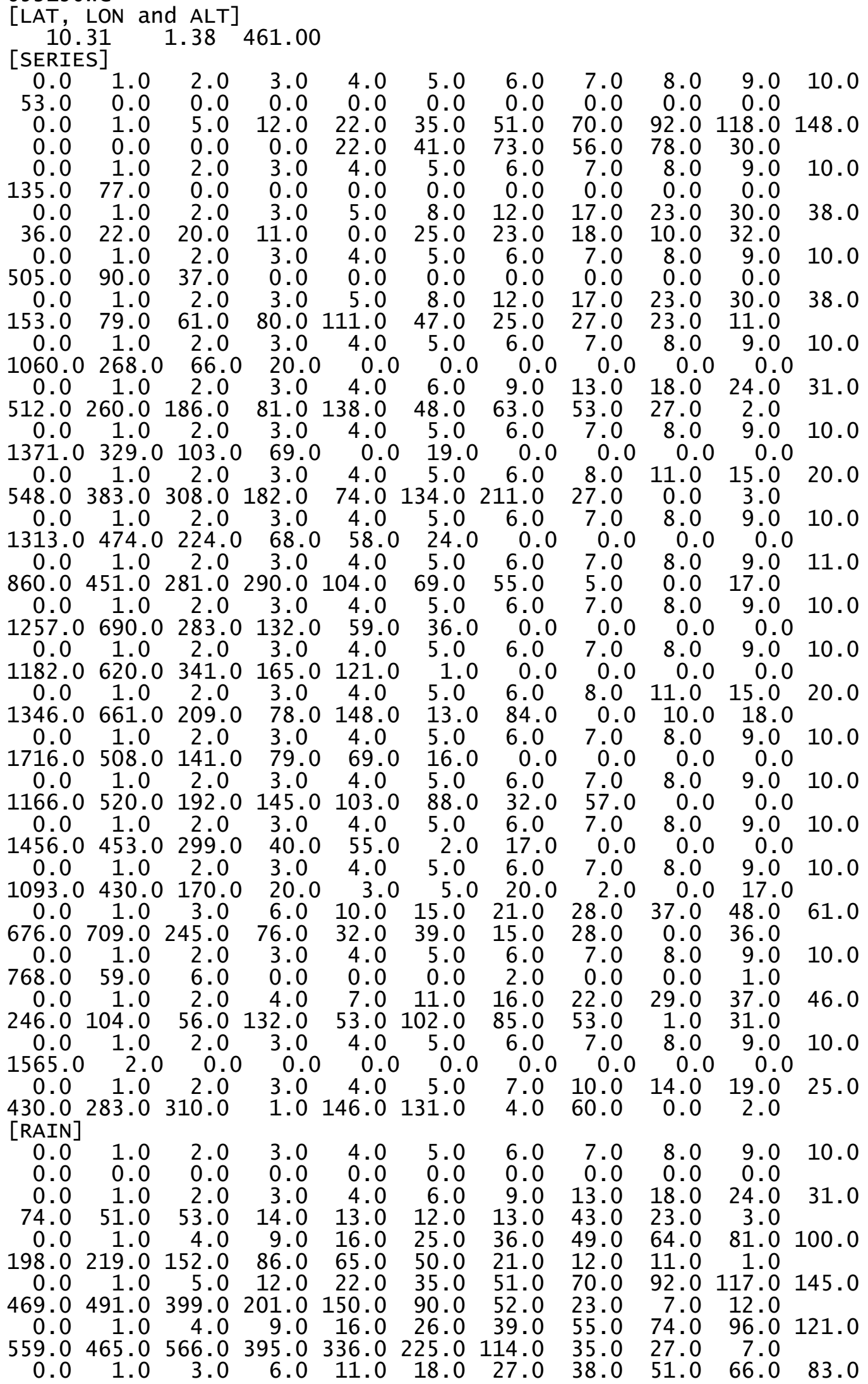


$\begin{array}{llllllllllll}699.0 & 647.0 & 367.0 & 612.0 & 520.0 & 310.0 & 276.0 & 121.0 & 50.0 & 15.0\end{array}$ $\begin{array}{lllllllllll}0.0 & 1.0 & 6.0 & 15.0 & 28.0 & 45.0 & 66.0 & 91.0 & 120.0 & 153.0 & 190.0\end{array}$ $\begin{array}{llllllllll}948.0 & 1071.0 & 1056.0 & 719.0 & 396.0 & 189.0 & 72.0 & 37.0 & 11.0 & 3.0\end{array}$ $\begin{array}{lllllllllllll}0.0 & 1.0 & 8.0 & 21.0 & 40.0 & 65.0 & 97.0 & 136.0 & 182.0 & 235.0 & 295.0\end{array}$

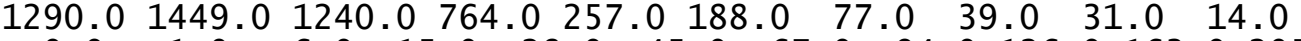
$\begin{array}{lllllllllllll}0.0 & 1.0 & 6.0 & 15.0 & 28.0 & 45.0 & 67.0 & 94.0 & 126.0 & 163.0 & 205.0\end{array}$

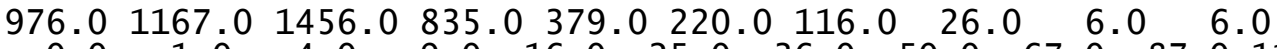
$\begin{array}{lllllllllll}0.0 & 1.0 & 4.0 & 9.0 & 16.0 & 25.0 & 36.0 & 50.0 & 67.0 & 87.0 & 110.0\end{array}$

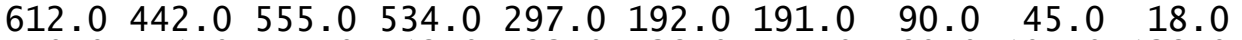

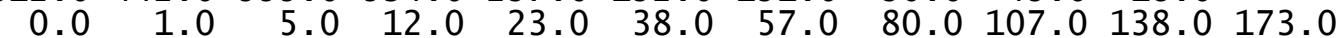

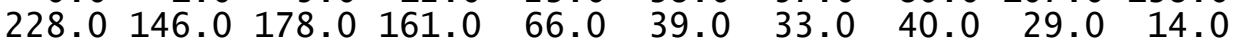

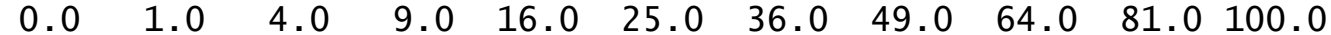

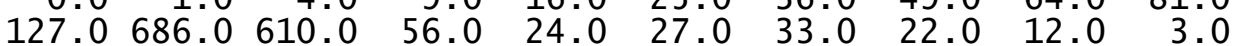

[END] 
[NAME]

C-6

653300WG

\begin{tabular}{|c|c|c|c|c|c|c|c|c|c|c|}
\hline $\begin{array}{l}\text {-AT, } \\
9 . \\
\text { SERIE }\end{array}$ & $\begin{array}{l}\text { LON a } \\
35 \\
\text { S] }\end{array}$ & $\begin{array}{l}\text { a } \\
2.61\end{array}$ & 393.00 & & & & & & & \\
\hline 0.0 & 1.0 & 2.0 & 3.0 & 4.0 & 5.0 & 6.0 & 7.0 & 8.0 & 9.0 & 10.0 \\
\hline 48.0 & 210.0 & & & & & & & & & \\
\hline 0.0 & 1.0 & 4. & & 17 & 28 & 42 & 59 & 79. & 102.0 & 128.0 \\
\hline & 76.0 & 134. & 142 & 80 & & & & 67. & 56.0 & \\
\hline $\begin{array}{r}0.0 \\
37.0\end{array}$ & $\begin{array}{r}1.0 \\
26.0\end{array}$ & & & $\begin{array}{l}4 . \\
0 .\end{array}$ & $\begin{array}{l}5 . \\
0 .\end{array}$ & & $\begin{array}{l}7 \\
0\end{array}$ & & $\begin{array}{l}9.0 \\
0.0\end{array}$ & 10.0 \\
\hline 0 & 1.0 & & & 16. & 25. & & 52. & 70.0 & 91.0 & 115.0 \\
\hline 39 & 30.0 & 83. & & & & & & & & \\
\hline & 1.0 & & & & & & & & & 10.0 \\
\hline 3.0 & 82.0 & 10. & & & & 0 & 0 & 0 & & \\
\hline & 1.0 & & & & & & & & & 38.0 \\
\hline 45.0 & 49.0 & 85.0 & 63. & 67.0 & 22. & & & & 10 & \\
\hline & 1.0 & & & & & & & & & 10.0 \\
\hline 8 & 172.0 & 13. & & & & & & & & \\
\hline & 1.0 & 2. & & 4 & & & & & 24 & 31.0 \\
\hline 50 & 119.0 & 119.0 & 101.0 & 161.0 & 128.0 & 57.0 & 41.0 & 13 & & \\
\hline & 1.0 & 2.0 & 3.0 & 4.0 & 5.0 & 6.0 & 7.0 & 8.0 & 9.0 & 10.0 \\
\hline 479 & 370.0 & 116.0 & 36.0 & 20.0 & 0.0 & 0.0 & 0.0 & 0.0 & 0.0 & \\
\hline & 1.0 & 2.0 & 3. & 4.0 & 5.0 & 6.0 & 7.0 & 9.0 & 12 & 16.0 \\
\hline 47 & 448.0 & 220.0 & 187.0 & 130.0 & 40.0 & 61.0 & & & & \\
\hline & 1.0 & 2.0 & & 4.0 & 5.0 & 6.0 & 7.0 & 8.0 & 9.0 & 10.0 \\
\hline 319.0 & 610.0 & 194.0 & 62.0 & 19.0 & 7.0 & 0.0 & 0.0 & 0.0 & 0.0 & 0 \\
\hline 5.0 & 502.0 & 325.0 & 154.0 & 174.0 & 49.0 & & & 46.0 & 27 & \\
\hline & 1.0 & 2.0 & 3.0 & 4.0 & 5.0 & 6.0 & 7.0 & 8.0 & 9.0 & 10.0 \\
\hline $\begin{array}{l}089.0 \\
0.0\end{array}$ & $\begin{array}{r}566.0 \\
1.0\end{array}$ & $\begin{array}{r}251.0 \\
2.0\end{array}$ & $\begin{array}{c}146.0 \\
3.0\end{array}$ & $\begin{array}{l}42.0 \\
4.0\end{array}$ & & $\begin{array}{l}11.0 \\
6.0\end{array}$ & $\begin{array}{l}0.0 \\
7.0\end{array}$ & $\begin{array}{l}0.0 \\
8.0\end{array}$ & 0.0 & 0 \\
\hline 29. & 624.0 & 240. & 270. & 78.0 & 64.0 & & & 4.0 & 11 . & \\
\hline 0 & 1.0 & 2.0 & 3. & 4.0 & 5.0 & 6.0 & 7.0 & 8.0 & 9.0 & 10. \\
\hline 0 & $\begin{array}{c}642.0 \\
1.0\end{array}$ & $\begin{array}{r}252.0 \\
2.0\end{array}$ & & $\begin{array}{l}36.0 \\
4.0\end{array}$ & $\begin{array}{l}19.0 \\
5.0\end{array}$ & $\begin{array}{l}15.0 \\
6.0\end{array}$ & $\begin{array}{l}34.0 \\
7.0\end{array}$ & $\begin{array}{l}16.0 \\
8.0\end{array}$ & $\begin{array}{l}0.0 \\
9.0\end{array}$ & 0 \\
\hline 18 & 615.0 & 219.0 & 198.0 & 71.0 & 34.0 & 52.0 & 0.0 & 13.0 & 1.0 & \\
\hline & 1.0 & 2.0 & & 4.0 & & & 7.0 & 8.0 & 9.0 & 10.0 \\
\hline 0. & $\begin{array}{c}581.0 \\
1.0\end{array}$ & $\begin{array}{r}252.0 \\
2.0\end{array}$ & $\begin{array}{l}82.0 \\
3.0\end{array}$ & $\begin{array}{l}89.0 \\
4.0\end{array}$ & $\begin{array}{l}19.0 \\
5.0\end{array}$ & $\begin{array}{l}73.0 \\
6.0\end{array}$ & 7.0 & 8.0 & $\begin{array}{l}0.0 \\
9.0\end{array}$ & 10.0 \\
\hline 103 & 405.0 & 358.0 & 102.0 & 17.0 & & & & & 0.0 & \\
\hline & & & $\begin{array}{l}3 . \\
26\end{array}$ & & & & & $\begin{array}{r}8.0 \\
0.0\end{array}$ & $\begin{array}{c}9.0 \\
0.0\end{array}$ & 10.0 \\
\hline & 1.0 & & & & & & & & & 70. \\
\hline & 404.0 & 362. & 148 & & & & & & & \\
\hline & & & & & & & & & & 10. \\
\hline & 159. & & & & & & & & & \\
\hline & & & & & & & & & & 46. \\
\hline & 43.0 & 156 & & & & & & & & \\
\hline & & & & & & & & & & 10. \\
\hline & & & & & & & & & & \\
\hline & & & & & & & & & & 25. \\
\hline & & & & & & & & & & \\
\hline RA & & & & & & & & & & \\
\hline & & & & & & & & & & 16. \\
\hline & 161 & & & & & & & & & \\
\hline & & & & & & & & & & 46. \\
\hline & $4 \overline{7}$. & & & & & & & & & \\
\hline & & & & & & & & & & 91. \\
\hline & 142.0 & 111 & & & & & & & & \\
\hline & & & & & & & & & 117 & 145. \\
\hline & & & & & & & & & & \\
\hline & & & & & & & & & 153 & 190.0 \\
\hline & & & & & & & & & & \\
\hline & & & & & & & & & & 173 \\
\hline
\end{tabular}




$\begin{array}{rrrrrrrrrrrr}788.0 & 681.0 & 921.0 & 456.0 & 331.0 & 194.0 & 57.0 & 38.0 & 13.0 & 20.0 & \\ 0.0 & 1.0 & 5.0 & 12.0 & 22.0 & 35.0 & 52.0 & 73.0 & 98.0 & 127.0 & 160.0 \\ 1062.0 & 714.0 & 1050.0 & 437.0 & 466.0 & 251.0 & 89.0 & 105.0 & 19.0 & 25.0 \\ 0.0 & 1.0 & 5.0 & 13.0 & 25.0 & 41.0 & 61.0 & 85.0 & 113.0 & 145.0 & 181.0 \\ 1010.0 & 745.0 & 1020.0 & 711.0 & 439.0 & 229.0 & 142.0 & 62.0 & 34.0 & 29.0 \\ 0.0 & 1.0 & 4.0 & 10.0 & 19.0 & 31.0 & 46.0 & 64.0 & 85.0 & 109.0 & 136.0 \\ 882.0 & 751.0 & 857.0 & 869.0 & 639.0 & 333.0 & 96.0 & 88.0 & 73.0 & 15.0 & \\ 0.0 & 1.0 & 4.0 & 9.0 & 16.0 & 25.0 & 36.0 & 49.0 & 64.0 & 81.0 & 100.0 \\ 571.0 & 484.0 & 582.0 & 480.0 & 403.0 & 164.0 & 67.0 & 5.0 & 20.0 & 40.0 & \\ 0.0 & 1.0 & 4.0 & 9.0 & 16.0 & 25.0 & 36.0 & 49.0 & 64.0 & 81.0 & 100.0 \\ 311.0 & 146.0 & 214.0 & 164.0 & 140.0 & 30.0 & 17.0 & 13.0 & 19.0 & 21.0 & \\ 0.0 & 1.0 & 2.0 & 4.0 & 7.0 & 11.0 & 16.0 & 22.0 & 29.0 & 37.0 & 46.0 \\ 247.0 & 187.0 & 43.0 & 246.0 & 111.0 & 22.0 & 12.0 & 7.0 & 3.0 & 6.0 & \\ \text { [END] } & & & & & & & & & & \end{array}$


[NAME]

C-8

653350WG

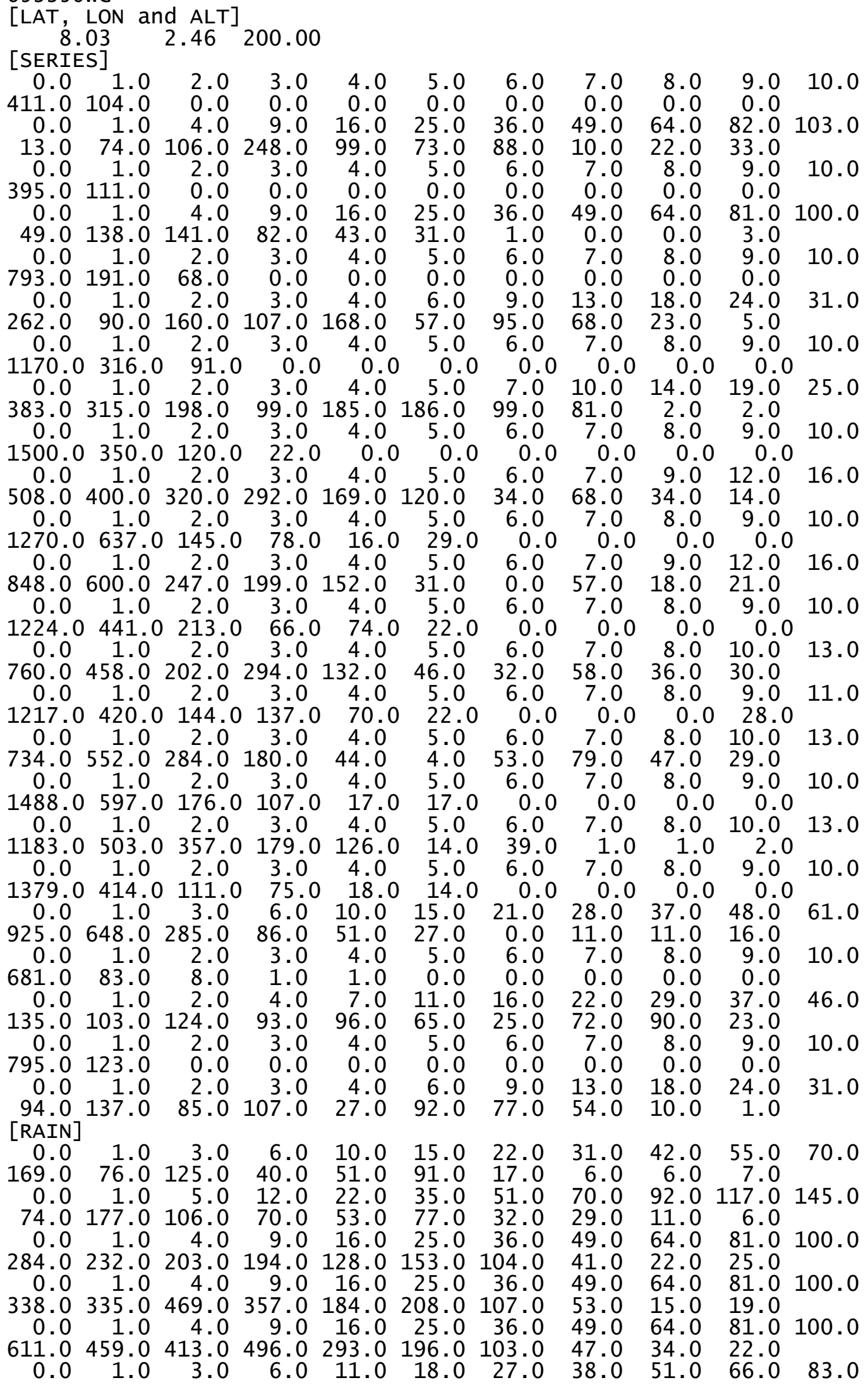


$\begin{array}{llllllllllll}923.0 & 422.0 & 415.0 & 557.0 & 469.0 & 282.0 & 221.0 & 117.0 & 79.0 & 20.0\end{array}$ $\begin{array}{lllllllllll}0.0 & 1.0 & 5.0 & 12.0 & 22.0 & 35.0 & 51.0 & 70.0 & 92.0 & 117.0 & 145.0\end{array}$ $\begin{array}{llllllllllll}1052.0 & 594.0 & 636.0 & 505.0 & 300.0 & 223.0 & 135.0 & 60.0 & 11.0 & 7.0\end{array}$

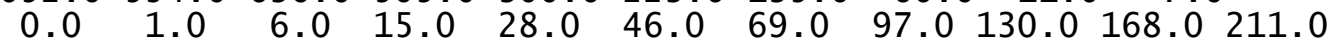

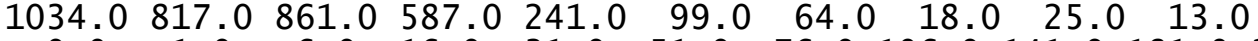
$\begin{array}{lllllllllll}0.0 & 1.0 & 6.0 & 16.0 & 31.0 & 51.0 & 76.0 & 106.0 & 141.0 & 181.0 & 226.0\end{array}$

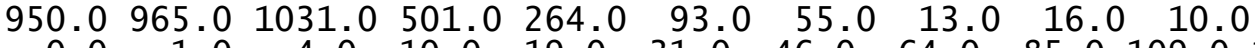

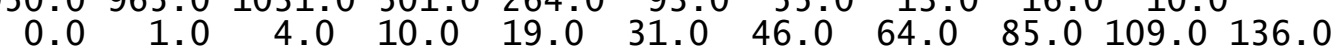
$\begin{array}{llllllllll}500.0 & 650.0 & 626.0 & 490.0 & 396.0 & 201.0 & 84.0 & 40.0 & 10.0 & 4.0\end{array}$

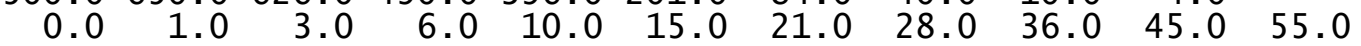
$\begin{array}{llllllllll}118.0 & 152.0 & 109.0 & 124.0 & 138.0 & 94.0 & 54.0 & 35.0 & 16.0 & 4.0\end{array}$

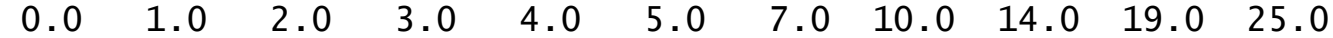
$\begin{array}{llllllllll}213.0 & 81.0 & 26.0 & 42.0 & 161.0 & 130.0 & 147.0 & 167.0 & 86.0 & 13.0\end{array}$

[END] 
[NAME]

C-10

653380WG

\begin{tabular}{|c|c|c|c|c|c|c|c|c|c|c|}
\hline AT, & $\begin{array}{l}\text { LON } \\
16 \\
\text { ES] }\end{array}$ & $\begin{array}{l}\text { nd } \mathrm{ALT}] \\
2.06\end{array}$ & 167. & & & & & & & \\
\hline 0.0 & 1.0 & 2.0 & & 4.0 & 5.0 & 6.0 & 7.0 & 8.0 & 9.0 & 10. \\
\hline 53.0 & 0.0 & & & & & & & & & \\
\hline 69 & $\begin{array}{l}1.0 \\
0.1 .0\end{array}$ & 3. & & 11. & 18 & & & & & 3.0 \\
\hline 69. & 94.0 & 21. & 194 & 130. & 119 & & & & 0 & \\
\hline 50 & $\frac{1}{6}$. & & & 4 & & & & & & 0.0 \\
\hline & 68. & & & & & & & & & \\
\hline 43. & 31.0 & 109. & & 79. & & & & & & \\
\hline 0.0 & 1.0 & & & & & & & & & 0.0 \\
\hline 0. & 213.0 & & & & & & & & & \\
\hline 0. & 1.0 & & & & & & & & & 1.0 \\
\hline 45.0 & 234.0 & 170.0 & 82. & 195.0 & 76.0 & 115.0 & & & 0 & \\
\hline & $341^{1.0}$ & 2.0 & 3.0 & 4.0 & 5.0 & 6.0 & 7.0 & 8.0 & 9.0 & 0.0 \\
\hline 346.0 & 341.0 & & $\frac{14}{3}$ & 0.0 & 0.0 & 0.0 & & 0.0 & 0.0 & \\
\hline 0.0 & 316.0 & 225.0 & 261.0 & 127.0 & 100.0 & 88.0 & 74. & & 57 & 0.1 \\
\hline 0. & 1.0 & 2.0 & 3.0 & 4.0 & 5.0 & 6.0 & 7.0 & 8.0 & 9.0 & 10.0 \\
\hline 0.0 & $\begin{array}{r}438.0 \\
1.0\end{array}$ & $\begin{array}{l}95.0 \\
2.0\end{array}$ & $\begin{array}{l}53 \\
3 .\end{array}$ & $\begin{array}{l}15.0 \\
4.0\end{array}$ & $\begin{array}{l}0.0 \\
5.0\end{array}$ & 6.0 & 7.0 & 9.0 & 0 & 6.1 \\
\hline 73.0 & $42 \overline{3} .0$ & 336.0 & 220. & 174.0 & 67.0 & & & & & \\
\hline 0.0 & 1.0 & 2.0 & 3.0 & 4.0 & 5.0 & 6.0 & & 8.0 & 9.0 & 10. \\
\hline+95.0 & 528. & 0 & 45.0 & & & 0.0 & 12.0 & 0.0 & 0.0 & \\
\hline 8 & $\begin{array}{r}1.0 \\
637.0\end{array}$ & 2. & $\begin{array}{r}3 . \\
174 .\end{array}$ & $\begin{array}{r}4 . \\
146 .\end{array}$ & $\begin{array}{r}5 . \\
39 .\end{array}$ & $\begin{array}{r}6 . \\
26 .\end{array}$ & & $\begin{array}{r}9.0 \\
15.0\end{array}$ & & 6. \\
\hline 0. & 1.0 & 2.0 & 3.0 & 4.0 & 5.0 & 6.0 & 7.0 & 8.0 & 9 & 10. \\
\hline 498 & 511.0 & 0182.0 & 49 & 52.0 & 0.0 & 0.0 & 0.0 & 0.0 & .0 & \\
\hline & 1.0 & 2. & 3 & 4. & 5.0 & 6.0 & 7.0 & 9.0 & & 16. \\
\hline 2. & 564.0 & 348. & 111. & 169. & 71.0 & 21. & & 14 & & \\
\hline & & & & & & & & & & 0.0 \\
\hline 96 & 534.0 & 185 & & & 23 & & & & & \\
\hline & & & & & & & & & & 0. \\
\hline 74. & 318.0 & 305. & 211. & 117. & 81.0 & & & & & \\
\hline & & & & & 5.0 & 6.0 & & 8.0 & 9.0 & 10.0 \\
\hline 276 & $\begin{array}{r}584.0 \\
1.0\end{array}$ & $\begin{array}{r}157.0 \\
2.0\end{array}$ & $\begin{array}{l}36 \\
3 .\end{array}$ & $\begin{array}{l}61.0 \\
4.0\end{array}$ & $\begin{array}{l}1.0 \\
5.0\end{array}$ & $\begin{array}{l}0.0 \\
6.0\end{array}$ & & & $\begin{array}{l}0.0 \\
9.0\end{array}$ & 1. \\
\hline 33 & 515.0 & 275.0 & 82. & 102.0 & 101.0 & & & 14 & 14 & \\
\hline 0 & 1.0 & 2.0 & & & 5.0 & 6.0 & 7 & 8 & 9 & 10.0 \\
\hline 7 & 358.0 & $\begin{array}{ll}0 & 163 \\
0\end{array}$ & & & 16 & & & & 0 & \\
\hline & 548. & 230 & & 142 & & & & & & \\
\hline & & & & & & & & & & 10. \\
\hline 2 & & & & & & & & & & \\
\hline & & & & & & & & & & 46. \\
\hline 6 & 86. & 102. & 252. & & 108 & 16 & & & & \\
\hline & 1.0 & 2. & & & & & & & & 10. \\
\hline 18 & & & & & & & & & & \\
\hline & 1.0 & 2. & 3 & & & & & & & 31.0 \\
\hline & & & & & & & & & & \\
\hline & 0 & & & & & & & & & 56. \\
\hline & 123. & 100 & & & & & & & & \\
\hline & & & & & & & & & & 70. \\
\hline 7 & 83. & & & & & & & & & \\
\hline & 1. & & & & & & & & & 100 \\
\hline 58 & 200 & 241 & & & & & & & & \\
\hline & 0 & & & & & & & & & 121. \\
\hline & 333.0 & & & & & & & & & \\
\hline & & & & & & & & & & 106.0 \\
\hline & & 413 & & & & & & & & \\
\hline & 1 & & & & & & & & 0 & 15. \\
\hline
\end{tabular}




$\begin{array}{rrrrrrrrrrr}788.0 & 522.0 & 579.0 & 446.0 & 457.0 & 277.0 & 123.0 & 30.0 & 55.0 & 21.0 & \\ 0.0 & 1.0 & 4.0 & 9.0 & 16.0 & 25.0 & 37.0 & 52.0 & 70.0 & 91.0 & 115.0 \\ 1072.0 & 429.0 & 621.0 & 406.0 & 392.0 & 156.0 & 199.0 & 124.0 & 82.0 & 42.0 & \\ 0.0 & 1.0 & 4.0 & 9.0 & 16.0 & 25.0 & 36.0 & 49.0 & 64.0 & 81.0 & 100.0 \\ 1008.0 & 577.0 & 540.0 & 449.0 & 273.0 & 229.0 & 154.0 & 109.0 & 67.0 & 14.0 & \\ 0.0 & 1.0 & 3.0 & 6.0 & 10.0 & 16.0 & 24.0 & 34.0 & 46.0 & 60.0 & 76.0 \\ 1107.0 & 367.0 & 301.0 & 473.0 & 349.0 & 350.0 & 202.0 & 148.0 & 100.0 & 40.0 & \\ 0.0 & 1.0 & 4.0 & 10.0 & 19.0 & 31.0 & 46.0 & 64.0 & 85.0 & 109.0 & 136.0 \\ 791.0 & 361.0 & 454.0 & 518.0 & 370.0 & 208.0 & 127.0 & 53.0 & 24.0 & 8.0 & \\ 0.0 & 1.0 & 6.0 & 15.0 & 28.0 & 45.0 & 66.0 & 91.0 & 120.0 & 153.0 & 190.0 \\ 363.0 & 222.0 & 158.0 & 140.0 & 77.0 & 61.0 & 46.0 & 30.0 & 29.0 & 13.0 & \\ 0.0 & 1.0 & 5.0 & 12.0 & 22.0 & 35.0 & 51.0 & 70.0 & 92.0 & 117.0 & 145.0 \\ 215.0 & 276.0 & 285.0 & 191.0 & 118.0 & 43.0 & 35.0 & 46.0 & 22.0 & 13.0 & \\ \text { [END] } & & & & & & & & & & \end{array}$


[NAME]

C-12

653440WG

\begin{tabular}{|c|c|c|c|c|c|c|c|c|c|c|}
\hline $\begin{array}{r}6 \\
\text { ERIE }\end{array}$ & $\begin{array}{l}.35 \\
\text { ES] }\end{array}$ & 2.38 & 9.00 & & & & & & & \\
\hline 0 & 1.0 & 2.0 & 3.0 & 4.0 & 5.0 & 6.0 & 7.0 & 8.0 & 9.0 & 10.0 \\
\hline 36 & 44.0 & & & & 0.0 & & & 0.0 & 0.0 & \\
\hline & $\begin{array}{r}1.0 \\
0.0\end{array}$ & & & 13. & 21. & 31. & 43 & 57.0 & 73.0 & 91.0 \\
\hline & $\begin{array}{r}90.0 \\
1.0\end{array}$ & $\begin{array}{r}115 \\
?\end{array}$ & 88 & 0 & 52. & & & & 2.0 & n \\
\hline 3 & 76.0 & & & 0. & 0. & 0 & & $\begin{array}{l}0 . \\
0 .\end{array}$ & $\begin{array}{l}9.0 \\
0.0\end{array}$ & \\
\hline & 1.0 & & & 13 & 21. & 31. & 43. & 57 & 73.0 & 1.0 \\
\hline & 89.0 & & 101. & 60. & 50. & 25 & 0 . & 0 & & \\
\hline & 1.0 & & & & & & & & 0 & 0.0 \\
\hline & $19 \overline{3}$. & & & 0 . & & 0. & 0. & 0.0 & 0.0 & \\
\hline & 1.0 & & & & 8.0 & 12.0 & 17. & 23 & 30 & 38.0 \\
\hline 1 & 139.0 & 87. & 110. & 180.0 & 139.0 & & 40 & & & \\
\hline 0 & 1.0 & 2.0 & 3.0 & 4.0 & 5.0 & 6.0 & 7.0 & 8.0 & 9.0 & 0.0 \\
\hline 192 & 0293.0 & 55.0 & 36.0 & 0.0 & 0.0 & 0.0 & 0.0 & 0.0 & 0.0 & \\
\hline 70 & $\begin{array}{r}1.0 \\
324.0\end{array}$ & $\begin{array}{r}2.0 \\
218.0\end{array}$ & 3. & $\begin{array}{r}4.0 \\
107.0\end{array}$ & $\begin{array}{r}5.0 \\
123.0\end{array}$ & $\begin{array}{r}7.0 \\
46.0\end{array}$ & $\begin{array}{r}10.0 \\
107.0\end{array}$ & $\begin{array}{l}14.0 \\
33.0\end{array}$ & $\begin{array}{r}19.0 \\
1.0\end{array}$ & 25.0 \\
\hline 0 & 1.0 & 2.0 & 3.0 & 4.0 & 5.0 & 6.0 & 7.0 & 8.0 & 9.0 & 10.0 \\
\hline 50 & $\begin{array}{ll}0 & 423.0\end{array}$ & 71.0 & 49.0 & 29.0 & 0.0 & 15.0 & 0.0 & 0.0 & 0.0 & \\
\hline 8 & $\begin{array}{l}1 . \\
646 .\end{array}$ & 523. & $\begin{array}{r}3 . \\
158 .\end{array}$ & $\begin{array}{r}4.0 \\
73.0\end{array}$ & $\begin{array}{r}5 . \\
64 .\end{array}$ & $\begin{array}{r}6.0 \\
36.0\end{array}$ & $\begin{array}{r}7 \\
35\end{array}$ & $\begin{array}{r}8.0 \\
39.0\end{array}$ & $\begin{array}{l}0 \\
0\end{array}$ & $3 .($ \\
\hline & 1.0 & & & 4. & & & & & 0 & 0.0 \\
\hline 8 & 592.0 & 259. & & 75. & 64. & & & & & \\
\hline & 1.0 & & & 4. & & & & & 19 & 5.0 \\
\hline 1. & 553.0 & 253 & & 78. & 112 & 14. & & & 11 & \\
\hline & & & & 4. & & & & & & 1.0 \\
\hline 3 & 416.0 & 127. & & 43. & & & & & & \\
\hline & 1.0 & & & 4. & 6.0 & & & & & 1.0 \\
\hline & 297.0 & 335.0 & 154 & 137.0 & 73.0 & 119.0 & & & 36 & \\
\hline 0 . & 1.0 & 2.0 & & 4.0 & 5.0 & 6.0 & 7.0 & 8.0 & 9.0 & 11.0 \\
\hline 295 & $\begin{array}{lll}0 & 179.0\end{array}$ & : & & 13.0 & 1.0 & 0.0 & 0.0 & 17.0 & 1.0 & \\
\hline 48 & 387.0 & 196. & 140. & $\begin{array}{r}4.0 \\
128.0\end{array}$ & $\begin{array}{r}0.0 \\
122.0\end{array}$ & 39. & & & $\begin{array}{l}24 \\
17\end{array}$ & \\
\hline & 1.0 & 2.0 & 3. & 4.0 & 5.0 & 6. & 7 & & 9 & 10.0 \\
\hline 0 & 493.0 & 0245.0 & 18 & 1.0 & 0.0 & & & & & \\
\hline & 1.0 & 2. & & 4.0 & 5. & 6. & & & 15 & 20. \\
\hline 4 & 395.0 & 366. & 107 & 135. & 59. & 48 & 43 & & & \\
\hline & & & & 4.0 & & 6 & 7 & 8.0 & & 10.0 \\
\hline 0 & $\begin{array}{lc}0 & 451 \\
1\end{array}$ & $\begin{array}{ll}0 & 169\end{array}$ & & 0 & 14.0 & & 0 & 0.0 & 0 & . \\
\hline 12 & 381.0 & 233. & 221 & 203 & $47^{\circ}$ & & $1 /$. & & & \\
\hline & 1. & & & & & & & & & 10. \\
\hline 56 & $24 \overline{7} .0$ & & & & & & & & & \\
\hline & 1.0 & & & & & & & & & 46.0 \\
\hline 36 & $17 \overline{6}$. & 169. & 162 & 195 & & & & & & \\
\hline & & & & & & & & & & $10 .($ \\
\hline & 217. & & & & & & & & & \\
\hline & & & & & & & & & & 31.0 \\
\hline & 149.0 & 170 & & 77.0 & 116.0 & & & & & \\
\hline & & & & & & & & & & \\
\hline 11 & 88. & & & & & & & & & \\
\hline & 1. & & & & & & & & & 101. \\
\hline L3 & 61.0 & & & & & & & & & \\
\hline & 1.0 & & & & & & & & 189 & $35 .(1-x)$ \\
\hline & 240.0 & 274.0 & 19 & & & & & & & \\
\hline & 1. & & & & & & & & & $16 .(1+2 y$ \\
\hline & 560.0 & 516.0 & 344 & 172 & & & & & & \\
\hline & & & & & & & & & & 218.0 \\
\hline & $58 \overline{5} .0$ & & & & & & & & & \\
\hline & 10 & & & & & & & 0 & & 8. \\
\hline
\end{tabular}




$\begin{array}{rrrrrrrrrrrrr}1084.0 & 1031.0 & 902.0 & 536.0 & 372.0 & 182.0 & 117.0 & 60.0 & 29.0 & 21.0 \\ 0.0 & 1.0 & 6.0 & 15.0 & 28.0 & 45.0 & 66.0 & 91.0 & 121.0 & 156.0 & 196.0 \\ 910.0 & 708.0 & 582.0 & 288.0 & 182.0 & 100.0 & 60.0 & 63.0 & 29.0 & 12.0 & \\ 0.0 & 1.0 & 4.0 & 10.0 & 19.0 & 31.0 & 46.0 & 64.0 & 85.0 & 109.0 & 136.0 \\ 987.0 & 413.0 & 389.0 & 247.0 & 161.0 & 98.0 & 63.0 & 48.0 & 36.0 & 30.0 & \\ 0.0 & 1.0 & 4.0 & 9.0 & 16.0 & 25.0 & 36.0 & 50.0 & 67.0 & 87.0 & 110.0 \\ 1004.0 & 543.0 & 397.0 & 396.0 & 322.0 & 177.0 & 59.0 & 108.0 & 89.0 & 16.0 & \\ 0.0 & 1.0 & 4.0 & 9.0 & 17.0 & 28.0 & 42.0 & 59.0 & 79.0 & 102.0 & 128.0 \\ 786.0 & 547.0 & 622.0 & 421.0 & 406.0 & 187.0 & 82.0 & 48.0 & 74.0 & 11.0 & \\ 0.0 & 1.0 & 6.0 & 15.0 & 29.0 & 48.0 & 72.0 & 101.0 & 135.0 & 174.0 & 218.0 \\ 360.0 & 350.0 & 318.0 & 207.0 & 89.0 & 48.0 & 17.0 & 13.0 & 3.0 & 3.0 & \\ 0.0 & 1.0 & 9.0 & 24.0 & 47.0 & 78.0 & 117.0 & 164.0 & 219.0 & 282.0 & 353.0 \\ 553.0 & 309.0 & 202.0 & 144.0 & 81.0 & 35.0 & 14.0 & 16.0 & 21.0 & 17.0 & \\ \text { [END] } & & & & & & & & & \end{array}$


[NAME]

C-14

653520WG

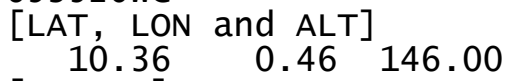

[SERIES]

$\begin{array}{rrrrrrrrrrr} & & & & & & & & & & \\ 0.0 & 1.0 & 2.0 & 3.0 & 4.0 & 5.0 & 6.0 & 7.0 & 8.0 & 9.0 & 10.0 \\ 849.0 & 0.0 & 0.0 & 0.0 & 0.0 & 0.0 & 0.0 & 0.0 & 0.0 & 0.0 & \\ 0.0 & 1.0 & 4.0 & 9.0 & 16.0 & 25.0 & 36.0 & 49.0 & 64.0 & 81.0 & 100.0 \\ 133.0 & 194.0 & 284.0 & 169.0 & 80.0 & 24.0 & 72.0 & 58.0 & 18.0 & 28.0 & \\ 0.0 & 1.0 & 2.0 & 3.0 & 4.0 & 5.0 & 6.0 & 7.0 & 8.0 & 9.0 & 10.0 \\ 509.0 & 0.0 & 0.0 & 0.0 & 0.0 & 0.0 & 0.0 & 0.0 & 0.0 & 0.0 & \\ 0.0 & 1.0 & 3.0 & 6.0 & 10.0 & 16.0 & 24.0 & 34.0 & 46.0 & 60.0 & 76.0 \\ 100.0 & 106.0 & 89.0 & 80.0 & 60.0 & 53.0 & 15.0 & 3.0 & 3.0 & 1.0 & \\ 0.0 & 1.0 & 2.0 & 3.0 & 4.0 & 5.0 & 6.0 & 7.0 & 8.0 & 9.0 & 10.0 \\ 915.0 & 184.0 & 0.0 & 0.0 & 0.0 & 0.0 & 0.0 & 0.0 & 0.0 & 0.0 & \\ 0.0 & 1.0 & 2.0 & 3.0 & 4.0 & 6.0 & 9.0 & 13.0 & 18.0 & 24.0 & 31.0\end{array}$

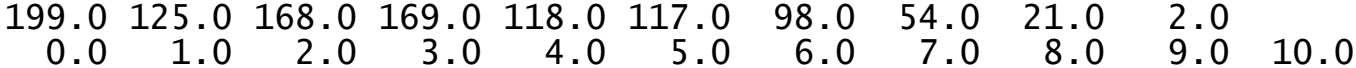

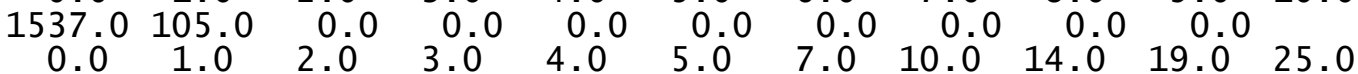

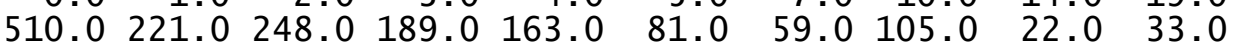

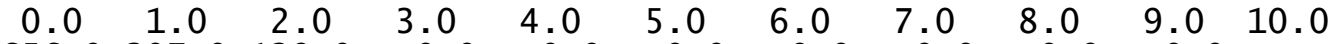

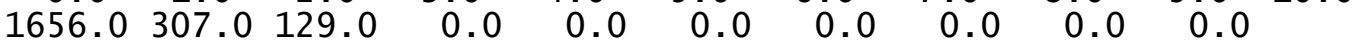

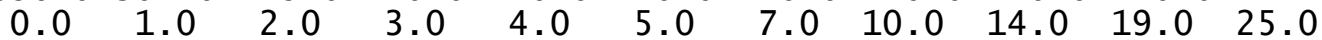

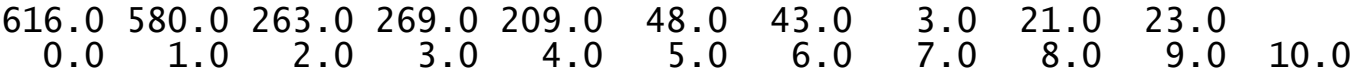

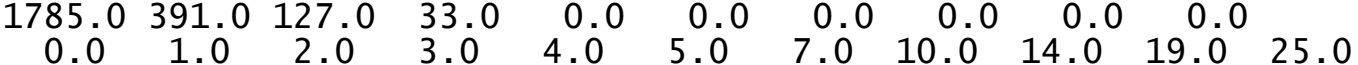

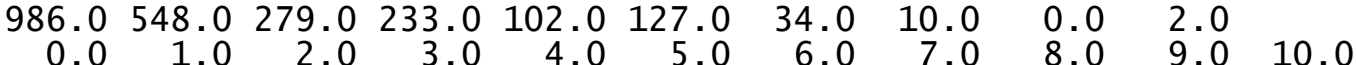

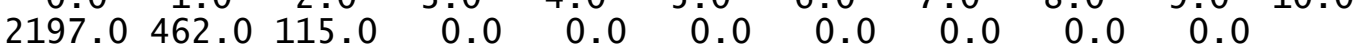
$\begin{array}{ccccccccccc}0.0 & 1.0 & 2.0 & 3.0 & 4.0 & 5.0 & 6.0 & 7.0 & 8.0 & 9.0 & 10.0\end{array}$ $\begin{array}{ccccccccccc}1328.0 & 697.0 & 437.0 & 109.0 & 96.0 & 2.0 & 32.0 & 47.0 & 19.0 & 0.0 & \\ 0.0 & 1.0 & 2.0 & 3.0 & 4.0 & 5.0 & 6.0 & 7.0 & 8.0 & 9.0 & 10.0\end{array}$

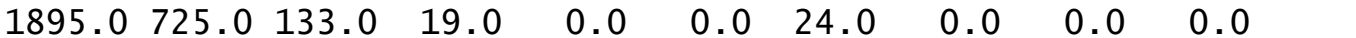

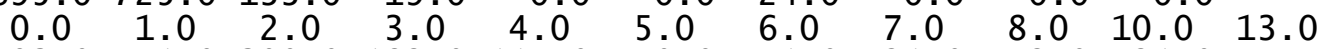

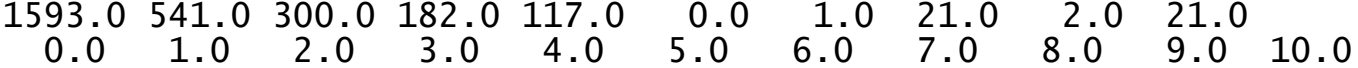
$\begin{array}{ccccccccccc}1446.0 & 626.0 & 343.0 & 58.0 & 0.0 & 26.0 & 0.0 & 0.0 & 0.0 & 0.0 & \\ 0.0 & 1.0 & 2.0 & 3.0 & 4.0 & 5.0 & 6.0 & 7.0 & 8.0 & 10.0 & 13.0\end{array}$

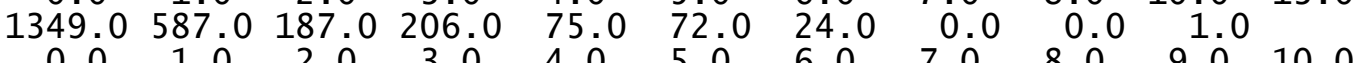

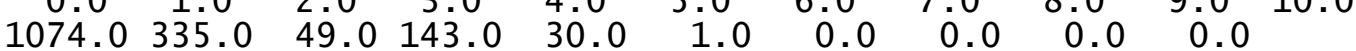
$\begin{array}{lllllllllll}0.0 & 1.0 & 3.0 & 6.0 & 10.0 & 15.0 & 22.0 & 31.0 & 42.0 & 55.0 & 70.0\end{array}$

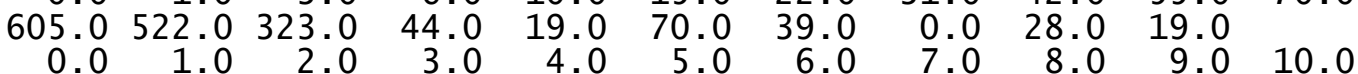
$\begin{array}{llllllllll}1027.0 & 146.0 & 0.0 & 4.0 & 0.0 & 0.0 & 0.0 & 0.0 & 0.0 & 0.0\end{array}$

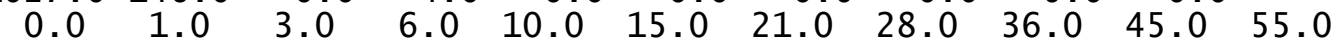
$\begin{array}{rrrrrrrrrrr}500.0 & 207.0 & 176.0 & 163.0 & 56.0 & 31.0 & 69.0 & 0.0 & 0.0 & 9.0 & \\ 0.0 & 1.0 & 2.0 & 3.0 & 4.0 & 5.0 & 6.0 & 7.0 & 8.0 & 9.0 & 10.0\end{array}$ $\begin{array}{ccccccccccc}1721.0 & 6.0 & 0.0 & 0.0 & 0.0 & 0.0 & 0.0 & 0.0 & 0.0 & 0.0 & \\ 0.0 & 1.0 & 2.0 & 3.0 & 4.0 & 6.0 & 9.0 & 13.0 & 18.0 & 24.0 & 31.0\end{array}$

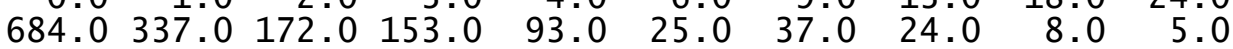

[RAIN]

$\begin{array}{rrrrrrrrrrr} & \\ 0.0 & 1.0 & 3.0 & 6.0 & 10.0 & 15.0 & 21.0 & 28.0 & 36.0 & 45.0 & 55.0 \\ 105.0 & 0.0 & 0.0 & 0.0 & 8.0 & 74.0 & 32.0 & 14.0 & 63.0 & 508.0 & \\ 0.0 & 1.0 & 3.0 & 7.0 & 13.0 & 21.0 & 31.0 & 43.0 & 57.0 & 73.0 & 91.0 \\ 145.0 & 34.0 & 49.0 & 97.0 & 81.0 & 64.0 & 36.0 & 5.0 & 7.0 & 8.0 & \\ 0.0 & 1.0 & 6.0 & 16.0 & 31.0 & 51.0 & 76.0 & 106.0 & 141.0 & 181.0 & 226.0 \\ 310.0 & 297.0 & 201.0 & 193.0 & 89.0 & 74.0 & 57.0 & 39.0 & 27.0 & 15.0 & \\ 0.0 & 1.0 & 4.0 & 9.0 & 17.0 & 28.0 & 42.0 & 59.0 & 79.0 & 102.0 & 128.0 \\ 400.0 & 383.0 & 266.0 & 238.0 & 223.0 & 126.0 & 63.0 & 32.0 & 11.0 & 6.0 & \\ 0.0 & 1.0 & 4.0 & 9.0 & 16.0 & 25.0 & 37.0 & 52.0 & 70.0 & 91.0 & 115.0 \\ 606.0 & 438.0 & 482.0 & 392.0 & 281.0 & 243.0 & 141.0 & 49.0 & 30.0 & 9.0 & \\ 0.0 & 1.0 & 5.0 & 13.0 & 25.0 & 41.0 & 61.0 & 85.0 & 113.0 & 145.0 & 181.0\end{array}$




$\begin{array}{rrrrrrrrrrr}656.0 & 464.0 & 770.0 & 560.0 & 274.0 & 164.0 & 75.0 & 60.0 & 51.0 & 18.0 & \\ 0.0 & 1.0 & 5.0 & 12.0 & 22.0 & 35.0 & 51.0 & 70.0 & 92.0 & 117.0 & 145.0 \\ 755.0 & 425.0 & 826.0 & 600.0 & 409.0 & 258.0 & 113.0 & 62.0 & 31.0 & 10.0 & \\ 0.0 & 1.0 & 3.0 & 7.0 & 13.0 & 21.0 & 31.0 & 43.0 & 57.0 & 73.0 & 91.0 \\ 729.0 & 524.0 & 634.0 & 810.0 & 509.0 & 326.0 & 223.0 & 108.0 & 89.0 & 22.0 & \\ 0.0 & 1.0 & 3.0 & 6.0 & 10.0 & 16.0 & 24.0 & 34.0 & 46.0 & 60.0 & 76.0 \\ 810.0 & 619.0 & 432.0 & 716.0 & 423.0 & 375.0 & 348.0 & 207.0 & 136.0 & 27.0 & \\ 0.0 & 1.0 & 4.0 & 9.0 & 16.0 & 25.0 & 36.0 & 49.0 & 64.0 & 81.0 & 100.0 \\ 580.0 & 390.0 & 341.0 & 488.0 & 350.0 & 212.0 & 134.0 & 58.0 & 15.0 & 10.0 & \\ 0.0 & 1.0 & 5.0 & 12.0 & 22.0 & 35.0 & 51.0 & 70.0 & 93.0 & 120.0 & 151.0 \\ 316.0 & 303.0 & 148.0 & 193.0 & 174.0 & 39.0 & 22.0 & 26.0 & 82.0 & 50.0 & \\ 0.0 & 1.0 & 4.0 & 9.0 & 16.0 & 25.0 & 36.0 & 49.0 & 64.0 & 81.0 & 100.0 \\ 117.0 & 153.0 & 148.0 & 170.0 & 65.0 & 0.0 & 408.0 & 607.0 & 51.0 & 32.0 & \\ \text { [END] } & & & & & & & & & & \end{array}$


[NAME]

C-16

653550WG

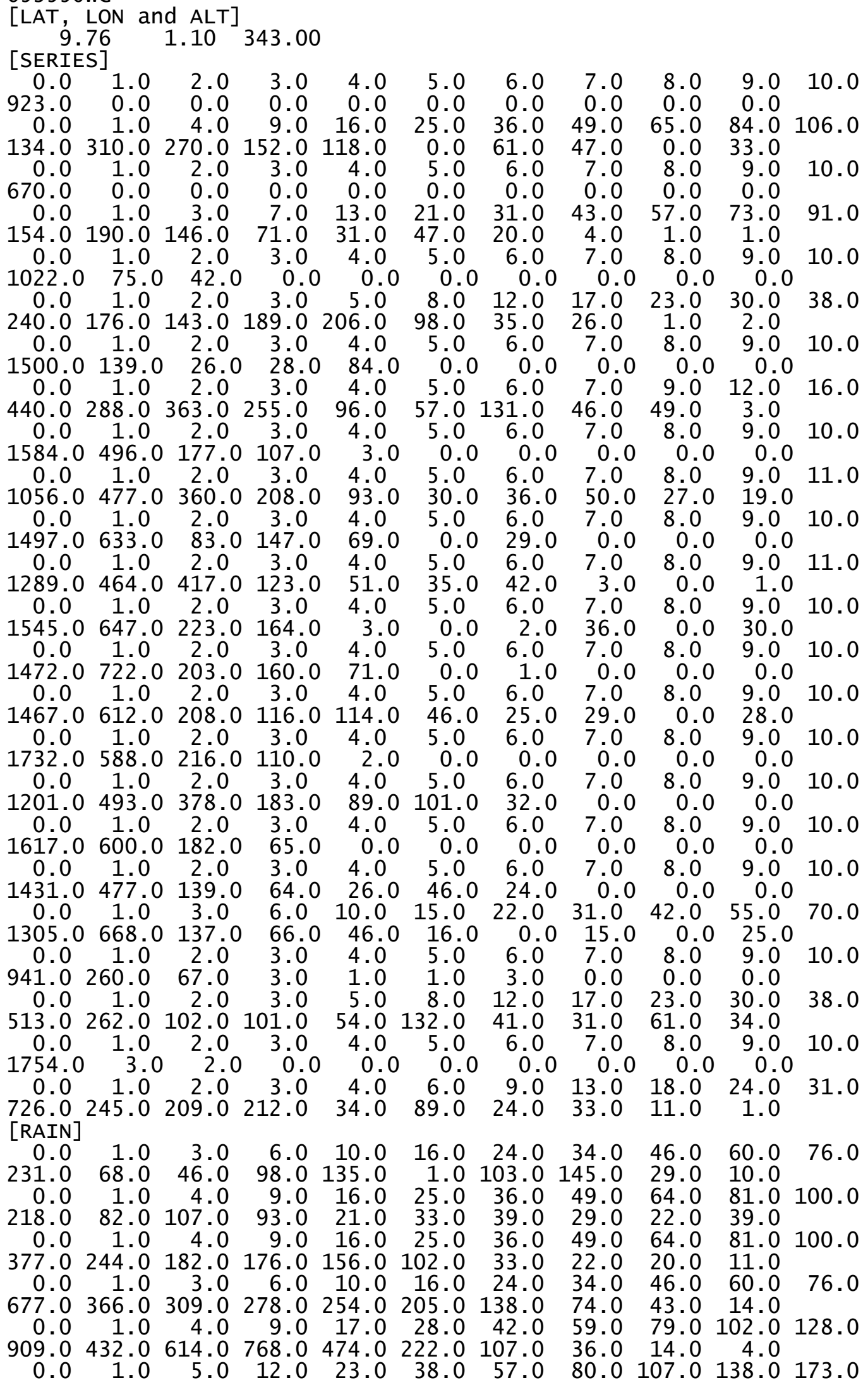


$\begin{array}{lllllllllll}1009.0 & 699.0 & 736.0 & 767.0 & 431.0 & 223.0 & 107.0 & 68.0 & 49.0 & 23.0\end{array}$ $\begin{array}{lllllllllll}0.0 & 1.0 & 6.0 & 15.0 & 28.0 & 45.0 & 66.0 & 92.0 & 123.0 & 159.0 & 200.0\end{array}$ $\begin{array}{llllllllllll}1100.0 & 1204.0 & 873.0 & 583.0 & 383.0 & 281.0 & 189.0 & 82.0 & 37.0 & 9.0\end{array}$ $\begin{array}{llllllllllll}0.0 & 1.0 & 9.0 & 24.0 & 47.0 & 78.0 & 117.0 & 164.0 & 219.0 & 282.0 & 353.0\end{array}$

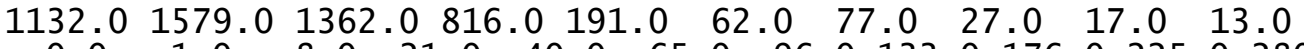
$\begin{array}{lllllllllllll}0.0 & 1.0 & 8.0 & 21.0 & 40.0 & 65.0 & 96.0 & 133.0 & 176.0 & 225.0 & 280.0\end{array}$ $\begin{array}{llllllllll}914.0 & 1503.0 & 1663.0 & 869.0 & 264.0 & 92.0 & 35.0 & 22.0 & 11.0 & 7.0\end{array}$

$\begin{array}{lllllllllll}0.0 & 1.0 & 4.0 & 9.0 & 17.0 & 28.0 & 42.0 & 59.0 & 79.0 & 102.0 & 128.0\end{array}$

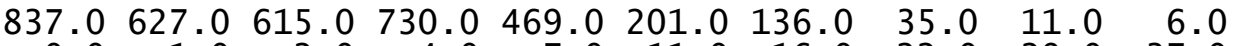

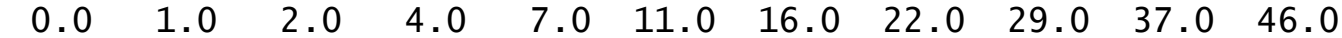

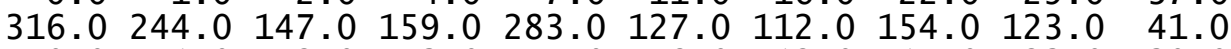

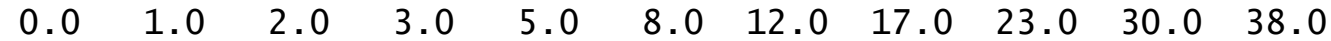
$\begin{array}{llllllllll}693.0 & 82.0 & 52.0 & 87.0 & 289.0 & 137.0 & 72.0 & 215.0 & 168.0 & 16.0\end{array}$ [END] 
[NAME]

C-18

653570WG

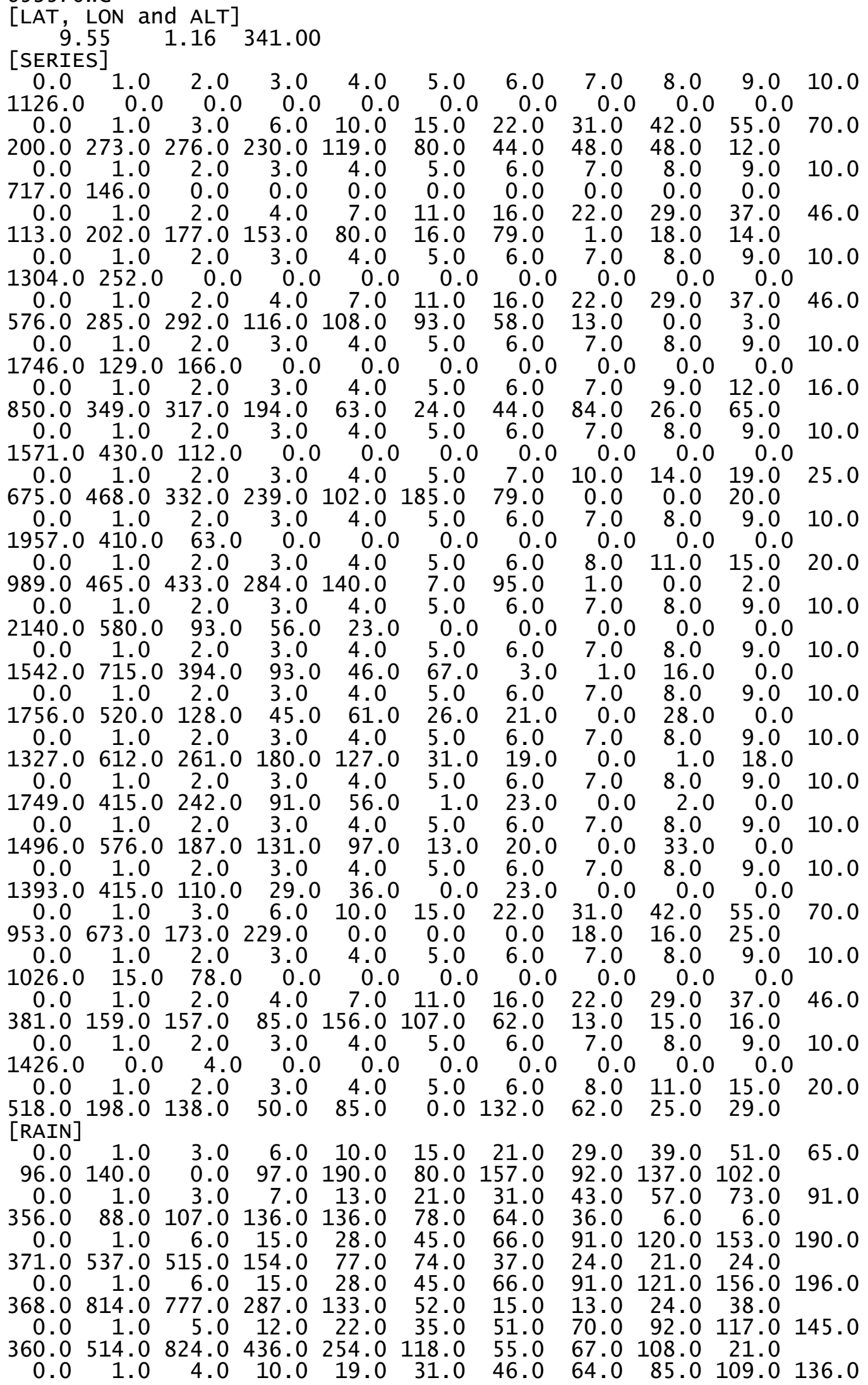




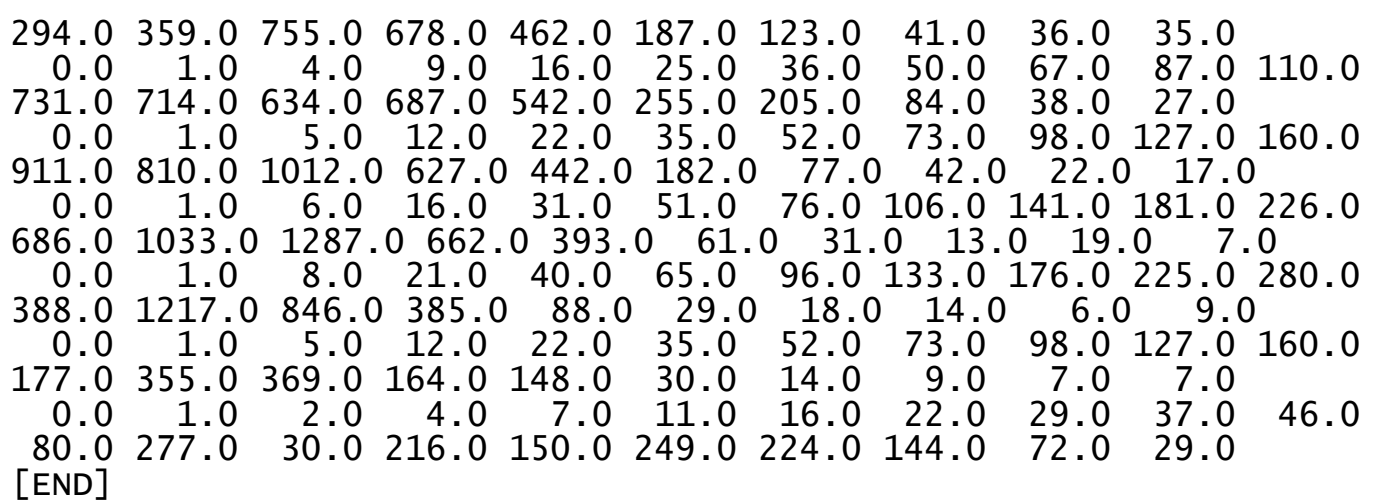


[NAME]

C-20

653610WG

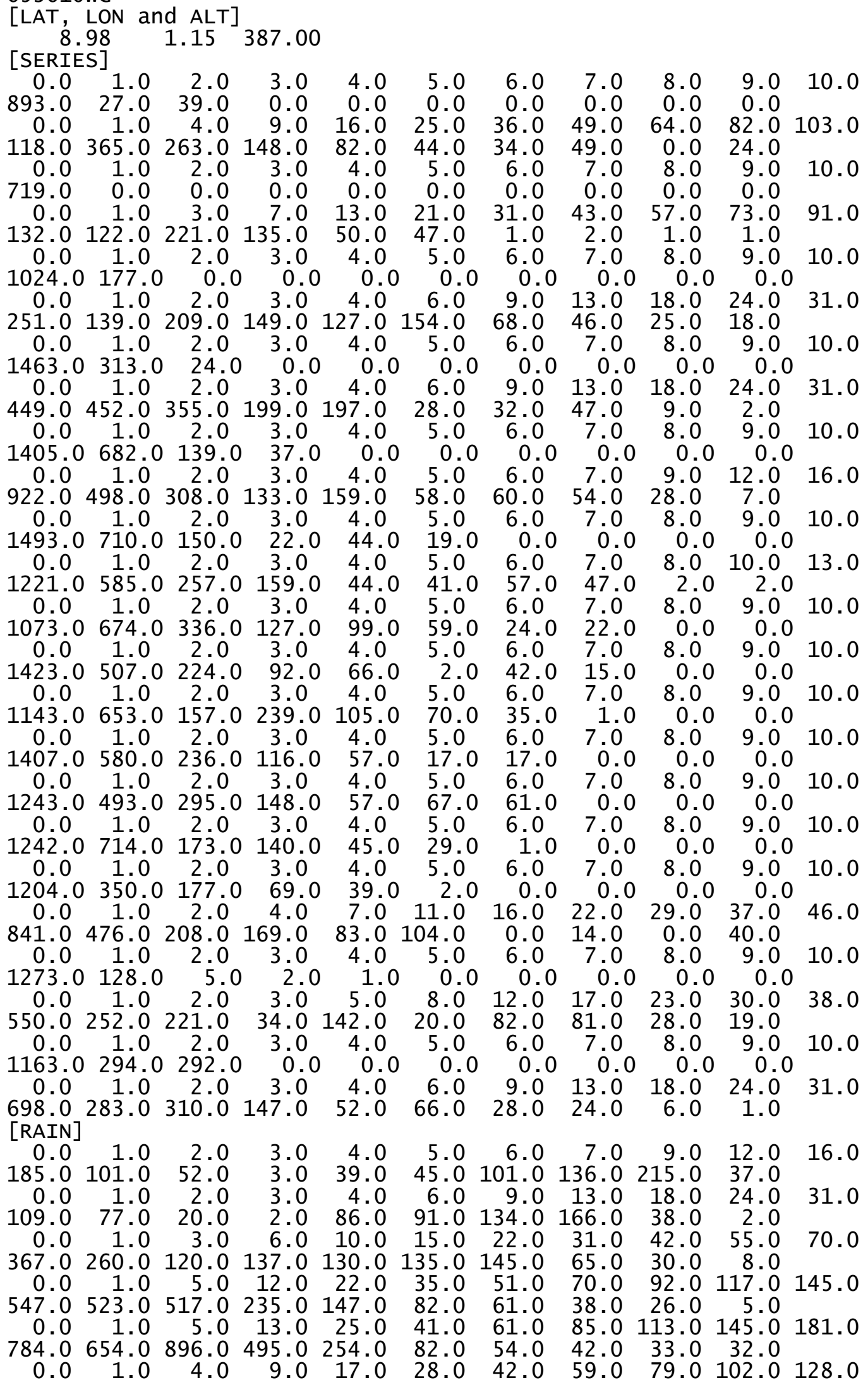


$\begin{array}{llllllllllll}734.0 & 685.0 & 689.0 & 757.0 & 439.0 & 222.0 & 132.0 & 79.0 & 42.0 & 11.0\end{array}$

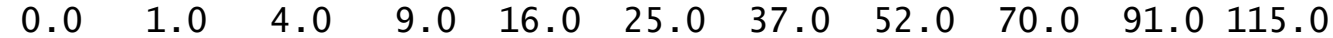
$\begin{array}{llllllllll}1112.0 & 988.0 & 915.0 & 686.0 & 544.0 & 460.0 & 181.0 & 83.0 & 49.0 & 10.0\end{array}$

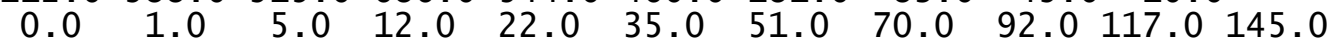

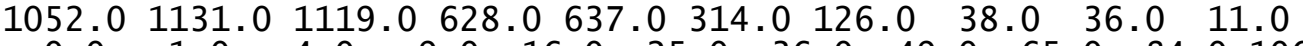
$\begin{array}{llllllllllll}0.0 & 1.0 & 4.0 & 9.0 & 16.0 & 25.0 & 36.0 & 49.0 & 65.0 & 84.0 & 106.0\end{array}$ $\begin{array}{llllllllll}1004.0 & 796.0 & 773.0 & 752.0 & 667.0 & 414.0 & 204.0 & 133.0 & 50.0 & 9.0\end{array}$ $\begin{array}{lllllllllll}0.0 & 1.0 & 4.0 & 10.0 & 19.0 & 31.0 & 46.0 & 64.0 & 85.0 & 109.0 & 136.0\end{array}$ $\begin{array}{llllllllll}586.0 & 400.0 & 780.0 & 450.0 & 441.0 & 182.0 & 70.0 & 38.0 & 18.0 & 10.0\end{array}$

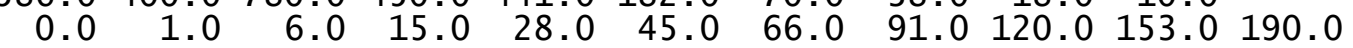

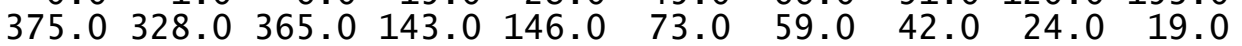

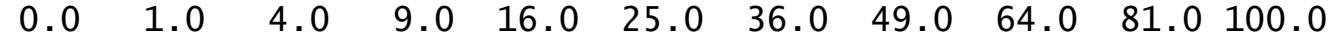

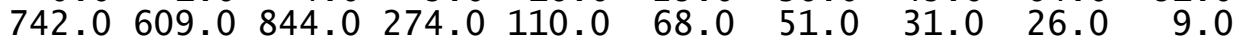
[END] 
[NAME]

C-22

653760WG

[LAT, LON and ALT] 702.00

[SERIES]

$\begin{array}{rrrrrrrrrrr}0.0 & 1.0 & 2.0 & 3.0 & 4.0 & 5.0 & 6.0 & 7.0 & 8.0 & 9.0 & 10.0 \\ 831.0 & 127.0 & 0.0 & 0.0 & 0.0 & 0.0 & 0.0 & 0.0 & 0.0 & 0.0 & \\ 0.0 & 1.0 & 3.0 & 7.0 & 13.0 & 21.0 & 31.0 & 43.0 & 57.0 & 73.0 & 91.0 \\ 139.0 & 219.0 & 261.0 & 288.0 & 99.0 & 18.0 & 56.0 & 11.0 & 36.0 & 34.0 & \\ 0.0 & 1.0 & 2.0 & 3.0 & 4.0 & 5.0 & 6.0 & 7.0 & 8.0 & 9.0 & 10.0 \\ 626.0 & 119.0 & 0.0 & 0.0 & 0.0 & 0.0 & 0.0 & 0.0 & 0.0 & 0.0 & \\ 0.0 & 1.0 & 3.0 & 7.0 & 13.0 & 21.0 & 31.0 & 43.0 & 57.0 & 73.0 & 91.0 \\ 109.0 & 179.0 & 228.0 & 118.0 & 90.0 & 19.0 & 1.0 & 0.0 & 1.0 & 2.0 & \\ 0.0 & 1.0 & 2.0 & 3.0 & 4.0 & 5.0 & 6.0 & 7.0 & 8.0 & 9.0 & 10.0\end{array}$

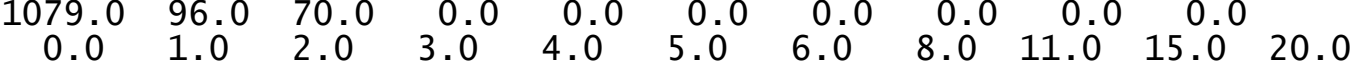

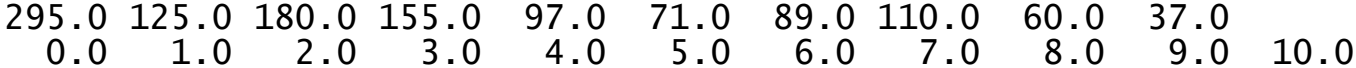

$\begin{array}{cccccccccc}1314.0 & 302.0 & 66.0 & 0.0 & 26.0 & 0.0 & 0.0 & 0.0 & 0.0 & 0.0\end{array}$

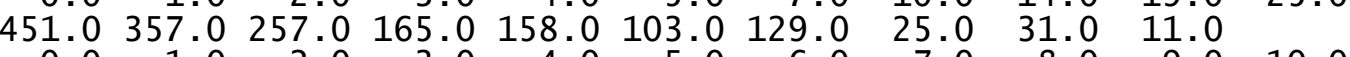

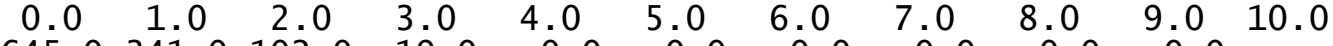

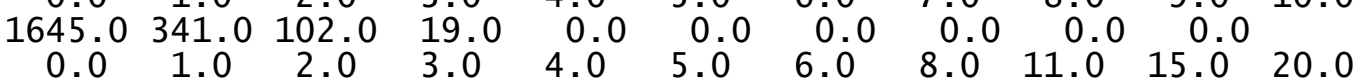

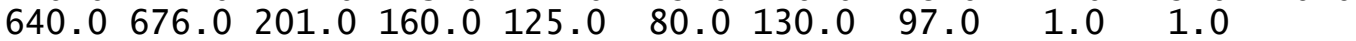

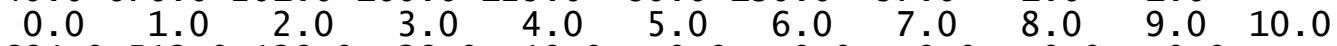

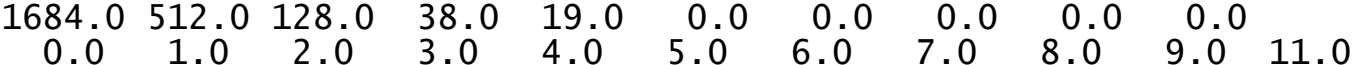

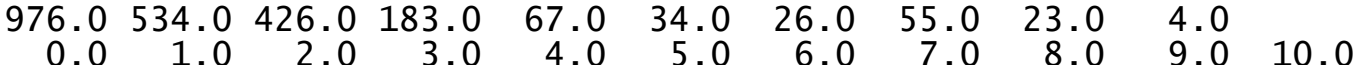

$\begin{array}{llllllllll}1308.0 & 483.0 & 215.0 & 83.0 & 139.0 & 35.0 & 0.0 & 0.0 & 0.0 & 0.0\end{array}$ $\begin{array}{lllllllllll}0.0 & 1.0 & 2.0 & 3.0 & 4.0 & 5.0 & 6.0 & 7.0 & 9.0 & 12.0 & 16.0\end{array}$

$\begin{array}{llllllllll}1029.0 & 525.0 & 288.0 & 182.0 & 102.0 & 47.0 & 33.0 & 36.0 & 15.0 & 10.0\end{array}$

$\begin{array}{lllllllllll}0.0 & 1.0 & 2.0 & 3.0 & 4.0 & 5.0 & 6.0 & 7.0 & 8.0 & 9.0 & 10.0\end{array}$

$\begin{array}{ccccccccccc}1552.0 & 652.0 & 127.0 & 84.0 & 68.0 & 3.0 & 0.0 & 13.0 & 0.0 & 0.0 & \\ 0.0 & 1.0 & 2.0 & 3.0 & 4.0 & 5.0 & 6.0 & 7.0 & 8.0 & 10.0 & 13.0\end{array}$

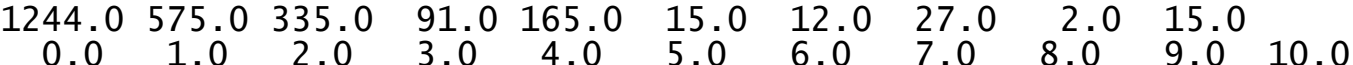

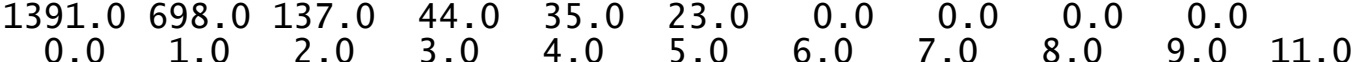

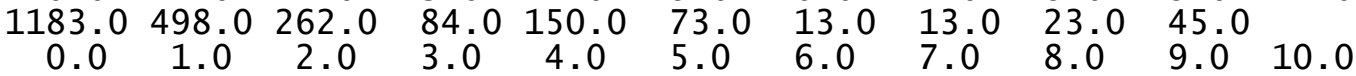

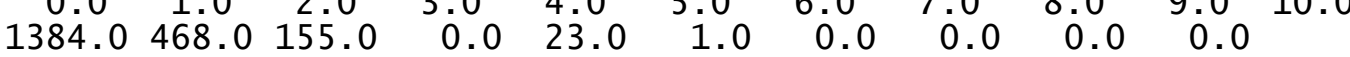
$\begin{array}{lllllllllll}0.0 & 1.0 & 3.0 & 6.0 & 10.0 & 15.0 & 21.0 & 28.0 & 36.0 & 45.0 & 55.0\end{array}$

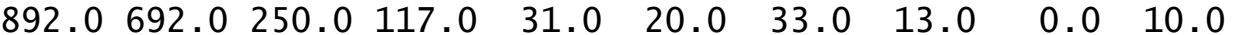

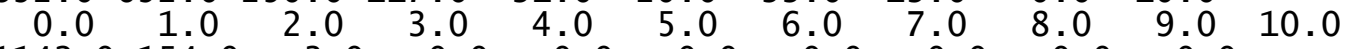

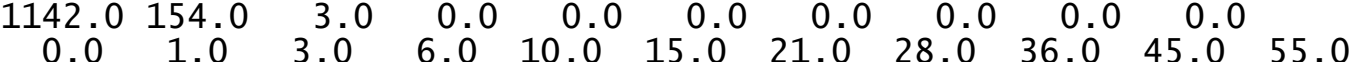

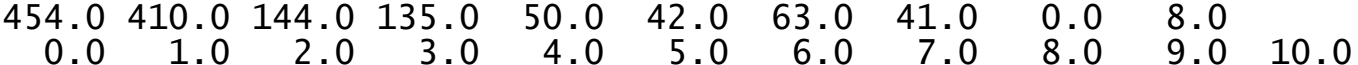

$\begin{array}{llllllllll}1718.0 & 4.0 & 0.0 & 0.0 & 0.0 & 0.0 & 0.0 & 0.0 & 0.0 & 0.0\end{array}$

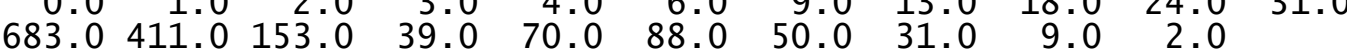

[RAIN]

$\begin{array}{lllllllllll}0.0 & 1.0 & 3.0 & 6.0 & 10.0 & 15.0 & 21.0 & 28.0 & 37.0 & 48.0 & 61.0\end{array}$

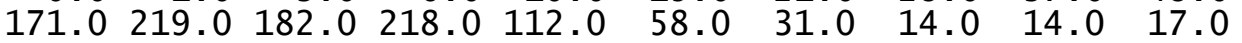

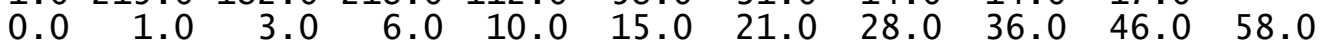

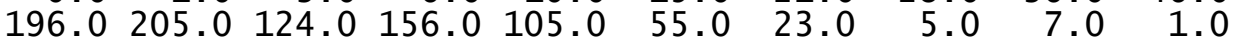

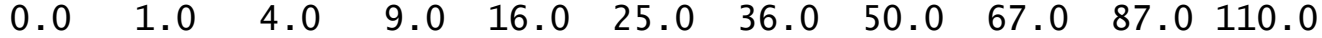

$\begin{array}{llllllllll}340.0 & 262.0 & 292.0 & 212.0 & 183.0 & 91.0 & 42.0 & 29.0 & 23.0 & 15.0\end{array}$

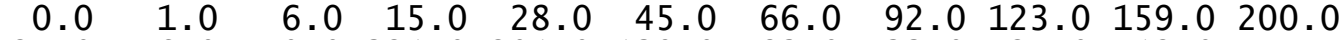

$\begin{array}{llllllllll}427.0 & 576.0 & 450.0 & 321.0 & 201.0 & 139.0 & 83.0 & 33.0 & 24.0 & 12.0\end{array}$

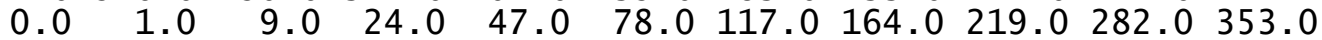

$\begin{array}{llllllllll}444.0 & 897.0 & 655.0 & 333.0 & 167.0 & 78.0 & 45.0 & 40.0 & 35.0 & 26.0\end{array}$

$\begin{array}{llllllllllll}0.0 & 1.0 & 7.0 & 18.0 & 34.0 & 55.0 & 81.0 & 113.0 & 151.0 & 195.0 & 245.0\end{array}$ 


$\begin{array}{rrrrrrrrrrr}581.0 & 870.0 & 836.0 & 435.0 & 293.0 & 138.0 & 101.0 & 36.0 & 27.0 & 23.0 & \\ 0.0 & 1.0 & 4.0 & 9.0 & 16.0 & 25.0 & 36.0 & 49.0 & 64.0 & 81.0 & 101.0 \\ 931.0 & 739.0 & 672.0 & 640.0 & 430.0 & 261.0 & 195.0 & 112.0 & 55.0 & 75.0 & \\ 0.0 & 1.0 & 3.0 & 7.0 & 13.0 & 21.0 & 31.0 & 43.0 & 57.0 & 73.0 & 91.0 \\ 914.0 & 593.0 & 523.0 & 672.0 & 432.0 & 389.0 & 230.0 & 120.0 & 94.0 & 55.0 & \\ 0.0 & 1.0 & 3.0 & 7.0 & 13.0 & 21.0 & 31.0 & 43.0 & 57.0 & 73.0 & 91.0 \\ 647.0 & 566.0 & 572.0 & 673.0 & 499.0 & 305.0 & 201.0 & 131.0 & 83.0 & 46.0 & \\ 0.0 & 1.0 & 3.0 & 6.0 & 10.0 & 15.0 & 22.0 & 31.0 & 42.0 & 55.0 & 70.0 \\ 604.0 & 466.0 & 281.0 & 479.0 & 340.0 & 210.0 & 224.0 & 116.0 & 89.0 & 96.0 & \\ 0.0 & 1.0 & 3.0 & 6.0 & 11.0 & 18.0 & 27.0 & 38.0 & 51.0 & 66.0 & 83.0 \\ 464.0 & 223.0 & 123.0 & 289.0 & 115.0 & 117.0 & 57.0 & 26.0 & 36.0 & 8.0 & \\ 0.0 & 1.0 & 4.0 & 9.0 & 16.0 & 25.0 & 37.0 & 52.0 & 70.0 & 91.0 & 115.0 \\ 332.0 & 315.0 & 406.0 & 180.0 & 154.0 & 151.0 & 94.0 & 35.0 & 32.0 & 48.0 & \\ \text { [END] } & & & & & & & & & & \end{array}$


[NAME]

C-24

653800WG

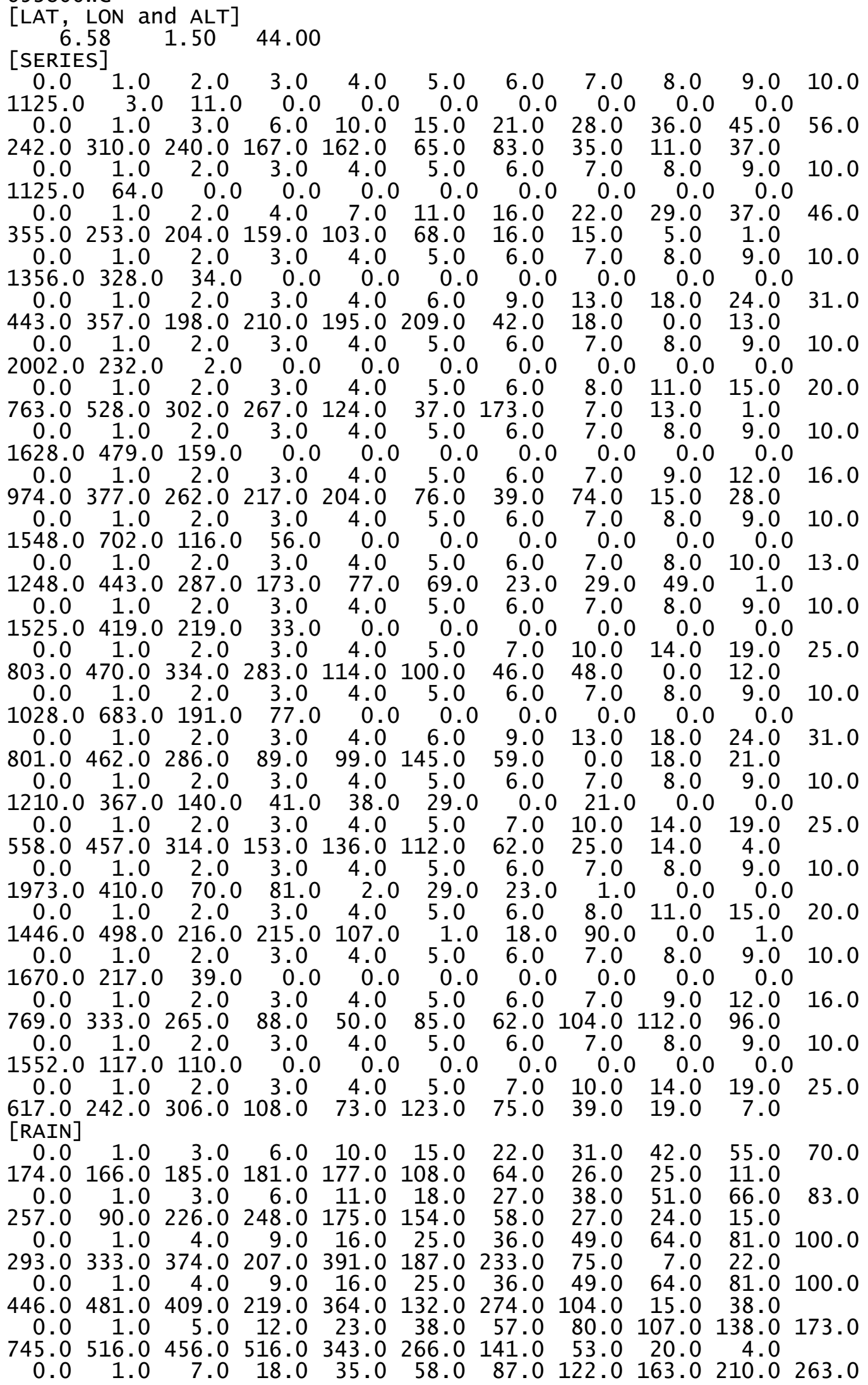


$\begin{array}{lllllllllll}884.0 & 959.0 & 712.0 & 443.0 & 215.0 & 132.0 & 81.0 & 51.0 & 34.0 & 24.0\end{array}$ $\begin{array}{lllllllllll}0.0 & 1.0 & 5.0 & 12.0 & 22.0 & 35.0 & 52.0 & 73.0 & 98.0 & 127.0 & 160.0\end{array}$ $\begin{array}{llllllllll}1113.0 & 649.0 & 525.0 & 324.0 & 216.0 & 117.0 & 112.0 & 41.0 & 21.0 & 16.0\end{array}$ $\begin{array}{lllllllllll}0.0 & 1.0 & 4.0 & 9.0 & 16.0 & 25.0 & 36.0 & 49.0 & 64.0 & 81.0 & 100.0\end{array}$

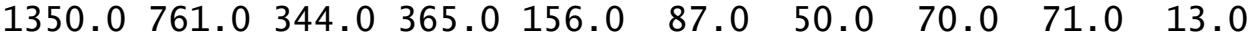

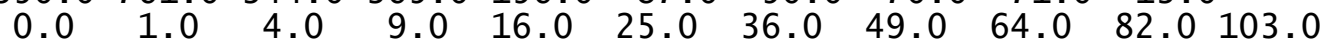

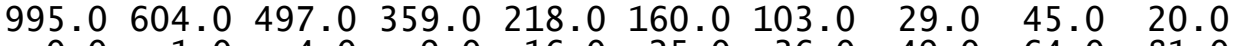

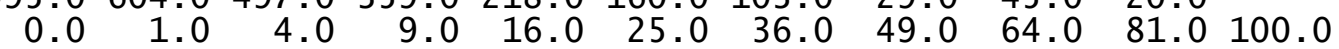

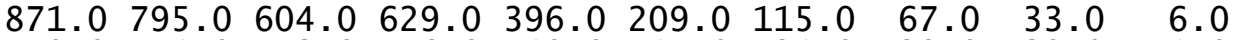

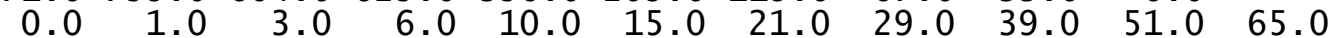
$\begin{array}{llllllllll}740.0 & 300.0 & 201.0 & 237.0 & 222.0 & 186.0 & 128.0 & 99.0 & 76.0 & 19.0\end{array}$

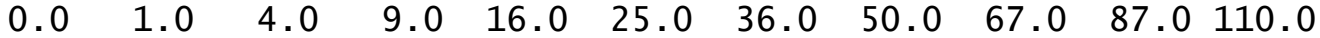

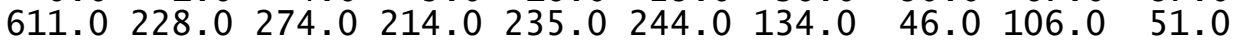
[END] 
[NAME]

C-26

653870WG

\begin{tabular}{|c|c|c|c|c|c|c|c|c|c|c|}
\hline $\begin{array}{r}\text { AT, } \\
6 \\
\text { SERIE }\end{array}$ & $\begin{array}{l}\text { LON } \\
16 \\
\text { ES] }\end{array}$ & $\begin{array}{l}\text { nd } A L T] \\
1.25\end{array}$ & 25.00 & & & & & & & \\
\hline & 1.0 & 2.0 & & 4.0 & 5.0 & 6.0 & 7.0 & 8.0 & 9.0 & 10. \\
\hline 2 & 85.0 & & & & & & & & & \\
\hline & 1.0 & & & 13. & 21 & & & & & 91.0 \\
\hline & 99.0 & 164.0 & 140. & 121.0 & & & & & 0 & \\
\hline & 1.0 & & & & & & & & & 0.0 \\
\hline 8 & 25.0 & & & 0 & 0 & & & & & \\
\hline & 1.0 & & & 10. & & & & & & 0.0 \\
\hline 8.0 & 18.0 & 71. & 114. & 70. & 26. & & & & & \\
\hline & 1.0 & & & & & & & & & 0.0 \\
\hline 7 & 9.0 & & & & & & & & & \\
\hline & 1.0 & & & & & & & & 19 & 5.0 \\
\hline & 21.0 & 56. & 124 & & 142.0 & 157 & & & & \\
\hline 7 & $\begin{array}{r}1.0 \\
56.0\end{array}$ & $\begin{array}{r}2 \\
54\end{array}$ & $\begin{array}{r}3 \\
15\end{array}$ & & & & & & & 0.0 \\
\hline & & & & & & & & & & 5.0 \\
\hline 4 & 80.0 & 160.0 & 138.0 & 135. & 145.0 & 199.0 & & & & \\
\hline & 1.0 & 2.0 & 3 & 4. & 5.0 & 6.0 & 7.0 & 8.0 & 9 & 0.0 \\
\hline 29 & 0393.0 & 0139.0 & 50.0 & 55.0 & 0.0 & 0.0 & 0.0 & 0.0 & 0.0 & \\
\hline & & 2.0 & 3. & 4. & 5.0 & 7.0 & 10.0 & 14.0 & 19.0 & $5 .($ \\
\hline & $\begin{array}{r}400.0 \\
1.0\end{array}$ & $\begin{array}{r}269.0 \\
2.0\end{array}$ & & $\begin{array}{r}82.0 \\
4.0\end{array}$ & $\begin{array}{r}102.0 \\
5.0\end{array}$ & & & & & ( \\
\hline 5 & 391.0 & $\begin{array}{ll}0 & 157.0\end{array}$ & 57.0 & 20.0 & 0.0 & 13.0 & 13.0 & 0.0 & 0.0 & \\
\hline & 1.0 & & 3. & 4.0 & 5.0 & 6.0 & 8.0 & 11. & 15 & 20. \\
\hline 36 & 429.0 & 295.0 & 211. & 104.0 & 135.0 & & & & & \\
\hline 0 & 1.0 & 2.0 & 3.0 & 4.0 & 5.0 & 6.0 & 7.0 & 8.0 & 9 & 10.0 \\
\hline & C $\begin{array}{ll}0 & 450.0 \\
1 & 0\end{array}$ & 63.0 & 2.0 & 16.0 & 22.0 & $9^{1.0}$ & $13^{0.0}$ & 18.0 & 0 & 18 \\
\hline & 346.0 & 245.0 & 121.0 & 219.0 & 146.0 & 77.0 & & & 11 & \\
\hline & 1.0 & 2.0 & 3. & 4.0 & 5.0 & 6.0 & 7.0 & 8.0 & 9.0 & $10 .($ \\
\hline & $\begin{array}{lc}0 & 148.0 \\
1 & 0\end{array}$ & $\begin{array}{lll}0 & 156.0 \\
2 & 0\end{array}$ & 29 & 5.0 & ${ }_{8.0}^{0.0}$ & 120.0 & $17^{0.0}$ & 0.0 & & 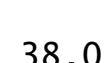 \\
\hline 21 & 303.0 & 203.0 & 172.0 & 143.0 & 129.0 & & & & 12 & \\
\hline & & & 3.0 & 4.0 & 5.0 & 6.0 & & 8.0 & & $10 .($ \\
\hline 6 & $0 \quad 400.0$ & 0159.0 & 23.0 & 0.0 & 0.0 & 0.0 & 0.0 & 0.0 & .0 & \\
\hline & $7 \frac{1}{6}$ & 185 & 276 & 92 & 131 & & & & & \\
\hline & 1.0 & 2.0 & & & 5.0 & & & & & 0. \\
\hline 6 & 271.0 & 0141.0 & 20 & & 0.0 & & & & & \\
\hline & 1.0 & & & 7. & 11. & 16. & & & & 46. \\
\hline & 392.0 & 307. & 248 & 155 & & & & & & \\
\hline & 1.0 & & & & & & & & & 10. \\
\hline & 153. & 46 & & & & & & & & \\
\hline & & & & & & & & & & 46. \\
\hline & 153. & 211.0 & 237 & 109 & & & & & & \\
\hline & 1. & 2.0 & & & & & & & & 0.0 \\
\hline & 148 & & & & & & & & & \\
\hline & & 2.0 & 3 & & & 7.0 & & & & 25. \\
\hline & & & & & & & & & & \\
\hline & & & & & & & & & & 58.0 \\
\hline & 127 & 11 & & & & & & & & \\
\hline & & & & & & & & & & 145. \\
\hline & 233. & 131 & & & & & & & & \\
\hline & & & & & & & & & 168 & 211. \\
\hline & 282. & & & & & & & & & \\
\hline & & & & & & & & & 289 & $361 .($ \\
\hline & & & & & & & & & & \\
\hline & & & & & & & & & 189 & 235. \\
\hline & 39 & & & & & & & & & \\
\hline & & & & & & & & & 0 & 45 \\
\hline
\end{tabular}




$\begin{array}{rrrrrrrrrrr}706.0 & 608.0 & 508.0 & 448.0 & 283.0 & 273.0 & 124.0 & 67.0 & 58.0 & 6.0 & \\ 0.0 & 1.0 & 4.0 & 9.0 & 16.0 & 25.0 & 37.0 & 52.0 & 70.0 & 91.0 & 115.0 \\ 785.0 & 508.0 & 238.0 & 295.0 & 117.0 & 150.0 & 143.0 & 52.0 & 23.0 & 51.0 & \\ 0.0 & 1.0 & 4.0 & 9.0 & 16.0 & 25.0 & 36.0 & 50.0 & 67.0 & 87.0 & 110.0 \\ 952.0 & 489.0 & 205.0 & 172.0 & 76.0 & 62.0 & 54.0 & 29.0 & 12.0 & 21.0 & \\ 0.0 & 1.0 & 5.0 & 12.0 & 22.0 & 35.0 & 51.0 & 70.0 & 92.0 & 117.0 & 145.0 \\ 887.0 & 565.0 & 462.0 & 222.0 & 121.0 & 80.0 & 62.0 & 28.0 & 5.0 & 12.0 & \\ 0.0 & 1.0 & 4.0 & 9.0 & 16.0 & 26.0 & 39.0 & 55.0 & 74.0 & 96.0 & 121.0 \\ 689.0 & 391.0 & 451.0 & 323.0 & 203.0 & 128.0 & 57.0 & 38.0 & 9.0 & 9.0 & \\ 0.0 & 1.0 & 3.0 & 6.0 & 10.0 & 16.0 & 24.0 & 34.0 & 46.0 & 60.0 & 76.0 \\ 423.0 & 204.0 & 134.0 & 166.0 & 273.0 & 151.0 & 28.0 & 26.0 & 22.0 & 8.0 & \\ 0.0 & 1.0 & 3.0 & 6.0 & 11.0 & 18.0 & 27.0 & 38.0 & 51.0 & 66.0 & 83.0 \\ 499.0 & 239.0 & 148.0 & 349.0 & 190.0 & 112.0 & 42.0 & 47.0 & 54.0 & 25.0 & \\ \text { [END] } & & & & & & & & & & \end{array}$


[NAME]

C-28

654720WG

[LAT, LON and ALT] 69.00

[SERIES]

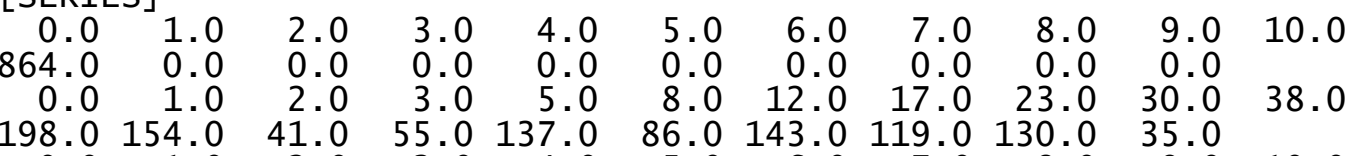

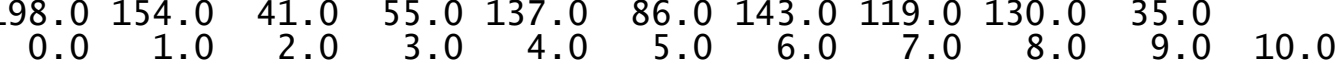

$\begin{array}{rrrrrrrrrrr}1142.0 & 0.0 & 0.0 & 0.0 & 0.0 & 0.0 & 0.0 & 0.0 & 0.0 & 0.0 & \\ 0.0 & 1.0 & 2.0 & 3.0 & 5.0 & 8.0 & 12.0 & 17.0 & 23.0 & 30.0 & 38.0\end{array}$

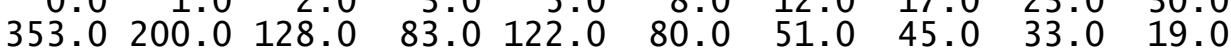

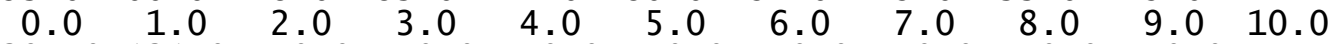

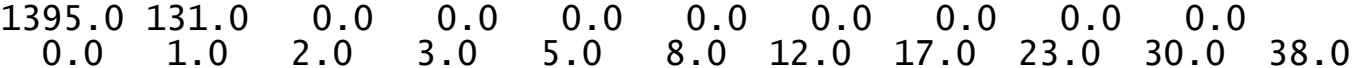

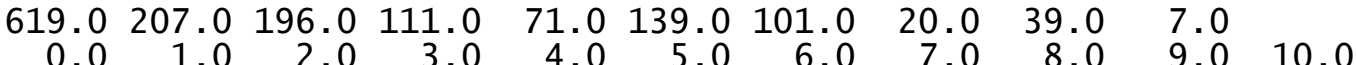

$\begin{array}{llllllllll}1485.0 & 267.0 & 0.0 & 35.0 & 0.0 & 0.0 & 0.0 & 0.0 & 0.0 & 0.0\end{array}$

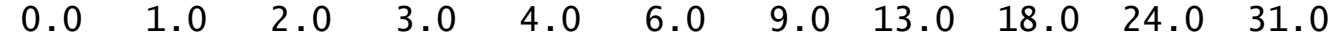

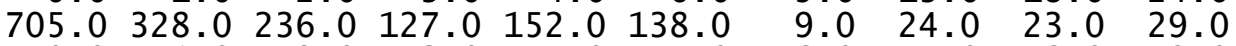

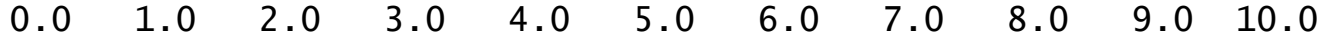

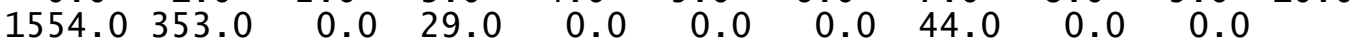

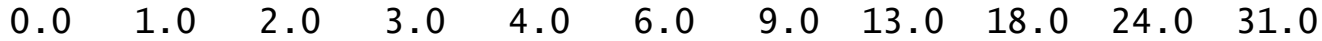

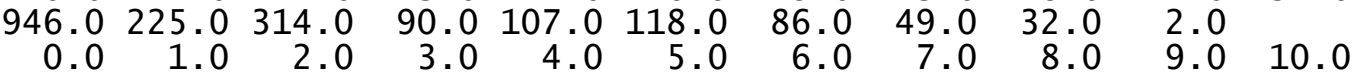

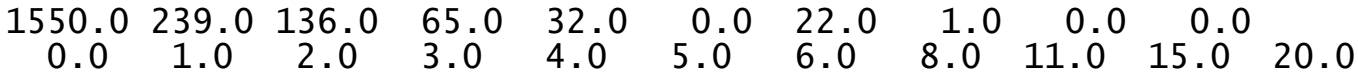

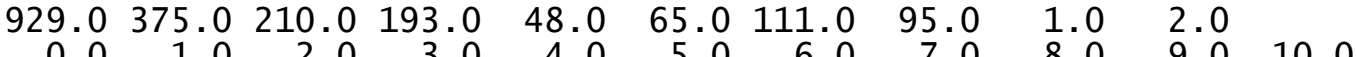
$\begin{array}{llllllllll}1049.0 & 343.0 & 53.0 & 118.0 & 37.0 & 0.0 & 0.0 & 0.0 & 0.0 & 0.0\end{array}$

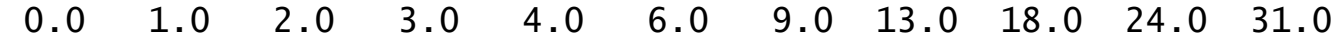

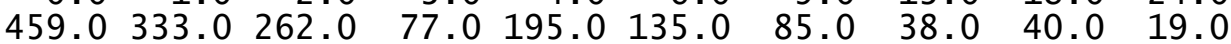

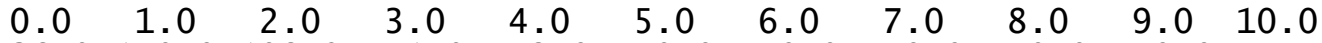

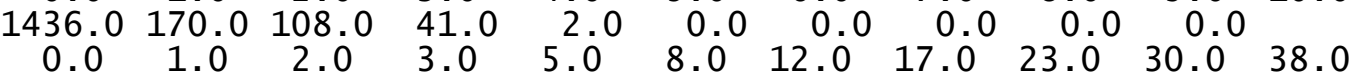

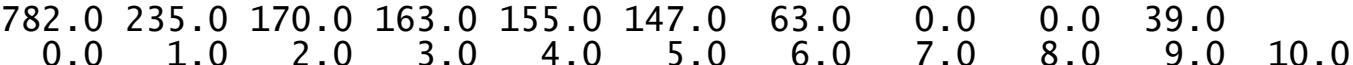

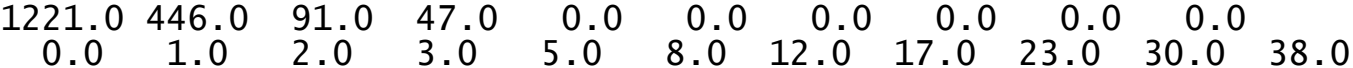

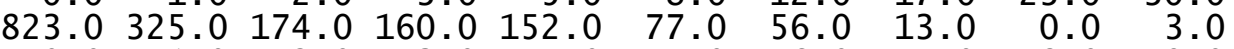

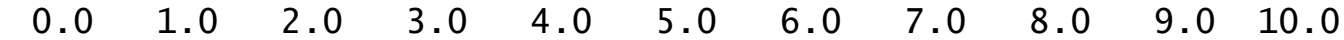
$\begin{array}{lllllllllll}1375.0 & 394.0 & 86.0 & 35.0 & 0.0 & 0.0 & 0.0 & 0.0 & 0.0 & 0.0 & \\ 0.0 & 1.0 & 0.0 & 38.0\end{array}$

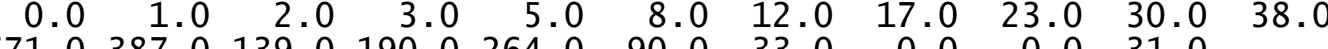

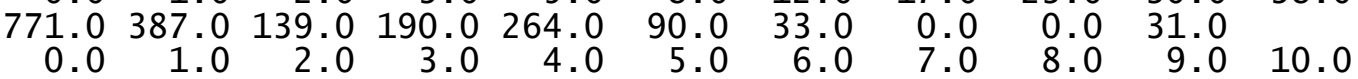
$\begin{array}{ccccccccccc}1395.0 & 115.0 & 2.0 & 3.0 & 0.0 & 0.0 & 0.0 & 0.0 & 0.0 & 0.0 & \\ 0.0 & 1.0 & 2.0 & 3.0 & 5.0 & 8.0 & 12.0 & 17.0 & 23.0 & 30.0 & 38.0\end{array}$

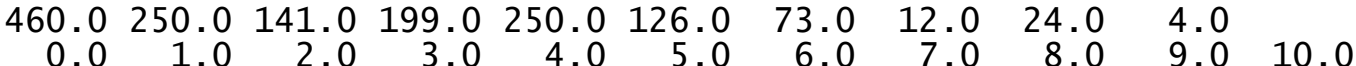

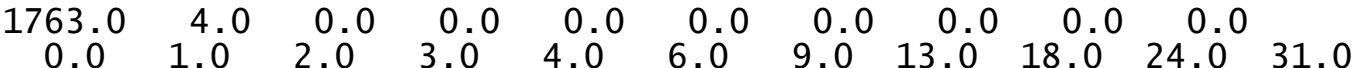

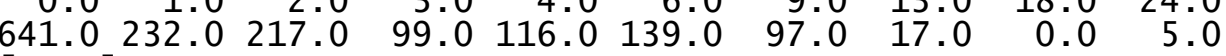

[RAIN]

$\begin{array}{lllllllllll}0.0 & 1.0 & 3.0 & 6.0 & 10.0 & 15.0 & 21.0 & 28.0 & 36.0 & 45.0 & 56.0\end{array}$

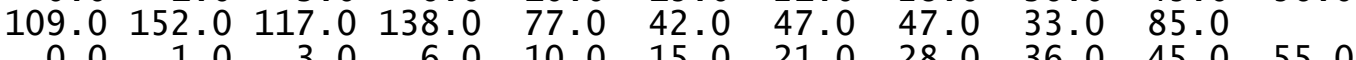

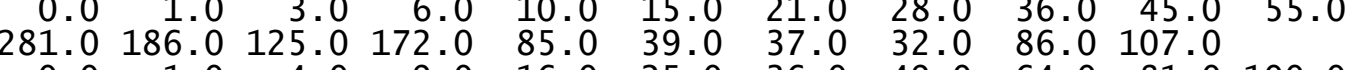

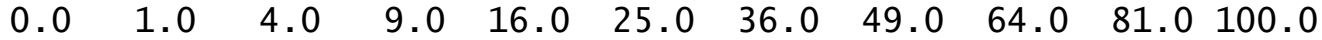
$\begin{array}{llllllllll}270.0 & 320.0 & 255.0 & 119.0 & 148.0 & 232.0 & 161.0 & 105.0 & 36.0 & 18.0\end{array}$

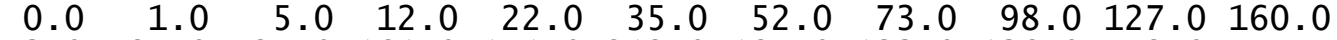
$\begin{array}{llllllllll}243.0 & 487.0 & 424.0 & 161.0 & 151.0 & 212.0 & 187.0 & 133.0 & 136.0 & 42.0\end{array}$

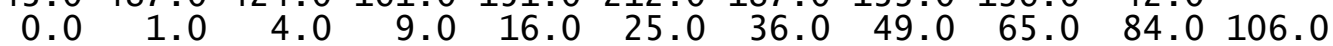
$\begin{array}{rrrrrrrrrr}375.0 & 294.0 & 421.0 & 410.0 & 373.0 & 348.0 & 182.0 & 141.0 & 115.0 & 39.0\end{array}$

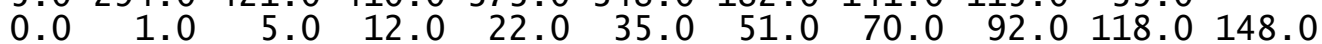


$\begin{array}{lllllllllll}947.0 & 396.0 & 558.0 & 370.0 & 327.0 & 218.0 & 127.0 & 42.0 & 28.0 & 15.0\end{array}$ $\begin{array}{lllllllllll}0.0 & 1.0 & 6.0 & 15.0 & 28.0 & 46.0 & 69.0 & 97.0 & 130.0 & 168.0 & 211.0\end{array}$ $\begin{array}{llllllllll}1067.0 & 418.0 & 503.0 & 185.0 & 147.0 & 119.0 & 60.0 & 35.0 & 13.0 & 12.0\end{array}$ $\begin{array}{lllllllllll}0.0 & 1.0 & 5.0 & 12.0 & 22.0 & 35.0 & 51.0 & 71.0 & 95.0 & 123.0 & 155.0\end{array}$ $\begin{array}{llllllllll}1372.0 & 382.0 & 228.0 & 137.0 & 77.0 & 37.0 & 15.0 & 14.0 & 12.0 & 9.0\end{array}$ $\begin{array}{lllllllllll}0.0 & 1.0 & 7.0 & 18.0 & 34.0 & 56.0 & 84.0 & 118.0 & 158.0 & 204.0 & 256.0\end{array}$

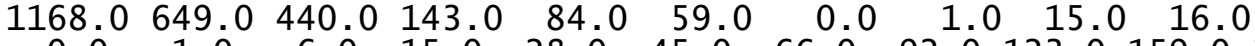
$\begin{array}{lllllllllll}0.0 & 1.0 & 6.0 & 15.0 & 28.0 & 45.0 & 66.0 & 92.0 & 123.0 & 159.0 & 200.0\end{array}$

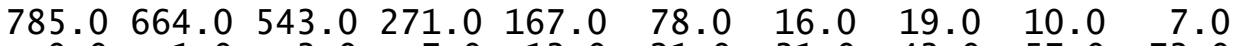

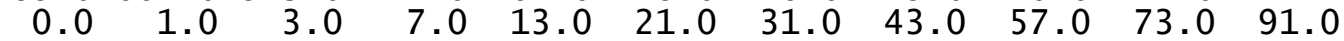

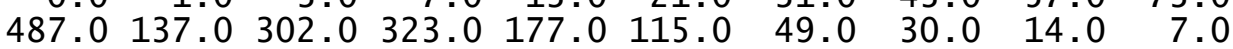

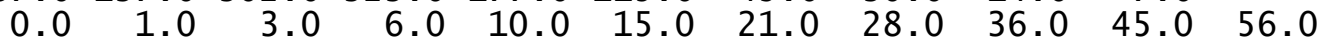

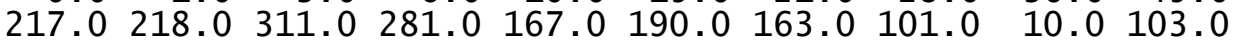
[END] 
[NAME]

C-30

655010WG

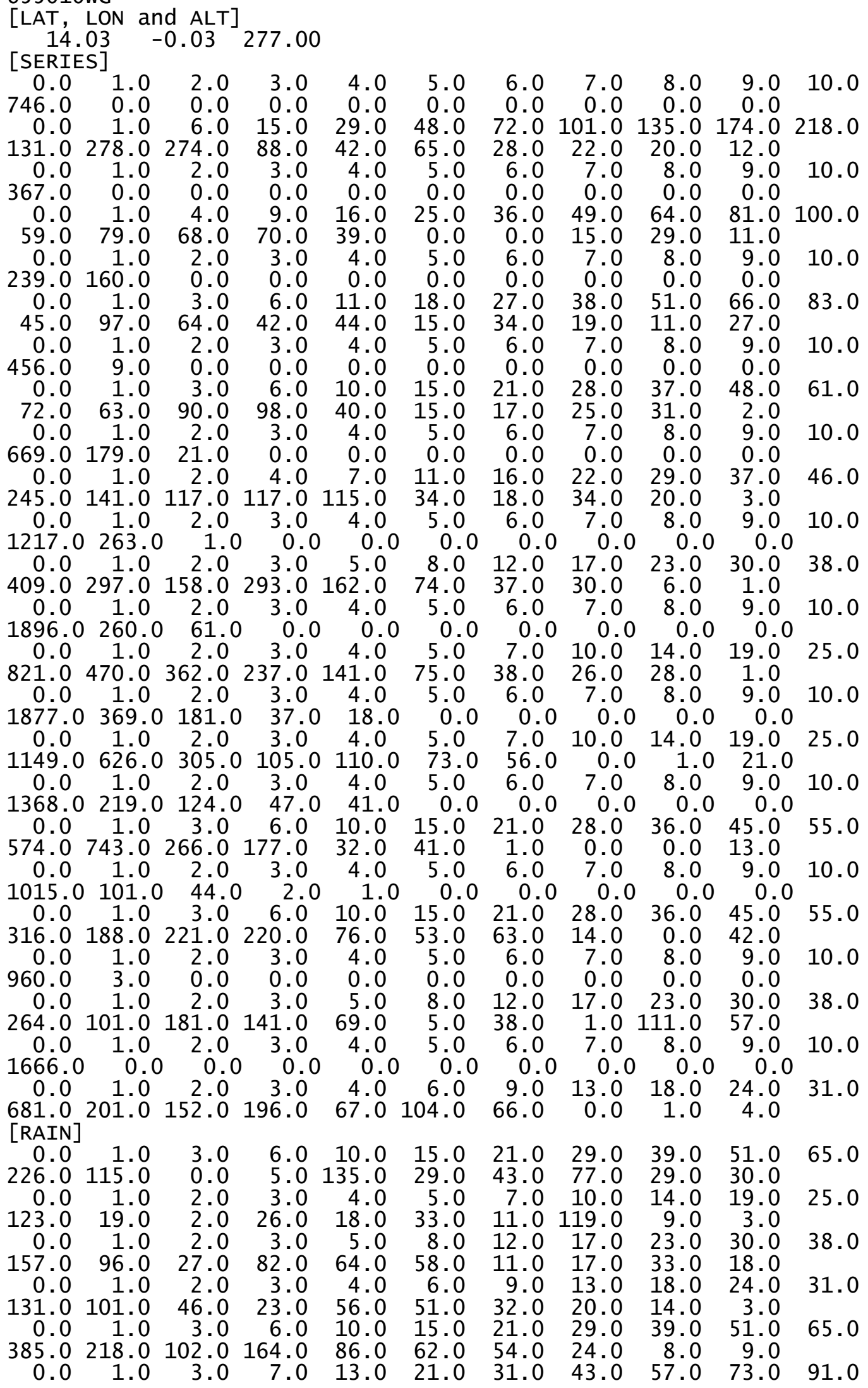




$\begin{array}{rrrrrrrrrrr}464.0 & 258.0 & 240.0 & 347.0 & 170.0 & 127.0 & 105.0 & 17.0 & 19.0 & 11.0 & \\ 0.0 & 1.0 & 4.0 & 9.0 & 16.0 & 25.0 & 36.0 & 49.0 & 64.0 & 81.0 & 100.0 \\ 639.0 & 370.0 & 528.0 & 386.0 & 230.0 & 187.0 & 167.0 & 65.0 & 22.0 & 22.0 & \\ 0.0 & 1.0 & 4.0 & 9.0 & 16.0 & 25.0 & 36.0 & 49.0 & 64.0 & 81.0 & 100.0 \\ 780.0 & 485.0 & 451.0 & 525.0 & 463.0 & 328.0 & 202.0 & 91.0 & 52.0 & 15.0 & \\ 0.0 & 1.0 & 4.0 & 9.0 & 16.0 & 25.0 & 36.0 & 49.0 & 65.0 & 84.0 & 106.0 \\ 560.0 & 525.0 & 456.0 & 383.0 & 342.0 & 150.0 & 86.0 & 43.0 & 10.0 & 5.0 & \\ 0.0 & 1.0 & 5.0 & 12.0 & 22.0 & 36.0 & 54.0 & 76.0 & 102.0 & 132.0 & 166.0 \\ 465.0 & 283.0 & 327.0 & 68.0 & 66.0 & 48.0 & 36.0 & 17.0 & 15.0 & 12.0 & \\ 0.0 & 1.0 & 4.0 & 9.0 & 16.0 & 25.0 & 36.0 & 50.0 & 67.0 & 87.0 & 110.0 \\ 294.0 & 199.0 & 92.0 & 0.0 & 35.0 & 39.0 & 114.0 & 107.0 & 83.0 & 8.0 & \\ 0.0 & 1.0 & 4.0 & 9.0 & 16.0 & 26.0 & 39.0 & 55.0 & 74.0 & 96.0 & 121.0 \\ 295.0 & 303.0 & 0.0 & 0.0 & 0.0 & 69.0 & 361.0 & 448.0 & 145.0 & 83.0 & \\ \text { [END] } & & & & & & & & & & \end{array}$


[NAME]

C-32

655020WG

\begin{tabular}{|c|c|c|c|c|c|c|c|c|c|c|}
\hline $\begin{array}{r}A T, \\
13 \\
\text { SERI }\end{array}$ & $\begin{array}{l}\text { LON a } \\
.56 \\
\text { ES] }\end{array}$ & $\begin{array}{l}A L 1] \\
.41\end{array}$ & 336.00 & & & & & & & \\
\hline 0.0 & 1.0 & 2.0 & & 4.0 & 5.0 & 6.0 & 7.0 & 8.0 & 9.0 & 10.0 \\
\hline 65.0 & 0.0 & & & & & & & & & \\
\hline $\begin{array}{l}0 \\
0\end{array}$ & 1.0 & & 18 & 34 & 55 & 81 & 112 & 149 & 192.0 & 241.0 \\
\hline & 0.0 & & & $\begin{array}{r}112 \\
4\end{array}$ & & & & & $\begin{array}{l}3.0 \\
9.0\end{array}$ & 10 \\
\hline & 0.0 & & & & 0 & & & & 0 & \\
\hline & 1.0 & & & & 21 & & & & 73 & 91.0 \\
\hline & 34.0 & 75. & 120 & & 26 & & & & & \\
\hline & 1.0 & & & & & & & & & 10.0 \\
\hline & 0.0 & & & 0 & 0 & & & & & \\
\hline & 1.0 & & & 10 & & & & & & 70.0 \\
\hline & 152.0 & 37. & & 81 & & & & & & \\
\hline & 1.0 & & & & & & & & & 10.0 \\
\hline 37 & 24.0 & & & & & & & & & \\
\hline & 1.0 & & & 10 & & & & & & 55.0 \\
\hline & 119.0 & 91. & & 69. & & & & & 11 & \\
\hline & $\begin{array}{l}1.0 \\
152.0\end{array}$ & $\begin{array}{l}2.0 \\
23.0\end{array}$ & $\begin{array}{c}3.0 \\
0.0\end{array}$ & $\begin{array}{r}4.0 \\
0.0\end{array}$ & $\begin{array}{l}5.0 \\
0.0\end{array}$ & $\begin{array}{c}6.0 \\
0.0\end{array}$ & $\begin{array}{l}7.0 \\
0.0\end{array}$ & $\begin{array}{c}8.0 \\
0.0\end{array}$ & $\begin{array}{c}9.0 \\
0.0\end{array}$ & 10.0 \\
\hline & 1.0 & 2.0 & 3.0 & 4.0 & 6.0 & 9.0 & 13. & 18.0 & 24 & 31.0 \\
\hline 34 & 250.0 & 69.0 & 159. & 151.0 & 115.0 & & & & & \\
\hline & $\begin{array}{l}1.0 \\
281.0\end{array}$ & $\begin{array}{l}2.0 \\
66.0\end{array}$ & & $\begin{array}{r}4.0 \\
0.0\end{array}$ & $\begin{array}{l}5.0 \\
0.0\end{array}$ & $\begin{array}{r}6.0 \\
0.0\end{array}$ & $\begin{array}{l}7.0 \\
0.0\end{array}$ & $\begin{array}{c}8.0 \\
0.0\end{array}$ & $\begin{array}{l}9.0 \\
0.0\end{array}$ & 10.0 \\
\hline & 1.0 & 2.0 & 3. & 4.0 & 5.0 & 7.0 & 10. & 14. & 19 & 25.0 \\
\hline & 432.0 & 301.0 & 161. & 96.0 & 109.0 & & & & & \\
\hline & & $\begin{array}{l}2.0 \\
97.0\end{array}$ & & $\begin{array}{l}4.0 \\
32.0\end{array}$ & & $\begin{array}{r}6.0 \\
0.0\end{array}$ & $\begin{array}{c}7.0 \\
0.0\end{array}$ & $\begin{array}{r}8.0 \\
0.0\end{array}$ & $\begin{array}{r}9.0 \\
0.0\end{array}$ & 10.0 \\
\hline & $759^{1}$ & $\begin{array}{r}2.0 \\
358.0\end{array}$ & 3. & 4.0 & 5.0 & 6. & 8 & 11.0 & 15 & 20 \\
\hline & 759. & 358. & 168. & 44. & 87. & 52. & 47 & 2 & 2 & \\
\hline & $\begin{array}{c}1.0 \\
439.0\end{array}$ & $\begin{array}{r}2.0 \\
132.0\end{array}$ & $\begin{array}{l}3.0 \\
44.0\end{array}$ & $\begin{array}{l}4.0 \\
56.0\end{array}$ & $\begin{array}{l}5.0 \\
45.0\end{array}$ & $\begin{array}{r}6.0 \\
0.0\end{array}$ & $\begin{array}{c}7.0 \\
0.0\end{array}$ & $\begin{array}{c}8.0 \\
0.0\end{array}$ & $\begin{array}{c}9.0 \\
0.0\end{array}$ & \\
\hline & & $\begin{array}{r}2.0 \\
150.0\end{array}$ & & & & & & 8.0 & 9.0 & 10.0 \\
\hline & $\begin{array}{l}1.0 \\
2970\end{array}$ & $\begin{array}{l}2.0 \\
179.0\end{array}$ & 3. & $\begin{array}{l}4.0 \\
180\end{array}$ & & & & 8.0 & 9.0 & 10. \\
\hline & $\begin{array}{r}297 \\
1 .\end{array}$ & $\begin{array}{r}179 \\
2 .\end{array}$ & $\begin{array}{l}43 \\
3 .\end{array}$ & $\begin{array}{l}18 \\
4 .\end{array}$ & 5.0 & & & 9. & 12 & 16. \\
\hline & 594. & 338.0 & 190. & 127.0 & 26.0 & 27 & & & & \\
\hline & 234 & $\begin{array}{l}2.0 \\
69.0\end{array}$ & & & $\begin{array}{r}5.0 \\
0.0\end{array}$ & & & 0.0 & $\begin{array}{c}9.0 \\
0.0\end{array}$ & 10.0 \\
\hline & & & 6. & 11. & & & & & 66 & 3. \\
\hline & 364. & 245. & 206 & & & & & & & \\
\hline & & & & & & & & & & 10. \\
\hline & 299 & & & & & & & & & \\
\hline & & & & & & & & & & 55. \\
\hline & 244.0 & 173. & & & & & & & & \\
\hline & 1.0 & 2.0 & & & & & & & & 10.0 \\
\hline $\begin{array}{r}41 \\
0\end{array}$ & & 0 & & & & & & & & \\
\hline & & & & & 116 & & & & & $\perp$. \\
\hline & & & & & & & & & & \\
\hline & & & & & & & & & & 10. \\
\hline & & & & & & & & & & \\
\hline & & & & & & & & & & 65. \\
\hline & & & & & & & & & & \\
\hline & & & & & & & & & & 65. \\
\hline & 79 & & & & & & & & & \\
\hline & $\perp$ & & & & & & & & & 103.0 \\
\hline & 143 & & & & & & & & & \\
\hline & & & & & & & 127 & 169 & 217 & 271.0 \\
\hline & 478 & & & & & & & & & \\
\hline & & & & & & & 106 & 141.0 & 81 & 226. \\
\hline
\end{tabular}




$\begin{array}{rrrrrrrrrrr}585.0 & 528.0 & 585.0 & 256.0 & 150.0 & 103.0 & 41.0 & 12.0 & 13.0 & 9.0 & \\ 0.0 & 1.0 & 4.0 & 9.0 & 16.0 & 25.0 & 37.0 & 52.0 & 70.0 & 91.0 & 115.0 \\ 592.0 & 496.0 & 648.0 & 576.0 & 346.0 & 306.0 & 212.0 & 96.0 & 27.0 & 9.0 & \\ 0.0 & 1.0 & 4.0 & 9.0 & 17.0 & 28.0 & 42.0 & 59.0 & 79.0 & 102.0 & 128.0 \\ 805.0 & 474.0 & 756.0 & 820.0 & 567.0 & 308.0 & 129.0 & 67.0 & 33.0 & 14.0 & \\ 0.0 & 1.0 & 4.0 & 9.0 & 16.0 & 25.0 & 36.0 & 49.0 & 65.0 & 84.0 & 106.0 \\ 727.0 & 481.0 & 630.0 & 589.0 & 373.0 & 255.0 & 96.0 & 39.0 & 26.0 & 17.0 & \\ 0.0 & 1.0 & 3.0 & 6.0 & 11.0 & 18.0 & 27.0 & 38.0 & 51.0 & 66.0 & 83.0 \\ 532.0 & 320.0 & 198.0 & 246.0 & 244.0 & 131.0 & 36.0 & 23.0 & 27.0 & 8.0 & \\ 0.0 & 1.0 & 3.0 & 6.0 & 10.0 & 15.0 & 22.0 & 31.0 & 42.0 & 55.0 & 70.0 \\ 171.0 & 414.0 & 100.0 & 194.0 & 57.0 & 43.0 & 24.0 & 61.0 & 108.0 & 52.0 & \\ 0.0 & 1.0 & 2.0 & 3.0 & 5.0 & 8.0 & 12.0 & 17.0 & 23.0 & 30.0 & 38.0 \\ 64.0 & 1092.0 & 38.0 & 95.0 & 52.0 & 16.0 & 26.0 & 30.0 & 44.0 & 15.0 & \\ \text { [END] } & & & & & & & & & & \end{array}$


[NAME]

C-34

655030WG

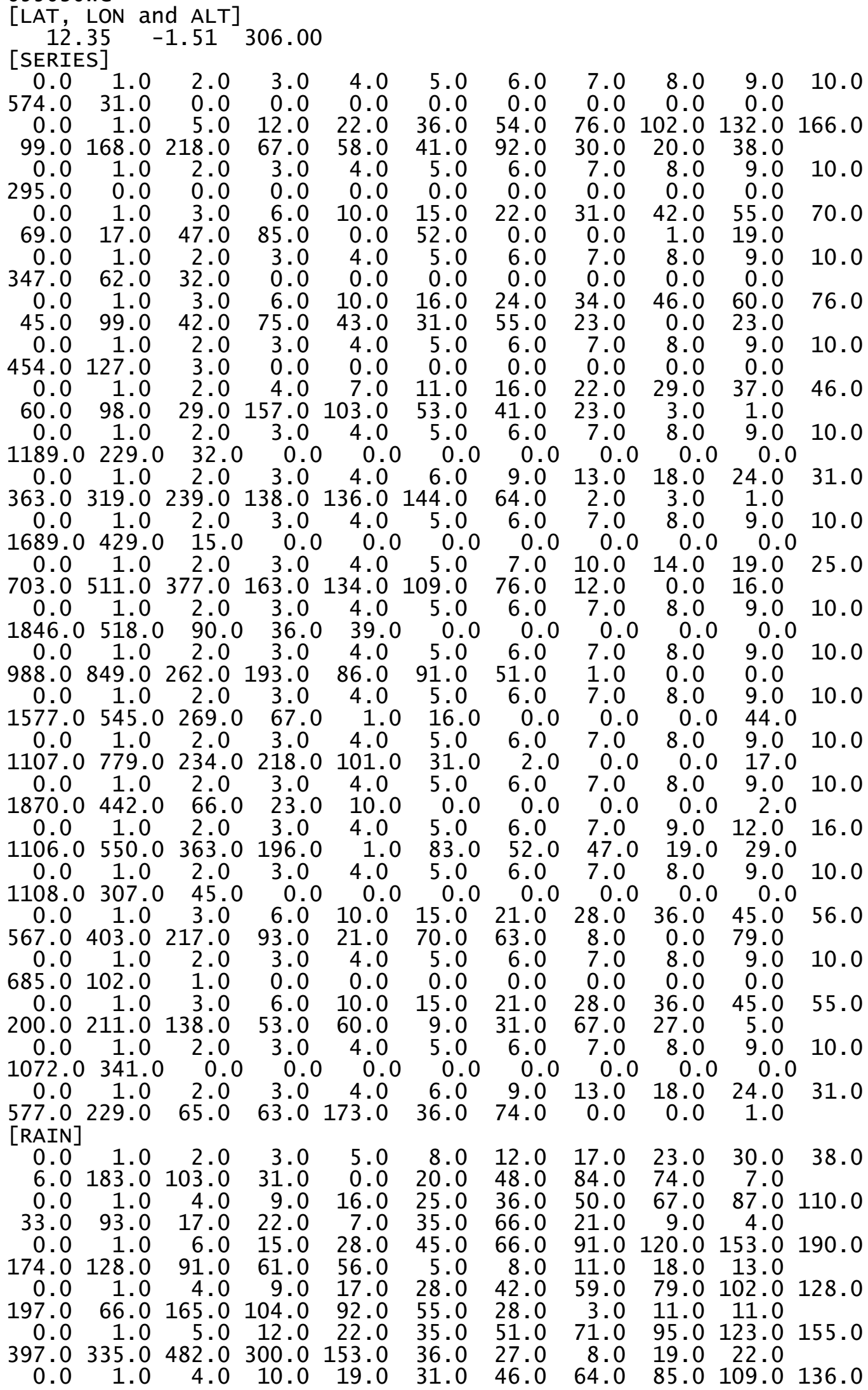




$\begin{array}{rrrrrrrrrrr}522.0 & 368.0 & 726.0 & 503.0 & 226.0 & 134.0 & 63.0 & 27.0 & 16.0 & 4.0 & \\ 0.0 & 1.0 & 4.0 & 9.0 & 16.0 & 25.0 & 36.0 & 49.0 & 64.0 & 81.0 & 100.0 \\ 651.0 & 423.0 & 819.0 & 520.0 & 470.0 & 257.0 & 200.0 & 113.0 & 41.0 & 20.0 & \\ 0.0 & 1.0 & 5.0 & 12.0 & 22.0 & 35.0 & 51.0 & 70.0 & 92.0 & 117.0 & 145.0 \\ 792.0 & 618.0 & 1033.0 & 754.0 & 448.0 & 278.0 & 161.0 & 65.0 & 24.0 & 5.0 & \\ 0.0 & 1.0 & 6.0 & 15.0 & 29.0 & 48.0 & 72.0 & 101.0 & 135.0 & 174.0 & 218.0 \\ 769.0 & 599.0 & 788.0 & 595.0 & 215.0 & 92.0 & 66.0 & 44.0 & 14.0 & 16.0 & \\ 0.0 & 1.0 & 5.0 & 12.0 & 22.0 & 35.0 & 51.0 & 70.0 & 93.0 & 120.0 & 151.0 \\ 462.0 & 471.0 & 506.0 & 274.0 & 66.0 & 11.0 & 11.0 & 15.0 & 7.0 & 5.0 & \\ 0.0 & 1.0 & 3.0 & 6.0 & 10.0 & 15.0 & 22.0 & 31.0 & 42.0 & 55.0 & 70.0 \\ 245.0 & 235.0 & 112.0 & 116.0 & 58.0 & 9.0 & 4.0 & 92.0 & 3.0 & 7.0 & \\ 0.0 & 1.0 & 2.0 & 4.0 & 7.0 & 11.0 & 16.0 & 22.0 & 29.0 & 37.0 & 46.0 \\ 111.0 & 511.0 & 538.0 & 11.0 & 90.0 & 53.0 & 0.0 & 94.0 & 202.0 & 205.0 & \\ \text { [END] } & & & & & & & & & & \end{array}$


[NAME]

C-36

655050WG

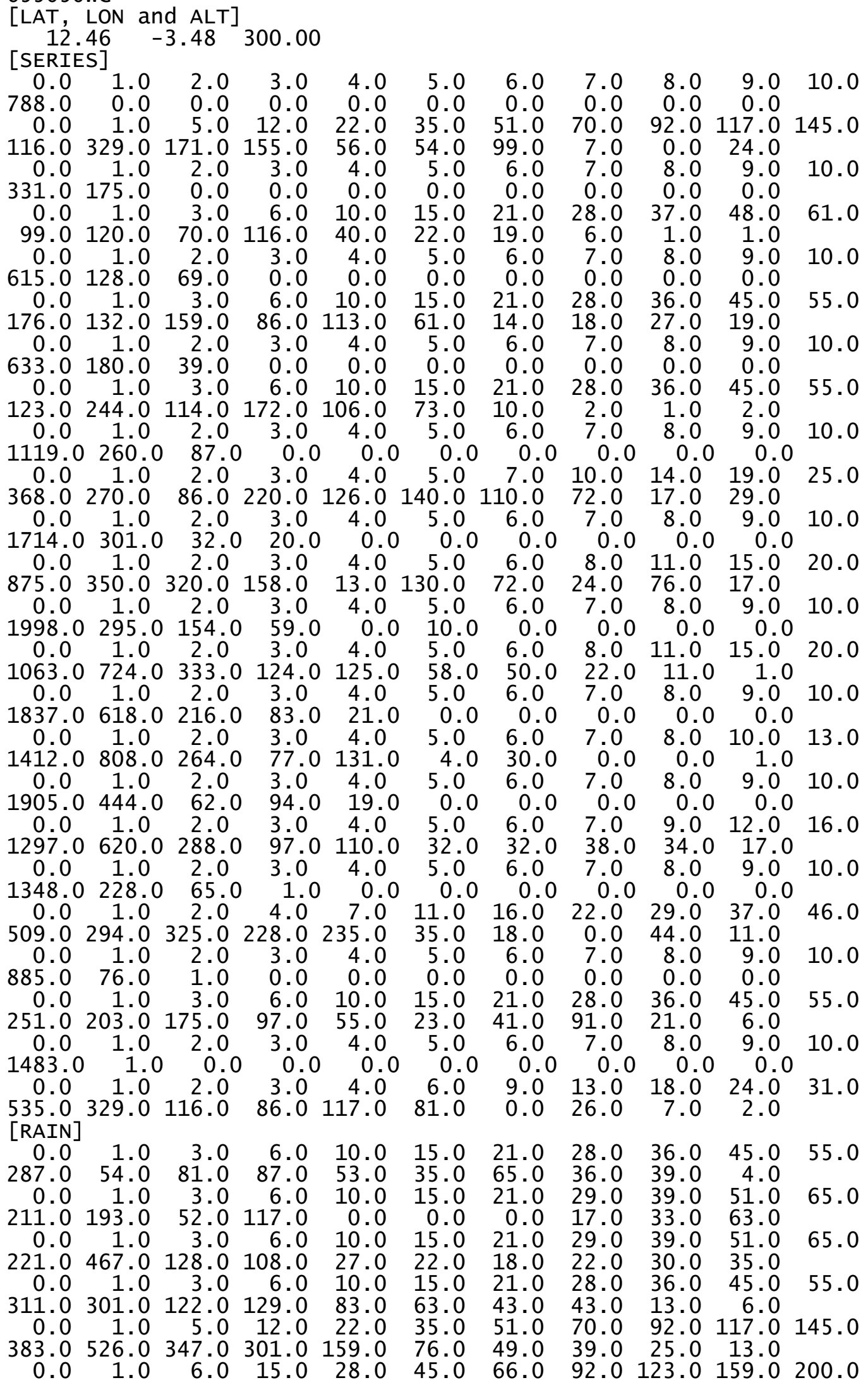




$\begin{array}{rrrrrrrrrrr}410.0 & 598.0 & 721.0 & 360.0 & 156.0 & 83.0 & 68.0 & 29.0 & 48.0 & 36.0 & \\ 0.0 & 1.0 & 5.0 & 12.0 & 22.0 & 35.0 & 51.0 & 71.0 & 95.0 & 123.0 & 155.0 \\ 485.0 & 558.0 & 805.0 & 623.0 & 419.0 & 264.0 & 147.0 & 30.0 & 18.0 & 10.0 & \\ 0.0 & 1.0 & 4.0 & 9.0 & 16.0 & 25.0 & 36.0 & 49.0 & 64.0 & 81.0 & 100.0 \\ 595.0 & 552.0 & 659.0 & 896.0 & 670.0 & 409.0 & 223.0 & 108.0 & 33.0 & 12.0 & \\ 0.0 & 1.0 & 3.0 & 6.0 & 10.0 & 16.0 & 24.0 & 34.0 & 46.0 & 60.0 & 76.0 \\ 668.0 & 585.0 & 407.0 & 455.0 & 501.0 & 356.0 & 274.0 & 129.0 & 62.0 & 11.0 & \\ 0.0 & 1.0 & 4.0 & 9.0 & 16.0 & 26.0 & 39.0 & 55.0 & 74.0 & 96.0 & 121.0 \\ 393.0 & 506.0 & 328.0 & 257.0 & 221.0 & 133.0 & 90.0 & 27.0 & 21.0 & 6.0 & \\ 0.0 & 1.0 & 6.0 & 15.0 & 28.0 & 45.0 & 66.0 & 91.0 & 121.0 & 156.0 & 196.0 \\ 302.0 & 217.0 & 122.0 & 95.0 & 61.0 & 41.0 & 51.0 & 37.0 & 45.0 & 32.0 & \\ 0.0 & 1.0 & 5.0 & 12.0 & 22.0 & 35.0 & 51.0 & 70.0 & 92.0 & 117.0 & 145.0 \\ 374.0 & 93.0 & 235.0 & 186.0 & 144.0 & 216.0 & 150.0 & 86.0 & 36.0 & 16.0 & \\ \text { [END] } & & & & & & & & & & \end{array}$


[NAME]

C-38

655070WG

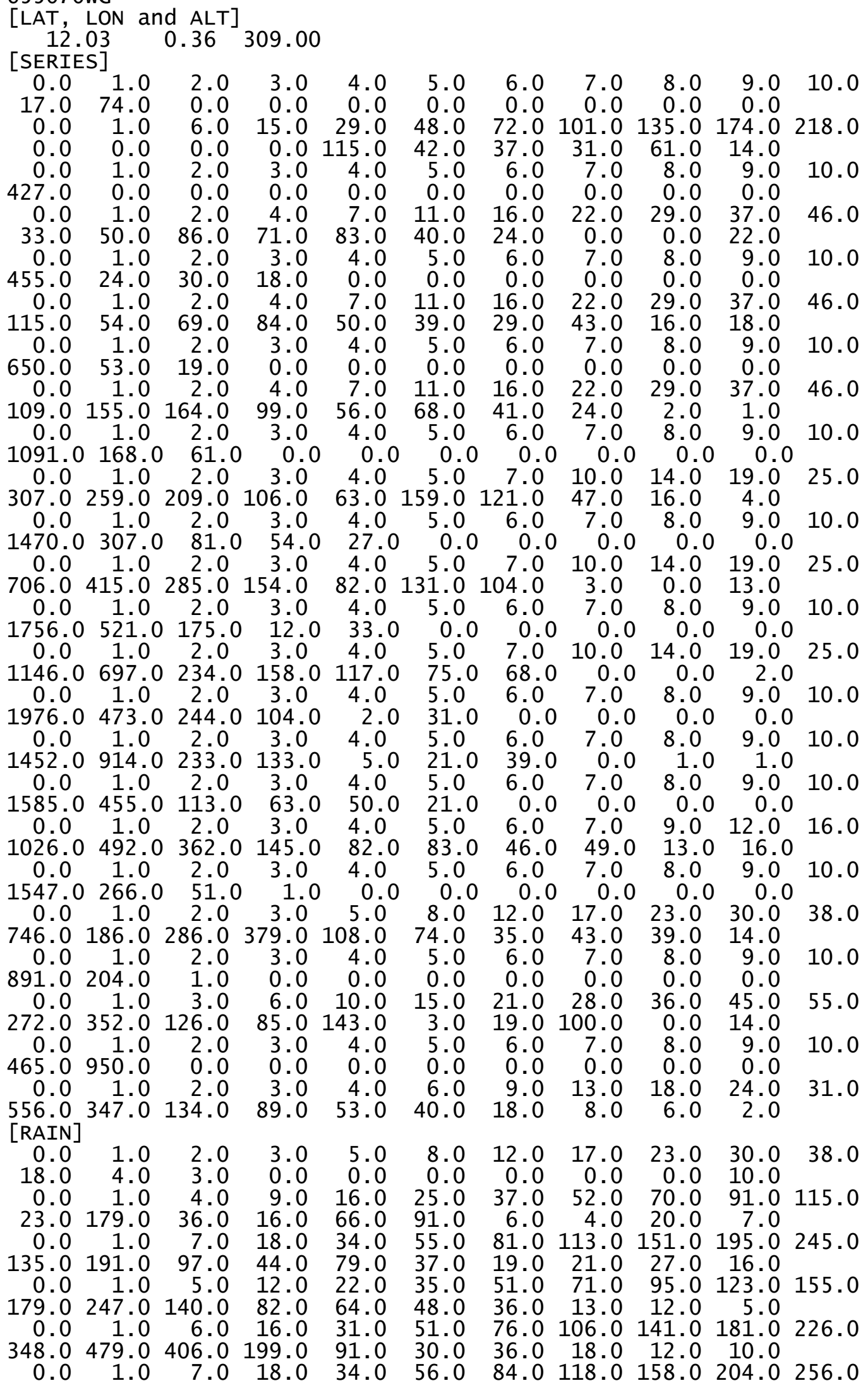




$\begin{array}{rrrrrrrrrrr}389.0 & 819.0 & 684.0 & 376.0 & 178.0 & 99.0 & 78.0 & 27.0 & 9.0 & 19.0 & \\ 0.0 & 1.0 & 6.0 & 15.0 & 28.0 & 45.0 & 66.0 & 91.0 & 121.0 & 156.0 & 196.0 \\ 605.0 & 846.0 & 841.0 & 537.0 & 350.0 & 241.0 & 96.0 & 26.0 & 14.0 & 7.0 & \\ 0.0 & 1.0 & 8.0 & 21.0 & 40.0 & 65.0 & 97.0 & 136.0 & 182.0 & 235.0 & 295.0 \\ 717.0 & 1394.0 & 1078.0 & 620.0 & 228.0 & 125.0 & 38.0 & 28.0 & 15.0 & 9.0 \\ 0.0 & 1.0 & 6.0 & 16.0 & 31.0 & 51.0 & 76.0 & 106.0 & 141.0 & 181.0 & 226.0 \\ 677.0 & 680.0 & 1030.0 & 647.0 & 254.0 & 83.0 & 20.0 & 16.0 & 16.0 & 10.0 & \\ 0.0 & 1.0 & 4.0 & 9.0 & 16.0 & 26.0 & 39.0 & 55.0 & 74.0 & 96.0 & 121.0 \\ 574.0 & 320.0 & 501.0 & 438.0 & 242.0 & 87.0 & 54.0 & 23.0 & 6.0 & 6.0 & \\ 0.0 & 1.0 & 3.0 & 6.0 & 11.0 & 18.0 & 27.0 & 38.0 & 51.0 & 66.0 & 83.0 \\ 344.0 & 145.0 & 104.0 & 265.0 & 91.0 & 49.0 & 48.0 & 23.0 & 51.0 & 147.0 & \\ 0.0 & 1.0 & 3.0 & 6.0 & 10.0 & 16.0 & 24.0 & 34.0 & 46.0 & 60.0 & 76.0 \\ 910.0 & 505.0 & 2.0 & 162.0 & 6.0 & 0.0 & 28.0 & 254.0 & 367.0 & 243.0 & \\ \text { [END] } & & & & & & & & & & \\ \end{array}$


[NAME]

C-40

655100WG

\begin{tabular}{|c|c|c|c|c|c|c|c|c|c|c|}
\hline $\begin{array}{l}\text { AT, } \\
11 . \\
\text { ERIE }\end{array}$ & $\begin{array}{l}\text { LON an } \\
16 \\
\text { S] }\end{array}$ & $\begin{array}{l}\text { Id ALT] } \\
4.31\end{array}$ & 460.0 & & & & & & & \\
\hline & 1.0 & 2.0 & 3.0 & 4.0 & 5.0 & 6.0 & 7.0 & 8.0 & 9.0 & 10.0 \\
\hline 1 & 0.0 & & & & & & & & & \\
\hline & 1.0 & & & 16. & & & 49. & 65 & 84 & 06.0 \\
\hline 7 & 41.0 & 169.0 & 185. & 16. & & & 0. & & 0.0 & \\
\hline & 1.0 & & & & & & & & & 10.0 \\
\hline & 97.0 & & & & 0 & & & & 0 & \\
\hline & 1.0 & & & & & & & 65 & 84 & 106.0 \\
\hline & 96.0 & 97. & & & & & & & & \\
\hline & 1.0 & & & & & & & & & 10.0 \\
\hline 7 & 167.0 & 18. & & & & & & & & \\
\hline & 1.0 & & & & & & & & & 46.0 \\
\hline 5 & 73.0 & 85.0 & 158. & 96. & & & & & & \\
\hline & 1.0 & & & & & & & & & 10. \\
\hline 6 & 182.0 & 63. & & & & & & & & \\
\hline & 1.0 & & & & & & & & & 46.0 \\
\hline 5 & 169.0 & 221.0 & 222.0 & 109.0 & & & & & & \\
\hline & 1.0 & 2.0 & 3.0 & 4.0 & 5.0 & 6.0 & 7.0 & & & 10.0 \\
\hline $\begin{array}{l}62.0 \\
0.0\end{array}$ & & $\begin{array}{l}43.0 \\
2.0\end{array}$ & $\begin{array}{l}0.0 \\
3.0\end{array}$ & $\begin{array}{l}0.0 \\
4.0\end{array}$ & $\begin{array}{l}0.0 \\
5.0\end{array}$ & $\begin{array}{l}0.0 \\
7.0\end{array}$ & $\begin{array}{r}0.0 \\
10.0\end{array}$ & $\begin{array}{r}0.0 \\
14.0\end{array}$ & $\begin{array}{r}0.0 \\
19.0\end{array}$ & 25.0 \\
\hline 50 & 361.0 & 309.0 & 134.0 & 187.0 & 115.0 & 93.0 & & & & \\
\hline & 1.0 & & 3 & 4.0 & 5.0 & 6.0 & 7.0 & 8.0 & & 10.0 \\
\hline 45.0 & $\begin{array}{r}579.0 \\
10\end{array}$ & 112.0 & $\begin{array}{l}79 \\
3\end{array}$ & 0.0 & 0.0 & 0.0 & 0.0 & 0.0 & 0.0 & ) \\
\hline 6 & 435.0 & 300.0 & 182.0 & 216.0 & 98.0 & & & & & \\
\hline 0.0 & 1.0 & 2.0 & 3.0 & 4.0 & 5.0 & 6.0 & 7.0 & 8.0 & 0 & 10. \\
\hline $\begin{array}{l}315.0 \\
0.0\end{array}$ & $\begin{array}{c}457.0 \\
1.0\end{array}$ & $\begin{array}{r}256.0 \\
2.0\end{array}$ & $\begin{array}{l}19.0 \\
3.0\end{array}$ & $\begin{array}{r}0.0 \\
4.0\end{array}$ & $\begin{array}{c}0.0 \\
5.0\end{array}$ & $\begin{array}{c}0.0 \\
6.0\end{array}$ & $\begin{array}{l}0.0 \\
7.0\end{array}$ & $\begin{array}{l}0.0 \\
9.0\end{array}$ & 0 & 16.0 \\
\hline 129 & 662.0 & 259.0 & 243.0 & 84.0 & 78.0 & 37.0 & & 0.0 & 11.0 & \\
\hline $\begin{array}{r}0 \\
39\end{array}$ & $\begin{array}{c}1.0 \\
640.0\end{array}$ & $\begin{array}{c}2.0 \\
177.0\end{array}$ & 3. & $\begin{array}{l}4.0 \\
98.0\end{array}$ & 14. & & & 8.0 & 9.0 & 10. \\
\hline 0 & 1.0 & 2.0 & 3. & 4.0 & 5.0 & 6.0 & 7.0 & 8.0 & 9.0 & 10. \\
\hline 0 & 761.0 & 279.0 & 69 & 26.0 & 37.0 & 0.0 & 1.0 & 1.0 & 0.0 & \\
\hline 423 & $\begin{array}{r}1.0 \\
662.0\end{array}$ & $\begin{array}{c}2.0 \\
221.0\end{array}$ & $\begin{array}{l}3.0 \\
87.0\end{array}$ & $\begin{array}{l}4.0 \\
80.0\end{array}$ & $\begin{array}{l}5.0 \\
2.0\end{array}$ & $\begin{array}{l}6.0 \\
3.0\end{array}$ & $\begin{array}{l}7.0 \\
0.0\end{array}$ & $\begin{array}{c}8.0 \\
0.0\end{array}$ & $\begin{array}{c}9.0 \\
0.0\end{array}$ & ) \\
\hline & 1.0 & $271^{0}$ & $\begin{array}{l}3.0 \\
1780\end{array}$ & $\begin{array}{l}4.0 \\
690\end{array}$ & 5.0 & & $\begin{array}{l}7.0 \\
0.0\end{array}$ & $\begin{array}{c}8.0 \\
0.0\end{array}$ & $\begin{array}{l}9.0 \\
9.0\end{array}$ & 10.0 \\
\hline & $\begin{array}{c}013.0 \\
1.0\end{array}$ & 2.0 & $\begin{array}{r}1 / 8.0 \\
3.0\end{array}$ & 4.0 & 5.0 & 6.0 & 7.0 & 8.0 & 9.0 & 10.0 \\
\hline$j^{0}$ & $\begin{array}{r}260.0 \\
1.0\end{array}$ & $\begin{array}{l}97.0 \\
3.0\end{array}$ & & 10.0 & $\begin{array}{r}0.0 \\
15.0\end{array}$ & & & $\begin{array}{r}0.0 \\
36.0\end{array}$ & & 8. \\
\hline & 474.0 & 347.0 & 140. & 106 & & & & & & \\
\hline & & & & & & & & & & 10. \\
\hline & 289 & & & & & & & & & \\
\hline & & & & & & & & & & 38. \\
\hline & 179.0 & 62. & 112. & 152 & 162 & & & 104 & & \\
\hline & 1.0 & 2.0 & 3.0 & 4.0 & 5.0 & 6.0 & & 8.0 & 0 & 10.0 \\
\hline 0 & $\begin{array}{l}7.0 \\
1.0\end{array}$ & 2.0 & & & & $0^{0}$ & & $\begin{array}{r}0.0 \\
18.0\end{array}$ & & 31.0 \\
\hline & 238.0 & & & 115 & & & & & & \\
\hline & & & & & & & & & & \\
\hline & & & & & & & & & & 40. \\
\hline & & & & & & & & & & 55. \\
\hline & 11 & & 104 & & & & & & & \\
\hline & & 3 & & & & & & & & 70. \\
\hline & 163.0 & & & & & & & & & \\
\hline & & & & & & & & & & 91. \\
\hline & & & & & & & & & & \\
\hline & & & & & & & & & & 115. \\
\hline & & & & & & & & & & \\
\hline & & & & & & & & & & 8 \\
\hline
\end{tabular}




$\begin{array}{rrrrrrrrrrr}764.0 & 510.0 & 779.0 & 551.0 & 339.0 & 172.0 & 48.0 & 30.0 & 32.0 & 19.0 & \\ 0.0 & 1.0 & 6.0 & 15.0 & 28.0 & 46.0 & 69.0 & 97.0 & 130.0 & 168.0 & 211.0 \\ 617.0 & 697.0 & 959.0 & 765.0 & 320.0 & 148.0 & 25.0 & 10.0 & 16.0 & 20.0 & \\ 0.0 & 1.0 & 6.0 & 16.0 & 31.0 & 51.0 & 76.0 & 106.0 & 141.0 & 181.0 & 226.0 \\ 954.0 & 806.0 & 1308.0 & 968.0 & 514.0 & 184.0 & 74.0 & 8.0 & 23.0 & 22.0 & \\ 0.0 & 1.0 & 5.0 & 12.0 & 22.0 & 35.0 & 52.0 & 73.0 & 98.0 & 127.0 & 160.0 \\ 819.0 & 916.0 & 996.0 & 696.0 & 434.0 & 238.0 & 89.0 & 32.0 & 25.0 & 8.0 & \\ 0.0 & 1.0 & 3.0 & 6.0 & 10.0 & 15.0 & 22.0 & 31.0 & 42.0 & 55.0 & 70.0 \\ 460.0 & 307.0 & 293.0 & 286.0 & 285.0 & 262.0 & 151.0 & 89.0 & 50.0 & 15.0 & \\ 0.0 & 1.0 & 3.0 & 6.0 & 10.0 & 15.0 & 22.0 & 31.0 & 42.0 & 55.0 & 70.0 \\ 301.0 & 302.0 & 71.0 & 157.0 & 86.0 & 112.0 & 64.0 & 62.0 & 74.0 & 27.0 & \\ 0.0 & 1.0 & 2.0 & 4.0 & 7.0 & 11.0 & 16.0 & 22.0 & 29.0 & 37.0 & 46.0 \\ 112.0 & 279.0 & 48.0 & 169.0 & 363.0 & 265.0 & 174.0 & 140.0 & 43.0 & 1.0 & \\ \text { [END] } & & & & & & & & & & \end{array}$


[NAME]

C-42

655160WG

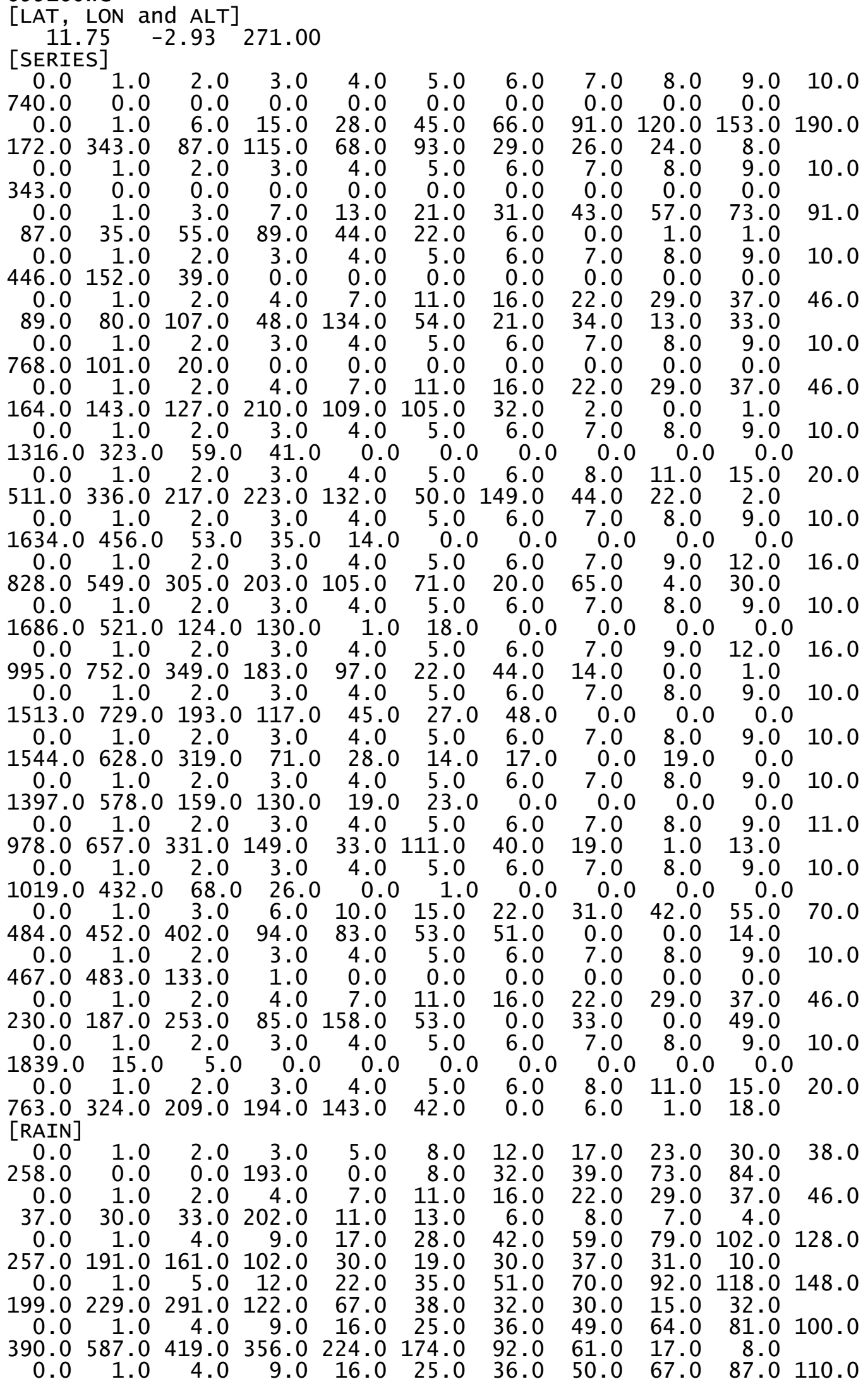




$\begin{array}{rrrrrrrrrrr}538.0 & 456.0 & 538.0 & 484.0 & 321.0 & 270.0 & 173.0 & 76.0 & 36.0 & 10.0 & \\ 0.0 & 1.0 & 6.0 & 15.0 & 28.0 & 45.0 & 66.0 & 92.0 & 123.0 & 159.0 & 200.0 \\ 808.0 & 828.0 & 905.0 & 599.0 & 299.0 & 120.0 & 97.0 & 42.0 & 26.0 & 18.0 & \\ 0.0 & 1.0 & 5.0 & 12.0 & 22.0 & 35.0 & 51.0 & 70.0 & 92.0 & 117.0 & 145.0 \\ 796.0 & 927.0 & 1048.0 & 825.0 & 596.0 & 276.0 & 131.0 & 81.0 & 28.0 & 5.0 & \\ 0.0 & 1.0 & 4.0 & 9.0 & 17.0 & 28.0 & 42.0 & 59.0 & 79.0 & 102.0 & 128.0 \\ 786.0 & 505.0 & 846.0 & 763.0 & 427.0 & 248.0 & 117.0 & 48.0 & 39.0 & 14.0 & \\ 0.0 & 1.0 & 3.0 & 7.0 & 13.0 & 21.0 & 31.0 & 43.0 & 57.0 & 73.0 & 91.0 \\ 386.0 & 318.0 & 479.0 & 518.0 & 236.0 & 144.0 & 83.0 & 21.0 & 9.0 & 7.0 & \\ 0.0 & 1.0 & 2.0 & 4.0 & 7.0 & 11.0 & 16.0 & 22.0 & 29.0 & 37.0 & 46.0 \\ 496.0 & 246.0 & 224.0 & 197.0 & 151.0 & 218.0 & 149.0 & 65.0 & 53.0 & 12.0 & \\ 0.0 & 1.0 & 3.0 & 6.0 & 10.0 & 15.0 & 21.0 & 29.0 & 39.0 & 51.0 & 65.0 \\ 803.0 & 127.0 & 99.0 & 0.0 & 0.0 & 48.0 & 122.0 & 200.0 & 342.0 & 180.0 & \\ \text { [END] } & & & & & & & & & & \end{array}$


[NAME]

C-44

655180WG

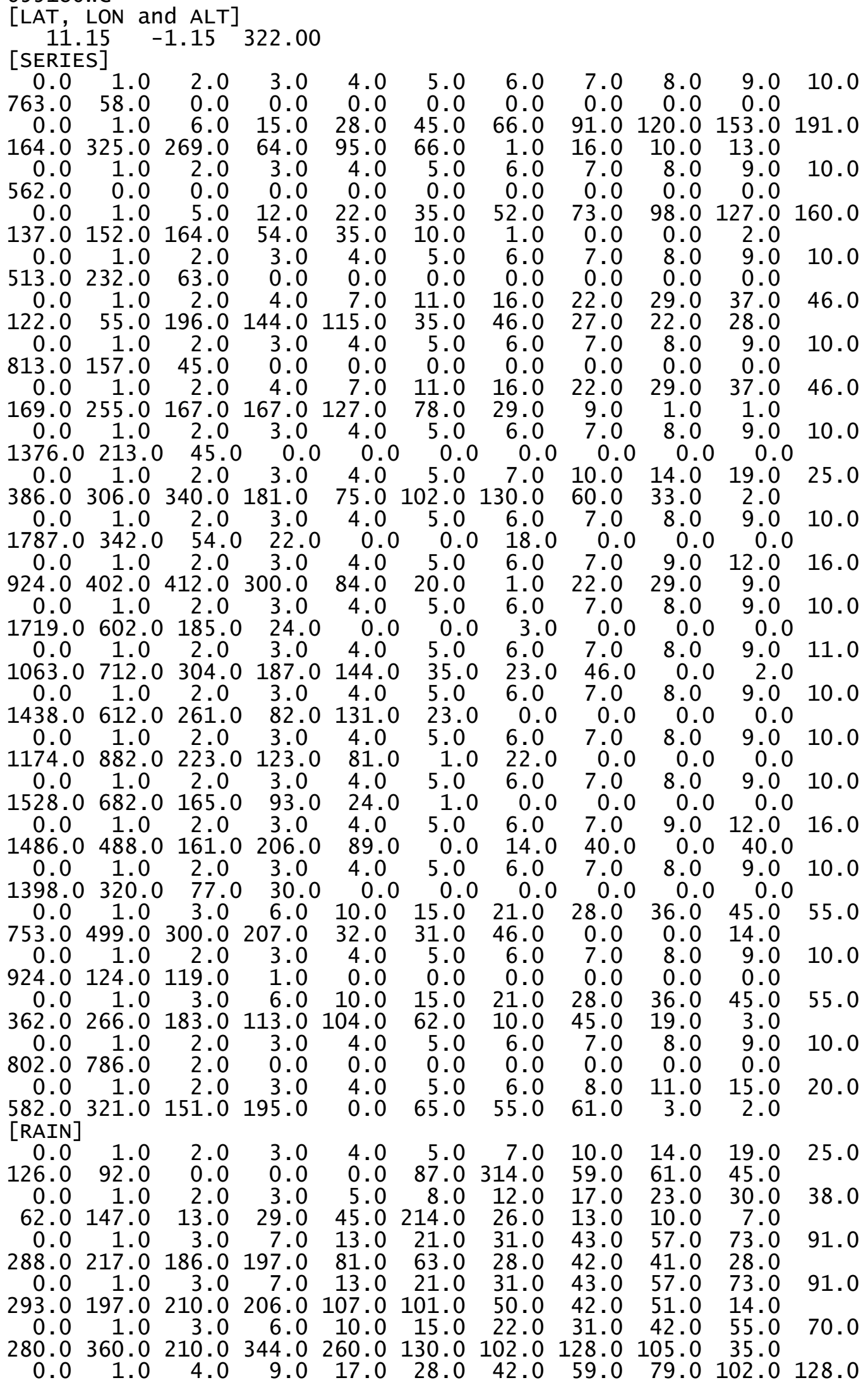




$\begin{array}{rrrrrrrrrrr}488.0 & 406.0 & 558.0 & 523.0 & 368.0 & 245.0 & 174.0 & 77.0 & 18.0 & 13.0 & \\ 0.0 & 1.0 & 6.0 & 15.0 & 28.0 & 45.0 & 66.0 & 91.0 & 120.0 & 154.0 & 193.0 \\ 655.0 & 746.0 & 818.0 & 635.0 & 372.0 & 141.0 & 90.0 & 51.0 & 42.0 & 29.0 & \\ 0.0 & 1.0 & 4.0 & 10.0 & 19.0 & 31.0 & 46.0 & 64.0 & 85.0 & 109.0 & 136.0 \\ 812.0 & 616.0 & 812.0 & 933.0 & 653.0 & 319.0 & 186.0 & 128.0 & 60.0 & 16.0 & \\ 0.0 & 1.0 & 4.0 & 9.0 & 17.0 & 28.0 & 42.0 & 59.0 & 79.0 & 102.0 & 128.0 \\ 558.0 & 735.0 & 785.0 & 802.0 & 592.0 & 243.0 & 98.0 & 42.0 & 52.0 & 19.0 & \\ 0.0 & 1.0 & 3.0 & 7.0 & 13.0 & 21.0 & 31.0 & 43.0 & 57.0 & 73.0 & 91.0 \\ 475.0 & 342.0 & 445.0 & 472.0 & 336.0 & 172.0 & 74.0 & 20.0 & 16.0 & 10.0 & \\ 0.0 & 1.0 & 2.0 & 3.0 & 5.0 & 8.0 & 12.0 & 17.0 & 23.0 & 30.0 & 38.0 \\ 250.0 & 62.0 & 92.0 & 154.0 & 297.0 & 314.0 & 140.0 & 95.0 & 104.0 & 9.0 & \\ 0.0 & 1.0 & 2.0 & 3.0 & 5.0 & 8.0 & 12.0 & 17.0 & 23.0 & 30.0 & 38.0 \\ 407.0 & 5.0 & 68.0 & 5.0 & 255.0 & 684.0 & 272.0 & 149.0 & 378.0 & 243.0 & \\ \text { [END] } & & & & & & & & & & \end{array}$


[NAME]

C-46

655220WG

\begin{tabular}{|c|c|c|c|c|c|c|c|c|c|c|}
\hline $\begin{array}{l}\text { AT, } \\
10 . \\
\text { SERIE }\end{array}$ & $\begin{array}{l}\text { LON an } \\
33 \\
\text { S] }\end{array}$ & 3.18 & 335.0 & & & & & & & \\
\hline & 1.0 & 2.0 & & 4.0 & 5.0 & 6.0 & 7.0 & 8.0 & 9.0 & 10. \\
\hline 74 & 130.0 & & & & & & & & & \\
\hline & 1.0 & & & 17 & & 42 & & & 102.0 & 28.0 \\
\hline & 14.0 & 200 & 146 & & & & & 1.0 & 32.0 & \\
\hline 8 & $\begin{array}{l}1.0 \\
2.0\end{array}$ & & & & & & & & & 10.0 \\
\hline & 1.0 & & & 10. & & & & & & 76.0 \\
\hline 87. & 37.0 & 71. & & & & & & & & \\
\hline & 1.0 & & & & & & & & & 10.0 \\
\hline 32. & 95.0 & & & & & & & & & \\
\hline & 1.0 & & & & & & & & & 38.0 \\
\hline 68 & 56.0 & 77.0 & 136. & 112.0 & 101.0 & & & & & \\
\hline 04 & $\begin{array}{l}1.0 \\
2350\end{array}$ & $\begin{array}{l}2.0 \\
20.0\end{array}$ & $\begin{array}{r}3.0 \\
0.0\end{array}$ & $\begin{array}{r}4.0 \\
0.0\end{array}$ & $\begin{array}{r}5.0 \\
0.0\end{array}$ & $\begin{array}{c}6.0 \\
0.0\end{array}$ & $\begin{array}{l}7.0 \\
0.0\end{array}$ & $\begin{array}{r}8.0 \\
0.0\end{array}$ & $\begin{array}{r}9.0 \\
0.0\end{array}$ & 10.0 \\
\hline & 1.0 & 2.0 & 3. & 4.0 & 5.0 & 6.0 & 8.0 & 11.0 & 15 & 20.0 \\
\hline L8 & 251.0 & 154.0 & 160. & 84.0 & 90.0 & 95.0 & 47.0 & & 14 & \\
\hline $\begin{array}{r}0 \\
+8\end{array}$ & 265.0 & $\begin{array}{r}2.0 \\
135.0\end{array}$ & $\begin{array}{l}3.0 \\
14.0\end{array}$ & $\begin{array}{r}4.0 \\
0.0\end{array}$ & $\begin{array}{r}5.0 \\
0.0\end{array}$ & $\begin{array}{c}6.0 \\
0.0\end{array}$ & $\begin{array}{l}7.0 \\
0.0\end{array}$ & $\begin{array}{c}8.0 \\
0.0\end{array}$ & $\begin{array}{c}9.0 \\
0.0\end{array}$ & 10.0 \\
\hline & & & 33. & 4.0 & 5.0 & 6.0 & $\begin{array}{r}7.0 \\
\end{array}$ & 9.0 & 12.0 & 16.0 \\
\hline & $\begin{array}{l}3.0 \\
1.0\end{array}$ & $\begin{array}{r}201.0 \\
2.0\end{array}$ & 3. & $\begin{array}{r}208.0 \\
4.0\end{array}$ & & $\begin{array}{l}2.0 \\
6.0\end{array}$ & & $\begin{array}{r}89.0 \\
8.0\end{array}$ & & \\
\hline 508 & 414.0 & 85.0 & 19 & 0.0 & & 0.0 & 0.0 & 0.0 & 0.0 & \\
\hline & $\begin{array}{r}1.0 \\
07\end{array}$ & & 3 & 4.0 & 5.0 & 6.0 & 7.0 & 8.0 & & 13. \\
\hline 0.0 & $\begin{array}{r}97.0 \\
10\end{array}$ & 268.0 & 241. & 87. & 59.0 & 3.0 & & & 2.0 & \\
\hline 332.0 & 524.0 & 292.0 & 83 & 38.0 & 0.0 & 0.0 & 0.0 & 0.0 & 0.0 & ) \\
\hline 0 & $\begin{array}{l}1.0 \\
7030\end{array}$ & $\begin{array}{r}2.0 \\
338\end{array}$ & & & 5.0 & 6.0 & 7.0 & 8.0 & 10.0 & 13. \\
\hline 0 & 1.0 & $\begin{array}{l}350.0 \\
2.0\end{array}$ & $\begin{array}{r}185 \\
3 .\end{array}$ & 4.0 & 5.0 & 6.0 & $\begin{array}{l}18.0 \\
7.0\end{array}$ & $\begin{array}{l}11.0 \\
8.0\end{array}$ & 0 & 10. \\
\hline 510 & 676.0 & 225.0 & & 76.0 & & 0.0 & 0.0 & 0.0 & 0 & \\
\hline 37 & $\begin{array}{r}1.0 \\
698.0\end{array}$ & 327.0 & 15 & & $\begin{array}{r}5.0 \\
0.0\end{array}$ & $\begin{array}{l}6.0 \\
1.0\end{array}$ & & $\begin{array}{c}8.0 \\
0.0\end{array}$ & 0 & 10. \\
\hline & 1.0 & 2.0 & 3 & 4.0 & 5.0 & 6.0 & 7.0 & 8.0 & 9.0 & 10. \\
\hline 0 & 430.0 & 153.0 & 137 & 38.0 & 41.0 & 0.0 & 0.0 & 0.0 & 0.0 & \\
\hline 0 & 662.0 & 263.0 & 172 & 101 & & 0.0 & & 0.0 & $\begin{array}{l}9.0 \\
0.0\end{array}$ & \\
\hline & & $\begin{array}{l}2.0 \\
99.0\end{array}$ & & & & $\begin{array}{r}6.0 \\
0.0\end{array}$ & & $\begin{array}{r}8.0 \\
0.0\end{array}$ & $\begin{array}{c}9.0 \\
0.0\end{array}$ & 10. \\
\hline & 1.0 & 3.0 & 6. & & 15.0 & & & & & 5 \\
\hline & 578.0 & 220.0 & 207. & & & & & & & \\
\hline & 1.0 & 2.0 & & & 5.0 & 6.0 & & 0 & 0 & 10.0 \\
\hline & 1.0 & 2.0 & & & 11.0 & & & 29. & & 46. \\
\hline & 167.0 & 172. & 117 & & 133 & & & & & \\
\hline & 1.0 & 2.0 & 3. & 4.0 & 5.0 & 6.0 & & 8.0 & 9.0 & 10.0 \\
\hline & $\begin{array}{r}509.0 \\
1.0\end{array}$ & 2.0 & & & 5. & 7.0 & & & & 25. \\
\hline & & & & & & & & & & \\
\hline & & & & & & & & & & \\
\hline & & & & & & & & & & \\
\hline & & & & & & & & & & 65. \\
\hline & & & & & & & & & & \\
\hline & & & & & & & & & & 70. \\
\hline & 88. & & & & & & & & & \\
\hline & & & & & & & & & & 115. \\
\hline & & & & & & & & & & \\
\hline & & & & & & & & & & 160. \\
\hline & & & & & & & & & & \\
\hline & & & & & & & & & & 9 \\
\hline
\end{tabular}




$\begin{array}{rrrrrrrrrrr}419.0 & 590.0 & 835.0 & 480.0 & 274.0 & 125.0 & 36.0 & 9.0 & 4.0 & 16.0 & \\ 0.0 & 1.0 & 5.0 & 12.0 & 22.0 & 36.0 & 54.0 & 76.0 & 102.0 & 132.0 & 166.0 \\ 898.0 & 608.0 & 859.0 & 692.0 & 449.0 & 265.0 & 98.0 & 60.0 & 12.0 & 21.0 & \\ 0.0 & 1.0 & 5.0 & 12.0 & 22.0 & 35.0 & 52.0 & 73.0 & 98.0 & 127.0 & 160.0 \\ 900.0 & 767.0 & 969.0 & 687.0 & 488.0 & 285.0 & 141.0 & 47.0 & 30.0 & 21.0 & \\ 0.0 & 1.0 & 4.0 & 9.0 & 17.0 & 28.0 & 42.0 & 59.0 & 79.0 & 102.0 & 128.0 \\ 609.0 & 612.0 & 850.0 & 876.0 & 633.0 & 288.0 & 104.0 & 47.0 & 25.0 & 8.0 & \\ 0.0 & 1.0 & 3.0 & 6.0 & 10.0 & 15.0 & 22.0 & 31.0 & 42.0 & 55.0 & 70.0 \\ 457.0 & 480.0 & 366.0 & 415.0 & 297.0 & 312.0 & 206.0 & 107.0 & 49.0 & 26.0 & \\ 0.0 & 1.0 & 3.0 & 6.0 & 10.0 & 15.0 & 21.0 & 28.0 & 36.0 & 45.0 & 55.0 \\ 498.0 & 243.0 & 150.0 & 132.0 & 115.0 & 94.0 & 89.0 & 49.0 & 8.0 & 1.0 & \\ 0.0 & 1.0 & 3.0 & 7.0 & 13.0 & 21.0 & 31.0 & 43.0 & 57.0 & 73.0 & 91.0 \\ 1017.0 & 70.0 & 61.0 & 64.0 & 107.0 & 219.0 & 338.0 & 169.0 & 107.0 & 82.0 & \\ \text { [END] } & & & & & & & & & & \end{array}$


[NAME]

C-48

655280WG

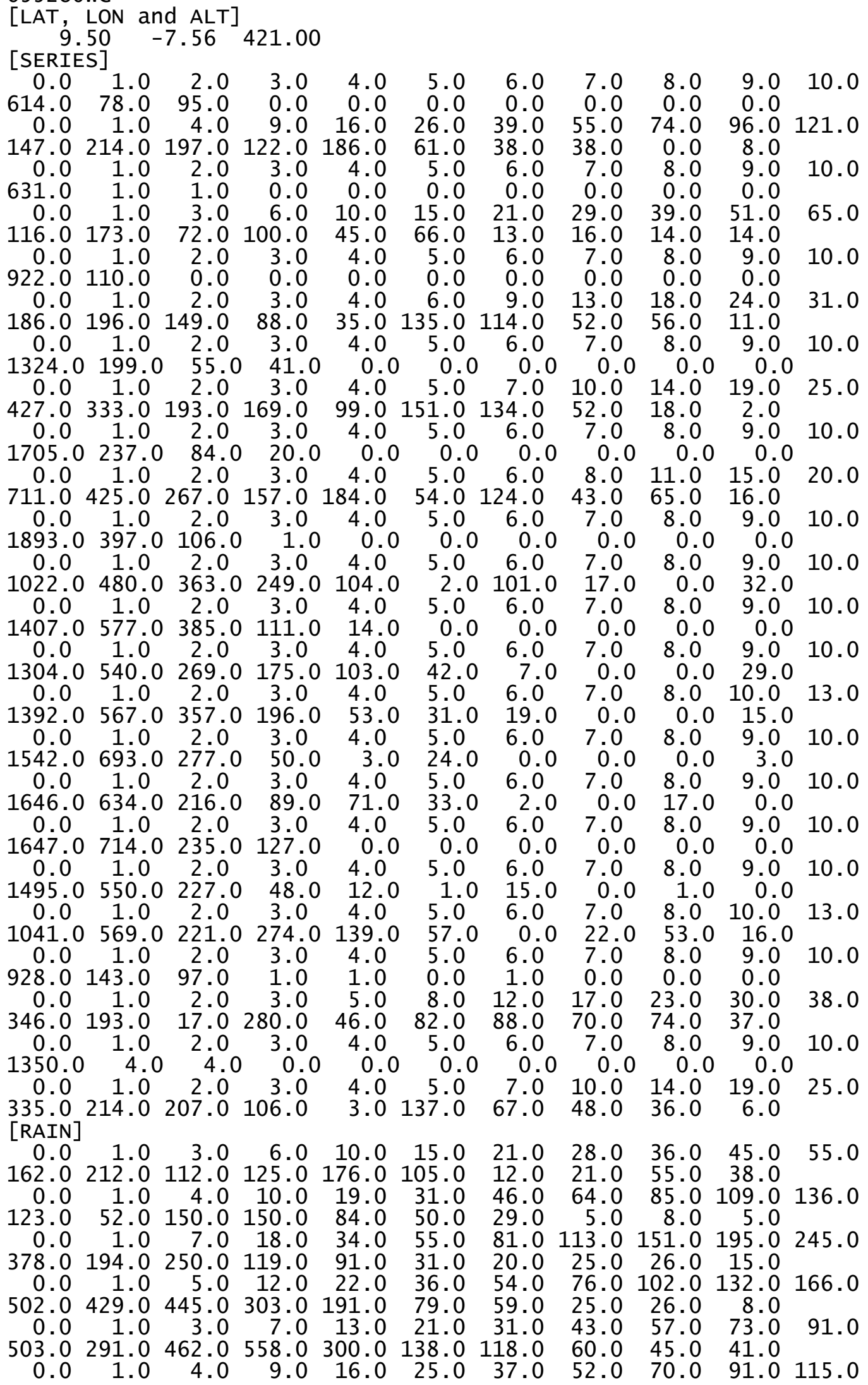




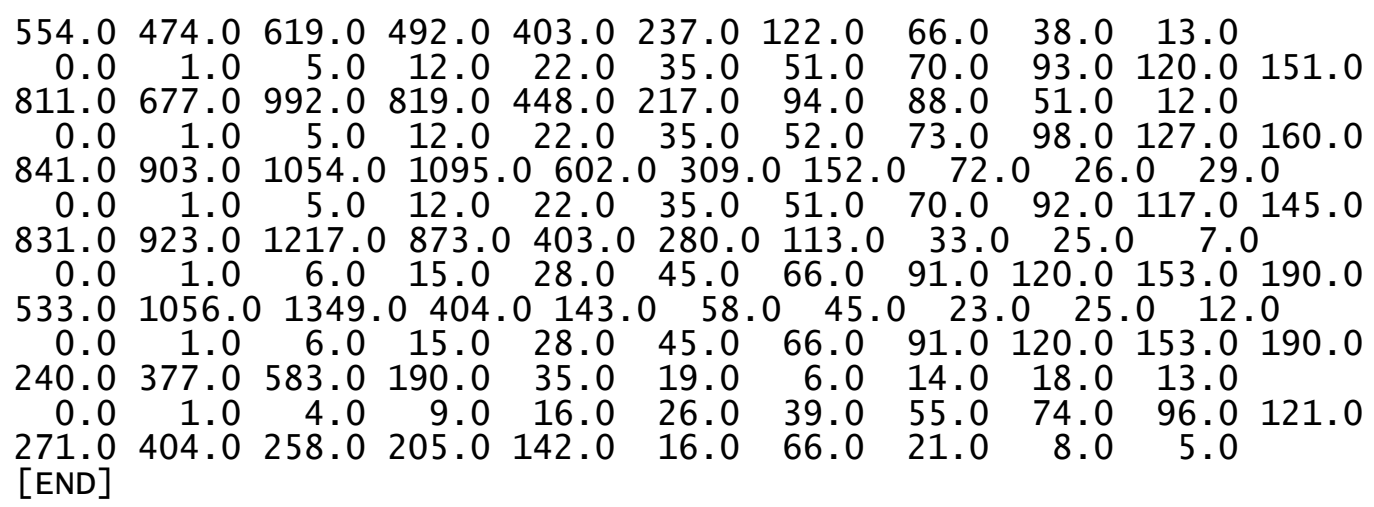


[NAME]

C-50

655360WG

\begin{tabular}{|c|c|c|c|c|c|c|c|c|c|c|}
\hline $\begin{array}{r}9 \\
\text { SERIE }\end{array}$ & $\begin{array}{l}41 \\
\text { EST }\end{array}$ & -5.61 & 381.00 & & & & & & & \\
\hline . & 1.0 & & & & & & & & & 10.0 \\
\hline U & $\begin{array}{r}195.0 \\
1.0\end{array}$ & $\begin{array}{l}0.0 \\
4.0\end{array}$ & & $\begin{array}{r}0.0 \\
16.0\end{array}$ & $\begin{array}{r}0.0 \\
25.0\end{array}$ & $\begin{array}{r}0.0 \\
37.0\end{array}$ & $\begin{array}{r}0.0 \\
52.0\end{array}$ & $\begin{array}{r}0.0 \\
70.0\end{array}$ & 91.0 & 115.0 \\
\hline & 221.0 & 175.0 & 162. & 67.0 & & & & & 37.0 & \\
\hline & 1.0 & & & & & & & & & 10.0 \\
\hline & 29.0 & & & 0 & 0 & 0 & 0 & 0 & 0.0 & \\
\hline & 1.0 & 4 & & 16 & 25 & & 49 & 64 & 81.0 & 100.0 \\
\hline & 47.0 & 106.0 & & & & & & & 1.0 & \\
\hline & 1.0 & 2.0 & & & & & & & 9 & 10.0 \\
\hline & 5.0 & & & & & & & & 0 & \\
\hline & 1.0 & 2.0 & & 7.0 & 11. & & 22 & 29. & 37 & 46.0 \\
\hline & 71.0 & 202.0 & 152. & 68.0 & 112. & & & & 0 & \\
\hline & & $\begin{array}{l}2.0 \\
18.0\end{array}$ & $\begin{array}{l}3.0 \\
15.0\end{array}$ & $\begin{array}{r}4.0 \\
0.0\end{array}$ & $\begin{array}{r}5.0 \\
0.0\end{array}$ & $\begin{array}{l}6.0 \\
0.0\end{array}$ & $\begin{array}{l}7.0 \\
0.0\end{array}$ & $\begin{array}{l}8.0 \\
0.0\end{array}$ & $\begin{array}{l}9.0 \\
0.0\end{array}$ & 10.0 \\
\hline & 1.0 & 2.0 & 4.0 & 7.0 & 11.0 & 16.0 & 22.0 & 29.0 & 37.0 & 46.0 \\
\hline & 37.0 & 352.0 & 297. & 80.0 & 63. & & & 0 & & \\
\hline & $\begin{array}{l}1.0 \\
400.0\end{array}$ & $\begin{array}{r}2.0 \\
0 \\
02.0\end{array}$ & $\begin{array}{l}3.0 \\
25.0\end{array}$ & $\begin{array}{l}4.0 \\
23.0\end{array}$ & $\begin{array}{l}5.0 \\
0.0\end{array}$ & $\begin{array}{c}6.0 \\
0.0\end{array}$ & $\begin{array}{l}7.0 \\
0.0\end{array}$ & $\begin{array}{l}8.0 \\
0.0\end{array}$ & $\begin{array}{r}9.0 \\
0.0\end{array}$ & 10.0 \\
\hline & 1.0 & 2.0 & 3.0 & 4.0 & 5.0 & 6.0 & 7.0 & 9.0 & 12.0 & 16.0 \\
\hline & 343.0 & 302.0 & 184.0 & 151.0 & 22.0 & 81.0 & 91. & 42.0 & 37 & \\
\hline & $\begin{array}{l}1.0 \\
688.0\end{array}$ & $\begin{array}{l}2.0 \\
14.0\end{array}$ & $\begin{array}{l}3.0 \\
19.0\end{array}$ & & $\begin{array}{l}5.0 \\
0.0\end{array}$ & $\begin{array}{c}6.0 \\
0.0\end{array}$ & $\begin{array}{r}7.0 \\
0.0\end{array}$ & $\begin{array}{l}8.0 \\
0.0\end{array}$ & $\begin{array}{c}9.0 \\
0.0\end{array}$ & 10.0 \\
\hline & 1.0 & 2.0 & 3.0 & 4.0 & 5.0 & 6.0 & 7.0 & 9.0 & 12.0 & 16.0 \\
\hline 0. & $\begin{array}{r}2.0 \\
1.0\end{array}$ & $\begin{array}{r}328.0 \\
2.0\end{array}$ & $\begin{array}{r}19.0 \\
3.0\end{array}$ & $\begin{array}{r}108.0 \\
4.0\end{array}$ & $\begin{array}{r}58.0 \\
5.0\end{array}$ & $\begin{array}{r}49.0 \\
6.0\end{array}$ & $\begin{array}{r}17.0 \\
7.0\end{array}$ & $\begin{array}{r}37.0 \\
8.0\end{array}$ & $\begin{array}{r}15.0 \\
9.0\end{array}$ & 10.0 \\
\hline & $\begin{array}{c}534.0 \\
1.0\end{array}$ & $\begin{array}{c}197.0 \\
2.0\end{array}$ & $\begin{array}{l}83.0 \\
3.0\end{array}$ & $\begin{array}{r}0.0 \\
4.0\end{array}$ & $\begin{array}{l}0.0 \\
5.0\end{array}$ & $\begin{array}{l}0.0 \\
6.0\end{array}$ & $\begin{array}{l}0.0 \\
7.0\end{array}$ & $\begin{array}{l}0.0 \\
8.0\end{array}$ & 0.0 & 10. \\
\hline & 715.0 & 411.0 & 145.0 & 153.0 & 43.0 & 35.0 & 57 & 9 & & \\
\hline & & $\begin{array}{r}2.0 \\
218.0\end{array}$ & $\begin{array}{l}3.0 \\
110.0\end{array}$ & $\begin{array}{l}4.0 \\
37.0\end{array}$ & $\begin{array}{l}5.0 \\
35.0\end{array}$ & $\begin{array}{l}6.0 \\
0.0\end{array}$ & $\begin{array}{l}7.0 \\
0.0\end{array}$ & $\begin{array}{l}8.0 \\
0.0\end{array}$ & $\begin{array}{r}9.0 \\
0.0\end{array}$ & 10.0 \\
\hline & 1.0 & 2.0 & 3. & 4.0 & 5.0 & 6.0 & 7. & 8.0 & & 11. \\
\hline & 711 & 415 & 109 & & 3.0 & & & .0 & & \\
\hline & & 270 & & & & & & 8.0 & 0 & 10.0 \\
\hline & 1.0 & 2. & 3 & 4.0 & 5.0 & 6. & & & & 10. \\
\hline 32 & 732.0 & 369 & 108 & 41.0 & & & & & .0 & \\
\hline & 1.0 & 2.0 & & 4.0 & & & & 8.0 & 9.0 & 10.0 \\
\hline & 1.0 & $\begin{array}{r}141 \\
2 .\end{array}$ & 3. & 5.0 & 8.0 & 12.0 & 17 & 23 & & 38. \\
\hline & 503. & 248 & 185 & 153 & 68 & & & & & \\
\hline & & & 3 & & & & & & & 10.0 \\
\hline & 1 & & & & & & & & $\begin{array}{r}0 \\
45\end{array}$ & 55.0 \\
\hline & 210 & 249 & 210 & & & & & & & \\
\hline & & & & & & & & & & 10. \\
\hline & $28 \overline{5}$ & & & & & & & & & \\
\hline & & & & & & & & & & 31.0 \\
\hline & & & & & 108 & & & & & \\
\hline & & & & & & & & & & 46 \\
\hline & & & 25 & & & & & & & \\
\hline & & & & & & & & & & 76. \\
\hline & 75.0 & 35 & & & & & & & & \\
\hline & & & & & & & & & & 115. \\
\hline & 07.0 & 200 & 13 & & & & & & & \\
\hline & & & & & & & & & & 145.0 \\
\hline & & $40 \bar{\varepsilon}$ & $20\}$ & & & & & & & \\
\hline & & & & & & & & & & 190.0 \\
\hline & & 704 & & & & & & & & \\
\hline & & & & & & & & 10 & 0 & 166. \\
\hline
\end{tabular}




$\begin{array}{rrrrrrrrrrr}450.0 & 477.0 & 752.0 & 637.0 & 439.0 & 138.0 & 57.0 & 17.0 & 22.0 & 22.0 & \\ 0.0 & 1.0 & 5.0 & 13.0 & 25.0 & 41.0 & 61.0 & 85.0 & 113.0 & 145.0 & 181.0 \\ 623.0 & 462.0 & 916.0 & 666.0 & 470.0 & 208.0 & 84.0 & 18.0 & 11.0 & 15.0 & \\ 0.0 & 1.0 & 6.0 & 15.0 & 28.0 & 45.0 & 66.0 & 91.0 & 121.0 & 156.0 & 196.0 \\ 666.0 & 820.0 & 1134.0 & 800.0 & 442.0 & 246.0 & 61.0 & 21.0 & 11.0 & 16.0 & \\ 0.0 & 1.0 & 4.0 & 10.0 & 19.0 & 31.0 & 46.0 & 64.0 & 85.0 & 109.0 & 136.0 \\ 695.0 & 642.0 & 778.0 & 876.0 & 632.0 & 245.0 & 82.0 & 36.0 & 25.0 & 10.0 & \\ 0.0 & 1.0 & 4.0 & 9.0 & 16.0 & 25.0 & 36.0 & 49.0 & 64.0 & 81.0 & 100.0 \\ 790.0 & 538.0 & 701.0 & 490.0 & 294.0 & 193.0 & 34.0 & 25.0 & 23.0 & 15.0 & \\ 0.0 & 1.0 & 3.0 & 6.0 & 10.0 & 16.0 & 24.0 & 34.0 & 46.0 & 60.0 & 76.0 \\ 414.0 & 256.0 & 102.0 & 198.0 & 93.0 & 84.0 & 77.0 & 31.0 & 25.0 & 3.0 & \\ 0.0 & 1.0 & 3.0 & 6.0 & 10.0 & 15.0 & 21.0 & 28.0 & 36.0 & 45.0 & 55.0 \\ 268.0 & 184.0 & 76.0 & 216.0 & 165.0 & 166.0 & 138.0 & 118.0 & 117.0 & 87.0 & \\ \text { [END] } & & & & & & & & & & \end{array}$


[NAME]

C-52

655450WG

\begin{tabular}{|c|c|c|c|c|c|c|c|c|c|c|}
\hline $\begin{array}{r}\text { AT, } \\
8 . \\
\text { SERIE }\end{array}$ & $\begin{array}{l}\text { LON } \\
05 \\
\text { ES] }\end{array}$ & -2.78 & 370.0 & & & & & & & \\
\hline & 1.0 & 2.0 & 3.0 & 4.0 & 5.0 & 6.0 & 7.0 & 8.0 & 9.0 & 10. \\
\hline & 69.0 & & & & & & & & & \\
\hline & 1.0 & & & 13. & 21 & 31 & & & & 91.0 \\
\hline & 52.0 & 226 & 177 & 157. & & & & & & \\
\hline 5 & $\begin{array}{r}1.0 \\
27.0\end{array}$ & $\begin{array}{r}2.0 \\
33.0\end{array}$ & & $\begin{array}{l}4 . \\
0 .\end{array}$ & & & & & & 10.0 \\
\hline & 1.0 & & & 11 . & & & & & & 33.0 \\
\hline & 32.0 & 104. & 136 & & & & & & & \\
\hline & 1.0 & $\begin{array}{l}2.0 \\
53.0\end{array}$ & $\begin{array}{l}3.0 \\
0.0\end{array}$ & $\begin{array}{l}4.0 \\
0.0\end{array}$ & $\begin{array}{l}5.0 \\
0.0\end{array}$ & $\begin{array}{l}6.0 \\
0.0\end{array}$ & $\begin{array}{l}7.0 \\
0.0\end{array}$ & $\begin{array}{l}8.0 \\
0.0\end{array}$ & $\begin{array}{l}9.0 \\
0.0\end{array}$ & 0.0 \\
\hline & 1.0 & 2.0 & 3.0 & 5.0 & 8.0 & 12.0 & 17.0 & 23.0 & 30.0 & 38.0 \\
\hline & 229.0 & 115.0 & 215.0 & 183.0 & 134.0 & 22.0 & 12.0 & & 10 & \\
\hline 0 & $\begin{array}{l}1.0 \\
96.0\end{array}$ & $\begin{array}{ll}2.0 \\
2.0\end{array}$ & $\begin{array}{r}3.0 \\
0.0\end{array}$ & $\begin{array}{l}4.0 \\
0.0\end{array}$ & $\begin{array}{l}5.0 \\
0.0\end{array}$ & $\begin{array}{l}6.0 \\
0.0\end{array}$ & $\begin{array}{l}7.0 \\
0.0\end{array}$ & $\begin{array}{l}8.0 \\
0.0\end{array}$ & $\begin{array}{l}9.0 \\
0.0\end{array}$ & 10.0 \\
\hline & 1.0 & 2.0 & 3. & 4.0 & 5.0 & 7.0 & 10. & 14.0 & 19 & 5.0 \\
\hline 1 & 413.0 & 372.0 & 195. & 63.0 & 111.0 & 60.0 & & & & \\
\hline & $\begin{array}{l}1.0 \\
330.0\end{array}$ & $\begin{array}{r}2.0 \\
115.0\end{array}$ & $\begin{array}{l}3.0 \\
107.0\end{array}$ & $\begin{array}{c}4.0 \\
0.0\end{array}$ & $\begin{array}{l}5.0 \\
0.0\end{array}$ & $\begin{array}{c}6.0 \\
0.0\end{array}$ & $\begin{array}{l}7.0 \\
0.0\end{array}$ & $\begin{array}{l}8.0 \\
18.0\end{array}$ & $\begin{array}{c}9.0 \\
0.0\end{array}$ & 10.0 \\
\hline & & 2.0 & 3. & 4.0 & 5.0 & 6.0 & 7.0 & 8.0 & 10 & 13.0 \\
\hline & 459.0 & 350.0 & 205. & 49.0 & 72.0 & & 45.0 & & 46 & \\
\hline & 0 & $\begin{array}{cc}2.0 \\
0 & 169.0\end{array}$ & $\begin{array}{l}3.0 \\
19.0\end{array}$ & $\begin{array}{l}4.0 \\
12.0\end{array}$ & $\begin{array}{c}5.0 \\
0.0\end{array}$ & $\begin{array}{r}6.0 \\
0.0\end{array}$ & $\begin{array}{l}7.0 \\
0.0\end{array}$ & $\begin{array}{l}8.0 \\
1.0\end{array}$ & 0 & 10. \\
\hline & 1.0 & 2.0 & 3. & 4.0 & 5.0 & 6.0 & 7.0 & 8.0 & 10 & 13.0 \\
\hline & 669.0 & 277.0 & 183.0 & 145.0 & 94.0 & 40.0 & 12.0 & & & \\
\hline & & $\begin{array}{c}2.0 \\
0 \\
120.0\end{array}$ & $\begin{array}{l}3.0 \\
43.0\end{array}$ & $\begin{array}{l}4.0 \\
26.0\end{array}$ & $\begin{array}{c}5.0 \\
0.0\end{array}$ & $\begin{array}{c}6.0 \\
0.0\end{array}$ & & $\begin{array}{r}8.0 \\
0.0\end{array}$ & 0 & 10.0 \\
\hline & 1.0 & & 3. & 5.0 & 8.0 & 12.0 & 17.0 & 23.0 & 30 & $38 .($ \\
\hline & 482.0 & 299.0 & 264.0 & 143.0 & 35.0 & & 11.0 & & & \\
\hline 6 & & $\begin{array}{cc}2.0 \\
0 & 167.0\end{array}$ & $\begin{array}{l}3.0 \\
63.0\end{array}$ & $\begin{array}{r}4.0 \\
4.0\end{array}$ & & $\begin{array}{c}6.0 \\
0.0\end{array}$ & & $\begin{array}{l}8.0 \\
0.0\end{array}$ & & 10. \\
\hline & & 2.0 & & 4.0 & 5.0 & 6.0 & 7.0 & 9.0 & 12 & 16. \\
\hline & 427.0 & 341.0 & 239.0 & 128.0 & 57.0 & & & 42 & & \\
\hline & & 2.0 & & 4.0 & & & & & 0 & $10 .($ \\
\hline 0 & $\begin{array}{c}0374.0 \\
1.0\end{array}$ & $\begin{array}{c}187.0 \\
2.0\end{array}$ & $\begin{array}{l}86 \\
3 .\end{array}$ & $\begin{array}{l}51.0 \\
4.0\end{array}$ & & $\begin{array}{l}23.0 \\
6.0\end{array}$ & & $\begin{array}{l}0.0 \\
8.0\end{array}$ & 0 & 10. \\
\hline & 512.0 & 234.0 & 244. & 122.0 & & & & & & \\
\hline & 0 & 153 & & & & $\begin{array}{l}6.0 \\
1.0\end{array}$ & & & 0 & $10 .($ \\
\hline & & & & & & & & & & 5. \\
\hline & 426.0 & 354. & 191. & 101 & & & & & & \\
\hline & & & & & & & & & & 10. \\
\hline & 60 . & 31 & & & & & & & & \\
\hline & & & & & & & & & & 31. \\
\hline & & 186 & & 184 & & & & & & \\
\hline & & & & & & & & & & 10. \\
\hline & & & & & & & & & & \\
\hline & & & & & & & & & & 31. \\
\hline & & 161. & 118 & & 115 & & & & & \\
\hline & & & & & & & & & & \\
\hline & & & & & & & & & & \\
\hline & & & & & & & & & & 155. \\
\hline & & & & & & & & & & \\
\hline & & & & & & & & & & 181. \\
\hline & & & & & & & & & & \\
\hline & & & & & & & & & & 308. \\
\hline & & & & & & & & & & \\
\hline & & & & & & & & & & $181 .($ \\
\hline & & & & & & & & & & \\
\hline & & & & & & & & & & 2 \\
\hline
\end{tabular}




$\begin{array}{rrrrrrrrrrr}574.0 & 472.0 & 576.0 & 538.0 & 475.0 & 369.0 & 112.0 & 76.0 & 38.0 & 16.0 & \\ 0.0 & 1.0 & 4.0 & 9.0 & 16.0 & 26.0 & 39.0 & 55.0 & 74.0 & 96.0 & 121.0 \\ 747.0 & 552.0 & 374.0 & 364.0 & 310.0 & 202.0 & 140.0 & 84.0 & 44.0 & 10.0 & \\ 0.0 & 1.0 & 5.0 & 12.0 & 23.0 & 38.0 & 57.0 & 80.0 & 107.0 & 138.0 & 173.0 \\ 898.0 & 532.0 & 472.0 & 347.0 & 317.0 & 89.0 & 64.0 & 45.0 & 31.0 & 23.0 & \\ 0.0 & 1.0 & 4.0 & 10.0 & 19.0 & 31.0 & 46.0 & 64.0 & 85.0 & 109.0 & 136.0 \\ 919.0 & 487.0 & 909.0 & 691.0 & 544.0 & 203.0 & 73.0 & 21.0 & 14.0 & 12.0 & \\ 0.0 & 1.0 & 3.0 & 7.0 & 13.0 & 21.0 & 31.0 & 43.0 & 57.0 & 73.0 & 91.0 \\ 726.0 & 502.0 & 501.0 & 532.0 & 373.0 & 221.0 & 115.0 & 34.0 & 29.0 & 6.0 & \\ 0.0 & 1.0 & 3.0 & 6.0 & 10.0 & 15.0 & 21.0 & 28.0 & 36.0 & 45.0 & 56.0 \\ 389.0 & 269.0 & 245.0 & 200.0 & 158.0 & 88.0 & 67.0 & 53.0 & 34.0 & 10.0 & \\ 0.0 & 1.0 & 3.0 & 6.0 & 10.0 & 15.0 & 21.0 & 29.0 & 39.0 & 51.0 & 65.0 \\ 489.0 & 202.0 & 267.0 & 140.0 & 126.0 & 98.0 & 230.0 & 169.0 & 89.0 & 38.0 & \\ \text { [END] } & & & & & & & & & & \end{array}$


[NAME]

C-54

655480WG

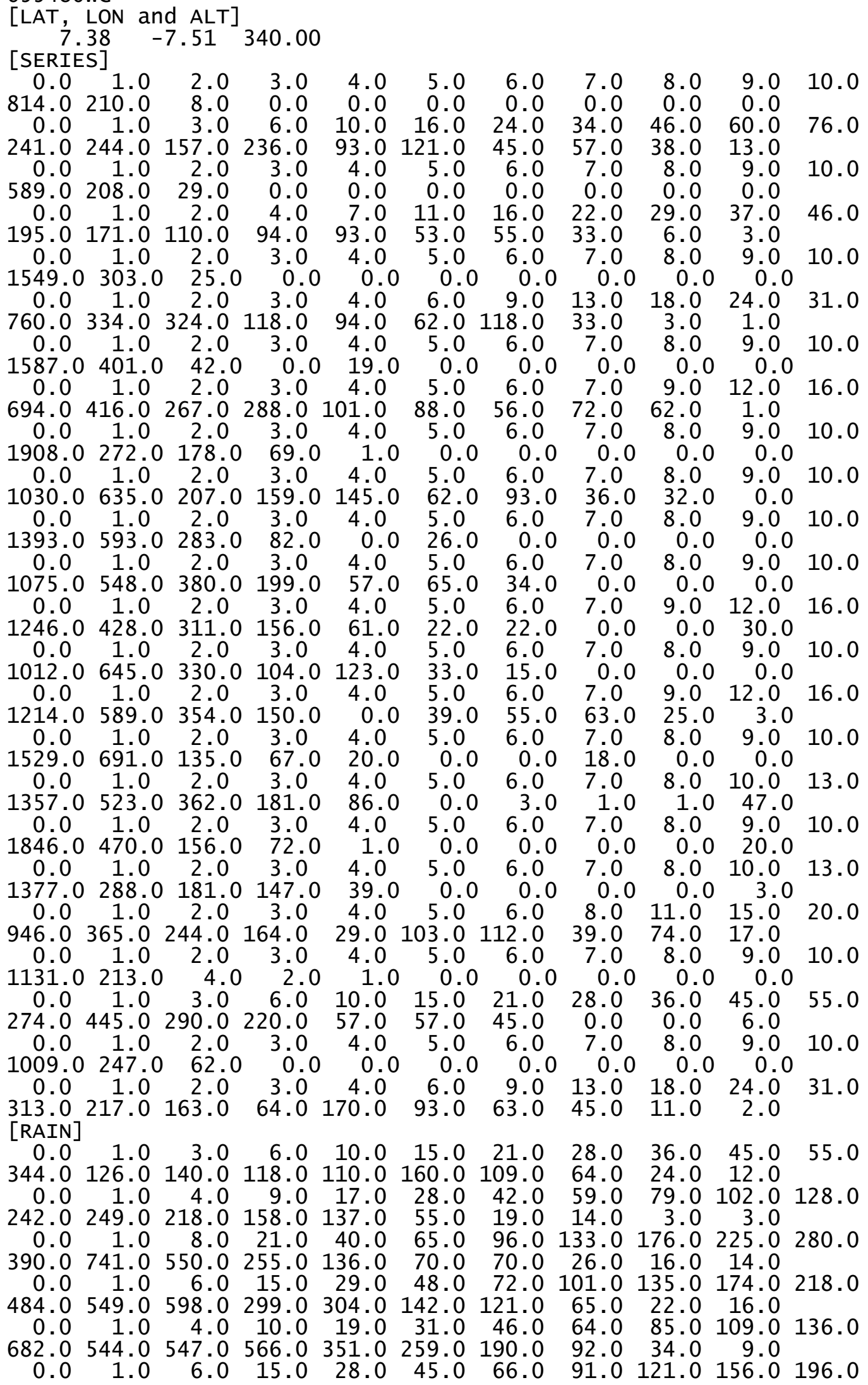


$\begin{array}{lllllllllll}805.0 & 842.0 & 906.0 & 610.0 & 393.0 & 139.0 & 124.0 & 33.0 & 28.0 & 23.0\end{array}$ $\begin{array}{lllllllllll}0.0 & 1.0 & 6.0 & 15.0 & 28.0 & 45.0 & 67.0 & 94.0 & 126.0 & 163.0 & 205.0\end{array}$ $\begin{array}{llllllllll}1296.0 & 1133.0 & 998.0 & 579.0 & 339.0 & 76.0 & 148.0 & 15.0 & 19.0 & 15.0\end{array}$ $\begin{array}{lllllllllll}0.0 & 1.0 & 6.0 & 15.0 & 28.0 & 45.0 & 67.0 & 94.0 & 126.0 & 163.0 & 205.0\end{array}$ $\begin{array}{lllllllllll}1074.0 & 1589.0 & 1227.0 & 728.0 & 426.0 & 180.0 & 157.0 & 35.0 & 28.0 & 20.0\end{array}$ $\begin{array}{lllllllllll}0.0 & 1.0 & 5.0 & 12.0 & 22.0 & 35.0 & 51.0 & 70.0 & 92.0 & 117.0 & 146.0\end{array}$

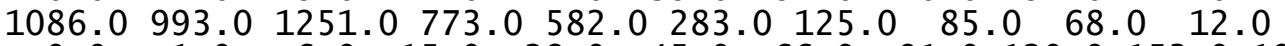
$\begin{array}{lllllllllll}0.0 & 1.0 & 6.0 & 15.0 & 28.0 & 45.0 & 66.0 & 91.0 & 120.0 & 153.0 & 190.0\end{array}$ $\begin{array}{llllllllll}636.0 & 753.0 & 983.0 & 499.0 & 291.0 & 132.0 & 44.0 & 11.0 & 12.0 & 16.0\end{array}$

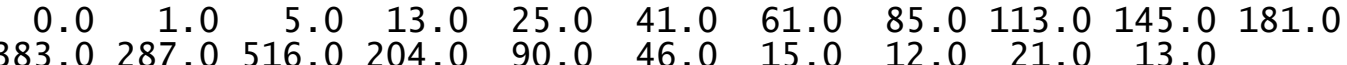

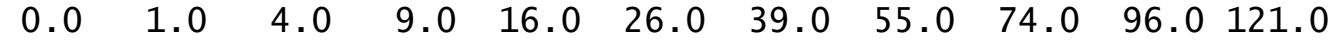

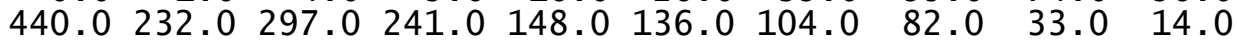
[END] 
[NAME]

C-56

655550WG

\begin{tabular}{|c|c|c|c|c|c|c|c|c|c|c|}
\hline $\begin{array}{r}7 \\
7\end{array}$ & $\begin{array}{l}.73 \\
\text { EST }\end{array}$ & -5.06 & 376.00 & & & & & & & \\
\hline Lin & 1.0 & & & & & & $\begin{array}{l}7.0 \\
0.0\end{array}$ & & & 10.0 \\
\hline 0 & $\begin{array}{r}63.0 \\
1.0\end{array}$ & 3.0 & & 10.0 & $\begin{array}{r}15.0 \\
0\end{array}$ & 21.0 & 29 & 39 & $\begin{array}{r}0 \\
51\end{array}$ & 65.0 \\
\hline & 62.0 & 157.0 & 168. & 104.0 & 130.0 & & & & & \\
\hline & 1.0 & & & 4. & & & & & & 10.0 \\
\hline & 93.0 & & & 0 & & & & & & \\
\hline & 1.0 & & & 10 & & & & & & 61.0 \\
\hline & 73.0 & 115. & 144 & & & & & & & \\
\hline & 1.0 & $\begin{array}{l}2.0 \\
28.0\end{array}$ & $\begin{array}{l}3.0 \\
25.0\end{array}$ & 4.0 & 5.0 & $\begin{array}{r}6.0 \\
0.0\end{array}$ & $\begin{array}{l}7.0 \\
0.0\end{array}$ & $\begin{array}{c}8.0 \\
0.0\end{array}$ & $\begin{array}{l}9.0 \\
0.0\end{array}$ & 10.0 \\
\hline & 1.0 & 2.0 & 3.0 & 4.0 & 6.0 & 9.0 & 13.0 & 18.0 & 24 & 31.0 \\
\hline & 378.0 & 144.0 & 190.0 & 184.0 & 116.0 & 122.0 & & & & \\
\hline & $\begin{array}{r}1.0 \\
433.0\end{array}$ & $\begin{array}{ll}2.0 \\
0 & 24.0\end{array}$ & $\begin{array}{l}3.0 \\
26.0\end{array}$ & $\begin{array}{r}4.0 \\
0.0\end{array}$ & $\begin{array}{r}5.0 \\
0.0\end{array}$ & $\begin{array}{c}6.0 \\
0.0\end{array}$ & $\begin{array}{r}7.0 \\
0.0\end{array}$ & $\begin{array}{r}8.0 \\
0.0\end{array}$ & $\begin{array}{c}9.0 \\
0.0\end{array}$ & 10. \\
\hline & & $\begin{array}{r}2.0 \\
271.0\end{array}$ & $\begin{array}{r}3.0 \\
182.0\end{array}$ & $\begin{array}{r}4.0 \\
94.0\end{array}$ & $\begin{array}{r}5.0 \\
135.0\end{array}$ & $\begin{array}{r}6.0 \\
370\end{array}$ & $\begin{array}{r}7.0 \\
57.0\end{array}$ & 9.0 & 12 & 16.0 \\
\hline & $\begin{array}{r}1.0 \\
353.0\end{array}$ & $\begin{array}{r}1.0 \\
2.0 \\
92.0\end{array}$ & 3.0 & 4.0 & 5.0 & 6.0 & 7.0 & 8.0 & $\begin{array}{c}59.0 \\
9.0\end{array}$ & 10.0 \\
\hline & 1.0 & 2.0 & 3.0 & 4.0 & 5.0 & 6.0 & 7. & 9.0 & 12 & 16.0 \\
\hline & 449.0 & 322.0 & 233.0 & 171.0 & 100.0 & 91.0 & 64 & 38. & 22 & \\
\hline & $\begin{array}{r}1.0 \\
550.0\end{array}$ & $\begin{array}{l}2.0 \\
29.0\end{array}$ & $\begin{array}{l}3.0 \\
0.0\end{array}$ & $\begin{array}{r}4.0 \\
0.0\end{array}$ & & $\begin{array}{r}6.0 \\
0.0\end{array}$ & $\begin{array}{r}7.0 \\
0.0\end{array}$ & $\begin{array}{c}8.0 \\
0.0\end{array}$ & $\begin{array}{r}9.0 \\
0.0\end{array}$ & 10. \\
\hline & & 2.0 & 3.0 & $\begin{array}{r}4.0 \\
172.0\end{array}$ & $\begin{array}{r}5.0 \\
108.0\end{array}$ & 6.0 & & 9.0 & 12 & 16 \\
\hline & $\begin{array}{l}2.0 \\
1.0\end{array}$ & 2 & 3. & $\begin{array}{r}172.0 \\
4.0\end{array}$ & $\begin{array}{r}108.0 \\
5.0\end{array}$ & $\begin{array}{r}35.0 \\
6.0\end{array}$ & & $\begin{array}{r}43.0 \\
8.0\end{array}$ & 9.0 & 0. \\
\hline & 425.0 & 101.0 & 38 & 29.0 & 0.0 & & & & & \\
\hline & & 148 & $\begin{array}{r}3 . \\
181\end{array}$ & $\begin{array}{r}4.0 \\
131\end{array}$ & $\begin{array}{r}5.0 \\
67.0\end{array}$ & & 8. & & & 20. \\
\hline & 1.0 & $\begin{array}{r}1+0.0 \\
2.0\end{array}$ & $\begin{array}{r}3.0 \\
3.0\end{array}$ & $\begin{array}{r}4.0 \\
4.0\end{array}$ & 5.0 & 6.0 & 7.0 & 0 & & 10. \\
\hline & $\begin{array}{r}524.0 \\
1.0\end{array}$ & $\begin{array}{rr}0 & 136 \\
2 .\end{array}$ & $\begin{array}{ll}0 & 42 \\
& 3\end{array}$ & $\begin{array}{l}1.0 \\
4.0\end{array}$ & $\begin{array}{l}24.0 \\
5.0\end{array}$ & 6 . & 0 & $0^{0}$ & 15.0 & +0 \\
\hline & 559.0 & 259.0 & 158. & 98.0 & 48.0 & 46. & 59 & & & \\
\hline & & 2.0 & & 4.0 & & & 7.0 & & 0 & 10. \\
\hline & & 2 & 3 & 4.0 & & & & & & 10. \\
\hline & 412 & $0 \quad 419$ & 157 & 20 & & & & & & \\
\hline & & & & 4 ; & & & & & 0 & 10. \\
\hline & 1.0 & 3. & & 10. & 15. & & & & & 55. \\
\hline & & 372 & & & & & & & & \\
\hline & & & & & & & & & & 10. \\
\hline & 1. & & & & & & & & $\begin{array}{r}0 \\
45\end{array}$ & 55. \\
\hline & 328.0 & 169. & & 127.0 & & & & & & \\
\hline & 135 & 2.0 & & & & & & & & 10.0 \\
\hline & & 2 & & & & & & & & 31.0 \\
\hline & & ז & 110 & & $1 / 4$ & & & & & \\
\hline & & & & & & & & & & \\
\hline & 48. & 63 & & & & & & & & \\
\hline & & & & & & & & & & 160. \\
\hline & 39. & 10 & & & & & & & & \\
\hline & & & & & & & & & & 238. \\
\hline & 70. & 16 & 19 & 11 & & & & & & \\
\hline & & & & & & & & & & 173.0 \\
\hline & & & & 34 & & & & & & \\
\hline & & & & & & & & & & 100.0 \\
\hline & & 5 & & & & & & & & \\
\hline & & & & & & & & & 0 & 0.1 \\
\hline
\end{tabular}




$\begin{array}{rrrrrrrrrrr}588.0 & 550.0 & 551.0 & 445.0 & 297.0 & 246.0 & 119.0 & 42.0 & 23.0 & 53.0 & \\ 0.0 & 1.0 & 4.0 & 9.0 & 16.0 & 25.0 & 36.0 & 49.0 & 64.0 & 81.0 & 100.0 \\ 796.0 & 470.0 & 395.0 & 428.0 & 292.0 & 239.0 & 117.0 & 68.0 & 17.0 & 30.0 & \\ 0.0 & 1.0 & 4.0 & 9.0 & 16.0 & 25.0 & 36.0 & 49.0 & 64.0 & 81.0 & 100.0 \\ 907.0 & 463.0 & 599.0 & 414.0 & 352.0 & 209.0 & 227.0 & 45.0 & 3.0 & 71.0 & \\ 0.0 & 1.0 & 4.0 & 9.0 & 16.0 & 25.0 & 36.0 & 50.0 & 67.0 & 87.0 & 110.0 \\ 891.0 & 588.0 & 639.0 & 652.0 & 444.0 & 199.0 & 146.0 & 80.0 & 19.0 & 61.0 & \\ 0.0 & 1.0 & 4.0 & 9.0 & 16.0 & 26.0 & 39.0 & 55.0 & 74.0 & 96.0 & 121.0 \\ 476.0 & 468.0 & 576.0 & 496.0 & 392.0 & 125.0 & 84.0 & 38.0 & 63.0 & 35.0 & \\ 0.0 & 1.0 & 3.0 & 7.0 & 13.0 & 21.0 & 31.0 & 43.0 & 57.0 & 73.0 & 91.0 \\ 358.0 & 262.0 & 300.0 & 252.0 & 97.0 & 63.0 & 80.0 & 22.0 & 24.0 & 8.0 & \\ 0.0 & 1.0 & 4.0 & 9.0 & 16.0 & 25.0 & 36.0 & 49.0 & 64.0 & 82.0 & 103.0 \\ 317.0 & 184.0 & 380.0 & 183.0 & 73.0 & 79.0 & 80.0 & 62.0 & 43.0 & 81.0 & \\ \text { [END] } & & & & & & & & & & \end{array}$


[NAME]

C-58

655570WG

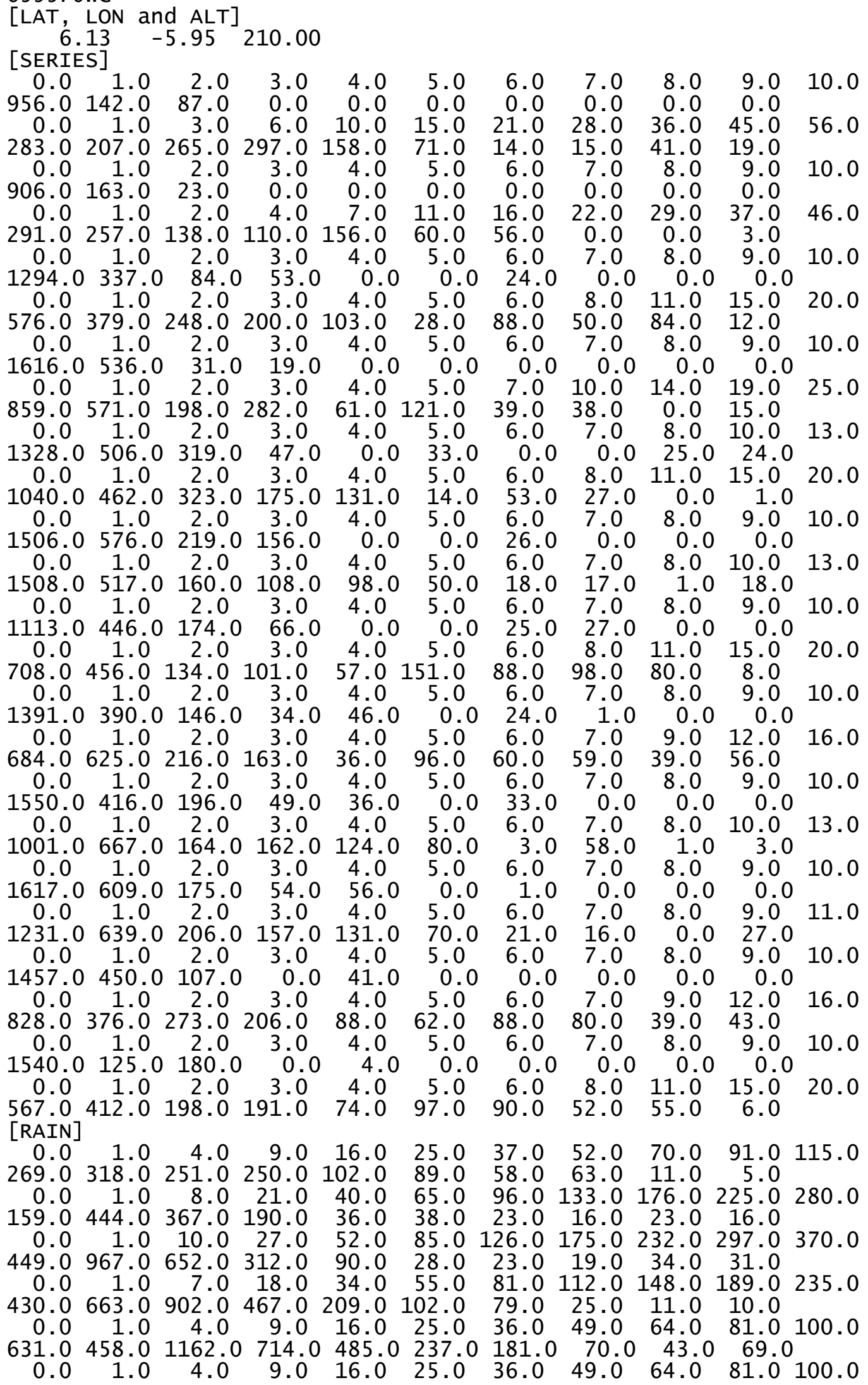




$\begin{array}{rrrrrrrrrrr}780.0 & 676.0 & 1180.0 & 674.0 & 400.0 & 184.0 & 238.0 & 59.0 & 39.0 & 29.0 & \\ 0.0 & 1.0 & 3.0 & 6.0 & 11.0 & 18.0 & 27.0 & 38.0 & 51.0 & 66.0 & 83.0 \\ 847.0 & 630.0 & 519.0 & 419.0 & 292.0 & 183.0 & 119.0 & 69.0 & 37.0 & 12.0 & \\ 0.0 & 1.0 & 4.0 & 9.0 & 16.0 & 25.0 & 36.0 & 49.0 & 64.0 & 81.0 & 100.0 \\ 1121.0 & 809.0 & 447.0 & 305.0 & 181.0 & 104.0 & 92.0 & 49.0 & 30.0 & 22.0 & \\ 0.0 & 1.0 & 4.0 & 9.0 & 16.0 & 25.0 & 36.0 & 50.0 & 67.0 & 87.0 & 110.0 \\ 1019.0 & 602.0 & 793.0 & 469.0 & 243.0 & 77.0 & 168.0 & 77.0 & 88.0 & 58.0 & \\ 0.0 & 1.0 & 4.0 & 9.0 & 16.0 & 26.0 & 39.0 & 55.0 & 74.0 & 96.0 & 121.0 \\ 882.0 & 646.0 & 1044.0 & 567.0 & 384.0 & 149.0 & 82.0 & 54.0 & 59.0 & 31.0 & \\ 0.0 & 1.0 & 4.0 & 9.0 & 16.0 & 25.0 & 36.0 & 50.0 & 67.0 & 87.0 & 110.0 \\ 630.0 & 551.0 & 723.0 & 427.0 & 301.0 & 108.0 & 77.0 & 7.0 & 20.0 & 20.0 & \\ 0.0 & 1.0 & 3.0 & 7.0 & 13.0 & 21.0 & 31.0 & 43.0 & 57.0 & 73.0 & 91.0 \\ 672.0 & 332.0 & 381.0 & 412.0 & 290.0 & 153.0 & 95.0 & 4.0 & 45.0 & 23.0 & \\ \text { [END] } & & & & & & & & & \end{array}$


[NAME]

C-60

655600WG

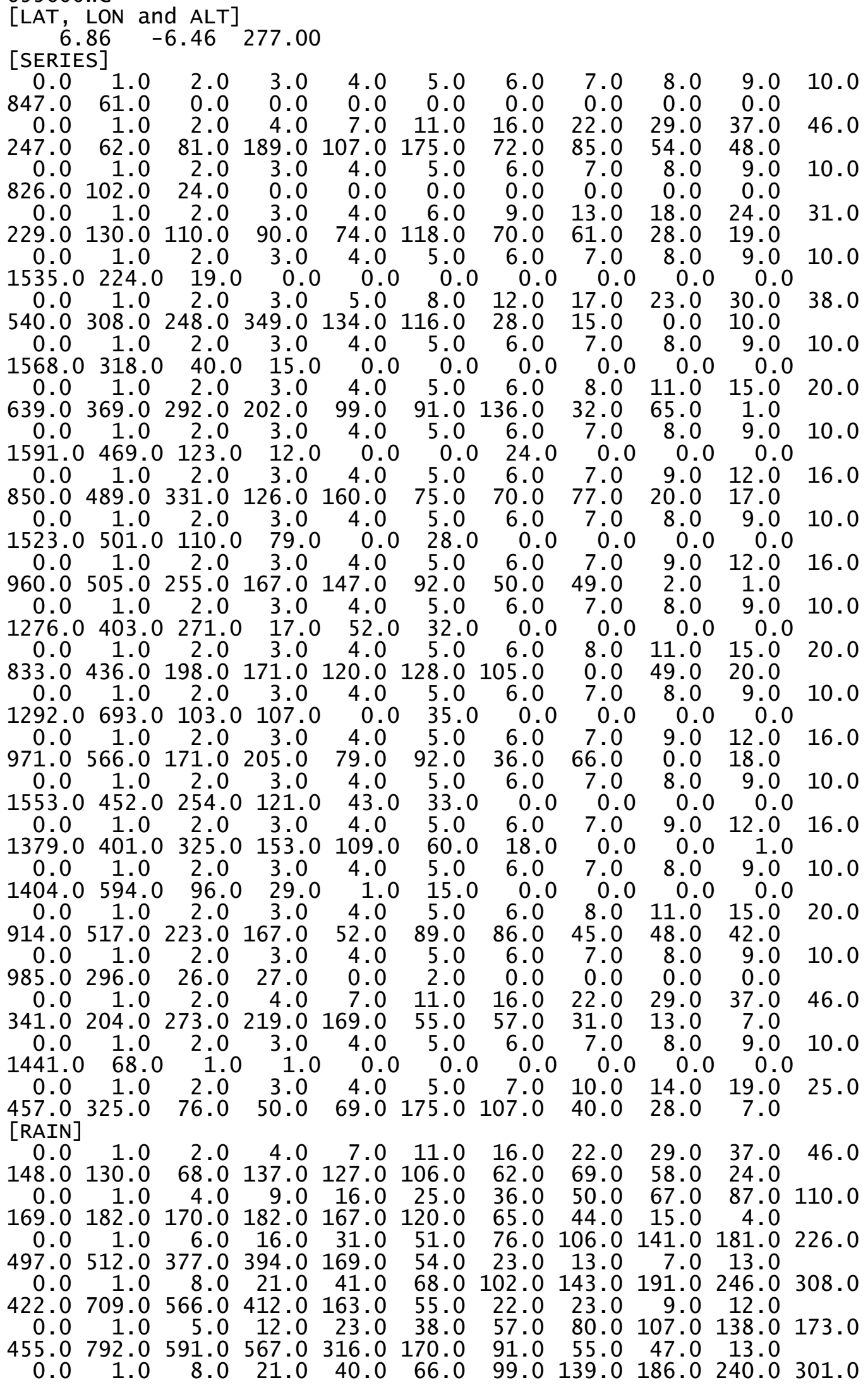


$\begin{array}{llllllllll}736.0 & 1361.0 & 627.0 & 268.0 & 188.0 & 77.0 & 35.0 & 34.0 & 14.0 & 10.0\end{array}$

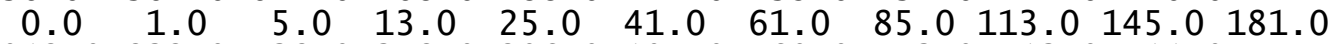
$\begin{array}{llllllllll}1012.0 & 832.0 & 738.0 & 358.0 & 206.0 & 107.0 & 60.0 & 43.0 & 13.0 & 11.0\end{array}$

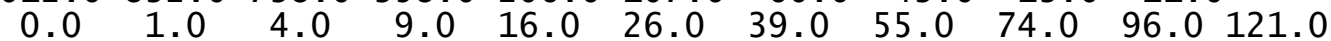

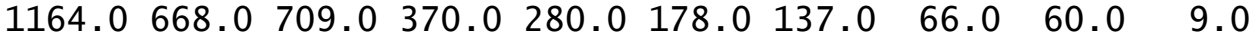

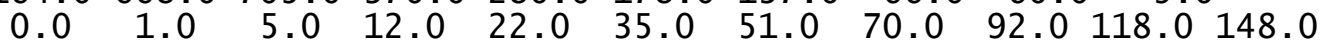
$\begin{array}{lllllllllll}1144.0 & 1047.0 & 839.0 & 575.0 & 246.0 & 77.0 & 89.0 & 43.0 & 34.0 & 17.0\end{array}$

$\begin{array}{lllllllllll}0.0 & 1.0 & 4.0 & 10.0 & 19.0 & 31.0 & 46.0 & 64.0 & 85.0 & 109.0 & 136.0\end{array}$ $\begin{array}{llllllllll}761.0 & 672.0 & 697.0 & 555.0 & 236.0 & 107.0 & 36.0 & 23.0 & 19.0 & 7.0\end{array}$

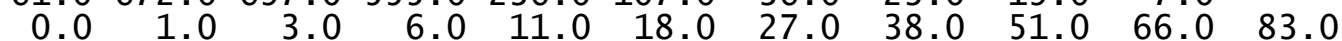

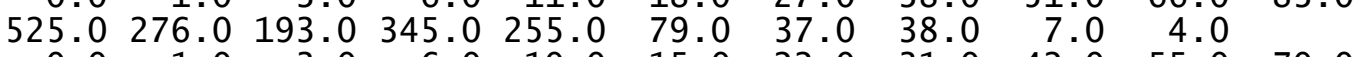

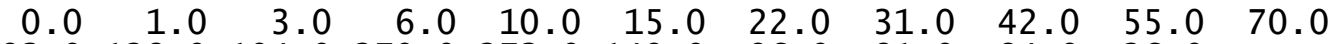

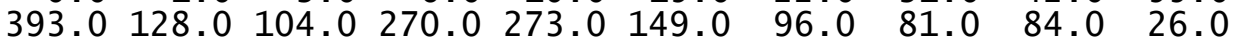
[END] 
[NAME]

C-62

655620WG

\begin{tabular}{|c|c|c|c|c|c|c|c|c|c|c|}
\hline $\begin{array}{r}A T, \\
6 . \\
6 F R T\end{array}$ & $\begin{array}{l}\text { LON an } \\
.65 \\
\text { EST }\end{array}$ & $\begin{array}{l}\text { nd } \mathrm{ALT}] \\
-4.70\end{array}$ & 92.00 & & & & & & & \\
\hline DLN & 1.0 & 2.0 & 3.0 & 4.0 & 5.0 & 6.0 & 7.0 & 8.0 & 9.0 & 10.0 \\
\hline & 3.0 & & & & & & & & & \\
\hline & 56.0 & 190. & 127. & $\begin{array}{r}10.0 \\
194.0\end{array}$ & $\begin{array}{r}15 . \\
115 .\end{array}$ & & & $\begin{array}{l}42 \\
35\end{array}$ & $\begin{array}{l}55 \\
18\end{array}$ & 70.0 \\
\hline & 1.0 & & & 4.0 & & & & & & 10.0 \\
\hline & 59.0 & & & & & & & & & \\
\hline & 1.0 & & & 10 & & & & & & 70.0 \\
\hline & 55.0 & 144. & 161. & & & & & & & \\
\hline & $\begin{array}{l}1.0 \\
351.0\end{array}$ & $\begin{array}{l}2.0 \\
54.0\end{array}$ & 3.0 & $\begin{array}{l}4.0 \\
22.0\end{array}$ & $\begin{array}{l}5.0 \\
20.0\end{array}$ & $\begin{array}{l}6.0 \\
0.0\end{array}$ & 7.0 & 8.0 & $\begin{array}{l}9.0 \\
0.0\end{array}$ & 10.0 \\
\hline & & & $\begin{array}{r}3.0 \\
214.0\end{array}$ & $\begin{array}{r}5.0 \\
187.0\end{array}$ & 8. & 12.0 & & 23.0 & 30 & 38.0 \\
\hline & 1.0 & $\begin{array}{r}2.0 \\
2.0\end{array}$ & 3.0 & 4.0 & 5.0 & $\begin{array}{r}4.0 \\
6.0\end{array}$ & $\begin{array}{r}17.0 \\
7.0\end{array}$ & $\begin{array}{r}10.0 \\
8.0\end{array}$ & $\begin{array}{r}16.0 \\
9.0\end{array}$ & 10.0 \\
\hline & $\begin{array}{c}201.0 \\
1.0\end{array}$ & $\begin{array}{c}114.0 \\
2.0\end{array}$ & $\begin{array}{l}0.0 \\
3.0\end{array}$ & $\begin{array}{r}1.0 \\
5.0\end{array}$ & 8.0 & $\begin{array}{l}0.0 \\
12.0\end{array}$ & $\begin{array}{l}0.0 \\
17.0\end{array}$ & $\begin{array}{r}0.0 \\
23.0\end{array}$ & & +0.0 \\
\hline & 376.0 & 319.0 & 239.0 & 137.0 & 127.0 & & & & & \\
\hline & $\begin{array}{l}1.0 \\
595.0\end{array}$ & $\begin{array}{c}2.0 \\
0246.0\end{array}$ & & $\begin{array}{r}4.0 \\
0.0\end{array}$ & $\begin{array}{c}5.0 \\
0.0\end{array}$ & $\begin{array}{l}6.0 \\
0.0\end{array}$ & $\begin{array}{l}7.0 \\
0.0\end{array}$ & $\begin{array}{l}8.0 \\
0.0\end{array}$ & $\begin{array}{r}9.0 \\
0.0\end{array}$ & 10. \\
\hline & 1.0 & 2. & 3. & 4.0 & 5.0 & 6.0 & 7. & 8 & & 11.0 \\
\hline 16 & 423.0 & 308. & 214. & 96.0 & 101.0 & 14. & 32 & 70 & & \\
\hline 0 & $\begin{array}{l}1.0 \\
425.0\end{array}$ & $\begin{array}{l}2 . \\
0 \quad 326\end{array}$ & $\begin{array}{l}3.0 \\
63.0\end{array}$ & $\begin{array}{r}4.0 \\
0.0\end{array}$ & $\begin{array}{ll} & 5.0 \\
0 & 29.0\end{array}$ & $\begin{array}{c}6.0 \\
0.0\end{array}$ & 7. & 8 & $\begin{array}{l}9 \\
2\end{array}$ & 1 \\
\hline & & $\begin{array}{c}2.0 \\
0\end{array}$ & $\begin{array}{r}31 \\
0 \quad 217\end{array}$ & $\begin{array}{l}4.0 \\
42.0\end{array}$ & & & & & $\begin{array}{c}10.0 \\
12.0\end{array}$ & 13. \\
\hline & $\begin{array}{l}1.0 \\
474.0\end{array}$ & $\begin{array}{c}2.0 \\
0 \\
146.0\end{array}$ & $\begin{array}{l}3 . \\
0 \quad 28\end{array}$ & $\begin{array}{l}4.0 \\
24.0\end{array}$ & $\begin{array}{l}5.0 \\
1.0\end{array}$ & & $\begin{array}{l}7.0 \\
0.0\end{array}$ & $\begin{array}{l}8.0 \\
0.0\end{array}$ & $\begin{array}{c}9.0 \\
0.0\end{array}$ & 10. \\
\hline & & & & 4.0 & 5.0 & & & & 19 & 25 \\
\hline & 1. & 2. & 3. & $\begin{array}{r}69.0 \\
4.0\end{array}$ & $\begin{array}{l}5.0 \\
5.0\end{array}$ & $\begin{array}{r}12 \angle .0 \\
6.0\end{array}$ & 7.0 & $\begin{array}{r}29.0 \\
8.0\end{array}$ & & 0.5 \\
\hline & $\begin{array}{rr}0 & 428 \\
& 1 .\end{array}$ & $\begin{array}{r}235 \\
2 .\end{array}$ & $\begin{array}{l}28 \\
3 .\end{array}$ & $\begin{array}{r}0.0 \\
4.0\end{array}$ & 0.0 & $\begin{array}{l}0.0 \\
7.0\end{array}$ & 10 & & .0 & 3 \\
\hline & 278. & 191. & 201 & 68.0 & 206.0 & 105. & & & & \\
\hline & & 2 & 3 & & & & 7.0 & 8.0 & 0 & 10. \\
\hline & 1. & & 3. & 4.0 & 5. & 6 & 7 & & 9 & 11. \\
\hline & 07 & 229. & 138. & 128.0 & & & & & 29 & \\
\hline & & 2. & 3 & 4.0 & 5.0 & & 7.0 & & 0 & 10. \\
\hline & & & & 5.0 & & 12 & 17 & 23 & & 38. \\
\hline & 405 & 299. & 263. & 140.0 & & & & & & \\
\hline & 224 & 2. & & 4.0 & & 6.0 & & 8.0 & 9.0 & 10. \\
\hline & 1. & & & 7.0 & 11. & & & & & 46. \\
\hline & & 330 & 203 & 174.0 & 114 & & & & & \\
\hline & & & & & & 6 & 7 & & & 10.0 \\
\hline & & & & & & & & & & \\
\hline & 56. & $2 \angle 5$ & & & 161 & 130 & & & & \\
\hline & & & & & & & & & & 65. \\
\hline & 29 & & 9 & & & & & & & \\
\hline & 1. & & & & & & & & & 70. \\
\hline & 141. & & 19 & & & & & & & \\
\hline & & & & & & & & & & 10 \\
\hline & 102. & 334 & & & & & & & & \\
\hline & & & & & & & & & & 110. \\
\hline & 7. & & & & & & & & & \\
\hline & & & & & & & & & & 181. \\
\hline & . & $4 \varepsilon$ & & & & & & & & \\
\hline & & & & & & & & & 0 & 5. \\
\hline
\end{tabular}




$\begin{array}{rrrrrrrrrrr}928.0 & 1125.0 & 929.0 & 405.0 & 252.0 & 110.0 & 30.0 & 26.0 & 20.0 & 16.0 & \\ 0.0 & 1.0 & 6.0 & 15.0 & 28.0 & 45.0 & 66.0 & 91.0 & 120.0 & 153.0 & 190.0 \\ 953.0 & 741.0 & 683.0 & 295.0 & 160.0 & 88.0 & 25.0 & 19.0 & 2.0 & 4.0 & \\ 0.0 & 1.0 & 5.0 & 12.0 & 22.0 & 35.0 & 51.0 & 70.0 & 92.0 & 117.0 & 145.0 \\ 1177.0 & 636.0 & 513.0 & 273.0 & 142.0 & 70.0 & 38.0 & 21.0 & 9.0 & 2.0 & \\ 0.0 & 1.0 & 6.0 & 15.0 & 28.0 & 46.0 & 69.0 & 97.0 & 130.0 & 168.0 & 211.0 \\ 1079.0 & 829.0 & 744.0 & 405.0 & 207.0 & 109.0 & 46.0 & 25.0 & 36.0 & 32.0 & \\ 0.0 & 1.0 & 5.0 & 12.0 & 22.0 & 35.0 & 51.0 & 70.0 & 92.0 & 117.0 & 145.0 \\ 948.0 & 624.0 & 638.0 & 489.0 & 298.0 & 201.0 & 109.0 & 39.0 & 19.0 & 15.0 & \\ 0.0 & 1.0 & 3.0 & 6.0 & 10.0 & 15.0 & 21.0 & 28.0 & 37.0 & 48.0 & 61.0 \\ 404.0 & 189.0 & 183.0 & 311.0 & 176.0 & 119.0 & 92.0 & 110.0 & 68.0 & 22.0 & \\ 0.0 & 1.0 & 3.0 & 6.0 & 10.0 & 15.0 & 22.0 & 31.0 & 42.0 & 55.0 & 70.0 \\ 282.0 & 235.0 & 86.0 & 305.0 & 186.0 & 128.0 & 169.0 & 70.0 & 43.0 & 47.0 & \\ \text { [END] } & & & & & & & & & & \\ \end{array}$


[NAME]

C-64

655630WG

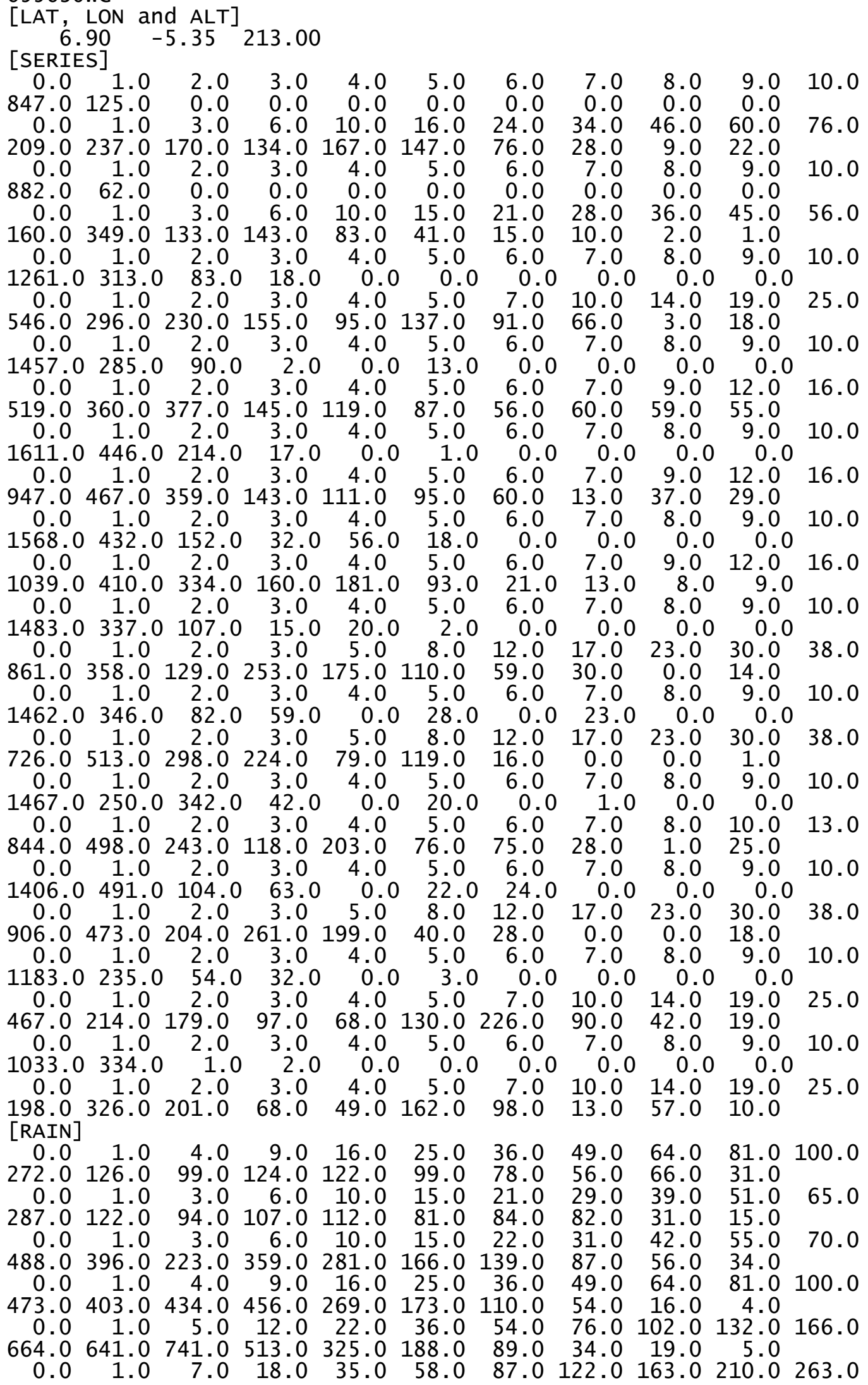




$\begin{array}{rrrrrrrrrrr}661.0 & 1040.0 & 889.0 & 480.0 & 169.0 & 73.0 & 35.0 & 20.0 & 21.0 & 17.0 & \\ 0.0 & 1.0 & 5.0 & 12.0 & 23.0 & 38.0 & 57.0 & 80.0 & 107.0 & 138.0 & 173.0 \\ 747.0 & 511.0 & 629.0 & 311.0 & 225.0 & 132.0 & 37.0 & 27.0 & 9.0 & 7.0 & \\ 0.0 & 1.0 & 4.0 & 9.0 & 16.0 & 25.0 & 36.0 & 49.0 & 64.0 & 81.0 & 100.0 \\ 1035.0 & 561.0 & 408.0 & 379.0 & 168.0 & 152.0 & 149.0 & 79.0 & 26.0 & 22.0 & \\ 0.0 & 1.0 & 4.0 & 9.0 & 16.0 & 25.0 & 36.0 & 49.0 & 64.0 & 81.0 & 100.0 \\ 995.0 & 645.0 & 595.0 & 453.0 & 238.0 & 191.0 & 45.0 & 79.0 & 22.0 & 36.0 & \\ 0.0 & 1.0 & 4.0 & 9.0 & 16.0 & 25.0 & 36.0 & 49.0 & 64.0 & 82.0 & 103.0 \\ 625.0 & 763.0 & 746.0 & 605.0 & 256.0 & 88.0 & 50.0 & 65.0 & 26.0 & 18.0 & \\ 0.0 & 1.0 & 4.0 & 9.0 & 16.0 & 25.0 & 36.0 & 49.0 & 64.0 & 81.0 & 100.0 \\ 366.0 & 519.0 & 462.0 & 309.0 & 149.0 & 36.0 & 38.0 & 24.0 & 30.0 & 12.0 & \\ 0.0 & 1.0 & 3.0 & 6.0 & 10.0 & 15.0 & 22.0 & 31.0 & 42.0 & 55.0 & 70.0 \\ 379.0 & 480.0 & 206.0 & 187.0 & 119.0 & 104.0 & 56.0 & 91.0 & 84.0 & 30.0 & \\ \text { [END] } & & & & & & & & & & \end{array}$


[NAME]

C-66

655780WG

\begin{tabular}{|c|c|c|c|c|c|c|c|c|c|c|}
\hline $\begin{array}{r}\text {-AT, } \\
5 . \\
\text { SERIE }\end{array}$ & $\begin{array}{l}\text { LON an } \\
.25 \\
\text { ES] }\end{array}$ & $\begin{array}{l}\text { nd } \mathrm{ALT}] \\
-3.93\end{array}$ & 8.00 & & & & & & & \\
\hline 0 & 1.0 & 2.0 & & & & & & & & 10.0 \\
\hline 08. & $\begin{array}{r}201.0 \\
1.0\end{array}$ & 3. & $\frac{1}{6}$. & $\begin{array}{r}0.0 \\
10.0\end{array}$ & 15. & 21 & & 37 & $\begin{array}{r}0.0 \\
48.0\end{array}$ & 61.0 \\
\hline 79.0 & 139.0 & 92. & 76 . & 133.0 & & & & & & \\
\hline & 1.0 & & & & & & & & & 10.0 \\
\hline & 75.0 & & & & & & & & & \\
\hline & $\begin{array}{r}1.0 \\
166.0\end{array}$ & $\begin{array}{r}3 . \\
51 .\end{array}$ & $\begin{array}{r}6 . \\
123 .\end{array}$ & $\begin{array}{l}10.0 \\
90.0\end{array}$ & & & & & $\begin{array}{r}45.0 \\
1.0\end{array}$ & 55.0 \\
\hline & & $\begin{array}{l}2.0 \\
67.0\end{array}$ & $\begin{array}{l}3.0 \\
1.0\end{array}$ & $\begin{array}{r}4.0 \\
0.0\end{array}$ & $\begin{array}{l}5.0 \\
0.0\end{array}$ & $\begin{array}{c}6.0 \\
0.0\end{array}$ & & $\begin{array}{l}8.0 \\
0.0\end{array}$ & $\begin{array}{l}9.0 \\
0.0\end{array}$ & 10.0 \\
\hline & & 2. & 3. & 4.0 & 5.0 & 7.0 & 10.0 & 14.0 & 19.0 & 25.0 \\
\hline & 348.0 & 175. & 193. & 202.0 & 147.0 & 119.0 & & & & \\
\hline & $\begin{array}{ll}1.0 & 312.0\end{array}$ & $\begin{array}{l}2.0 \\
72.0\end{array}$ & $\begin{array}{ll} & 3.0 \\
& 56.0\end{array}$ & $\begin{array}{l}4.0 \\
26.0\end{array}$ & $\begin{array}{c}5.0 \\
0.0\end{array}$ & $\begin{array}{c}6.0 \\
0.0\end{array}$ & $\begin{array}{c}7.0 \\
0.0\end{array}$ & $\begin{array}{r}8.0 \\
0.0\end{array}$ & $\begin{array}{c}9.0 \\
0.0\end{array}$ & 10.0 \\
\hline & & 2. & 3. & 4.0 & 5.0 & 7.0 & 10.0 & 14.0 & 19.0 & 25.0 \\
\hline & 450.0 & 285. & 172. & 227.0 & 182. & 42.0 & & & 2.0 & \\
\hline & $\begin{array}{l}1.0 \\
497.0\end{array}$ & 0246.0 & $\begin{array}{l}3.0 \\
85.0\end{array}$ & $\begin{array}{l}4.0 \\
39.0\end{array}$ & $\begin{array}{l}5.0 \\
15.0\end{array}$ & $\begin{array}{l}6.0 \\
19.0\end{array}$ & $\begin{array}{l}8.0 \\
37.0\end{array}$ & $\begin{array}{r}11.0 \\
20.0\end{array}$ & $\begin{array}{cc}15.0 \\
0 & 16.0\end{array}$ & $20 .($ \\
\hline & $\begin{array}{l}1.0 \\
447.0\end{array}$ & $\begin{array}{l}29 \\
029\end{array}$ & $\begin{array}{l}3 . \\
84\end{array}$ & $\begin{array}{r}4 \\
110\end{array}$ & & & & $\begin{array}{l}9.0 \\
2.0\end{array}$ & $0^{12.0}$ & 16.0 \\
\hline & 1.0 & 2.0 & 3. & 4. & & & 7 & 8.0 & 10.0 & 13.0 \\
\hline & 500. & 377. & 178. & 76. & 94. & & & 17 & & \\
\hline & $\begin{array}{l}1 \\
387\end{array}$ & $\begin{array}{l}21.0 \\
021.0\end{array}$ & $\begin{array}{l}3.0 \\
78.0\end{array}$ & $\begin{array}{l}4.0 \\
20.0\end{array}$ & $\begin{array}{r}5.0 \\
0.0\end{array}$ & 6 & 7. & $\begin{array}{l}8.0 \\
48.0\end{array}$ & $\begin{array}{c}10.0 \\
31.0\end{array}$ & 13.0 \\
\hline & & 2. & 3. & 4. & 5. & 6 & 7 & & 10 & 13.0 \\
\hline & 466. & 105. & 89. & 78 & & & & & & \\
\hline & & & & & & & & & & 16.0 \\
\hline & 67. & 147. & 120. & 175 & 87 & & & & & \\
\hline & 1.0 & & & & & & & & & 10. \\
\hline & 90. & 110 & & & & & & & & \\
\hline & & & & & & & & & & 31. \\
\hline & 51. & 146. & 85 & 149. & 103.0 & 116. & & & & \\
\hline & & & & 4.0 & & 6.0 & & & 9.0 & 10. \\
\hline & 1. & $\begin{array}{l}218 \\
2 .\end{array}$ & 3. & $\begin{array}{l}20.0 \\
4.0\end{array}$ & 5.0 & 6. & & & 15.0 & 20. \\
\hline & 467. & 234. & 152. & 101. & 65.0 & & & & & \\
\hline & 503 & 285 & & $\begin{array}{l}4.0 \\
20.0\end{array}$ & & 6 & $\begin{array}{l}7.0 \\
0.0\end{array}$ & $\begin{array}{c}8.0 \\
0.0\end{array}$ & $\begin{array}{r}10 \\
26\end{array}$ & 13. \\
\hline & & & & & 5 & & 10 & 14 & & 5. \\
\hline & $11 \overline{5}$ & 320 & 135 & 68.0 & 171.0 & & & & & \\
\hline 0 & $\begin{array}{c}1.0 \\
539.0\end{array}$ & $\begin{array}{r}2 . \\
187\end{array}$ & 3. & $\begin{array}{l}4.0 \\
26.0\end{array}$ & 5.0 & 6.0 & 7.0 & 8.0 & 9.0 & 10.0 \\
\hline & & & & & & & & & & 20. \\
\hline & 417. & 252. & & 138. & & & & & & \\
\hline 0 & 562 & $\begin{array}{l}2 . \\
89\end{array}$ & 17 & $\begin{array}{l}4.0 \\
2.0\end{array}$ & & & $\begin{array}{l}7.0 \\
0.0\end{array}$ & $\begin{array}{c}8.0 \\
0.0\end{array}$ & $\begin{array}{c}9.0 \\
0.0\end{array}$ & 10.0 \\
\hline & & & & & & & & & & 20.1 \\
\hline & & & & & & & & & & \\
\hline & & & & & & & & & & 3. \\
\hline & & & & & & & & & & \\
\hline & & & & & & & & & & 115. \\
\hline & & 150 & & & & & & & & \\
\hline & & & & & & & & 113 & 14 & 181.0 \\
\hline & & & & & & & & & & \\
\hline & & & & & & & & & 127 & 160.0 \\
\hline & & & & & & & & & & \\
\hline & & & & & & & & & & \\
\hline & & & & & & & & & & \\
\hline & & & & & & & & & & \\
\hline
\end{tabular}


$\begin{array}{lllllllllll}981.0 & 1385.0 & 1028.0 & 708.0 & 489.0 & 226.0 & 106.0 & 18.0 & 31.0 & 8.0\end{array}$ $\begin{array}{lllllllllll}0.0 & 1.0 & 6.0 & 16.0 & 31.0 & 51.0 & 76.0 & 106.0 & 141.0 & 181.0 & 226.0\end{array}$ $\begin{array}{llllllllll}954.0 & 826.0 & 559.0 & 306.0 & 194.0 & 99.0 & 64.0 & 48.0 & 53.0 & 14.0\end{array}$ $\begin{array}{lllllllllll}0.0 & 1.0 & 6.0 & 16.0 & 31.0 & 51.0 & 76.0 & 106.0 & 141.0 & 181.0 & 226.0\end{array}$

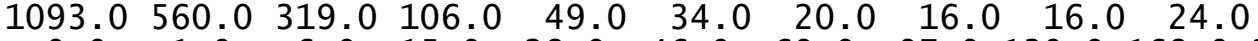
$\begin{array}{lllllllllll}0.0 & 1.0 & 6.0 & 15.0 & 28.0 & 46.0 & 69.0 & 97.0 & 130.0 & 168.0 & 211.0\end{array}$

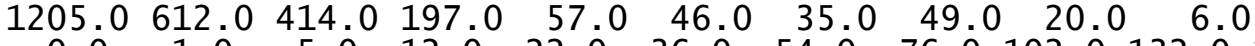
$\begin{array}{lllllllllll}0.0 & 1.0 & 5.0 & 12.0 & 22.0 & 36.0 & 54.0 & 76.0 & 102.0 & 132.0 & 166.0\end{array}$ $\begin{array}{lllllllllll}1190.0 & 920.0 & 756.0 & 273.0 & 239.0 & 177.0 & 93.0 & 62.0 & 12.0 & 14.0\end{array}$

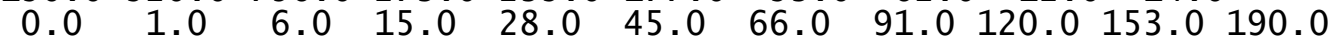

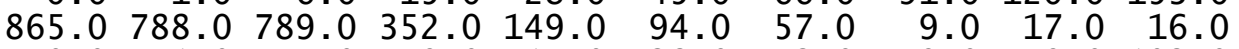

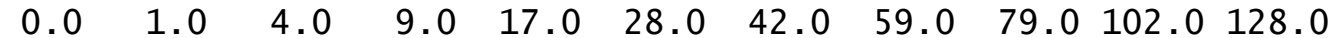

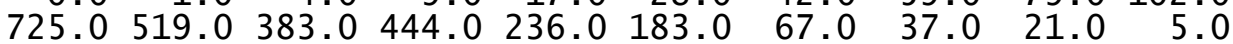
[END] 
[NAME]

C-68

655850WG

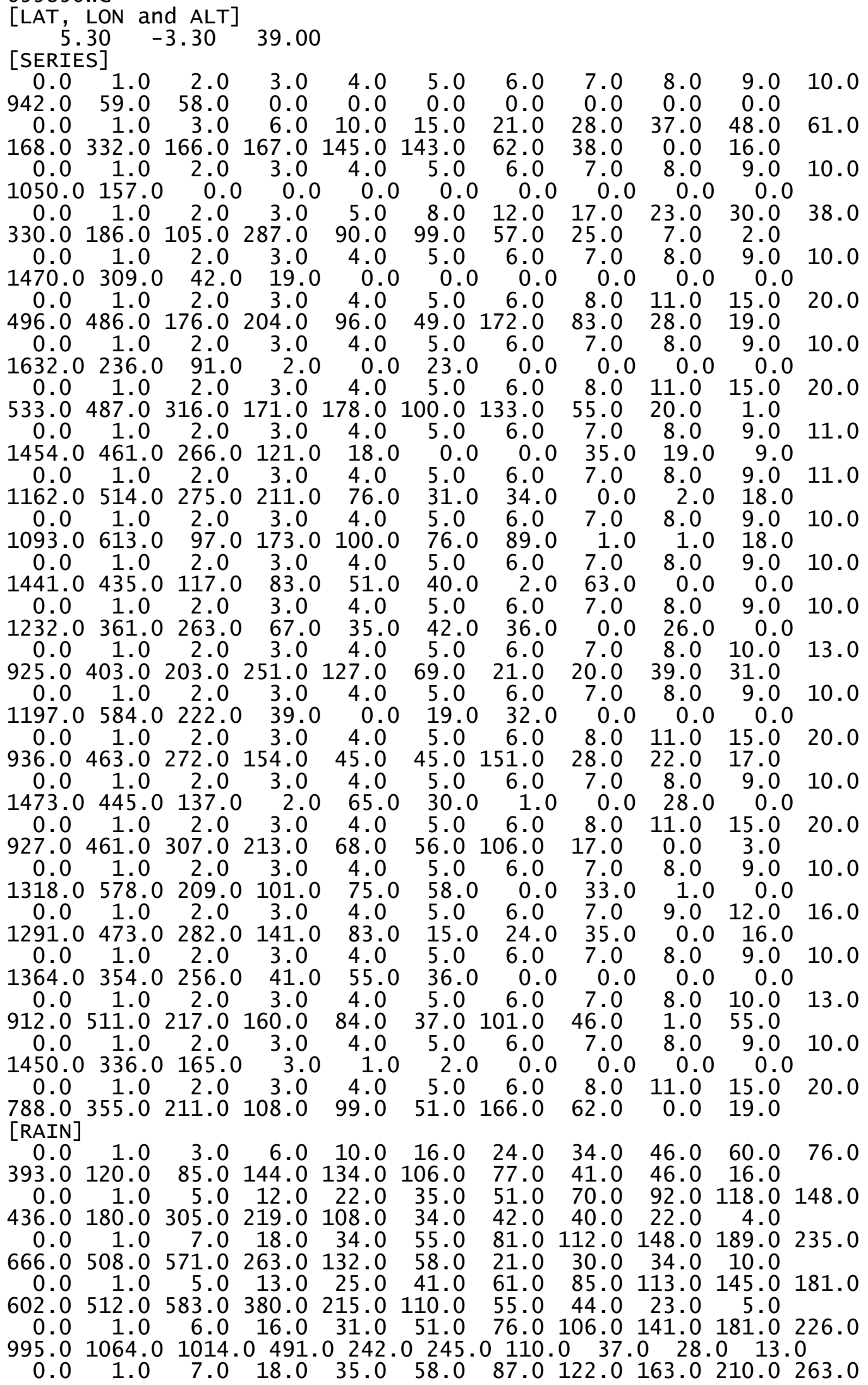


$\begin{array}{lllllllllll}996.0 & 1436.0 & 984.0 & 711.0 & 321.0 & 271.0 & 197.0 & 17.0 & 35.0 & 23.0\end{array}$

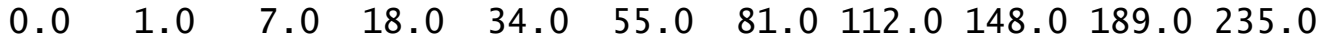

$\begin{array}{llllllllllll}1490.0 & 1047.0 & 702.0 & 360.0 & 143.0 & 94.0 & 87.0 & 32.0 & 29.0 & 14.0\end{array}$

$\begin{array}{llllllllllll}0.0 & 1.0 & 6.0 & 15.0 & 28.0 & 45.0 & 67.0 & 94.0 & 126.0 & 163.0 & 205.0\end{array}$

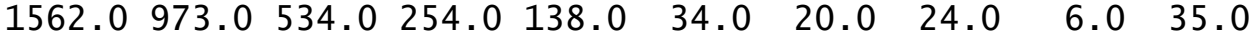

$\begin{array}{lllllllllll}0.0 & 1.0 & 6.0 & 15.0 & 28.0 & 46.0 & 69.0 & 97.0 & 130.0 & 168.0 & 211.0\end{array}$

$\begin{array}{llllllllll}1500.0 & 963.0 & 465.0 & 325.0 & 203.0 & 29.0 & 10.0 & 15.0 & 0.0 & 14.0\end{array}$

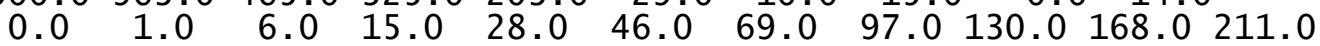
$\begin{array}{lllllllllllll}1509.0 & 1018.0 & 934.0 & 453.0 & 317.0 & 133.0 & 72.0 & 27.0 & 7.0 & 10.0\end{array}$

$\begin{array}{lllllllllll}0.0 & 1.0 & 5.0 & 12.0 & 22.0 & 35.0 & 51.0 & 71.0 & 95.0 & 123.0 & 155.0\end{array}$ $\begin{array}{llllllllll}1128.0 & 627.0 & 904.0 & 401.0 & 220.0 & 120.0 & 55.0 & 37.0 & 20.0 & 8.0\end{array}$

$\begin{array}{lllllllllll}0.0 & 1.0 & 3.0 & 6.0 & 10.0 & 16.0 & 24.0 & 34.0 & 46.0 & 60.0 & 76.0\end{array}$

$\begin{array}{llllllllll}1003.0 & 301.0 & 340.0 & 304.0 & 268.0 & 228.0 & 155.0 & 72.0 & 36.0 & 8.0\end{array}$

[END] 
[NAME]

C-70

655920WG

[LAT, LON and ALT]

[SERIES]

$4.41-7.36 \quad 21.00$

$\begin{array}{rrrrrrrrrrr}0.0 & 1.0 & 2.0 & 3.0 & 4.0 & 5.0 & 6.0 & 7.0 & 8.0 & 9.0 & 10.0 \\ 967.0 & 378.0 & 73.0 & 4.0 & 0.0 & 0.0 & 0.0 & 0.0 & 0.0 & 0.0 & \\ 0.0 & 1.0 & 2.0 & 4.0 & 7.0 & 11.0 & 16.0 & 22.0 & 29.0 & 37.0 & 46.0 \\ 409.0 & 185.0 & 365.0 & 256.0 & 166.0 & 65.0 & 60.0 & 38.0 & 23.0 & 14.0 & \\ 0.0 & 1.0 & 2.0 & 3.0 & 4.0 & 5.0 & 6.0 & 7.0 & 8.0 & 9.0 & 10.0 \\ 1008.0 & 109.0 & 32.0 & 0.0 & 0.0 & 0.0 & 0.0 & 0.0 & 0.0 & 0.0 & \\ 0.0 & 1.0 & 2.0 & 3.0 & 5.0 & 8.0 & 12.0 & 17.0 & 23.0 & 30.0 & 38.0\end{array}$

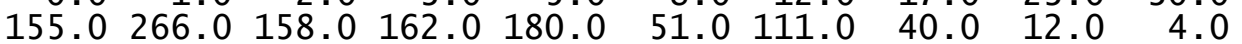

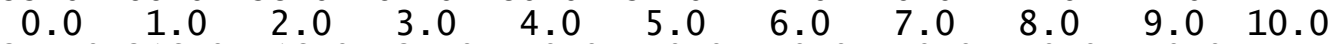

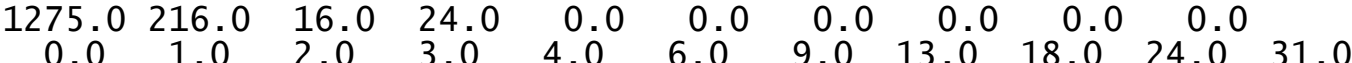

$\begin{array}{llllllllll}428.0 & 220.0 & 144.0 & 126.0 & 256.0 & 172.0 & 74.0 & 82.0 & 19.0 & 10.0\end{array}$

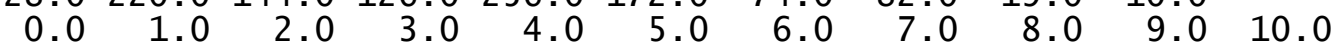

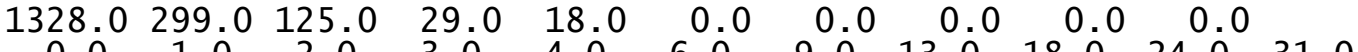

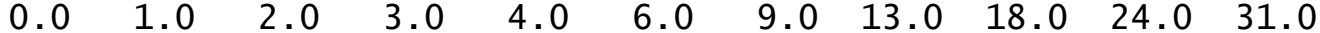

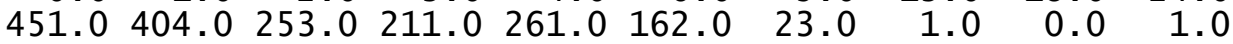

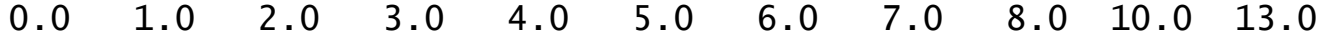

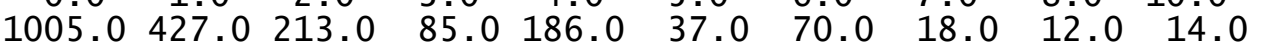

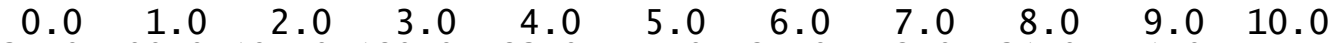

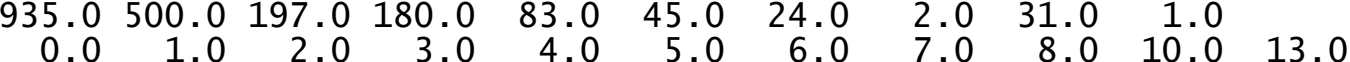

$\begin{array}{ccccccccccc}1054.0 & 597.0 & 292.0 & 109.0 & 61.0 & 57.0 & 43.0 & 1.0 & 0.0 & 25.0 & \\ 0.0 & 1.0 & 2.0 & 3.0 & 4.0 & 5.0 & 6.0 & 7.0 & 8.0 & 9.0 & 10.0\end{array}$

$\begin{array}{ccccccccccc}1284.0 & 501.0 & 307.0 & 99.0 & 19.0 & 2.0 & 49.0 & 0.0 & 1.0 & 0.0 & \\ 0.0 & 1.0 & 2.0 & 3.0 & 4.0 & 5.0 & 6.0 & 7.0 & 8.0 & 9.0 & 10.0\end{array}$

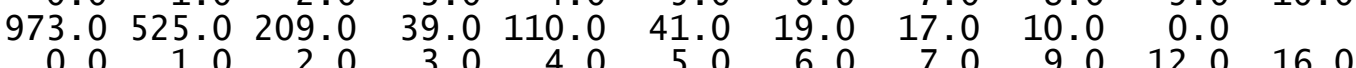

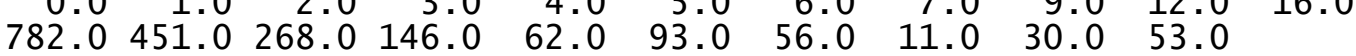

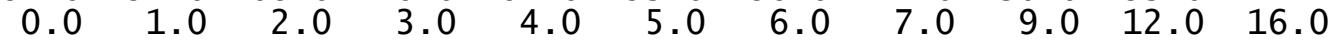

$\begin{array}{ccccccccccc}1139.0 & 463.0 & 255.0 & 100.0 & 72.0 & 62.0 & 35.0 & 22.0 & 15.0 & 10.0 & \\ 0.0 & 1.0 & 2.0 & 3.0 & 4.0 & 5.0 & 6.0 & 7.0 & 9.0 & 12.0 & 16.0\end{array}$

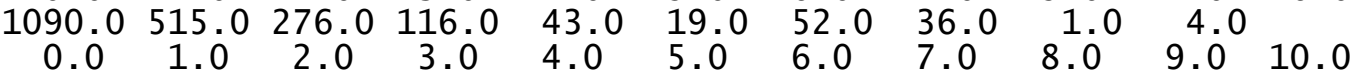

$\begin{array}{ccccccccccc}1170.0 & 531.0 & 235.0 & 152.0 & 43.0 & 112.0 & 20.0 & 79.0 & 0.0 & 0.0 & \\ 0.0 & 1.0 & 2.0 & 3.0 & 4.0 & 5.0 & 6.0 & 7.0 & 8.0 & 9.0 & 10.0\end{array}$

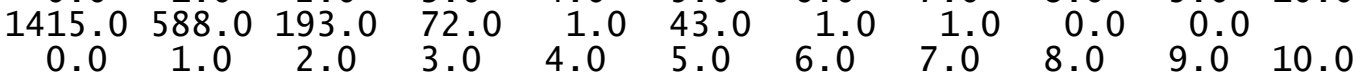

$\begin{array}{ccccccccccc}1150.0 & 525.0 & 244.0 & 118.0 & 100.0 & 25.0 & 14.0 & 39.0 & 0.0 & 0.0 & \\ 0.0 & 1.0 & 2.0 & 3.0 & 4.0 & 5.0 & 6.0 & 7.0 & 8.0 & 10.0 & 13.0\end{array}$

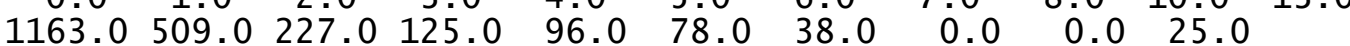

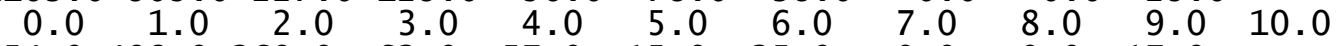

$\begin{array}{rrrrrrrrrrr}954.0 & 498.0 & 269.0 & 63.0 & 57.0 & 15.0 & 35.0 & 0.0 & 0.0 & 17.0 & \\ 0.0 & 1.0 & 2.0 & 3.0 & 4.0 & 5.0 & 6.0 & 8.0 & 11.0 & 15.0 & 20.0\end{array}$

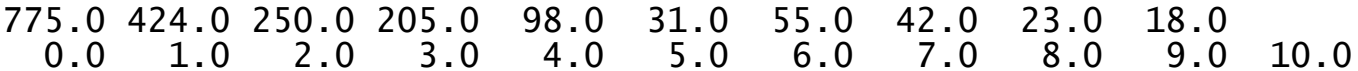

$\begin{array}{rrrrrrrrrr}1531.0 & 464.0 & 150.0 & 23.0 & 3.0 & 1.0 & 2.0 & 0.0 & 0.0 & 0.0\end{array}$

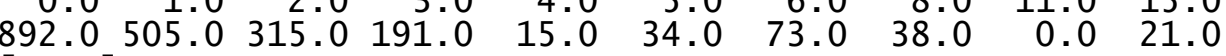

[RAIN]

$\begin{array}{lllllllllll}0.0 & 1.0 & 3.0 & 7.0 & 13.0 & 21.0 & 31.0 & 43.0 & 57.0 & 73.0 & 91.0\end{array}$

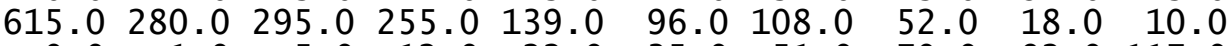

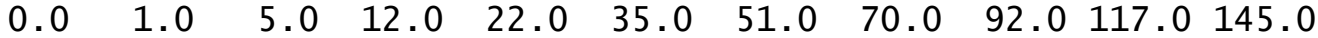

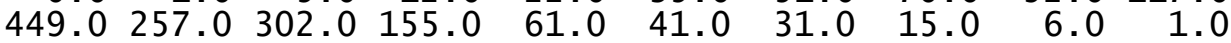

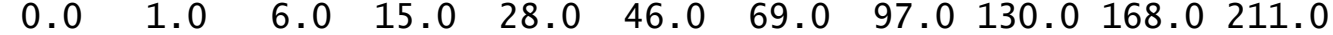

$\begin{array}{llllllllll}497.0 & 401.0 & 428.0 & 214.0 & 157.0 & 54.0 & 34.0 & 26.0 & 28.0 & 19.0\end{array}$

$\begin{array}{lllllllllll}0.0 & 1.0 & 5.0 & 12.0 & 22.0 & 36.0 & 54.0 & 76.0 & 102.0 & 132.0 & 166.0\end{array}$

$\begin{array}{llllllllll}703.0 & 416.0 & 566.0 & 269.0 & 271.0 & 149.0 & 75.0 & 29.0 & 26.0 & 12.0\end{array}$

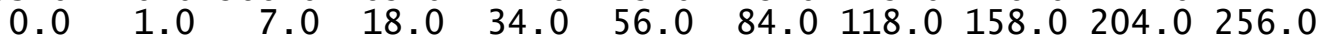

$\begin{array}{llllllllll}960.0 & 1127.0 & 1085.0 & 524.0 & 494.0 & 301.0 & 143.0 & 44.0 & 46.0 & 23.0\end{array}$

$\begin{array}{llllllllllll}0.0 & 1.0 & 7.0 & 19.0 & 37.0 & 61.0 & 91.0 & 127.0 & 169.0 & 217.0 & 271.0\end{array}$ 
$\begin{array}{lllllllllll}980.0 & 1074.0 & 1061.0 & 660.0 & 599.0 & 243.0 & 167.0 & 48.0 & 43.0 & 27.0\end{array}$

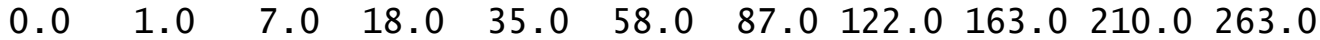
$\begin{array}{llllllllllll}1252.0 & 1012.0 & 872.0 & 347.0 & 224.0 & 128.0 & 64.0 & 37.0 & 31.0 & 17.0\end{array}$ $\begin{array}{lllllllllll}0.0 & 1.0 & 6.0 & 15.0 & 28.0 & 45.0 & 66.0 & 91.0 & 121.0 & 156.0 & 196.0\end{array}$ $\begin{array}{lllllllllll}1544.0 & 1139.0 & 995.0 & 456.0 & 239.0 & 145.0 & 58.0 & 31.0 & 24.0 & 8.0\end{array}$ $\begin{array}{llllllllllll}0.0 & 1.0 & 7.0 & 18.0 & 35.0 & 58.0 & 87.0 & 122.0 & 163.0 & 210.0 & 263.0\end{array}$ $\begin{array}{llllllllll}1693.0 & 1652.0 & 1045.0 & 332.0 & 203.0 & 113.0 & 83.0 & 31.0 & 34.0 & 20.0\end{array}$ $\begin{array}{lllllllllll}0.0 & 1.0 & 6.0 & 15.0 & 28.0 & 45.0 & 66.0 & 91.0 & 120.0 & 153.0 & 190.0\end{array}$

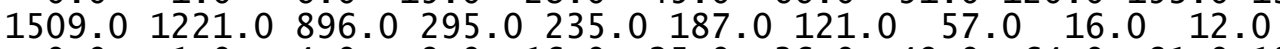
$\begin{array}{llllllllllll}0.0 & 1.0 & 4.0 & 9.0 & 16.0 & 25.0 & 36.0 & 49.0 & 64.0 & 81.0 & 100.0\end{array}$ $\begin{array}{lllllllllll}1196.0 & 721.0 & 630.0 & 537.0 & 260.0 & 206.0 & 121.0 & 63.0 & 42.0 & 17.0\end{array}$ $\begin{array}{lllllllllll}0.0 & 1.0 & 4.0 & 10.0 & 19.0 & 31.0 & 46.0 & 64.0 & 85.0 & 109.0 & 136.0\end{array}$

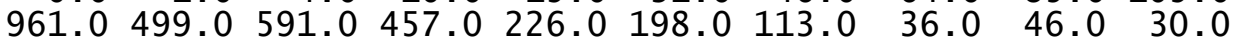
[END] 
[NAME]

C-72

655940WG

\begin{tabular}{|c|c|c|c|c|c|c|c|c|c|c|}
\hline $\begin{array}{r}\text { AT, } \\
4 \\
\text { SERIE }\end{array}$ & $\begin{array}{l}\text { LON } \\
.75 \\
\text { ES] }\end{array}$ & $\begin{array}{l}\text { nd } A L T] \\
-6.65\end{array}$ & 30.00 & & & & & & & \\
\hline & 1.0 & 2.0 & 3.0 & 4.0 & & 6.0 & 7.0 & 8.0 & 9.0 & 10. \\
\hline & 42.0 & 54.0 & & & & & & & & \\
\hline & 1.0 & 2. & & & 11. & & & & & 6.0 \\
\hline & 99.0 & 238. & 170 & 165 . & 118. & 10 & & & & \\
\hline & $\begin{array}{r}1.0 \\
45.0\end{array}$ & $\begin{array}{l}2.0 \\
3.0\end{array}$ & & $\begin{array}{l}4 \\
0\end{array}$ & & & & & & 10.0 \\
\hline & 1.0 & 3 . & & 10.0 & & & & & & 5.0 \\
\hline & 27.0 & 229.0 & 66. & 109.0 & & & & & & \\
\hline & 1.0 & 2.0 & & 4.0 & & & & & & 0.0 \\
\hline $\begin{array}{l}46.0 \\
0.0\end{array}$ & $\begin{array}{l}94.0 \\
1.0\end{array}$ & $\begin{array}{ll}0 & 0.0 \\
2.0\end{array}$ & $\begin{array}{l}0.0 \\
3.0\end{array}$ & $\begin{array}{l}0.0 \\
5.0\end{array}$ & $\begin{array}{l}0.0 \\
8.0\end{array}$ & $12.0^{0.0}$ & 17.0 & $23.0^{0.0}$ & $\begin{array}{r}0.0 \\
30.0^{0}\end{array}$ & 8.0 \\
\hline & 123.0 & 142.0 & 262.0 & 147.0 & 147.0 & & & 10.0 & & \\
\hline & $\begin{array}{c}1.0 \\
329.0\end{array}$ & $\begin{array}{ll} & 2.0 \\
0 & 67.0\end{array}$ & $\begin{array}{l}3 . \\
44\end{array}$ & $\begin{array}{r}4.0 \\
0.0\end{array}$ & $\begin{array}{c}5.0 \\
0.0\end{array}$ & $\begin{array}{c}6.0 \\
0.0\end{array}$ & $\begin{array}{l}7.0 \\
0.0\end{array}$ & $\begin{array}{r}8.0 \\
0.0\end{array}$ & $\begin{array}{c}9.0 \\
0.0\end{array}$ & 10.0 \\
\hline & 1.0 & 2.0 & 3. & 4.0 & 5.0 & 6.0 & 8. & 11.0 & 15 & 0.0 \\
\hline & 354.0 & 297.0 & 161. & 179. & 34.0 & 131.0 & & 17.0 & & \\
\hline & 332.0 & $\begin{array}{l}23.0 \\
0232.0\end{array}$ & $\begin{array}{r}3 . \\
127\end{array}$ & $\begin{array}{l}4.0 \\
23.0\end{array}$ & $\begin{array}{l}5.0 \\
56.0\end{array}$ & $\begin{array}{c}6.0 \\
0.0\end{array}$ & $\begin{array}{l}7.0 \\
18.0\end{array}$ & $\begin{array}{c}9.0 \\
0.0\end{array}$ & $\begin{array}{l}0 \\
.0\end{array}$ & 16.0 \\
\hline & & 2.0 & 3. & 4. & & 6.0 & & 11.0 & & 20 \\
\hline & 568. & 123 & 103 & 91. & 88. & 97.0 & & & & \\
\hline & & & & 4. & 5 & 6.0 & & 9.0 & & $16 .($ \\
\hline 0 & $\begin{array}{ll}0 & 619.0 \\
1.0\end{array}$ & $\begin{array}{lll}0 & 228.0 \\
2 & 0\end{array}$ & $\begin{array}{c}195 \\
3\end{array}$ & & $\begin{array}{l}55.0 \\
5.0\end{array}$ & 6.0 & $\begin{array}{l}40.0 \\
7.0\end{array}$ & 9.0 & $12^{1.0}$ & ) \\
\hline & 691.0 & 0125.0 & 119 & 35.0 & 45.0 & 1.0 & i. 0 & 14.0 & 6.0 & \\
\hline & 1.0 & 2.0 & 3. & & & 6.0 & $\begin{array}{c}7.0 \\
0.0\end{array}$ & 8.0 & 9.0 & 10. \\
\hline 0 & $\begin{array}{c}532.0 \\
1.0\end{array}$ & $\begin{array}{ll}0 & 152.0 \\
2.0\end{array}$ & 26 & & $\begin{array}{l}28.0 \\
5.0\end{array}$ & $\begin{array}{l}0.0 \\
6.0\end{array}$ & $\begin{array}{l}0.0 \\
7.0\end{array}$ & $\begin{array}{l}1.0 \\
9.0\end{array}$ & $0^{0}$ & 6. \\
\hline & 420.0 & 333. & 163. & 83. & 114.0 & & & & & \\
\hline 13 & 1.0 & 2.0 & 3. & 4.0 & 5.0 & $\begin{array}{c}6.0 \\
0.0\end{array}$ & & $\begin{array}{r}8.0 \\
0.0\end{array}$ & 0 & 10. \\
\hline & $\begin{array}{c}555.0 \\
1.0\end{array}$ & $\begin{array}{c}188 \\
2 .\end{array}$ & $\begin{array}{l}63 \\
3\end{array}$ & & $\begin{array}{l}0.0 \\
5.0\end{array}$ & 7.0 & 10. & 14 & 19 & 5. \\
\hline & 375.0 & 237. & 187. & 87. & 95. & & & & 21 & \\
\hline & & 2.0 & & & 5.0 & & & 8.0 & & $10 .($ \\
\hline $\begin{array}{r}37 \\
0\end{array}$ & $\begin{array}{c}360.0 \\
1.0\end{array}$ & $\begin{array}{ll}0 & 95.0 \\
2.0\end{array}$ & $\begin{array}{l}74 \\
3 .\end{array}$ & $\begin{array}{l}28 \\
4 .\end{array}$ & $\begin{array}{l}24.0 \\
5.0\end{array}$ & $\begin{array}{l}0.0 \\
6.0\end{array}$ & & $\begin{array}{r}0.0 \\
11.0\end{array}$ & 0 & 0 . \\
\hline & 458.0 & 299.0 & 128. & & 54. & 120.0 & & & & \\
\hline & & & 3.0 & & 5.0 & $\begin{array}{r}6.0 \\
0.0\end{array}$ & & & 0 & 10. \\
\hline & 1. & $\begin{array}{r}10 / \\
2 .\end{array}$ & 3. & & & & & & & 0. \\
\hline & 100 & 321. & 224. & & & & & & & \\
\hline & 0 & 0226 & & & & & & & & 10. \\
\hline & & & & & & & & & & 1 . \\
\hline & 10 & 250 & 185 & 147 & 109 & & & & & \\
\hline & & 106 & $\begin{array}{l}3.0 \\
22.0\end{array}$ & 4.0 & $\begin{array}{l}5.0 \\
0.0\end{array}$ & $\begin{array}{c}6.0 \\
0.0\end{array}$ & & 0 & 0 & 10.0 \\
\hline & & & & & & & & 18.0 & & 31. \\
\hline & & & & & 135 & & & & & \\
\hline & & & & & & & & & & 0. \\
\hline & & & & & & & & & & \\
\hline & & & & & & & & & & 91. \\
\hline & & & & & & & & & & \\
\hline & & & & & & & & & & 136. \\
\hline & & & & & & & & & & \\
\hline & & & & & & & & & 18 & 226. \\
\hline & & & & & & & & & & \\
\hline & & & & & & 111 & 154 & & & \\
\hline & & & & & & & & & & \\
\hline & & & & & & & & & & \\
\hline
\end{tabular}


$\begin{array}{llllllllll}975.0 & 1293.0 & 999.0 & 592.0 & 397.0 & 186.0 & 89.0 & 77.0 & 28.0 & 5.0\end{array}$

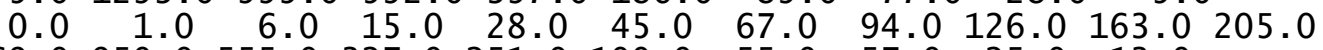
$\begin{array}{llllllllll}969.0 & 950.0 & 555.0 & 327.0 & 251.0 & 100.0 & 55.0 & 57.0 & 25.0 & 13.0\end{array}$

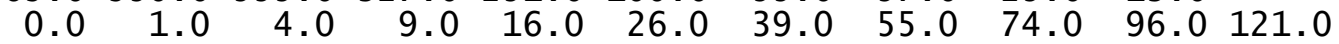

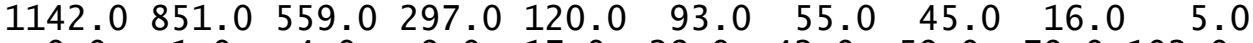
$\begin{array}{lllllllllll}0.0 & 1.0 & 4.0 & 9.0 & 17.0 & 28.0 & 42.0 & 59.0 & 79.0 & 102.0 & 128.0\end{array}$ $\begin{array}{llllllllll}1201.0 & 800.0 & 564.0 & 270.0 & 96.0 & 61.0 & 34.0 & 37.0 & 31.0 & 12.0\end{array}$ $\begin{array}{lllllllllll}0.0 & 1.0 & 5.0 & 12.0 & 22.0 & 35.0 & 52.0 & 73.0 & 98.0 & 127.0 & 160.0\end{array}$ $\begin{array}{llllllllll}1323.0 & 890.0 & 590.0 & 249.0 & 145.0 & 178.0 & 52.0 & 23.0 & 23.0 & 31.0\end{array}$

$\begin{array}{lllllllllll}0.0 & 1.0 & 5.0 & 12.0 & 22.0 & 35.0 & 51.0 & 70.0 & 92.0 & 117.0 & 145.0\end{array}$

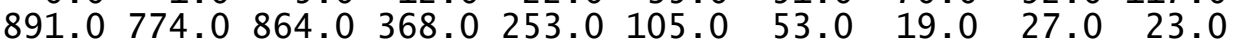

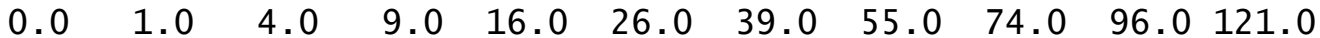

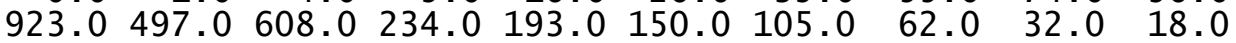
[END] 
[NAME]

C-74

655990WG

\begin{tabular}{|c|c|c|c|c|c|c|c|c|c|c|}
\hline $\begin{array}{r}\text {-AT, } \\
4 \\
\text { SERIE }\end{array}$ & $\begin{array}{l}\text { LON an } \\
.95 \\
\text { ES }\end{array}$ & $\begin{array}{l}\text { nd } A L T] \\
-6.08\end{array}$ & 66.00 & & & & & & & \\
\hline . & 1.0 & 2.0 & 3.0 & 4.0 & 5.0 & 6.0 & 7.0 & 8.0 & 9.0 & 10.0 \\
\hline & 09.0 & 60.0 & & 0.0 & & & & & & \\
\hline U & $\begin{array}{r}1.0 \\
12.0\end{array}$ & $\begin{array}{r}3.0 \\
163.0\end{array}$ & $\begin{array}{r}6.0 \\
208.0\end{array}$ & $\begin{array}{r}10.0 \\
170.0\end{array}$ & $\begin{array}{l}15 \\
77\end{array}$ & $\begin{array}{l}21 \\
56\end{array}$ & & & $\begin{array}{l}45 \\
27\end{array}$ & 55.0 \\
\hline & 1.0 & 2.0 & 3. & & & & & & & 10.0 \\
\hline & & & & & & & & & & \\
\hline & 1.0 & 3. & & 10 & & & & & & 56.0 \\
\hline & 1.0 & 263.0 & 123. & & & & & & & \\
\hline & $\begin{array}{l}1.0 \\
82.0\end{array}$ & $\begin{array}{l}2.0 \\
0.0\end{array}$ & $\begin{array}{c}3.0 \\
0.0\end{array}$ & $\begin{array}{r}4.0 \\
0.0\end{array}$ & $\begin{array}{l}5.0 \\
37.0\end{array}$ & $\begin{array}{r}6.0 \\
0.0\end{array}$ & $\begin{array}{l}7.0 \\
0.0\end{array}$ & $\begin{array}{l}8.0 \\
0.0\end{array}$ & $\begin{array}{l}9.0 \\
0.0\end{array}$ & 10.0 \\
\hline & & $\begin{array}{r}2.0 \\
173.0\end{array}$ & $\begin{array}{r}3.0 \\
173.0\end{array}$ & $\begin{array}{r}4.0 \\
147.0\end{array}$ & & $\begin{array}{r}7.0 \\
89.0\end{array}$ & 10.0 & $\begin{array}{r}14.0 \\
104.0\end{array}$ & 19.0 & 25.0 \\
\hline & 1.0 & 2.0 & 3.0 & 4.0 & 5.0 & 6.0 & 7.0 & 8.0 & 9.0 & 10.0 \\
\hline 46 & $\begin{array}{l}87.0 \\
1.0\end{array}$ & $\begin{array}{l}91.0 \\
2.0\end{array}$ & $\begin{array}{l}0.0 \\
3.0\end{array}$ & $\begin{array}{r}0.0 \\
4.0\end{array}$ & $\begin{array}{l}23.0 \\
5.0\end{array}$ & $\begin{array}{l}0.0 \\
7.0\end{array}$ & $\begin{array}{r}0.0 \\
10.0\end{array}$ & $\begin{array}{r}0.0 \\
14.0\end{array}$ & $\begin{array}{r}0.0 \\
19.0\end{array}$ & 0 \\
\hline & 30.0 & 263.0 & 180.0 & 125.0 & 155.0 & 164.0 & & & & \\
\hline & & $\begin{array}{r}2.0 \\
264.0\end{array}$ & & $\begin{array}{l}4.0 \\
85.0\end{array}$ & $\begin{array}{l}5.0 \\
23.0\end{array}$ & $\begin{array}{l}6.0 \\
11.0\end{array}$ & $\begin{array}{c}7.0 \\
0.0\end{array}$ & $\begin{array}{c}8.0 \\
0.0\end{array}$ & $\begin{array}{c}10.0 \\
16.0\end{array}$ & 13.0 \\
\hline & & & $\begin{array}{r}3 . \\
161 .\end{array}$ & $\begin{array}{r}4.0 \\
87.0\end{array}$ & 5.0 & & $\begin{array}{r}7.0 \\
35.0\end{array}$ & $\begin{array}{r}9.0 \\
53.0\end{array}$ & & 16.0 \\
\hline & & 2.0 & 3 & $\begin{array}{r}8.0 \\
4.0\end{array}$ & $\begin{array}{r}36.0 \\
5.0\end{array}$ & $\begin{array}{r}47.0 \\
6.0\end{array}$ & 7.0 & & 9.0 & 10.0 \\
\hline 5 & $\begin{array}{c}684.0 \\
1.0 \\
5000\end{array}$ & $\begin{array}{l}0294.0 \\
2.0 \\
0\end{array}$ & $\begin{array}{r}170.0 \\
3.0\end{array}$ & $\begin{array}{l}21.0 \\
4.0\end{array}$ & $\begin{array}{l}41.0 \\
5.0\end{array}$ & $\begin{array}{l}1.0 \\
6.0\end{array}$ & 7.0 & $\begin{array}{l}0.0 \\
8.0\end{array}$ & 9.0 & 10.0 \\
\hline & $\begin{array}{c}500.0 \\
1.0 \\
488.0\end{array}$ & $\begin{array}{cc}0 & 247.0 \\
2.0 \\
0 \\
0 \\
152.0\end{array}$ & $\begin{array}{c}124.0 \\
3.0 \\
42.0\end{array}$ & $\begin{array}{l}72.0 \\
4.0 \\
70.0\end{array}$ & 0 & $\begin{array}{c}22.0 \\
6.0 \\
0.0\end{array}$ & $\begin{array}{l}21.0 \\
7.0 \\
0.0\end{array}$ & $\begin{array}{c}7.0 \\
8.0 \\
0.0\end{array}$ & $\begin{array}{c}0.0 \\
9.0 \\
0.0\end{array}$ & 0.0 \\
\hline & & 2.0 & 3. & 4.0 & 6.0 & 9.0 & 13 & 18.0 & 24. & 31. \\
\hline & & 252.0 & 165. & 94.0 & 199.0 & & & & 21 & \\
\hline & & & & $\begin{array}{l}4.0 \\
88.0\end{array}$ & $\begin{array}{l}5.0 \\
1.0\end{array}$ & $\begin{array}{c}6.0 \\
0.0\end{array}$ & $\begin{array}{c}7.0 \\
0.0\end{array}$ & $\begin{array}{l}8.0 \\
0.0\end{array}$ & $\begin{array}{r}9.0 \\
0.0\end{array}$ & 10. \\
\hline & & 2.0 & 3. & 4.0 & 50 & 6. & 8. & 11 & 15 & -0 \\
\hline & 20 & 329. & 117. & 82.0 & 62.0 & 81. & 119. & & & \\
\hline & & 138 & & $\begin{array}{l}4.0 \\
1.0\end{array}$ & & & $\begin{array}{l}7.0 \\
0.0\end{array}$ & 8.0 & 0 & 10. \\
\hline & & 2. & 3. & 4.0 & 5.0 & & & & & 20. \\
\hline & $=0$ & 231. & 201 & 148.0 & & & & & & \\
\hline & & $\begin{array}{r}2.0 \\
189^{\circ} \mathrm{C}\end{array}$ & & 4.0 & & & 7.0 & & 9.0 & 10. \\
\hline & & 2. & & 4.0 & & & 13. & & & 31. \\
\hline & & 277.0 & & 265.0 & & & & & & \\
\hline & & $\begin{array}{l}2.0 \\
67.0\end{array}$ & & $\begin{array}{r}4.0 \\
0.0\end{array}$ & & & $\begin{array}{c}7.0 \\
0.0\end{array}$ & $\begin{array}{l}8.0 \\
0.0\end{array}$ & $\begin{array}{r}9.0 \\
0.0\end{array}$ & 10.0 \\
\hline & & 2.0 & & 4.0 & & & & & & 20. \\
\hline & & 306. & 190. & 145.0 & & & & & & \\
\hline & 30 & 156 & & & & & $\begin{array}{c}7.0 \\
0.0\end{array}$ & & & 10.0 \\
\hline & & 2. & & & & & & & & 20. \\
\hline & & & & & & & 50.0 & & & \\
\hline & & & & & & & & & & \\
\hline & 17 & 58 & 5 & & & & & & & \\
\hline & & & & & & & & & & 115. \\
\hline & 46. & 295 & & & & & & & & \\
\hline & & & & & & & & & & 83. \\
\hline & & 307 & & & & & & & & \\
\hline & & & & & & & & & & 121.0 \\
\hline & & & & & & & & & & \\
\hline & $\perp$ & & & & & & & & & 151.0 \\
\hline & & & & & & & & & & \\
\hline & & & & & & & & & 0 & 3. \\
\hline
\end{tabular}


$\begin{array}{llllllllllll}861.0 & 912.0 & 860.0 & 570.0 & 485.0 & 390.0 & 138.0 & 131.0 & 32.0 & 34.0\end{array}$

$\begin{array}{lllllllllll}0.0 & 1.0 & 5.0 & 12.0 & 22.0 & 35.0 & 51.0 & 71.0 & 95.0 & 123.0 & 155.0\end{array}$ $\begin{array}{llllllllll}1046.0 & 618.0 & 625.0 & 318.0 & 195.0 & 153.0 & 44.0 & 71.0 & 50.0 & 30.0\end{array}$

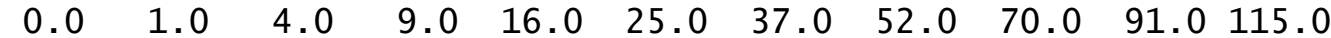
$\begin{array}{llllllllll}1241.0 & 612.0 & 440.0 & 215.0 & 84.0 & 66.0 & 25.0 & 12.0 & 58.0 & 34.0\end{array}$ $\begin{array}{lllllllllll}0.0 & 1.0 & 4.0 & 9.0 & 16.0 & 25.0 & 37.0 & 52.0 & 70.0 & 91.0 & 115.0\end{array}$

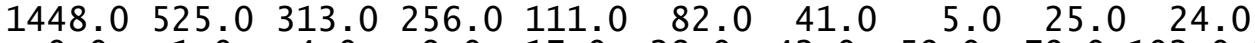
$\begin{array}{lllllllllll}0.0 & 1.0 & 4.0 & 9.0 & 17.0 & 28.0 & 42.0 & 59.0 & 79.0 & 102.0 & 128.0\end{array}$ $\begin{array}{lllllllllll}1062.0 & 640.0 & 558.0 & 377.0 & 215.0 & 132.0 & 70.0 & 28.0 & 29.0 & 14.0\end{array}$

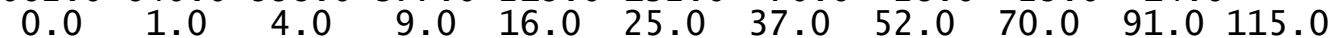
$\begin{array}{llllllllll}706.0 & 502.0 & 461.0 & 389.0 & 165.0 & 128.0 & 119.0 & 94.0 & 63.0 & 18.0\end{array}$

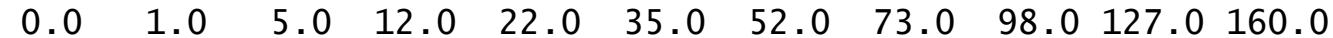

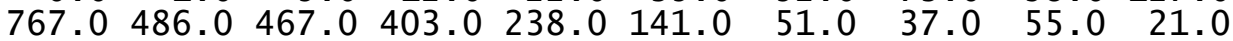
[END] 

Appendix D: Conditioned 1st-Order Markov Transition Probabilities 


\begin{tabular}{|c|c|c|c|c|c|c|c|c|}
\hline \multirow[t]{2}{*}{ Gauge } & \multicolumn{8}{|c|}{$\begin{array}{l}\text { 1st-Order Conditioned Markov Transition Pro } \\
\text { Cold Pacific Sea Surface Temperature Event }\end{array}$} \\
\hline & $\mathbf{P}_{\mathrm{ww}}$ & $\mathbf{P}_{\mathrm{wd}}$ & $\mathbf{P}_{\mathrm{dw}}$ & $\mathbf{P}_{\mathrm{dd}}$ & $\mathbf{P}_{\mathrm{ww}}$ & $\mathbf{P}_{\mathrm{wd}}$ & $\mathbf{P}_{\mathrm{dw}}$ & $\mathbf{P}_{\mathrm{dd}}$ \\
\hline 653060 & 0.000 & 1.000 & 0.000 & 1.000 & 0.000 & 0.000 & 0.005 & 0.995 \\
\hline 653190 & 0.000 & 0.000 & 0.000 & 1.000 & 0.000 & 0.000 & 0.000 & 1.000 \\
\hline 653300 & 0.333 & 0.667 & 0.009 & 0.991 & 0.000 & 1.000 & 0.005 & 0.995 \\
\hline 653350 & 0.000 & 1.000 & 0.005 & 0.995 & 0.000 & 1.000 & 0.019 & 0.981 \\
\hline 653380 & 0.000 & 1.000 & 0.014 & 0.986 & 0.500 & 0.500 & 0.013 & 0.987 \\
\hline 653440 & 0.167 & 0.833 & 0.024 & 0.976 & 0.000 & 1.000 & 0.045 & 0.955 \\
\hline 653520 & 0.000 & 0.000 & 0.000 & 1.000 & 0.000 & 0.000 & 0.000 & 1.000 \\
\hline 653550 & 0.000 & 0.000 & 0.000 & 1.000 & 0.000 & 1.000 & 0.029 & 0.971 \\
\hline 653570 & 0.000 & 0.000 & 0.009 & 0.991 & 0.000 & 0.000 & 0.000 & 1.000 \\
\hline 653610 & 0.000 & 1.000 & 0.022 & 0.978 & 0.000 & 1.000 & 0.014 & 0.986 \\
\hline 653760 & 0.000 & 1.000 & 0.028 & 0.972 & 0.167 & 0.833 & 0.049 & 0.951 \\
\hline 653800 & 0.000 & 1.000 & 0.017 & 0.983 & 0.200 & 0.800 & 0.051 & 0.949 \\
\hline 653870 & 0.167 & 0.833 & 0.021 & 0.979 & 0.000 & 1.000 & 0.028 & 0.972 \\
\hline 654720 & 0.000 & 1.000 & 0.011 & 0.989 & 0.000 & 0.000 & 0.017 & 0.983 \\
\hline 655010 & 0.000 & 1.000 & 0.004 & 0.996 & 0.000 & 1.000 & 0.012 & 0.988 \\
\hline 655020 & 0.000 & 0.000 & 0.000 & 1.000 & 0.000 & 0.000 & 0.000 & 1.000 \\
\hline 655030 & 0.000 & 0.000 & 0.000 & 1.000 & 0.000 & 0.000 & 0.000 & 1.000 \\
\hline 655050 & 0.000 & 0.000 & 0.000 & 1.000 & 0.000 & 0.000 & 0.000 & 1.000 \\
\hline 655070 & 0.000 & 0.000 & 0.000 & 1.000 & 0.000 & 1.000 & 0.000 & 1.000 \\
\hline 655100 & 0.000 & 0.000 & 0.000 & 1.000 & 0.000 & 1.000 & 0.005 & 0.995 \\
\hline 655160 & 0.000 & 0.000 & 0.000 & 1.000 & 0.000 & 0.000 & 0.000 & 1.000 \\
\hline 655180 & 0.000 & 0.000 & 0.000 & 1.000 & 0.000 & 0.000 & 0.000 & 1.000 \\
\hline 655220 & 0.000 & 1.000 & 0.004 & 0.996 & 0.000 & 1.000 & 0.019 & 0.981 \\
\hline 655280 & 0.000 & 1.000 & 0.007 & 0.993 & 0.000 & 1.000 & 0.007 & 0.993 \\
\hline 655360 & 0.250 & 0.750 & 0.013 & 0.987 & 0.000 & 1.000 & 0.022 & 0.978 \\
\hline 655450 & 0.250 & 0.750 & 0.022 & 0.978 & 0.000 & 1.000 & 0.010 & 0.990 \\
\hline 655480 & 0.250 & 0.750 & 0.025 & 0.975 & 0.400 & 0.600 & 0.057 & 0.943 \\
\hline 655550 & 0.222 & 0.778 & 0.028 & 0.972 & 0.250 & 0.750 & 0.019 & 0.981 \\
\hline 655570 & 0.250 & 0.750 & 0.060 & 0.940 & 0.182 & 0.818 & 0.099 & 0.901 \\
\hline 655600 & 0.000 & 1.000 & 0.048 & 0.952 & 0.167 & 0.833 & 0.048 & 0.952 \\
\hline 655620 & 0.000 & 1.000 & 0.054 & 0.946 & 0.000 & 1.000 & 0.042 & 0.958 \\
\hline 655630 & 0.200 & 0.800 & 0.045 & 0.955 & 0.000 & 1.000 & 0.038 & 0.962 \\
\hline 655780 & 0.313 & 0.688 & 0.050 & 0.950 & 0.308 & 0.692 & 0.061 & 0.939 \\
\hline 655850 & 0.286 & 0.714 & 0.068 & 0.932 & 0.143 & 0.857 & 0.048 & 0.952 \\
\hline 655920 & 0.148 & 0.852 & 0.155 & 0.845 & 0.200 & 0.800 & 0.070 & 0.930 \\
\hline 655940 & 0.125 & 0.875 & 0.071 & 0.929 & 0.000 & 1.000 & 0.048 & 0.952 \\
\hline 655990 & 0.333 & 0.667 & 0.034 & 0.966 & 0.000 & 1.000 & 0.040 & 0.960 \\
\hline
\end{tabular}




\begin{tabular}{|c|c|c|c|c|c|c|c|c|}
\hline \multirow[t]{2}{*}{ Gauge } & \multicolumn{8}{|c|}{$\begin{array}{l}\text { 1st-Order Conditioned Markov Transition Probabilities } \\
\text { Cold Pacific Sea Surface Temperature Event }\end{array}$} \\
\hline & $\mathbf{P}_{\mathrm{ww}}$ & $\mathbf{P}_{\mathrm{wd}}$ & $\mathbf{P}_{\mathrm{dw}}$ & $\mathbf{P}_{\mathrm{dd}}$ & $\mathbf{P}_{\mathrm{ww}}$ & $\mathbf{P}_{\mathrm{wd}}$ & $\mathbf{P}_{\mathrm{dw}}$ & $\mathbf{P}_{\mathrm{dd}}$ \\
\hline 653060 & 0.000 & 1.000 & 0.013 & 0.987 & 0.000 & 1.000 & 0.039 & 0.961 \\
\hline 653190 & 0.286 & 0.714 & 0.025 & 0.975 & 0.188 & 0.813 & 0.140 & 0.860 \\
\hline 653300 & 0.200 & 0.800 & 0.060 & 0.940 & 0.333 & 0.667 & 0.085 & 0.915 \\
\hline 653350 & 0.188 & 0.813 & 0.119 & 0.881 & 0.375 & 0.625 & 0.129 & 0.871 \\
\hline 653380 & 0.167 & 0.833 & 0.131 & 0.869 & 0.211 & 0.789 & 0.193 & 0.807 \\
\hline 653440 & 0.192 & 0.808 & 0.136 & 0.864 & 0.190 & 0.810 & 0.192 & 0.808 \\
\hline 653520 & 0.000 & 1.000 & 0.011 & 0.989 & 0.000 & 1.000 & 0.076 & 0.924 \\
\hline 653550 & 0.333 & 0.667 & 0.051 & 0.949 & 0.000 & 1.000 & 0.107 & 0.893 \\
\hline 653570 & 0.000 & 1.000 & 0.053 & 0.947 & 0.000 & 1.000 & 0.057 & 0.943 \\
\hline 653610 & 0.091 & 0.909 & 0.071 & 0.929 & 0.176 & 0.824 & 0.255 & 0.745 \\
\hline 653760 & 0.059 & 0.941 & 0.136 & 0.864 & 0.273 & 0.727 & 0.180 & 0.820 \\
\hline 653800 & 0.167 & 0.833 & 0.190 & 0.810 & 0.167 & 0.833 & 0.208 & 0.792 \\
\hline 653870 & 0.263 & 0.737 & 0.107 & 0.893 & 0.125 & 0.875 & 0.106 & 0.894 \\
\hline 654720 & 0.000 & 1.000 & 0.028 & 0.972 & 0.000 & 1.000 & 0.119 & 0.881 \\
\hline 655010 & 0.000 & 1.000 & 0.005 & 0.995 & 0.000 & 0.000 & 0.000 & 1.000 \\
\hline 655020 & 0.000 & 1.000 & 0.022 & 0.978 & 0.000 & 1.000 & 0.008 & 0.992 \\
\hline 655030 & 0.000 & 1.000 & 0.025 & 0.975 & 0.200 & 0.800 & 0.022 & 0.978 \\
\hline 655050 & 0.333 & 0.667 & 0.023 & 0.977 & 0.333 & 0.667 & 0.039 & 0.961 \\
\hline 655070 & 0.000 & 1.000 & 0.017 & 0.983 & 0.000 & 1.000 & 0.033 & 0.967 \\
\hline 655100 & 0.250 & 0.750 & 0.037 & 0.963 & 0.000 & 1.000 & 0.037 & 0.963 \\
\hline 655160 & 0.400 & 0.600 & 0.023 & 0.977 & 0.273 & 0.727 & 0.078 & 0.922 \\
\hline 655180 & 0.333 & 0.667 & 0.044 & 0.956 & 0.000 & 1.000 & 0.056 & 0.944 \\
\hline 655220 & 0.300 & 0.700 & 0.042 & 0.958 & 0.200 & 0.800 & 0.113 & 0.887 \\
\hline 655280 & 0.167 & 0.833 & 0.038 & 0.962 & 0.500 & 0.500 & 0.058 & 0.942 \\
\hline 655360 & 0.091 & 0.909 & 0.072 & 0.928 & 0.000 & 1.000 & 0.104 & 0.896 \\
\hline 655450 & 0.083 & 0.917 & 0.133 & 0.867 & 0.313 & 0.688 & 0.154 & 0.846 \\
\hline 655480 & 0.182 & 0.818 & 0.170 & 0.830 & 0.167 & 0.833 & 0.098 & 0.902 \\
\hline 655550 & 0.167 & 0.833 & 0.139 & 0.861 & 0.000 & 1.000 & 0.070 & 0.930 \\
\hline 655570 & 0.190 & 0.810 & 0.163 & 0.837 & 0.111 & 0.889 & 0.146 & 0.854 \\
\hline 655600 & 0.143 & 0.857 & 0.140 & 0.860 & 0.000 & 1.000 & 0.098 & 0.902 \\
\hline 655620 & 0.214 & 0.786 & 0.130 & 0.870 & 0.167 & 0.833 & 0.076 & 0.924 \\
\hline 655630 & 0.263 & 0.737 & 0.188 & 0.813 & 0.400 & 0.600 & 0.059 & 0.941 \\
\hline 655780 & 0.273 & 0.727 & 0.123 & 0.877 & 0.188 & 0.813 & 0.140 & 0.860 \\
\hline 655850 & 0.278 & 0.722 & 0.165 & 0.835 & 0.000 & 1.000 & 0.132 & 0.868 \\
\hline 655920 & 0.217 & 0.783 & 0.146 & 0.854 & 0.154 & 0.846 & 0.156 & 0.844 \\
\hline 655940 & 0.000 & 1.000 & 0.111 & 0.889 & 0.125 & 0.875 & 0.105 & 0.895 \\
\hline 655990 & 0.000 & 1.000 & 0.068 & 0.932 & 0.000 & 1.000 & 0.063 & 0.938 \\
\hline
\end{tabular}




\begin{tabular}{|c|c|c|c|c|c|c|c|c|}
\hline \multirow{3}{*}{$\begin{array}{l}\text { Gauge } \\
653060\end{array}$} & \multicolumn{8}{|c|}{$\begin{array}{l}\text { 1st-Order Conditioned Markov Transition Probabilities } \\
\text { Cold Pacific Sea Surface Temperature Event }\end{array}$} \\
\hline & $\mathbf{P}_{\mathbf{w w}}$ & $\begin{array}{l}\mathbf{P}_{\mathbf{w d}} \\
0765\end{array}$ & $\begin{array}{l}\mathbf{P}_{\mathbf{d w}} \\
0.093\end{array}$ & $\begin{array}{l}\mathbf{P}_{\text {dd }} \\
0.907\end{array}$ & $\mathbf{P}_{\mathbf{w w}}$ & $\begin{array}{l}\mathbf{P}_{\mathbf{w d}} \\
0730\end{array}$ & $\mathbf{P}_{\mathbf{d w}}$ & $\mathbf{P}_{\text {dd }}$ \\
\hline & $\begin{array}{l}0.235 \\
0.375\end{array}$ & $\begin{array}{l}0.165 \\
0.625\end{array}$ & $\begin{array}{l}0.093 \\
0.144\end{array}$ & 0.856 & 0.270 & 0.556 & $\begin{array}{l}0.291 \\
0.293\end{array}$ & $\begin{array}{l}0.103 \\
0.707\end{array}$ \\
\hline 653300 & 0.250 & 0.750 & 0.203 & 0.797 & 0.432 & 0.568 & 0.286 & 0.714 \\
\hline 653350 & 0.192 & 0.808 & 0.196 & 0.804 & 0.579 & 0.421 & 0.247 & 0.753 \\
\hline 653380 & 0.367 & 0.633 & 0.164 & 0.836 & 0.316 & 0.684 & 0.347 & 0.653 \\
\hline 653440 & 0.243 & 0.757 & 0.250 & 0.750 & 0.423 & 0.577 & 0.484 & 0.516 \\
\hline 653520 & 0.167 & 0.833 & 0.169 & 0.831 & 0.250 & 0.750 & 0.349 & 0.651 \\
\hline 653550 & 0.182 & 0.818 & 0.323 & 0.677 & 0.571 & 0.429 & 0.313 & 0.688 \\
\hline 653570 & 0.167 & 0.833 & 0.125 & 0.875 & 0.000 & 1.000 & 0.250 & 0.750 \\
\hline 653610 & 0.300 & 0.700 & 0.179 & 0.821 & 0.370 & 0.630 & 0.358 & 0.642 \\
\hline 653760 & 0.118 & 0.882 & 0.173 & 0.827 & 0.429 & 0.571 & 0.349 & 0.651 \\
\hline 653800 & 0.214 & 0.786 & 0.186 & 0.814 & 0.200 & 0.800 & 0.250 & 0.750 \\
\hline 653870 & 0.314 & 0.686 & 0.214 & 0.786 & 0.200 & 0.800 & 0.315 & 0.685 \\
\hline 654720 & 0.500 & 0.500 & 0.030 & 0.970 & 0.571 & 0.429 & 0.222 & 0.778 \\
\hline 655010 & 0.167 & 0.833 & 0.018 & 0.982 & 0.133 & 0.867 & 0.122 & 0.878 \\
\hline 655020 & 0.167 & 0.833 & 0.030 & 0.970 & 0.118 & 0.882 & 0.150 & 0.850 \\
\hline 655030 & 0.200 & 0.800 & 0.122 & 0.878 & 0.182 & 0.818 & 0.238 & 0.762 \\
\hline 655050 & 0.333 & 0.667 & 0.087 & 0.913 & 0.188 & 0.813 & 0.172 & 0.828 \\
\hline 655070 & 0.091 & 0.909 & 0.088 & 0.912 & 0.345 & 0.655 & 0.222 & 0.778 \\
\hline 655100 & 0.148 & 0.852 & 0.159 & 0.841 & 0.388 & 0.612 & 0.319 & 0.681 \\
\hline 655160 & 0.263 & 0.737 & 0.100 & 0.900 & 0.313 & 0.688 & 0.235 & 0.765 \\
\hline 655180 & 0.154 & 0.846 & 0.126 & 0.874 & 0.375 & 0.625 & 0.381 & 0.619 \\
\hline 655220 & 0.154 & 0.846 & 0.164 & 0.836 & 0.333 & 0.667 & 0.348 & 0.652 \\
\hline 655280 & 0.000 & 1.000 & 0.073 & 0.927 & 0.375 & 0.625 & 0.233 & 0.767 \\
\hline 655360 & 0.267 & 0.733 & 0.233 & 0.767 & 0.313 & 0.688 & 0.208 & 0.792 \\
\hline 655450 & 0.063 & 0.938 & 0.197 & 0.803 & 0.071 & 0.929 & 0.378 & 0.622 \\
\hline 655480 & 0.292 & 0.708 & 0.225 & 0.775 & 0.333 & 0.667 & 0.271 & 0.729 \\
\hline 655550 & 0.067 & 0.933 & 0.127 & 0.873 & 0.154 & 0.846 & 0.191 & 0.809 \\
\hline 655570 & 0.370 & 0.630 & 0.235 & 0.765 & 0.500 & 0.500 & 0.219 & 0.781 \\
\hline 655600 & 0.176 & 0.824 & 0.213 & 0.787 & 0.455 & 0.545 & 0.206 & 0.794 \\
\hline 655620 & 0.238 & 0.762 & 0.210 & 0.790 & 0.375 & 0.625 & 0.222 & 0.778 \\
\hline 655630 & 0.190 & 0.810 & 0.250 & 0.750 & 0.500 & 0.500 & 0.189 & 0.811 \\
\hline 655780 & 0.413 & 0.587 & 0.272 & 0.728 & 0.509 & 0.491 & 0.676 & 0.324 \\
\hline 655850 & 0.400 & 0.600 & 0.352 & 0.648 & 0.524 & 0.476 & 0.478 & 0.522 \\
\hline 655920 & 0.515 & 0.485 & 0.254 & 0.746 & 0.607 & 0.393 & 0.321 & 0.679 \\
\hline 655940 & 0.444 & 0.556 & 0.283 & 0.717 & 0.593 & 0.407 & 0.500 & 0.500 \\
\hline 655990 & 0.522 & 0.478 & 0.224 & 0.776 & 0.500 & 0.500 & 0.500 & 0.500 \\
\hline
\end{tabular}




\begin{tabular}{|c|c|c|c|c|c|c|c|c|}
\hline \multirow[t]{2}{*}{ Gauge } & \multicolumn{8}{|l|}{ Cold } \\
\hline & $\mathbf{P}_{\mathrm{ww}}$ & $\mathbf{P}_{\mathrm{wd}}$ & $\mathbf{P}_{\mathrm{dw}}$ & $\mathbf{P}_{\mathrm{dd}}$ & $\mathbf{P}_{\mathrm{ww}}$ & $P_{w d}$ & $\mathbf{P}_{\mathrm{dw}}$ & $\mathbf{P}_{\mathrm{dd}}$ \\
\hline 653060 & 0.438 & 0.563 & 0.358 & 0.642 & 0.500 & 0.500 & 0.457 & 0.543 \\
\hline 653190 & 0.610 & 0.390 & 0.478 & 0.522 & 0.657 & 0.343 & 0.483 & 0.517 \\
\hline 653300 & 0.444 & 0.556 & 0.400 & 0.600 & 0.541 & 0.459 & 0.455 & 0.545 \\
\hline 653350 & 0.455 & 0.545 & 0.309 & 0.691 & 0.588 & 0.412 & 0.205 & 0.795 \\
\hline 653380 & 0.394 & 0.606 & 0.310 & 0.690 & 0.333 & & & 0.854 \\
\hline 653440 & 0.500 & & & & 0.286 & & & 0.892 \\
\hline 653520 & 0.286 & 0.7 & 0.3 & & 0.267 & 0.733 & 0.257 & 0.743 \\
\hline 653550 & 0.529 & 0.4 & .368 & 632 & .545 & & 417 & 0.583 \\
\hline 653570 & 0.455 & 0.5 & .227 & & .000 & & & 0.462 \\
\hline 653610 & 0.556 & 0.4 & 0.476 & 0.524 & 0.565 & & .615 & 0.385 \\
\hline 653760 & 0.516 & 0.484 & 0.350 & 0.650 & 0.294 & 0.706 & 0.278 & 0.722 \\
\hline 653800 & 0.267 & 0.733 & 0.189 & 0.811 & 0.333 & 0.667 & 0.219 & 0.781 \\
\hline 653870 & 0.526 & 0.474 & 0.101 & 0.899 & 0.000 & 1.000 & 0.067 & 0.933 \\
\hline 654720 & 0.400 & 0.600 & 0.090 & 0.910 & 0.333 & & 0.100 & 0.900 \\
\hline 655010 & 0.111 & 0.889 & 0.316 & 0.684 & 0.357 & 0.6 & 0.292 & 0.708 \\
\hline 655020 & 0.361 & 0.639 & 0.296 & 0.704 & 0.280 & 0.720 & 0.327 & 0.673 \\
\hline 655030 & 0.273 & 0.727 & 0.371 & 0.6 & 0.281 & & & 0.566 \\
\hline 655050 & 0.261 & & & & 0.556 & & & 0.583 \\
\hline 655070 & 0.158 & & & & .419 & & & 0.550 \\
\hline 655100 & 0.422 & 0.578 & 0.400 & & 0.537 & & 0.474 & 0.526 \\
\hline 655160 & 0.425 & 0.575 & 0.362 & 0.638 & 0.487 & & 0.526 & 0.474 \\
\hline 655180 & 0.462 & 0.5 & 0.333 & 0.667 & 0.462 & & 0.433 & 0.567 \\
\hline 655220 & 0.395 & 0.605 & 0.368 & 0.632 & 0.382 & 0.6 & 0.457 & 0.543 \\
\hline 655280 & 0.414 & 0.586 & 0.333 & 0.667 & 0.688 & 0.313 & 0.464 & 0.536 \\
\hline 655360 & 0.375 & 0.625 & 0.382 & 0.618 & 0.536 & 0.464 & 0.457 & 0.543 \\
\hline 655450 & 0.333 & 0.667 & 0.196 & 0.804 & 0.111 & 0.889 & 0.162 & 0.838 \\
\hline 655480 & 0.659 & 0.341 & 0.405 & 0.595 & 0.622 & 0.378 & 0.500 & 0.500 \\
\hline 655550 & 0.333 & 0.667 & 0.148 & 0.852 & 0.455 & & 0.219 & 0.781 \\
\hline 655570 & 0.600 & 0.4 & 0.150 & 0.850 & 0.412 & & 0.228 & 0.772 \\
\hline 655600 & 0.593 & 0.4 & & & 0.375 & & 0.263 & 0.737 \\
\hline 655620 & 0.577 & & & & & & & 0.780 \\
\hline 655630 & 0.417 & & & & & & & 0.676 \\
\hline 655780 & 0.250 & 0.750 & 0.149 & 0.851 & 0.267 & 0.733 & 0.130 & 0.870 \\
\hline 655850 & 0.500 & 0.500 & 0.267 & 0.733 & 0.111 & 0.889 & 0.156 & 0.844 \\
\hline 655920 & 0.467 & 0.533 & 0.273 & 0.727 & 0.581 & 0.419 & 0.333 & 0.667 \\
\hline 655940 & 0.522 & 0.478 & 0.233 & 0.767 & 0.571 & 0.429 & 0.159 & 0.841 \\
\hline 655990 & 0.579 & 0.421 & 0.140 & 0.860 & 0.400 & 0.600 & 0.111 & 0.889 \\
\hline
\end{tabular}

1st-Order Conditioned Markov Transition Probabilities

Cold Pacific Sea Surface Temperature Event 


\begin{tabular}{|c|c|c|c|c|c|c|c|c|}
\hline \multirow{3}{*}{ Gauge } & \multicolumn{8}{|c|}{$\begin{array}{l}\text { 1st-Order Conditioned Markov Transition Probabilities } \\
\text { Cold Pacific Sea Surface Temperature Event }\end{array}$} \\
\hline & \multicolumn{4}{|c|}{ Sep } & \multicolumn{4}{|c|}{ Oct } \\
\hline & $\mathbf{P}_{\mathrm{ww}}$ & $\mathbf{P}_{\mathrm{wd}}$ & $\mathbf{P}_{\mathrm{dw}}$ & $\mathbf{P}_{\mathrm{dd}}$ & $\mathbf{P}_{\mathrm{ww}}$ & $\mathbf{P}_{\mathrm{wd}}$ & $\mathbf{P}_{\mathrm{dw}}$ & $\mathbf{P}_{\mathrm{dd}}$ \\
\hline 653060 & 0.358 & 0.642 & 0.444 & 0.556 & 0.267 & 0.733 & 0.099 & 0.901 \\
\hline 653190 & 0.635 & 0.365 & 0.511 & 0.489 & 0.490 & 0.510 & 0.162 & 0.838 \\
\hline 653300 & 0.500 & 0.500 & 0.418 & 0.582 & 0.400 & 0.600 & 0.210 & 0.790 \\
\hline 653350 & 0.419 & 0.581 & 0.395 & 0.605 & 0.283 & 0.717 & 0.248 & 0.752 \\
\hline 653380 & 0.450 & 0.550 & 0.327 & 0.673 & 0.358 & 0.642 & 0.273 & 0.727 \\
\hline 653440 & 0.362 & 0.638 & 0.225 & 0.775 & 0.375 & 0.625 & 0.237 & 0.763 \\
\hline 653520 & 0.460 & 0.540 & 0.403 & 0.597 & 0.517 & 0.483 & 0.067 & 0.933 \\
\hline 653550 & 0.733 & 0.267 & 0.462 & 0.538 & 0.393 & 0.607 & 0.286 & 0.714 \\
\hline 653570 & 0.447 & 0.553 & 0.392 & 0.608 & 0.400 & 0.600 & 0.133 & 0.867 \\
\hline 653610 & 0.625 & 0.375 & 0.469 & 0.531 & 0.429 & 0.571 & 0.233 & 0.767 \\
\hline 653760 & 0.473 & 0.527 & 0.330 & 0.670 & 0.283 & 0.717 & 0.254 & 0.746 \\
\hline 653800 & 0.333 & 0.667 & 0.301 & 0.699 & 0.449 & 0.551 & 0.358 & 0.642 \\
\hline 653870 & 0.333 & 0.667 & 0.189 & 0.811 & 0.205 & 0.795 & 0.180 & 0.820 \\
\hline 654720 & 0.444 & 0.556 & 0.125 & 0.875 & 0.250 & 0.750 & 0.167 & 0.833 \\
\hline 655010 & 0.319 & 0.681 & 0.232 & 0.768 & 0.100 & 0.900 & 0.035 & 0.965 \\
\hline 655020 & 0.233 & 0.767 & 0.273 & 0.727 & 0.200 & 0.800 & 0.079 & 0.921 \\
\hline 655030 & 0.114 & 0.886 & 0.261 & 0.739 & 0.172 & 0.828 & 0.099 & 0.901 \\
\hline 655050 & 0.289 & 0.711 & 0.472 & 0.528 & 0.120 & 0.880 & 0.142 & 0.858 \\
\hline 655070 & 0.424 & 0.576 & 0.269 & 0.731 & 0.211 & 0.789 & 0.075 & 0.925 \\
\hline 655100 & 0.486 & 0.514 & 0.435 & 0.565 & 0.413 & 0.587 & 0.119 & 0.881 \\
\hline 655160 & 0.393 & 0.607 & 0.302 & 0.698 & 0.188 & 0.813 & 0.138 & 0.862 \\
\hline 655180 & 0.469 & 0.531 & 0.409 & 0.591 & 0.167 & 0.833 & 0.123 & 0.877 \\
\hline 655220 & 0.435 & 0.565 & 0.409 & 0.591 & 0.294 & 0.706 & 0.192 & 0.808 \\
\hline 655280 & 0.455 & 0.545 & 0.517 & 0.483 & 0.409 & 0.591 & 0.227 & 0.773 \\
\hline 655360 & 0.353 & 0.647 & 0.439 & 0.561 & 0.533 & 0.467 & 0.173 & 0.827 \\
\hline 655450 & 0.406 & 0.594 & 0.305 & 0.695 & 0.273 & 0.727 & 0.224 & 0.776 \\
\hline 655480 & 0.594 & 0.406 & 0.571 & 0.429 & 0.490 & 0.510 & 0.240 & 0.760 \\
\hline 655550 & 0.532 & 0.468 & 0.303 & 0.697 & 0.280 & 0.720 & 0.172 & 0.828 \\
\hline 655570 & 0.344 & 0.656 & 0.326 & 0.674 & 0.389 & 0.611 & 0.349 & 0.651 \\
\hline 655600 & 0.419 & 0.581 & 0.302 & 0.698 & 0.323 & 0.677 & 0.247 & 0.753 \\
\hline 655620 & 0.438 & 0.563 & 0.222 & 0.778 & 0.341 & 0.659 & 0.266 & 0.734 \\
\hline 655630 & 0.500 & 0.500 & 0.217 & 0.783 & 0.219 & 0.781 & 0.291 & 0.709 \\
\hline 655780 & 0.391 & 0.609 & 0.223 & 0.777 & 0.400 & 0.600 & 0.215 & 0.785 \\
\hline 655850 & 0.433 & 0.567 & 0.386 & 0.614 & 0.480 & 0.520 & 0.400 & 0.600 \\
\hline 655920 & 0.709 & 0.291 & 0.361 & 0.639 & 0.579 & 0.421 & 0.378 & 0.622 \\
\hline 655940 & 0.542 & 0.458 & 0.273 & 0.727 & 0.408 & 0.592 & 0.444 & 0.556 \\
\hline 655990 & 0.381 & 0.619 & 0.195 & 0.805 & 0.333 & 0.667 & 0.225 & 0.775 \\
\hline
\end{tabular}




\begin{tabular}{|c|c|c|c|c|c|c|c|c|}
\hline \multirow[t]{2}{*}{ Gauge } & \multicolumn{8}{|c|}{$\begin{array}{l}\text { 1st-Order Conditioned Markov Transition Pro } \\
\text { Cold Pacific Sea Surface Temperature Event }\end{array}$} \\
\hline & $\mathbf{P}_{\mathrm{ww}}$ & $\mathbf{P}_{\mathrm{wd}}$ & $\mathbf{P}_{\mathrm{dw}}$ & $\mathbf{P}_{\mathrm{dd}}$ & $\mathbf{P}_{\mathrm{ww}}$ & $\mathbf{P}_{\mathbf{w d}}$ & $\mathbf{P}_{d w}$ & $\mathbf{P}_{\mathrm{dd}}$ \\
\hline 653060 & 0.000 & 1.000 & 0.004 & 0.996 & 0.000 & 0.000 & 0.000 & 1.000 \\
\hline 653190 & 0.000 & 1.000 & 0.018 & 0.982 & 0.000 & 0.000 & 0.000 & 1.000 \\
\hline 653300 & 0.250 & 0.750 & 0.009 & 0.991 & 0.000 & 1.000 & 0.022 & 0.978 \\
\hline 653350 & 0.000 & 1.000 & 0.037 & 0.963 & 0.000 & 1.000 & 0.021 & 0.979 \\
\hline 653380 & 0.250 & 0.750 & 0.055 & 0.945 & 0.000 & 1.000 & 0.079 & 0.921 \\
\hline 653440 & 0.320 & 0.680 & 0.099 & 0.901 & 0.176 & 0.824 & 0.055 & 0.945 \\
\hline 653520 & 0.000 & 1.000 & 0.006 & 0.994 & 0.000 & 0.000 & 0.000 & 1.000 \\
\hline 653550 & 0.200 & 0.800 & 0.059 & 0.941 & 0.000 & 1.000 & 0.014 & 0.986 \\
\hline 653570 & 0.000 & 1.000 & 0.018 & 0.982 & 0.000 & 1.000 & 0.009 & 0.991 \\
\hline 653610 & 0.167 & 0.833 & 0.024 & 0.976 & 0.000 & 1.000 & 0.016 & 0.984 \\
\hline 653760 & 0.167 & 0.833 & 0.054 & 0.946 & 0.000 & 1.000 & 0.030 & 0.970 \\
\hline 653800 & 0.067 & 0.933 & 0.103 & 0.897 & 0.000 & 1.000 & 0.048 & 0.952 \\
\hline 653870 & 0.200 & 0.800 & 0.066 & 0.934 & 0.182 & 0.818 & 0.035 & 0.965 \\
\hline 654720 & 0.000 & 1.000 & 0.069 & 0.931 & 0.000 & 1.000 & 0.037 & 0.963 \\
\hline 655010 & 0.000 & 0.000 & 0.000 & 1.000 & 0.000 & 0.000 & 0.000 & 1.000 \\
\hline 655020 & 0.000 & 1.000 & 0.005 & 0.995 & 0.000 & 0.000 & 0.000 & 1.000 \\
\hline 655030 & 0.000 & 1.000 & 0.018 & 0.982 & 0.333 & 0.667 & 0.004 & 0.996 \\
\hline 655050 & 0.250 & 0.750 & 0.020 & 0.980 & 0.000 & 0.000 & 0.000 & 1.000 \\
\hline 655070 & 0.000 & 1.000 & 0.010 & 0.990 & 0.000 & 1.000 & 0.005 & 0.995 \\
\hline 655100 & 0.250 & 0.750 & 0.017 & 0.983 & 0.000 & 1.000 & 0.018 & 0.982 \\
\hline 655160 & 0.250 & 0.750 & 0.014 & 0.986 & 0.000 & 0.000 & 0.000 & 1.000 \\
\hline 655180 & 0.250 & 0.750 & 0.027 & 0.973 & 0.000 & 1.000 & 0.006 & 0.994 \\
\hline 655220 & 0.000 & 1.000 & 0.028 & 0.972 & 0.250 & 0.750 & 0.014 & 0.986 \\
\hline 655280 & 0.091 & 0.909 & 0.070 & 0.930 & 0.500 & 0.500 & 0.007 & 0.993 \\
\hline 655360 & 0.077 & 0.923 & 0.067 & 0.933 & 0.500 & 0.500 & 0.005 & 0.995 \\
\hline 655450 & 0.182 & 0.818 & 0.165 & 0.835 & 0.250 & 0.750 & 0.031 & 0.969 \\
\hline 655480 & 0.200 & 0.800 & 0.079 & 0.921 & 0.182 & 0.818 & 0.049 & 0.951 \\
\hline 655550 & 0.083 & 0.917 & 0.059 & 0.941 & 0.100 & 0.900 & 0.046 & 0.954 \\
\hline 655570 & 0.511 & 0.489 & 0.275 & 0.725 & 0.211 & 0.789 & 0.128 & 0.872 \\
\hline 655600 & 0.188 & 0.813 & 0.098 & 0.902 & 0.143 & 0.857 & 0.085 & 0.915 \\
\hline 655620 & 0.385 & 0.615 & 0.108 & 0.892 & 0.071 & 0.929 & 0.080 & 0.920 \\
\hline 655630 & 0.280 & 0.720 & 0.144 & 0.856 & 0.222 & 0.778 & 0.043 & 0.957 \\
\hline 655780 & 0.282 & 0.718 & 0.340 & 0.660 & 0.328 & 0.672 & 0.170 & 0.830 \\
\hline 655850 & 0.500 & 0.500 & 0.301 & 0.699 & 0.400 & 0.600 & 0.170 & 0.830 \\
\hline 655920 & 0.554 & 0.446 & 0.330 & 0.670 & 0.383 & 0.617 & 0.176 & 0.824 \\
\hline 655940 & 0.444 & 0.556 & 0.358 & 0.642 & 0.222 & 0.778 & 0.130 & 0.870 \\
\hline 655990 & 0.324 & 0.676 & 0.269 & 0.731 & 0.250 & 0.750 & 0.112 & 0.888 \\
\hline
\end{tabular}




\begin{tabular}{|c|c|c|c|c|c|c|c|c|}
\hline \multirow[t]{2}{*}{ Gauge } & \multicolumn{8}{|c|}{$\begin{array}{l}\text { 1st-Order Conditioned Markov Transition Probal } \\
\text { Neutral Pacific Sea Surface Temperature Event }\end{array}$} \\
\hline & $\mathbf{P}_{\mathrm{ww}}$ & $\mathbf{P}_{\mathrm{wd}}$ & $\mathbf{P}_{\mathbf{d w}}$ & $\mathbf{P}_{\mathrm{dd}}$ & $\mathbf{P}_{\mathbf{w w}}$ & $\mathbf{P}_{\text {wd }}$ & $\mathbf{P}_{\mathbf{d w}}$ & $\mathbf{P}_{\text {dd }}$ \\
\hline $\begin{array}{l}653060 \\
653190\end{array}$ & $\begin{array}{l}0.000 \\
0.000\end{array}$ & $\begin{array}{l}0.000 \\
1.000\end{array}$ & $\begin{array}{l}0.000 \\
0.007\end{array}$ & $\begin{array}{l}1.000 \\
0.993\end{array}$ & 0.400 & 0.600 & 0.011 & $\begin{array}{l}0.983 \\
0.989\end{array}$ \\
\hline 653300 & 0.500 & 0.500 & 0.006 & 0.994 & 0.000 & 1.000 & 0.009 & 0.991 \\
\hline 653350 & 0.125 & 0.875 & 0.023 & 0.977 & 0.214 & 0.786 & 0.035 & 0.965 \\
\hline 653380 & 0.000 & 1.000 & 0.023 & 0.977 & 0.105 & 0.895 & 0.054 & 0.946 \\
\hline 653440 & 0.167 & 0.833 & 0.049 & 0.951 & 0.207 & 0.793 & 0.062 & 0.938 \\
\hline 653520 & 0.000 & 1.000 & 0.005 & 0.995 & 0.000 & 1.000 & 0.008 & 0.992 \\
\hline 653550 & 0.000 & 0.000 & 0.006 & 0.994 & 0.000 & 1.000 & 0.031 & 0.969 \\
\hline 653570 & 0.500 & 0.500 & 0.005 & 0.995 & 0.167 & 0.833 & 0.023 & 0.977 \\
\hline 653610 & 0.000 & 0.000 & 0.005 & 0.995 & 0.000 & 1.000 & 0.019 & 0.981 \\
\hline 653760 & 0.000 & 1.000 & 0.009 & 0.991 & 0.083 & 0.917 & 0.050 & 0.950 \\
\hline 653800 & 0.000 & 0.000 & 0.009 & 0.991 & 0.263 & 0.737 & 0.094 & 0.906 \\
\hline 653870 & 0.182 & 0.818 & 0.028 & 0.972 & 0.111 & 0.889 & 0.054 & 0.946 \\
\hline 654720 & 0.000 & 1.000 & 0.043 & 0.957 & 0.091 & 0.909 & 0.085 & 0.915 \\
\hline 655010 & 0.000 & 0.000 & 0.000 & 1.000 & 0.000 & 0.000 & 0.000 & 1.000 \\
\hline 655020 & 0.000 & 0.000 & 0.000 & 1.000 & 0.000 & 1.000 & 0.003 & 0.997 \\
\hline 655030 & 0.000 & 1.000 & 0.000 & 1.000 & 0.000 & 1.000 & 0.006 & 0.994 \\
\hline 655050 & 0.000 & 0.000 & 0.000 & 1.000 & 0.000 & 1.000 & 0.013 & 0.987 \\
\hline 655070 & 0.000 & 0.000 & 0.000 & 1.000 & 0.000 & 1.000 & 0.007 & 0.993 \\
\hline 655100 & 0.000 & 1.000 & 0.007 & 0.993 & 0.200 & 0.800 & 0.012 & 0.988 \\
\hline 655160 & 0.000 & 0.000 & 0.000 & 1.000 & 0.000 & 1.000 & 0.003 & 0.997 \\
\hline 655180 & 0.000 & 0.000 & 0.000 & 1.000 & 0.000 & 0.000 & 0.013 & 0.987 \\
\hline 655220 & 0.000 & 1.000 & 0.012 & 0.988 & 0.000 & 1.000 & 0.014 & 0.986 \\
\hline 655280 & 0.200 & 0.800 & 0.016 & 0.984 & 0.000 & 1.000 & 0.021 & 0.979 \\
\hline 655360 & 0.000 & 1.000 & 0.000 & 1.000 & 0.143 & 0.857 & 0.029 & 0.971 \\
\hline 655450 & 0.000 & 1.000 & 0.008 & 0.992 & 0.294 & 0.706 & 0.042 & 0.958 \\
\hline 655480 & 0.000 & 1.000 & 0.004 & 0.996 & 0.138 & 0.862 & 0.087 & 0.913 \\
\hline 655550 & 0.000 & 1.000 & 0.021 & 0.979 & 0.091 & 0.909 & 0.048 & 0.952 \\
\hline 655570 & 0.167 & 0.833 & 0.035 & 0.965 & 0.140 & 0.860 & 0.156 & 0.844 \\
\hline 655600 & 0.000 & 1.000 & 0.013 & 0.987 & 0.125 & 0.875 & 0.119 & 0.881 \\
\hline 655620 & 0.000 & 1.000 & 0.023 & 0.977 & 0.080 & 0.920 & 0.094 & 0.906 \\
\hline 655630 & 0.000 & 1.000 & 0.022 & 0.978 & 0.050 & 0.950 & 0.088 & 0.912 \\
\hline 655780 & 0.167 & 0.833 & 0.068 & 0.932 & 0.250 & 0.750 & 0.109 & 0.891 \\
\hline 655850 & 0.118 & 0.882 & 0.064 & 0.936 & 0.171 & 0.829 & 0.191 & 0.809 \\
\hline 655920 & 0.325 & 0.675 & 0.095 & 0.905 & 0.140 & 0.860 & 0.145 & 0.855 \\
\hline 655940 & 0.286 & 0.714 & 0.030 & 0.970 & 0.083 & 0.917 & 0.038 & 0.962 \\
\hline 655990 & 0.267 & 0.733 & 0.037 & 0.963 & 0.125 & 0.875 & 0.093 & 0.907 \\
\hline
\end{tabular}




\begin{tabular}{|c|c|c|c|c|c|c|c|c|}
\hline \multirow{3}{*}{ Gauge } & \multicolumn{8}{|c|}{$\begin{array}{l}\text { 1st-Order Conditioned Markov Transition Proba } \\
\text { Neutral Pacific Sea Surface Temperature Event }\end{array}$} \\
\hline & \multicolumn{4}{|c|}{ Mar } & \multicolumn{4}{|c|}{ Apr } \\
\hline & $\mathbf{P}_{\mathrm{ww}}$ & $\mathbf{P}_{\mathrm{wd}}$ & $\mathbf{P}_{\mathrm{dw}}$ & $\mathbf{P}_{\mathrm{dd}}$ & $\mathbf{P}_{\mathrm{ww}}$ & $\mathbf{P}_{\mathrm{wd}}$ & $\mathbf{P}_{\mathrm{dw}}$ & $\mathbf{P}_{\mathrm{dd}}$ \\
\hline 653060 & 0.143 & 0.857 & 0.031 & 0.969 & 0.227 & 0.773 & 0.101 & 0.899 \\
\hline 653190 & 0.270 & 0.730 & 0.060 & 0.940 & 0.330 & 0.670 & 0.225 & 0.775 \\
\hline 653300 & 0.209 & 0.791 & 0.093 & 0.907 & 0.213 & 0.788 & 0.168 & 0.832 \\
\hline 653350 & 0.250 & 0.750 & 0.138 & 0.862 & 0.312 & 0.688 & 0.256 & 0.744 \\
\hline 653380 & 0.152 & 0.848 & 0.195 & 0.805 & 0.300 & 0.700 & 0.292 & 0.708 \\
\hline 653440 & 0.233 & 0.767 & 0.136 & 0.864 & 0.292 & 0.708 & 0.245 & 0.755 \\
\hline 653520 & 0.286 & 0.714 & 0.038 & 0.962 & 0.152 & 0.848 & 0.131 & 0.869 \\
\hline 653550 & 0.050 & 0.950 & 0.106 & 0.894 & 0.268 & 0.732 & 0.247 & 0.753 \\
\hline 653570 & 0.200 & 0.800 & 0.068 & 0.932 & 0.264 & 0.736 & 0.199 & 0.801 \\
\hline 653610 & 0.128 & 0.872 & 0.124 & 0.876 & 0.247 & 0.753 & 0.241 & 0.759 \\
\hline 653760 & 0.235 & 0.765 & 0.103 & 0.897 & 0.282 & 0.718 & 0.255 & 0.745 \\
\hline 653800 & 0.154 & 0.846 & 0.074 & 0.926 & 0.152 & 0.848 & 0.223 & 0.777 \\
\hline 653870 & 0.170 & 0.830 & 0.108 & 0.892 & 0.302 & 0.698 & 0.204 & 0.796 \\
\hline 654720 & 0.150 & 0.850 & 0.097 & 0.903 & 0.214 & 0.786 & 0.157 & 0.843 \\
\hline 655010 & 0.250 & 0.750 & 0.017 & 0.983 & 0.000 & 1.000 & 0.031 & 0.969 \\
\hline 655020 & 0.000 & 1.000 & 0.018 & 0.982 & 0.056 & 0.944 & 0.048 & 0.952 \\
\hline 655030 & 0.250 & 0.750 & 0.031 & 0.969 & 0.114 & 0.886 & 0.076 & 0.924 \\
\hline 655050 & 0.333 & 0.667 & 0.039 & 0.961 & 0.304 & 0.696 & 0.076 & 0.924 \\
\hline 655070 & 0.350 & 0.650 & 0.039 & 0.961 & 0.159 & 0.841 & 0.112 & 0.888 \\
\hline 655100 & 0.207 & 0.793 & 0.064 & 0.936 & 0.268 & 0.732 & 0.148 & 0.852 \\
\hline 655160 & 0.190 & 0.810 & 0.040 & 0.960 & 0.224 & 0.776 & 0.104 & 0.896 \\
\hline 655180 & 0.364 & 0.636 & 0.048 & 0.952 & 0.310 & 0.690 & 0.127 & 0.873 \\
\hline 655220 & 0.222 & 0.778 & 0.069 & 0.931 & 0.211 & 0.789 & 0.172 & 0.828 \\
\hline 655280 & 0.115 & 0.885 & 0.094 & 0.906 & 0.273 & 0.727 & 0.224 & 0.776 \\
\hline 655360 & 0.200 & 0.800 & 0.095 & 0.905 & 0.211 & 0.789 & 0.201 & 0.799 \\
\hline 655450 & 0.268 & 0.732 & 0.128 & 0.872 & 0.224 & 0.776 & 0.223 & 0.777 \\
\hline 655480 & 0.268 & 0.732 & 0.218 & 0.782 & 0.242 & 0.758 & 0.292 & 0.708 \\
\hline 655550 & 0.171 & 0.829 & 0.147 & 0.853 & 0.270 & 0.730 & 0.256 & 0.744 \\
\hline 655570 & 0.416 & 0.584 & 0.248 & 0.752 & 0.288 & 0.712 & 0.332 & 0.668 \\
\hline 655600 & 0.203 & 0.797 & 0.219 & 0.781 & 0.205 & 0.795 & 0.258 & 0.742 \\
\hline 655620 & 0.299 & 0.701 & 0.196 & 0.804 & 0.241 & 0.759 & 0.250 & 0.750 \\
\hline 655630 & 0.259 & 0.741 & 0.207 & 0.793 & 0.189 & 0.811 & 0.290 & 0.710 \\
\hline 655780 & 0.233 & 0.767 & 0.186 & 0.814 & 0.324 & 0.676 & 0.286 & 0.714 \\
\hline 655850 & 0.314 & 0.686 & 0.212 & 0.788 & 0.333 & 0.667 & 0.311 & 0.689 \\
\hline 655920 & 0.173 & 0.827 & 0.127 & 0.873 & 0.395 & 0.605 & 0.242 & 0.758 \\
\hline 655940 & 0.077 & 0.923 & 0.090 & 0.910 & 0.167 & 0.833 & 0.165 & 0.835 \\
\hline 655990 & 0.094 & 0.906 & 0.103 & 0.897 & 0.250 & 0.750 & 0.192 & 0.808 \\
\hline
\end{tabular}




\begin{tabular}{|c|c|c|c|c|c|c|c|c|}
\hline \multirow[t]{2}{*}{ Gauge } & May & & & & Jun & & & \\
\hline & $\mathbf{P}_{\mathrm{ww}}$ & $\mathbf{P}_{\mathrm{wd}}$ & $\mathbf{P}_{\mathrm{dw}}$ & $\mathbf{P}_{\mathrm{dd}}$ & $\mathbf{P}_{\mathrm{ww}}$ & $\mathbf{P}_{\mathrm{wd}}$ & $\mathbf{P}_{\mathrm{dw}}$ & $\mathbf{P}_{\mathrm{dd}}$ \\
\hline 653060 & 0.228 & 0.772 & 0.289 & 0.711 & 0.290 & 0.710 & 0.308 & 0.692 \\
\hline 653190 & 0.333 & 0.667 & 0.242 & 0.758 & 0.375 & 0.625 & 0.370 & 0.630 \\
\hline 653300 & 0.240 & 0.760 & 0.359 & 0.641 & 0.350 & 0.650 & 0.399 & 0.601 \\
\hline 653350 & 0.303 & 0.697 & 0.312 & 0.688 & 0.470 & 0.530 & 0.398 & 0.602 \\
\hline 653380 & 0.308 & 0.692 & 0.324 & 0.676 & 0.382 & 0.618 & 0.390 & 0.610 \\
\hline 653440 & 0.368 & 0.632 & 0.367 & 0.633 & 0.545 & 0.455 & 0.411 & 0.589 \\
\hline 653520 & 0.341 & 0.659 & 0.166 & 0.834 & 0.321 & 0.679 & 0.222 & 0.778 \\
\hline 653550 & 0.455 & 0.545 & 0.271 & 0.729 & 0.377 & 0.623 & 0.341 & 0.659 \\
\hline 653570 & 0.344 & 0.656 & 0.258 & 0.742 & 0.316 & 0.684 & 0.333 & 0.667 \\
\hline 653610 & 0.262 & 0.738 & 0.332 & 0.668 & 0.412 & 0.588 & 0.439 & 0.561 \\
\hline 653760 & 0.297 & 0.703 & 0.291 & 0.709 & 0.442 & 0.558 & 0.379 & 0.621 \\
\hline 653800 & 0.283 & 0.717 & 0.241 & 0.759 & 0.333 & 0.667 & 0.327 & 0.673 \\
\hline 653870 & 0.233 & 0.767 & 0.330 & 0.670 & 0.415 & 0.585 & 0.328 & 0.672 \\
\hline 654720 & 0.310 & 0.690 & 0.146 & 0.854 & 0.373 & 0.627 & 0.303 & 0.697 \\
\hline 655010 & 0.200 & 0.800 & 0.089 & 0.911 & 0.196 & 0.804 & 0.184 & 0.816 \\
\hline 655020 & 0.211 & 0.789 & 0.122 & 0.878 & 0.177 & 0.823 & 0.253 & 0.747 \\
\hline 655030 & 0.227 & 0.773 & 0.181 & 0.819 & 0.243 & 0.757 & 0.309 & 0.691 \\
\hline 655050 & 0.250 & 0.750 & 0.174 & 0.826 & 0.250 & 0.750 & 0.235 & 0.765 \\
\hline 655070 & 0.154 & 0.846 & 0.212 & 0.788 & 0.314 & 0.686 & 0.282 & 0.718 \\
\hline 655100 & 0.207 & 0.793 & 0.247 & 0.753 & 0.256 & 0.744 & 0.335 & 0.665 \\
\hline 655160 & 0.278 & 0.722 & 0.230 & 0.770 & 0.229 & 0.771 & 0.327 & 0.673 \\
\hline 655180 & 0.237 & 0.763 & 0.214 & 0.786 & 0.234 & 0.766 & 0.360 & 0.640 \\
\hline 655220 & 0.297 & 0.703 & 0.250 & 0.750 & 0.205 & 0.795 & 0.295 & 0.705 \\
\hline 655280 & 0.197 & 0.803 & 0.315 & 0.685 & 0.189 & 0.811 & 0.335 & 0.665 \\
\hline 655360 & 0.232 & 0.768 & 0.309 & 0.691 & 0.185 & 0.815 & 0.297 & 0.703 \\
\hline 655450 & 0.273 & 0.727 & 0.308 & 0.692 & 0.336 & 0.664 & 0.335 & 0.665 \\
\hline 655480 & 0.325 & 0.675 & 0.322 & 0.678 & 0.379 & 0.621 & 0.363 & 0.637 \\
\hline 655550 & 0.281 & 0.719 & 0.232 & 0.768 & 0.341 & 0.659 & 0.339 & 0.661 \\
\hline 655570 & 0.479 & 0.521 & 0.444 & 0.556 & 0.514 & 0.486 & 0.432 & 0.568 \\
\hline 655600 & 0.253 & 0.747 & 0.361 & 0.639 & 0.414 & 0.586 & 0.385 & 0.615 \\
\hline 655620 & 0.344 & 0.656 & 0.350 & 0.650 & 0.388 & 0.612 & 0.364 & 0.636 \\
\hline 655630 & 0.308 & 0.692 & 0.310 & 0.690 & 0.435 & 0.565 & 0.309 & 0.691 \\
\hline 655780 & 0.466 & 0.534 & 0.437 & 0.563 & 0.584 & 0.416 & 0.459 & 0.541 \\
\hline 655850 & 0.489 & 0.511 & 0.490 & 0.510 & 0.647 & 0.353 & 0.465 & 0.535 \\
\hline 655920 & 0.576 & 0.424 & 0.479 & 0.521 & 0.643 & 0.357 & 0.481 & 0.519 \\
\hline 655940 & 0.357 & 0.643 & 0.345 & 0.655 & 0.625 & 0.375 & 0.447 & 0.553 \\
\hline 655990 & 0.519 & 0.481 & 0.371 & 0.629 & 0.517 & 0.483 & 0.462 & 0.538 \\
\hline
\end{tabular}

1st-Order Conditioned Markov Transition Probabilities Neutral Pacific Sea Surface Temperature Event 


\begin{tabular}{|c|c|c|c|c|c|c|c|c|}
\hline \multirow[t]{2}{*}{ Gauge } & \\
\hline & $\mathbf{P}_{\mathrm{ww}}$ & $\mathbf{P}_{\mathrm{wd}}$ & $\mathbf{P}_{\mathrm{dw}}$ & $\mathbf{P}_{\mathrm{dd}}$ & $\mathbf{P}_{\mathrm{ww}}$ & $\mathbf{P}_{\mathrm{wd}}$ & $\mathbf{P}_{\mathrm{dw}}$ & $\mathbf{P}_{\mathrm{dd}}$ \\
\hline 653060 & 0.406 & 0.594 & 0.402 & 0.598 & 0.513 & 0.487 & 0.455 & 0.545 \\
\hline 653190 & 0.528 & 0.472 & 0.464 & 0.536 & 0.563 & 0.437 & 0.580 & 0.420 \\
\hline 653300 & 0.558 & 0.442 & 0.391 & 0.609 & 0.512 & 0.488 & 0.436 & 0.564 \\
\hline 653350 & 0.525 & 0.475 & 0.289 & 0.711 & 0.431 & 0.569 & 0.303 & 0.697 \\
\hline 653380 & 0.423 & 0.577 & 0.328 & 0.672 & 0.420 & 0.580 & 0.255 & 0.745 \\
\hline 653440 & 0.417 & 0.583 & 0.197 & 0.803 & 0.385 & 0.615 & 0.162 & 0.838 \\
\hline 653520 & 0.348 & 0.652 & 0.318 & 0.682 & 0.360 & 40 & 0.389 & 0.611 \\
\hline 653550 & 0.470 & 0.530 & 0.405 & 0.595 & 0.596 & 0.404 & 0.627 & 0.373 \\
\hline 653570 & 0.390 & 0.610 & 0.453 & 0.547 & 0.496 & 0.504 & 0.449 & 0.551 \\
\hline 653610 & 0.571 & 0.429 & 0.535 & 0.465 & 0.629 & 0.371 & 0.472 & 0.528 \\
\hline 653760 & 0.549 & 0.451 & 0.390 & 0.610 & 0.458 & 0.542 & 0.406 & 0.594 \\
\hline 653800 & 0.393 & 0.607 & 0.181 & 0.819 & 0.397 & 0.603 & 0.140 & 0.860 \\
\hline 653870 & 0.340 & 0.660 & 0.157 & 0.842 & 0.275 & 0.725 & 0.160 & 0.840 \\
\hline 654720 & 0.300 & 0.70 & 0.12 & 0.8 & 0.200 & & 0.157 & 0.843 \\
\hline 655010 & 0.175 & 0.8 & 0.3 & & 0.338 & & 0.353 & 0.647 \\
\hline 655020 & 0.323 & 0.6 & 0.3 & 0.6 & 0.415 & & 0.474 & 0.526 \\
\hline 655030 & 0.293 & 0.707 & 0.4 & 0.5 & 0.460 & & 0.472 & .528 \\
\hline 655050 & 0.338 & 0.662 & 0.388 & 0.612 & 0.456 & & 0.500 & 0.500 \\
\hline 655070 & 0.351 & 0.649 & 0.412 & 0.588 & 0.472 & 0.528 & 0.503 & 0.497 \\
\hline 655100 & 0.351 & 0.649 & 0.405 & 0.595 & 0.523 & 0.477 & 0.529 & 0.471 \\
\hline 655160 & 0.352 & 0.648 & 0.390 & 0.610 & 0.518 & 0.482 & 0.502 & 0.498 \\
\hline 655180 & 0.308 & 0.692 & 0.410 & 0.590 & 0.527 & 0.473 & 0.516 & 0.484 \\
\hline 655220 & 0.387 & 0.613 & 0.378 & 0.622 & 0.482 & 0.518 & 0.435 & 0.565 \\
\hline 655280 & 0.475 & 0.525 & 0.393 & 0.607 & 0.585 & 0.415 & 0.532 & 0.468 \\
\hline 655360 & 0.336 & 0.664 & 0.315 & 0.685 & 0.437 & 0.563 & 0.355 & 0.645 \\
\hline 655450 & 0.326 & 0.674 & 0.215 & 0.785 & 0.372 & & 0.201 & 0.799 \\
\hline 655480 & 0.571 & & & & 0.584 & & 0.576 & 0.424 \\
\hline 655550 & 0.349 & & & & 0.4 & & & 0.750 \\
\hline 655570 & 0.441 & 0.55 & 0.204 & 0.796 & 0.451 & 0.549 & 0.300 & 0.700 \\
\hline 655600 & 0.470 & 0.530 & 0.246 & 0.754 & 0.469 & 0.531 & 0.371 & 0.629 \\
\hline 655620 & 0.341 & 0.659 & 0.204 & 0.796 & 0.426 & 0.574 & 0.207 & 0.793 \\
\hline 655630 & 0.308 & 0.692 & 0.232 & 0.768 & 0.402 & 0.598 & 0.231 & 0.769 \\
\hline 655780 & 0.492 & 0.508 & 0.163 & 0.837 & 0.382 & 0.618 & 0.153 & 0.847 \\
\hline 655850 & 0.564 & 0.436 & 0.279 & 0.721 & 0.526 & 0.474 & 0.289 & 0.711 \\
\hline 655920 & 0.525 & 0.475 & 0.249 & 0.751 & 0.612 & 0.388 & 0.433 & 0.567 \\
\hline 655940 & 0.412 & 0.587 & 0.215 & 0.785 & 0.471 & 0.529 & 0.220 & 0.780 \\
\hline 655990 & 0.425 & 0.575 & 0.186 & 0.814 & 0.432 & 0.568 & 0.199 & 0.801 \\
\hline
\end{tabular}

1st-Order Conditioned Markov Transition Probabilities Neutral Pacific Sea Surface Temperature Event 
1st-Order Conditioned Markov Transition Probabilities Neutral Pacific Sea Surface Temperature Event

\begin{tabular}{|c|c|c|c|c|c|c|c|c|}
\hline Gauge & Sep & & & & Oct & & & \\
\hline & $\mathbf{P}_{\mathrm{ww}}$ & $\mathbf{P}_{\mathbf{w d}}$ & $\mathbf{P}_{\mathrm{dw}}$ & $\mathbf{P}_{\text {dd }}$ & $\begin{array}{l}\mathbf{P}_{\mathrm{ww}} \\
0.281\end{array}$ & $\begin{array}{l}\mathbf{P}_{\mathbf{w d}} \\
0.71\end{array}$ & $\begin{array}{l}\mathbf{P}_{\mathrm{dw}} \\
0.090\end{array}$ & $\begin{array}{l}\mathbf{P}_{\text {dd }} \\
0.910\end{array}$ \\
\hline 653190 & $\begin{array}{l}0.408 \\
0.605\end{array}$ & 0.395 & 0.612 & 0.388 & 0.376 & 0.624 & 0.219 & 0.781 \\
\hline 653300 & 0.566 & 0.434 & 0.531 & 0.469 & 0.419 & 0.581 & 0.206 & 0.794 \\
\hline 653350 & 0.439 & 0.561 & 0.432 & 0.568 & 0.385 & 0.615 & 0.285 & 0.715 \\
\hline 653380 & 0.463 & 0.537 & 0.394 & 0.606 & 0.352 & 0.648 & 0.271 & 0.729 \\
\hline 653440 & 0.402 & 0.598 & 0.336 & 0.664 & 0.383 & 0.617 & 0.318 & 0.682 \\
\hline 653520 & 0.500 & 0.500 & 0.462 & 0.538 & 0.200 & 0.800 & 0.138 & 0.862 \\
\hline 653550 & 0.545 & 0.455 & 0.500 & 0.500 & 0.270 & 0.730 & 0.278 & 0.722 \\
\hline 653570 & 0.440 & 0.560 & 0.463 & 0.537 & 0.333 & 0.667 & 0.234 & 0.766 \\
\hline 653610 & 0.488 & 0.512 & 0.574 & 0.426 & 0.373 & 0.627 & 0.195 & 0.805 \\
\hline 653760 & 0.400 & 0.600 & 0.386 & 0.614 & 0.400 & 0.600 & 0.249 & 0.751 \\
\hline 653800 & 0.444 & 0.556 & 0.269 & 0.731 & 0.220 & 0.780 & 0.364 & 0.636 \\
\hline 653870 & 0.364 & 0.636 & 0.231 & 0.769 & 0.290 & 0.710 & 0.211 & 0.789 \\
\hline 54720 & 0.435 & 0.565 & 0.147 & 0.853 & .438 & 0.563 & 0.182 & 0.818 \\
\hline 655010 & 0.276 & 0.724 & 0.225 & 0.775 & .125 & 0.875 & 0.049 & 0.951 \\
\hline 655020 & 0.320 & 0.680 & 0.308 & 0.692 & .243 & 0.757 & 0.110 & 0.890 \\
\hline 655030 & 0.297 & 0.703 & 0.391 & 0.609 & 0.300 & 0.700 & 0.064 & 0.936 \\
\hline 655050 & 0.271 & 0.729 & 0.358 & 0.642 & 0.217 & 0.783 & 0.139 & 0.861 \\
\hline 655070 & 0.373 & 0.627 & 0.400 & 0.600 & 0.176 & 0.824 & 0.109 & 0.891 \\
\hline 655100 & 0.497 & 0.503 & 0.463 & 0.537 & 0.241 & 0.759 & 0.163 & 0.837 \\
\hline 655160 & 0.434 & 0.566 & 0.372 & 0.628 & 0.244 & 0.756 & 0.115 & 0.885 \\
\hline 655180 & 0.338 & 0.662 & 0.396 & 0.604 & 0.294 & 0.706 & 0.085 & 0.915 \\
\hline 655220 & 0.441 & 0.559 & 0.485 & 0.515 & 0.250 & 0.750 & 0.217 & 0.783 \\
\hline 655280 & 0.480 & 0.520 & 0.602 & 0.398 & 0.350 & 0.650 & 0.368 & 0.632 \\
\hline 655360 & 0.410 & 0.590 & 0.508 & 0.492 & 0.329 & 0.671 & 0.247 & 0.753 \\
\hline 655450 & 0.509 & 0.491 & 0.431 & 0.569 & 0.425 & 0.575 & 0.267 & 0.733 \\
\hline 655480 & 0.519 & 0.481 & 0.598 & 0.402 & 0.437 & 0.563 & 0.265 & 0.735 \\
\hline 655550 & 0.379 & 0.621 & 0.379 & 0.621 & 0.300 & 0.700 & 0.272 & 0.728 \\
\hline 655570 & 0.545 & 0.455 & 0.351 & 0.649 & 0.453 & 0.547 & 0.428 & 0.572 \\
\hline 655600 & 0.464 & 0.536 & 0.469 & 0.531 & 0.342 & 0.658 & 0.267 & 0.733 \\
\hline 655620 & 0.471 & 0.529 & 0.291 & 0.709 & 0.412 & 0.588 & 0.311 & 0.689 \\
\hline 655630 & 0.448 & 0.552 & 0.371 & 0.629 & 0.465 & 0.535 & 0.316 & 0.684 \\
\hline 655780 & 0.385 & 0.615 & 0.254 & 0.746 & 0.567 & 0.433 & 0.299 & 0.701 \\
\hline 655850 & 0.490 & 0.510 & 0.355 & 0.645 & 0.556 & 0.444 & 0.401 & 0.599 \\
\hline 655920 & 0.633 & 0.367 & 0.462 & 0.538 & 0.585 & 0.415 & 0.389 & 0.611 \\
\hline 655940 & 0.388 & 0.612 & 0.260 & 0.740 & 0.480 & & 0.232 & 0.768 \\
\hline 655990 & 0.333 & 0.667 & 0.173 & 0.827 & 0.468 & 0.532 & 0.270 & 0.730 \\
\hline
\end{tabular}




\begin{tabular}{|c|c|c|c|c|c|c|c|c|}
\hline \multirow{3}{*}{ Gauge } & \multicolumn{8}{|c|}{$\begin{array}{l}\text { 1st-Order Conditioned Markov Transition Proba } \\
\text { Neutral Pacific Sea Surface Temperature Event }\end{array}$} \\
\hline & \multicolumn{4}{|c|}{ Nov } & \multicolumn{4}{|c|}{ Dec } \\
\hline & $\mathbf{P}_{\mathrm{ww}}$ & $\mathbf{P}_{\mathrm{wd}}$ & $\mathbf{P}_{\mathrm{dw}}$ & $\mathbf{P}_{\mathrm{dd}}$ & $\mathbf{P}_{\mathrm{ww}}$ & $\mathbf{P}_{\mathrm{wd}}$ & $\mathbf{P}_{\mathrm{dw}}$ & $\mathbf{P}_{\mathrm{dd}}$ \\
\hline 653060 & 0.000 & 0.000 & 0.000 & 1.000 & 0.000 & 1.000 & 0.004 & 0.996 \\
\hline 653190 & 0.333 & 0.667 & 0.015 & 0.985 & 0.000 & 1.000 & 0.007 & 0.993 \\
\hline 653300 & 0.333 & 0.667 & 0.014 & 0.986 & 0.000 & 1.000 & 0.009 & 0.991 \\
\hline 653350 & 0.176 & 0.824 & 0.046 & 0.954 & 0.333 & 0.667 & 0.007 & 0.993 \\
\hline 653380 & 0.227 & 0.773 & 0.061 & 0.939 & 0.000 & 1.000 & 0.016 & 0.984 \\
\hline 653440 & 0.320 & 0.680 & 0.117 & 0.883 & 0.000 & 1.000 & 0.050 & 0.950 \\
\hline 653520 & 0.200 & 0.800 & 0.017 & 0.983 & 0.000 & 1.000 & 0.005 & 0.995 \\
\hline 653550 & 0.200 & 0.800 & 0.037 & 0.963 & 0.000 & 1.000 & 0.024 & 0.976 \\
\hline 653570 & 0.300 & 0.700 & 0.036 & 0.964 & 0.000 & 1.000 & 0.015 & 0.985 \\
\hline 653610 & 0.176 & 0.824 & 0.048 & 0.952 & 0.375 & 0.625 & 0.023 & 0.977 \\
\hline 653760 & 0.000 & 1.000 & 0.076 & 0.924 & 0.000 & 1.000 & 0.021 & 0.979 \\
\hline 653800 & 0.172 & 0.828 & 0.142 & 0.858 & 0.250 & 0.750 & 0.031 & 0.969 \\
\hline 653870 & 0.154 & 0.846 & 0.087 & 0.913 & 0.000 & 1.000 & 0.027 & 0.973 \\
\hline 654720 & 0.200 & 0.800 & 0.114 & 0.886 & 0.000 & 1.000 & 0.055 & 0.945 \\
\hline 655010 & 0.000 & 1.000 & 0.008 & 0.992 & 0.000 & 1.000 & 0.014 & 0.986 \\
\hline 655020 & 0.250 & 0.750 & 0.007 & 0.993 & 0.000 & 1.000 & 0.004 & 0.996 \\
\hline 655030 & 0.286 & 0.714 & 0.010 & 0.990 & 0.000 & 1.000 & 0.006 & 0.994 \\
\hline 655050 & 0.000 & 1.000 & 0.012 & 0.988 & 0.000 & 1.000 & 0.012 & 0.988 \\
\hline 655070 & 0.000 & 1.000 & 0.012 & 0.988 & 0.400 & 0.600 & 0.011 & 0.989 \\
\hline 655100 & 0.294 & 0.706 & 0.031 & 0.969 & 0.000 & 1.000 & 0.009 & 0.991 \\
\hline 655160 & 0.556 & 0.444 & 0.012 & 0.988 & 0.000 & 1.000 & 0.007 & 0.993 \\
\hline 655180 & 0.286 & 0.714 & 0.026 & 0.974 & 0.500 & 0.500 & 0.000 & 1.000 \\
\hline 655220 & 0.333 & 0.667 & 0.024 & 0.976 & 0.000 & 1.000 & 0.008 & 0.992 \\
\hline 655280 & 0.188 & 0.813 & 0.101 & 0.899 & 0.167 & 0.833 & 0.019 & 0.981 \\
\hline 655360 & 0.087 & 0.913 & 0.068 & 0.932 & 0.000 & 1.000 & 0.014 & 0.986 \\
\hline 655450 & 0.273 & 0.727 & 0.066 & 0.934 & 0.273 & 0.727 & 0.033 & 0.967 \\
\hline 655480 & 0.214 & 0.786 & 0.109 & 0.891 & 0.214 & 0.786 & 0.050 & 0.950 \\
\hline 655550 & 0.143 & 0.857 & 0.063 & 0.937 & 0.083 & 0.917 & 0.043 & 0.957 \\
\hline 655570 & 0.226 & 0.774 & 0.316 & 0.684 & 0.282 & 0.718 & 0.109 & 0.891 \\
\hline 655600 & 0.447 & 0.553 & 0.089 & 0.911 & 0.083 & 0.917 & 0.050 & 0.950 \\
\hline 655620 & 0.324 & 0.676 & 0.096 & 0.904 & 0.000 & 1.000 & 0.054 & 0.946 \\
\hline 655630 & 0.417 & 0.583 & 0.096 & 0.904 & 0.190 & 0.810 & 0.078 & 0.922 \\
\hline 655780 & 0.489 & 0.511 & 0.375 & 0.625 & 0.304 & 0.696 & 0.171 & 0.829 \\
\hline 655850 & 0.466 & 0.534 & 0.385 & 0.615 & 0.288 & 0.712 & 0.203 & 0.797 \\
\hline 655920 & 0.520 & 0.480 & 0.400 & 0.600 & 0.341 & 0.659 & 0.287 & 0.713 \\
\hline 655940 & 0.402 & 0.598 & 0.329 & 0.671 & 0.333 & 0.667 & 0.166 & 0.834 \\
\hline 655990 & 0.414 & 0.586 & 0.329 & 0.671 & 0.326 & 0.674 & 0.148 & 0.852 \\
\hline
\end{tabular}




\begin{tabular}{|c|c|c|c|c|c|c|c|c|}
\hline \multirow[t]{2}{*}{ Gauge } & \multicolumn{4}{|l|}{ Jan } & \multicolumn{4}{|c|}{ Feb } \\
\hline & $\mathbf{P}_{\mathrm{ww}}$ & $\mathbf{P}_{\mathrm{wd}}$ & $\mathbf{P}_{\mathrm{dw}}$ & $\mathbf{P}_{\mathrm{dd}}$ & $\mathbf{P}_{\mathrm{ww}}$ & $\mathbf{P}_{\mathrm{wd}}$ & $\mathbf{P}_{\mathrm{dw}}$ & $\mathbf{P}_{\mathrm{dd}}$ \\
\hline 653060 & 0.000 & 0.000 & 0.000 & 1.000 & 0.000 & 0.000 & 0.000 & 1.000 \\
\hline 653190 & 0.000 & 1.000 & 0.004 & 0.996 & 0.000 & 1.000 & 0.010 & 0.990 \\
\hline 653300 & 0.000 & 1.000 & 0.008 & 0.992 & 0.250 & 0.750 & 0.022 & 0.978 \\
\hline 653350 & 0.000 & 1.000 & 0.000 & 1.000 & 0.250 & 0.750 & 0.037 & 0.963 \\
\hline 653380 & 0.000 & 1.000 & 0.014 & 0.986 & 0.118 & 0.882 & 0.076 & 0.924 \\
\hline 653440 & 0.000 & 1.000 & 0.035 & 0.965 & 0.077 & 0.923 & 0.052 & 0.948 \\
\hline 653520 & 0.000 & 0.000 & 0.000 & 1.000 & 0.000 & 1.000 & 0.022 & 0.978 \\
\hline 653550 & 0.000 & 0.000 & 0.000 & 1.000 & 0.333 & 0.667 & 0.023 & 0.977 \\
\hline 653570 & 0.000 & 1.000 & 0.006 & 0.994 & 0.000 & 0.000 & 0.000 & 1.000 \\
\hline 653610 & 0.000 & 1.000 & 0.011 & 0.989 & 0.000 & 1.000 & 0.012 & 0.988 \\
\hline 653760 & 0.000 & 1.000 & 0.016 & 0.984 & 0.143 & 0.857 & 0.050 & 0.950 \\
\hline 653800 & 0.000 & 1.000 & 0.000 & 1.000 & 0.000 & 1.000 & 0.038 & 0.962 \\
\hline 653870 & 0.000 & 1.000 & 0.008 & 0.992 & 0.100 & 0.900 & 0.046 & 0.954 \\
\hline 654720 & 0.333 & 0.667 & 0.013 & 0.987 & 0.000 & 1.000 & 0.046 & 0.954 \\
\hline 655010 & 0.000 & 0.000 & 0.000 & 1.000 & 0.000 & 1.000 & 0.005 & 0.995 \\
\hline 655020 & 0.000 & 1.000 & 0.005 & 0.995 & 0.000 & 0.000 & 0.000 & 1.000 \\
\hline 655030 & 0.000 & 0.000 & 0.000 & 1.000 & 0.000 & 1.000 & 0.008 & 0.992 \\
\hline 655050 & 0.000 & 1.000 & 0.008 & 0.992 & 0.000 & 0.000 & 0.000 & 1.000 \\
\hline 655070 & 0.000 & 0.000 & 0.000 & 1.000 & 0.000 & 0.000 & 0.000 & 1.000 \\
\hline 655100 & 0.000 & 1.000 & 0.004 & 0.996 & 0.000 & 1.000 & 0.004 & 0.996 \\
\hline 655160 & 0.000 & 1.000 & 0.014 & 0.986 & 0.000 & 0.000 & 0.000 & 1.000 \\
\hline 655180 & 0.000 & 0.000 & 0.000 & 1.000 & 0.000 & 1.000 & 0.008 & 0.992 \\
\hline 655220 & 0.000 & 1.000 & 0.018 & 0.982 & 0.000 & 1.000 & 0.005 & 0.995 \\
\hline 655280 & 0.250 & 0.750 & 0.018 & 0.982 & 0.000 & 1.000 & 0.013 & 0.987 \\
\hline 655360 & 0.000 & 1.000 & 0.010 & 0.990 & 0.000 & 1.000 & 0.039 & 0.961 \\
\hline 655450 & 0.000 & 1.000 & 0.021 & 0.979 & 0.200 & 0.800 & 0.079 & 0.921 \\
\hline 655480 & 0.250 & 0.750 & 0.024 & 0.976 & 0.400 & 0.600 & 0.040 & 0.960 \\
\hline 655550 & 0.000 & 0.000 & 0.011 & 0.989 & 0.125 & 0.875 & 0.041 & 0.959 \\
\hline 655570 & 0.222 & 0.778 & 0.036 & 0.964 & 0.364 & 0.636 & 0.074 & 0.926 \\
\hline 655600 & 0.000 & 1.000 & 0.025 & 0.975 & 0.222 & 0.778 & 0.080 & 0.920 \\
\hline 655620 & 0.000 & 1.000 & 0.005 & 0.995 & 0.188 & 0.813 & 0.083 & 0.917 \\
\hline 655630 & 0.000 & 1.000 & 0.007 & 0.993 & 0.154 & 0.846 & 0.060 & 0.940 \\
\hline 655780 & 0.250 & 0.750 & 0.029 & 0.971 & 0.000 & 1.000 & 0.081 & 0.919 \\
\hline 655850 & 0.000 & 1.000 & 0.015 & 0.985 & 0.211 & 0.789 & 0.090 & 0.910 \\
\hline 655920 & 0.231 & 0.769 & 0.053 & 0.947 & 0.360 & 0.640 & 0.088 & 0.912 \\
\hline 655940 & 0.000 & 1.000 & 0.016 & 0.984 & 0.000 & 1.000 & 0.050 & 0.950 \\
\hline 655990 & 0.000 & 1.000 & 0.011 & 0.989 & 0.100 & 0.900 & 0.054 & 0.946 \\
\hline
\end{tabular}

1st-Order Conditioned Markov Transition Probabilities Warm Pacific Sea Surface Temperature Event 


\begin{tabular}{|c|c|c|c|c|c|c|c|c|}
\hline \multirow[t]{2}{*}{ Gauge } & \multicolumn{8}{|c|}{$\begin{array}{l}\text { 1st-Order Conditioned Markov Transition Prob } \\
\text { Warm Pacific Sea Surface Temperature Event }\end{array}$} \\
\hline & $\mathbf{P}_{\mathrm{ww}}$ & $P_{w d}$ & $\mathbf{P}_{\mathrm{dw}}$ & $\mathbf{P}_{\mathrm{dd}}$ & $\mathbf{P}_{\mathrm{ww}}$ & $\mathbf{P}_{\mathrm{wd}}$ & $\mathbf{P}_{d w}$ & $\mathbf{P}_{\mathrm{dd}}$ \\
\hline 653060 & 0.333 & 0.667 & 0.005 & 0.995 & 0.083 & 0.917 & 0.086 & 0.914 \\
\hline 653190 & 0.111 & 0.889 & 0.045 & 0.955 & 0.182 & 0.818 & 0.162 & 0.838 \\
\hline 653300 & 0.083 & 0.917 & 0.066 & 0.934 & 0.100 & 0.900 & 0.140 & 0.860 \\
\hline 653350 & 0.250 & 0.750 & 0.067 & 0.933 & 0.103 & 0.897 & 0.221 & 0.779 \\
\hline 653380 & 0.182 & 0.818 & 0.121 & 0.879 & 0.265 & 0.735 & 0.233 & 0.767 \\
\hline 653440 & 0.222 & 0.778 & 0.105 & 0.895 & 0.160 & 0.840 & 0.168 & 0.832 \\
\hline 653520 & 0.125 & 0.875 & 0.051 & 0.949 & 0.000 & 1.000 & 0.103 & 0.897 \\
\hline 653550 & 0.071 & 0.929 & 0.090 & 0.910 & 0.154 & 0.846 & 0.176 & 0.824 \\
\hline 653570 & 0.000 & 0.000 & 0.022 & 0.978 & 0.200 & 0.800 & 0.185 & 0.815 \\
\hline 653610 & 0.111 & 0.889 & 0.059 & 0.941 & 0.111 & 0.889 & 0.167 & 0.833 \\
\hline 653760 & 0.167 & 0.833 & 0.119 & 0.881 & 0.238 & 0.762 & 0.159 & 0.841 \\
\hline 653800 & 0.250 & 0.750 & 0.165 & 0.835 & 0.000 & 1.000 & 0.261 & 0.739 \\
\hline 653870 & 0.304 & 0.696 & 0.089 & 0.911 & 0.333 & 0.667 & 0.139 & 0.861 \\
\hline 654720 & 0.133 & 0.867 & 0.094 & 0.906 & 0.000 & 1.000 & 0.053 & 0.947 \\
\hline 655010 & 0.000 & 1.000 & 0.005 & 0.995 & 0.000 & 1.000 & 0.007 & 0.993 \\
\hline 655020 & 0.250 & 0.750 & 0.015 & 0.985 & 0.000 & 1.000 & 0.013 & 0.987 \\
\hline 655030 & 0.250 & 0.750 & 0.009 & 0.991 & 0.250 & 0.750 & 0.045 & 0.955 \\
\hline 655050 & 0.250 & 0.750 & 0.033 & 0.967 & 0.000 & 1.000 & 0.060 & 0.940 \\
\hline 655070 & 0.000 & 1.000 & 0.029 & 0.971 & 0.333 & 0.667 & 0.038 & 0.962 \\
\hline 655100 & 0.273 & 0.727 & 0.038 & 0.962 & 0.250 & 0.750 & 0.112 & 0.888 \\
\hline 655160 & 0.250 & 0.750 & 0.015 & 0.985 & 0.063 & 0.938 & 0.113 & 0.887 \\
\hline 655180 & 0.333 & 0.667 & 0.033 & 0.967 & 0.125 & 0.875 & 0.053 & 0.947 \\
\hline 655220 & 0.333 & 0.667 & 0.036 & 0.964 & 0.158 & 0.842 & 0.128 & 0.872 \\
\hline 655280 & 0.125 & 0.875 & 0.061 & 0.939 & 0.048 & 0.952 & 0.162 & 0.838 \\
\hline 655360 & 0.125 & 0.875 & 0.055 & 0.945 & 0.261 & 0.739 & 0.140 & 0.860 \\
\hline 655450 & 0.167 & 0.833 & 0.034 & 0.966 & 0.130 & 0.870 & 0.179 & 0.821 \\
\hline 655480 & 0.208 & 0.792 & 0.151 & 0.849 & 0.231 & 0.769 & 0.238 & 0.762 \\
\hline 655550 & 0.176 & 0.824 & 0.111 & 0.889 & 0.214 & 0.786 & 0.198 & 0.802 \\
\hline 655570 & 0.324 & 0.676 & 0.175 & 0.825 & 0.311 & 0.689 & 0.337 & 0.663 \\
\hline 655600 & 0.125 & 0.875 & 0.202 & 0.798 & 0.278 & 0.722 & 0.300 & 0.700 \\
\hline 655620 & 0.387 & 0.613 & 0.162 & 0.838 & 0.313 & 0.688 & 0.194 & 0.806 \\
\hline 655630 & 0.304 & 0.696 & 0.116 & 0.884 & 0.308 & 0.692 & 0.247 & 0.753 \\
\hline 655780 & 0.308 & 0.692 & 0.177 & 0.823 & 0.293 & 0.707 & 0.230 & 0.770 \\
\hline 655850 & 0.125 & 0.875 & 0.192 & 0.808 & 0.208 & 0.792 & 0.253 & 0.747 \\
\hline 655920 & 0.296 & 0.704 & 0.156 & 0.844 & 0.400 & 0.600 & 0.295 & 0.705 \\
\hline 655940 & 0.133 & 0.867 & 0.086 & 0.914 & 0.424 & 0.576 & 0.189 & 0.811 \\
\hline 655990 & 0.375 & 0.625 & 0.051 & 0.949 & 0.484 & 0.516 & 0.173 & 0.827 \\
\hline
\end{tabular}




\begin{tabular}{|c|c|c|c|c|c|c|c|c|}
\hline \multirow[t]{2}{*}{ Gauge } & \multicolumn{8}{|c|}{$\begin{array}{l}\text { 1st-Order Conditioned Markov Transition Prob } \\
\text { Warm Pacific Sea Surface Temperature Event }\end{array}$} \\
\hline & $\mathbf{P}_{\mathrm{ww}}$ & $\mathbf{P}_{\mathrm{wd}}$ & $\mathbf{P}_{\mathrm{dw}}$ & $\mathbf{P}_{\mathrm{dd}}$ & $\mathbf{P}_{\mathrm{ww}}$ & $\mathbf{P}_{\mathrm{wd}}$ & $\mathbf{P}_{\mathrm{dw}}$ & $P_{d d}$ \\
\hline 653060 & 0.265 & 0.735 & 0.213 & 0.787 & 0.310 & 0.690 & 0.369 & 0.631 \\
\hline 653190 & 0.296 & 0.704 & 0.343 & 0.657 & 0.465 & 0.535 & 0.412 & 0.588 \\
\hline 653300 & 0.284 & 0.716 & 0.340 & 0.660 & 0.440 & 0.560 & 0.373 & 0.627 \\
\hline 653350 & 0.259 & 0.741 & 0.265 & 0.735 & 0.333 & 0.667 & 0.440 & 0.560 \\
\hline 653380 & 0.416 & 0.584 & 0.304 & 0.696 & 0.349 & 0.651 & 0.347 & 0.653 \\
\hline 653440 & 0.360 & 0.640 & 0.407 & 0.593 & 0.576 & 0.424 & 0.429 & 0.571 \\
\hline 653520 & 0.257 & 0.743 & 0.276 & 0.724 & 0.207 & 0.793 & 0.267 & 0.733 \\
\hline 653550 & 0.375 & 0.625 & 0.392 & 0.608 & 0.519 & 0.481 & 0.400 & 0.600 \\
\hline 653570 & 0.321 & 0.679 & 0.228 & 0.772 & 0.250 & 0.750 & 0.215 & 0.785 \\
\hline 653610 & 0.373 & 0.627 & 0.339 & 0.661 & 0.404 & 0.596 & 0.400 & 0.600 \\
\hline 653760 & 0.417 & 0.583 & 0.207 & 0.793 & 0.295 & 0.705 & 0.296 & 0.704 \\
\hline 653800 & 0.333 & 0.667 & 0.290 & 0.710 & 0.415 & 0.585 & 0.308 & 0.692 \\
\hline 653870 & 0.467 & 0.533 & 0.283 & 0.717 & 0.500 & 0.500 & 0.336 & 0.664 \\
\hline 654720 & 0.219 & 0.781 & 0.217 & 0.783 & 0.531 & 0.469 & 0.205 & 0.795 \\
\hline 655010 & 0.200 & 0.800 & 0.096 & 0.904 & 0.097 & 0.903 & 0.186 & 0.814 \\
\hline 655020 & 0.172 & 0.828 & 0.132 & 0.868 & 0.260 & 0.740 & 0.261 & 0.739 \\
\hline 655030 & 0.159 & 0.841 & 0.205 & 0.795 & 0.186 & 0.814 & 0.318 & 0.682 \\
\hline 655050 & 0.250 & 0.750 & 0.151 & 0.849 & 0.152 & 0.848 & 0.345 & 0.655 \\
\hline 655070 & 0.294 & 0.706 & 0.230 & 0.770 & 0.235 & 0.765 & 0.304 & 0.696 \\
\hline 655100 & 0.237 & 0.763 & 0.267 & 0.733 & 0.375 & 0.625 & 0.346 & 0.654 \\
\hline 655160 & 0.239 & 0.761 & 0.224 & 0.776 & 0.308 & 0.692 & 0.352 & 0.648 \\
\hline 655180 & 0.250 & 0.750 & 0.202 & 0.798 & 0.206 & 0.794 & 0.338 & 0.663 \\
\hline 655220 & 0.260 & 0.740 & 0.244 & 0.756 & 0.259 & 0.741 & 0.299 & 0.701 \\
\hline 655280 & 0.241 & 0.759 & 0.270 & 0.730 & 0.250 & 0.750 & 0.394 & 0.606 \\
\hline 655360 & 0.291 & 0.709 & 0.240 & 0.760 & 0.333 & 0.667 & 0.351 & 0.649 \\
\hline 655450 & 0.450 & 0.550 & 0.329 & 0.671 & 0.389 & 0.611 & 0.372 & 0.628 \\
\hline 655480 & 0.284 & 0.716 & 0.306 & 0.694 & 0.472 & 0.528 & 0.396 & 0.604 \\
\hline 655550 & 0.290 & 0.710 & 0.296 & 0.704 & 0.225 & 0.775 & 0.247 & 0.753 \\
\hline 655570 & 0.500 & 0.500 & 0.390 & 0.610 & 0.533 & 0.467 & 0.410 & 0.590 \\
\hline 655600 & 0.400 & 0.600 & 0.336 & 0.664 & 0.409 & 0.591 & 0.305 & 0.695 \\
\hline 655620 & 0.403 & 0.597 & 0.358 & 0.642 & 0.468 & 0.532 & 0.400 & 0.600 \\
\hline 655630 & 0.342 & 0.658 & 0.371 & 0.629 & 0.450 & 0.550 & 0.300 & 0.700 \\
\hline 655780 & 0.595 & 0.405 & 0.437 & 0.563 & 0.633 & 0.367 & 0.413 & 0.587 \\
\hline 655850 & 0.510 & 0.490 & 0.445 & 0.555 & 0.708 & 0.292 & 0.484 & 0.516 \\
\hline 655920 & 0.705 & 0.295 & 0.494 & 0.506 & 0.600 & 0.400 & 0.549 & 0.451 \\
\hline 655940 & 0.606 & 0.394 & 0.377 & 0.623 & 0.500 & 0.500 & 0.500 & 0.500 \\
\hline 655990 & 0.563 & 0.438 & 0.342 & 0.658 & 0.642 & 0.358 & 0.345 & 0.655 \\
\hline
\end{tabular}




\begin{tabular}{|c|c|c|c|c|c|c|c|c|}
\hline \multirow[t]{2}{*}{ Gauge } & \multicolumn{4}{|l|}{ Jul } & \multicolumn{4}{|l|}{ Aug } \\
\hline & $\mathbf{P}_{\mathrm{ww}}$ & $\mathbf{P}_{\mathrm{wd}}$ & $P_{d w}$ & $\mathbf{P}_{\mathrm{dd}}$ & $\mathbf{P}_{\mathbf{w w}}$ & $\mathbf{P}_{\mathbf{w d}}$ & $\mathbf{P}_{\mathrm{dw}}$ & $\mathbf{P}_{\mathrm{dd}}$ \\
\hline 653060 & 0.400 & 0.600 & 0.480 & 0.520 & 0.407 & $\begin{array}{l}0.593 \\
0.415\end{array}$ & $\begin{array}{l}0.520 \\
0630\end{array}$ & 0.480 \\
\hline 653190 & 0.358 & 0.642 & 0.531 & 0.469 & 0.585 & & 0.630 & 0.370 \\
\hline 653300 & 0.439 & 0.561 & 0.398 & 0.602 & 0.581 & 0.419 & 0.400 & 0.600 \\
\hline 653350 & 0.321 & 0.679 & 0.291 & 0.709 & 0.500 & 0.500 & 0.314 & 0.686 \\
\hline 653380 & 0.386 & 0.614 & 0.325 & 0.675 & 0.529 & 0.471 & 0.248 & 0.752 \\
\hline 653440 & 0.429 & 0.571 & 0.222 & 0.778 & 0.387 & 0.613 & 0.227 & 0.773 \\
\hline 653520 & 0.279 & 0.721 & 0.426 & 0.574 & 0.343 & 0.657 & 0.400 & 0.600 \\
\hline 653550 & 0.530 & 0.470 & 0.606 & 0.394 & 0.616 & 0.384 & 0.600 & 0.400 \\
\hline 653570 & 0.349 & 0.651 & 0.483 & 0.517 & 0.581 & 0.419 & 0.326 & 0.674 \\
\hline 653610 & 0.571 & 0.429 & 0.472 & 0.528 & 0.559 & 0.441 & 0.492 & 0.508 \\
\hline 653760 & 0.534 & 0.466 & 0.337 & 0.663 & 0.455 & 0.545 & 0.395 & 0.605 \\
\hline 653800 & 0.276 & 0.724 & 0.263 & 0.737 & 0.444 & 0.556 & 0.154 & 0.846 \\
\hline 653870 & 0.265 & 0.735 & 0.149 & 0.851 & 0.333 & 0.667 & 0.158 & 0.842 \\
\hline 654720 & 0.514 & 0.486 & 0.132 & 0.868 & 0.286 & 0.714 & 0.138 & 0.862 \\
\hline 655010 & 0.159 & 0.841 & 0.303 & 0.697 & 0.246 & 0.754 & 0.396 & 0.604 \\
\hline 655020 & 0.333 & 0.667 & 0.304 & 0.696 & 0.403 & 0.597 & 0.477 & 0.523 \\
\hline 655030 & 0.375 & 0.625 & 0.447 & 0.553 & 0.453 & 0.547 & 0.457 & 0.543 \\
\hline 655050 & 0.310 & 0.690 & 0.390 & 0.610 & 0.365 & 0.635 & 0.514 & 0.486 \\
\hline 655070 & 0.400 & 0.600 & 0.404 & 0.596 & 0.373 & 0.627 & 0.511 & 0.489 \\
\hline 655100 & 0.392 & 0.608 & 0.415 & 0.585 & 0.515 & 0.485 & 0.566 & 0.434 \\
\hline 655160 & 0.348 & 0.652 & 0.526 & 0.474 & 0.563 & 0.437 & 0.563 & 0.437 \\
\hline 655180 & 0.333 & 0.667 & 0.317 & 0.683 & 0.571 & 0.429 & 0.563 & 0.438 \\
\hline 655220 & 0.371 & 0.629 & 0.500 & 0.500 & 0.419 & 0.581 & 0.523 & 0.477 \\
\hline 655280 & 0.480 & 0.520 & 0.457 & 0.543 & 0.475 & 0.525 & 0.619 & 0.381 \\
\hline 655360 & 0.302 & 0.698 & 0.349 & 0.651 & 0.465 & 0.535 & 0.569 & 0.431 \\
\hline 655450 & 0.351 & 0.649 & 0.208 & 0.792 & 0.357 & 0.643 & 0.271 & 0.729 \\
\hline 655480 & 0.554 & 0.446 & 0.425 & 0.575 & 0.662 & 0.338 & 0.667 & 0.333 \\
\hline 655550 & 0.389 & 0.611 & 0.180 & 0.820 & 0.323 & 0.677 & 0.287 & 0.712 \\
\hline 655570 & 0.313 & 0.688 & 0.258 & 0.742 & 0.447 & 0.553 & 0.252 & 0.748 \\
\hline 655600 & 0.392 & 0.608 & 0.267 & 0.733 & 0.463 & 0.537 & 0.356 & 0.644 \\
\hline 655620 & 0.442 & 0.558 & 0.216 & 0.784 & 0.385 & 0.615 & 0.265 & 0.735 \\
\hline 655630 & 0.314 & 0.686 & 0.175 & 0.825 & 0.371 & 0.629 & 0.218 & 0.782 \\
\hline 655780 & 0.476 & 0.524 & 0.207 & 0.793 & 0.431 & 0.569 & 0.192 & 0.808 \\
\hline 655850 & 0.460 & 0.540 & 0.324 & 0.676 & 0.492 & 0.508 & 0.295 & 0.705 \\
\hline 655920 & 0.651 & 0.349 & 0.322 & 0.678 & 0.626 & 0.374 & 0.522 & 0.478 \\
\hline 655940 & 0.520 & 0.480 & 0.227 & 0.773 & 0.396 & 0.604 & 0.306 & 0.694 \\
\hline 655990 & 0.447 & 0.553 & 0.183 & 0.817 & 0.453 & 0.547 & 0.300 & 0.700 \\
\hline
\end{tabular}

1st-Order Conditioned Markov Transition Probabilities Warm Pacific Sea Surface Temperature Event 


\begin{tabular}{|c|c|c|c|c|c|c|c|c|}
\hline \multirow[b]{2}{*}{ Gauge } & \multicolumn{8}{|c|}{$\begin{array}{l}\text { 1st-Order Conditioned Markov Transition Probabilities } \\
\text { Warm Pacific Sea Surface Temperature Event }\end{array}$} \\
\hline & \multicolumn{4}{|c|}{ Sep } & \multicolumn{4}{|c|}{ Oct } \\
\hline & $\mathbf{P}_{\mathbf{w w}}$ & $\mathbf{P}_{\mathrm{wd}}$ & $\mathbf{P}_{\mathrm{dw}}$ & $P_{d d}$ & $\mathbf{P}_{\mathbf{w w}}$ & $\mathbf{P}_{\mathbf{w d}}$ & $\mathbf{P}_{\mathrm{dw}}$ & $\mathbf{P}_{\text {dd }}$ \\
\hline 653060 & 0.438 & 0.562 & 0.376 & 0.624 & 0.227 & & & 0.856 \\
\hline 653190 & 0.567 & 0.433 & 0.575 & 0.425 & 0.506 & 0.494 & 0.341 & 0.659 \\
\hline 653300 & 0.500 & 0.500 & 0.495 & 0.505 & 0.458 & 0.542 & 0.235 & 0.765 \\
\hline 653350 & 0.387 & 0.613 & 0.404 & 0.596 & 0.426 & 0.574 & 0.377 & 0.623 \\
\hline 653380 & 0.411 & 0.589 & 0.258 & 0.742 & 0.383 & 0.617 & 0.311 & 0.689 \\
\hline 653440 & 0.489 & 0.511 & 0.257 & 0.743 & 0.391 & 0.609 & 0.369 & 0.631 \\
\hline 653520 & 0.574 & 0.426 & 0.408 & 0.592 & 0.425 & 0.575 & 0.245 & 0.755 \\
\hline 653550 & 0.587 & 0.413 & 0.627 & 0.373 & 0.538 & 0.463 & 0.471 & 0.529 \\
\hline 653570 & 0.580 & 0.420 & 0.378 & 0.622 & 0.529 & 0.471 & 0.327 & 0.673 \\
\hline 653610 & 0.542 & 0.458 & 0.478 & 0.522 & 0.515 & 0.485 & 0.309 & 0.691 \\
\hline 653760 & 0.369 & 0.631 & 0.336 & 0.664 & 0.365 & 0.635 & 0.310 & 0.690 \\
\hline 653800 & 0.387 & 0.613 & 0.167 & 0.833 & 0.278 & 0.722 & 0.342 & 0.658 \\
\hline 653870 & 0.294 & 0.706 & 0.201 & 0.799 & 0.342 & 0.658 & 0.257 & 0.743 \\
\hline 654720 & 0.241 & 0.759 & 0.135 & 0.865 & 0.342 & 0.658 & 0.182 & 0.818 \\
\hline 655010 & 0.269 & 0.731 & 0.228 & 0.772 & 0.250 & 0.750 & 0.070 & 0.930 \\
\hline 655020 & 0.386 & 0.614 & 0.297 & 0.703 & 0.176 & 0.824 & 0.123 & 0.877 \\
\hline 655030 & 0.406 & 0.594 & 0.355 & 0.645 & 0.145 & 0.855 & 0.198 & 0.802 \\
\hline 655050 & 0.348 & 0.652 & 0.320 & 0.680 & 0.320 & 0.680 & 0.148 & 0.852 \\
\hline 655070 & 0.449 & 0.551 & 0.352 & 0.648 & 0.190 & 0.810 & 0.212 & 0.788 \\
\hline 655100 & 0.378 & 0.622 & 0.476 & 0.524 & 0.232 & 0.768 & 0.236 & 0.764 \\
\hline 655160 & 0.480 & 0.520 & 0.376 & 0.624 & 0.260 & 0.740 & 0.193 & 0.807 \\
\hline 655180 & 0.364 & 0.636 & 0.463 & 0.537 & 0.360 & 0.640 & 0.197 & 0.803 \\
\hline 655220 & 0.417 & 0.583 & 0.528 & 0.472 & 0.329 & 0.671 & 0.336 & 0.664 \\
\hline 655280 & 0.528 & 0.472 & 0.520 & 0.480 & 0.451 & 0.549 & 0.411 & 0.589 \\
\hline 655360 & 0.455 & 0.545 & 0.375 & 0.625 & 0.466 & 0.534 & 0.347 & 0.653 \\
\hline 655450 & 0.435 & 0.565 & 0.398 & 0.602 & 0.459 & 0.541 & 0.288 & 0.712 \\
\hline 655480 & 0.632 & 0.368 & 0.469 & 0.531 & 0.500 & 0.500 & 0.340 & 0.660 \\
\hline 655550 & 0.449 & 0.551 & 0.268 & 0.732 & 0.378 & 0.622 & 0.223 & 0.777 \\
\hline 655570 & 0.517 & 0.483 & 0.379 & 0.621 & 0.291 & 0.709 & 0.455 & 0.545 \\
\hline 655600 & 0.587 & 0.413 & 0.262 & 0.738 & 0.302 & 0.698 & 0.318 & 0.682 \\
\hline 655620 & 0.426 & 0.574 & 0.315 & 0.685 & 0.344 & 0.656 & 0.315 & 0.685 \\
\hline 655630 & 0.333 & 0.667 & 0.301 & 0.699 & 0.320 & 0.680 & 0.312 & 0.688 \\
\hline 655780 & 0.397 & 0.603 & 0.186 & 0.814 & 0.512 & 0.488 & 0.361 & 0.639 \\
\hline 655850 & 0.507 & 0.493 & 0.374 & 0.626 & 0.522 & 0.478 & 0.463 & 0.537 \\
\hline 655920 & 0.702 & 0.298 & 0.530 & 0.470 & 0.536 & 0.464 & 0.429 & 0.571 \\
\hline 655940 & 0.511 & 0.489 & 0.224 & 0.776 & 0.489 & 0.511 & 0.281 & 0.719 \\
\hline 655990 & 0.433 & 0.567 & 0.230 & 0.770 & 0.440 & 0.560 & 0.281 & 0.719 \\
\hline
\end{tabular}




\begin{tabular}{|c|c|c|c|c|c|c|c|c|}
\hline \multirow{3}{*}{ Gauge } & \multicolumn{8}{|c|}{$\begin{array}{l}\text { 1st-Order Conditioned Markov Transition Prob } \\
\text { Warm Pacific Sea Surface Temperature Event }\end{array}$} \\
\hline & \multicolumn{4}{|c|}{ Nov } & \multicolumn{4}{|c|}{ Dec } \\
\hline & $\mathbf{P}_{\mathrm{ww}}$ & $\mathbf{P}_{\mathrm{wd}}$ & $\mathbf{P}_{\mathrm{dw}}$ & $\mathbf{P}_{\mathrm{dd}}$ & $\mathbf{P}_{\text {ww }}$ & $P_{\text {wd }}$ & $\mathbf{P}_{\mathrm{dw}}$ & $\mathbf{P}_{\mathrm{dd}}$ \\
\hline 653060 & 0.400 & 0.600 & 0.011 & 0.989 & 0.000 & 0.000 & 0.000 & 1.000 \\
\hline 653190 & 0.357 & 0.643 & 0.032 & 0.968 & 0.000 & 0.000 & 0.000 & 1.000 \\
\hline 653300 & 0.250 & 0.750 & 0.030 & 0.970 & 0.000 & 0.000 & 0.000 & 1.000 \\
\hline 653350 & 0.200 & 0.800 & 0.020 & 0.980 & 0.000 & 1.000 & 0.004 & 0.996 \\
\hline 653380 & 0.261 & 0.739 & 0.071 & 0.929 & 0.000 & 1.000 & 0.008 & 0.992 \\
\hline 653440 & 0.200 & 0.800 & 0.121 & 0.879 & 0.125 & 0.875 & 0.022 & 0.978 \\
\hline 653520 & 0.143 & 0.857 & 0.043 & 0.957 & 0.000 & 1.000 & 0.006 & 0.994 \\
\hline 653550 & 0.471 & 0.529 & 0.042 & 0.958 & 0.000 & 0.000 & 0.000 & 1.000 \\
\hline 653570 & 0.000 & 1.000 & 0.020 & 0.980 & 0.000 & 0.000 & 0.000 & 1.000 \\
\hline 653610 & 0.333 & 0.667 & 0.047 & 0.953 & 0.000 & 0.000 & 0.000 & 1.000 \\
\hline 653760 & 0.091 & 0.909 & 0.042 & 0.958 & 0.000 & 1.000 & 0.011 & 0.989 \\
\hline 653800 & 0.333 & 0.667 & 0.075 & 0.925 & 0.000 & 0.000 & 0.000 & 1.000 \\
\hline 653870 & 0.125 & 0.875 & 0.079 & 0.921 & 0.000 & 1.000 & 0.035 & 0.965 \\
\hline 654720 & 0.000 & 1.000 & 0.071 & 0.929 & 0.000 & 1.000 & 0.033 & 0.967 \\
\hline 655010 & 0.000 & 0.000 & 0.000 & 1.000 & 0.000 & 1.000 & 0.004 & 0.996 \\
\hline 655020 & 0.000 & 0.000 & 0.000 & 1.000 & 0.000 & 1.000 & 0.004 & 0.996 \\
\hline 655030 & 0.000 & 1.000 & 0.014 & 0.986 & 0.000 & 1.000 & 0.003 & 0.997 \\
\hline 655050 & 0.250 & 0.750 & 0.015 & 0.985 & 0.000 & 1.000 & 0.007 & 0.993 \\
\hline 655070 & 0.250 & 0.750 & 0.010 & 0.990 & 0.000 & 0.000 & 0.000 & 1.000 \\
\hline 655100 & 0.231 & 0.769 & 0.037 & 0.963 & 0.000 & 0.000 & 0.000 & 1.000 \\
\hline 655160 & 0.250 & 0.750 & 0.013 & 0.987 & 0.000 & 0.000 & 0.000 & 1.000 \\
\hline 655180 & 0.400 & 0.600 & 0.031 & 0.969 & 0.000 & 0.000 & 0.000 & 1.000 \\
\hline 655220 & 0.000 & 1.000 & 0.049 & 0.951 & 0.000 & 0.000 & 0.000 & 1.000 \\
\hline 655280 & 0.450 & 0.550 & 0.055 & 0.945 & 0.000 & 1.000 & 0.011 & 0.989 \\
\hline 655360 & 0.350 & 0.650 & 0.056 & 0.944 & 0.000 & 0.000 & 0.000 & 1.000 \\
\hline 655450 & 0.222 & 0.778 & 0.056 & 0.944 & 0.200 & 0.800 & 0.018 & 0.982 \\
\hline 655480 & 0.240 & 0.760 & 0.107 & 0.893 & 0.000 & 1.000 & 0.006 & 0.994 \\
\hline 655550 & 0.286 & 0.714 & 0.062 & 0.938 & 0.000 & 1.000 & 0.012 & 0.988 \\
\hline 655570 & 0.304 & 0.696 & 0.253 & 0.747 & 0.176 & 0.824 & 0.065 & 0.935 \\
\hline 655600 & 0.259 & 0.741 & 0.110 & 0.890 & 0.250 & 0.750 & 0.030 & 0.970 \\
\hline 655620 & 0.172 & 0.828 & 0.124 & 0.876 & 0.000 & 1.000 & 0.020 & 0.980 \\
\hline 655630 & 0.115 & 0.885 & 0.177 & 0.823 & 0.167 & 0.833 & 0.013 & 0.987 \\
\hline 655780 & 0.382 & 0.618 & 0.247 & 0.753 & 0.341 & 0.659 & 0.097 & 0.903 \\
\hline 655850 & 0.333 & 0.667 & 0.372 & 0.628 & 0.161 & 0.839 & 0.114 & 0.886 \\
\hline 655920 & 0.485 & 0.515 & 0.343 & 0.657 & 0.333 & 0.667 & 0.194 & 0.806 \\
\hline 655940 & 0.457 & 0.543 & 0.268 & 0.732 & 0.192 & 0.808 & 0.157 & 0.843 \\
\hline 655990 & 0.250 & 0.750 & 0.224 & 0.776 & 0.261 & 0.739 & 0.075 & 0.925 \\
\hline
\end{tabular}



Appendix E: Gamma Distribution Parameters for Wet Day Amounts 


\begin{tabular}{|c|c|c|c|c|c|c|c|c|}
\hline \multirow[b]{2}{*}{ Gauges } & \multicolumn{8}{|c|}{ Gamma Parameters } \\
\hline & $\begin{array}{l}\text { Jan } \\
\text { alpha }\end{array}$ & beta & $\begin{array}{l}\text { Feb } \\
\text { alpha }\end{array}$ & beta & $\begin{array}{l}\text { Mar } \\
\text { alpha }\end{array}$ & beta & $\begin{array}{l}\text { Apr } \\
\text { alpha }\end{array}$ & beta \\
\hline 653060 & 0.000 & 0.000 & 1.101 & 0.956 & 1.003 & 0.444 & 0.675 & 0.703 \\
\hline 653190 & 0.399 & 1.427 & 0.848 & 0.298 & 0.728 & 0.497 & 0.665 & 0.786 \\
\hline 653300 & 0.726 & 0.377 & 0.864 & 0.386 & 0.676 & 0.626 & 0.617 & 0.965 \\
\hline 653350 & 0.628 & 0.568 & 0.595 & 1.429 & 0.708 & 0.784 & 0.753 & 0.716 \\
\hline 653380 & 0.397 & 2.799 & 0.819 & 0.514 & 0.707 & 0.644 & 0.730 & 0.753 \\
\hline 653440 & 0.449 & 1.200 & 0.608 & 1.076 & 0.589 & 0.968 & 0.622 & 1.398 \\
\hline 653520 & 0.000 & 0.200 & 0.862 & 0.315 & 0.459 & 1.989 & 0.818 & 0.453 \\
\hline 653550 & 0.000 & 1.100 & 0.458 & 1.627 & 0.603 & 0.667 & 0.709 & 0.554 \\
\hline 653570 & 0.481 & 1.605 & 0.721 & 0.509 & 0.482 & 1.188 & 0.972 & 0.424 \\
\hline 653610 & 0.879 & 0.538 & 0.980 & 0.347 & 0.869 & 0.448 & 0.675 & 0.621 \\
\hline 653760 & 0.878 & 0.251 & 0.860 & 0.375 & 0.574 & 0.828 & 0.693 & 0.795 \\
\hline 653800 & 0.892 & 1.191 & 0.389 & 1.923 & 0.801 & 0.634 & 0.719 & 0.886 \\
\hline 653870 & 0.755 & 0.550 & 0.492 & 1.131 & 0.647 & 0.850 & 0.542 & 1.295 \\
\hline 654720 & 0.578 & 0.588 & 0.523 & 0.825 & 0.705 & 0.682 & 0.554 & 1.655 \\
\hline 655010 & 0.000 & 0.470 & 3.908 & 0.302 & 0.776 & 0.353 & 1.506 & 0.119 \\
\hline 655020 & 0.000 & 0.080 & 0.000 & 2.400 & 0.639 & 0.773 & 0.908 & 0.277 \\
\hline 655030 & 0.000 & 0.000 & 0.763 & 0.681 & 0.448 & 1.301 & 0.868 & 0.539 \\
\hline 655050 & 0.000 & 0.020 & 1.027 & 1.305 & 0.726 & 0.412 & 0.599 & 0.541 \\
\hline 655070 & 0.000 & 0.000 & 0.795 & 0.842 & 0.464 & 2.645 & 0.753 & 0.428 \\
\hline 655100 & 0.903 & 0.753 & 0.725 & 0.536 & 0.728 & 0.357 & 0.702 & 0.533 \\
\hline 655160 & 1.803 & 0.094 & 0.000 & 0.160 & 0.507 & 0.948 & 0.599 & 0.882 \\
\hline 655180 & 0.000 & 0.000 & 1.138 & 0.161 & 0.573 & 0.769 & 0.620 & 0.651 \\
\hline 655220 & 0.515 & 1.697 & 0.749 & 0.437 & 0.854 & 0.657 & 0.678 & 0.707 \\
\hline 655280 & 1.228 & 0.236 & 0.611 & 0.888 & 0.477 & 0.988 & 0.770 & 0.604 \\
\hline 655360 & 0.868 & 0.417 & 0.982 & 0.913 & 0.653 & 0.898 & 0.756 & 0.776 \\
\hline 655450 & 0.469 & 1.845 & 0.632 & 0.893 & 0.633 & 0.840 & 0.676 & 0.980 \\
\hline 655480 & 0.627 & 0.834 & 0.723 & 0.451 & 0.632 & 1.068 & 0.636 & 1.211 \\
\hline 655550 & 0.586 & 1.563 & 0.678 & 0.889 & 0.538 & 1.520 & 0.717 & 1.169 \\
\hline 655570 & 0.862 & 0.500 & 0.671 & 1.022 & 0.607 & 1.168 & 0.718 & 0.932 \\
\hline 655600 & 1.114 & 0.202 & 0.722 & 0.773 & 0.628 & 0.777 & 0.737 & 0.911 \\
\hline 655620 & 0.550 & 0.711 & 1.210 & 0.333 & 0.638 & 0.752 & 0.708 & 0.946 \\
\hline 655630 & 0.584 & 1.233 & 0.768 & 0.415 & 0.827 & 0.419 & 0.874 & 0.574 \\
\hline 655780 & 0.593 & 0.712 & 0.630 & 0.650 & 0.637 & 0.856 & 0.650 & 0.913 \\
\hline 655850 & 0.726 & 0.556 & 0.589 & 0.934 & 0.566 & 0.888 & 0.628 & 0.900 \\
\hline 655920 & 0.642 & 0.600 & 0.656 & 0.532 & 0.617 & 0.820 & 0.636 & 0.884 \\
\hline 655940 & 0.781 & 0.427 & 0.623 & 0.472 & 0.625 & 0.750 & 0.637 & 0.954 \\
\hline 655990 & 0.593 & 0.516 & 0.857 & 0.591 & 0.693 & 0.637 & 0.663 & 1.100 \\
\hline
\end{tabular}




\begin{tabular}{|c|c|c|c|c|c|c|c|c|}
\hline \multirow[b]{2}{*}{ Gauges } & \multicolumn{8}{|c|}{ Gamma Parameters } \\
\hline & $\begin{array}{l}\text { May } \\
\text { alpha }\end{array}$ & beta & $\begin{array}{l}\text { Jun } \\
\text { alpha }\end{array}$ & beta & $\begin{array}{l}\text { Jul } \\
\text { alpha }\end{array}$ & beta & $\begin{array}{l}\text { Aug } \\
\text { alpha }\end{array}$ & beta \\
\hline 653060 & 0.771 & 0.695 & 0.777 & 0.763 & 0.748 & 0.887 & 0.696 & 0.851 \\
\hline 653190 & 0.731 & 0.620 & 0.821 & 0.546 & 0.770 & 0.565 & 0.711 & 0.789 \\
\hline 653300 & 0.833 & 0.584 & 0.665 & 0.772 & 0.637 & 0.949 & 0.651 & 0.893 \\
\hline 653350 & 0.755 & 0.653 & 0.708 & 0.593 & 0.572 & 0.996 & 0.604 & 0.796 \\
\hline 653380 & 0.776 & 0.641 & 0.699 & 0.780 & 0.541 & 1.013 & 0.614 & 0.674 \\
\hline 653440 & 0.660 & 1.031 & 0.544 & 1.481 & 0.496 & 0.907 & 0.452 & 0.853 \\
\hline 653520 & 0.778 & 0.654 & 0.582 & 1.145 & 0.589 & 1.069 & 0.816 & 0.617 \\
\hline 653550 & 0.790 & 0.508 & 0.667 & 0.683 & 0.574 & 1.051 & 0.701 & 0.968 \\
\hline 653570 & 0.804 & 0.673 & 0.729 & 0.913 & 0.792 & 0.573 & 0.654 & 0.743 \\
\hline 653610 & 0.711 & 0.615 & 0.812 & 0.506 & 0.679 & 0.734 & 0.783 & 0.593 \\
\hline 653760 & 0.642 & 0.996 & 0.679 & 0.908 & 0.714 & 0.671 & 0.633 & 0.810 \\
\hline 653800 & 0.663 & 1.014 & 0.569 & 1.104 & 0.650 & 0.535 & 0.445 & 0.816 \\
\hline 653870 & 0.563 & 1.093 & 0.597 & 0.922 & 0.503 & 0.891 & 0.589 & 0.373 \\
\hline 654720 & 0.874 & 0.732 & 0.544 & 0.955 & 0.436 & 1.161 & 0.659 & 0.197 \\
\hline 655010 & 0.645 & 0.425 & 0.714 & 0.526 & 0.708 & 0.691 & 0.737 & 0.769 \\
\hline 655020 & 0.551 & 0.984 & 0.709 & 0.685 & 0.855 & 0.595 & 0.790 & 0.624 \\
\hline 655030 & 0.683 & 0.689 & 0.797 & 0.550 & 0.743 & 0.760 & 0.805 & 0.650 \\
\hline 655050 & 0.757 & 0.573 & 0.685 & 0.840 & 0.922 & 0.630 & 0.852 & 0.643 \\
\hline 655070 & 0.594 & 1.013 & 0.718 & 0.878 & 0.778 & 0.800 & 0.646 & 1.035 \\
\hline 655100 & 0.716 & 0.619 & 0.767 & 0.611 & 0.743 & 0.679 & 0.738 & 0.846 \\
\hline 655160 & 0.789 & 0.510 & 0.898 & 0.481 & 0.785 & 0.703 & 0.852 & 0.646 \\
\hline 655180 & 0.824 & 0.486 & 0.839 & 0.665 & 0.615 & 1.050 & 0.772 & 0.801 \\
\hline 655220 & 0.677 & 0.780 & 0.823 & 0.702 & 0.749 & 0.752 & 0.788 & 0.736 \\
\hline 655280 & 0.835 & 0.598 & 0.925 & 0.517 & 0.612 & 1.249 & 0.711 & 0.876 \\
\hline 655360 & 0.742 & 0.752 & 0.838 & 0.702 & 0.813 & 0.733 & 0.710 & 0.921 \\
\hline 655450 & 0.723 & 0.822 & 0.812 & 0.706 & 0.634 & 0.705 & 0.540 & 1.048 \\
\hline 655480 & 0.751 & 0.703 & 0.559 & 1.350 & 0.550 & 0.911 & 0.595 & 1.115 \\
\hline 655550 & 0.849 & 0.626 & 0.746 & 0.684 & 0.492 & 1.589 & 0.551 & 1.109 \\
\hline 655570 & 0.873 & 0.562 & 0.757 & 0.606 & 0.767 & 0.388 & 0.629 & 0.477 \\
\hline 655600 & 0.851 & 0.561 & 0.548 & 1.122 & 0.649 & 0.451 & 0.597 & 0.645 \\
\hline 655620 & 0.722 & 0.721 & 0.630 & 0.781 & 0.631 & 0.530 & 0.459 & 0.834 \\
\hline 655630 & 0.736 & 0.727 & 0.711 & 0.670 & 0.566 & 0.833 & 0.612 & 0.586 \\
\hline 655780 & 0.567 & 1.174 & 0.576 & 1.684 & 0.524 & 0.841 & 0.466 & 0.642 \\
\hline 655850 & 0.612 & 1.026 & 0.629 & 1.349 & 0.462 & 1.096 & 0.578 & 0.387 \\
\hline 655920 & 0.614 & 1.230 & 0.528 & 1.707 & 0.508 & 1.093 & 0.588 & 0.626 \\
\hline 655940 & 0.608 & 1.209 & 0.613 & 1.122 & 0.506 & 0.998 & 0.692 & 0.295 \\
\hline 655990 & 0.764 & 0.912 & 0.652 & 1.110 & 0.496 & 0.859 & 0.579 & 0.385 \\
\hline
\end{tabular}




\begin{tabular}{|c|c|c|c|c|c|c|c|c|}
\hline \multirow[b]{2}{*}{ Gauges } & \multicolumn{8}{|c|}{ Gamma Parameters } \\
\hline & $\begin{array}{l}\text { Sep } \\
\text { alpha }\end{array}$ & beta & $\begin{array}{l}\text { Oct } \\
\text { alpha }\end{array}$ & beta & $\begin{array}{l}\text { Nov } \\
\text { alpha }\end{array}$ & beta & $\begin{array}{l}\text { Dec } \\
\text { alpha }\end{array}$ & beta \\
\hline 653060 & 0.780 & 0.677 & 0.826 & 0.394 & 0.690 & 0.169 & 0.000 & 2.130 \\
\hline 653190 & 0.902 & 0.560 & 0.854 & 0.425 & 1.016 & 0.360 & 4.764 & 0.045 \\
\hline 653300 & 0.782 & 0.673 & 0.818 & 0.540 & 0.586 & 0.944 & 2.119 & 0.073 \\
\hline 653350 & 0.691 & 0.645 & 0.801 & 0.527 & 0.911 & 0.422 & 1.741 & 0.126 \\
\hline 653380 & 0.651 & 0.675 & 0.721 & 0.686 & 0.508 & 0.905 & 0.873 & 1.005 \\
\hline 653440 & 0.585 & 0.724 & 0.666 & 0.792 & 0.773 & 0.480 & 0.375 & 2.098 \\
\hline 653520 & 0.817 & 0.549 & 0.831 & 0.507 & 0.387 & 3.090 & 7.355 & 0.185 \\
\hline 653550 & 0.845 & 0.590 & 0.723 & 0.523 & 0.925 & 0.581 & 0.537 & 0.437 \\
\hline 653570 & 0.837 & 0.647 & 1.138 & 0.403 & 1.520 & 0.301 & 0.859 & 0.457 \\
\hline 653610 & 0.758 & 0.605 & 0.834 & 0.511 & 0.541 & 1.357 & 1.133 & 0.124 \\
\hline 653760 & 0.767 & 0.702 & 0.841 & 0.514 & 0.733 & 0.358 & 0.603 & 1.094 \\
\hline 653800 & 0.569 & 0.745 & 0.746 & 0.515 & 0.751 & 0.400 & 0.610 & 1.557 \\
\hline 653870 & 0.593 & 0.581 & 0.681 & 0.636 & 0.836 & 0.310 & 0.613 & 0.574 \\
\hline 654720 & 0.451 & 0.811 & 0.612 & 0.590 & 0.765 & 0.494 & 0.957 & 0.467 \\
\hline 655010 & 0.805 & 0.442 & 0.447 & 0.997 & 0.330 & 3.716 & 1.210 & 1.997 \\
\hline 655020 & 0.747 & 0.628 & 0.857 & 0.302 & 0.588 & 1.208 & 8.654 & 0.007 \\
\hline 655030 & 0.650 & 0.913 & 0.708 & 0.444 & 0.481 & 1.352 & 0.739 & 0.947 \\
\hline 655050 & 0.832 & 0.521 & 0.805 & 0.407 & 0.791 & 0.303 & 2.631 & 0.688 \\
\hline 655070 & 0.802 & 0.565 & 0.833 & 0.341 & 0.591 & 0.533 & 0.467 & 2.617 \\
\hline 655100 & 0.758 & 0.640 & 0.826 & 0.449 & 0.719 & 0.550 & 0.976 & 0.190 \\
\hline 655160 & 0.778 & 0.547 & 0.895 & 0.413 & 0.781 & 0.393 & 0.466 & 2.662 \\
\hline 655180 & 0.954 & 0.459 & 0.997 & 0.343 & 1.168 & 0.217 & 32.153 & 0.018 \\
\hline 655220 & 0.988 & 0.477 & 0.851 & 0.458 & 0.508 & 0.744 & 0.331 & 2.087 \\
\hline 655280 & 0.849 & 0.657 & 0.784 & 0.634 & 0.785 & 0.613 & 0.683 & 0.568 \\
\hline 655360 & 0.906 & 0.568 & 0.700 & 0.587 & 0.621 & 0.506 & 1.134 & 0.845 \\
\hline 655450 & 0.747 & 0.578 & 0.803 & 0.562 & 0.839 & 0.337 & 0.724 & 0.471 \\
\hline 655480 & 0.829 & 0.623 & 0.764 & 0.626 & 0.836 & 0.428 & 0.558 & 1.189 \\
\hline 655550 & 0.745 & 0.711 & 0.837 & 0.570 & 0.720 & 0.536 & 0.608 & 0.948 \\
\hline 655570 & 0.654 & 0.657 & 0.815 & 0.471 & 0.874 & 0.408 & 0.788 & 0.528 \\
\hline 655600 & 0.682 & 0.648 & 0.757 & 0.479 & 0.942 & 0.300 & 0.860 & 0.743 \\
\hline 655620 & 0.611 & 0.684 & 0.710 & 0.577 & 0.744 & 0.498 & 0.848 & 0.663 \\
\hline 655630 & 0.667 & 0.568 & 0.750 & 0.492 & 0.962 & 0.360 & 0.805 & 0.411 \\
\hline 655780 & 0.380 & 1.486 & 0.535 & 0.848 & 0.786 & 0.519 & 0.559 & 1.005 \\
\hline 655850 & 0.521 & 0.661 & 0.576 & 0.840 & 0.656 & 0.574 & 0.523 & 1.005 \\
\hline 655920 & 0.601 & 0.754 & 0.492 & 1.016 & 0.634 & 0.582 & 0.539 & 1.008 \\
\hline 655940 & 0.640 & 0.389 & 0.562 & 0.552 & 0.637 & 0.666 & 0.598 & 0.863 \\
\hline 655990 & 0.615 & 0.373 & 0.649 & 0.535 & 0.681 & 0.564 & 0.707 & 0.654 \\
\hline
\end{tabular}




\section{Appendix F: Mixed Exponential Distribution Parameters for Wet Day Amounts}




\begin{tabular}{lllllll}
\multicolumn{7}{l}{ Mixed Exponential Parameters } \\
Gauges & Jan & & \multicolumn{3}{l}{ Feb } \\
& alpha & beta 1 & beta 2 & alpha & beta 1 & beta 2 \\
$\mathbf{6 5 3 0 6 0}$ & 1.000 & 0.000 & 0.000 & 0.918 & 1.143 & 0.040 \\
$\mathbf{6 5 3 1 9 0}$ & 0.352 & 1.563 & 0.030 & 0.304 & 0.069 & 0.333 \\
$\mathbf{6 5 3 3 0 0}$ & 0.471 & 0.507 & 0.066 & 0.818 & 0.398 & 0.042 \\
$\mathbf{6 5 3 3 5 0}$ & 0.373 & 0.026 & 0.553 & 0.650 & 0.212 & 2.038 \\
$\mathbf{6 5 3 3 8 0}$ & 0.484 & 0.048 & 2.107 & 0.332 & 0.790 & 0.238 \\
$\mathbf{6 5 3 4 4 0}$ & 0.469 & 1.103 & 0.041 & 0.460 & 0.115 & 1.114 \\
$\mathbf{6 5 3 5 2 0}$ & 1.000 & 0.200 & 29.407 & 0.767 & 0.346 & 0.029 \\
$\mathbf{6 5 3 5 5 0}$ & 1.000 & 1.100 & 39.622 & 0.425 & 0.044 & 1.263 \\
$\mathbf{6 5 3 5 7 0}$ & 0.561 & 1.338 & 0.049 & 0.606 & 0.579 & 0.040 \\
$\mathbf{6 5 3 6 1 0}$ & 0.222 & 0.071 & 0.588 & 0.866 & 0.390 & 0.014 \\
$\mathbf{6 5 3 7 6 0}$ & 0.164 & 0.777 & 0.111 & 0.541 & 0.153 & 0.522 \\
$\mathbf{6 5 3 8 0 0}$ & 0.764 & 1.342 & 0.157 & 0.742 & 0.124 & 2.542 \\
$\mathbf{6 5 3 8 7 0}$ & 0.843 & 0.487 & 0.029 & 0.795 & 0.160 & 2.095 \\
$\mathbf{6 5 4 7 2 0}$ & 0.535 & 0.048 & 0.675 & 0.738 & 0.117 & 1.319 \\
$\mathbf{6 5 5 0 1 0}$ & 0.000 & 0.471 & 0.470 & 1.000 & 1.180 & 23.921 \\
$\mathbf{6 5 5 0 2 0}$ & 1.000 & 0.080 & 128 & 0.999 & 2.400 & 2.400 \\
$\mathbf{6 5 5 0 3 0}$ & 1.000 & 0.000 & 0.000 & 0.587 & 0.140 & 1.059 \\
$\mathbf{6 5 5 0 5 0}$ & 1.000 & 0.020 & 108 & 0.253 & 0.242 & 1.712 \\
$\mathbf{6 5 5 0 7 0}$ & 1.000 & 0.000 & 0.000 & 0.379 & 0.080 & 1.029 \\
$\mathbf{6 5 5 1 0 0}$ & 0.320 & 0.174 & 0.918 & 0.318 & 0.847 & 0.175 \\
$\mathbf{6 5 5 1 6 0}$ & 0.000 & 499 & 0.170 & 0.000 & 0.162 & 0.160 \\
$\mathbf{6 5 5 1 8 0}$ & 1.000 & 0.000 & 0.000 & 0.295 & 0.061 & 0.235 \\
$\mathbf{6 5 5 2 2 0}$ & 0.606 & 0.148 & 1.989 & 0.543 & 0.548 & 0.065 \\
$\mathbf{6 5 5 2 8 0}$ & 1.000 & 0.290 & 18.883 & 0.298 & 0.029 & 0.760 \\
$\mathbf{6 5 5 3 6 0}$ & 0.381 & 0.719 & 0.143 & 0.911 & 0.974 & 0.105 \\
$\mathbf{6 5 5 4 5 0}$ & 0.408 & 0.032 & 1.440 & 0.835 & 0.274 & 2.031 \\
$\mathbf{6 5 5 4 8 0}$ & 0.636 & 0.801 & 0.037 & 0.817 & 0.394 & 0.023 \\
$\mathbf{6 5 5 5 5 0}$ & 0.409 & 1.866 & 0.258 & 0.844 & 0.602 & 0.033 \\
$\mathbf{6 5 5 5 7 0}$ & 0.535 & 0.201 & 0.696 & 0.936 & 0.423 & 4.551 \\
$\mathbf{6 5 5 6 0 0}$ & 0.214 & 0.147 & 0.247 & 0.224 & 0.056 & 0.703 \\
$\mathbf{6 5 5 6 2 0}$ & 0.624 & 0.612 & 0.024 & 0.000 & 0.403 & 0.403 \\
$\mathbf{6 5 5 6 3 0}$ & 0.246 & 0.028 & 0.947 & 0.609 & 0.470 & 0.082 \\
$\mathbf{6 5 5 7 8 0}$ & 0.503 & 0.081 & 0.769 & 0.950 & 0.264 & 3.158 \\
$\mathbf{6 5 5 8 5 0}$ & 0.291 & 0.060 & 0.545 & 0.380 & 0.066 & 0.847 \\
$\mathbf{6 5 5 9 2 0}$ & 0.577 & 0.106 & 0.766 & 0.336 & 0.047 & 0.502 \\
$\mathbf{6 5 5 9 4 0}$ & 0.343 & 0.100 & 0.456 & 0.904 & 0.157 & 1.576 \\
$\mathbf{6 5 5 9 9 0}$ & 0.600 & 0.482 & 0.042 & 0.953 & 0.403 & 2.574
\end{tabular}




\begin{tabular}{lllllll}
\multicolumn{7}{l}{ Mixed Exponential Parameters } \\
Gauges & Mar & & \multicolumn{3}{l}{ Apr } & \\
& alpha & beta 1 & beta 2 & alpha & beta 1 & beta 2 \\
$\mathbf{6 5 3 0 6 0}$ & 0.592 & 0.555 & 0.285 & 0.509 & 0.143 & 0.819 \\
$\mathbf{6 5 3 1 9 0}$ & 0.439 & 0.090 & 0.575 & 0.559 & 0.850 & 0.107 \\
$\mathbf{6 5 3 3 0 0}$ & 0.541 & 0.115 & 0.787 & 0.292 & 0.062 & 0.815 \\
$\mathbf{6 5 3 3 5 0}$ & 0.779 & 0.697 & 0.053 & 0.724 & 0.706 & 0.102 \\
$\mathbf{6 5 3 3 8 0}$ & 0.234 & 0.051 & 0.579 & 0.285 & 0.097 & 0.730 \\
$\mathbf{6 5 3 4 4 0}$ & 0.318 & 0.053 & 0.811 & 0.932 & 0.606 & 4.505 \\
$\mathbf{6 5 3 5 2 0}$ & 0.623 & 1.436 & 0.050 & 0.275 & 0.077 & 0.482 \\
$\mathbf{6 5 3 5 5 0}$ & 0.493 & 0.742 & 0.071 & 0.704 & 0.535 & 0.054 \\
$\mathbf{6 5 3 5 7 0}$ & 0.882 & 0.206 & 3.311 & 0.481 & 0.231 & 0.581 \\
$\mathbf{6 5 3 6 1 0}$ & 0.216 & 0.060 & 0.480 & 0.386 & 0.866 & 0.138 \\
$\mathbf{6 5 3 7 6 0}$ & 0.577 & 0.773 & 0.069 & 0.432 & 0.132 & 0.870 \\
$\mathbf{6 5 3 8 0 0}$ & 0.727 & 0.664 & 0.090 & 0.662 & 0.906 & 0.111 \\
$\mathbf{6 5 3 8 7 0}$ & 0.403 & 1.115 & 0.168 & 0.580 & 0.167 & 1.441 \\
$\mathbf{6 5 4 7 2 0}$ & 0.374 & 0.085 & 0.717 & 0.595 & 0.185 & 1.992 \\
$\mathbf{6 5 5 0 1 0}$ & 0.403 & 0.509 & 0.114 & 1.000 & 0.179 & 1057 \\
$\mathbf{6 5 5 0 2 0}$ & 0.520 & 0.847 & 0.112 & 0.479 & 0.106 & 0.385 \\
$\mathbf{6 5 5 0 3 0}$ & 0.177 & 2.586 & 0.154 & 0.120 & 0.046 & 0.525 \\
$\mathbf{6 5 5 0 5 0}$ & 0.145 & 1.240 & 0.140 & 0.543 & 0.069 & 0.627 \\
$\mathbf{6 5 5 0 7 0}$ & 0.439 & 2.589 & 0.159 & 0.455 & 0.089 & 0.517 \\
$\mathbf{6 5 5 1 0 0}$ & 0.444 & 0.065 & 0.416 & 0.455 & 0.089 & 0.613 \\
$\mathbf{6 5 5 1 6 0}$ & 0.865 & 0.141 & 2.659 & 0.783 & 0.219 & 1.642 \\
$\mathbf{6 5 5 1 8 0}$ & 0.258 & 1.258 & 0.156 & 0.451 & 0.754 & 0.117 \\
$\mathbf{6 5 5 2 2 0}$ & 0.104 & 0.061 & 0.619 & 0.612 & 0.724 & 0.091 \\
$\mathbf{6 5 5 2 8 0}$ & 0.948 & 0.220 & 5.052 & 0.705 & 0.617 & 0.102 \\
$\mathbf{6 5 5 3 6 0}$ & 0.471 & 1.074 & 0.152 & 0.447 & 0.187 & 0.911 \\
$\mathbf{6 5 5 4 5 0}$ & 0.435 & 0.085 & 0.876 & 0.885 & 0.434 & 2.427 \\
$\mathbf{6 5 5 4 8 0}$ & 0.851 & 0.369 & 2.425 & 0.432 & 0.167 & 1.228 \\
$\mathbf{6 5 5 5 5 0}$ & 0.828 & 0.316 & 3.230 & 0.051 & 6.053 & 0.556 \\
$\mathbf{6 5 5 5 7 0}$ & 0.968 & 0.435 & 8.924 & 0.989 & 0.579 & 9.058 \\
$\mathbf{6 5 5 6 0 0}$ & 0.347 & 0.070 & 0.710 & 0.010 & 9.485 & 0.577 \\
$\mathbf{6 5 5 6 2 0}$ & 0.362 & 0.081 & 0.706 & 0.240 & 1.455 & 0.423 \\
$\mathbf{6 5 5 6 3 0}$ & 0.706 & 0.455 & 0.085 & 0.319 & 0.802 & 0.362 \\
$\mathbf{6 5 5 7 8 0}$ & 0.543 & 0.145 & 1.021 & 0.278 & 0.063 & 0.797 \\
$\mathbf{6 5 5 8 5 0}$ & 0.469 & 0.091 & 0.865 & 0.442 & 0.122 & 0.916 \\
$\mathbf{6 5 5 9 2 0}$ & 0.598 & 0.161 & 1.021 & 0.557 & 0.924 & 0.107 \\
$\mathbf{6 5 5 9 4 0}$ & 0.568 & 0.770 & 0.074 & 0.388 & 0.102 & 0.928 \\
$\mathbf{6 5 5 9 9 0}$ & 0.540 & 0.720 & 0.115 & 0.541 & 0.262 & 1.279
\end{tabular}




\begin{tabular}{lllllll}
$\mathbf{6}$ & \multicolumn{7}{l}{ Mixed Exponential Parameters } \\
Gauges & May & & \multicolumn{3}{l}{ Jun } & \\
& alpha & beta 1 & beta 2 & alpha & beta 1 & beta 2 \\
$\mathbf{6 5 3 0 6 0}$ & 0.751 & 0.686 & 0.083 & 0.024 & 2.747 & 0.540 \\
$\mathbf{6 5 3 1 9 0}$ & 0.252 & 0.065 & 0.585 & 0.283 & 0.117 & 0.579 \\
$\mathbf{6 5 3 3 0 0}$ & 0.987 & 0.448 & 3.396 & 0.658 & 0.732 & 0.093 \\
$\mathbf{6 5 3 3 5 0}$ & 0.811 & 0.595 & 0.059 & 0.705 & 0.569 & 0.063 \\
$\mathbf{6 5 3 3 8 0}$ & 0.802 & 0.605 & 0.066 & 0.762 & 0.698 & 0.054 \\
$\mathbf{6 5 3 4 4 0}$ & 0.303 & 0.094 & 0.936 & 0.639 & 1.217 & 0.077 \\
$\mathbf{6 5 3 5 2 0}$ & 0.846 & 0.594 & 0.043 & 0.884 & 0.357 & 3.020 \\
$\mathbf{6 5 3 5 5 0}$ & 0.159 & 0.030 & 0.472 & 0.318 & 0.061 & 0.640 \\
$\mathbf{6 5 3 5 7 0}$ & 0.169 & 1.469 & 0.352 & 0.455 & 0.191 & 1.063 \\
$\mathbf{6 5 3 6 1 0}$ & 0.814 & 0.275 & 1.146 & 0.839 & 0.480 & 0.050 \\
$\mathbf{6 5 3 7 6 0}$ & 0.945 & 0.447 & 3.973 & 0.591 & 0.229 & 1.175 \\
$\mathbf{6 5 3 8 0 0}$ & 0.687 & 0.941 & 0.081 & 0.283 & 0.050 & 0.857 \\
$\mathbf{6 5 3 8 7 0}$ & 0.429 & 0.093 & 1.008 & 0.420 & 0.078 & 0.891 \\
$\mathbf{6 5 4 7 2 0}$ & 0.887 & 0.710 & 0.093 & 0.603 & 0.833 & 0.045 \\
$\mathbf{6 5 5 0 1 0}$ & 0.559 & 0.448 & 0.053 & 0.554 & 0.595 & 0.104 \\
$\mathbf{6 5 5 0 2 0}$ & 0.846 & 0.219 & 2.311 & 0.329 & 1.015 & 0.226 \\
$\mathbf{6 5 5 0 3 0}$ & 0.955 & 0.344 & 3.185 & 0.234 & 0.966 & 0.278 \\
$\mathbf{6 5 5 0 5 0}$ & 0.422 & 0.124 & 0.659 & 0.956 & 0.415 & 4.093 \\
$\mathbf{6 5 5 0 7 0}$ & 0.197 & 1.957 & 0.269 & 0.891 & 0.432 & 2.257 \\
$\mathbf{6 5 5 1 0 0}$ & 0.549 & 0.693 & 0.138 & 0.260 & 0.085 & 0.603 \\
$\mathbf{6 5 5 1 6 0}$ & 0.639 & 0.564 & 0.115 & 0.862 & 0.492 & 0.061 \\
$\mathbf{6 5 5 1 8 0}$ & 0.744 & 0.502 & 0.103 & 0.914 & 0.608 & 0.023 \\
$\mathbf{6 5 5 2 2 0}$ & 0.273 & 0.069 & 0.701 & 0.014 & 4.191 & 0.528 \\
$\mathbf{6 5 5 2 8 0}$ & 0.505 & 0.761 & 0.233 & 0.120 & 0.112 & 0.528 \\
$\mathbf{6 5 5 3 6 0}$ & 0.308 & 0.123 & 0.751 & 0.074 & 0.033 & 0.633 \\
$\mathbf{6 5 5 4 5 0}$ & 0.850 & 0.693 & 0.033 & 0.876 & 0.646 & 0.055 \\
$\mathbf{6 5 5 4 8 0}$ & 0.199 & 0.058 & 0.644 & 0.067 & 4.552 & 0.482 \\
$\mathbf{6 5 5 5 5 0}$ & 0.433 & 0.241 & 0.753 & 0.256 & 0.083 & 0.658 \\
$\mathbf{6 5 5 5 7 0}$ & 0.682 & 0.625 & 0.202 & 0.473 & 0.173 & 0.715 \\
$\mathbf{6 5 5 6 0 0}$ & 0.651 & 0.644 & 0.168 & 0.731 & 0.206 & 1.725 \\
$\mathbf{6 5 5 6 2 0}$ & 0.270 & 0.075 & 0.685 & 0.522 & 0.827 & 0.126 \\
$\mathbf{6 5 5 6 3 0}$ & 0.670 & 0.738 & 0.122 & 0.017 & 5.386 & 0.390 \\
$\mathbf{6 5 5 7 8 0}$ & 0.318 & 0.058 & 0.950 & 0.635 & 1.461 & 0.119 \\
$\mathbf{6 5 5 8 5 0}$ & 0.598 & 0.982 & 0.100 & 0.412 & 0.166 & 1.328 \\
$\mathbf{6 5 5 9 2 0}$ & 0.384 & 0.126 & 1.148 & 0.597 & 1.444 & 0.097 \\
$\mathbf{6 5 5 9 4 0}$ & 0.339 & 0.097 & 1.061 & 0.498 & 0.155 & 1.216 \\
$\mathbf{6 5 5 9 9 0}$ & 0.257 & 0.132 & 0.892 & 0.658 & 1.038 & 0.118
\end{tabular}




\begin{tabular}{|c|c|c|c|c|c|c|}
\hline \multirow[b]{2}{*}{ Gauges } & \multicolumn{6}{|c|}{ Mixed Exponential Parameters } \\
\hline & $\begin{array}{l}\text { Jul } \\
\text { alpha }\end{array}$ & beta 1 & beta 2 & $\begin{array}{l}\text { Aug } \\
\text { alpha }\end{array}$ & beta 1 & beta 2 \\
\hline 653060 & 0.842 & 0.774 & 0.069 & 0.768 & 0.754 & 0.055 \\
\hline 653190 & 0.795 & 0.534 & 0.052 & 0.190 & 0.052 & 0.681 \\
\hline 653300 & 0.431 & 0.137 & 0.960 & 0.667 & 0.823 & 0.096 \\
\hline 653350 & 0.642 & 0.855 & 0.058 & 0.426 & 0.087 & 0.773 \\
\hline 653380 & 0.423 & 0.058 & & 0.473 & 0.766 & 0.098 \\
\hline 653440 & 0.343 & 1.136 & 0.092 & 0.638 & 0.057 & 0.962 \\
\hline 653520 & 0.519 & 1.074 & 0.150 & 0.787 & 0.613 & 0.098 \\
\hline 653550 & 0.436 & 0.090 & 1.0 & 0.972 & 0.551 & 5.085 \\
\hline 653570 & 0.603 & 0.658 & 0.1 & 0.674 & 0.689 & 0.065 \\
\hline 653610 & 0.614 & 0.745 & 0.1 & 0.244 & 0.0 & 0.588 \\
\hline 653760 & 0.554 & 0.7 & & 0.4 & & 0.825 \\
\hline 653800 & 0.406 & 0.06 & 0.5 & 0.837 & 01 & 1.703 \\
\hline 653870 & 0.456 & 0.9 & & 0.785 & & 0.753 \\
\hline 654720 & 0.453 & 1.0 & 0.0 & 699 & & .342 \\
\hline 655010 & 0.258 & 0.0 & & 193 & & 0.690 \\
\hline 655020 & 0.086 & 0.033 & 0.5 & 0.190 & 0.0 & 0.593 \\
\hline 655030 & 0.215 & 0.07 & 0.7 & 0.130 & 0.0 & 0.595 \\
\hline 655050 & 0.079 & 0.078 & 0.624 & 0.116 & 0.046 & 0.613 \\
\hline 655070 & 0.240 & 0.119 & 0.782 & 0.900 & 0.437 & 2.763 \\
\hline 655100 & 0.786 & 0.624 & 0.066 & 0.816 & 0.753 & 0.054 \\
\hline 655160 & 0.161 & 0.060 & 0.647 & 0.888 & 0.613 & 0.056 \\
\hline 655180 & 0.677 & 0.918 & 0.072 & 0.839 & 0.727 & 0.055 \\
\hline 655220 & 0.241 & 0.091 & 0.714 & 0.216 & 0.101 & 0.711 \\
\hline 655280 & 0.852 & & & 0.978 & & 5.506 \\
\hline & 0.124 & & & & & 0.574 \\
\hline 655450 & 0.364 & 0.069 & 0.664 & 0.386 & 0.047 & 0.892 \\
\hline 655480 & 0.589 & 0.808 & 0.060 & 0.507 & 1.171 & 0.142 \\
\hline 655550 & 0.605 & 1.253 & 0.063 & 0.399 & 0.079 & 0.964 \\
\hline 655570 & 0.473 & 0.104 & 0.471 & 0.647 & 0.092 & 0.681 \\
\hline 655600 & 0.488 & 0.529 & 0.067 & 0.525 & 0.078 & 0.725 \\
\hline 655620 & 0.614 & 0.511 & 0.053 & 0.623 & 0.061 & 0.916 \\
\hline 655630 & 0.412 & 0.979 & 0.116 & 0.540 & 0.613 & 0.061 \\
\hline 655780 & 0.688 & 0.102 & 1.189 & 0.130 & 1.698 & 0.091 \\
\hline 655850 & 0.649 & 0.080 & 1.294 & 0.134 & 1.041 & 0.097 \\
\hline 655920 & 0.201 & 2.050 & 0.179 & 0.606 & 0.091 & 0.795 \\
\hline 655940 & 0.730 & & & 0.206 & 0.643 & 0.091 \\
\hline 655990 & 0.702 & 0.091 & 1.214 & 0.848 & 0.092 & 0.955 \\
\hline
\end{tabular}




\begin{tabular}{|c|c|c|c|c|c|c|}
\hline \multirow[b]{2}{*}{ Gauges } & \multicolumn{6}{|c|}{ Mixed Exponential Parameters } \\
\hline & $\begin{array}{l}\text { Sep } \\
\text { alpha }\end{array}$ & beta 1 & beta 2 & $\begin{array}{l}\text { Oct } \\
\text { alpha }\end{array}$ & beta 1 & beta 2 \\
\hline 653060 & 0.856 & 0.608 & 0.052 & 0.271 & 0.082 & 0.416 \\
\hline 653190 & 0.066 & 0.037 & 0.538 & 0.300 & 0.129 & 0.463 \\
\hline 653300 & 0.794 & 0.642 & 0.081 & 0.234 & 0.084 & 0.551 \\
\hline 653350 & 0.586 & 0.679 & 0.116 & 0.237 & 0.081 & 0.527 \\
\hline 653380 & 0.281 & 0.047 & & 0.203 & 0.043 & 0.610 \\
\hline 653440 & 0.598 & 0.672 & 0.053 & 0.278 & 0.063 & 0.706 \\
\hline 653520 & 0.226 & 0.087 & 0.5 & 0.860 & 0.483 & 0.043 \\
\hline 653550 & 0.982 & 0.448 & 3.3 & 0.716 & 0.507 & 0.054 \\
\hline 653570 & 0.983 & 0.489 & 3.5 & 0.000 & 171 & 0.459 \\
\hline 653610 & 0.788 & 0.5 & 0.0 & 0.252 & 0.119 & 0.530 \\
\hline 653760 & 0.820 & 0.6 & & 0.808 & & 0.089 \\
\hline 653800 & 0.509 & 0.079 & 0.7 & 0.705 & 14 & 0.072 \\
\hline 653870 & 0.547 & 0.0 & 0.6 & 643 & & 0.081 \\
\hline 654720 & 0.060 & 3.9 & & 647 & 25 & 0.794 \\
\hline 655010 & 0.289 & 0.086 & & .740 & 0.0 & 1.462 \\
\hline 655020 & 0.255 & 0.067 & 0.6 & 0.417 & 0.1 & 0.370 \\
\hline 655030 & 0.040 & 4.819 & 0.420 & 0.927 & 0.227 & 1.416 \\
\hline 655050 & 0.768 & 0.535 & 0.098 & 0.416 & 0.092 & 0.495 \\
\hline 655070 & 0.165 & 0.048 & 0.533 & 0.765 & 0.355 & 0.056 \\
\hline 655100 & 0.767 & 0.611 & 0.07 & 0.829 & 0.437 & 0.048 \\
\hline 655160 & 0.194 & 0.061 & 0.513 & 0.436 & 0.174 & 0.521 \\
\hline 655180 & 0.954 & 0.400 & 1.237 & 0.858 & 0.385 & 0.081 \\
\hline 655220 & 0.935 & 0.498 & 0.072 & 0.197 & 0.082 & 0.465 \\
\hline 655280 & 0.296 & & & 0.016 & & 0.357 \\
\hline & 0.919 & & & & & 0.300 \\
\hline 655450 & 0.201 & 0.046 & 0.528 & 0.364 & 0.781 & 0.262 \\
\hline 655480 & 0.193 & 0.094 & 0.618 & 0.792 & 0.308 & 1.126 \\
\hline 655550 & 0.796 & 0.646 & 0.075 & 0.759 & 0.297 & 1.045 \\
\hline 655570 & 0.531 & 0.719 & 0.10 & 0.392 & 0.131 & 0.547 \\
\hline 655600 & 0.778 & 0.223 & 1.205 & 0.314 & 0.076 & 0.493 \\
\hline 655620 & 0.992 & 0.335 & 10.442 & 0.680 & 0.572 & 0.064 \\
\hline 655630 & 0.496 & 0.093 & 0.660 & 0.362 & 0.087 & 0.530 \\
\hline 655780 & 0.256 & 1.988 & 0.076 & 0.555 & 0.078 & 0.922 \\
\hline 655850 & 0.349 & 0.855 & 0.070 & 0.451 & 0.083 & 0.813 \\
\hline 655920 & 0.807 & 0.199 & 1.517 & 0.621 & 0.094 & 1.164 \\
\hline 655940 & 0.859 & & & 0.306 & 0.812 & 0.089 \\
\hline 655990 & 0.372 & 0.519 & 0.058 & 0.537 & 0.094 & 0.640 \\
\hline
\end{tabular}




\begin{tabular}{lllllll}
\multicolumn{7}{l}{ Mixed Exponential Parameters } \\
Gauges & Nov & & \multicolumn{3}{l}{ Dec } \\
& alpha & beta 1 & beta 2 & alpha & beta 1 & beta 2 \\
$\mathbf{6 5 3 0 6 0}$ & 0.234 & 0.378 & 0.037 & 1.000 & 2.130 & 6.459 \\
$\mathbf{6 5 3 1 9 0}$ & 0.514 & 0.250 & 0.487 & 1.000 & 0.215 & 16.842 \\
$\mathbf{6 5 3 3 0 0}$ & 0.249 & 0.030 & 0.726 & 0.000 & 30.935 & 0.154 \\
$\mathbf{6 5 3 3 5 0}$ & 0.920 & 0.416 & 0.027 & 0.000 & 0.219 & 0.219 \\
$\mathbf{6 5 3 3 8 0}$ & 0.439 & 0.047 & 0.784 & 0.788 & 1.065 & 0.179 \\
$\mathbf{6 5 3 4 4 0}$ & 0.263 & 0.068 & 0.479 & 0.620 & 0.054 & 1.979 \\
$\mathbf{6 5 3 5 2 0}$ & 0.199 & 5.148 & 0.212 & 0.000 & 1.360 & 1.360 \\
$\mathbf{6 5 3 5 5 0}$ & 0.930 & 0.576 & 0.039 & 0.555 & 0.411 & 0.015 \\
$\mathbf{6 5 3 5 7 0}$ & 0.000 & 0.457 & 0.457 & 0.685 & 0.546 & 0.058 \\
$\mathbf{6 5 3 6 1 0}$ & 0.696 & 0.208 & 1.942 & 0.111 & 0.043 & 0.152 \\
$\mathbf{6 5 3 7 6 0}$ & 0.388 & 0.531 & 0.093 & 0.866 & 0.302 & 2.973 \\
$\mathbf{6 5 3 8 0 0}$ & 0.631 & 0.441 & 0.060 & 0.293 & 0.065 & 1.316 \\
$\mathbf{6 5 3 8 7 0}$ & 0.795 & 0.316 & 0.039 & 0.521 & 0.065 & 0.663 \\
$\mathbf{6 5 4 7 2 0}$ & 0.224 & 0.053 & 0.472 & 0.769 & 0.318 & 0.877 \\
$\mathbf{6 5 5 0 1 0}$ & 0.506 & 2.413 & 0.010 & 0.093 & 0.120 & 2.653 \\
$\mathbf{6 5 5 0 2 0}$ & 0.310 & 2.002 & 0.130 & 0.000 & 0.060 & 0.060 \\
$\mathbf{6 5 5 0 3 0}$ & 0.408 & 0.042 & 1.070 & 0.493 & 0.106 & 1.278 \\
$\mathbf{6 5 5 0 5 0}$ & 0.414 & 0.051 & 0.373 & 1.000 & 1.810 & 590 \\
$\mathbf{6 5 5 0 7 0}$ & 0.308 & 0.017 & 0.448 & 0.533 & 2.254 & 0.046 \\
$\mathbf{6 5 5 1 0 0}$ & 0.448 & 0.100 & 0.635 & 0.220 & 0.027 & 0.226 \\
$\mathbf{6 5 5 1 6 0}$ & 0.613 & 0.462 & 0.060 & 0.524 & 2.330 & 0.040 \\
$\mathbf{6 5 5 1 8 0}$ & 0.000 & 86.429 & 0.253 & 1.000 & 0.570 & 160 \\
$\mathbf{6 5 5 2 2 0}$ & 0.671 & 0.071 & 1.004 & 0.653 & 0.025 & 1.944 \\
$\mathbf{6 5 5 2 8 0}$ & 0.042 & 3.967 & 0.330 & 0.675 & 0.153 & 0.874 \\
$\mathbf{6 5 5 3 6 0}$ & 0.465 & 0.050 & 0.545 & 0.000 & 4.032 & 0.958 \\
$\mathbf{6 5 5 4 5 0}$ & 0.312 & 0.554 & 0.160 & 0.802 & 0.420 & 0.022 \\
$\mathbf{6 5 5 4 8 0}$ & 0.027 & 3.663 & 0.266 & 0.319 & 0.045 & 0.953 \\
$\mathbf{6 5 5 5 5 0}$ & 0.284 & 0.904 & 0.180 & 0.665 & 0.187 & 1.349 \\
$\mathbf{6 5 5 5 7 0}$ & 0.555 & 0.197 & 0.556 & 0.692 & 0.562 & 0.088 \\
$\mathbf{6 5 5 6 0 0}$ & 0.153 & 0.069 & 0.321 & 0.094 & 0.039 & 0.702 \\
$\mathbf{6 5 5 6 2 0}$ & 0.819 & 0.444 & 0.036 & 0.902 & 0.618 & 0.045 \\
$\mathbf{6 5 5 6 3 0}$ & 0.058 & 1.376 & 0.283 & 0.371 & 0.080 & 0.480 \\
$\mathbf{6 5 5 7 8 0}$ & 0.799 & 0.495 & 0.063 & 0.547 & 0.957 & 0.084 \\
$\mathbf{6 5 5 8 5 0}$ & 0.398 & 0.076 & 0.575 & 0.989 & 0.350 & 16.914 \\
$\mathbf{6 5 5 9 2 0}$ & 0.536 & 0.622 & 0.076 & 0.493 & 0.084 & 0.990 \\
$\mathbf{6 5 5 9 4 0}$ & 0.629 & 0.146 & 0.895 & 0.413 & 0.075 & 0.827 \\
$\mathbf{6 5 5 9 9 0}$ & 0.494 & 0.663 & 0.112 & 0.341 & 0.069 & 0.666
\end{tabular}



Appendix G: Conditioned Mixed Exponential Distribution Parameters for Wet Day Amounts 


\begin{tabular}{lllllll}
\multicolumn{7}{c}{ Mixed Exponential Parameters } \\
Gauges & Cold Pacific Sea Surface Temperature Event & \\
& Jalpha & beta 1 & beta 2 & alpha & beta 1 & beta 2 \\
$\mathbf{6 5 3 0 6 0}$ & 0.813 & 9.709 & 4.391 & 1.000 & 0.750 & 8.822 \\
$\mathbf{6 5 3 1 9 0}$ & 0.569 & 5.967 & 17.466 & 0.362 & 1.468 & 2.151 \\
$\mathbf{6 5 3 3 0 0}$ & 0.000 & 5.607 & 0.140 & 0.000 & 0.115 & 0.120 \\
$\mathbf{6 5 3 3 5 0}$ & 0.000 & 77.895 & 0.160 & 0.000 & 64.795 & 2.007 \\
$\mathbf{6 5 3 3 8 0}$ & 0.679 & 4.423 & 0.030 & 0.675 & 0.763 & 0.158 \\
$\mathbf{6 5 3 4 4 0}$ & 0.652 & 0.049 & 0.787 & 0.427 & 0.044 & 1.418 \\
$\mathbf{6 5 3 5 2 0}$ & 0.393 & 8.587 & 5.115 & 0.054 & 8.451 & 6.413 \\
$\mathbf{6 5 3 5 5 0}$ & 0.652 & 0.684 & 5.273 & 0.496 & 0.010 & 3.873 \\
$\mathbf{6 5 3 5 7 0}$ & 1.000 & 0.430 & 161 & 0.910 & 1.959 & 2.243 \\
$\mathbf{6 5 3 6 1 0}$ & 0.613 & 0.814 & 0.062 & 1.000 & 0.410 & 25.065 \\
$\mathbf{6 5 3 7 6 0}$ & 0.749 & 0.069 & 0.959 & 0.481 & 0.123 & 0.888 \\
$\mathbf{6 5 3 8 0 0}$ & 1.000 & 1.390 & 60.811 & 0.788 & 0.105 & 1.963 \\
$\mathbf{6 5 3 8 7 0}$ & 0.864 & 0.753 & 0.010 & 0.606 & 0.047 & 0.395 \\
$\mathbf{6 5 4 7 2 0}$ & 1.000 & 0.030 & 1.449 & 0.000 & 10.609 & 1.610 \\
$\mathbf{6 5 5 0 1 0}$ & 0.000 & 0.470 & 0.470 & 1.000 & 1.535 & 868 \\
$\mathbf{6 5 5 0 2 0}$ & 0.387 & 4.820 & 10.092 & 0.896 & 9.015 & 0.603 \\
$\mathbf{6 5 5 0 3 0}$ & 0.645 & 6.917 & 10.251 & 0.154 & 1.112 & 0.762 \\
$\mathbf{6 5 5 0 5 0}$ & 0.416 & 3.619 & 17.417 & 0.438 & 2.419 & 14.936 \\
$\mathbf{6 5 5 0 7 0}$ & 0.674 & 5.715 & 17.575 & 0.696 & 4.515 & 15.094 \\
$\mathbf{6 5 5 1 0 0}$ & 0.932 & 7.812 & 17.733 & 0.000 & 0.120 & 0.120 \\
$\mathbf{6 5 5 1 6 0}$ & 0.216 & 9.119 & 11.907 & 0.626 & 6.064 & 19.663 \\
$\mathbf{6 5 5 1 8 0}$ & 0.474 & 1.215 & 12.065 & 0.910 & 7.370 & 13.837 \\
$\mathbf{6 5 5 2 2 0}$ & 1.000 & 0.390 & 15.225 & 0.560 & 0.077 & 0.887 \\
$\mathbf{6 5 5 2 8 0}$ & 0.000 & 12.038 & 0.080 & 1.000 & 0.310 & 3.384 \\
$\mathbf{6 5 5 3 6 0}$ & 0.000 & 0.003 & 0.157 & 0.630 & 0.080 & 2.096 \\
$\mathbf{6 5 5 4 5 0}$ & 0.223 & 0.020 & 1.552 & 1.000 & 0.310 & 33.152 \\
$\mathbf{6 5 5 4 8 0}$ & 0.244 & 0.043 & 0.647 & 0.657 & 0.326 & 0.024 \\
$\mathbf{6 5 5 5 5 0}$ & 0.512 & 1.435 & 0.054 & 0.519 & 0.106 & 0.727 \\
$\mathbf{6 5 5 5 7 0}$ & 0.000 & 15.373 & 0.754 & 0.128 & 2.805 & 0.243 \\
$\mathbf{6 5 5 6 0 0}$ & 1.000 & 0.178 & 23.255 & 0.000 & 69.003 & 0.407 \\
$\mathbf{6 5 5 6 2 0}$ & 0.329 & 0.020 & 0.779 & 0.682 & 0.158 & 1.164 \\
$\mathbf{6 5 5 6 3 0}$ & 0.723 & 0.677 & 0.020 & 0.708 & 0.910 & 0.020 \\
$\mathbf{6 5 5 7 8 0}$ & 0.763 & 0.701 & 0.039 & 0.086 & 5.942 & 0.380 \\
$\mathbf{6 5 5 8 5 0}$ & 0.258 & 0.052 & 0.546 & 0.396 & 0.018 & 0.355 \\
$\mathbf{6 5 5 9 2 0}$ & 0.629 & 0.092 & 1.310 & 0.117 & 0.060 & 0.496 \\
$\mathbf{6 5 5 9 4 0}$ & 0.763 & 0.395 & 0.115 & 0.620 & 0.204 & 1.700 \\
$\mathbf{6 5 5 9 9 0}$ & 0.696 & 0.584 & 0.011 & 0.272 & 3.414 & 0.157 \\
& & & & & &
\end{tabular}




\begin{tabular}{|c|c|c|c|c|c|c|}
\hline \multirow[b]{2}{*}{ Gauges } & \multicolumn{6}{|c|}{$\begin{array}{l}\text { Mixed Exponential Parameters } \\
\text { Cold Pacific Sea Surface Temperature Event }\end{array}$} \\
\hline & $\begin{array}{l}\text { Mar } \\
\text { alpha }\end{array}$ & beta 1 & beta 2 & $\begin{array}{l}\text { Apr } \\
\text { alpha }\end{array}$ & beta 1 & beta 2 \\
\hline 653060 & 0.201 & 0.203 & 1.232 & 0.818 & 1.220 & 0.010 \\
\hline 653190 & 0.431 & 0.103 & 0.616 & 0.567 & 0.101 & 0.687 \\
\hline 653300 & 1.000 & 0.598 & 20.599 & 0.932 & 0.425 & 0.014 \\
\hline 653350 & 0.842 & 1.114 & 0.108 & 0.602 & 0.218 & 1.164 \\
\hline 653380 & 0.465 & 0.903 & & & & 0.492 \\
\hline 653440 & 0.366 & 0.0 & 0.7 & 0.726 & & 0.108 \\
\hline 653520 & 0.000 & 42.707 & 0.1 & 0.339 & & 0.670 \\
\hline 653550 & 0.630 & 0.5 & 0.0 & 1.0 & & 1.863 \\
\hline 653570 & 0.653 & & & 1.0 & & 122 \\
\hline 653610 & 0.491 & & & & & 0.055 \\
\hline 653760 & 0.649 & 0.3 & & & & 0.851 \\
\hline 653800 & 0.737 & 0.6 & & & & 32 \\
\hline 653870 & 0.611 & & & 0.8 & & 0.028 \\
\hline 654720 & 0.596 & & & & & \\
\hline 655010 & 0.000 & 57. & & & & 571 \\
\hline 655020 & 0.547 & 1.7 & & & & 60 \\
\hline 655030 & 0.341 & & & & & \\
\hline 655050 & 1.000 & 0.0 & & & & 292 \\
\hline 655070 & 0.410 & & & & & 53.624 \\
\hline 655100 & 0.078 & 0.0 & & 0.0 & & 0.474 \\
\hline 655160 & 0.400 & 0.0 & 0.2 & 0.2 & & 0.265 \\
\hline 655180 & 0.051 & 0.022 & & & & \\
\hline 655220 & 0.740 & & & & & \\
\hline 655280 & 0.6 & & & & & \\
\hline 655360 & 0.193 & 1.2 & & 0.0 & & 0.832 \\
\hline 655450 & 0.744 & & & 0.0 & & 0.522 \\
\hline 655480 & 0.289 & & 0.486 & 0.325 & & 1.001 \\
\hline 655550 & 0.164 & & 0.222 & 0.000 & & 0.510 \\
\hline 655570 & 0.176 & 0.035 & 0.496 & 0.011 & 0.015 & 0.201 \\
\hline 655600 & 0.880 & 0.697 & 0.038 & 0.110 & 0.010 & 0.415 \\
\hline 655620 & 0.148 & 0.0 & 0.6 & 0.432 & & \\
\hline 655630 & 0.31 & & & & & \\
\hline 655780 & 0.472 & & & & & 1.627 \\
\hline 655850 & 0.199 & 0.028 & 0.652 & 0.182 & 0.052 & 0.466 \\
\hline 655920 & 0.355 & 0.794 & 0.105 & 0.315 & & 2.036 \\
\hline 655940 & 0.655 & 0.072 & 1.071 & 0.457 & & 0.027 \\
\hline 655990 & 0.595 & 0.088 & 0.749 & 0.000 & 0.046 & 3.910 \\
\hline
\end{tabular}




\begin{tabular}{|c|c|c|c|c|c|c|}
\hline \multirow[b]{2}{*}{ Gauges } & \multicolumn{5}{|c|}{$\begin{array}{l}\text { Mixed Exponential Parameters } \\
\text { Cold Pacific Sea Surface Temperature Event }\end{array}$} & \multirow[b]{2}{*}{ beta 2} \\
\hline & $\begin{array}{l}\text { May } \\
\text { alpha }\end{array}$ & beta 1 & beta 2 & $\begin{array}{l}\text { Jun } \\
\text { alpha }\end{array}$ & beta 1 & \\
\hline 653060 & 0.256 & 0.051 & 0.493 & 0.892 & 0.678 & 0.063 \\
\hline 653190 & 0.361 & 0.064 & 0.670 & 0.535 & 0.718 & 0.172 \\
\hline 653300 & 0.717 & 0.563 & 0.111 & 0.413 & 0.177 & 0.943 \\
\hline 653350 & 0.894 & 0.472 & 0.028 & 0.366 & 0.113 & 0.631 \\
\hline 653380 & 0.365 & 1.224 & & & & 0.707 \\
\hline 653440 & 0.766 & 1.170 & 0.0 & 0.692 & & 0.044 \\
\hline 653520 & 0.243 & 0.0 & 3 & 0.155 & 18 & 0.619 \\
\hline 653550 & 0.256 & 0.0 & & 0.737 & & 026 \\
\hline 653570 & 0.802 & 0.2 & 2.9 & 0.194 & & 0.658 \\
\hline 653610 & 0.226 & 0.02 & 0.6 & 0.942 & & 0.107 \\
\hline 653760 & 0.521 & 0.6 & 0.1 & 0.852 & & 0.036 \\
\hline 653800 & 0.794 & .1 & 0.0 & 0.372 & & 0.502 \\
\hline 653870 & 0.254 & & & & & 0.551 \\
\hline 654720 & 1.000 & & & & & 996 \\
\hline 655010 & 0.069 & & & 0.342 & & 043 \\
\hline 655020 & 0.704 & & & 0.462 & & 42 \\
\hline 655030 & 0.024 & & & 0.926 & & 82 \\
\hline 655050 & 0.820 & 0.3 & 0.0 & 0.075 & & 0.484 \\
\hline 655070 & 0.336 & & & 0.214 & & 0.692 \\
\hline 655100 & 0.843 & 0.2 & 10 & 0.798 & & 0.069 \\
\hline 655160 & 0.626 & 0.1 & 0.4 & 0.722 & & 0.055 \\
\hline 655180 & 0.137 & & & & & 0.573 \\
\hline 655220 & 0.829 & & & 37 & & 0.732 \\
\hline 655280 & 0.706 & & & & & \\
\hline 655360 & 0.278 & & 0.728 & 0.750 & & 019 \\
\hline 655450 & 0.127 & & 0.7 & 0.333 & & 0.865 \\
\hline 655480 & 0.586 & & 0.060 & 0.931 & & 14.609 \\
\hline 655550 & 1.000 & 0.63 & & 0.449 & & 0.638 \\
\hline 655570 & 0.783 & 0.475 & 0.095 & 0.820 & 0.363 & 0.015 \\
\hline 655600 & 1.000 & 0.34 & 30.026 & 0.449 & 0.032 & 0.466 \\
\hline 655620 & 0.884 & 0.6 & & 0.708 & & 0.049 \\
\hline 655630 & 0.325 & & & & & 0.038 \\
\hline 655780 & 0.227 & & & & & 1.289 \\
\hline 655850 & 0.807 & 0.241 & 2.519 & 0.432 & 2.326 & 0.309 \\
\hline 655920 & 0.268 & 0.105 & 0.752 & 0.937 & 0.734 & 0.038 \\
\hline 655940 & 0.793 & 0.409 & 0.029 & 0.256 & 0.072 & 0.847 \\
\hline 655990 & 0.000 & 10.609 & 0.573 & 0.895 & 1.137 & 0.076 \\
\hline
\end{tabular}




\begin{tabular}{|c|c|c|c|c|c|c|}
\hline \multirow[b]{2}{*}{ Gauges } & \multicolumn{6}{|c|}{$\begin{array}{l}\text { Mixed Exponential Parameters } \\
\text { Cold Pacific Sea Surface Temperature Event }\end{array}$} \\
\hline & $\begin{array}{l}\text { Jul } \\
\text { alpha }\end{array}$ & beta 1 & beta 2 & $\begin{array}{l}\text { Aug } \\
\text { alpha }\end{array}$ & beta 1 & beta 2 \\
\hline 653060 & 0.678 & 0.984 & 0.173 & 0.225 & 0.031 & 0.674 \\
\hline 653190 & 0.698 & 0.537 & 0.131 & 0.807 & 0.948 & 0.041 \\
\hline 653300 & 0.273 & 0.097 & 0.999 & 0.620 & 1.225 & 0.094 \\
\hline 653350 & 0.537 & 1.162 & 0.047 & 0.889 & 0.969 & 0.023 \\
\hline 653380 & 0.475 & 0.053 & 1.125 & 0.199 & & 1.007 \\
\hline 653440 & 0.173 & & & 0.791 & & 1.424 \\
\hline 653520 & 0.770 & 0.6 & 6 & 0.287 & 0.252 & 0.915 \\
\hline 653550 & 0.667 & 0.0 & & 0.334 & & 0.926 \\
\hline 653570 & 0.046 & 0.059 & & 0.214 & & 0.628 \\
\hline 653610 & 0.498 & 0.082 & & 0.819 & & 0.037 \\
\hline 653760 & 0.505 & 0.224 & & 0.544 & & 0.696 \\
\hline 653800 & 0.259 & 0.942 & 0.1 & 0.050 & & 0.206 \\
\hline 653870 & 0.518 & 0.0 & & & & 0.030 \\
\hline 654720 & 0.676 & & & & & 609 \\
\hline 655010 & 0.130 & 0.0 & & 0.229 & & 0.687 \\
\hline 655020 & 0.256 & & & 0.870 & & 0.021 \\
\hline 655030 & 0.532 & & & 0.108 & & 780 \\
\hline 655050 & 0.253 & 0.0 & 0.7 & 0.060 & & 0.579 \\
\hline 655070 & 0.743 & & & 1.000 & & 0.576 \\
\hline 655100 & 0.834 & 0.5 & & 0.818 & & 0.164 \\
\hline 655160 & 0.928 & 0.5 & & 0.097 & & 0.756 \\
\hline 655180 & 0.472 & & & 0.524 & & 0.090 \\
\hline 655220 & 0.891 & & & 0.708 & & 1.925 \\
\hline 655280 & 0.673 & & & & & 0.772 \\
\hline 655360 & 0.726 & 0.9 & & 0.000 & & 0.615 \\
\hline 655450 & 0.361 & 0.02 & & 0.646 & & \\
\hline 655480 & 0.033 & 5.828 & 0.365 & 0.655 & & 0.055 \\
\hline 655550 & 0.581 & 1.168 & 0.026 & 0.532 & 0.452 & 0.026 \\
\hline 655570 & 0.649 & 0.091 & 0.240 & 0.570 & 0.035 & 0.504 \\
\hline 655600 & 0.515 & 0.144 & 0.324 & 0.077 & 2.565 & 0.099 \\
\hline 655620 & 0.541 & 0.0 & & 0.719 & & 0.349 \\
\hline 655630 & 0.682 & & & & & 0.062 \\
\hline 655780 & 0.557 & & & & & 0.075 \\
\hline 655850 & 0.766 & 0.089 & 1.894 & 0.984 & 0.051 & 0.017 \\
\hline 655920 & 0.446 & 0.075 & 1.078 & 0.549 & 0.040 & 0.373 \\
\hline 655940 & 0.195 & 0.726 & 0.215 & 0.910 & 0.079 & 1.121 \\
\hline 655990 & 0.170 & 0.566 & 0.142 & 0.730 & 0.061 & 0.811 \\
\hline
\end{tabular}




\begin{tabular}{|c|c|c|c|c|c|c|}
\hline \multirow[b]{2}{*}{ Gauges } & \multicolumn{5}{|c|}{ Mixed Exponential Parameters } & \\
\hline & $\begin{array}{l}\text { Sep } \\
\text { alpha }\end{array}$ & beta 1 & beta 2 & $\begin{array}{l}\text { Oct } \\
\text { alpha }\end{array}$ & beta 1 & beta 2 \\
\hline 653060 & 0.909 & 0.433 & 2.399 & 0.327 & 0.055 & 0.387 \\
\hline 653190 & 0.915 & 0.560 & 0.036 & 0.839 & 0.418 & 0.023 \\
\hline 653300 & 0.186 & 0.057 & 0.612 & 1.000 & 0.515 & 34.765 \\
\hline 653350 & 1.000 & 0.391 & 0.390 & 0.828 & & 0.104 \\
\hline 653380 & 0.178 & 0.032 & & 0.814 & & 0.040 \\
\hline 653440 & 0.373 & 0.06 & 0.5 & 0.679 & & 0.070 \\
\hline 653520 & 0.814 & 0.5 & 0.1 & 0.906 & & 0.074 \\
\hline 653550 & 0.716 & 0.5 & 0. & 0.944 & & 51 \\
\hline 653570 & 0.516 & 0.6 & & 0.000 & & 0.367 \\
\hline 653610 & 0.143 & 0.0 & & 0.788 & & 0.027 \\
\hline 653760 & 0.283 & 0.144 & & 0.550 & & 0.151 \\
\hline 653800 & 0.705 & 0.6 & 0.0 & 0.2 & & 0.386 \\
\hline 653870 & 0.465 & 0.8 & & 0.273 & & 0.321 \\
\hline 654720 & 0.294 & & & & & 98 \\
\hline 655010 & 0.765 & 0.4 & & 0.5 & & 719 \\
\hline 655020 & 0.880 & 0.6 & & 0.7 & & 33 \\
\hline 655030 & 0.097 & 0.0 & & $0 . c$ & & 426 \\
\hline 655050 & 0.862 & 0.5 & 0.0 & 0.683 & & 0.142 \\
\hline 655070 & 0.135 & 0.0 & & 0.877 & & 0.028 \\
\hline 655100 & 0.302 & 0.079 & & 0.615 & & 0.553 \\
\hline 655160 & 0.204 & 0.0 & 0.5 & 0.660 & & 0.117 \\
\hline 655180 & 0.481 & 0.148 & 0.778 & 1.0 & & 179 \\
\hline 655220 & 0.089 & & & & & 0.568 \\
\hline 655280 & 0.37 & & & & & \\
\hline 655360 & 0.924 & 0.4 & 0.0 & 0.1 & & 0.414 \\
\hline 655450 & 0.691 & 0.7 & & 0.6 & & 0.569 \\
\hline 655480 & 0.663 & 0.650 & 0.098 & 0.900 & & 0.017 \\
\hline 655550 & 0.363 & 0.047 & 0.618 & 0.177 & 1.431 & 0.270 \\
\hline 655570 & 0.901 & 0.182 & 1.400 & 0.295 & 0.229 & 0.321 \\
\hline 655600 & 0.789 & 0.209 & 1.894 & 0.047 & 0.032 & 0.397 \\
\hline 655620 & 0.954 & 0.264 & 11.5 & 0.441 & & 0.092 \\
\hline 655630 & 0.412 & & & & & \\
\hline 655780 & 0.368 & & & & & 0.786 \\
\hline 655850 & 0.469 & 0.038 & 0.260 & 0.328 & 0.029 & 0.430 \\
\hline 655920 & 0.523 & 0.962 & 0.099 & 0.514 & 0.083 & 0.873 \\
\hline 655940 & 0.158 & 0.500 & 0.089 & 0.780 & & 0.996 \\
\hline 655990 & 0.880 & 0.071 & 0.348 & 0.298 & 0.049 & 0.452 \\
\hline
\end{tabular}




\begin{tabular}{lllllll}
\multicolumn{7}{c}{ Mixed Exponential Parameters } \\
Gauges & Cold Pacific Sea Surface & Nemperature Event \\
& alpha & beta 1 & beta 2 & alpha & beta 1 & beta 2 \\
$\mathbf{6 5 3 0 6 0}$ & 0.000 & 0.020 & 0.020 & 0.080 & 6.019 & 14.051 \\
$\mathbf{6 5 3 1 9 0}$ & 1.000 & 0.073 & 1657 & 0.980 & 8.769 & 11.296 \\
$\mathbf{6 5 3 3 0 0}$ & 0.417 & 0.195 & 0.015 & 1.000 & 0.104 & 205 \\
$\mathbf{6 5 3 3 5 0}$ & 0.844 & 0.497 & 0.047 & 0.940 & 0.243 & 0.020 \\
$\mathbf{6 5 3 3 8 0}$ & 0.851 & 0.481 & 0.065 & 0.212 & 0.102 & 1.050 \\
$\mathbf{6 5 3 4 4 0}$ & 0.595 & 0.805 & 0.128 & 0.352 & 0.730 & 0.046 \\
$\mathbf{6 5 3 5 2 0}$ & 1.000 & 0.160 & 252 & 0.085 & 3.107 & 19.811 \\
$\mathbf{6 5 3 5 5 0}$ & 0.000 & 0.748 & 0.756 & 1.000 & 0.710 & 0.710 \\
$\mathbf{6 5 3 5 7 0}$ & 0.000 & 54.818 & 0.730 & 0.000 & 0.080 & 0.080 \\
$\mathbf{6 5 3 6 1 0}$ & 1.000 & 0.196 & 76.421 & 0.000 & 22.364 & 0.210 \\
$\mathbf{6 5 3 7 6 0}$ & 0.387 & 0.048 & 0.322 & 1.000 & 0.162 & 109 \\
$\mathbf{6 5 3 8 0 0}$ & 0.421 & 0.029 & 0.609 & 0.036 & 0.119 & 0.874 \\
$\mathbf{6 5 3 8 7 0}$ & 0.716 & 0.357 & 0.046 & 1.000 & 0.353 & 122 \\
$\mathbf{6 5 4 7 2 0}$ & 0.000 & 79.823 & 0.220 & 1.000 & 1.090 & 1.090 \\
$\mathbf{6 5 5 0 1 0}$ & 0.143 & 9.061 & 8.083 & 0.883 & 2.412 & 17.502 \\
$\mathbf{6 5 5 0 2 0}$ & 1.000 & 0.080 & 1533 & 0.141 & 4.509 & 17.660 \\
$\mathbf{6 5 5 0 3 0}$ & 0.520 & 0.195 & 0.044 & 1.000 & 0.100 & 0.100 \\
$\mathbf{6 5 5 0 5 0}$ & 0.382 & 0.029 & 0.407 & 0.199 & 2.105 & 12.308 \\
$\mathbf{6 5 5 0 7 0}$ & 0.521 & 1.325 & 0.020 & 1.000 & 2.360 & 0.012 \\
$\mathbf{6 5 5 1 0 0}$ & 0.359 & 1.445 & 0.238 & 0.000 & 0.215 & 0.215 \\
$\mathbf{6 5 5 1 6 0}$ & 1.000 & 0.433 & 0.040 & 0.748 & 4.686 & 13.257 \\
$\mathbf{6 5 5 1 8 0}$ & 0.000 & 0.330 & 0.332 & 0.000 & 28.552 & 0.670 \\
$\mathbf{6 5 5 2 2 0}$ & 0.184 & 3.941 & 0.094 & 0.274 & 0.969 & 0.030 \\
$\mathbf{6 5 5 2 8 0}$ & 1.000 & 0.276 & 6.145 & 1.000 & 0.215 & 319 \\
$\mathbf{6 5 5 3 6 0}$ & 0.366 & 0.063 & 0.555 & 0.540 & 1.429 & 0.040 \\
$\mathbf{6 5 5 4 5 0}$ & 0.530 & 0.550 & 0.116 & 0.761 & 0.374 & 0.024 \\
$\mathbf{6 5 5 4 8 0}$ & 0.929 & 0.293 & 5.452 & 0.701 & 0.482 & 0.023 \\
$\mathbf{6 5 5 5 5 0}$ & 0.322 & 1.190 & 0.104 & 0.300 & 2.166 & 0.237 \\
$\mathbf{6 5 5 5 7 0}$ & 0.122 & 1.357 & 0.362 & 0.354 & 0.055 & 0.491 \\
$\mathbf{6 5 5 6 0 0}$ & 0.292 & 0.078 & 0.497 & 0.651 & 0.968 & 0.179 \\
$\mathbf{6 5 5 6 2 0}$ & 0.074 & 0.026 & 0.278 & 0.493 & 0.094 & 0.861 \\
$\mathbf{6 5 5 6 3 0}$ & 0.666 & 0.468 & 0.085 & 0.393 & 0.650 & 0.193 \\
$\mathbf{6 5 5 7 8 0}$ & 0.315 & 0.992 & 0.163 & 0.533 & 0.781 & 0.067 \\
$\mathbf{6 5 5 8 5 0}$ & 0.266 & 0.027 & 0.492 & 0.720 & 0.545 & 0.026 \\
$\mathbf{6 5 5 9 2 0}$ & 0.689 & 0.113 & 0.604 & 0.559 & 0.133 & 0.773 \\
$\mathbf{6 5 5 9 4 0}$ & 0.597 & 0.119 & 0.662 & 0.087 & 2.867 & 0.272 \\
$\mathbf{6 5 5 9 9 0}$ & 0.596 & 0.105 & 0.846 & 0.812 & 0.551 & 0.052 \\
& & & & & &
\end{tabular}




\begin{tabular}{lllllll}
\multicolumn{7}{c}{ Mixed Exponential Parameters } \\
Gauges & Neutral Pacific Sea Surface Temperature Event \\
& Jlpha & beta 1 & beta 2 & Feb \\
$\mathbf{6 5 3 0 6 0}$ & 0.339 & 8.562 & 8.690 & 0.872 & beta 1 & beta 2 \\
$\mathbf{6 5 3 1 9 0}$ & 0.463 & 0.040 & 1.539 & 0.623 & 0.402 & 0.040 \\
$\mathbf{6 5 3 3 0 0}$ & 0.391 & 1.083 & 0.059 & 1.000 & 0.657 & 0.657 \\
$\mathbf{6 5 3 3 5 0}$ & 0.558 & 0.664 & 0.025 & 0.470 & 2.036 & 0.119 \\
$\mathbf{6 5 3 3 8 0}$ & 0.471 & 0.076 & 1.188 & 0.751 & 0.254 & 1.190 \\
$\mathbf{6 5 3 4 4 0}$ & 0.528 & 0.041 & 1.143 & 0.466 & 0.111 & 1.186 \\
$\mathbf{6 5 3 5 2 0}$ & 0.000 & 0.200 & 0.200 & 0.000 & 0.497 & 0.495 \\
$\mathbf{6 5 3 5 5 0}$ & 0.000 & 1.101 & 1.100 & 0.453 & 1.173 & 0.046 \\
$\mathbf{6 5 3 5 7 0}$ & 1.000 & 0.050 & 12340 & 0.394 & 0.040 & 0.579 \\
$\mathbf{6 5 3 6 1 0}$ & 1.000 & 0.750 & 18.878 & 0.328 & 0.015 & 0.377 \\
$\mathbf{6 5 3 7 6 0}$ & 1.000 & 0.220 & 983 & 1.000 & 0.265 & 154 \\
$\mathbf{6 5 3 8 0 0}$ & 1.000 & 0.080 & 130 & 0.726 & 0.097 & 3.049 \\
$\mathbf{6 5 3 8 7 0}$ & 0.938 & 0.276 & 0.032 & 0.655 & 0.126 & 2.203 \\
$\mathbf{6 5 4 7 2 0}$ & 0.358 & 1.003 & 0.244 & 0.746 & 0.104 & 1.468 \\
$\mathbf{6 5 5 0 1 0}$ & 0.706 & 9.997 & 2.265 & 0.733 & 0.474 & 13.949 \\
$\mathbf{6 5 5 0 2 0}$ & 0.964 & 2.093 & 2.423 & 0.000 & 13.038 & 2.400 \\
$\mathbf{6 5 5 0 3 0}$ & 0.735 & 8.795 & 9.589 & 1.000 & 0.960 & 36.010 \\
$\mathbf{6 5 5 0 5 0}$ & 0.993 & 0.892 & 9.747 & 0.253 & 0.242 & 1.712 \\
$\mathbf{6 5 5 0 7 0}$ & 0.277 & 2.198 & 3.921 & 0.621 & 1.029 & 0.080 \\
$\mathbf{6 5 5 1 0 0}$ & 1.000 & 0.980 & 0.980 & 0.162 & 0.011 & 0.571 \\
$\mathbf{6 5 5 1 6 0}$ & 0.207 & 3.746 & 8.491 & 1.000 & 0.160 & 163 \\
$\mathbf{6 5 5 1 8 0}$ & 0.465 & 5.843 & 8.649 & 1.000 & 0.255 & 37.835 \\
$\mathbf{6 5 5 2 2 0}$ & 0.964 & 1.906 & 0.458 & 0.490 & 0.271 & 0.059 \\
$\mathbf{6 5 5 2 8 0}$ & 1.000 & 0.426 & 44.373 & 0.650 & 0.997 & 0.024 \\
$\mathbf{6 5 5 3 6 0}$ & 0.604 & 0.657 & 18.653 & 1.000 & 0.941 & 156 \\
$\mathbf{6 5 5 4 5 0}$ & 0.471 & 0.040 & 1.940 & 0.547 & 0.229 & 1.700 \\
$\mathbf{6 5 5 4 8 0}$ & 0.000 & 70.683 & 0.040 & 0.795 & 0.392 & 0.124 \\
$\mathbf{6 5 5 5 5 0}$ & 0.693 & 1.780 & 0.250 & 0.238 & 0.023 & 0.892 \\
$\mathbf{6 5 5 5 7 0}$ & 0.779 & 0.265 & 0.975 & 0.967 & 0.476 & 6.102 \\
$\mathbf{6 5 5 6 0 0}$ & 0.745 & 0.499 & 0.020 & 0.308 & 0.041 & 0.907 \\
$\mathbf{6 5 5 6 2 0}$ & 0.434 & 0.020 & 0.444 & 0.000 & 0.573 & 0.421 \\
$\mathbf{6 5 5 6 3 0}$ & 0.737 & 0.201 & 2.830 & 0.639 & 0.475 & 0.106 \\
$\mathbf{6 5 5 7 8 0}$ & 0.676 & 0.101 & 0.763 & 0.286 & 0.078 & 0.354 \\
$\mathbf{6 5 5 8 5 0}$ & 0.245 & 0.062 & 0.476 & 0.438 & 0.107 & 1.092 \\
$\mathbf{6 5 5 9 2 0}$ & 0.306 & 0.074 & 0.463 & 0.564 & 0.573 & 0.061 \\
$\mathbf{6 5 5 9 4 0}$ & 0.758 & 0.318 & 0.016 & 0.651 & 0.168 & 0.038 \\
$\mathbf{6 5 5 9 9 0}$ & 0.569 & 0.078 & 0.510 & 0.950 & 0.490 & 0.039 \\
& & & & & &
\end{tabular}




\begin{tabular}{lllllll}
\multicolumn{7}{c}{ Mixed Exponential Parameters } \\
Gauges & \multicolumn{2}{l}{ Neutral Pacific Sea Surface Temperature Event } \\
& Mlpha & beta 1 & beta 2 & alpha & beta 1 & beta 2 \\
$\mathbf{6 5 3 0 6 0}$ & 0.000 & 4.041 & 0.364 & 0.289 & 1.082 & 0.204 \\
$\mathbf{6 5 3 1 9 0}$ & 0.483 & 0.105 & 0.653 & 0.666 & 0.735 & 0.101 \\
$\mathbf{6 5 3 3 0 0}$ & 0.582 & 0.104 & 0.671 & 0.348 & 0.071 & 0.985 \\
$\mathbf{6 5 3 3 5 0}$ & 0.745 & 0.637 & 0.048 & 0.249 & 0.072 & 0.674 \\
$\mathbf{6 5 3 3 8 0}$ & 0.274 & 0.060 & 0.493 & 0.347 & 0.126 & 0.796 \\
$\mathbf{6 5 3 4 4 0}$ & 0.621 & 0.958 & 0.076 & 0.030 & 5.981 & 0.643 \\
$\mathbf{6 5 3 5 2 0}$ & 0.469 & 2.448 & 0.129 & 0.856 & 0.460 & 0.090 \\
$\mathbf{6 5 3 5 5 0}$ & 0.276 & 0.090 & 0.392 & 0.752 & 0.561 & 0.040 \\
$\mathbf{6 5 3 5 7 0}$ & 0.924 & 0.168 & 5.331 & 0.447 & 0.233 & 0.607 \\
$\mathbf{6 5 3 6 1 0}$ & 0.769 & 0.522 & 0.061 & 0.581 & 0.130 & 0.868 \\
$\mathbf{6 5 3 7 6 0}$ & 0.450 & 0.078 & 1.108 & 0.562 & 0.759 & 0.134 \\
$\mathbf{6 5 3 8 0 0}$ & 0.000 & 45.656 & 0.235 & 0.374 & 0.130 & 0.988 \\
$\mathbf{6 5 3 8 7 0}$ & 0.680 & 0.211 & 1.203 & 0.222 & 2.314 & 0.263 \\
$\mathbf{6 5 4 7 2 0}$ & 0.481 & 0.121 & 0.777 & 0.604 & 0.208 & 2.010 \\
$\mathbf{6 5 5 0 1 0}$ & 0.495 & 0.505 & 0.141 & 0.000 & 184 & 0.189 \\
$\mathbf{6 5 5 0 2 0}$ & 0.000 & 0.375 & 0.346 & 0.432 & 0.090 & 0.401 \\
$\mathbf{6 5 5 0 3 0}$ & 0.678 & 0.118 & 2.155 & 0.878 & 0.485 & 0.044 \\
$\mathbf{6 5 5 0 5 0}$ & 0.132 & 1.740 & 0.137 & 0.561 & 0.449 & 0.033 \\
$\mathbf{6 5 5 0 7 0}$ & 0.456 & 0.204 & 2.797 & 0.537 & 0.097 & 0.575 \\
$\mathbf{6 5 5 1 0 0}$ & 0.607 & 0.454 & 0.061 & 0.507 & 0.082 & 0.611 \\
$\mathbf{6 5 5 1 6 0}$ & 0.803 & 0.125 & 2.728 & 0.427 & 0.830 & 0.142 \\
$\mathbf{6 5 5 1 8 0}$ & 0.134 & 2.298 & 0.211 & 0.341 & 0.075 & 0.569 \\
$\mathbf{6 5 5 2 2 0}$ & 0.226 & 0.109 & 0.741 & 0.644 & 0.690 & 0.075 \\
$\mathbf{6 5 5 2 8 0}$ & 0.572 & 0.064 & 0.339 & 0.259 & 0.078 & 0.513 \\
$\mathbf{6 5 5 3 6 0}$ & 0.491 & 0.170 & 1.062 & 0.500 & 0.905 & 0.193 \\
$\mathbf{6 5 5 4 5 0}$ & 0.465 & 0.960 & 0.095 & 0.813 & 0.391 & 2.233 \\
$\mathbf{6 5 5 4 8 0}$ & 0.858 & 0.386 & 2.589 & 0.457 & 0.180 & 1.112 \\
$\mathbf{6 5 5 5 5 0}$ & 0.884 & 0.373 & 4.543 & 0.051 & 7.208 & 0.589 \\
$\mathbf{6 5 5 5 7 0}$ & 0.044 & 9.461 & 0.399 & 0.986 & 0.619 & 9.301 \\
$\mathbf{6 5 5 6 0 0}$ & 0.422 & 0.107 & 0.852 & 0.889 & 0.713 & 0.089 \\
$\mathbf{6 5 5 6 2 0}$ & 0.575 & 0.791 & 0.071 & 0.905 & 0.580 & 2.273 \\
$\mathbf{6 5 5 6 3 0}$ & 0.281 & 0.062 & 0.439 & 0.107 & 0.968 & 0.437 \\
$\mathbf{6 5 5 7 8 0}$ & 0.540 & 0.955 & 0.135 & 0.274 & 0.067 & 0.805 \\
$\mathbf{6 5 5 8 5 0}$ & 0.568 & 0.788 & 0.062 & 0.562 & 0.937 & 0.141 \\
$\mathbf{6 5 5 9 2 0}$ & 0.168 & 2.299 & 0.217 & 0.330 & 0.067 & 0.711 \\
$\mathbf{6 5 5 9 4 0}$ & 0.505 & 0.064 & 0.491 & 0.412 & 0.129 & 1.102 \\
$\mathbf{6 5 5 9 9 0}$ & 0.307 & 0.961 & 0.162 & 0.226 & 0.058 & 0.858
\end{tabular}




\begin{tabular}{lllllll}
\multicolumn{7}{c}{ Mixed Exponential Parameters } \\
Gauges & Meutral Pacific Sea Surface Temperature Event \\
& May & \multicolumn{5}{c}{ Jun } \\
& alpha & beta 1 & beta 2 & alpha & beta 1 & beta 2 \\
$\mathbf{6 5 3 0 6 0}$ & 0.754 & 0.782 & 0.090 & 0.937 & 0.554 & 0.038 \\
$\mathbf{6 5 3 1 9 0}$ & 0.767 & 0.620 & 0.042 & 0.258 & 0.091 & 0.630 \\
$\mathbf{6 5 3 3 0 0}$ & 0.107 & 0.042 & 0.549 & 0.715 & 0.752 & 0.068 \\
$\mathbf{6 5 3 3 5 0}$ & 0.373 & 0.162 & 0.771 & 0.688 & 0.591 & 0.061 \\
$\mathbf{6 5 3 3 8 0}$ & 0.743 & 0.626 & 0.067 & 0.800 & 0.764 & 0.049 \\
$\mathbf{6 5 3 4 4 0}$ & 0.425 & 0.190 & 0.965 & 0.590 & 1.041 & 0.089 \\
$\mathbf{6 5 3 5 2 0}$ & 0.754 & 0.697 & 0.074 & 0.974 & 0.378 & 7.199 \\
$\mathbf{6 5 3 5 5 0}$ & 0.217 & 0.056 & 0.502 & 0.368 & 0.062 & 0.650 \\
$\mathbf{6 5 3 5 7 0}$ & 0.489 & 0.663 & 0.265 & 0.307 & 0.088 & 0.837 \\
$\mathbf{6 5 3 6 1 0}$ & 0.165 & 0.955 & 0.231 & 0.877 & 0.414 & 0.036 \\
$\mathbf{6 5 3 7 6 0}$ & 0.851 & 0.339 & 2.849 & 0.765 & 0.295 & 1.541 \\
$\mathbf{6 5 3 8 0 0}$ & 0.706 & 1.020 & 0.097 & 0.592 & 0.836 & 0.196 \\
$\mathbf{6 5 3 8 7 0}$ & 0.957 & 0.386 & 4.750 & 0.422 & 0.081 & 0.827 \\
$\mathbf{6 5 4 7 2 0}$ & 0.873 & 0.839 & 0.062 & 0.397 & 0.033 & 0.685 \\
$\mathbf{6 5 5 0 1 0}$ & 0.555 & 0.051 & 0.514 & 0.255 & 0.787 & 0.201 \\
$\mathbf{6 5 5 0 2 0}$ & 0.226 & 2.580 & 0.205 & 0.326 & 0.180 & 0.506 \\
$\mathbf{6 5 5 0 3 0}$ & 0.974 & 0.357 & 3.713 & 0.833 & 0.289 & 1.123 \\
$\mathbf{6 5 5 0 5 0}$ & 0.713 & 0.639 & 0.104 & 0.761 & 0.509 & 0.113 \\
$\mathbf{6 5 5 0 7 0}$ & 0.374 & 0.124 & 0.607 & 0.969 & 0.503 & 0.075 \\
$\mathbf{6 5 5 1 0 0}$ & 0.186 & 0.050 & 0.534 & 0.238 & 0.104 & 0.620 \\
$\mathbf{6 5 5 1 6 0}$ & 0.196 & 0.051 & 0.536 & 0.119 & 0.066 & 0.496 \\
$\mathbf{6 5 5 1 8 0}$ & 0.292 & 0.107 & 0.447 & 0.000 & 0.253 & 0.629 \\
$\mathbf{6 5 5 2 2 0}$ & 0.694 & 0.748 & 0.072 & 0.434 & 0.323 & 0.641 \\
$\mathbf{6 5 5 2 8 0}$ & 0.200 & 0.125 & 0.643 & 0.024 & 0.032 & 0.499 \\
$\mathbf{6 5 5 3 6 0}$ & 0.026 & 4.297 & 0.532 & 0.934 & 0.629 & 0.099 \\
$\mathbf{6 5 5 4 5 0}$ & 0.169 & 0.045 & 0.648 & 0.251 & 0.899 & 0.386 \\
$\mathbf{6 5 5 4 8 0}$ & 0.102 & 0.035 & 0.595 & 0.308 & 0.068 & 0.841 \\
$\mathbf{6 5 5 5 5 0}$ & 0.963 & 0.473 & 0.103 & 0.802 & 0.642 & 0.057 \\
$\mathbf{6 5 5 5 7 0}$ & 0.385 & 0.224 & 0.632 & 0.257 & 0.105 & 0.617 \\
$\mathbf{6 5 5 6 0 0}$ & 0.331 & 0.110 & 0.641 & 0.543 & 0.144 & 0.788 \\
$\mathbf{6 5 5 6 2 0}$ & 0.349 & 0.086 & 0.715 & 0.627 & 0.652 & 0.097 \\
$\mathbf{6 5 5 6 3 0}$ & 0.150 & 1.347 & 0.330 & 0.221 & 0.084 & 0.485 \\
$\mathbf{6 5 5 7 8 0}$ & 0.317 & 0.054 & 0.991 & 0.624 & 1.333 & 0.126 \\
$\mathbf{6 5 5 8 5 0}$ & 0.318 & 0.095 & 0.801 & 0.446 & 0.180 & 1.314 \\
$\mathbf{6 5 5 9 2 0}$ & 0.312 & 0.070 & 0.915 & 0.565 & 1.720 & 0.117 \\
$\mathbf{6 5 5 9 4 0}$ & 0.218 & 0.060 & 0.731 & 0.514 & 1.276 & 0.171 \\
$\mathbf{6 5 5 9 9 0}$ & 0.775 & 0.714 & 0.086 & 0.601 & 1.097 & 0.140
\end{tabular}




\begin{tabular}{lllllll}
\multicolumn{7}{c}{ Mixed Exponential Parameters } \\
Gauges & \multicolumn{2}{l}{ Neutral Pacific Sea Surface Temperature Event } \\
& Julpha & beta 1 & beta 2 & alpha & beta 1 & beta 2 \\
$\mathbf{6 5 3 0 6 0}$ & 0.831 & 0.813 & 0.046 & 0.262 & 0.064 & 0.834 \\
$\mathbf{6 5 3 1 9 0}$ & 0.768 & 0.562 & 0.041 & 0.989 & 0.468 & 5.853 \\
$\mathbf{6 5 3 3 0 0}$ & 0.470 & 1.087 & 0.160 & 0.806 & 0.697 & 0.048 \\
$\mathbf{6 5 3 3 5 0}$ & 0.662 & 0.818 & 0.055 & 0.590 & 0.636 & 0.080 \\
$\mathbf{6 5 3 3 8 0}$ & 0.570 & 0.918 & 0.065 & 0.605 & 0.119 & 0.790 \\
$\mathbf{6 5 3 4 4 0}$ & 0.697 & 0.087 & 0.798 & 0.397 & 0.951 & 0.057 \\
$\mathbf{6 5 3 5 2 0}$ & 0.651 & 1.054 & 0.037 & 0.156 & 0.072 & 0.606 \\
$\mathbf{6 5 3 5 5 0}$ & 0.353 & 0.053 & 0.772 & 0.163 & 0.049 & 0.815 \\
$\mathbf{6 5 3 5 7 0}$ & 0.632 & 0.213 & 0.835 & 0.252 & 0.042 & 0.658 \\
$\mathbf{6 5 3 6 1 0}$ & 0.665 & 0.634 & 0.093 & 0.769 & 0.578 & 0.083 \\
$\mathbf{6 5 3 7 6 0}$ & 0.437 & 0.144 & 0.779 & 0.527 & 0.097 & 0.928 \\
$\mathbf{6 5 3 8 0 0}$ & 0.421 & 0.079 & 0.535 & 0.750 & 0.073 & 1.503 \\
$\mathbf{6 5 3 8 7 0}$ & 0.552 & 0.061 & 0.711 & 0.184 & 0.614 & 0.074 \\
$\mathbf{6 5 4 7 2 0}$ & 0.405 & 0.804 & 0.039 & 0.306 & 0.319 & 0.034 \\
$\mathbf{6 5 5 0 1 0}$ & 0.247 & 0.070 & 0.606 & 0.835 & 0.640 & 0.066 \\
$\mathbf{6 5 5 0 2 0}$ & 0.068 & 0.025 & 0.540 & 0.217 & 0.095 & 0.569 \\
$\mathbf{6 5 5 0 3 0}$ & 0.189 & 0.068 & 0.689 & 0.853 & 0.553 & 0.037 \\
$\mathbf{6 5 5 0 5 0}$ & 0.891 & 0.590 & 0.097 & 0.138 & 0.047 & 0.591 \\
$\mathbf{6 5 5 0 7 0}$ & 0.637 & 0.856 & 0.180 & 0.325 & 0.096 & 0.748 \\
$\mathbf{6 5 5 1 0 0}$ & 0.763 & 0.636 & 0.078 & 0.172 & 0.054 & 0.758 \\
$\mathbf{6 5 5 1 6 0}$ & 0.165 & 0.076 & 0.588 & 0.868 & 0.607 & 0.043 \\
$\mathbf{6 5 5 1 8 0}$ & 0.402 & 0.092 & 1.046 & 0.850 & 0.652 & 0.049 \\
$\mathbf{6 5 5 2 2 0}$ & 0.726 & 0.768 & 0.091 & 0.189 & 0.090 & 0.632 \\
$\mathbf{6 5 5 2 8 0}$ & 0.391 & 1.199 & 0.313 & 0.789 & 0.671 & 0.099 \\
$\mathbf{6 5 5 3 6 0}$ & 0.906 & 0.670 & 0.045 & 0.173 & 0.057 & 0.619 \\
$\mathbf{6 5 5 4 5 0}$ & 0.370 & 0.088 & 0.807 & 0.630 & 0.837 & 0.045 \\
$\mathbf{6 5 5 4 8 0}$ & 0.594 & 0.937 & 0.060 & 0.558 & 0.186 & 1.325 \\
$\mathbf{6 5 5 5 5 0}$ & 0.905 & 0.465 & 5.480 & 0.941 & 0.504 & 4.629 \\
$\mathbf{6 5 5 5 7 0}$ & 0.415 & 0.084 & 0.475 & 0.661 & 0.113 & 0.760 \\
$\mathbf{6 5 5 6 0 0}$ & 0.481 & 0.506 & 0.069 & 0.452 & 0.077 & 0.742 \\
$\mathbf{6 5 5 6 2 0}$ & 0.679 & 0.165 & 0.808 & 0.481 & 0.506 & 0.042 \\
$\mathbf{6 5 5 6 3 0}$ & 0.568 & 0.100 & 1.161 & 0.463 & 0.061 & 0.634 \\
$\mathbf{6 5 5 7 8 0}$ & 0.294 & 1.324 & 0.104 & 0.105 & 1.724 & 0.113 \\
$\mathbf{6 5 5 8 5 0}$ & 0.398 & 1.090 & 0.064 & 0.713 & 0.063 & 0.408 \\
$\mathbf{6 5 5 9 2 0}$ & 0.804 & 0.160 & 2.070 & 0.408 & 0.797 & 0.086 \\
$\mathbf{6 5 5 9 4 0}$ & 0.793 & 0.118 & 2.005 & 0.833 & 0.108 & 0.619 \\
$\mathbf{6 5 5 9 9 0}$ & 0.694 & 0.081 & 1.053 & 0.844 & 0.102 & 0.499
\end{tabular}




\begin{tabular}{lllllll}
\multicolumn{7}{c}{ Mixed Exponential Parameters } \\
Gauges & Neutral Pacific Sea Surface Temperature Event \\
& Sep & \multicolumn{5}{c}{ Oct } \\
$\mathbf{6 5 3 0 6 0}$ & 0.067 & beta 1 & beta 2 & alpha & beta 1 & beta 2 \\
$\mathbf{6 5 3 1 9 0}$ & 0.107 & 0.034 & 0.633 & 0.727 & 0.248 & 0.675 \\
$\mathbf{6 5 3 3 0 0}$ & 0.773 & 0.655 & 0.089 & 0.767 & 0.455 & 0.085 \\
$\mathbf{6 5 3 3 5 0}$ & 0.397 & 0.094 & 0.663 & 0.155 & 0.487 & 0.066 \\
$\mathbf{6 5 3 3 8 0}$ & 0.262 & 0.039 & 0.574 & 0.852 & 0.538 & 0.492 \\
$\mathbf{6 5 3 4 4 0}$ & 0.560 & 0.587 & 0.043 & 0.292 & 0.043 & 0.687 \\
$\mathbf{6 5 3 5 2 0}$ & 0.173 & 0.047 & 0.514 & 0.175 & 0.035 & 0.350 \\
$\mathbf{6 5 3 5 5 0}$ & 0.101 & 0.820 & 0.414 & 0.753 & 0.472 & 0.050 \\
$\mathbf{6 5 3 5 7 0}$ & 0.000 & 0.708 & 0.512 & 0.020 & 0.146 & 0.519 \\
$\mathbf{6 5 3 6 1 0}$ & 0.148 & 0.042 & 0.507 & 0.899 & 0.486 & 0.109 \\
$\mathbf{6 5 3 7 6 0}$ & 0.735 & 0.668 & 0.090 & 0.809 & 0.563 & 0.073 \\
$\mathbf{6 5 3 8 0 0}$ & 0.509 & 0.928 & 0.061 & 0.313 & 0.066 & 0.554 \\
$\mathbf{6 5 3 8 7 0}$ & 0.516 & 0.058 & 0.453 & 0.718 & 0.192 & 1.032 \\
$\mathbf{6 5 4 7 2 0}$ & 0.353 & 0.037 & 0.229 & 0.771 & 0.259 & 0.022 \\
$\mathbf{6 5 5 0 1 0}$ & 0.796 & 0.417 & 0.061 & 0.916 & 0.069 & 1.206 \\
$\mathbf{6 5 5 0 2 0}$ & 0.739 & 0.539 & 0.070 & 0.732 & 0.185 & 0.676 \\
$\mathbf{6 5 5 0 3 0}$ & 0.055 & 3.577 & 0.359 & 0.188 & 0.101 & 0.235 \\
$\mathbf{6 5 5 0 5 0}$ & 0.584 & 0.597 & 0.116 & 0.534 & 0.562 & 0.063 \\
$\mathbf{6 5 5 0 7 0}$ & 0.819 & 0.527 & 0.045 & 0.843 & 0.291 & 0.060 \\
$\mathbf{6 5 5 1 0 0}$ & 0.145 & 0.054 & 0.572 & 0.801 & 0.448 & 0.027 \\
$\mathbf{6 5 5 1 6 0}$ & 0.025 & 2.343 & 0.455 & 0.358 & 0.536 & 0.232 \\
$\mathbf{6 5 5 1 8 0}$ & 0.000 & 0.414 & 0.414 & 0.181 & 0.096 & 0.498 \\
$\mathbf{6 5 5 2 2 0}$ & 0.936 & 0.521 & 0.082 & 0.408 & 0.092 & 0.410 \\
$\mathbf{6 5 5 2 8 0}$ & 0.793 & 0.659 & 0.120 & 0.984 & 0.324 & 5.616 \\
$\mathbf{6 5 5 3 6 0}$ & 0.117 & 0.090 & 0.630 & 0.952 & 0.308 & 3.120 \\
$\mathbf{6 5 5 4 5 0}$ & 0.892 & 0.529 & 0.043 & 0.808 & 0.592 & 0.049 \\
$\mathbf{6 5 5 4 8 0}$ & 0.336 & 0.840 & 0.365 & 0.744 & 0.346 & 0.912 \\
$\mathbf{6 5 5 5 5 0}$ & 0.113 & 2.098 & 0.403 & 0.926 & 0.407 & 1.072 \\
$\mathbf{6 5 5 5 7 0}$ & 0.462 & 0.111 & 0.747 & 0.235 & 0.096 & 0.530 \\
$\mathbf{6 5 5 6 0 0}$ & 0.503 & 0.154 & 0.532 & 0.263 & 0.068 & 0.363 \\
$\mathbf{6 5 5 6 2 0}$ & 0.786 & 0.502 & 0.062 & 0.177 & 0.057 & 0.502 \\
$\mathbf{6 5 5 6 3 0}$ & 0.567 & 0.663 & 0.101 & 0.440 & 0.088 & 0.722 \\
$\mathbf{6 5 5 7 8 0}$ & 0.827 & 0.113 & 3.471 & 0.587 & 0.092 & 1.019 \\
$\mathbf{6 5 5 8 5 0}$ & 0.315 & 0.976 & 0.080 & 0.381 & 0.097 & 0.694 \\
$\mathbf{6 5 5 9 2 0}$ & 0.167 & 1.748 & 0.200 & 0.400 & 1.448 & 0.090 \\
$\mathbf{6 5 5 9 4 0}$ & 0.846 & 0.132 & 1.083 & 0.308 & 0.667 & 0.119 \\
$\mathbf{6 5 5 9 9 0}$ & 0.502 & 0.668 & 0.053 & 0.629 & 0.130 & 0.811
\end{tabular}




\begin{tabular}{lllllll}
\multicolumn{7}{c}{ Mixed Exponential Parameters } \\
Gauges & Neutral Pacific Sea Surface Temperature Event \\
& Novha & beta 1 & beta 2 & alpha & beta 1 & beta 2 \\
$\mathbf{6 5 3 0 6 0}$ & 0.844 & 2.722 & 11.412 & 0.000 & 72.452 & 2.130 \\
$\mathbf{6 5 3 1 9 0}$ & 0.000 & 0.295 & 0.264 & 0.000 & 0.696 & 0.215 \\
$\mathbf{6 5 3 3 0 0}$ & 0.824 & 0.324 & 0.039 & 0.000 & 0.237 & 0.237 \\
$\mathbf{6 5 3 3 5 0}$ & 0.932 & 0.409 & 0.028 & 1.000 & 0.210 & 99.502 \\
$\mathbf{6 5 3 3 8 0}$ & 0.930 & 0.236 & 2.646 & 0.595 & 1.434 & 0.223 \\
$\mathbf{6 5 3 4 4 0}$ & 0.227 & 0.053 & 0.454 & 0.687 & 0.064 & 1.256 \\
$\mathbf{6 5 3 5 2 0}$ & 0.000 & 70.560 & 0.168 & 1.000 & 1.850 & 47.379 \\
$\mathbf{6 5 3 5 5 0}$ & 0.846 & 0.492 & 0.010 & 0.443 & 0.155 & 0.015 \\
$\mathbf{6 5 3 5 7 0}$ & 0.000 & 2.352 & 0.412 & 0.800 & 0.611 & 0.040 \\
$\mathbf{6 5 3 6 1 0}$ & 0.222 & 3.729 & 0.211 & 0.216 & 0.045 & 0.133 \\
$\mathbf{6 5 3 7 6 0}$ & 0.747 & 0.096 & 0.904 & 0.709 & 0.322 & 3.178 \\
$\mathbf{6 5 3 8 0 0}$ & 0.328 & 0.518 & 0.112 & 0.602 & 1.702 & 0.034 \\
$\mathbf{6 5 3 8 7 0}$ & 0.797 & 0.339 & 0.029 & 0.736 & 0.038 & 1.904 \\
$\mathbf{6 5 4 7 2 0}$ & 0.357 & 0.037 & 0.540 & 1.000 & 0.317 & 17.885 \\
$\mathbf{6 5 5 0 1 0}$ & 0.506 & 2.413 & 0.010 & 0.000 & 2.990 & 2.990 \\
$\mathbf{6 5 5 0 2 0}$ & 0.430 & 1.936 & 0.155 & 1.000 & 0.040 & 19128 \\
$\mathbf{6 5 5 0 3 0}$ & 0.520 & 1.351 & 0.084 & 0.000 & 13.499 & 1.590 \\
$\mathbf{6 5 5 0 5 0}$ & 1.000 & 0.140 & 289 & 1.000 & 2.400 & 2.400 \\
$\mathbf{6 5 5 0 7 0}$ & 0.242 & 0.010 & 0.188 & 0.427 & 2.273 & 0.046 \\
$\mathbf{6 5 5 1 0 0}$ & 0.904 & 0.570 & 0.085 & 0.432 & 0.308 & 0.024 \\
$\mathbf{6 5 5 1 6 0}$ & 0.777 & 0.120 & 1.058 & 0.524 & 2.330 & 0.040 \\
$\mathbf{6 5 5 1 8 0}$ & 1.000 & 0.155 & 115 & 0.000 & 39.017 & 0.470 \\
$\mathbf{6 5 5 2 2 0}$ & 0.822 & 0.415 & 0.071 & 0.495 & 0.010 & 2.963 \\
$\mathbf{6 5 5 2 8 0}$ & 0.899 & 0.292 & 3.484 & 0.300 & 1.228 & 0.131 \\
$\mathbf{6 5 5 3 6 0}$ & 0.484 & 0.040 & 0.536 & 0.000 & 1.044 & 1.043 \\
$\mathbf{6 5 5 4 5 0}$ & 0.159 & 0.743 & 0.205 & 0.205 & 0.014 & 0.404 \\
$\mathbf{6 5 5 4 8 0}$ & 0.107 & 0.566 & 0.193 & 0.718 & 1.232 & 0.044 \\
$\mathbf{6 5 5 5 5 0}$ & 0.161 & 0.149 & 0.444 & 0.684 & 0.436 & 0.075 \\
$\mathbf{6 5 5 5 7 0}$ & 0.505 & 0.204 & 0.509 & 0.812 & 0.587 & 0.071 \\
$\mathbf{6 5 5 6 0 0}$ & 0.000 & 2.195 & 0.278 & 1.000 & 0.712 & 0.712 \\
$\mathbf{6 5 5 6 2 0}$ & 0.666 & 0.573 & 0.122 & 0.949 & 0.652 & 0.018 \\
$\mathbf{6 5 5 6 3 0}$ & 0.045 & 2.333 & 0.298 & 0.387 & 0.061 & 0.507 \\
$\mathbf{6 5 5 7 8 0}$ & 0.939 & 0.430 & 0.043 & 0.981 & 0.472 & 14.366 \\
$\mathbf{6 5 5 8 5 0}$ & 0.066 & 1.195 & 0.277 & 0.595 & 0.492 & 0.082 \\
$\mathbf{6 5 5 9 2 0}$ & 0.531 & 0.720 & 0.090 & 0.285 & 0.047 & 0.982 \\
$\mathbf{6 5 5 9 4 0}$ & 0.330 & 1.215 & 0.173 & 0.561 & 0.908 & 0.106 \\
$\mathbf{6 5 5 9 9 0}$ & 0.490 & 0.102 & 0.512 & 0.343 & 0.048 & 0.738
\end{tabular}




\begin{tabular}{lllllll}
\multicolumn{7}{c}{ Mixed Exponential Parameters } \\
Gauges & Warm Pacific Sea Surface Temperature Event \\
& Jalpha & beta 1 & beta 2 & alpha & beta 1 & beta 2 \\
$\mathbf{6 5 3 0 6 0}$ & 0.021 & 2.190 & 4.620 & 0.054 & 8.451 & 6.413 \\
$\mathbf{6 5 3 1 9 0}$ & 0.000 & 0.019 & 0.020 & 0.040 & 0.042 & 0.181 \\
$\mathbf{6 5 3 3 0 0}$ & 0.838 & 0.225 & 0.041 & 0.628 & 0.337 & 0.038 \\
$\mathbf{6 5 3 3 5 0}$ & 0.599 & 3.569 & 12.893 & 1.000 & 0.268 & 5.952 \\
$\mathbf{6 5 3 3 8 0}$ & 0.644 & 0.015 & 0.666 & 0.933 & 0.338 & 0.020 \\
$\mathbf{6 5 3 4 4 0}$ & 0.444 & 0.029 & 1.166 & 0.485 & 0.183 & 0.758 \\
$\mathbf{6 5 3 5 2 0}$ & 0.026 & 9.279 & 10.380 & 0.500 & 0.029 & 0.217 \\
$\mathbf{6 5 3 5 5 0}$ & 0.284 & 1.375 & 10.538 & 1.000 & 0.375 & 2.356 \\
$\mathbf{6 5 3 5 7 0}$ & 0.000 & 2.560 & 2.560 & 0.630 & 3.635 & 17.886 \\
$\mathbf{6 5 3 6 1 0}$ & 1.000 & 0.235 & 10.525 & 1.000 & 0.475 & 88.716 \\
$\mathbf{6 5 3 7 6 0}$ & 0.000 & 0.063 & 0.100 & 0.767 & 0.326 & 0.039 \\
$\mathbf{6 5 3 8 0 0}$ & 0.789 & 4.714 & 10.072 & 0.000 & 38.870 & 0.413 \\
$\mathbf{6 5 3 8 7 0}$ & 0.575 & 0.797 & 0.040 & 0.986 & 0.202 & 0.017 \\
$\mathbf{6 5 4 7 2 0}$ & 0.000 & 70.613 & 0.033 & 0.913 & 0.164 & 0.010 \\
$\mathbf{6 5 5 0 1 0}$ & 0.009 & 6.746 & 15.432 & 1.000 & 0.470 & 920 \\
$\mathbf{6 5 5 0 2 0}$ & 1.000 & 0.080 & 10848 & 0.509 & 4.264 & 6.875 \\
$\mathbf{6 5 5 0 3 0}$ & 0.529 & 0.528 & 9.057 & 0.000 & 0.931 & 0.080 \\
$\mathbf{6 5 5 0 5 0}$ & 0.000 & 22.029 & 0.020 & 0.081 & 6.465 & 8.531 \\
$\mathbf{6 5 5 0 7 0}$ & 0.048 & 4.310 & 2.682 & 0.852 & 3.167 & 15.698 \\
$\mathbf{6 5 5 1 0 0}$ & 1.000 & 0.080 & 38.336 & 0.000 & 76.796 & 0.200 \\
$\mathbf{6 5 5 1 6 0}$ & 0.000 & 0.162 & 0.170 & 0.320 & 8.530 & 1.291 \\
$\mathbf{6 5 5 1 8 0}$ & 0.882 & 8.197 & 17.804 & 0.000 & 53.643 & 0.040 \\
$\mathbf{6 5 5 2 2 0}$ & 1.000 & 0.053 & 176 & 1.000 & 0.670 & 111 \\
$\mathbf{6 5 5 2 8 0}$ & 1.000 & 0.173 & 34.840 & 0.397 & 0.040 & 0.596 \\
$\mathbf{6 5 5 3 6 0}$ & 0.253 & 0.121 & 0.856 & 0.000 & 112 & 0.865 \\
$\mathbf{6 5 5 4 5 0}$ & 0.616 & 0.035 & 0.690 & 0.000 & 3.937 & 0.229 \\
$\mathbf{6 5 5 4 8 0}$ & 0.656 & 0.963 & 0.030 & 0.267 & 0.010 & 0.575 \\
$\mathbf{6 5 5 5 5 0}$ & 1.000 & 0.665 & 65.564 & 1.000 & 0.574 & 32.659 \\
$\mathbf{6 5 5 5 7 0}$ & 1.000 & 0.156 & 0.156 & 0.069 & 5.941 & 0.418 \\
$\mathbf{6 5 5 6 0 0}$ & 0.000 & 0.179 & 0.183 & 0.152 & 0.140 & 0.503 \\
$\mathbf{6 5 5 6 2 0}$ & 1.000 & 0.080 & 1419 & 0.000 & 0.353 & 0.353 \\
$\mathbf{6 5 5 6 3 0}$ & 1.000 & 1.220 & 43.111 & 0.576 & 0.238 & 0.075 \\
$\mathbf{6 5 5 7 8 0}$ & 0.535 & 0.052 & 1.004 & 0.755 & 0.110 & 1.196 \\
$\mathbf{6 5 5 8 5 0}$ & 0.412 & 1.136 & 0.066 & 0.362 & 0.040 & 0.572 \\
$\mathbf{6 5 5 9 2 0}$ & 0.583 & 0.257 & 0.084 & 0.458 & 0.042 & 0.470 \\
$\mathbf{6 5 5 9 4 0}$ & 0.939 & 0.745 & 0.168 & 0.385 & 0.113 & 0.250 \\
$\mathbf{6 5 5 9 9 0}$ & 0.428 & 0.020 & 0.414 & 1.000 & 0.358 & 8.197
\end{tabular}




\begin{tabular}{lllllll}
\multicolumn{7}{c}{ Mixed Exponential Parameters } \\
Gauges & Marm Pacific Sea Surface Temperature Event \\
& Marpha & beta 1 & beta 2 & alpha & beta 1 & beta 2 \\
$\mathbf{6 5 3 0 6 0}$ & 1.000 & 0.395 & 1.888 & 0.377 & 0.070 & 0.487 \\
$\mathbf{6 5 3 1 9 0}$ & 0.375 & 0.040 & 0.356 & 0.634 & 0.091 & 1.619 \\
$\mathbf{6 5 3 3 0 0}$ & 0.597 & 0.095 & 1.281 & 0.783 & 0.545 & 0.039 \\
$\mathbf{6 5 3 3 5 0}$ & 0.101 & 0.017 & 0.450 & 0.589 & 0.281 & 0.974 \\
$\mathbf{6 5 3 3 8 0}$ & 0.821 & 0.791 & 0.039 & 0.249 & 0.044 & 0.772 \\
$\mathbf{6 5 3 4 4 0}$ & 0.802 & 0.582 & 0.027 & 0.790 & 0.276 & 4.887 \\
$\mathbf{6 5 3 5 2 0}$ & 0.707 & 0.642 & 0.026 & 0.608 & 0.067 & 0.221 \\
$\mathbf{6 5 3 5 5 0}$ & 0.356 & 1.458 & 0.056 & 0.738 & 0.102 & 0.561 \\
$\mathbf{6 5 3 5 7 0}$ & 0.000 & 0.439 & 0.450 & 1.000 & 0.325 & 0.325 \\
$\mathbf{6 5 3 6 1 0}$ & 0.000 & 0.478 & 0.478 & 0.907 & 0.194 & 2.424 \\
$\mathbf{6 5 3 7 6 0}$ & 0.827 & 0.341 & 0.072 & 0.470 & 1.487 & 0.146 \\
$\mathbf{6 5 3 8 0 0}$ & 0.787 & 0.860 & 0.088 & 0.690 & 0.571 & 0.067 \\
$\mathbf{6 5 3 8 7 0}$ & 0.446 & 0.088 & 0.749 & 0.512 & 0.071 & 1.178 \\
$\mathbf{6 5 4 7 2 0}$ & 0.330 & 0.065 & 0.742 & 0.148 & 0.010 & 0.425 \\
$\mathbf{6 5 5 0 1 0}$ & 1.000 & 0.040 & 57.886 & 1.000 & 0.040 & 1218 \\
$\mathbf{6 5 5 0 2 0}$ & 0.567 & 0.439 & 0.020 & 1.000 & 0.140 & 0.140 \\
$\mathbf{6 5 5 0 3 0}$ & 1.000 & 0.210 & 219 & 0.109 & 0.022 & 0.490 \\
$\mathbf{6 5 5 0 5 0}$ & 0.430 & 0.533 & 0.219 & 0.253 & 1.872 & 0.292 \\
$\mathbf{6 5 5 0 7 0}$ & 1.000 & 0.128 & 0.128 & 0.249 & 0.031 & 0.441 \\
$\mathbf{6 5 5 1 0 0}$ & 0.815 & 0.065 & 0.335 & 0.376 & 0.141 & 0.684 \\
$\mathbf{6 5 5 1 6 0}$ & 1.000 & 0.130 & 0.129 & 0.914 & 0.229 & 3.647 \\
$\mathbf{6 5 5 1 8 0}$ & 0.773 & 0.834 & 0.010 & 0.383 & 1.310 & 0.175 \\
$\mathbf{6 5 5 2 2 0}$ & 1.000 & 0.489 & 19.124 & 0.537 & 0.132 & 0.601 \\
$\mathbf{6 5 5 2 8 0}$ & 0.263 & 0.025 & 0.390 & 0.468 & 0.151 & 1.233 \\
$\mathbf{6 5 5 3 6 0}$ & 0.509 & 0.105 & 1.232 & 0.343 & 0.141 & 0.920 \\
$\mathbf{6 5 5 4 5 0}$ & 0.243 & 0.195 & 0.849 & 0.000 & 27.503 & 0.449 \\
$\mathbf{6 5 5 4 8 0}$ & 0.377 & 0.081 & 1.307 & 0.422 & 0.175 & 1.806 \\
$\mathbf{6 5 5 5 5 0}$ & 0.617 & 1.790 & 0.048 & 0.107 & 1.795 & 0.431 \\
$\mathbf{6 5 5 5 7 0}$ & 0.076 & 0.051 & 0.626 & 0.665 & 0.642 & 0.300 \\
$\mathbf{6 5 5 6 0 0}$ & 0.386 & 0.029 & 0.427 & 0.035 & 10.066 & 0.450 \\
$\mathbf{6 5 5 6 2 0}$ & 0.396 & 0.760 & 0.181 & 0.852 & 0.315 & 1.619 \\
$\mathbf{6 5 5 6 3 0}$ & 0.430 & 0.144 & 0.539 & 0.703 & 0.626 & 0.088 \\
$\mathbf{6 5 5 7 8 0}$ & 0.315 & 0.925 & 0.153 & 0.125 & 0.049 & 0.611 \\
$\mathbf{6 5 5 8 5 0}$ & 0.920 & 0.322 & 3.409 & 0.560 & 0.096 & 1.085 \\
$\mathbf{6 5 5 9 2 0}$ & 0.055 & 0.015 & 0.567 & 0.376 & 0.975 & 0.149 \\
$\mathbf{6 5 5 9 4 0}$ & 0.045 & 0.417 & 0.875 & 0.211 & 0.039 & 0.591 \\
$\mathbf{6 5 5 9 9 0}$ & 0.130 & 0.045 & 0.698 & 0.604 & 0.253 & 1.081
\end{tabular}




\begin{tabular}{lllllll}
\multicolumn{7}{c}{ Mixed Exponential Parameters } \\
Gauges & Marm Pacific Sea Surface Temperature Event \\
& Malpha & beta 1 & beta 2 & alpha & beta 1 & beta 2 \\
$\mathbf{6 5 3 0 6 0}$ & 0.217 & 0.079 & 0.556 & 0.221 & 0.026 & 0.899 \\
$\mathbf{6 5 3 1 9 0}$ & 0.417 & 0.145 & 0.597 & 0.132 & 0.069 & 0.424 \\
$\mathbf{6 5 3 3 0 0}$ & 0.020 & 5.392 & 0.397 & 0.365 & 0.085 & 0.554 \\
$\mathbf{6 5 3 3 5 0}$ & 0.281 & 0.063 & 0.581 & 0.735 & 0.516 & 0.058 \\
$\mathbf{6 5 3 3 8 0}$ & 0.915 & 0.530 & 0.039 & 0.815 & 0.527 & 0.046 \\
$\mathbf{6 5 3 4 4 0}$ & 0.294 & 0.034 & 0.926 & 0.248 & 0.043 & 1.517 \\
$\mathbf{6 5 3 5 2 0}$ & 0.111 & 0.013 & 0.571 & 0.711 & 0.215 & 2.722 \\
$\mathbf{6 5 3 5 5 0}$ & 0.935 & 0.470 & 0.019 & 0.314 & 0.075 & 0.634 \\
$\mathbf{6 5 3 5 7 0}$ & 0.790 & 0.369 & 1.733 & 0.759 & 0.338 & 2.361 \\
$\mathbf{6 5 3 6 1 0}$ & 0.954 & 0.431 & 3.480 & 0.379 & 0.091 & 0.529 \\
$\mathbf{6 5 3 7 6 0}$ & 0.890 & 0.617 & 0.038 & 0.372 & 0.121 & 1.193 \\
$\mathbf{6 5 3 8 0 0}$ & 0.659 & 0.728 & 0.063 & 0.622 & 0.142 & 1.743 \\
$\mathbf{6 5 3 8 7 0}$ & 0.531 & 0.067 & 1.077 & 0.441 & 0.086 & 1.134 \\
$\mathbf{6 5 4 7 2 0}$ & 0.555 & 0.609 & 0.274 & 0.425 & 0.075 & 1.065 \\
$\mathbf{6 5 5 0 1 0}$ & 0.335 & 0.654 & 0.124 & 0.239 & 0.037 & 0.552 \\
$\mathbf{6 5 5 0 2 0}$ & 0.848 & 0.231 & 0.949 & 0.690 & 0.246 & 1.618 \\
$\mathbf{6 5 5 0 3 0}$ & 0.886 & 0.267 & 2.597 & 0.592 & 0.191 & 0.905 \\
$\mathbf{6 5 5 0 5 0}$ & 0.855 & 0.226 & 1.565 & 0.873 & 0.377 & 3.696 \\
$\mathbf{6 5 5 0 7 0}$ & 0.186 & 3.186 & 0.263 & 0.620 & 0.188 & 2.085 \\
$\mathbf{6 5 5 1 0 0}$ & 0.657 & 0.146 & 1.095 & 0.291 & 0.060 & 0.631 \\
$\mathbf{6 5 5 1 6 0}$ & 0.534 & 0.629 & 0.134 & 0.901 & 0.488 & 0.057 \\
$\mathbf{6 5 5 1 8 0}$ & 0.126 & 0.069 & 0.593 & 0.139 & 0.042 & 0.586 \\
$\mathbf{6 5 5 2 2 0}$ & 0.260 & 0.053 & 0.544 & 0.962 & 0.558 & 4.489 \\
$\mathbf{6 5 5 2 8 0}$ & 0.198 & 1.002 & 0.278 & 0.288 & 0.117 & 0.616 \\
$\mathbf{6 5 5 3 6 0}$ & 0.314 & 0.072 & 0.639 & 0.043 & 0.029 & 0.580 \\
$\mathbf{6 5 5 4 5 0}$ & 0.149 & 0.029 & 0.734 & 0.137 & 0.055 & 0.753 \\
$\mathbf{6 5 5 4 8 0}$ & 0.402 & 0.118 & 0.770 & 0.267 & 0.068 & 0.877 \\
$\mathbf{6 5 5 5 5 0}$ & 0.613 & 0.211 & 1.167 & 0.146 & 1.596 & 0.315 \\
$\mathbf{6 5 5 5 7 0}$ & 0.772 & 0.400 & 1.010 & 0.755 & 0.224 & 1.111 \\
$\mathbf{6 5 5 6 0 0}$ & 0.391 & 0.228 & 0.715 & 0.201 & 3.749 & 0.294 \\
$\mathbf{6 5 5 6 2 0}$ & 0.184 & 0.042 & 0.643 & 0.856 & 0.276 & 2.301 \\
$\mathbf{6 5 5 6 3 0}$ & 0.718 & 0.785 & 0.112 & 0.944 & 0.364 & 4.653 \\
$\mathbf{6 5 5 7 8 0}$ & 0.403 & 0.090 & 1.038 & 0.312 & 0.134 & 1.729 \\
$\mathbf{6 5 5 8 5 0}$ & 0.525 & 1.199 & 0.110 & 0.339 & 0.126 & 1.223 \\
$\mathbf{6 5 5 9 2 0}$ & 0.401 & 0.158 & 1.443 & 0.448 & 0.085 & 1.314 \\
$\mathbf{6 5 5 9 4 0}$ & 0.573 & 1.553 & 0.145 & 0.620 & 0.162 & 1.407 \\
$\mathbf{6 5 5 9 9 0}$ & 0.255 & 0.178 & 1.184 & 0.674 & 0.951 & 0.100
\end{tabular}




\begin{tabular}{lllllll}
\multicolumn{7}{c}{ Mixed Exponential Parameters } \\
Gauges & Warm Pacific Sea Surface Temperature Event \\
& Julpha & beta 1 & beta 2 & alpha & beta 1 & beta 2 \\
$\mathbf{6 5 3 0 6 0}$ & 0.100 & 0.164 & 0.641 & 0.169 & 0.041 & 0.620 \\
$\mathbf{6 5 3 1 9 0}$ & 0.757 & 0.529 & 0.130 & 0.794 & 0.695 & 0.053 \\
$\mathbf{6 5 3 3 0 0}$ & 0.679 & 0.752 & 0.123 & 0.477 & 0.113 & 0.907 \\
$\mathbf{6 5 3 3 5 0}$ & 0.319 & 0.067 & 0.778 & 0.553 & 0.115 & 1.002 \\
$\mathbf{6 5 3 3 8 0}$ & 0.614 & 0.787 & 0.051 & 0.503 & 0.083 & 0.722 \\
$\mathbf{6 5 3 4 4 0}$ & 0.578 & 0.071 & 1.296 & 0.669 & 0.052 & 0.938 \\
$\mathbf{6 5 3 5 2 0}$ & 0.992 & 0.526 & 0.205 & 0.229 & 0.080 & 0.466 \\
$\mathbf{6 5 3 5 5 0}$ & 0.540 & 0.156 & 1.343 & 0.984 & 0.477 & 12.438 \\
$\mathbf{6 5 3 5 7 0}$ & 0.707 & 0.678 & 0.096 & 0.340 & 1.032 & 0.122 \\
$\mathbf{6 5 3 6 1 0}$ & 0.767 & 0.576 & 0.117 & 0.681 & 0.642 & 0.094 \\
$\mathbf{6 5 3 7 6 0}$ & 0.422 & 0.109 & 0.519 & 0.137 & 0.095 & 0.693 \\
$\mathbf{6 5 3 8 0 0}$ & 0.637 & 0.556 & 0.026 & 0.922 & 0.108 & 2.129 \\
$\mathbf{6 5 3 8 7 0}$ & 0.458 & 0.036 & 1.067 & 0.267 & 0.993 & 0.074 \\
$\mathbf{6 5 4 7 2 0}$ & 0.723 & 0.106 & 2.089 & 0.858 & 0.081 & 0.682 \\
$\mathbf{6 5 5 0 1 0}$ & 0.355 & 0.059 & 0.725 & 0.738 & 0.838 & 0.040 \\
$\mathbf{6 5 5 0 2 0}$ & 0.037 & 0.023 & 0.476 & 0.247 & 0.049 & 0.657 \\
$\mathbf{6 5 5 0 3 0}$ & 0.270 & 0.067 & 0.621 & 0.087 & 0.053 & 0.619 \\
$\mathbf{6 5 5 0 5 0}$ & 0.963 & 0.636 & 1.754 & 0.898 & 0.668 & 0.041 \\
$\mathbf{6 5 5 0 7 0}$ & 0.127 & 0.050 & 0.804 & 0.127 & 4.915 & 0.451 \\
$\mathbf{6 5 5 1 0 0}$ & 0.072 & 2.255 & 0.405 & 0.244 & 0.050 & 0.793 \\
$\mathbf{6 5 5 1 6 0}$ & 0.814 & 0.795 & 0.029 & 0.589 & 0.321 & 0.830 \\
$\mathbf{6 5 5 1 8 0}$ & 0.697 & 0.547 & 1.451 & 0.940 & 0.882 & 0.017 \\
$\mathbf{6 5 5 2 2 0}$ & 0.641 & 0.737 & 0.133 & 0.840 & 0.654 & 0.068 \\
$\mathbf{6 5 5 2 8 0}$ & 0.944 & 0.488 & 9.784 & 0.962 & 0.467 & 8.190 \\
$\mathbf{6 5 5 3 6 0}$ & 0.078 & 0.042 & 0.557 & 0.023 & 12.334 & 0.647 \\
$\mathbf{6 5 5 4 5 0}$ & 0.252 & 0.038 & 0.395 & 0.396 & 0.061 & 1.007 \\
$\mathbf{6 5 5 4 8 0}$ & 0.366 & 0.040 & 0.460 & 0.548 & 0.142 & 1.217 \\
$\mathbf{6 5 5 5 5 0}$ & 0.683 & 0.631 & 0.041 & 0.490 & 0.848 & 0.056 \\
$\mathbf{6 5 5 5 7 0}$ & 0.667 & 0.201 & 0.633 & 0.694 & 0.073 & 0.612 \\
$\mathbf{6 5 5 6 0 0}$ & 0.519 & 0.633 & 0.036 & 0.546 & 0.071 & 0.559 \\
$\mathbf{6 5 5 6 2 0}$ & 0.574 & 0.556 & 0.042 & 0.911 & 0.193 & 6.459 \\
$\mathbf{6 5 5 6 3 0}$ & 0.713 & 0.412 & 0.040 & 0.273 & 0.056 & 0.467 \\
$\mathbf{6 5 5 7 8 0}$ & 0.294 & 1.122 & 0.105 & 0.731 & 0.050 & 0.650 \\
$\mathbf{6 5 5 8 5 0}$ & 0.683 & 0.105 & 1.598 & 0.204 & 1.403 & 0.114 \\
$\mathbf{6 5 5 9 2 0}$ & 0.823 & 0.195 & 2.253 & 0.640 & 0.124 & 0.927 \\
$\mathbf{6 5 5 9 4 0}$ & 0.613 & 0.086 & 1.377 & 0.733 & 0.069 & 0.692 \\
$\mathbf{6 5 5 9 9 0}$ & 0.401 & 1.494 & 0.071 & 0.792 & 0.066 & 1.289 \\
& & & & & &
\end{tabular}




\begin{tabular}{lllllll}
\multicolumn{7}{c}{ Mixed Exponential Parameters } \\
Gauges & Wep Pacific Sea Surface Temperature Event \\
& Slpha & beta 1 & beta 2 & Oct \\
& alpha & beta 1 & beta 2 \\
$\mathbf{6 5 3 0 6 0}$ & 0.588 & 0.576 & 0.088 & 0.708 & 0.435 & 0.074 \\
$\mathbf{6 5 3 1 9 0}$ & 0.947 & 0.523 & 0.331 & 0.664 & 0.468 & 0.153 \\
$\mathbf{6 5 3 3 0 0}$ & 0.713 & 0.700 & 0.135 & 0.442 & 0.103 & 0.799 \\
$\mathbf{6 5 3 3 5 0}$ & 0.540 & 0.102 & 0.971 & 0.334 & 0.088 & 0.618 \\
$\mathbf{6 5 3 3 8 0}$ & 0.447 & 0.735 & 0.097 & 0.320 & 0.065 & 0.742 \\
$\mathbf{6 5 3 4 4 0}$ & 0.655 & 0.792 & 0.061 & 0.616 & 0.878 & 0.161 \\
$\mathbf{6 5 3 5 2 0}$ & 0.604 & 0.666 & 0.103 & 0.123 & 0.038 & 0.501 \\
$\mathbf{6 5 3 5 5 0}$ & 0.035 & 3.472 & 0.434 & 0.372 & 0.057 & 0.559 \\
$\mathbf{6 5 3 5 7 0}$ & 0.200 & 1.895 & 0.377 & 1.000 & 0.445 & 226 \\
$\mathbf{6 5 3 6 1 0}$ & 0.391 & 0.081 & 0.739 & 0.457 & 0.729 & 0.220 \\
$\mathbf{6 5 3 7 6 0}$ & 0.878 & 0.575 & 0.034 & 0.879 & 0.327 & 0.681 \\
$\mathbf{6 5 3 8 0 0}$ & 0.801 & 0.102 & 0.690 & 0.299 & 0.054 & 0.616 \\
$\mathbf{6 5 3 8 7 0}$ & 0.444 & 0.857 & 0.090 & 0.766 & 0.707 & 0.038 \\
$\mathbf{6 5 4 7 2 0}$ & 0.639 & 0.055 & 0.289 & 0.511 & 0.539 & 0.121 \\
$\mathbf{6 5 5 0 1 0}$ & 0.555 & 0.144 & 0.666 & 0.310 & 0.030 & 0.393 \\
$\mathbf{6 5 5 0 2 0}$ & 0.663 & 0.686 & 0.054 & 0.283 & 0.057 & 0.291 \\
$\mathbf{6 5 5 0 3 0}$ & 0.978 & 0.441 & 10.185 & 0.665 & 0.489 & 0.034 \\
$\mathbf{6 5 5 0 5 0}$ & 0.060 & 0.038 & 0.513 & 0.267 & 0.094 & 0.571 \\
$\mathbf{6 5 5 0 7 0}$ & 0.162 & 0.038 & 0.599 & 0.420 & 0.075 & 0.449 \\
$\mathbf{6 5 5 1 0 0}$ & 0.905 & 0.302 & 1.847 & 0.861 & 0.466 & 0.061 \\
$\mathbf{6 5 5 1 6 0}$ & 0.630 & 0.464 & 0.082 & 0.694 & 0.575 & 0.145 \\
$\mathbf{6 5 5 1 8 0}$ & 0.756 & 0.529 & 0.191 & 0.792 & 0.369 & 0.059 \\
$\mathbf{6 5 5 2 2 0}$ & 0.000 & 0.486 & 0.486 & 0.094 & 0.037 & 0.595 \\
$\mathbf{6 5 5 2 8 0}$ & 0.370 & 0.216 & 0.774 & 0.985 & 0.424 & 17.054 \\
$\mathbf{6 5 5 3 6 0}$ & 0.708 & 0.381 & 0.677 & 0.458 & 0.745 & 0.139 \\
$\mathbf{6 5 5 4 5 0}$ & 0.324 & 0.053 & 0.455 & 0.549 & 0.414 & 0.586 \\
$\mathbf{6 5 5 4 8 0}$ & 0.162 & 0.036 & 0.643 & 0.701 & 0.198 & 1.246 \\
$\mathbf{6 5 5 5 5 0}$ & 0.111 & 0.022 & 0.585 & 0.388 & 1.067 & 0.160 \\
$\mathbf{6 5 5 5 7 0}$ & 0.385 & 0.070 & 0.666 & 0.252 & 0.897 & 0.176 \\
$\mathbf{6 5 5 6 0 0}$ & 0.555 & 0.136 & 1.078 & 0.488 & 0.085 & 0.798 \\
$\mathbf{6 5 5 6 2 0}$ & 0.442 & 0.053 & 0.441 & 0.603 & 0.616 & 0.059 \\
$\mathbf{6 5 5 6 3 0}$ & 0.422 & 0.725 & 0.077 & 0.868 & 0.311 & 0.063 \\
$\mathbf{6 5 5 7 8 0}$ & 0.234 & 1.553 & 0.062 & 0.607 & 0.095 & 0.997 \\
$\mathbf{6 5 5 8 5 0}$ & 0.428 & 0.845 & 0.058 & 0.494 & 1.142 & 0.078 \\
$\mathbf{6 5 5 9 2 0}$ & 0.824 & 0.195 & 1.402 & 0.751 & 0.117 & 1.097 \\
$\mathbf{6 5 5 9 4 0}$ & 0.912 & 0.134 & 1.568 & 0.319 & 0.999 & 0.050 \\
$\mathbf{6 5 5 9 9 0}$ & 0.559 & 0.046 & 0.307 & 0.625 & 0.093 & 0.668
\end{tabular}




\begin{tabular}{lllllll}
\multicolumn{7}{c}{ Mixed Exponential Parameters } \\
Gauges & Warm Pacific Sea Surface Temperature Event \\
& Nolpha & beta 1 & beta 2 & alpha & beta 1 & beta 2 \\
$\mathbf{6 5 3 0 6 0}$ & 0.291 & 0.367 & 0.041 & 0.568 & 2.682 & 4.712 \\
$\mathbf{6 5 3 1 9 0}$ & 1.000 & 0.531 & 8.456 & 0.391 & 7.803 & 19.910 \\
$\mathbf{6 5 3 3 0 0}$ & 0.486 & 1.371 & 0.322 & 0.701 & 8.319 & 8.100 \\
$\mathbf{6 5 3 3 5 0}$ & 0.923 & 0.367 & 0.010 & 0.000 & 210.610 & 0.200 \\
$\mathbf{6 5 3 3 8 0}$ & 0.616 & 0.040 & 1.336 & 1.000 & 0.905 & 227 \\
$\mathbf{6 5 3 4 4 0}$ & 0.189 & 0.065 & 0.333 & 0.350 & 0.035 & 3.676 \\
$\mathbf{6 5 3 5 2 0}$ & 0.681 & 0.240 & 5.030 & 0.000 & 0.870 & 0.870 \\
$\mathbf{6 5 3 5 5 0}$ & 0.840 & 0.590 & 0.063 & 0.367 & 6.093 & 18.345 \\
$\mathbf{6 5 3 5 7 0}$ & 1.000 & 0.435 & 114 & 0.625 & 8.189 & 18.503 \\
$\mathbf{6 5 3 6 1 0}$ & 0.642 & 1.014 & 0.147 & 0.396 & 4.891 & 5.669 \\
$\mathbf{6 5 3 7 6 0}$ & 0.907 & 0.257 & 0.123 & 1.000 & 0.665 & 556 \\
$\mathbf{6 5 3 8 0 0}$ & 0.220 & 0.065 & 0.422 & 0.916 & 8.673 & 19.294 \\
$\mathbf{6 5 3 8 7 0}$ & 0.850 & 0.263 & 0.052 & 0.361 & 0.343 & 0.074 \\
$\mathbf{6 5 4 7 2 0}$ & 0.933 & 0.497 & 0.126 & 0.292 & 0.020 & 0.337 \\
$\mathbf{6 5 5 0 1 0}$ & 0.429 & 9.510 & 1.085 & 1.000 & 0.120 & 0.120 \\
$\mathbf{6 5 5 0 2 0}$ & 0.687 & 1.607 & 1.243 & 1.000 & 0.080 & 64.595 \\
$\mathbf{6 5 5 0 3 0}$ & 0.765 & 1.389 & 0.010 & 1.000 & 0.120 & 9810 \\
$\mathbf{6 5 5 0 5 0}$ & 0.397 & 0.636 & 0.040 & 1.000 & 0.630 & 26.172 \\
$\mathbf{6 5 5 0 7 0}$ & 0.201 & 0.020 & 0.261 & 0.463 & 9.165 & 19.386 \\
$\mathbf{6 5 5 1 0 0}$ & 0.485 & 0.084 & 0.174 & 0.721 & 1.261 & 19.544 \\
$\mathbf{6 5 5 1 6 0}$ & 1.000 & 0.138 & 213 & 0.467 & 8.753 & 12.694 \\
$\mathbf{6 5 5 1 8 0}$ & 0.828 & 0.344 & 0.043 & 0.725 & 0.850 & 12.852 \\
$\mathbf{6 5 5 2 2 0}$ & 0.191 & 0.432 & 0.046 & 0.009 & 2.157 & 7.026 \\
$\mathbf{6 5 5 2 8 0}$ & 1.000 & 0.386 & 4.125 & 0.547 & 0.614 & 0.020 \\
$\mathbf{6 5 5 3 6 0}$ & 0.579 & 0.068 & 0.605 & 0.222 & 5.011 & 5.770 \\
$\mathbf{6 5 5 4 5 0}$ & 1.000 & 0.164 & 25.675 & 0.733 & 0.608 & 0.071 \\
$\mathbf{6 5 5 4 8 0}$ & 0.503 & 0.182 & 0.475 & 1.000 & 0.120 & 0.120 \\
$\mathbf{6 5 5 5 5 0}$ & 0.906 & 0.183 & 1.721 & 0.519 & 1.485 & 0.020 \\
$\mathbf{6 5 5 5 7 0}$ & 0.193 & 0.046 & 0.309 & 0.370 & 0.638 & 0.140 \\
$\mathbf{6 5 5 6 0 0}$ & 0.723 & 0.299 & 0.058 & 0.781 & 0.476 & 0.022 \\
$\mathbf{6 5 5 6 2 0}$ & 0.791 & 0.479 & 0.034 & 1.000 & 0.618 & 97.956 \\
$\mathbf{6 5 5 6 3 0}$ & 0.367 & 0.147 & 0.376 & 0.578 & 0.320 & 0.059 \\
$\mathbf{6 5 5 7 8 0}$ & 0.672 & 0.550 & 0.080 & 0.711 & 0.551 & 0.055 \\
$\mathbf{6 5 5 8 5 0}$ & 0.556 & 0.750 & 0.043 & 0.957 & 0.367 & 17.455 \\
$\mathbf{6 5 5 9 2 0}$ & 0.596 & 0.591 & 0.057 & 0.732 & 0.081 & 1.222 \\
$\mathbf{6 5 5 9 4 0}$ & 0.478 & 0.130 & 0.532 & 0.449 & 0.038 & 0.768 \\
$\mathbf{6 5 5 9 9 0}$ & 0.373 & 0.133 & 0.722 & 0.511 & 0.127 & 0.658
\end{tabular}



Appendix H: Domestic Rainwater Harvesting Reliabilities Using the $1^{\text {st }}$-Order Markov Model 


\begin{tabular}{|c|c|c|c|c|c|c|c|c|c|c|c|}
\hline $\begin{array}{l}\text { 1st-O } \\
\text { Janua }\end{array}$ & \multirow{2}{*}{\multicolumn{11}{|c|}{ per capita roof area and storage }} \\
\hline \multirow[b]{2}{*}{ Gauge } & & & & & & & & & & & \\
\hline & sq. m & 0 & 40 & 80 & 120 & 160 & 200 & 400 & 600 & 800 & 1000 \\
\hline \multirow[t]{2}{*}{653060} & 2 & 0.0 & 0.0 & 0.1 & 0.1 & 0.1 & 0.1 & 0.1 & 0.1 & 0.1 & 0.1 \\
\hline & 10 & 0.1 & 0.2 & 0.3 & 0.4 & 0.5 & 0.5 & 0.8 & 1.2 & 1.4 & 1.5 \\
\hline \multirow[t]{2}{*}{653190} & 2 & 0.1 & 0.2 & 0.3 & 0.3 & 0.4 & 0.4 & 0.4 & 0.4 & 0.4 & 0.4 \\
\hline & 10 & 0.2 & 0.3 & 0.6 & 0.8 & 1.0 & 1.1 & 1.4 & 1.5 & 1.7 & 2.6 \\
\hline \multirow[t]{2}{*}{653300} & 2 & 0.2 & 0.3 & 0.3 & 0.3 & 0.3 & 0.3 & 0.3 & 0.3 & 0.3 & 0.3 \\
\hline & 10 & 0.6 & 0.9 & 1.4 & 1.7 & 1.9 & 2.0 & 2.2 & 2.2 & 2.3 & 3.1 \\
\hline \multirow[t]{2}{*}{653350} & 2 & 0.5 & 0.7 & 0.9 & 0.9 & 0.9 & 0.9 & 0.9 & 0.9 & 0.9 & 0.9 \\
\hline & 10 & 1.0 & 1.9 & 3.0 & 3.8 & 4.5 & 4.8 & 5.3 & 5.3 & 5.4 & 6.5 \\
\hline \multirow[t]{2}{*}{653380} & 2 & 0.7 & 1.3 & 2.1 & 2.5 & 2.9 & 3.0 & 3.1 & 3.1 & 3.1 & 3.1 \\
\hline & 10 & 1.0 & 1.9 & 3.6 & 5.2 & 7.3 & 8.6 & 13.2 & 16.7 & 19.8 & 25.9 \\
\hline \multirow[t]{2}{*}{653440} & 2 & 1.1 & 1.9 & 2.7 & 3.1 & 3.4 & 3.6 & 3.7 & 3.8 & 3.8 & 3.8 \\
\hline & 10 & 1.9 & 3.5 & 6.0 & 8.1 & 10.6 & 12.0 & 17.4 & 21.6 & 26.3 & 34.1 \\
\hline \multirow[t]{2}{*}{653520} & 2 & 0.2 & 0.3 & 0.4 & 0.4 & 0.5 & 0.5 & 0.5 & 0.5 & 0.5 & 0.6 \\
\hline & 10 & 0.2 & 0.5 & 0.8 & 1.1 & 1.5 & 1.8 & 2.6 & 3.2 & 3.8 & 5.2 \\
\hline \multirow[t]{2}{*}{653550} & 2 & 0.1 & 0.2 & 0.3 & 0.3 & 0.3 & 0.3 & 0.3 & 0.3 & 0.3 & 0.3 \\
\hline & 10 & 0.2 & 0.4 & 0.7 & 1.0 & 1.3 & 1.4 & 1.7 & 1.7 & 2.7 & 7.3 \\
\hline \multirow[t]{2}{*}{653570} & 2 & 0.4 & 0.6 & 0.9 & 1.0 & 1.1 & 1.1 & 1.1 & 1.1 & 1.1 & 1.1 \\
\hline & 10 & 0.5 & 1.0 & 1.6 & 2.2 & 2.9 & 3.3 & 4.2 & 4.5 & 4.7 & 6.0 \\
\hline \multirow[t]{2}{*}{653610} & 2 & 0.4 & 0.5 & 0.6 & 0.6 & 0.6 & 0.6 & 0.6 & 0.6 & 0.6 & 0.6 \\
\hline & 10 & 1.0 & 1.7 & 2.7 & 3.3 & 3.9 & 4.0 & 4.2 & 4.3 & 4.9 & 7.7 \\
\hline \multirow[t]{2}{*}{653760} & 2 & 0.3 & 0.4 & 0.5 & 0.5 & 0.5 & 0.6 & 0.6 & 0.6 & 0.7 & 0.7 \\
\hline & 10 & 1.3 & 2.1 & 3.0 & 3.7 & 4.2 & 4.5 & 5.4 & 6.4 & 7.4 & 9.4 \\
\hline \multirow[t]{2}{*}{653800} & 2 & 0.9 & 1.4 & 2.0 & 2.3 & 2.5 & 2.6 & 2.6 & 2.6 & 2.6 & 2.6 \\
\hline & 10 & 1.3 & 2.5 & 4.6 & 6.4 & 8.6 & 9.8 & 14.1 & 17.6 & 20.2 & 24.1 \\
\hline \multirow[t]{2}{*}{653870} & 2 & 0.6 & 0.9 & 1.0 & 1.1 & 1.1 & 1.1 & 1.1 & 1.1 & 1.1 & 1.1 \\
\hline & 10 & 1.8 & 3.1 & 4.7 & 5.8 & 6.9 & 7.4 & 8.7 & 9.3 & 9.6 & 10.3 \\
\hline \multirow[t]{2}{*}{654720} & 2 & 0.5 & 0.8 & 1.0 & 1.1 & 1.1 & 1.1 & 1.1 & 1.1 & 1.1 & 1.1 \\
\hline & 10 & 1.2 & 2.2 & 3.7 & 4.9 & 6.2 & 6.9 & 8.8 & 9.7 & 10.0 & 10.6 \\
\hline 655010 & 2 & 0.0 & 0.1 & 0.1 & 0.2 & 0.3 & 0.3 & 0.5 & 0.6 & 0.6 & 0.6 \\
\hline & 10 & 0.1 & 0.2 & 0.4 & 0.7 & 1.0 & 1.3 & 2.9 & 4.8 & 6.0 & 6.6 \\
\hline 655020 & 2 & 0.0 & 0.0 & 0.0 & 0.0 & 0.0 & 0.0 & 0.0 & 0.0 & 0.0 & 0.0 \\
\hline & 10 & 0.0 & 0.1 & 0.1 & 0.1 & 0.1 & 0.1 & 0.1 & 0.1 & 0.1 & 0.2 \\
\hline 655030 & 2 & 0.0 & 0.0 & 0.0 & 0.0 & 0.0 & 0.0 & 0.0 & 0.0 & 0.0 & 0.0 \\
\hline & 10 & 0.0 & 0.0 & 0.1 & 0.1 & 0.2 & 0.3 & 0.8 & 1.2 & 1.4 & 1.8 \\
\hline 655050 & 2 & 0.0 & 0.0 & 0.1 & 0.1 & 0.1 & 0.2 & 0.2 & 0.2 & 0.2 & 0.2 \\
\hline & 10 & 0.0 & 0.0 & 0.1 & 0.3 & 0.5 & 0.7 & 1.9 & 3.3 & 4.0 & 4.5 \\
\hline 655070 & 2 & 0.0 & 0.0 & 0.1 & 0.1 & 0.2 & 0.2 & 0.3 & 0.3 & 0.3 & 0.3 \\
\hline & 10 & 0.0 & 0.0 & 0.1 & 0.2 & 0.5 & 0.7 & 1.8 & 3.2 & 4.2 & 4.7 \\
\hline 655100 & 2 & 0.2 & 0.3 & 0.4 & 0.4 & 0.4 & 0.4 & 0.4 & 0.4 & 0.4 & 0.4 \\
\hline & 10 & 0.3 & 0.6 & 1.0 & 1.3 & 1.7 & 1.8 & 2.1 & 2.2 & 2.3 & 2.9 \\
\hline 655160 & 2 & 0.0 & 0.0 & 0.0 & 0.1 & 0.1 & 0.1 & 0.1 & 0.1 & 0.1 & 0.1 \\
\hline & 10 & 0.2 & 0.4 & 0.5 & 0.6 & 0.7 & 0.7 & 1.1 & 1.6 & 1.9 & 2.2 \\
\hline 655180 & 2 & 0.0 & 0.0 & 0.0 & 0.0 & 0.0 & 0.0 & 0.0 & 0.0 & 0.0 & 0.0 \\
\hline & 10 & 0.0 & 0.0 & 0.1 & 0.2 & 0.4 & 0.6 & 1.1 & 1.5 & 1.6 & 1.8 \\
\hline 655220 & 2 & 0.5 & 0.9 & 1.3 & 1.5 & 1.7 & 1.7 & 1.8 & 1.8 & 1.8 & 1.8 \\
\hline & 10 & 1.0 & 1.7 & 2.7 & 3.5 & 4.5 & 5.1 & 6.7 & 7.7 & 8.3 & 9.5 \\
\hline 655280 & 2 & 0.6 & 0.9 & 1.0 & 1.1 & 1.3 & 1.4 & 2.1 & 2.7 & 2.9 & 3.1 \\
\hline & 10 & 1.7 & 2.8 & 4.3 & 5.4 & 6.4 & 6.9 & 8.8 & 11.3 & 14.4 & 20.0 \\
\hline 655360 & 2 & 0.3 & 0.4 & 0.4 & 0.5 & 0.5 & 0.5 & 0.5 & 0.5 & 0.5 & 0.5 \\
\hline & 10 & 0.8 & 1.4 & 2.1 & 2.7 & 3.3 & 3.6 & 4.7 & 5.7 & 6.6 & 9.0 \\
\hline
\end{tabular}




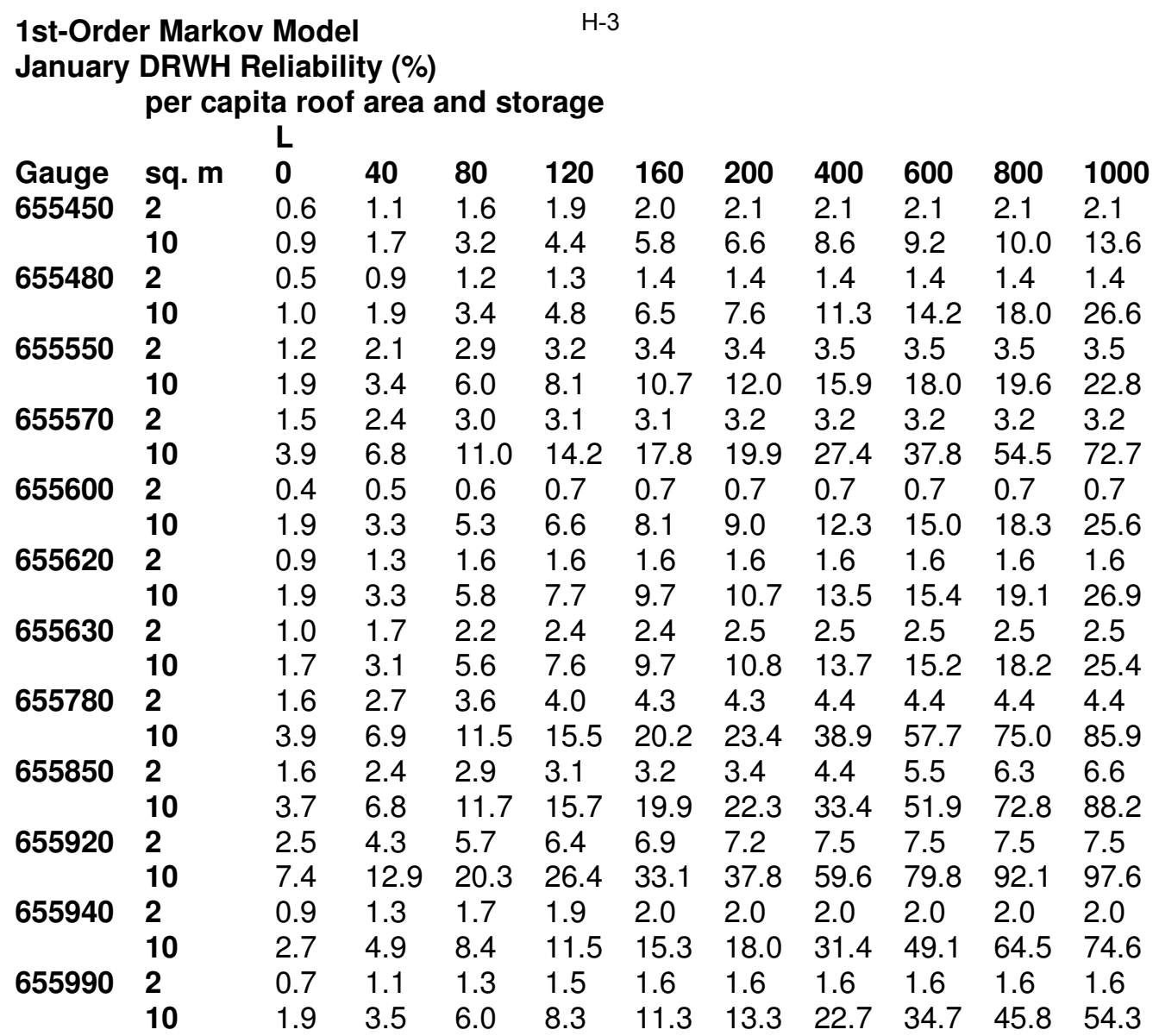




\begin{tabular}{|c|c|c|c|c|c|c|c|c|c|c|c|}
\hline \multicolumn{12}{|c|}{$\begin{array}{l}\text { 1st-Order Markov Model } \\
\text { February DRWH Reliability (\%) } \\
\text { per capita roof area and storage } \\
\text { L }\end{array}$} \\
\hline Gauge & sq. $\mathrm{m}$ & 0 & 40 & 80 & 120 & 160 & 200 & 400 & 600 & 800 & 100 \\
\hline \multirow[t]{2}{*}{653060} & 2 & 0.5 & 0.9 & 1.2 & 1.3 & 1.4 & 1.4 & 1.4 & 1.4 & 1.4 & 1.4 \\
\hline & 10 & 0.8 & 1.5 & 2.6 & 3.4 & 4.3 & 4.8 & 5.8 & 5.9 & 6.0 & 6.0 \\
\hline \multirow[t]{2}{*}{653190} & 2 & 0.4 & 0.6 & 0.6 & 0.7 & 0.7 & 0.7 & 0.7 & 0.7 & 0.7 & 0.7 \\
\hline & 10 & 1.2 & 2.0 & 2.9 & 3.6 & 4.2 & 4.5 & 5.1 & 5.4 & 5.5 & 5.6 \\
\hline \multirow[t]{2}{*}{653300} & 2 & 0.3 & 0.5 & 0.5 & 0.5 & 0.5 & 0.5 & 0.5 & 0.5 & 0.5 & 0.5 \\
\hline & 10 & 1.0 & 1.8 & 2.8 & 3.3 & 3.8 & 4.0 & 4.3 & 4.3 & 4.3 & 4.4 \\
\hline \multirow[t]{2}{*}{653350} & 2 & 1.7 & 2.8 & 3.9 & 4.5 & 4.8 & 4.9 & 5.0 & 5.0 & 5.0 & 5.0 \\
\hline & 10 & 3.3 & 5.7 & 9.2 & 11.7 & 14.2 & 15.5 & 18.5 & 18.9 & 19.0 & 19.0 \\
\hline \multirow[t]{2}{*}{653380} & 2 & 1.7 & 2.5 & 3.0 & 3.2 & 3.3 & 3.4 & 3.6 & 3.7 & 3.7 & 3.7 \\
\hline & 10 & 4.4 & 7.4 & 11.3 & 14.0 & 16.4 & 17.6 & 21.1 & 23.3 & 24.7 & 26.0 \\
\hline \multirow[t]{2}{*}{653440} & 2 & 2.2 & 3.6 & 5.0 & 5.6 & 5.9 & 5.9 & 6.0 & 6.0 & 6.0 & 6.0 \\
\hline & 10 & 4.2 & 7.4 & 12.0 & 15.5 & 19.2 & 21.1 & 26.1 & 28.4 & 30.1 & 32.3 \\
\hline \multirow[t]{2}{*}{653520} & 2 & 0.2 & 0.2 & 0.2 & 0.2 & 0.3 & 0.3 & 0.3 & 0.3 & 0.3 & 0.3 \\
\hline & 10 & 0.5 & 0.9 & 1.4 & 1.7 & 2.0 & 2.1 & 2.6 & 2.9 & 3.0 & 3.2 \\
\hline \multirow[t]{2}{*}{653550} & 2 & 1.2 & 2.0 & 2.7 & 3.0 & 3.2 & 3.2 & 3.2 & 3.2 & & 3.2 \\
\hline & 10 & 1.8 & 3. & 5.8 & 7.7 & 9.7 & 10.7 & 12.9 & 13.3 & 13.3 & 13.3 \\
\hline \multirow[t]{2}{*}{653570} & 2 & 0.3 & 0.5 & 0.6 & 0.6 & 0.7 & 0.7 & 0.7 & 0.7 & 0.7 & 0.7 \\
\hline & 10 & 0.7 & 1.3 & 2.1 & 2.7 & 3.4 & 3.7 & 4.9 & 5.8 & 6.2 & 6.4 \\
\hline \multirow[t]{2}{*}{653610} & 2 & 0.3 & 0.5 & 0.5 & 0.5 & 0.5 & 0.5 & 0.5 & 0.5 & 0.5 & 0.5 \\
\hline & 10 & 1.0 & 1.7 & 2.8 & 3.4 & 4.0 & 4.3 & 4.7 & 4.7 & 4.7 & 4.7 \\
\hline \multirow[t]{2}{*}{653760} & 2 & 1.0 & 1.3 & 1.5 & 1.5 & 1.5 & 1.5 & 1.5 & 1.5 & 1.5 & 1.5 \\
\hline & 10 & 3.3 & 5. & 8. & 9.7 & 10.9 & 11.4 & 12.3 & 12.4 & 12.6 & 12.9 \\
\hline \multirow[t]{2}{*}{653800} & 2 & $1 . \varepsilon$ & 2.6 & 3.5 & 4.1 & 4.7 & 5.1 & 5.8 & 5.8 & 5. & 5.8 \\
\hline & 10 & 5.3 & 9.0 & 13.6 & 16.5 & 19.0 & 20.3 & 23.7 & 25 & 26.0 & 27.2 \\
\hline \multirow[t]{2}{*}{653870} & 2 & 0.9 & 1. & 2.2 & 2.6 & 2.9 & 3.0 & 3.2 & 3.2 & 3.2 & 3.2 \\
\hline & 10 & 3.0 & 4.9 & 7.3 & 8.9 & 10.4 & 11.3 & 13.6 & 14.1 & 14.2 & 14.3 \\
\hline \multirow[t]{2}{*}{654720} & 2 & 0.9 & 1.6 & 2.2 & 2.5 & 2.6 & 2.6 & 2.7 & 2.7 & 2.7 & 2.7 \\
\hline & 10 & 3.0 & 4.9 & 7.3 & 8.9 & 10.6 & 11.5 & 13.8 & 14.2 & 14.2 & 14.3 \\
\hline \multirow[t]{2}{*}{655010} & 2 & 0.3 & 0.4 & 0.6 & 0.7 & 0.7 & 0.7 & 0.7 & 0.7 & 0.7 & 0.8 \\
\hline & 10 & 0.4 & 0.7 & 1.2 & 1.7 & 2.2 & 2.4 & 2.9 & 3.0 & 3.2 & 3.7 \\
\hline \multirow[t]{2}{*}{655020} & 2 & 0.1 & 0.2 & 0.3 & 0.3 & 0.4 & 0.4 & 0.4 & 0.4 & 0.4 & 0.4 \\
\hline & 10 & 0.1 & 0. & 0.4 & 0.6 & 0.8 & 0.9 & 1.1 & 1.2 & 1.2 & 1.2 \\
\hline 655030 & 2 & & 0.2 & 0. & 0.4 & 0.4 & 0. & 0 & & 0 & 0.4 \\
\hline & 10 & 0.4 & 0.6 & 1.0 & 1.2 & 1.5 & 1.6 & 1.8 & 1.8 & 1.8 & 1.9 \\
\hline 655050 & 2 & 0.2 & 0. & 0. & 0.8 & 0.8 & 0. & 0. & & 0. & 0.9 \\
\hline & 10 & 0.5 & 0.8 & 1.2 & 1.5 & 1.9 & 2.1 & 2.7 & 2.8 & 2.9 & 3.2 \\
\hline 655070 & 2 & 0.2 & 0. & 0. & 0.6 & 0.7 & 0.7 & 0.7 & 0. & 0.7 & 0.7 \\
\hline & 10 & 0.4 & 0.7 & 1.2 & 1.6 & 2.0 & 2.2 & 2.7 & 2.7 & 3.0 & 3.4 \\
\hline 655100 & 2 & 0.2 & 0.3 & 0.4 & 0.4 & 0.4 & 0.4 & 0.4 & 0.4 & 0.4 & 0.4 \\
\hline & 10 & 0.6 & 1.0 & 1.5 & 1.9 & 2.3 & 2.5 & 3.0 & 3.2 & 3.3 & 3.4 \\
\hline 655160 & 2 & 0.0 & 0.0 & 0.0 & 0.0 & 0.0 & 0.0 & 0.0 & 0.0 & 0.0 & 0.0 \\
\hline & 10 & 0.1 & 0.1 & 0.2 & 0.2 & 0.2 & 0.2 & 0.2 & 0.2 & 0.3 & 0.4 \\
\hline 655180 & 2 & 0.1 & 0.1 & 0.1 & 0.1 & 0.1 & 0.1 & 0.1 & 0.1 & 0.1 & 0.1 \\
\hline & 10 & 0.4 & 0.6 & 0.8 & 0.9 & 1.0 & 1.0 & 1. & $1 .($ & 1. & 1.1 \\
\hline 655220 & 2 & 0.4 & 0.6 & 0.7 & 0.8 & $0 . \varepsilon$ & 0.8 & 0. & 0.8 & 0.8 & 0.8 \\
\hline & 10 & 0.9 & 1.5 & 2.5 & 3.2 & 4.0 & 4.5 & 6.1 & 7.4 & 8.1 & 8.5 \\
\hline 655280 & 2 & 0.6 & 0.9 & 1.1 & 1.1 & 1. & 1.2 & 1.2 & 1.2 & & 1.3 \\
\hline & 10 & 1.0 & 1.9 & 3.3 & 4.3 & 5.5 & 6.0 & 7.2 & 7.5 & 8.2 & 9.7 \\
\hline 655360 & 2 & 1.6 & 2.5 & 3.5 & 3.8 & 3.9 & 3.9 & 3.9 & 3.9 & 3.9 & 3.9 \\
\hline & 10 & 2.5 & 4.6 & 7.8 & 10.3 & 12.8 & 14.0 & 16.3 & 16.7 & 16.8 & 16. \\
\hline
\end{tabular}




\begin{tabular}{|c|c|c|c|c|c|c|c|c|c|c|c|}
\hline \multirow{2}{*}{\multicolumn{12}{|c|}{$\begin{array}{l}\text { 1st-Order Markov Model } \\
\text { February DRWH Reliability (\%) } \\
\text { per capita roof area and storage } \\
\text { L }\end{array}$}} \\
\hline & & & & & & & & & & & \\
\hline Gauge & sq. $m$ & 0 & 40 & 80 & 120 & 160 & 200 & 400 & 600 & 800 & 1000 \\
\hline \multirow[t]{2}{*}{655450} & 2 & 1.6 & 2.6 & 3.6 & 4.1 & 4.6 & 4.8 & 5.0 & 5.0 & 5.0 & 5.0 \\
\hline & 10 & 4.3 & 7.2 & 10.9 & 13.3 & 15.6 & 16.9 & 20.4 & 22.0 & 22.7 & 22.9 \\
\hline \multirow[t]{2}{*}{655480} & 2 & 2.0 & 2.8 & 3.0 & 3.1 & 3.1 & 3.1 & 3.1 & 3.1 & 3.1 & 3.1 \\
\hline & 10 & 5.2 & 9.0 & 13.7 & 16.7 & 19.0 & 20.0 & 21.9 & 22.4 & 23.0 & 24.2 \\
\hline \multirow[t]{2}{*}{655550} & 2 & 1.5 & 2.4 & 3.0 & 3.1 & 3.2 & 3.2 & 3.2 & 3.2 & 3.2 & 3.2 \\
\hline & 10 & 2.7 & 5.0 & 8.6 & 11.2 & 13.9 & 15.3 & 19.3 & 21.6 & 22.6 & 23.4 \\
\hline \multirow[t]{2}{*}{655570} & 2 & 4.7 & 7.1 & 8.7 & 9.6 & 10.2 & 10.5 & 11.1 & 11.2 & 11.2 & 11.2 \\
\hline & 10 & 10.8 & 18.5 & 28.5 & 34.7 & 39.8 & 42.4 & 47.4 & 48.9 & 51.8 & 58.0 \\
\hline \multirow[t]{2}{*}{655600} & 2 & 4.2 & 6.4 & 7.9 & 8.3 & 8.3 & 8.4 & 8.4 & 8.4 & 8.4 & 8.4 \\
\hline & 10 & 7.8 & 13.9 & 22.3 & 28.0 & 32.7 & 34.8 & 38.2 & 38.4 & 38.8 & 39.8 \\
\hline \multirow[t]{2}{*}{655620} & 2 & 2.4 & 3.3 & 3.6 & 3.6 & 3.6 & 3.6 & 3.6 & 3.6 & 3.6 & 3.6 \\
\hline & 10 & 6.6 & 11.4 & 17.8 & 21.5 & 24.3 & 25.5 & 27.5 & 27.8 & 28.0 & 28.8 \\
\hline \multirow[t]{2}{*}{655630} & 2 & 1.7 & 2.4 & 2.8 & 2.9 & 3.0 & 3.0 & 3.0 & 3.0 & 3.0 & 3.0 \\
\hline & 10 & 4.6 & 7.8 & 12.1 & 14.9 & 17.4 & 18.6 & 21.6 & 22.9 & 23.4 & 24.0 \\
\hline \multirow[t]{2}{*}{655780} & 2 & 1.9 & 2.7 & 3.3 & 3.7 & 4.0 & 4.1 & 4.3 & 4.3 & 4.3 & 4.3 \\
\hline & 10 & 7.3 & 12.2 & 17.9 & 21.2 & 23.9 & 25.3 & 29.1 & 32.7 & 41.4 & 55.5 \\
\hline \multirow[t]{2}{*}{655850} & 2 & 4.4 & 7.1 & 8.9 & 9.4 & 9.5 & 9.6 & 9.6 & 9.6 & 9.8 & 10.1 \\
\hline & 10 & 8.6 & 15.2 & 24.3 & 30.6 & 35.9 & 38.7 & 43.9 & 45.9 & 52.0 & 63.8 \\
\hline \multirow[t]{2}{*}{655920} & 2 & 3.5 & 5.4 & 6.5 & 6.7 & 6.7 & 6.7 & 6.7 & 6.7 & 6.7 & 6.7 \\
\hline & 10 & 7.6 & 13.5 & 21.5 & 27.0 & 31.8 & 34.6 & 42.1 & 50.3 & 65.7 & 81.6 \\
\hline \multirow[t]{2}{*}{655940} & 2 & 0.8 & 1.2 & 1.4 & 1.5 & 1.5 & 1.5 & 1.5 & 1.5 & 1.5 & 1.5 \\
\hline & 10 & 2.5 & 4.1 & 6.3 & 7.8 & 9.1 & 9.8 & 10.9 & 12.2 & 18.7 & 30.9 \\
\hline \multirow[t]{2}{*}{655990} & 2 & 2.0 & 2.9 & 3.4 & 3.7 & 3.8 & 3.9 & 4.0 & 4.0 & 4.0 & 4.0 \\
\hline & 10 & 5.3 & 9.2 & 14.4 & 17.6 & 20.2 & 21.3 & 23.3 & 24.0 & 26.7 & 32.1 \\
\hline
\end{tabular}




\begin{tabular}{|c|c|c|c|c|c|c|c|c|c|c|c|}
\hline \multicolumn{12}{|c|}{$\begin{array}{l}\text { 1st-Order Markov Model } \\
\text { March DRWH Reliability (\%) } \\
\text { per capita roof area and storage } \\
\text { L }\end{array}$} \\
\hline Gauge & sq. m & 0 & 40 & 80 & 120 & 160 & 200 & 400 & 600 & 800 & 1000 \\
\hline \multirow[t]{2}{*}{653060} & 2 & 0.8 & 1.1 & 1.3 & 1.3 & 1.3 & 1.3 & 1.3 & 1.3 & 1.3 & 1.3 \\
\hline & 10 & 2.0 & 3.5 & 5.5 & 6.9 & 8.2 & 8.7 & 10.1 & 10.9 & 11.2 & 11.2 \\
\hline \multirow[t]{2}{*}{653190} & 2 & 1.4 & 2.1 & 2.4 & 2.4 & 2.4 & 2.4 & 2.4 & 2.4 & 2.4 & 2.4 \\
\hline & 10 & 4.2 & 7.1 & 10.5 & 12.7 & 14.6 & 15.4 & 17.0 & 17.2 & 17.2 & 17.2 \\
\hline \multirow[t]{2}{*}{653300} & 2 & 2.2 & 3.4 & 4.2 & 4.4 & 4.5 & 4.5 & 4.5 & 4.5 & 4.5 & 4.5 \\
\hline & 10 & 5.9 & 10.0 & 15.1 & 18.5 & 21.4 & 22.8 & 25.2 & 25.4 & 25.4 & 25.4 \\
\hline \multirow[t]{2}{*}{653350} & 2 & 5.0 & 7.8 & 9.5 & 10.0 & 10.2 & 10.3 & 10.4 & 10.4 & 10.4 & 10.4 \\
\hline & 10 & 9.5 & 16.6 & 26.3 & 33.0 & 38.9 & 42.0 & 49.1 & 51.7 & 52.3 & 52.5 \\
\hline \multirow[t]{2}{*}{653380} & 2 & 5.3 & 8.0 & 9.3 & 9.6 & 9.7 & 9.7 & 9.7 & 9.7 & 9.7 & 9.7 \\
\hline & 10 & 10.9 & 19.6 & 31.0 & 38.4 & 44.0 & 46.9 & 52.1 & 53.1 & 53.7 & 54.2 \\
\hline \multirow[t]{2}{*}{653440} & 2 & 5.4 & 8.9 & 11.5 & 12.4 & 12.8 & 12.9 & 13.0 & 13.0 & 13.0 & 13.0 \\
\hline & 10 & 9.6 & 17.2 & 27.4 & 34.6 & 41.1 & 44.6 & 52.9 & 55.5 & 56.5 & 57.1 \\
\hline \multirow[t]{2}{*}{653520} & 2 & 1.9 & 3.3 & 4.7 & 5.5 & 5.9 & 6.1 & 6.2 & 6.2 & 6.2 & 6.2 \\
\hline & 10 & 3.0 & 5.3 & 8.8 & 11.5 & 14.5 & 16.1 & 19.7 & 20.4 & 20.4 & 20.5 \\
\hline \multirow[t]{2}{*}{653550} & 2 & 2.2 & 3.5 & 4.3 & 4.6 & 4.6 & 4.7 & 4.7 & 4.7 & 4.7 & 4.7 \\
\hline & 10 & 5.4 & 9.3 & 14.6 & 18.3 & 21.6 & 23.3 & 27.6 & 29.3 & 29.8 & 29.9 \\
\hline \multirow[t]{2}{*}{653570} & 2 & 1.1 & 1.7 & 2.4 & 2.9 & 3.3 & 3.5 & 3.8 & 3.9 & 3.9 & 3.9 \\
\hline & 10 & 4.5 & 7.5 & 10.8 & 12.7 & 14.3 & 15.0 & 17.0 & 17.5 & 17.6 & 17.7 \\
\hline \multirow[t]{2}{*}{653610} & 2 & 2.6 & 3.6 & 4.0 & 4.1 & 4.1 & 4.1 & 4.1 & 4.1 & 4.1 & 4.1 \\
\hline & 10 & 6.3 & 11.0 & 17.1 & 20.9 & 23.8 & 25.0 & 26.7 & 26.8 & 26.8 & 26.9 \\
\hline \multirow{2}{*}{653760} & 2 & 3.4 & 5.5 & 7.0 & 7.4 & 7.6 & 7.6 & 7.6 & 7.6 & 7.6 & 7.6 \\
\hline & 10 & 7.5 & 12.9 & 20.1 & 25.1 & 29.3 & 31.5 & 36.0 & 36.4 & 36.4 & 36.5 \\
\hline \multirow[t]{2}{*}{653800} & 2 & 4.8 & 7.4 & 8.9 & 9.3 & 9.5 & 9.6 & 10.2 & 10.6 & 10.7 & 10.7 \\
\hline & 10 & 10.2 & 17.8 & 27.9 & 34.5 & 40.1 & 42.8 & 48.9 & 51.0 & 52.0 & 52.3 \\
\hline \multirow[t]{2}{*}{653870} & 2 & 3.5 & 5.8 & 8.1 & 9.2 & 9.8 & 10.0 & 10.3 & 10.4 & 10.4 & 10.4 \\
\hline & 10 & 8.3 & 14.1 & 21.6 & 26.5 & 31.0 & 33.5 & 39.6 & 41.8 & 42.6 & 42.7 \\
\hline \multirow[t]{2}{*}{654720} & 2 & 2.7 & 4.2 & 5.3 & 5.6 & 5.7 & 5.7 & 5.8 & 5.8 & 5.8 & 5.8 \\
\hline & 10 & 5.6 & 9.9 & 15.9 & 20.1 & 23.9 & 25.9 & 30.6 & 32.2 & 32.6 & 32.7 \\
\hline \multirow[t]{2}{*}{655010} & 2 & 0.2 & 0.3 & 0.3 & 0.4 & 0.4 & 0.4 & 0.4 & 0.4 & 0.4 & 0.4 \\
\hline & 10 & 0.8 & 1.3 & 1.8 & 2.2 & 2.6 & 2.8 & 3.5 & 4.0 & 4.1 & 4.2 \\
\hline \multirow[t]{2}{*}{655020} & 2 & 0.6 & 0.9 & 1.2 & 1.3 & 1.3 & 1.3 & 1.4 & 1.4 & 1.4 & 1.4 \\
\hline & 10 & 1.3 & 2.2 & 3.4 & 4.3 & 5.2 & 5.7 & 6.8 & 7.3 & 7.5 & 7.5 \\
\hline 655030 & 2 & 0.5 & 0.9 & 1.4 & 1.7 & 2.0 & 2.2 & 2.3 & 2.3 & 2.3 & 2.3 \\
\hline & 10 & 1.8 & 2.9 & 4.3 & 5.1 & 6.1 & 6.6 & 8.2 & 8.7 & 8.8 & 8.8 \\
\hline 655050 & 2 & 0.5 & 0.9 & 1.2 & 1.3 & 1.4 & 1.4 & 1.5 & 1.5 & 1.5 & 1.5 \\
\hline & 10 & 2.7 & 4.3 & 5.8 & 6.7 & 7.5 & 8.0 & 9.4 & 10.2 & 10.5 & 10.6 \\
\hline 655070 & 2 & 1.4 & 2.6 & 4.0 & 4.9 & 5.5 & 5.8 & 6.3 & 6.3 & 6.3 & 6.3 \\
\hline & 10 & 3.0 & 5.1 & 7.8 & 9.9 & 12.2 & 13.4 & 16.8 & 17.9 & 18.1 & 18.1 \\
\hline 655100 & 2 & 1.1 & 1.5 & 1.7 & 1.7 & 1.7 & 1.7 & 1.7 & 1.7 & 1.7 & 1.7 \\
\hline & 10 & 3.6 & 6.0 & 8.7 & 10.3 & 11.7 & 12.2 & 13.1 & 13.3 & 13.4 & 13.4 \\
\hline 655160 & 2 & 0.5 & 0.9 & 1.4 & 1.7 & 1.9 & 2.0 & 2.2 & 2.2 & 2.2 & 2.2 \\
\hline & 10 & 2.3 & 3.7 & 5.1 & 5.9 & 6.6 & 7.1 & 8.4 & 8.7 & 8.7 & 8.7 \\
\hline 655180 & 2 & 1.2 & 2.1 & 2.7 & 3.0 & 3.2 & 3.2 & 3.3 & 3.3 & 3.3 & 3.3 \\
\hline & 10 & 4.0 & 6.4 & 9.1 & 10.9 & 12.5 & 13.4 & 15.4 & 15.6 & 15.6 & 15.6 \\
\hline 655220 & 2 & 2.7 & 4.2 & 4.9 & 5.1 & 5.1 & 5.1 & 5.1 & 5.1 & 5.1 & 5.1 \\
\hline & 10 & 5.4 & 9.3 & 14.7 & 18.5 & 22.2 & 23.8 & 26.6 & 27.0 & 27.2 & 27.5 \\
\hline 655280 & 2 & 1.3 & 1.7 & 2.2 & 2.5 & 2.8 & 2.9 & 3.3 & 3.4 & 3.4 & 3.4 \\
\hline & 10 & 5.4 & 8.9 & 12.9 & 15.1 & 16.6 & 17.3 & 18.9 & 19.5 & 19.6 & 19.6 \\
\hline 655360 & 2 & 2.5 & 4.1 & 5.5 & 6.1 & 6.3 & 6.4 & 6.4 & 6.4 & 6.4 & 6.4 \\
\hline & 10 & 6.3 & 10.9 & 16.8 & 20.9 & 24.9 & 27.1 & 32.8 & 34.7 & 35.2 & 35.3 \\
\hline
\end{tabular}




\begin{tabular}{|c|c|c|c|c|c|c|c|c|c|c|c|}
\hline \multirow{2}{*}{\multicolumn{12}{|c|}{$\begin{array}{l}\text { 1st-Order Markov Model } \\
\text { March DRWH Reliability (\%) } \\
\text { per capita roof area and storage } \\
\text { L }\end{array}$}} \\
\hline & & & & & & & & & & & \\
\hline Gauge & sq. m & 0 & 40 & 80 & 120 & 160 & 200 & 400 & 600 & 800 & 1000 \\
\hline \multirow{2}{*}{655450} & 2 & 3.9 & 6.4 & 8.3 & 8.9 & 9.2 & 9.3 & 9.5 & 9.5 & 9.5 & 9.5 \\
\hline & 10 & 7.7 & 13.5 & 21.4 & 27.2 & 32.4 & 35.3 & 42.2 & 45.0 & 46.2 & 46.6 \\
\hline \multirow[t]{2}{*}{655480} & 2 & 6.9 & 11.2 & 15.1 & 16.9 & 17.9 & 18.4 & 18.8 & 18.8 & 18.8 & 18.8 \\
\hline & 10 & 16.2 & 27.7 & 41.6 & 50.0 & 56.3 & 59.5 & 65.1 & 65.6 & 65.6 & 65.7 \\
\hline \multirow[t]{2}{*}{655550} & 2 & 4.2 & 6.6 & 9.2 & 10.9 & 12.1 & 12.8 & 13.9 & 13.9 & 13.9 & 13.9 \\
\hline & 10 & 10.5 & 18.1 & 27.7 & 33.9 & 38.9 & 41.5 & 47.4 & 48.7 & 49.1 & 49.4 \\
\hline \multirow[t]{2}{*}{655570} & 2 & 8.7 & 13.4 & 15.9 & 17.2 & 18.2 & 19.0 & 21.0 & 21.5 & 21.5 & 21.5 \\
\hline & 10 & 20.9 & 34.7 & 50.8 & 60.3 & 67.4 & 70.9 & 78.2 & 80.0 & 80.6 & 81.6 \\
\hline \multirow[t]{2}{*}{655600} & 2 & 6.2 & 9.8 & 12.2 & 12.8 & 13.0 & 13.0 & 13.0 & 13.0 & 13.0 & 13.0 \\
\hline & 10 & 13.0 & 23.2 & 36.2 & 44.9 & 51.9 & 55.9 & 65.1 & 67.1 & 67.3 & 67.4 \\
\hline \multirow[t]{2}{*}{655620} & 2 & 6.7 & 10.8 & 13.2 & 13.8 & 14.0 & 14.1 & 14.1 & 14.1 & 14.1 & 14.1 \\
\hline & 10 & 13.9 & 24.0 & 36.4 & 44.5 & 51.0 & 54.4 & 60.1 & 60.7 & 60.7 & 60.7 \\
\hline \multirow[t]{2}{*}{655630} & 2 & 4.6 & 6.6 & 7.2 & 7.3 & 7.3 & 7.3 & 7.3 & 7.3 & 7.3 & 7.3 \\
\hline & 10 & 13.2 & 22.7 & 33.5 & 40.0 & 44.6 & 47.0 & 50.9 & 51.4 & 51.7 & 51.8 \\
\hline \multirow[t]{2}{*}{655780} & 2 & 5.4 & 9.1 & 11.9 & 13.1 & 13.6 & 13.8 & 14.1 & 14.2 & 14.2 & 14.2 \\
\hline & 10 & 12.8 & 21.9 & 32.9 & 40.3 & 46.3 & 49.5 & 55.7 & 57.3 & 58.6 & 61.3 \\
\hline \multirow[t]{2}{*}{655850} & 2 & 6.5 & 10.9 & 13.8 & 14.7 & 15.0 & 15.1 & 15.2 & 15.2 & 15.2 & 15.2 \\
\hline & 10 & 13.9 & 24.6 & 37.7 & 46.5 & 53.7 & 58.0 & 68.0 & 70.4 & 71.4 & 73.7 \\
\hline \multirow[t]{2}{*}{655920} & 2 & 4.0 & 6.6 & 8.6 & 9.4 & 9.7 & 9.7 & 9.8 & 9.8 & 9.8 & 9.8 \\
\hline & 10 & 10.0 & 17.2 & 26.5 & 32.9 & 38.6 & 42.0 & 50.8 & 54.3 & 58.5 & 66.3 \\
\hline \multirow[t]{2}{*}{655940} & 2 & 3.0 & 4.7 & 5.9 & 6.3 & 6.4 & 6.4 & 6.4 & 6.4 & 6.4 & 6.4 \\
\hline & 10 & 6.3 & 11.1 & 17.7 & 22.2 & 26.1 & 28.0 & 32.0 & 32.6 & 32.7 & 33.3 \\
\hline \multirow[t]{2}{*}{655990} & 2 & 2.8 & 4.3 & 5.4 & 5.6 & 5.7 & 5.8 & 5.8 & 5.8 & 5.8 & 5.8 \\
\hline & 10 & 6.5 & 11.2 & 17.6 & 22.1 & 26.2 & 28.3 & 33.5 & 34.9 & 35.4 & 36.0 \\
\hline
\end{tabular}




\begin{tabular}{|c|c|c|c|c|c|c|c|c|c|c|c|}
\hline \multicolumn{12}{|c|}{$\begin{array}{l}\text { 1st-Order Markov Model } \\
\text { April DRWH Reliability (\%) } \\
\text { per capita roof area and storage } \\
\text { L }\end{array}$} \\
\hline Gauge & sq. m & 0 & 40 & 80 & 120 & 160 & 200 & 400 & 600 & 800 & \\
\hline \multirow[t]{2}{*}{653060} & 2 & 2.5 & 4.0 & 5.0 & 5.3 & 5.4 & 5.4 & 5.4 & 5.4 & 5.4 & \\
\hline & 10 & 6.6 & 11.2 & 17.1 & 21.0 & 24.3 & 26.0 & 29.4 & 29.8 & 29.9 & 29. \\
\hline \multirow[t]{2}{*}{653190} & 2 & 6.6 & 10.9 & 13.6 & 14.4 & 14.7 & 14.7 & 14.7 & 14.7 & 14.7 & 4. \\
\hline & 10 & 15.1 & 26.1 & 38.9 & 46.9 & 52.9 & 56.1 & 61.3 & 61.7 & 61.7 & 61. \\
\hline \multirow[t]{2}{*}{653300} & 2 & 6.0 & 9.7 & 12.4 & 13.1 & 13.3 & 13.4 & 13.4 & 13.4 & 13.4 & 13 \\
\hline & 10 & 11.1 & 19.8 & 31.4 & 39.2 & 45.9 & 49.3 & 56.6 & 58.0 & 58.2 & 8 \\
\hline \multirow[t]{2}{*}{653350} & 2 & 9.0 & 14.6 & 18.1 & 19.1 & 19.4 & 19.4 & 19.5 & 19.5 & 19.5 & 9 \\
\hline & 10 & 18.2 & 31.6 & 48.0 & 58.1 & 65.9 & 69.9 & 78.8 & 80.7 & 81.3 & . \\
\hline \multirow[t]{2}{*}{653380} & 2 & 10.0 & 16.4 & 20.3 & 21.4 & 21.6 & 21.7 & 21.7 & 21.7 & 21.7 & 21 \\
\hline & 10 & 20.3 & 35.4 & 53 & 63.4 & 70.7 & 74.7 & 82.4 & 83.7 & 83.9 & 84 \\
\hline \multirow[t]{2}{*}{653440} & 2 & 10. & 16.9 & 1.6 & 23.7 & 25.0 & 25.8 & 26.8 & & 26.9 & 26. \\
\hline & 10 & & 33.9 & & 62.4 & 70.5 & 74 & 82.9 & & 5.6 & 85. \\
\hline \multirow[t]{2}{*}{653520} & 2 & 3.1 & 4.5 & 5.2 & 5.4 & 5.6 & 5.6 & 5.8 & 5.8 & 5.8 & \\
\hline & 10 & 7.9 & 13.7 & & 26.1 & 30.2 & 32 & .9 & & & 4 \\
\hline \multirow[t]{2}{*}{653550} & 2 & 6.0 & 9.0 & 10.4 & 10.7 & 10.7 & 10.7 & 10.7 & 10 & 10.7 & 10. \\
\hline & 10 & 13.6 & 24.0 & 36.7 & 44.6 & 50.5 & 53.6 & 59.4 & 60.5 & 61.0 & 61. \\
\hline \multirow[t]{2}{*}{653570} & 2 & 4.9 & 7.0 & 8.0 & 8.2 & 8.4 & 8.5 & 8.8 & 8.9 & 9.0 & \\
\hline & 10 & 14.2 & 24.2 & 35.8 & 42.3 & 46.9 & 49.1 & 53.2 & & 55.5 & \\
\hline \multirow[t]{2}{*}{653610} & 2 & 5.3 & 8.6 & 10.9 & 11.5 & 11.7 & 11.7 & 11.7 & 11 & 11.7 & 11 \\
\hline & 10 & 14.5 & 25.1 & 37 & 44.6 & 50.2 & 53 & 59.0 & & 59 & 59 \\
\hline \multirow[t]{2}{*}{653760} & 2 & 7.8 & 12.8 & 16 & 17.1 & 17.5 & 17 & 17.7 & & 17.7 & \\
\hline & 10 & 16 & 28.6 & & 52.2 & 59.3 & 63.2 & 71.3 & & 73.0 & 73. \\
\hline \multirow[t]{2}{*}{653800} & 2 & & 12.5 & & 17.5 & 17.9 & 18 & & & & 18 \\
\hline & 10 & 14.4 & 25.9 & 40.7 & 50.4 & 58.2 & 62 & 72.5 & & & 7 \\
\hline \multirow[t]{2}{*}{653870} & 2 & 6.4 & 11.4 & 16.0 & 18.4 & 19.8 & 20.5 & 21.1 & 21 & 21.2 & 1. \\
\hline & 10 & 14.4 & 24.4 & 36.8 & 45.1 & 52.3 & 56.5 & 67.5 & 71 & 72.3 & 72 \\
\hline \multirow[t]{2}{*}{654720} & 2 & 4.8 & 8.4 & 12.4 & 14.5 & 15.8 & 16.3 & 16.9 & 16.9 & 16.9 & 16. \\
\hline & 10 & 9.7 & 16.9 & 26.6 & 33.6 & 40.3 & 44.1 & 53.4 & 55 & 56.5 & 56 \\
\hline \multirow[t]{2}{*}{655010} & 2 & 0.1 & 0.1 & 0.1 & 0.1 & 0.1 & 0.1 & 0.1 & 0.1 & 0.1 & 0.1 \\
\hline & 10 & 1.1 & 1.8 & 2.4 & 2.6 & 2.7 & 2.8 & 2.9 & 3. & 3.0 & 3.1 \\
\hline \multirow[t]{2}{*}{655020} & 2 & 0.4 & 0.5 & 0.6 & 0.6 & 0.6 & 0.6 & 0. & & & 0.6 \\
\hline & 10 & 2.0 & 3. & & 5.5 & 6.2 & 6.6 & 7.5 & & 8.2 & 8 \\
\hline 655030 & 2 & & 3. & & 3.8 & & & & & & 4.1 \\
\hline & 10 & 5 & 9.0 & 14.2 & 17.5 & 20.3 & 21 & 24.4 & & 27.0 & 27. \\
\hline 655050 & 2 & & 2.3 & & 2.9 & 2.9 & 2. & 2 & & & \\
\hline & 10 & 4.0 & 6.8 & 10.2 & 12.6 & 14.7 & 15.8 & 18.2 & 19 & 19.5 & 19 \\
\hline 655070 & 2 & 1.8 & 2.6 & 3.1 & 3.3 & 3.6 & 3.8 & 4.3 & 4. & 4. & \\
\hline & 10 & 5.5 & 9.3 & 14.1 & 17.2 & 19.9 & 21.4 & 26.9 & 31 & 33.7 & \\
\hline 655100 & 2 & 3.4 & 5.2 & 6.1 & 6.4 & 6.4 & 6.4 & 6.4 & 6.4 & 6.4 & \\
\hline & 10 & 8.9 & 15.0 & 22.7 & 27.8 & 31.9 & 33.9 & 37.4 & 37.7 & 37.8 & 37 \\
\hline 655160 & 2 & 2.8 & 4.4 & 5.9 & 6.7 & 7.2 & 7.4 & 7.7 & 7.7 & 7.7 & \\
\hline & 10 & 8.3 & 14.0 & 20.8 & 24.8 & 28.3 & 30.1 & 34.5 & 36.1 & 36.8 & 36. \\
\hline 655180 & 2 & 2.6 & 4.3 & 5.6 & 6.0 & 6.2 & 6.3 & 6.3 & 6.3 & 6.3 & 6.3 \\
\hline & 10 & 7.1 & 11.9 & 17.8 & 21.9 & 25.6 & 27.6 & 33.3 & 35.3 & 35.9 & 36 \\
\hline 655220 & 2 & 5.0 & 7.9 & 9.8 & 10.3 & 10.4 & 10.4 & 10.4 & 10 & 10.4 & 10. \\
\hline & 10 & 10.9 & 19.1 & 29.8 & 37.0 & 43.0 & 46 & 53.1 & 54 & 4.8 & 54 \\
\hline 655280 & 2 & 6.1 & 9.4 & 11.0 & 11.3 & 11.5 & 11.5 & 11.9 & 12.2 & 12.3 & 12. \\
\hline & 10 & & 24.4 & 36.8 & 44.3 & 49.8 & 52.6 & 57.1 & 58.1 & 58.4 & 58 \\
\hline j55360 & 2 & 5.9 & 9.5 & 12.3 & 13.2 & 13.6 & 13.7 & 13.7 & 13.7 & 13.7 & 13. \\
\hline & 10 & 13.8 & 24.0 & 36.5 & 44.2 & 50.5 & 53.8 & 61.7 & 63.9 & 64.6 & \\
\hline
\end{tabular}




\begin{tabular}{|c|c|c|c|c|c|c|c|c|c|c|c|}
\hline \multicolumn{12}{|c|}{$\begin{array}{l}\text { 1st-Order Markov Model } \\
\text { April DRWH Reliability (\%) } \\
\text { per capita roof area and storage } \\
\text { L }\end{array}$} \\
\hline Gauge & sq. m & 0 & 40 & 80 & 120 & 160 & 200 & 400 & 600 & 800 & \\
\hline \multirow[t]{2}{*}{655450} & 2 & 8.7 & 13.2 & 16.3 & 17.5 & 18.2 & 18.5 & 19.0 & 19.0 & 19.0 & \\
\hline & 10 & 17.8 & 31.1 & 47.5 & 57.3 & 64.8 & 68.5 & 76.3 & 78.4 & 79.2 & $y$ \\
\hline \multirow[t]{2}{*}{655480} & 2 & 10.2 & 17.9 & 24.7 & 27.7 & 29.3 & 30.0 & 31.0 & 31.1 & 31.1 & \\
\hline & 10 & 19.4 & 34.1 & 52.1 & 63.4 & 72.0 & 76.7 & 87.8 & 90.2 & 90.4 & \\
\hline \multirow[t]{2}{*}{655550} & 2 & 9.3 & 14.7 & 18.5 & 20.3 & 21.6 & 22.5 & 24.7 & 25.3 & 25.4 & \\
\hline & 10 & 18.0 & 31.2 & 47.9 & 58.0 & 65.8 & 69.6 & 78.2 & 81.0 & 81.6 & \\
\hline \multirow[t]{2}{*}{655570} & 2 & 11.4 & 18.5 & 23.9 & 26.4 & 27.9 & 28.8 & 31.6 & 33.0 & 33.4 & \\
\hline & 10 & 24. & 42.6 & 62.7 & 73.9 & 81.4 & 85.5 & 93.3 & 94.7 & & \\
\hline \multirow[t]{2}{*}{655600} & 2 & 1 & 15.7 & 18 & 19.8 & 20.2 & 20.4 & 20.9 & 20.9 & & \\
\hline & 10 & & 36.0 & & 65.4 & 73.3 & 77.3 & 6.1 & 88.1 & .5 & \\
\hline \multirow[t]{2}{*}{655620} & 2 & 10 & 16.8 & 21 & 22.4 & 22.7 & 22.8 & 2.9 & 22.9 & 2.9 & \\
\hline & 10 & 17 & 31.9 & 49 & 61.1 & 69.9 & 74.5 & 85.1 & 87.0 & 87.2 & 87 \\
\hline \multirow[t]{2}{*}{655630} & 2 & 9.1 & 13.7 & 16. & 16.4 & 16.5 & 16.5 & 16.5 & 16.5 & 16.5 & \\
\hline & 10 & 19 & 33.5 & 50.6 & 60.7 & 67.9 & 71.6 & 78.1 & 78.8 & 78.9 & \\
\hline \multirow[t]{2}{*}{655780} & 2 & 10.4 & 17.6 & 22.8 & 24.6 & 25.4 & 25.7 & 25.9 & 25.9 & 26.0 & \\
\hline & 10 & 18.8 & 33.3 & 50.8 & 61.6 & 69.8 & 74.3 & 84.2 & 86.3 & 86.9 & \\
\hline \multirow[t]{2}{*}{655850} & 2 & 9.2 & 15.7 & 20.5 & 22.2 & 22.8 & 23.0 & 23.2 & 23.2 & 23.2 & \\
\hline & 10 & 20.3 & 35.5 & 52.6 & 63.0 & 70.6 & 75.0 & 85.7 & 88.3 & 89.0 & \\
\hline \multirow[t]{2}{*}{655920} & 2 & 8.5 & 14.5 & 18.7 & 20.1 & 20.7 & 21.0 & 21.1 & 21.1 & 21.1 & \\
\hline & 10 & 18.3 & 31.4 & 46.4 & 56.0 & 63.3 & 67.5 & 76.6 & 79.1 & 80.2 & \\
\hline \multirow[t]{2}{*}{655940} & 2 & 6.5 & 10.8 & 14.0 & 15.1 & 15.5 & 15.6 & 15.7 & 15.7 & 15.7 & \\
\hline & 10 & 13.0 & 22.6 & 34.9 & 43.3 & 50.4 & 54.3 & 62.9 & 64.7 & 65.0 & \\
\hline \multirow[t]{2}{*}{655990} & 2 & 8.8 & 14.8 & 19.2 & 20.8 & 21 & 21.6 & 21.7 & 21.7 & 21.7 & \\
\hline & 10 & 15.0 & 26.1 & 40.9 & 50.5 & 58.4 & 62.4 & 70.3 & 71.6 & 71.9 & \\
\hline
\end{tabular}




\begin{tabular}{|c|c|c|c|c|c|c|c|c|c|c|c|}
\hline \multicolumn{12}{|c|}{$\begin{array}{l}\text { 1st-Order Markov Model } \\
\text { May DRWH Reliability (\%) } \\
\text { per capita roof area and storage } \\
\text { L }\end{array}$} \\
\hline Gauge & sq. m & 0 & 40 & 80 & 120 & 160 & 200 & 400 & 600 & 800 & \\
\hline \multirow[t]{2}{*}{653060} & 2 & 7.9 & 12.4 & 15.0 & 15.6 & 15.8 & 15.8 & 15.9 & 15.9 & 15.9 & \\
\hline & 10 & 16.3 & 28.6 & 43.5 & 52.7 & 59.6 & 63.1 & 69.5 & 70.8 & 71.0 & \\
\hline \multirow[t]{2}{*}{653190} & 2 & 8.5 & 13.5 & 16.0 & 16.7 & 16.9 & 17.0 & 17.0 & 17.0 & 17.0 & 17 \\
\hline & 10 & 18.4 & 32.4 & 48.8 & 59.2 & 67.0 & 71.6 & 82.7 & 85.0 & 85.2 & \\
\hline \multirow[t]{2}{*}{653300} & 2 & 10.4 & 15.5 & 17.9 & 18.6 & 18.9 & 19.1 & 19.3 & 19.3 & 19.3 & \\
\hline & 10 & 24.4 & 42.4 & 61.9 & 72.2 & 78.9 & 82.4 & 88.7 & 90.1 & 90.3 & \\
\hline \multirow[t]{2}{*}{653350} & 2 & 9.2 & 14.5 & 17.4 & 18.2 & 18.4 & 18.5 & 18.5 & 18.5 & 18.5 & \\
\hline & 10 & 19.9 & 35.0 & 53.0 & 64.2 & 72.6 & 77.4 & 89.6 & 92.6 & 93.1 & \\
\hline \multirow[t]{2}{*}{653380} & 2 & 10.6 & 16.8 & 20.2 & 21.1 & 21.4 & 21.5 & 21.5 & 21.5 & 21.5 & \\
\hline & 10 & 22.3 & 39.0 & 58.1 & 69.6 & 78.0 & 82.6 & 93.2 & 95.0 & 5.2 & \\
\hline \multirow[t]{2}{*}{653440} & 2 & 14.2 & 24.8 & & 35.9 & 37.5 & 38.4 & 0.8 & 41.5 & 1.6 & \\
\hline & 10 & 25.8 & 45.2 & & 77.6 & 5.3 & 89.3 & 6.5 & 97.6 & 7.8 & \\
\hline \multirow[t]{2}{*}{653520} & 2 & 7.2 & 11.0 & & 13.3 & 13.3 & 13.4 & 3.4 & 13.4 & & \\
\hline & 10 & 15.2 & 26.6 & 40.6 & 49.4 & 56.2 & 59.8 & 65.9 & 67.4 & 68.4 & \\
\hline \multirow[t]{2}{*}{653550} & 2 & 10.0 & 15.2 & 16.9 & 17.1 & 17.2 & 17.2 & 17.2 & 17.2 & 17.2 & \\
\hline & 10 & 24.3 & 42.1 & 60.2 & 70.3 & 77.0 & 80.5 & 86.6 & 87.5 & 87.6 & \\
\hline \multirow[t]{2}{*}{653570} & 2 & 8.2 & 13.0 & 16.1 & 17.4 & 17.9 & 18.1 & 18.3 & 18.3 & 18.3 & \\
\hline & 10 & 19.9 & 33.7 & 49.7 & 59.4 & 66.6 & 70.5 & 78.3 & 79.6 & 80.1 & \\
\hline \multirow[t]{2}{*}{653610} & 2 & 7.8 & 12.3 & 15.1 & 16.1 & 16.6 & 16.7 & 16.8 & 16.8 & 16.8 & \\
\hline & 10 & 22.8 & 38.8 & 55 & 65.3 & 72 & 75 & & .2 & & \\
\hline \multirow[t]{2}{*}{653760} & 2 & 10.1 & 15.7 & 19 & 21.1 & 22.2 & 22.8 & & 23.8 & & \\
\hline & 10 & 21 & 37. & & 66.8 & 75.0 & 79.4 & 9.4 & 91.6 & 1.9 & \\
\hline \multirow[t]{2}{*}{653800} & 2 & 10. & 17.9 & 23 & 25.4 & 26.2 & 26.5 & & 26.7 & & \\
\hline & 10 & 18. & 33.0 & 51.1 & 62.6 & 71.6 & 76.6 & 88.2 & 90.8 & 91.4 & \\
\hline \multirow[t]{2}{*}{653870} & 2 & 10.4 & 18.3 & 24.1 & 26.5 & 27.7 & 28.4 & 29.4 & 29.4 & 29.4 & \\
\hline & 10 & 20.6 & 36.2 & 53.9 & 64.9 & 72.9 & 77.5 & 88.1 & 91.4 & 92.3 & \\
\hline \multirow[t]{2}{*}{654720} & 2 & 7.7 & 12.2 & 15.1 & 16.1 & 16.7 & 17.1 & 17.8 & 18.0 & 18.0 & \\
\hline & 10 & 14.3 & 25.1 & 39.6 & 49.1 & 57.2 & 61.6 & 74.0 & 79.1 & 80.4 & \\
\hline \multirow[t]{2}{*}{655010} & 2 & 1.6 & 2.2 & 2.4 & 2.4 & 2.4 & 2.4 & 2.4 & 2.4 & & \\
\hline & 10 & 4.6 & 7.8 & 11. & 14.2 & 16. & 16 & 17.7 & 17.7 & 17.7 & \\
\hline \multirow[t]{2}{*}{655020} & 2 & 2.3 & 3.7 & & 5.9 & & 6 & & & & \\
\hline & 10 & 8. & 13.7 & 19 & 23.1 & 25.9 & 27.3 & 30.5 & 30.9 & 31.0 & \\
\hline 655030 & 2 & & & & 8.4 & & & & & & \\
\hline & 10 & 13 & 23.8 & 35 & 42.4 & 47. & 49 & 54 & 54.8 & & \\
\hline 655050 & 2 & 4. & 7.4 & 9. & 9. & 9. & 9 & & & & \\
\hline & 10 & 11 & 19.5 & & 36.0 & 4 & 44 & & 50.3 & & \\
\hline 655070 & 2 & 5.6 & 8.8 & 11.6 & 13.1 & 14.0 & 14.4 & 14.9 & 15.0 & 15.0 & \\
\hline & 10 & 14.8 & 25.3 & 37.7 & 45.0 & 50.6 & 53.4 & 58.7 & 59.8 & 61.0 & \\
\hline 655100 & 2 & 6.3 & 9.8 & 11.8 & 12.2 & 12.4 & 12.4 & 12.4 & 12.4 & 12.4 & \\
\hline & 10 & 16. & 28.1 & 42.1 & 50.6 & 56.8 & 60.1 & 66.8 & 67.9 & 68.0 & \\
\hline 655160 & 2 & 6.0 & 9.3 & 10.9 & 11.4 & 11.6 & 11.7 & 11.8 & 11.8 & 11.8 & \\
\hline & 10 & 15.0 & 25.9 & 39.0 & 47.1 & 53.3 & 56.6 & 64.1 & 66.6 & 67.4 & \\
\hline 655180 & 2 & 5.7 & 8.2 & 9.2 & 9.4 & 9.4 & 9.4 & & 9.5 & 9 & \\
\hline & 10 & 14.3 & 25.1 & 38.1 & 46.0 & 51.8 & 54.9 & 61. & 63.5 & 64.1 & \\
\hline 655220 & 2 & 7. & 12.2 & 15.2 & 15.9 & 16.1 & 16 & 16.2 & 16.2 & 16.2 & \\
\hline & 10 & & 28.1 & 43.0 & 52.7 & 60.3 & 64.5 & 74.2 & 76.8 & 77.3 & \\
\hline 655280 & 2 & 7.4 & 11.6 & 14.2 & 14.9 & 15.1 & 15.1 & 15.1 & 15.1 & 15.2 & \\
\hline & 10 & 18.0 & 31.6 & 47.9 & 57.7 & 65 & 69.4 & 78.8 & 80.7 & 81.1 & \\
\hline 655360 & 2 & 9.6 & 15.6 & 19 & 20.6 & 20.9 & 21.0 & 21.1 & 21.1 & 21.1 & \\
\hline & 10 & 19.9 & 34.9 & 52.5 & 63.2 & 71.0 & 75.4 & 84.8 & 87.1 & 87.6 & \\
\hline
\end{tabular}




\begin{tabular}{|c|c|c|c|c|c|c|c|c|c|c|c|}
\hline \multirow{2}{*}{\multicolumn{12}{|c|}{$\begin{array}{l}\text { 1st-Order Markov Model } \\
\text { May DRWH Reliability (\%) } \\
\text { per capita roof area and storage }\end{array}$}} \\
\hline & & & & & & & & & & & \\
\hline Gauge & sq. $m$ & 0 & 40 & 80 & 120 & 160 & 200 & 400 & 600 & 800 & 1000 \\
\hline \multirow[t]{2}{*}{655450} & 2 & 12.1 & 19.7 & 24.1 & 25.4 & 26.0 & 26.4 & 27.3 & 27.5 & 27.5 & 27.5 \\
\hline & 10 & 22.7 & 40.1 & 59.7 & 71.2 & 79.3 & 83.6 & 92.1 & 93.7 & 94.1 & 94.3 \\
\hline \multirow[t]{2}{*}{655480} & 2 & 10.7 & 17.2 & 21.3 & 22.8 & 23.4 & 23.8 & 24.5 & 24.5 & 24.5 & 24.5 \\
\hline & 10 & 21.2 & 37.7 & 56.9 & 68.6 & 77.2 & 82.1 & 93.9 & 96.7 & 97.4 & 97.4 \\
\hline \multirow[t]{2}{*}{655550} & 2 & 7.8 & 12.4 & 15.4 & 16.5 & 17.1 & 17.5 & 19.4 & 20.7 & 21.1 & 21.2 \\
\hline & 10 & 17.5 & 30.7 & 47.4 & 58.0 & 66.7 & 71.9 & 85.7 & 89.9 & 91.1 & 91.4 \\
\hline \multirow[t]{2}{*}{655570} & 2 & 13.7 & 22.6 & 27.1 & 28.7 & 29.6 & 30.1 & 31.4 & 32.1 & 32.8 & 33.2 \\
\hline & 10 & 32.9 & 54.0 & 74.1 & 84.3 & 90.3 & 93.3 & 98.4 & 99.2 & 99.4 & 99.4 \\
\hline \multirow[t]{2}{*}{655600} & 2 & 9.9 & 15.7 & 18.7 & 19.5 & 19.8 & 20.0 & 20.7 & 21.3 & 21.4 & 21.5 \\
\hline & 10 & 24.3 & 42.3 & 61.7 & 72.7 & 80.3 & 84.8 & 94.3 & 96.0 & 96.3 & 96.4 \\
\hline \multirow[t]{2}{*}{655620} & 2 & 11.9 & 19.7 & 24.2 & 25.5 & 25.9 & 26.1 & 26.2 & 26.2 & 26.2 & 26.2 \\
\hline & 10 & 24.3 & 42.5 & 62.1 & 73.4 & 81.0 & 85.3 & 94.4 & 96.4 & 96.8 & 96.8 \\
\hline \multirow[t]{2}{*}{655630} & 2 & 11.1 & 18.3 & 22.5 & 23.7 & 24.0 & 24.0 & 24.1 & 24.1 & 24.1 & 24.1 \\
\hline & 10 & 23.9 & 41.8 & 61.4 & 72.7 & 80.3 & 84.5 & 92.8 & 93.8 & 93.9 & 93.9 \\
\hline \multirow[t]{2}{*}{655780} & 2 & 18.6 & 33.0 & 42.8 & 46.8 & 48.7 & 49.5 & 50.0 & 50.0 & 50.0 & 50.0 \\
\hline & 10 & 31.3 & 54.1 & 74.3 & 84.4 & 90.2 & 93.1 & 97.7 & 98.5 & 98.7 & 98.7 \\
\hline \multirow[t]{2}{*}{655850} & 2 & 17.0 & 30.5 & 39.3 & 43.0 & 44.7 & 45.4 & 45.8 & 45.8 & 45.8 & 45.8 \\
\hline & 10 & 32.9 & 56.2 & 76.3 & 85.8 & 91.0 & 93.5 & 97.9 & 98.7 & 98.8 & 98.9 \\
\hline \multirow[t]{2}{*}{655920} & 2 & 20.9 & 38.4 & 50.1 & 55.7 & 58.5 & 59.7 & 60.5 & 60.5 & 60.5 & 60.5 \\
\hline & 10 & 39.2 & 62.1 & 80.2 & 88.4 & 92.8 & 94.8 & 97.9 & 98.3 & 98.5 & 98.6 \\
\hline \multirow[t]{2}{*}{655940} & 2 & 16.2 & 29.2 & 38.3 & 42.3 & 44.2 & 44.9 & 45.4 & 45.4 & 45.4 & 45.4 \\
\hline & 10 & 29.4 & 49.1 & 68.1 & 78.3 & 84.6 & 87.7 & 93.0 & 94.1 & 94.3 & 94.3 \\
\hline \multirow[t]{2}{*}{655990} & 2 & 17.5 & 30.9 & 39.8 & 43.4 & 45.1 & 45.8 & 46.2 & 46.2 & 46.2 & 46.2 \\
\hline & 10 & 32.7 & 52.8 & 72.2 & 82.2 & 88.3 & 91.0 & 95.8 & 96.7 & 96.8 & 96.8 \\
\hline
\end{tabular}




\begin{tabular}{|c|c|c|c|c|c|c|c|c|c|c|c|}
\hline \multirow{2}{*}{ Gauge } & $\begin{array}{l}\text { r Marko } \\
\text { WH Re } \\
\text { per ca }\end{array}$ & \multicolumn{2}{|c|}{$\begin{array}{l}\text { per capita roof area and storage } \\
L\end{array}$} & \multicolumn{3}{|c|}{$\mathrm{H}-12$} & \multirow[b]{2}{*}{200} & \multirow[b]{2}{*}{400} & \multirow[b]{2}{*}{600} & \multirow[b]{2}{*}{800} & \\
\hline & sq. m & 0 & 40 & 80 & 120 & 160 & & & & & \\
\hline \multirow[t]{2}{*}{653060} & 2 & 13.6 & 22.0 & & 28.1 & 28.4 & 28.5 & 28.5 & 28.5 & & \\
\hline & 10 & 25.2 & 44.4 & 65.5 & 76.8 & 84.1 & 87.8 & 94.0 & 94.7 & 4.8 & \\
\hline \multirow[t]{2}{*}{653190} & 2 & 12.5 & 20.2 & 23.7 & 24.5 & 24.7 & 24.7 & 24.8 & 24.8 & 24.8 & \\
\hline & 10 & 29.5 & 49.7 & 69.4 & 79.6 & 85.8 & 89.1 & 95.3 & 96.6 & 96.8 & \\
\hline \multirow[t]{2}{*}{653300} & 2 & 12.7 & 21.5 & 26.1 & 27.5 & 28.0 & 28.2 & 28.5 & 28.6 & 8.6 & \\
\hline & 10 & 27.2 & 47.2 & 67.6 & 78.7 & 85.8 & 89.8 & 96.7 & 97.6 & 97.7 & \\
\hline \multirow[t]{2}{*}{653350} & 2 & 11.9 & 19.3 & 22.4 & 23.1 & 23.3 & 23.3 & 23.3 & 23.3 & 23.3 & \\
\hline & 10 & 27.5 & 47.7 & 67.0 & 77.5 & 84.1 & 87.9 & 95.4 & 97.4 & 97.9 & \\
\hline \multirow[t]{2}{*}{653380} & 2 & 13.8 & 22.9 & 27.8 & 29.2 & 29.6 & 29.7 & 29.8 & 29.8 & 29.8 & \\
\hline & 10 & 27.0 & 47.7 & 68.6 & 79.8 & 86.7 & 90.4 & 97.2 & 98.6 & & \\
\hline \multirow[t]{2}{*}{653440} & 2 & 0. & 37.2 & 49 & 55.3 & 58.4 & 59.9 & 61.3 & 61.7 & & \\
\hline & 10 & 33 & 58.0 & & 88.3 & 93 & & & 99.8 & & \\
\hline \multirow[t]{2}{*}{653520} & 2 & & 13 & & 19.3 & 20 & 21.3 & 22.2 & 22.2 & 22.2 & \\
\hline & 10 & & 35.4 & & 62.7 & 70.1 & 74.4 & 4.3 & 86.5 & & \\
\hline 653550 & 2 & 14.0 & 23.5 & 28 & 29.8 & 30.2 & 30.3 & 30.3 & .3 & & \\
\hline & 10 & 28.4 & 49.6 & 70.1 & 80.9 & 87.4 & 91.0 & 96.9 & 97.6 & 97.7 & \\
\hline 653570 & 2 & 11.3 & 19.0 & 24.6 & 26.6 & 27.5 & 27.9 & 28.2 & 28.2 & 28.2 & \\
\hline & 10 & 23.5 & 40.5 & 59.9 & 71.0 & 78.9 & 83.1 & 92.1 & 93.8 & 94.1 & \\
\hline 653610 & 2 & 13.0 & 20.6 & 23.6 & 24.2 & 24.4 & 24.5 & 24.5 & 24.5 & 24.5 & \\
\hline & 10 & 30.0 & 52.0 & 72.1 & 82.0 & 87.6 & 90.8 & 95.9 & 96.7 & 96.9 & \\
\hline 653760 & 2 & 11.9 & 21.2 & 28 & 30.9 & 32.3 & 33.1 & 34.6 & 35.0 & & \\
\hline & 10 & & 46.8 & & 77.3 & & 88.6 & & 97.6 & & \\
\hline 653800 & 2 & 9 & 16.7 & 22 & 25.2 & 26.6 & 27.2 & 27.7 & 27.7 & 7.7 & \\
\hline & 10 & 24. & 41.0 & 59 & 70.2 & 78.2 & 83.0 & & & & \\
\hline 653870 & 2 & 11.4 & 20.1 & 26 & 28.6 & 29.7 & 30.2 & 30.7 & 30.7 & & \\
\hline & 10 & 23.2 & 40.8 & 59.6 & 70.9 & 78.9 & 83.8 & 94.7 & 97.3 & 98.0 & \\
\hline 654720 & 2 & 9. & 15.6 & 19.5 & 20.8 & 21.2 & 21.3 & 21.4 & 21.4 & 21.4 & \\
\hline & 10 & 16.9 & 30.7 & 46.6 & 57.3 & 65.7 & 70.7 & 83.4 & 87.9 & 89.9 & \\
\hline 655010 & 2 & 4.5 & 6.6 & 7.5 & 7.6 & 7. & 7.6 & 7. & 7. & & \\
\hline & 10 & 12.1 & 21.0 & 31.8 & 38.2 & 42.8 & 45.1 & 48 & .7 & .7 & \\
\hline 655020 & 2 & & 10.4 & 13.2 & 14.3 & 14.8 & 15.0 & 15.4 & 15.5 & 5.5 & \\
\hline & 10 & 17. & 30.1 & 44.3 & 52.6 & 58.5 & 61.7 & 68 & 70.5 & .9 & \\
\hline 655030 & 2 & & 11 & 13 & 14.4 & & & & & & \\
\hline & 10 & 21 & 37.1 & 54 & 63.4 & & 3.1 & 9.6 & .7 & & \\
\hline 655050 & 2 & & & & 17 & & & & & & \\
\hline & 10 & & 35 & 53 & 63.4 & & 74.0 & & 82.2 & & \\
\hline 655070 & 2 & & 16.9 & 20 & 22.7 & & 24.6 & & & & \\
\hline & 10 & 24 & 41.1 & 60.1 & 70.6 & 77.6 & 81.2 & 87.7 & 89.1 & 89.3 & \\
\hline 655100 & 2 & 10.8 & 17.3 & 20.3 & 21.0 & 21.1 & 21.2 & 21.2 & 21.2 & 21.2 & \\
\hline & 10 & 24.2 & 42.4 & 61.5 & 72.0 & 78.9 & 82.7 & 89.5 & 90.6 & 90.8 & \\
\hline 655160 & 2 & 9.9 & 14.9 & 16.7 & 17.0 & 17.0 & 17.0 & 17.0 & 17.0 & 17.0 & \\
\hline & 10 & 23.0 & 40.6 & 59.5 & 69.7 & 76.4 & 80.1 & 87.1 & 88.8 & 89.4 & \\
\hline 655180 & 2 & 13.1 & 20.7 & 24.6 & 25.4 & 25.6 & 25.6 & 25.6 & 25.6 & 5.6 & \\
\hline & 10 & 25.4 & 45.0 & 65.9 & 76.6 & 83.1 & 86.1 & 90.9 & 91.6 & 91.8 & \\
\hline 655220 & 2 & 12 & 18.7 & 22.2 & 23.2 & 23 & 23.9 & 24 & 24.1 & 24.1 & \\
\hline & 10 & 25.2 & 43.6 & 64.0 & 75.0 & 82.0 & 85.7 & 92.5 & 93.9 & 94.2 & \\
\hline 655280 & 2 & 10. & 16.1 & 19. & 19.7 & 19 & 19.9 & & 19.9 & 19.9 & \\
\hline & 10 & & 42.5 & 62.2 & 73.0 & 80.0 & 83.9 & 91.9 & 93.6 & 93.9 & \\
\hline & 2 & 12.0 & 18.8 & 22.8 & 24.2 & 24.8 & 25.0 & 25.2 & 25.2 & 25.2 & \\
\hline & 10 & 25.0 & 43.3 & 63.9 & 75.4 & 83.2 & 87.2 & 95.1 & 96.7 & 97.0 & \\
\hline
\end{tabular}




\begin{tabular}{|c|c|c|c|c|c|c|c|c|c|c|c|}
\hline June & $\begin{array}{l}\text { Mar } \\
\text { NH R }\end{array}$ & Mode & ) & I & $\mathrm{H}-1$ & & & & & & \\
\hline Gauge & sa. m & 0 & 40 & 80 & 120 & 160 & 200 & 400 & 600 & 800 & 1000 \\
\hline 655450 & 2 & 14.3 & 23.4 & 28.2 & 29.7 & 30.1 & 30.2 & 30.4 & 30.4 & 30.5 & 30.5 \\
\hline & 10 & 27.1 & 47.4 & 68.8 & 80.2 & 87.5 & 91.3 & 97.9 & 98.9 & 99.1 & 99.1 \\
\hline 655480 & 2 & 14.2 & 23.2 & 29.0 & 31.7 & 33.4 & 34.4 & 35.8 & 35.8 & 35.8 & 35.8 \\
\hline & 10 & 30.9 & 51.2 & 71.6 & 82.0 & 88.5 & 91.8 & 97.7 & 99.2 & 99.5 & 99.5 \\
\hline 655550 & 2 & 10.0 & 16.0 & 19.3 & 20.2 & 20.4 & 20.5 & 20.5 & 20.7 & 21.1 & 21.5 \\
\hline & 10 & 20.2 & 35.9 & 54.2 & 65.4 & 73.4 & 78.0 & 89.0 & 93.3 & 95.0 & 95.5 \\
\hline 655570 & 2 & 13.3 & 22.9 & 27.8 & 29.3 & 29.8 & 29.9 & 30.0 & 30.1 & 30.2 & 30.5 \\
\hline & 10 & 33.7 & 56.1 & 76.1 & 86.2 & 92.2 & 95.2 & 99.5 & 99.9 & 99.9 & 99.9 \\
\hline 655600 & 2 & 9.6 & 17.3 & 23.8 & 27.1 & 28.8 & 29.6 & 30.4 & 30.5 & 30.7 & 30.8 \\
\hline & 10 & 26.1 & 44.4 & 62.8 & 73.6 & 81.1 & 85.7 & 95.6 & 97.7 & 98.2 & 98.2 \\
\hline 655620 & 2 & 10.8 & 18.6 & 23.2 & 24.7 & 25.3 & 25.5 & 25.6 & 25.6 & 25.6 & 25.6 \\
\hline & 10 & 25.9 & 44.8 & 64.0 & 75.2 & 82.7 & 87.3 & 96.8 & 98.6 & 99.0 & 99.0 \\
\hline 655630 & 2 & 10.6 & 16.4 & 19.0 & 20.0 & 20.6 & 21.0 & 21.5 & 21.5 & 21.5 & 21.5 \\
\hline & 10 & 28.2 & 46.5 & 65.6 & 76.5 & 83.8 & 88.0 & 96.7 & 98.2 & 98.4 & 98.4 \\
\hline 655780 & 2 & 23.9 & 44.6 & 59.4 & 67.0 & 71.1 & 73.4 & 75.5 & 75.5 & 75.5 & 75.5 \\
\hline & 10 & 41.3 & 66.3 & 85.5 & 93.3 & 96.9 & 98.5 & 99.9 & 100.0 & 100.0 & 100.0 \\
\hline 655850 & 2 & 23.6 & 44.5 & 58.0 & 64.6 & 68.1 & 69.8 & 71.2 & 71.2 & 71.2 & 71.2 \\
\hline & 10 & 44.8 & 69.9 & 87.7 & 94.7 & 97.7 & 98.9 & 99.9 & 100.0 & 100.0 & 100.0 \\
\hline 655920 & 2 & 23.1 & 43.6 & 58.7 & 66.6 & 71.5 & 74.5 & 78.7 & 78.8 & 78.8 & 78.8 \\
\hline & 10 & 39.2 & 64.9 & 84.4 & 92.8 & 96.7 & 98.3 & 99.9 & 100.0 & 100.0 & 100.0 \\
\hline 655940 & 2 & 18.4 & 34.8 & 46.3 & 52.1 & 55.3 & 57.2 & 59.3 & 59.3 & 59.3 & 59.3 \\
\hline & 10 & 38.2 & 62.6 & 81.7 & 90.4 & 94.9 & 97.0 & 99.6 & 99.7 & 99.8 & 99.8 \\
\hline 655990 & 2 & 19.7 & 36.7 & 49.0 & 55.3 & 58.8 & 60.7 & 62.5 & 62.5 & 62.5 & 62.5 \\
\hline & 10 & 36.0 & 59.2 & 79.5 & 89.1 & 94.4 & 96.9 & 99.7 & 99.9 & 99.9 & 99.9 \\
\hline
\end{tabular}




\begin{tabular}{|c|c|c|c|c|c|c|c|c|c|c|c|}
\hline \multicolumn{12}{|c|}{$\begin{array}{l}\text { 1st-Order Markov Model } \\
\text { July DRWH Reliability (\%) } \\
\text { per capita roof area and storage } \\
\text { L }\end{array}$} \\
\hline Gauge & sq. m & 0 & 40 & 80 & 120 & 160 & 200 & 400 & 600 & 800 & 1000 \\
\hline \multirow[t]{2}{*}{653060} & 2 & 17.6 & 30.0 & 37.2 & 39.7 & 40.5 & 40.8 & 40.9 & 40.9 & 40.9 & 40.9 \\
\hline & 10 & 31.4 & 54.2 & 76.2 & 86.6 & 92.5 & 95.2 & 99.1 & 99.5 & 99.5 & 99.5 \\
\hline \multirow[t]{2}{*}{653190} & 2 & 15.6 & 25.4 & 29.4 & 30.4 & 30.6 & 30.7 & 30.7 & 30.7 & 30.7 & 30.7 \\
\hline & 10 & 34.5 & 59.5 & 79.9 & 89.0 & 93.8 & 96.1 & 99.3 & 99.6 & 99.7 & 99.7 \\
\hline \multirow[t]{2}{*}{653300} & 2 & 15.2 & 27.4 & 35.3 & 38.4 & 39.8 & 40.4 & 40.7 & 40.7 & 40.7 & 40.7 \\
\hline & 10 & 31.8 & 53.7 & 74.0 & 84.4 & 90.6 & 93.8 & 99.0 & 99.5 & 99.6 & 99.6 \\
\hline \multirow[t]{2}{*}{653350} & 2 & 12.6 & 22.0 & 28.1 & 30.4 & 31.3 & 31.6 & 31.9 & 31.9 & 31.9 & 31.9 \\
\hline & 10 & 23.1 & 40.7 & 59.8 & 72.0 & 81.0 & 86.1 & 96.7 & 98.6 & 99.0 & 99.1 \\
\hline \multirow[t]{2}{*}{653380} & 2 & 11.7 & 20.6 & 26.6 & 28.8 & 29.7 & 30.1 & 30.3 & 30.3 & 30.3 & 30.3 \\
\hline & 10 & 21.4 & 39.1 & 58.7 & 71.0 & 80.0 & 85.5 & 97.0 & 99.2 & 99.6 & 99.6 \\
\hline \multirow[t]{2}{*}{653440} & 2 & 5.9 & 11.6 & 16.8 & 20.3 & 23.0 & 25.0 & 30.1 & 31.0 & 31.2 & 31.2 \\
\hline & 10 & 14.3 & 25.7 & 39.7 & 50.8 & 61.1 & 68.3 & 91.5 & 99.1 & 99.9 & 100.0 \\
\hline \multirow[t]{2}{*}{653520} & 2 & 14.5 & 24.6 & 31.5 & 34.2 & 35.5 & 36.2 & 37.6 & 37.8 & 37.8 & 37.8 \\
\hline & 10 & 24.5 & 44.1 & 65.2 & 76.8 & 84.1 & 88.0 & 95.2 & 96.7 & 97.0 & 97.0 \\
\hline \multirow[t]{2}{*}{653550} & 2 & 15.9 & 29.3 & 37.9 & 41.3 & 42.8 & 43.5 & 44.0 & 44.0 & 44.0 & 44.0 \\
\hline & 10 & 31.5 & 55.5 & 76.2 & 86.3 & 92.0 & 95.0 & 99.2 & 99.6 & 99.7 & 99.7 \\
\hline \multirow[t]{2}{*}{653570} & 2 & 12.7 & 20.8 & 24.9 & 26.0 & 26.5 & 26.7 & 26.9 & 26.9 & 26.9 & 26.9 \\
\hline & 10 & 30.1 & 52.4 & 73.1 & 83.2 & 89.2 & 92.4 & 97.9 & 98.8 & 99.0 & 99.0 \\
\hline \multirow[t]{2}{*}{653610} & 2 & 16.8 & 29.8 & 36.2 & 38.3 & 39.1 & 39.3 & 39.3 & 39.3 & 39.3 & 39.3 \\
\hline & 10 & 38.2 & 64.4 & 83.7 & 91.7 & 95.6 & 97.5 & 99.6 & 99.8 & 99.8 & 99.8 \\
\hline \multirow[t]{2}{*}{653760} & 2 & 12.9 & 22.7 & 28.4 & 30.7 & 31.8 & 32.4 & 33.3 & 33.5 & 33.6 & 33.6 \\
\hline & 10 & 31.5 & 52.2 & 71.3 & 81.8 & 88.3 & 91.9 & 98.3 & 99.5 & 99.6 & 99.7 \\
\hline \multirow[t]{2}{*}{653800} & 2 & 5.1 & 8.0 & 9.7 & 10.4 & 11.0 & 11.3 & 12.0 & 12.0 & 12.0 & 12.0 \\
\hline & 10 & 13.3 & 23.8 & 36.5 & 45.7 & 53.8 & 59.7 & 81.6 & 92.8 & 96.0 & 96.7 \\
\hline \multirow[t]{2}{*}{653870} & 2 & 5.0 & 8.7 & 11.6 & 12.9 & 13.5 & 13.8 & 14.1 & 14.1 & 14.1 & 14.1 \\
\hline & 10 & 10.3 & 18.7 & 30.2 & 39.5 & 48.6 & 55.0 & 79.3 & 92.5 & 96.6 & 97.6 \\
\hline \multirow[t]{2}{*}{654720} & 2 & 4.6 & 8.3 & 11.2 & 12.5 & 13.1 & 13.3 & 13.5 & 13.5 & 13.5 & 13.5 \\
\hline & 10 & 8.5 & 15.8 & 25.8 & 34.1 & 42.3 & 48.0 & 69.2 & 81.4 & 86.2 & 87.8 \\
\hline \multirow[t]{2}{*}{655010} & 2 & 9.2 & 14.4 & 17.1 & 17.7 & 17.8 & 17.8 & 17.8 & 17.8 & 17.8 & 17.8 \\
\hline & 10 & 18.9 & 34.7 & 53.0 & 63.4 & 70.2 & 74.0 & 80.3 & 80.9 & 80.9 & 80.9 \\
\hline \multirow[t]{2}{*}{655020} & 2 & 12.5 & 19.5 & 22.8 & 23.6 & 23.8 & 23.9 & 23.9 & 23.9 & 23.9 & 23.9 \\
\hline & 10 & 26.1 & 45.4 & 65.5 & 76.1 & 82.6 & 85.9 & 92.0 & 93.0 & 93.3 & 93.4 \\
\hline 655030 & 2 & 14.4 & 23.7 & 28.7 & 30.1 & 30.5 & 30.6 & 30.7 & 30.7 & 30.7 & 30.7 \\
\hline & 10 & 28.1 & 50.1 & 71.8 & 82.1 & 88.0 & 91.0 & 95.7 & 96.3 & 96.4 & 96.4 \\
\hline 655050 & 2 & 15.1 & 24.5 & 29.5 & 31.1 & 31.8 & 32.3 & 33.8 & 34.3 & 34.4 & 34.4 \\
\hline & 10 & 29.9 & 51.7 & 73.3 & 83.6 & 89.4 & 92.3 & 96.5 & 97.1 & 97.2 & 97.2 \\
\hline 655070 & 2 & 14.6 & 24.7 & 31.1 & 33.3 & 34.3 & 34.8 & 35.7 & 36.0 & 36.0 & 36.0 \\
\hline & 10 & 28.9 & 50.7 & 72.3 & 83.1 & 89.5 & 92.7 & 97.8 & 98.4 & 98.5 & 98.6 \\
\hline 655100 & 2 & 13.8 & 22.4 & 26.6 & 27.6 & 27.9 & 28.0 & 28.0 & 28.0 & 28.0 & 28.0 \\
\hline & 10 & 28.5 & 50.2 & 71.3 & 82.1 & 88.6 & 92.0 & 97.6 & 98.3 & 98.3 & 98.3 \\
\hline 655160 & 2 & 15.4 & 25.3 & 30.3 & 31.6 & 32.0 & 32.1 & 32.1 & 32.1 & 32.1 & 32.1 \\
\hline & 10 & 30.2 & 52.8 & 74.5 & 84.8 & 90.5 & 93.3 & 97.4 & 97.9 & 98.0 & 98.1 \\
\hline 655180 & 2 & 14.5 & 25.4 & 32.9 & 35.8 & 36.9 & 37.3 & 37.5 & 37.5 & 37.5 & 37.5 \\
\hline & 10 & 25.9 & 46.1 & 67.7 & 79.6 & 87.3 & 91.4 & 98.1 & 98.6 & 98.7 & 98.7 \\
\hline 655220 & 2 & 14.6 & 24.5 & 29.9 & 31.5 & 32.1 & 32.4 & 32.9 & 32.9 & 33.0 & 33.0 \\
\hline & 10 & 29.3 & 51.1 & 72.6 & 83.4 & 89.7 & 93.0 & 98.0 & 98.8 & 98.8 & 98.9 \\
\hline 655280 & 2 & 15.4 & 26.2 & 33.7 & 37.6 & 39.9 & 41.2 & 42.8 & 42.8 & 42.8 & 42.8 \\
\hline & 10 & 34.5 & 56.8 & 77.3 & 86.8 & 92.1 & 94.6 & 98.4 & 99.0 & 99.1 & 99.1 \\
\hline 655360 & 2 & 13.8 & 22.5 & 27.4 & 28.8 & 29.4 & 29.6 & 29.8 & 29.8 & 29.8 & 29.8 \\
\hline & 10 & 26.0 & 45.5 & 66.7 & 78.4 & 86.1 & 90.1 & 97.6 & 98.8 & 99.1 & 99.1 \\
\hline
\end{tabular}




\begin{tabular}{|c|c|c|c|c|c|c|c|c|c|c|c|}
\hline \multicolumn{12}{|c|}{$\begin{array}{l}\text { 1st-Order Markov Model } \\
\text { July DRWH Reliability (\%) } \\
\qquad \begin{array}{l}\mathrm{H}-15 \\
\text { per capita roof area and storage }\end{array}\end{array}$} \\
\hline & & & & & & & & & & & \\
\hline Gauge & sq. m & 0 & 40 & 80 & 120 & 160 & 200 & 400 & 600 & 800 & 1000 \\
\hline \multirow[t]{2}{*}{655450} & 2 & 7.0 & 11.6 & 14.2 & 15.0 & 15.3 & 15.4 & 15.4 & 15.4 & 15.4 & 15.4 \\
\hline & 10 & 15.4 & 27.8 & 43.5 & 54.7 & 64.5 & 71.0 & 91.4 & 98.0 & 99.1 & 99.2 \\
\hline \multirow[t]{2}{*}{655480} & 2 & 15.2 & 27.1 & 33.7 & 36.4 & 38.1 & 39.2 & 42.8 & 43.8 & 43.9 & 43.9 \\
\hline & 10 & 30.6 & 54.2 & 74.1 & 84.5 & 90.7 & 94.2 & 99.2 & 99.8 & 99.9 & 99.9 \\
\hline \multirow[t]{2}{*}{655550} & 2 & 9.1 & 16.0 & 22.1 & 24.8 & 26.1 & 26.6 & 27.0 & 27.0 & 27.0 & 27.0 \\
\hline & 10 & 15.3 & 27.1 & 42.9 & 54.4 & 64.4 & 70.5 & 87.5 & 93.7 & 95.9 & 96.8 \\
\hline \multirow[t]{2}{*}{655570} & 2 & 4.7 & 7.5 & 8.9 & 9.4 & 9.6 & 9.7 & 9.8 & 9.8 & 9.8 & 9.9 \\
\hline & 10 & 16.8 & 29.1 & 43.1 & 53.1 & 61.9 & 68.5 & 91.2 & 98.7 & 99.8 & 99.9 \\
\hline \multirow[t]{2}{*}{655600} & 2 & 6.2 & 10.2 & 12.1 & 13.1 & 13.9 & 14.5 & 16.1 & 16.3 & 16.3 & 16.4 \\
\hline & 10 & 17.7 & 31.6 & 46.2 & 56.3 & 64.5 & 70.4 & 89.0 & 96.2 & 97.9 & 98.2 \\
\hline \multirow[t]{2}{*}{655620} & 2 & 6.0 & 9.4 & 11.0 & 11.5 & 11.8 & 11.8 & 11.9 & 11.9 & 11.9 & 11.9 \\
\hline & 10 & 14.8 & 26.8 & 41.0 & 51.2 & 59.9 & 65.9 & 87.0 & 96.2 & 98.5 & 98.9 \\
\hline \multirow[t]{2}{*}{655630} & 2 & 5.4 & 9.6 & 12.5 & 13.7 & 14.3 & 14.6 & 15.6 & 16.1 & 16.3 & 16.3 \\
\hline & 10 & 14.6 & 25.6 & 39.5 & 49.4 & 58.4 & 64.6 & 86.3 & 95.5 & 97.4 & 97.7 \\
\hline \multirow[t]{2}{*}{655780} & 2 & 5.6 & 11.4 & 17.3 & 21.8 & 25.9 & 29.5 & 41.4 & 44.6 & 44.8 & 44.8 \\
\hline & 10 & 14.7 & 26.2 & 40.2 & 51.5 & 62.0 & 69.5 & 92.6 & 99.5 & 100.0 & 100.0 \\
\hline \multirow[t]{2}{*}{655850} & 2 & 9.0 & 18.0 & 26.0 & 31.2 & 35.4 & 38.8 & 48.0 & 49.4 & 49.4 & 49.4 \\
\hline & 10 & 20.1 & 36.8 & 54.3 & 66.8 & 76.6 & 83.1 & 97.8 & 99.9 & 100.0 & 100.0 \\
\hline \multirow[t]{2}{*}{655920} & 2 & 8.0 & 16.5 & 24.7 & 30.6 & 35.5 & 39.6 & 53.5 & 57.1 & 57.3 & 57.3 \\
\hline & 10 & 25.8 & 43.4 & 60.5 & 71.7 & 80.4 & 86.0 & 98.2 & 99.9 & 99.9 & 100.0 \\
\hline \multirow[t]{2}{*}{655940} & 2 & 5.6 & 11.3 & 16.8 & 20.5 & 23.1 & 25.0 & 29.5 & 30.3 & 30.3 & 30.3 \\
\hline & 10 & 16.8 & 29.3 & 43.7 & 54.6 & 64.5 & 71.6 & 93.3 & 99.3 & 99.9 & 99.9 \\
\hline \multirow[t]{2}{*}{655990} & 2 & 5.6 & 10.9 & 15.9 & 19.3 & 22.1 & 24.1 & 29.6 & 30.7 & 30.8 & 30.8 \\
\hline & 10 & 13.7 & 24.5 & 37.8 & 48.7 & 59.0 & 66.4 & 90.8 & 99.1 & 99.9 & 100.0 \\
\hline
\end{tabular}




\begin{tabular}{|c|c|c|c|c|c|c|c|c|c|c|c|}
\hline \multicolumn{12}{|c|}{$\begin{array}{l}\text { 1st-Order Markov Model } \\
\text { August DRWH Reliability (\%) } \\
\text { per capita roof area and storage } \\
\text { L }\end{array}$} \\
\hline Gauge & sq. m & 0 & 40 & 80 & 120 & 160 & 200 & 400 & 600 & 800 & 1000 \\
\hline \multirow[t]{2}{*}{653060} & 2 & 18.5 & 32.0 & 40.0 & 42.9 & 44.2 & 44.8 & 45.4 & 45.4 & 45.4 & 45.4 \\
\hline & 10 & 34.3 & 59.5 & 81.0 & 90.3 & 95.1 & 97.3 & 99.8 & 99.9 & 100.0 & 100.0 \\
\hline \multirow[t]{2}{*}{653190} & 2 & 22.2 & 38.3 & 46.0 & 48.5 & 49.4 & 49.6 & 49.7 & 49.7 & 49.7 & 49.7 \\
\hline & 10 & 41.6 & 71.0 & 89.5 & 95.6 & 98.1 & 99.0 & 99.9 & 100.0 & 100.0 & 100.0 \\
\hline \multirow[t]{2}{*}{653300} & 2 & 17.4 & 31.0 & 39.0 & 42.2 & 43.8 & 44.6 & 45.5 & 45.5 & 45.5 & 45.5 \\
\hline & 10 & 34.0 & 57.9 & 78.4 & 88.3 & 93.7 & 96.4 & 99.7 & 99.9 & 100.0 & 100.0 \\
\hline \multirow[t]{2}{*}{653350} & 2 & 10.6 & 18.2 & 22.7 & 24.4 & 25.2 & 25.6 & 26.0 & 26.1 & 26.1 & 26.1 \\
\hline & 10 & 23.0 & 39.9 & 58.0 & 69.5 & 78.0 & 83.3 & 95.8 & 98.8 & 99.4 & 99.5 \\
\hline \multirow[t]{2}{*}{653380} & 2 & 8.0 & 14.0 & 17.8 & 19.3 & 20.1 & 20.4 & 20.7 & 20.7 & 20.7 & 20.7 \\
\hline & 10 & 20.0 & 34.3 & 49.9 & 60.7 & 69.6 & 75.4 & 91.9 & 97.8 & 99.3 & 99.6 \\
\hline \multirow[t]{2}{*}{653440} & 2 & 4.5 & 7.9 & 10.4 & 11.4 & 11.8 & 12.0 & 12.4 & 12.6 & 12.7 & 12.8 \\
\hline & 10 & 9.8 & 17.7 & 27.4 & 34.8 & 41.7 & 46.5 & 64.7 & 81.5 & 93.5 & 98.7 \\
\hline \multirow{2}{*}{653520} & 2 & 12.4 & 20.1 & 24.2 & 25.5 & 26.1 & 26.4 & 26.9 & 27.0 & 27.0 & 27.0 \\
\hline & 10 & 26.8 & 46.8 & 67.5 & 79.1 & 86.6 & 90.7 & 98.5 & 99.5 & 99.6 & 99.6 \\
\hline \multirow[t]{2}{*}{653550} & 2 & 24.1 & 41.3 & 49.9 & 53.7 & 55.9 & 57.1 & 58.4 & 58.4 & 58.4 & 58.4 \\
\hline & 10 & 49.1 & 75.9 & 92.5 & 97.1 & 98.7 & 99.3 & 99.9 & 100.0 & 100.0 & 100.0 \\
\hline \multirow[t]{2}{*}{653570} & 2 & 14.8 & 25.1 & 30.1 & 31.5 & 31.9 & 32.1 & 32.1 & 32.1 & 32.1 & 32.1 \\
\hline & 10 & 31.3 & 54.6 & 75.3 & 85.6 & 91.3 & 94.5 & 99.2 & 99.7 & 99.8 & 99.9 \\
\hline \multirow[t]{2}{*}{653610} & 2 & 17.9 & 30.9 & 36.8 & 38.8 & 39.7 & 40.1 & 40.5 & 40.5 & 40.5 & 40.5 \\
\hline & 10 & 40.3 & 66.3 & 85.4 & 93.3 & 96.9 & 98.5 & 100.0 & 100.0 & 100.0 & 100.0 \\
\hline \multirow[t]{2}{*}{653760} & 2 & 12.2 & 21.5 & 27.2 & 29.2 & 30.0 & 30.4 & 30.7 & 30.7 & 30.7 & 30.7 \\
\hline & 10 & 27.5 & 47.9 & 68.0 & 79.3 & 86.7 & 90.9 & 98.7 & 99.8 & 99.9 & 100.0 \\
\hline \multirow[t]{2}{*}{653800} & 2 & 2.5 & 4.9 & 6.8 & 7.7 & 8.2 & 8.4 & 8.7 & 8.7 & 8.7 & 8.7 \\
\hline & 10 & 10.1 & 16.8 & 23.6 & 29.0 & 34.0 & 37.7 & 51.9 & 67.3 & 81.6 & 89.4 \\
\hline \multirow[t]{2}{*}{653870} & 2 & 1.9 & 3.2 & 4.1 & 4.4 & 4.5 & 4.6 & 4.6 & 4.6 & 4.6 & 4.6 \\
\hline & 10 & 7.2 & 12.0 & 17.7 & 22.1 & 26.4 & 29.4 & 43.1 & 59.5 & 76.4 & 87.3 \\
\hline \multirow[t]{2}{*}{654720} & 2 & 1.0 & 1.4 & 1.7 & 1.9 & 2.0 & 2.1 & 2.1 & 2.1 & 2.1 & 2.1 \\
\hline & 10 & 4.7 & 8.0 & 11.9 & 14.6 & 17.4 & 19.6 & 30.8 & 45.2 & 59.2 & 68.3 \\
\hline \multirow[t]{2}{*}{655010} & 2 & 13.0 & 21.3 & 26.0 & 27.2 & 27.5 & 27.6 & 27.7 & 27.7 & 27.7 & 27.7 \\
\hline & 10 & 24.7 & 44.1 & 65.0 & 76.5 & 83.8 & 87.8 & 95.2 & 96.1 & 96.1 & 96.2 \\
\hline \multirow[t]{2}{*}{655020} & 2 & 15.4 & 25.3 & 30.2 & 31.4 & 31.7 & 31.8 & 31.8 & 31.8 & 31.8 & 31.8 \\
\hline & 10 & 31.7 & 55.4 & 77.0 & 87.0 & 92.2 & 95.0 & 98.9 & 99.2 & 99.3 & 99.3 \\
\hline 655030 & 2 & 16.7 & 27.6 & 32.7 & 34.3 & 34.8 & 35.0 & 35.1 & 35.1 & 35.1 & 35.1 \\
\hline & 10 & 34.0 & 58.6 & 80.1 & 89.6 & 94.4 & 96.7 & 99.6 & 99.8 & 99.8 & 99.8 \\
\hline 655050 & 2 & 18.9 & 31.5 & 37.8 & 39.6 & 40.3 & 40 & 40.6 & 40.9 & 41 & 41.0 \\
\hline & 10 & 37.0 & 63.0 & 84.2 & 92.5 & 96.4 & 98.1 & 99.8 & 99.9 & 99.9 & 99.9 \\
\hline 655070 & 2 & 16.6 & 27.8 & 34.8 & 38.3 & 40.3 & 41.4 & 42.6 & 42.7 & 42.7 & 42.7 \\
\hline & 10 & 37.7 & 63.2 & 84.0 & 92.2 & 96.1 & 97.9 & 99.8 & 99.9 & 99.9 & 99.9 \\
\hline 655100 & 2 & 22.1 & 38.7 & 47.6 & 50.7 & 51.8 & 52.2 & 52.4 & 52.4 & 52.4 & 52.4 \\
\hline & 10 & 40.2 & 67.6 & 87.3 & 94.3 & 97.3 & 98.5 & 99.8 & 99.9 & 99.9 & 99.9 \\
\hline 655160 & 2 & 19.7 & 33.3 & 39.7 & 41.7 & 42.4 & 42.6 & 42.7 & 42.7 & 42.7 & 42.7 \\
\hline & 10 & 39.8 & 66.1 & 86.0 & 93.5 & 96.9 & 98.4 & 99.8 & 99.9 & 99.9 & 99.9 \\
\hline 655180 & 2 & 22.2 & 38.7 & 48.1 & 51.6 & 53.2 & 54.0 & 54.6 & 54.6 & 54.6 & 54.6 \\
\hline & 10 & 39.4 & 66.0 & 86.0 & 93.4 & 96.8 & 98.2 & 99.7 & 99.9 & 99.9 & 99.9 \\
\hline 655220 & 2 & 17.6 & 30.4 & 37.5 & 39.8 & 40.7 & 41.0 & 41.2 & 41.3 & 41.3 & 41.3 \\
\hline & 10 & 34.1 & 58.2 & 79.2 & 88.9 & 94.1 & 96.7 & 99.7 & 99.9 & 99.9 & 99.9 \\
\hline 655280 & 2 & 22.3 & 37.9 & 45.5 & 48.8 & 51.2 & 52.9 & 57.3 & 57.9 & 57.9 & 57.9 \\
\hline & 10 & 46.9 & 73.0 & 90.9 & 96.4 & 98.6 & 99.3 & 99.9 & 100.0 & 100.0 & 100.0 \\
\hline & 2 & 18.4 & 30.4 & 36.8 & 39.0 & 40.1 & 40.7 & 41.4 & 41.4 & 41.4 & 41.4 \\
\hline & 10 & 36.6 & 59.9 & 81.1 & 90.2 & 94.9 & 96.9 & 99.6 & 99.9 & 99.9 & 99.9 \\
\hline
\end{tabular}




\begin{tabular}{|c|c|c|c|c|c|c|c|c|c|c|c|}
\hline \multicolumn{12}{|c|}{$\begin{array}{l}\text { 1st-Order Markov Model } \\
\text { August DRWH Reliability (\%) } \\
\text { per capita roof area and storage }\end{array}$} \\
\hline Gauge & sq. m & 0 & 40 & 80 & 120 & 160 & 200 & 400 & 600 & 800 & 1000 \\
\hline \multirow[t]{2}{*}{655450} & 2 & 8.9 & 15.1 & 19.4 & 20.9 & 21.5 & 21.6 & 21.7 & 21.7 & 21.7 & 21.7 \\
\hline & 10 & 15.3 & 28.0 & 43.5 & 54.2 & 63.0 & 68.3 & 83.2 & 92.2 & 97.2 & 98.6 \\
\hline \multirow[t]{2}{*}{655480} & 2 & 19.8 & 37.7 & 48.7 & 53.6 & 56.0 & 57.0 & 58.1 & 58.8 & 59.1 & 59.2 \\
\hline & 10 & 43.8 & 71.8 & 89.5 & 95.4 & 97.7 & 98.7 & 99.9 & 100.0 & 100.0 & 100.0 \\
\hline \multirow[t]{2}{*}{655550} & 2 & 10.3 & 18.0 & 23.6 & 26.0 & 27.3 & 27.9 & 28.7 & 28.7 & 28.7 & 28.7 \\
\hline & 10 & 19.3 & 34.2 & 51.3 & 62.6 & 71.3 & 76.5 & 89.9 & 95.2 & 97.3 & 98.1 \\
\hline \multirow[t]{2}{*}{655570} & 2 & 5.5 & 9.1 & 10.7 & 11.1 & 11.2 & 11.2 & 11.2 & 11.2 & 11.2 & 11.2 \\
\hline & 10 & 18.1 & 31.3 & 44.0 & 52.1 & 58.1 & 62.1 & 75.3 & 88.5 & 96.8 & 99.3 \\
\hline \multirow[t]{2}{*}{655600} & 2 & 9.0 & 15.2 & 18.1 & 18.9 & 19.2 & 19.2 & 19.2 & 19.2 & 19.2 & 19.3 \\
\hline & 10 & 21.6 & 38.6 & 55.3 & 65.4 & 72.3 & 76.7 & 87.5 & 93.8 & 97.2 & 98.3 \\
\hline \multirow[t]{2}{*}{655620} & 2 & 5.5 & 9.6 & 12.1 & 13.0 & 13.3 & 13.4 & 13.5 & 13.5 & 13.5 & 13.5 \\
\hline & 10 & 13.2 & 23.5 & 35.0 & 43.4 & 50.4 & 55.1 & 70.2 & 82.8 & 92.2 & 96.3 \\
\hline \multirow[t]{2}{*}{655630} & 2 & 6.9 & 11.4 & 13.9 & 14.7 & 15.0 & 15.1 & 15.1 & 15.2 & 15.3 & 15.5 \\
\hline & 10 & 15.6 & 28.0 & 41.8 & 51.1 & 58.5 & 63.2 & 77.3 & 87.4 & 93.8 & 96.0 \\
\hline \multirow[t]{2}{*}{655780} & 2 & 2.2 & 4. & 6.7 & 7.9 & 8.6 & 8. & 9. & 10.5 & 11.3 & 11.5 \\
\hline & 10 & 9.2 & 15.4 & 21.6 & 26.7 & 31.9 & 35.9 & 54.9 & 75.6 & 91.4 & 98.4 \\
\hline \multirow[t]{2}{*}{655850} & 2 & 3.5 & 6.6 & 8.7 & 9.7 & 10.4 & 10.8 & 12.2 & 13.5 & 14.1 & 14.1 \\
\hline & 10 & 16.4 & 28.0 & 38.5 & 46.1 & 52.7 & 58.1 & 80.3 & 93.9 & 98.8 & 99.9 \\
\hline \multirow[t]{2}{*}{655920} & 2 & 10.5 & 19.3 & 23.8 & 25.9 & 27.1 & 28.0 & 31.0 & 34.2 & 35.9 & 36.1 \\
\hline & 10 & 31.0 & 53.5 & 70.5 & 79.7 & 85.4 & 89.0 & 96.8 & 99.3 & 99.9 & 100.0 \\
\hline \multirow[t]{2}{*}{655940} & 2 & 2.8 & 4.7 & 5.8 & 6.4 & 6.8 & 7.1 & 7.8 & 8.1 & 8.2 & 8.2 \\
\hline & 10 & 14.7 & 24.7 & 33.5 & 39.6 & 44.9 & 49.0 & 67.4 & 85.2 & 95.7 & 99.3 \\
\hline \multirow[t]{2}{*}{655990} & 2 & 2.4 & 4.3 & 5.8 & 6.5 & 6.8 & 7.0 & 7.2 & 7.4 & 7.5 & 7.5 \\
\hline & 10 & 12.4 & 20.4 & 27.7 & 33.0 & 37.9 & 41.7 & 59.0 & 77.9 & 92.3 & 98.5 \\
\hline
\end{tabular}




\begin{tabular}{|c|c|c|c|c|c|c|c|c|c|c|c|}
\hline \multicolumn{12}{|c|}{$\begin{array}{l}\text { 1st-Order Markov Model } \\
\text { September DRWH Reliability (\%) } \\
\text { per capita roof area and storage } \\
\text { L }\end{array}$} \\
\hline Gauge & sq. m & 0 & 40 & 80 & 120 & 160 & 200 & 400 & 600 & 800 & 1000 \\
\hline \multirow[t]{2}{*}{653060} & 2 & 15.5 & 25.9 & 31.5 & 33.6 & 34.5 & 35.0 & 35.6 & 35.6 & 35.6 & 35.6 \\
\hline & 10 & 30.8 & 53.8 & 75.9 & 86.9 & 93.0 & 95.9 & 99.8 & 100.0 & 100.0 & 100.0 \\
\hline \multirow[t]{2}{*}{653190} & 2 & 21.7 & 36.9 & 43.5 & 45.9 & 47.1 & 47.6 & 48.0 & 48.0 & 48.0 & 48.0 \\
\hline & 10 & 46.8 & 74.7 & 92.0 & 97.2 & 99.0 & 99.6 & 100.0 & 100.0 & 100.0 & 100.0 \\
\hline \multirow[t]{2}{*}{653300} & 2 & 18.8 & 32.4 & 39.1 & 41.5 & 42.6 & 43.2 & 44.0 & 44.0 & 44.1 & 44.1 \\
\hline & 10 & 38.6 & 64.7 & 84.6 & 92.7 & 96.4 & 98.1 & 99.9 & 100.0 & 100.0 & 100.0 \\
\hline \multirow[t]{2}{*}{653350} & 2 & 13.1 & 21.9 & 26.4 & 27.7 & 28.1 & 28.3 & 28.4 & 28.4 & 28.4 & 28.4 \\
\hline & 10 & 29.9 & 51.7 & 72.0 & 82.2 & 88.3 & 91.6 & 97.8 & 99.5 & 99.8 & 99.9 \\
\hline \multirow[t]{2}{*}{653380} & 2 & 11.4 & 18.6 & 21.9 & 22.8 & 23.1 & 23.2 & 23.3 & 23.3 & 23.3 & 23.3 \\
\hline & 10 & 24.4 & 43.2 & 61.8 & 72.5 & 79.7 & 83.9 & 92.9 & 97.2 & 99.1 & 99.7 \\
\hline \multirow[t]{2}{*}{653440} & 2 & 9.1 & 15.0 & 18.1 & 19.0 & 19.2 & 19.3 & 19.3 & 19.3 & 19.3 & 19.3 \\
\hline & 10 & 19.5 & 34.6 & 50.6 & 60.4 & 67.3 & 71.3 & 80.4 & 85.2 & 90.9 & 95.7 \\
\hline \multirow[t]{2}{*}{653520} & 2 & 14.2 & 23.2 & 26.8 & 27.6 & 27.7 & 27.8 & 27.8 & 27.8 & 27.8 & 27.8 \\
\hline & 10 & 34.0 & 57.0 & 77.0 & 86.5 & 92.0 & 94.7 & 99.2 & 99.8 & 9.9 & 99.9 \\
\hline \multirow[t]{2}{*}{653550} & 2 & 20.3 & 34.3 & 40.1 & 43.0 & 45.0 & 46.6 & 51.1 & 51.7 & 51.7 & 51.7 \\
\hline & 10 & 47.8 & 74.5 & 91.8 & 97.3 & 99.2 & 99.7 & 100.0 & 100.0 & 100.0 & 100.0 \\
\hline \multirow[t]{2}{*}{653570} & 2 & 15.8 & 27.4 & 33.5 & 35.5 & 36.3 & 36.6 & 36.8 & 36.8 & 36.8 & 36.8 \\
\hline & 10 & 36.1 & 60.4 & 81.0 & 90.0 & 94.8 & 97.0 & 99.9 & 100.0 & 100.0 & 100.0 \\
\hline \multirow[t]{2}{*}{653610} & 2 & 18.4 & 31.3 & 37.0 & 38.8 & 39.5 & 39.7 & 39.9 & 40.0 & 40.0 & 40.0 \\
\hline & 10 & 39.1 & 66.2 & 85.8 & 93.5 & 97.2 & 98.7 & 100.0 & 100.0 & 100.0 & 100.0 \\
\hline \multirow[t]{2}{*}{653760} & 2 & 14.1 & 23.3 & 28.5 & 30.2 & 30.8 & 31.1 & 31.3 & 31.3 & 31.3 & 31.3 \\
\hline & 10 & 28.5 & 48.8 & 69.7 & 80.9 & 87.9 & 91.7 & 98.7 & 99.8 & 100.0 & 100.0 \\
\hline \multirow[t]{2}{*}{653800} & 2 & 7.9 & 13.3 & 16.4 & 17.5 & 18.0 & 18.2 & 18.5 & 18.5 & 18.5 & 18.5 \\
\hline & 10 & 18.2 & 31.3 & 45.3 & 54.2 & 60.6 & 64.3 & 72.7 & 76.9 & 81.8 & 87.4 \\
\hline \multirow[t]{2}{*}{653870} & 2 & 5.4 & 9.1 & 11.2 & 11.8 & 11.9 & 12.0 & 12.0 & 12.0 & 12.0 & 12.0 \\
\hline & 10 & 14.2 & 24.6 & 35.8 & 43.1 & 48.6 & 51.9 & 58.3 & 61.5 & 68.1 & 76.9 \\
\hline \multirow[t]{2}{*}{654720} & 2 & 1.5 & 2.8 & 4.2 & 5.0 & 5.6 & 5.9 & 6.4 & 6.4 & 6.4 & 6.4 \\
\hline & 10 & 9.7 & 15.6 & 20.5 & 23.2 & 25.3 & 26.6 & 29.5 & 31.0 & 36.2 & 44.5 \\
\hline \multirow[t]{2}{*}{655010} & 2 & 6.1 & 9.3 & 10.6 & 11.0 & 11.2 & 11.2 & 11.3 & 11.3 & 11.3 & 11.3 \\
\hline & 10 & 17.1 & 30.0 & 45.8 & 56.6 & 65.6 & 71.7 & 90.8 & 96.4 & 97.1 & 97.2 \\
\hline \multirow[t]{2}{*}{655020} & 2 & 10.1 & 16.4 & 20.0 & 21.0 & 21.4 & 21.5 & 21.6 & 21.6 & 21.6 & 21.6 \\
\hline & 10 & 21.5 & 38.5 & 58.4 & 71.0 & 80.3 & 86.0 & 97.9 & 99.6 & 99.7 & 99.7 \\
\hline 655030 & 2 & 11.4 & 18.0 & 21.9 & 23.7 & 24.8 & 25.5 & 26.6 & 26.6 & 26.6 & 26.6 \\
\hline & 10 & 27.5 & 47.8 & 69.5 & 81.2 & 88.8 & 92.9 & 99.5 & 100.0 & 100.0 & 100.0 \\
\hline 655050 & 2 & 11.2 & 17. & 20 & 21.8 & 22.2 & 22.4 & 22.5 & 22.5 & 22.6 & 22.6 \\
\hline & 10 & 26.8 & 47.7 & 69.5 & 81.4 & 88.8 & 93.0 & 99.6 & 100.0 & 100.0 & 100.0 \\
\hline 655070 & 2 & 12.3 & 19.5 & 23.1 & 24.5 & 25.6 & 26.6 & 29.8 & 30.4 & & 30 \\
\hline & 10 & 27.2 & 47.3 & 68.4 & 80.3 & 88.2 & 92.6 & 99.5 & 100.0 & 100.0 & 100.0 \\
\hline 655100 & 2 & 15.6 & 26.3 & 32.2 & 34.5 & 35.7 & 36.5 & 37.3 & 37.3 & 37.3 & 37.3 \\
\hline & 10 & 33.1 & 57.6 & 79.4 & 89.4 & 94.7 & 97.2 & 99.9 & 100.0 & 100.0 & 100.0 \\
\hline 655160 & 2 & 12.2 & 19.8 & 23.2 & 24.2 & 24.6 & 24.7 & 24.9 & 24.9 & 24.9 & 24.9 \\
\hline & 10 & 29.0 & 50.0 & 71.1 & 82.7 & 89.9 & 93.8 & 99.7 & 100.0 & 100.0 & 100.0 \\
\hline 655180 & 2 & 14.0 & 22.6 & 27.1 & 29.0 & 30.2 & 31.0 & 32.0 & 32.1 & 32.1 & 32.1 \\
\hline & 10 & 33.9 & 57.2 & 79.2 & 89.2 & 94.7 & 97.1 & 99.9 & 100.0 & 100.0 & 100.0 \\
\hline 655220 & 2 & 15.7 & 25.3 & 29.5 & 30.8 & 31.5 & 31.8 & 32.1 & 32.1 & 32.2 & 32.2 \\
\hline & 10 & 36.0 & 60.9 & 82.2 & 91.2 & 95.7 & 97.7 & 99.9 & 100.0 & 100.0 & 100.0 \\
\hline 655280 & 2 & 19.0 & 33.1 & 40.7 & 43.8 & 45.5 & 46.7 & 50.7 & 52.2 & 52.4 & 52.4 \\
\hline & 10 & 40.9 & 68.7 & 88.7 & 95.6 & 98.4 & 99.4 & 100.0 & 100.0 & 100.0 & 100.0 \\
\hline 655360 & 2 & 16.4 & 26.9 & 31.9 & 33.6 & 34.4 & 34.9 & 37.1 & 37.9 & 38.0 & 38.0 \\
\hline & 10 & 34.8 & 59.4 & 81.4 & 90.9 & 95.8 & 97.8 & 99.9 & 100.0 & 100.0 & 100.0 \\
\hline
\end{tabular}




\begin{tabular}{|c|c|c|c|c|c|c|c|c|c|c|c|}
\hline \multirow{2}{*}{\multicolumn{12}{|c|}{$\begin{array}{l}\text { 1st-Order Markov Model } \\
\text { September DRWH Reliability (\%) } \\
\text { per capita roof area and storage } \\
\text { L }\end{array}$}} \\
\hline & & & & & & & & & & & \\
\hline Gauge & sq. m & 0 & 40 & 80 & 120 & 160 & 200 & 400 & 600 & 800 & 1000 \\
\hline \multirow[t]{2}{*}{655450} & 2 & 13.5 & 21.7 & 25.2 & 26.2 & 26.5 & 26.7 & 26.8 & 26.8 & 26.8 & 26.8 \\
\hline & 10 & 29.6 & 51.2 & 71.2 & 81.2 & 87.2 & 90.2 & 95.7 & 97.6 & 98.7 & 99.4 \\
\hline \multirow[t]{2}{*}{655480} & 2 & 21.1 & 36.8 & 44.9 & 48.8 & 51.4 & 53.4 & 56.9 & 57.2 & 57.5 & 57.5 \\
\hline & 10 & 43.1 & 71.1 & 89.8 & 96.1 & 98.6 & 99.4 & 100.0 & 100.0 & 100.0 & 100.0 \\
\hline \multirow[t]{2}{*}{655550} & 2 & 13.1 & 21.8 & 26.4 & 27.9 & 28.5 & 28.7 & 29.1 & 29.1 & 29.1 & 29.1 \\
\hline & 10 & 27.7 & 47.1 & 66.8 & 77.8 & 84.9 & 88.8 & 96.1 & 98.2 & 99.0 & 99.4 \\
\hline \multirow[t]{2}{*}{655570} & 2 & 12.7 & 22.2 & 27.5 & 29.1 & 29.7 & 29.9 & 30.0 & 30.0 & 30.0 & 30.0 \\
\hline & 10 & 28.7 & 48.9 & 67.3 & 77.2 & 83.1 & 86.3 & 92.0 & 94.4 & 97.2 & 99.0 \\
\hline \multirow[t]{2}{*}{655600} & 2 & 10.3 & 18.1 & 22.7 & 24.4 & 25.1 & 25.3 & 25.4 & 25.4 & 25.4 & 25.4 \\
\hline & 10 & 30.5 & 51.1 & 69.6 & 79.2 & 85.3 & 88.8 & 95.9 & 97.7 & 98.7 & 99.3 \\
\hline \multirow[t]{2}{*}{655620} & 2 & 9.3 & 13.6 & 15.1 & 15.7 & 16.1 & 16.4 & 16.9 & 16.9 & 16.9 & 16.9 \\
\hline & 10 & 27.9 & 45.5 & 62.7 & 71.9 & 77.9 & 81.1 & 87.9 & 91.3 & 94.4 & 96.9 \\
\hline \multirow[t]{2}{*}{655630} & 2 & 8.4 & 14.0 & 16.7 & 17.4 & 17.6 & 17.6 & 17.7 & 17.7 & 17.7 & 17.7 \\
\hline & 10 & 22.3 & 38.7 & 55.2 & 65.2 & 72.3 & 76.8 & 87.0 & 91.1 & 94.5 & 96.7 \\
\hline \multirow[t]{2}{*}{655780} & 2 & 5.3 & 10.6 & 15.5 & 18.3 & 20.1 & 21.0 & 22.3 & 22.3 & 22.3 & 22.3 \\
\hline & 10 & 13.6 & 23.4 & 33.6 & 40.9 & 47.0 & 51.2 & 62.5 & 69.1 & 78.4 & 88.2 \\
\hline \multirow[t]{2}{*}{655850} & 2 & 7.8 & 14.1 & 17.7 & 18.9 & 19.3 & 19.5 & 19.6 & 19.6 & 19.6 & 19.7 \\
\hline & 10 & 20.7 & 36.9 & 51.8 & 60.9 & 67.2 & 71.2 & 80.5 & 88.4 & 95.2 & 98.6 \\
\hline \multirow[t]{2}{*}{655920} & 2 & 11.9 & 22.8 & 29.5 & 32.7 & 34.4 & 35.2 & 36.0 & 36.4 & 36.8 & 37.2 \\
\hline & 10 & 40.9 & 66.6 & 83.0 & 90.4 & 94.3 & 96.3 & 99.4 & 99.8 & 100.0 & 100.0 \\
\hline \multirow[t]{2}{*}{655940} & 2 & 3.1 & 5.6 & 7.0 & 7.4 & 7.6 & 7.6 & 7.7 & 7.7 & 7.7 & 7.7 \\
\hline & 10 & 16.6 & 27.7 & 36.9 & 42.5 & 46.6 & 49.6 & 58.6 & 68.2 & 80.8 & 91.3 \\
\hline \multirow[t]{2}{*}{655990} & 2 & 3.5 & 5.3 & 6.1 & 6.3 & 6.4 & 6.4 & 6.4 & 6.4 & 6.4 & 6.4 \\
\hline & 10 & 11.0 & 19.2 & 27.8 & 33.3 & 37.6 & 40.4 & 48.8 & 57.3 & 70.2 & 83.4 \\
\hline
\end{tabular}




\begin{tabular}{|c|c|c|c|c|c|c|c|c|c|c|c|}
\hline \multicolumn{12}{|c|}{$\begin{array}{l}\text { 1st-Order Markov Model } \\
\text { October DRWH Reliability (\%) } \\
\text { per capita roof area and storage } \\
\text { L }\end{array}$} \\
\hline Gauge & sq. m & 0 & 40 & 80 & 120 & 160 & 200 & 400 & 600 & 800 & 1000 \\
\hline \multirow[t]{2}{*}{653060} & 2 & 2.7 & 4.2 & 5.1 & 5.5 & 5.7 & 5.8 & 5.9 & 5.9 & 5.9 & 5.9 \\
\hline & 10 & 8.6 & 15.4 & 25.5 & 33.8 & 42.6 & 49.2 & 78.4 & 96.5 & 99.8 & 100.0 \\
\hline \multirow[t]{2}{*}{653190} & 2 & 7.4 & 11.9 & 14.0 & 14.8 & 15.2 & 15.3 & 15.5 & 15.5 & 15.5 & 15.5 \\
\hline & 10 & 21.5 & 37.1 & 55.1 & 67.3 & 77.0 & 83.1 & 97.9 & 99.9 & 100.0 & 100.0 \\
\hline \multirow[t]{2}{*}{653300} & 2 & 8.6 & 14.1 & 17.0 & 18.0 & 18.4 & 18.6 & 18.8 & 18.8 & 18.8 & 18.8 \\
\hline & 10 & 19.7 & 34.1 & 51.8 & 64.2 & 74.6 & 81.0 & 96.9 & 99.8 & 100.0 & 100.0 \\
\hline \multirow[t]{2}{*}{653350} & 2 & 9.6 & 15.1 & 17.5 & 18.1 & 18.3 & 18.4 & 18.5 & 18.5 & 18.5 & 18.5 \\
\hline & 10 & 23.5 & 41.1 & 60.3 & 72.0 & 80.5 & 85.7 & 97.3 & 99.5 & 99.8 & 99.9 \\
\hline \multirow[t]{2}{*}{653380} & 2 & 10.7 & 17.0 & 20.2 & 21.1 & 21.3 & 21.4 & 21.4 & 21.4 & 21.4 & 21.4 \\
\hline & 10 & 21.7 & 38.6 & 57.8 & 69.5 & 78.2 & 83.3 & 95.2 & 98.2 & 99.3 & 99.8 \\
\hline \multirow[t]{2}{*}{653440} & 2 & 12.0 & 19.9 & 24.3 & 25.6 & 26.0 & 26.2 & 26.2 & 26.2 & 26.2 & 26.2 \\
\hline & 10 & 23.5 & 41.1 & 60.5 & 71.8 & 79.8 & 84.4 & 93.6 & 95.7 & 96.9 & 98.1 \\
\hline \multirow[t]{2}{*}{653520} & 2 & 6.1 & 9.5 & 11.0 & 11.4 & 11.5 & 11.6 & 11.6 & 11.6 & 11.6 & 11.6 \\
\hline & 10 & 14.0 & 24.7 & 38.9 & 49.7 & 60.0 & 66.8 & 89.7 & 98.5 & 99.7 & 99.8 \\
\hline \multirow[t]{2}{*}{653550} & 2 & 10.9 & 17.5 & 20.5 & 21.4 & 22.0 & 22.4 & 24.6 & 26.6 & 27.3 & 27.4 \\
\hline & 10 & 26.2 & 46.8 & 67.7 & 79.6 & 87.5 & 92.0 & 99.5 & 100.0 & 100.0 & 100.0 \\
\hline \multirow[t]{2}{*}{653570} & 2 & 9.0 & 13.9 & 16.4 & 17.2 & 17.5 & 17.7 & 17.9 & 17.9 & 17.9 & 17.9 \\
\hline & 10 & 21.3 & 35.7 & 53.9 & 66.0 & 76.2 & 82.2 & 97.0 & 99.8 & 100.0 & 100.0 \\
\hline \multirow[t]{2}{*}{653610} & 2 & 8.8 & 14.2 & 16.8 & 17.6 & 17.9 & 18.0 & 18.1 & 18.1 & 18.1 & 18.1 \\
\hline & 10 & 22.3 & 37.9 & 56.5 & 68.8 & 78.4 & 84.3 & 97.9 & 99.9 & 100.0 & 100.0 \\
\hline \multirow[t]{2}{*}{653760} & 2 & 9.3 & 14.8 & 17.4 & 18.1 & 18.4 & 18.5 & 18.6 & 18.6 & 18.6 & 18.6 \\
\hline & 10 & 21.6 & 37.9 & 56.7 & 68.6 & 77.7 & 83.3 & 96.6 & 99.5 & 99.9 & 100.0 \\
\hline \multirow[t]{2}{*}{653800} & 2 & 9.2 & 14.1 & 16.2 & 16.6 & 16.8 & 16.9 & 16.9 & 16.9 & 16.9 & 16.9 \\
\hline & 10 & 23.1 & 41.3 & 59.9 & 70.3 & 77.2 & 81.4 & 90.2 & 92.4 & 93.5 & 94.8 \\
\hline \multirow[t]{2}{*}{653870} & 2 & 6.8 & 10.8 & 13.2 & 13.9 & 14.1 & 14.1 & 14.2 & 14.2 & 14.2 & 14.2 \\
\hline & 10 & 15.4 & 27.0 & 41.2 & 50.5 & 58.1 & 62.7 & 74.4 & 77.9 & 79.7 & 82.3 \\
\hline \multirow[t]{2}{*}{654720} & 2 & 4.6 & 7.4 & 9.1 & 9.7 & 10.0 & 10.2 & 10.9 & 11.0 & 11.0 & 11.0 \\
\hline & 10 & 13.1 & 22.2 & 32.0 & 38.5 & 43.7 & 46.7 & 54.3 & 57.1 & 58.2 & 59.4 \\
\hline \multirow[t]{2}{*}{655010} & 2 & 1.0 & 1.8 & 2.7 & 3.2 & 3.4 & 3.5 & 3.5 & 3.5 & 3.5 & 3.5 \\
\hline & 10 & 3.2 & 5.7 & 9.5 & 13.1 & 17.3 & 20.6 & 38.0 & 60.7 & 77.2 & 83.8 \\
\hline \multirow[t]{2}{*}{655020} & 2 & 1.9 & 2.7 & 3.2 & 3.3 & 3.4 & 3.4 & 3.5 & 3.5 & 3.5 & 3.5 \\
\hline & 10 & 7.5 & 13.1 & 20.7 & 26.7 & 33.0 & 37.8 & 63.6 & 86.9 & 96.4 & 98.3 \\
\hline 655030 & 2 & 2.0 & 3.1 & 4.0 & 4.5 & 5.0 & 5.4 & 7.3 & 8.3 & 8.6 & 8.6 \\
\hline & 10 & 9.0 & 15.8 & 24.9 & 31.8 & 39.2 & 44.8 & 73.4 & 93.9 & 99.2 & 99.8 \\
\hline 655050 & 2 & $3 .($ & 4.6 & 5.4 & 5.6 & 5. & 5. & 5. & & 5. & 5.7 \\
\hline & 10 & 9.7 & 17.2 & 27.4 & 35.5 & 43.5 & 49.5 & 77.2 & 95.0 & 99.4 & 99.9 \\
\hline 655070 & 2 & 2.6 & 3.7 & 4.2 & 4.4 & 4.4 & 4.4 & 4.5 & 4.7 & 4.8 & 4.8 \\
\hline & 10 & 8.8 & 16.0 & 25.9 & 33.4 & 40.9 & 46.7 & 74.9 & 94.2 & 99.2 & 99.9 \\
\hline 655100 & 2 & 5.0 & 7.5 & 8.8 & 9.1 & 9.3 & 9.4 & 9.5 & 9.5 & 9.5 & 9.5 \\
\hline & 10 & 13.4 & 24.0 & 38.2 & 48.7 & 58.5 & 65.3 & 89.9 & 99.0 & 100.0 & 100.0 \\
\hline 655160 & 2 & 4.0 & 6.2 & 7.4 & 7.6 & 7.7 & 7.7 & 7.7 & 7.7 & 7.7 & 7.7 \\
\hline & 10 & 11.6 & 20.5 & 32.6 & 41.7 & 50.7 & 57.0 & 83.1 & 96.8 & 99.6 & 100.0 \\
\hline 655180 & 2 & 3.5 & 5.0 & 5.6 & 5.8 & 5.8 & 5.9 & 5.9 & 6.0 & 6.0 & 6.0 \\
\hline & 10 & 10.3 & 18.3 & 29.9 & 39.0 & 48.3 & 54.8 & 82.8 & 97.7 & 99.9 & 100.0 \\
\hline 655220 & 2 & 7.1 & 10.8 & 12.4 & 12.7 & 12.8 & 12.8 & 12.9 & 12.9 & 12.9 & 12.9 \\
\hline & 10 & 18.8 & 33.1 & 50.9 & 62.8 & 72.5 & 78.9 & 96.3 & 99.8 & 100.0 & 100.0 \\
\hline 655280 & 2 & 10.2 & 15.7 & 18.8 & 20.3 & 21.4 & 22.1 & 24.1 & 25.7 & 26.5 & 26.7 \\
\hline & 10 & 29.0 & 49.5 & 70.5 & 81.9 & 89.3 & 93.4 & 99.7 & 100.0 & 100.0 & 100.0 \\
\hline 655360 & 2 & 7.6 & 11.7 & 13.7 & 14.7 & 15.3 & 15.5 & 16.0 & 16.6 & 17.2 & 17.5 \\
\hline & 10 & 24.1 & 40.3 & 58.3 & 69.8 & 78.7 & 84.3 & 97.8 & 99.9 & 100.0 & 100.0 \\
\hline
\end{tabular}




\begin{tabular}{|c|c|c|c|c|c|c|c|c|c|c|c|}
\hline \multicolumn{12}{|c|}{$\begin{array}{l}\text { 1st-Order Markov Model } \\
\text { October DRWH Reliability (\%) } \\
\text { per capita roof area and storage } \\
\text { L }\end{array}$} \\
\hline Gauge & sq. m & 0 & 40 & 80 & 120 & 160 & 200 & 400 & 600 & 800 & 1000 \\
\hline \multirow[t]{2}{*}{655450} & 2 & 9.2 & 15.0 & 17.9 & 18.7 & 18.9 & 18.9 & 18.9 & 18.9 & 18.9 & 18.9 \\
\hline & 10 & 23.3 & 39.6 & 58.1 & 69.9 & 78.8 & 84.3 & 96.7 & 98.9 & 99.4 & 99.7 \\
\hline \multirow[t]{2}{*}{655480} & 2 & 10.3 & 17.8 & 22.5 & 24.4 & 25.3 & 25.7 & 27.0 & 27.5 & 27.6 & 27.8 \\
\hline & 10 & 26.6 & 44.4 & 64.0 & 76.0 & 84.9 & 89.9 & 99.1 & 100.0 & 100.0 & 100.0 \\
\hline \multirow[t]{2}{*}{655550} & 2 & 7.1 & 11.3 & 14.0 & 15.1 & 15.5 & 15.7 & 15.7 & 15.7 & 15.7 & 15.7 \\
\hline & 10 & 18.8 & 32.5 & 49.0 & 60.0 & 69.3 & 75.3 & 92.9 & 98.0 & 99.2 & 99.5 \\
\hline \multirow[t]{2}{*}{655570} & 2 & 10.1 & 16.7 & 20.1 & 21.2 & 21.7 & 22.0 & 22.2 & 22.2 & 22.2 & 22.2 \\
\hline & 10 & 29.8 & 51.5 & 71.2 & 81.4 & 87.7 & 91.5 & 98.2 & 99.1 & 99.4 & 99.7 \\
\hline \multirow[t]{2}{*}{655600} & 2 & 7.9 & 12.0 & 13.8 & 14.4 & 14.7 & 14.9 & 15.0 & 15.0 & 15.0 & 15.0 \\
\hline & 10 & 21.8 & 37.9 & 55.7 & 67.0 & 75.3 & 80.7 & 95.0 & 98.3 & 99.1 & 99.5 \\
\hline \multirow[t]{2}{*}{655620} & 2 & 9.5 & 15.2 & 17.7 & 18.3 & 18.5 & 18.7 & 19.7 & 20.4 & 20.7 & 20.7 \\
\hline & 10 & 21.8 & 38.7 & 57.3 & 68.7 & 77.1 & 82.2 & 93.3 & 95.8 & 97.1 & 98.1 \\
\hline \multirow[t]{2}{*}{655630} & 2 & 8.5 & 13.4 & 15.5 & 15.9 & 16.1 & 16.1 & 16.1 & 16.1 & 16.1 & 16.1 \\
\hline & 10 & 22.3 & 39.1 & 56 & 67.3 & 74.8 & 79.6 & 91.3 & 94.7 & 96.3 & 97.5 \\
\hline \multirow[t]{2}{*}{655780} & 2 & 9.6 & 17.5 & 22.9 & 25.3 & 26.7 & 27.5 & 29.2 & 29.6 & 29.6 & 29.6 \\
\hline & 10 & 22.3 & 38.4 & 54.1 & 63.9 & 71.1 & 75.7 & 87.0 & 90.6 & 92.6 & 94.4 \\
\hline \multirow[t]{2}{*}{655850} & 2 & 14.2 & 24.9 & 30.5 & 32.4 & 33.2 & 33.5 & 33.7 & 33.7 & 33.7 & 33.7 \\
\hline & 10 & 30.5 & 53.0 & 71.9 & 81.6 & 87.3 & 90.4 & 96.0 & 97.3 & 98.4 & 99.3 \\
\hline \multirow[t]{2}{*}{655920} & 2 & 12.3 & 23.9 & 31.7 & 35.5 & 37.6 & 38.8 & 40.5 & 40.6 & 40.7 & 40.7 \\
\hline & 10 & 28.8 & 51.0 & 70.3 & 81.5 & 88.5 & 92.7 & 99.4 & 99.9 & 100.0 & 100.0 \\
\hline \multirow[t]{2}{*}{655940} & 2 & 5.8 & 10.9 & 14.2 & 15.6 & 16.2 & 16.4 & 16.6 & 16.6 & 16.6 & 16.6 \\
\hline & 10 & 18.7 & 32.1 & 44.6 & 52.9 & 59.0 & 63.0 & 72.8 & 76.7 & 81.3 & 87.2 \\
\hline \multirow[t]{2}{*}{655990} & 2 & 7.0 & 11.2 & 13.1 & 13.5 & 13.6 & 13.6 & 13.6 & 13.6 & 13.6 & 13.6 \\
\hline & 10 & 19.8 & 33.8 & 47.9 & 56.5 & 62.7 & 66.3 & 72.8 & 75.1 & 78.4 & 83.6 \\
\hline
\end{tabular}




\begin{tabular}{|c|c|c|c|c|c|c|c|c|c|c|c|}
\hline $\begin{array}{l}\text { Tst-U } \\
\text { Nover }\end{array}$ & $\begin{array}{l}\text { r Mark } \\
\text { er DRV } \\
\text { per ca }\end{array}$ & $\begin{array}{l}\text { Me } \\
\text { Re } \\
\text { r r }\end{array}$ & ity & hd s & age & & & & & & \\
\hline Gauge & sq. m & 0 & 40 & 80 & 120 & 160 & 200 & 400 & 600 & 800 & 1000 \\
\hline 653060 & 2 & 0.0 & 0.1 & 0.1 & 0.1 & 0.1 & 0.1 & 0.1 & 0.1 & 0.1 & 0.1 \\
\hline & 10 & 0.2 & 0.6 & 1.4 & 2.4 & 3.5 & 4.3 & 8.8 & 24.6 & 53.8 & 81.1 \\
\hline 653190 & 2 & 0.8 & 1.4 & 1.8 & 2.1 & 2.3 & 2.4 & 2.5 & 2.6 & 2.6 & 2.6 \\
\hline & 10 & 2.4 & 4.6 & 8.5 & 12.3 & 17.2 & 20.8 & 43.3 & 73.2 & 92.4 & 99.0 \\
\hline 653300 & 2 & 1.0 & 1.7 & 2.3 & 2.5 & 2.6 & 2.6 & 2.6 & 2.6 & 2.6 & 2.6 \\
\hline & 10 & 1.8 & 3.7 & 7.7 & 11.7 & 17.0 & 21.0 & 43.5 & 72.6 & 91.6 & 98.5 \\
\hline 653350 & 2 & 1.2 & 1.8 & 2.2 & 2.3 & 2.3 & 2.3 & 2.3 & 2.3 & 2.3 & 2.3 \\
\hline & 10 & 3.3 & 6.4 & 11.9 & 17.0 & 22.9 & 27.5 & 52.8 & 80.4 & 94.5 & 98.5 \\
\hline 653380 & 2 & 2.3 & 3.9 & 5.1 & 5.5 & 5.7 & 5.7 & 5.8 & 5.8 & 5.8 & 5.8 \\
\hline & 10 & 4.4 & 8.4 & 15.2 & 21.5 & 28.8 & 34.1 & 59.5 & 82.4 & 93.7 & 97.6 \\
\hline 653440 & 2 & 3.5 & 5.4 & 6.5 & 6.9 & 7.1 & 7.1 & 7.2 & 7.2 & 7.2 & 7.2 \\
\hline & 10 & 9.3 & 16.6 & 27.0 & 35.3 & 43.7 & 49.6 & 74.7 & 89.8 & 94.3 & 96.3 \\
\hline 653520 & 2 & 0.6 & 1.2 & 1.9 & 2.4 & 2.9 & 3.1 & 3.7 & 3.8 & 3.8 & 3.8 \\
\hline & 10 & 1.7 & 3.4 & 6.4 & 9.4 & 13.2 & 15.9 & 30.9 & 55.3 & 79.1 & 93.1 \\
\hline 653550 & 2 & 2.8 & 4.5 & 5.5 & 5.8 & 5.9 & 5.9 & 5.9 & 6.1 & 6.4 & 6.7 \\
\hline & 10 & 5.8 & 10.2 & 17.6 & 24.5 & 32.6 & 38.4 & 66.3 & 90.0 & 98.6 & 99.9 \\
\hline 653570 & 2 & 1.3 & 2.0 & 2.5 & 2.6 & 2.6 & 2.6 & 2.6 & 2.6 & 2.6 & 2.6 \\
\hline & 10 & 3.0 & 5.8 & 10.9 & 15.6 & 21.4 & 25.5 & 48.2 & 76.4 & 93.1 & 98.7 \\
\hline 653610 & 2 & 1.4 & 2.4 & 3.6 & 4.2 & 4.7 & 4.9 & 5.1 & 5.1 & 5.1 & 5.1 \\
\hline & 10 & 3.5 & 6.6 & 11.9 & 16.8 & 23.0 & 27.6 & 52.2 & 79.9 & 94.8 & 99.3 \\
\hline 653760 & 2 & 1.1 & 1.8 & 2.3 & 2.4 & 2.4 & 2.4 & 2.4 & 2.4 & 2.4 & 2.4 \\
\hline & 10 & 3.4 & 6.4 & 11.6 & 16.4 & 22.1 & 26.5 & 51.4 & 79.1 & 94.2 & 98.9 \\
\hline 653800 & 2 & 2.5 & 3.5 & 4.0 & 4.1 & 4.1 & 4.1 & 4.1 & 4.1 & 4.1 & 4.1 \\
\hline & 10 & 7.4 & 13.4 & 22.0 & 28.8 & 35.7 & 40.8 & 63.9 & 79.1 & 84.3 & 87.2 \\
\hline 653870 & 2 & 1.6 & 2.2 & 2.5 & 2.6 & 2.7 & 2.7 & 2.7 & 2.7 & 2.7 & 2.7 \\
\hline & 10 & 5.4 & 9.7 & 15.7 & 20.2 & 24.9 & 28.2 & 44.0 & 56.2 & 61.8 & 65.8 \\
\hline 654720 & 2 & 2.2 & 3.1 & 3.5 & 3.7 & 3.7 & 3.7 & 3.7 & 3.7 & 3.7 & 3.7 \\
\hline & 10 & 5.7 & 10.3 & 16.8 & 21.5 & 25.9 & 28.7 & 39.4 & 46.2 & 49.2 & 50.9 \\
\hline 655010 & 2 & 0.1 & 0.2 & 0.4 & 0.6 & 0.7 & 0.8 & 0.9 & 0.9 & 0.9 & 0.9 \\
\hline & 10 & 0.1 & 0.3 & 0.7 & 1.2 & 2.0 & 2.6 & 5.2 & 9.1 & 18.3 & 32.9 \\
\hline 655020 & 2 & 0.1 & 0.3 & 0.4 & 0.5 & 0.5 & 0.6 & 0.6 & 0.6 & 0.6 & 0.6 \\
\hline & 10 & 0.4 & 0.8 & 1.6 & 2.4 & 3.3 & 3.8 & 6.2 & 15.1 & 37.4 & 63.5 \\
\hline 655030 & 2 & 0.6 & 1.0 & 1.4 & 1.6 & 1.7 & 1.7 & 1.7 & 1.7 & 1.9 & 2.0 \\
\hline & 10 & 0.9 & 1.8 & 3.6 & 5.1 & 6.9 & 8.0 & 12.6 & 26.1 & 52.4 & 78.1 \\
\hline 655050 & 2 & 0.3 & 0.4 & 0.5 & 0.5 & 0.5 & 0.5 & 0.5 & 0.5 & 0.5 & 0.5 \\
\hline & 10 & 0.9 & 1.9 & 3.5 & 5.0 & 6.6 & 7.7 & 13.6 & 29.9 & 57.1 & 81.7 \\
\hline 655070 & 2 & 0.2 & 0.3 & 0.4 & 0.4 & 0.4 & 0.4 & 0.4 & 0.4 & 0.4 & 0.4 \\
\hline & 10 & 0.6 & 1.3 & 2.6 & 3.8 & 5.1 & 5.8 & 9.7 & 24.1 & 51.8 & 78.9 \\
\hline 655100 & 2 & 1.0 & 1.5 & 1.9 & 1.9 & 2.0 & 2.0 & 2.0 & 2.0 & 2.0 & 2.0 \\
\hline & 10 & 2.6 & 4.7 & 8.1 & 11.2 & 14.7 & 17.0 & 29.8 & 53.9 & 79.1 & 94.4 \\
\hline 655160 & 2 & 0.5 & 0.8 & 1.0 & 1.0 & 1.0 & 1.0 & 1.0 & 1.0 & 1.0 & 1.0 \\
\hline & 10 & 1.4 & 2.7 & 4.6 & 6.6 & 9.1 & 10.8 & 20.1 & 40.4 & 67.0 & 87.7 \\
\hline 655180 & 2 & 0.5 & 0.7 & 0.7 & 0.7 & 0.7 & 0.7 & 0.7 & 0.7 & 0.7 & 0.7 \\
\hline & 10 & 2.7 & 4.7 & 7.2 & 9.2 & 11.2 & 12.5 & 19.5 & 38.5 & 66.8 & 88.9 \\
\hline 655220 & 2 & 0.7 & 1.2 & 1.8 & 2.0 & 2.1 & 2.1 & 2.1 & 2.1 & 2.1 & 2.1 \\
\hline & 10 & 1.8 & 3.5 & 6.9 & 10.5 & 15.0 & 18.5 & 39.4 & 68.5 & 89.8 & 98.2 \\
\hline 655280 & 2 & 2.3 & 3.4 & 4.2 & 4.7 & 5.2 & 5.6 & 7.4 & 8.7 & 9.6 & 10.0 \\
\hline & 10 & 7.6 & 13.5 & 22.3 & 29.4 & 37.1 & 42.8 & 71.3 & 93.1 & 99.1 & 100.0 \\
\hline 655360 & 2 & 1.5 & 2.2 & 2.7 & 2.9 & 3.0 & 3.1 & 3.4 & 3.5 & 3.5 & 3.6 \\
\hline & 10 & 3.9 & 7.5 & 13.4 & 18.5 & 24.2 & 28.4 & 52.5 & 79.9 & 94.8 & 99.3 \\
\hline
\end{tabular}




\begin{tabular}{|c|c|c|c|c|c|c|c|c|c|c|c|}
\hline \multirow{2}{*}{\multicolumn{12}{|c|}{$\begin{array}{l}\text { 1st-Order Markov Model } \\
\text { November DRWH Reliability (\%) } \\
\quad \text { per capita roof area and storage }\end{array}$}} \\
\hline & & & & & & & & & & & \\
\hline Gauge & sq. m & 0 & 40 & 80 & 120 & 160 & 200 & 400 & 600 & 800 & 1000 \\
\hline \multirow[t]{2}{*}{655450} & 2 & 1.7 & 2.6 & 3.1 & 3.3 & 3.4 & 3.4 & 3.4 & 3.4 & 3.4 & 3.4 \\
\hline & 10 & 6.6 & 11.5 & 18.3 & 23.9 & 30.1 & 34.9 & 60.0 & 83.7 & 94.4 & 97.8 \\
\hline \multirow[t]{2}{*}{655480} & 2 & 2.2 & 3.2 & 4.0 & 4.5 & 4.9 & 5.1 & 5.4 & 5.4 & 5.5 & 5.5 \\
\hline & 10 & 8.7 & 15.4 & 24.4 & 31.1 & 37.9 & 43.2 & 70.4 & 91.7 & 98.7 & 99.9 \\
\hline \multirow[t]{2}{*}{655550} & 2 & 1.6 & 2.5 & 3.2 & 3.5 & 3.7 & 3.8 & 3.8 & 3.8 & 3.8 & 3.8 \\
\hline & 10 & 5.5 & 9.6 & 15.5 & 20.2 & 25.6 & 29.4 & 49.6 & 73.5 & 89.2 & 95.9 \\
\hline \multirow[t]{2}{*}{655570} & 2 & 6.4 & 9.7 & 11.1 & 11.4 & 11.5 & 11.5 & 11.6 & 11.6 & 11.6 & 11.6 \\
\hline & 10 & 20.8 & 36.1 & 53.1 & 63.7 & 71.8 & 77.5 & 94.0 & 98.6 & 99.3 & 99.7 \\
\hline \multirow[t]{2}{*}{655600} & 2 & 2.4 & 3.2 & 3.5 & 3.6 & 3.6 & 3.6 & 3.6 & 3.6 & 3.6 & 3.6 \\
\hline & 10 & 8.9 & 15.4 & 23.7 & 29.8 & 35.8 & 40.2 & 61.7 & 82.1 & 93.0 & 97.1 \\
\hline \multirow[t]{2}{*}{655620} & 2 & 3.5 & 5.2 & 6.0 & 6.2 & 6.2 & 6.2 & 6.2 & 6.4 & 6.7 & 7.0 \\
\hline & 10 & 8.9 & 16.0 & 25.9 & 33.6 & 41.3 & 46.5 & 69.7 & 85.3 & 91.5 & 94.5 \\
\hline \multirow[t]{2}{*}{655630} & 2 & 2.9 & 4.2 & 4.9 & 5.2 & 5.3 & 5.3 & 5.3 & 5.3 & 5.3 & 5.3 \\
\hline & 10 & 11.4 & 19.4 & 29.5 & 36.6 & 43.5 & 48.3 & 69.6 & 83.9 & 90.4 & 93.5 \\
\hline \multirow[t]{2}{*}{655780} & 2 & 9.2 & 15.1 & 18.7 & 20.1 & 20.7 & 21.0 & 21.3 & 21.4 & 21.4 & 21.4 \\
\hline & 10 & 26.1 & 44.1 & 62.2 & 72.7 & 79.9 & 84.2 & 94.2 & 97.0 & 97.8 & 98.3 \\
\hline \multirow[t]{2}{*}{655850} & 2 & 9.2 & 15.2 & 18.2 & 19.1 & 19.6 & 19.8 & 20.0 & 20.0 & 20.0 & 20.0 \\
\hline & 10 & 25.0 & 43.9 & 62.8 & 74.4 & 82.4 & 87.6 & 98.0 & 99.3 & 99.6 & 99.8 \\
\hline \multirow[t]{2}{*}{655920} & 2 & 10.1 & 17.3 & 21.2 & 22.8 & 23.8 & 24.5 & 26.0 & 26.2 & 26.2 & 26.2 \\
\hline & 10 & 26.6 & 46.3 & 64.6 & 75.6 & 83.1 & 87.9 & 97.9 & 99.7 & 100.0 & 100.0 \\
\hline \multirow[t]{2}{*}{655940} & 2 & 8.5 & 14.7 & 18.5 & 19.8 & 20.4 & 20.6 & 20.8 & 20.8 & 20.8 & 20.8 \\
\hline & 10 & 24.1 & 41.0 & 57.5 & 67.2 & 74.0 & 78.2 & 88.2 & 91.0 & 92.3 & 93.8 \\
\hline \multirow[t]{2}{*}{655990} & 2 & 7.6 & 12.3 & 14.3 & 14.8 & 14.9 & 14.9 & 14.9 & 14.9 & 14.9 & 14.9 \\
\hline & 10 & 20.1 & 34.8 & 50.7 & 60.8 & 68.3 & 73.1 & 84.3 & 86.9 & 88.1 & 89.9 \\
\hline
\end{tabular}




\begin{tabular}{|c|c|c|c|c|c|c|c|c|c|c|c|}
\hline \multicolumn{12}{|c|}{$\begin{array}{l}\text { 1st-Order Markov Model } \\
\text { December DRWH Reliability (\%) } \\
\text { per capita roof area and storage } \\
\text { L }\end{array}$} \\
\hline Gauge & sq. m & 0 & 40 & 80 & 120 & 160 & 200 & 400 & 600 & 800 & 100 \\
\hline \multirow[t]{2}{*}{653060} & 2 & 0.1 & 0.2 & 0.3 & 0.4 & 0.4 & 0.4 & 0.5 & 0.5 & 0.5 & \\
\hline & 10 & 0.1 & 0.3 & 0.5 & 0.7 & 0.9 & 1.1 & 1.5 & 1.6 & 2.6 & 8.1 \\
\hline \multirow[t]{2}{*}{653190} & 2 & 0.0 & 0.0 & 0.1 & 0.1 & 0.2 & 0.2 & 0.3 & 0.4 & 0.4 & 0.5 \\
\hline & 10 & 0.2 & 0.4 & 0.7 & 0.9 & 1.2 & 1.5 & 2.5 & 5.5 & 19.3 & 44.2 \\
\hline \multirow[t]{2}{*}{653300} & 2 & 0.0 & 0.1 & 0.1 & 0.1 & 0.1 & 0.1 & 0.1 & 0.1 & 0.1 & 0.1 \\
\hline & 10 & 0.6 & 0.9 & 1.3 & 1.6 & 2.0 & 2.3 & 3.5 & 6.5 & 20.3 & 45. \\
\hline \multirow[t]{2}{*}{653350} & 2 & 0.1 & 0.1 & 0.2 & 0.2 & 0.2 & 0.2 & 0.2 & 0.2 & 0.2 & 0.2 \\
\hline & 10 & 0.7 & 1.2 & 2.0 & 2.5 & 3.1 & 3.4 & 4.4 & 8.9 & 26.9 & \\
\hline \multirow[t]{2}{*}{653380} & 2 & 1.7 & 2.7 & 3.8 & 4.2 & 4.4 & 4.4 & 4.4 & 4.4 & 4.4 & 4.4 \\
\hline & 10 & 2.6 & 4.9 & 8.7 & 11.9 & 15.4 & 17.4 & 23.6 & 31.9 & 49.5 & 70. \\
\hline \multirow[t]{2}{*}{653440} & 2 & 1.3 & 2.3 & 3.6 & 4.2 & 4.7 & 4.9 & 5.1 & 5.1 & 5.1 & 5.1 \\
\hline & 10 & 2.1 & 3.9 & 7. & 9.9 & 13.3 & 15.5 & 24.7 & 39.0 & 58.0 & 74. \\
\hline \multirow{2}{*}{653520} & 2 & 0.2 & 0.4 & 0.7 & 0.8 & 1.0 & 1.1 & 1.5 & 1.8 & 2.0 & 2.0 \\
\hline & 10 & 0.3 & 0.7 & 1. & 1.9 & 2.6 & 3.1 & 5. & 8.0 & 16.2 & 33.1 \\
\hline \multirow[t]{2}{*}{653550} & 2 & 0. & 0. & 0. & 0.4 & 0.4 & 0.4 & 0.4 & 0. & 0. & \\
\hline & 10 & 0. & 1.0 & 2. & 3.0 & 4.3 & 5.3 & 9.5 & 20.3 & 43.6 & 70 \\
\hline \multirow[t]{2}{*}{653570} & 2 & 0. & 0.4 & 0. & 0.4 & 0.4 & 0.4 & 0. & 0.4 & 0.4 & 0.4 \\
\hline & 10 & 0.5 & 1.0 & 1.9 & 2.6 & 3.4 & 3.9 & 5.4 & 9. & 25.4 & 50. \\
\hline \multirow[t]{2}{*}{653610} & 2 & 0.1 & 0.1 & 0.2 & 0.3 & 0.4 & 0.5 & 0.6 & 0.7 & 0.7 & 0.7 \\
\hline & 10 & 0.9 & 1.6 & 2.2 & 2.7 & 3.3 & 3.8 & 6.9 & 13.2 & 31.0 & 56. \\
\hline \multirow[t]{2}{*}{653760} & 2 & 0.5 & 0.8 & 1.1 & 1.3 & 1.5 & 1.5 & 1.6 & 1.6 & 1.6 & 1.6 \\
\hline & 10 & 1.5 & 2.7 & 4.2 & 5.3 & 6.5 & 7.1 & 9.0 & 13.5 & 30.8 & 56. \\
\hline \multirow[t]{2}{*}{653800} & 2 & 1.5 & 2.5 & 3.5 & 4.0 & 4.2 & 4.3 & 4.3 & 4.3 & 4.3 & 4.3 \\
\hline & 10 & 2.2 & 4. & 7. & 10.1 & 13.3 & 15.1 & 20.9 & 29.4 & 42.7 & 55. \\
\hline \multirow[t]{2}{*}{653870} & 2 & 0.6 & $1 .($ & 1. & 1.4 & 1.4 & 1.4 & 1.4 & 1. & 1.4 & 1.4 \\
\hline & 10 & 2.0 & 3.3 & 5.3 & 6.8 & 8.3 & 9. & 10.8 & 13 & 19 & 26. \\
\hline \multirow[t]{2}{*}{654720} & 2 & 1. & 1. & 2. & 2.3 & 2. & 2. & 2. & & & \\
\hline & 10 & 3.3 & 6.0 & 9.7 & 12.3 & 14.8 & 16.0 & 19.2 & 21.6 & 24.8 & 27. \\
\hline \multirow[t]{2}{*}{655010} & 2 & 0.5 & 0.9 & 1.4 & 1.8 & 2.1 & 2.2 & 2.4 & 2.4 & 2.4 & 2.4 \\
\hline & 10 & 0.6 & 1.2 & 2.1 & 2.9 & 3.8 & 4.4 & 5.9 & 6.7 & 7.4 & 9.0 \\
\hline \multirow[t]{2}{*}{655020} & 2 & 0.0 & 0.0 & 0.0 & 0.0 & 0.1 & 0.1 & 0.1 & 0. & 0.1 & 0.1 \\
\hline & 10 & 0.1 & 0.1 & 0.1 & 0.2 & 0.2 & 0.3 & 0.6 & 1.0 & 1.6 & 4.2 \\
\hline 655030 & 2 & 0.2 & 0.4 & 0.6 & 0.7 & 0.7 & 0.7 & 0.7 & 0. & 0.7 & 0.7 \\
\hline & 10 & 0.5 & 0.8 & 1. & 1.9 & 2.6 & $3 .($ & 4.7 & 5.8 & 7. & 13. \\
\hline 655050 & 2 & & 0.9 & 1. & 1.5 & 1.7 & 1.7 & 1. & 1. & 1 & 1.8 \\
\hline & 10 & 0.6 & 1. & 2.2 & 3.1 & 4.2 & 4.7 & 5. & 6. & 8 & 15 \\
\hline 655070 & 2 & & 0. & & 1.3 & 1.5 & 1. & 1. & & & \\
\hline & 10 & 0.5 & 1.0 & 1.6 & 2.2 & 2.9 & 3.5 & 4.3 & 4. & 5 & 10. \\
\hline 655100 & 2 & 0.1 & 0.1 & 0. & 0.1 & 0. & 0. & 0. & 0. & 0.1 & 0.1 \\
\hline & 10 & 0.5 & 0.8 & 1.3 & 1.7 & 2.2 & 2.5 & 3.8 & 5. & 12.8 & 29 \\
\hline 655160 & 2 & 0.1 & 0.2 & 0.4 & 0.5 & 0.5 & 0.5 & 0.6 & 0.6 & 0.6 & 0.6 \\
\hline & 10 & 0.2 & 0.3 & 0.7 & 1.0 & 1.4 & 1.6 & 2.4 & 3.2 & 7.1 & 18. \\
\hline 655180 & 2 & 0.5 & 0.7 & 0.9 & 1.0 & 1.0 & 1.0 & 1.0 & 1.0 & 1.0 & 1.0 \\
\hline & 10 & 0.8 & 1.4 & 2.4 & 3.2 & 4.1 & 4.5 & 5.3 & 5.8 & 9.0 & 20. \\
\hline 655220 & 2 & 0.3 & 0.5 & 0.7 & 0.9 & 1.0 & 1.1 & 1.1 & 1.1 & 1.1 & 1.1 \\
\hline & 10 & 0.3 & 0.7 & 1.3 & 1.8 & 2.6 & 3.1 & 5.0 & 7.7 & 19.3 & 42. \\
\hline 655280 & 2 & 0.5 & 1.0 & 1.6 & 2.1 & 2.7 & 3.0 & 3.9 & 4.2 & 4.5 & 4.8 \\
\hline & 10 & 1.3 & 2.4 & 4.1 & 5.6 & 7.4 & 8.5 & 13.7 & 26.4 & 51.2 & 76. \\
\hline 655360 & 2 & 0.6 & 0.9 & 1.3 & 1.4 & 1.5 & 1.5 & 1.5 & 1.5 & 1.5 & \\
\hline & 10 & 0.8 & 1.5 & 2.8 & 4.0 & 5.5 & 6.3 & 9.0 & 14.5 & 32.0 & 57 \\
\hline
\end{tabular}




\begin{tabular}{|c|c|c|c|c|c|c|c|c|c|c|c|}
\hline \multirow{2}{*}{\multicolumn{12}{|c|}{$\begin{array}{l}\text { 1st-Order Markov Model } \\
\text { December DRWH Reliability (\%) } \\
\text { per capita roof area and storage } \\
\text { L }\end{array}$}} \\
\hline & & & & & & & & & & & \\
\hline Gauge & sq. m & 0 & 40 & 80 & 120 & 160 & 200 & 400 & 600 & 800 & 1000 \\
\hline \multirow{2}{*}{655450} & 2 & 0.8 & 1.1 & 1.3 & 1.3 & 1.3 & 1.3 & 1.3 & 1.3 & 1.3 & 1.3 \\
\hline & 10 & 2.2 & 3.9 & 6.4 & 8.1 & 9.7 & 10.6 & 13.5 & 21.1 & 40.7 & 64.6 \\
\hline \multirow[t]{2}{*}{655480} & 2 & 1.9 & 3.1 & 3.9 & 4.3 & 4.4 & 4.5 & 4.7 & 4.8 & 4.8 & 4.8 \\
\hline & 10 & 3.3 & 6.0 & 10.3 & 13.7 & 17.1 & 18.8 & 24.8 & 37.1 & 59.8 & 81.8 \\
\hline \multirow[t]{2}{*}{655550} & 2 & 1.0 & 1.6 & 2.3 & 2.6 & 2.8 & 2.8 & 2.9 & 2.9 & 2.9 & 2.9 \\
\hline & 10 & 2.7 & 4.7 & 7.5 & 9.5 & 11.6 & 12.8 & 16.7 & 22.2 & 36.5 & 57.5 \\
\hline \multirow[t]{2}{*}{655570} & 2 & 3.5 & 5.5 & 6.6 & 6.9 & 7.0 & 7.0 & 7.0 & 7.0 & 7.0 & 7.0 \\
\hline & 10 & 8.1 & 14.5 & 23.5 & 30.6 & 37.5 & 42.0 & 62.2 & 81.4 & 92.5 & 97.0 \\
\hline \multirow[t]{2}{*}{655600} & 2 & 2.4 & 3.7 & 4.5 & 4.7 & 4.8 & 4.8 & 4.8 & 4.8 & 4.8 & 4.8 \\
\hline & 10 & 4.3 & 8.0 & 13.6 & 17.6 & 21.6 & 23.6 & 29.1 & 37.6 & 54.5 & 72.9 \\
\hline \multirow[t]{2}{*}{655620} & 2 & 1.9 & 2.8 & 3.3 & 3.4 & 3.5 & 3.5 & 3.5 & 3.5 & 3.5 & 3.5 \\
\hline & 10 & 3.6 & 6.8 & 11.7 & 15.4 & 19.3 & 21.3 & 28.6 & 40.0 & 56.4 & 71.8 \\
\hline \multirow[t]{2}{*}{655630} & 2 & 1.4 & 2.0 & 2.4 & 2.4 & 2.5 & 2.5 & 2.5 & 2.5 & 2.5 & 2.5 \\
\hline & 10 & 4.1 & 7.1 & 11.2 & 14.2 & 17.1 & 18.7 & 25.2 & 36.7 & 53.1 & 68.7 \\
\hline \multirow[t]{2}{*}{655780} & 2 & 6.0 & 10.3 & 13.6 & 14.8 & 15.2 & 15.4 & 15.5 & 15.5 & 15.5 & 15.5 \\
\hline & 10 & 12.0 & 21.3 & 34.0 & 43.9 & 53.4 & 59.6 & 81.6 & 92.5 & 96.1 & 97.4 \\
\hline \multirow[t]{2}{*}{655850} & 2 & 4.6 & 6.6 & 7.8 & 8.4 & 9.0 & 9.3 & 10.4 & 10.5 & 10.5 & 10.5 \\
\hline & 10 & 14.1 & 24.5 & 37.5 & 46.4 & 54.4 & 59.9 & 82.0 & 94.6 & 98.5 & 99.4 \\
\hline \multirow[t]{2}{*}{655920} & 2 & 8.2 & 14.7 & 19.8 & 21.9 & 22.8 & 23.2 & 23.5 & 23.5 & 23.5 & 23.5 \\
\hline & 10 & 16.8 & 29.7 & 45.6 & 56.9 & 66.2 & 72.5 & 90.9 & 97.9 & 99.7 & 100.0 \\
\hline \multirow[t]{2}{*}{655940} & 2 & 5.5 & 9.3 & 12.0 & 12.9 & 13.3 & 13.5 & 13.6 & 13.6 & 13.6 & 13.6 \\
\hline & 10 & 12.1 & 21.5 & 34.2 & 43.8 & 52.7 & 58.6 & 79.1 & 88.4 & 91.6 & 93.4 \\
\hline \multirow[t]{2}{*}{655990} & 2 & 4.4 & 7.3 & 9.3 & 10.0 & 10.2 & 10.2 & 10.3 & 10.3 & 10.3 & 10.3 \\
\hline & 10 & 8.7 & 15.7 & 25.8 & 33.9 & 41.9 & 47.2 & 66.4 & 76.8 & 81.0 & 83.5 \\
\hline
\end{tabular}





\section{Appendix I: Domestic Rainwater Harvesting Reliabilities Using the LARS-WG Spell-length Model}




\begin{tabular}{|c|c|c|c|c|c|c|c|c|c|c|c|}
\hline \multicolumn{12}{|c|}{$\begin{array}{l}\text { LARS-WG Model } \\
\text { January DRWH Reliability (\%) } \\
\text { per capita roof area and storage } \\
\text { L }\end{array}$} \\
\hline Gauge & sq. m & 0 & 40 & 80 & 120 & 160 & 200 & 400 & 600 & 800 & \\
\hline \multirow[t]{2}{*}{653060} & 2 & 5.5 & 8.2 & 8.7 & 9.0 & 9.2 & 9.2 & 9.2 & 9.2 & 9.2 & \\
\hline & 10 & 6.8 & 14.1 & 26.3 & 36.2 & 46.4 & 52.6 & 77.2 & 90.9 & 91.2 & \\
\hline \multirow[t]{2}{*}{653190} & 2 & 3.3 & 4.1 & 4.4 & 4.4 & 4.4 & 4.4 & 4.4 & 4.4 & 4.4 & \\
\hline & 10 & 3.6 & 7.7 & 15.3 & 22.1 & 27.3 & 29.4 & 33.8 & 39.1 & 48.9 & \\
\hline \multirow[t]{2}{*}{653300} & 2 & 1.6 & 2.5 & 2.6 & 2.6 & 2.6 & 2.6 & 2.6 & 2.6 & 2.6 & \\
\hline & 10 & 4.8 & 9.7 & 14.4 & 17.5 & 20.4 & 21.7 & 23.4 & 26.2 & 33.4 & 45 \\
\hline \multirow[t]{2}{*}{653350} & 2 & 3.8 & 4.7 & 5.2 & 5.2 & 5.2 & 5.2 & 5.2 & 5.2 & 5.2 & 5.2 \\
\hline & 10 & 5.9 & 10.9 & 19.6 & 25.9 & 29.6 & 30.8 & 31.6 & 33.0 & 37.9 & 2 \\
\hline \multirow[t]{2}{*}{653380} & 2 & 4.3 & 7.9 & 12.2 & 14.5 & 16.9 & 18.0 & 19.0 & 19.1 & 19.1 & 19 \\
\hline & 10 & 6.3 & 12.3 & 22.5 & 31.0 & 40.9 & 47.4 & 70.8 & 82.7 & 88.0 & \\
\hline \multirow[t]{2}{*}{653440} & 2 & 2.5 & 4.3 & 6.6 & 8.1 & 9.3 & 10.0 & 12.6 & 13.4 & 13.4 & \\
\hline & 10 & 4. & 7.7 & & 18.9 & 24.2 & 27.6 & 42.2 & 55.2 & 66.7 & \\
\hline \multirow[t]{2}{*}{653520} & 2 & 8.7 & 15.8 & 23.6 & 24.9 & 25.3 & 25.5 & 25.9 & 26.1 & 26.1 & 26 \\
\hline & 10 & 10 & 20 & 36.2 & 48.5 & 62.6 & 69.8 & 7 & 97.4 & 8.2 & 98 \\
\hline \multirow[t]{2}{*}{653550} & 2 & 6.6 & 10.9 & 11.5 & 11.5 & 11.5 & 11.5 & 11.5 & 11.5 & 11.5 & \\
\hline & 10 & 8.7 & 17.2 & 30.2 & 40.8 & 52.6 & 57.9 & 68.0 & 72.1 & 78.3 & 85 \\
\hline \multirow[t]{2}{*}{653570} & 2 & 6.4 & 12.2 & 17.0 & 17.7 & 17.9 & 18.0 & 18.0 & 18.0 & 18.0 & \\
\hline & 10 & 7.9 & 16.1 & 28.1 & 37.5 & 47.9 & 54.3 & 69.3 & 74.0 & 79.6 & 86 \\
\hline \multirow[t]{2}{*}{653610} & 2 & 3.6 & 5.4 & 5.8 & 5.8 & $5 . \varepsilon$ & 5.8 & 5. & 5.8 & & \\
\hline & 10 & 6.9 & 14.4 & 24.4 & 31.0 & 36.3 & 39.1 & 47.6 & 58.5 & 70.9 & \\
\hline \multirow[t]{2}{*}{653760} & 2 & 2.7 & 4. & 6.0 & 6.4 & 6. & 6.6 & 6.7 & 6. & & 6.7 \\
\hline & 10 & 8. & & 23 & 29.7 & & 39.3 & & & & \\
\hline \multirow[t]{2}{*}{653800} & 2 & 6.0 & & 16 & 19.7 & 20 & 21.0 & 21.3 & 21.3 & 1.3 & 21 \\
\hline & 10 & 9.3 & 18 & 31 & 41.2 & 52 & 59.1 & 82.0 & 92.8 & 96.2 & 97. \\
\hline \multirow[t]{2}{*}{653870} & 2 & 2.0 & 3. & 4.6 & 4.8 & 4. & 4. & & & & \\
\hline & 10 & 5.5 & 10. & 17.1 & 21.3 & 25.6 & 28.2 & 37.8 & 43.7 & 47.8 & 53 \\
\hline \multirow[t]{2}{*}{654720} & 2 & 2.1 & 3.5 & 4.8 & 4.8 & 4.8 & 4.8 & 4.8 & 4.8 & 4. & 4.8 \\
\hline & 10 & 5.3 & 10.3 & 17.4 & 21.9 & 26.7 & 30.3 & 45.6 & 54.0 & 59.2 & 63 \\
\hline \multirow[t]{2}{*}{655010} & 2 & 7.8 & 12.8 & 17.0 & 19.2 & 21.1 & 22.5 & 25.1 & 25.3 & 25.3 & 5 \\
\hline & 10 & 8.2 & 16.8 & 31.2 & 43.3 & 56.8 & 64.0 & 87.2 & 97.5 & 98.0 & 8. \\
\hline \multirow[t]{2}{*}{655020} & 2 & 3.0 & 3.8 & & 3.8 & 3.8 & 3. & & & & 3.8 \\
\hline & 10 & 3.4 & 6.6 & 11.2 & 13.9 & 15.0 & 15.0 & 15 & & 18.4 & 25 \\
\hline 655050 & 2 & 0.4 & 1.1 & 2. & 3.2 & 3.8 & 4.2 & 4 & 4 & & 4.5 \\
\hline & 10 & 5.1 & 10 & 15 & 19.5 & 25.1 & 29.6 & 54.1 & 5.8 & 82.2 & 84 \\
\hline 655070 & 2 & & 0. & 2. & 3. & 3. & 4.2 & & 7 & & 4.7 \\
\hline & 10 & 0. & 0. & 3. & 7. & 13 & 18 & 43 & 71.3 & 82 & 85. \\
\hline 655100 & 2 & 2. & 5. & 5. & 5. & 5. & 5. & 5 & & & \\
\hline & 10 & 5.1 & 10.7 & 18.7 & 25.3 & 31.8 & 34.9 & 39 & 40.5 & 44.0 & 51. \\
\hline 655160 & 2 & 2.0 & 2.1 & 2.4 & 2.5 & 2.6 & 2.6 & 2.6 & 2.6 & 2.6 & \\
\hline & 10 & 5.2 & 9.9 & 16. & 22.2 & 27.6 & 31.8 & 55 & 72.9 & 79.9 & 8 \\
\hline 655180 & 2 & 0.0 & 0.0 & 0.0 & 0.0 & 0.0 & 0.0 & 0.0 & 0.0 & 0.0 & 0.0 \\
\hline & 10 & 0.4 & 1.4 & 5 & 9.6 & 16.4 & 21.5 & 44.1 & 64.8 & 74.1 & 80 \\
\hline 655220 & 2 & 3.2 & 6.3 & 9.7 & 12.2 & 13.0 & 13.2 & 13.2 & 13.2 & 13.2 & 13. \\
\hline & 10 & 5.7 & 11.2 & 20.1 & 27.4 & 35.9 & 41.7 & 62.0 & 74.0 & 79.0 & 83. \\
\hline 655280 & 2 & 5.0 & 8.5 & 12.5 & 15.2 & 18.2 & 20.5 & 30 & 33.0 & 33.5 & 33. \\
\hline & 10 & 8.1 & 15.4 & 26.3 & 35.3 & 45.1 & 51.4 & 75.5 & 88.4 & 92.9 & 96. \\
\hline 655360 & 2 & 3.7 & 5.9 & 6.5 & 6.6 & 6.6 & 6.6 & 6.6 & 6.6 & 6.6 & 6.6 \\
\hline & 10 & $6 . \varsigma$ & 13.4 & 22.6 & 29.8 & 37.4 & 42.7 & 62.7 & 73.1 & 78.0 & 83 \\
\hline 655450 & 2 & 4. & 8.7 & 14.0 & 16.1 & 16.9 & 17.0 & 17.1 & 17.1 & 17.1 & 17. \\
\hline & 10 & 7.4 & 14.5 & 25.7 & 34.2 & 43.3 & 49.5 & 69.5 & 77.2 & 81.8 & 87 \\
\hline
\end{tabular}




\begin{tabular}{|c|c|c|c|c|c|c|c|c|c|c|c|}
\hline \multirow{2}{*}{\multicolumn{12}{|c|}{$\begin{array}{l}\text { LARS-WG Markov Model } \\
\text { January DRWH Reliability (\%) } \\
\text { per capita roof area and storage }\end{array}$}} \\
\hline & & & & & & & & & & & \\
\hline Gauge & sq. m & 0 & 40 & 80 & 120 & 160 & 200 & 400 & 600 & 800 & 1000 \\
\hline \multirow{2}{*}{655480} & 2 & 5.2 & 7.6 & 9.6 & 9.8 & 9.8 & 9.8 & 9.9 & 9.9 & 9.9 & 9.9 \\
\hline & 10 & 7.8 & 15.4 & 26.6 & 35.2 & 43.1 & 47.8 & 64.5 & 73.8 & 80.5 & 87.3 \\
\hline \multirow[t]{2}{*}{655550} & 2 & 5.2 & 8.0 & 11.4 & 13.8 & 14.9 & 15.1 & 15.2 & 15.2 & 15.2 & 15.2 \\
\hline & 10 & 9.6 & 18.3 & 30.5 & 38.5 & 46.1 & 50.7 & 66.3 & 73.8 & 78.4 & 83.9 \\
\hline \multirow[t]{2}{*}{655570} & 2 & 4.3 & 6.8 & 8.9 & 9.4 & 9.5 & 9.5 & 9.5 & 9.5 & 9.5 & 9.5 \\
\hline & 10 & 10.9 & 19.6 & 31.0 & 39.0 & 46.7 & 51.7 & 70.3 & 84.9 & 93.6 & 97.8 \\
\hline \multirow[t]{2}{*}{655600} & 2 & 2.2 & 3.1 & 3.4 & 3.4 & 3.4 & 3.4 & 3.4 & 3.4 & 3.4 & 3.4 \\
\hline & 10 & 5.6 & 11.0 & 18.2 & 23.3 & 28.5 & 31.9 & 45.4 & 55.2 & 65.0 & 76.3 \\
\hline \multirow[t]{2}{*}{655620} & 2 & 3.4 & 5.5 & 6.3 & 6.3 & 6.4 & 6.4 & 6.4 & 6.4 & 6.4 & 6.4 \\
\hline & 10 & 6.9 & 13.6 & 22.8 & 29.5 & 36.3 & 40.6 & 54.9 & 65.3 & 74.8 & 83.9 \\
\hline \multirow[t]{2}{*}{655630} & 2 & 4.0 & 7.0 & 10.0 & 11.2 & 11.4 & 11.5 & 11.5 & 11.5 & 11.5 & 11.5 \\
\hline & 10 & 7.2 & 13.7 & 23.0 & 30.1 & 37.7 & 42.5 & 57.8 & 65.9 & 73.8 & 82.4 \\
\hline \multirow[t]{2}{*}{655780} & 2 & 2.2 & 3.8 & 5.0 & 5.4 & 5.4 & 5.4 & 5.4 & 5.4 & 5.4 & 5.4 \\
\hline & 10 & 5.1 & 9.7 & 16.7 & 22.5 & 29.2 & 34.0 & 56.8 & 78.8 & 90.9 & 95.9 \\
\hline \multirow[t]{2}{*}{655850} & 2 & 4.1 & 6.4 & 8.2 & 8.8 & 9.2 & 9.4 & 9.6 & 9.6 & 9.6 & 9.6 \\
\hline & 10 & 9.0 & 17.1 & 28.1 & 35.8 & 42.7 & 47.6 & 68.1 & 85.4 & 94.9 & 98.5 \\
\hline \multirow[t]{2}{*}{655920} & 2 & 5.3 & 8.9 & 11.7 & 13.1 & 13.9 & 14.3 & 15.0 & 15.0 & 15.0 & 15.0 \\
\hline & 10 & 12.2 & 23.1 & 36.4 & 45.7 & 54.2 & 60.3 & 82.8 & 94.4 & 98.5 & 99.7 \\
\hline \multirow[t]{2}{*}{655940} & 2 & 3.1 & 5.5 & 6.5 & 6.7 & 6.7 & 6.7 & 6.7 & 6.7 & 6.7 & 6.7 \\
\hline & 10 & 8.5 & 16.2 & 25.4 & 32.0 & 38.7 & 43.6 & 64.4 & 80.7 & 89.5 & 93.7 \\
\hline \multirow[t]{2}{*}{655990} & 2 & 3.4 & 4.9 & 6.2 & 6.8 & 7.0 & 7.1 & 7.2 & 7.2 & 7.2 & 7.2 \\
\hline & 10 & 8.4 & 15.5 & 24.8 & 31.6 & 38.1 & 42.7 & 63.8 & 80.7 & 88.8 & 92.4 \\
\hline
\end{tabular}




\begin{tabular}{|c|c|c|c|c|c|c|c|c|c|c|c|}
\hline \multicolumn{12}{|c|}{$\begin{array}{l}\text { LARS-WG Model } \\
\text { February DRWH Reliability (\%) } \\
\text { per capita roof area and storage } \\
\text { L }\end{array}$} \\
\hline Gauge & sq. m & 0 & 40 & 80 & 120 & 160 & 200 & 400 & 600 & 800 & \\
\hline \multirow[t]{2}{*}{653060} & 2 & 2.6 & 4.3 & 5.3 & 5.3 & 5.3 & 5.3 & 5.3 & 5.3 & 5.3 & \\
\hline & 10 & 3.6 & 7.2 & 13.2 & 17.7 & 22.1 & 23.9 & 28.2 & 41.6 & 61.9 & \\
\hline \multirow[t]{2}{*}{653190} & 2 & 1.6 & 2.2 & 2.2 & 2.2 & 2.2 & 2.2 & 2.2 & 2.2 & 2.2 & 2.2 \\
\hline & 10 & 3.6 & 6.5 & 10.1 & 12.3 & 14.4 & 15.7 & 19.0 & 20.3 & 22.6 & \\
\hline \multirow[t]{2}{*}{653300} & 2 & 1.5 & 1.9 & 1.9 & 1.9 & 1.9 & 1.9 & 1.9 & 1.9 & 1.9 & \\
\hline & 10 & 3.3 & 6.3 & 10.2 & 12.7 & 14.8 & 15.7 & 17.5 & 17.9 & 18.6 & \\
\hline \multirow[t]{2}{*}{653350} & 2 & 3.5 & 6.2 & 8.8 & 10.1 & 10.8 & 10.9 & 11.0 & 11.0 & 11.0 & \\
\hline & 10 & 6.8 & 11.8 & 18.2 & 22.5 & 27.0 & 29.6 & 36.4 & 37.4 & 37.9 & \\
\hline \multirow[t]{2}{*}{653380} & 2 & 2.8 & 4.3 & 5.4 & 5.8 & 6.2 & 6.4 & 6.7 & 6.8 & 6.8 & \\
\hline & 10 & 6.1 & 11.3 & 17.8 & 21.9 & 26.0 & 28.5 & 37.7 & 50.0 & & \\
\hline \multirow[t]{2}{*}{653440} & 2 & 2.5 & 4.3 & 5.8 & 6. & 6.6 & 6.7 & 6.7 & 7 & & \\
\hline & 10 & 4.5 & 8.3 & & 16.6 & 20.3 & 22.5 & 8.8 & & 3.7 & \\
\hline \multirow[t]{2}{*}{653520} & 2 & 4.0 & 5.9 & 6.5 & 6.5 & 6.5 & 6.5 & 6.5 & 6.6 & 6 & \\
\hline & 10 & 7.1 & 14.5 & 23.5 & 29.7 & 35.0 & 38.4 & 53.2 & 70.9 & 86.2 & \\
\hline \multirow[t]{2}{*}{653550} & 2 & 2.8 & 4.7 & 7.1 & 8.1 & 8.3 & 8.4 & 8.4 & 8.4 & 8. & \\
\hline & 10 & 5.8 & 11.1 & 17.3 & 21.6 & 25.8 & 28.7 & 41.2 & 49.3 & 55.4 & \\
\hline \multirow[t]{2}{*}{653570} & 2 & 4.6 & 7.6 & 8.7 & 8.9 & 8.9 & 8.9 & 8.9 & 8.9 & 8. & \\
\hline & 10 & 6.3 & 13.0 & 21.4 & 28.0 & 33.9 & 37.8 & 52.8 & 63.5 & 69 & \\
\hline \multirow[t]{2}{*}{653610} & 2 & 3.7 & 4.1 & 4.1 & 4.1 & 4.1 & 4.1 & 4.1 & 4.1 & & \\
\hline & 10 & 7.3 & 14.3 & 23.2 & 29.0 & 33 & 35 & 40.9 & 44.1 & 50 & \\
\hline \multirow[t]{2}{*}{653760} & 2 & 2.3 & 3. & 3.8 & 3.8 & 3. & 3.8 & & & & \\
\hline & 10 & 7. & 13 & 20 & 23.8 & 26.4 & 27.9 & 2.1 & 8.4 & 6.7 & \\
\hline \multirow[t]{2}{*}{653800} & 2 & 6. & 11 & 15.2 & 17.6 & 19.8 & 21 & & & & \\
\hline & 10 & 11. & 20.7 & 31.0 & 37.7 & 42.9 & 46.0 & 58.3 & 72.8 & 85.7 & \\
\hline \multirow[t]{2}{*}{653870} & 2 & 1.5 & 2.5 & 3.5 & 4.2 & 4.7 & 4.8 & 4. & 4.8 & 4. & \\
\hline & 10 & 4.2 & 7.5 & 11.4 & 13.9 & 16.2 & 17.6 & 22.3 & 25.2 & 27.9 & \\
\hline \multirow[t]{2}{*}{654720} & 2 & 2.8 & 5.8 & 7.9 & 8.0 & 8.0 & 8.0 & 8.0 & 8.0 & 8. & \\
\hline & 10 & 7.2 & 13.7 & 20.0 & 23.8 & 27.3 & 29.7 & 37 & 40.2 & 44 & \\
\hline \multirow[t]{2}{*}{655010} & 2 & 5.6 & 10.2 & 11.5 & 11.5 & 11.5 & 11.5 & 11.5 & 11.5 & 11.5 & \\
\hline & 10 & 6.4 & 12.6 & 21.3 & 27.6 & 34.7 & 37.9 & 46.9 & 63.0 & 80 & \\
\hline \multirow[t]{2}{*}{655020} & 2 & 5.5 & 11.0 & 16. & 17.1 & 17 & 17.3 & 1 & & & \\
\hline & 10 & 6. & 12.7 & 22.3 & 29.6 & 38.5 & 42.9 & 49.9 & 50.5 & 50.5 & \\
\hline 655050 & 2 & 2.6 & 5.6 & 8.5 & 8.9 & 8.9 & 9.0 & .0 & 9.0 & 0 & 9.0 \\
\hline & 10 & 4.2 & 8.4 & 13.5 & 17.4 & 21.7 & 24.1 & 28.3 & 31.4 & 46.2 & 61 \\
\hline 655070 & 2 & 4.7 & 8. & & 10.7 & 10.7 & 10.7 & & .7 & & \\
\hline & 10 & 5.7 & 10.8 & 18.0 & 23.4 & 28.3 & 30.3 & 33.1 & 33.2 & 45.4 & \\
\hline 655100 & 2 & $0 . \varepsilon$ & 1.5 & $2 .($ & $2 .($ & 2.0 & 2. & 2. & & & 2.0 \\
\hline & 10 & 2.9 & 5. & 7. & 9 & 11 & 12 & 18 & & .9 & \\
\hline 655160 & 2 & & 0. & & 0. & 0. & 0.6 & 0 & & & \\
\hline & 10 & 4. & 5.8 & 6. & 7. & 7. & 7. & 7 & 12.7 & 27.4 & 40 \\
\hline 655180 & 2 & 0.5 & 0.7 & 0.7 & 0.7 & 0.7 & 0.7 & 0.7 & 0.7 & 0.7 & \\
\hline & 10 & 2.5 & 5.0 & 7.5 & 8.1 & 8.2 & 8.2 & 8.2 & 8.2 & 18.8 & \\
\hline 655220 & 2 & 2.5 & 4.0 & 4.8 & 5.0 & 5.0 & 5.0 & 5.0 & 5.0 & & \\
\hline & 10 & 4.3 & 8.2 & 13.2 & 16.9 & 20.6 & 22.9 & 31.7 & 42.5 & 54.2 & 64 \\
\hline 655280 & 2 & 3.0 & 4.5 & 5.6 & 5.8 & 5.8 & 5.8 & 5.9 & 6.3 & 7.2 & \\
\hline & 10 & 5.6 & 11.4 & 18.6 & 23.3 & 27.3 & 29.6 & 37.6 & 52.3 & 70.8 & \\
\hline 655360 & 2 & 3.6 & 6.6 & 8.9 & 9.2 & 9.2 & 9.2 & 9.2 & 9.2 & 9.2 & 9.2 \\
\hline & 10 & 5.8 & 11.3 & 18.3 & 22.9 & 27.4 & 29.9 & 36.1 & 42.3 & 51.6 & \\
\hline 655450 & 2 & 4.2 & 7.4 & 10. & 11.6 & 12 & 13.0 & 13.2 & 13.2 & 13.2 & \\
\hline & 10 & 8.9 & 16.1 & 24.2 & 29.8 & 34.7 & 37.4 & 48.1 & 59.6 & 69.1 & \\
\hline
\end{tabular}




\begin{tabular}{|c|c|c|c|c|c|c|c|c|c|c|c|}
\hline \multicolumn{12}{|c|}{$\begin{array}{l}\text { LARS-WG Markov Model } \\
\text { February DRWH Reliability (\%) } \\
\text { per capita roof area and storage }\end{array}$} \\
\hline Gauge & sq. m & 0 & 40 & 80 & 120 & 160 & 200 & 400 & 600 & 800 & \\
\hline 655480 & $\begin{array}{l}2 \\
10\end{array}$ & $\begin{array}{l}3.8 \\
8.7\end{array}$ & & & & & $\begin{array}{l}6.8 \\
36.0\end{array}$ & $\begin{array}{l}6.8 \\
44.3\end{array}$ & $\begin{array}{l}6.8 \\
52.6\end{array}$ & $\begin{array}{l}6.8 \\
61.6\end{array}$ & $\begin{array}{l}6.8 \\
71.3\end{array}$ \\
\hline \multirow[t]{2}{*}{655550} & 2 & 4.2 & 6.8 & & 10.6 & 11.2 & 11.3 & 11.4 & 11.4 & 1.4 & 10 \\
\hline & 10 & & & & 31.8 & 37.5 & 40.8 & 51.9 & 62.1 & 0.4 & 6. \\
\hline \multirow[t]{2}{*}{655570} & 2 & & 10. & 12.9 & 14.4 & 15.5 & 16.1 & 16.5 & 16.5 & 16.5 & 6. \\
\hline & 10 & 13 & 25.0 & 37.2 & 44.6 & 50.6 & 53.8 & 62.6 & 70.8 & 80.9 & 89. \\
\hline \multirow[t]{2}{*}{655600} & 2 & 5.5 & 8.5 & 10.3 & 10.6 & 10.6 & 10.6 & 10.6 & 10.6 & 10.6 & 10. \\
\hline & 10 & 9.7 & 18.1 & 28.8 & 35.1 & 40.1 & 42.4 & 45.2 & 47.4 & 52.8 & 60. \\
\hline \multirow[t]{2}{*}{655620} & 2 & 4. & 6.1 & 6. & 6.5 & 6. & 6.5 & 6.5 & 6.5 & & 6.5 \\
\hline & 10 & 11. & 21. & 32.0 & 37.7 & 42.3 & 44.5 & 49.6 & 54.1 & 61 & 70. \\
\hline \multirow[t]{2}{*}{655630} & 2 & 3.4 & 5.3 & 6. & 6.5 & 6.6 & 6.6 & 6.6 & 6.6 & & 6.6 \\
\hline & 10 & & & & 27.5 & & 34.7 & 44 & & 60 & \\
\hline \multirow[t]{2}{*}{655780} & 2 & 2.6 & 4.0 & 5.1 & 5.7 & 6.1 & 6.2 & 6.3 & 6.3 & 6.3 & 6.3 \\
\hline & 10 & & 12 & & 22.1 & 24 & 26.5 & & 38.2 & & 73. \\
\hline \multirow[t]{2}{*}{655850} & 2 & & 8. & & 12.4 & 12 & 12 & 12 & 12.7 & & 12. \\
\hline & 10 & 11 & 21.3 & 32 & 39.8 & & 48 & 56 & 66.1 & & 89. \\
\hline \multirow[t]{2}{*}{655920} & 2 & 5.0 & 7.6 & 9.6 & 10.3 & 10.4 & 10.5 & 10.5 & 10.5 & 10.5 & 10. \\
\hline & 10 & 11.3 & 21.3 & 33. & 40.4 & 46.1 & 49.6 & 62.7 & 76.3 & 88.9 & 96. \\
\hline \multirow[t]{2}{*}{655940} & 2 & 2.0 & 2.9 & 4.0 & 4.1 & 4. & 4.1 & 4.1 & 4.1 & 4. & 4.1 \\
\hline & 10 & 5.4 & 10.3 & 16.4 & 19.7 & 22.3 & 24.2 & 32.0 & 42.2 & 58.2 & 73.7 \\
\hline \multirow[t]{2}{*}{655990} & 2 & 4.2 & 6.2 & 7.5 & 8.2 & 8.3 & 8.4 & 8.4 & 8.4 & 8.4 & 8.4 \\
\hline & & 10.4 & 19.2 & & & 39.3 & 41.6 & 48.1 & 56.8 & 69.5 & \\
\hline
\end{tabular}




\begin{tabular}{|c|c|c|c|c|c|c|c|c|c|c|c|}
\hline \multicolumn{12}{|c|}{$\begin{array}{l}\text { LARS-WG Model } \\
\text { March DRWH Reliability (\%) } \\
\text { per capita roof area and storage } \\
\text { L }\end{array}$} \\
\hline Gauge & sq. m & 0 & 40 & 80 & 120 & 160 & 200 & 400 & 600 & 800 & 1000 \\
\hline \multirow[t]{2}{*}{653060} & 2 & 2.1 & 3.3 & 4.1 & 4.1 & 4.1 & 4.1 & 4.1 & 4.1 & 4.1 & 4.1 \\
\hline & 10 & 5.8 & 10.5 & 16.0 & 19.5 & 22.7 & 24.8 & 31.9 & 34.5 & 37.9 & 44.0 \\
\hline \multirow[t]{2}{*}{653190} & 2 & 2.8 & 4.3 & 4.9 & 4.9 & 4.9 & 4.9 & 4.9 & 4.9 & 4.9 & 4.9 \\
\hline & 10 & 6.9 & 12.4 & 18.4 & 22.0 & 24.7 & 26.1 & 28.5 & 29.0 & 29.4 & 30.2 \\
\hline \multirow[t]{2}{*}{653300} & 2 & 2.6 & 4.1 & 5.0 & 5.1 & 5.1 & 5.1 & 5.1 & 5.1 & 5.1 & 5.1 \\
\hline & 10 & 5.9 & 10.5 & 16.2 & 19.8 & 22.7 & 24.2 & 26.8 & 27.0 & 27.1 & 27.3 \\
\hline \multirow[t]{2}{*}{653350} & 2 & 5.9 & 9.9 & 12.6 & 13.5 & 13.8 & 13.9 & 14.1 & 14.1 & 14.1 & 14.1 \\
\hline & 10 & 10.7 & 19.7 & 31.1 & 38.4 & 44.4 & 48.0 & 57.7 & 61.6 & 62.5 & 62.8 \\
\hline \multirow[t]{2}{*}{653380} & 2 & 5.4 & 8.8 & 10.5 & 10.9 & 11.0 & 11.0 & 11.0 & 11.0 & 11.0 & 11.0 \\
\hline & 10 & 11.8 & 22.1 & 33.5 & 40.2 & 45.3 & 48.1 & 54.2 & 57.7 & 61.7 & 67.7 \\
\hline \multirow[t]{2}{*}{653440} & 2 & 5.8 & 9.6 & 12.5 & 13.8 & 14.4 & 14.5 & 14.6 & 14.6 & 14.6 & 14.6 \\
\hline & 10 & 10.2 & 18.7 & 29.4 & 36.7 & 42.6 & 45.9 & 53.6 & 56.5 & 58.3 & 60.9 \\
\hline \multirow{2}{*}{653520} & 2 & 8.5 & 14.1 & 20.0 & 22.6 & 24.3 & 25.3 & 26.1 & 26.1 & 26.1 & 26.1 \\
\hline & 10 & 13.1 & 24.0 & 38.8 & 47.9 & 54.7 & 58.2 & 65.7 & 70.2 & 75.7 & 82.4 \\
\hline \multirow[t]{2}{*}{653550} & 2 & 4.0 & 5.9 & 7.3 & 8.0 & 8.1 & 8.1 & 8.1 & 8.1 & 8.1 & 8.1 \\
\hline & 10 & 7.7 & 14.3 & 23.2 & 29.2 & 33.9 & 36.5 & 44.8 & 50.3 & 53.6 & 57.7 \\
\hline \multirow[t]{2}{*}{653570} & 2 & 4.8 & 8.1 & 11.3 & 13.1 & 14.3 & 14.8 & 15.2 & 15.2 & 15.2 & 15.2 \\
\hline & 10 & 12.1 & 21.8 & 31.9 & 37.8 & 42.8 & 45.9 & 55.8 & 61.7 & 66.6 & 71.6 \\
\hline \multirow[t]{2}{*}{653610} & 2 & 4.8 & 6.7 & 7.2 & 7.2 & 7.2 & 7.2 & 7.2 & 7.2 & 7.2 & 7.2 \\
\hline & 10 & 9.8 & 18.7 & 29.8 & 36.6 & 41.2 & 43.2 & 46.4 & 47.8 & 49.9 & 53.6 \\
\hline \multirow[t]{2}{*}{653760} & 2 & 5.3 & 8.3 & 10.6 & 11.5 & 11.7 & 11.7 & 11.7 & 11.7 & 11.7 & 11.7 \\
\hline & 10 & 11.9 & 22.1 & 33.5 & 40.2 & 44.7 & 47.3 & 52.6 & 53.9 & 55.5 & 58.5 \\
\hline \multirow[t]{2}{*}{653800} & 2 & 10.4 & 18.5 & 25.5 & 28.9 & 31.3 & 32.9 & 37.5 & 38.3 & 38.4 & 38.4 \\
\hline & 10 & 18.1 & 33.4 & 52.0 & 63.6 & 71.7 & 76.3 & 86.0 & 88.9 & 91.5 & 94.5 \\
\hline \multirow[t]{2}{*}{653870} & 2 & 4.9 & 8.1 & 11.0 & 12.3 & 13.1 & 13.5 & 13.8 & 13.8 & 13.8 & 13.8 \\
\hline & 10 & 10.6 & 19.1 & 29.5 & 36.0 & 41.2 & 43.9 & 49.7 & 51.8 & 52.8 & 53.5 \\
\hline \multirow[t]{2}{*}{654720} & 2 & 5.5 & 10.2 & 13.0 & 13.2 & 13.3 & 13.3 & 13.3 & 13.3 & 13.3 & 13.3 \\
\hline & 10 & 10.4 & 20.1 & 31.1 & 38.2 & 44.7 & 48.8 & 60.3 & 62.9 & 63.9 & 64.9 \\
\hline \multirow[t]{2}{*}{655010} & 2 & 1.6 & 2.8 & 3.1 & 3.1 & 3.1 & 3.1 & 3.1 & 3.1 & 3.1 & 3.1 \\
\hline & 10 & 4.2 & 7.8 & 12.7 & 16.1 & 19.7 & 22.6 & 34.5 & 43.5 & 51.2 & 60.2 \\
\hline \multirow[t]{2}{*}{655020} & 2 & 2.6 & 4.1 & 5.4 & 5.6 & 5.6 & 5.6 & 5.6 & 5.6 & 5.6 & 5.6 \\
\hline & 10 & 5.4 & 9.8 & 15.6 & 20.1 & 24.7 & 27.7 & 41.7 & 51.0 & 54.1 & 54.5 \\
\hline 655050 & 2 & 1.9 & 3.6 & 4.8 & 4.9 & 4.9 & 4.9 & 4.9 & 4.9 & 4.9 & 4.9 \\
\hline & 10 & 6.4 & 11.1 & 16.4 & 19.5 & 22.8 & 25.4 & 36.8 & 42.2 & 44.4 & 47.2 \\
\hline 655070 & 2 & 3.2 & 6.1 & 8.8 & 10.4 & 11.7 & 12.4 & 13.4 & 13.4 & 13.4 & 13.4 \\
\hline & 10 & 6.1 & 10.7 & 16.5 & 20.9 & 25.5 & 28.5 & 39.0 & 43.8 & 45.4 & 47.3 \\
\hline 655100 & 2 & 2.0 & 2.7 & 2.9 & 2.9 & 2.9 & 2.9 & 2.9 & 2.9 & 2.9 & 2.9 \\
\hline & 10 & 4.8 & 8.7 & 13.0 & 15.8 & 17.9 & 18.8 & 21.2 & 22.0 & 22.4 & 22.9 \\
\hline 655160 & 2 & 1.5 & 2.8 & 4.4 & 5.1 & 5.3 & 5.3 & 5.3 & 5.3 & 5.3 & 5.3 \\
\hline & 10 & 6.3 & 10.9 & 15.2 & 17.5 & 19.6 & 20.9 & 24.1 & 24.3 & 24.5 & 26.0 \\
\hline 655180 & 2 & 2.6 & 4.8 & 6.3 & 6.8 & 6.8 & 6.9 & 6.9 & 6.9 & 6.9 & 6.9 \\
\hline & 10 & 7.9 & 14.6 & 21.6 & 25.3 & 28.4 & 30.0 & 32.7 & 32.8 & 32.8 & 33.0 \\
\hline 655220 & 2 & 4.5 & 7.0 & 8.3 & 8.5 & 8.5 & 8.5 & 8.5 & 8.5 & 8.5 & 8.5 \\
\hline & 10 & 9.1 & 16.5 & 26.1 & 31.9 & 36.7 & 39.2 & 44.4 & 46.9 & 50.2 & 55.0 \\
\hline 655280 & 2 & 3.9 & 6.2 & 8.3 & 9.3 & 10.1 & 10.6 & 11.1 & 11.1 & 11.1 & 11.1 \\
\hline & 10 & 8.1 & 15.3 & 24.3 & 29.3 & 33.1 & 35.4 & 42.3 & 46.2 & 51.5 & 61.1 \\
\hline 655360 & 2 & 4.4 & 7.2 & 10.1 & 11.2 & 11.4 & 11.5 & 11.5 & 11.5 & 11.5 & 11.5 \\
\hline & 10 & 9.5 & 17.2 & 26.3 & 32.0 & 37.1 & 40.2 & 50.4 & 54.6 & 56.6 & 59.3 \\
\hline 655450 & 2 & 6.5 & 12.0 & 16.6 & 18.7 & 19.7 & 20.0 & 20.5 & 20.5 & 20.5 & 20.5 \\
\hline & 10 & 11.9 & 21.9 & 33.8 & 41.8 & 48.6 & 52.7 & 63.8 & 68.7 & 72.3 & 76.3 \\
\hline
\end{tabular}




\begin{tabular}{|c|c|c|c|c|c|c|c|c|c|c|c|}
\hline \multirow{2}{*}{\multicolumn{12}{|c|}{$\begin{array}{l}\text { LARS-WG Markov Model } \\
\text { March DRWH Reliability (\%) } \\
\text { per capita roof area and storage }\end{array}$}} \\
\hline & & & & & & & & & & & \\
\hline Gauge & sq. m & 0 & 40 & 80 & 120 & 160 & 200 & 400 & 600 & 800 & 1000 \\
\hline \multirow{2}{*}{655480} & 2 & 9.3 & 15.3 & 20.2 & 22.5 & 23.8 & 24.5 & 25.1 & 25.1 & 25.1 & 25.1 \\
\hline & 10 & 18.3 & 33.3 & 48.9 & 57.0 & 62.4 & 65.5 & 71.5 & 73.3 & 75.5 & 78.6 \\
\hline \multirow[t]{2}{*}{655550} & 2 & 7.8 & 12.4 & 16.7 & 19.5 & 21.5 & 22.5 & 23.4 & 23.4 & 23.4 & 23.4 \\
\hline & 10 & 15.4 & 28.1 & 42.7 & 50.9 & 56.9 & 60.1 & 68.9 & 73.4 & 77.3 & 81.0 \\
\hline \multirow[t]{2}{*}{655570} & 2 & 10.8 & 18.3 & 23.4 & 25.6 & 27.0 & 28.0 & 31.2 & 31.4 & 31.4 & 31.4 \\
\hline & 10 & 22.1 & 39.2 & 56.1 & 65.4 & 72.4 & 76.2 & 84.8 & 87.6 & 89.9 & 92.4 \\
\hline \multirow[t]{2}{*}{655600} & 2 & 7.9 & 12.4 & 15.3 & 16.4 & 17.1 & 17.5 & 17.8 & 17.8 & 17.8 & 17.8 \\
\hline & 10 & 15.3 & 28.1 & 43.1 & 52.6 & 59.7 & 64.1 & 74.7 & 76.7 & 77.4 & 79.0 \\
\hline \multirow[t]{2}{*}{655620} & 2 & 8.7 & 14.4 & 17.8 & 18.5 & 18.6 & 18.6 & 18.6 & 18.6 & 18.6 & 18.6 \\
\hline & 10 & 16.9 & 31.5 & 46.9 & 55.8 & 62.5 & 66.3 & 73.3 & 74.6 & 76.1 & 78.8 \\
\hline \multirow[t]{2}{*}{655630} & 2 & 6.7 & 9.8 & 10.7 & 10.7 & 10.7 & 10.7 & 10.7 & 10.7 & 10.7 & 10.7 \\
\hline & 10 & 16.1 & 30.1 & 44.2 & 51.6 & 56.7 & 59.4 & 64.9 & 67.5 & 70.0 & 73.6 \\
\hline \multirow[t]{2}{*}{655780} & 2 & 7.3 & 12.5 & 16.5 & 17.8 & 18.4 & 18.7 & 19.3 & 19.3 & 19.3 & 19.3 \\
\hline & 10 & 15.3 & 27.6 & 42.0 & 50.2 & 56.0 & 59.2 & 65.6 & 67.4 & 69.3 & 73.3 \\
\hline \multirow[t]{2}{*}{655850} & 2 & 8.2 & 13.0 & 16.0 & 17.5 & 18.7 & 19.4 & 20.2 & 20.2 & 20.2 & 20.2 \\
\hline & 10 & 16.4 & 30.2 & 46.4 & 56.1 & 63.2 & 67.4 & 77.1 & 80.8 & 84.0 & 88.4 \\
\hline \multirow[t]{2}{*}{655920} & 2 & 6.6 & 11.1 & 13.8 & 15.2 & 15.9 & 16.3 & 16.7 & 16.7 & 16.7 & 16.7 \\
\hline & 10 & 14.5 & 27.1 & 41.2 & 49.8 & 56.6 & 60.5 & 70.5 & 76.7 & 83.3 & 90.1 \\
\hline \multirow[t]{2}{*}{655940} & 2 & 4.8 & 7.2 & 9.5 & 10.1 & 10.2 & 10.2 & 10.2 & 10.2 & 10.2 & 10.2 \\
\hline & 10 & 8.9 & 16.6 & 26.8 & 33.6 & 38.1 & 40.6 & 46.7 & 49.2 & 52.6 & 59.2 \\
\hline \multirow[t]{2}{*}{655990} & 2 & 5.6 & 9.0 & 11.8 & 12.4 & 12.6 & 12.6 & 12.6 & 12.6 & 12.6 & 12.6 \\
\hline & 10 & 11.5 & 21.3 & 33.5 & 40.9 & 46.9 & 50.6 & 59.9 & 63.7 & 67.5 & 73.2 \\
\hline
\end{tabular}




\begin{tabular}{|c|c|c|c|c|c|c|c|c|c|c|c|}
\hline \multicolumn{12}{|c|}{$\begin{array}{l}\text { LARS-WG Model } \\
\text { April DRWH Reliability (\%) } \\
\text { per capita roof area and storage } \\
\text { L }\end{array}$} \\
\hline Gauge & sq. m & 0 & 40 & 80 & 120 & 160 & 200 & 400 & 600 & 800 & \\
\hline \multirow[t]{2}{*}{653060} & 2 & 3.6 & 6.0 & 7.8 & 8.2 & 8.3 & 8.3 & 8.3 & 8.3 & 8.3 & \\
\hline & 10 & 7.8 & 14.6 & & 27.5 & 31.5 & 33.6 & 38.7 & 41.0 & 42.6 & \\
\hline \multirow[t]{2}{*}{653190} & 2 & 6.8 & 11.0 & 13.8 & 14.7 & 15.0 & 15.1 & 15.2 & 15.2 & 15.2 & 5. \\
\hline & 10 & 14.7 & 26.6 & 39.1 & 46.5 & 52.1 & 55.1 & 61.4 & 62.4 & 62.5 & 2 \\
\hline \multirow[t]{2}{*}{653300} & 2 & 5.6 & 8.8 & 11.8 & 12.8 & 13.0 & 13.1 & 13.1 & 13.1 & 13.1 & 3 \\
\hline & 10 & 11.5 & 20.9 & 32.1 & 38.9 & 43.6 & 46.3 & 52.2 & 53.5 & 53.6 & 3 \\
\hline \multirow[t]{2}{*}{653350} & 2 & 9.6 & 16.0 & 19.7 & 20.5 & 20.6 & 20.7 & 20.7 & 20.7 & 20.7 & 0 \\
\hline & 10 & 19.4 & 34.9 & 51.9 & 61.7 & 68.2 & 72.0 & 80.3 & 83.1 & 84.2 & \\
\hline \multirow[t]{2}{*}{653380} & 2 & 10.2 & 16.7 & 20.5 & 21.5 & 21.8 & 21.9 & 21.9 & 21.9 & 21.9 & \\
\hline & 10 & 20 & 36.5 & 55.0 & 65.8 & 73.1 & 77.1 & 84.6 & 86.1 & 87.1 & \\
\hline \multirow[t]{2}{*}{653440} & 2 & 11 & 20.1 & & 30.0 & 31.8 & 32.8 & 34.1 & 34.1 & 4.1 & \\
\hline & 10 & 19 & 34 & 52 & 63.6 & 71.4 & 75.6 & 4.8 & 87.0 & 7.7 & \\
\hline \multirow[t]{2}{*}{653520} & 2 & 8.2 & 13.5 & 17.3 & 19.4 & 20.9 & 21.9 & 24.4 & 24.6 & 24.6 & \\
\hline & 10 & 14.6 & 28.9 & 45.5 & 56.2 & 64.7 & 70.1 & 84.4 & 8 & 90.2 & 1 \\
\hline \multirow[t]{2}{*}{653550} & 2 & 8.5 & 12.7 & 14.3 & 14.5 & 14.5 & 14.5 & 14.5 & 14.5 & 14.5 & \\
\hline & 10 & 16.5 & 30.1 & 46.0 & 55.2 & 61.4 & 64.8 & 71.0 & 73.4 & 75.2 & \\
\hline \multirow[t]{2}{*}{653570} & 2 & 7.6 & 12.3 & 15.5 & 16.7 & 17.5 & 17.9 & 18.5 & 18.5 & 18.5 & \\
\hline & 10 & 19.0 & 34.4 & 50.9 & 59.8 & 66.4 & 70.4 & 80.1 & 83.9 & 85.6 & \\
\hline \multirow[t]{2}{*}{653610} & 2 & 7.3 & 12.0 & 15.1 & 16.0 & 16.2 & 16.3 & 16.3 & & 16.3 & \\
\hline & 10 & 18.3 & 33. & 48 & 57.1 & 62.9 & 66 & 72 & & & \\
\hline \multirow[t]{2}{*}{653760} & 2 & & 17.9 & 23 & 26.1 & 26.7 & 26 & 26 & & & \\
\hline & 10 & & 35.9 & & 64.0 & 70.8 & 74.9 & 83.2 & 84.8 & 5.3 & 85 \\
\hline \multirow[t]{2}{*}{653800} & 2 & 11 & 19.4 & 25 & 26.3 & 26.5 & & 26.9 & & & \\
\hline & 10 & 19.6 & 37.1 & 57.3 & 69.0 & 77.5 & 82.5 & 93.2 & .5 & 97.6 & \\
\hline \multirow[t]{2}{*}{653870} & 2 & 8.4 & 14.2 & 19.5 & 22.3 & 23.7 & 24.4 & 25.6 & .7 & 25.7 & 5. \\
\hline & 10 & 15.8 & 28.7 & 44.1 & 54.1 & 61.8 & 66.2 & 77.3 & 80.5 & 81.6 & 8 \\
\hline \multirow[t]{2}{*}{654720} & 2 & 7.6 & 14.5 & 21.4 & 24.8 & 26.5 & 27.1 & 27.3 & 27.3 & 27.3 & 7 \\
\hline & 10 & 15.0 & 27.6 & 41.6 & 50.2 & 58.0 & 62.8 & 76.6 & 81.1 & 82.2 & 8 \\
\hline \multirow[t]{2}{*}{655010} & 2 & 0.8 & 0.9 & 0.9 & 0.9 & 0.9 & 0. & 0.9 & 0. & 0. & \\
\hline & 10 & 3.6 & 6.4 & 9.5 & 11.0 & 12.0 & 12.4 & 14.1 & 17 & 23.7 & 32 \\
\hline \multirow[t]{2}{*}{655020} & 2 & 2.0 & 3.1 & 3. & 3.8 & 3.8 & 3. & & & & \\
\hline & 10 & 5 & 9.3 & 14.8 & 18.3 & 21.2 & 23.0 & 28.4 & & 40.0 & \\
\hline 655050 & 2 & & 4. & & 4.7 & & 4 & & & & 4.7 \\
\hline & 10 & 6 & 12 & 20 & 24.7 & 28 & & 3.5 & & & 44 \\
\hline 655070 & 2 & & 5. & 7. & 7. & & 8. & 10.0 & & 10.3 & U \\
\hline & 10 & 7.8 & 14.1 & 21.7 & 26.5 & 30.8 & 33.6 & 43.3 & 49.8 & 53.6 & 55 \\
\hline 655100 & 2 & 4.0 & 6.1 & 7.0 & 7.1 & 7.2 & 7.2 & 7.2 & 7. & 7.2 & 7.2 \\
\hline & 10 & 9.1 & 16.2 & 24.8 & 30.1 & 34.3 & 36.5 & 40.0 & 40.5 & 40.7 & \\
\hline 655160 & 2 & 3.4 & 5.5 & 7.3 & 8.5 & 9.3 & 9.5 & 9.6 & 9. & 9. & \\
\hline & 10 & 8.8 & 15.8 & 23.9 & 28.8 & 32.8 & 35.0 & 41.3 & 44.6 & 45.2 & \\
\hline 655180 & 2 & 3.6 & 6.2 & 8.4 & 9.3 & 9.4 & 9.5 & 9.5 & 9.5 & 9.5 & \\
\hline & 10 & 9.3 & 17.3 & 26.1 & 31.2 & 35.9 & 38.8 & 47.8 & 51.1 & 51.6 & 51 \\
\hline 655220 & 2 & 6.0 & 10.1 & 12.3 & 12.7 & 12.7 & 12.7 & 12.7 & 12.7 & 12.7 & 12. \\
\hline & 10 & 12.2 & 22.9 & 35.8 & 44.0 & 50.4 & 54.2 & 63.4 & 65.9 & 67.0 & 68 \\
\hline 655280 & 2 & 7.7 & 12. & 15.5 & 17.0 & 18 & 18.5 & 19.5 & 19.6 & .6 & 19 \\
\hline & 10 & 15. & 27.8 & 42.5 & 51.7 & 58.8 & 63.1 & 72.5 & 74.9 & 76.4 & 78 \\
\hline 655360 & 2 & $7 .($ & 11.5 & 14.7 & 15.8 & 16.3 & 16.3 & 16.4 & 16.4 & 16.4 & 16. \\
\hline & 10 & & 28 & 42.5 & 50.9 & 56.7 & 60.1 & 68.6 & 72.4 & 74.2 & 15 \\
\hline j55450 & 2 & 10.8 & 17.7 & 23.7 & 27.0 & 29.0 & 30.2 & 31.7 & 31.7 & 31.7 & 31. \\
\hline & 10 & 19.4 & 35.9 & 54.4 & 64.5 & 71.6 & 75.6 & 85.5 & 89.0 & 90.5 & \\
\hline
\end{tabular}




\begin{tabular}{|c|c|c|c|c|c|c|c|c|c|c|c|}
\hline \multirow{2}{*}{\multicolumn{12}{|c|}{$\begin{array}{l}\text { LARS-WG Markov Model } \\
\text { April DRWH Reliability (\%) } \\
\text { per capita roof area and storage }\end{array}$}} \\
\hline & & & & & & & & & & & \\
\hline Gauge & sq. m & 0 & 40 & 80 & 120 & 160 & 200 & 400 & 600 & 800 & 1000 \\
\hline \multirow{2}{*}{655480} & 2 & 12.0 & 21.4 & 29.1 & 33.1 & 35.4 & 36.8 & 39.3 & 39.4 & 39.4 & 39.4 \\
\hline & 10 & 22.3 & 41.2 & 62.4 & 74.4 & 82.4 & 86.8 & 95.2 & 96.3 & 96.5 & 96.8 \\
\hline \multirow[t]{2}{*}{655550} & 2 & 11.9 & 18.8 & 23.5 & 26.1 & 27.8 & 28.9 & 31.1 & 31.3 & 31.3 & 31.3 \\
\hline & 10 & 21.9 & 39.5 & 60.3 & 72.0 & 80.2 & 84.3 & 92.3 & 94.2 & 95.0 & 95.7 \\
\hline \multirow[t]{2}{*}{655570} & 2 & 13.5 & 22.9 & 30.5 & 34.6 & 37.4 & 39.4 & 44.8 & 46.4 & 46.5 & 46.5 \\
\hline & 10 & 26.3 & 48.0 & 68.7 & 79.1 & 86.1 & 90.0 & 96.9 & 98.2 & 98.6 & 98.9 \\
\hline \multirow[t]{2}{*}{655600} & 2 & 11.5 & 18.7 & 23.5 & 24.9 & 25.6 & 26.1 & 27.2 & 27.2 & 27.2 & 27.2 \\
\hline & 10 & 20.8 & 38.1 & 58.0 & 68.5 & 76.1 & 80.2 & 89.1 & 91.4 & 92.1 & 92.6 \\
\hline \multirow[t]{2}{*}{655620} & 2 & 11.3 & 18.1 & 23.5 & 26.0 & 27.3 & 27.9 & 28.3 & 28.3 & 28.3 & 28.3 \\
\hline & 10 & 20.0 & 36.8 & 56.7 & 67.5 & 75.2 & 79.4 & 88.9 & 91.1 & 91.7 & 92.3 \\
\hline \multirow[t]{2}{*}{655630} & 2 & 9.9 & 15.0 & 17.5 & 18.0 & 18.1 & 18.1 & 18.1 & 18.1 & 18.1 & 18.1 \\
\hline & 10 & 20.7 & 39.1 & 59.3 & 69.4 & 76.7 & 80.8 & 88.4 & 89.6 & 90.2 & 91.1 \\
\hline \multirow[t]{2}{*}{655780} & 2 & 11.5 & 19.6 & 25.2 & 27.8 & 28.9 & 29.6 & 30.4 & 30.4 & 30.4 & 30.4 \\
\hline & 10 & 20.9 & 38.2 & 57.1 & 68.4 & 75.8 & 80.2 & 89.3 & 91.3 & 91.7 & 92.2 \\
\hline \multirow[t]{2}{*}{655850} & 2 & 11.3 & 18.4 & 23.3 & 25.5 & 26.7 & 27.5 & 29.1 & 29.1 & 29.1 & 29.1 \\
\hline & 10 & 21.9 & 40.2 & 60.0 & 71.4 & 79.1 & 83.3 & 91.9 & 93.7 & 94.8 & 95.8 \\
\hline \multirow[t]{2}{*}{655920} & 2 & 10.7 & 19.1 & 25.2 & 27.9 & 29.5 & 30.4 & 31.4 & 31.4 & 31.4 & 31.4 \\
\hline & 10 & 21.8 & 39.4 & 57.3 & 67.5 & 74.7 & 78.7 & 87.4 & 90.5 & 92.5 & 94.6 \\
\hline \multirow[t]{2}{*}{655940} & 2 & 8.8 & 15.0 & 19.5 & 21.1 & 21.8 & 22.1 & 22.3 & 22.3 & 22.3 & 22.3 \\
\hline & 10 & 17.5 & 31.7 & 48.8 & 58.3 & 65.2 & 69.2 & 77.5 & 79.5 & 80.1 & 81.1 \\
\hline \multirow[t]{2}{*}{655990} & 2 & 10.7 & 18.1 & 24.1 & 26.7 & 27.8 & 28.1 & 28.3 & 28.3 & 28.3 & 28.3 \\
\hline & 10 & 19.4 & 35.3 & 53.1 & 63.2 & 70.7 & 74.7 & 83.2 & 85.6 & 86.8 & 88.1 \\
\hline
\end{tabular}




\begin{tabular}{|c|c|c|c|c|c|c|c|c|c|c|c|}
\hline \multicolumn{12}{|c|}{$\begin{array}{l}\text { LARS-WG Model } \\
\text { May DRWH Reliability (\%) } \\
\text { per capita roof area and storage } \\
\text { L }\end{array}$} \\
\hline Gauge & sq. m & 0 & 40 & 80 & 120 & 160 & 200 & 400 & 600 & 800 & \\
\hline \multirow[t]{2}{*}{653060} & 2 & 9.2 & & 19.3 & 20.1 & 20.3 & 20.3 & 20.3 & 20.3 & & \\
\hline & 10 & 16.8 & 30.9 & 47.2 & 56.2 & 62.2 & 65.5 & 72.3 & 73.9 & 74.5 & \\
\hline \multirow[t]{2}{*}{653190} & 2 & 9.3 & 15.1 & 17.9 & 18.6 & 18.8 & 18.9 & 19.0 & 19.0 & 19.0 & \\
\hline & 10 & 20.7 & 37.3 & 54.6 & 64.7 & 72.2 & 76.4 & 85.5 & 87.4 & 87.7 & \\
\hline \multirow[t]{2}{*}{653300} & 2 & 10.7 & 16.7 & 20.1 & 21.5 & 22.1 & 22.5 & 22.7 & 22.7 & 22.7 & \\
\hline & 10 & 22.9 & 41.9 & 61.4 & 71.0 & 76.6 & 79.6 & 85.6 & 86.8 & 87.0 & \\
\hline \multirow[t]{2}{*}{653350} & 2 & 10.5 & 16.7 & 20.1 & 21.1 & 21.4 & 21.5 & 21.5 & 21.5 & 21.5 & \\
\hline & 10 & 21.9 & 40.2 & 60.8 & 72.4 & 80.2 & 84.8 & 94.3 & 96.0 & 96.5 & \\
\hline \multirow[t]{2}{*}{653380} & 2 & 12.4 & 19.9 & 23.4 & 24.7 & 25.1 & 25.2 & 25.2 & 25.2 & 25.2 & \\
\hline & 10 & 23.0 & 41.8 & 62.8 & 74.6 & 82.3 & 86.8 & 95.7 & 97.0 & 97 & \\
\hline \multirow[t]{2}{*}{653440} & 2 & 16 & 29.5 & 40 & 46.3 & 49.8 & 52.1 & 56.4 & 56.8 & 6.8 & \\
\hline & 10 & 27 & 49 & & 82.3 & 88.5 & 91.8 & 97.8 & 3.6 & & \\
\hline \multirow[t]{2}{*}{653520} & 2 & 10 & 17 & & 22.4 & 22.5 & 22.5 & 22.5 & 22.5 & 2.5 & \\
\hline & 10 & 20 & 38 & & 68.2 & 74.7 & 78.4 & 3.0 & .4 & & \\
\hline \multirow[t]{2}{*}{653550} & 2 & 12.2 & 18 & 21 & 21.4 & 21.4 & 21.4 & 1.4 & .4 & & \\
\hline & 10 & 25.1 & 46.1 & 65.7 & 75.9 & 82.5 & 86.2 & 92.9 & 93.9 & & \\
\hline \multirow[t]{2}{*}{653570} & 2 & 10.2 & 16.6 & 20.9 & 22.7 & 23.1 & 23.1 & 23.1 & 23.1 & 23.1 & \\
\hline & 10 & 25.2 & 45.3 & 65.3 & 75.2 & 81.6 & 85.2 & 92.3 & 94.5 & 95.3 & \\
\hline \multirow[t]{2}{*}{653610} & 2 & 10.8 & 17.6 & 21.2 & 22.7 & 23.7 & 24.3 & 24.6 & 24.6 & 24.6 & \\
\hline & 10 & 24.9 & 45.8 & 66.0 & 76.1 & 82.4 & 85.9 & 92.6 & 93.6 & 93.7 & \\
\hline \multirow[t]{2}{*}{653760} & 2 & 12.3 & 21. & 28.6 & 32.9 & 35.8 & 37.6 & 40.5 & 40.6 & & \\
\hline & 10 & & 42 & & 74.4 & & 86.5 & & & & \\
\hline \multirow[t]{2}{*}{653800} & 2 & 14 & 26 & & 38.6 & & 39.7 & .7 & & & \\
\hline & 10 & 23 & 44 & & 78.2 & & 89.9 & .6 & & .5 & \\
\hline \multirow[t]{2}{*}{653870} & 2 & 11.3 & 20 & 27. & 30.4 & 32.4 & 33.5 & 35.8 & & & \\
\hline & 10 & 22.5 & 41.6 & 60.2 & 70.9 & 78.4 & 82.8 & 92.8 & 95.2 & & \\
\hline \multirow[t]{2}{*}{654720} & 2 & 11.0 & 19.3 & 25.5 & 28.0 & 29.4 & 30.2 & 31.2 & 31.2 & 31.2 & \\
\hline & 10 & 19.6 & 35.4 & 51.7 & 61.3 & 69.9 & 74.9 & 87.5 & 92.4 & 93.8 & \\
\hline \multirow[t]{2}{*}{655010} & 2 & 2.4 & 3.5 & $3 . \subseteq$ & 3.9 & 3.9 & 3.9 & 3.9 & 3.9 & & \\
\hline & 10 & 7.0 & 13.1 & 19.3 & 22.6 & 24.9 & 25.8 & 26.9 & 27.0 & 6 & \\
\hline 655020 & 2 & 4.9 & 7.7 & 10.1 & 11.4 & 12.1 & 12.6 & 13.3 & 13.3 & & \\
\hline & 10 & 11. & 20. & 30.0 & 35.8 & 39.9 & 42.4 & 47.6 & 49.1 & 0.5 & \\
\hline 655050 & 2 & 7. & 11 & & 15.4 & 15.6 & 15 & & 6 & & \\
\hline & 10 & 15 & 27.2 & 40.6 & 48.8 & 54.6 & 57.7 & 63.0 & 64.0 & 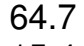 & \\
\hline 655070 & 2 & & & & 14.0 & & & & & & \\
\hline & 10 & 14 & 26 & $3 s$ & 47.2 & 52.5 & 55 & 62 & & & \\
\hline 655100 & 2 & & 10 & 13 & 13 & 14 & 14 & 14.0 & & & \\
\hline & 10 & 17 & 31. & 46.9 & 55.7 & 61.7 & 65.3 & 72.1 & 73.2 & 73.3 & \\
\hline 655160 & 2 & 7.4 & 12.1 & 14.5 & 15.2 & 15.5 & 15.6 & 15.6 & 15.6 & 15.6 & \\
\hline & 10 & 15. & 29.6 & 44.3 & 53.2 & 59.4 & 62.8 & 69.9 & 72.2 & 2 & \\
\hline 655180 & 2 & 6.7 & 10.4 & 12.1 & 12.2 & 12.3 & 12.3 & 12.3 & 12.3 & 12.3 & \\
\hline & 10 & 15.6 & 29.1 & 44.4 & 52.1 & 57.4 & 60.5 & 68.2 & 71.2 & 72.1 & \\
\hline 655220 & 2 & 9.3 & 15.7 & 20.2 & 21.5 & 22.0 & 22.2 & 22.2 & 22.2 & 22.2 & \\
\hline & 10 & 19.1 & 35.2 & 53.4 & 64.0 & 71.2 & 75.4 & 84.7 & 86.8 & 87.5 & \\
\hline 655280 & 2 & 9.0 & 14.5 & 17.8 & 18.6 & 18 & 18.7 & 18.7 & 18.7 & 3.7 & \\
\hline & 10 & 21. & 40.2 & 59.2 & 68.8 & 75.5 & 79.4 & 88.4 & 91.2 & 92.0 & \\
\hline 655360 & 2 & 11 & 18.9 & 23.0 & 24.6 & 25.4 & 25.8 & 26.0 & 26.0 & 26.0 & \\
\hline & 10 & 23.5 & 42.7 & 63.0 & 73.8 & 80.9 & 84.7 & 91.9 & 93.4 & 93.9 & \\
\hline & 2 & 14. & 25.1 & 32.8 & 36.3 & 38.4 & 39.7 & 42.4 & 42.6 & 42.6 & \\
\hline & 10 & 25.9 & 47.1 & 68.3 & 78.8 & 85.9 & 89.8 & 96.8 & 98.3 & 98.7 & \\
\hline
\end{tabular}




\begin{tabular}{|c|c|c|c|c|c|c|c|c|c|c|c|}
\hline \multirow{2}{*}{\multicolumn{12}{|c|}{$\begin{array}{l}\text { LARS-WG Markov Model } \\
\text { May DRWH Reliability (\%) } \\
\text { per capita roof area and storage }\end{array}$}} \\
\hline & & & & & & & & & & & \\
\hline Gauge & sq. m & 0 & 40 & 80 & 120 & 160 & 200 & 400 & 600 & 800 & 1000 \\
\hline \multirow{2}{*}{655480} & 2 & 13.7 & 23.2 & 28.9 & 31.0 & 32.1 & 32.8 & 34.2 & 34.4 & 34.4 & 34.4 \\
\hline & 10 & 25.4 & 46.8 & 67.6 & 79.6 & 86.8 & 90.8 & 98.1 & 99.2 & 99.4 & 99.5 \\
\hline \multirow[t]{2}{*}{655550} & 2 & 11.6 & 19.0 & 23.9 & 25.9 & 27.0 & 27.5 & 28.3 & 28.4 & 28.4 & 28.4 \\
\hline & 10 & 22.5 & 40.9 & 62.3 & 74.2 & 82.5 & 87.2 & 96.8 & 98.4 & 98.9 & 99.0 \\
\hline \multirow[t]{2}{*}{655570} & 2 & 15.5 & 25.6 & 30.5 & 32.2 & 32.8 & 33.1 & 34.2 & 35.4 & 35.9 & 36.0 \\
\hline & 10 & 35.2 & 60.6 & 79.5 & 88.3 & 92.8 & 95.1 & 98.7 & 99.6 & 99.8 & 99.9 \\
\hline \multirow[t]{2}{*}{655600} & 2 & 14.2 & 24.2 & 31.8 & 35.3 & 37.3 & 38.6 & 41.0 & 41.3 & 41.3 & 41.3 \\
\hline & 10 & 25.8 & 47.0 & 68.9 & 80.3 & 87.5 & 91.3 & 97.7 & 98.7 & 98.9 & 98.9 \\
\hline \multirow[t]{2}{*}{655620} & 2 & 13.9 & 22.3 & 27.0 & 28.9 & 29.9 & 30.5 & 31.2 & 31.2 & 31.2 & 31.2 \\
\hline & 10 & 26.4 & 48.0 & 68.7 & 79.9 & 86.5 & 90.1 & 96.7 & 98.2 & 98.5 & 98.6 \\
\hline \multirow[t]{2}{*}{655630} & 2 & 12.2 & 20.3 & 25.0 & 26.6 & 27.2 & 27.3 & 27.3 & 27.3 & 27.3 & 27.3 \\
\hline & 10 & 25.1 & 45.9 & 66.1 & 76.5 & 83.5 & 87.4 & 95.2 & 96.7 & 96.9 & 97.1 \\
\hline \multirow[t]{2}{*}{655780} & 2 & 18.9 & 33.8 & 43.7 & 47.9 & 50.0 & 51.1 & 52.5 & 52.5 & 52.5 & 52.5 \\
\hline & 10 & 32.4 & 56.6 & 76.0 & 85.2 & 90.8 & 93.6 & 98.3 & 99.0 & 99.2 & 99.2 \\
\hline \multirow[t]{2}{*}{655850} & 2 & 18.8 & 33.0 & 41.9 & 46.1 & 48.3 & 49.6 & 51.2 & 51.3 & 51.3 & 51.3 \\
\hline & 10 & 35.0 & 61.2 & 80.4 & 88.5 & 93.0 & 95.3 & 98.9 & 99.3 & 99.4 & 99.5 \\
\hline \multirow[t]{2}{*}{655920} & 2 & 22.3 & 40.8 & 52.6 & 58.1 & 60.8 & 62.2 & 63.4 & 63.4 & 63.4 & 63.4 \\
\hline & 10 & 38.8 & 64.9 & 82.8 & 90.7 & 94.6 & 96.4 & 99.0 & 99.3 & 99.5 & 99.6 \\
\hline \multirow[t]{2}{*}{655940} & 2 & 16.9 & 29.8 & 37.9 & 41.5 & 43.4 & 44.4 & 45.8 & 45.8 & 45.8 & 45.8 \\
\hline & 10 & 29.9 & 52.1 & 70.2 & 79.8 & 86.1 & 89.3 & 95.6 & 96.7 & 97.0 & 97.2 \\
\hline \multirow[t]{2}{*}{655990} & 2 & 18.5 & 33.3 & 42.9 & 47.4 & 49.8 & 51.1 & 52.2 & 52.2 & 52.2 & 52.2 \\
\hline & 10 & 33.5 & 56.7 & 75.6 & 84.8 & 90.3 & 93.0 & 97.9 & 98.6 & 98.8 & 98.9 \\
\hline
\end{tabular}




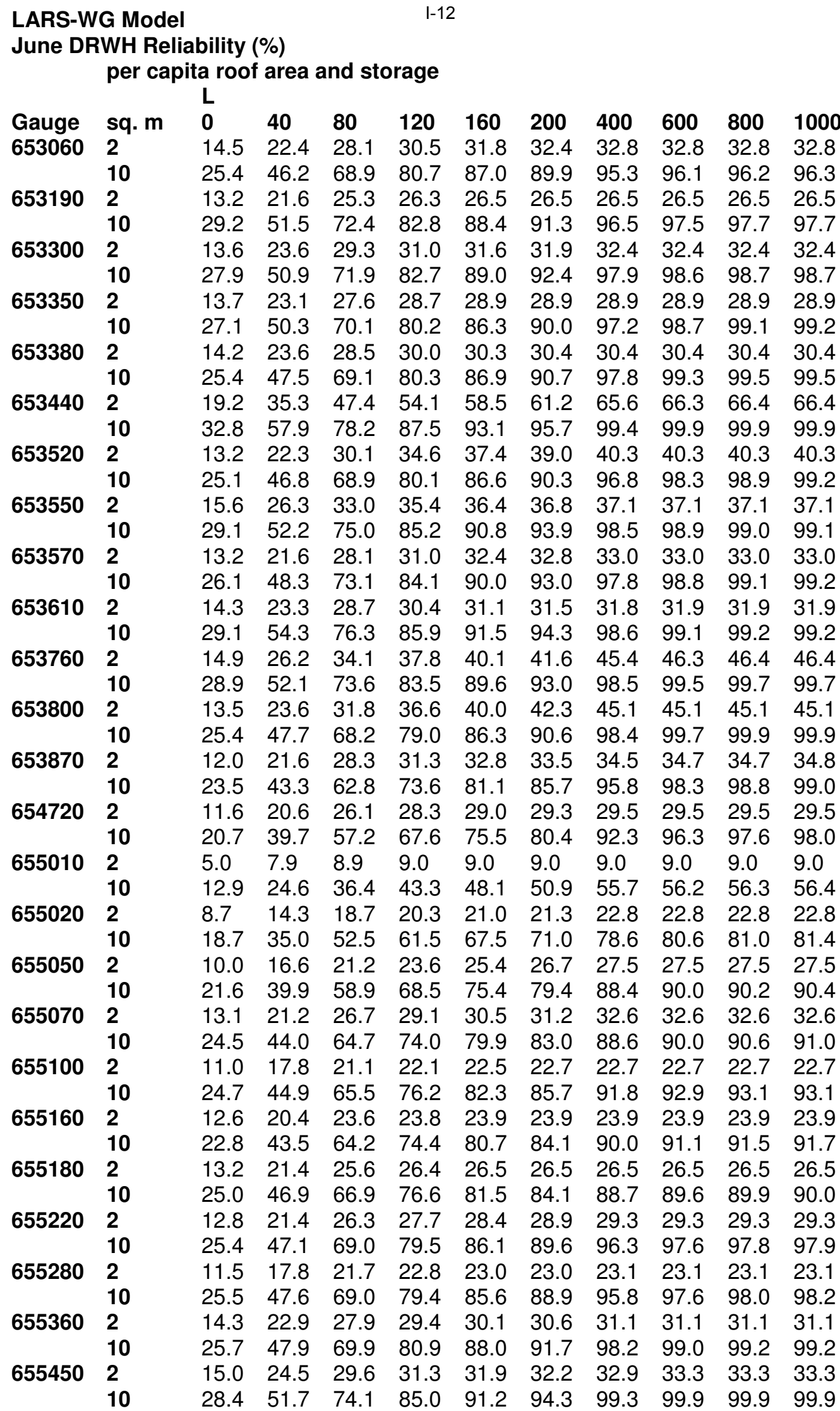




\begin{tabular}{|c|c|c|c|c|c|c|c|c|c|c|c|}
\hline \multicolumn{12}{|c|}{$\begin{array}{l}\text { LARS-WG Markov Model } \\
\text { June DRWH Reliability (\%) } \\
\text { per capita roof area and storage } \\
\text { L }\end{array}$} \\
\hline Gauge & sa. $\mathrm{m}$ & 0 & 40 & 80 & 120 & 160 & 200 & 400 & 600 & 800 & 1000 \\
\hline \multirow[t]{2}{*}{655480} & 2 & 16.9 & 29.0 & 36.6 & 39.6 & 41.2 & 42.0 & 42.8 & 42.8 & 42.9 & 42.9 \\
\hline & 10 & 30.5 & 55.3 & 77.4 & 87.8 & 93.1 & 95.7 & 99.4 & 99.9 & 99.9 & 100.0 \\
\hline \multirow[t]{2}{*}{655550} & 2 & 12.6 & 20.9 & 25.1 & 26.5 & 27.0 & 27.0 & 27.1 & 27.1 & 27.1 & 27.1 \\
\hline & 10 & 24.1 & 45.0 & 65.5 & 76.4 & 83.7 & 88.0 & 97.2 & 99.2 & 99.5 & 99.6 \\
\hline \multirow[t]{2}{*}{655570} & 2 & 13.8 & 23.9 & 29.1 & 30.9 & 31.6 & 31.8 & 31.9 & 32.0 & 32.2 & 32.3 \\
\hline & 10 & 33.7 & 59.8 & 78.8 & 87.9 & 93.4 & 96.2 & 99.7 & 100.0 & 100.0 & 100.0 \\
\hline \multirow[t]{2}{*}{655600} & 2 & 12.6 & 22.3 & 29.9 & 33.9 & 36.5 & 38.1 & 40.2 & 40.5 & 40.5 & 40.5 \\
\hline & 10 & 26.2 & 47.5 & 67.9 & 79.8 & 87.4 & 91.8 & 98.9 & 99.7 & 99.9 & 99.9 \\
\hline \multirow[t]{2}{*}{655620} & 2 & 13.6 & 23.0 & 28.6 & 30.7 & 31.8 & 32.5 & 33.5 & 33.5 & 33.5 & 33.5 \\
\hline & 10 & 28.4 & 51.5 & 72.7 & 83.3 & 89.7 & 93.4 & 99.1 & 99.8 & 99.9 & 99.9 \\
\hline \multirow[t]{2}{*}{655630} & 2 & 13.6 & 22.1 & 27.0 & 29.4 & 30.8 & 31.7 & 32.9 & 32.9 & 32.9 & 32.9 \\
\hline & 10 & 27.0 & 48.1 & 68.7 & 80.2 & 87.0 & 90.9 & 98.0 & 99.1 & 99.3 & 99.4 \\
\hline \multirow[t]{2}{*}{655780} & 2 & 23.4 & 44.4 & 58.3 & 65.3 & 69.6 & 72.0 & 75.2 & 75.4 & 75.4 & 75.4 \\
\hline & 10 & 39.3 & 67.0 & 83.8 & 90.4 & 94.8 & 96.9 & 99.9 & 100.0 & 100.0 & 100.0 \\
\hline \multirow[t]{2}{*}{655850} & 2 & 24.2 & 44.9 & 57.8 & 64.4 & 68.2 & 70.3 & 73.2 & 73.2 & 73.2 & 73.2 \\
\hline & 10 & 43.2 & 72.3 & 88.6 & 95.1 & 98.1 & 99.2 & 100.0 & 100.0 & 100.0 & 100.0 \\
\hline \multirow[t]{2}{*}{655920} & 2 & 23.9 & 44.9 & 59.6 & 67.6 & 72.7 & 76.0 & 80.5 & 80.7 & 80.7 & 80.7 \\
\hline & 10 & 39.7 & 69.3 & 88.3 & 94.9 & 97.9 & 99.2 & 100.0 & 100.0 & 100.0 & 100.0 \\
\hline \multirow[t]{2}{*}{655940} & 2 & 19.6 & 36.3 & 47.0 & 53.1 & 57.0 & 59.6 & 64.2 & 64.5 & 64.5 & 64.5 \\
\hline & 10 & 36.6 & 64.3 & 82.6 & 90.6 & 94.7 & 96.6 & 99.4 & 99.8 & 99.9 & 99.9 \\
\hline \multirow[t]{2}{*}{655990} & 2 & 19.8 & 36.5 & 47.9 & 53.7 & 57.3 & 59.7 & 62.9 & 63.0 & 63.0 & 63.0 \\
\hline & & 35.5 & 62.3 & 81.4 & 89.8 & 94.4 & 96.5 & 99.7 & 100.0 & 100.0 & \\
\hline
\end{tabular}




\begin{tabular}{|c|c|c|c|c|c|c|c|c|c|c|c|}
\hline \multicolumn{12}{|c|}{$\begin{array}{l}\text { LARS-WG Model } \\
\text { July DRWH Reliability (\%) } \\
\text { per capita roof area and storage } \\
\text { L }\end{array}$} \\
\hline Gauge & sq. m & 0 & 40 & 80 & 120 & 160 & 200 & 400 & 600 & 800 & 1000 \\
\hline \multirow[t]{2}{*}{653060} & 2 & 18.5 & 31.0 & 38.0 & 40.3 & 41.4 & 42.0 & 42.7 & 42.7 & 42.7 & 42.7 \\
\hline & 10 & 32.5 & 58.2 & 80.7 & 89.9 & 94.5 & 96.6 & 99.5 & 99.8 & 99.8 & 99.9 \\
\hline \multirow[t]{2}{*}{653190} & 2 & 18.0 & 31.1 & 37.2 & 38.7 & 39.0 & 39.1 & 39.1 & 39.1 & 39.1 & 39.1 \\
\hline & 10 & 33.5 & 60.5 & 81.6 & 90.9 & 95.2 & 97.2 & 99.6 & 99.8 & 99.8 & 99.8 \\
\hline \multirow[t]{2}{*}{653300} & 2 & 16.8 & 29.1 & 37.1 & 40.7 & 42.4 & 43.3 & 44.3 & 44.3 & 44.3 & 44.3 \\
\hline & 10 & 31.5 & 55.7 & 76.8 & 87.0 & 92.4 & 95.2 & 99.3 & 99.8 & 99.8 & 99.8 \\
\hline \multirow[t]{2}{*}{653350} & 2 & 13.1 & 24.1 & 31.7 & 35.0 & 36.5 & 37.1 & 37.5 & 37.5 & 37.5 & 37.5 \\
\hline & 10 & 24.9 & 45.9 & 65.7 & 78.2 & 85.8 & 90.4 & 98.5 & 99.6 & 99.7 & 99.8 \\
\hline \multirow[t]{2}{*}{653380} & 2 & 12.9 & 22.7 & 29.5 & 32.4 & 33.5 & 33.8 & 34.0 & 34.0 & 34.0 & 34.0 \\
\hline & 10 & 23.7 & 44.6 & 65.1 & 76.3 & 83.8 & 88.4 & 97.5 & 99.4 & 99.8 & 99.9 \\
\hline \multirow[t]{2}{*}{653440} & 2 & 8.5 & 15.2 & 20.9 & 24.5 & 27.6 & 30.0 & 38.2 & 40.9 & 41.3 & 41.3 \\
\hline & 10 & 17.0 & 31.9 & 48.8 & 59.1 & 68.2 & 74.6 & 93.2 & 99.1 & 99.9 & 100.0 \\
\hline \multirow[t]{2}{*}{653520} & 2 & 15.4 & 25.9 & 33.8 & 37.1 & 39.3 & 41.0 & 44.3 & 44.5 & 44.5 & 44.5 \\
\hline & 10 & 27.2 & 51.7 & 74.4 & 84.4 & 90.5 & 93.9 & 99.1 & 99.7 & 99.8 & 99.9 \\
\hline \multirow[t]{2}{*}{653550} & 2 & 19.1 & 35.3 & 46.4 & 51.0 & 53.1 & 54.2 & 55.1 & 55.2 & 55.2 & 55.2 \\
\hline & 10 & 33.3 & 60.7 & 82.0 & 91.0 & 95.2 & 97.3 & 99.8 & 99.9 & 99.9 & 99.9 \\
\hline \multirow[t]{2}{*}{653570} & 2 & 15.5 & 24.6 & 29.3 & 30.9 & 31.6 & 31.9 & 32.1 & 32.1 & 32.1 & 32.1 \\
\hline & 10 & 30.0 & 56.2 & 78.7 & 88.3 & 93.3 & 96.1 & 99.5 & 99.8 & 99.9 & 99.9 \\
\hline \multirow[t]{2}{*}{653610} & 2 & 17.9 & 31.1 & 38.2 & 41.0 & 42.2 & 42.7 & 42.9 & 42.9 & 42.9 & 42.9 \\
\hline & 10 & 36.6 & 64.8 & 84.9 & 92.3 & 96.1 & 97.9 & 99.8 & 99.9 & 99.9 & 99.9 \\
\hline \multirow[t]{2}{*}{653760} & 2 & 15.4 & 27.3 & 35.0 & 38.6 & 40.5 & 41.6 & 44.0 & 44.8 & 45.1 & 45.1 \\
\hline & 10 & 30.9 & 55.0 & 75.3 & 85.4 & 91.2 & 94.4 & 99.5 & 99.9 & 100.0 & 100.0 \\
\hline \multirow[t]{2}{*}{653800} & 2 & 8.6 & 15.3 & 20.9 & 23.5 & 25.0 & 26.1 & 29.5 & 30.1 & 30.2 & 30.2 \\
\hline & 10 & 17.4 & 33.1 & 51.2 & 61.9 & 70.2 & 76.1 & 93.0 & 98.4 & 99.7 & 99.9 \\
\hline \multirow[t]{2}{*}{653870} & 2 & 6.7 & 12.0 & 15.9 & 17.8 & 18.8 & 19.1 & 19.4 & 19.5 & 19.5 & 19.5 \\
\hline & 10 & 14.2 & 26.8 & 42.0 & 52.4 & 61.3 & 67.6 & 87.6 & 96.2 & 98.5 & 99.1 \\
\hline \multirow[t]{2}{*}{654720} & 2 & 6.0 & 11.1 & 15.3 & 17.7 & 19.4 & 20.4 & 21.5 & 21.6 & 21.6 & 21.6 \\
\hline & 10 & 12.2 & 23.8 & 37.6 & 47.1 & 55.9 & 62.4 & 84.2 & 94.3 & 97.4 & 98.3 \\
\hline \multirow[t]{2}{*}{655010} & 2 & 9.9 & 16.3 & 19.3 & 19.8 & 19.9 & 19.9 & 19.9 & 19.9 & 19.9 & 19.9 \\
\hline & 10 & 20.0 & 38.2 & 57.1 & 66.8 & 72.7 & 76.3 & 82.7 & 83.5 & 83.6 & 83.6 \\
\hline \multirow[t]{2}{*}{655020} & 2 & 13.7 & 23.5 & 30.0 & 32.3 & 33.5 & 34.0 & 34.6 & 34.6 & 34.6 & 34.6 \\
\hline & 10 & 26.2 & 48.2 & 69.2 & 79.7 & 86.0 & 89.3 & 94.9 & 96.3 & 96.5 & 96.6 \\
\hline 655050 & 2 & & 27.2 & 33 & 35.7 & 37.0 & 37.9 & 39.4 & 39.4 & 39.4 & 39.4 \\
\hline & 10 & 30.3 & 54.8 & 75.5 & 84.4 & 89.9 & 92.7 & 97.5 & 98.5 & 98.6 & 98.6 \\
\hline 655070 & 2 & 15.1 & 27.1 & 35.3 & 38.2 & 39.6 & 40.4 & 42.0 & 42.0 & 42.0 & 42.0 \\
\hline & 10 & 30.0 & 54.5 & 76.1 & 85.6 & 91.1 & 93.9 & 97.7 & 98.5 & 98.6 & 98.7 \\
\hline 655100 & 2 & 15.5 & 25.4 & 29.8 & 30.9 & 31.3 & 31.6 & 31.8 & 31.8 & 31.8 & 31.8 \\
\hline & 10 & 30.3 & 55.3 & 77.3 & 87.1 & 92.5 & 95.2 & 98.9 & 99.3 & 99.3 & 99.3 \\
\hline 655160 & 2 & 15.6 & 26.6 & 33.8 & 36.8 & 38.2 & 38.9 & 39.2 & 39.2 & 39.2 & 39.2 \\
\hline & 10 & 29.4 & 53.7 & 76.4 & 86.6 & 91.9 & 94.6 & 98.3 & 98.6 & 98.7 & 98.7 \\
\hline 655180 & 2 & 17.3 & 30.1 & 39.4 & 43.1 & 44.9 & 45.7 & 46.2 & 46.2 & 46.2 & 46.2 \\
\hline & 10 & 29.3 & 53.7 & 76.8 & 87.1 & 92.1 & 94.5 & 97.6 & 97.9 & 97.9 & 97.9 \\
\hline 655220 & 2 & 16.2 & 28.0 & 34.6 & 36.7 & 37.6 & 38.1 & 38.6 & 38.7 & 38.7 & 38.7 \\
\hline & 10 & 31.6 & 57.4 & 79.8 & 88.4 & 93.2 & 95.7 & 99.3 & 99.7 & 99.7 & 99.8 \\
\hline 655280 & 2 & 17.6 & 29.3 & 36.6 & 39.8 & 40.9 & 41.1 & 41.2 & 41.2 & 41.2 & 41.2 \\
\hline & 10 & 34.5 & 61.0 & 82.1 & 90.8 & 94.9 & 96.9 & 99.4 & 99.8 & 99.8 & 99.8 \\
\hline 655360 & 2 & 15.8 & 26.7 & 33.6 & 35.6 & 36.3 & 36.5 & 36.7 & 36.7 & 36.7 & 36.7 \\
\hline & 10 & 27.2 & 49.6 & 73.0 & 83.6 & 90.1 & 93.5 & 99.0 & 99.8 & 99.9 & 99.9 \\
\hline 655450 & 2 & 9.6 & 16.2 & 20.6 & 22.2 & 22.7 & 22.9 & 23.0 & 23.0 & 23.0 & 23.0 \\
\hline & 10 & 19.2 & 36.3 & 55.1 & 66.8 & 75.2 & 80.7 & 95.5 & 99.4 & 99.9 & 99.9 \\
\hline
\end{tabular}




\begin{tabular}{|c|c|c|c|c|c|c|c|c|c|c|c|}
\hline \multirow{2}{*}{\multicolumn{12}{|c|}{$\begin{array}{l}\text { LARS-WG Markov Model } \\
\text { July DRWH Reliability (\%) } \\
\text { per capita roof area and storage } \\
\text { L }\end{array}$}} \\
\hline & & & & & & & & & & & \\
\hline Gauge & sq. m & 0 & 40 & 80 & 120 & 160 & 200 & 400 & 600 & 800 & 1000 \\
\hline \multirow{2}{*}{655480} & 2 & 17.4 & 29.8 & 36.7 & 40.1 & 41.9 & 43.1 & 45.6 & 45.7 & 45.7 & 45.7 \\
\hline & 10 & 33.6 & 60.0 & 80.5 & 89.9 & 94.5 & 96.9 & 99.8 & 100.0 & 100.0 & 100.0 \\
\hline \multirow[t]{2}{*}{655550} & 2 & 12.9 & 22.1 & 29.1 & 32.3 & 34.1 & 35.3 & 36.7 & 36.7 & 36.7 & 36.7 \\
\hline & 10 & 21.7 & 40.3 & 60.5 & 72.4 & 80.6 & 85.6 & 96.9 & 99.4 & 99.8 & 99.9 \\
\hline \multirow[t]{2}{*}{655570} & 2 & 6.3 & 10.4 & 12.2 & 12.6 & 12.7 & 12.7 & 12.8 & 12.8 & 12.8 & 12.8 \\
\hline & 10 & 18.9 & 35.2 & 50.7 & 60.9 & 69.5 & 75.8 & 94.8 & 99.5 & 100.0 & 100.0 \\
\hline \multirow[t]{2}{*}{655600} & 2 & 8.7 & 15.2 & 19.3 & 21.4 & 22.6 & 23.5 & 26.1 & 26.7 & 26.7 & 26.7 \\
\hline & 10 & 18.9 & 35.7 & 52.1 & 63.3 & 72.0 & 78.2 & 94.9 & 99.1 & 99.8 & 99.9 \\
\hline \multirow[t]{2}{*}{655620} & 2 & 7.2 & 12.3 & 16.0 & 17.8 & 18.9 & 19.6 & 21.2 & 21.4 & 21.4 & 21.4 \\
\hline & 10 & 17.9 & 34.6 & 51.3 & 61.8 & 70.2 & 76.3 & 94.3 & 99.1 & 99.8 & 99.9 \\
\hline \multirow[t]{2}{*}{655630} & 2 & 8.2 & 13.4 & 16.8 & 18.3 & 19.2 & 19.8 & 22.1 & 22.3 & 22.3 & 22.3 \\
\hline & 10 & 17.3 & 32.6 & 49.0 & 59.7 & 68.2 & 74.2 & 92.5 & 98.3 & 99.4 & 99.5 \\
\hline \multirow[t]{2}{*}{655780} & 2 & 8.2 & 15.9 & 23.0 & 28.0 & 32.3 & 35.9 & 47.3 & 50.2 & 50.4 & 50.4 \\
\hline & 10 & 18.2 & 33.6 & 50.4 & 61.8 & 71.3 & 77.9 & 95.7 & 99.7 & 100.0 & 100.0 \\
\hline \multirow[t]{2}{*}{655850} & 2 & 10.9 & 20.8 & 28.5 & 33.9 & 38.3 & 41.8 & 51.9 & 53.5 & 53.6 & 53.6 \\
\hline & 10 & 23.9 & 44.6 & 62.4 & 74.1 & 82.6 & 88.0 & 99.0 & 100.0 & 100.0 & 100.0 \\
\hline \multirow[t]{2}{*}{655920} & 2 & 13.4 & 24.4 & 33.9 & 40.0 & 45.2 & 49.8 & 65.0 & 68.6 & 68.8 & 68.8 \\
\hline & 10 & 29.0 & 52.0 & 72.2 & 82.5 & 89.3 & 93.2 & 99.6 & 100.0 & 100.0 & 100.0 \\
\hline \multirow[t]{2}{*}{655940} & 2 & 9.9 & 18.5 & 25.0 & 29.1 & 32.4 & 34.9 & 42.3 & 44.3 & 44.5 & 44.5 \\
\hline & 10 & 23.4 & 42.8 & 61.3 & 72.7 & 81.1 & 86.6 & 98.3 & 99.9 & 100.0 & 100.0 \\
\hline \multirow[t]{2}{*}{655990} & 2 & 7.6 & 14.3 & 19.6 & 23.1 & 25.8 & 27.9 & 33.2 & 34.2 & 34.3 & 34.3 \\
\hline & 10 & 18.3 & 34.3 & 50.9 & 62.0 & 71.4 & 78.0 & 95.6 & 99.6 & 100.0 & 100.0 \\
\hline
\end{tabular}




\begin{tabular}{|c|c|c|c|c|c|c|c|c|c|c|c|}
\hline \multicolumn{12}{|c|}{$\begin{array}{l}\text { LARS-WG Model } \\
\text { August DRWH Reliability (\%) } \\
\text { per capita roof area and storage } \\
\text { L }\end{array}$} \\
\hline Gauge & sq. m & 0 & 40 & 80 & 120 & 160 & 200 & 400 & 600 & 800 & 1000 \\
\hline \multirow[t]{2}{*}{653060} & 2 & 19.1 & 34.2 & 42.1 & 45.0 & 46.4 & 47.2 & 48.5 & 48.5 & 48.5 & 48.5 \\
\hline & 10 & 34.8 & 62.6 & 83.5 & 92.0 & 96.2 & 98.1 & 99.9 & 100.0 & 100.0 & 100.0 \\
\hline \multirow[t]{2}{*}{653190} & 2 & 22.9 & 40.6 & 50.9 & 56.2 & 59.2 & 60.8 & 62.1 & 62.1 & 62.1 & 62.1 \\
\hline & 10 & 40.8 & 71.0 & 89.2 & 95.7 & 98.1 & 99.1 & 100.0 & 100.0 & 100.0 & 100.0 \\
\hline \multirow[t]{2}{*}{653300} & 2 & 18.7 & 33.6 & 42.5 & 46.8 & 49.3 & 50.8 & 53.5 & 53.7 & 53.7 & 53.7 \\
\hline & 10 & 35.5 & 62.7 & 83.0 & 91.6 & 95.7 & 97.6 & 99.9 & 100.0 & 100.0 & 100.0 \\
\hline \multirow[t]{2}{*}{653350} & 2 & 12.4 & 20.7 & 25.6 & 27.7 & 28.8 & 29.6 & 30.8 & 30.8 & 30.8 & 30.8 \\
\hline & 10 & 26.0 & 46.9 & 67.0 & 77.5 & 84.8 & 89.2 & 98.0 & 99.6 & 99.9 & 99.9 \\
\hline \multirow[t]{2}{*}{653380} & 2 & 9.6 & 16.8 & 21.4 & 23.4 & 24.0 & 24.3 & 24.6 & 24.6 & 24.6 & 24.6 \\
\hline & 10 & 20.7 & 38.8 & 56.9 & 68.4 & 76.4 & 81.7 & 95.2 & 98.9 & 99.7 & 99.8 \\
\hline \multirow[t]{2}{*}{653440} & 2 & 6.5 & 11.4 & 15.5 & 17.7 & 19.1 & 20.0 & 21.9 & 22.8 & 23.4 & 23.6 \\
\hline & 10 & 13.3 & 25.1 & 37.4 & 45.4 & 51.9 & 56.5 & 73.4 & 87.3 & 96.0 & 99.2 \\
\hline \multirow[t]{2}{*}{653520} & 2 & 14.8 & 25.8 & 32.4 & 35.6 & 37.6 & 38.9 & 41.8 & 42.3 & 42.3 & 42.3 \\
\hline & 10 & 29.0 & 55.1 & 76.6 & 86.6 & 91.8 & 94.7 & 99.5 & 99.9 & 100.0 & 100.0 \\
\hline \multirow[t]{2}{*}{653550} & 2 & 24.3 & 43.5 & 55.0 & 61.8 & 66.5 & 69.4 & 72.8 & 72.8 & 72 & 72.8 \\
\hline & 10 & 43.5 & 74.8 & 91.5 & 96.4 & 98.4 & 99.3 & 100.0 & 100.0 & 100.0 & 100.0 \\
\hline \multirow[t]{2}{*}{653570} & 2 & 15.1 & 26.2 & 31.4 & 33.4 & 34.3 & 34.7 & 34.8 & 34.9 & 34.9 & 34.9 \\
\hline & 10 & 32.3 & 57.9 & 78.4 & 87.9 & 93.2 & 95.9 & 99.7 & 99.9 & 100.0 & 100.0 \\
\hline \multirow[t]{2}{*}{653610} & 2 & 19.0 & 34.1 & 42.2 & 45.7 & 47.6 & 48.7 & 50.0 & 50.1 & 50.1 & 50.1 \\
\hline & 10 & 37.6 & 66.4 & 86.3 & 93.9 & 97.4 & 98.8 & 100.0 & 100.0 & 100.0 & 100.0 \\
\hline \multirow[t]{2}{*}{653760} & 2 & 15.3 & 27.1 & 34.7 & 37.9 & 39.6 & 40.7 & 42.0 & 42.3 & 42.5 & 42.5 \\
\hline & 10 & 30.5 & 55.5 & 76.5 & 86.5 & 91.8 & 94.8 & 99.5 & 99.9 & 100.0 & 100.0 \\
\hline \multirow[t]{2}{*}{653800} & 2 & 6.7 & 11.8 & 16.2 & 18.8 & 20.4 & 21.3 & 22.7 & 22.9 & 23.0 & 23.0 \\
\hline & 10 & 15.8 & 29.6 & 43.4 & 52.2 & 59.3 & 64.6 & 82.1 & 92.3 & 97.5 & 99.4 \\
\hline \multirow[t]{2}{*}{653870} & 2 & 3.4 & 5.6 & 7.0 & 7.6 & 7.9 & 7.9 & 7. & 7.9 & 7 & 7.9 \\
\hline & 10 & 9.0 & 16.9 & 25.9 & 31.7 & 36.8 & 40.7 & 58.1 & 75.6 & 89.0 & 95.4 \\
\hline \multirow[t]{2}{*}{654720} & 2 & 2.7 & 4.3 & 5.3 & 5.8 & 6.1 & 6.3 & 6.7 & 6.8 & 6. & 6.8 \\
\hline & 10 & 7.1 & 14.3 & 21.7 & 26.4 & 30.5 & 33.7 & 49.2 & 67.1 & 82.8 & 91.6 \\
\hline \multirow[t]{2}{*}{655010} & 2 & 14.5 & 24.0 & 28.7 & 29.9 & 30.2 & 30.3 & 30.3 & 30.3 & 30.3 & 30.3 \\
\hline & 10 & 26.0 & 49.4 & 71.4 & 81.5 & 87.7 & 91.1 & 96.8 & 97.4 & 97.5 & 97.5 \\
\hline \multirow[t]{2}{*}{655020} & 2 & 16.2 & 26.7 & 31.9 & 33.6 & 34.3 & 34.4 & 34.5 & 34.5 & 34.5 & 34.5 \\
\hline & 10 & 31.5 & 57.6 & 79.1 & 88.7 & 93.6 & 96.2 & 99.5 & 99.8 & 99.8 & 99.8 \\
\hline 655050 & 2 & 20.2 & 33.6 & 40.3 & 42.3 & 42.8 & 43.0 & 43.3 & 43.3 & 43.4 & 43.4 \\
\hline & 10 & 35.8 & 65.2 & 86.2 & 93.9 & 97.0 & 98.3 & 99.8 & 99.9 & 100.0 & 100.0 \\
\hline 655070 & 2 & 17.2 & 30 & 39. & 42.1 & 43. & 44.6 & 45 & 45 & & 45.5 \\
\hline & 10 & 35.7 & 63.7 & 85.2 & 92.8 & 96.3 & 98.0 & 99.8 & 100.0 & 100.0 & 100.0 \\
\hline 655100 & 2 & 23.1 & 39. & 47.7 & 50.4 & 51.4 & 51.9 & 52.5 & 52.5 & 52 & 52.5 \\
\hline & 10 & 39.4 & 68.1 & 88.2 & 94.8 & 97.6 & 98.8 & 99.9 & 99.9 & 100.0 & 100.0 \\
\hline 655160 & 2 & 20.7 & 35.4 & 43.1 & 46.0 & 47.3 & 48.0 & 48.8 & 48.8 & 48.8 & 48.8 \\
\hline & 10 & 38.2 & 67.3 & 87.8 & 94.3 & 97.5 & 98.7 & 99.9 & 100.0 & 100.0 & 100.0 \\
\hline 655180 & 2 & 22.7 & 38.7 & 49.1 & 53.7 & 56.2 & 57.6 & 59.4 & 59.4 & 59.4 & 59.4 \\
\hline & 10 & 38.3 & 67.2 & 88.7 & 95.4 & 98.1 & 99.0 & 99.9 & 99.9 & 100.0 & 100.0 \\
\hline 655220 & 2 & 18.5 & 31.9 & 39.1 & 41.9 & 43.0 & 43.7 & 44.1 & 44.1 & 44.1 & 44.1 \\
\hline & 10 & 35.3 & 63.0 & 85.3 & 93.4 & 96.9 & 98.4 & 99.9 & 100.0 & 100.0 & 100.0 \\
\hline 655280 & 2 & 22.8 & 38.5 & 46.8 & 50.5 & 52.6 & 53.6 & 54.4 & 54.4 & 54.4 & 54.4 \\
\hline & 10 & 42.8 & 72.4 & 91.3 & 96.5 & 98.5 & 99.3 & 100.0 & 100.0 & 100.0 & 100.0 \\
\hline 655360 & 2 & 19.0 & 31.8 & 40.2 & 43.3 & 44.8 & 45.5 & 45.9 & 45.9 & 45.9 & 45.9 \\
\hline & 10 & 33.1 & 58.6 & 81.7 & 90.0 & 94.5 & 96.6 & 99.6 & 99.9 & 100.0 & 100.0 \\
\hline & 2 & 10.8 & 17.9 & 22.9 & 25.6 & 27.1 & 27.8 & 28.2 & 28.2 & 28.2 & 28.2 \\
\hline & 10 & 19.4 & 36.7 & 55.2 & 65.9 & 73.4 & 78.0 & 89.5 & 95.7 & 98.9 & 99.8 \\
\hline
\end{tabular}




\begin{tabular}{|c|c|c|c|c|c|c|c|c|c|c|c|}
\hline \multirow{2}{*}{\multicolumn{12}{|c|}{$\begin{array}{l}\text { LARS-WG Markov Model } \\
\text { August DRWH Reliability (\%) } \\
\text { per capita roof area and storage }\end{array}$}} \\
\hline & & & & & & & & & & & \\
\hline Gauge & sq. m & 0 & 40 & 80 & 120 & 160 & 200 & 400 & 600 & 800 & 1000 \\
\hline \multirow{2}{*}{655480} & 2 & 22.2 & 40.5 & 51.0 & 55.9 & 58.3 & 59.6 & 61.8 & 62.0 & 62.0 & 62.0 \\
\hline & 10 & 42.3 & 73.3 & 90.9 & 96.2 & 98.3 & 99.1 & 100.0 & 100.0 & 100.0 & 100.0 \\
\hline \multirow[t]{2}{*}{655550} & 2 & 12.1 & 21.1 & 27.6 & 30.4 & 32.0 & 33.0 & 35.5 & 35.7 & 35.8 & 35.8 \\
\hline & 10 & 23.5 & 42.9 & 63.0 & 73.7 & 80.8 & 85.3 & 96.0 & 99.0 & 99.8 & 99.9 \\
\hline \multirow[t]{2}{*}{655570} & 2 & 7.0 & 11.6 & 13.5 & 14.0 & 14.1 & 14.1 & 14.1 & 14.1 & 14.1 & 14.1 \\
\hline & 10 & 19.0 & 35.6 & 50.5 & 58.9 & 65.2 & 69.3 & 83.0 & 93.9 & 98.6 & 99.8 \\
\hline \multirow[t]{2}{*}{655600} & 2 & 10.2 & 17.6 & 21.0 & 22.0 & 22.1 & 22.1 & 22.2 & 22.2 & 22.3 & 22.3 \\
\hline & 10 & 22.4 & 43.1 & 59.4 & 69.2 & 75.6 & 79.9 & 91.1 & 97.0 & 99.3 & 99.8 \\
\hline \multirow[t]{2}{*}{655620} & 2 & 6.1 & 9.7 & 11.6 & 12.1 & 12.2 & 12.2 & 12.2 & 12.3 & 12.3 & 12.3 \\
\hline & 10 & 15.2 & 28.6 & 42.9 & 51.2 & 57.4 & 61.6 & 77.4 & 90.8 & 97.5 & 99.4 \\
\hline \multirow[t]{2}{*}{655630} & 2 & 8.5 & 14.7 & 19.1 & 20.9 & 21.8 & 22.3 & 23.6 & 23.7 & 23.8 & 23.8 \\
\hline & 10 & 18.2 & 34.2 & 50.4 & 59.9 & 66.8 & 71.4 & 84.3 & 92.7 & 97.3 & 98.9 \\
\hline \multirow[t]{2}{*}{655780} & 2 & 4.0 & 6.9 & 9.6 & 11.2 & 12.6 & 13.8 & 16.4 & 18.3 & 19.4 & 19.7 \\
\hline & 10 & 11.0 & 20.5 & 30.2 & 36.8 & 42.8 & 47.5 & 68.7 & 86.8 & 96.4 & 99.4 \\
\hline \multirow[t]{2}{*}{655850} & 2 & 6.1 & 10.7 & 13.6 & 15.4 & 17.0 & 18.0 & 20.7 & 22.8 & 23.6 & 23.8 \\
\hline & 10 & 18.2 & 34.2 & 48.6 & 57.3 & 64.3 & 69.8 & 88.6 & 97.6 & 99.7 & 100.0 \\
\hline \multirow[t]{2}{*}{655920} & 2 & 11.0 & 20.3 & 26.4 & 29.3 & 31.2 & 32.5 & 38.1 & 43.7 & 46.0 & 46.3 \\
\hline & 10 & 29.5 & 54.1 & 72.1 & 81.4 & 87.3 & 90.9 & 98.4 & 99.9 & 100.0 & 100.0 \\
\hline \multirow[t]{2}{*}{655940} & 2 & 4.3 & 7.4 & 9.4 & 10.2 & 10.7 & 11.1 & 12.3 & 13.4 & 13.9 & 14.1 \\
\hline & 10 & 15.9 & 29.4 & 41.4 & 49.3 & 56.0 & 61.4 & 82.9 & 95.3 & 99.3 & 99.9 \\
\hline \multirow[t]{2}{*}{655990} & 2 & 3.8 & 6.2 & 8.2 & 9.4 & 10.0 & 10.2 & 10.6 & 11.0 & 11.1 & 11.2 \\
\hline & 10 & 14.5 & 26.9 & 37.3 & 44.0 & 49.4 & 53.8 & 73.0 & 88.8 & 97.1 & 99.5 \\
\hline
\end{tabular}




\begin{tabular}{|c|c|c|c|c|c|c|c|c|c|c|c|}
\hline \multicolumn{12}{|c|}{$\begin{array}{l}\text { LARS-WG Model } \\
\text { September DRWH Reliability (\%) } \\
\text { per capita roof area and storage } \\
\text { L }\end{array}$} \\
\hline Gauge & sq. m & 0 & 40 & 80 & 120 & 160 & 200 & 400 & 600 & 800 & 1000 \\
\hline \multirow[t]{2}{*}{653060} & 2 & 15.1 & 27.0 & 33.0 & 35.2 & 36.3 & 37.1 & 38.1 & 38.2 & 38.2 & 38.2 \\
\hline & 10 & 32.0 & 58.2 & 79.7 & 89.2 & 94.1 & 96.6 & 99.7 & 100.0 & 100.0 & 100.0 \\
\hline \multirow[t]{2}{*}{653190} & 2 & 20.7 & 36.8 & 45.2 & 49.5 & 52.4 & 54.6 & 59.0 & 59.2 & 59.2 & 59.2 \\
\hline & 10 & 44.1 & 75.0 & 91.4 & 96.8 & 98.9 & 99.6 & 100.0 & 100.0 & 100.0 & 100.0 \\
\hline \multirow[t]{2}{*}{653300} & 2 & 20.2 & 34.7 & 42.1 & 45.7 & 47.8 & 49.0 & 51.7 & 52.2 & 52.2 & 52.2 \\
\hline & 10 & 37.4 & 66.6 & 86.4 & 93.6 & 97.2 & 98.7 & 99.9 & 100.0 & 100.0 & 100.0 \\
\hline \multirow[t]{2}{*}{653350} & 2 & 13.7 & 23.3 & 28.4 & 30.2 & 31.0 & 31.5 & 32.5 & 32.5 & 32.5 & 32.5 \\
\hline & 10 & 30.0 & 54.4 & 75.8 & 85.5 & 90.7 & 93.6 & 98.8 & 99.8 & 99.9 & 100.0 \\
\hline \multirow[t]{2}{*}{653380} & 2 & 12.1 & 20.9 & 25.3 & 26.3 & 26.6 & 26.6 & 26.7 & 26.7 & 26.7 & 26.7 \\
\hline & 10 & 24.9 & 46.0 & 65.0 & 75.5 & 82.8 & 87.1 & 95.9 & 98.8 & 99.7 & 99.9 \\
\hline \multirow[t]{2}{*}{653440} & 2 & 11.3 & 19.6 & 25.4 & 28.1 & 29.8 & 31.0 & 33.3 & 33.5 & 33.6 & 33.7 \\
\hline & 10 & 21.0 & 39.2 & 56.3 & 66.1 & 72.9 & 77.1 & 86.9 & 91.4 & 95.1 & 97.8 \\
\hline \multirow[t]{2}{*}{653520} & 2 & 14.8 & 25.1 & 28.9 & 29.5 & 29.7 & 29.8 & 30.1 & 30.4 & 30.5 & 30.5 \\
\hline & 10 & 32.8 & 61.2 & 81.9 & 90.5 & 94.7 & 96.8 & 99.7 & 100.0 & 100.0 & 100.0 \\
\hline \multirow[t]{2}{*}{653550} & 2 & 22.4 & 38.6 & 46.8 & 50.9 & 53.9 & 56.3 & 64.4 & 65.6 & 65.6 & 65.6 \\
\hline & 10 & 44.8 & 75.2 & 92.9 & 97.4 & 99.0 & 99.6 & 100.0 & 100.0 & 100.0 & 100.0 \\
\hline \multirow[t]{2}{*}{653570} & 2 & 16.0 & 28.9 & 34.6 & 36.6 & 37.8 & 38.6 & 39.3 & 39.3 & 39.3 & 39.3 \\
\hline & 10 & 36.4 & 64.4 & 83.8 & 92.0 & 96.0 & 97.9 & 99.9 & 100.0 & 100.0 & 100.0 \\
\hline \multirow[t]{2}{*}{653610} & 2 & 18.5 & 31.5 & 37.2 & 39.1 & 40.0 & 40.3 & 40.9 & 41.0 & 41.0 & 41.0 \\
\hline & 10 & 38.3 & 67.7 & 87.7 & 94.8 & 97.8 & 99.0 & 100.0 & 100.0 & 100.0 & 100.0 \\
\hline \multirow[t]{2}{*}{653760} & 2 & 15.3 & 25.7 & 32.6 & 35.6 & 37.4 & 38.4 & 40.0 & 40.1 & 40.1 & 40.1 \\
\hline & 10 & 28.7 & 52.9 & 74.5 & 85.0 & 91.4 & 94.8 & 99.5 & 99.9 & 100.0 & 100.0 \\
\hline \multirow[t]{2}{*}{653800} & 2 & 8.4 & 14.9 & 19.4 & 21.4 & 22.6 & 23.3 & 24.9 & 25.1 & 25.1 & 25.1 \\
\hline & 10 & 19.2 & 35 & & 61.6 & 68.6 & 73.1 & 84.4 & 91.1 & 95.7 & 98.3 \\
\hline \multirow[t]{2}{*}{653870} & 2 & 6.0 & 10. & 12.6 & 13.3 & 13.5 & 13.6 & 13.7 & 13.7 & 13.7 & 13.7 \\
\hline & 10 & 15.7 & 28.8 & 42.0 & 49.5 & 54.6 & 57.7 & 64.9 & 70.7 & 79.3 & 87.8 \\
\hline \multirow[t]{2}{*}{654720} & 2 & 4.2 & 6.8 & 8.8 & 10.0 & 10.8 & 11.3 & 12.0 & 12.0 & 12.0 & 12.0 \\
\hline & 10 & 12.2 & 22.5 & 32.6 & 38.1 & 42.0 & 44.5 & 50.6 & 55.8 & 65.0 & 76.5 \\
\hline \multirow[t]{2}{*}{655010} & 2 & 8.7 & 13.7 & 16.0 & 16.3 & 16.3 & 16.4 & 16.4 & 16.4 & 16.4 & 16.4 \\
\hline & 10 & 19.0 & 35.7 & 53.9 & 64.8 & 73.6 & 79.4 & 94.9 & 98.8 & 99.2 & 99.2 \\
\hline \multirow[t]{2}{*}{655020} & 2 & 12.2 & 19.1 & 22.4 & 23.5 & 24.1 & 24.3 & 24.4 & 24.4 & 24.4 & 24.4 \\
\hline & 10 & 24.4 & 45.4 & 67.5 & 79.2 & 86.7 & 91.3 & 99.3 & 99.9 & 100.0 & 100.0 \\
\hline 655050 & 2 & 12.5 & 20.1 & 23.4 & 24.1 & 24.4 & 24.5 & 24.6 & 24.6 & 24.6 & 24.6 \\
\hline & 10 & 26.8 & 51.2 & 73.1 & 83.5 & 90.1 & 94.0 & 99.6 & 100.0 & 100.0 & 100.0 \\
\hline 655070 & 2 & 13.8 & 23.1 & 28. & & 32.2 & 33 & 34 & 34.8 & 34.9 & 34.9 \\
\hline & 10 & 27.2 & 50.7 & 72.9 & 84.3 & 91.0 & 94.6 & 99.7 & 100.0 & 100.0 & 100.0 \\
\hline 655100 & 2 & 17.0 & 28 & 35 & 38.3 & 40.1 & 41 & 43 & 43 & 43 & 43.1 \\
\hline & 10 & 34.3 & 61.4 & 83.7 & 92.6 & 96.7 & 98.5 & 100.0 & 100.0 & 100.0 & 100.0 \\
\hline 655160 & 2 & 13.5 & 21.6 & 25.9 & 27.5 & 28.3 & 28.6 & 29.0 & 29.0 & 29.0 & 29.0 \\
\hline & 10 & 30.5 & 54.6 & 76.7 & 86.9 & 93.0 & 96.1 & 99.9 & 100.0 & 100.0 & 100.0 \\
\hline 655180 & 2 & 14.6 & 23.7 & 28.1 & 30.1 & 31.2 & 31.9 & 33.5 & 33.7 & 33.7 & 33.7 \\
\hline & 10 & 31.7 & 58.0 & 78.5 & 88.2 & 93.3 & 95.9 & 99.8 & 100.0 & 100.0 & 100.0 \\
\hline 655220 & 2 & 17.1 & 27.7 & 32.2 & 33.8 & 34.7 & 35.2 & 35.8 & 35.8 & 35.8 & 35.8 \\
\hline & 10 & 34.7 & 62.8 & 84.5 & 93.4 & 97.1 & 98.7 & 100.0 & 100.0 & 100.0 & 100.0 \\
\hline 655280 & 2 & 19.4 & 34.2 & 42.9 & 46.4 & 48.4 & 49.6 & 51.2 & 51.3 & 51.3 & 51.3 \\
\hline & 10 & 40.5 & 70.5 & 91.1 & 97.1 & 99.0 & 99.7 & 100.0 & 100.0 & 100.0 & 100.0 \\
\hline 655360 & 2 & 18.9 & 31.3 & 38.2 & 40.7 & 42.1 & 42.8 & 43.9 & 43.9 & 43.9 & 43.9 \\
\hline & 10 & 34.8 & 62.4 & 85.7 & 93.2 & 96.8 & 98.5 & 100.0 & 100.0 & 100.0 & 100.0 \\
\hline 655450 & 2 & 15.2 & 24.1 & 28.7 & 30.3 & 31.1 & 31.6 & 32.2 & 32.2 & 32.2 & 32.2 \\
\hline & 10 & 31.0 & 55.4 & 75.4 & 84.7 & 90.1 & 93.1 & 98.0 & 99.1 & 99.6 & 99.9 \\
\hline
\end{tabular}




\begin{tabular}{|c|c|c|c|c|c|c|c|c|c|c|c|}
\hline \multicolumn{12}{|c|}{$\begin{array}{l}\text { LARS-WG Markov Model } \\
\text { September DRWH Reliability (\%) } \\
\text { per capita roof area and storage } \\
\text { L }\end{array}$} \\
\hline Gauqe & sa. m & 0 & 40 & 80 & 120 & 160 & 200 & 400 & 600 & 800 & 1000 \\
\hline \multirow[t]{2}{*}{655480} & 2 & 21.5 & 39.4 & 49.5 & 54.7 & 58.3 & 61.0 & 65.9 & 66.3 & 66.4 & 66.4 \\
\hline & 10 & 42.3 & 73.5 & 90.7 & 96.0 & 98.3 & 99.3 & 100.0 & 100.0 & 100.0 & 100.0 \\
\hline \multirow[t]{2}{*}{655550} & 2 & 13.8 & 22.7 & 27.9 & 30.1 & 31.4 & 31.9 & 32.4 & 32.5 & 32.6 & 32.6 \\
\hline & 10 & 28.5 & 51.0 & 72.0 & 82.1 & 88.5 & 91.8 & 98.0 & 99.4 & 99.8 & 99.9 \\
\hline \multirow[t]{2}{*}{655570} & 2 & 11.9 & 20.8 & 25.7 & 27.8 & 28.4 & 28.5 & 28.6 & 28.6 & 28.6 & 28.6 \\
\hline & 10 & 28.0 & 50.7 & 68.7 & 77.9 & 83.8 & 87.0 & 93.5 & 96.3 & 98.5 & 99.6 \\
\hline \multirow[t]{2}{*}{655600} & 2 & 12.0 & 20.5 & 25.4 & 27.6 & 28.7 & 29.1 & 29.2 & 29.2 & 29.2 & 29.2 \\
\hline & 10 & 29.9 & 53.5 & 72.5 & 82.4 & 88.1 & 91.4 & 97.4 & 98.9 & 99.6 & 99.9 \\
\hline \multirow[t]{2}{*}{655620} & 2 & 11.1 & 19.0 & 23.6 & 25.7 & 26.8 & 27.5 & 28.4 & 28.4 & 28.4 & 28.4 \\
\hline & 10 & 24.1 & 44.1 & 61.6 & 71.5 & 77.9 & 81.5 & 88.5 & 92.8 & 96.5 & 98.7 \\
\hline \multirow[t]{2}{*}{655630} & 2 & 9.6 & 15.5 & 18.6 & 19.7 & 20.0 & 20.0 & 20.1 & 20.1 & 20.1 & 20.1 \\
\hline & 10 & 23.6 & 43.4 & 61.2 & 71.7 & 78.4 & 82.7 & 92.4 & 95.9 & 97.8 & 98.9 \\
\hline \multirow[t]{2}{*}{655780} & 2 & 6.5 & 11.2 & 15.9 & 18.6 & 20.3 & 21.4 & 24.2 & 24.6 & 24.7 & 24.8 \\
\hline & 10 & 15.2 & 28.4 & 41.1 & 48.7 & 54.0 & 57.2 & 66.3 & 75.1 & 85.3 & 93.8 \\
\hline \multirow[t]{2}{*}{655850} & 2 & 8.8 & 15.2 & 18.4 & 19.9 & 20.9 & 21.6 & 22.5 & 22.6 & 22.8 & 23.0 \\
\hline & 10 & 22.3 & 41.4 & 57.0 & 65.9 & 71.9 & 75.9 & 86.8 & 94.0 & 98.1 & 99.6 \\
\hline \multirow[t]{2}{*}{655920} & 2 & 14.6 & 26.1 & 32.9 & 36.4 & 38.3 & 39.4 & 40.5 & 41.7 & 43.0 & 43.7 \\
\hline & 10 & 36.2 & 64.2 & 82.3 & 90.1 & 94.2 & 96.4 & 99.5 & 99.9 & 100.0 & 100.0 \\
\hline \multirow[t]{2}{*}{655940} & 2 & 4.4 & 7.5 & 9.7 & 10.8 & 11.0 & 11.0 & 11.0 & 11.0 & 11.1 & 11.1 \\
\hline & 10 & 18.3 & 33.3 & 45.0 & 51.5 & 56.0 & 59.1 & 70.4 & 82.8 & 93.0 & 98.0 \\
\hline \multirow[t]{2}{*}{655990} & 2 & 4.6 & 7.1 & 8.3 & 9.0 & 9.1 & 9.1 & 9.2 & 9.2 & 9.2 & 9.2 \\
\hline & 10 & 14.7 & 27.4 & 38.8 & 45.2 & 49.7 & 52.8 & 63.0 & 74.0 & 85.8 & 94.3 \\
\hline
\end{tabular}




\begin{tabular}{|c|c|c|c|c|c|c|c|c|c|c|c|}
\hline \multicolumn{12}{|c|}{$\begin{array}{l}\text { LARS-WG Model } \\
\text { October DRWH Reliability (\%) } \\
\text { per capita roof area and storage } \\
\text { L }\end{array}$} \\
\hline Gauge & sq. m & 0 & 40 & 80 & 120 & 160 & 200 & 400 & 600 & 800 & 1000 \\
\hline \multirow[t]{2}{*}{653060} & 2 & 5.5 & 8.3 & 9.7 & 10.2 & 10.5 & 10.7 & 10.9 & 10.9 & 10.9 & 10.9 \\
\hline & 10 & 14.2 & 26.9 & 42.0 & 52.2 & 61.2 & 67.5 & 89.2 & 98.3 & 99.8 & 100.0 \\
\hline \multirow[t]{2}{*}{653190} & 2 & 10.9 & 18.4 & 22.6 & 23.6 & 24.0 & 24.3 & 26.3 & 27.2 & 27.4 & 27.4 \\
\hline & 10 & 23.6 & 43.3 & 63.0 & 73.6 & 81.4 & 86.1 & 97.1 & 99.8 & 100.0 & 100.0 \\
\hline \multirow[t]{2}{*}{653300} & 2 & 10.3 & 16.5 & 19.6 & 21.1 & 21.7 & 22.0 & 22.9 & 23.2 & 23.3 & 23.4 \\
\hline & 10 & 21.7 & 39.2 & 56.7 & 67.9 & 77.0 & 82.4 & 95.9 & 99.7 & 100.0 & 100.0 \\
\hline \multirow[t]{2}{*}{653350} & 2 & 10.7 & 18.3 & 23.1 & 25.4 & 26.7 & 27.5 & 28.7 & 28.8 & 28.8 & 28.8 \\
\hline & 10 & 23.0 & 42.9 & 61.8 & 72.9 & 80.9 & 85.8 & 97.0 & 99.7 & 100.0 & 100.0 \\
\hline \multirow[t]{2}{*}{653380} & 2 & 12.6 & 21.0 & 25.8 & 26.8 & 27.1 & 27.1 & 27.1 & 27.1 & 27.1 & 27.1 \\
\hline & 10 & 22.3 & 41.8 & 62.0 & 73.0 & 80.9 & 85.5 & 96.2 & 99.0 & 99.7 & 99.9 \\
\hline \multirow[t]{2}{*}{653440} & 2 & 11.3 & 18.6 & 23.2 & 25.3 & 26.1 & 26.4 & 26.7 & 26.9 & 27.0 & 27.0 \\
\hline & 10 & 24.0 & 43.8 & 62.4 & 73.3 & 80.6 & 85.0 & 94.8 & 97.3 & 98.3 & 99.0 \\
\hline \multirow[t]{2}{*}{653520} & 2 & 10.5 & 17.1 & 20.1 & 20.4 & 20.5 & 20.5 & 20.5 & 20.5 & 20.6 & 20.6 \\
\hline & 10 & 20.1 & 37.7 & 56.8 & 67.7 & 76.1 & 81.5 & 95.5 & 99.6 & 100.0 & 100.0 \\
\hline \multirow[t]{2}{*}{653550} & 2 & 11.5 & 18.7 & 21.6 & 22.9 & 23.9 & 24.7 & 28.9 & 32.3 & 33.2 & 33.3 \\
\hline & 10 & 27.0 & 50.0 & 67.4 & 77.2 & 84.4 & 88.8 & 98.1 & 99.9 & 100.0 & 100.0 \\
\hline \multirow[t]{2}{*}{653570} & 2 & 12.4 & 20.1 & 23.7 & 25.1 & 25.8 & 26.2 & 27.0 & 27.0 & 27.0 & 27.0 \\
\hline & 10 & 26.1 & 46.6 & 66.3 & 76.7 & 84.5 & 88.9 & 97.4 & 99.7 & 100.0 & 100.0 \\
\hline \multirow[t]{2}{*}{653610} & 2 & 12.4 & 20.3 & 24.0 & 25.1 & 25.5 & 25.7 & 25.8 & 25.8 & 25.8 & 25.8 \\
\hline & 10 & 25.0 & 46.6 & 64.9 & 75.4 & 83.3 & 87.8 & 97.7 & 99.9 & 100.0 & 100.0 \\
\hline \multirow[t]{2}{*}{653760} & 2 & 10.9 & 18.0 & 21.5 & 22.2 & 22.4 & 22.5 & 22.9 & 22.9 & 22.9 & 22.9 \\
\hline & 10 & 23.2 & 44.0 & 63.1 & 73.4 & 81.0 & 85.6 & 96.5 & 99.6 & 100.0 & 100.0 \\
\hline \multirow[t]{2}{*}{653800} & 2 & 9.8 & 15.1 & 17.7 & 18.5 & 18.7 & 18.7 & 18.8 & 18.8 & 18.8 & 18.8 \\
\hline & 10 & 22.8 & 44.1 & 62.8 & 72.7 & 79.3 & 83.7 & 93.6 & 96.3 & 97.7 & 98.8 \\
\hline \multirow[t]{2}{*}{653870} & 2 & 7.2 & 11.7 & 14.5 & 15.6 & 16.1 & 16.3 & 16.4 & 16.4 & 16.4 & 16.4 \\
\hline & 10 & 16.7 & 30.7 & 45.9 & 55.5 & 63.3 & 68.4 & 81.1 & 84.7 & 87.1 & 90.2 \\
\hline \multirow[t]{2}{*}{654720} & 2 & 5.7 & 9.1 & 11.0 & 12.2 & 13.2 & 13.9 & 15.6 & 15.7 & 15.7 & 15.7 \\
\hline & 10 & 17.2 & 31.0 & 45.4 & 53.7 & 60.0 & 64.1 & 73.6 & 76.7 & 78.9 & 82.1 \\
\hline \multirow[t]{2}{*}{655010} & 2 & 3.8 & 6.6 & 9.2 & 10.5 & 11.2 & 11.5 & 11.6 & 11.6 & 11.6 & 11.6 \\
\hline & 10 & 9.0 & 17.2 & 27.6 & 35.0 & 42.5 & 47.9 & 70.4 & 86.9 & 94.2 & 96.4 \\
\hline \multirow[t]{2}{*}{655020} & 2 & 3.8 & 5.4 & 6.2 & 6.4 & 6.6 & 6.6 & 6.7 & 6.7 & 6.7 & 6.7 \\
\hline & 10 & 10.9 & 21.0 & 32.9 & 41.0 & 48.3 & 54.0 & 79.1 & 94.7 & 99.1 & 99.8 \\
\hline 655050 & 2 & 4.9 & 7.3 & 7.9 & 7.9 & 7.9 & 7.9 & 7.9 & 7.9 & 7.9 & 7.9 \\
\hline & 10 & 13.0 & 24.7 & 38.5 & 48.2 & 56.7 & 63.1 & 87.1 & 97.7 & 99.7 & 100.0 \\
\hline 655070 & 2 & 4.6 & 6.5 & 6.9 & 7.0 & 7.0 & 7.0 & 7. & 7.1 & & 7. \\
\hline & 10 & 13.3 & 25.8 & 40.3 & 49.6 & 57.7 & 63.6 & 87.2 & 97.8 & 99 & 100.0 \\
\hline 655100 & 2 & 6.8 & 10.1 & 11.4 & 11.8 & 11.9 & 12.0 & 12.4 & 12.4 & 12 & 12.5 \\
\hline & 10 & 14. & 27.6 & 43.5 & 54.4 & 64.0 & 70.5 & 91.9 & 99.2 & 100.0 & 100.0 \\
\hline 655160 & 2 & 5.5 & 8.9 & 10.6 & 11.2 & 11.3 & 11.4 & 11.5 & 11.5 & 11.5 & 11.5 \\
\hline & 10 & 15.5 & 29.7 & 45.3 & 55.5 & 64.2 & 70.4 & 91.3 & 98.9 & 100.0 & 100.0 \\
\hline 655180 & 2 & 6.7 & 9.1 & 9.8 & 9.9 & 10.0 & 10.0 & 10.0 & 10.1 & 10.1 & 10.1 \\
\hline & 10 & 17.9 & 34.1 & 51.0 & 60.9 & 69.2 & 75.0 & 92.8 & 99.0 & 99.9 & 100.0 \\
\hline 655220 & 2 & 9.2 & 14.5 & 16.7 & 17.1 & 17.2 & 17.2 & 17.2 & 17.3 & 17.3 & 17.3 \\
\hline & 10 & 20.3 & 38.5 & 57.2 & 68.0 & 76.9 & 82.6 & 96.7 & 99.8 & 100.0 & 100.0 \\
\hline 655280 & 2 & 11.3 & 19.0 & 23.7 & 26.0 & 27.3 & 28.1 & 29.1 & 29.2 & 29.2 & 29.2 \\
\hline & 10 & 32.4 & 57.1 & 78.2 & 88.7 & 94.0 & 96.8 & 99.9 & 100.0 & 100.0 & 100.0 \\
\hline 655360 & 2 & 9.7 & 16.4 & 20.2 & 22.0 & 23.2 & 23.8 & 24.3 & 24.3 & 24.3 & 24.3 \\
\hline & 10 & 23.1 & 42.2 & 60.9 & 71.4 & 79.8 & 85.1 & 96.9 & 99.8 & 100.0 & 100.0 \\
\hline 655450 & 2 & 10.0 & 16.7 & 19.8 & 20.8 & 21.2 & 21.3 & 21.4 & 21.4 & 21.4 & 21.4 \\
\hline & 10 & 25.0 & 45.0 & 64.4 & 75.1 & 82.6 & 87.3 & 97.5 & 99.5 & 99.8 & 99.9 \\
\hline
\end{tabular}




\begin{tabular}{|c|c|c|c|c|c|c|c|c|c|c|c|}
\hline \multicolumn{12}{|c|}{$\begin{array}{l}\text { LARS-WG Markov Model } \\
\text { October DRWH Reliability (\%) } \\
\text { per capita roof area and storage }\end{array}$} \\
\hline Gauge & sq. m & 0 & 40 & 80 & 120 & 160 & 200 & 400 & 600 & 800 & 1000 \\
\hline \multirow[t]{2}{*}{655480} & 2 & 12.6 & 21.7 & 27.5 & 30.2 & 31.9 & 33.0 & 37.0 & 38.6 & 38.9 & 38.9 \\
\hline & 10 & 28.2 & 49.2 & 69.8 & 80.2 & 87.8 & 92.0 & 99.4 & 100.0 & 100.0 & 100.0 \\
\hline \multirow[t]{2}{*}{655550} & 2 & 8.9 & 13.9 & 17.1 & 18.6 & 19.3 & 19.7 & 19.9 & 20.0 & 20.0 & 20.0 \\
\hline & 10 & 21.3 & 38.4 & 57.0 & 68.3 & 76.7 & 81.9 & 95.5 & 99.2 & 99.8 & 99.9 \\
\hline \multirow[t]{2}{*}{655570} & 2 & 10.9 & 17.7 & 21.6 & 23.3 & 24.1 & 24.4 & 24.5 & 24.5 & 24.5 & 24.5 \\
\hline & 10 & 29.5 & 53.6 & 73.8 & 83.6 & 89.8 & 93.3 & 98.7 & 99.4 & 99.7 & 99.9 \\
\hline \multirow[t]{2}{*}{655600} & 2 & 8.2 & 12.6 & 14.8 & 15.9 & 16.6 & 17.1 & 17.4 & 17.4 & 17.4 & 17.4 \\
\hline & 10 & 22.6 & 41.4 & 59.6 & 70.5 & 78.3 & 83.5 & 96.4 & 99.1 & 99.7 & 99.9 \\
\hline \multirow[t]{2}{*}{655620} & 2 & 11.6 & 20.1 & 26.4 & 29.7 & 32.1 & 33.8 & 38.3 & 39.0 & 39.1 & 39.1 \\
\hline & 10 & 22.6 & 42.6 & 62.7 & 74.1 & 82.0 & 86.8 & 96.2 & 98.1 & 98.9 & 99.5 \\
\hline \multirow[t]{2}{*}{655630} & 2 & 9.0 & 13.4 & 15.9 & 16.8 & 17.0 & 17.1 & 17.1 & 17.1 & 17.1 & 17.1 \\
\hline & 10 & 23.0 & 42.3 & 60.3 & 70.9 & 78.0 & 82.6 & 93.9 & 97.4 & 98.7 & 99.3 \\
\hline \multirow[t]{2}{*}{655780} & 2 & 10.9 & 19.6 & 25.8 & 29.0 & 31.1 & 32.6 & 36.6 & 37.6 & 37.7 & 37.7 \\
\hline & 10 & 24.1 & 43.6 & 60.3 & 69.7 & 76.6 & 80.9 & 90.4 & 92.9 & 94.6 & 96.4 \\
\hline \multirow[t]{2}{*}{655850} & 2 & 13.3 & 23.2 & 28.8 & 31.1 & 32.3 & 32.9 & 34.1 & 34.2 & 34.2 & 34.2 \\
\hline & 10 & 29.6 & 53.8 & 72.9 & 82.3 & 87.7 & 91.0 & 96.6 & 98.1 & 99.1 & 99.7 \\
\hline \multirow[t]{2}{*}{655920} & 2 & 13.8 & 25.2 & 32.9 & 37.1 & 40.0 & 41.8 & 44.9 & 45.1 & 45.4 & 45.8 \\
\hline & 10 & 30.5 & 56.3 & 75.7 & 85.8 & 91.8 & 95.2 & 99.7 & 100.0 & 100.0 & 100.0 \\
\hline \multirow[t]{2}{*}{655940} & 2 & 6.6 & 12.0 & 15.4 & 17.1 & 18.3 & 18.8 & 19.1 & 19.1 & 19.1 & 19.1 \\
\hline & 10 & 20.6 & 37.3 & 52.3 & 60.5 & 66.1 & 69.9 & 80.0 & 85.2 & 90.4 & 95.3 \\
\hline \multirow[t]{2}{*}{655990} & 2 & 7.8 & 12.9 & 15.1 & 15.7 & 15.8 & 15.8 & 15.8 & 15.8 & 15.8 & 15.8 \\
\hline & 10 & 20.4 & 384 & 53.9 & 62.7 & 68.6 & 72.2 & 80.0 & 83.6 & 87.7 & 92.6 \\
\hline
\end{tabular}




\begin{tabular}{|c|c|c|c|c|c|c|c|c|c|c|c|}
\hline \multicolumn{12}{|c|}{$\begin{array}{l}\text { LARS-WG Model } \\
\text { November DRWH Reliability (\%) } \\
\text { per capita roof area and storage } \\
\text { L }\end{array}$} \\
\hline Gauge & sq. $\mathrm{m}$ & 0 & 40 & 80 & 120 & 160 & 200 & 400 & 600 & 800 & 1000 \\
\hline \multirow[t]{2}{*}{653060} & 2 & 1.9 & 2.3 & 2.4 & 2.4 & 2.4 & 2.4 & 2.4 & 2.4 & 2.4 & 2.4 \\
\hline & 10 & 4.7 & 9.1 & 14.5 & 17.7 & 20.1 & 21.8 & 34.8 & 57.7 & 80.3 & 93.8 \\
\hline \multirow[t]{2}{*}{653190} & 2 & 5.7 & 9.4 & 13.5 & 16.2 & 18.0 & 18.8 & 19.5 & 19.6 & 19.6 & 19.6 \\
\hline & 10 & 10.7 & 19.4 & 31.4 & 40.1 & 48.4 & 54.1 & 75.8 & 89.0 & 95.5 & 98.7 \\
\hline \multirow[t]{2}{*}{653300} & 2 & 4.2 & 6.3 & 7.9 & 8.5 & 8.7 & 8.7 & 8.7 & 8.7 & 8.7 & 8.7 \\
\hline & 10 & 9.3 & 17.4 & 28.0 & 35.5 & 42.3 & 46.9 & 66.6 & 82.8 & 92.8 & 98.0 \\
\hline \multirow[t]{2}{*}{653350} & 2 & 3.1 & 4.4 & 4.7 & 4.7 & 4.7 & 4.7 & 4.7 & 4.7 & 4.7 & 4.7 \\
\hline & 10 & 8.5 & 15.8 & 25.6 & 32.7 & 39.6 & 44.6 & 67.9 & 87.0 & 95.8 & 99.1 \\
\hline \multirow[t]{2}{*}{653380} & 2 & 5.4 & 9.2 & 12.9 & 14.9 & 16.1 & 16.6 & 16.9 & 16.9 & 16.9 & 16.9 \\
\hline & 10 & 10.4 & 19.8 & 32.6 & 41.8 & 50.9 & 57.1 & 81.4 & 94.2 & 98.1 & 99.5 \\
\hline \multirow[t]{2}{*}{653440} & 2 & 6.3 & 9.8 & 12.2 & 13.0 & 13.4 & 13.4 & 13.5 & 13.5 & 13.6 & 13.6 \\
\hline & 10 & 12.5 & 23.5 & 37.1 & 46.4 & 54.9 & 60.8 & 82.3 & 93.0 & 96.7 & 98.1 \\
\hline \multirow[t]{2}{*}{653520} & 2 & 5.3 & 9.4 & 14.9 & 18.3 & 20.9 & 22.1 & 23.1 & 23.1 & 23.1 & 23.1 \\
\hline & 10 & 9.0 & 16.2 & 28.0 & 36.6 & 45.3 & 51.1 & 72.5 & 86.3 & 94.3 & 98.4 \\
\hline \multirow[t]{2}{*}{653550} & 2 & 5.0 & 8.2 & 9.4 & 9.5 & 9.6 & 9.6 & 9.7 & 9.9 & 10.3 & 10.6 \\
\hline & 10 & 11.6 & 22.0 & 32.9 & 41.0 & 49.0 & 54.4 & 75.4 & 89.8 & 96.6 & 99.2 \\
\hline \multirow[t]{2}{*}{653570} & 2 & 5.0 & 6.9 & 7.1 & 7.2 & 7.2 & 7.2 & 7.2 & 7.2 & 7.2 & 7.2 \\
\hline & 10 & 10.8 & 20.3 & 32.2 & 40.6 & 48.7 & 54.0 & 75.2 & 88.8 & 95.5 & 98.9 \\
\hline \multirow[t]{2}{*}{653610} & 2 & 8.0 & 14.0 & 19.5 & 22.0 & 23.5 & 24.2 & 24.7 & 24.7 & 24.7 & 24.7 \\
\hline & 10 & 14.2 & 26.5 & 41.5 & 51.3 & 60.3 & 66.0 & 84.1 & 93.3 & 97.8 & 99.6 \\
\hline \multirow[t]{2}{*}{653760} & 2 & 3.6 & 5.5 & 6.8 & 6.9 & 6.9 & 6.9 & 6.9 & 6.9 & 6.9 & 6.9 \\
\hline & 10 & 8.6 & 16.9 & 27.6 & 35.1 & 42.4 & 47.8 & 71.5 & 88.2 & 95.9 & 99.1 \\
\hline \multirow[t]{2}{*}{653800} & 2 & 6.4 & 9.4 & 10.6 & 10.7 & 10.7 & 10.7 & 10.7 & 10.7 & 10.7 & 10.7 \\
\hline & 10 & 14.3 & 27.8 & 43.6 & 53.4 & 61.2 & 66.7 & 85.7 & 93.8 & 96.7 & 98.0 \\
\hline \multirow[t]{2}{*}{653870} & 2 & 3.8 & 5.2 & 6.1 & 6.3 & 6.3 & 6.3 & 6.3 & 6.3 & 6.3 & 6.3 \\
\hline & 10 & 9.6 & 18.1 & 28.4 & 35.5 & 41.4 & 45.5 & 62.7 & 73.6 & 79.0 & 82.9 \\
\hline \multirow[t]{2}{*}{654720} & 2 & 4.4 & 6.6 & 8.0 & 8.4 & 8.6 & 8.6 & 8.6 & 8.7 & 8.7 & 8.7 \\
\hline & 10 & 12.2 & 22.8 & 34.1 & 41.2 & 47.5 & 51.6 & 65.4 & 72.4 & 75.8 & 78.4 \\
\hline \multirow[t]{2}{*}{655010} & 2 & 3.8 & 7.5 & 12.6 & 14.5 & 15.0 & 15.1 & 15.2 & 15.2 & 15.2 & 15.2 \\
\hline & 10 & 6.3 & 11.7 & 18.7 & 23.6 & 28.2 & 30.9 & 40.3 & 53.3 & 69.6 & 82.4 \\
\hline \multirow[t]{2}{*}{655020} & 2 & 3.3 & 6.1 & 8.6 & 8.9 & 9.0 & 9.0 & 9.0 & 9.0 & 9.0 & 9.0 \\
\hline & 10 & 6.2 & 11.9 & 19.4 & 24.3 & 28.2 & 30.7 & 39.5 & 55.0 & 75.4 & 90.4 \\
\hline 655050 & 2 & 2.9 & 4.1 & 4.3 & 4.3 & 4.3 & 4.3 & 4.3 & 4.3 & 4.3 & 4.3 \\
\hline & 10 & 6.5 & 12.5 & 20.1 & 24.6 & 28.4 & 30.5 & 41.8 & 62.4 & 83.3 & 95.4 \\
\hline 655070 & 2 & 2.5 & 4.3 & 4.9 & 4.9 & 4.9 & 4.9 & 4.9 & 4.9 & 4.9 & 4.9 \\
\hline & 10 & 8.0 & 14.8 & 22.4 & 26.9 & 30.4 & 32.6 & 43.4 & 63.8 & 84.5 & 95.9 \\
\hline 655100 & 2 & 3.4 & 5.0 & 5.8 & 5.9 & 5.9 & 5.9 & 5.9 & 5.9 & 5.9 & 5.9 \\
\hline & 10 & 7.3 & 13.7 & 22.0 & 28.2 & 33.8 & 37.4 & 53.5 & 73.7 & 90.0 & 97.7 \\
\hline 655160 & 2 & 3.2 & 5.4 & 6.4 & 6.4 & 6.4 & 6.4 & 6.4 & 6.4 & 6.4 & 6.4 \\
\hline & 10 & 10.2 & 19.6 & 29.8 & 36.1 & 41.7 & 45.4 & 61.0 & 78.2 & 91.7 & 97.9 \\
\hline 655180 & 2 & 3.1 & 4.0 & 4.0 & 4.0 & 4.0 & 4.0 & 4.0 & 4.0 & 4.0 & 4.0 \\
\hline & 10 & 13.8 & 24.8 & 36.3 & 42.5 & 47.2 & 50.2 & 65.2 & 82.3 & 93.7 & 98.5 \\
\hline 655220 & 2 & 4.0 & 6.3 & 8.5 & 9.5 & 9.8 & 9.8 & 9.8 & 9.8 & 9.8 & 9.8 \\
\hline & 10 & 8.1 & 15.5 & 25.9 & 33.5 & 40.9 & 46.1 & 69.1 & 87.0 & 95.7 & 99.1 \\
\hline 655280 & 2 & 6.2 & 9.2 & 10.8 & 11.6 & 12.1 & 12.6 & 13.4 & 13.4 & 13.4 & 13.4 \\
\hline & 10 & 14.8 & 27.7 & 43.0 & 53.3 & 62.1 & 68.1 & 89.8 & 98.8 & 99.9 & 100.0 \\
\hline 655360 & 2 & 3.5 & 5.5 & 6.7 & 7.1 & 7.4 & 7.5 & 7.7 & 7.7 & 7.7 & 7.7 \\
\hline & 10 & 9.0 & 17.6 & 27.5 & 34.8 & 41.4 & 46.3 & 69.3 & 87.7 & 96.5 & 99.4 \\
\hline 655450 & 2 & 3.9 & 5.8 & 6.8 & 7.1 & 7.2 & 7.2 & 7.2 & 7.2 & 7.2 & 7.2 \\
\hline & 10 & 11.8 & 21.7 & 33.0 & 40.9 & 48.1 & 53.6 & 76.7 & 92.3 & 98.1 & 99.5 \\
\hline
\end{tabular}




\begin{tabular}{|c|c|c|c|c|c|c|c|c|c|c|c|}
\hline \multirow{2}{*}{\multicolumn{12}{|c|}{$\begin{array}{l}\text { LARS-WG Markov Model } \\
\text { November DRWH Reliability (\%) } \\
\text { per capita roof area and storage } \\
\text { L }\end{array}$}} \\
\hline & & & & & & & & & & & \\
\hline Gauge & sq. $\mathrm{m}$ & 0 & 40 & 80 & 120 & 160 & 200 & 400 & 600 & 800 & 1000 \\
\hline \multirow{2}{*}{655480} & 2 & 4.2 & 6.6 & 8.1 & 8.8 & 9.3 & 9.7 & 10.4 & 10.8 & 11.0 & 11.1 \\
\hline & 10 & 14.1 & 25.6 & 39.3 & 48.0 & 56.0 & 61.6 & 84.7 & 96.8 & 99.6 & 100.0 \\
\hline \multirow[t]{2}{*}{655550} & 2 & 3.4 & 5.5 & 6.8 & 7.5 & 7.7 & 7.7 & 7.7 & 7.7 & 7.7 & 7.7 \\
\hline & 10 & 9.8 & 18.2 & 28.0 & 35.1 & 41.5 & 46.2 & 67.8 & 85.7 & 95.1 & 98.8 \\
\hline \multirow[t]{2}{*}{655570} & 2 & 8.2 & 12.5 & 14.3 & 14.9 & 15.1 & 15.1 & 15.2 & 15.2 & 15.2 & 15.2 \\
\hline & 10 & 24.0 & 43.6 & 62.4 & 73.0 & 80.5 & 85.5 & 97.2 & 99.3 & 99.7 & 99.9 \\
\hline \multirow[t]{2}{*}{655600} & 2 & 4.5 & 6.2 & 6.8 & 7.0 & 7.0 & 7.0 & 7.1 & 7.1 & 7.1 & 7.1 \\
\hline & 10 & 13.2 & 24.7 & 36.9 & 44.7 & 51.2 & 5.8 & 76.0 & 90.7 & 97.2 & 99.1 \\
\hline \multirow[t]{2}{*}{655620} & 2 & 5.3 & 8.9 & 10.3 & 10.4 & 10.4 & 10.4 & 10.8 & 11.4 & 11.6 & 11.6 \\
\hline & 10 & 13.3 & 25.5 & 39.4 & 48.8 & 56.7 & 62.5 & 83.4 & 94.0 & 97.7 & 98.9 \\
\hline \multirow[t]{2}{*}{655630} & 2 & 4.9 & 7.1 & 8.6 & 9.2 & 9.3 & 9.3 & 9.3 & 9.3 & 9.3 & 9.3 \\
\hline & 10 & 15.5 & 28.8 & 42.9 & 51.3 & 58.4 & 63.5 & 81.7 & 91.3 & 95.8 & 97.9 \\
\hline \multirow[t]{2}{*}{655780} & 2 & 10.5 & 17.2 & 21.0 & 22.6 & 23.5 & 24.3 & 26.0 & 26.8 & 27.0 & 27.1 \\
\hline & 10 & 25.3 & 46.0 & 64.9 & 75.3 & 82.2 & 86.5 & 95.6 & 97.9 & 98.6 & 99.0 \\
\hline \multirow[t]{2}{*}{655850} & 2 & 9.9 & 16.8 & 20.6 & 22.5 & 23.8 & 24.5 & 26.0 & 26.0 & 26.1 & 26.1 \\
\hline & 10 & 25.4 & 47.1 & 66.2 & 76.6 & 83.8 & 88.4 & 97.9 & 99.4 & 99.7 & 99.8 \\
\hline \multirow[t]{2}{*}{655920} & 2 & 10.1 & 18.2 & 23.2 & 25.6 & 27.1 & 28.1 & 31.0 & 31.9 & 32.0 & 32.1 \\
\hline & 10 & 26.1 & 47.9 & 66.5 & 77.0 & 84.2 & 88.8 & 98.4 & 99.9 & 100.0 & 100.0 \\
\hline \multirow[t]{2}{*}{655940} & 2 & 9.0 & 15.8 & 19.7 & 21.3 & 22.4 & 23.1 & 23.6 & 23.6 & 23.6 & 23.6 \\
\hline & 10 & 24.2 & 44.0 & 60.8 & 70.5 & 77.2 & 81.3 & 90.5 & 93.8 & 95.7 & 97.3 \\
\hline \multirow[t]{2}{*}{655990} & 2 & 8.8 & 14.5 & 17.3 & 17.9 & 18.0 & 18.0 & 18.0 & 18.0 & 18.0 & 18.0 \\
\hline & 10 & 20.7 & 39.2 & 57.4 & 67.4 & 74.4 & 78.8 & 89.0 & 91.9 & 93.7 & 95.5 \\
\hline
\end{tabular}




\begin{tabular}{|c|c|c|c|c|c|c|c|c|c|c|c|}
\hline \multicolumn{12}{|c|}{$\begin{array}{l}\text { LARS-WG Model } \\
\text { December DRWH Reliability (\%) } \\
\text { per capita roof area and storage } \\
\text { L }\end{array}$} \\
\hline Gauge & sq. m & 0 & 40 & 80 & 120 & 160 & 200 & 400 & 600 & 800 & \\
\hline \multirow[t]{2}{*}{653060} & 2 & 12.3 & 24.3 & 33.1 & 34.6 & 34.7 & 34.7 & 34.7 & 34.7 & 34.7 & \\
\hline & 10 & 12.4 & 24.5 & 40.2 & 49.6 & 55.1 & 57.8 & 60.1 & 61.9 & 67.6 & 6. \\
\hline \multirow[t]{2}{*}{653190} & 2 & 3.3 & 3.9 & 4.4 & 4.7 & 4.8 & 4.9 & 5.1 & 5.1 & 5.1 & \\
\hline & 10 & 12.7 & 21.8 & 31.6 & 35.6 & 38.1 & 40.0 & 49.8 & 65.0 & & \\
\hline \multirow[t]{2}{*}{653300} & 2 & 1.5 & 1.8 & 2.0 & 2.1 & 2.1 & 2.1 & 2.1 & 2.1 & & \\
\hline & 10 & 8.5 & 15.2 & 21.0 & 23.9 & 26.3 & 28.1 & 37.4 & 50.5 & 66.8 & 0 \\
\hline \multirow[t]{2}{*}{653350} & 2 & 2.0 & 2.1 & 2.1 & 2.1 & 2.1 & 2.1 & 2.1 & 2.1 & 2.1 & \\
\hline & 10 & 10.2 & 17.9 & 26.3 & 29.6 & 31.3 & 32.2 & 36.3 & 48.1 & 67.9 & $\varepsilon$ \\
\hline \multirow[t]{2}{*}{653380} & 2 & 7.7 & 12.9 & 17.5 & 19.9 & 21.1 & 21.4 & 21.7 & 21.8 & 21.8 & \\
\hline & 10 & 12.1 & 22.8 & & 47.8 & 55.2 & 58.9 & 68.8 & 80 & 91.2 & \\
\hline \multirow[t]{2}{*}{653440} & 2 & 4.7 & 8.7 & & 14.0 & 15.2 & 15.9 & 17.3 & 17.4 & 7.4 & 7. \\
\hline & 10 & 8.0 & 15 & & 33.2 & 40.8 & 45 & 2.7 & & 7.2 & 3. \\
\hline \multirow[t]{2}{*}{653520} & 2 & 13.1 & 23.5 & 34 & 37.4 & 39.4 & 40.3 & 41.8 & & 1.9 & \\
\hline & 10 & 14.8 & 28.5 & 45.9 & 54.5 & 62.0 & 65.4 & 74.2 & 82.4 & 89.1 & 3. \\
\hline \multirow[t]{2}{*}{653550} & 2 & 5.9 & 6.1 & 6.7 & 6.1 & 6.1 & 6. & 6. & & & \\
\hline & 10 & 10.6 & 21.5 & 32.7 & 39.5 & 44.4 & 47.0 & 57.2 & 69 & 82.5 & \\
\hline \multirow[t]{2}{*}{653570} & 2 & 6.3 & 8.3 & 8.5 & 8.5 & 8.5 & 8.5 & 8.5 & 8.5 & 8.5 & \\
\hline & 10 & 8.4 & 17.3 & 30.5 & 38.7 & 45.0 & 48.0 & 56.9 & 69 & 82.8 & \\
\hline \multirow[t]{2}{*}{653610} & 2 & 4.6 & 6.8 & 8.5 & 9.4 & 10.0 & 10.4 & 11.1 & 11.2 & 11.2 & \\
\hline & 10 & 11.6 & 22.2 & 34 & 40.8 & 46.4 & 50 & 67.4 & & 90.6 & \\
\hline \multirow[t]{2}{*}{653760} & 2 & 4.6 & 7.2 & 10 & 12.2 & 12.8 & 12 & 12.9 & & 2.9 & \\
\hline & 10 & 10 & 19. & & 35.3 & 40.0 & 42.5 & 50.1 & 61.8 & 77.3 & 89 \\
\hline \multirow[t]{2}{*}{653800} & 2 & 11 & 19 & & 27.5 & 28.2 & & & & & 28 \\
\hline & 10 & 15. & 30.7 & 50 & 60.9 & 70.1 & 75 & 86.5 & 92 & & 9 \\
\hline \multirow[t]{2}{*}{653870} & 2 & 3.5 & 5.0 & 6. & 6.8 & 6.9 & 6. & 6.9 & 6. & 6. & \\
\hline & 10 & 9.0 & 17.5 & 28.3 & 33.9 & 38.0 & 40 & 47.7 & & 63.1 & 7 \\
\hline \multirow[t]{2}{*}{654720} & 2 & 5.5 & 8.3 & 9. & 10.0 & 10.0 & 10.0 & 10.0 & 10.0 & 10.0 & \\
\hline & 10 & 14.7 & 27.6 & 41.8 & 48.9 & 54.5 & 57.7 & 65.9 & 71 & 75 & \\
\hline \multirow[t]{2}{*}{655010} & 2 & 11.7 & 23.2 & 39.0 & 45.5 & 49.2 & 49.9 & 50.3 & 50 & 50.3 & 50 \\
\hline & 10 & 12.3 & 23.9 & 40.8 & 51.4 & 61.4 & 65.5 & 75.7 & 80.6 & 83.2 & \\
\hline \multirow[t]{2}{*}{655020} & 2 & 0.1 & 0.5 & 0. & 0.7 & 0.7 & 0. & 0. & & & \\
\hline & 10 & 0.1 & 0.5 & 1.7 & 3.4 & 5. & 8.2 & 20.6 & 29.4 & 37.8 & 49 \\
\hline 655050 & 2 & & 19 & & 32.4 & & & & & & 33 \\
\hline & 10 & 14 & 28.4 & & 52.0 & 58.5 & & & & & 84 \\
\hline 655070 & 2 & 12 & 24.5 & & 37.7 & 39.0 & 39 & 39.3 & & & 39 \\
\hline & 10 & 13.5 & 26.9 & 41.5 & 50.9 & 58.5 & 62.7 & 70.4 & 72.7 & 77.7 & 85 \\
\hline 655100 & 2 & 2.9 & 3.1 & 3.2 & 3.2 & 3.2 & 3.2 & 3.2 & 3. & & \\
\hline & 10 & 8.3 & 16.2 & 26.1 & 30.3 & 32.3 & 33 & 38.9 & & 59 & \\
\hline 655160 & 2 & 7.8 & 15.7 & 24.1 & 25.2 & 25.3 & 25.4 & 25.4 & 25.4 & 25.4 & \\
\hline & 10 & 9.4 & 19.8 & 35.2 & 44.0 & 51.2 & 55.5 & 65.7 & 71.9 & 80.1 & \\
\hline 655180 & 2 & 8.9 & 15.1 & 15.8 & 15.8 & 15.8 & 15.8 & 15.8 & 15.8 & 15.8 & \\
\hline & 10 & 25.6 & 43.6 & 60.6 & 67.9 & 72.7 & 74.3 & 76.8 & 81.2 & 87.8 & 93 \\
\hline 655220 & 2 & 5.5 & 10.7 & 15.3 & 17.4 & 17.7 & 17.8 & 17.8 & 17.8 & 17.8 & 17. \\
\hline & 10 & 8.2 & 15.8 & 25.5 & 32.6 & 38.3 & 41.9 & 51.1 & 62.6 & 77.3 & 89. \\
\hline 655280 & 2 & 8.4 & 14.4 & 21.3 & 24.4 & 26.5 & 27 & 30 & & 30.6 & 30. \\
\hline & 10 & 14.5 & 27.8 & 43.8 & 52.7 & 59.7 & 63 & 74.0 & 86.5 & 96.1 & 99 \\
\hline 655360 & 2 & 7.9 & 12.9 & 16 & 16.4 & 16.4 & 16 & 16.4 & 16.4 & 3.4 & 16. \\
\hline & 10 & 12.0 & 22 & & 43.3 & 49 & & 60.3 & 68 & 81.4 & 91 \\
\hline 55450 & 2 & 6.4 & 10.5 & 11. & 11.6 & 11.6 & 11 & 11.6 & 11.6 & 11.6 & 11. \\
\hline & 10 & 12.7 & 22.8 & 34.6 & 41.9 & 47.7 & 50.6 & 58.4 & 70.8 & 85.5 & \\
\hline
\end{tabular}




\begin{tabular}{|c|c|c|c|c|c|c|c|c|c|c|c|}
\hline \multicolumn{12}{|c|}{$\begin{array}{l}\text { LARS-WG Markov Model } \\
\text { December DRWH Reliability (\%) } \\
\text { per capita roof area and storage }\end{array}$} \\
\hline Gauge & $\mathrm{sa} \cdot \mathrm{m}$ & 0 & 40 & 80 & 120 & 160 & 200 & 400 & 600 & 800 & 1000 \\
\hline \multirow{2}{*}{655480} & 2 & 5.7 & 9.3 & 13.2 & 14.6 & 15.2 & 15.3 & 15.4 & 15.4 & 15.4 & 15.5 \\
\hline & 10 & 11.7 & 22.1 & 34.7 & 42.2 & 47.8 & 51.2 & 62.8 & 76.5 & 89.7 & 97.0 \\
\hline \multirow[t]{2}{*}{655550} & 2 & 5.3 & 8.8 & 12.0 & 13.4 & 13.7 & 13.7 & 13.7 & 13.7 & 13.7 & 13.7 \\
\hline & 10 & 13.5 & 25.1 & 36.8 & 43.4 & 48.8 & 51.7 & 59.7 & 68.7 & 80.8 & 90.8 \\
\hline \multirow[t]{2}{*}{655570} & 2 & 7.2 & 11.5 & 13.6 & 14.5 & 14.7 & 14.7 & 14.7 & 14.7 & 14.7 & 14.7 \\
\hline & 10 & 18.3 & 33.6 & 49.5 & 59.8 & 67.9 & 73.1 & 89.6 & 96.9 & 99.2 & 99.8 \\
\hline \multirow[t]{2}{*}{655600} & 2 & 5.8 & 8.8 & 10.5 & 10.6 & 10.6 & 10.6 & 10.6 & 10.6 & 10.6 & 10.6 \\
\hline & 10 & 11.9 & 23.7 & 37.2 & 45.4 & 51.8 & 55.1 & 64.4 & 74.6 & 86.2 & 94.5 \\
\hline \multirow[t]{2}{*}{655620} & 2 & 6.0 & 9.2 & 11.2 & 11.3 & 11.3 & 11.3 & 11.3 & 11.3 & 11.3 & 11.3 \\
\hline & 10 & 11.1 & 22.0 & 36.1 & 44.8 & 52.0 & 56.2 & 69.2 & 80.5 & 90.0 & 95.7 \\
\hline \multirow[t]{2}{*}{655630} & 2 & 4.9 & 7.8 & 9.5 & 9.6 & 9.6 & 9.6 & 9.6 & 9.6 & 9.6 & 9.6 \\
\hline & 10 & 9.8 & 19.0 & 30.0 & 37.0 & 42.9 & 46.5 & 60.3 & 74.6 & 86.0 & 92.9 \\
\hline \multirow[t]{2}{*}{655780} & 2 & 8.3 & 14.4 & 17.6 & 18.6 & 19.0 & 19.3 & 19.6 & 19.6 & 19.7 & 19.8 \\
\hline & 10 & 17.9 & 34.3 & 50.8 & 62.3 & 71.2 & 77.1 & 92.7 & 97.5 & 98.8 & 99.2 \\
\hline \multirow[t]{2}{*}{655850} & 2 & 8.4 & 12.8 & 15.2 & 16.5 & 17.4 & 18.0 & 18.4 & 18.5 & 18.5 & 18.5 \\
\hline & 10 & 18.3 & 34.8 & 52.5 & 63.4 & 71.9 & 77.3 & 92.9 & 98.2 & 99.6 & 99.9 \\
\hline \multirow[t]{2}{*}{655920} & 2 & 10.2 & 18.5 & 24.5 & 27.3 & 28.9 & 29.7 & 30.3 & 30.5 & 30.6 & 30.6 \\
\hline & 10 & 22.2 & 41.6 & 61.3 & 71.9 & 79.5 & 84.5 & 95.9 & 99.2 & 99.9 & 100.0 \\
\hline \multirow[t]{2}{*}{655940} & 2 & 7.5 & 13.2 & 17.0 & 18.5 & 19.3 & 19.5 & 19.7 & 19.7 & 19.7 & 19.7 \\
\hline & 10 & 18.0 & 33.6 & 49.7 & 59.9 & 68.4 & 74.1 & 89.4 & 94.6 & 96.5 & 97.5 \\
\hline \multirow[t]{2}{*}{655990} & 2 & 8.8 & 14.7 & 18.3 & 19.7 & 20.5 & 20.9 & 21.1 & 21.1 & 21.1 & 21.1 \\
\hline & 10 & 17.4 & 32.5 & 49.8 & 61.1 & 70.4 & 76.1 & 90.6 & 94.8 & 96.1 & 97.1 \\
\hline
\end{tabular}



Appendix J: Domestic Rainwater Harvesting Reliabilities Using the Conditioned $1^{\text {st }}$-Order Markov Model 


\begin{tabular}{|c|c|c|c|c|c|c|c|c|c|c|c|}
\hline \multicolumn{12}{|c|}{$\begin{array}{l}\text { Conditioned 1st-Order Markov Model } \\
\text { January DRWH Reliability (\%) } \\
\text { per capita roof area and storage } \\
\text { L }\end{array}$} \\
\hline Gauge & sq. m & 0 & 40 & 80 & 120 & 160 & 200 & 400 & 600 & 800 & 1000 \\
\hline \multirow{2}{*}{653060} & 2 & 0.0 & 0.0 & 0.0 & 0.0 & 0.1 & 0.1 & 0.1 & 0.1 & 0.1 & 0.1 \\
\hline & 10 & 0.1 & 0.2 & 0.3 & 0.4 & 0.4 & 0.5 & 0.8 & 1.0 & 1.2 & 1.2 \\
\hline \multirow[t]{2}{*}{653190} & 2 & 0.1 & 0.2 & 0.3 & 0.3 & 0.3 & 0.3 & 0.3 & 0.3 & 0.3 & 0.3 \\
\hline & 10 & 0.1 & 0.3 & 0.5 & 0.6 & 0.8 & 0.9 & 1.1 & 1.2 & 1.4 & 2.4 \\
\hline \multirow[t]{2}{*}{653300} & 2 & 0.2 & 0.3 & 0.3 & 0.4 & 0.4 & 0.4 & 0.4 & 0.4 & 0.4 & 0.4 \\
\hline & 10 & 0.6 & 1.0 & 1.4 & 1.6 & 1.8 & 1.9 & 2.1 & 2.1 & 2.4 & 3.3 \\
\hline \multirow[t]{2}{*}{653350} & 2 & 0.5 & 0.7 & 0.8 & 0.9 & 0.9 & 0.9 & 0.9 & 0.9 & 0.9 & 0.9 \\
\hline & 10 & 0.9 & 1.6 & 2.6 & 3.2 & 3.9 & 4.2 & 4.8 & 4.8 & 4.9 & 5.9 \\
\hline \multirow[t]{2}{*}{653380} & 2 & 0.6 & 1.1 & 1.7 & 2.1 & 2.4 & 2.6 & 2.8 & 2.8 & 2.8 & 2.8 \\
\hline & 10 & 0.9 & 1.7 & 3.4 & 4.9 & 6.9 & 8.1 & 12.6 & 16.1 & 19.2 & 24.8 \\
\hline \multirow[t]{2}{*}{653440} & 2 & 1.2 & 2.1 & 3.0 & 3.4 & 3.7 & 3.9 & 4.3 & 4.5 & 4.6 & 4.6 \\
\hline & 10 & 2.0 & 3.6 & 6.3 & 8.5 & 11.1 & 12.6 & 17.4 & 21.0 & 24.9 & 31.7 \\
\hline \multirow[t]{2}{*}{653520} & 2 & 0.2 & 0.3 & 0.5 & 0.6 & 0.7 & 0.7 & 0.7 & 0.7 & 0.8 & 0.8 \\
\hline & 10 & 0.3 & 0.6 & 1.0 & 1.3 & 1.8 & 2.0 & 3.0 & 3.6 & 4.4 & 5.9 \\
\hline \multirow[t]{2}{*}{653550} & 2 & 0.2 & 0.2 & 0.4 & 0.4 & 0.4 & 0.4 & 0.4 & 0.4 & 0.4 & 0.4 \\
\hline & 10 & 0.2 & 0.4 & 0.7 & 1.0 & 1.4 & 1.6 & 2.0 & 2.2 & 3.2 & 7.4 \\
\hline \multirow[t]{2}{*}{653570} & 2 & 0.2 & 0.4 & 0.7 & 0.8 & 1.0 & 1.0 & 1.1 & 1.1 & 1.1 & 1.1 \\
\hline & 10 & 0.5 & 0.9 & 1.5 & 1.9 & 2.5 & 2.7 & 3.5 & 3.8 & 4.0 & 5.2 \\
\hline \multirow[t]{2}{*}{653610} & 2 & 0.4 & 0.5 & 0.6 & 0.7 & 0.7 & 0.7 & 0.7 & 0.7 & 0.7 & 0.7 \\
\hline & 10 & 1.0 & 1.8 & 2.7 & 3.3 & 3.8 & 4.0 & 4.4 & 4.4 & 5.2 & 7.9 \\
\hline \multirow[t]{2}{*}{653760} & 2 & 0.3 & 0.4 & 0.5 & 0.6 & 0.6 & 0.6 & 0.7 & 0.8 & 0.8 & 0.8 \\
\hline & 10 & 1.2 & 1.9 & 2.9 & 3.6 & 4.2 & 4.5 & 5.5 & 6.5 & 7.3 & 9.0 \\
\hline \multirow{2}{*}{653800} & 2 & 0.6 & 1.1 & 1.6 & 1.9 & 2.0 & 2.1 & 2.1 & 2.1 & 2.1 & 2.1 \\
\hline & 10 & 1.2 & 2.2 & 3.8 & 5.3 & 7.1 & 8.2 & 11.8 & 14.6 & 16.9 & 20.4 \\
\hline \multirow[t]{2}{*}{653870} & 2 & 0.6 & 0.9 & 1.1 & 1.1 & 1.1 & 1.1 & 1.2 & 1.2 & 1.2 & 1.2 \\
\hline & 10 & 1.8 & 3.1 & 4.8 & 6.0 & 7.0 & 7.5 & 8.7 & 9.4 & 9.8 & 10.5 \\
\hline \multirow[t]{2}{*}{654720} & 2 & 0.5 & 0.7 & 0.9 & 1.0 & 1.0 & 1.0 & 1.0 & 1.0 & 1.0 & 1.0 \\
\hline & 10 & 1.2 & 2.1 & 3.5 & 4.5 & 5.6 & 6.3 & 8.1 & 9.2 & 9.8 & 10.9 \\
\hline \multirow[t]{2}{*}{655010} & 2 & 0.0 & 0.1 & 0.1 & 0.2 & 0.2 & 0.3 & 0.4 & 0.4 & 0.4 & 0.4 \\
\hline & 10 & 0.1 & 0.2 & 0.4 & 0.5 & 0.8 & 1.0 & 2.2 & 3.8 & 5.1 & 5.8 \\
\hline \multirow[t]{2}{*}{655020} & 2 & 0.0 & 0.0 & 0.0 & 0.0 & 0.0 & 0.0 & 0.0 & 0.0 & 0.0 & 0.0 \\
\hline & 10 & 0.1 & 0.1 & 0.1 & 0.1 & 0.1 & 0.1 & 0.1 & 0.1 & 0.1 & 0.2 \\
\hline 655030 & 2 & 0.0 & 0.0 & 0.0 & 0.0 & 0.0 & 0.0 & 0.0 & 0.0 & 0.0 & 0.0 \\
\hline & 10 & 0.0 & 0.0 & 0.0 & 0.1 & 0.2 & 0.2 & 0.5 & 0.8 & 1.0 & 1.5 \\
\hline 655050 & 2 & 0.0 & 0.0 & 0.0 & 0.1 & 0.1 & 0.1 & 0.2 & 0.2 & 0.2 & 0.2 \\
\hline & 10 & 0.0 & 0.0 & 0.1 & 0.2 & 0.5 & 0.6 & 1.6 & 2.9 & 3.7 & 4.2 \\
\hline 655070 & 2 & 0.0 & 0.0 & 0.0 & 0.1 & 0.1 & 0.1 & 0.2 & 0.3 & 0.3 & 0.3 \\
\hline & 10 & 0.0 & 0.0 & 0.1 & 0.2 & 0.4 & 0.5 & 1.5 & 2.7 & 3.6 & 4.0 \\
\hline 655100 & 2 & 0.2 & 0.3 & 0.3 & 0.4 & 0.4 & 0.4 & 0.4 & 0.4 & 0.4 & 0.4 \\
\hline & 10 & 0.3 & 0.5 & 0.9 & 1.1 & 1.4 & 1.5 & 1.7 & 1.8 & 2.0 & 2.6 \\
\hline 655160 & 2 & 0.0 & 0.0 & 0.0 & 0.0 & 0.0 & 0.0 & 0.1 & 0.1 & 0.1 & 0.1 \\
\hline & 10 & 0.3 & 0.4 & 0.6 & 0.6 & 0.7 & 0.7 & 0.9 & 1.2 & 1.4 & 1.8 \\
\hline 655180 & 2 & 0.0 & 0.0 & 0.0 & 0.0 & 0.0 & 0.0 & 0.0 & 0.0 & 0.0 & 0.0 \\
\hline & 10 & 0.0 & 0.0 & 0.1 & 0.2 & 0.4 & 0.5 & 1.0 & 1.3 & 1.4 & 1.6 \\
\hline 655220 & 2 & 0.5 & 0.7 & 1.1 & 1.3 & 1.4 & 1.5 & 1.6 & 1.6 & 1.7 & 1.7 \\
\hline & 10 & 0.8 & 1.2 & 2.0 & 2.7 & 3.4 & 3.9 & 5.1 & 5.9 & 6.4 & 7.2 \\
\hline 655280 & 2 & 0.6 & 0.9 & 1.1 & 1.2 & 1.3 & 1.4 & 2.0 & 2.7 & 3.2 & 3.4 \\
\hline & 10 & 1.6 & 2.7 & 4.1 & 5.1 & 6.2 & 6.7 & 8.4 & 10.1 & 12.3 & 17.4 \\
\hline 655360 & 2 & 0.2 & 0.4 & 0.4 & 0.5 & 0.5 & 0.5 & 0.5 & 0.5 & 0.5 & 0.5 \\
\hline & 10 & 0.8 & 1.4 & 2.1 & 2.6 & 3.1 & 3.4 & 4.3 & 5.0 & 5.7 & 7.9 \\
\hline
\end{tabular}




\begin{tabular}{|c|c|c|c|c|c|c|c|c|c|c|c|}
\hline \multirow{2}{*}{\multicolumn{12}{|c|}{$\begin{array}{l}\text { Conditioned 1st-Order Markov Model J-3 } \\
\text { January DRWH Reliability (\%) } \\
\text { per capita roof area and storage } \\
\text { L }\end{array}$}} \\
\hline & & & & & & & & & & & \\
\hline Gauge & sq. m & 0 & 40 & 80 & 120 & 160 & 200 & 400 & 600 & 800 & 1000 \\
\hline \multirow[t]{2}{*}{655450} & 2 & 0.7 & 1.2 & 1.8 & 2.1 & 2.2 & 2.3 & 2.3 & 2.3 & 2.3 & 2.3 \\
\hline & 10 & 1.0 & 1.9 & 3.4 & 4.7 & 6.1 & 6.9 & 8.9 & 9.4 & 10.3 & 13.7 \\
\hline \multirow[t]{2}{*}{655480} & 2 & 0.7 & 1.1 & 1.4 & 1.6 & 1.6 & 1.7 & 1.7 & 1.7 & 1.7 & 1.7 \\
\hline & 10 & 1.2 & 2.2 & 3.8 & 5.3 & 7.0 & 8.0 & 11.3 & 14.0 & 17.4 & 24.8 \\
\hline \multirow[t]{2}{*}{655550} & 2 & 1.2 & 2.0 & 2.8 & 3.2 & 3.4 & 3.4 & 3.5 & 3.5 & 3.5 & 3.5 \\
\hline & 10 & 1.8 & 3.4 & 5.9 & 8.0 & 10.3 & 11.6 & 15.0 & 16.6 & 18.0 & 21.1 \\
\hline \multirow[t]{2}{*}{655570} & 2 & 1.9 & 2.9 & 3.6 & 3.8 & 3.9 & 4.0 & 4.0 & 4.0 & 4.0 & 4.0 \\
\hline & 10 & 4.4 & 7.6 & 12.2 & 15.8 & 19.4 & 21.4 & 28.1 & 36.5 & 50.9 & 67.6 \\
\hline \multirow[t]{2}{*}{655600} & 2 & 0.4 & 0.6 & 0.7 & 0.8 & 0.8 & 0.8 & 0.8 & 0.8 & 0.8 & 0.8 \\
\hline & 10 & 2.0 & 3.4 & 5.2 & 6.5 & 7.9 & 8.8 & 12.4 & 15.4 & 18.5 & 25.0 \\
\hline \multirow[t]{2}{*}{655620} & 2 & 0.9 & 1.4 & 1.7 & 1.8 & 1.8 & 1.8 & 1.8 & 1.8 & 1.8 & 1.8 \\
\hline & 10 & 1.9 & 3.5 & 6.1 & 8.0 & 9.9 & 10.9 & 13.9 & 15.8 & 19.0 & 25.2 \\
\hline \multirow[t]{2}{*}{655630} & 2 & 0.9 & 1.5 & 2.0 & 2.2 & 2.4 & 2.4 & 2.5 & 2.5 & 2.5 & 2.5 \\
\hline & 10 & 1.8 & 3.4 & 5.7 & 7.5 & 9.4 & 10.4 & 13.0 & 14.3 & 16.9 & 22.9 \\
\hline \multirow[t]{2}{*}{655780} & 2 & 1.7 & 2.8 & 3.6 & 3.9 & 4.1 & 4.1 & 4.5 & 5.0 & 5.3 & 5.4 \\
\hline & 10 & 3.8 & 6.9 & 11.5 & 15.6 & 20.3 & 23.4 & 37.0 & 53.8 & 70.1 & 81.8 \\
\hline \multirow[t]{2}{*}{655850} & 2 & 1.6 & 2.4 & 3.0 & 3.3 & 3.4 & 3.5 & 4.0 & 4.7 & 5.2 & 5.4 \\
\hline & 10 & 3.9 & 7.0 & 11.7 & 15.5 & 19.6 & 22.2 & 33.7 & 50.6 & 69.8 & 85.0 \\
\hline \multirow[t]{2}{*}{655920} & 2 & 2.5 & 4.1 & 5.6 & 6.4 & 6.9 & 7.2 & 7.7 & 7.7 & 7.7 & 7.7 \\
\hline & 10 & 7.3 & 12.8 & 19.9 & 25.7 & 31.9 & 36.2 & 56.0 & 74.8 & 88.1 & 95.7 \\
\hline \multirow[t]{2}{*}{655940} & 2 & 0.9 & 1.3 & 1.7 & 1.8 & 2.0 & 2.0 & 2.2 & 2.3 & 2.3 & 2.3 \\
\hline & 10 & 2.6 & 4.9 & 8.3 & 11.2 & 14.4 & 16.6 & 27.6 & 42.5 & 57.4 & 68.4 \\
\hline \multirow[t]{2}{*}{655990} & 2 & 0.7 & 1.1 & 1.4 & 1.6 & 1.7 & 1.8 & 1.9 & 1.9 & 1.9 & 1.9 \\
\hline & 10 & 2.0 & 3.7 & 6.3 & 8.5 & 11.2 & 12.9 & 21.2 & 31.8 & 42.3 & 50.2 \\
\hline
\end{tabular}




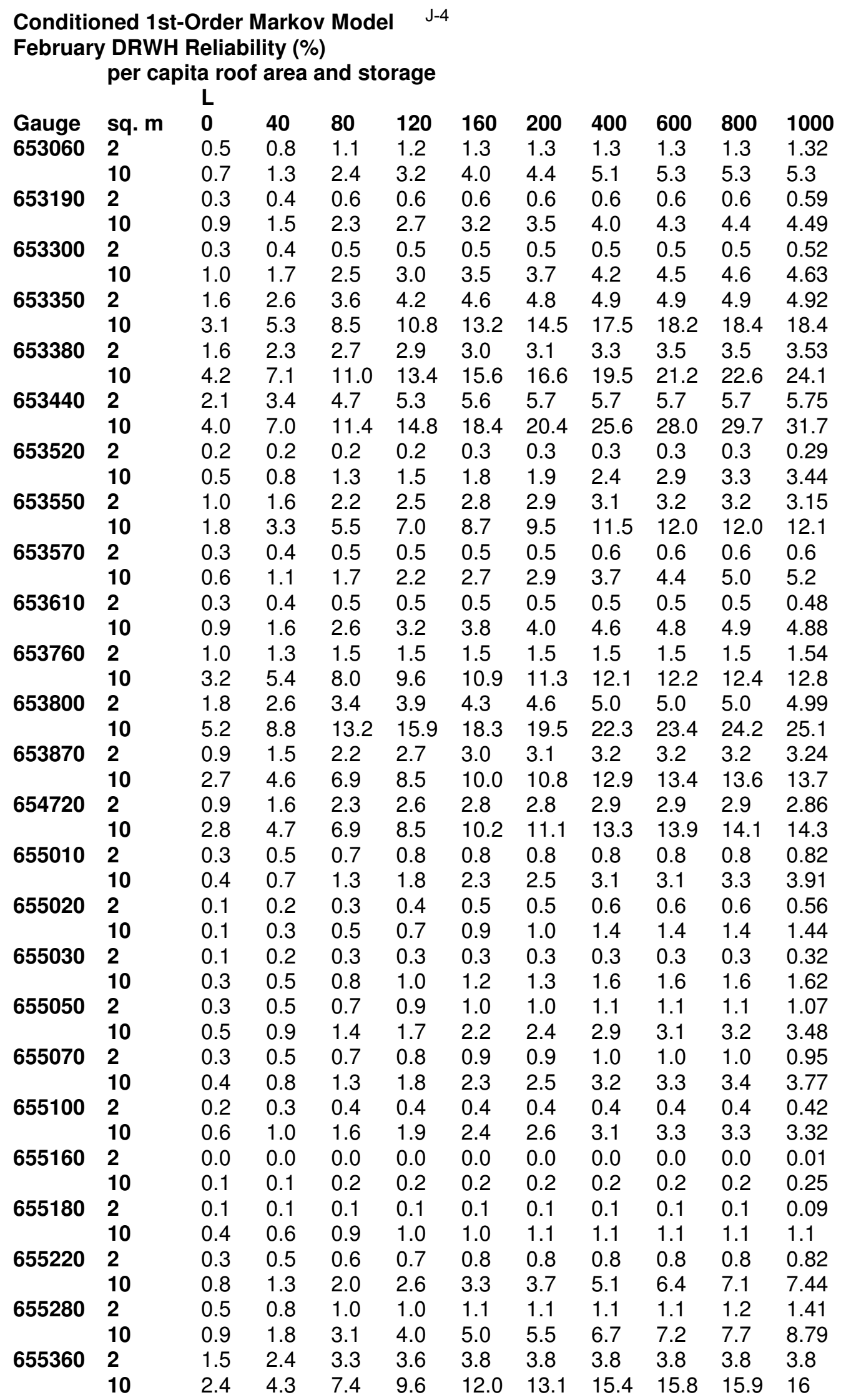




\begin{tabular}{|c|c|c|c|c|c|c|c|c|c|c|c|}
\hline \multicolumn{12}{|c|}{$\begin{array}{l}\text { Conditioned 1st-Order Markov Model } \\
\text { February DRWH Reliability (\%) } \\
\text { per capita roof area and storage } \\
\text { L }\end{array}$} \\
\hline Gauge & sq. m & 0 & 40 & 80 & 120 & 160 & 200 & 400 & 600 & 800 & 1000 \\
\hline \multirow[t]{2}{*}{655450} & 2 & 1.5 & 2.4 & 3.3 & 3.9 & 4.3 & 4.5 & 4.8 & 4.8 & 4.8 & 4.77 \\
\hline & 10 & 3.8 & 6.4 & 9.6 & 11.7 & 13.9 & 15.1 & 18.7 & 20.4 & 21.1 & 21.4 \\
\hline \multirow[t]{2}{*}{655480} & 2 & 1.8 & 2.6 & 2.8 & 2.9 & 2.9 & 2.9 & 2.9 & 2.9 & 2.9 & 2.88 \\
\hline & 10 & 5.2 & 8.9 & 13.3 & 16.1 & 18.4 & 19.4 & 21.5 & 22.4 & 23.0 & 24.3 \\
\hline \multirow[t]{2}{*}{655550} & 2 & 1.6 & 2.4 & 3.0 & 3.3 & 3.4 & 3.4 & 3.5 & 3.5 & 3.5 & 3.48 \\
\hline & 10 & 2.8 & 5.0 & 8.5 & 11.0 & 13.7 & 15.1 & 19.1 & 21.4 & 22.4 & 23 \\
\hline \multirow[t]{2}{*}{655570} & 2 & 4.3 & 6.5 & 8.1 & 8.9 & 9.5 & 9.8 & 10.4 & 10.5 & 10.5 & 10.5 \\
\hline & 10 & 10.2 & 17.3 & 26.4 & 32.2 & 37.2 & 39.7 & 45.2 & 47.1 & 49.9 & 55.6 \\
\hline \multirow[t]{2}{*}{655600} & 2 & 3.7 & 5.7 & 7.1 & 7.5 & 7.7 & 7.7 & 7.7 & 7.7 & 7.7 & 7.73 \\
\hline & 10 & 7.4 & 13.2 & 21.0 & 26.1 & 30.3 & 32.2 & 35.3 & 35.6 & 36.1 & 37.2 \\
\hline \multirow[t]{2}{*}{655620} & 2 & 2.2 & 3.0 & 3.3 & 3.4 & 3.4 & 3.4 & 3.4 & 3.4 & 3.4 & 3.41 \\
\hline & 10 & 6.2 & 10.8 & 16.6 & 20.2 & 22.9 & 24.1 & 26.3 & 26.9 & 27.3 & 28.2 \\
\hline \multirow{2}{*}{655630} & 2 & 1.5 & 2.1 & 2.6 & 2.7 & 2.8 & 2.8 & 2.9 & 3.0 & 3.0 & 2.99 \\
\hline & 10 & 4.3 & 7.3 & 11.2 & 13.6 & 15.8 & 16.9 & 19.8 & 21.2 & 22.0 & 22.8 \\
\hline \multirow[t]{2}{*}{655780} & 2 & 2.0 & 2.8 & 3.5 & 3.8 & 4.0 & 4.2 & 4.5 & 4.5 & 4.6 & 4.77 \\
\hline & 10 & 6.5 & 10.9 & 16.1 & 19.5 & 22.5 & 24.1 & 28.5 & 32.1 & 39.7 & 51.7 \\
\hline \multirow[t]{2}{*}{655850} & 2 & 3.8 & 6.2 & 8.1 & 8.8 & 9.1 & 9.2 & 9.3 & 9.3 & 9.4 & 9.69 \\
\hline & 10 & 8.1 & 14.3 & 22.2 & 27.5 & 31.8 & 34.1 & 38.9 & 41.3 & 47.4 & 58.7 \\
\hline \multirow[t]{2}{*}{655920} & 2 & 3.3 & 5.1 & 6.1 & 6.4 & 6.5 & 6.6 & 6.6 & 6.6 & 6.6 & 6.63 \\
\hline & 10 & 7.3 & 13.1 & 20.7 & 26.0 & 30.7 & 33.2 & 40.2 & 48.0 & 61.4 & 76.5 \\
\hline \multirow[t]{2}{*}{655940} & 2 & 0.6 & 0.9 & 1.2 & 1.3 & 1.3 & 1.3 & 1.4 & 1.4 & 1.4 & 1.38 \\
\hline & 10 & 2.6 & 4.2 & 6.1 & 7.3 & 8.3 & 8.8 & 10.1 & 11.4 & 16.5 & 26.5 \\
\hline \multirow[t]{2}{*}{655990} & 2 & 2.0 & 2.9 & 3.5 & 3.8 & 3.9 & 4.0 & 4.1 & 4.1 & 4.2 & 4.15 \\
\hline & 10 & 5.0 & 8.7 & 13.5 & 16.6 & 19.3 & 20.5 & 23.0 & 23.8 & 26.4 & 31 \\
\hline
\end{tabular}




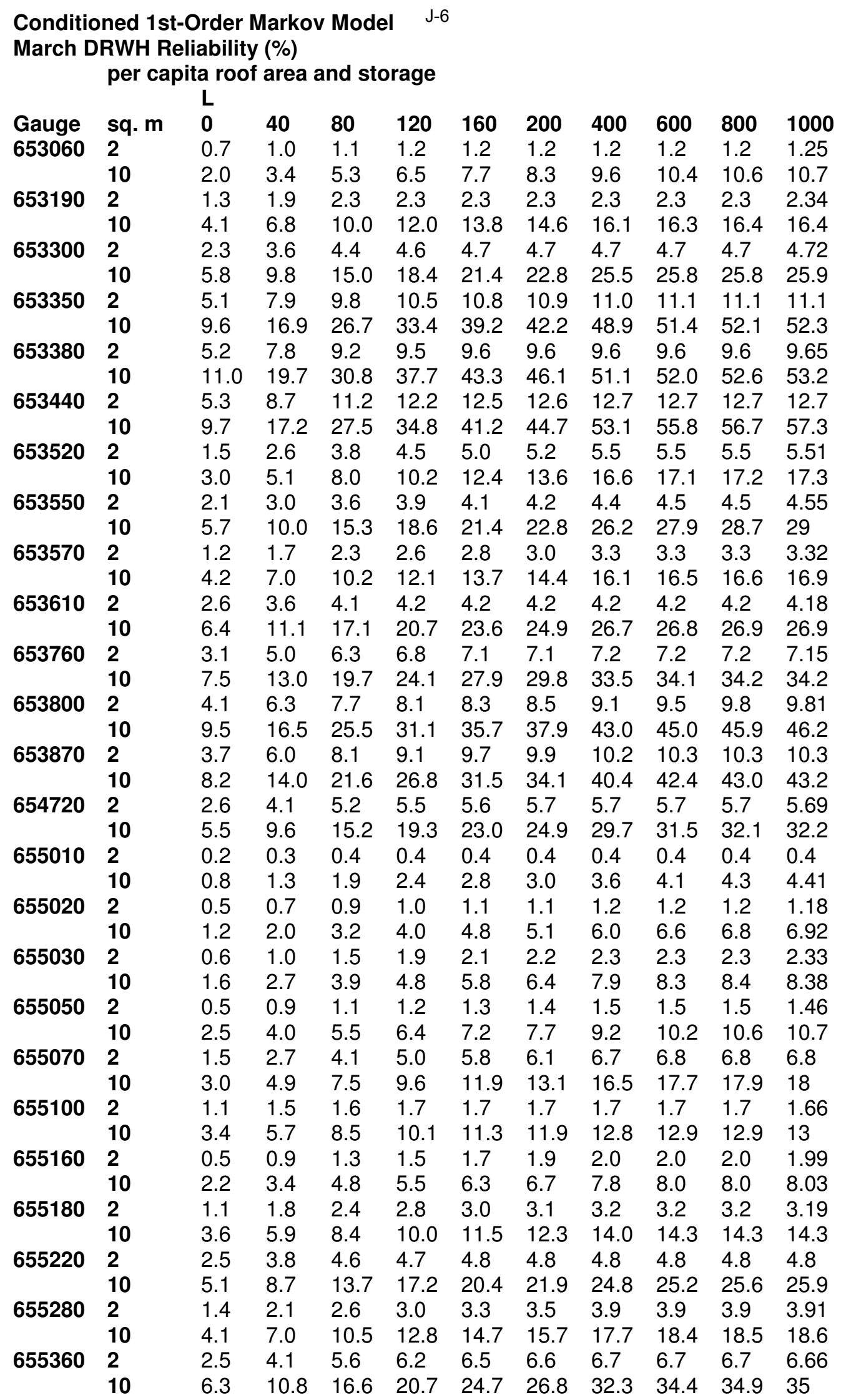




\begin{tabular}{|c|c|c|c|c|c|c|c|c|c|c|c|}
\hline \multirow{2}{*}{\multicolumn{12}{|c|}{$\begin{array}{l}\text { Conditioned 1st-Order Markov Model J-7 } \\
\text { March DRWH Reliability (\%) } \\
\text { per capita roof area and storage }\end{array}$}} \\
\hline & & & & & & & & & & & \\
\hline \multirow{3}{*}{655450} & sq. m & 0 & 40 & 80 & 120 & 160 & 200 & 400 & 600 & 800 & 1000 \\
\hline & & 3.9 & 6.4 & 8.3 & 9.0 & 9.4 & 9.5 & 9.7 & 9.7 & 9.7 & 9.71 \\
\hline & 10 & 7.7 & 13.6 & 21.7 & 27.3 & 32.5 & 35.3 & 41.8 & 44.3 & 45.5 & 46 \\
\hline \multirow[t]{2}{*}{655480} & 2 & 7.1 & 11.5 & 15.1 & 16.8 & 17.7 & 18.2 & 18.6 & 18.7 & 18.7 & 18.7 \\
\hline & 10 & 15.5 & 26.8 & 40.5 & 48.8 & 55.2 & 58.5 & 63.9 & 64.4 & 64.5 & 64.6 \\
\hline \multirow[t]{2}{*}{655550} & 2 & 4.5 & 7.2 & 10.0 & 11.6 & 12.7 & 13.3 & 14.2 & 14.3 & 14.3 & 14.3 \\
\hline & 10 & 10.2 & 17.8 & 27.5 & 33.8 & 39.1 & 41.9 & 48.3 & 49.9 & 50.4 & 50.8 \\
\hline \multirow[t]{2}{*}{655570} & 2 & 8.2 & 12.8 & 15.6 & 17.0 & 18.0 & 18.7 & 20.5 & 21.0 & 21.1 & 21.1 \\
\hline & 10 & 20.0 & 33.3 & 48.6 & 57.6 & 64.4 & 67.8 & 75.2 & 77.3 & 78.0 & 79 \\
\hline \multirow[t]{2}{*}{655600} & 2 & 5.7 & 9.1 & 11.3 & 11.9 & 12.1 & 12.1 & 12.2 & 12.2 & 12.2 & 12.2 \\
\hline & 10 & 12.8 & 22.8 & 35.3 & 43.3 & 49.8 & 53.5 & 61.9 & 63.9 & 64.2 & 64.3 \\
\hline \multirow[t]{2}{*}{655620} & 2 & 6.3 & 10.3 & 12.8 & 13.5 & 13.7 & 13.7 & 13.7 & 13.8 & 13.8 & 13.8 \\
\hline & 10 & 13.7 & 23.7 & 36.0 & 44.0 & 50.3 & 53.6 & 59.4 & 59.9 & 60.0 & 60.1 \\
\hline \multirow[t]{2}{*}{655630} & 2 & 4.6 & 6.5 & 7.1 & 7.2 & 7.2 & 7.2 & 7.2 & 7.2 & 7.2 & 7.2 \\
\hline & 10 & 13.4 & 23.0 & 33.9 & 40.2 & 44.7 & 46.9 & 50.9 & 51.6 & 51.9 & 52.2 \\
\hline \multirow[t]{2}{*}{655780} & 2 & 5.4 & 9.0 & 11.9 & 13.0 & 13.5 & 13.6 & 13.9 & 14.1 & 14.1 & 14.2 \\
\hline & 10 & 12.9 & 22.0 & 33.1 & 40.3 & 46.3 & 49.6 & 56.2 & 57.6 & 58.8 & 61 \\
\hline \multirow[t]{2}{*}{655850} & 2 & 6.4 & 10.2 & 12.7 & 13.6 & 14.1 & 14.3 & 14.6 & 14.6 & 14.6 & 14.6 \\
\hline & 10 & 14.3 & 25.4 & 38.8 & 47.3 & 54.0 & 57.9 & 67.2 & 69.6 & 70.6 & 72.2 \\
\hline \multirow[t]{2}{*}{655920} & 2 & 3.9 & 6.2 & 8.0 & 8.7 & 9.2 & 9.4 & 9.6 & 9.6 & 9.6 & 9.6 \\
\hline & 10 & 10.5 & 17.9 & 27.0 & 33.1 & 38.3 & 41.4 & 49.3 & 52.6 & 56.7 & 63.3 \\
\hline \multirow[t]{2}{*}{655940} & 2 & 2.9 & 4.4 & 5.6 & 6.1 & 6.2 & 6.3 & 6.3 & 6.3 & 6.3 & 6.31 \\
\hline & 10 & 6.2 & 10.8 & 17.0 & 21.1 & 24.7 & 26.4 & 29.8 & 30.6 & 30.9 & 31.4 \\
\hline \multirow[t]{2}{*}{655990} & 2 & 2.6 & 4.1 & 5.2 & 5.6 & 5.7 & 5.8 & 5.9 & 6.0 & 6.0 & 5.98 \\
\hline & 10 & 6.4 & 11.0 & 17.0 & 21.3 & 25.3 & 27.5 & 32.8 & 34.4 & 35.1 & 35.8 \\
\hline
\end{tabular}




\begin{tabular}{|c|c|c|c|c|c|c|c|c|c|c|c|}
\hline \multirow{2}{*}{\multicolumn{12}{|c|}{$\begin{array}{l}\text { Conditioned 1st-Order Markov Model } \\
\text { April DRWH Reliability (\%) } \\
\text { per capita roof area and storage } \\
\text { L }\end{array}$}} \\
\hline & & & & & & & & & & & \\
\hline \multirow{3}{*}{$\begin{array}{l}\text { Gauge } \\
653060\end{array}$} & sq. m & 0 & 40 & 80 & 120 & 160 & 200 & 400 & 600 & 800 & 1000 \\
\hline & 2 & 2.4 & 3.7 & 4.7 & 5.1 & 5.3 & 5.3 & 5.4 & 5.4 & 5.4 & 5.37 \\
\hline & 10 & 6.5 & 11.0 & 16.7 & 20.4 & 23.5 & 25.0 & 27.9 & 28.5 & 28.7 & 28.8 \\
\hline \multirow[t]{2}{*}{653190} & 2 & 6.7 & 11.0 & 13.8 & 14.7 & 15.0 & 15.1 & 15.2 & 15.2 & 15.2 & 15.2 \\
\hline & 10 & 15.1 & 26.0 & 38.5 & 46.1 & 51.9 & 54.8 & 59.9 & 60.5 & 60.6 & 60.6 \\
\hline \multirow[t]{2}{*}{653300} & 2 & 5.6 & 9.0 & 11.6 & 12.5 & 12.9 & 13.0 & 13.0 & 13.0 & 13.0 & 13 \\
\hline & 10 & 11.0 & 19.4 & 30.7 & 38.2 & 44.5 & 47.8 & 55.2 & 56.6 & 56.8 & 56.9 \\
\hline \multirow[t]{2}{*}{653350} & 2 & 9.0 & 14.6 & 18.2 & 19.3 & 19.7 & 19.8 & 19.9 & 19.9 & 19.9 & 19.9 \\
\hline & 10 & 18.2 & 31.8 & 48.1 & 58.1 & 65.7 & 69.7 & 78.2 & 80.4 & 81.1 & 81.3 \\
\hline \multirow[t]{2}{*}{653380} & 2 & 9.7 & 15.8 & 19.5 & 20.6 & 21.0 & 21.1 & 21.1 & 21.1 & 21.1 & 21.1 \\
\hline & 10 & 20.0 & 35.3 & 52.8 & 62.9 & 70.1 & 73.8 & 81.5 & 82.8 & 83.0 & 83.2 \\
\hline \multirow[t]{2}{*}{653440} & 2 & 10.0 & 16.3 & 21.2 & 23.5 & 24.8 & 25.5 & 26.8 & 26.9 & 26.9 & 26.9 \\
\hline & 10 & 18.9 & 32.7 & 49.8 & 60.1 & 68.0 & 72.1 & 81.5 & 83.9 & 84.7 & 85 \\
\hline \multirow{2}{*}{653520} & 2 & 3.0 & 4.3 & 5.1 & 5.3 & 5.5 & 5.6 & 6.0 & 6.1 & 6.1 & 6.11 \\
\hline & 10 & 7.6 & 13.2 & 20.2 & 24.6 & 28.3 & 30.2 & 35.1 & 38.4 & 39.8 & 40.1 \\
\hline \multirow[t]{2}{*}{653550} & 2 & 5.7 & 8.7 & 10.3 & 10.6 & 10.7 & 10.7 & 10.7 & 10.7 & 10.7 & 10.7 \\
\hline & 10 & 12.9 & 23.0 & 34.9 & 41.8 & 47.0 & 49.6 & 54.0 & 55.2 & 55.9 & 56.3 \\
\hline \multirow[t]{2}{*}{653570} & 2 & 4.2 & 6.1 & 7.0 & 7.2 & 7.3 & 7.4 & 7.7 & 7.9 & 8.0 & 8.04 \\
\hline & 10 & 12.9 & 21.9 & 32.0 & 37.5 & 41.4 & 43.3 & 46.7 & 47.9 & 48.4 & 48.5 \\
\hline \multirow[t]{2}{*}{653610} & 2 & 5.3 & 8.6 & 10.8 & 11.5 & 11.8 & 11.8 & 11.9 & 11.9 & 11.9 & 11.9 \\
\hline & 10 & 14.4 & 25.0 & 37.1 & 44.4 & 49.9 & 52.9 & 59.0 & 59.7 & 59.7 & 59.7 \\
\hline \multirow[t]{2}{*}{653760} & 2 & 7.5 & 12.5 & 15.9 & 17.0 & 17.5 & 17.6 & 17.7 & 17.7 & 17.7 & 17.7 \\
\hline & 10 & 16.2 & 28.1 & 42.1 & 51.0 & 58.0 & 61.7 & 69.6 & 71.3 & 71.6 & 71.6 \\
\hline \multirow{2}{*}{653800} & 2 & 7.3 & 12.0 & 15.8 & 17.2 & 17.7 & 17.9 & 18.1 & 18.1 & 18.2 & 18.4 \\
\hline & 10 & 14.3 & 25.6 & 40.4 & 49.9 & 57.5 & 61.7 & 70.9 & 72.9 & 73.7 & 74.1 \\
\hline \multirow[t]{2}{*}{653870} & 2 & 6.2 & 10.6 & 14.7 & 16.8 & 18.1 & 18.7 & 19.6 & 19.6 & 19.6 & 19.6 \\
\hline & 10 & 13.8 & 23.7 & 36.0 & 44.1 & 51.1 & 55.0 & 65.2 & 68.9 & 70.0 & 70.3 \\
\hline \multirow[t]{2}{*}{654720} & 2 & 4.9 & 8.6 & 12.6 & 14.8 & 16.3 & 16.9 & 17.6 & 17.7 & 17.7 & 17.7 \\
\hline & 10 & 9.7 & 17.0 & 26.9 & 33.7 & 40.1 & 43.6 & 52.4 & 54.8 & 55.6 & 55.9 \\
\hline \multirow[t]{2}{*}{655010} & 2 & 0.1 & 0.1 & 0.2 & 0.2 & 0.2 & 0.2 & 0.2 & 0.2 & 0.2 & 0.15 \\
\hline & 10 & 1.1 & 1.7 & 2.3 & 2.5 & 2.7 & 2.7 & 2.9 & 3.0 & 3.0 & 3.12 \\
\hline \multirow[t]{2}{*}{655020} & 2 & 0.5 & 0.6 & 0.7 & 0.7 & 0.7 & 0.7 & 0.7 & 0.7 & 0.7 & 0.73 \\
\hline & 10 & 2.0 & 3.2 & 4.7 & 5.6 & 6.3 & 6.6 & 7.4 & 7.9 & 8.2 & 8.34 \\
\hline 655030 & 2 & 2.3 & 3.2 & 3.8 & 3.9 & 4.0 & 4.1 & 4.2 & 4.3 & 4.3 & 4.31 \\
\hline & 10 & 4.9 & 8.7 & 13.9 & 17.2 & 20.1 & 21.4 & 24.3 & 25.9 & 26.7 & 26.9 \\
\hline 655050 & 2 & 1.3 & 1.9 & 2.3 & 2.4 & 2.5 & 2.5 & 2.5 & 2.5 & 2.5 & 2.55 \\
\hline & 10 & 3.7 & 6.5 & 9.9 & 12.0 & 13.7 & 14.5 & 16.4 & 17.2 & 17.7 & 18 \\
\hline 655070 & 2 & 1.8 & 2.6 & 3.2 & 3.4 & 3.7 & 3.9 & 4.6 & 4.9 & 5.0 & 4.99 \\
\hline & 10 & 5.4 & 9.3 & 14.0 & 17.0 & 19.6 & 21.1 & 26.2 & 30.7 & 33.1 & 33.8 \\
\hline 655100 & 2 & 3.3 & 5.1 & 6.1 & 6.3 & 6.3 & 6.3 & 6.3 & 6.3 & 6.3 & 6.32 \\
\hline & 10 & 8.5 & 14.7 & 22.2 & 27.1 & 30.9 & 32.8 & 36.2 & 36.6 & 36.6 & 36.6 \\
\hline 655160 & 2 & 2.7 & 4.3 & 5.6 & 6.2 & 6.7 & 6.9 & 7.3 & 7.3 & 7.3 & 7.35 \\
\hline & 10 & 8.0 & 13.5 & 20.0 & 24.0 & 27.2 & 28.9 & 33.0 & 34.5 & 35.1 & 35.2 \\
\hline 655180 & 2 & 2.9 & 4.5 & 5.5 & 5.8 & 6.0 & 6.1 & 6.2 & 6.3 & 6.3 & 6.29 \\
\hline & 10 & 6.9 & 12.1 & 18.2 & 22.4 & 25.9 & 27.8 & 32.2 & 34.0 & 34.7 & 35 \\
\hline 655220 & 2 & 5.0 & 7.8 & 9.8 & 10.3 & 10.5 & 10.6 & 10.6 & 10.6 & 10.6 & 10.6 \\
\hline & 10 & 10.9 & 19.1 & 29.7 & 36.6 & 42.5 & 45.7 & 52.7 & 54.2 & 54.4 & 54.4 \\
\hline 655280 & 2 & 6.0 & 9.2 & 10.9 & 11.3 & 11.5 & 11.6 & 11.9 & 12.1 & 12.2 & 12.2 \\
\hline & 10 & 14.3 & 24.9 & 37.0 & 44.3 & 49.8 & 52.6 & 57.2 & 58.3 & 58.8 & 58.9 \\
\hline 655360 & 2 & 6.0 & 9.7 & 12.5 & 13.4 & 13.8 & 13.9 & 14.0 & 14.0 & 14.1 & 14.1 \\
\hline & 10 & 13.6 & 23.9 & 36.6 & 44.4 & 50.7 & 54.1 & 62.1 & 64.6 & 65.3 & 65.5 \\
\hline
\end{tabular}




\begin{tabular}{|c|c|c|c|c|c|c|c|c|c|c|c|}
\hline \multirow{2}{*}{\multicolumn{12}{|c|}{$\begin{array}{l}\text { Conditioned 1st-Order Markov Model J-9 } \\
\text { April DRWH Reliability (\%) } \\
\text { per capita roof area and storage }\end{array}$}} \\
\hline & & & & & & & & & & & \\
\hline \multirow{3}{*}{$\begin{array}{l}\text { Gauge } \\
655450\end{array}$} & sq. m & 0 & 40 & 80 & 120 & 160 & 200 & 400 & 600 & 800 & 1000 \\
\hline & 2 & 8.1 & 12.6 & 15.9 & 17.3 & 18.1 & 18.5 & 18.9 & 18.9 & 18.9 & 18.9 \\
\hline & 10 & 17.1 & 29.9 & 45.6 & 55.2 & 62.4 & 66.1 & 74.4 & 76.9 & 77.7 & 78 \\
\hline \multirow[t]{2}{*}{655480} & 2 & 9.9 & 17.1 & 23.7 & 26.6 & 28.2 & 29.0 & 30.1 & 30.2 & 30.2 & 30.2 \\
\hline & 10 & 18.7 & 33.1 & 50.5 & 61.1 & 69.4 & 74.0 & 84.8 & 87.5 & 87.9 & 88 \\
\hline \multirow[t]{2}{*}{655550} & 2 & 9.4 & 14.9 & 18.9 & 20.7 & 22.0 & 22.9 & 25.0 & 25.7 & 25.8 & 25.8 \\
\hline & 10 & 17.9 & 31.3 & 47.6 & 57.1 & 64.2 & 67.7 & 75.9 & 79.2 & 80.2 & 80.4 \\
\hline \multirow[t]{2}{*}{655570} & 2 & 10.7 & 17.5 & 22.3 & 24.4 & 25.7 & 26.5 & 29.0 & 30.5 & 30.9 & 31 \\
\hline & 10 & 23.0 & 39.7 & 57.7 & 67.1 & 73.4 & 76.8 & 85.1 & 88.7 & 89.8 & 90.2 \\
\hline \multirow[t]{2}{*}{655600} & 2 & 9.3 & 14.6 & 17.9 & 18.9 & 19.4 & 19.6 & 20.0 & 20.0 & 20.0 & 20 \\
\hline & 10 & 18.5 & 32.9 & 49.9 & 59.8 & 66.9 & 70.6 & 79.2 & 82.1 & 82.8 & 82.9 \\
\hline \multirow[t]{2}{*}{655620} & 2 & 9.3 & 14.8 & 18.6 & 20.0 & 20.7 & 21.0 & 21.4 & 21.4 & 21.4 & 21.4 \\
\hline & 10 & 18.3 & 32.0 & 48.7 & 58.7 & 66.4 & 70.5 & 80.3 & 82.9 & 83.2 & 83.3 \\
\hline \multirow[t]{2}{*}{655630} & 2 & 8.1 & 12.3 & 14.5 & 15.1 & 15.4 & 15.5 & 15.5 & 15.5 & 15.5 & 15.5 \\
\hline & 10 & 18.6 & 32.6 & 48.4 & 57.3 & 63.7 & 66.9 & 73.4 & 74.5 & 74.7 & 74.8 \\
\hline \multirow[t]{2}{*}{655780} & 2 & 10.0 & 16.7 & 21.6 & 23.6 & 24.5 & 25.0 & 25.5 & 25.6 & 25.6 & 25.6 \\
\hline & 10 & 18.1 & 32.4 & 49.2 & 59.4 & 67.0 & 71.3 & 81.4 & 84.2 & 84.8 & 85.2 \\
\hline \multirow[t]{2}{*}{655850} & 2 & 8.5 & 14.5 & 18.9 & 20.6 & 21.4 & 21.7 & 22.1 & 22.2 & 22.2 & 22.2 \\
\hline & 10 & 20.0 & 34.8 & 50.7 & 59.9 & 66.3 & 70.2 & 79.9 & 83.5 & 84.6 & 85.4 \\
\hline \multirow[t]{2}{*}{655920} & 2 & 8.6 & 14.5 & 18.7 & 20.4 & 21.3 & 21.7 & 22.3 & 22.3 & 22.3 & 22.3 \\
\hline & 10 & 17.7 & 30.8 & 45.9 & 55.4 & 62.8 & 66.9 & 75.9 & 78.6 & 79.8 & 81.5 \\
\hline \multirow[t]{2}{*}{655940} & 2 & 6.3 & 10.4 & 13.7 & 14.9 & 15.4 & 15.5 & 15.7 & 15.7 & 15.7 & 15.7 \\
\hline & 10 & 12.1 & 21.4 & 33.6 & 41.8 & 48.6 & 52.3 & 60.5 & 62.5 & 62.9 & 63.1 \\
\hline \multirow[t]{2}{*}{655990} & 2 & 8.0 & 13.3 & 17.9 & 19.7 & 20.8 & 21.1 & 21.6 & 21.6 & 21.6 & 21.6 \\
\hline & 10 & 14.2 & 24.9 & 38.8 & 47.9 & 55.7 & 59.7 & 68.2 & 70.1 & 70.5 & 70.8 \\
\hline
\end{tabular}




\begin{tabular}{|c|c|c|c|c|c|c|c|c|c|c|c|}
\hline May $\mathrm{C}$ & $\begin{array}{l}\text { H Re } \\
\text { er C }\end{array}$ & lity & 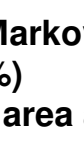 & 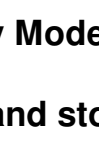 & & & & & & & \\
\hline & & $\begin{array}{l}L \\
0\end{array}$ & 40 & 80 & 120 & 160 & 200 & 400 & 600 & 800 & \\
\hline 653060 & & 7.8 & 12.3 & 14.9 & 15.6 & 15.8 & & 15.9 & 15.9 & & \\
\hline & 10 & 16.0 & 28.1 & 42.2 & 50.5 & 56.5 & 59.4 & 65.0 & 66.3 & 66.7 & \\
\hline 653190 & 2 & 7.9 & 12.6 & 15.1 & 15.8 & 16.1 & 16.2 & 16.3 & 16.3 & 16.3 & \\
\hline & 10 & 17.5 & 31.0 & 46.6 & 56.4 & 64.1 & 68.5 & 79.7 & 82.5 & 82.9 & \\
\hline 653300 & 2 & 9.7 & 14.7 & 17.3 & 18.0 & 18.3 & 18.5 & 18.8 & 18.8 & 18.8 & \\
\hline & 10 & 22.1 & 38.9 & 57.0 & 66.8 & 73.2 & 76.6 & 83.5 & 85.3 & 85.6 & \\
\hline 653350 & 2 & 8.6 & 13.6 & 16.6 & 17.4 & 17.7 & 17.8 & 17.9 & 17.9 & 17.9 & \\
\hline & 10 & 19.3 & 34.0 & 51.1 & 61.6 & 69.7 & 74.5 & 86.6 & 90.3 & 91.2 & \\
\hline 653380 & 2 & 9.8 & 15.7 & 19.2 & 20.4 & 20.8 & 21.0 & 21.1 & 21.2 & 21.2 & \\
\hline & 10 & 21.4 & 37.1 & 55.0 & 65.9 & 73.9 & 78.6 & 89.6 & 92.4 & 92.9 & \\
\hline 653440 & 2 & 13.6 & 23.9 & 31.6 & 34.9 & 36.7 & 37.6 & 39.9 & 40.8 & & \\
\hline & 10 & 24.6 & 43.6 & 64.1 & 75.5 & 83.1 & 87.2 & 94.9 & 96.5 & 96.8 & 7 \\
\hline 653520 & 2 & 6.9 & 10.6 & 12.6 & 13.0 & 13.1 & 13.1 & 13.1 & 13.1 & 13 & \\
\hline & 10 & 14.5 & & 38 & 47.0 & 53.6 & & & 5.0 & 66.2 & \\
\hline 653550 & 2 & 9.0 & & 14 & 15.2 & 15.2 & 3 & & 5.3 & & \\
\hline & 10 & 22.1 & 38 & 55. & 64.5 & 70.6 & & & & 6 & \\
\hline 653570 & 2 & 7.6 & 12 & 15. & 16.7 & 17.4 & & 18 & & & \\
\hline & 10 & 17.8 & 30. & 45.4 & 54.7 & 61.9 & 65.6 & 73 & 74.7 & 75.1 & \\
\hline 653610 & 2 & 7.9 & 12.2 & 14.5 & 15.3 & 15.8 & 16.0 & 16.2 & 16.3 & 16.3 & \\
\hline & 10 & 20.9 & 35.8 & 51.5 & 60.5 & 66.8 & 70.4 & 79.2 & 81.5 & 81.8 & \\
\hline 653760 & 2 & 9.2 & 14 & 18.8 & 20.6 & 21.6 & & & 22.9 & & \\
\hline & 10 & 20.2 & 34. & 51.8 & 62.1 & 70.1 & 74.5 & 85.3 & 88.5 & 89 & \\
\hline 653800 & 2 & 10.4 & 17. & 23.3 & 25.5 & 26.6 & 27.0 & & 27.3 & 27 & \\
\hline & 10 & 18.1 & & 49 & 60.9 & 70.0 & 74 & & .4 & 90.2 & \\
\hline 653870 & 2 & 9.9 & & 21 & 24.2 & 25.8 & 26 & 6 & 28.9 & 9 & \\
\hline & 10 & 21. & & 55.2 & 65.6 & 73.3 & 77.5 & 87 & 90.0 & 90.9 & \\
\hline 654720 & 2 & 6.9 & 11 & 13.8 & 15.1 & 15.8 & 16.2 & 17 & 17.6 & 17 & \\
\hline & 10 & 12.9 & 22. & 35.4 & 44.0 & 51.6 & 55.8 & 68.5 & 74.5 & 76.6 & \\
\hline 655010 & 2 & 1.5 & 2.1 & 2.4 & 2.4 & 2.4 & 2. & 2. & 2.4 & & \\
\hline & 10 & 4.5 & 7. & 11.2 & 13.4 & 15.1 & 15.8 & 17.0 & 17.0 & 17.0 & \\
\hline 655020 & 2 & 2.4 & 3. & 5.2 & 5.9 & 6.4 & 6.7 & & & & \\
\hline & 10 & 7.8 & 13.2 & 19.3 & 23.0 & 26.0 & 27.5 & 30.7 & 31.0 & & \\
\hline 655030 & 2 & 4.5 & 6.4 & 7.6 & 8.1 & 8.5 & 8.6 & 8.9 & 9.0 & & \\
\hline & 10 & 13.1 & 22.7 & 34.0 & 40.5 & 45.2 & 47.6 & 52.2 & 53.1 & 53.6 & \\
\hline 655050 & 2 & & & 8.0 & 8.5 & 8.7 & 8.7 & & & & \\
\hline & 10 & & & 28 & 34.8 & 39 & & & & 2 & \\
\hline 655070 & 2 & & & 11 & 12.3 & 12 & & & & & \\
\hline & 10 & 13.6 & & 35 & 42.6 & 47. & 50.7 & 55.7 & 56.9 & 58.5 & \\
\hline 655100 & 2 & 6.1 & 9. & 11.2 & 11.7 & 11.9 & 12.0 & 12 & 12.0 & 12.0 & \\
\hline & 10 & 15. & 27 & 40.9 & 49.0 & 55.1 & 58.4 & 65 & 66.5 & 66.7 & \\
\hline 655160 & 2 & 5.6 & 8.6 & 10.1 & 10.5 & 10.7 & 10.8 & 11.0 & 11.2 & 11.2 & \\
\hline & 10 & 14.2 & 24.7 & 36.9 & 44.2 & 49.7 & 52.7 & 59.4 & 61.7 & 62.5 & \\
\hline 655180 & 2 & 5.2 & 7.6 & 8.6 & 8.8 & 8.8 & 8.8 & 8.9 & 8.9 & 8.9 & \\
\hline & 10 & 13.8 & 24.1 & 36.2 & 43.4 & 48.7 & 51.4 & 57.4 & 59.2 & 59.9 & \\
\hline 655220 & 2 & 7.5 & 12.0 & 14.7 & 15.7 & 16.1 & 16.2 & 16.4 & 16.5 & 16.5 & \\
\hline & 10 & 15.7 & 27.9 & 42.9 & 52.5 & 60.1 & 64.2 & 73.9 & 76.4 & 76.9 & \\
\hline 655280 & 2 & 7.4 & 11. & 14.1 & 14.9 & 15.1 & 15.2 & 15.2 & 15.2 & 15.2 & \\
\hline & 10 & & 29.0 & 44.2 & 53.3 & 60.1 & 64.0 & 73.1 & 75.5 & 76.2 & \\
\hline & 2 & & 14 & 18.2 & 19.3 & 19.8 & 20.0 & 20.4 & 20.4 & 20.4 & \\
\hline & 10 & 20.0 & 35.1 & 52.5 & 62.7 & 70.2 & 74.3 & 83.4 & 85.9 & 86.6 & \\
\hline
\end{tabular}




\begin{tabular}{|c|c|c|c|c|c|c|c|c|c|c|c|}
\hline \multicolumn{12}{|c|}{$\begin{array}{l}\text { Conditioned 1st-Order Markov Model } \mathrm{J}-11 \\
\text { May DRWH Reliability (\%) } \\
\text { per capita roof area and storage }\end{array}$} \\
\hline & & $\mathrm{L}$ & 40 & 80 & 120 & 160 & 200 & 400 & 600 & 800 & 1000 \\
\hline \multirow{2}{*}{$\begin{array}{l}\text { Gauge } \\
655450\end{array}$} & 2 & 11.4 & 18.5 & 22.8 & 24.2 & 24.9 & 25.2 & 25.9 & 25.9 & 25.9 & 25.9 \\
\hline & 10 & 21.2 & 37.5 & 56.3 & 67.5 & 75.5 & 79.8 & 89.3 & 91.6 & 92.2 & 92.4 \\
\hline \multirow[t]{2}{*}{655480} & 2 & 9.9 & 16.2 & 20.3 & 22.0 & 23.0 & 23.6 & 24.7 & 25.0 & 25.0 & 25 \\
\hline & 10 & 21.2 & 37.0 & 55.0 & 66.0 & 74.2 & 79.0 & 91.0 & 94.9 & 95.9 & 96.2 \\
\hline \multirow[t]{2}{*}{655550} & 2 & 7.3 & 11.5 & 14.4 & 15.6 & 16.2 & 16.7 & 18.7 & 20.1 & 20.6 & 20.7 \\
\hline & 10 & 16.7 & 29.3 & 45.2 & 55.5 & 63.9 & 68.9 & 82.4 & 87.1 & 88.8 & 89.4 \\
\hline \multirow[t]{2}{*}{655570} & 2 & 12.8 & 20.7 & 24.8 & 26.4 & 27.2 & 27.8 & 29.4 & 30.1 & 30.8 & 31.3 \\
\hline & 10 & 30.5 & 50.3 & 69.3 & 78.9 & 85.0 & 88.0 & 94.4 & 95.9 & 96.6 & 96.9 \\
\hline \multirow[t]{2}{*}{655600} & 2 & 9.1 & 14.3 & 17.2 & 18.0 & 18.3 & 18.5 & 19.0 & 19.4 & 19.5 & 19.6 \\
\hline & 10 & 22.4 & 39.2 & 57.4 & 67.8 & 75.0 & 79.2 & 89.0 & 91.7 & 92.4 & 92.6 \\
\hline \multirow[t]{2}{*}{655620} & 2 & 11.3 & 18.5 & 22.7 & 24.0 & 24.6 & 24.8 & 25.4 & 25.5 & 25.5 & 25.5 \\
\hline & 10 & 22.9 & 40.3 & 59.3 & 70.3 & 78.0 & 82.2 & 91.1 & 93.4 & 94.1 & 94.3 \\
\hline \multirow[t]{2}{*}{655630} & 2 & 10.2 & 16.9 & 20.9 & 22.1 & 22.4 & 22.6 & 22.8 & 22.8 & 22.8 & 22.8 \\
\hline & 10 & 22.3 & 39.1 & 57.7 & 68.5 & 76.0 & 80.3 & 89.4 & 91.0 & 91.3 & 91.4 \\
\hline \multirow[t]{2}{*}{655780} & 2 & 16.9 & 30.0 & 38.8 & 42.8 & 44.8 & 45.9 & 47.1 & 47.2 & 47.2 & 47.2 \\
\hline & 10 & 30.9 & 52.8 & 72.7 & 82.6 & 88.5 & 91.4 & 96.6 & 97.6 & 97.8 & 98 \\
\hline \multirow[t]{2}{*}{655850} & 2 & 16.0 & 28.7 & 37.1 & 40.5 & 42.1 & 42.8 & 43.5 & 43.5 & 43.6 & 43.6 \\
\hline & 10 & 30.9 & 53.1 & 72.9 & 82.5 & 87.8 & 90.6 & 95.5 & 96.6 & 97.0 & 97.2 \\
\hline \multirow[t]{2}{*}{655920} & 2 & 20.2 & 36.5 & 46.6 & 50.9 & 53.0 & 53.9 & 54.7 & 54.8 & 54.8 & 54.8 \\
\hline & 10 & 36.3 & 58.6 & 76.2 & 84.4 & 89.2 & 91.6 & 96.3 & 97.2 & 97.5 & 97.7 \\
\hline \multirow[t]{2}{*}{655940} & 2 & 13.7 & 23.5 & 30.0 & 32.7 & 34.0 & 34.6 & 35.1 & 35.1 & 35.1 & 35.1 \\
\hline & 10 & 26.7 & 45.3 & 63.6 & 73.6 & 79.9 & 83.1 & 89.7 & 91.3 & 91.6 & 91.7 \\
\hline \multirow[t]{2}{*}{655990} & 2 & 16.7 & 28.7 & 36.6 & 39.7 & 41.2 & 41.9 & 42.9 & 43.0 & 43.1 & 43.1 \\
\hline & 10 & 31.2 & 50.5 & 69.7 & 79.6 & 86.1 & 89.1 & 94.9 & 96.0 & 96.2 & 96.3 \\
\hline
\end{tabular}




\begin{tabular}{|c|c|c|c|c|c|c|c|c|c|c|c|}
\hline Gauge & sq. m & 0 & 40 & 80 & 120 & 160 & 200 & 400 & 600 & 800 & \\
\hline \multirow[t]{2}{*}{653060} & & 13.3 & & & 27.4 & 27.8 & & 28.1 & 28.1 & 28.1 & \\
\hline & 10 & 24.7 & 43.8 & 64.6 & 75.9 & 83.0 & 86.4 & 92.3 & 93.0 & 93.1 & \\
\hline \multirow[t]{2}{*}{653190} & 2 & 12.3 & 19.9 & 23.2 & 23.9 & 24.1 & 24.2 & 24.2 & 24.2 & 24.2 & \\
\hline & 10 & 29.0 & 49.1 & 68.5 & 78.5 & 84.6 & 87.8 & 93.8 & 95.4 & 95.9 & \\
\hline \multirow[t]{2}{*}{653300} & 2 & 12.7 & 21.4 & 26.3 & 27.7 & 28.3 & 28.5 & 29.0 & 29.1 & 29.2 & \\
\hline & 10 & 26.9 & 47.0 & 67.1 & 78.1 & 85.0 & 88.7 & 95.2 & 96.2 & 96.5 & \\
\hline \multirow[t]{2}{*}{653350} & 2 & 11.6 & 18.8 & 21.8 & 22.5 & 22.8 & 22.8 & 22.9 & 22.9 & 22.9 & \\
\hline & 10 & 27.1 & 47.0 & 65.8 & 76.0 & 82.5 & 86.1 & 93.5 & 95.7 & 96.5 & \\
\hline \multirow[t]{2}{*}{653380} & 2 & 13.3 & 22.1 & 26.9 & 28.3 & 28.8 & 28.9 & 29.0 & 29.0 & 29.0 & 29 \\
\hline & 10 & 26.4 & 46.7 & 67.0 & 77.8 & 84.4 & 88.1 & 95.5 & 97.5 & 98 & 98 \\
\hline \multirow[t]{2}{*}{653440} & 2 & 19.9 & 36 & 47.1 & 52.2 & 54.9 & 56.3 & 57.6 & 58.0 & & \\
\hline & 10 & 33.5 & & & 87.6 & 92.9 & 95.6 & 99.3 & 99.7 & & \\
\hline \multirow[t]{2}{*}{653520} & 2 & 8.1 & & & 18.7 & 19.8 & 20.4 & 21.2 & 21.3 & .3 & \\
\hline & 10 & 19.6 & & & 61.3 & 68.9 & 3.2 & 83.2 & .2 & & \\
\hline \multirow[t]{2}{*}{653550} & 2 & 13.5 & & 27. & 29.4 & 29.9 & 30.0 & 30.1 & 30.1 & & \\
\hline & 10 & 27.3 & 47 & 67.0 & 77.7 & 84.2 & 87.8 & 94.2 & 95.2 & 95.4 & \\
\hline \multirow[t]{2}{*}{653570} & 2 & 11.0 & 18.0 & 22.7 & 24.5 & 25.4 & 25.9 & 26.8 & 26.9 & 26.9 & \\
\hline & 10 & 21.8 & 38.4 & 57.6 & 68.9 & 76.7 & 80.9 & 89.9 & 92.0 & 92.3 & \\
\hline \multirow[t]{2}{*}{653610} & 2 & 12.5 & 20.1 & 23.4 & 24.3 & 24.6 & 24.8 & 25.4 & 25.6 & 25.6 & \\
\hline & 10 & 29.9 & 51 & 71.6 & 81.5 & 87.1 & 90.0 & 94.7 & 95.7 & 95.9 & 6 \\
\hline \multirow[t]{2}{*}{653760} & 2 & 12.0 & 20 & 27.0 & 29.7 & 31.2 & 32.0 & 33.6 & 33.8 & 33.8 & \\
\hline & 10 & 26.9 & & & 75.9 & 82.9 & 86.7 & 94.2 & 96.1 & & \\
\hline \multirow[t]{2}{*}{653800} & 2 & 9.5 & & & 23.6 & 24.8 & 25.4 & 26.2 & 26.2 & .3 & \\
\hline & 10 & 22.0 & & & 65.8 & 73.7 & .4 & .7 & 1.9 & & \\
\hline \multirow[t]{2}{*}{653870} & 2 & 11. & & 24 & 26.4 & 27.6 & 28.3 & 29.7 & 30.2 & & \\
\hline & 10 & 22.6 & & 58. & 69.7 & 77.6 & 82.4 & 93.4 & 96.2 & .9 & \\
\hline \multirow[t]{2}{*}{654720} & 2 & & 14 & 18. & 19.9 & 20.3 & 20.5 & 20.7 & 20.7 & 20.7 & \\
\hline & 10 & 16.4 & 30.0 & 45.6 & 56.0 & 64.2 & 69.1 & 81.1 & 85.5 & 87.7 & \\
\hline \multirow[t]{2}{*}{655010} & 2 & 4.3 & 6. & 7.2 & 7.4 & 7.4 & 7.5 & 7.5 & 7. & & \\
\hline & 10 & 11.7 & 20 & 30.9 & 37.1 & 41 & 43 & 47 & & & \\
\hline \multirow[t]{2}{*}{655020} & 2 & & 10 & 13.0 & 14.0 & 14.5 & 14.8 & 15.4 & 15.5 & 15.5 & \\
\hline & 10 & 16.4 & 28 & 43.1 & 51.4 & 57.1 & 60.2 & 66.4 & 68.2 & .7 & \\
\hline 655030 & 2 & 7.5 & & 13.7 & 14.4 & 14 & 15.0 & 15.4 & 15.5 & .5 & \\
\hline & 10 & 20.4 & & 52.7 & 61.9 & 8.2 & 71.6 & 77.8 & 79.1 & 9.4 & \\
\hline 655050 & 2 & & & & & & & & & & \\
\hline & 10 & & & 51 & 60.7 & 67. & & & & & \\
\hline 655070 & 2 & & & & 23.1 & 24 & & 26 & & & \\
\hline & 10 & 22.5 & & 57. & 67.8 & 75.1 & 79.0 & 86.3 & 87.5 & 87.8 & \\
\hline 655100 & 2 & 10.7 & 16. & 19.8 & 20.5 & 20.7 & 20.7 & 20.8 & 20.8 & 20.8 & \\
\hline & 10 & 24.0 & & 61. & 72.2 & 79.0 & & 1 & 0.3 & & \\
\hline 655160 & 2 & 9.7 & 14 & 16.3 & 16.6 & 16.7 & 16.7 & 16.7 & 16.7 & 16.7 & \\
\hline & 10 & 22.7 & 40 & 58.6 & 68.7 & 75.2 & 78.7 & 85.3 & 86.7 & 87.1 & \\
\hline 655180 & 2 & 13.3 & 20.8 & 24.6 & 25.5 & 25.6 & 25.7 & 25.7 & 25.7 & 25.7 & \\
\hline & 10 & 25.5 & 45.1 & 65.9 & 76.4 & 82.6 & 85.4 & 89.7 & 90.3 & 90.5 & \\
\hline 655220 & 2 & 11.3 & 17 & 21. & 23.1 & 23.7 & 24.0 & 24.4 & 24.4 & 24.4 & \\
\hline & 10 & 23.9 & 41. & 61.7 & 72.7 & 79.8 & 83.6 & 91.2 & 93.0 & 93.3 & \\
\hline 655280 & 2 & 10. & 15 & 18.1 & 18.7 & 18.8 & 18.9 & 18.9 & 18.9 & 18.9 & \\
\hline & 10 & 23.5 & 41. & 59.9 & 70.4 & 77.4 & 81.2 & 89.0 & 90.9 & 91.4 & \\
\hline & 2 & 11.5 & 18 & 22.2 & 23.4 & 24.0 & 24.3 & 25.1 & 25.3 & 25.4 & \\
\hline & 10 & 23.3 & 40.8 & 60.6 & 71.8 & 79.7 & 83.9 & 92.9 & 95.1 & 95.6 & \\
\hline
\end{tabular}




\begin{tabular}{|c|c|c|c|c|c|c|c|c|c|c|c|}
\hline \multicolumn{12}{|c|}{$\begin{array}{l}\text { Conditioned 1st-Order Markov Model J-13 } \\
\text { June DRWH Reliability (\%) } \\
\text { per capita roof area and storage }\end{array}$} \\
\hline Gauge & & $\mathrm{L}$ & 40 & 80 & 120 & 160 & 200 & 400 & 600 & 800 & 1000 \\
\hline \multirow{2}{*}{655450} & 2 & 13.2 & 21.6 & 26.6 & 28.3 & 28.8 & 29.0 & 29.1 & 29.1 & 29.1 & 29.1 \\
\hline & 10 & 25.9 & 45.4 & 66.3 & 77.6 & 84.8 & 88.6 & 96.1 & 97.7 & 98.1 & 98.2 \\
\hline \multirow[t]{2}{*}{655480} & 2 & 14.5 & 25.1 & 31.9 & 34.5 & 35.8 & 36.4 & 37.1 & 37.2 & 37.3 & 37.3 \\
\hline & 10 & 27.0 & 46.7 & 67.0 & 78.2 & 85.5 & 89.2 & 96.3 & 98.3 & 98.9 & 99.1 \\
\hline \multirow[t]{2}{*}{655550} & 2 & 9.6 & 15.2 & 18.3 & 19.2 & 19.6 & 19.8 & 20.0 & 20.1 & 20.5 & 20.9 \\
\hline & 10 & 20.5 & 36.2 & 53.8 & 64.1 & 71.5 & 75.7 & 86.1 & 90.9 & 93.1 & 93.9 \\
\hline \multirow[t]{2}{*}{655570} & 2 & 12.7 & 21.4 & 25.6 & 27.1 & 27.7 & 27.9 & 28.1 & 28.3 & 28.5 & 28.7 \\
\hline & 10 & 31.1 & 52.4 & 71.4 & 80.9 & 86.7 & 89.9 & 96.7 & 98.6 & 99.1 & 99.3 \\
\hline \multirow[t]{2}{*}{655600} & 2 & 9.2 & 15.6 & 19.8 & 21.7 & 22.7 & 23.3 & 24.2 & 24.3 & 24.3 & 24.5 \\
\hline & 10 & 23.6 & 40.6 & 57.9 & 67.9 & 74.9 & 79.2 & 89.7 & 94.0 & 95.3 & 95.6 \\
\hline \multirow[t]{2}{*}{655620} & 2 & 10.3 & 17.0 & 20.8 & 22.3 & 22.9 & 23.3 & 23.6 & 23.6 & 23.6 & 23.6 \\
\hline & 10 & 25.0 & 43.1 & 61.9 & 72.6 & 80.1 & 84.6 & 94.6 & 97.3 & 97.9 & 98 \\
\hline \multirow[t]{2}{*}{655630} & 2 & 10.0 & 15.6 & 18.1 & 19.1 & 19.7 & 20.0 & 20.4 & 20.4 & 20.5 & 20.5 \\
\hline & 10 & 25.9 & 43.2 & 61.5 & 72.1 & 79.6 & 83.9 & 93.7 & 96.3 & 96.7 & 96.8 \\
\hline \multirow[t]{2}{*}{655780} & 2 & 23.4 & 43.7 & 57.9 & 64.9 & 68.8 & 70.9 & 73.2 & 73.4 & 73.5 & 73.5 \\
\hline & 10 & 40.9 & 65.9 & 85.1 & 93.0 & 96.7 & 98.1 & 99.7 & 99.9 & 99.9 & 99.9 \\
\hline \multirow[t]{2}{*}{655850} & 2 & 23.0 & 43.0 & 56.4 & 63.0 & 66.7 & 68.6 & 70.4 & 70.4 & 70.5 & 70.5 \\
\hline & 10 & 43.5 & 67.9 & 85.9 & 93.3 & 96.8 & 98.2 & 99.7 & 99.9 & 99.9 & 99.9 \\
\hline \multirow[t]{2}{*}{655920} & 2 & 22.8 & 42.4 & 56.2 & 63.5 & 68.0 & 70.7 & 74.6 & 74.9 & 74.9 & 74.9 \\
\hline & 10 & 38.8 & 62.9 & 82.0 & 90.5 & 95.1 & 97.1 & 99.5 & 99.8 & 99.8 & 99.9 \\
\hline \multirow[t]{2}{*}{655940} & 2 & 18.9 & 35.2 & 45.9 & 51.1 & 54.0 & 55.6 & 58.0 & 58.2 & 58.2 & 58.2 \\
\hline & 10 & 38.5 & 62.5 & 81.4 & 89.9 & 94.4 & 96.4 & 98.9 & 99.2 & 99.2 & 99.3 \\
\hline \multirow[t]{2}{*}{655990} & 2 & 19.7 & 36.5 & 48.8 & 54.8 & 58.1 & 59.9 & 61.9 & 62.0 & 62.1 & 62.1 \\
\hline & 10 & 36.1 & 59.4 & 79.4 & 88.9 & 94.0 & 96.3 & 99.5 & 99.8 & 99.8 & 99.8 \\
\hline
\end{tabular}




\begin{tabular}{|c|c|c|c|c|c|c|c|c|c|c|c|}
\hline \multicolumn{12}{|c|}{$\begin{array}{l}\text { Conditioned 1st-Order Markov Model } \\
\text { July DRWH Reliability (\%) } \\
\text { per capita roof area and storage } \\
\text { L }\end{array}$} \\
\hline Gauge & sq. m & 0 & 40 & 80 & 120 & 160 & 200 & 400 & 600 & 800 & \\
\hline \multirow[t]{2}{*}{653060} & 2 & 17.4 & 29.5 & 37.2 & 39.8 & 40.9 & 41.2 & 41.5 & 41.5 & 41.5 & \\
\hline & 10 & 31.6 & 54.5 & 76.2 & 86.2 & 91.9 & 94.7 & 98.8 & 99.4 & 99.4 & \\
\hline \multirow[t]{2}{*}{653190} & 2 & 15.1 & 25.2 & 29.2 & 30.2 & 30.5 & 30.6 & 30.6 & 30.6 & 30.6 & \\
\hline & 10 & 34.7 & 59.3 & 79.4 & 88.5 & 93.3 & 95.7 & 99.1 & 99.5 & 99.6 & \\
\hline \multirow[t]{2}{*}{653300} & 2 & 15.5 & 27.5 & 35.2 & 38.2 & 39.6 & 40.2 & 40.7 & 40.8 & 40.8 & \\
\hline & 10 & 32.0 & 54.1 & 74.3 & 84.5 & 90.7 & 93.9 & 98.8 & 99.5 & 99.6 & \\
\hline \multirow[t]{2}{*}{653350} & 2 & 12.7 & 22.2 & 28.4 & 30.7 & 31.7 & 32.0 & 32.2 & 32.2 & 32.2 & \\
\hline & 10 & 23.2 & 41.0 & 59.9 & 71.9 & 80.7 & 85.8 & 96.1 & 98.3 & 3.8 & \\
\hline \multirow[t]{2}{*}{653380} & 2 & 11.7 & 20.5 & 26.6 & 28.9 & 29.9 & 30.3 & 30.6 & 30.6 & & \\
\hline & 10 & 21.6 & & 58.8 & 71.1 & 79.8 & 85.1 & & 98.8 & & \\
\hline \multirow[t]{2}{*}{653440} & 2 & 5.7 & & 15.3 & 18.6 & 21.2 & 23.2 & .0 & 30.5 & & \\
\hline & 10 & 14.3 & & 39.1 & 49.8 & 59.9 & 67.1 & 90.9 & 3.9 & & 9 \\
\hline \multirow[t]{2}{*}{653520} & 2 & 14.1 & & 30.3 & 33.0 & 34.3 & 35.1 & 37.1 & 37.7 & & \\
\hline & 10 & 24.8 & 44.4 & 65.0 & 76.0 & 83.0 & 86.8 & 94.0 & 95.7 & & \\
\hline \multirow[t]{2}{*}{653550} & 2 & 14.1 & 25.5 & 32.8 & 35.7 & 37.0 & 37.6 & 38.0 & 38.0 & 38.0 & 8 \\
\hline & 10 & 28.7 & 50.9 & 70.2 & 80.3 & 86.6 & 90.4 & 97.8 & 99.1 & 99.2 & \\
\hline \multirow[t]{2}{*}{653570} & 2 & 11.7 & 19.2 & 23.1 & 24.3 & 24.9 & 25.2 & 25.8 & 25.9 & & \\
\hline & 10 & 29.5 & 51 & 70.9 & 80.5 & 86.3 & 89.6 & 96.3 & 97.8 & & \\
\hline \multirow[t]{2}{*}{653610} & 2 & 16.9 & 29.8 & 36.2 & 38.3 & 39.1 & 39.4 & 39.6 & 39.7 & 39.8 & \\
\hline & 10 & & & & 91.3 & 95.2 & 97.1 & & 99.6 & & \\
\hline \multirow[t]{2}{*}{653760} & 2 & 13 & & & 30.8 & 32.1 & 32.7 & 33.8 & 34.0 & & \\
\hline & 10 & 31.7 & 52 & 71. & 81.6 & 88.1 & & 98.0 & .2 & & \\
\hline \multirow[t]{2}{*}{653800} & 2 & & & 10. & 10.7 & 11.2 & 11.5 & 12.1 & 12.3 & & \\
\hline & 10 & 13.7 & 24 & 37.1 & 46.2 & 54.2 & 59.8 & 79.6 & 90.1 & .0 & \\
\hline \multirow[t]{2}{*}{653870} & 2 & 5.1 & 8. & 11.7 & 13.0 & 13.7 & 14.1 & 14.6 & 14.8 & 14.9 & \\
\hline & 10 & 10.4 & 19 & 30.4 & 39.4 & 48.3 & 54.6 & 77.7 & 90.9 & 95.2 & \\
\hline \multirow[t]{2}{*}{654720} & 2 & 4.0 & 7. & 9.6 & 10.8 & 11.5 & 11.9 & 12.3 & 12.3 & 12.3 & \\
\hline & 10 & 8.5 & & 24. & 32.1 & 39.7 & 5.1 & 65 & 78.3 & 83 & \\
\hline \multirow[t]{2}{*}{655010} & 2 & 9.1 & 14.4 & 17.2 & 17.8 & 17.9 & 17.9 & 17.9 & 17.9 & 17.9 & \\
\hline & 10 & 19.1 & 34 & 52.9 & 63.2 & 69.9 & 73.5 & 79.8 & 80.6 & .7 & \\
\hline \multirow[t]{2}{*}{655020} & 2 & 12.4 & & 22.9 & 23.7 & 24.1 & .3 & .5 & .5 & .5 & \\
\hline & 10 & & & & 76.0 & 82.4 & & & 2.4 & & 9 \\
\hline 655030 & 2 & & & 29 & 30.7 & 31 & & & & & \\
\hline & 10 & 28 & 50 & 72.3 & 82.2 & 87.9 & 90.8 & 95.4 & 95.9 & 96.1 & \\
\hline 655050 & 2 & 14. & 23 & 28.9 & 30.4 & 31.1 & 31.5 & 32.9 & 33.5 & & \\
\hline & 10 & 29.7 & 51.2 & 72.5 & 82.6 & 88.2 & 91.0 & 95.4 & 96.1 & 96.2 & \\
\hline 655070 & 2 & 13.9 & 23.7 & 29.9 & 32.1 & 33.1 & 33.6 & 34.6 & 35.0 & 35.1 & \\
\hline & 10 & 28.6 & 49. & 70.2 & 80.2 & 86.1 & 89.5 & 95.9 & 97.2 & 97.4 & \\
\hline 655100 & 2 & 13.4 & 21.7 & 25.9 & 27.1 & 27.5 & 27.7 & 27.9 & 27.9 & 27.9 & \\
\hline & 10 & 29.3 & 50.9 & 71.9 & 82.2 & 88.4 & 91.6 & 97.2 & 97.9 & 98.1 & \\
\hline 655160 & 2 & 15.2 & 25.2 & 30.3 & 31.5 & 31.9 & 32.0 & 32.1 & 32.1 & 32.1 & \\
\hline & 10 & 30.3 & 52.9 & 74.1 & 84.1 & 89.7 & 92.5 & 97.1 & 97.7 & 97.9 & \\
\hline 655180 & 2 & 14. & 25 & 32. & 35.5 & 36.8 & 37.3 & 37.6 & 37.6 & 37.6 & \\
\hline & 10 & 26. & 46 & 68.0 & 79.5 & 86.8 & 90.8 & 97.4 & 98.2 & 98.3 & \\
\hline 655220 & 2 & 14. & 24 & 29.8 & 31.6 & 32.3 & 32.6 & 33.2 & 33.3 & 33.3 & \\
\hline & 10 & & 50.9 & 71.9 & 82.4 & 88.8 & 92.2 & 97.8 & 98.7 & 98.9 & \\
\hline 655280 & 2 & & 26 & 33 & 37.2 & 38.9 & & 40.6 & 40.6 & & \\
\hline & 10 & & & 76 & 85.6 & 91.0 & & 97.8 & 98.6 & 98.8 & \\
\hline 655360 & 2 & 13.7 & 22.4 & 27.6 & 29.2 & 29.8 & 30.1 & 30.5 & 30.7 & 30.7 & \\
\hline & 10 & 25.9 & 45.3 & 66.5 & 78.0 & 85.6 & 89.5 & 97.0 & 98.7 & 99.1 & \\
\hline
\end{tabular}




\begin{tabular}{|c|c|c|c|c|c|c|c|c|c|c|c|}
\hline \multicolumn{12}{|c|}{$\begin{array}{l}\text { Conditioned 1st-Order Markov Model J-15 } \\
\text { July DRWH Reliability (\%) } \\
\text { per capita roof area and storage }\end{array}$} \\
\hline & & $\begin{array}{l}\mathrm{L} \\
0\end{array}$ & 40 & 80 & 120 & 160 & 200 & 400 & 600 & 800 & 1000 \\
\hline \multirow{2}{*}{$\begin{array}{l}\text { Gauge } \\
655450\end{array}$} & 2 & 6.8 & 10.9 & 13.6 & 14.6 & 15.0 & 15.2 & 15.3 & 15.3 & 15.3 & 15.3 \\
\hline & 10 & 15.4 & 27.6 & 42.9 & 53.6 & 62.9 & 69.1 & 89.1 & 96.5 & 98.0 & 98.2 \\
\hline \multirow[t]{2}{*}{655480} & 2 & 14.8 & 25.9 & 32.1 & 34.9 & 36.5 & 37.3 & 39.5 & 40.4 & 40.7 & 40.7 \\
\hline & 10 & 32.2 & 55.4 & 74.9 & 84.8 & 90.7 & 93.9 & 99.0 & 99.7 & 99.9 & 99.9 \\
\hline \multirow[t]{2}{*}{655550} & 2 & 8.5 & 14.0 & 18.2 & 20.3 & 21.7 & 22.5 & 23.8 & 23.9 & 23.9 & 24 \\
\hline & 10 & 17.3 & 29.8 & 45.8 & 56.4 & 65.6 & 71.0 & 85.4 & 91.0 & 93.4 & 94.6 \\
\hline \multirow[t]{2}{*}{655570} & 2 & 4.7 & 7.6 & 8.9 & 9.4 & 9.7 & 9.8 & 9.9 & 9.9 & 10.0 & 10 \\
\hline & 10 & 17.0 & 29.4 & 42.9 & 52.5 & 60.9 & 66.9 & 87.4 & 95.9 & 98.2 & 98.8 \\
\hline \multirow[t]{2}{*}{655600} & 2 & 6.0 & 9.7 & 11.6 & 12.4 & 13.0 & 13.4 & 15.5 & 16.5 & 16.7 & 16.7 \\
\hline & 10 & 18.3 & 32.2 & 46.5 & 56.1 & 64.0 & 69.4 & 85.8 & 92.2 & 94.8 & 95.8 \\
\hline \multirow[t]{2}{*}{655620} & 2 & 5.2 & 8.5 & 10.4 & 11.1 & 11.5 & 11.7 & 12.2 & 12.3 & 12.4 & 12.4 \\
\hline & 10 & 15.5 & 27.3 & 40.5 & 49.9 & 58.2 & 64.0 & 84.4 & 93.5 & 96.5 & 97.1 \\
\hline \multirow[t]{2}{*}{655630} & 2 & 5.8 & 10.0 & 13.2 & 14.4 & 15.1 & 15.4 & 16.3 & 16.7 & 16.9 & 16.9 \\
\hline & 10 & 14.6 & 25.9 & 39.7 & 49.6 & 58.5 & 64.4 & 83.9 & 92.7 & 95.3 & 95.7 \\
\hline \multirow[t]{2}{*}{655780} & 2 & 5.8 & 11.5 & 17.3 & 21.5 & 25.4 & 28.7 & 39.9 & 43.3 & 43.7 & 43.7 \\
\hline & 10 & 14.7 & 26.5 & 40.6 & 51.9 & 62.6 & 70.1 & 92.7 & 99.5 & 100.0 & 100 \\
\hline \multirow[t]{2}{*}{655850} & 2 & 8.9 & 17.7 & 25.7 & 31.0 & 35.2 & 38.6 & 48.7 & 50.7 & 50.7 & 50.7 \\
\hline & 10 & 20.1 & 36.9 & 54.4 & 66.8 & 76.7 & 83.3 & 97.9 & 99.9 & 100.0 & 100 \\
\hline \multirow[t]{2}{*}{655920} & 2 & 8.6 & 17.4 & 25.5 & 31.1 & 35.7 & 39.6 & 52.9 & 57.0 & 57.5 & 57.5 \\
\hline & 10 & 25.4 & 42.8 & 59.8 & 71.2 & 80.3 & 85.9 & 98.0 & 99.8 & 100.0 & 100 \\
\hline \multirow[t]{2}{*}{655940} & 2 & 5.8 & 11.3 & 16.7 & 20.3 & 23.0 & 25.0 & 30.0 & 31.2 & 31.4 & 31.4 \\
\hline & 10 & 17.0 & 29.7 & 44.2 & 55.2 & 65.2 & 72.3 & 93.5 & 99.2 & 99.7 & 99.7 \\
\hline \multirow[t]{2}{*}{655990} & 2 & 5.7 & 11.0 & 15.9 & 19.1 & 21.8 & 23.8 & 29.6 & 30.9 & 31.0 & 31.1 \\
\hline & 10 & 13.9 & 25.2 & 38.6 & 49.4 & 59.8 & 67.1 & 90.5 & 98.9 & 99.9 & 99.9 \\
\hline
\end{tabular}




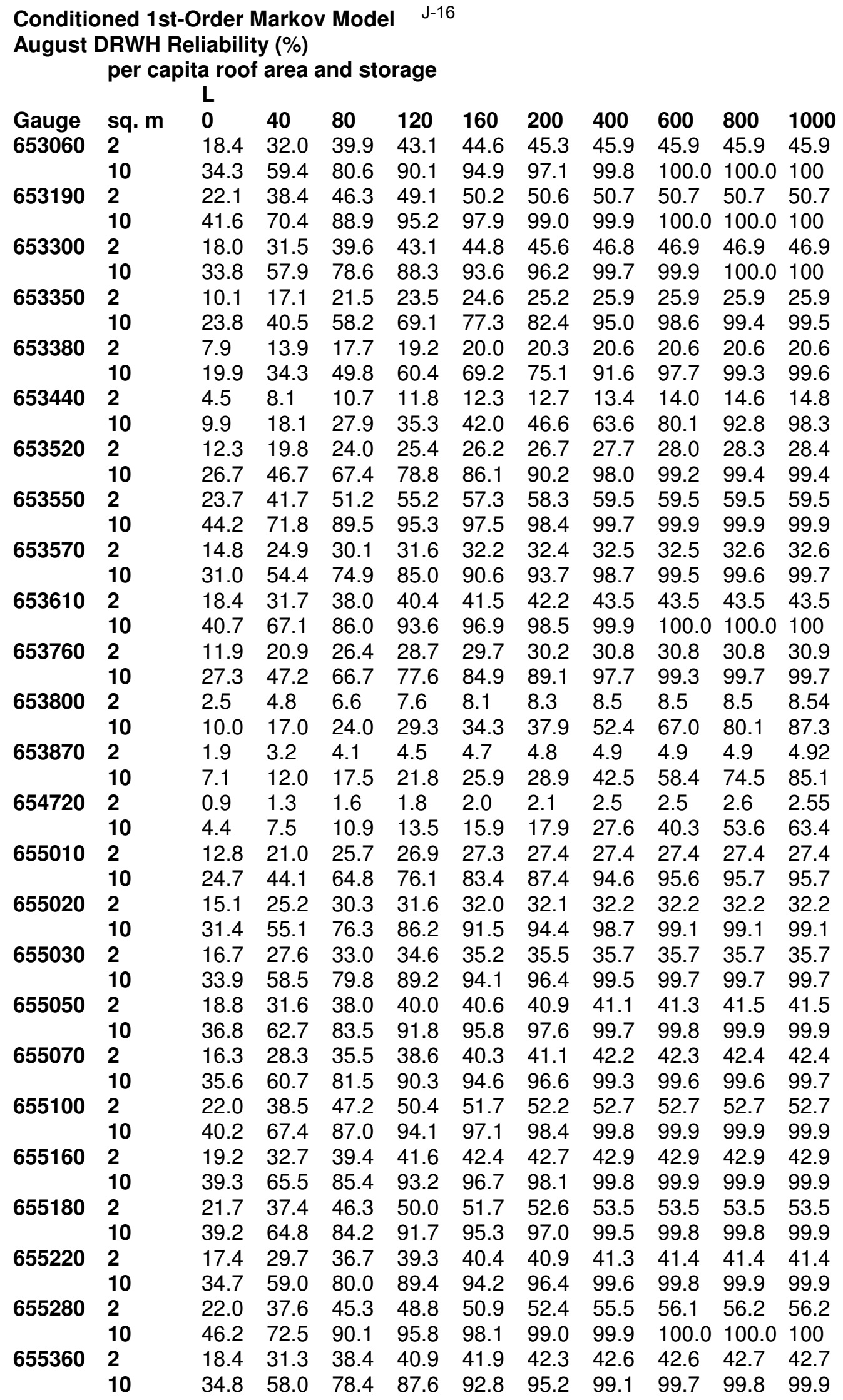




\begin{tabular}{|c|c|c|c|c|c|c|c|c|c|c|c|}
\hline \multicolumn{12}{|c|}{$\begin{array}{l}\text { Conditioned 1st-Order Markov Model J-17 } \\
\text { August DRWH Reliability (\%) } \\
\text { per capita roof area and storage }\end{array}$} \\
\hline & & $\mathrm{L}$ & 40 & 80 & 120 & 160 & 200 & 400 & 600 & 800 & 1000 \\
\hline \multirow[t]{2}{*}{655450} & & 8.5 & 14.5 & 18.8 & 20.5 & 21.2 & 21.4 & 21.7 & 21.7 & 21.7 & 21.7 \\
\hline & 10 & 14.8 & 27.1 & 42.0 & 52.3 & 60.6 & 65.7 & 80.3 & 89.6 & 95.3 & 97.3 \\
\hline \multirow[t]{2}{*}{655480} & 2 & 19.5 & 37.1 & 47.9 & 53.0 & 55.6 & 57.0 & 58.6 & 59.2 & 59.7 & 59.9 \\
\hline & 10 & 43.7 & 71.9 & 89.1 & 95.0 & 97.5 & 98.6 & 99.8 & 100.0 & 100.0 & 100 \\
\hline \multirow[t]{2}{*}{655550} & 2 & 9.7 & 15.8 & 19.6 & 21.5 & 22.8 & 23.8 & 26.6 & 27.9 & 28.2 & 28.2 \\
\hline & 10 & 20.9 & 35.6 & 52.3 & 62.6 & 70.4 & 74.9 & 87.1 & 92.5 & 95.0 & 96.2 \\
\hline \multirow[t]{2}{*}{655570} & 2 & 5.5 & 9.0 & 10.7 & 11.1 & 11.2 & 11.2 & 11.2 & 11.2 & 11.2 & 11.2 \\
\hline & 10 & 18.0 & 31.2 & 44.0 & 52.0 & 57.9 & 61.8 & 74.2 & 85.8 & 93.9 & 97.3 \\
\hline \multirow[t]{2}{*}{655600} & 2 & 8.7 & 14.8 & 18.0 & 18.9 & 19.3 & 19.4 & 19.5 & 19.6 & 19.9 & 20 \\
\hline & 10 & 21.7 & 38.4 & 54.2 & 63.7 & 70.1 & 74.2 & 85.1 & 91.6 & 95.0 & 96.4 \\
\hline \multirow[t]{2}{*}{655620} & 2 & 4.1 & 6.5 & 7.9 & 8.7 & 9.2 & 9.5 & 10.2 & 10.2 & 10.2 & 10.2 \\
\hline & 10 & 14.2 & 24.9 & 35.8 & 42.9 & 48.5 & 52.3 & 66.0 & 79.2 & 88.9 & 93.4 \\
\hline \multirow[t]{2}{*}{655630} & 2 & 6.8 & 11.4 & 14.1 & 15.0 & 15.4 & 15.5 & 15.8 & 15.8 & 15.9 & 16 \\
\hline & 10 & 15.5 & 27.7 & 41.4 & 50.6 & 58.0 & 62.7 & 77.0 & 86.5 & 92.4 & 94.6 \\
\hline \multirow[t]{2}{*}{655780} & 2 & 2.3 & 4.4 & 6.2 & 7.2 & 7.9 & 8.3 & 9.3 & 10.4 & 11.6 & 12.1 \\
\hline & 10 & 9.1 & 15.7 & 22.4 & 27.6 & 32.7 & 36.7 & 55.4 & 76.1 & 91.6 & 98.3 \\
\hline \multirow[t]{2}{*}{655850} & 2 & 3.7 & 6.4 & 8.4 & 9.6 & 10.4 & 10.9 & 12.5 & 14.2 & 14.8 & 15 \\
\hline & 10 & 15.3 & 26.9 & 37.8 & 45.6 & 52.1 & 57.4 & 79.3 & 93.5 & 98.8 & 99.9 \\
\hline \multirow[t]{2}{*}{655920} & 2 & 10.4 & 19.0 & 23.8 & 26.0 & 27.4 & 28.3 & 31.7 & 35.1 & 37.0 & 37.5 \\
\hline & 10 & 30.6 & 52.8 & 69.2 & 78.1 & 83.7 & 87.2 & 95.9 & 99.1 & 99.8 & 100 \\
\hline \multirow[t]{2}{*}{655940} & 2 & 2.8 & 4.6 & 5.7 & 6.3 & 6.7 & 7.0 & 7.8 & 8.2 & 8.3 & 8.37 \\
\hline & 10 & 14.5 & 24.6 & 33.6 & 39.5 & 44.5 & 48.5 & 66.5 & 84.4 & 95.2 & 98.9 \\
\hline \multirow[t]{2}{*}{655990} & 2 & 2.4 & 4.2 & 5.6 & 6.3 & 6.7 & 6.9 & 7.3 & 7.7 & 8.0 & 8.06 \\
\hline & 10 & 12.1 & 20.4 & 27.9 & 33.2 & 37.8 & 41.4 & 58.2 & 77.0 & 91.6 & 98.1 \\
\hline
\end{tabular}




\begin{tabular}{|c|c|c|c|c|c|c|c|c|c|c|c|}
\hline \multicolumn{12}{|c|}{$\begin{array}{c}\text { Conditioned 1st-Order Markov Model J-18 } \\
\text { September DRWH Reliability (\%) } \\
\text { per capita roof area and storage } \\
\text { L }\end{array}$} \\
\hline Gauge & sq. m & 0 & 40 & 80 & 120 & 160 & 200 & 400 & 600 & 800 & \\
\hline \multirow[t]{2}{*}{653060} & 2 & 14.3 & 23.9 & 29.4 & 31.6 & 32.7 & 33.3 & 34.1 & 34.1 & 34.1 & \\
\hline & 10 & 30.8 & & 74.8 & 85.5 & 91.7 & 95.0 & 99.6 & 100.0 & 100.0 & 100 \\
\hline \multirow[t]{2}{*}{653190} & 2 & 21.1 & 36.2 & 43.0 & 45.6 & 46.9 & 47.7 & 48.6 & 48.6 & 48.6 & 48.6 \\
\hline & 10 & 46.3 & 73.8 & 91.2 & 96.8 & 98.8 & 99.6 & 100.0 & 100.0 & 100.0 & 100 \\
\hline \multirow[t]{2}{*}{653300} & 2 & 18.1 & 31.1 & 37.9 & 40.4 & 41.6 & 42.2 & 43.2 & 43.3 & 43.3 & \\
\hline & 10 & 38.1 & 63.9 & 83.9 & 91.9 & 95.9 & 97.6 & 99.9 & 100.0 & 100.0 & 10 \\
\hline \multirow[t]{2}{*}{653350} & 2 & 12.7 & 21.2 & 25.4 & 26.8 & 27.4 & 27.8 & 28.3 & 28.3 & 28.4 & 28 \\
\hline & 10 & 29.6 & 51.1 & 70.6 & 80.6 & 86.6 & 90.2 & 97.1 & 99.1 & 99 & 99 \\
\hline \multirow[t]{2}{*}{653380} & 2 & 10.8 & 17.7 & 21.0 & 21.9 & 22.2 & 22.3 & 22.4 & 22.4 & 22.4 & \\
\hline & 10 & 24.1 & 42.2 & 59.7 & 69.8 & 76.7 & 80.8 & & 96.2 & & \\
\hline \multirow[t]{2}{*}{653440} & 2 & 9.0 & & & 18.5 & 18.8 & 18.9 & & 9.0 & & 9 \\
\hline & 10 & & & 49.5 & 59.0 & 65.8 & & & & & 4. \\
\hline \multirow[t]{2}{*}{653520} & 2 & & & 26.1 & 26.9 & 27.1 & & & & & \\
\hline & 10 & 33. & 56.5 & 76.3 & 85.9 & 91.3 & 94.2 & 98.9 & 99.8 & & \\
\hline \multirow[t]{2}{*}{653550} & 2 & 18. & 32.3 & 38.5 & 41.5 & 43.5 & 45.2 & 48.6 & 49.0 & 49.0 & 49 \\
\hline & 10 & 45.8 & 72.1 & 89.7 & 95.9 & 98.5 & 99.4 & 100.0 & 100.0 & 100.0 & 10 \\
\hline \multirow[t]{2}{*}{653570} & 2 & 15.4 & 26.0 & 31.8 & 33.9 & 34.9 & 35.4 & 35.7 & 35.7 & & 35 \\
\hline & 10 & 36. & 59.6 & 79 & 88.8 & 93.7 & 96.0 & 99.5 & 99.9 & & 100 \\
\hline \multirow[t]{2}{*}{653610} & 2 & 18.1 & 30.6 & 36.2 & 37.8 & 38.5 & 38.7 & 39.2 & 39.4 & & \\
\hline & 10 & & & & 93.2 & & & 100.0 & 100.0 & & 100 \\
\hline \multirow[t]{2}{*}{653760} & 2 & & & 27 & 28.7 & 29 & 29.7 & & 30.0 & & 30 \\
\hline & 10 & 28.3 & & 68 & 79.7 & 86.8 & & & & & 99. \\
\hline \multirow[t]{2}{*}{653800} & 2 & & 12 & 15 & 17.0 & 17.4 & 17.7 & & & & 18 \\
\hline & 10 & 17. & 30 & 43. & 51.6 & 57.4 & 60.6 & 68.3 & 72.8 & & \\
\hline \multirow[t]{2}{*}{653870} & 2 & 5. & & 10.6 & 11.3 & 11.5 & 11.6 & 11.7 & 11.7 & 11 & \\
\hline & 10 & 13.9 & 24. & 35.2 & 42.2 & 47.5 & 50.5 & 56.7 & 60.3 & 66.9 & \\
\hline \multirow[t]{2}{*}{654720} & 2 & 2.6 & 4. & 6.1 & 7.2 & 8.1 & 8. & & & & \\
\hline & 10 & 8.9 & 14.8 & 20. & 24.1 & 26. & 28 & & & & \\
\hline \multirow[t]{2}{*}{655010} & 2 & 5.7 & 8. & 10. & 10.7 & 10.9 & 11.0 & 11.0 & 11.0 & 11.0 & 11 \\
\hline & 10 & 16.8 & 29 & 44.9 & 55.2 & 63.9 & 69.9 & 89.6 & 5.8 & & \\
\hline \multirow[t]{2}{*}{655020} & 2 & & & & 20.2 & 20.6 & .7 & & & & \\
\hline & 10 & & & & 69.9 & 9.1 & 4.8 & 7.5 & .5 & & 99 \\
\hline 655030 & 2 & & & & 22.4 & & & & & & \\
\hline & 10 & & & 67. & 79.3 & 87.0 & 91.3 & 99.2 & & 0.0 & 10 \\
\hline 655050 & 2 & 10. & 17 & 20. & 21.2 & 21.6 & 21.8 & 21.9 & 22. & & 22 \\
\hline & 10 & 26.3 & 46.6 & 67.9 & 79.7 & 87.4 & 91.9 & 99.4 & 100.0 & 100.0 & 10 \\
\hline 655070 & 2 & 12.1 & 19.3 & 22.8 & 24.2 & 25.0 & 25.7 & 28.6 & 29.7 & & 29 \\
\hline & 10 & 26.8 & 46 & 67.5 & 79.5 & 87.4 & 91.8 & 99.2 & 99.9 & 100.0 & \\
\hline 655100 & 2 & 14.6 & 24.5 & 30.3 & 32.9 & 34.5 & 35.4 & 36.8 & 36.9 & & 36. \\
\hline & 10 & 33.7 & 58.0 & 79.4 & 89.2 & 94.5 & 97.0 & 99.9 & 100.0 & 100.0 & 100 \\
\hline 655160 & 2 & 11.3 & 18.1 & 21.4 & 22.6 & 23.3 & 23.6 & 24.0 & 24.0 & 24.0 & 24 \\
\hline & 10 & 29.1 & 49.7 & 70.1 & 81.4 & 88.6 & 92.7 & 99.5 & 100.0 & 100.0 & 10 \\
\hline 655180 & 2 & 13 & 22 & 26. & 28.8 & 30.2 & 31.2 & 33.2 & & & 33 \\
\hline & 10 & 33. & 56 & 78. & 88.4 & 94.1 & 96.7 & 99.8 & 0.0 & 100.0 & 10 \\
\hline 655220 & 2 & & 24. & 28 & 30.2 & 30 & 31.3 & 32.2 & & & 32 \\
\hline & 10 & & & 82. & 90.9 & & & & 100.0 & 100.0 & 10 \\
\hline 655280 & 2 & & & 40 & 43.0 & & & & & & \\
\hline & 10 & & & 88 & 95.3 & & & 100.0 & 100.0 & 100.0 & 10 \\
\hline 655360 & 2 & 15.8 & 25.8 & 30.9 & 32.6 & 33.5 & 34.1 & 35.7 & 36.2 & 36.2 & \\
\hline & 10 & 34.3 & 58.4 & 79.9 & 89.4 & 94.4 & 96.7 & 99.7 & 99.9 & 100.0 & \\
\hline
\end{tabular}




\begin{tabular}{|c|c|c|c|c|c|c|c|c|c|c|c|}
\hline \multicolumn{12}{|c|}{$\begin{array}{l}\text { Conditioned 1st-Order Markov Model J-19 } \\
\text { September DRWH Reliability (\%) } \\
\text { per capita roof area and storage }\end{array}$} \\
\hline & & $\mathrm{L}$ & 40 & 80 & 120 & 160 & 200 & 400 & 600 & 800 & 1000 \\
\hline \multirow{2}{*}{$\begin{array}{l}\text { Gauge } \\
655450\end{array}$} & 2 & 13.1 & 20.9 & 24.4 & 25.5 & 26.0 & 26.1 & 26.3 & 26.4 & 26.4 & 26.4 \\
\hline & 10 & 29.0 & 49.9 & 69.2 & 78.8 & 84.6 & 87.8 & 94.1 & 96.4 & 98.0 & 98.8 \\
\hline \multirow[t]{2}{*}{655480} & 2 & 20.2 & 35.3 & 43.1 & 46.7 & 49.1 & 50.9 & 54.5 & 55.1 & 55.5 & 55.8 \\
\hline & 10 & 42.4 & 70.2 & 89.0 & 95.5 & 98.2 & 99.3 & 100.0 & 100.0 & 100.0 & 100 \\
\hline \multirow[t]{2}{*}{655550} & 2 & 12.0 & 19.5 & 23.5 & 25.2 & 26.2 & 26.8 & 28.7 & 29.6 & 30.2 & 30.4 \\
\hline & 10 & 27.2 & 46.5 & 65.5 & 75.9 & 82.7 & 86.5 & 94.3 & 96.8 & 98.0 & 98.6 \\
\hline \multirow[t]{2}{*}{655570} & 2 & 12.2 & 21.3 & 26.5 & 28.4 & 29.1 & 29.5 & 29.7 & 29.7 & 29.7 & 29.7 \\
\hline & 10 & 28.1 & 48.0 & 66.2 & 76.0 & 81.9 & 85.2 & 91.1 & 93.7 & 96.4 & 98.3 \\
\hline \multirow[t]{2}{*}{655600} & 2 & 10.0 & 16.6 & 20.4 & 22.1 & 23.0 & 23.5 & 23.9 & 23.9 & 23.9 & 24 \\
\hline & 10 & 29.9 & 49.7 & 67.8 & 77.4 & 83.5 & 87.1 & 94.2 & 96.5 & 97.7 & 98.4 \\
\hline \multirow[t]{2}{*}{655620} & 2 & 9.1 & 13.9 & 15.8 & 16.5 & 17.0 & 17.5 & 19.1 & 19.8 & 20.1 & 20.1 \\
\hline & 10 & 24.3 & 40.8 & 57.2 & 66.4 & 72.4 & 75.6 & 82.1 & 86.0 & 90.4 & 94.1 \\
\hline \multirow[t]{2}{*}{655630} & 2 & 8.1 & 13.3 & 15.7 & 16.3 & 16.5 & 16.6 & 16.6 & 16.6 & 16.6 & 16.6 \\
\hline & 10 & 21.8 & 37.4 & 53.1 & 62.5 & 69.2 & 73.4 & 84.4 & 89.7 & 93.3 & 95.7 \\
\hline \multirow[t]{2}{*}{655780} & 2 & 5.2 & 10.1 & 14.8 & 17.3 & 18.9 & 19.8 & 21.3 & 21.6 & 21.8 & 21.9 \\
\hline & 10 & 13.8 & 23.6 & 33.9 & 41.0 & 47.0 & 51.0 & 61.4 & 68.1 & 77.6 & 87.5 \\
\hline \multirow[t]{2}{*}{655850} & 2 & 7.2 & 13.0 & 16.3 & 17.4 & 17.9 & 18.1 & 18.3 & 18.3 & 18.3 & 18.4 \\
\hline & 10 & 20.3 & 36.1 & 50.2 & 58.8 & 64.7 & 68.5 & 77.8 & 86.5 & 94.4 & 98.3 \\
\hline \multirow[t]{2}{*}{655920} & 2 & 12.3 & 23.3 & 29.9 & 33.0 & 34.5 & 35.3 & 36.0 & 36.5 & 37.2 & 37.9 \\
\hline & 10 & 39.4 & 64.8 & 81.4 & 89.0 & 93.1 & 95.3 & 98.8 & 99.6 & 99.9 & 100 \\
\hline \multirow[t]{2}{*}{655940} & 2 & 2.9 & 5.1 & 6.4 & 7.0 & 7.2 & 7.3 & 7.4 & 7.4 & 7.4 & 7.44 \\
\hline & 10 & 16.5 & 27.4 & 36.2 & 41.6 & 45.7 & 48.5 & 56.9 & 66.3 & 79.2 & 90.4 \\
\hline \multirow[t]{2}{*}{655990} & 2 & 3.3 & 4.9 & 5.7 & 5.9 & 5.9 & 6.0 & 6.0 & 6.0 & 6.0 & 6.03 \\
\hline & 10 & 10.6 & 18.4 & 26.4 & 31.6 & 35.5 & 38.0 & 45.5 & 53.5 & 66.4 & 80.9 \\
\hline
\end{tabular}




\begin{tabular}{|c|c|c|c|c|c|c|c|c|c|c|c|}
\hline $\begin{array}{l}\text { Condit } \\
\text { Octobe }\end{array}$ & $\begin{array}{l}\text { ned 1s } \\
\text { DRWH } \\
\text { per ca }\end{array}$ & $\begin{array}{l}\text { rder } \\
\text { eliab } \\
\text { a ro } \\
\text { L }\end{array}$ & rea & Mod & ige & & & & & & \\
\hline Gauge & sq. m & 0 & 40 & 80 & 120 & 160 & 200 & 400 & 600 & 800 & 1000 \\
\hline 653060 & 2 & 3.0 & 4.5 & 5.5 & 5.9 & 6.2 & 6.3 & 6.6 & 6.7 & 6.7 & 6.66 \\
\hline & 10 & 8.9 & 16.1 & 26.4 & 34.7 & 43.2 & 49.5 & 77.7 & 95.5 & 99.4 & 100 \\
\hline 653190 & 2 & 8.0 & 12.7 & 14.8 & 15.5 & 15.9 & 16.1 & 16.5 & 16.5 & 16.5 & 16.5 \\
\hline & 10 & 21.9 & 37.9 & 55.9 & 67.6 & 76.7 & 82.5 & 96.9 & 99.9 & 100.0 & 100 \\
\hline 653300 & 2 & 8.4 & 14.0 & 17.3 & 18.5 & 19.0 & 19.3 & 19.6 & 19.7 & 19.7 & 19.7 \\
\hline & 10 & 19.5 & 33.9 & 51.5 & 63.5 & 73.7 & 80.2 & 96.5 & 99.7 & 100.0 & 100 \\
\hline 653350 & 2 & 9.8 & 15.6 & 18.2 & 19.0 & 19.2 & 19.3 & 19.4 & 19.5 & 19.5 & 19.5 \\
\hline & 10 & 23.8 & 41.5 & 60.4 & 71.6 & 79.7 & 84.7 & 96.5 & 99.2 & 99.7 & 99.9 \\
\hline 653380 & 2 & 10.9 & 17.4 & 20.7 & 21.6 & 21.8 & 21.9 & 21.9 & 21.9 & 21.9 & 21.9 \\
\hline & 10 & 22.0 & 38.8 & 58.0 & 69.5 & 78.0 & 83.1 & 94.4 & 97.4 & 98.9 & 99.6 \\
\hline 653440 & 2 & 11.3 & 18.9 & 23.4 & 24.9 & 25.4 & 25.7 & 25.8 & 25.8 & 25.8 & 25.8 \\
\hline & 10 & 24.0 & 41.7 & 60.4 & 71.1 & 78.4 & 82.7 & 91.8 & 94.0 & 95.3 & 96.8 \\
\hline 653520 & 2 & 6.2 & 9.7 & 11.4 & 11.9 & 12.1 & 12.1 & 12.1 & 12.1 & 12.1 & 12.1 \\
\hline & 10 & 14.6 & 25.5 & 39.3 & 49.4 & 58.9 & 65.2 & 87.7 & 97.8 & 99.5 & 99.7 \\
\hline 653550 & 2 & 10.3 & 16.4 & 18.9 & 19.8 & 20.3 & 20.6 & 22.1 & 22.9 & 23.4 & 23.6 \\
\hline & 10 & 25.3 & 44.9 & 65.2 & 76.9 & 85.1 & 89.9 & 99.1 & 100.0 & 100.0 & 100 \\
\hline 653570 & 2 & 9.3 & 14.6 & 17.0 & 17.9 & 18.4 & 18.8 & 19.8 & 19.9 & 19.9 & 19.9 \\
\hline & 10 & 22.5 & 37.3 & 55.1 & 66.5 & 75.7 & 81.0 & 95.2 & 99.3 & 99.8 & 99.9 \\
\hline 653610 & 2 & 8.8 & 14.3 & 17.0 & 18.0 & 18.3 & 18.5 & 18.6 & 18.6 & 18.6 & 18.6 \\
\hline & 10 & 22.6 & 38.5 & 57.0 & 68.8 & 78.2 & 84.0 & 97.6 & 99.9 & 100.0 & 100 \\
\hline 653760 & 2 & 8.9 & 14.2 & 17.0 & 17.9 & 18.2 & 18.3 & 18.4 & 18.4 & 18.4 & 18.4 \\
\hline & 10 & 22.0 & 38.2 & 56.8 & 68.4 & 77.3 & 82.9 & 96.3 & 99.4 & 99.8 & 99.9 \\
\hline 653800 & 2 & 9.2 & 14.1 & 16.3 & 16.8 & 16.9 & 17.0 & 17.1 & 17.1 & 17.1 & 17.1 \\
\hline & 10 & 23.0 & 40.8 & 59.3 & 69.6 & 76.4 & 80.4 & 88.7 & 90.6 & 91.8 & 93.3 \\
\hline 653870 & 2 & 6.7 & 10.7 & 13.1 & 13.8 & 14.1 & 14.2 & 14.3 & 14.3 & 14.3 & 14.3 \\
\hline & 10 & 15.4 & 27.1 & 40.9 & 49.7 & 56.7 & 60.9 & 71.5 & 74.6 & 76.5 & 79.4 \\
\hline 654720 & 2 & 5.1 & 8.0 & 10.3 & 11.5 & 12.3 & 12.8 & 14.4 & 15.2 & 15.3 & 15.3 \\
\hline & 10 & 13.1 & 22.6 & 33.3 & 40.0 & 45.4 & 48.3 & 55.3 & 58.1 & 59.1 & 59.9 \\
\hline 655010 & 2 & 1.1 & 1.8 & 2.4 & 2.7 & 2.9 & 3.0 & 3.1 & 3.1 & 3.1 & 3.12 \\
\hline & 10 & 3.1 & 5.6 & 9.7 & 13.4 & 17.6 & 20.6 & 36.7 & 58.9 & 75.7 & 82.7 \\
\hline 655020 & 2 & 1.7 & 2.6 & 3.2 & 3.4 & 3.5 & 3.5 & 3.5 & 3.5 & 3.5 & 3.54 \\
\hline & 10 & 7.8 & 13.7 & 21.1 & 26.9 & 32.9 & 37.6 & 62.6 & 85.9 & 95.9 & 98.2 \\
\hline 655030 & 2 & 2.6 & 3.8 & 4.7 & 5.1 & 5.5 & 5.9 & 7. & 8.3 & 8 & 8.68 \\
\hline & 10 & 8.3 & 15.0 & 24.4 & 31.7 & 39.1 & 44.7 & 71.7 & 92.1 & 98.6 & 99.6 \\
\hline 655050 & 2 & 3.2 & 4.9 & 5.9 & 6.1 & 6.2 & 6.2 & 6.2 & 6.2 & 6.2 & 6.17 \\
\hline & 10 & 10.0 & 17.7 & 28.0 & 35.9 & 43.6 & 49.3 & 75.8 & 93.7 & 98.9 & 99.8 \\
\hline 655070 & 2 & 2.7 & 3.9 & 4.5 & 4.6 & 4.7 & 4.7 & 4.8 & 5.4 & 6.0 & 6.32 \\
\hline & 10 & 9.2 & 16.8 & 26.8 & 34.4 & 41.8 & 47.5 & 74.6 & 93.6 & 99.0 & 99.7 \\
\hline 655100 & 2 & 5.1 & 7.7 & 9.2 & 9.7 & 9.9 & 10.1 & 10.4 & 10.4 & 10.4 & 10.4 \\
\hline & 10 & 13.8 & 24.8 & 39.1 & 49.4 & 59.0 & 65.6 & 89.4 & 98.8 & 99.9 & 100 \\
\hline 655160 & 2 & 4.2 & 6.5 & 7.8 & 8.1 & 8.3 & 8.4 & 8.6 & 8.6 & 8.6 & 8.63 \\
\hline & 10 & 11.8 & 20.8 & 33.1 & 41.9 & 50.4 & 56.3 & 81.2 & 95.8 & 99.4 & 99.9 \\
\hline 655180 & 2 & 3.7 & 5.4 & 6.3 & 6.5 & 6.6 & 6.7 & 6.9 & 7.0 & 7.0 & 7.03 \\
\hline & 10 & 10.9 & 19.6 & 31.5 & 40.6 & 49.6 & 56.1 & 83.0 & 97.4 & 99.8 & 100 \\
\hline 655220 & 2 & 7.3 & 11.3 & 13.1 & 13.6 & 13.7 & 13.7 & 13.8 & 13.9 & 13.9 & 13.9 \\
\hline & 10 & 19.0 & 33.5 & 50.8 & 61.7 & 70.6 & 76.5 & 94.5 & 99.5 & 100.0 & 100 \\
\hline 655280 & 2 & 10.0 & 15.7 & 18.9 & 20.4 & 21.4 & 22.0 & 23.6 & 25.0 & 26.5 & 27.3 \\
\hline & 10 & 28.8 & 48.7 & 68.8 & 79.7 & 87.0 & 91.3 & 99.2 & 100.0 & 100.0 & 100 \\
\hline 655360 & 2 & 7.8 & 12.7 & 15.3 & 16.3 & 16.8 & 17.1 & 17.5 & 18.2 & 18.9 & 19.2 \\
\hline & 10 & 22.9 & 39.1 & 56.6 & 67.6 & 76.5 & 82.2 & 96.7 & 99.7 & 100.0 & 100 \\
\hline
\end{tabular}




\begin{tabular}{|c|c|c|c|c|c|c|c|c|c|c|c|}
\hline $\begin{array}{l}\text { Condi } \\
\text { Octob }\end{array}$ & $\begin{array}{l}\text { ned 1s } \\
\text { DRWh } \\
\text { per ce }\end{array}$ & $\begin{array}{l}\text { rde } \\
\text { aliak } \\
\text { a ro } \\
\text { L }\end{array}$ & $\left({ }^{\circ}\right.$ & d & ige & & & & & & \\
\hline Gauge & sq. $m$ & 0 & 40 & 80 & 120 & 160 & 200 & 400 & 600 & 800 & 1000 \\
\hline 655450 & 2 & 9.5 & 15.1 & 17.8 & 18.4 & 18.6 & 18.7 & 18.7 & 18.7 & 18.7 & 18.7 \\
\hline & 10 & 22.4 & 38.1 & 56.0 & 67.1 & 75.7 & 80.9 & 94.2 & 97.7 & 98.6 & 99.1 \\
\hline 655480 & 2 & 10.3 & 17.7 & 22.1 & 24.1 & 25.0 & 25.6 & 26.9 & 27.5 & 27.9 & 28.2 \\
\hline & 10 & 26.3 & 44.3 & 63.9 & 75.8 & 84.5 & 89.6 & 99.0 & 100.0 & 100.0 & 100 \\
\hline 655550 & 2 & 7.0 & 11.4 & 14.2 & 15.4 & 16.0 & 16.4 & 17.1 & 17.4 & 17.8 & 18 \\
\hline & 10 & 18.4 & 31.7 & 48.0 & 58.8 & 67.8 & 73.6 & 90.7 & 96.6 & 98.1 & 98.7 \\
\hline 655570 & 2 & 9.5 & 15.4 & 18.5 & 19.8 & 20.4 & 20.8 & 21.2 & 21.3 & 21.3 & 21.3 \\
\hline & 10 & 29.3 & 50.4 & 69.5 & 79.6 & 85.9 & 89.9 & 97.5 & 98.6 & 99.0 & 99.4 \\
\hline 655600 & 2 & 7.5 & 11.5 & 13.6 & 14.3 & 14.8 & 15.0 & 15.6 & 15.7 & 15.7 & 15.7 \\
\hline & 10 & 21.8 & 37.7 & 55.3 & 66.2 & 74.5 & 79.9 & 93.7 & 97.3 & 98.4 & 98.8 \\
\hline 655620 & 2 & 9.4 & 15.1 & 17.9 & 18.6 & 18.8 & 19.0 & 20.0 & 20.9 & 21.4 & 21.6 \\
\hline & 10 & 21.6 & 38.2 & 56.3 & 67.2 & 75.1 & 80.1 & 91.2 & 93.7 & 95.2 & 96.6 \\
\hline 655630 & 2 & 7.7 & 11.8 & 13.8 & 14.4 & 14.6 & 14.7 & 14.7 & 14.7 & 14.7 & 14.7 \\
\hline & 10 & 22.7 & 39.5 & 56.7 & 66.6 & 73.5 & 78.0 & 88.9 & 92.6 & 94.8 & 96.4 \\
\hline 655780 & 2 & 9.5 & 17.2 & 22.5 & 25.0 & 26.3 & 27.2 & 29.1 & 29.8 & 30.0 & 30.2 \\
\hline & 10 & 22.4 & 38.8 & 54.3 & 63.8 & 70.7 & 74.9 & 85.1 & 88.6 & 90.5 & 92.7 \\
\hline 655850 & 2 & 13.5 & 23.3 & 28.5 & 30.3 & 31.1 & 31.4 & 31.6 & 31.6 & 31.6 & 31.6 \\
\hline & 10 & 29.7 & 52.1 & 70.8 & 80.2 & 85.7 & 88.8 & 94.5 & 96.0 & 97.5 & 98.9 \\
\hline 655920 & 2 & 11.8 & 22.7 & 30.1 & 33.8 & 35.9 & 37.1 & 38.7 & 38.9 & 39.0 & 39.2 \\
\hline & 10 & 29.2 & 51.4 & 70.5 & 81.3 & 88.2 & 92.3 & 99.3 & 99.9 & 100.0 & 100 \\
\hline 655940 & 2 & 6.1 & 11.1 & 14.4 & 15.8 & 16.5 & 16.8 & 17.1 & 17.1 & 17.1 & 17.1 \\
\hline & 10 & 18.3 & 31.8 & 44.7 & 52.8 & 58.7 & 62.6 & 71.6 & 75.3 & 79.9 & 85.8 \\
\hline 655990 & 2 & 6.7 & 10.7 & 12.5 & 13.0 & 13.1 & 13.1 & 13.1 & 13.1 & 13.1 & 13.1 \\
\hline & 10 & 19.8 & 33.7 & 47.5 & 55.6 & 61.2 & 64.4 & 70.5 & 72.6 & 75.7 & 80.6 \\
\hline
\end{tabular}




\begin{tabular}{|c|c|c|c|c|c|c|c|c|c|c|c|}
\hline \multicolumn{12}{|c|}{$\begin{array}{l}\text { Conditioned 1st-Order Markov Model } \\
\text { November DRWH Reliability (\%) } \\
\text { per capita roof area and storage } \\
\text { L }\end{array}$} \\
\hline Gauge & sq. m & 0 & 40 & 80 & 120 & 160 & 200 & 400 & 600 & 800 & 1000 \\
\hline \multirow[t]{2}{*}{653060} & 2 & 0.1 & 0.1 & 0.1 & 0.1 & 0.1 & 0.1 & 0.1 & 0.1 & 0.1 & 0.13 \\
\hline & 10 & 0.2 & 0.6 & 1.5 & 2.5 & 3.8 & 4.7 & 10.0 & 26.1 & 54.3 & 80.3 \\
\hline \multirow[t]{2}{*}{653190} & 2 & 0.8 & 1.4 & 1.8 & 2.0 & 2.2 & 2.3 & 2.5 & 2.5 & 2.5 & 2.53 \\
\hline & 10 & 2.1 & 4.1 & 7.9 & 11.9 & 17.0 & 20.8 & 42.9 & 72.0 & 90.9 & 98.4 \\
\hline \multirow[t]{2}{*}{653300} & 2 & 0.8 & 1.4 & 2.0 & 2.3 & 2.5 & 2.5 & 2.6 & 2.6 & 2.6 & 2.59 \\
\hline & 10 & 1.7 & 3.5 & 7.1 & 10.9 & 15.9 & 19.7 & 41.4 & 70.5 & 90.2 & 98 \\
\hline \multirow[t]{2}{*}{653350} & 2 & 1.2 & 1.9 & 2.3 & 2.4 & 2.4 & 2.4 & 2.4 & 2.4 & 2.4 & 2.41 \\
\hline & 10 & 3.3 & 6.3 & 11.7 & 16.8 & 23.0 & 27.7 & 52.7 & 79.4 & 93.1 & 97.8 \\
\hline \multirow[t]{2}{*}{653380} & 2 & 1.8 & 3.0 & 4.2 & 4.6 & 4.9 & 5.0 & 5.1 & 5.1 & 5.1 & 5.12 \\
\hline & 10 & 4.7 & 8.7 & 15.1 & 21.0 & 27.9 & 33.0 & 58.7 & 81.9 & 92.6 & 96.7 \\
\hline \multirow{2}{*}{653440} & 2 & 3.4 & 5.2 & 6.4 & 6.9 & 7.1 & 7.2 & 7.4 & 7.4 & 7.4 & 7.36 \\
\hline & 10 & 9.1 & 16.3 & 26.2 & 34.1 & 42.0 & 47.7 & 72.5 & 87.4 & 92.2 & 94.4 \\
\hline \multirow[t]{2}{*}{653520} & 2 & 0.6 & 1.1 & 1.8 & 2.3 & 2.8 & 3.0 & 3.4 & 3.5 & 3.5 & 3.49 \\
\hline & 10 & 1.6 & 3.2 & 6.0 & 8.7 & 12.3 & 15.0 & 29.2 & 52.0 & 75.2 & 90.7 \\
\hline \multirow[t]{2}{*}{653550} & 2 & 2.6 & 4.1 & 5.1 & 5.4 & 5.5 & 5.5 & 5.6 & 5.6 & 5.7 & 5.97 \\
\hline & 10 & 5.1 & 9.3 & 16.5 & 23.0 & 30.7 & 36.2 & 63.0 & 87.5 & 97.5 & 99.7 \\
\hline \multirow[t]{2}{*}{653570} & 2 & 1.3 & 2.0 & 2.5 & 2.6 & 2.6 & 2.6 & 2.7 & 2.7 & 2.7 & 2.67 \\
\hline & 10 & 2.7 & 5.3 & 10.4 & 15.1 & 21.1 & 25.4 & 47.5 & 74.1 & 90.5 & 97.4 \\
\hline \multirow[t]{2}{*}{653610} & 2 & 1.4 & 2.4 & 3.5 & 4.1 & 4.5 & 4.7 & 5.0 & 5.0 & 5.0 & 5.01 \\
\hline & 10 & 3.5 & 6.5 & 11.7 & 16.5 & 22.5 & 26.9 & 51.0 & 78.7 & 94.2 & 99.1 \\
\hline \multirow{2}{*}{653760} & 2 & 1.0 & 1.6 & 2.1 & 2.2 & 2.3 & 2.3 & 2.3 & 2.3 & 2.3 & 2.29 \\
\hline & 10 & 3.5 & 6.4 & 11.5 & 16.2 & 21.7 & 26.0 & 50.7 & 78.1 & 93.3 & 98.3 \\
\hline \multirow[t]{2}{*}{653800} & 2 & 2.4 & 3.4 & 4.0 & 4.1 & 4.1 & 4.1 & 4.1 & 4.1 & 4.1 & 4.1 \\
\hline & 10 & 7.3 & 13.1 & 21.4 & 28.1 & 35.0 & 40.1 & 63.0 & 77.5 & 82.7 & 85.4 \\
\hline \multirow[t]{2}{*}{653870} & 2 & 1.5 & 2.1 & 2.5 & 2.7 & 2.7 & 2.7 & 2.8 & 2.8 & 2.8 & 2.76 \\
\hline & 10 & 5.2 & 9.4 & 15.3 & 19.6 & 24.2 & 27.5 & 42.5 & 54.1 & 59.4 & 62.9 \\
\hline \multirow[t]{2}{*}{654720} & 2 & 2.0 & 2.8 & 3.4 & 3.7 & 4.0 & 4.1 & 4.5 & 4.6 & 4.7 & 4.83 \\
\hline & 10 & 5.3 & 9.6 & 15.7 & 20.2 & 24.7 & 27.4 & 38.4 & 46.1 & 50.2 & 52.3 \\
\hline \multirow[t]{2}{*}{655010} & 2 & 0.1 & 0.2 & 0.4 & 0.5 & 0.7 & 0.7 & 0.9 & 1.0 & 1.0 & 1.01 \\
\hline & 10 & 0.1 & 0.3 & 0.8 & 1.3 & 2.0 & 2.6 & 4.8 & 8.0 & 16.3 & 30.1 \\
\hline \multirow[t]{2}{*}{655020} & 2 & 0.2 & 0.3 & 0.4 & 0.5 & 0.6 & 0.6 & 0.6 & 0.6 & 0.6 & 0.61 \\
\hline & 10 & 0.3 & 0.7 & 1.6 & 2.4 & 3.4 & 4.0 & 6.8 & 15.8 & 37.2 & 62.7 \\
\hline 655030 & 2 & 0.5 & 0.8 & 1.2 & 1.5 & 1.6 & 1.7 & 1.7 & 1.8 & 1.9 & 2.07 \\
\hline & 10 & 0.9 & 1.7 & 3.3 & 4.8 & 6.8 & 8.0 & 13.8 & 27.1 & 51.6 & 76.2 \\
\hline 655050 & 2 & 0.3 & 0.4 & 0.5 & 0.6 & 0.6 & 0.6 & 0.6 & 0.6 & 0.6 & 0.57 \\
\hline & 10 & 0.8 & 1.7 & 3.1 & 4.6 & 6.3 & 7.6 & 14.3 & 30.4 & 56.2 & 80 \\
\hline 655070 & 2 & 0.2 & 0.3 & 0.4 & 0.4 & 0.4 & 0.4 & 0.4 & 0.4 & 0.4 & 0.44 \\
\hline & 10 & 0.5 & 1.2 & 2.4 & 3.6 & 5.0 & 5.8 & 10.7 & 25.1 & 51.8 & 77.6 \\
\hline 655100 & 2 & 0.9 & 1.5 & 1.8 & 2.0 & 2.0 & 2.0 & 2.0 & 2.0 & 2.0 & 2.03 \\
\hline & 10 & 2.6 & 4.6 & 7.8 & 10.7 & 14.1 & 16.5 & 30.2 & 53.8 & 78.5 & 93.6 \\
\hline 655160 & 2 & 0.3 & 0.6 & 0.8 & 0.9 & 1.0 & 1.0 & 1.0 & 1.0 & 1.0 & 1.01 \\
\hline & 10 & 1.4 & 2.6 & 4.5 & 6.3 & 8.9 & 10.7 & 20.5 & 39.9 & 65.5 & 85.8 \\
\hline 655180 & 2 & 0.6 & 0.8 & 0.9 & 0.9 & 0.9 & 0.9 & 0.9 & 0.9 & 0.9 & 0.89 \\
\hline & 10 & 2.6 & 4.5 & 7.2 & 9.3 & 11.6 & 13.0 & 21.4 & 40.8 & 67.8 & 88.3 \\
\hline 655220 & 2 & 0.5 & 0.9 & 1.3 & 1.5 & 1.7 & 1.7 & 1.8 & 1.8 & 1.8 & 1.77 \\
\hline & 10 & 1.7 & 3.3 & 6.4 & 9.7 & 13.9 & 17.0 & 35.8 & 63.9 & 85.6 & 96.5 \\
\hline 655280 & 2 & 2.3 & 3.4 & 4.3 & 4.8 & 5.3 & 5.7 & 7.4 & 8.8 & 9.6 & 10.2 \\
\hline & 10 & 7.2 & 12.6 & 20.8 & 27.4 & 34.6 & 39.9 & 67.3 & 90.2 & 98.3 & 99.8 \\
\hline 655360 & 2 & 1.5 & 2.3 & 2.9 & 3.1 & 3.3 & 3.4 & 3.6 & 3.7 & 3.8 & 3.98 \\
\hline & 10 & 3.9 & 7.4 & 13.1 & 18.1 & 23.8 & 28.0 & 51.1 & 77.7 & 93.3 & 98.7 \\
\hline
\end{tabular}




\begin{tabular}{|c|c|c|c|c|c|c|c|c|c|c|c|}
\hline \multicolumn{12}{|c|}{$\begin{array}{c}\text { Conditioned 1st-Order Markov Model } \mathrm{J}-23 \\
\text { November DRWH Reliability (\%) } \\
\text { per capita roof area and storage } \\
\text { L }\end{array}$} \\
\hline Gauge & sq. m & 0 & 40 & 80 & 120 & 160 & 200 & 400 & 600 & 800 & 1000 \\
\hline \multirow[t]{2}{*}{655450} & 2 & 1.7 & 2.6 & 3.1 & 3.3 & 3.4 & 3.4 & 3.4 & 3.4 & 3.4 & 3.38 \\
\hline & 10 & 6.6 & 11.7 & 18.6 & 24.2 & 30.1 & 34.6 & 57.6 & 79.4 & 90.7 & 95.5 \\
\hline \multirow[t]{2}{*}{655480} & 2 & 2.2 & 3.4 & 4.2 & 4.8 & 5.3 & 5.5 & 6.1 & 6.2 & 6.3 & 6.37 \\
\hline & 10 & 8.5 & 14.9 & 23.6 & 30.3 & 37.3 & 42.7 & 69.9 & 91.3 & 98.5 & 99.9 \\
\hline \multirow[t]{2}{*}{655550} & 2 & 1.6 & 2.4 & 3.2 & 3.5 & 3.7 & 3.8 & 4.0 & 4.0 & 4.1 & 4.1 \\
\hline & 10 & 5.4 & 9.6 & 15.4 & 20.1 & 25.4 & 29.3 & 49.4 & 71.6 & 86.4 & 93.3 \\
\hline \multirow[t]{2}{*}{655570} & 2 & 6.7 & 9.9 & 11.4 & 11.9 & 12.2 & 12.4 & 12.6 & 12.7 & 12.7 & 12.7 \\
\hline & 10 & 21.1 & 36.4 & 53.1 & 63.5 & 71.2 & 76.3 & 92.3 & 97.6 & 98.7 & 99.2 \\
\hline \multirow[t]{2}{*}{655600} & 2 & 2.3 & 3.3 & 3.7 & 3.8 & 3.8 & 3.8 & 3.9 & 3.9 & 3.9 & 3.88 \\
\hline & 10 & 8.6 & 14.8 & 23.1 & 29.3 & 35.5 & 39.8 & 61.3 & 80.8 & 91.0 & 95.2 \\
\hline \multirow[t]{2}{*}{655620} & 2 & 3.2 & 4.9 & 5.8 & 6.0 & 6.0 & 6.0 & 6.1 & 6.2 & 6.5 & 6.91 \\
\hline & 10 & 8.8 & 15.7 & 25.1 & 32.3 & 39.7 & 45.0 & 68.1 & 83.2 & 88.9 & 91.8 \\
\hline \multirow[t]{2}{*}{655630} & 2 & 3.1 & 4.4 & 5.0 & 5.3 & 5.4 & 5.4 & 5.5 & 5.5 & 5.5 & 5.49 \\
\hline & 10 & 11.2 & 19.1 & 29.1 & 36.2 & 42.6 & 47.0 & 66.3 & 79.6 & 86.7 & 90.6 \\
\hline \multirow[t]{2}{*}{655780} & 2 & 9.5 & 15.2 & 18.2 & 19.4 & 20.1 & 20.5 & 21.2 & 21.4 & 21.6 & 21.6 \\
\hline & 10 & 25.8 & 43.4 & 60.9 & 70.8 & 77.6 & 81.9 & 93.0 & 96.2 & 97.2 & 97.8 \\
\hline \multirow[t]{2}{*}{655850} & 2 & 9.4 & 15.1 & 18.0 & 19.0 & 19.5 & 19.9 & 20.4 & 20.5 & 20.5 & 20.5 \\
\hline & 10 & 24.3 & 43.3 & 62.5 & 73.7 & 81.3 & 86.4 & 96.9 & 98.5 & 99.0 & 99.4 \\
\hline \multirow[t]{2}{*}{655920} & 2 & 9.7 & 16.8 & 20.7 & 22.5 & 23.6 & 24.4 & 26.1 & 26.4 & 26.4 & 26.4 \\
\hline & 10 & 26.2 & 45.6 & 63.2 & 73.9 & 81.3 & 86.1 & 97.2 & 99.7 & 100.0 & 100 \\
\hline \multirow[t]{2}{*}{655940} & 2 & 8.5 & 14.3 & 17.6 & 18.9 & 19.6 & 19.9 & 20.4 & 20.4 & 20.4 & 20.4 \\
\hline & 10 & 24.4 & 41.1 & 57.6 & 67.3 & 74.0 & 78.1 & 87.5 & 90.3 & 91.7 & 93.5 \\
\hline \multirow[t]{2}{*}{655990} & 2 & 7.6 & 11.9 & 13.9 & 14.4 & 14.5 & 14.6 & 14.6 & 14.6 & 14.6 & 14.6 \\
\hline & 10 & 19.6 & 34.3 & 50.3 & 60.3 & 67.6 & 72.1 & 83.2 & 85.8 & 86.8 & 88.4 \\
\hline
\end{tabular}




\begin{tabular}{|c|c|c|c|c|c|c|c|c|c|c|c|}
\hline \multicolumn{12}{|c|}{$\begin{array}{c}\text { Conditioned 1st-Order Markov Model J-24 } \\
\text { December DRWH Reliability (\%) } \\
\text { per capita roof area and storage } \\
\text { L }\end{array}$} \\
\hline Gauge & sq. m & 0 & 40 & 80 & 120 & 160 & 200 & 400 & 600 & 800 & 1000 \\
\hline \multirow[t]{2}{*}{653060} & 2 & 0.1 & 0.2 & 0.3 & 0.3 & 0.4 & 0.4 & 0.4 & 0.4 & 0.4 & 0.38 \\
\hline & 10 & 0.1 & 0.2 & 0.4 & 0.6 & 0.8 & 0.9 & 1.2 & 1.3 & 2.6 & 9.08 \\
\hline \multirow[t]{2}{*}{653190} & 2 & 0.0 & 0.0 & 0.1 & 0.1 & 0.1 & 0.2 & 0.4 & 0.5 & 0.6 & 0.62 \\
\hline & 10 & 0.2 & 0.3 & 0.6 & 0.8 & 1.2 & 1.4 & 2.6 & 5.5 & 19.4 & 43.6 \\
\hline \multirow[t]{2}{*}{653300} & 2 & 0.0 & 0.1 & 0.1 & 0.1 & 0.1 & 0.1 & 0.2 & 0.2 & 0.2 & 0.16 \\
\hline & 10 & 0.5 & 0.8 & 1.1 & 1.4 & 1.7 & 2.0 & 3.2 & 5.7 & 18.8 & 42.6 \\
\hline \multirow[t]{2}{*}{653350} & 2 & 0.1 & 0.1 & 0.1 & 0.1 & 0.1 & 0.1 & 0.1 & 0.1 & 0.1 & 0.14 \\
\hline & 10 & 0.8 & 1.3 & 2.0 & 2.4 & 3.0 & 3.2 & 4.2 & 8.7 & 26.8 & 53 \\
\hline \multirow[t]{2}{*}{653380} & 2 & 1.7 & 2.8 & 4.0 & 4.4 & 4.7 & 4.8 & 4.9 & 4.9 & 4.9 & 4.89 \\
\hline & 10 & 2.7 & 5.2 & 9.2 & 12.2 & 15.5 & 17.2 & 22.8 & 30.4 & 47.9 & 68.6 \\
\hline \multirow{2}{*}{653440} & 2 & 1.2 & 2.1 & 3.1 & 3.7 & 4.2 & 4.4 & 4.9 & 4.9 & 4.9 & 4.94 \\
\hline & 10 & 2.1 & 4.0 & 7.1 & 9.8 & 12.9 & 14.8 & 23.0 & 36.6 & 55.0 & 71.7 \\
\hline \multirow[t]{2}{*}{653520} & 2 & 0.2 & 0.4 & 0.6 & 0.8 & 1.0 & 1.2 & 1.7 & 2.0 & 2.2 & 2.2 \\
\hline & 10 & 0.3 & 0.7 & 1.3 & 1.9 & 2.6 & 3.0 & 4.9 & 7.8 & 15.9 & 31 \\
\hline \multirow[t]{2}{*}{653550} & 2 & 0.2 & 0.3 & 0.4 & 0.5 & 0.5 & 0.5 & 0.5 & 0.5 & 0.5 & 0.51 \\
\hline & 10 & 0.5 & 1.0 & 2.0 & 2.9 & 4.3 & 5.1 & 9.3 & 18.8 & 40.7 & 66.7 \\
\hline \multirow[t]{2}{*}{653570} & 2 & 0.2 & 0.3 & 0.3 & 0.3 & 0.4 & 0.4 & 0.4 & 0.4 & 0.4 & 0.35 \\
\hline & 10 & 0.4 & 0.8 & 1.5 & 2.1 & 2.8 & 3.3 & 4.8 & 8.8 & 24.7 & 49 \\
\hline \multirow[t]{2}{*}{653610} & 2 & 0.1 & 0.1 & 0.2 & 0.3 & 0.4 & 0.5 & 0.6 & 0.7 & 0.8 & 0.78 \\
\hline & 10 & 0.8 & 1.4 & 2.0 & 2.4 & 3.1 & 3.6 & 6.5 & 12.6 & 29.7 & 54.8 \\
\hline \multirow{2}{*}{653760} & 2 & 0.5 & 0.7 & 1.1 & 1.2 & 1.4 & 1.4 & 1.5 & 1.5 & 1.5 & 1.53 \\
\hline & 10 & 1.3 & 2.3 & 3.7 & 4.7 & 5.8 & 6.4 & 8.1 & 12.3 & 29.3 & 55 \\
\hline \multirow[t]{2}{*}{653800} & 2 & 1.3 & 2.2 & 3.0 & 3.4 & 3.6 & 3.6 & 3.7 & 3.7 & 3.7 & 3.7 \\
\hline & 10 & 2.0 & 3.9 & 7.1 & 9.8 & 12.6 & 14.2 & 19.4 & 27.5 & 39.9 & 52.2 \\
\hline \multirow[t]{2}{*}{653870} & 2 & 0.6 & 0.9 & 1.2 & 1.3 & 1.4 & 1.4 & 1.4 & 1.4 & 1.4 & 1.43 \\
\hline & 10 & 1.8 & 3.2 & 5.1 & 6.5 & 7.9 & 8.6 & 10.4 & 13.4 & 19.6 & 26.6 \\
\hline \multirow[t]{2}{*}{654720} & 2 & 1.3 & 1.9 & 2.3 & 2.5 & 2.5 & 2.5 & 2.6 & 2.6 & 2.6 & 2.57 \\
\hline & 10 & 3.0 & 5.5 & 9.2 & 11.7 & 14.1 & 15.3 & 18.3 & 21.0 & 25.5 & 30.4 \\
\hline \multirow[t]{2}{*}{655010} & 2 & 0.4 & 0.7 & 1.2 & 1.6 & 1.9 & 2.0 & 2.3 & 2.3 & 2.3 & 2.3 \\
\hline & 10 & 0.5 & 1.0 & 1.8 & 2.5 & 3.3 & 3.8 & 5.3 & 6.1 & 6.8 & 8.01 \\
\hline \multirow[t]{2}{*}{655020} & 2 & 0.0 & 0.0 & 0.0 & 0.1 & 0.1 & 0.1 & 0.1 & 0.1 & 0.1 & 0.09 \\
\hline & 10 & 0.1 & 0.1 & 0.1 & 0.2 & 0.3 & 0.3 & 0.7 & 1.1 & 2.0 & 4.92 \\
\hline 655030 & 2 & 0.2 & 0.3 & 0.4 & 0.5 & 0.5 & 0.6 & 0.6 & 0.6 & 0.6 & 0.59 \\
\hline & 10 & 0.3 & 0.6 & 1.0 & 1.4 & 1.9 & 2.2 & 3.4 & 4.7 & 7.1 & 13.7 \\
\hline 655050 & 2 & 0.5 & 0.8 & 1.2 & 1.4 & 1.6 & 1.7 & 1.8 & 1.8 & 1.8 & 1.78 \\
\hline & 10 & 0.6 & 1.2 & 2.2 & 3.0 & 3.9 & 4.4 & 5.6 & 6.1 & 8.5 & 16.4 \\
\hline 655070 & 2 & 0.3 & 0.6 & 1.0 & 1.2 & 1.4 & 1.5 & 1.5 & 1.6 & 1.6 & 1.55 \\
\hline & 10 & 0.5 & 0.9 & 1.5 & 2.0 & 2.6 & 3.0 & 4.1 & 4.6 & 5.9 & 11.9 \\
\hline 655100 & 2 & 0.1 & 0.1 & 0.1 & 0.1 & 0.2 & 0.2 & 0.2 & 0.2 & 0.2 & 0.16 \\
\hline & 10 & 0.4 & 0.8 & 1.3 & 1.6 & 1.9 & 2.2 & 3.4 & 5.4 & 13.1 & 30.2 \\
\hline 655160 & 2 & 0.1 & 0.2 & 0.3 & 0.3 & 0.4 & 0.4 & 0.4 & 0.4 & 0.4 & 0.42 \\
\hline & 10 & 0.1 & 0.3 & 0.5 & 0.8 & 1.1 & 1.3 & 2.0 & 2.8 & 7.1 & 18.8 \\
\hline 655180 & 2 & 0.3 & 0.5 & 0.7 & 0.7 & 0.7 & 0.7 & 0.7 & 0.7 & 0.7 & 0.72 \\
\hline & 10 & 0.6 & 1.2 & 2.0 & 2.7 & 3.4 & 3.8 & 4.5 & 5.1 & 9.2 & 21.4 \\
\hline 655220 & 2 & 0.2 & 0.4 & 0.6 & 0.7 & 0.8 & 0.8 & 0.9 & 1.0 & 1.0 & 0.99 \\
\hline & 10 & 0.3 & 0.6 & 1.1 & 1.5 & 2.1 & 2.4 & 3.6 & 5.5 & 16.6 & 37.6 \\
\hline 655280 & 2 & 0.6 & 1.0 & 1.5 & 1.9 & 2.3 & 2.5 & 3.3 & 3.6 & 4.0 & 4.56 \\
\hline & 10 & 1.3 & 2.3 & 3.8 & 5.2 & 6.9 & 8.0 & 12.7 & 24.1 & 47.5 & 72.9 \\
\hline 655360 & 2 & 0.4 & 0.7 & 1.0 & 1.1 & 1.2 & 1.2 & 1.2 & 1.2 & 1.2 & 1.21 \\
\hline & 10 & 0.6 & 1.3 & 2.4 & 3.5 & 4.9 & 5.6 & 8.2 & 13.7 & 30.5 & 55.4 \\
\hline
\end{tabular}




\begin{tabular}{|c|c|c|c|c|c|c|c|c|c|c|c|}
\hline \multirow{2}{*}{\multicolumn{12}{|c|}{$\begin{array}{l}\text { Conditioned 1st-Order Markov Model } \\
\text { December DRWH Reliability (\%) } \\
\text { per capita roof area and storage }\end{array}$}} \\
\hline & & & & & & & & & & & \\
\hline \multirow{3}{*}{$\begin{array}{l}\text { Gauge } \\
655450\end{array}$} & sq. m & 0 & 40 & 80 & 120 & 160 & 200 & 400 & 600 & 800 & 1000 \\
\hline & 2 & 0.8 & 1.1 & 1.3 & 1.3 & 1.3 & 1.3 & 1.3 & 1.3 & 1.3 & 1.31 \\
\hline & 10 & 2.1 & 3.8 & 6.1 & 7.8 & 9.4 & 10.3 & 13.4 & 21.0 & 39.0 & 61 \\
\hline \multirow[t]{2}{*}{655480} & 2 & 1.6 & 2.5 & 3.3 & 3.6 & 3.8 & 3.9 & 4.1 & 4.2 & 4.3 & 4.29 \\
\hline & 10 & 3.1 & 5.7 & 9.6 & 12.5 & 15.4 & 16.9 & 22.3 & 34.5 & 57.5 & 80.4 \\
\hline \multirow[t]{2}{*}{655550} & 2 & 1.0 & 1.5 & 2.1 & 2.3 & 2.5 & 2.5 & 2.6 & 2.6 & 2.6 & 2.63 \\
\hline & 10 & 2.4 & 4.3 & 7.0 & 8.9 & 10.9 & 12.0 & 15.5 & 21.0 & 35.0 & 54.5 \\
\hline \multirow[t]{2}{*}{655570} & 2 & 3.1 & 4.6 & 5.5 & 5.8 & 6.0 & 6.1 & 6.1 & 6.1 & 6.1 & 6.15 \\
\hline & 10 & 8.0 & 14.2 & 22.7 & 29.1 & 35.2 & 39.3 & 57.8 & 76.9 & 89.4 & 95.3 \\
\hline \multirow[t]{2}{*}{655600} & 2 & 2.2 & 3.5 & 4.4 & 4.6 & 4.7 & 4.8 & 4.8 & 4.8 & 4.8 & 4.77 \\
\hline & 10 & 4.5 & 8.2 & 13.5 & 17.4 & 21.2 & 23.1 & 28.4 & 36.8 & 53.1 & 70.9 \\
\hline \multirow[t]{2}{*}{655620} & 2 & 1.7 & 2.5 & 3.2 & 3.4 & 3.4 & 3.4 & 3.4 & 3.4 & 3.4 & 3.43 \\
\hline & 10 & 3.4 & 6.3 & 10.9 & 14.3 & 17.8 & 19.7 & 26.7 & 37.2 & 52.3 & 66.6 \\
\hline \multirow[t]{2}{*}{655630} & 2 & 1.2 & 1.8 & 2.1 & 2.1 & 2.2 & 2.2 & 2.3 & 2.3 & 2.3 & 2.29 \\
\hline & 10 & 3.6 & 6.4 & 10.0 & 12.7 & 15.4 & 17.0 & 23.3 & 33.6 & 48.3 & 63 \\
\hline \multirow[t]{2}{*}{655780} & 2 & 5.6 & 8.9 & 10.8 & 11.4 & 11.7 & 11.9 & 12.6 & 12.8 & 12.8 & 12.8 \\
\hline & 10 & 12.6 & 22.2 & 35.1 & 44.4 & 53.2 & 59.0 & 79.2 & 90.3 & 94.6 & 96.3 \\
\hline \multirow[t]{2}{*}{655850} & 2 & 4.9 & 7.7 & 9.7 & 10.4 & 10.8 & 11.0 & 11.5 & 11.7 & 11.7 & 11.7 \\
\hline & 10 & 11.9 & 21.3 & 33.5 & 42.5 & 50.6 & 56.3 & 78.9 & 92.4 & 97.1 & 98.4 \\
\hline \multirow[t]{2}{*}{655920} & 2 & 7.9 & 14.1 & 19.1 & 21.2 & 22.2 & 22.6 & 23.0 & 23.0 & 23.1 & 23.1 \\
\hline & 10 & 16.4 & 29.0 & 44.0 & 54.3 & 63.0 & 68.8 & 87.4 & 96.3 & 99.2 & 99.9 \\
\hline \multirow[t]{2}{*}{655940} & 2 & 4.7 & 7.7 & 10.1 & 11.0 & 11.4 & 11.6 & 12.0 & 12.0 & 12.0 & 12 \\
\hline & 10 & 11.5 & 20.5 & 32.3 & 41.0 & 49.0 & 54.6 & 74.9 & 85.3 & 88.9 & 90.8 \\
\hline \multirow[t]{2}{*}{655990} & 2 & 3.9 & 6.3 & 8.2 & 8.9 & 9.2 & 9.3 & 9.5 & 9.5 & 9.5 & 9.46 \\
\hline & 10 & 8.5 & 15.4 & 25.0 & 32.3 & 39.6 & 44.5 & 63.3 & 74.3 & 78.1 & 80.4 \\
\hline
\end{tabular}





\section{Appendix K: Statistical Downscaling Results}




\begin{tabular}{|c|c|c|c|c|c|c|c|}
\hline \multicolumn{8}{|c|}{$\begin{array}{l}\text { Observed } \\
\text { DRWH Reliability (\%) }\end{array}$} \\
\hline Gauge & sq. m & Jan & Feb & Mar & Apr & May & Jun \\
\hline \multirow[t]{2}{*}{655920} & 2 & 0.8 & 0.7 & 2.0 & 2.8 & 13.0 & 16.5 \\
\hline & 10 & 6.8 & 7.1 & 12.3 & 19.8 & 49.2 & 51.2 \\
\hline \multirow[t]{2}{*}{655940} & 2 & 0.2 & 0.3 & 1.4 & 3.7 & 8.2 & 11.2 \\
\hline & 10 & 2.3 & 3.0 & 7.8 & 17.0 & 35.5 & 48.0 \\
\hline \multirow[t]{2}{*}{655990} & 2 & 0.4 & 0.3 & 0.9 & 4.2 & 9.8 & 11.4 \\
\hline & 10 & 3.4 & 2.6 & 5.8 & 16.5 & 36.4 & 45.2 \\
\hline \multirow[t]{2}{*}{655780} & 2 & 0.6 & 0.9 & 2.3 & 3.8 & 6.9 & 16.9 \\
\hline & 10 & 4.2 & 7.4 & 15.7 & 22.6 & 39.3 & 51.4 \\
\hline \multirow[t]{2}{*}{655850} & 2 & 0.4 & 1.7 & 2.4 & 3.3 & 6.7 & 14.6 \\
\hline & 10 & 3.3 & 10.3 & 15.5 & 20.3 & 37.2 & 53.0 \\
\hline \multirow[t]{2}{*}{655480} & 2 & 0.1 & 0.8 & 4.2 & 6.7 & 5.5 & 8.2 \\
\hline & 10 & 1.3 & 6.1 & 17.7 & 24.1 & 26.9 & 34.9 \\
\hline \multirow[t]{2}{*}{655600} & 2 & 0.3 & 2.5 & 2.2 & 4.9 & 5.5 & 3.6 \\
\hline & 10 & 2.2 & 8.4 & 13.5 & 20.1 & 26.6 & 26.1 \\
\hline \multirow[t]{2}{*}{655570} & 2 & 0.9 & 3.3 & 4.8 & 7.5 & 9.1 & 7.5 \\
\hline & 10 & 4.0 & 11.3 & 20.5 & 26.4 & 37.6 & 39.1 \\
\hline \multirow[t]{2}{*}{655630} & 2 & 0.5 & 0.6 & 2.2 & 5.0 & 5.5 & 5.5 \\
\hline & 10 & 2.2 & 4.7 & 14.7 & 20.5 & 27.1 & 29.9 \\
\hline \multirow[t]{2}{*}{655620} & 2 & 0.4 & 1.4 & 2.6 & 4.4 & 6.6 & 4.5 \\
\hline & 10 & 2.5 & 6.2 & 15.9 & 19.2 & 28.8 & 29.1 \\
\hline \multirow[t]{2}{*}{655550} & 2 & 0.8 & 1.1 & 3.0 & 6.1 & 4.3 & 4.3 \\
\hline & 10 & 2.4 & 4.0 & 12.8 & 19.8 & 20.8 & 23.9 \\
\hline \multirow[t]{2}{*}{655450} & 2 & 0.2 & 0.8 & 1.5 & 5.6 & 6.6 & 7.9 \\
\hline & 10 & 1.2 & 4.8 & 8.6 & 19.1 & 27.4 & 31.4 \\
\hline \multirow[t]{2}{*}{654720} & 2 & 0.4 & 0.4 & 1.5 & 5.4 & 6.5 & 3.4 \\
\hline & 10 & 1.4 & 2.7 & 6.4 & 13.3 & 16.1 & 22.7 \\
\hline \multirow[t]{2}{*}{653870} & 2 & 0.0 & 0.7 & 1.8 & 2.7 & 4.2 & 5.9 \\
\hline & 10 & 0.8 & 3.6 & 10.3 & 15.7 & 25.8 & 28.4 \\
\hline \multirow[t]{2}{*}{653760} & 2 & 0.0 & 0.5 & 1.6 & 3.9 & 6.2 & 8.5 \\
\hline & 10 & 1.2 & 3.7 & 9.8 & 18.4 & 23.0 & 31.8 \\
\hline \multirow[t]{2}{*}{653800} & 2 & 0.2 & 0.7 & 4.0 & 4.4 & 4.8 & 3.9 \\
\hline & 10 & 0.7 & 4.5 & 13.8 & 18.6 & 23.1 & 25.2 \\
\hline \multirow[t]{2}{*}{653440} & 2 & 0.3 & 1.3 & 2.1 & 7.1 & 7.8 & 11.0 \\
\hline & 10 & 2.2 & 4.9 & 11.6 & 21.0 & 30.9 & 42.6 \\
\hline \multirow[t]{2}{*}{653380} & 2 & 0.0 & 0.8 & 2.0 & 5.0 & 5.8 & 5.3 \\
\hline & 10 & 0.5 & 4.3 & 13.0 & 22.3 & 26.4 & 30.7 \\
\hline \multirow[t]{2}{*}{653350} & 2 & 0.1 & 1.3 & 2.9 & 4.8 & 3.5 & 4.7 \\
\hline & 10 & 0.8 & 4.3 & 12.9 & 20.8 & 22.2 & 31.5 \\
\hline \multirow[t]{2}{*}{655280} & 2 & 0.3 & 0.6 & 0.6 & 2.9 & 3.8 & 5.4 \\
\hline & 10 & 1.6 & 1.6 & 4.9 & 17.2 & 19.0 & 27.3 \\
\hline
\end{tabular}




\begin{tabular}{|c|c|c|c|c|c|c|c|}
\hline \multicolumn{8}{|c|}{$\begin{array}{l}\text { Observed } \\
\text { DRWH Reliability (\%) }\end{array}$} \\
\hline Gauge & sq. $m$ & Jul & Aug & Sep & Oct & Nov & Dec \\
\hline \multirow[t]{2}{*}{655920} & 2 & 3.3 & 3.8 & 4.7 & 4.1 & 3.3 & 2.6 \\
\hline & 10 & 25.8 & 36.2 & 40.9 & 34.8 & 28.5 & 18.7 \\
\hline \multirow[t]{2}{*}{655940} & 2 & 2.1 & 1.3 & 1.3 & 2.1 & 3.6 & 1.7 \\
\hline & 10 & 18.5 & 16.3 & 15.6 & 20.7 & 27.2 & 13.5 \\
\hline \multirow[t]{2}{*}{655990} & 2 & 2.2 & 0.8 & 1.0 & 2.2 & 2.5 & 1.6 \\
\hline & 10 & 18.6 & 11.8 & 10.6 & 19.9 & 18.9 & 10.8 \\
\hline \multirow[t]{2}{*}{655780} & 2 & 2.2 & 0.7 & 1.2 & 3.4 & 4.3 & 2.4 \\
\hline & 10 & 19.1 & 10.6 & 14.4 & 27.4 & 27.6 & 16.1 \\
\hline \multirow[t]{2}{*}{655850} & 2 & 2.2 & 1.4 & 1.6 & 3.9 & 3.1 & 1.5 \\
\hline & 10 & 23.8 & 17.5 & 21.6 & 34.3 & 25.4 & 13.0 \\
\hline \multirow[t]{2}{*}{655480} & 2 & 4.1 & 9.4 & 10.5 & 5.6 & 1.4 & 1.0 \\
\hline & 10 & 34.0 & 50.7 & 49.6 & 29.7 & 8.4 & 4.4 \\
\hline \multirow[t]{2}{*}{655600} & 2 & 2.3 & 2.8 & 3.7 & 2.8 & 0.8 & 1.3 \\
\hline & 10 & 21.2 & 24.5 & 30.4 & 21.0 & 7.2 & 5.6 \\
\hline \multirow[t]{2}{*}{655570} & 2 & 2.0 & 1.5 & 3.9 & 5.6 & 3.4 & 0.9 \\
\hline & 10 & 17.2 & 19.1 & 27.6 & 33.0 & 20.9 & 7.1 \\
\hline \multirow[t]{2}{*}{655630} & 2 & 2.6 & 1.6 & 2.8 & 4.1 & 1.4 & 0.6 \\
\hline & 10 & 17.6 & 17.8 & 23.3 & 25.1 & 10.5 & 5.0 \\
\hline \multirow[t]{2}{*}{655620} & 2 & 2.0 & 1.0 & 3.2 & 4.5 & 1.1 & 1.3 \\
\hline & 10 & 18.2 & 13.8 & 24.4 & 26.6 & 8.7 & 4.9 \\
\hline \multirow[t]{2}{*}{655550} & 2 & 3.3 & 3.7 & 5.1 & 4.5 & 0.6 & 0.6 \\
\hline & 10 & 18.5 & 25.6 & 29.5 & 21.0 & 5.4 & 3.4 \\
\hline \multirow[t]{2}{*}{655450} & 2 & 2.4 & 2.1 & 6.4 & 4.3 & 0.6 & 0.3 \\
\hline & 10 & 17.5 & 18.8 & 34.7 & 25.5 & 6.5 & 2.6 \\
\hline \multirow[t]{2}{*}{654720} & 2 & 1.2 & 0.4 & 0.9 & 1.7 & 0.7 & 1.3 \\
\hline & 10 & 11.2 & 6.4 & 8.8 & 13.7 & 5.2 & 3.8 \\
\hline \multirow[t]{2}{*}{653870} & 2 & 1.6 & 0.6 & 1.6 & 1.9 & 0.4 & 0.4 \\
\hline & 10 & 13.9 & 7.9 & 15.7 & 15.7 & 5.0 & 3.3 \\
\hline \multirow[t]{2}{*}{653760} & 2 & 6.4 & 6.2 & 7.2 & 4.4 & 0.3 & 0.4 \\
\hline & 10 & 36.0 & 33.3 & 33.1 & 22.4 & 3.2 & 2.0 \\
\hline \multirow[t]{2}{*}{653800} & 2 & 1.8 & 0.7 & 2.4 & 4.6 & 0.7 & 0.9 \\
\hline & 10 & 18.2 & 10.3 & 23.0 & 29.0 & 8.0 & 3.4 \\
\hline \multirow[t]{2}{*}{653440} & 2 & 2.6 & 1.2 & 3.1 & 5.2 & 1.4 & 0.3 \\
\hline & 10 & 19.6 & 12.6 & 24.3 & 28.9 & 8.8 & 3.2 \\
\hline \multirow[t]{2}{*}{653380} & 2 & 4.1 & 3.4 & 3.3 & 4.2 & 0.4 & 1.0 \\
\hline & 10 & 27.8 & 24.7 & 26.7 & 24.9 & 4.4 & 3.4 \\
\hline \multirow[t]{2}{*}{653350} & 2 & 3.8 & 4.5 & 5.6 & 4.6 & 0.8 & 0.1 \\
\hline & 10 & 26.3 & 29.6 & 33.3 & 24.9 & 3.3 & 1.1 \\
\hline \multirow[t]{2}{*}{655280} & 2 & 9.7 & 12.7 & 8.8 & 6.0 & 1.2 & 0.1 \\
\hline & 10 & 38.7 & 52.5 & 45.6 & 30.1 & 7.2 & 1.1 \\
\hline
\end{tabular}




\begin{tabular}{|c|c|c|c|c|c|c|c|}
\hline \multicolumn{8}{|c|}{$\begin{array}{l}\text { Observed } \\
\text { DRWH Reliability (\%) }\end{array}$} \\
\hline \multirow[b]{2}{*}{ Gauge } & \multicolumn{7}{|c|}{ per capita roof area } \\
\hline & sq. m & Jan & Feb & Mar & Apr & May & Jun \\
\hline \multirow[t]{2}{*}{655360} & 2 & 0.1 & 1.2 & 1.9 & 3.7 & 4.7 & 7.5 \\
\hline & 10 & 0.8 & 3.0 & 7.8 & 15.3 & 22.8 & 28.4 \\
\hline \multirow[t]{2}{*}{655100} & 2 & 0.1 & 0.1 & 0.4 & 1.5 & 3.4 & 5.4 \\
\hline & 10 & 0.3 & 1.1 & 4.4 & 10.2 & 19.2 & 28.6 \\
\hline \multirow[t]{2}{*}{655220} & 2 & 0.2 & 0.2 & 1.3 & 2.1 & 3.5 & 8.1 \\
\hline & 10 & 1.0 & 1.3 & 5.4 & 13.5 & 20.0 & 26.9 \\
\hline \multirow[t]{2}{*}{655180} & 2 & 0.0 & 0.1 & 0.4 & 1.0 & 3.1 & 7.2 \\
\hline & 10 & 0.0 & 0.6 & 3.9 & 6.9 & 15.5 & 28.8 \\
\hline \multirow[t]{2}{*}{653520} & 2 & 0.2 & 0.1 & 0.7 & 0.9 & 3.2 & 5.8 \\
\hline & 10 & 0.2 & 0.7 & 3.3 & 7.8 & 17.8 & 23.2 \\
\hline \multirow[t]{2}{*}{653190} & 2 & 0.0 & 0.0 & 0.6 & 2.6 & 3.9 & 6.3 \\
\hline & 10 & 0.0 & 0.8 & 4.6 & 18.6 & 23.4 & 33.4 \\
\hline \multirow[t]{2}{*}{653550} & 2 & 0.0 & 0.4 & 1.0 & 1.5 & 4.0 & 6.6 \\
\hline & 10 & 0.0 & 2.7 & 6.4 & 13.5 & 26.8 & 35.5 \\
\hline \multirow[t]{2}{*}{653570} & 2 & 0.5 & 0.2 & 0.7 & 2.7 & 4.4 & 10.0 \\
\hline & 10 & 0.5 & 1.2 & 4.5 & 14.9 & 19.2 & 24.0 \\
\hline \multirow[t]{2}{*}{653610} & 2 & 0.1 & 0.4 & 1.2 & 2.4 & 3.8 & 6.4 \\
\hline & 10 & 0.7 & 1.6 & 8.4 & 16.8 & 25.5 & 33.5 \\
\hline \multirow[t]{2}{*}{653300} & 2 & 0.0 & 0.1 & 1.0 & 2.7 & 6.6 & 5.6 \\
\hline & 10 & 0.0 & 1.1 & 7.0 & 13.6 & 28.5 & 31.8 \\
\hline \multirow[t]{2}{*}{653060} & 2 & 0.0 & 0.7 & 0.6 & 1.3 & 3.4 & 7.4 \\
\hline & 10 & 0.0 & 0.7 & 2.1 & 8.2 & 18.1 & 28.1 \\
\hline \multirow[t]{2}{*}{655050} & 2 & 0.0 & 0.1 & 0.3 & 0.4 & 1.9 & 5.0 \\
\hline & 10 & 0.0 & 0.6 & 2.9 & 4.8 & 13.0 & 21.7 \\
\hline \multirow[t]{2}{*}{655160} & 2 & 0.0 & 0.0 & 0.4 & 2.0 & 3.1 & 5.7 \\
\hline & 10 & 0.0 & 0.2 & 3.1 & 10.0 & 18.1 & 26.6 \\
\hline \multirow[t]{2}{*}{655030} & 2 & 0.0 & 0.3 & 0.4 & 1.2 & 2.6 & 3.7 \\
\hline & 10 & 0.0 & 0.3 & 2.6 & 6.0 & 15.2 & 21.9 \\
\hline \multirow[t]{2}{*}{655020} & 2 & 0.0 & 0.2 & 0.1 & 0.5 & 1.1 & 2.6 \\
\hline & 10 & 0.0 & 0.2 & 1.2 & 2.8 & 9.0 & 17.4 \\
\hline \multirow[t]{2}{*}{655070} & 2 & 0.0 & 0.1 & 1.2 & 0.9 & 3.1 & 6.7 \\
\hline & 10 & 0.0 & 0.2 & 4.4 & 6.4 & 15.9 & 25.0 \\
\hline \multirow[t]{2}{*}{655010} & 2 & 0.0 & 0.0 & 0.1 & 0.1 & 0.5 & 1.6 \\
\hline & 10 & 0.1 & 0.1 & 0.8 & 0.9 & 4.5 & 12.9 \\
\hline
\end{tabular}




\begin{tabular}{|c|c|c|c|c|c|c|c|}
\hline \multicolumn{8}{|c|}{$\begin{array}{l}\text { Observed } \\
\text { DRWH Reliability (\%) }\end{array}$} \\
\hline Gauge & sq. $m$ & Jul & Aug & Sep & Oct & Nov & Dec \\
\hline \multirow[t]{2}{*}{655360} & 2 & 8.4 & 13.5 & 7.5 & 2.9 & 0.3 & 0.7 \\
\hline & 10 & 31.0 & 43.2 & 37.0 & 22.2 & 3.8 & 1.2 \\
\hline \multirow[t]{2}{*}{655100} & 2 & 7.8 & 12.8 & 7.4 & 2.7 & 0.3 & 0.2 \\
\hline & 10 & 32.7 & 49.7 & 39.1 & 15.8 & 2.5 & 0.5 \\
\hline \multirow[t]{2}{*}{655220} & 2 & 6.8 & 8.9 & 9.7 & 3.5 & 0.2 & 0.1 \\
\hline & 10 & 34.1 & 39.7 & 39.5 & 20.6 & 2.1 & 0.5 \\
\hline \multirow[t]{2}{*}{655180} & 2 & 7.3 & 11.1 & 8.8 & 1.6 & 0.4 & 0.8 \\
\hline & 10 & 31.0 & 45.7 & 37.2 & 11.8 & 2.9 & 0.9 \\
\hline \multirow[t]{2}{*}{653520} & 2 & 7.0 & 7.0 & 7.7 & 2.5 & 0.5 & 0.2 \\
\hline & 10 & 30.0 & 33.5 & 38.8 & 15.0 & 1.8 & 0.2 \\
\hline \multirow[t]{2}{*}{653190} & 2 & 7.5 & 8.1 & 12.9 & 3.4 & 0.5 & 0.0 \\
\hline & 10 & 40.7 & 49.1 & 54.9 & 23.3 & 2.4 & 0.3 \\
\hline \multirow[t]{2}{*}{653550} & 2 & 6.5 & 12.8 & 12.3 & 4.8 & 1.0 & 0.1 \\
\hline & 10 & 39.8 & 54.6 & 54.0 & 31.5 & 5.6 & 0.6 \\
\hline \multirow[t]{2}{*}{653570} & 2 & 6.1 & 6.6 & 8.4 & 5.1 & 0.6 & 0.4 \\
\hline & 10 & 33.2 & 34.9 & 38.5 & 21.2 & 2.8 & 0.7 \\
\hline \multirow[t]{2}{*}{653610} & 2 & 6.8 & 8.9 & 8.8 & 3.6 & 0.8 & 0.0 \\
\hline & 10 & 44.0 & 46.7 & 46.9 & 21.7 & 3.7 & 1.1 \\
\hline \multirow[t]{2}{*}{653300} & 2 & 6.7 & 7.9 & 9.8 & 4.3 & 0.2 & 0.0 \\
\hline & 10 & 38.4 & 39.7 & 44.7 & 23.3 & 1.3 & 0.8 \\
\hline \multirow[t]{2}{*}{653060} & 2 & 10.3 & 8.8 & 7.4 & 1.0 & 0.0 & 0.0 \\
\hline & 10 & 36.9 & 41.4 & 36.4 & 9.1 & 0.4 & 0.2 \\
\hline \multirow[t]{2}{*}{655050} & 2 & 8.1 & 12.6 & 5.6 & 1.4 & 0.1 & 0.8 \\
\hline & 10 & 30.2 & 41.1 & 30.3 & 11.9 & 1.3 & 0.8 \\
\hline \multirow[t]{2}{*}{655160} & 2 & 6.9 & 10.4 & 6.2 & 2.3 & 0.2 & 0.0 \\
\hline & 10 & 34.4 & 46.1 & 33.0 & 13.2 & 1.5 & 0.2 \\
\hline \multirow[t]{2}{*}{655030} & 2 & 7.7 & 8.9 & 4.7 & 1.1 & 0.1 & 0.2 \\
\hline & 10 & 32.7 & 39.0 & 27.0 & 9.7 & 1.1 & 0.8 \\
\hline \multirow[t]{2}{*}{655020} & 2 & 6.2 & 8.1 & 4.6 & 0.9 & 0.1 & 0.0 \\
\hline & 10 & 30.6 & 37.8 & 25.0 & 7.6 & 0.6 & 0.0 \\
\hline \multirow[t]{2}{*}{655070} & 2 & 9.4 & 10.5 & 7.2 & 1.1 & 0.1 & 0.2 \\
\hline & 10 & 34.2 & 43.0 & 31.0 & 9.9 & 0.8 & 0.8 \\
\hline \multirow[t]{2}{*}{655010} & 2 & 4.4 & 6.6 & 2.6 & 0.4 & 0.0 & 0.7 \\
\hline & 10 & 23.5 & 30.0 & 19.2 & 3.2 & 0.2 & 0.8 \\
\hline
\end{tabular}


GCM - CGCM3; Downscaling Method - Wilby et. al, 1998

SRES A1b (2046 - 2065)

DRWH Reliability (\%)

per capita roof area

\begin{tabular}{|c|c|c|c|c|c|c|c|}
\hline Gauge & sq. m & Jan & Feb & Mar & Apr & May & Jun \\
\hline \multirow[t]{2}{*}{655920} & 2 & 0.8 & 0.8 & 1.5 & 1.9 & 10.2 & 13.4 \\
\hline & 10 & 7.0 & 6.1 & 12.3 & 19.6 & 49.1 & 49.2 \\
\hline \multirow[t]{2}{*}{655940} & 2 & 0.3 & 0.3 & 1.3 & 3.8 & 6.2 & 9.6 \\
\hline & 10 & 2.5 & 2.7 & 7.7 & 16.2 & 32.4 & 45.1 \\
\hline \multirow[t]{2}{*}{655990} & 2 & 0.4 & 0.4 & 0.7 & 3.6 & 8.1 & 9.7 \\
\hline & 10 & 3.5 & 2.5 & 5.6 & 15.6 & 32.9 & 43.2 \\
\hline \multirow[t]{2}{*}{655780} & 2 & 0.7 & 0.9 & 1.9 & 3.9 & 5.8 & 15.0 \\
\hline & 10 & 4.5 & 6.6 & 13.9 & 21.9 & 38.3 & 50.7 \\
\hline \multirow[t]{2}{*}{655850} & 2 & 0.3 & 1.4 & 1.9 & 3.3 & 5.2 & 12.8 \\
\hline & 10 & 3.7 & 9.0 & 14.1 & 19.8 & 36.3 & 51.7 \\
\hline \multirow[t]{2}{*}{655480} & 2 & 0.1 & 0.8 & 4.4 & 5.0 & 4.6 & 6.6 \\
\hline & 10 & 1.2 & 6.4 & 17.8 & 22.3 & 26.0 & 35.8 \\
\hline \multirow[t]{2}{*}{655600} & 2 & 0.2 & 2.5 & 2.2 & 3.7 & 3.0 & 3.7 \\
\hline & 10 & 2.0 & 8.4 & 13.7 & 19.2 & 26.0 & 27.0 \\
\hline \multirow[t]{2}{*}{655570} & 2 & 0.6 & 3.3 & 4.9 & 5.7 & 5.1 & 7.8 \\
\hline & 10 & 3.8 & 11.3 & 21.3 & 25.3 & 37.4 & 39.6 \\
\hline \multirow[t]{2}{*}{655630} & 2 & 0.3 & 0.5 & 2.7 & 3.4 & 3.0 & 6.1 \\
\hline & 10 & 2.1 & 4.5 & 15.4 & 19.6 & 26.5 & 30.4 \\
\hline \multirow[t]{2}{*}{655620} & 2 & 0.2 & 1.3 & 2.4 & 3.9 & 3.8 & 4.0 \\
\hline & 10 & 2.3 & 6.0 & 16.5 & 18.1 & 28.8 & 28.9 \\
\hline \multirow[t]{2}{*}{655550} & 2 & 0.4 & 1.2 & 3.3 & 4.8 & 2.3 & 4.6 \\
\hline & 10 & 2.4 & 4.0 & 13.2 & 19.3 & 20.1 & 24.0 \\
\hline \multirow[t]{2}{*}{655450} & 2 & 0.1 & 0.7 & 1.6 & 4.0 & 4.5 & 8.3 \\
\hline & 10 & 1.2 & 4.6 & 8.6 & 18.2 & 26.7 & 33.3 \\
\hline \multirow[t]{2}{*}{654720} & 2 & 0.1 & 0.4 & 1.9 & 4.4 & 5.8 & 3.2 \\
\hline & 10 & 1.3 & 2.8 & 6.5 & 12.8 & 16.7 & 21.7 \\
\hline \multirow[t]{2}{*}{653870} & 2 & 0.0 & 0.6 & 2.7 & 2.2 & 3.8 & 5.7 \\
\hline & 10 & 0.4 & 3.7 & 10.7 & 13.9 & 25.6 & 27.6 \\
\hline \multirow[t]{2}{*}{653760} & 2 & 0.0 & 0.5 & 2.1 & 3.1 & 5.5 & 7.2 \\
\hline & 10 & 0.8 & 3.8 & 9.8 & 17.1 & 23.2 & 30.7 \\
\hline \multirow[t]{2}{*}{653800} & 2 & 0.1 & 0.6 & 4.5 & 2.8 & 2.8 & 3.3 \\
\hline & 10 & 0.6 & 4.5 & 13.7 & 18.0 & 21.9 & 24.6 \\
\hline \multirow[t]{2}{*}{653440} & 2 & 0.0 & 1.1 & 2.3 & 4.2 & 5.4 & 9.2 \\
\hline & 10 & 1.8 & 4.8 & 11.4 & 20.6 & 30.1 & 41.9 \\
\hline \multirow[t]{2}{*}{653380} & 2 & 0.0 & 0.8 & 2.5 & 2.6 & 3.5 & 4.4 \\
\hline & 10 & 0.5 & 4.3 & 12.7 & 21.1 & 26.0 & 29.6 \\
\hline \multirow[t]{2}{*}{653350} & 2 & 0.0 & 1.0 & 3.3 & 2.7 & 2.0 & 4.3 \\
\hline & 10 & 0.6 & 4.1 & 12.7 & 20.2 & 21.3 & 30.3 \\
\hline \multirow[t]{2}{*}{655280} & 2 & 0.1 & 0.5 & 0.4 & 1.8 & 2.5 & 3.7 \\
\hline & 10 & 1.6 & 1.6 & 4.8 & 15.4 & 19.5 & 28.5 \\
\hline
\end{tabular}


GCM - CGCM3; Downscaling Method - Wilby et. al, 1998

SRES A1b (2046 - 2065)

DRWH Reliability (\%)

\begin{tabular}{|c|c|c|c|c|c|c|c|}
\hline \multirow{2}{*}{ Gauge } & \multicolumn{7}{|c|}{ per capita roof area } \\
\hline & sq. $m$ & Jul & Aug & Sep & Oct & Nov & Dec \\
\hline \multirow[t]{2}{*}{655920} & 2 & 3.4 & 3.9 & 5.5 & 4.1 & 2.6 & 3.6 \\
\hline & 10 & 25.9 & 37.4 & 42.1 & 34.6 & 26.9 & 20.7 \\
\hline \multirow[t]{2}{*}{655940} & 2 & 2.4 & 1.5 & 1.4 & 2.3 & 2.7 & 2.8 \\
\hline & 10 & 20.0 & 16.7 & 17.2 & 21.8 & 27.2 & 15.3 \\
\hline \multirow[t]{2}{*}{655990} & 2 & 2.3 & 0.8 & 0.8 & 3.1 & 2.1 & 2.8 \\
\hline & 10 & 18.9 & 11.8 & 11.9 & 21.3 & 18.6 & 12.0 \\
\hline \multirow[t]{2}{*}{655780} & 2 & 3.0 & 0.7 & 1.7 & 2.9 & 3.6 & 3.5 \\
\hline & 10 & 22.2 & 10.8 & 16.7 & 29.2 & 26.4 & 17.8 \\
\hline \multirow[t]{2}{*}{655850} & 2 & 3.2 & 1.3 & 2.7 & 3.6 & 2.8 & 2.6 \\
\hline & 10 & 27.1 & 17.1 & 25.2 & 36.6 & 24.3 & 14.4 \\
\hline \multirow[t]{2}{*}{655480} & 2 & 4.1 & 11.8 & 10.8 & 3.9 & 0.7 & 0.4 \\
\hline & 10 & 38.1 & 52.4 & 51.0 & 26.2 & 7.9 & 4.3 \\
\hline \multirow[t]{2}{*}{655600} & 2 & 2.6 & 2.8 & 4.0 & 2.6 & 0.4 & 1.1 \\
\hline & 10 & 22.4 & 24.4 & 31.7 & 18.6 & 6.4 & 5.7 \\
\hline \multirow[t]{2}{*}{655570} & 2 & 2.2 & 1.7 & 4.3 & 4.7 & 1.9 & 0.6 \\
\hline & 10 & 18.4 & 18.2 & 28.9 & 29.7 & 19.7 & 6.4 \\
\hline \multirow[t]{2}{*}{655630} & 2 & 3.0 & 1.7 & 3.4 & 3.2 & 0.9 & 0.4 \\
\hline & 10 & 18.7 & 17.0 & 25.1 & 22.5 & 9.7 & 4.7 \\
\hline \multirow[t]{2}{*}{655620} & 2 & 2.2 & 0.9 & 3.5 & 3.9 & 0.8 & 1.1 \\
\hline & 10 & 19.3 & 13.5 & 25.1 & 23.8 & 8.4 & 4.9 \\
\hline \multirow[t]{2}{*}{655550} & 2 & 3.4 & 3.2 & 5.8 & 3.7 & 0.4 & 0.4 \\
\hline & 10 & 19.5 & 24.2 & 30.8 & 19.3 & 5.1 & 3.3 \\
\hline \multirow[t]{2}{*}{655450} & 2 & 2.9 & 1.8 & 6.7 & 3.0 & 0.6 & 0.1 \\
\hline & 10 & 20.0 & 17.0 & 35.7 & 22.4 & 5.9 & 2.8 \\
\hline \multirow[t]{2}{*}{654720} & 2 & 1.1 & 0.4 & 0.8 & 1.1 & 0.6 & 0.8 \\
\hline & 10 & 11.2 & 7.1 & 9.1 & 14.1 & 5.0 & 4.1 \\
\hline \multirow[t]{2}{*}{653870} & 2 & 1.5 & 0.6 & 1.9 & 1.4 & 0.4 & 0.3 \\
\hline & 10 & 13.6 & 8.1 & 16.2 & 16.1 & 4.4 & 3.6 \\
\hline \multirow[t]{2}{*}{653760} & 2 & 5.8 & 6.3 & 8.5 & 3.4 & 0.3 & 0.4 \\
\hline & 10 & 35.9 & 34.5 & 33.8 & 23.2 & 2.7 & 2.1 \\
\hline \multirow[t]{2}{*}{653800} & 2 & 1.6 & 0.8 & 2.9 & 3.4 & 0.6 & 1.0 \\
\hline & 10 & 17.3 & 12.4 & 23.7 & 29.6 & 7.1 & 3.5 \\
\hline \multirow[t]{2}{*}{653440} & 2 & 2.1 & 1.4 & 3.5 & 4.4 & 1.0 & 0.5 \\
\hline & 10 & 19.8 & 13.8 & 25.4 & 28.8 & 8.1 & 3.4 \\
\hline \multirow[t]{2}{*}{653380} & 2 & 3.9 & 3.4 & 3.7 & 3.3 & 0.4 & 1.4 \\
\hline & 10 & 28.2 & 26.8 & 27.8 & 24.9 & 3.9 & 3.5 \\
\hline \multirow[t]{2}{*}{653350} & 2 & 3.3 & 4.9 & 5.8 & 3.9 & 0.6 & 0.1 \\
\hline & 10 & 26.7 & 31.7 & 34.4 & 25.7 & 3.0 & 1.1 \\
\hline \multirow[t]{2}{*}{655280} & 2 & 9.0 & 11.5 & 8.4 & 4.2 & 1.0 & 0.1 \\
\hline & 10 & 39.5 & 50.4 & 45.9 & 29.8 & 7.1 & 1.1 \\
\hline
\end{tabular}


GCM - CGCM3; Downscaling Method - Wilby et. al, 1998

SRES A1b (2046 - 2065)

DRWH Reliability (\%)

\begin{tabular}{|c|c|c|c|c|c|c|c|}
\hline \multirow[b]{2}{*}{ Gauge } & \multicolumn{7}{|c|}{ per capita roof area } \\
\hline & sq. $m$ & Jan & Feb & Mar & Apr & May & Jun \\
\hline \multirow[t]{2}{*}{655360} & 2 & 0.2 & 1.6 & 1.0 & 4.0 & 3.1 & 5.3 \\
\hline & 10 & 0.8 & 3.0 & 7.7 & 15.4 & 21.1 & 29.8 \\
\hline \multirow[t]{2}{*}{655100} & 2 & 0.2 & 0.3 & 0.3 & 1.9 & 2.4 & 3.2 \\
\hline & 10 & 0.3 & 1.1 & 4.0 & 10.6 & 18.0 & 29.8 \\
\hline \multirow[t]{2}{*}{655220} & 2 & 0.3 & 0.2 & 1.0 & 2.6 & 2.5 & 5.5 \\
\hline & 10 & 0.9 & 1.2 & 5.2 & 13.5 & 17.9 & 27.3 \\
\hline \multirow[t]{2}{*}{655180} & 2 & 0.0 & 0.0 & 0.4 & 0.9 & 1.6 & 6.9 \\
\hline & 10 & 0.0 & 0.5 & 3.5 & 7.1 & 14.3 & 29.8 \\
\hline \multirow[t]{2}{*}{653520} & 2 & 0.2 & 0.0 & 0.5 & 0.9 & 1.9 & 5.1 \\
\hline & 10 & 0.2 & 0.6 & 3.2 & 8.1 & 16.7 & 24.2 \\
\hline \multirow[t]{2}{*}{653190} & 2 & 0.0 & 0.0 & 0.3 & 2.9 & 2.3 & 6.1 \\
\hline & 10 & 0.0 & 0.8 & 4.1 & 19.3 & 21.5 & 34.7 \\
\hline \multirow[t]{2}{*}{653550} & 2 & 0.0 & 0.3 & 0.6 & 1.5 & 2.6 & 6.4 \\
\hline & 10 & 0.0 & 2.6 & 6.0 & 13.8 & 24.7 & 36.3 \\
\hline \multirow[t]{2}{*}{653570} & 2 & 0.5 & 0.0 & 0.5 & 2.8 & 2.8 & 9.6 \\
\hline & 10 & 0.5 & 1.2 & 4.0 & 14.9 & 17.9 & 25.5 \\
\hline \multirow[t]{2}{*}{653610} & 2 & 0.2 & 0.2 & 0.8 & 2.3 & 2.2 & 5.9 \\
\hline & 10 & 0.6 & 1.6 & 7.3 & 16.5 & 23.8 & 34.7 \\
\hline \multirow[t]{2}{*}{653300} & 2 & 0.0 & 0.1 & 1.0 & 2.2 & 3.4 & 2.6 \\
\hline & 10 & 0.0 & 1.2 & 6.4 & 14.4 & 27.3 & 21.2 \\
\hline \multirow[t]{2}{*}{653060} & 2 & 0.0 & 0.6 & 0.7 & 0.9 & 1.8 & 3.6 \\
\hline & 10 & 0.0 & 0.7 & 2.2 & 8.6 & 17.0 & 18.0 \\
\hline \multirow[t]{2}{*}{655050} & 2 & 0.0 & 0.2 & 0.2 & 0.5 & 1.6 & 4.6 \\
\hline & 10 & 0.0 & 0.5 & 2.7 & 4.6 & 12.1 & 21.4 \\
\hline \multirow[t]{2}{*}{655160} & 2 & 0.0 & 0.0 & 0.5 & 1.5 & 2.4 & 5.4 \\
\hline & 10 & 0.0 & 0.2 & 3.1 & 9.3 & 17.0 & 26.5 \\
\hline \multirow[t]{2}{*}{655030} & 2 & 0.0 & 0.2 & 0.3 & 1.2 & 1.9 & 3.4 \\
\hline & 10 & 0.0 & 0.3 & 2.5 & 5.9 & 14.0 & 21.6 \\
\hline \multirow[t]{2}{*}{655020} & 2 & 0.0 & 0.2 & 0.1 & 0.2 & 1.2 & 2.1 \\
\hline & 10 & 0.0 & 0.2 & 1.0 & 2.6 & 8.6 & 16.9 \\
\hline \multirow{2}{*}{655070} & 2 & 0.0 & 0.1 & 1.4 & 0.7 & 1.7 & 6.2 \\
\hline & 10 & 0.0 & 0.2 & 4.3 & 6.7 & 13.5 & 24.9 \\
\hline \multirow[t]{2}{*}{655010} & 2 & 0.0 & 0.0 & 0.1 & 0.1 & 0.2 & 1.7 \\
\hline & 10 & 0.2 & 0.2 & 0.8 & 1.0 & 3.5 & 10.9 \\
\hline
\end{tabular}


GCM - CGCM3; Downscaling Method - Wilby et. al, 1998

SRES A1b (2046 - 2065)

DRWH Reliability (\%)

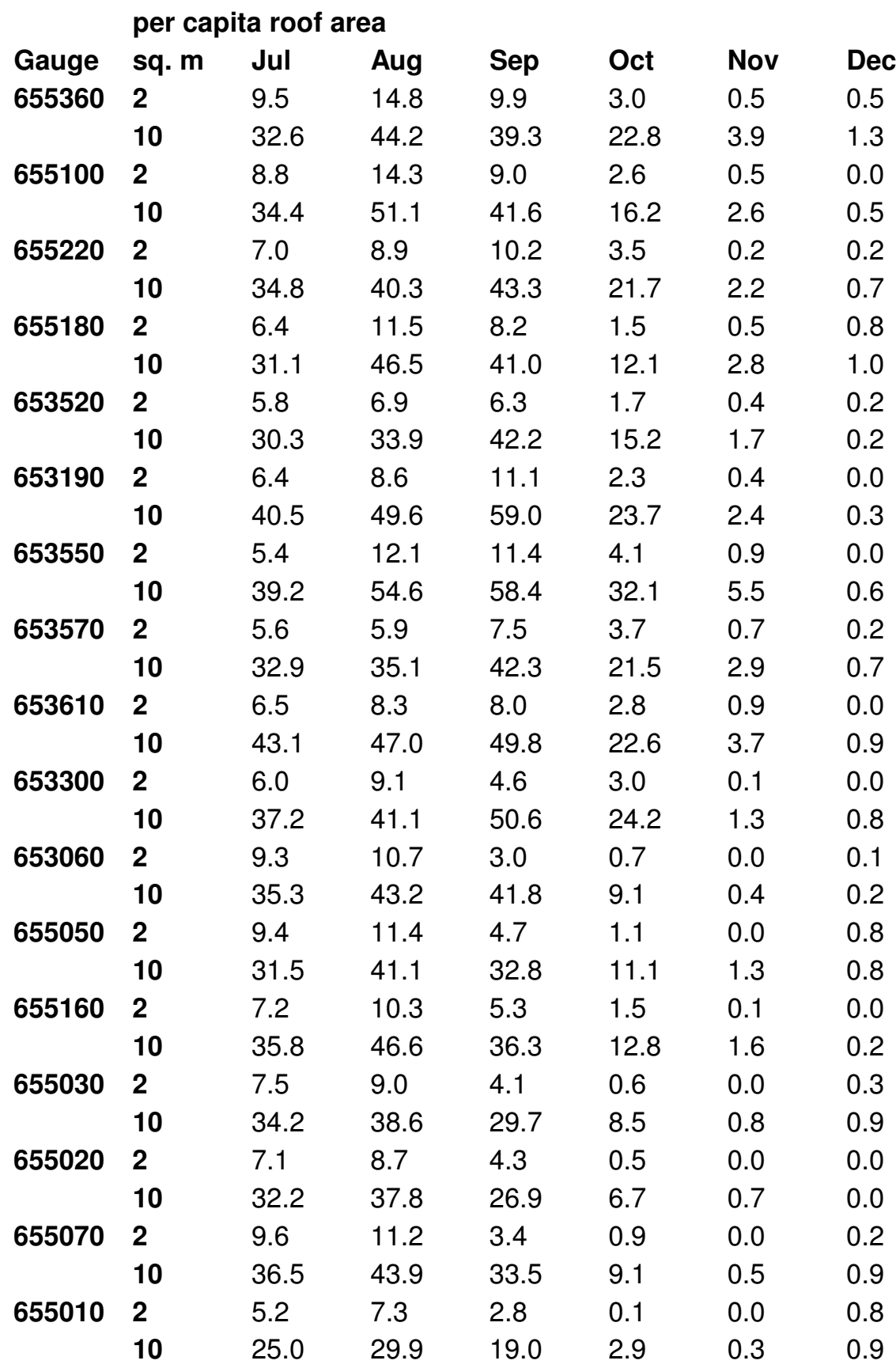


GCM - CGCM3; Downscaling Method - Wilby et. al, 1998

SRES A2 (2046 - 2065)

DRWH Reliability (\%)

per capita roof area

$\begin{array}{llllllll}\text { Gauge } & \text { sq. } \mathbf{m} & \text { Jan } & \text { Feb } & \text { Mar } & \text { Apr } & \text { May } & \text { Jun } \\ \mathbf{6 5 5 9 2 0} & \mathbf{2} & 0.9 & 0.6 & 1.7 & 2.4 & 12.3 & 15.6\end{array}$

$\begin{array}{llllllll}655920 & 2 & 0.9 & 0.6 & 1.7 & 2.4 & 12.3 & 15.6 \\ & 10 & 7.6 & 6.2 & 12.5 & 19.0 & 50.1 & 50.9\end{array}$

$\begin{array}{llllllll}655940 & 2 & 0.2 & 0.3 & 1.1 & 3.0 & 5.6 & 10.6\end{array}$

$\begin{array}{lllllll}10 & 2.7 & 2.9 & 8.0 & 15.8 & 33.3 & 47.9\end{array}$

$\begin{array}{llllllll}655990 & 2 & 0.6 & 0.2 & 0.7 & 3.3 & 7.5 & 10.7\end{array}$

$\begin{array}{lllllll}10 & 3.7 & 2.6 & 5.9 & 15.5 & 33.7 & 45.4\end{array}$

$\begin{array}{llllllll}655780 & 2 & 0.8 & 0.8 & 2.0 & 2.8 & 5.8 & 15.3\end{array}$

$\begin{array}{llllllll}10 & 4.7 & 6.8 & 15.2 & 20.3 & 39.1 & 49.1\end{array}$

$\begin{array}{llllllll}655850 & 2 & 0.4 & 1.3 & 1.7 & 2.7 & 5.4 & 13.3\end{array}$

$\begin{array}{lllllll}10 & 3.6 & 9.1 & 15.0 & 18.6 & 36.6 & 51.1\end{array}$

$\begin{array}{llllllll}655480 & 2 & 0.2 & 0.8 & 4.1 & 5.8 & 5.2 & 7.2\end{array}$

$\begin{array}{llllllll} & \mathbf{1 0} & 1.5 & 6.3 & 17.9 & 24.5 & 24.9 & 33.2 \\ \mathbf{6 5 5 6 0 0} & \mathbf{2} & 0.4 & 2.4 & 2.1 & 4.5 & 4.5 & 3.0\end{array}$

$\begin{array}{llllllll}10 & 2.4 & 8.5 & 13.8 & 20.2 & 27.0 & 27.0\end{array}$

$\begin{array}{llllllll}655570 & 2 & 1.0 & 3.3 & 5.3 & 6.5 & 7.1 & 6.2\end{array}$

$\begin{array}{lllllll}10 & 4.3 & 11.3 & 21.3 & 26.0 & 38.1 & 40.0\end{array}$

$\begin{array}{llllllll}655630 & 2 & 0.7 & 0.4 & 2.5 & 3.8 & 4.6 & 5.2\end{array}$

$\begin{array}{lllllll}10 & 2.2 & 4.5 & 15.1 & 20.7 & 26.8 & 30.9\end{array}$

$\begin{array}{llllllll}655620 & 2 & 0.4 & 1.2 & 2.9 & 4.0 & 5.1 & 3.6\end{array}$

$\begin{array}{lllllll}10 & 2.5 & 6.1 & 16.9 & 19.2 & 29.1 & 29.4\end{array}$

$\begin{array}{llllllll}655550 & 2 & 0.8 & 1.0 & 3.2 & 5.6 & 3.3 & 3.9\end{array}$

$\begin{array}{lllllll}10 & 2.5 & 4.0 & 13.0 & 19.8 & 20.8 & 24.5\end{array}$

$\begin{array}{llllllll}655450 & 2 & 0.2 & 0.9 & 1.4 & 3.6 & 4.8 & 7.4\end{array}$

$\begin{array}{lllllll}10 & 1.3 & 4.6 & 8.8 & 17.7 & 27.0 & 32.9\end{array}$

$\begin{array}{llllllll}654720 & 2 & 0.4 & 0.5 & 1.8 & 4.1 & 6.2 & 3.1\end{array}$

$\begin{array}{lllllll}10 & 1.4 & 2.9 & 6.6 & 12.8 & 16.2 & 22.4\end{array}$

$\begin{array}{llllllll}653870 & 2 & 0.0 & 0.8 & 2.5 & 2.0 & 4.9 & 5.7\end{array}$

$\begin{array}{lllllll}10 & 0.7 & 3.8 & 10.8 & 14.1 & 24.3 & 28.1\end{array}$

$\begin{array}{llllllll}653760 & 2 & 0.0 & 0.5 & 2.1 & 2.9 & 6.2 & 7.4\end{array}$

$\begin{array}{lllllll}10 & 1.4 & 4.1 & 9.9 & 16.9 & 22.1 & 30.9\end{array}$

$\begin{array}{llllllll}653800 & 2 & 0.2 & 0.6 & 3.4 & 3.2 & 4.6 & 3.1\end{array}$

$\begin{array}{lllllll}10 & 0.7 & 4.7 & 13.9 & 19.0 & 21.9 & 24.6\end{array}$

$\begin{array}{llllllll}653440 & 2 & 0.3 & 1.3 & 1.9 & 4.8 & 6.8 & 9.4\end{array}$

$\begin{array}{lllllll}10 & 2.2 & 5.0 & 11.8 & 21.1 & 30.1 & 42.8\end{array}$

$\begin{array}{llllllll}653380 & 2 & 0.1 & 0.8 & 1.8 & 2.8 & 5.2 & 4.6\end{array}$

$\begin{array}{lllllll}10 & 0.7 & 4.3 & 13.4 & 22.0 & 25.8 & 30.2\end{array}$

$\begin{array}{llllllll}653350 & 2 & 0.1 & 1.5 & 2.5 & 3.1 & 2.9 & 4.4\end{array}$

$\begin{array}{lllllll}10 & 0.8 & 4.4 & 12.9 & 21.2 & 20.8 & 30.6\end{array}$

$\begin{array}{llllllll}655280 & 2 & 0.1 & 0.6 & 0.6 & 1.3 & 2.1 & 3.3\end{array}$

$\begin{array}{lllllll}10 & 1.6 & 1.6 & 5.2 & 15.2 & 18.4 & 28.6\end{array}$ 
GCM - CGCM3; Downscaling Method - Wilby et. al, 1998

SRES A2 (2046 - 2065)

DRWH Reliability (\%)

\begin{tabular}{|c|c|c|c|c|c|c|c|}
\hline \multirow{2}{*}{ Gauge } & \multicolumn{7}{|c|}{ per capita roof area } \\
\hline & sq. $m$ & Jul & Aug & Sep & Oct & Nov & Dec \\
\hline \multirow[t]{2}{*}{655920} & 2 & 3.9 & 4.1 & 5.5 & 3.5 & 2.4 & 4.5 \\
\hline & 10 & 26.8 & 35.4 & 42.7 & 34.3 & 28.4 & 21.8 \\
\hline \multirow[t]{2}{*}{655940} & 2 & 2.5 & 1.2 & 1.6 & 3.1 & 2.4 & 4.2 \\
\hline & 10 & 19.8 & 15.8 & 18.5 & 21.1 & 26.9 & 16.4 \\
\hline \multirow[t]{2}{*}{655990} & 2 & 2.4 & 0.9 & 0.9 & 3.2 & 1.9 & 4.5 \\
\hline & 10 & 19.4 & 11.2 & 12.8 & 20.1 & 19.0 & 12.6 \\
\hline \multirow[t]{2}{*}{655780} & 2 & 2.6 & 0.5 & 1.7 & 3.1 & 3.1 & 4.8 \\
\hline & 10 & 20.9 & 9.6 & 16.3 & 27.9 & 26.4 & 18.6 \\
\hline \multirow[t]{2}{*}{655850} & 2 & 3.0 & 1.0 & 2.1 & 3.9 & 2.2 & 3.1 \\
\hline & 10 & 25.6 & 15.8 & 24.3 & 34.4 & 25.1 & 15.4 \\
\hline \multirow[t]{2}{*}{655480} & 2 & 4.4 & 10.7 & 11.3 & 1.4 & 1.0 & 0.7 \\
\hline & 10 & 34.9 & 51.6 & 50.6 & 6.6 & 8.1 & 4.6 \\
\hline \multirow[t]{2}{*}{655600} & 2 & 2.5 & 2.4 & 4.2 & 3.0 & 0.4 & 2.9 \\
\hline & 10 & 22.0 & 22.9 & 31.5 & 18.5 & 6.7 & 5.9 \\
\hline \multirow[t]{2}{*}{655570} & 2 & 2.2 & 1.7 & 4.1 & 5.8 & 2.5 & 2.4 \\
\hline & 10 & 18.0 & 17.7 & 28.3 & 29.9 & 20.8 & 7.7 \\
\hline \multirow[t]{2}{*}{655630} & 2 & 3.0 & 1.7 & 3.3 & 3.6 & 1.0 & 1.8 \\
\hline & 10 & 18.5 & 16.4 & 24.8 & 22.2 & 9.9 & 5.3 \\
\hline \multirow[t]{2}{*}{655620} & 2 & 2.0 & 1.0 & 3.9 & 4.2 & 1.0 & 2.5 \\
\hline & 10 & 18.9 & 13.4 & 25.4 & 23.4 & 8.9 & 5.2 \\
\hline \multirow[t]{2}{*}{655550} & 2 & 3.2 & 3.2 & 5.7 & 4.1 & 0.4 & 1.4 \\
\hline & 10 & 19.4 & 23.4 & 30.8 & 18.5 & 5.0 & 3.6 \\
\hline \multirow[t]{2}{*}{655450} & 2 & 2.7 & 1.7 & 6.6 & 3.8 & 0.7 & 1.1 \\
\hline & 10 & 18.8 & 16.1 & 35.0 & 21.3 & 6.3 & 2.8 \\
\hline \multirow[t]{2}{*}{654720} & 2 & 1.1 & 0.3 & 0.8 & 1.2 & 0.8 & 2.5 \\
\hline & 10 & 10.5 & 6.3 & 8.4 & 14.5 & 5.3 & 4.2 \\
\hline \multirow[t]{2}{*}{653870} & 2 & 1.6 & 0.4 & 1.7 & 1.3 & 0.5 & 1.4 \\
\hline & 10 & 13.3 & 7.2 & 15.4 & 16.2 & 5.1 & 3.7 \\
\hline \multirow[t]{2}{*}{653760} & 2 & 5.8 & 5.2 & 8.0 & 3.5 & 0.3 & 1.1 \\
\hline & 10 & 35.2 & 32.0 & 32.7 & 23.0 & 3.3 & 2.3 \\
\hline \multirow[t]{2}{*}{653800} & 2 & 1.7 & 0.8 & 2.5 & 3.3 & 0.9 & 1.7 \\
\hline & 10 & 17.5 & 11.1 & 23.2 & 29.2 & 8.4 & 3.8 \\
\hline \multirow[t]{2}{*}{653440} & 2 & 2.3 & 1.2 & 3.3 & 3.9 & 1.6 & 1.2 \\
\hline & 10 & 19.6 & 13.2 & 24.7 & 28.1 & 8.5 & 3.8 \\
\hline \multirow[t]{2}{*}{653380} & 2 & 4.2 & 3.4 & 3.3 & 3.2 & 0.5 & 2.1 \\
\hline & 10 & 28.1 & 25.6 & 27.5 & 24.1 & 4.1 & 3.8 \\
\hline \multirow[t]{2}{*}{653350} & 2 & 4.0 & 4.3 & 5.7 & 3.5 & 0.8 & 0.4 \\
\hline & 10 & 26.3 & 30.4 & 34.2 & 24.9 & 3.2 & 1.3 \\
\hline \multirow[t]{2}{*}{655280} & 2 & 8.5 & 11.3 & 8.7 & 6.9 & 2.3 & 0.1 \\
\hline & 10 & 38.0 & 54.9 & 46.6 & 30.8 & 7.4 & 1.2 \\
\hline
\end{tabular}


GCM - CGCM3; Downscaling Method - Wilby et. al, 1998

SRES A2 (2046 - 2065)

DRWH Reliability (\%)

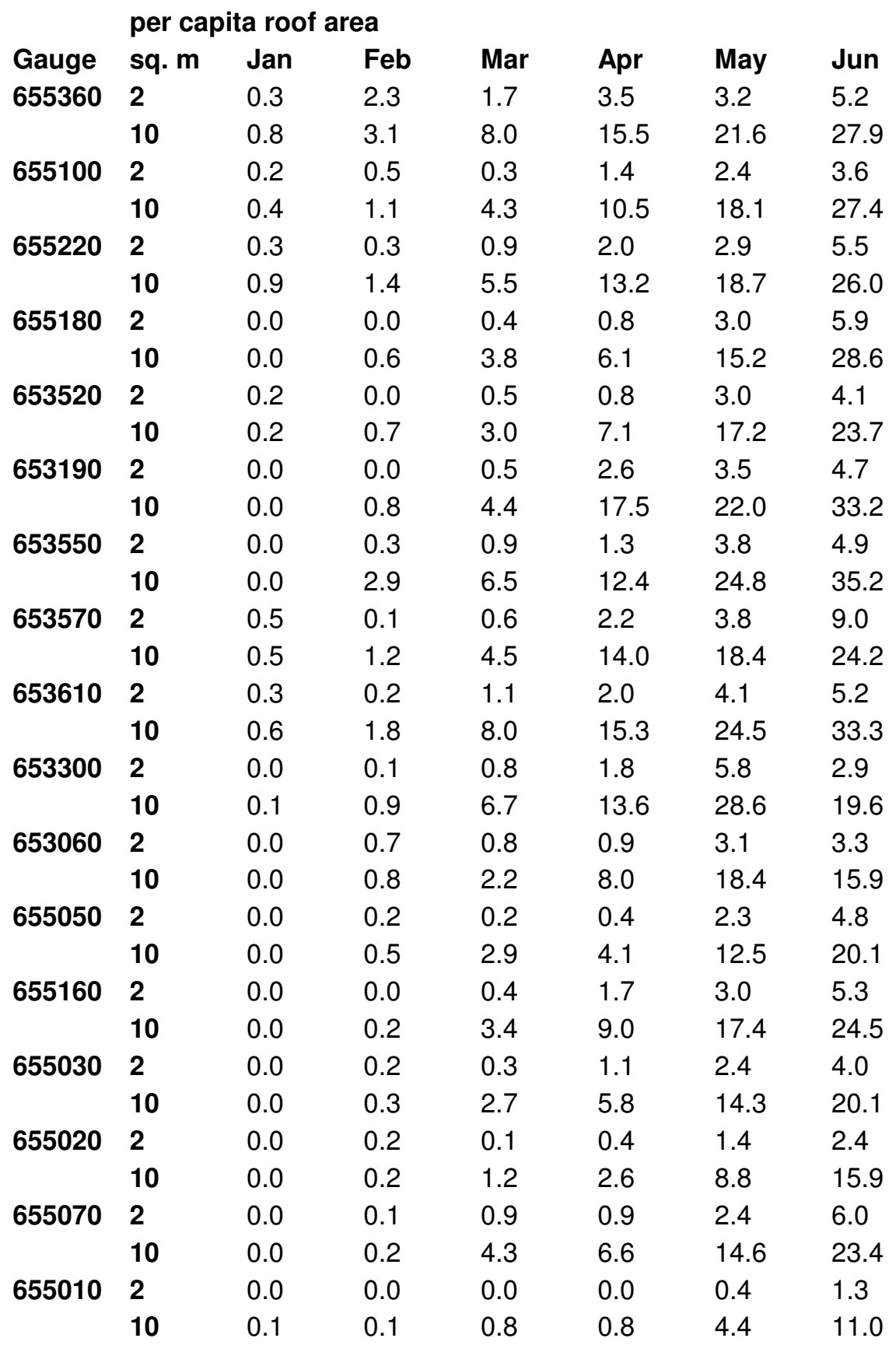


GCM - CGCM3; Downscaling Method - Wilby et. al, 1998

SRES B1 (2046 - 2065)

DRWH Reliability (\%)

\begin{tabular}{|c|c|c|c|c|c|c|c|}
\hline \multirow{2}{*}{ Gauge } & \multicolumn{7}{|c|}{ per capita roof area } \\
\hline & sq. $m$ & Jan & Feb & Mar & Apr & May & Jun \\
\hline \multirow[t]{2}{*}{655920} & 2 & 1.0 & 0.7 & 1.7 & 2.0 & 10.2 & 13.2 \\
\hline & 10 & 8.1 & 6.8 & 13.6 & 20.5 & 49.7 & 49.9 \\
\hline \multirow[t]{2}{*}{655940} & 2 & 0.2 & 0.4 & 1.5 & 2.6 & 6.2 & 8.4 \\
\hline & 10 & 2.6 & 3.0 & 9.2 & 16.8 & 33.5 & 45.6 \\
\hline \multirow[t]{2}{*}{655990} & 2 & 0.7 & 0.4 & 0.6 & 2.7 & 7.6 & 9.0 \\
\hline & 10 & 3.8 & 2.7 & 6.3 & 16.4 & 34.3 & 44.1 \\
\hline \multirow[t]{2}{*}{655780} & 2 & 0.8 & 1.0 & 2.4 & 2.5 & 4.0 & 13.0 \\
\hline & 10 & 4.8 & 7.4 & 16.9 & 21.3 & 38.7 & 50.2 \\
\hline \multirow[t]{2}{*}{655850} & 2 & 0.4 & 1.7 & 2.1 & 2.0 & 3.5 & 11.6 \\
\hline & 10 & 3.7 & 10.2 & 16.9 & 19.8 & 36.0 & 51.1 \\
\hline \multirow[t]{2}{*}{655480} & 2 & 0.0 & 0.6 & 4.9 & 5.8 & 4.3 & 7.4 \\
\hline & 10 & 1.2 & 5.6 & 18.7 & 23.2 & 27.0 & 33.8 \\
\hline \multirow[t]{2}{*}{655600} & 2 & 0.0 & 2.8 & 2.3 & 3.3 & 3.6 & 3.5 \\
\hline & 10 & 1.7 & 8.1 & 14.6 & 19.6 & 27.7 & 27.1 \\
\hline \multirow[t]{2}{*}{655570} & 2 & 0.1 & 3.4 & 5.3 & 5.7 & 6.5 & 7.4 \\
\hline & 10 & 3.6 & 10.8 & 22.1 & 26.5 & 39.4 & 39.8 \\
\hline \multirow[t]{2}{*}{655630} & 2 & 0.1 & 0.5 & 2.6 & 3.6 & 3.1 & 6.0 \\
\hline & 10 & 1.8 & 4.3 & 16.2 & 20.2 & 28.1 & 30.6 \\
\hline \multirow[t]{2}{*}{655620} & 2 & 0.0 & 1.3 & 2.7 & 3.8 & 4.2 & 4.4 \\
\hline & 10 & 1.9 & 5.9 & 17.2 & 18.9 & 30.3 & 29.7 \\
\hline \multirow[t]{2}{*}{655550} & 2 & 0.1 & 1.2 & 3.3 & 5.3 & 3.1 & 4.2 \\
\hline & 10 & 2.3 & 3.9 & 13.7 & 20.0 & 21.5 & 24.6 \\
\hline \multirow[t]{2}{*}{655450} & 2 & 0.0 & 0.8 & 1.2 & 3.5 & 5.2 & 9.1 \\
\hline & 10 & 0.9 & 4.4 & 8.8 & 17.7 & 27.4 & 33.2 \\
\hline \multirow[t]{2}{*}{654720} & 2 & 0.0 & 0.4 & 1.8 & 4.4 & 6.9 & 4.6 \\
\hline & 10 & 1.3 & 2.9 & 6.9 & 12.8 & 15.9 & 23.7 \\
\hline \multirow[t]{2}{*}{653870} & 2 & 0.0 & 0.6 & 2.1 & 1.9 & 5.7 & 6.4 \\
\hline & 10 & 0.4 & 3.5 & 10.7 & 14.5 & 25.1 & 29.2 \\
\hline \multirow[t]{2}{*}{653760} & 2 & 0.0 & 0.3 & 1.7 & 3.2 & 6.8 & 9.8 \\
\hline & 10 & 0.6 & 3.7 & 10.2 & 17.6 & 22.0 & 32.6 \\
\hline \multirow[t]{2}{*}{653800} & 2 & 0.0 & 0.9 & 4.3 & 3.2 & 5.2 & 3.5 \\
\hline & 10 & 0.7 & 4.1 & 14.4 & 18.5 & 23.2 & 25.7 \\
\hline \multirow[t]{2}{*}{653440} & 2 & 0.1 & 1.3 & 2.0 & 5.7 & 8.5 & 10.6 \\
\hline & 10 & 1.9 & 4.7 & 11.9 & 20.9 & 31.5 & 42.6 \\
\hline \multirow[t]{2}{*}{653380} & 2 & 0.0 & 0.9 & 2.2 & 3.0 & 6.3 & 5.3 \\
\hline & 10 & 0.5 & 4.2 & 13.6 & 21.8 & 27.2 & 30.9 \\
\hline \multirow[t]{2}{*}{653350} & 2 & 0.0 & 1.3 & 2.8 & 3.3 & 4.1 & 5.0 \\
\hline & 10 & 0.6 & 4.2 & 13.2 & 20.7 & 22.6 & 31.9 \\
\hline \multirow[t]{2}{*}{655280} & 2 & 0.1 & 0.0 & 0.5 & 1.6 & 2.2 & 4.5 \\
\hline & 10 & 1.5 & 1.5 & 4.9 & 15.8 & 19.0 & 27.5 \\
\hline
\end{tabular}


GCM - CGCM3; Downscaling Method - Wilby et. al, 1998

SRES A2 (2046 - 2065)

DRWH Reliability (\%)

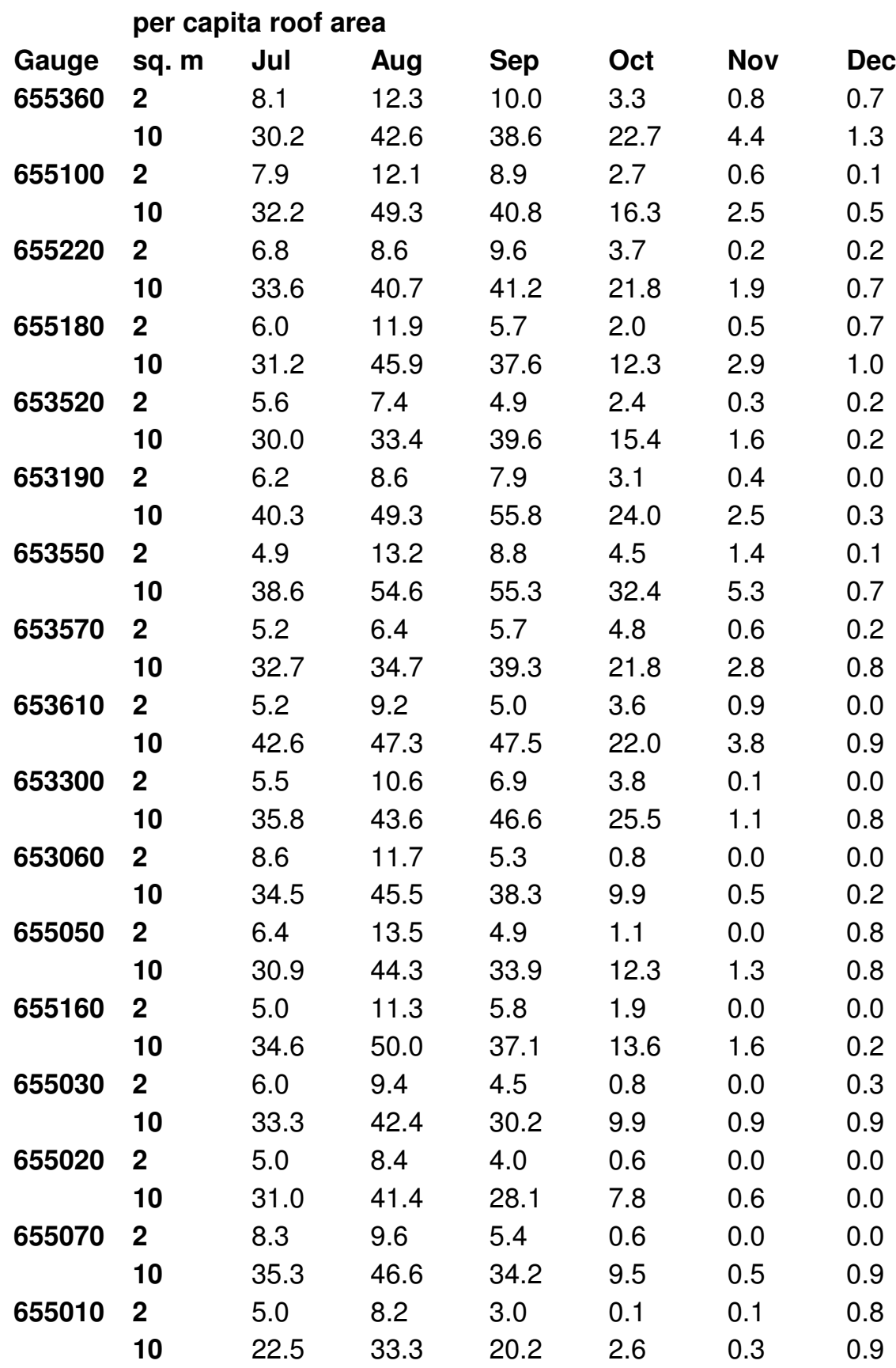


GCM - CGCM3; Downscaling Method - Wilby et. al, 1998

SRES B1 (2046 - 2065)

DRWH Reliability (\%)

\begin{tabular}{|c|c|c|c|c|c|c|c|}
\hline \multirow{2}{*}{ Gauge } & \multicolumn{7}{|c|}{ per capita roof area } \\
\hline & sq. $m$ & Jul & Aug & Sep & Oct & Nov & Dec \\
\hline \multirow[t]{2}{*}{655920} & 2 & 2.7 & 4.3 & 4.0 & 3.2 & 2.7 & 4.1 \\
\hline & 10 & 23.7 & 37.5 & 39.3 & 34.8 & 28.6 & 20.9 \\
\hline \multirow[t]{2}{*}{655940} & 2 & 1.9 & 1.5 & 1.6 & 1.9 & 3.4 & 3.8 \\
\hline & 10 & 18.3 & 16.4 & 17.9 & 21.2 & 29.5 & 14.8 \\
\hline \multirow[t]{2}{*}{655990} & 2 & 1.9 & 1.1 & 0.9 & 2.3 & 2.3 & 4.1 \\
\hline & 10 & 17.2 & 11.9 & 12.1 & 20.5 & 20.8 & 11.8 \\
\hline \multirow[t]{2}{*}{655780} & 2 & 2.4 & 0.8 & 1.4 & 2.5 & 3.6 & 3.8 \\
\hline & 10 & 20.2 & 10.7 & 16.5 & 28.2 & 27.9 & 17.4 \\
\hline \multirow[t]{2}{*}{655850} & 2 & 2.7 & 1.3 & 2.4 & 3.1 & 2.2 & 2.7 \\
\hline & 10 & 24.8 & 16.9 & 24.4 & 35.3 & 26.2 & 14.8 \\
\hline \multirow[t]{2}{*}{655480} & 2 & 4.9 & 10.9 & 10.4 & 3.1 & 0.9 & 0.4 \\
\hline & 10 & 42.6 & 53.2 & 49.3 & 24.3 & 8.3 & 4.3 \\
\hline \multirow[t]{2}{*}{655600} & 2 & 2.2 & 2.9 & 5.0 & 3.2 & 0.3 & 2.3 \\
\hline & 10 & 20.3 & 26.1 & 33.2 & 18.9 & 6.5 & 5.8 \\
\hline \multirow[t]{2}{*}{655570} & 2 & 1.7 & 1.9 & 4.8 & 5.6 & 1.8 & 2.0 \\
\hline & 10 & 15.7 & 19.3 & 29.9 & 30.0 & 20.7 & 7.3 \\
\hline \multirow[t]{2}{*}{655630} & 2 & 2.4 & 1.9 & 3.9 & 3.8 & 0.8 & 1.3 \\
\hline & 10 & 16.9 & 18.5 & 25.8 & 22.6 & 10.1 & 4.9 \\
\hline \multirow[t]{2}{*}{655620} & 2 & 1.9 & 1.3 & 4.1 & 4.0 & 0.7 & 2.1 \\
\hline & 10 & 17.0 & 15.2 & 26.3 & 23.5 & 8.7 & 5.0 \\
\hline \multirow[t]{2}{*}{655550} & 2 & 2.5 & 4.1 & 6.2 & 4.0 & 0.3 & 0.8 \\
\hline & 10 & 18.0 & 26.2 & 31.5 & 18.9 & 5.0 & 3.3 \\
\hline \multirow[t]{2}{*}{655450} & 2 & 2.3 & 2.3 & 7.4 & 3.1 & 0.6 & 0.4 \\
\hline & 10 & 17.9 & 17.9 & 35.7 & 22.4 & 6.1 & 2.9 \\
\hline \multirow[t]{2}{*}{654720} & 2 & 1.0 & 0.4 & 0.8 & 2.0 & 0.6 & 1.6 \\
\hline & 10 & 10.8 & 6.4 & 8.6 & 12.6 & 5.1 & 4.0 \\
\hline \multirow[t]{2}{*}{653870} & 2 & 1.7 & 0.6 & 1.6 & 2.2 & 0.5 & 0.5 \\
\hline & 10 & 13.4 & 8.3 & 15.5 & 15.1 & 5.1 & 3.5 \\
\hline \multirow[t]{2}{*}{653760} & 2 & 6.3 & 6.3 & 8.0 & 4.3 & 0.2 & 0.5 \\
\hline & 10 & 35.4 & 33.7 & 32.2 & 21.0 & 3.2 & 2.1 \\
\hline \multirow[t]{2}{*}{653800} & 2 & 2.0 & 0.8 & 2.7 & 5.0 & 0.8 & 0.8 \\
\hline & 10 & 19.0 & 10.8 & 22.3 & 27.0 & 7.8 & 3.6 \\
\hline \multirow[t]{2}{*}{653440} & 2 & 2.6 & 1.3 & 3.1 & 5.2 & 1.3 & 0.4 \\
\hline & 10 & 21.2 & 13.4 & 23.9 & 26.7 & 8.6 & 3.3 \\
\hline \multirow[t]{2}{*}{653380} & 2 & 5.1 & 3.1 & 3.5 & 4.2 & 0.5 & 1.0 \\
\hline & 10 & 29.7 & 26.3 & 26.6 & 22.6 & 3.9 & 3.5 \\
\hline \multirow[t]{2}{*}{653350} & 2 & 4.4 & 4.4 & 5.2 & 5.1 & 0.6 & 0.2 \\
\hline & 10 & 27.9 & 30.6 & 33.0 & 23.4 & 3.2 & 1.2 \\
\hline \multirow[t]{2}{*}{655280} & 2 & 8.1 & 11.5 & 6.9 & 7.4 & 0.7 & 0.1 \\
\hline & 10 & 37.2 & 51.5 & 45.4 & 30.7 & 7.2 & 1.2 \\
\hline
\end{tabular}


GCM - CGCM3; Downscaling Method - Wilby et. al, 1998

SRES B1 (2046 - 2065)

DRWH Reliability (\%)

\begin{tabular}{|c|c|c|c|c|c|c|c|}
\hline \multirow{2}{*}{ Gauge } & \multicolumn{7}{|c|}{ per capita roof area } \\
\hline & sq. $m$ & Jan & Feb & Mar & Apr & May & Jun \\
\hline \multirow[t]{2}{*}{655360} & 2 & 0.1 & 0.5 & 1.9 & 3.9 & 4.9 & 5.0 \\
\hline & 10 & 0.9 & 3.0 & 8.2 & 15.6 & 22.6 & 29.3 \\
\hline \multirow[t]{2}{*}{655100} & 2 & 0.1 & 0.0 & 0.4 & 1.5 & 3.1 & 3.0 \\
\hline & 10 & 0.3 & 1.1 & 4.5 & 10.3 & 19.4 & 29.6 \\
\hline \multirow[t]{2}{*}{655220} & 2 & 0.3 & 0.0 & 1.0 & 2.5 & 4.3 & 7.5 \\
\hline & 10 & 0.9 & 1.0 & 5.2 & 13.4 & 19.6 & 28.1 \\
\hline \multirow[t]{2}{*}{655180} & 2 & 0.0 & 0.0 & 0.4 & 1.1 & 4.2 & 8.8 \\
\hline & 10 & 0.0 & 0.6 & 3.8 & 6.6 & 15.3 & 29.6 \\
\hline \multirow[t]{2}{*}{653520} & 2 & 0.2 & 0.0 & 0.4 & 1.0 & 4.1 & 6.3 \\
\hline & 10 & 0.2 & 0.5 & 3.2 & 8.1 & 17.6 & 24.5 \\
\hline \multirow[t]{2}{*}{653190} & 2 & 0.0 & 0.0 & 0.5 & 2.8 & 5.3 & 8.1 \\
\hline & 10 & 0.0 & 0.8 & 4.2 & 18.5 & 22.9 & 34.5 \\
\hline \multirow[t]{2}{*}{653550} & 2 & 0.0 & 0.2 & 0.8 & 1.6 & 5.2 & 8.4 \\
\hline & 10 & 0.0 & 2.7 & 6.1 & 13.6 & 25.8 & 36.5 \\
\hline \multirow[t]{2}{*}{653570} & 2 & 0.4 & 0.0 & 0.4 & 2.8 & 5.2 & 11.8 \\
\hline & 10 & 0.5 & 1.2 & 4.4 & 14.7 & 18.9 & 25.4 \\
\hline \multirow[t]{2}{*}{653610} & 2 & 0.2 & 0.1 & 1.0 & 2.3 & 5.4 & 8.2 \\
\hline & 10 & 0.5 & 1.4 & 7.9 & 16.3 & 25.3 & 34.8 \\
\hline \multirow[t]{2}{*}{653300} & 2 & 0.0 & 0.1 & 0.8 & 2.0 & 6.8 & 3.8 \\
\hline & 10 & 0.0 & 1.1 & 6.8 & 13.6 & 28.7 & 20.1 \\
\hline \multirow[t]{2}{*}{653060} & 2 & 0.0 & 0.7 & 0.5 & 1.0 & 3.6 & 4.7 \\
\hline & 10 & 0.0 & 0.7 & 2.2 & 8.2 & 18.0 & 16.8 \\
\hline \multirow[t]{2}{*}{655050} & 2 & 0.0 & 0.1 & 0.3 & 0.4 & 2.5 & 6.5 \\
\hline & 10 & 0.0 & 0.5 & 2.8 & 4.1 & 12.5 & 20.1 \\
\hline \multirow[t]{2}{*}{655160} & 2 & 0.0 & 0.0 & 0.4 & 1.4 & 3.1 & 7.3 \\
\hline & 10 & 0.0 & 0.2 & 3.2 & 9.0 & 17.5 & 25.4 \\
\hline \multirow[t]{2}{*}{655030} & 2 & 0.0 & 0.3 & 0.2 & 1.1 & 2.6 & 4.8 \\
\hline & 10 & 0.0 & 0.3 & 2.3 & 5.5 & 14.7 & 20.3 \\
\hline \multirow[t]{2}{*}{655020} & 2 & 0.0 & 0.2 & 0.2 & 0.3 & 1.3 & 3.4 \\
\hline & 10 & 0.0 & 0.2 & 1.2 & 2.5 & 8.8 & 16.3 \\
\hline \multirow[t]{2}{*}{655070} & 2 & 0.0 & 0.1 & 1.0 & 0.8 & 2.3 & 8.1 \\
\hline & 10 & 0.0 & 0.2 & 4.3 & 6.4 & 14.8 & 24.4 \\
\hline \multirow[t]{2}{*}{655010} & 2 & 0.0 & 0.0 & 0.0 & 0.0 & 0.2 & 2.0 \\
\hline & 10 & 0.2 & 0.2 & 0.8 & 1.1 & 4.2 & 11.2 \\
\hline
\end{tabular}


GCM - CGCM3; Downscaling Method - Wilby et. al, 1998

SRES B1 (2046 - 2065)

DRWH Reliability (\%)

\begin{tabular}{llllllll}
\multicolumn{6}{c}{ per capita roof area } & & \\
Gauge & sq. $\mathbf{m}$ & Jul & Aug & Sep & Oct & Nov & Dec \\
$\mathbf{6 5 5 3 6 0}$ & $\mathbf{2}$ & 6.9 & 14.7 & 8.9 & 4.5 & 0.5 & 0.6 \\
& $\mathbf{1 0}$ & 30.2 & 46.3 & 40.4 & 23.2 & 4.2 & 1.3 \\
$\mathbf{6 5 5 1 0 0}$ & $\mathbf{2}$ & 6.2 & 14.1 & 8.5 & 4.1 & 0.3 & 0.1 \\
& $\mathbf{1 0}$ & 31.9 & 53.0 & 42.9 & 16.2 & 2.6 & 0.5 \\
$\mathbf{6 5 5 2 2 0}$ & $\mathbf{2}$ & 6.4 & 9.9 & 7.5 & 4.9 & 0.2 & 0.1 \\
& $\mathbf{1 0}$ & 32.5 & 41.7 & 40.1 & 22.2 & 1.9 & 0.5 \\
$\mathbf{6 5 5 1 8 0}$ & $\mathbf{2}$ & 6.2 & 12.4 & 6.8 & 2.2 & 0.3 & 0.5 \\
& $\mathbf{1 0}$ & 29.9 & 45.5 & 35.2 & 12.5 & 2.7 & 1.0 \\
$\mathbf{6 5 3 5 2 0}$ & $\mathbf{2}$ & 5.9 & 7.4 & 6.0 & 2.8 & 0.3 & 0.2 \\
& $\mathbf{1 0}$ & 29.2 & 33.0 & 36.7 & 15.3 & 1.6 & 0.2 \\
$\mathbf{6 5 3 1 9 0}$ & $\mathbf{2}$ & 6.5 & 8.4 & 10.7 & 3.7 & 0.4 & 0.0 \\
& $\mathbf{1 0}$ & 38.6 & 49.5 & 53.3 & 24.0 & 2.3 & 0.3 \\
$\mathbf{6 5 3 5 5 0}$ & $\mathbf{2}$ & 5.4 & 13.1 & 11.0 & 5.5 & 0.9 & 0.0 \\
& $\mathbf{1 0}$ & 37.5 & 53.8 & 52.2 & 33.0 & 5.2 & 0.5 \\
$\mathbf{6 5 3 5 7 0}$ & $\mathbf{2}$ & 5.3 & 6.3 & 6.8 & 5.2 & 0.6 & 0.0 \\
& $\mathbf{1 0}$ & 31.4 & 34.7 & 36.8 & 22.0 & 2.8 & 0.8 \\
$\mathbf{6 5 3 6 1 0}$ & $\mathbf{2}$ & 6.2 & 9.2 & 7.2 & 4.3 & 0.6 & 0.0 \\
& $\mathbf{1 0}$ & 42.2 & 46.4 & 44.2 & 22.6 & 3.7 & 0.7 \\
$\mathbf{6 5 3 3 0 0}$ & $\mathbf{2}$ & 5.5 & 10.0 & 6.6 & 3.4 & 0.1 & 0.0 \\
& $\mathbf{1 0}$ & 35.4 & 41.1 & 43.9 & 25.8 & 1.0 & 0.6 \\
$\mathbf{6 5 3 0 6 0}$ & $\mathbf{2}$ & 7.9 & 11.0 & 5.6 & 0.7 & 0.0 & 0.0 \\
& $\mathbf{1 0}$ & 34.1 & 42.9 & 35.8 & 9.8 & 0.3 & 0.2 \\
$\mathbf{6 5 5 0 5 0}$ & $\mathbf{2}$ & 7.4 & 11.5 & 5.3 & 1.5 & 0.0 & 0.8 \\
& $\mathbf{1 0}$ & 30.7 & 42.1 & 32.7 & 12.5 & 1.1 & 0.8 \\
$\mathbf{6 5 5 1 6 0}$ & $\mathbf{2}$ & 6.0 & 10.4 & 6.1 & 2.3 & 0.0 & 0.0 \\
& $\mathbf{1 0}$ & 35.0 & 47.8 & 35.8 & 13.6 & 1.4 & 0.2 \\
$\mathbf{6 5 5 0 3 0}$ & $\mathbf{2}$ & 7.3 & 9.1 & 4.6 & 0.8 & 0.0 & 0.3 \\
& $\mathbf{1 0}$ & 33.7 & 40.2 & 29.1 & 9.6 & 0.6 & 0.9 \\
$\mathbf{6 5 5 0 2 0}$ & $\mathbf{2}$ & 6.3 & 8.2 & 4.8 & 0.6 & 0.0 & 0.0 \\
& $\mathbf{1 0}$ & 31.1 & 39.1 & 26.6 & 7.8 & 0.6 & 0.0 \\
$\mathbf{6 5 5 0 7 0}$ & $\mathbf{2}$ & 10.1 & 9.8 & 4.4 & 0.9 & 0.0 & 0.0 \\
& $\mathbf{1 0}$ & 37.2 & 44.6 & 32.0 & 9.6 & 0.4 & 1.0 \\
$\mathbf{6 5 5 0 1 0}$ & $\mathbf{2}$ & 4.7 & 8.4 & 2.9 & 0.2 & 0.0 & 0.7 \\
& $\mathbf{1 0}$ & 23.3 & 30.9 & 18.2 & 2.9 & 0.3 & 0.9
\end{tabular}


GCM - MIROC; Downscaling Method - Wilby et. al, 1998

SRES A1b (2046 - 2065)

DRWH Reliability (\%)

\section{per capita roof area}

$\begin{array}{llllllll}\text { Gauge } & \text { sq. } \mathbf{m} & \text { Jan } & \text { Feb } & \text { Mar } & \text { Apr } & \text { May } & \text { Jun } \\ \mathbf{6 5 5 9 2 0} & \mathbf{2} & 1.2 & 0.0 & 1.6 & 2.0 & 13.1 & 13.0 \\ & \mathbf{1 0} & 8.2 & 6.4 & 10.9 & 17.7 & 48.6 & 49.6 \\ \mathbf{6 5 5 9 4 0} & \mathbf{2} & 0.8 & 0.1 & 1.0 & 2.6 & 8.4 & 8.8 \\ & \mathbf{1 0} & 3.1 & 2.2 & 7.5 & 15.2 & 35.9 & 45.9 \\ \mathbf{6 5 5 9 9 0} & \mathbf{2} & 1.4 & 0.1 & 0.8 & 3.0 & 10.4 & 9.1 \\ & \mathbf{1 0} & 4.3 & 2.1 & 5.6 & 15.0 & 36.5 & 43.6 \\ \mathbf{6 5 5 7 8 0} & \mathbf{2} & 1.4 & 0.2 & 2.0 & 2.6 & 6.3 & 13.2 \\ & \mathbf{1 0} & 5.0 & 6.1 & 14.6 & 19.4 & 38.5 & 50.8 \\ \mathbf{6 5 5 8 5 0} & \mathbf{2} & 0.8 & 0.4 & 1.9 & 2.4 & 5.9 & 11.7 \\ & \mathbf{1 0} & 4.2 & 9.6 & 14.4 & 18.0 & 35.7 & 51.7 \\ \mathbf{6 5 5 4 8 0} & \mathbf{2} & 0.1 & 0.4 & 3.0 & 4.2 & 4.5 & 5.8 \\ & \mathbf{1 0} & 1.5 & 5.9 & 16.5 & 22.3 & 25.9 & 32.8 \\ \mathbf{6 5 5 6 0 0} & \mathbf{2} & 0.4 & 2.1 & 1.7 & 3.5 & 5.0 & 2.4 \\ & \mathbf{1 0} & 2.5 & 8.0 & 12.5 & 18.3 & 26.2 & 23.3 \\ \mathbf{6 5 5 5 7 0} & \mathbf{2} & 1.3 & 2.4 & 4.1 & 5.7 & 8.3 & 5.1 \\ & \mathbf{1 0} & 4.6 & 10.4 & 19.1 & 24.4 & 37.2 & 35.7 \\ \mathbf{6 5 5 6 3 0} & \mathbf{2} & 0.6 & 0.4 & 1.6 & 3.7 & 4.6 & 4.3 \\ & \mathbf{1 0} & 2.4 & 4.1 & 13.3 & 19.1 & 26.2 & 27.5 \\ \mathbf{6 5 5 6 2 0} & \mathbf{2} & 0.4 & 1.1 & 2.1 & 3.8 & 5.8 & 2.6 \\ & \mathbf{1 0} & 2.7 & 5.7 & 15.0 & 17.6 & 28.3 & 25.4 \\ \mathbf{6 5 5 5 5 0} & \mathbf{2} & 1.0 & 0.9 & 2.2 & 5.2 & 4.1 & 2.9 \\ & \mathbf{1 0} & 2.7 & 3.8 & 11.6 & 18.8 & 20.2 & 21.8 \\ \mathbf{6 5 5 4 5 0} & \mathbf{2} & 0.2 & 0.3 & 1.5 & 4.0 & 7.8 & 5.7 \\ & \mathbf{1 0} & 1.3 & 4.2 & 8.8 & 17.9 & 27.4 & 29.5 \\ \mathbf{6 5 4 7 2 0} & \mathbf{2} & 0.1 & 0.0 & 1.8 & 3.4 & 5.6 & 2.6 \\ & \mathbf{1 0} & 1.5 & 1.6 & 6.4 & 12.2 & 15.6 & 21.4 \\ \mathbf{6 5 5 3 3 5 0} & \mathbf{2} & 0.0 & 0.1 & 2.9 & 2.8 & 2.9 & 4.6 \\ & \mathbf{1 0} & 0.6 & 3.5 & 12.4 & 18.5 & 20.6 & 30.3 \\ \mathbf{6 5 5 3 8 0 0} & \mathbf{2} & 0.0 & 0.1 & 0.8 & 1.7 & 2.4 & 2.9 \\ & \mathbf{1 0} & 1.2 & 1.3 & 4.9 & 15.6 & 17.2 & 23.0\end{array}$


GCM - MIROC; Downscaling Method - Wilby et. al, 1998

SRES A1b (2046 - 2065)

DRWH Reliability (\%)

\begin{tabular}{|c|c|c|c|c|c|c|c|}
\hline \multirow{2}{*}{ Gauge } & \multicolumn{7}{|c|}{ per capita roof area } \\
\hline & sq. $m$ & Jul & Aug & Sep & Oct & Nov & Dec \\
\hline \multirow[t]{2}{*}{655920} & 2 & 2.4 & 2.8 & 3.8 & 3.0 & 4.6 & 3.8 \\
\hline & 10 & 23.7 & 31.7 & 37.0 & 29.4 & 32.8 & 21.5 \\
\hline \multirow[t]{2}{*}{655940} & 2 & 2.0 & 0.7 & 1.0 & 1.4 & 5.7 & 2.8 \\
\hline & 10 & 18.5 & 12.9 & 14.4 & 18.0 & 31.0 & 15.9 \\
\hline \multirow[t]{2}{*}{655990} & 2 & 1.9 & 0.4 & 0.6 & 1.4 & 3.9 & 2.9 \\
\hline & 10 & 17.9 & 9.4 & 9.3 & 17.5 & 21.8 & 12.5 \\
\hline \multirow[t]{2}{*}{655780} & 2 & 1.6 & 0.6 & 1.0 & 2.7 & 6.1 & 3.4 \\
\hline & 10 & 17.9 & 8.8 & 13.7 & 26.2 & 30.0 & 18.4 \\
\hline \multirow[t]{2}{*}{655850} & 2 & 2.0 & 1.0 & 1.6 & 3.9 & 3.7 & 2.2 \\
\hline & 10 & 22.6 & 13.8 & 20.5 & 33.2 & 28.2 & 15.4 \\
\hline \multirow[t]{2}{*}{655480} & 2 & 3.7 & 5.7 & 7.6 & 4.5 & 1.4 & 1.2 \\
\hline & 10 & 30.8 & 44.3 & 45.7 & 27.6 & 9.0 & 4.8 \\
\hline \multirow[t]{2}{*}{655600} & 2 & 2.2 & 1.8 & 2.9 & 2.3 & 0.8 & 1.0 \\
\hline & 10 & 19.9 & 20.2 & 27.5 & 19.6 & 8.0 & 6.2 \\
\hline \multirow[t]{2}{*}{655570} & 2 & 1.5 & 1.2 & 3.2 & 4.5 & 3.9 & 0.5 \\
\hline & 10 & 15.6 & 15.1 & 25.4 & 30.8 & 22.7 & 7.5 \\
\hline \multirow[t]{2}{*}{655630} & 2 & 2.3 & 1.1 & 2.1 & 2.9 & 1.9 & 0.3 \\
\hline & 10 & 16.8 & 14.2 & 21.7 & 23.4 & 11.3 & 4.9 \\
\hline \multirow[t]{2}{*}{655620} & 2 & 1.5 & 0.8 & 2.4 & 3.1 & 1.6 & 0.9 \\
\hline & 10 & 16.5 & 11.5 & 22.3 & 24.9 & 9.8 & 5.0 \\
\hline \multirow[t]{2}{*}{655550} & 2 & 2.5 & 2.3 & 3.8 & 3.4 & 0.7 & 0.4 \\
\hline & 10 & 17.6 & 22.0 & 27.6 & 19.9 & 5.9 & 3.4 \\
\hline \multirow[t]{2}{*}{655450} & 2 & 2.3 & 1.8 & 5.4 & 3.7 & 1.0 & 0.2 \\
\hline & 10 & 16.9 & 15.6 & 33.9 & 25.0 & 7.6 & 2.5 \\
\hline \multirow[t]{2}{*}{654720} & 2 & 1.0 & 0.3 & 0.7 & 1.6 & 0.8 & 0.4 \\
\hline & 10 & 10.5 & 4.7 & 8.5 & 13.5 & 5.9 & 3.8 \\
\hline \multirow[t]{2}{*}{653870} & 2 & 1.2 & 0.3 & 1.6 & 1.5 & 0.7 & 0.1 \\
\hline & 10 & 12.4 & 6.2 & 15.3 & 15.2 & 6.0 & 2.7 \\
\hline \multirow[t]{2}{*}{653760} & 2 & 5.1 & 3.5 & 7.0 & 3.8 & 0.4 & 0.1 \\
\hline & 10 & 33.9 & 28.7 & 31.6 & 22.2 & 3.6 & 1.9 \\
\hline \multirow[t]{2}{*}{653800} & 2 & 1.5 & 0.5 & 2.0 & 5.5 & 0.9 & 0.6 \\
\hline & 10 & 15.7 & 8.7 & 20.9 & 30.7 & 9.1 & 3.4 \\
\hline \multirow[t]{2}{*}{653440} & 2 & 1.8 & 0.7 & 2.6 & 6.2 & 1.4 & 0.2 \\
\hline & 10 & 17.6 & 10.4 & 22.4 & 29.5 & 9.5 & 3.0 \\
\hline \multirow[t]{2}{*}{653380} & 2 & 3.3 & 2.1 & 2.8 & 5.2 & 0.5 & 0.7 \\
\hline & 10 & 25.9 & 21.2 & 24.7 & 25.6 & 4.8 & 3.5 \\
\hline \multirow[t]{2}{*}{653350} & 2 & 2.8 & 2.9 & 3.9 & 5.6 & 0.9 & 0.1 \\
\hline & 10 & 24.4 & 25.5 & 31.1 & 26.0 & 3.5 & 1.1 \\
\hline \multirow[t]{2}{*}{655280} & 2 & 8.2 & 12.9 & 9.9 & 5.6 & 1.9 & 0.3 \\
\hline & 10 & 37.4 & 50.5 & 46.6 & 29.4 & 8.0 & 1.2 \\
\hline
\end{tabular}


GCM - MIROC; Downscaling Method - Wilby et. al, 1998

SRES A1b (2046 - 2065)

DRWH Reliability (\%)

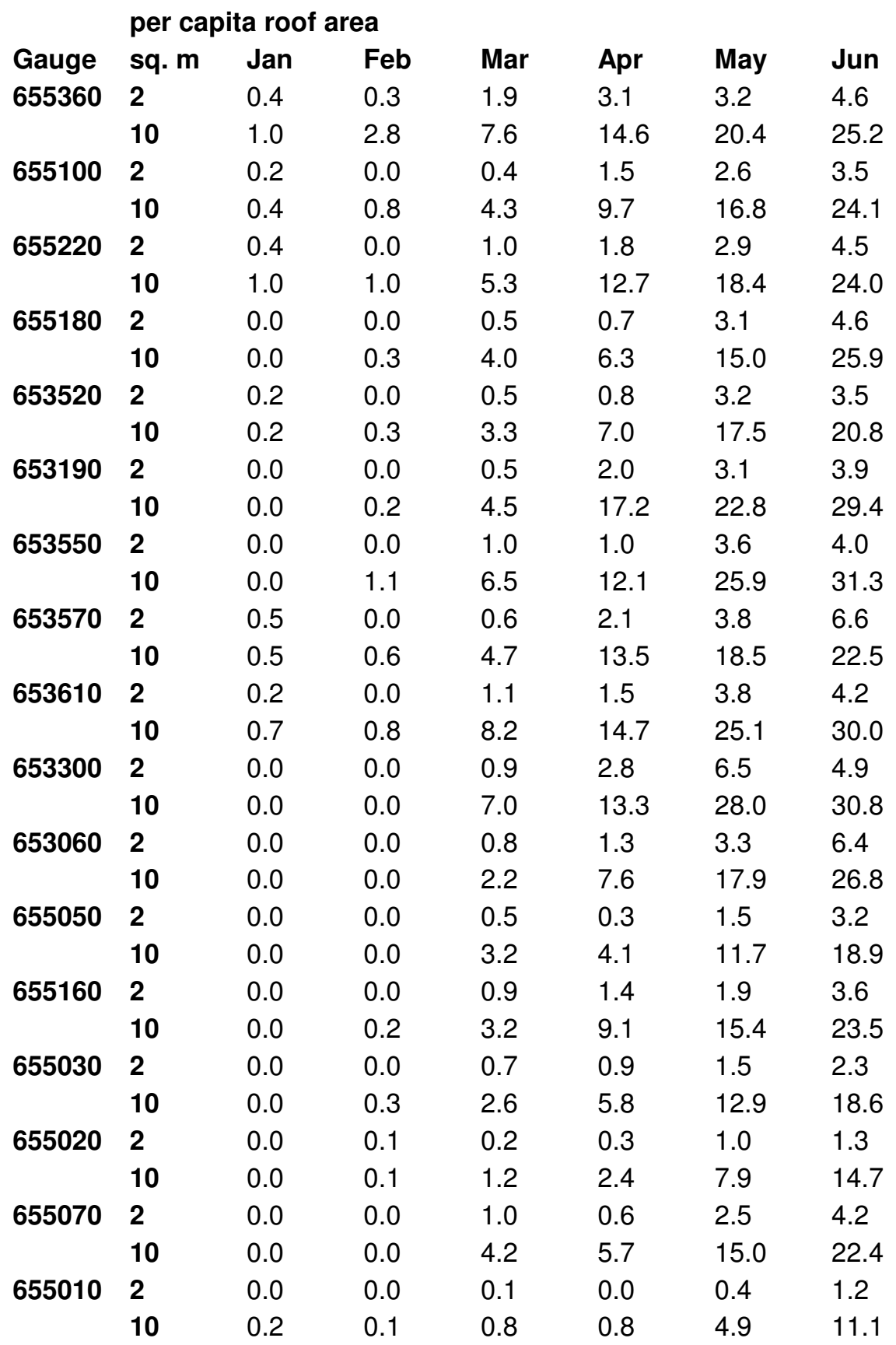


GCM - MIROC; Downscaling Method - Wilby et. al, 1998

SRES A1b (2046 - 2065)

DRWH Reliability (\%)

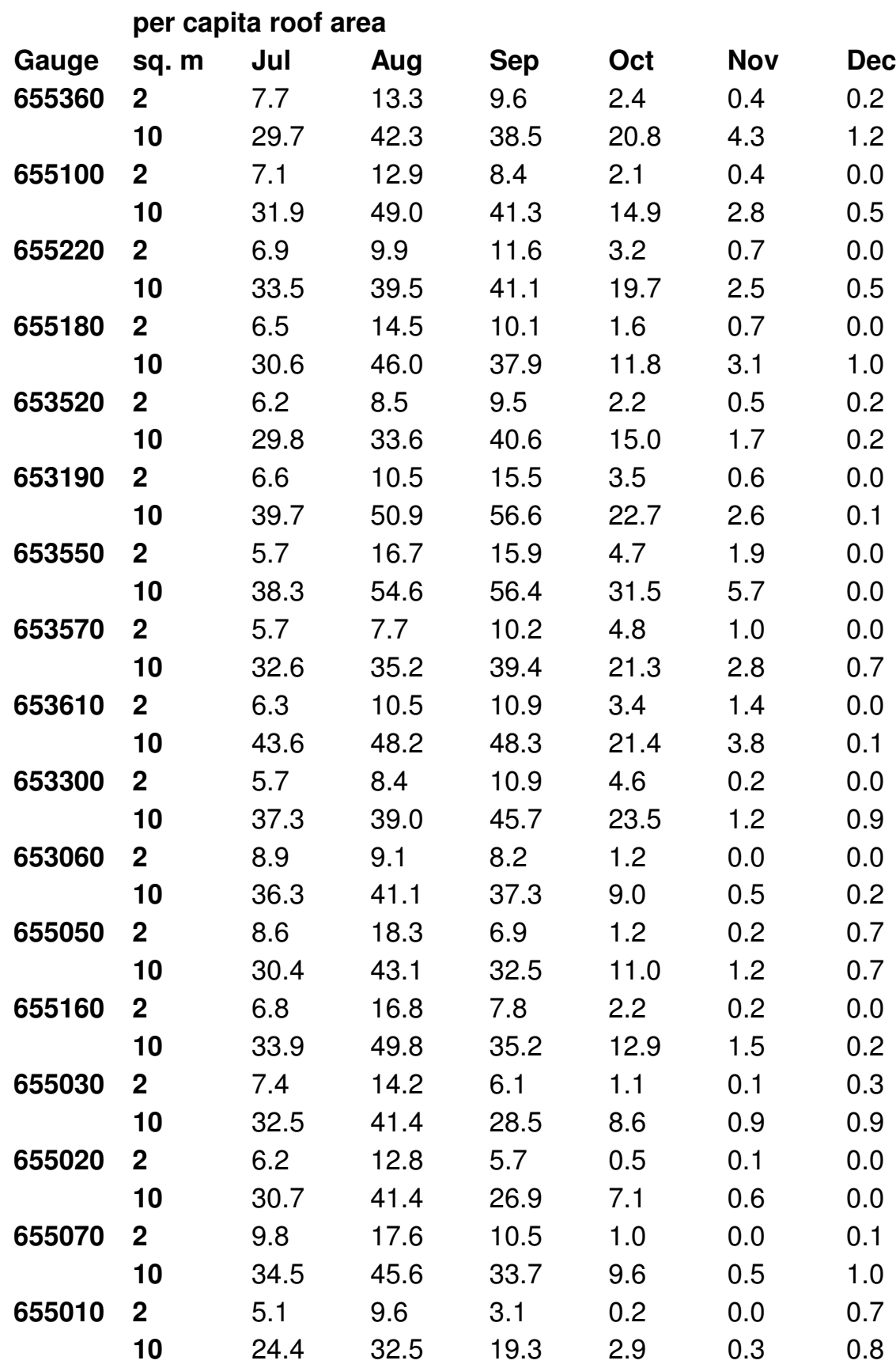


GCM - MIROC; Downscaling Method - Wilby et. al, 1998

SRES A2 (2046 - 2065)

DRWH Reliability (\%)

per capita roof area

\begin{tabular}{|c|c|c|c|c|c|c|c|}
\hline Gauge & sq. m & Jan & Feb & Mar & Apr & May & Jun \\
\hline \multirow[t]{2}{*}{655920} & 2 & 1.4 & 0.7 & 1.5 & 1.5 & 11.2 & 11.9 \\
\hline & 10 & 8.2 & 6.8 & 11.7 & 16.1 & 47.1 & 50.0 \\
\hline \multirow[t]{2}{*}{655940} & 2 & 0.8 & 0.3 & 1.3 & 2.5 & 5.8 & 8.6 \\
\hline & 10 & 2.9 & 3.1 & 7.6 & 15.0 & 33.1 & 46.0 \\
\hline \multirow[t]{2}{*}{655990} & 2 & 1.2 & 0.3 & 0.9 & 2.3 & 7.0 & 9.6 \\
\hline & 10 & 4.0 & 2.7 & 5.5 & 14.7 & 34.3 & 44.0 \\
\hline \multirow[t]{2}{*}{655780} & 2 & 1.1 & 1.2 & 1.6 & 2.5 & 5.1 & 13.1 \\
\hline & 10 & 4.8 & 7.7 & 14.5 & 19.3 & 35.7 & 49.8 \\
\hline \multirow[t]{2}{*}{655850} & 2 & 0.7 & 2.1 & 1.6 & 2.6 & 4.8 & 10.9 \\
\hline & 10 & 3.9 & 10.6 & 14.5 & 17.5 & 33.1 & 50.4 \\
\hline \multirow[t]{2}{*}{655480} & 2 & 0.1 & 0.4 & 3.1 & 3.3 & 3.5 & 6.7 \\
\hline & 10 & 1.4 & 5.9 & 16.3 & 20.6 & 24.2 & 34.2 \\
\hline \multirow[t]{2}{*}{655600} & 2 & 0.0 & 1.2 & 1.6 & 2.7 & 3.8 & 2.5 \\
\hline & 10 & 2.2 & 8.2 & 12.1 & 17.3 & 24.4 & 24.4 \\
\hline \multirow[t]{2}{*}{655570} & 2 & 0.0 & 1.5 & 3.7 & 4.2 & 6.7 & 5.4 \\
\hline & 10 & 4.2 & 10.8 & 18.9 & 23.1 & 34.9 & 37.2 \\
\hline \multirow[t]{2}{*}{655630} & 2 & 0.0 & 0.1 & 1.9 & 2.7 & 3.5 & 4.7 \\
\hline & 10 & 2.2 & 4.3 & 13.5 & 17.5 & 24.2 & 28.7 \\
\hline \multirow[t]{2}{*}{655620} & 2 & 0.0 & 0.5 & 2.1 & 2.4 & 4.6 & 3.1 \\
\hline & 10 & 2.2 & 5.7 & 14.9 & 16.3 & 27.0 & 26.6 \\
\hline \multirow[t]{2}{*}{655550} & 2 & 0.0 & 0.4 & 2.2 & 3.8 & 2.8 & 3.7 \\
\hline & 10 & 2.7 & 3.9 & 11.8 & 18.0 & 19.0 & 22.7 \\
\hline \multirow[t]{2}{*}{655450} & 2 & 0.0 & 0.3 & 1.4 & 3.5 & 5.9 & 6.3 \\
\hline & 10 & 0.7 & 4.3 & 8.5 & 17.6 & 26.2 & 29.9 \\
\hline \multirow[t]{2}{*}{654720} & 2 & 0.0 & 0.0 & 1.5 & 4.5 & 4.7 & 2.9 \\
\hline & 10 & 0.8 & 2.2 & 6.1 & 12.7 & 15.4 & 21.7 \\
\hline \multirow[t]{2}{*}{653870} & 2 & 0.0 & 0.1 & 1.7 & 2.1 & 3.0 & 5.0 \\
\hline & 10 & 0.1 & 3.0 & 9.8 & 13.9 & 23.2 & 27.7 \\
\hline \multirow[t]{2}{*}{653760} & 2 & 0.0 & 0.0 & 1.4 & 3.0 & 4.4 & 6.7 \\
\hline & 10 & 0.0 & 2.8 & 9.2 & 17.2 & 21.3 & 30.9 \\
\hline \multirow[t]{2}{*}{653800} & 2 & 0.0 & 0.0 & 3.1 & 3.3 & 3.6 & 3.6 \\
\hline & 10 & 0.6 & 2.3 & 13.2 & 17.1 & 21.8 & 25.2 \\
\hline \multirow[t]{2}{*}{653440} & 2 & 0.0 & 0.0 & 1.9 & 5.3 & 6.6 & 10.1 \\
\hline & 10 & 1.5 & 3.0 & 10.8 & 20.0 & 29.8 & 41.9 \\
\hline \multirow[t]{2}{*}{653380} & 2 & 0.0 & 0.0 & 1.7 & 3.3 & 4.8 & 5.0 \\
\hline & 10 & 0.2 & 2.4 & 12.2 & 20.2 & 25.6 & 30.3 \\
\hline \multirow[t]{2}{*}{653350} & 2 & 0.0 & 0.0 & 2.6 & 3.3 & 2.8 & 4.7 \\
\hline & 10 & 0.4 & 2.9 & 11.9 & 19.1 & 20.5 & 31.0 \\
\hline \multirow[t]{2}{*}{655280} & 2 & 0.0 & 0.7 & 0.6 & 1.8 & 2.0 & 3.3 \\
\hline & 10 & 1.4 & 1.5 & 4.7 & 15.0 & 16.6 & 24.1 \\
\hline
\end{tabular}


GCM - MIROC; Downscaling Method - Wilby et. al, 1998

SRES A2 (2046 - 2065)

DRWH Reliability (\%)

\begin{tabular}{|c|c|c|c|c|c|c|c|}
\hline \multirow{2}{*}{ Gauge } & \multicolumn{7}{|c|}{ per capita roof area } \\
\hline & sq. $m$ & Jul & Aug & Sep & Oct & Nov & Dec \\
\hline \multirow[t]{2}{*}{655920} & 2 & 3.0 & 2.1 & 3.8 & 3.4 & 4.1 & 3.6 \\
\hline & 10 & 24.6 & 29.8 & 36.9 & 31.0 & 31.8 & 21.1 \\
\hline \multirow[t]{2}{*}{655940} & 2 & 2.5 & 0.8 & 0.8 & 1.2 & 4.7 & 2.3 \\
\hline & 10 & 18.5 & 13.1 & 14.2 & 17.5 & 30.4 & 15.6 \\
\hline \multirow[t]{2}{*}{655990} & 2 & 2.0 & 0.5 & 0.6 & 1.7 & 3.2 & 2.6 \\
\hline & 10 & 18.2 & 9.1 & 9.6 & 17.1 & 21.3 & 12.6 \\
\hline \multirow[t]{2}{*}{655780} & 2 & 1.8 & 0.5 & 0.9 & 3.0 & 4.8 & 2.7 \\
\hline & 10 & 17.8 & 9.0 & 13.6 & 25.6 & 28.9 & 17.5 \\
\hline \multirow[t]{2}{*}{655850} & 2 & 1.9 & 1.0 & 1.2 & 3.5 & 3.2 & 1.7 \\
\hline & 10 & 22.0 & 14.3 & 20.2 & 32.4 & 27.7 & 14.5 \\
\hline \multirow[t]{2}{*}{655480} & 2 & 3.5 & 5.1 & 8.7 & 5.2 & 1.3 & 0.5 \\
\hline & 10 & 31.4 & 42.6 & 47.1 & 29.1 & 9.0 & 4.6 \\
\hline \multirow[t]{2}{*}{655600} & 2 & 2.2 & 1.9 & 2.8 & 2.3 & 1.0 & 0.9 \\
\hline & 10 & 19.8 & 20.4 & 26.8 & 20.5 & 7.9 & 5.7 \\
\hline \multirow[t]{2}{*}{655570} & 2 & 1.5 & 1.2 & 3.0 & 5.1 & 4.3 & 0.5 \\
\hline & 10 & 15.2 & 15.9 & 25.0 & 32.6 & 22.7 & 7.0 \\
\hline \multirow[t]{2}{*}{655630} & 2 & 2.2 & 1.1 & 1.9 & 3.7 & 1.8 & 0.4 \\
\hline & 10 & 16.2 & 14.2 & 21.2 & 24.0 & 11.3 & 4.9 \\
\hline \multirow[t]{2}{*}{655620} & 2 & 1.7 & 0.7 & 2.2 & 3.8 & 1.5 & 1.0 \\
\hline & 10 & 16.5 & 11.8 & 22.0 & 26.2 & 9.5 & 5.1 \\
\hline \multirow[t]{2}{*}{655550} & 2 & 2.2 & 2.3 & 4.0 & 3.9 & 0.7 & 0.3 \\
\hline & 10 & 16.8 & 21.0 & 27.7 & 20.7 & 5.9 & 3.2 \\
\hline \multirow[t]{2}{*}{655450} & 2 & 2.1 & 1.7 & 3.7 & 3.8 & 1.2 & 0.3 \\
\hline & 10 & 15.9 & 15.8 & 30.7 & 25.7 & 7.8 & 2.7 \\
\hline \multirow[t]{2}{*}{654720} & 2 & 0.8 & 0.2 & 0.4 & 1.9 & 1.0 & 0.9 \\
\hline & 10 & 10.0 & 4.6 & 7.0 & 14.0 & 6.0 & 4.0 \\
\hline \multirow[t]{2}{*}{653870} & 2 & 1.3 & 0.5 & 0.9 & 2.0 & 0.8 & 0.3 \\
\hline & 10 & 12.4 & 5.8 & 12.6 & 16.1 & 5.9 & 3.0 \\
\hline \multirow[t]{2}{*}{653760} & 2 & 4.9 & 3.6 & 4.5 & 4.6 & 0.4 & 0.2 \\
\hline & 10 & 33.5 & 28.4 & 28.9 & 22.6 & 3.6 & 1.9 \\
\hline \multirow[t]{2}{*}{653800} & 2 & 1.1 & 0.4 & 1.8 & 5.2 & 1.1 & 1.0 \\
\hline & 10 & 15.0 & 8.5 & 19.2 & 29.9 & 9.0 & 3.5 \\
\hline \multirow[t]{2}{*}{653440} & 2 & 1.4 & 0.7 & 1.8 & 6.2 & 1.7 & 0.4 \\
\hline & 10 & 16.9 & 10.2 & 20.3 & 29.3 & 9.6 & 3.4 \\
\hline \multirow[t]{2}{*}{653380} & 2 & 3.0 & 2.0 & 2.2 & 4.9 & 0.4 & 1.3 \\
\hline & 10 & 24.8 & 21.1 & 21.5 & 25.1 & 4.6 & 3.6 \\
\hline \multirow[t]{2}{*}{653350} & 2 & 2.8 & 2.4 & 3.1 & 5.5 & 0.9 & 0.1 \\
\hline & 10 & 23.4 & 25.1 & 27.8 & 26.1 & 3.4 & 1.0 \\
\hline \multirow[t]{2}{*}{655280} & 2 & 7.5 & 11.5 & 9.8 & 6.2 & 1.5 & 0.1 \\
\hline & 10 & 37.6 & 49.1 & 47.1 & 30.5 & 7.6 & 1.0 \\
\hline
\end{tabular}


GCM - MIROC; Downscaling Method - Wilby et. al, 1998

SRES A2 (2046 - 2065)

DRWH Reliability (\%)

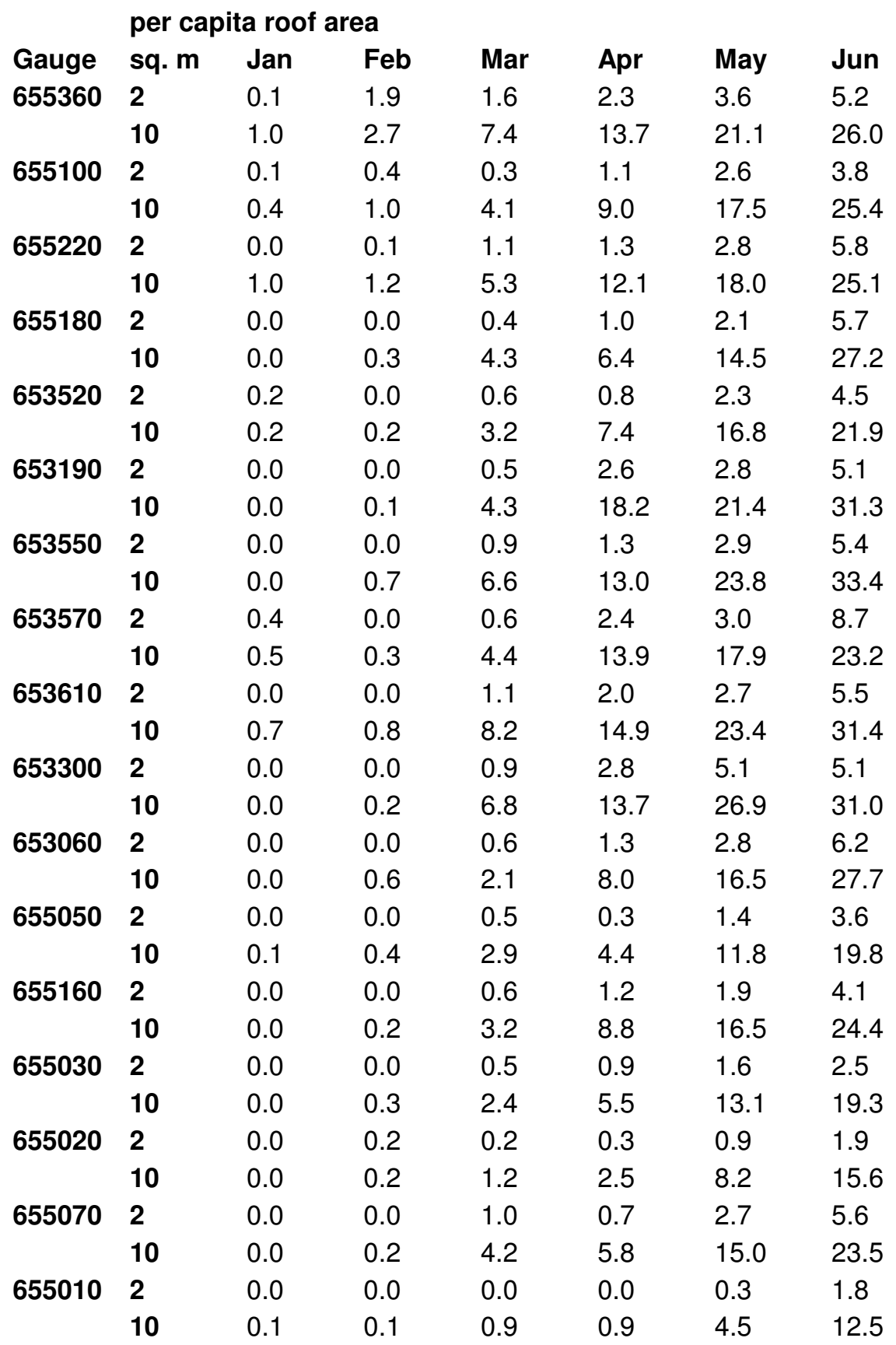


GCM - MIROC; Downscaling Method - Wilby et. al, 1998

SRES A2 (2046 - 2065)

DRWH Reliability (\%)

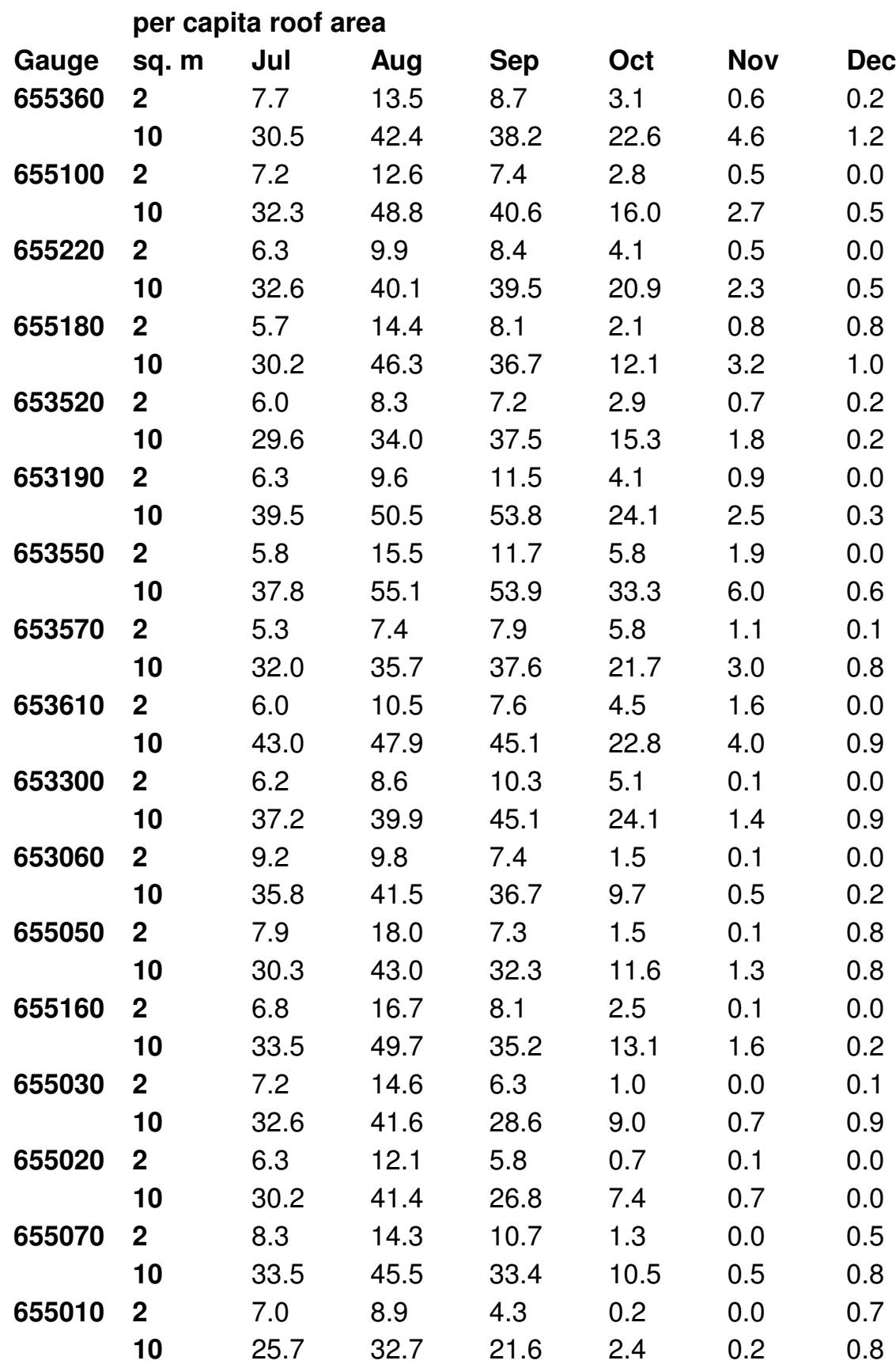


GCM - MIROC; Downscaling Method - Wilby et. al, 1998

SRES B1 (2046 - 2065)

DRWH Reliability (\%)

\section{per capita roof area}

\begin{tabular}{|c|c|c|c|c|c|c|c|}
\hline Gauge & sq. m & Jan & Feb & Mar & Apr & May & Jun \\
\hline \multirow[t]{2}{*}{655920} & 2 & 1.2 & 0.6 & 1.4 & 1.9 & 13.5 & 14.4 \\
\hline & 10 & 8.2 & 6.5 & 10.7 & 16.9 & 48.7 & 50.0 \\
\hline \multirow[t]{2}{*}{655940} & 2 & 0.8 & 0.2 & 1.1 & 2.9 & 5.9 & 8.9 \\
\hline & 10 & 3.1 & 2.9 & 7.6 & 15.4 & 33.6 & 45.2 \\
\hline \multirow[t]{2}{*}{655990} & 2 & 1.4 & 0.2 & 0.8 & 3.1 & 7.8 & 8.5 \\
\hline & 10 & 4.1 & 2.4 & 5.5 & 15.2 & 34.9 & 43.1 \\
\hline \multirow[t]{2}{*}{655780} & 2 & 1.4 & 1.0 & 1.9 & 2.8 & 5.5 & 11.7 \\
\hline & 10 & 5.0 & 7.5 & 14.7 & 19.6 & 36.8 & 48.7 \\
\hline \multirow[t]{2}{*}{655850} & 2 & 0.9 & 1.9 & 1.8 & 1.9 & 4.7 & 9.7 \\
\hline & 10 & 4.0 & 11.0 & 14.4 & 17.7 & 34.5 & 49.4 \\
\hline \multirow[t]{2}{*}{655480} & 2 & 0.4 & 0.9 & 2.7 & 4.1 & 4.5 & 6.3 \\
\hline & 10 & 1.5 & 6.0 & 16.4 & 22.2 & 26.2 & 32.8 \\
\hline \multirow[t]{2}{*}{655600} & 2 & 0.4 & 2.7 & 1.5 & 3.3 & 5.3 & 2.9 \\
\hline & 10 & 2.4 & 8.3 & 12.4 & 18.6 & 26.7 & 24.8 \\
\hline \multirow[t]{2}{*}{655570} & 2 & 0.6 & 3.6 & 3.8 & 5.4 & 9.3 & 5.8 \\
\hline & 10 & 4.5 & 11.0 & 19.5 & 24.3 & 38.1 & 36.8 \\
\hline \multirow[t]{2}{*}{655630} & 2 & 0.5 & 0.6 & 1.8 & 3.1 & 5.4 & 4.9 \\
\hline & 10 & 2.2 & 4.5 & 13.7 & 18.9 & 26.9 & 28.6 \\
\hline \multirow[t]{2}{*}{655620} & 2 & 0.2 & 1.5 & 2.1 & 3.5 & 6.7 & 3.6 \\
\hline & 10 & 2.5 & 5.8 & 15.1 & 17.4 & 29.5 & 27.0 \\
\hline \multirow[t]{2}{*}{655550} & 2 & 0.8 & 1.0 & 2.4 & 4.8 & 4.5 & 3.6 \\
\hline & 10 & 2.7 & 4.0 & 12.2 & 18.7 & 20.8 & 22.5 \\
\hline \multirow[t]{2}{*}{655450} & 2 & 0.0 & 0.0 & 1.1 & 4.1 & 6.1 & 6.2 \\
\hline & 10 & 1.2 & 4.0 & 8.3 & 17.8 & 27.1 & 30.0 \\
\hline \multirow[t]{2}{*}{654720} & 2 & 0.0 & 0.0 & 1.9 & 4.4 & 6.4 & 2.8 \\
\hline & 10 & 1.1 & 2.3 & 6.6 & 12.7 & 15.8 & 21.1 \\
\hline \multirow[t]{2}{*}{653870} & 2 & 0.0 & 0.1 & 2.2 & 1.9 & 4.4 & 4.5 \\
\hline & 10 & 0.1 & 3.3 & 10.2 & 14.2 & 24.9 & 27.1 \\
\hline \multirow[t]{2}{*}{653760} & 2 & 0.0 & 0.0 & 2.1 & 3.2 & 5.4 & 6.4 \\
\hline & 10 & 0.3 & 3.2 & 10.2 & 16.9 & 22.3 & 30.5 \\
\hline \multirow[t]{2}{*}{653800} & 2 & 0.1 & 0.5 & 5.0 & 3.3 & 4.9 & 3.6 \\
\hline & 10 & 0.7 & 3.7 & 14.1 & 17.0 & 22.3 & 25.1 \\
\hline \multirow[t]{2}{*}{653440} & 2 & 0.1 & 0.9 & 2.8 & 4.4 & 7.8 & 10.4 \\
\hline & 10 & 2.2 & 4.3 & 12.4 & 19.7 & 30.6 & 42.3 \\
\hline \multirow[t]{2}{*}{653380} & 2 & 0.0 & 0.5 & 2.8 & 3.3 & 6.1 & 5.1 \\
\hline & 10 & 0.5 & 3.7 & 13.8 & 19.8 & 26.7 & 30.5 \\
\hline \multirow[t]{2}{*}{653350} & 2 & 0.0 & 0.8 & 3.4 & 3.1 & 3.4 & 4.9 \\
\hline & 10 & 0.7 & 3.8 & 13.4 & 18.8 & 22.1 & 31.1 \\
\hline \multirow[t]{2}{*}{655280} & 2 & 0.0 & 0.7 & 0.7 & 2.2 & 2.5 & 3.7 \\
\hline & 10 & 0.8 & 1.4 & 4.8 & 16.4 & 17.6 & 24.5 \\
\hline
\end{tabular}


GCM - MIROC; Downscaling Method - Wilby et. al, 1998

SRES B1 (2046 - 2065)

DRWH Reliability (\%)

\begin{tabular}{|c|c|c|c|c|c|c|c|}
\hline \multirow{2}{*}{ Gauge } & \multicolumn{7}{|c|}{ per capita roof area } \\
\hline & sq. $m$ & Jul & Aug & Sep & Oct & Nov & Dec \\
\hline \multirow[t]{2}{*}{655920} & 2 & 2.3 & 2.8 & 3.6 & 2.8 & 3.8 & 3.2 \\
\hline & 10 & 23.2 & 33.1 & 37.3 & 29.9 & 30.1 & 20.1 \\
\hline \multirow[t]{2}{*}{655940} & 2 & 1.9 & 1.0 & 0.9 & 1.7 & 3.8 & 1.8 \\
\hline & 10 & 18.2 & 14.5 & 13.7 & 19.0 & 28.4 & 14.2 \\
\hline \multirow[t]{2}{*}{655990} & 2 & 1.8 & 0.7 & 0.5 & 2.3 & 2.7 & 2.0 \\
\hline & 10 & 16.9 & 10.3 & 9.1 & 18.7 & 19.7 & 11.4 \\
\hline \multirow[t]{2}{*}{655780} & 2 & 1.6 & 0.7 & 0.9 & 3.7 & 4.4 & 2.3 \\
\hline & 10 & 17.6 & 9.8 & 13.1 & 27.6 & 28.6 & 16.5 \\
\hline \multirow[t]{2}{*}{655850} & 2 & 1.8 & 1.2 & 1.5 & 4.7 & 3.2 & 1.7 \\
\hline & 10 & 21.7 & 16.0 & 20.2 & 34.2 & 26.9 & 13.3 \\
\hline \multirow[t]{2}{*}{655480} & 2 & 3.3 & 6.5 & 7.7 & 5.7 & 1.2 & 0.8 \\
\hline & 10 & 31.7 & 45.9 & 46.0 & 28.9 & 8.5 & 4.5 \\
\hline \multirow[t]{2}{*}{655600} & 2 & 1.9 & 2.0 & 2.9 & 2.5 & 0.8 & 1.1 \\
\hline & 10 & 19.5 & 22.4 & 26.7 & 19.7 & 7.5 & 5.8 \\
\hline \multirow[t]{2}{*}{655570} & 2 & 1.5 & 1.4 & 2.8 & 4.6 & 3.4 & 0.7 \\
\hline & 10 & 15.5 & 16.8 & 24.1 & 31.5 & 21.8 & 7.3 \\
\hline \multirow[t]{2}{*}{655630} & 2 & 2.1 & 1.3 & 2.0 & 2.9 & 1.7 & 0.5 \\
\hline & 10 & 15.9 & 15.8 & 20.6 & 23.5 & 10.6 & 5.1 \\
\hline \multirow[t]{2}{*}{655620} & 2 & 1.4 & 0.7 & 2.2 & 3.4 & 1.5 & 0.9 \\
\hline & 10 & 16.4 & 12.2 & 21.0 & 25.4 & 9.2 & 5.0 \\
\hline \multirow[t]{2}{*}{655550} & 2 & 2.2 & 2.8 & 3.5 & 3.3 & 0.6 & 0.5 \\
\hline & 10 & 16.7 & 22.8 & 26.5 & 20.7 & 5.5 & 3.4 \\
\hline \multirow[t]{2}{*}{655450} & 2 & 1.9 & 1.8 & 5.0 & 3.9 & 1.1 & 0.3 \\
\hline & 10 & 16.3 & 17.3 & 31.5 & 25.2 & 7.3 & 2.7 \\
\hline \multirow[t]{2}{*}{654720} & 2 & 1.1 & 0.3 & 0.7 & 1.8 & 1.1 & 0.9 \\
\hline & 10 & 9.8 & 5.6 & 8.4 & 14.1 & 5.8 & 3.6 \\
\hline \multirow[t]{2}{*}{653870} & 2 & 1.1 & 0.5 & 1.4 & 2.1 & 0.8 & 0.4 \\
\hline & 10 & 12.3 & 6.6 & 14.8 & 16.3 & 5.8 & 2.8 \\
\hline \multirow[t]{2}{*}{653760} & 2 & 4.7 & 4.5 & 6.3 & 4.8 & 0.4 & 0.3 \\
\hline & 10 & 32.9 & 30.7 & 31.7 & 22.8 & 3.6 & 1.8 \\
\hline \multirow[t]{2}{*}{653800} & 2 & 1.2 & 0.5 & 2.0 & 5.4 & 1.2 & 1.0 \\
\hline & 10 & 16.0 & 8.5 & 20.8 & 30.6 & 9.2 & 3.4 \\
\hline \multirow[t]{2}{*}{653440} & 2 & 1.9 & 0.9 & 2.5 & 6.0 & 1.9 & 0.3 \\
\hline & 10 & 18.1 & 10.9 & 22.2 & 29.1 & 9.7 & 3.1 \\
\hline \multirow[t]{2}{*}{653380} & 2 & 3.2 & 1.9 & 2.9 & 5.4 & 0.5 & 1.0 \\
\hline & 10 & 25.5 & 21.3 & 24.5 & 25.2 & 5.1 & 3.5 \\
\hline \multirow[t]{2}{*}{653350} & 2 & 2.8 & 2.6 & 4.6 & 5.7 & 1.0 & 0.1 \\
\hline & 10 & 24.3 & 25.7 & 31.4 & 25.9 & 3.6 & 1.1 \\
\hline \multirow[t]{2}{*}{655280} & 2 & 8.5 & 13.3 & 9.4 & 6.4 & 1.3 & 0.4 \\
\hline & 10 & 38.1 & 51.5 & 46.8 & 30.7 & 7.2 & 1.2 \\
\hline
\end{tabular}


GCM - MIROC; Downscaling Method - Wilby et. al, 1998

SRES B1 (2046 - 2065)

DRWH Reliability (\%)

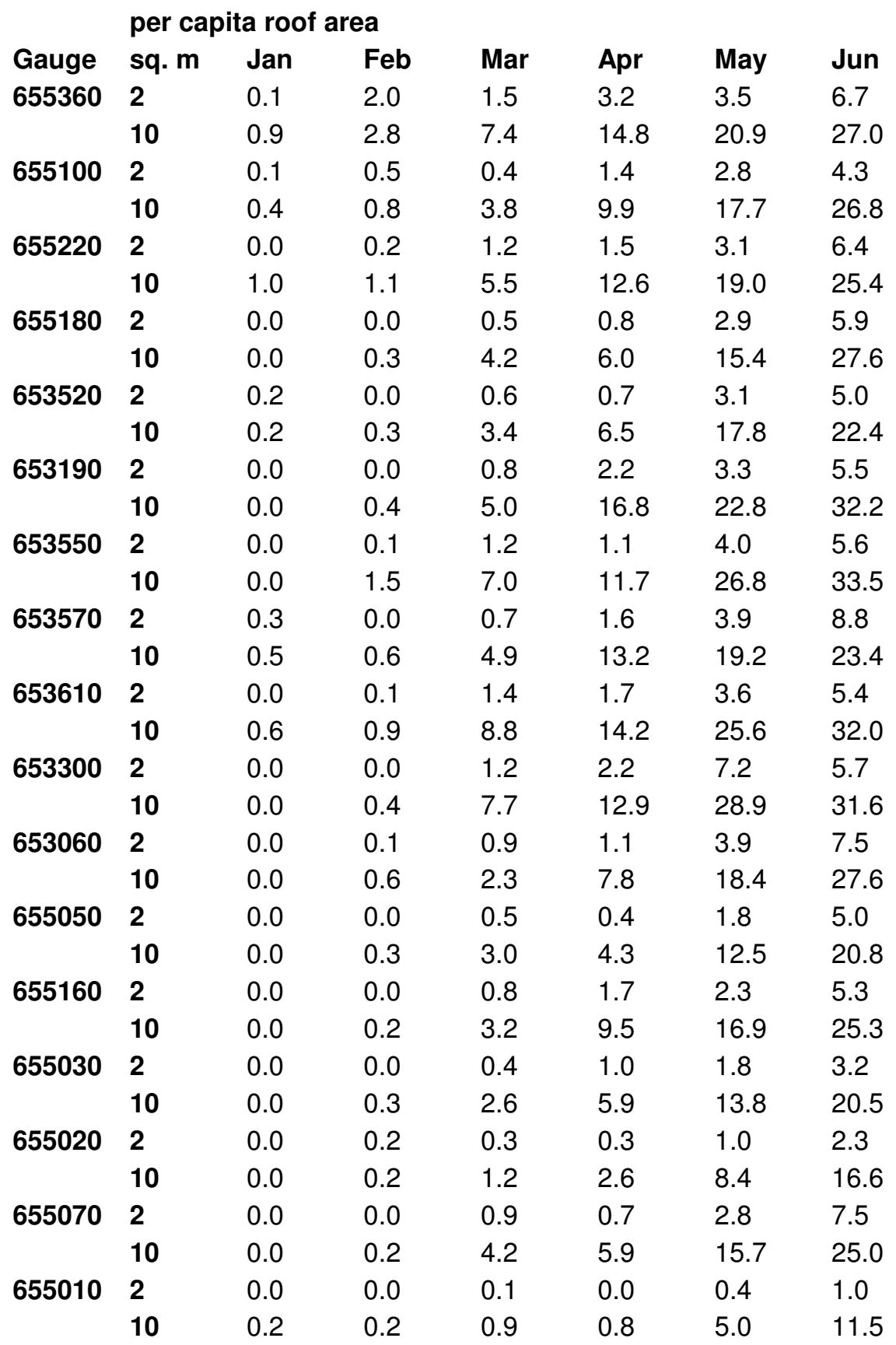


GCM - MIROC; Downscaling Method - Wilby et. al, 1998

SRES B1 (2046 - 2065)

DRWH Reliability (\%)

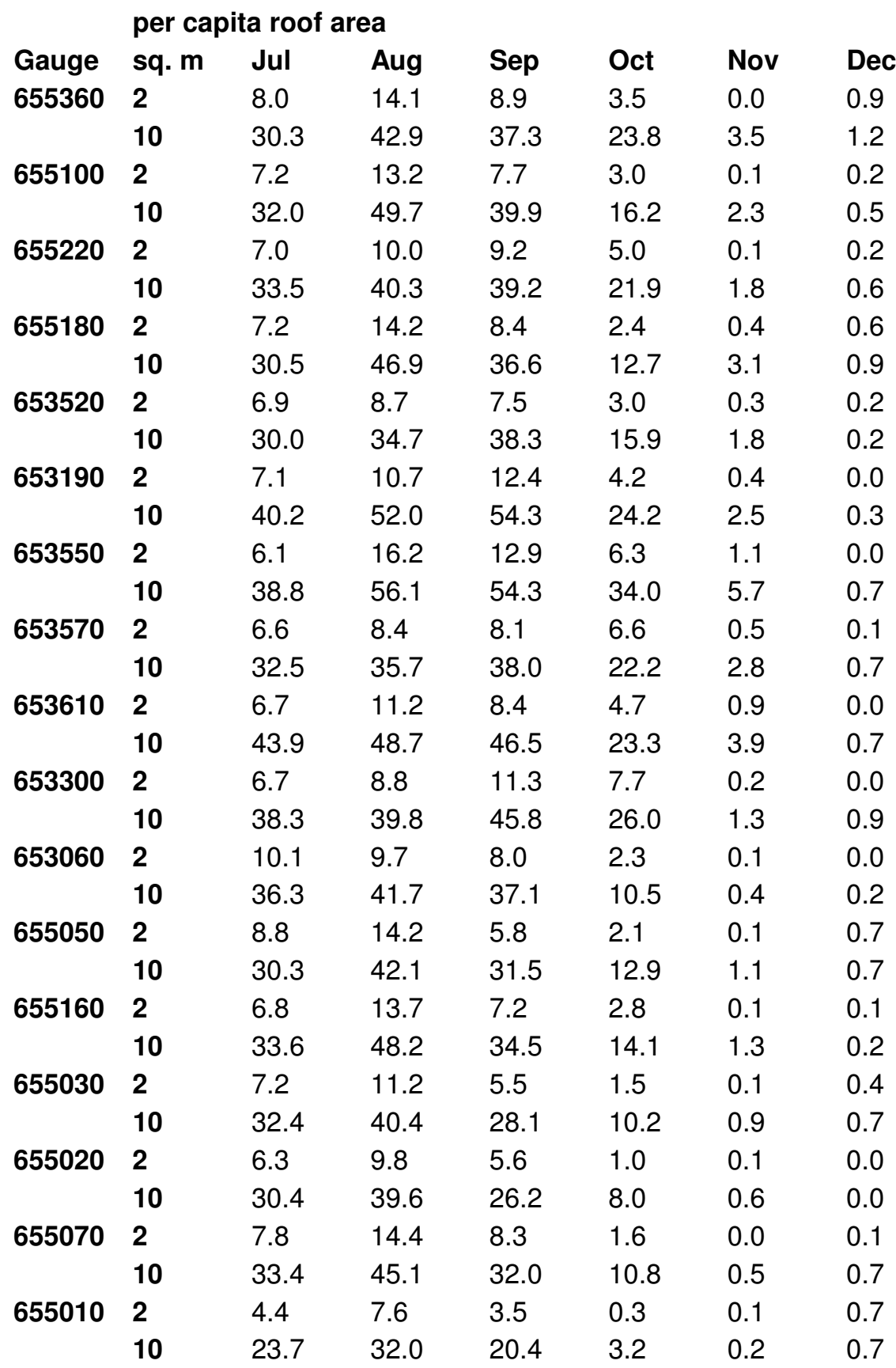


GCM - MRI; Downscaling Method - Wilby et. al, 1998

SRES A1b (2046 - 2065)

DRWH Reliability (\%)

per capita roof area

\begin{tabular}{|c|c|c|c|c|c|c|c|}
\hline Gauge & sq. m & Jan & Feb & Mar & Apr & May & Jun \\
\hline \multirow[t]{2}{*}{655920} & 2 & 0.4 & 0.8 & 0.9 & 3.4 & 9.2 & 13.0 \\
\hline & 10 & 6.1 & 7.7 & 10.8 & 20.0 & 47.8 & 50.0 \\
\hline \multirow[t]{2}{*}{655940} & 2 & 0.1 & 0.3 & 0.5 & 4.7 & 6.2 & 8.7 \\
\hline & 10 & 2.1 & 3.2 & 6.9 & 17.0 & 34.9 & 45.2 \\
\hline \multirow[t]{2}{*}{655990} & 2 & 0.2 & 0.3 & 0.2 & 4.7 & 8.1 & 8.8 \\
\hline & 10 & 3.3 & 2.7 & 4.7 & 16.4 & 35.9 & 43.0 \\
\hline \multirow[t]{2}{*}{655780} & 2 & 0.4 & 0.8 & 1.1 & 3.8 & 5.0 & 13.4 \\
\hline & 10 & 3.8 & 7.4 & 13.6 & 21.9 & 38.8 & 48.9 \\
\hline \multirow[t]{2}{*}{655850} & 2 & 0.1 & 1.5 & 0.8 & 2.8 & 4.8 & 11.8 \\
\hline & 10 & 2.7 & 10.6 & 13.2 & 19.4 & 36.3 & 50.4 \\
\hline \multirow[t]{2}{*}{655480} & 2 & 0.0 & 0.5 & 2.4 & 5.9 & 4.6 & 7.0 \\
\hline & 10 & 1.1 & 6.4 & 17.1 & 23.5 & 25.8 & 34.2 \\
\hline \multirow[t]{2}{*}{655600} & 2 & 0.1 & 2.2 & 1.2 & 4.2 & 4.5 & 3.3 \\
\hline & 10 & 2.2 & 8.7 & 13.8 & 19.8 & 24.9 & 26.2 \\
\hline \multirow[t]{2}{*}{655570} & 2 & 0.2 & 3.1 & 3.5 & 6.8 & 7.8 & 6.8 \\
\hline & 10 & 4.1 & 11.4 & 21.1 & 26.2 & 36.4 & 38.6 \\
\hline \multirow[t]{2}{*}{655630} & 2 & 0.3 & 0.5 & 1.4 & 3.7 & 4.7 & 5.6 \\
\hline & 10 & 2.2 & 4.8 & 14.9 & 20.1 & 25.6 & 29.6 \\
\hline \multirow[t]{2}{*}{655620} & 2 & 0.1 & 1.2 & 1.3 & 4.0 & 5.7 & 4.0 \\
\hline & 10 & 2.4 & 6.3 & 16.0 & 18.6 & 27.9 & 28.4 \\
\hline \multirow[t]{2}{*}{655550} & 2 & 0.3 & 0.9 & 1.8 & 5.8 & 3.7 & 3.8 \\
\hline & 10 & 2.5 & 4.1 & 12.7 & 19.6 & 19.6 & 23.3 \\
\hline \multirow[t]{2}{*}{655450} & 2 & 0.2 & 0.8 & 1.0 & 5.3 & 6.2 & 7.1 \\
\hline & 10 & 1.2 & 4.6 & 8.4 & 19.1 & 26.6 & 30.5 \\
\hline \multirow[t]{2}{*}{654720} & 2 & 0.4 & 0.2 & 1.3 & 5.0 & 5.2 & 3.1 \\
\hline & 10 & 1.3 & 2.7 & 6.5 & 13.2 & 15.6 & 21.1 \\
\hline \multirow[t]{2}{*}{653870} & 2 & 0.0 & 0.5 & 2.0 & 2.1 & 3.0 & 4.5 \\
\hline & 10 & 0.7 & 3.7 & 10.2 & 15.2 & 24.9 & 26.4 \\
\hline \multirow[t]{2}{*}{653760} & 2 & 0.0 & 0.3 & 1.5 & 3.7 & 4.5 & 6.2 \\
\hline & 10 & 1.1 & 3.9 & 9.8 & 17.9 & 21.9 & 30.1 \\
\hline \multirow[t]{2}{*}{653800} & 2 & 0.2 & 0.6 & 3.9 & 4.4 & 4.9 & 3.4 \\
\hline & 10 & 0.7 & 4.5 & 14.1 & 18.2 & 21.8 & 25.6 \\
\hline \multirow[t]{2}{*}{653440} & 2 & 0.3 & 1.3 & 2.3 & 7.1 & 8.1 & 10.1 \\
\hline & 10 & 2.1 & 5.0 & 11.9 & 20.6 & 30.4 & 43.1 \\
\hline \multirow[t]{2}{*}{653380} & 2 & 0.0 & 0.9 & 2.4 & 4.6 & 6.1 & 4.7 \\
\hline & 10 & 0.5 & 4.3 & 13.1 & 21.2 & 26.1 & 31.4 \\
\hline \multirow[t]{2}{*}{653350} & 2 & 0.1 & 1.4 & 3.0 & 4.4 & 3.4 & 4.7 \\
\hline & 10 & 0.8 & 4.2 & 13.3 & 20.1 & 21.6 & 32.1 \\
\hline \multirow[t]{2}{*}{655280} & 2 & 0.0 & 0.3 & 0.3 & 2.5 & 4.1 & 6.2 \\
\hline & 10 & 1.5 & 1.5 & 5.2 & 17.0 & 19.0 & 28.1 \\
\hline
\end{tabular}


GCM - MRI; Downscaling Method - Wilby et. al, 1998

SRES A1b (2046 - 2065)

DRWH Reliability (\%)

\begin{tabular}{|c|c|c|c|c|c|c|c|}
\hline \multirow{2}{*}{ Gauge } & \multicolumn{7}{|c|}{ per capita roof area } \\
\hline & sq. $m$ & Jul & Aug & Sep & Oct & Nov & Dec \\
\hline \multirow[t]{2}{*}{655920} & 2 & 3.0 & 7.9 & 9.0 & 4.8 & 2.7 & 2.1 \\
\hline & 10 & 27.6 & 39.9 & 44.3 & 36.8 & 28.3 & 17.8 \\
\hline \multirow[t]{2}{*}{655940} & 2 & 2.7 & 4.6 & 2.7 & 2.3 & 3.2 & 1.2 \\
\hline & 10 & 19.7 & 18.8 & 18.7 & 22.4 & 27.6 & 12.5 \\
\hline \multirow[t]{2}{*}{655990} & 2 & 2.4 & 3.0 & 1.9 & 2.9 & 2.2 & 1.2 \\
\hline & 10 & 19.1 & 14.3 & 12.8 & 21.9 & 19.0 & 10.6 \\
\hline \multirow[t]{2}{*}{655780} & 2 & 3.3 & 2.0 & 3.0 & 6.0 & 5.6 & 1.5 \\
\hline & 10 & 20.3 & 11.6 & 17.0 & 30.6 & 28.5 & 14.6 \\
\hline \multirow[t]{2}{*}{655850} & 2 & 3.1 & 3.3 & 4.3 & 7.5 & 4.3 & 1.0 \\
\hline & 10 & 24.8 & 19.1 & 24.9 & 37.6 & 27.3 & 11.4 \\
\hline \multirow[t]{2}{*}{655480} & 2 & 5.2 & 14.5 & 14.9 & 2.9 & 1.0 & 1.1 \\
\hline & 10 & 34.8 & 55.0 & 51.2 & 27.2 & 8.1 & 4.2 \\
\hline \multirow[t]{2}{*}{655600} & 2 & 2.6 & 6.3 & 6.0 & 1.1 & 0.5 & 1.1 \\
\hline & 10 & 21.9 & 29.1 & 32.2 & 19.8 & 6.5 & 5.4 \\
\hline \multirow[t]{2}{*}{655570} & 2 & 2.1 & 4.5 & 5.7 & 2.6 & 2.3 & 0.5 \\
\hline & 10 & 17.7 & 22.9 & 28.8 & 31.8 & 19.4 & 6.7 \\
\hline \multirow[t]{2}{*}{655630} & 2 & 2.8 & 4.4 & 4.2 & 1.8 & 0.8 & 0.5 \\
\hline & 10 & 18.5 & 20.9 & 25.4 & 24.4 & 9.5 & 4.4 \\
\hline \multirow[t]{2}{*}{655620} & 2 & 2.0 & 3.0 & 4.8 & 2.0 & 0.7 & 1.0 \\
\hline & 10 & 19.0 & 17.5 & 25.6 & 25.7 & 8.2 & 4.6 \\
\hline \multirow[t]{2}{*}{655550} & 2 & 3.1 & 7.8 & 7.9 & 2.0 & 0.4 & 0.5 \\
\hline & 10 & 19.1 & 28.9 & 31.0 & 20.8 & 5.2 & 2.9 \\
\hline \multirow[t]{2}{*}{655450} & 2 & 2.5 & 5.7 & 11.6 & 4.1 & 0.6 & 0.0 \\
\hline & 10 & 19.0 & 21.6 & 37.6 & 25.6 & 6.2 & 2.4 \\
\hline \multirow[t]{2}{*}{654720} & 2 & 1.5 & 1.2 & 1.2 & 2.1 & 0.7 & 0.9 \\
\hline & 10 & 11.7 & 8.3 & 9.6 & 13.5 & 5.3 & 3.9 \\
\hline \multirow[t]{2}{*}{653870} & 2 & 2.1 & 1.9 & 3.0 & 2.1 & 0.6 & 0.3 \\
\hline & 10 & 14.0 & 10.0 & 17.9 & 15.6 & 5.2 & 3.1 \\
\hline \multirow[t]{2}{*}{653760} & 2 & 7.1 & 14.7 & 11.3 & 4.6 & 0.3 & 0.3 \\
\hline & 10 & 36.9 & 37.4 & 35.1 & 21.7 & 3.2 & 1.9 \\
\hline \multirow[t]{2}{*}{653800} & 2 & 2.1 & 2.2 & 3.9 & 4.4 & 0.8 & 0.8 \\
\hline & 10 & 19.4 & 13.1 & 24.6 & 29.1 & 7.7 & 3.3 \\
\hline \multirow[t]{2}{*}{653440} & 2 & 2.8 & 3.4 & 4.4 & 4.7 & 1.2 & 0.2 \\
\hline & 10 & 20.5 & 15.8 & 26.4 & 27.8 & 8.4 & 3.1 \\
\hline \multirow[t]{2}{*}{653380} & 2 & 4.8 & 7.9 & 4.8 & 4.0 & 0.4 & 0.8 \\
\hline & 10 & 29.9 & 27.8 & 29.1 & 24.1 & 4.3 & 3.6 \\
\hline \multirow[t]{2}{*}{653350} & 2 & 4.4 & 10.4 & 7.5 & 4.4 & 0.7 & 0.1 \\
\hline & 10 & 28.1 & 32.9 & 35.2 & 25.0 & 3.1 & 1.2 \\
\hline \multirow[t]{2}{*}{655280} & 2 & 10.1 & 11.1 & 10.3 & 7.0 & 0.3 & 0.3 \\
\hline & 10 & 38.8 & 51.7 & 43.3 & 30.8 & 7.0 & 1.0 \\
\hline
\end{tabular}


GCM - MRI; Downscaling Method - Wilby et. al, 1998

SRES A1b (2046 - 2065)

DRWH Reliability (\%)

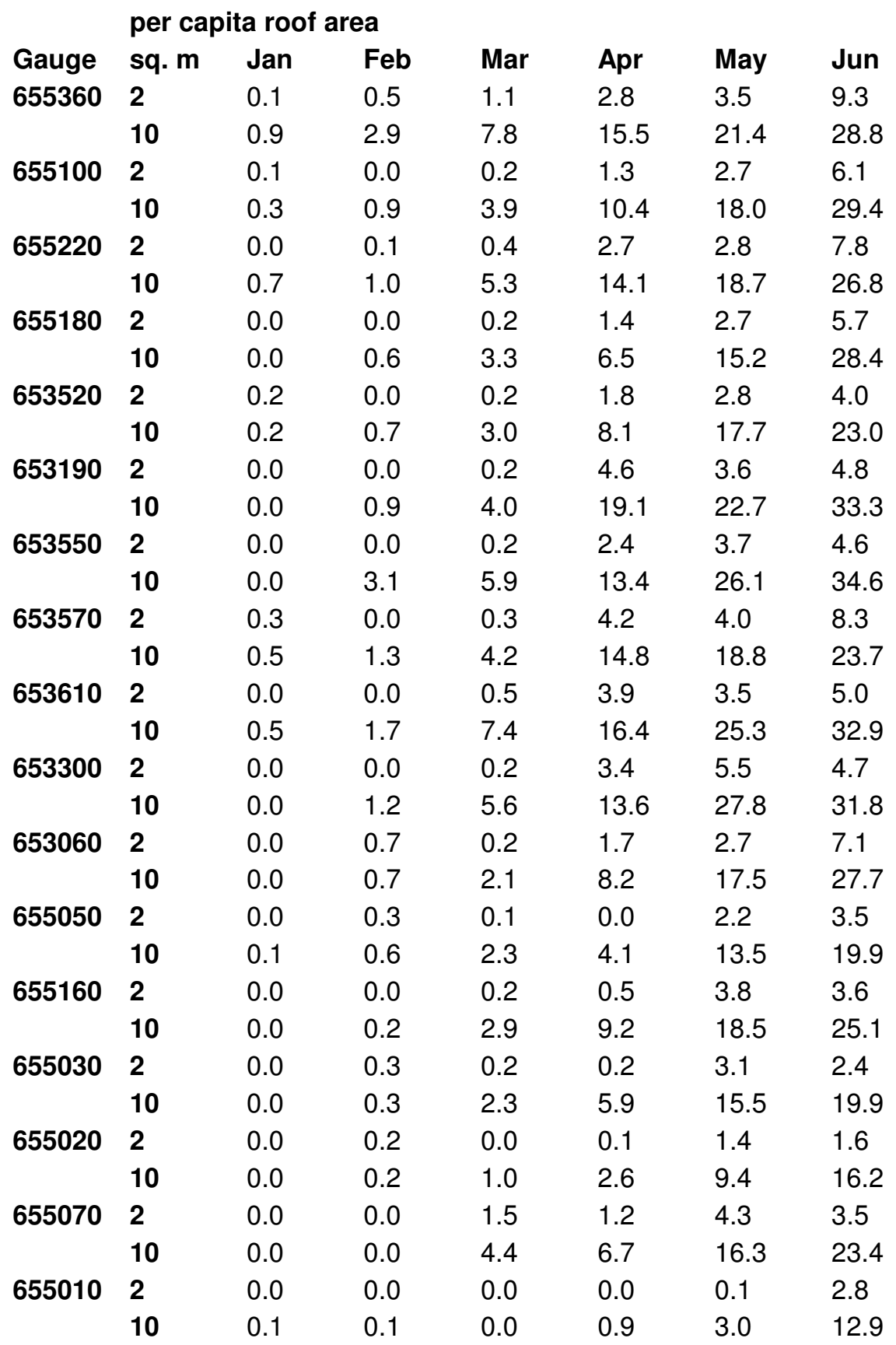


GCM - MRI; Downscaling Method - Wilby et. al, 1998

SRES A1b (2046 - 2065)

DRWH Reliability (\%)

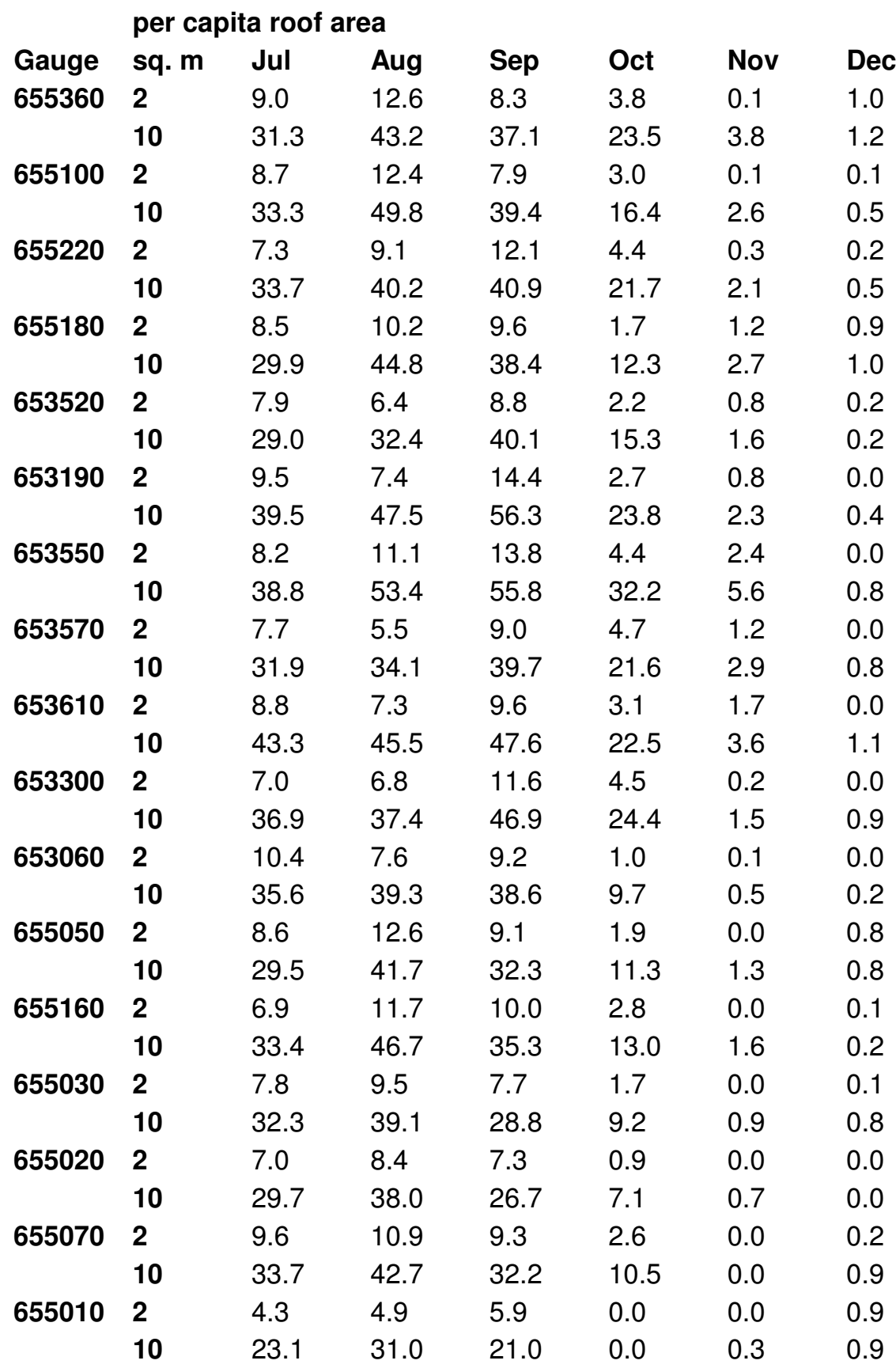


GCM - MRI; Downscaling Method - Wilby et. al, 1998

SRES A2 (2046 - 2065)

DRWH Reliability (\%)

\begin{tabular}{|c|c|c|c|c|c|c|c|}
\hline \multirow{2}{*}{ Gauge } & \multicolumn{7}{|c|}{ per capita roof area } \\
\hline & sq. $m$ & Jan & Feb & Mar & Apr & May & Jun \\
\hline \multirow[t]{2}{*}{655920} & 2 & 0.6 & 0.4 & 1.6 & 4.5 & 11.5 & 15.1 \\
\hline & 10 & 6.7 & 6.3 & 11.5 & 20.0 & 47.9 & 52.2 \\
\hline \multirow[t]{2}{*}{655940} & 2 & 0.1 & 0.2 & 1.0 & 4.0 & 7.6 & 8.7 \\
\hline & 10 & 2.2 & 2.7 & 7.7 & 16.9 & 35.7 & 47.7 \\
\hline \multirow[t]{2}{*}{655990} & 2 & 0.2 & 0.3 & 0.5 & 4.5 & 9.1 & 9.6 \\
\hline & 10 & 2.9 & 2.5 & 5.4 & 16.8 & 36.4 & 45.0 \\
\hline \multirow[t]{2}{*}{655780} & 2 & 0.3 & 0.5 & 2.1 & 3.5 & 6.7 & 14.4 \\
\hline & 10 & 3.5 & 6.7 & 14.8 & 22.3 & 39.2 & 49.8 \\
\hline \multirow[t]{2}{*}{655850} & 2 & 0.1 & 0.8 & 2.0 & 3.1 & 6.2 & 12.6 \\
\hline & 10 & 2.7 & 9.7 & 14.8 & 20.5 & 36.6 & 51.2 \\
\hline \multirow[t]{2}{*}{655480} & 2 & 0.1 & 0.8 & 4.5 & 6.8 & 3.8 & 8.3 \\
\hline & 10 & 1.3 & 6.5 & 18.3 & 24.2 & 26.5 & 34.9 \\
\hline \multirow[t]{2}{*}{655600} & 2 & 0.2 & 3.5 & 1.8 & 4.1 & 4.5 & 3.6 \\
\hline & 10 & 2.2 & 9.0 & 13.8 & 20.1 & 25.6 & 25.4 \\
\hline \multirow[t]{2}{*}{655570} & 2 & 0.8 & 4.1 & 4.6 & 6.8 & 7.4 & 6.8 \\
\hline & 10 & 4.2 & 12.0 & 20.9 & 26.5 & 35.8 & 38.2 \\
\hline \multirow[t]{2}{*}{655630} & 2 & 0.4 & 0.8 & 1.9 & 3.9 & 4.3 & 5.8 \\
\hline & 10 & 2.1 & 5.1 & 15.7 & 20.3 & 25.8 & 29.5 \\
\hline \multirow[t]{2}{*}{655620} & 2 & 0.2 & 1.7 & 2.3 & 4.1 & 5.8 & 4.0 \\
\hline & 10 & 2.4 & 6.5 & 16.9 & 19.2 & 28.2 & 27.9 \\
\hline \multirow[t]{2}{*}{655550} & 2 & 0.4 & 1.5 & 2.6 & 5.7 & 3.6 & 4.3 \\
\hline & 10 & 2.4 & 4.3 & 13.2 & 20.0 & 19.8 & 22.8 \\
\hline \multirow[t]{2}{*}{655450} & 2 & 0.4 & 0.7 & 1.7 & 4.7 & 6.0 & 7.1 \\
\hline & 10 & 1.2 & 4.8 & 8.8 & 18.6 & 26.3 & 31.0 \\
\hline \multirow[t]{2}{*}{654720} & 2 & 0.4 & 0.3 & 1.4 & 4.7 & 6.1 & 3.5 \\
\hline & 10 & 1.4 & 2.9 & 6.6 & 12.9 & 15.7 & 22.2 \\
\hline \multirow[t]{2}{*}{653870} & 2 & 0.0 & 0.5 & 2.0 & 2.2 & 4.2 & 5.7 \\
\hline & 10 & 0.6 & 3.7 & 10.5 & 14.5 & 24.9 & 27.9 \\
\hline \multirow[t]{2}{*}{653760} & 2 & 0.0 & 0.2 & 1.5 & 3.5 & 5.7 & 8.3 \\
\hline & 10 & 1.1 & 3.8 & 10.1 & 17.6 & 22.1 & 31.3 \\
\hline \multirow[t]{2}{*}{653800} & 2 & 0.1 & 0.8 & 3.5 & 4.4 & 4.3 & 4.0 \\
\hline & 10 & 0.7 & 4.6 & 14.2 & 18.1 & 23.0 & 25.9 \\
\hline \multirow[t]{2}{*}{653440} & 2 & 0.1 & 1.3 & 1.9 & 6.4 & 7.6 & 11.8 \\
\hline & 10 & 2.1 & 4.9 & 11.8 & 20.6 & 31.3 & 43.3 \\
\hline \multirow[t]{2}{*}{653380} & 2 & 0.0 & 0.8 & 2.0 & 4.3 & 5.6 & 5.3 \\
\hline & 10 & 0.4 & 4.4 & 13.6 & 21.5 & 27.2 & 31.4 \\
\hline \multirow[t]{2}{*}{653350} & 2 & 0.0 & 1.6 & 2.9 & 4.4 & 3.3 & 5.7 \\
\hline & 10 & 0.7 & 4.4 & 13.5 & 20.0 & 22.1 & 32.4 \\
\hline \multirow[t]{2}{*}{655280} & 2 & 0.2 & 0.9 & 1.1 & 3.0 & 3.4 & 5.2 \\
\hline & 10 & 1.5 & 1.6 & 5.4 & 17.0 & 18.5 & 26.5 \\
\hline
\end{tabular}


GCM - MRI; Downscaling Method - Wilby et. al, 1998

SRES A2 (2046 - 2065)

DRWH Reliability (\%)

\begin{tabular}{|c|c|c|c|c|c|c|c|}
\hline \multirow{2}{*}{ Gauge } & \multicolumn{7}{|c|}{ per capita roof area } \\
\hline & sq. $m$ & Jul & Aug & Sep & Oct & Nov & Dec \\
\hline \multirow[t]{2}{*}{655920} & 2 & 2.5 & 5.0 & 7.0 & 5.4 & 2.9 & 2.7 \\
\hline & 10 & 26.6 & 38.4 & 43.4 & 37.4 & 27.4 & 18.3 \\
\hline \multirow[t]{2}{*}{655940} & 2 & 2.1 & 2.4 & 1.8 & 3.0 & 3.6 & 1.5 \\
\hline & 10 & 18.8 & 17.5 & 17.9 & 23.0 & 26.8 & 13.0 \\
\hline \multirow[t]{2}{*}{655990} & 2 & 1.8 & 1.6 & 1.1 & 3.4 & 2.5 & 1.6 \\
\hline & 10 & 18.2 & 13.1 & 11.8 & 22.1 & 18.5 & 10.7 \\
\hline \multirow[t]{2}{*}{655780} & 2 & 2.3 & 1.2 & 2.3 & 6.4 & 5.3 & 2.5 \\
\hline & 10 & 19.2 & 11.6 & 15.4 & 30.6 & 27.3 & 16.0 \\
\hline \multirow[t]{2}{*}{655850} & 2 & 2.1 & 2.0 & 3.0 & 7.7 & 4.1 & 2.0 \\
\hline & 10 & 23.6 & 18.5 & 23.0 & 37.7 & 26.1 & 12.6 \\
\hline \multirow[t]{2}{*}{655480} & 2 & 5.5 & 13.1 & 12.8 & 2.2 & 0.9 & 1.0 \\
\hline & 10 & 35.2 & 52.5 & 50.8 & 25.2 & 8.0 & 4.3 \\
\hline \multirow[t]{2}{*}{655600} & 2 & 2.5 & 6.1 & 4.8 & 1.5 & 0.7 & 0.9 \\
\hline & 10 & 21.9 & 28.7 & 31.9 & 19.4 & 6.8 & 5.7 \\
\hline \multirow[t]{2}{*}{655570} & 2 & 2.2 & 4.5 & 4.8 & 2.6 & 3.1 & 0.5 \\
\hline & 10 & 17.5 & 22.5 & 28.9 & 30.5 & 20.9 & 6.9 \\
\hline \multirow[t]{2}{*}{655630} & 2 & 3.1 & 4.4 & 3.7 & 2.0 & 1.5 & 0.3 \\
\hline & 10 & 18.1 & 20.8 & 24.6 & 22.8 & 10.3 & 4.5 \\
\hline \multirow[t]{2}{*}{655620} & 2 & 1.9 & 2.9 & 4.2 & 1.9 & 1.2 & 0.8 \\
\hline & 10 & 19.0 & 17.8 & 24.9 & 24.0 & 8.5 & 4.8 \\
\hline \multirow[t]{2}{*}{655550} & 2 & 3.3 & 7.7 & 6.4 & 2.3 & 0.5 & 0.2 \\
\hline & 10 & 19.4 & 28.2 & 30.9 & 20.2 & 5.2 & 3.3 \\
\hline \multirow[t]{2}{*}{655450} & 2 & 2.4 & 5.0 & 9.9 & 2.7 & 0.6 & 0.3 \\
\hline & 10 & 18.8 & 21.7 & 37.8 & 24.1 & 6.2 & 3.0 \\
\hline \multirow[t]{2}{*}{654720} & 2 & 1.7 & 1.1 & 1.2 & 1.6 & 0.5 & 0.4 \\
\hline & 10 & 12.4 & 8.5 & 10.0 & 13.7 & 5.3 & 3.9 \\
\hline \multirow[t]{2}{*}{653870} & 2 & 2.0 & 1.9 & 2.5 & 2.0 & 0.4 & 0.1 \\
\hline & 10 & 15.3 & 10.5 & 18.4 & 15.5 & 5.3 & 3.1 \\
\hline \multirow[t]{2}{*}{653760} & 2 & 8.2 & 14.1 & 11.2 & 4.0 & 0.2 & 0.2 \\
\hline & 10 & 38.8 & 37.2 & 35.2 & 22.1 & 3.0 & 2.0 \\
\hline \multirow[t]{2}{*}{653800} & 2 & 3.0 & 2.6 & 3.7 & 4.1 & 0.8 & 0.6 \\
\hline & 10 & 20.4 & 14.3 & 24.6 & 29.0 & 8.0 & 3.3 \\
\hline \multirow[t]{2}{*}{653440} & 2 & 4.4 & 3.6 & 4.7 & 4.5 & 1.0 & 0.3 \\
\hline & 10 & 22.3 & 16.0 & 26.4 & 28.0 & 8.8 & 3.2 \\
\hline \multirow[t]{2}{*}{653380} & 2 & 7.9 & 9.2 & 4.3 & 4.1 & 0.5 & 0.8 \\
\hline & 10 & 31.0 & 29.3 & 29.0 & 24.3 & 4.3 & 3.6 \\
\hline \multirow[t]{2}{*}{653350} & 2 & 7.1 & 11.9 & 6.6 & 4.2 & 0.6 & 0.1 \\
\hline & 10 & 29.8 & 34.4 & 36.0 & 24.6 & 3.4 & 1.1 \\
\hline \multirow[t]{2}{*}{655280} & 2 & 9.2 & 10.8 & 9.7 & 6.0 & 0.7 & 0.1 \\
\hline & 10 & 36.1 & 51.1 & 44.2 & 29.9 & 7.4 & 0.9 \\
\hline
\end{tabular}


GCM - MRI; Downscaling Method - Wilby et. al, 1998

SRES A2 (2046 - 2065)

DRWH Reliability (\%)

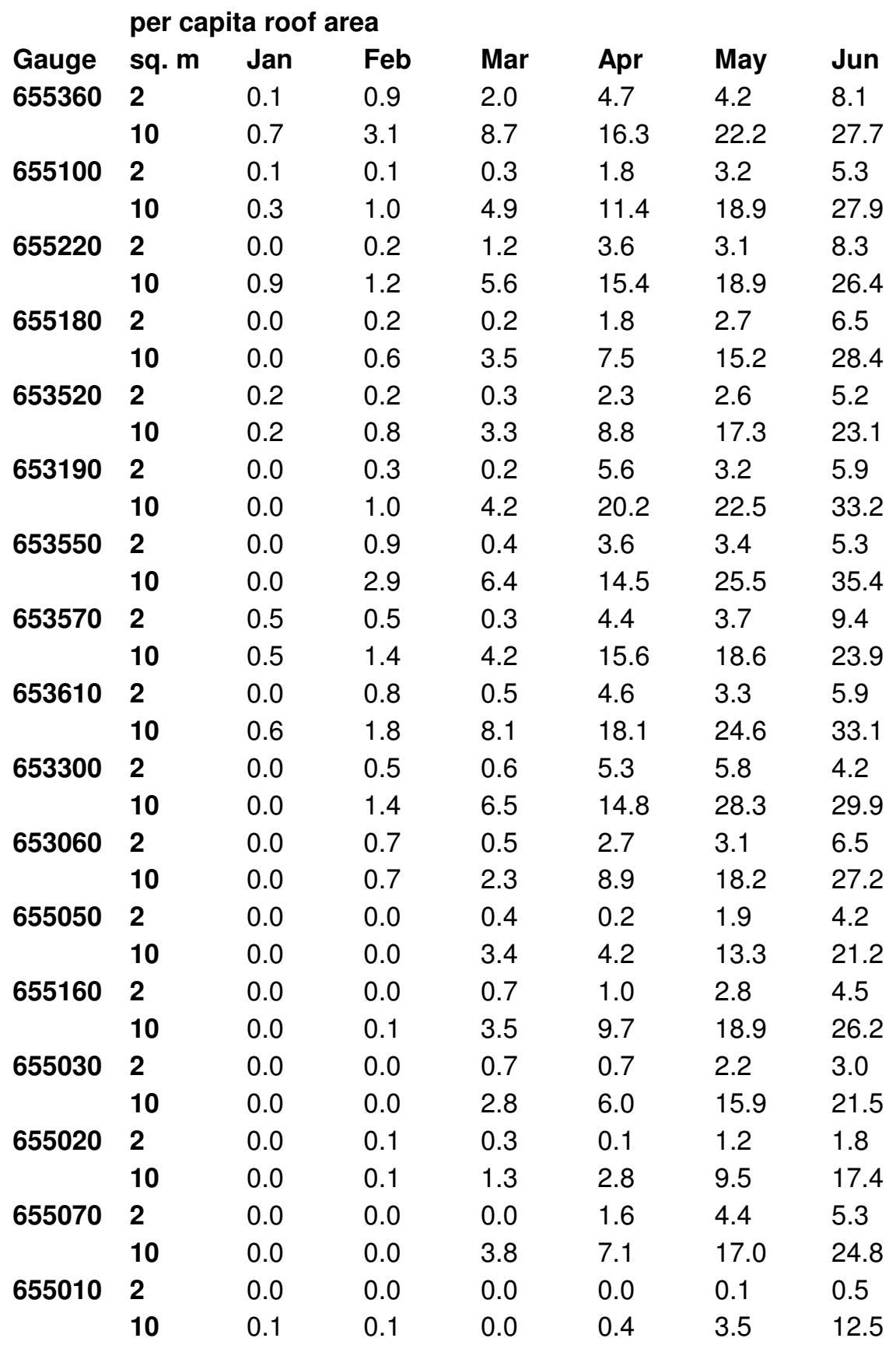


GCM - MRI; Downscaling Method - Wilby et. al, 1998

SRES A2 (2046 - 2065)

DRWH Reliability (\%)

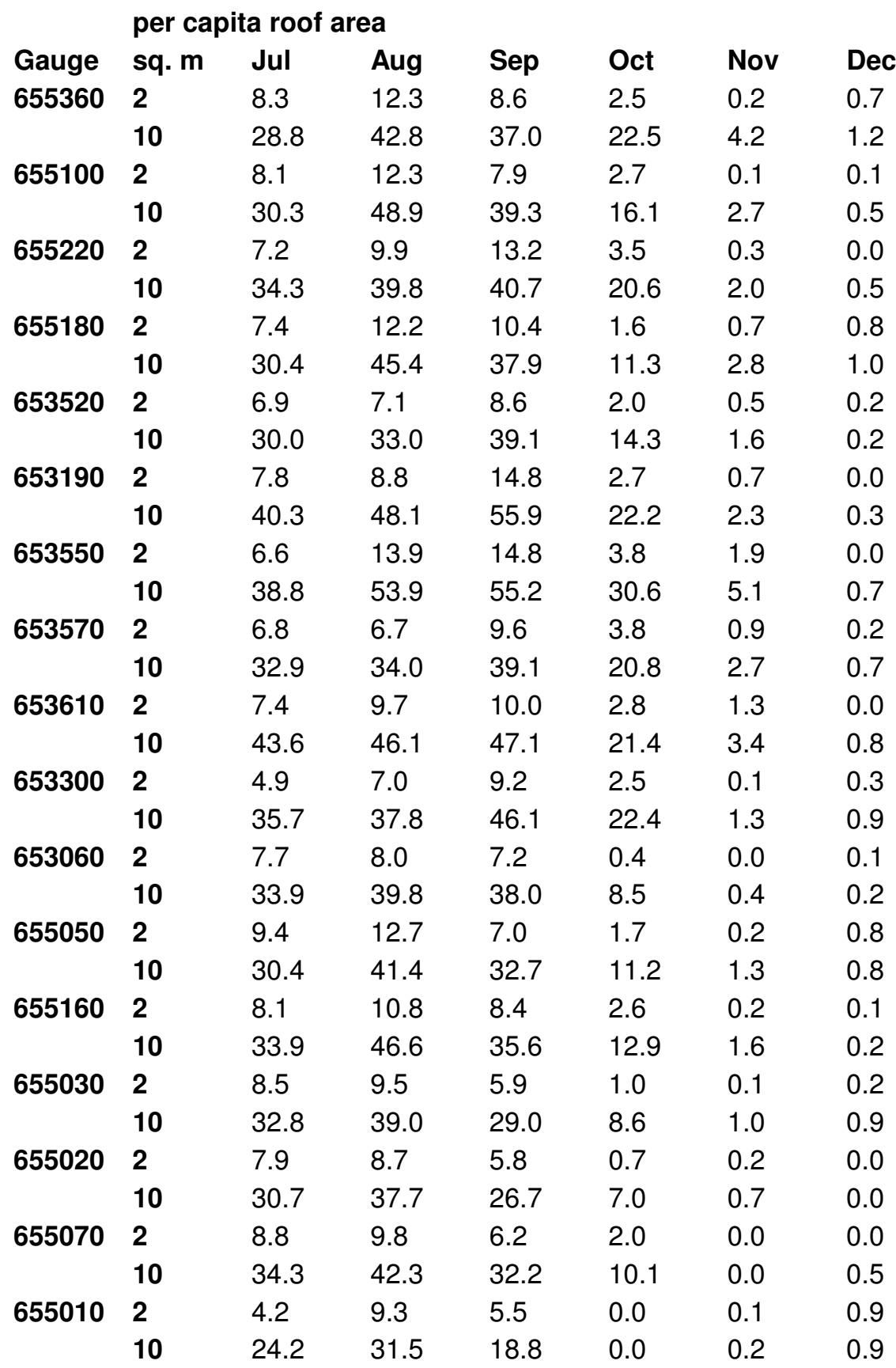


GCM - MRI; Downscaling Method - Wilby et. al, 1998

SRES B1 (2046 - 2065)

DRWH Reliability (\%)

\begin{tabular}{|c|c|c|c|c|c|c|c|}
\hline \multirow{2}{*}{ Gauge } & \multicolumn{7}{|c|}{ per capita roof area } \\
\hline & sq. $m$ & Jan & Feb & Mar & Apr & May & Jun \\
\hline \multirow[t]{2}{*}{655920} & 2 & 0.5 & 0.5 & 0.8 & 3.9 & 9.1 & 15.5 \\
\hline & 10 & 6.5 & 7.3 & 11.0 & 19.7 & 47.1 & 51.9 \\
\hline \multirow[t]{2}{*}{655940} & 2 & 0.2 & 0.3 & 0.9 & 4.8 & 5.5 & 10.4 \\
\hline & 10 & 2.3 & 3.1 & 7.6 & 16.6 & 34.5 & 49.7 \\
\hline \multirow[t]{2}{*}{655990} & 2 & 0.3 & 0.2 & 0.4 & 4.8 & 7.6 & 11.8 \\
\hline & 10 & 3.2 & 2.7 & 5.2 & 16.5 & 35.2 & 46.7 \\
\hline \multirow[t]{2}{*}{655780} & 2 & 0.4 & 0.5 & 1.5 & 3.9 & 6.6 & 15.7 \\
\hline & 10 & 3.8 & 7.8 & 14.6 & 21.9 & 38.9 & 51.2 \\
\hline \multirow[t]{2}{*}{655850} & 2 & 0.2 & 1.7 & 1.4 & 3.7 & 6.7 & 12.8 \\
\hline & 10 & 2.7 & 10.6 & 14.4 & 20.4 & 36.7 & 52.8 \\
\hline \multirow[t]{2}{*}{655480} & 2 & 0.0 & 0.4 & 4.1 & 8.2 & 4.2 & 10.4 \\
\hline & 10 & 1.1 & 6.1 & 17.7 & 25.2 & 25.8 & 36.0 \\
\hline \multirow[t]{2}{*}{655600} & 2 & 0.1 & 2.9 & 1.6 & 4.8 & 4.8 & 3.8 \\
\hline & 10 & 2.1 & 8.5 & 13.4 & 20.0 & 24.9 & 26.4 \\
\hline \multirow[t]{2}{*}{655570} & 2 & 0.4 & 3.7 & 4.1 & 7.6 & 7.7 & 8.4 \\
\hline & 10 & 3.9 & 11.4 & 20.9 & 26.2 & 35.0 & 39.0 \\
\hline \multirow[t]{2}{*}{655630} & 2 & 0.2 & 0.6 & 1.8 & 5.0 & 4.6 & 6.6 \\
\hline & 10 & 1.9 & 4.6 & 14.6 & 20.6 & 25.2 & 30.0 \\
\hline \multirow[t]{2}{*}{655620} & 2 & 0.1 & 1.5 & 2.2 & 4.9 & 5.8 & 4.9 \\
\hline & 10 & 2.2 & 6.1 & 16.1 & 19.0 & 27.6 & 29.1 \\
\hline \multirow[t]{2}{*}{655550} & 2 & 0.3 & 1.3 & 2.7 & 7.0 & 3.9 & 5.1 \\
\hline & 10 & 2.4 & 4.2 & 12.8 & 19.9 & 19.4 & 24.0 \\
\hline \multirow[t]{2}{*}{655450} & 2 & 0.3 & 0.7 & 1.3 & 5.9 & 6.0 & 10.1 \\
\hline & 10 & 1.4 & 4.5 & 8.5 & 19.6 & 26.9 & 32.5 \\
\hline \multirow[t]{2}{*}{654720} & 2 & 0.4 & 0.2 & 1.3 & 6.4 & 5.8 & 4.8 \\
\hline & 10 & 1.4 & 2.7 & 6.3 & 13.5 & 16.0 & 24.0 \\
\hline \multirow[t]{2}{*}{653870} & 2 & 0.0 & 0.5 & 1.6 & 3.0 & 3.3 & 8.0 \\
\hline & 10 & 0.7 & 3.7 & 9.8 & 16.1 & 25.3 & 29.9 \\
\hline \multirow[t]{2}{*}{653760} & 2 & 0.0 & 0.2 & 1.4 & 4.7 & 4.8 & 11.1 \\
\hline & 10 & 1.3 & 3.8 & 9.4 & 18.6 & 22.5 & 32.7 \\
\hline \multirow[t]{2}{*}{653800} & 2 & 0.2 & 0.8 & 4.4 & 5.5 & 4.0 & 6.7 \\
\hline & 10 & 0.7 & 4.7 & 14.0 & 18.9 & 22.3 & 28.3 \\
\hline \multirow[t]{2}{*}{653440} & 2 & 0.4 & 1.8 & 2.2 & 7.5 & 7.0 & 17.3 \\
\hline & 10 & 2.2 & 4.9 & 12.1 & 21.1 & 30.4 & 45.1 \\
\hline \multirow[t]{2}{*}{653380} & 2 & 0.2 & 1.2 & 2.2 & 5.0 & 5.1 & 9.1 \\
\hline & 10 & 0.6 & 4.4 & 13.7 & 22.2 & 26.6 & 32.5 \\
\hline \multirow[t]{2}{*}{653350} & 2 & 0.1 & 1.7 & 3.2 & 5.5 & 2.8 & 8.4 \\
\hline & 10 & 0.6 & 4.3 & 13.3 & 21.0 & 21.5 & 33.8 \\
\hline \multirow[t]{2}{*}{655280} & 2 & 0.1 & 0.3 & 1.5 & 3.2 & 3.6 & 6.5 \\
\hline & 10 & 1.4 & 1.5 & 5.6 & 16.7 & 19.4 & 27.6 \\
\hline
\end{tabular}


GCM - MRI; Downscaling Method - Wilby et. al, 1998

SRES B1 (2046 - 2065)

DRWH Reliability (\%)

\begin{tabular}{|c|c|c|c|c|c|c|c|}
\hline \multirow{2}{*}{ Gauge } & \multicolumn{7}{|c|}{ per capita roof area } \\
\hline & sq. $m$ & Jul & Aug & Sep & Oct & Nov & Dec \\
\hline \multirow[t]{2}{*}{655920} & 2 & 3.3 & 3.4 & 2.8 & 4.4 & 2.4 & 3.8 \\
\hline & 10 & 26.4 & 35.6 & 38.8 & 35.1 & 28.9 & 18.9 \\
\hline \multirow[t]{2}{*}{655940} & 2 & 1.6 & 1.8 & 0.8 & 1.2 & 3.3 & 1.9 \\
\hline & 10 & 18.3 & 16.7 & 15.8 & 20.5 & 27.0 & 13.7 \\
\hline \multirow[t]{2}{*}{655990} & 2 & 1.7 & 1.2 & 0.5 & 1.7 & 2.1 & 2.3 \\
\hline & 10 & 18.0 & 12.6 & 10.3 & 19.9 & 19.5 & 11.0 \\
\hline \multirow[t]{2}{*}{655780} & 2 & 1.9 & 0.7 & 0.9 & 3.4 & 4.6 & 3.1 \\
\hline & 10 & 19.0 & 10.8 & 14.9 & 28.1 & 27.4 & 16.2 \\
\hline \multirow[t]{2}{*}{655850} & 2 & 2.2 & 1.2 & 1.3 & 3.9 & 3.3 & 2.1 \\
\hline & 10 & 22.9 & 16.8 & 22.6 & 35.5 & 25.3 & 13.3 \\
\hline \multirow[t]{2}{*}{655480} & 2 & 5.4 & 13.3 & 13.8 & 2.9 & 0.7 & 1.3 \\
\hline & 10 & 34.7 & 53.1 & 50.2 & 26.6 & 7.7 & 4.1 \\
\hline \multirow[t]{2}{*}{655600} & 2 & 2.5 & 5.5 & 4.1 & 1.5 & 0.5 & 2.1 \\
\hline & 10 & 21.8 & 27.5 & 30.4 & 19.6 & 6.4 & 5.5 \\
\hline \multirow[t]{2}{*}{655570} & 2 & 1.9 & 3.7 & 4.0 & 3.0 & 2.4 & 1.5 \\
\hline & 10 & 17.6 & 21.6 & 27.5 & 31.2 & 20.4 & 6.8 \\
\hline \multirow[t]{2}{*}{655630} & 2 & 3.0 & 3.8 & 3.1 & 2.0 & 0.7 & 1.2 \\
\hline & 10 & 18.1 & 20.1 & 23.7 & 23.1 & 9.6 & 4.8 \\
\hline \multirow[t]{2}{*}{655620} & 2 & 1.8 & 2.2 & 3.4 & 2.2 & 0.8 & 1.7 \\
\hline & 10 & 18.3 & 16.4 & 24.0 & 25.2 & 8.5 & 4.7 \\
\hline \multirow[t]{2}{*}{655550} & 2 & 2.9 & 6.6 & 5.2 & 2.6 & 0.4 & 0.8 \\
\hline & 10 & 19.1 & 27.0 & 29.6 & 20.4 & 4.9 & 3.1 \\
\hline \multirow[t]{2}{*}{655450} & 2 & 2.5 & 4.0 & 8.2 & 3.3 & 0.4 & 0.0 \\
\hline & 10 & 18.5 & 20.8 & 37.0 & 24.0 & 6.2 & 2.3 \\
\hline \multirow[t]{2}{*}{654720} & 2 & 1.7 & 1.1 & 0.7 & 1.3 & 0.4 & 0.6 \\
\hline & 10 & 11.8 & 7.9 & 8.9 & 13.1 & 5.1 & 3.6 \\
\hline \multirow[t]{2}{*}{653870} & 2 & 2.3 & 1.5 & 2.2 & 1.2 & 0.3 & 0.2 \\
\hline & 10 & 14.9 & 9.8 & 16.6 & 15.0 & 5.3 & 2.8 \\
\hline \multirow[t]{2}{*}{653760} & 2 & 8.3 & 12.5 & 8.9 & 3.2 & 0.2 & 0.2 \\
\hline & 10 & 38.0 & 35.9 & 33.6 & 21.7 & 3.1 & 1.9 \\
\hline \multirow[t]{2}{*}{653800} & 2 & 3.4 & 1.8 & 3.0 & 4.1 & 0.5 & 0.5 \\
\hline & 10 & 19.2 & 12.6 & 22.8 & 28.5 & 6.9 & 3.3 \\
\hline \multirow[t]{2}{*}{653440} & 2 & 4.5 & 3.0 & 3.6 & 4.7 & 0.7 & 0.2 \\
\hline & 10 & 21.4 & 14.7 & 25.2 & 27.3 & 7.8 & 2.9 \\
\hline \multirow[t]{2}{*}{653380} & 2 & 7.3 & 7.0 & 3.7 & 3.7 & 0.3 & 0.6 \\
\hline & 10 & 30.0 & 28.0 & 27.4 & 23.8 & 3.5 & 3.5 \\
\hline \multirow[t]{2}{*}{653350} & 2 & 6.8 & 8.9 & 6.0 & 4.2 & 0.4 & 0.0 \\
\hline & 10 & 29.0 & 32.5 & 34.3 & 24.2 & 3.0 & 1.0 \\
\hline \multirow[t]{2}{*}{655280} & 2 & 9.5 & 10.7 & 11.2 & 6.0 & 1.2 & 0.2 \\
\hline & 10 & 37.2 & 50.1 & 46.5 & 31.4 & 7.6 & 0.9 \\
\hline
\end{tabular}


GCM - MRI; Downscaling Method - Wilby et. al, 1998

SRES B1 (2046 - 2065)

DRWH Reliability (\%)

\begin{tabular}{|c|c|c|c|c|c|c|c|}
\hline \multirow{3}{*}{$\begin{array}{l}\text { Gauge } \\
655360\end{array}$} & \multicolumn{7}{|c|}{ per capita roof area } \\
\hline & sq. $\mathrm{m}$ & Jan & Feb & Mar & Apr & May & Jun \\
\hline & 2 & 0.1 & 0.7 & 1.5 & 4.4 & 3.6 & 7.6 \\
\hline \multirow{3}{*}{655100} & 10 & 0.8 & 2.9 & 8.2 & 16.0 & 21.7 & 27.5 \\
\hline & 2 & 0.1 & 0.1 & 0.2 & 2.0 & 2.8 & 5.7 \\
\hline & 10 & 0.3 & 0.9 & 4.5 & 11.0 & 18.4 & 27.7 \\
\hline \multirow[t]{2}{*}{655220} & 2 & 0.0 & 0.3 & 0.9 & 2.5 & 3.0 & 6.5 \\
\hline & 10 & 0.4 & 0.9 & 5.6 & 14.3 & 19.6 & 26.6 \\
\hline \multirow[t]{2}{*}{655180} & 2 & 0.0 & 0.2 & 0.2 & 1.3 & 3.1 & 5.7 \\
\hline & 10 & 0.0 & 0.6 & 4.0 & 6.6 & 15.4 & 29.2 \\
\hline \multirow[t]{2}{*}{653520} & 2 & 0.2 & 0.1 & 0.2 & 1.5 & 3.0 & 4.4 \\
\hline & 10 & 0.2 & 0.6 & 3.2 & 7.9 & 17.9 & 23.8 \\
\hline \multirow[t]{2}{*}{653190} & 2 & 0.0 & 0.1 & 0.2 & 4.3 & 3.4 & 4.6 \\
\hline & 10 & 0.0 & 0.9 & 4.5 & 18.7 & 23.3 & 34.1 \\
\hline \multirow[t]{2}{*}{653550} & 2 & 0.0 & 0.4 & 0.3 & 2.3 & 4.0 & 4.6 \\
\hline & 10 & 0.0 & 2.8 & 6.3 & 13.6 & 26.4 & 35.9 \\
\hline \multirow[t]{2}{*}{653570} & 2 & 0.5 & 0.3 & 0.4 & 3.5 & 4.5 & 9.1 \\
\hline & 10 & 0.5 & 1.3 & 4.3 & 14.4 & 19.0 & 24.7 \\
\hline \multirow[t]{2}{*}{653610} & 2 & 0.0 & 0.4 & 0.6 & 3.3 & 3.6 & 4.9 \\
\hline & 10 & 0.7 & 1.7 & 7.9 & 16.0 & 25.5 & 33.7 \\
\hline \multirow[t]{2}{*}{653300} & 2 & 0.0 & 0.0 & 0.4 & 3.5 & 5.9 & 4.2 \\
\hline & 10 & 0.1 & 1.0 & 6.2 & 14.2 & 28.9 & 29.7 \\
\hline \multirow[t]{2}{*}{653060} & 2 & 0.0 & 0.6 & 0.3 & 1.9 & 2.9 & 6.1 \\
\hline & 10 & 0.0 & 0.6 & 2.3 & 8.5 & 18.2 & 26.6 \\
\hline \multirow[t]{2}{*}{655050} & 2 & 0.0 & 0.2 & 1.2 & 0.3 & 3.2 & 6.9 \\
\hline & 10 & 0.0 & 0.5 & 3.4 & 4.3 & 14.4 & 22.5 \\
\hline \multirow[t]{2}{*}{655160} & 2 & 0.0 & 0.0 & 1.3 & 1.2 & 3.9 & 7.7 \\
\hline & 10 & 0.0 & 0.2 & 3.3 & 9.5 & 19.4 & 28.1 \\
\hline \multirow[t]{2}{*}{655030} & 2 & 0.0 & 0.2 & 1.1 & 1.0 & 3.1 & 4.7 \\
\hline & 10 & 0.0 & 0.3 & 2.4 & 5.9 & 16.5 & 23.3 \\
\hline \multirow[t]{2}{*}{655020} & 2 & 0.0 & 0.2 & 0.5 & 0.2 & 1.7 & 3.6 \\
\hline & 10 & 0.0 & 0.2 & 1.3 & 2.7 & 10.1 & 18.3 \\
\hline \multirow[t]{2}{*}{655070} & 2 & 0.0 & 0.0 & 1.6 & 2.2 & 4.6 & 8.3 \\
\hline & 10 & 0.0 & 0.0 & 4.1 & 6.9 & 17.3 & 25.9 \\
\hline \multirow[t]{2}{*}{655010} & 2 & 0.0 & 0.0 & 0.0 & 0.0 & 0.1 & 3.7 \\
\hline & 10 & 0.1 & 0.1 & 0.0 & 0.3 & 2.9 & 13.7 \\
\hline
\end{tabular}


GCM - MRI; Downscaling Method - Wilby et. al, 1998

SRES B1 (2046 - 2065)

DRWH Reliability (\%)

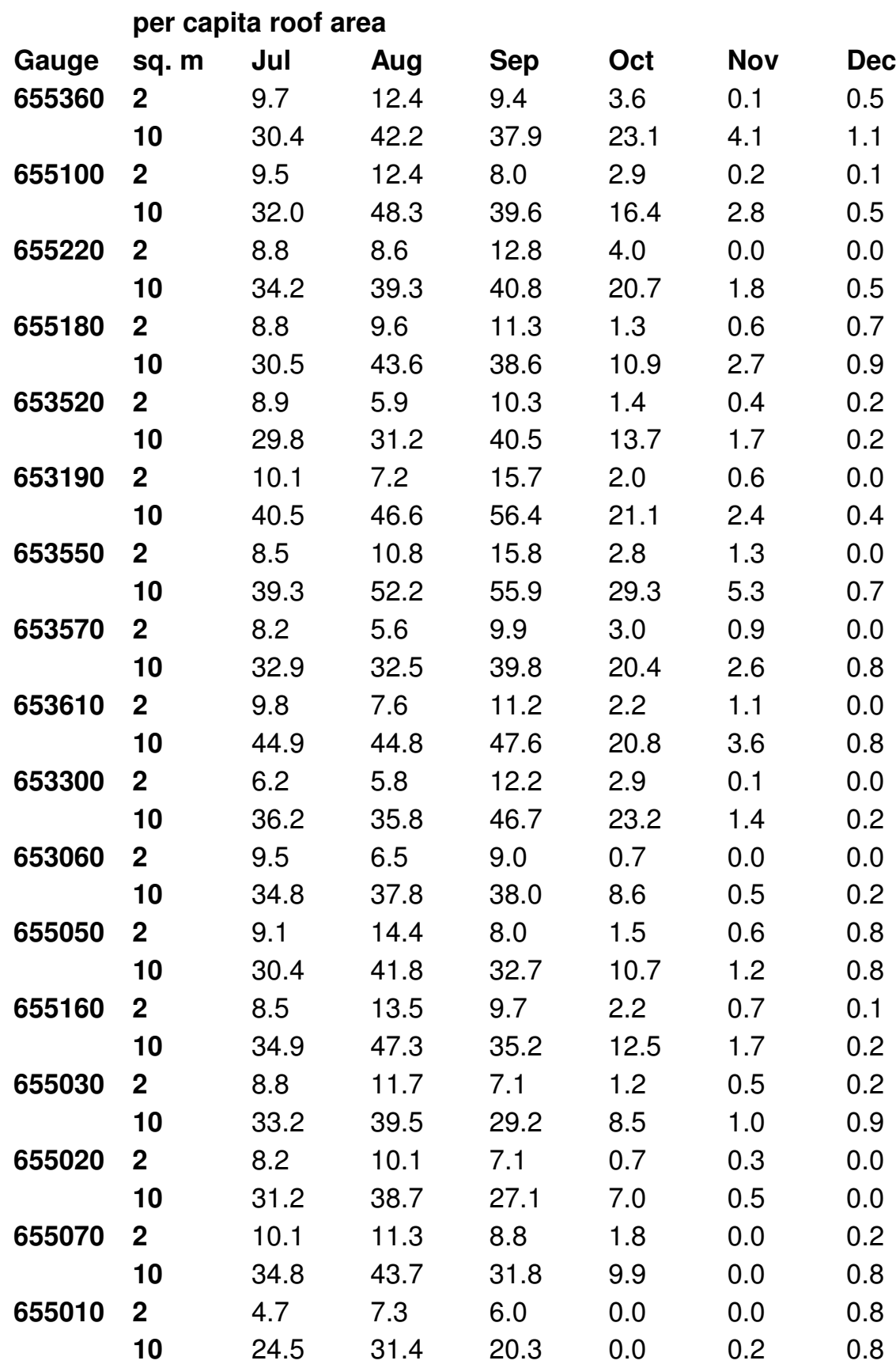


GCM - CGCM3; Downscaling Method - LARS-WG

SRES A1b (2046 - 2065)

DRWH Reliability (\%)

per capita roof area

\begin{tabular}{|c|c|c|c|c|c|c|c|}
\hline Gauge & sq. m & Jan & Feb & Mar & Apr & May & Jun \\
\hline \multirow[t]{2}{*}{655920} & 2 & 4.7 & 4.2 & 7.1 & 9.3 & 18.9 & 24.0 \\
\hline & 10 & 11.8 & 9.8 & 15.6 & 19.5 & 32.5 & 42.2 \\
\hline \multirow[t]{2}{*}{655940} & 2 & 4.0 & 3.1 & 6.6 & 10.1 & 17.1 & 17.6 \\
\hline & 10 & 9.6 & 6.9 & 13.4 & 19.5 & 32.9 & 35.8 \\
\hline \multirow[t]{2}{*}{655990} & 2 & 4.7 & 3.5 & 6.1 & 9.6 & 15.8 & 19.1 \\
\hline & 10 & 10.5 & 9.1 & 16.4 & 18.2 & 33.6 & 34.8 \\
\hline \multirow[t]{2}{*}{655780} & 2 & 2.7 & 3.1 & 7.0 & 12.2 & 15.2 & 22.0 \\
\hline & 10 & 6.9 & 5.9 & 17.9 & 24.9 & 20.5 & 34.2 \\
\hline \multirow[t]{2}{*}{655850} & 2 & 5.1 & 7.5 & 8.5 & 11.2 & 14.6 & 20.1 \\
\hline & 10 & 10.6 & 11.3 & 20.4 & 24.2 & 21.3 & 33.3 \\
\hline \multirow[t]{2}{*}{655480} & 2 & 9.7 & 10.8 & 19.9 & 18.8 & 14.1 & 16.7 \\
\hline & 10 & 23.9 & 24.4 & 32.6 & 38.9 & 25.8 & 34.9 \\
\hline \multirow[t]{2}{*}{655600} & 2 & 2.5 & 6.0 & 9.4 & 11.3 & 12.8 & 12.1 \\
\hline & 10 & 6.8 & 12.8 & 19.6 & 23.5 & 26.4 & 24.8 \\
\hline \multirow[t]{2}{*}{655570} & 2 & 4.0 & 7.0 & 13.5 & 12.0 & 12.5 & 13.6 \\
\hline & 10 & 10.4 & 14.9 & 27.9 & 29.8 & 35.5 & 31.3 \\
\hline \multirow[t]{2}{*}{655630} & 2 & 4.5 & 3.5 & 6.8 & 7.7 & 11.2 & 14.8 \\
\hline & 10 & 7.3 & 8.9 & 21.6 & 22.1 & 26.3 & 27.1 \\
\hline \multirow[t]{2}{*}{655620} & 2 & 2.8 & 4.3 & 9.8 & 10.5 & 13.9 & 13.7 \\
\hline & 10 & 6.4 & 11.5 & 22.2 & 22.1 & 27.4 & 25.7 \\
\hline \multirow[t]{2}{*}{655550} & 2 & 4.5 & 5.5 & 9.6 & 11.3 & 9.4 & 11.4 \\
\hline & 10 & 8.8 & 11.3 & 22.7 & 24.1 & 21.9 & 21.9 \\
\hline \multirow[t]{2}{*}{655450} & 2 & 4.2 & 4.6 & 8.2 & 12.5 & 18.1 & 15.5 \\
\hline & 10 & 7.2 & 9.8 & 15.8 & 20.1 & 42.2 & 32.2 \\
\hline \multirow[t]{2}{*}{654720} & 2 & 2.8 & 4.7 & 9.8 & 11.2 & 15.6 & 12.2 \\
\hline & 10 & 6.1 & 10.0 & 16.6 & 19.6 & 27.6 & 20.3 \\
\hline \multirow[t]{2}{*}{653870} & 2 & 1.1 & 2.0 & 7.1 & 7.9 & 11.9 & 12.3 \\
\hline & 10 & 3.6 & 4.5 & 13.8 & 13.2 & 25.4 & 21.0 \\
\hline \multirow[t]{2}{*}{653760} & 2 & 1.6 & 1.6 & 6.6 & 9.5 & 13.2 & 12.9 \\
\hline & 10 & 7.0 & 6.3 & 14.9 & 17.0 & 27.1 & 24.2 \\
\hline \multirow[t]{2}{*}{653800} & 2 & 3.1 & 5.7 & 13.2 & 12.3 & 15.0 & 13.2 \\
\hline & 10 & 8.7 & 11.7 & 21.7 & 21.2 & 27.5 & 25.5 \\
\hline \multirow[t]{2}{*}{653440} & 2 & 1.8 & 2.3 & 6.4 & 11.4 & 15.8 & 19.1 \\
\hline & 10 & 3.2 & 3.8 & 11.2 & 20.0 & 28.9 & 31.2 \\
\hline \multirow[t]{2}{*}{653380} & 2 & 1.2 & 2.3 & 6.9 & 8.2 & 11.6 & 11.3 \\
\hline & 10 & 3.4 & 4.7 & 13.4 & 19.3 & 26.7 & 23.3 \\
\hline \multirow[t]{2}{*}{653350} & 2 & 0.7 & 3.1 & 6.9 & 7.2 & 7.7 & 9.8 \\
\hline & 10 & 3.9 & 5.1 & 12.4 & 18.0 & 21.3 & 22.9 \\
\hline \multirow[t]{2}{*}{655280} & 2 & 3.0 & 3.5 & 4.5 & 6.4 & 6.8 & 11.4 \\
\hline & 10 & 7.8 & 6.8 & 7.8 & 14.0 & 23.5 & 27.3 \\
\hline
\end{tabular}


GCM - CGCM3; Downscaling Method - LARS-WG

SRES A1b (2046 - 2065)

DRWH Reliability (\%)

per capita roof area

$\begin{array}{llllllll}\text { Gauge } & \text { sq. } \mathbf{m} & \text { Jul } & \text { Aug } & \text { Sep } & \text { Oct } & \text { Nov } & \text { Dec } \\ \mathbf{6 5 5 9 2 0} & \mathbf{2} & 12.2 & 12.3 & 15.4 & 11.7 & 8.9 & 12.5\end{array}$

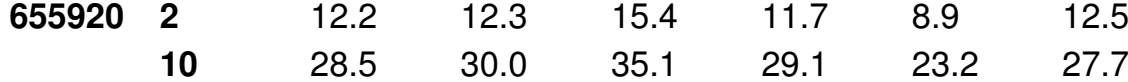

$\begin{array}{llllllll}655940 & 2 & 10.6 & 4.1 & 5.4 & 8.0 & 8.6 & 9.4\end{array}$

$\begin{array}{lllllll}10 & 27.5 & 17.5 & 20.2 & 20.8 & 23.3 & 22.9\end{array}$

$\begin{array}{llllllll}655990 & 2 & 9.9 & 4.0 & 4.6 & 8.7 & 7.6 & 12.8\end{array}$

$\begin{array}{lllllll}10 & 24.2 & 14.0 & 14.1 & 20.9 & 18.7 & 25.1\end{array}$

$\begin{array}{llllllll}655780 & 2 & 10.8 & 4.0 & 6.9 & 9.4 & 8.5 & 8.8\end{array}$

$\begin{array}{lllllll}10 & 23.5 & 12.8 & 15.7 & 22.9 & 22.8 & 20.1\end{array}$

$\begin{array}{llllllll}655850 & 2 & 12.0 & 6.7 & 10.1 & 13.5 & 8.9 & 8.4\end{array}$

$\begin{array}{lllllll}10 & 28.3 & 20.3 & 21.0 & 26.6 & 23.5 & 19.3\end{array}$

$\begin{array}{llllllll}655480 & 2 & 14.0 & 23.4 & 31.4 & 29.3 & 25.7 & 16.4\end{array}$

$\begin{array}{lllllll}10 & 33.7 & 60.2 & 67.6 & 57.9 & 43.9 & 33.9\end{array}$

$\begin{array}{llllllll}655600 & 2 & 11.0 & 10.2 & 12.2 & 8.6 & 3.9 & 5.5\end{array}$

$\begin{array}{lllllll}10 & 25.4 & 25.4 & 29.6 & 19.3 & 10.1 & 9.5\end{array}$

$\begin{array}{llllllll}655570 & 2 & 7.3 & 6.3 & 10.7 & 11.0 & 7.8 & 6.2\end{array}$

$\begin{array}{lllllll}10 & 22.5 & 18.0 & 26.2 & 26.1 & 19.9 & 13.7\end{array}$

$\begin{array}{llllllll}655630 & 2 & 9.4 & 7.8 & 10.2 & 8.4 & 4.2 & 3.5\end{array}$

$\begin{array}{lllllll}10 & 22.4 & 18.0 & 24.1 & 20.3 & 12.1 & 8.6\end{array}$

$\begin{array}{llllllll}655620 & 2 & 7.6 & 6.2 & 11.5 & 11.7 & 4.4 & 5.2\end{array}$

$\begin{array}{lllllll}10 & 22.5 & 16.1 & 24.0 & 21.1 & 10.4 & 9.7\end{array}$

$\begin{array}{llllllll}655550 & 2 & 12.1 & 12.2 & 14.4 & 9.1 & 3.2 & 5.1\end{array}$

$\begin{array}{lllllll}10 & 23.7 & 26.3 & 29.1 & 17.5 & 8.5 & 9.8\end{array}$

$\begin{array}{llllllll}655450 & 2 & 9.9 & 9.5 & 14.1 & 7.0 & 2.6 & 8.6\end{array}$

$\begin{array}{lllllll}10 & 20.4 & 21.7 & 29.6 & 26.6 & 13.0 & 16.1\end{array}$

$\begin{array}{llllllll}654720 & 2 & 5.9 & 2.9 & 6.2 & 7.0 & 4.2 & 7.2\end{array}$

$\begin{array}{lllllll}10 & 15.1 & 7.7 & 14.1 & 19.8 & 11.4 & 15.0\end{array}$

$\begin{array}{llllllll}653870 & 2 & 6.5 & 3.7 & 6.3 & 5.8 & 3.5 & 5.1\end{array}$

$\begin{array}{lllllll}10 & 14.4 & 9.2 & 15.9 & 16.6 & 9.1 & 11.6\end{array}$

$\begin{array}{llllllll}653760 & 2 & 13.4 & 13.5 & 14.2 & 9.8 & 3.8 & 7.1\end{array}$

$\begin{array}{llllllll} & \mathbf{1 0} & 30.3 & 29.9 & 28.1 & 23.7 & 9.2 & 14.5 \\ \mathbf{6 5 3 8 0 0} & \mathbf{2} & 12.1 & 6.2 & 9.5 & 11.7 & 5.2 & 12.4 \\ & \mathbf{1 0} & 30.5 & 15.5 & 20.5 & 25.0 & 10.6 & 18.4 \\ \mathbf{6 5 3 4 4 0} & \mathbf{2} & 11.8 & 7.9 & 11.2 & 11.0 & 4.6 & 5.9 \\ & \mathbf{1 0} & 29.6 & 16.8 & 20.1 & 21.1 & 9.1 & 9.6 \\ \mathbf{6 5 3 3 8 0} & \mathbf{2} & 13.7 & 11.2 & 11.8 & 13.5 & 4.7 & 5.3 \\ & \mathbf{1 0} & 38.0 & 24.7 & 22.4 & 20.4 & 6.8 & 10.6 \\ \mathbf{6 5 3 3 5 0} & \mathbf{2} & 14.0 & 14.8 & 15.1 & 10.9 & 3.3 & 0.7 \\ & \mathbf{1 0} & 38.8 & 29.7 & 29.4 & 21.3 & 6.1 & 7.6 \\ \mathbf{6 5 5 2 8 0} & \mathbf{2} & 17.3 & 22.8 & 15.5 & 9.2 & 5.2 & 5.6 \\ & \mathbf{1 0} & 35.6 & 45.3 & 41.1 & 34.6 & 18.4 & 12.4\end{array}$


GCM - CGCM3; Downscaling Method - LARS-WG

SRES A1b (2046 - 2065)

DRWH Reliability (\%)

\begin{tabular}{|c|c|c|c|c|c|c|c|}
\hline \multirow{3}{*}{$\begin{array}{l}\text { Gauge } \\
655360\end{array}$} & \multicolumn{7}{|c|}{ per capita roof area } \\
\hline & sq. $\mathrm{m}$ & Jan & Feb & Mar & Apr & May & Jun \\
\hline & 2 & 3.9 & 3.8 & 4.5 & 7.9 & 9.8 & 13.2 \\
\hline \multirow{3}{*}{655100} & 10 & 7.7 & 5.3 & 10.1 & 16.8 & 22.0 & 27.2 \\
\hline & 2 & 4.5 & 1.5 & 2.1 & 5.5 & 7.8 & 11.9 \\
\hline & 10 & 6.5 & 3.4 & 6.0 & 11.8 & 18.2 & 25.9 \\
\hline \multirow[t]{2}{*}{655220} & 2 & 5.9 & 2.2 & 3.3 & 7.9 & 9.8 & 11.1 \\
\hline & 10 & 6.6 & 4.0 & 9.8 & 15.6 & 21.8 & 29.9 \\
\hline \multirow[t]{2}{*}{655180} & 2 & 3.3 & 0.8 & 3.1 & 4.7 & 7.4 & 15.2 \\
\hline & 10 & 6.1 & 4.6 & 7.9 & 9.7 & 20.3 & 26.3 \\
\hline \multirow[t]{2}{*}{653520} & 2 & 6.6 & 3.0 & 6.6 & 7.0 & 11.6 & 17.2 \\
\hline & 10 & 6.6 & 5.3 & 11.0 & 12.8 & 24.9 & 25.9 \\
\hline \multirow[t]{2}{*}{653190} & 2 & 0.0 & 1.1 & 2.7 & 7.1 & 9.8 & 13.3 \\
\hline & 10 & 0.0 & 2.8 & 6.5 & 16.5 & 26.5 & 26.2 \\
\hline \multirow[t]{2}{*}{653550} & 2 & 4.6 & 3.7 & 5.4 & 7.1 & 13.7 & 19.9 \\
\hline & 10 & 6.0 & 5.9 & 10.1 & 16.5 & 32.9 & 29.6 \\
\hline \multirow[t]{2}{*}{653570} & 2 & 7.6 & 4.7 & 5.1 & 8.8 & 11.7 & 19.0 \\
\hline & 10 & 9.7 & 8.0 & 14.8 & 21.6 & 31.5 & 30.1 \\
\hline \multirow[t]{2}{*}{653610} & 2 & 3.1 & 3.5 & 5.7 & 6.9 & 10.7 & 14.8 \\
\hline & 10 & 8.2 & 6.7 & 10.3 & 16.6 & 31.0 & 28.1 \\
\hline \multirow[t]{2}{*}{653300} & 2 & 0.0 & 0.3 & 1.8 & 4.7 & 7.6 & 9.7 \\
\hline & 10 & 3.3 & 1.6 & 5.0 & 8.8 & 26.1 & 31.5 \\
\hline \multirow[t]{2}{*}{653060} & 2 & 0.0 & 1.7 & 1.8 & 3.3 & 6.6 & 9.1 \\
\hline & 10 & 0.0 & 2.6 & 5.1 & 6.2 & 17.9 & 32.2 \\
\hline \multirow[t]{2}{*}{655050} & 2 & 5.2 & 2.5 & 1.7 & 2.2 & 6.4 & 11.2 \\
\hline & 10 & 6.7 & 6.5 & 6.4 & 6.2 & 14.6 & 27.4 \\
\hline \multirow[t]{2}{*}{655160} & 2 & 3.7 & 0.6 & 2.2 & 3.7 & 8.7 & 12.7 \\
\hline & 10 & 6.5 & 4.6 & 6.2 & 9.0 & 20.0 & 29.6 \\
\hline \multirow[t]{2}{*}{655030} & 2 & 3.6 & 2.8 & 2.4 & 2.8 & 5.9 & 7.9 \\
\hline & 10 & 7.3 & 4.0 & 4.6 & 5.9 & 17.1 & 26.8 \\
\hline \multirow[t]{2}{*}{655020} & 2 & 0.0 & 4.2 & 2.3 & 1.7 & 5.3 & 9.8 \\
\hline & 10 & 0.0 & 4.9 & 4.0 & 4.0 & 12.9 & 23.5 \\
\hline \multirow[t]{2}{*}{655070} & 2 & 0.4 & 3.0 & 4.2 & 4.2 & 6.6 & 12.3 \\
\hline & 10 & 0.7 & 4.7 & 7.2 & 8.1 & 16.5 & 21.5 \\
\hline \multirow[t]{2}{*}{655010} & 2 & 4.0 & 1.1 & 1.4 & 0.6 & 2.3 & 5.2 \\
\hline & 10 & 6.7 & 2.3 & 4.4 & 2.6 & 5.5 & 12.7 \\
\hline
\end{tabular}


GCM - CGCM3; Downscaling Method - LARS-WG

SRES A1b (2046 - 2065)

DRWH Reliability (\%)

\begin{tabular}{|c|c|c|c|c|c|c|c|}
\hline \multirow{2}{*}{ Gauge } & \multicolumn{7}{|c|}{ per capita roof area } \\
\hline & sq. $m$ & Jul & Aug & Sep & Oct & Nov & Dec \\
\hline \multirow[t]{2}{*}{655360} & 2 & 16.4 & 20.6 & 18.3 & 9.2 & 3.5 & 13.9 \\
\hline & 10 & 30.5 & 38.6 & 36.6 & 20.8 & 8.6 & 22.7 \\
\hline \multirow[t]{2}{*}{655100} & 2 & 16.6 & 24.4 & 17.3 & 8.0 & 4.6 & 10.5 \\
\hline & 10 & 32.4 & 43.0 & 38.2 & 15.6 & 8.5 & 22.8 \\
\hline \multirow[t]{2}{*}{655220} & 2 & 15.7 & 17.6 & 15.4 & 8.8 & 4.1 & 14.2 \\
\hline & 10 & 34.4 & 35.7 & 39.2 & 21.2 & 9.1 & 16.0 \\
\hline \multirow[t]{2}{*}{655180} & 2 & 14.7 & 16.9 & 14.8 & 6.6 & 6.9 & 11.9 \\
\hline & 10 & 35.1 & 37.2 & 38.5 & 15.5 & 13.0 & 13.0 \\
\hline \multirow[t]{2}{*}{653520} & 2 & 15.5 & 11.5 & 15.4 & 9.6 & 6.9 & 9.0 \\
\hline & 10 & 33.8 & 30.0 & 35.6 & 16.7 & 10.6 & 10.0 \\
\hline \multirow[t]{2}{*}{653190} & 2 & 17.9 & 19.8 & 18.8 & 9.5 & 5.2 & 4.8 \\
\hline & 10 & 38.3 & 41.5 & 47.4 & 20.0 & 7.7 & 9.2 \\
\hline \multirow[t]{2}{*}{653550} & 2 & 19.8 & 22.6 & 21.1 & 12.1 & 7.4 & 6.5 \\
\hline & 10 & 36.4 & 44.1 & 48.3 & 24.6 & 13.1 & 8.9 \\
\hline \multirow[t]{2}{*}{653570} & 2 & 15.1 & 11.9 & 15.2 & 12.2 & 6.2 & 7.0 \\
\hline & 10 & 34.4 & 29.5 & 39.2 & 25.6 & 11.8 & 9.0 \\
\hline \multirow[t]{2}{*}{653610} & 2 & 15.6 & 16.4 & 19.2 & 11.0 & 8.1 & 8.5 \\
\hline & 10 & 39.2 & 37.8 & 41.4 & 22.1 & 13.5 & 13.8 \\
\hline \multirow[t]{2}{*}{653300} & 2 & 16.2 & 19.0 & 18.8 & 8.9 & 4.4 & 1.0 \\
\hline & 10 & 48.1 & 38.6 & 43.0 & 20.8 & 8.1 & 6.8 \\
\hline \multirow[t]{2}{*}{653060} & 2 & 15.5 & 18.8 & 13.4 & 4.1 & 1.2 & 6.4 \\
\hline & 10 & 52.3 & 37.7 & 38.7 & 12.7 & 3.1 & 7.1 \\
\hline \multirow[t]{2}{*}{655050} & 2 & 16.3 & 18.5 & 12.1 & 6.1 & 8.3 & 15.5 \\
\hline & 10 & 35.7 & 34.9 & 33.3 & 12.5 & 12.8 & 17.7 \\
\hline \multirow[t]{2}{*}{655160} & 2 & 14.7 & 18.5 & 12.5 & 5.3 & 10.4 & 12.7 \\
\hline & 10 & 34.8 & 37.3 & 39.0 & 14.8 & 20.6 & 16.9 \\
\hline \multirow[t]{2}{*}{655030} & 2 & 15.4 & 14.7 & 12.9 & 3.9 & 3.3 & 9.7 \\
\hline & 10 & 35.5 & 33.0 & 32.7 & 11.5 & 8.0 & 22.9 \\
\hline \multirow[t]{2}{*}{655020} & 2 & 14.0 & 16.0 & 11.9 & 4.1 & 5.1 & 2.9 \\
\hline & 10 & 32.9 & 31.7 & 32.5 & 10.4 & 14.2 & 20.1 \\
\hline \multirow[t]{2}{*}{655070} & 2 & 16.6 & 17.2 & 12.1 & 5.1 & 7.4 & 16.0 \\
\hline & 10 & 32.5 & 38.7 & 35.4 & 16.9 & 13.3 & 22.9 \\
\hline \multirow[t]{2}{*}{655010} & 2 & 12.1 & 14.9 & 8.9 & 2.3 & 11.0 & 19.1 \\
\hline & 10 & 29.1 & 24.2 & 21.3 & 7.3 & 17.6 & 25.2 \\
\hline
\end{tabular}


GCM - CGCM3; Downscaling Method - LARS-WG

SRES A2 (2046 - 2065)

DRWH Reliability (\%)

per capita roof area

\begin{tabular}{|c|c|c|c|c|c|c|c|}
\hline Gauge & sq. m & Jan & Feb & Mar & Apr & May & Jun \\
\hline \multirow[t]{2}{*}{655920} & 2 & 4.9 & 3.1 & 7.6 & 9.1 & 20.3 & 25.5 \\
\hline & 10 & 11.5 & 8.8 & 16.0 & 18.8 & 33.3 & 43.2 \\
\hline \multirow[t]{2}{*}{655940} & 2 & 4.1 & 3.2 & 7.3 & 10.3 & 16.2 & 20.4 \\
\hline & 10 & 8.4 & 7.2 & 14.5 & 19.3 & 30.1 & 43.9 \\
\hline \multirow[t]{2}{*}{655990} & 2 & 4.0 & 3.2 & 5.6 & 8.9 & 14.8 & 21.0 \\
\hline & 10 & 8.9 & 8.8 & 17.2 & 17.2 & 30.4 & 41.9 \\
\hline \multirow[t]{2}{*}{655780} & 2 & 2.5 & 2.6 & 5.9 & 9.1 & 17.7 & 20.8 \\
\hline & 10 & 5.6 & 6.1 & 15.6 & 18.1 & 27.1 & 33.5 \\
\hline \multirow[t]{2}{*}{655850} & 2 & 4.5 & 5.9 & 8.1 & 9.0 & 15.4 & 19.6 \\
\hline & 10 & 8.3 & 10.5 & 17.6 & 18.3 & 26.6 & 33.7 \\
\hline \multirow[t]{2}{*}{655480} & 2 & 20.6 & 18.0 & 22.7 & 22.3 & 25.6 & 28.8 \\
\hline & 10 & 40.9 & 41.2 & 46.8 & 50.0 & 44.3 & 45.8 \\
\hline \multirow[t]{2}{*}{655600} & 2 & 6.5 & 7.2 & 10.5 & 11.2 & 15.6 & 10.5 \\
\hline & 10 & 11.4 & 11.2 & 21.3 & 20.7 & 36.6 & 27.2 \\
\hline \multirow[t]{2}{*}{655570} & 2 & 8.4 & 8.1 & 13.1 & 12.6 & 13.4 & 11.5 \\
\hline & 10 & 17.3 & 13.4 & 28.1 & 27.0 & 46.7 & 35.9 \\
\hline \multirow[t]{2}{*}{655630} & 2 & 7.7 & 4.3 & 7.8 & 8.0 & 12.0 & 12.0 \\
\hline & 10 & 12.2 & 7.9 & 22.9 & 19.9 & 34.9 & 30.9 \\
\hline \multirow[t]{2}{*}{655620} & 2 & 6.0 & 6.1 & 11.3 & 10.1 & 14.7 & 12.3 \\
\hline & 10 & 10.9 & 10.8 & 23.0 & 19.1 & 37.2 & 30.0 \\
\hline \multirow[t]{2}{*}{655550} & 2 & 8.4 & 5.4 & 8.5 & 10.9 & 10.9 & 10.1 \\
\hline & 10 & 14.3 & 9.0 & 21.0 & 20.6 & 31.4 & 23.6 \\
\hline \multirow[t]{2}{*}{655450} & 2 & 6.7 & 5.0 & 10.0 & 12.3 & 19.5 & 15.6 \\
\hline & 10 & 10.8 & 9.4 & 17.7 & 19.4 & 41.9 & 28.9 \\
\hline \multirow[t]{2}{*}{654720} & 2 & 3.9 & 5.4 & 8.4 & 10.6 & 13.2 & 12.5 \\
\hline & 10 & 9.1 & 11.4 & 14.4 & 18.5 & 22.0 & 21.3 \\
\hline \multirow[t]{2}{*}{653870} & 2 & 1.6 & 2.4 & 5.7 & 7.5 & 10.4 & 12.5 \\
\hline & 10 & 4.5 & 5.9 & 11.5 & 12.4 & 20.4 & 22.7 \\
\hline \multirow[t]{2}{*}{653760} & 2 & 2.8 & 1.2 & 5.2 & 8.1 & 12.4 & 14.2 \\
\hline & 10 & 9.0 & 6.8 & 11.8 & 14.8 & 21.4 & 26.4 \\
\hline \multirow[t]{2}{*}{653800} & 2 & 5.9 & 6.2 & 11.6 & 11.5 & 16.2 & 12.9 \\
\hline & 10 & 12.4 & 13.0 & 18.9 & 24.5 & 26.9 & 20.9 \\
\hline \multirow[t]{2}{*}{653440} & 2 & 2.9 & 3.6 & 5.5 & 11.4 & 18.1 & 17.9 \\
\hline & 10 & 4.2 & 5.6 & 10.0 & 25.1 & 31.2 & 25.6 \\
\hline \multirow[t]{2}{*}{653380} & 2 & 2.2 & 2.6 & 5.3 & 8.8 & 13.8 & 12.2 \\
\hline & 10 & 5.2 & 5.9 & 12.2 & 23.3 & 26.8 & 20.2 \\
\hline \multirow[t]{2}{*}{653350} & 2 & 2.5 & 4.2 & 7.5 & 8.7 & 9.0 & 11.8 \\
\hline & 10 & 5.0 & 7.0 & 12.9 & 24.3 & 21.6 & 20.2 \\
\hline \multirow[t]{2}{*}{655280} & 2 & 3.5 & 4.7 & 5.3 & 6.7 & 6.3 & 11.1 \\
\hline & 10 & 8.5 & 8.4 & 9.3 & 16.1 & 19.9 & 23.6 \\
\hline
\end{tabular}


GCM - CGCM3; Downscaling Method - LARS-WG

SRES A2 (2046 - 2065)

DRWH Reliability (\%)

\begin{tabular}{|c|c|c|c|c|c|c|c|}
\hline \multirow{2}{*}{ Gauge } & \multicolumn{7}{|c|}{ per capita roof area } \\
\hline & sq. $m$ & Jul & Aug & Sep & Oct & Nov & Dec \\
\hline \multirow[t]{2}{*}{655920} & 2 & 13.1 & 13.5 & 16.2 & 11.9 & 8.8 & 14.5 \\
\hline & 10 & 25.4 & 32.2 & 34.6 & 28.6 & 23.5 & 28.2 \\
\hline \multirow[t]{2}{*}{655940} & 2 & 10.4 & 4.8 & 5.9 & 8.3 & 8.7 & 11.2 \\
\hline & 10 & 23.0 & 17.2 & 19.6 & 20.0 & 27.4 & 27.4 \\
\hline \multirow[t]{2}{*}{655990} & 2 & 9.9 & 4.7 & 5.3 & 8.2 & 8.6 & 16.4 \\
\hline & 10 & 20.8 & 14.4 & 14.5 & 18.8 & 21.5 & 30.7 \\
\hline \multirow[t]{2}{*}{655780} & 2 & 9.6 & 4.5 & 6.7 & 9.7 & 9.2 & 10.8 \\
\hline & 10 & 22.5 & 12.0 & 14.3 & 27.1 & 27.3 & 20.6 \\
\hline \multirow[t]{2}{*}{655850} & 2 & 12.1 & 6.9 & 9.6 & 13.4 & 9.0 & 9.0 \\
\hline & 10 & 28.2 & 19.0 & 20.8 & 31.6 & 27.7 & 18.9 \\
\hline \multirow[t]{2}{*}{655480} & 2 & 23.6 & 24.8 & 32.7 & 29.9 & 28.7 & 22.5 \\
\hline & 10 & 42.9 & 61.5 & 70.6 & 66.9 & 60.5 & 49.5 \\
\hline \multirow[t]{2}{*}{655600} & 2 & 9.5 & 9.4 & 16.0 & 7.6 & 4.9 & 9.3 \\
\hline & 10 & 24.0 & 20.6 & 27.7 & 11.3 & 9.2 & 11.8 \\
\hline \multirow[t]{2}{*}{655570} & 2 & 6.9 & 7.0 & 12.8 & 10.4 & 9.3 & 11.0 \\
\hline & 10 & 21.6 & 16.9 & 22.4 & 14.7 & 20.6 & 18.1 \\
\hline \multirow[t]{2}{*}{655630} & 2 & 8.0 & 7.5 & 12.4 & 7.9 & 4.5 & 5.6 \\
\hline & 10 & 20.2 & 15.6 & 21.4 & 11.6 & 11.4 & 12.8 \\
\hline \multirow[t]{2}{*}{655620} & 2 & 7.0 & 6.0 & 13.5 & 9.1 & 6.6 & 9.7 \\
\hline & 10 & 21.3 & 14.5 & 21.4 & 12.4 & 11.6 & 12.8 \\
\hline \multirow[t]{2}{*}{655550} & 2 & 10.0 & 11.5 & 16.8 & 7.6 & 4.2 & 7.9 \\
\hline & 10 & 21.0 & 21.7 & 25.3 & 9.9 & 8.3 & 11.1 \\
\hline \multirow[t]{2}{*}{655450} & 2 & 10.0 & 8.5 & 14.4 & 8.4 & 3.5 & 10.7 \\
\hline & 10 & 19.6 & 15.4 & 25.7 & 14.2 & 10.6 & 19.7 \\
\hline \multirow[t]{2}{*}{654720} & 2 & 7.6 & 2.6 & 8.2 & 4.8 & 4.4 & 10.3 \\
\hline & 10 & 13.7 & 5.7 & 14.7 & 6.5 & 7.1 & 18.3 \\
\hline \multirow[t]{2}{*}{653870} & 2 & 7.1 & 3.3 & 8.8 & 4.3 & 3.3 & 8.7 \\
\hline & 10 & 13.8 & 7.1 & 16.2 & 5.7 & 5.4 & 14.1 \\
\hline \multirow[t]{2}{*}{653760} & 2 & 13.6 & 11.9 & 20.3 & 5.8 & 4.5 & 10.1 \\
\hline & 10 & 26.8 & 21.2 & 30.1 & 7.1 & 7.5 & 17.3 \\
\hline \multirow[t]{2}{*}{653800} & 2 & 12.7 & 6.1 & 8.7 & 12.5 & 8.4 & 17.3 \\
\hline & 10 & 28.2 & 15.7 & 16.0 & 20.2 & 14.5 & 22.3 \\
\hline \multirow[t]{2}{*}{653440} & 2 & 12.3 & 7.4 & 9.5 & 10.5 & 5.6 & 8.9 \\
\hline & 10 & 28.0 & 16.7 & 15.6 & 16.3 & 10.3 & 12.5 \\
\hline \multirow[t]{2}{*}{653380} & 2 & 14.2 & 10.9 & 12.3 & 11.6 & 5.1 & 10.2 \\
\hline & 10 & 36.5 & 24.1 & 18.6 & 14.8 & 8.0 & 15.5 \\
\hline \multirow[t]{2}{*}{653350} & 2 & 14.5 & 14.3 & 14.5 & 10.8 & 3.9 & 4.7 \\
\hline & 10 & 36.6 & 29.5 & 24.5 & 18.1 & 8.2 & 11.8 \\
\hline \multirow[t]{2}{*}{655280} & 2 & 17.0 & 17.7 & 14.8 & 10.0 & 7.8 & 5.8 \\
\hline & 10 & 31.8 & 48.0 & 40.0 & 35.6 & 17.2 & 10.9 \\
\hline
\end{tabular}


GCM - CGCM3; Downscaling Method - LARS-WG

SRES A2 (2046 - 2065)

DRWH Reliability (\%)

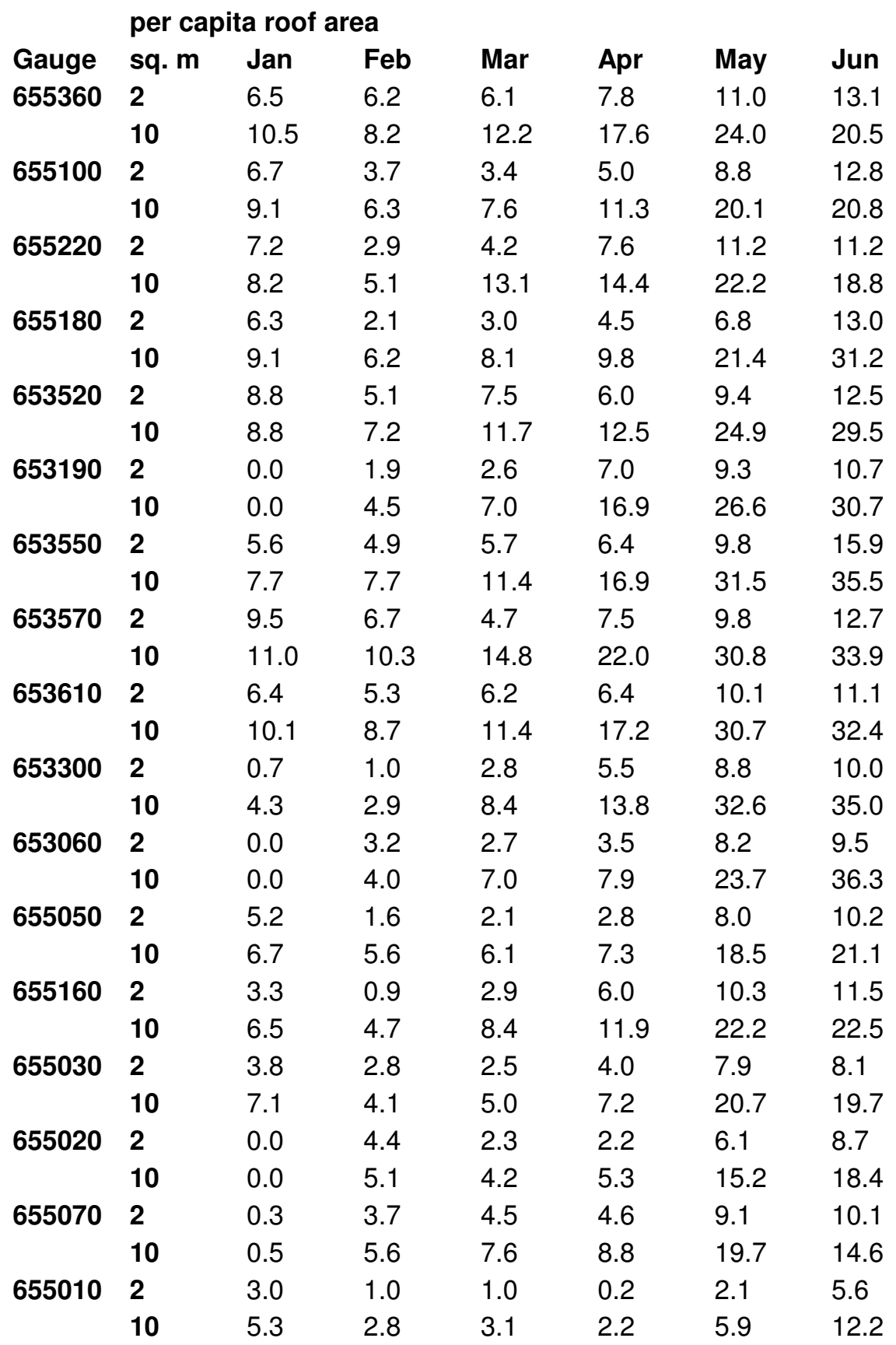


GCM - CGCM3; Downscaling Method - LARS-WG

SRES A2 (2046 - 2065)

DRWH Reliability (\%)

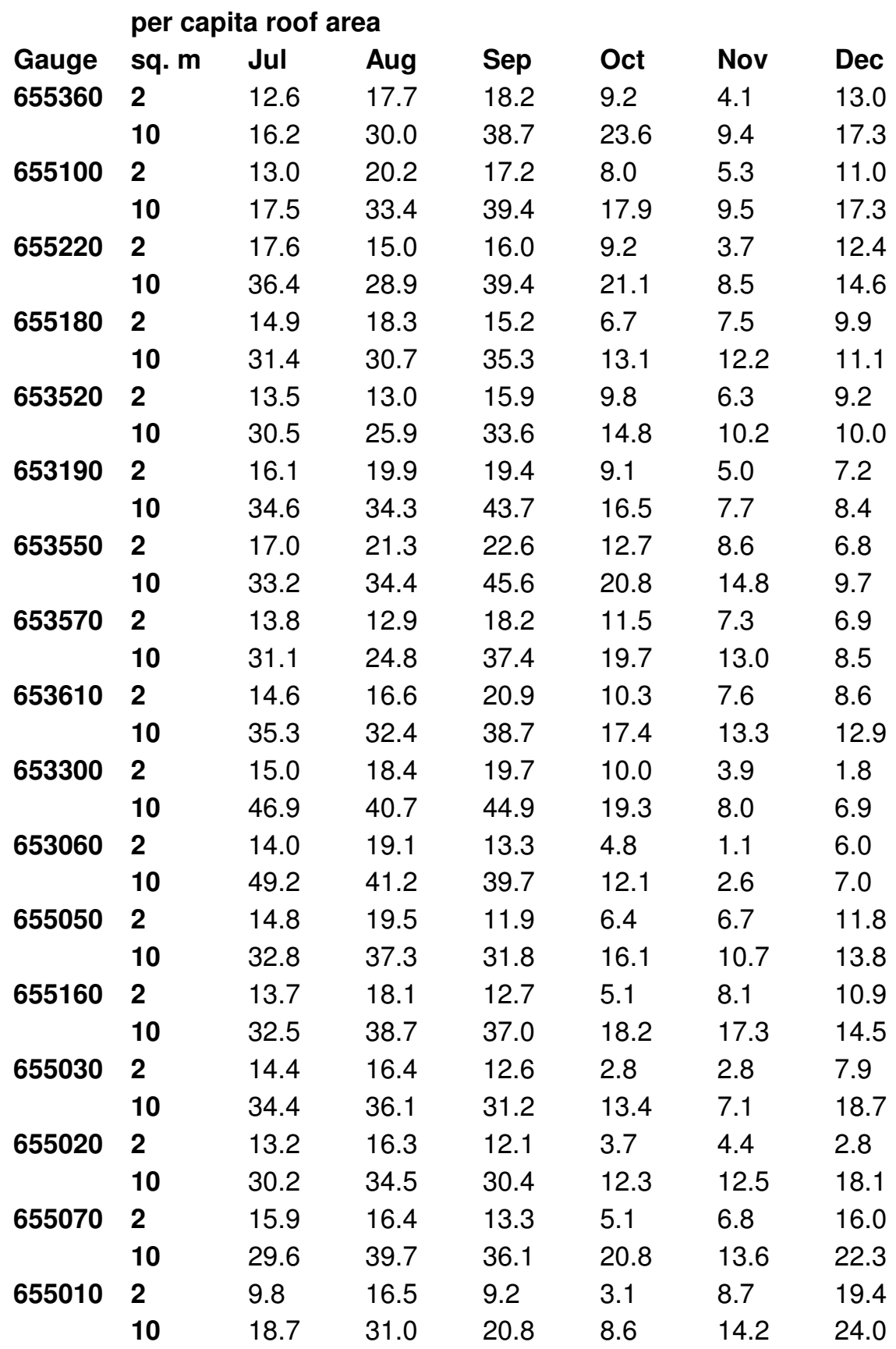


GCM - CGCM3; Downscaling Method - LARS-WG

SRES B1 (2046 - 2065)

DRWH Reliability (\%)

per capita roof area

\begin{tabular}{|c|c|c|c|c|c|c|c|}
\hline Gauge & sq. m & Jan & Feb & Mar & Apr & May & Jun \\
\hline \multirow[t]{2}{*}{655920} & 2 & 6.2 & 3.1 & 6.5 & 10.2 & 18.9 & 25.2 \\
\hline & 10 & 14.0 & 9.5 & 18.5 & 24.2 & 37.6 & 48.9 \\
\hline \multirow[t]{2}{*}{655940} & 2 & 4.7 & 2.5 & 6.3 & 10.9 & 15.0 & 18.4 \\
\hline & 10 & 10.8 & 6.9 & 17.5 & 19.3 & 24.1 & 38.3 \\
\hline \multirow[t]{2}{*}{655990} & 2 & 5.9 & 3.4 & 6.5 & 10.0 & 14.1 & 19.2 \\
\hline & 10 & 12.2 & 9.3 & 21.1 & 18.6 & 24.2 & 38.4 \\
\hline \multirow[t]{2}{*}{655780} & 2 & 2.7 & 3.0 & 6.8 & 10.5 & 17.0 & 20.1 \\
\hline & 10 & 7.3 & 7.5 & 17.5 & 22.6 & 30.1 & 31.0 \\
\hline \multirow[t]{2}{*}{655850} & 2 & 5.1 & 6.0 & 8.1 & 9.4 & 14.9 & 18.2 \\
\hline & 10 & 10.3 & 11.7 & 19.9 & 22.0 & 29.5 & 29.8 \\
\hline \multirow[t]{2}{*}{655480} & 2 & 13.5 & 12.5 & 21.3 & 22.7 & 21.2 & 22.9 \\
\hline & 10 & 34.5 & 35.2 & 44.2 & 44.1 & 44.7 & 44.6 \\
\hline \multirow[t]{2}{*}{655600} & 2 & 3.4 & 5.5 & 9.2 & 12.4 & 17.9 & 11.9 \\
\hline & 10 & 8.0 & 9.2 & 21.3 & 21.3 & 34.6 & 24.4 \\
\hline \multirow[t]{2}{*}{655570} & 2 & 4.4 & 6.3 & 13.1 & 13.2 & 15.0 & 14.3 \\
\hline & 10 & 10.6 & 11.2 & 30.5 & 26.8 & 44.1 & 31.6 \\
\hline \multirow[t]{2}{*}{655630} & 2 & 4.6 & 3.5 & 7.5 & 8.9 & 13.7 & 15.0 \\
\hline & 10 & 7.6 & 6.8 & 23.9 & 19.7 & 31.6 & 25.8 \\
\hline \multirow[t]{2}{*}{655620} & 2 & 3.3 & 4.4 & 10.9 & 11.3 & 17.1 & 14.2 \\
\hline & 10 & 7.0 & 9.2 & 25.8 & 19.1 & 33.9 & 25.2 \\
\hline \multirow[t]{2}{*}{655550} & 2 & 4.9 & 4.0 & 9.1 & 11.9 & 12.1 & 11.8 \\
\hline & 10 & 9.2 & 7.8 & 23.7 & 21.2 & 27.4 & 21.4 \\
\hline \multirow[t]{2}{*}{655450} & 2 & 3.8 & 3.9 & 8.1 & 10.3 & 17.8 & 16.6 \\
\hline & 10 & 7.2 & 7.6 & 15.8 & 19.7 & 37.4 & 26.8 \\
\hline \multirow[t]{2}{*}{654720} & 2 & 2.7 & 4.4 & 10.4 & 10.9 & 16.3 & 14.4 \\
\hline & 10 & 6.6 & 9.2 & 16.8 & 18.1 & 29.2 & 24.2 \\
\hline \multirow[t]{2}{*}{653870} & 2 & 1.3 & 2.0 & 6.6 & 7.7 & 12.8 & 14.0 \\
\hline & 10 & 3.9 & 4.4 & 13.3 & 12.1 & 27.5 & 24.5 \\
\hline \multirow[t]{2}{*}{653760} & 2 & 1.9 & 1.4 & 7.3 & 8.9 & 14.4 & 15.3 \\
\hline & 10 & 6.8 & 5.9 & 15.7 & 15.6 & 29.5 & 28.5 \\
\hline \multirow[t]{2}{*}{653800} & 2 & 3.4 & 5.2 & 12.1 & 11.5 & 17.9 & 14.0 \\
\hline & 10 & 9.8 & 12.0 & 23.4 & 21.7 & 30.6 & 23.1 \\
\hline \multirow[t]{2}{*}{653440} & 2 & 2.2 & 2.4 & 6.5 & 12.2 & 19.2 & 18.4 \\
\hline & 10 & 3.7 & 4.0 & 12.8 & 23.3 & 34.6 & 27.1 \\
\hline \multirow[t]{2}{*}{653380} & 2 & 1.4 & 2.4 & 6.4 & 9.1 & 14.7 & 12.6 \\
\hline & 10 & 3.9 & 5.4 & 14.9 & 21.4 & 31.3 & 22.0 \\
\hline \multirow[t]{2}{*}{653350} & 2 & 1.3 & 3.4 & 7.0 & 8.1 & 10.4 & 11.7 \\
\hline & 10 & 4.1 & 5.6 & 14.3 & 21.6 & 26.9 & 21.8 \\
\hline \multirow[t]{2}{*}{655280} & 2 & 2.2 & 2.0 & 5.1 & 7.4 & 7.3 & 11.2 \\
\hline & 10 & 6.7 & 5.0 & 8.2 & 15.4 & 20.4 & 28.1 \\
\hline
\end{tabular}


GCM - CGCM3; Downscaling Method - LARS-WG

SRES B1 (2046 - 2065)

DRWH Reliability (\%)

per capita roof area

$\begin{array}{llllllll}\text { Gauge } & \text { sq. } \mathbf{m} & \text { Jul } & \text { Aug } & \text { Sep } & \text { Oct } & \text { Nov } & \text { Dec } \\ 655920 & \mathbf{2} & 11.6 & 11.5 & 12.6 & 11.5 & 9.6 & 13.0\end{array}$

$\begin{array}{llllllll}655920 & 10 & 30.8 & 28.8 & 33.8 & 30.3 & 23.3 & 24.2\end{array}$

$\begin{array}{llllllll}655940 & 2 & 10.1 & 4.9 & 4.9 & 8.1 & 8.5 & 9.5\end{array}$

$\begin{array}{lllllll}10 & 25.0 & 17.2 & 19.1 & 20.8 & 21.5 & 19.9\end{array}$

$\begin{array}{llllllll}655990 & 2 & 9.4 & 4.2 & 4.6 & 8.1 & 8.3 & 13.3\end{array}$

$\begin{array}{lllllll}10 & 22.8 & 13.9 & 14.4 & 21.1 & 16.6 & 21.3\end{array}$

$\begin{array}{llllllll}655780 & 2 & 9.1 & 4.1 & 6.9 & 10.8 & 9.7 & 9.5\end{array}$

$\begin{array}{lllllll}10 & 18.9 & 14.1 & 14.4 & 26.5 & 18.8 & 18.6\end{array}$

$\begin{array}{llllllll}655850 & 2 & 10.5 & 8.2 & 9.2 & 13.8 & 8.2 & 8.9\end{array}$

$\begin{array}{lllllll}10 & 22.4 & 22.2 & 19.8 & 30.2 & 19.8 & 18.2\end{array}$

$\begin{array}{llllllll}655480 & 2 & 18.4 & 23.5 & 36.9 & 49.7 & 37.1 & 21.9\end{array}$

$\begin{array}{lllllll}10 & 41.1 & 60.3 & 70.4 & 70.7 & 59.1 & 47.5\end{array}$

$\begin{array}{llllllll}655600 & 2 & 9.8 & 10.9 & 14.6 & 8.4 & 4.6 & 8.4\end{array}$

$\begin{array}{lllllll}10 & 21.6 & 24.3 & 27.4 & 12.7 & 9.2 & 12.7\end{array}$

$\begin{array}{llllllll}655570 & 2 & 6.9 & 7.0 & 12.6 & 11.4 & 8.9 & 8.9\end{array}$

$\begin{array}{lllllll}10 & 19.5 & 17.7 & 24.1 & 16.7 & 20.1 & 17.4\end{array}$

$\begin{array}{llllllll}655630 & 2 & 8.1 & 7.8 & 11.5 & 9.2 & 4.9 & 5.4\end{array}$

$\begin{array}{lllllll}10 & 18.1 & 17.1 & 21.2 & 13.8 & 12.3 & 11.8\end{array}$

$\begin{array}{llllllll}655620 & 2 & 7.4 & 5.7 & 12.1 & 9.9 & 5.7 & 6.9\end{array}$

$\begin{array}{lllllll}10 & 18.0 & 14.7 & 20.9 & 13.9 & 10.7 & 12.1\end{array}$

$\begin{array}{llllllll}655550 & 2 & 10.6 & 12.8 & 16.9 & 8.9 & 4.1 & 6.1\end{array}$

$\begin{array}{lllllll}10 & 20.0 & 24.2 & 26.7 & 12.2 & 8.9 & 11.2\end{array}$

$\begin{array}{llllllll}655450 & 2 & 10.4 & 10.2 & 15.1 & 8.9 & 3.2 & 10.7\end{array}$

$\begin{array}{lllllll}10 & 18.0 & 19.9 & 26.7 & 17.8 & 10.4 & 19.9\end{array}$

$\begin{array}{llllllll}654720 & 2 & 8.7 & 3.3 & 6.3 & 10.2 & 6.5 & 9.4\end{array}$

$\begin{array}{lllllll}10 & 12.8 & 6.6 & 13.2 & 14.4 & 11.4 & 19.4\end{array}$

$\begin{array}{llllllll}653870 & 2 & 7.7 & 3.8 & 6.7 & 9.5 & 5.9 & 6.4\end{array}$

$\begin{array}{lllllll}10 & 12.9 & 7.9 & 14.7 & 12.7 & 9.7 & 14.0\end{array}$

$\begin{array}{llllllll}653760 & 2 & 15.0 & 14.7 & 18.0 & 13.4 & 4.7 & 8.2\end{array}$

$\begin{array}{lllllll}10 & 25.1 & 23.3 & 30.6 & 18.5 & 8.7 & 16.9\end{array}$

$\begin{array}{llllllll}653800 & 2 & 14.3 & 6.8 & 9.6 & 12.3 & 7.4 & 12.4\end{array}$

$\begin{array}{lllllll}10 & 30.2 & 16.0 & 16.7 & 19.8 & 13.5 & 17.9\end{array}$

$\begin{array}{llllllll}653440 & 2 & 13.5 & 8.1 & 9.4 & 10.9 & 5.1 & 6.8\end{array}$

$\begin{array}{lllllll}10 & 30.3 & 17.6 & 15.3 & 17.2 & 9.6 & 9.9\end{array}$

$\begin{array}{llllllll}653380 & 2 & 16.5 & 11.9 & 11.9 & 11.4 & 5.1 & 6.2\end{array}$

$\begin{array}{lllllll}10 & 38.1 & 24.4 & 17.5 & 15.0 & 7.4 & 11.2\end{array}$

$\begin{array}{llllllll}653350 & 2 & 16.3 & 15.4 & 14.9 & 11.1 & 3.8 & 1.8\end{array}$

$\begin{array}{lllllll}10 & 38.5 & 29.4 & 23.1 & 17.4 & 7.3 & 8.8\end{array}$

$\begin{array}{llllllll}655280 & 2 & 16.8 & 20.8 & 14.6 & 12.0 & 4.7 & 4.6\end{array}$

$\begin{array}{lllllll}10 & 33.9 & 48.5 & 43.6 & 30.3 & 13.6 & 11.1\end{array}$


GCM - CGCM3; Downscaling Method - LARS-WG

SRES B1 (2046 - 2065)

DRWH Reliability (\%)

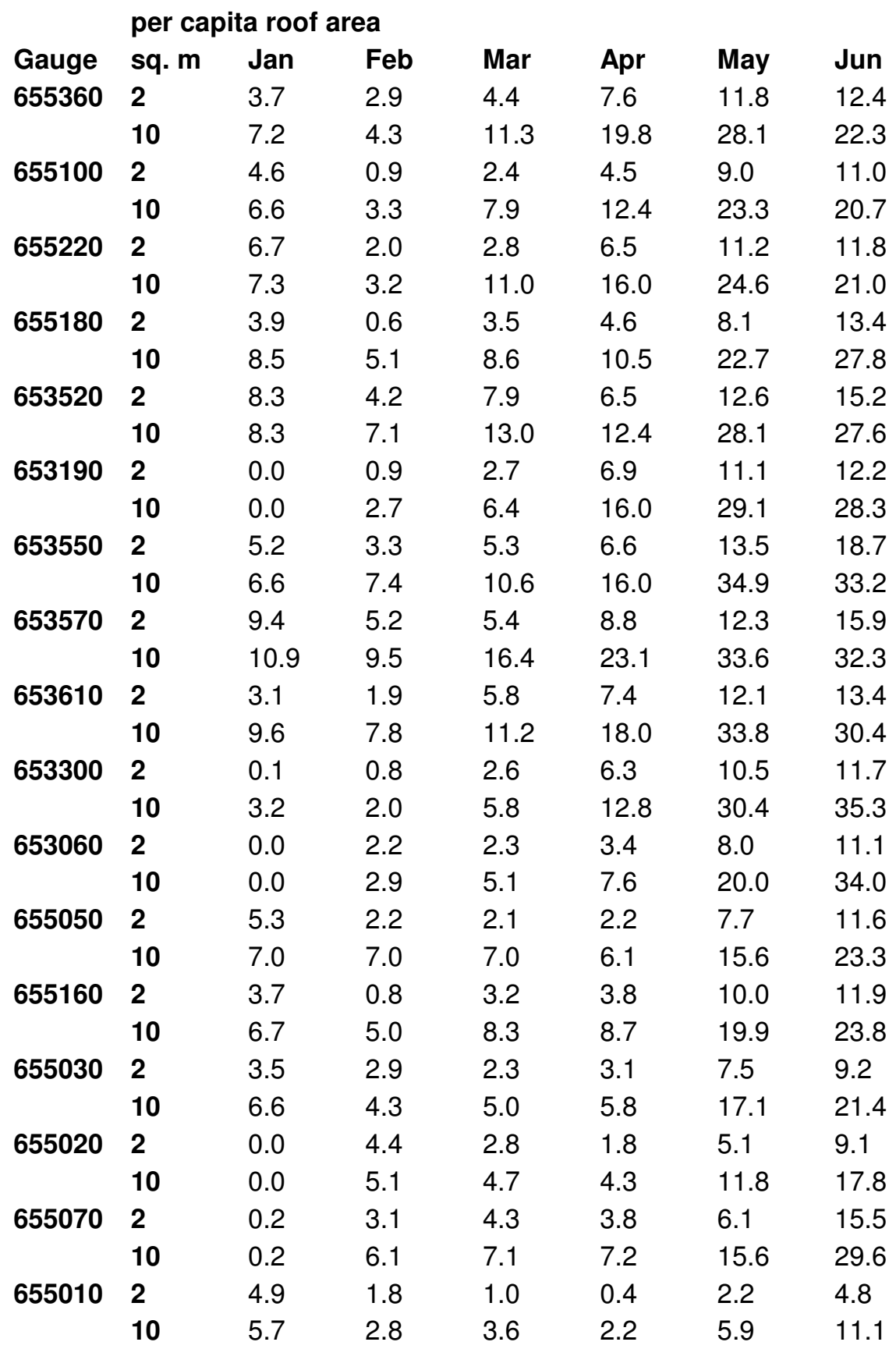


GCM - CGCM3; Downscaling Method - LARS-WG

SRES B1 (2046 - 2065)

DRWH Reliability (\%)

\begin{tabular}{|c|c|c|c|c|c|c|c|}
\hline \multirow{3}{*}{$\begin{array}{l}\text { Gauge } \\
655360\end{array}$} & \multicolumn{7}{|c|}{ per capita roof area } \\
\hline & sq. $m$ & Jul & Aug & Sep & Oct & Nov & Dec \\
\hline & 2 & 14.1 & 19.9 & 17.5 & 10.1 & 3.1 & 12.8 \\
\hline \multirow{3}{*}{655100} & 10 & 26.3 & 39.9 & 36.3 & 24.2 & 8.1 & 19.8 \\
\hline & 2 & 13.8 & 22.8 & 16.1 & 8.4 & 4.0 & 8.7 \\
\hline & 10 & 27.7 & 45.6 & 37.3 & 17.3 & 7.0 & 18.0 \\
\hline \multirow[t]{2}{*}{655220} & 2 & 16.0 & 16.6 & 17.0 & 10.1 & 4.5 & 12.0 \\
\hline & 10 & 33.1 & 31.0 & 32.8 & 20.1 & 8.9 & 13.6 \\
\hline \multirow[t]{2}{*}{655180} & 2 & 15.4 & 20.5 & 16.5 & 8.0 & 5.7 & 12.3 \\
\hline & 10 & 32.9 & 34.6 & 29.5 & 14.2 & 12.8 & 12.9 \\
\hline \multirow[t]{2}{*}{653520} & 2 & 14.3 & 13.9 & 15.1 & 10.5 & 6.6 & 9.2 \\
\hline & 10 & 31.3 & 29.3 & 27.9 & 15.8 & 10.8 & 10.5 \\
\hline \multirow[t]{2}{*}{653190} & 2 & 17.8 & 22.7 & 20.8 & 11.0 & 4.8 & 4.2 \\
\hline & 10 & 36.4 & 38.4 & 38.4 & 19.4 & 7.6 & 9.1 \\
\hline \multirow[t]{2}{*}{653550} & 2 & 18.9 & 23.4 & 23.9 & 14.6 & 6.9 & 6.0 \\
\hline & 10 & 35.5 & 39.1 & 40.3 & 23.6 & 13.1 & 8.0 \\
\hline \multirow[t]{2}{*}{653570} & 2 & 14.4 & 15.1 & 17.0 & 11.8 & 5.8 & 6.8 \\
\hline & 10 & 31.9 & 28.9 & 31.2 & 20.9 & 11.4 & 8.8 \\
\hline \multirow[t]{2}{*}{653610} & 2 & 16.0 & 17.7 & 19.4 & 10.9 & 6.7 & 6.8 \\
\hline & 10 & 37.1 & 35.6 & 32.7 & 18.9 & 13.2 & 11.5 \\
\hline \multirow[t]{2}{*}{653300} & 2 & 17.0 & 20.7 & 19.3 & 9.5 & 3.3 & 0.5 \\
\hline & 10 & 42.2 & 33.6 & 37.6 & 23.5 & 8.6 & 6.0 \\
\hline \multirow[t]{2}{*}{653060} & 2 & 16.6 & 20.1 & 14.8 & 3.8 & 1.1 & 6.1 \\
\hline & 10 & 43.6 & 32.3 & 32.6 & 13.1 & 3.4 & 6.3 \\
\hline \multirow[t]{2}{*}{655050} & 2 & 16.3 & 18.9 & 13.2 & 6.2 & 5.5 & 12.2 \\
\hline & 10 & 38.7 & 34.7 & 30.9 & 12.8 & 9.2 & 13.7 \\
\hline \multirow[t]{2}{*}{655160} & 2 & 14.8 & 17.5 & 13.8 & 6.0 & 6.3 & 10.2 \\
\hline & 10 & 37.1 & 35.9 & 36.8 & 17.2 & 14.0 & 12.7 \\
\hline \multirow[t]{2}{*}{655030} & 2 & 15.3 & 16.0 & 13.3 & 4.3 & 2.2 & 7.0 \\
\hline & 10 & 39.0 & 32.4 & 30.5 & 13.0 & 6.4 & 18.5 \\
\hline \multirow[t]{2}{*}{655020} & 2 & 15.4 & 16.1 & 12.4 & 4.4 & 3.2 & 1.9 \\
\hline & 10 & 36.2 & 30.9 & 30.0 & 11.1 & 8.6 & 16.2 \\
\hline \multirow[t]{2}{*}{655070} & 2 & 22.1 & 19.3 & 12.6 & 5.1 & 4.5 & 11.8 \\
\hline & 10 & 31.3 & 38.8 & 39.7 & 20.2 & 10.4 & 15.4 \\
\hline \multirow[t]{2}{*}{655010} & 2 & 11.1 & 15.6 & 9.0 & 2.9 & 6.6 & 16.2 \\
\hline & 10 & 22.8 & 26.1 & 21.8 & 9.0 & 9.1 & 20.0 \\
\hline
\end{tabular}


GCM - MIROC; Downscaling Method - LARS-WG

SRES A1b (2046 - 2065)

DRWH Reliability (\%)

per capita roof area

$\begin{array}{llllllll}\text { Gauge } & \text { sq. } \mathbf{m} & \text { Jan } & \text { Feb } & \text { Mar } & \text { Apr } & \text { May } & \text { Jun } \\ \mathbf{6 5 5 9 2 0} & \mathbf{2} & 8.1 & 2.3 & 5.1 & 8.2 & 20.6 & 24.1\end{array}$

$\begin{array}{llllllll}655920 & \mathbf{2} & 8.1 & 2.3 & 5.1 & 8.2 & 20.6 & 24.1 \\ & \mathbf{1 0} & 16.8 & 6.5 & 11.3 & 16.6 & 35.5 & 38.0\end{array}$

$\begin{array}{llllllll}655940 & 2 & 6.3 & 2.4 & 4.0 & 9.8 & 15.8 & 19.3\end{array}$

$\begin{array}{lllllll}10 & 13.3 & 5.1 & 7.6 & 17.3 & 28.0 & 37.6\end{array}$

$\begin{array}{llllllll}655990 & 2 & 7.9 & 2.2 & 4.3 & 8.6 & 16.2 & 19.5\end{array}$

$\begin{array}{lllllll}10 & 16.4 & 7.0 & 10.1 & 16.7 & 30.8 & 37.5\end{array}$

$\begin{array}{llllllll}655780 & 2 & 3.8 & 2.8 & 5.3 & 7.8 & 16.4 & 26.2\end{array}$

$\begin{array}{lllllll}10 & 8.8 & 6.5 & 12.8 & 15.0 & 30.0 & 45.5\end{array}$

$\begin{array}{llllllll}655850 & 2 & 7.1 & 5.1 & 7.6 & 7.2 & 14.5 & 21.7\end{array}$

$\begin{array}{lllllll}10 & 13.1 & 10.4 & 14.8 & 14.7 & 30.0 & 42.6\end{array}$

$\begin{array}{llllllll}655480 & 2 & 6.4 & 2.8 & 7.0 & 10.7 & 13.3 & 15.9\end{array}$

$\begin{array}{lllllll}10 & 11.5 & 7.0 & 14.4 & 18.8 & 22.7 & 32.7\end{array}$

$\begin{array}{llllllll}655600 & 2 & 5.2 & 5.6 & 7.5 & 9.7 & 14.2 & 11.2\end{array}$

$\begin{array}{lllllll}10 & 10.6 & 11.0 & 12.8 & 15.8 & 27.5 & 26.8\end{array}$

$\begin{array}{llllllll}655570 & 2 & 8.7 & 5.9 & 10.9 & 12.1 & 13.6 & 12.8\end{array}$

$\begin{array}{lllllll}10 & 16.7 & 12.4 & 19.0 & 20.4 & 36.8 & 35.1\end{array}$

$\begin{array}{llllllll}655630 & 2 & 8.3 & 3.8 & 5.8 & 9.1 & 12.0 & 13.7\end{array}$

$\begin{array}{lllllll}10 & 10.8 & 7.9 & 13.3 & 16.0 & 26.8 & 30.3\end{array}$

$\begin{array}{llllllll}655620 & 2 & 5.9 & 4.1 & 8.1 & 9.3 & 14.2 & 12.8\end{array}$

$\begin{array}{lllllll}10 & 10.4 & 10.2 & 14.8 & 13.8 & 27.3 & 27.9\end{array}$

$\begin{array}{llllllll}655550 & 2 & 8.5 & 4.4 & 7.3 & 10.9 & 9.5 & 10.5\end{array}$

$\begin{array}{lllllll}10 & 13.3 & 8.7 & 13.6 & 16.1 & 22.0 & 23.6\end{array}$

$\begin{array}{llllllll}655450 & 2 & 8.3 & 3.5 & 5.2 & 9.4 & 14.5 & 16.0\end{array}$

$\begin{array}{lllllll}10 & 11.5 & 6.6 & 9.1 & 14.2 & 23.5 & 27.9\end{array}$

$\begin{array}{llllllll}654720 & 2 & 3.4 & 2.6 & 6.3 & 9.7 & 13.6 & 14.5\end{array}$

$\begin{array}{lllllll}10 & 9.3 & 5.8 & 10.3 & 15.6 & 20.8 & 23.9\end{array}$

$\begin{array}{llllllll}653870 & 2 & 0.9 & 1.0 & 4.2 & 6.2 & 11.1 & 13.1\end{array}$

$\begin{array}{lllllll}10 & 5.5 & 2.8 & 7.7 & 10.0 & 19.7 & 23.9\end{array}$

$\begin{array}{llllllll}653760 & 2 & 1.5 & 0.6 & 4.9 & 9.1 & 13.0 & 16.6\end{array}$

$\begin{array}{lllllll}10 & 10.8 & 3.8 & 9.3 & 14.8 & 21.5 & 28.9\end{array}$

$\begin{array}{llllllll}653800 & 2 & 3.1 & 1.5 & 8.0 & 11.2 & 12.9 & 14.3\end{array}$

$\begin{array}{lllllll}10 & 11.4 & 6.9 & 13.2 & 18.5 & 18.8 & 25.4\end{array}$

$\begin{array}{llllllll}653440 & 2 & 2.9 & 1.5 & 3.3 & 8.9 & 14.2 & 20.5\end{array}$

$\begin{array}{lllllll}10 & 4.6 & 2.7 & 5.1 & 13.6 & 20.6 & 31.3\end{array}$

$\begin{array}{llllllll}653380 & 2 & 1.0 & 0.7 & 4.1 & 8.2 & 11.5 & 13.2\end{array}$

$\begin{array}{llllllll} & \mathbf{1 0} & 4.8 & 2.9 & 6.8 & 14.4 & 17.9 & 23.7 \\ \mathbf{6 5 3 3 5 5 0} & \mathbf{2} & 0.9 & 1.3 & 4.5 & 7.8 & 10.0 & 13.1 \\ & \mathbf{1 0} & 5.9 & 3.1 & 6.7 & 14.8 & 15.9 & 24.0 \\ \mathbf{6 5 5 2 8 0} & \mathbf{2} & 3.0 & 1.8 & 3.8 & 6.0 & 9.5 & 12.8 \\ & \mathbf{1 0} & 8.6 & 6.3 & 6.4 & 11.9 & 18.6 & 23.4\end{array}$


GCM - MIROC; Downscaling Method - LARS-WG

SRES A1b (2046 - 2065)

DRWH Reliability (\%)

per capita roof area

$\begin{array}{llllllll}\text { Gauge } & \text { sq. } \mathbf{m} & \text { Jul } & \text { Aug } & \text { Sep } & \text { Oct } & \text { Nov } & \text { Dec } \\ \mathbf{6 5 5 9 2 0} & \mathbf{2} & 11.7 & 10.0 & 11.5 & 10.6 & 11.0 & 16.1\end{array}$

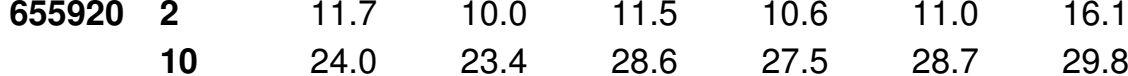

$\begin{array}{llllllll}655940 & 2 & 8.9 & 4.1 & 3.9 & 7.2 & 11.1 & 10.3\end{array}$

$\begin{array}{lllllll}10 & 19.3 & 14.1 & 14.9 & 18.5 & 30.9 & 25.3\end{array}$

$\begin{array}{llllllll}655990 & 2 & 8.6 & 3.4 & 3.7 & 7.6 & 9.6 & 15.2\end{array}$

$\begin{array}{lllllll}10 & 17.6 & 11.3 & 10.7 & 19.0 & 22.3 & 27.9\end{array}$

$\begin{array}{llllllll}655780 & 2 & 8.6 & 3.2 & 4.6 & 8.0 & 10.6 & 10.4\end{array}$

$\begin{array}{lllllll}10 & 18.9 & 8.3 & 10.8 & 22.4 & 28.8 & 23.5\end{array}$

$\begin{array}{llllllll}655850 & 2 & 11.0 & 4.9 & 6.6 & 11.5 & 10.9 & 10.3\end{array}$

$\begin{array}{lllllll}10 & 24.3 & 13.4 & 15.4 & 26.6 & 28.9 & 23.8\end{array}$

$\begin{array}{llllllll}655480 & 2 & 15.0 & 18.1 & 19.2 & 11.9 & 7.0 & 10.2\end{array}$

$\begin{array}{llllllll} & \mathbf{1 0} & 26.5 & 36.2 & 37.8 & 23.7 & 15.9 & 21.4 \\ \mathbf{6 5 5 6 0 0} & \mathbf{2} & 9.0 & 8.4 & 10.7 & 8.1 & 5.8 & 7.8\end{array}$

$\begin{array}{lllllll}10 & 18.1 & 18.3 & 27.7 & 19.5 & 16.3 & 15.1\end{array}$

$\begin{array}{llllllll}655570 & 2 & 7.2 & 6.3 & 9.8 & 12.0 & 11.8 & 8.5\end{array}$

$\begin{array}{llllllll} & \mathbf{1 0} & 16.1 & 14.0 & 25.3 & 27.8 & 30.0 & 18.1 \\ \mathbf{6 5 5 6 3 0} & \mathbf{2} & 8.8 & 7.3 & 8.7 & 8.9 & 6.8 & 5.8 \\ & \mathbf{1 0} & 15.4 & 14.0 & 22.0 & 21.5 & 19.8 & 13.0\end{array}$

$\begin{array}{llllllll}655620 & 2 & 8.2 & 5.4 & 9.9 & 11.8 & 7.0 & 8.0\end{array}$

$\begin{array}{lllllll}10 & 15.8 & 11.7 & 23.3 & 21.1 & 17.1 & 15.6\end{array}$

$\begin{array}{llllllll}655550 & 2 & 10.1 & 10.3 & 13.4 & 9.8 & 5.2 & 8.1\end{array}$

$\begin{array}{llllllll}10 & 16.4 & 18.7 & 28.0 & 19.2 & 14.7 & 16.7\end{array}$

$\begin{array}{llllllll}655450 & 2 & 9.4 & 8.2 & 13.7 & 9.5 & 5.8 & 10.2\end{array}$

$\begin{array}{lllllll}10 & 16.2 & 13.9 & 25.5 & 19.9 & 13.7 & 18.1\end{array}$

$\begin{array}{llllllll}654720 & 2 & 7.2 & 2.9 & 4.7 & 7.0 & 6.2 & 6.1\end{array}$

$\begin{array}{lllllll}10 & 12.1 & 6.2 & 12.2 & 17.8 & 14.9 & 18.4\end{array}$

$\begin{array}{llllllll}653870 & 2 & 7.5 & 3.3 & 5.5 & 6.4 & 5.3 & 4.5\end{array}$

$\begin{array}{lllllll}10 & 13.3 & 7.7 & 14.2 & 15.4 & 12.0 & 15.8\end{array}$

$\begin{array}{llllllll}653760 & 2 & 14.0 & 13.3 & 14.8 & 10.6 & 5.0 & 7.2\end{array}$

$\begin{array}{llllllll} & \mathbf{1 0} & 25.9 & 22.9 & 28.6 & 20.5 & 11.5 & 17.4 \\ \mathbf{6 5 3 8 0 0} & \mathbf{2} & 9.9 & 5.3 & 7.6 & 12.7 & 7.6 & 13.5 \\ & \mathbf{1 0} & 19.0 & 12.1 & 17.7 & 28.0 & 16.2 & 20.6\end{array}$

$\begin{array}{llllllll}653440 & 2 & 9.1 & 5.7 & 9.2 & 12.8 & 6.6 & 7.0\end{array}$

$\begin{array}{lllllll}10 & 19.1 & 11.1 & 17.1 & 25.1 & 12.9 & 11.5\end{array}$

$\begin{array}{llllllll}653380 & 2 & 12.4 & 8.3 & 10.6 & 15.3 & 7.0 & 6.1\end{array}$

$\begin{array}{llllllll} & \mathbf{1 0} & 23.9 & 17.6 & 21.2 & 24.0 & 10.2 & 13.1 \\ \mathbf{6 5 3 3 5 0} & \mathbf{2} & 11.9 & 11.2 & 12.9 & 12.4 & 4.2 & 1.1 \\ & \mathbf{1 0} & 23.0 & 21.1 & 27.0 & 24.6 & 8.8 & 11.7 \\ \mathbf{6 5 5 2 8 0} & \mathbf{2} & 18.7 & 23.3 & 19.0 & 13.0 & 9.3 & 4.8 \\ & \mathbf{1 0} & 35.3 & 42.0 & 36.9 & 33.4 & 16.6 & 10.9\end{array}$


GCM - MIROC; Downscaling Method - LARS-WG

SRES A1b (2046 - 2065)

DRWH Reliability (\%)

\begin{tabular}{|c|c|c|c|c|c|c|c|}
\hline \multirow[b]{2}{*}{ Gauge } & \multicolumn{7}{|c|}{ per capita roof area } \\
\hline & sq. $m$ & Jan & Feb & Mar & Apr & May & Jun \\
\hline \multirow[t]{2}{*}{655360} & 2 & 4.6 & 2.9 & 4.2 & 6.6 & 11.2 & 13.4 \\
\hline & 10 & 8.2 & 4.8 & 7.5 & 12.4 & 18.8 & 18.2 \\
\hline \multirow[t]{2}{*}{655100} & 2 & 5.0 & 0.9 & 2.5 & 4.3 & 8.1 & 12.1 \\
\hline & 10 & 6.5 & 3.1 & 5.1 & 7.8 & 15.1 & 17.2 \\
\hline \multirow[t]{2}{*}{655220} & 2 & 4.3 & 0.9 & 2.9 & 5.8 & 9.4 & 12.5 \\
\hline & 10 & 4.8 & 2.2 & 6.1 & 9.1 & 17.3 & 22.0 \\
\hline \multirow[t]{2}{*}{655180} & 2 & 1.3 & 0.2 & 2.1 & 3.2 & 6.4 & 11.1 \\
\hline & 10 & 6.2 & 1.9 & 4.9 & 6.8 & 12.6 & 20.6 \\
\hline \multirow[t]{2}{*}{653520} & 2 & 6.7 & 1.3 & 3.8 & 5.0 & 9.4 & 12.2 \\
\hline & 10 & 6.7 & 2.5 & 6.4 & 8.8 & 16.9 & 22.1 \\
\hline \multirow[t]{2}{*}{653190} & 2 & 0.0 & 0.0 & 1.8 & 4.8 & 8.9 & 11.6 \\
\hline & 10 & 0.0 & 0.6 & 4.3 & 10.2 & 16.4 & 24.0 \\
\hline \multirow[t]{2}{*}{653550} & 2 & 4.2 & 0.8 & 2.9 & 5.1 & 11.6 & 16.6 \\
\hline & 10 & 6.1 & 1.9 & 6.7 & 10.8 & 20.6 & 28.0 \\
\hline \multirow[t]{2}{*}{653570} & 2 & 7.4 & 1.6 & 3.1 & 6.9 & 10.0 & 13.5 \\
\hline & 10 & 9.1 & 3.5 & 9.8 & 17.0 & 19.9 & 24.5 \\
\hline \multirow[t]{2}{*}{653610} & 2 & 0.6 & 0.0 & 3.7 & 5.7 & 11.9 & 13.1 \\
\hline & 10 & 6.4 & 2.8 & 7.5 & 13.3 & 21.4 & 26.1 \\
\hline \multirow[t]{2}{*}{653300} & 2 & 0.0 & 0.1 & 0.7 & 2.9 & 9.8 & 11.1 \\
\hline & 10 & 1.2 & 0.4 & 1.3 & 5.1 & 17.0 & 21.4 \\
\hline \multirow[t]{2}{*}{653060} & 2 & 0.0 & 0.2 & 0.9 & 2.2 & 6.7 & 11.9 \\
\hline & 10 & 0.0 & 0.5 & 1.8 & 3.7 & 10.6 & 17.9 \\
\hline \multirow[t]{2}{*}{655050} & 2 & 3.5 & 0.3 & 1.5 & 2.0 & 5.5 & 7.6 \\
\hline & 10 & 5.1 & 1.8 & 5.4 & 5.5 & 12.3 & 15.8 \\
\hline \multirow[t]{2}{*}{655160} & 2 & 2.6 & 0.4 & 2.6 & 3.5 & 7.4 & 9.7 \\
\hline & 10 & 5.2 & 2.1 & 6.0 & 8.2 & 15.8 & 17.7 \\
\hline \multirow[t]{2}{*}{655030} & 2 & 2.6 & 0.5 & 2.2 & 2.7 & 4.9 & 6.7 \\
\hline & 10 & 5.9 & 2.3 & 3.8 & 4.8 & 12.8 & 15.9 \\
\hline \multirow[t]{2}{*}{655020} & 2 & 0.0 & 0.4 & 2.1 & 1.5 & 4.0 & 6.6 \\
\hline & 10 & 0.0 & 3.7 & 3.3 & 3.5 & 8.9 & 13.0 \\
\hline \multirow[t]{2}{*}{655070} & 2 & 0.4 & 0.6 & 2.8 & 3.5 & 5.2 & 9.7 \\
\hline & 10 & 0.4 & 1.9 & 5.0 & 6.5 & 11.2 & 19.7 \\
\hline \multirow[t]{2}{*}{655010} & 2 & 4.3 & 0.2 & 1.1 & 0.5 & 1.8 & 3.1 \\
\hline & 10 & 5.2 & 1.4 & 3.2 & 2.1 & 4.5 & 7.6 \\
\hline
\end{tabular}


GCM - MIROC; Downscaling Method - LARS-WG

SRES A1b (2046 - 2065)

DRWH Reliability (\%)

\begin{tabular}{|c|c|c|c|c|c|c|c|}
\hline \multirow{2}{*}{ Gauge } & \multicolumn{7}{|c|}{ per capita roof area } \\
\hline & sq. $\mathrm{m}$ & Jul & Aug & Sep & Oct & Nov & Dec \\
\hline \multirow[t]{2}{*}{655360} & 2 & 15.4 & 21.4 & 18.4 & 8.7 & 4.7 & 6.4 \\
\hline & 10 & 20.5 & 34.2 & 35.4 & 21.8 & 9.6 & 10.6 \\
\hline \multirow[t]{2}{*}{655100} & 2 & 16.0 & 25.4 & 17.7 & 8.2 & 5.4 & 6.5 \\
\hline & 10 & 21.8 & 39.9 & 36.1 & 17.1 & 9.8 & 10.9 \\
\hline \multirow[t]{2}{*}{655220} & 2 & 16.7 & 19.6 & 18.4 & 8.5 & 4.2 & 8.0 \\
\hline & 10 & 25.6 & 33.5 & 35.8 & 19.8 & 9.1 & 9.8 \\
\hline \multirow[t]{2}{*}{655180} & 2 & 17.3 & 22.6 & 17.8 & 8.5 & 6.5 & 3.6 \\
\hline & 10 & 26.8 & 34.4 & 34.6 & 17.3 & 12.7 & 20.7 \\
\hline \multirow[t]{2}{*}{653520} & 2 & 16.1 & 16.8 & 16.3 & 11.3 & 7.3 & 16.6 \\
\hline & 10 & 26.3 & 28.7 & 33.1 & 18.4 & 10.5 & 19.8 \\
\hline \multirow[t]{2}{*}{653190} & 2 & 18.4 & 23.9 & 23.8 & 12.1 & 5.7 & 2.1 \\
\hline & 10 & 31.0 & 38.1 & 44.2 & 21.7 & 8.0 & 10.8 \\
\hline \multirow[t]{2}{*}{653550} & 2 & 18.2 & 24.3 & 26.1 & 14.1 & 8.1 & 4.5 \\
\hline & 10 & 31.5 & 38.9 & 45.0 & 25.8 & 13.5 & 12.1 \\
\hline \multirow[t]{2}{*}{653570} & 2 & 16.5 & 16.6 & 19.4 & 13.7 & 6.3 & 6.9 \\
\hline & 10 & 28.7 & 27.9 & 36.1 & 26.7 & 12.1 & 14.9 \\
\hline \multirow[t]{2}{*}{653610} & 2 & 18.0 & 19.3 & 21.5 & 12.6 & 9.8 & 4.7 \\
\hline & 10 & 34.0 & 35.1 & 37.9 & 22.8 & 13.5 & 15.7 \\
\hline \multirow[t]{2}{*}{653300} & 2 & 14.4 & 18.7 & 21.1 & 9.4 & 4.0 & 0.9 \\
\hline & 10 & 26.2 & 30.4 & 35.4 & 17.6 & 7.0 & 4.0 \\
\hline \multirow[t]{2}{*}{653060} & 2 & 16.5 & 18.8 & 16.0 & 4.8 & 1.6 & 0.7 \\
\hline & 10 & 25.2 & 29.4 & 30.3 & 10.9 & 3.2 & 10.1 \\
\hline \multirow[t]{2}{*}{655050} & 2 & 15.4 & 27.8 & 17.4 & 5.8 & 4.4 & 6.6 \\
\hline & 10 & 27.0 & 41.0 & 32.0 & 11.5 & 7.4 & 7.9 \\
\hline \multirow[t]{2}{*}{655160} & 2 & 16.7 & 26.8 & 20.1 & 5.4 & 5.8 & 5.6 \\
\hline & 10 & 28.3 & 43.5 & 38.3 & 14.1 & 12.9 & 7.2 \\
\hline \multirow[t]{2}{*}{655030} & 2 & 15.4 & 24.8 & 18.2 & 3.9 & 1.8 & 4.3 \\
\hline & 10 & 27.6 & 38.8 & 30.7 & 11.7 & 5.0 & 9.4 \\
\hline \multirow[t]{2}{*}{655020} & 2 & 13.7 & 22.7 & 17.2 & 4.3 & 2.9 & 1.2 \\
\hline & 10 & 24.9 & 36.1 & 30.7 & 10.5 & 7.3 & 7.0 \\
\hline \multirow[t]{2}{*}{655070} & 2 & 16.9 & 24.9 & 19.9 & 6.3 & 3.3 & 14.4 \\
\hline & 10 & 28.9 & 41.2 & 32.7 & 14.1 & 7.2 & 16.6 \\
\hline \multirow[t]{2}{*}{655010} & 2 & 11.5 & 21.4 & 10.3 & 2.8 & 3.4 & 14.4 \\
\hline & 10 & 21.0 & 32.7 & 23.3 & 7.1 & 4.7 & 16.2 \\
\hline
\end{tabular}


GCM - MIROC; Downscaling Method - LARS-WG

SRES A2 (2046 - 2065)

DRWH Reliability (\%)

\section{per capita roof area}

\begin{tabular}{|c|c|c|c|c|c|c|c|}
\hline Gauge & sq. m & Jan & Feb & Mar & Apr & May & Jun \\
\hline \multirow[t]{2}{*}{655920} & 2 & 8.4 & 4.0 & 5.3 & 7.3 & 17.3 & 24.4 \\
\hline & 10 & 18.6 & 7.5 & 9.5 & 14.1 & 32.6 & 40.1 \\
\hline \multirow[t]{2}{*}{655940} & 2 & 5.6 & 3.0 & 4.4 & 7.1 & 14.3 & 18.7 \\
\hline & 10 & 12.4 & 5.6 & 7.4 & 14.3 & 28.2 & 36.3 \\
\hline \multirow[t]{2}{*}{655990} & 2 & 6.7 & 2.6 & 4.4 & 6.6 & 14.1 & 18.8 \\
\hline & 10 & 14.2 & 6.3 & 9.6 & 13.5 & 29.6 & 35.6 \\
\hline \multirow[t]{2}{*}{655780} & 2 & 3.1 & 3.4 & 6.0 & 7.4 & 16.1 & 23.6 \\
\hline & 10 & 7.8 & 7.2 & 13.2 & 15.6 & 29.7 & 39.8 \\
\hline \multirow[t]{2}{*}{655850} & 2 & 6.3 & 6.1 & 8.2 & 7.2 & 13.2 & 21.0 \\
\hline & 10 & 12.2 & 10.5 & 15.3 & 14.4 & 28.0 & 39.8 \\
\hline \multirow[t]{2}{*}{655480} & 2 & 6.4 & 4.0 & 6.7 & 9.2 & 12.3 & 15.7 \\
\hline & 10 & 11.9 & 8.3 & 11.8 & 15.9 & 22.8 & 35.0 \\
\hline \multirow[t]{2}{*}{655600} & 2 & 3.9 & 5.0 & 5.6 & 7.7 & 11.8 & 11.0 \\
\hline & 10 & 11.2 & 9.1 & 9.2 & 13.5 & 25.7 & 26.8 \\
\hline \multirow[t]{2}{*}{655570} & 2 & 6.1 & 5.8 & 8.7 & 10.0 & 11.7 & 12.6 \\
\hline & 10 & 15.5 & 11.6 & 14.6 & 19.4 & 34.2 & 34.6 \\
\hline \multirow[t]{2}{*}{655630} & 2 & 7.4 & 3.6 & 5.6 & 6.5 & 10.4 & 14.3 \\
\hline & 10 & 11.8 & 7.3 & 11.5 & 14.1 & 24.6 & 30.3 \\
\hline \multirow[t]{2}{*}{655620} & 2 & 4.4 & 3.7 & 7.7 & 6.6 & 11.8 & 13.7 \\
\hline & 10 & 9.9 & 8.7 & 12.8 & 12.2 & 24.3 & 29.4 \\
\hline \multirow[t]{2}{*}{655550} & 2 & 7.2 & 4.7 & 6.4 & 8.3 & 8.6 & 10.4 \\
\hline & 10 & 14.5 & 8.9 & 10.2 & 14.5 & 20.8 & 23.4 \\
\hline \multirow[t]{2}{*}{655450} & 2 & 5.5 & 3.8 & 4.4 & 9.9 & 14.9 & 14.3 \\
\hline & 10 & 11.3 & 7.3 & 7.9 & 15.0 & 24.8 & 24.7 \\
\hline \multirow[t]{2}{*}{654720} & 2 & 2.6 & 3.0 & 6.2 & 9.5 & 12.6 & 12.3 \\
\hline & 10 & 8.2 & 6.6 & 10.1 & 16.6 & 20.6 & 20.9 \\
\hline \multirow[t]{2}{*}{653870} & 2 & 1.0 & 1.9 & 5.0 & 6.4 & 10.2 & 13.3 \\
\hline & 10 & 4.1 & 4.2 & 8.6 & 10.6 & 19.2 & 22.8 \\
\hline \multirow[t]{2}{*}{653760} & 2 & 1.1 & 0.9 & 5.4 & 8.5 & 12.0 & 15.9 \\
\hline & 10 & 7.6 & 4.5 & 10.8 & 14.3 & 21.0 & 27.6 \\
\hline \multirow[t]{2}{*}{653800} & 2 & 2.8 & 1.7 & 7.8 & 11.0 & 13.7 & 14.3 \\
\hline & 10 & 9.4 & 7.1 & 12.5 & 18.5 & 20.9 & 25.6 \\
\hline \multirow[t]{2}{*}{653440} & 2 & 2.2 & 1.5 & 3.7 & 10.1 & 14.0 & 20.4 \\
\hline & 10 & 3.8 & 2.8 & 5.6 & 15.7 & 22.3 & 31.9 \\
\hline \multirow[t]{2}{*}{653380} & 2 & 0.8 & 0.7 & 4.5 & 8.6 & 11.6 & 13.5 \\
\hline & 10 & 3.8 & 3.6 & 6.9 & 16.1 & 20.3 & 24.1 \\
\hline \multirow[t]{2}{*}{653350} & 2 & 0.5 & 1.5 & 4.5 & 8.1 & 9.4 & 12.0 \\
\hline & 10 & 4.4 & 3.4 & 7.1 & 15.9 & 16.9 & 24.0 \\
\hline \multirow[t]{2}{*}{655280} & 2 & 7.6 & 4.4 & 3.5 & 4.1 & 7.2 & 11.4 \\
\hline & 10 & 13.0 & 6.2 & 6.1 & 9.9 & 18.6 & 22.6 \\
\hline
\end{tabular}


GCM - MIROC; Downscaling Method - LARS-WG

SRES A2 (2046 - 2065)

DRWH Reliability (\%)

per capita roof area

$\begin{array}{llllllll}\text { Gauge } & \text { sq. } \mathbf{m} & \text { Jul } & \text { Aug } & \text { Sep } & \text { Oct } & \text { Nov } & \text { Dec } \\ \mathbf{6 5 5 9 2 0} & \mathbf{2} & 12.4 & 9.7 & 12.0 & 11.3 & 10.7 & 14.6\end{array}$

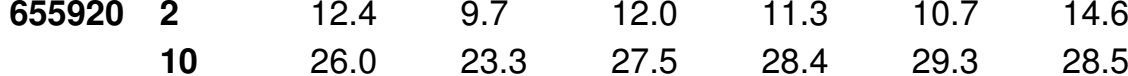

$\begin{array}{llllllll}655940 & 2 & 9.1 & 3.6 & 3.6 & 7.3 & 10.0 & 9.5\end{array}$

$\begin{array}{lllllll}10 & 19.5 & 13.1 & 13.7 & 19.3 & 29.7 & 22.9\end{array}$

$\begin{array}{llllllll}655990 & 2 & 8.3 & 3.3 & 3.7 & 7.8 & 9.4 & 13.0\end{array}$

$\begin{array}{lllllll}10 & 17.9 & 11.0 & 10.9 & 18.8 & 22.4 & 24.7\end{array}$

$\begin{array}{llllllll}655780 & 2 & 8.4 & 3.5 & 4.6 & 8.3 & 9.6 & 8.8\end{array}$

$\begin{array}{lllllll}10 & 19.2 & 8.8 & 11.2 & 21.8 & 28.3 & 21.2\end{array}$

$\begin{array}{llllllll}655850 & 2 & 10.1 & 5.0 & 6.9 & 11.8 & 10.5 & 8.5\end{array}$

$\begin{array}{lllllll}10 & 22.8 & 13.7 & 15.2 & 26.6 & 29.0 & 20.2\end{array}$

$\begin{array}{llllllll}655480 & 2 & 14.4 & 16.5 & 18.6 & 12.7 & 5.5 & 7.3\end{array}$

$\begin{array}{lllllll}10 & 31.8 & 35.3 & 38.5 & 23.4 & 14.8 & 17.9\end{array}$

$\begin{array}{llllllll}655600 & 2 & 8.7 & 8.3 & 10.0 & 8.0 & 4.5 & 5.0\end{array}$

$\begin{array}{lllllll}10 & 18.5 & 18.1 & 26.5 & 22.8 & 13.0 & 12.5\end{array}$

$\begin{array}{llllllll}655570 & 2 & 7.0 & 5.2 & 8.7 & 11.0 & 9.9 & 5.7\end{array}$

$\begin{array}{lllllll}10 & 17.4 & 13.6 & 22.9 & 29.7 & 25.5 & 15.5\end{array}$

$\begin{array}{llllllll}655630 & 2 & 8.3 & 6.5 & 7.5 & 8.3 & 5.0 & 4.1\end{array}$

$\begin{array}{lllllll}10 & 16.5 & 13.3 & 20.2 & 24.9 & 15.6 & 10.5\end{array}$

$\begin{array}{llllllll}655620 & 2 & 7.0 & 5.1 & 8.7 & 11.9 & 5.4 & 5.3\end{array}$

$\begin{array}{lllllll}10 & 16.6 & 12.1 & 20.6 & 23.4 & 13.0 & 12.1\end{array}$

$\begin{array}{llllllll}655550 & 2 & 10.5 & 10.2 & 11.4 & 9.3 & 3.8 & 5.9\end{array}$

$\begin{array}{lllllll}10 & 18.0 & 19.4 & 25.7 & 21.7 & 10.9 & 13.5\end{array}$

$\begin{array}{llllllll}655450 & 2 & 10.0 & 8.0 & 11.5 & 9.7 & 5.2 & 7.0\end{array}$

$\begin{array}{lllllll}10 & 17.6 & 13.1 & 25.3 & 21.8 & 11.4 & 13.2\end{array}$

$\begin{array}{llllllll}654720 & 2 & 7.5 & 2.5 & 4.0 & 7.3 & 6.1 & 6.4\end{array}$

$\begin{array}{lllllll}10 & 13.2 & 6.3 & 10.7 & 17.2 & 12.3 & 18.7\end{array}$

$\begin{array}{llllllll}653870 & 2 & 7.6 & 2.9 & 4.0 & 6.6 & 5.2 & 5.2\end{array}$

$\begin{array}{lllllll}10 & 13.7 & 7.2 & 11.4 & 14.8 & 9.7 & 15.8\end{array}$

$\begin{array}{llllllll}653760 & 2 & 15.2 & 12.6 & 11.5 & 10.5 & 4.3 & 6.6\end{array}$

$\begin{array}{lllllll}10 & 27.5 & 24.3 & 24.5 & 20.5 & 9.7 & 16.3\end{array}$

$\begin{array}{llllllll}653800 & 2 & 9.1 & 5.6 & 6.6 & 13.6 & 7.2 & 13.3\end{array}$

$\begin{array}{lllllll}10 & 16.6 & 12.9 & 15.8 & 29.4 & 13.6 & 19.5\end{array}$

$\begin{array}{llllllll}653440 & 2 & 8.5 & 5.7 & 8.5 & 12.9 & 5.6 & 6.8\end{array}$

$\begin{array}{lllllll}10 & 17.1 & 11.2 & 16.6 & 25.5 & 10.1 & 10.8\end{array}$

$\begin{array}{llllllll}653380 & 2 & 12.1 & 8.8 & 9.1 & 16.0 & 5.8 & 6.4\end{array}$

$\begin{array}{lllllll}10 & 21.5 & 17.0 & 18.7 & 24.7 & 8.3 & 12.3\end{array}$

$\begin{array}{llllllll}653350 & 2 & 12.0 & 11.8 & 11.0 & 12.3 & 4.2 & 0.9\end{array}$

$\begin{array}{lllllll}10 & 21.1 & 20.8 & 24.5 & 25.8 & 7.2 & 9.3\end{array}$

$\begin{array}{llllllll}655280 & 2 & 18.6 & 20.6 & 19.3 & 14.2 & 5.8 & 4.7\end{array}$

$\begin{array}{lllllll}10 & 37.5 & 34.5 & 43.2 & 33.0 & 14.5 & 11.2\end{array}$ 
GCM - MIROC; Downscaling Method - LARS-WG

SRES A2 (2046 - 2065)

DRWH Reliability (\%)

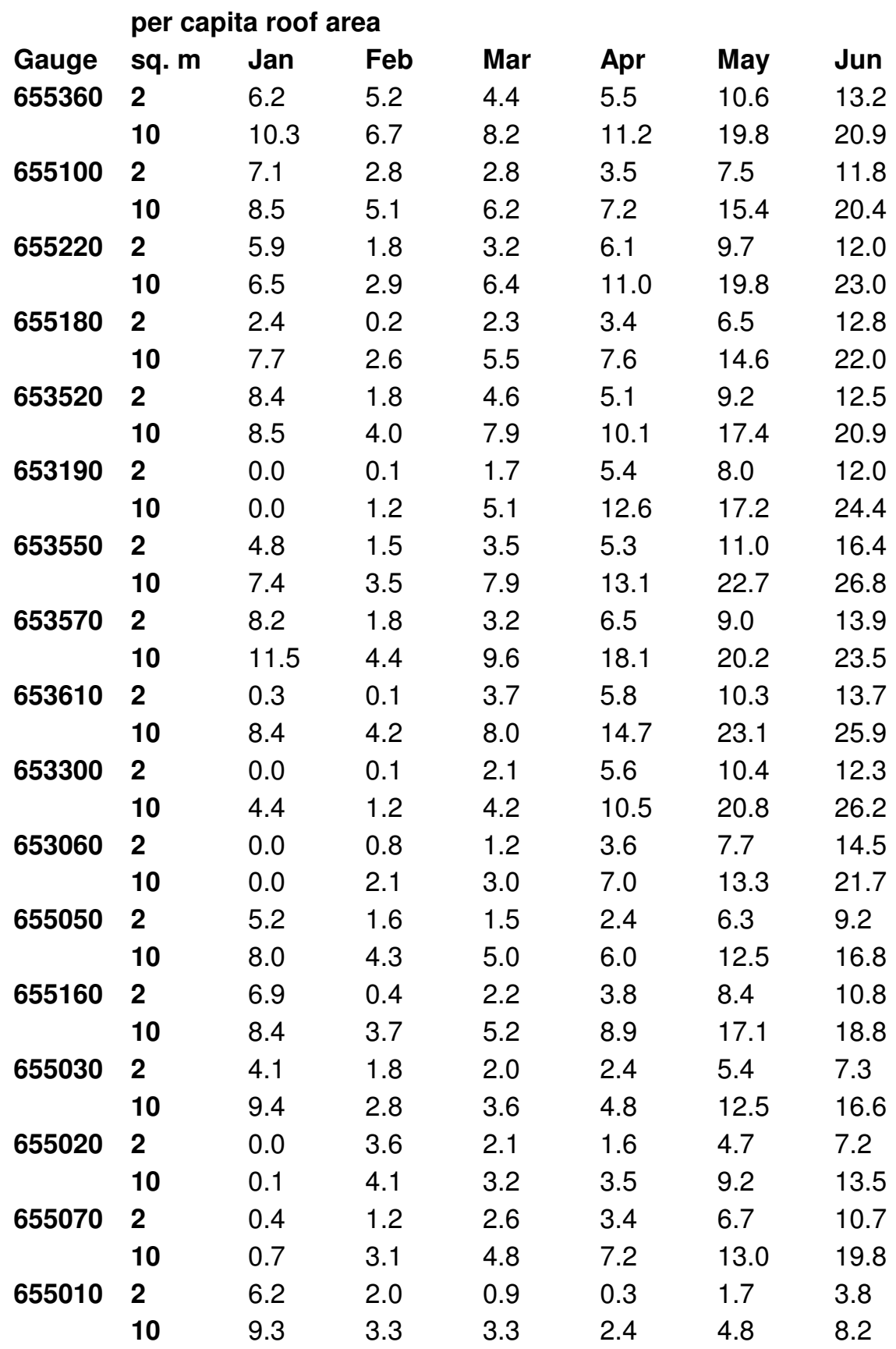


GCM - MIROC; Downscaling Method - LARS-WG

SRES A2 (2046 - 2065)

DRWH Reliability (\%)

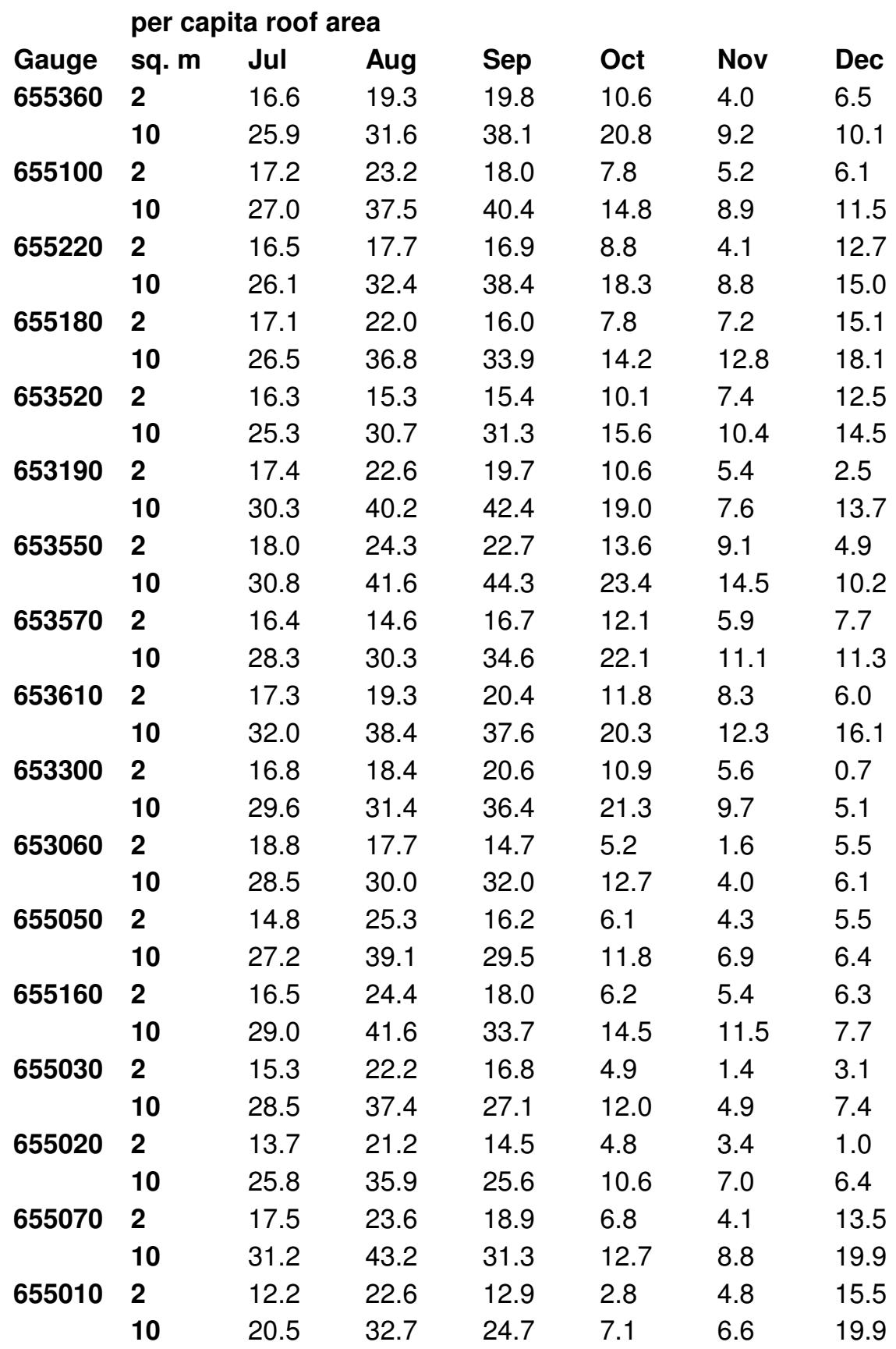


GCM - MIROC; Downscaling Method - LARS-WG

SRES B1 (2046 - 2065)

DRWH Reliability (\%)

per capita roof area

$\begin{array}{llllllll}\text { Gauge } & \text { sq. } \mathbf{m} & \text { Jan } & \text { Feb } & \text { Mar } & \text { Apr } & \text { May } & \text { Jun } \\ 655920 & \mathbf{2} & 7.7 & 3.2 & 5.4 & 7.9 & 20.2 & 24.0\end{array}$

$\begin{array}{lllllll}10 & 17.1 & 8.5 & 10.6 & 16.3 & 35.1 & 35.7\end{array}$

$\begin{array}{llllllll}655940 & 2 & 5.0 & 2.6 & 4.8 & 8.6 & 16.0 & 19.1\end{array}$

$\begin{array}{lllllll}10 & 11.5 & 6.3 & 8.5 & 16.6 & 30.6 & 36.2\end{array}$

$\begin{array}{llllllll}655990 & 2 & 6.8 & 2.8 & 4.1 & 7.8 & 14.2 & 19.3\end{array}$

$\begin{array}{lllllll}10 & 13.9 & 8.1 & 10.7 & 15.4 & 30.2 & 35.5\end{array}$

$\begin{array}{llllllll}655780 & 2 & 3.4 & 3.7 & 7.0 & 8.1 & 16.1 & 24.2\end{array}$

$\begin{array}{lllllll}10 & 7.7 & 7.3 & 15.2 & 16.4 & 30.7 & 40.3\end{array}$

$\begin{array}{llllllll}655850 & 2 & 7.0 & 7.2 & 9.3 & 8.3 & 13.8 & 20.3\end{array}$

$\begin{array}{lllllll}10 & 12.4 & 11.8 & 16.7 & 16.4 & 29.4 & 38.0\end{array}$

$\begin{array}{llllllll}655480 & 2 & 6.4 & 3.8 & 7.6 & 10.4 & 14.0 & 17.1\end{array}$

$\begin{array}{lllllll}10 & 11.2 & 9.0 & 16.1 & 21.6 & 24.3 & 33.8\end{array}$

$\begin{array}{llllllll}655600 & 2 & 4.5 & 6.0 & 7.1 & 9.9 & 13.7 & 12.4\end{array}$

$\begin{array}{lllllll}10 & 8.9 & 11.1 & 12.4 & 17.5 & 27.0 & 26.3\end{array}$

$\begin{array}{llllllll}655570 & 2 & 7.5 & 6.7 & 9.9 & 11.4 & 14.0 & 15.1\end{array}$

$\begin{array}{lllllll}10 & 14.1 & 13.7 & 18.1 & 22.5 & 34.9 & 34.6\end{array}$

$\begin{array}{llllllll}655630 & 2 & 7.4 & 5.5 & 6.3 & 7.6 & 12.1 & 14.5\end{array}$

$\begin{array}{lllllll}10 & 9.4 & 9.7 & 14.1 & 16.7 & 26.2 & 28.2\end{array}$

$\begin{array}{llllllll}655620 & 2 & 5.2 & 4.8 & 7.4 & 9.3 & 14.1 & 14.5\end{array}$

$\begin{array}{lllllll}10 & 8.8 & 11.5 & 14.3 & 16.0 & 26.2 & 28.6\end{array}$

$\begin{array}{llllllll}655550 & 2 & 7.8 & 5.2 & 7.1 & 10.1 & 10.0 & 11.9\end{array}$

$\begin{array}{lllllll}10 & 11.7 & 10.1 & 12.5 & 17.8 & 22.2 & 23.3\end{array}$

$\begin{array}{llllllll}655450 & 2 & 5.8 & 3.7 & 6.8 & 10.8 & 16.7 & 14.9\end{array}$

$\begin{array}{lllllll}10 & 9.6 & 8.4 & 12.2 & 17.3 & 28.8 & 24.8\end{array}$

$\begin{array}{llllllll}654720 & 2 & 3.2 & 4.0 & 8.0 & 10.6 & 14.0 & 13.0\end{array}$

$\begin{array}{lllllll}10 & 8.7 & 8.8 & 13.2 & 17.6 & 21.4 & 21.5\end{array}$

$\begin{array}{llllllll}653870 & 2 & 1.0 & 1.7 & 5.7 & 6.7 & 11.2 & 12.6\end{array}$

$\begin{array}{lllllll}10 & 4.5 & 4.6 & 10.2 & 10.9 & 20.9 & 21.8\end{array}$

$\begin{array}{llllllll}653760 & 2 & 1.7 & 1.0 & 7.0 & 9.8 & 12.7 & 15.3\end{array}$

$\begin{array}{lllllll}10 & 8.7 & 5.6 & 12.7 & 15.7 & 21.5 & 26.2\end{array}$

$\begin{array}{llllllll}653800 & 2 & 3.6 & 2.8 & 9.0 & 10.6 & 14.0 & 14.6\end{array}$

$\begin{array}{lllllll}10 & 12.0 & 8.8 & 14.7 & 18.1 & 20.9 & 25.1\end{array}$

$\begin{array}{llllllll}653440 & 2 & 3.0 & 2.2 & 4.3 & 9.8 & 14.6 & 20.8\end{array}$

$\begin{array}{lllllll}10 & 4.6 & 3.8 & 7.1 & 15.5 & 22.7 & 31.2\end{array}$

$\begin{array}{llllllll}653380 & 2 & 1.5 & 1.2 & 6.3 & 8.5 & 12.2 & 13.1\end{array}$

$\begin{array}{llllllll}10 & 5.3 & 4.3 & 10.2 & 16.2 & 20.2 & 23.9\end{array}$

$\begin{array}{llllllll}653350 & 2 & 2.2 & 2.2 & 5.9 & 7.0 & 9.4 & 12.9\end{array}$

$\begin{array}{lllllll}10 & 5.7 & 4.7 & 9.4 & 15.9 & 17.2 & 23.9\end{array}$

$\begin{array}{llllllll}655280 & 2 & 2.1 & 3.4 & 4.5 & 6.5 & 8.2 & 12.0\end{array}$

$\begin{array}{lllllll}10 & 7.4 & 6.9 & 7.7 & 14.2 & 19.5 & 24.8\end{array}$ 
GCM - MIROC; Downscaling Method - LARS-WG

SRES B1 (2046 - 2065)

DRWH Reliability (\%)

\section{per capita roof area}

$\begin{array}{llllllll}\text { Gauge } & \text { sq. } \mathbf{m} & \text { Jul } & \text { Aug } & \text { Sep } & \text { Oct } & \text { Nov } & \text { Dec } \\ 655920 & \mathbf{2} & 12.4 & 9.8 & 12.7 & 11.6 & 9.3 & 12.7\end{array}$

$\begin{array}{lllllll}10 & 26.0 & 23.1 & 28.1 & 28.1 & 26.2 & 25.4\end{array}$

$\begin{array}{llllllll}655940 & 2 & 9.5 & 3.6 & 3.9 & 7.6 & 9.0 & 8.5\end{array}$

$\begin{array}{lllllll}10 & 21.3 & 14.0 & 15.6 & 17.7 & 25.2 & 21.1\end{array}$

$\begin{array}{lllllllll}655990 & 2 & 8.5 & 3.3 & 4.0 & 7.7 & 7.7 & 10.8\end{array}$

$\begin{array}{lllllll}10 & 18.6 & 11.4 & 11.3 & 17.1 & 19.3 & 21.5\end{array}$

$\begin{array}{llllllll}655780 & 2 & 8.5 & 3.8 & 4.9 & 9.1 & 9.7 & 8.7\end{array}$

$\begin{array}{lllllll}10 & 18.9 & 9.1 & 10.5 & 21.8 & 27.8 & 19.8\end{array}$

$\begin{array}{llllllll}655850 & 2 & 10.7 & 6.2 & 7.1 & 13.4 & 10.2 & 8.1\end{array}$

$\begin{array}{lllllll}10 & 23.7 & 14.9 & 15.5 & 25.8 & 27.5 & 19.8\end{array}$

$\begin{array}{llllllll}655480 & 2 & 13.6 & 17.5 & 20.3 & 11.8 & 5.3 & 7.7\end{array}$

$\begin{array}{lllllll}10 & 28.3 & 39.7 & 39.5 & 18.1 & 12.5 & 18.5\end{array}$

$\begin{array}{llllllll}655600 & 2 & 8.5 & 8.7 & 10.3 & 8.0 & 3.6 & 5.7\end{array}$

$\begin{array}{lllllll}10 & 17.1 & 21.2 & 28.3 & 19.1 & 11.7 & 13.8\end{array}$

$\begin{array}{llllllll}655570 & 2 & 6.8 & 5.8 & 8.8 & 10.6 & 8.2 & 6.7\end{array}$

$\begin{array}{llllllll} & 10 & 16.2 & 15.1 & 25.4 & 26.0 & 24.6 & 16.9 \\ \mathbf{6 5 5 6 3 0} & \mathbf{2} & 8.4 & 6.8 & 8.8 & 8.8 & 3.9 & 4.8\end{array}$

$\begin{array}{llllllll} & \mathbf{1 0} & 14.1 & 15.2 & 22.4 & 21.9 & 14.9 & 11.7 \\ \mathbf{6 5 5 6 2 0} & \mathbf{2} & 7.7 & 5.3 & 10.4 & 11.2 & 5.4 & 5.5\end{array}$

$\begin{array}{llllllll} & \mathbf{1 0} & 14.8 & 13.8 & 23.7 & 20.9 & 13.5 & 12.4 \\ \mathbf{6 5 5 5 5 0} & \mathbf{2} & 10.3 & 11.5 & 12.8 & 9.1 & 3.6 & 6.4 \\ & \mathbf{1 0} & 15.8 & 22.8 & 28.1 & 18.6 & 10.6 & 14.4\end{array}$

$\begin{array}{llllllll}655450 & 2 & 9.3 & 10.0 & 13.9 & 9.3 & 4.9 & 6.6\end{array}$

$\begin{array}{lllllll}10 & 17.7 & 18.4 & 27.3 & 21.1 & 11.8 & 12.9\end{array}$

$\begin{array}{llllllll}654720 & 2 & 7.1 & 2.6 & 4.4 & 7.3 & 6.1 & 6.4\end{array}$

$\begin{array}{llllllll} & \mathbf{1 0} & 13.0 & 6.2 & 12.1 & 17.2 & 12.1 & 16.8 \\ \mathbf{6 5 3 8 7 0} & \mathbf{2} & 7.1 & 3.1 & 5.1 & 6.3 & 5.1 & 4.6 \\ & \mathbf{1 0} & 13.7 & 7.4 & 13.7 & 14.0 & 10.0 & 13.2 \\ \mathbf{6 5 3 7 6 0} & \mathbf{2} & 13.9 & 13.8 & 13.6 & 10.1 & 4.5 & 6.3 \\ & \mathbf{1 0} & 26.8 & 25.0 & 27.8 & 19.1 & 9.8 & 14.5 \\ \mathbf{6 5 3 8 0 0} & \mathbf{2} & 8.3 & 5.7 & 7.8 & 12.7 & 7.7 & 11.7 \\ & \mathbf{1 0} & 16.0 & 14.4 & 17.0 & 28.1 & 13.7 & 17.4 \\ \mathbf{6 5 3 4 4 0} & \mathbf{2} & 8.2 & 6.1 & 9.4 & 13.3 & 6.1 & 6.0 \\ & \mathbf{1 0} & 16.3 & 13.1 & 17.9 & 25.4 & 10.5 & 9.0 \\ \mathbf{6 5 3 3 8 0} & \mathbf{2} & 11.4 & 10.0 & 10.5 & 15.3 & 5.8 & 5.8 \\ & \mathbf{1 0} & 21.2 & 21.1 & 20.3 & 23.4 & 8.0 & 10.5 \\ \mathbf{6 5 3 3 5 0} & \mathbf{2} & 12.0 & 11.8 & 14.0 & 12.7 & 4.4 & 1.7 \\ & \mathbf{1 0} & 21.6 & 23.9 & 27.3 & 25.5 & 7.7 & 8.4 \\ \mathbf{6 5 5 2 8 0} & \mathbf{2} & 20.2 & 20.6 & 20.8 & 13.7 & 3.7 & 5.1 \\ & \mathbf{1 0} & 40.1 & 33.9 & 45.7 & 27.2 & 12.7 & 11.6\end{array}$


GCM - MIROC; Downscaling Method - LARS-WG

SRES B1 (2046 - 2065)

DRWH Reliability (\%)

\begin{tabular}{|c|c|c|c|c|c|c|c|}
\hline \multirow{3}{*}{$\begin{array}{l}\text { Gauge } \\
655360\end{array}$} & \multicolumn{7}{|c|}{ per capita roof area } \\
\hline & sq. $\mathrm{m}$ & Jan & Feb & Mar & Apr & May & Jun \\
\hline & 2 & 4.2 & 4.5 & 4.2 & 6.3 & 10.8 & 14.4 \\
\hline \multirow{3}{*}{655100} & 10 & 6.9 & 6.6 & 8.0 & 12.6 & 20.0 & 21.8 \\
\hline & 2 & 4.4 & 2.2 & 2.4 & 4.5 & 7.6 & 13.4 \\
\hline & 10 & 5.7 & 4.2 & 5.3 & 8.9 & 15.7 & 21.4 \\
\hline \multirow[t]{2}{*}{655220} & 2 & 4.3 & 2.0 & 3.7 & 6.3 & 9.6 & 12.7 \\
\hline & 10 & 4.6 & 4.2 & 7.9 & 11.1 & 18.9 & 22.4 \\
\hline \multirow[t]{2}{*}{655180} & 2 & 0.8 & 0.5 & 4.1 & 3.5 & 6.6 & 12.4 \\
\hline & 10 & 6.0 & 3.8 & 9.1 & 7.7 & 14.2 & 21.6 \\
\hline \multirow[t]{2}{*}{653520} & 2 & 7.0 & 1.8 & 7.7 & 5.5 & 9.9 & 13.8 \\
\hline & 10 & 7.2 & 4.5 & 12.4 & 10.2 & 18.6 & 24.1 \\
\hline \multirow[t]{2}{*}{653190} & 2 & 0.0 & 0.1 & 3.0 & 5.6 & 8.9 & 12.1 \\
\hline & 10 & 0.0 & 0.9 & 6.9 & 11.6 & 19.2 & 25.7 \\
\hline \multirow[t]{2}{*}{653550} & 2 & 3.2 & 1.9 & 5.9 & 5.4 & 11.9 & 17.6 \\
\hline & 10 & 5.7 & 4.5 & 10.9 & 12.5 & 24.2 & 29.9 \\
\hline \multirow[t]{2}{*}{653570} & 2 & 5.9 & 2.2 & 6.9 & 6.8 & 10.9 & 15.3 \\
\hline & 10 & 9.1 & 6.8 & 17.1 & 18.2 & 22.8 & 26.4 \\
\hline \multirow[t]{2}{*}{653610} & 2 & 0.0 & 0.4 & 6.9 & 5.8 & 11.3 & 14.0 \\
\hline & 10 & 5.9 & 6.1 & 12.5 & 14.3 & 24.5 & 27.5 \\
\hline \multirow[t]{2}{*}{653300} & 2 & 0.0 & 0.2 & 3.0 & 5.2 & 12.6 & 13.9 \\
\hline & 10 & 1.1 & 1.2 & 6.1 & 9.6 & 21.1 & 23.0 \\
\hline \multirow[t]{2}{*}{653060} & 2 & 0.0 & 0.5 & 2.4 & 3.4 & 8.7 & 14.3 \\
\hline & 10 & 0.0 & 2.6 & 5.4 & 6.8 & 14.0 & 20.3 \\
\hline \multirow[t]{2}{*}{655050} & 2 & 4.0 & 0.6 & 1.5 & 2.3 & 6.1 & 10.0 \\
\hline & 10 & 5.5 & 3.9 & 5.2 & 5.6 & 12.9 & 17.8 \\
\hline \multirow[t]{2}{*}{655160} & 2 & 3.9 & 0.8 & 2.4 & 3.5 & 7.8 & 10.6 \\
\hline & 10 & 7.1 & 3.0 & 5.4 & 7.5 & 16.5 & 17.9 \\
\hline \multirow[t]{2}{*}{655030} & 2 & 2.8 & 0.8 & 2.1 & 2.6 & 5.0 & 7.7 \\
\hline & 10 & 7.8 & 3.1 & 3.4 & 5.0 & 12.3 & 15.6 \\
\hline \multirow[t]{2}{*}{655020} & 2 & 0.0 & 0.6 & 1.9 & 1.7 & 3.8 & 7.7 \\
\hline & 10 & 0.0 & 4.6 & 3.2 & 3.6 & 8.3 & 13.3 \\
\hline \multirow[t]{2}{*}{655070} & 2 & 0.1 & 1.0 & 4.1 & 3.4 & 6.0 & 11.5 \\
\hline & 10 & 0.3 & 3.6 & 7.3 & 7.1 & 11.8 & 20.1 \\
\hline \multirow[t]{2}{*}{655010} & 2 & 3.1 & 0.4 & 0.9 & 0.5 & 2.8 & 3.5 \\
\hline & 10 & 5.3 & 2.8 & 3.3 & 3.3 & 7.5 & 9.5 \\
\hline
\end{tabular}


GCM - MIROC; Downscaling Method - LARS-WG

SRES B1 (2046 - 2065)

DRWH Reliability (\%)

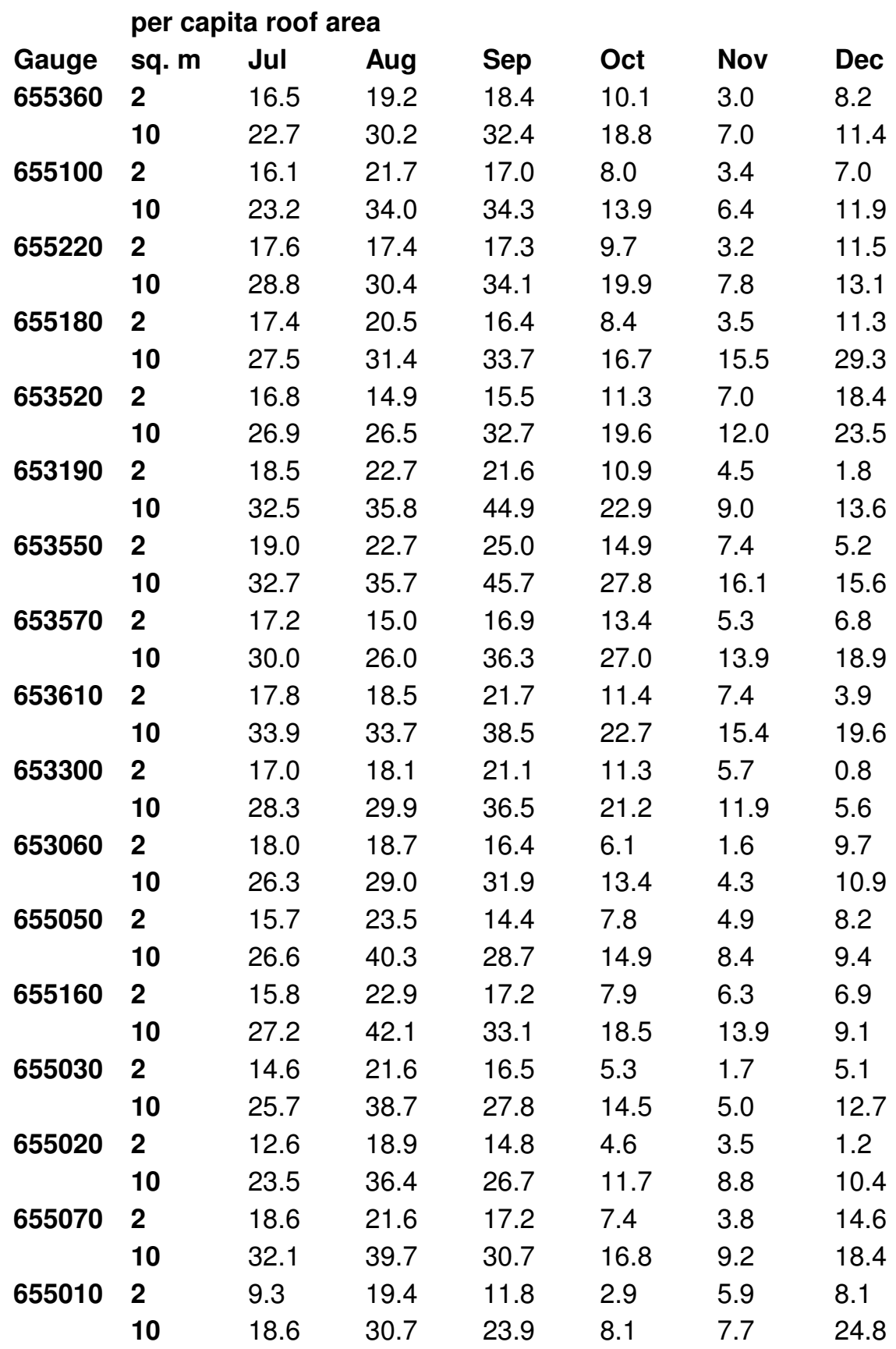


GCM - MRI; Downscaling Method - LARS-WG

SRES A1b (2046 - 2065)

DRWH Reliability (\%)

per capita roof area

$\begin{array}{llllllll}\text { Gauge } & \text { sq. } \mathbf{m} & \text { Jan } & \text { Feb } & \text { Mar } & \text { Apr } & \text { May } & \text { Jun } \\ \mathbf{6 5 5 9 2 0} & \mathbf{2} & 4.6 & 3.7 & 5.6 & 9.7 & 18.3 & 24.6 \\ & \mathbf{1 0} & 11.3 & 9.9 & 11.9 & 20.3 & 33.5 & 44.1\end{array}$

$\begin{array}{llllllll}655940 & 2 & 3.1 & 3.1 & 5.3 & 9.6 & 15.5 & 17.3\end{array}$

$\begin{array}{lllllll}10 & 8.2 & 6.7 & 8.8 & 17.2 & 28.5 & 32.6\end{array}$

$\begin{array}{llllllll}655990 & 2 & 4.2 & 3.0 & 4.6 & 8.7 & 15.4 & 18.1\end{array}$

$\begin{array}{lllllll}10 & 9.8 & 8.6 & 10.1 & 15.3 & 29.0 & 32.5\end{array}$

$\begin{array}{llllllll}655780 & 2 & 2.1 & 3.3 & 6.0 & 9.3 & 16.4 & 22.5\end{array}$

$\begin{array}{lllllll}10 & 5.3 & 7.5 & 11.5 & 19.0 & 28.7 & 35.7\end{array}$

$\begin{array}{llllllll}655850 & 2 & 3.6 & 5.9 & 7.7 & 9.1 & 14.8 & 19.9\end{array}$

$\begin{array}{lllllll}10 & 8.2 & 11.2 & 12.5 & 19.0 & 28.7 & 33.7\end{array}$

$\begin{array}{llllllll}655480 & 2 & 3.8 & 4.4 & 9.5 & 11.2 & 13.9 & 15.9\end{array}$

$\begin{array}{llllllll}10 & 8.8 & 10.4 & 20.8 & 21.3 & 24.2 & 29.9\end{array}$

$\begin{array}{llllllll}655600 & 2 & 2.4 & 6.0 & 8.2 & 12.4 & 12.8 & 11.3\end{array}$

$\begin{array}{lllllll}10 & 6.5 & 11.4 & 16.1 & 21.3 & 22.5 & 26.7\end{array}$

$\begin{array}{llllllll}655570 & 2 & 3.3 & 7.5 & 12.3 & 13.7 & 14.8 & 12.9\end{array}$

$\begin{array}{lllllll}10 & 9.5 & 14.6 & 24.9 & 26.3 & 30.5 & 33.8\end{array}$

$\begin{array}{llllllll}655630 & 2 & 4.0 & 3.6 & 6.3 & 9.9 & 11.5 & 13.6\end{array}$

$\begin{array}{lllllll}10 & 6.7 & 8.6 & 18.4 & 20.0 & 21.8 & 27.8\end{array}$

$\begin{array}{llllllll}655620 & 2 & 2.8 & 4.7 & 8.9 & 11.4 & 13.7 & 12.9\end{array}$

$\begin{array}{lllllll}10 & 5.8 & 11.3 & 18.4 & 18.9 & 22.8 & 26.6\end{array}$

$\begin{array}{llllllll}655550 & 2 & 4.1 & 4.7 & 7.1 & 12.7 & 10.2 & 10.8\end{array}$

$\begin{array}{lllllll}10 & 8.7 & 9.3 & 17.3 & 21.4 & 18.1 & 22.1\end{array}$

$\begin{array}{llllllll}655450 & 2 & 4.6 & 4.2 & 6.5 & 12.7 & 13.6 & 14.2\end{array}$

$\begin{array}{lllllll}10 & 7.2 & 7.6 & 12.1 & 20.8 & 21.8 & 24.4\end{array}$

$\begin{array}{llllllll}654720 & 2 & 2.8 & 4.0 & 8.7 & 13.4 & 13.4 & 11.6\end{array}$

$\begin{array}{lllllll}10 & 7.2 & 8.8 & 14.7 & 22.1 & 19.3 & 18.3\end{array}$

$\begin{array}{llllllll}653870 & 2 & 1.1 & 1.9 & 5.6 & 8.8 & 11.6 & 12.7\end{array}$

$\begin{array}{lllllll}10 & 4.1 & 3.7 & 11.2 & 15.1 & 18.7 & 19.9\end{array}$

$\begin{array}{llllllll}653760 & 2 & 1.7 & 1.7 & 5.7 & 11.2 & 13.1 & 15.1\end{array}$

$\begin{array}{lllllll}10 & 8.7 & 5.5 & 12.9 & 19.1 & 20.5 & 23.7\end{array}$

$\begin{array}{llllllll}653800 & 2 & 3.0 & 3.7 & 10.2 & 13.1 & 14.2 & 13.8\end{array}$

$\begin{array}{lllllll}10 & 9.6 & 8.3 & 17.1 & 21.9 & 21.5 & 24.2\end{array}$

$\begin{array}{llllllll}653440 & 2 & 2.1 & 1.3 & 4.7 & 11.8 & 14.1 & 19.4\end{array}$

$\begin{array}{lllllll}10 & 3.3 & 2.3 & 8.4 & 18.6 & 23.2 & 29.6\end{array}$

$\begin{array}{llllllll}653380 & 2 & 1.0 & 1.8 & 4.8 & 9.8 & 11.5 & 12.9\end{array}$

$\begin{array}{lllllll}10 & 3.7 & 3.9 & 9.6 & 18.6 & 20.5 & 23.4\end{array}$

$\begin{array}{llllllll}653350 & 2 & 1.4 & 2.1 & 5.6 & 7.8 & 8.4 & 11.1\end{array}$

$\begin{array}{lllllll}10 & 4.7 & 3.5 & 10.1 & 17.7 & 16.7 & 22.1\end{array}$

$\begin{array}{llllllll}655280 & 2 & 1.6 & 2.3 & 4.8 & 8.9 & 9.3 & 13.4\end{array}$

$\begin{array}{lllllll}10 & 6.4 & 4.4 & 8.6 & 13.3 & 21.9 & 27.2\end{array}$ 
GCM - MRI; Downscaling Method - LARS-WG

SRES A1b (2046 - 2065)

DRWH Reliability (\%)

per capita roof area

Gauge sq. m Jul Aug Sep Oct Nov Dec

$\begin{array}{llllllll}655920 & 2 & 14.4 & 15.1 & 18.4 & 13.6 & 9.5 & 8.8\end{array}$

$\begin{array}{llllllll} & \mathbf{1 0} & 29.8 & 32.7 & 37.4 & 32.3 & 23.3 & 17.2 \\ \mathbf{6 5 5 5 9 4 0} & \mathbf{2} & 10.2 & 7.0 & 5.5 & 8.9 & 9.0 & 6.6 \\ & \mathbf{1 0} & 18.5 & 18.0 & 19.6 & 24.2 & 25.1 & 15.3\end{array}$

$\begin{array}{llllllll}655990 & 2 & 9.2 & 5.9 & 6.2 & 9.0 & 9.0 & 9.2\end{array}$

$\begin{array}{llllllll}655780 & 10 & 16.5 & 13.9 & 15.9 & 23.6 & 20.7 & 16.3\end{array}$

$\begin{array}{lllllll}2 & 9.1 & 5.3 & 7.4 & 12.6 & 12.0 & 6.9\end{array}$

$\begin{array}{llllllll}655850 & 2 & 11.2 & 8.5 & 10.8 & 17.2 & 10.3 & 6.0\end{array}$

$\begin{array}{llllllll}10 & 21.4 & 18.6 & 22.8 & 34.8 & 22.3 & 13.6\end{array}$

$\begin{array}{llllllll}655480 & 2 & 16.4 & 24.9 & 23.2 & 12.2 & 6.4 & 5.9\end{array}$

$\begin{array}{lllllll}10 & 32.4 & 48.3 & 38.4 & 23.0 & 12.1 & 10.3\end{array}$

$\begin{array}{llllllll}655600 & 2 & 10.4 & 12.8 & 13.4 & 7.0 & 3.0 & 4.4\end{array}$

$\begin{array}{lllllll}10 & 26.0 & 28.6 & 31.6 & 20.3 & 11.0 & 11.3\end{array}$

$\begin{array}{llllllll}655570 & 2 & 6.8 & 9.3 & 12.0 & 10.3 & 6.7 & 4.9\end{array}$

$\begin{array}{lllllll}10 & 21.3 & 21.8 & 28.9 & 29.4 & 22.4 & 13.5\end{array}$

$\begin{array}{llllllll}655630 & 2 & 9.3 & 10.6 & 10.7 & 6.1 & 3.4 & 3.0\end{array}$

$\begin{array}{lllllll}10 & 22.3 & 22.1 & 25.0 & 21.4 & 13.4 & 8.5\end{array}$

$\begin{array}{llllllll}655620 & 2 & 7.7 & 7.6 & 12.6 & 10.6 & 3.9 & 4.6\end{array}$

$\begin{array}{lllllll}10 & 21.5 & 19.2 & 26.2 & 21.7 & 11.9 & 10.0\end{array}$

$\begin{array}{llllllll}655550 & 2 & 10.9 & 15.7 & 16.0 & 7.7 & 3.1 & 4.4\end{array}$

$\begin{array}{lllllll}10 & 21.4 & 28.4 & 30.6 & 19.5 & 9.3 & 10.8\end{array}$

$\begin{array}{llllllll}655450 & 2 & 10.8 & 14.0 & 18.5 & 9.8 & 3.4 & 6.6\end{array}$

$\begin{array}{lllllll}10 & 21.3 & 23.9 & 34.3 & 23.0 & 12.0 & 14.2\end{array}$

$\begin{array}{llllllll}654720 & 2 & 7.7 & 4.4 & 7.6 & 7.9 & 5.6 & 6.6\end{array}$

$\begin{array}{lllllll}10 & 16.1 & 11.1 & 18.4 & 16.3 & 13.4 & 17.4\end{array}$

$\begin{array}{llllllll}653870 & 2 & 8.6 & 5.2 & 8.0 & 7.2 & 3.9 & 4.8\end{array}$

$\begin{array}{lllllll}10 & 16.5 & 13.4 & 20.4 & 14.2 & 10.8 & 14.1\end{array}$

$\begin{array}{llllllll}653760 & 2 & 16.1 & 22.3 & 18.8 & 11.8 & 3.8 & 6.5\end{array}$

$\begin{array}{llllllll} & \mathbf{1 0} & 31.6 & 41.0 & 36.8 & 19.7 & 9.6 & 15.5 \\ \mathbf{6 5 3 8 0 0} & \mathbf{2} & 11.0 & 8.5 & 10.0 & 12.2 & 6.2 & 9.9 \\ & \mathbf{1 0} & 24.0 & 21.9 & 25.6 & 22.6 & 13.9 & 19.7\end{array}$

$\begin{array}{llllllll}653440 & 2 & 10.4 & 9.8 & 13.4 & 11.2 & 4.6 & 6.2\end{array}$

$\begin{array}{lllllll}10 & 24.1 & 21.6 & 25.3 & 19.3 & 10.7 & 12.2\end{array}$

$\begin{array}{llllllll}653380 & 2 & 14.7 & 15.8 & 14.3 & 11.9 & 5.1 & 4.6\end{array}$

$\begin{array}{lllllll}10 & 31.5 & 32.8 & 28.7 & 17.2 & 8.6 & 13.5\end{array}$

$\begin{array}{llllllll}653350 & 2 & 14.3 & 19.7 & 17.1 & 11.1 & 3.3 & 0.5\end{array}$

$\begin{array}{lllllll}10 & 31.2 & 37.5 & 36.4 & 20.1 & 7.5 & 11.6\end{array}$

$\begin{array}{llllllll}655280 & 2 & 18.8 & 22.9 & 20.1 & 12.1 & 3.0 & 3.4\end{array}$

$\begin{array}{lllllll}10 & 34.9 & 40.8 & 37.3 & 28.7 & 13.0 & 8.4\end{array}$


GCM - MRI; Downscaling Method - LARS-WG

SRES A1b (2046 - 2065)

DRWH Reliability (\%)

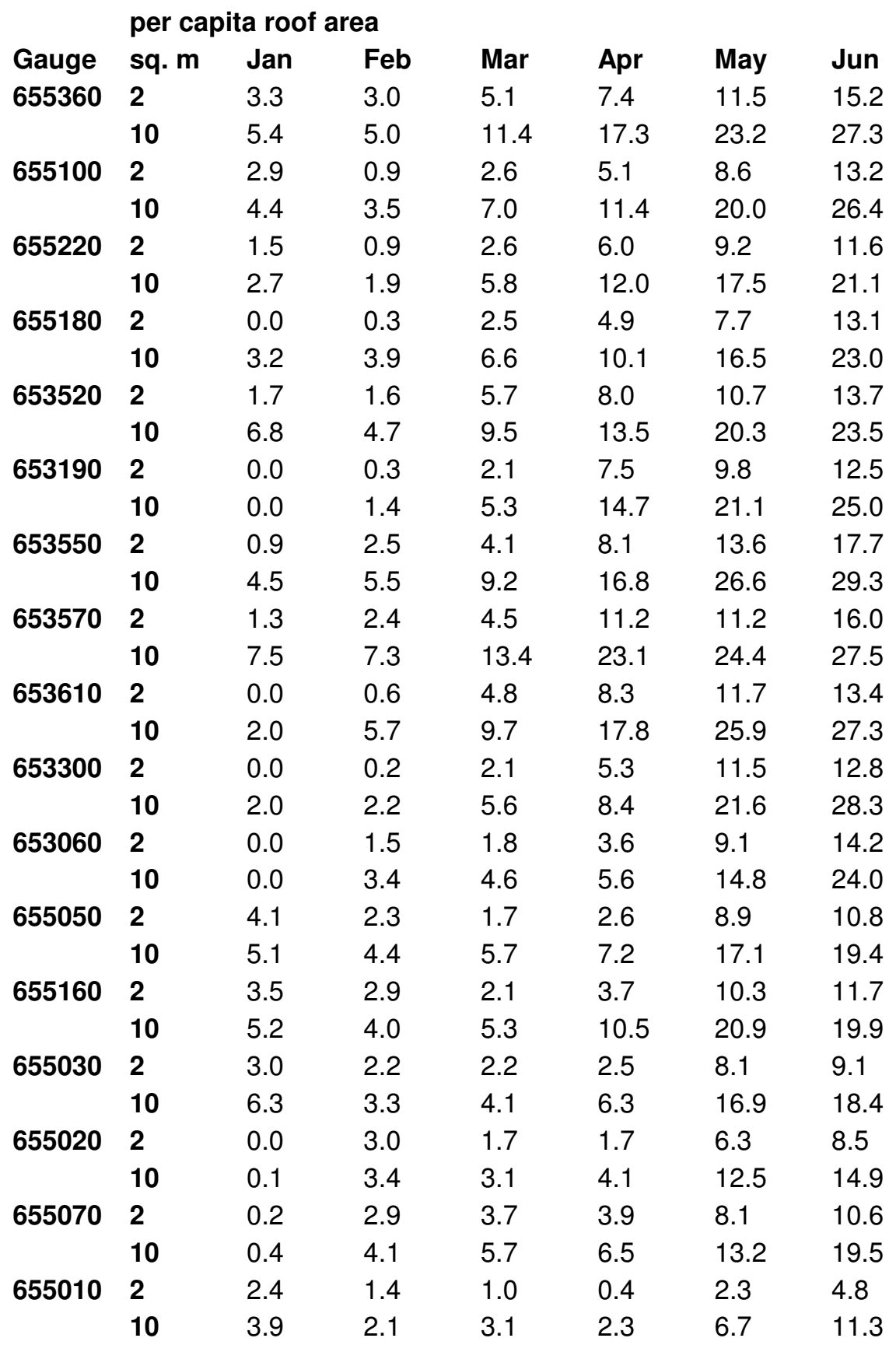


GCM - MRI; Downscaling Method - LARS-WG

SRES A1b (2046 - 2065)

DRWH Reliability (\%)

\begin{tabular}{|c|c|c|c|c|c|c|c|}
\hline \multirow{2}{*}{ Gauge } & \multicolumn{7}{|c|}{ per capita roof area } \\
\hline & sq. $m$ & Jul & Aug & Sep & Oct & Nov & Dec \\
\hline \multirow[t]{2}{*}{655360} & 2 & 16.1 & 19.5 & 18.2 & 9.9 & 2.9 & 5.8 \\
\hline & 10 & 28.9 & 34.4 & 34.4 & 20.1 & 6.7 & 7.3 \\
\hline \multirow[t]{2}{*}{655100} & 2 & 15.9 & 23.5 & 16.7 & 7.8 & 3.4 & 6.1 \\
\hline & 10 & 29.7 & 40.0 & 35.6 & 14.2 & 6.3 & 8.2 \\
\hline \multirow[t]{2}{*}{655220} & 2 & 16.0 & 16.9 & 18.4 & 9.2 & 5.9 & 4.5 \\
\hline & 10 & 32.1 & 31.6 & 37.4 & 19.3 & 12.0 & 5.9 \\
\hline \multirow[t]{2}{*}{655180} & 2 & 17.4 & 20.6 & 15.3 & 6.4 & 6.0 & 1.9 \\
\hline & 10 & 28.4 & 35.9 & 45.3 & 19.8 & 16.4 & 23.1 \\
\hline \multirow[t]{2}{*}{653520} & 2 & 16.6 & 14.3 & 17.9 & 11.1 & 9.4 & 4.1 \\
\hline & 10 & 27.4 & 30.0 & 43.0 & 20.8 & 13.9 & 33.4 \\
\hline \multirow[t]{2}{*}{653190} & 2 & 18.3 & 21.7 & 21.0 & 11.6 & 7.2 & 3.7 \\
\hline & 10 & 32.5 & 39.1 & 54.3 & 25.8 & 9.9 & 8.2 \\
\hline \multirow[t]{2}{*}{653550} & 2 & 18.9 & 23.3 & 25.3 & 12.7 & 8.3 & 2.6 \\
\hline & 10 & 33.1 & 39.5 & 55.3 & 29.7 & 15.0 & 14.5 \\
\hline \multirow[t]{2}{*}{653570} & 2 & 16.6 & 14.5 & 18.6 & 11.2 & 6.4 & 2.4 \\
\hline & 10 & 30.3 & 30.0 & 47.0 & 28.4 & 14.0 & 21.8 \\
\hline \multirow[t]{2}{*}{653610} & 2 & 17.8 & 18.2 & 22.0 & 13.5 & 11.0 & 6.2 \\
\hline & 10 & 35.2 & 37.0 & 48.6 & 28.7 & 16.9 & 11.5 \\
\hline \multirow[t]{2}{*}{653300} & 2 & 15.6 & 18.4 & 24.1 & 9.2 & 4.3 & 0.5 \\
\hline & 10 & 29.3 & 33.0 & 47.6 & 17.1 & 8.6 & 4.5 \\
\hline \multirow[t]{2}{*}{653060} & 2 & 18.2 & 17.8 & 17.1 & 4.8 & 1.6 & 4.9 \\
\hline & 10 & 29.6 & 32.1 & 43.0 & 11.5 & 3.8 & 5.2 \\
\hline \multirow[t]{2}{*}{655050} & 2 & 16.4 & 20.3 & 15.8 & 7.4 & 6.8 & 10.2 \\
\hline & 10 & 28.6 & 39.5 & 31.6 & 15.3 & 10.9 & 11.9 \\
\hline \multirow[t]{2}{*}{655160} & 2 & 15.4 & 21.4 & 18.4 & 7.3 & 8.5 & 8.4 \\
\hline & 10 & 27.4 & 42.0 & 36.0 & 18.2 & 17.4 & 10.5 \\
\hline \multirow[t]{2}{*}{655030} & 2 & 15.7 & 20.7 & 17.1 & 5.0 & 2.4 & 6.1 \\
\hline & 10 & 29.3 & 40.0 & 29.2 & 13.9 & 7.3 & 14.9 \\
\hline \multirow[t]{2}{*}{655020} & 2 & 13.2 & 17.0 & 16.3 & 5.3 & 4.6 & 1.7 \\
\hline & 10 & 25.1 & 36.4 & 29.6 & 12.2 & 10.8 & 11.6 \\
\hline \multirow[t]{2}{*}{655070} & 2 & 15.6 & 19.3 & 17.0 & 8.0 & 8.3 & 16.4 \\
\hline & 10 & 30.1 & 35.0 & 31.4 & 17.4 & 20.0 & 22.6 \\
\hline \multirow[t]{2}{*}{655010} & 2 & 9.0 & 16.1 & 23.3 & 4.8 & 9.8 & 22.3 \\
\hline & 10 & 17.8 & 26.5 & 36.5 & 6.9 & 11.6 & 24.9 \\
\hline
\end{tabular}


GCM - MRI; Downscaling Method - LARS-WG

SRES A2 (2046 - 2065)

DRWH Reliability (\%)

per capita roof area

$\begin{array}{llllllll}\text { Gauge } & \text { sq. } \mathbf{m} & \text { Jan } & \text { Feb } & \text { Mar } & \text { Apr } & \text { May } & \text { Jun } \\ \mathbf{6 5 5 9 2 0} & \mathbf{2} & 4.8 & 3.1 & 6.3 & 10.0 & 20.5 & 25.6\end{array}$

$\begin{array}{lllllll}10 & 12.7 & 7.8 & 12.8 & 20.1 & 35.6 & 45.1\end{array}$

$\begin{array}{llllllll}655940 & 2 & 3.7 & 2.7 & 5.2 & 10.4 & 16.8 & 18.9\end{array}$

$\begin{array}{lllllll}10 & 9.1 & 6.6 & 8.5 & 18.8 & 29.6 & 37.8\end{array}$

$\begin{array}{llllllll}655990 & 2 & 3.9 & 2.9 & 4.9 & 8.3 & 15.4 & 19.8\end{array}$

$\begin{array}{lllllll}10 & 9.8 & 8.3 & 10.5 & 16.9 & 30.9 & 37.5\end{array}$

$\begin{array}{llllllll}655780 & 2 & 1.7 & 2.5 & 6.5 & 11.2 & 18.3 & 22.3\end{array}$

$\begin{array}{lllllll}10 & 4.5 & 5.7 & 12.5 & 21.9 & 30.7 & 34.6\end{array}$

$\begin{array}{llllllll}655850 & 2 & 3.8 & 5.3 & 8.9 & 9.6 & 15.8 & 20.3\end{array}$

$\begin{array}{lllllll}10 & 7.8 & 10.6 & 14.0 & 21.0 & 29.7 & 33.6\end{array}$

$\begin{array}{llllllll}655480 & 2 & 4.2 & 4.8 & 11.1 & 12.7 & 12.8 & 17.1\end{array}$

$\begin{array}{lllllll}10 & 9.9 & 15.0 & 21.6 & 22.5 & 22.6 & 32.9\end{array}$

$\begin{array}{llllllll}655600 & 2 & 2.8 & 7.0 & 8.9 & 11.7 & 12.8 & 10.4\end{array}$

$\begin{array}{lllllll}10 & 8.0 & 13.4 & 18.3 & 20.5 & 23.1 & 22.8\end{array}$

$\begin{array}{llllllll}655570 & 2 & 4.2 & 8.7 & 12.7 & 13.2 & 14.7 & 13.6\end{array}$

$\begin{array}{lllllll}10 & 11.7 & 17.9 & 26.2 & 25.8 & 31.1 & 31.1\end{array}$

$\begin{array}{llllllll}655630 & 2 & 4.6 & 4.5 & 7.5 & 8.9 & 11.9 & 14.0\end{array}$

$\begin{array}{lllllll}10 & 8.3 & 10.3 & 20.5 & 20.0 & 22.7 & 24.8\end{array}$

$\begin{array}{llllllll}655620 & 2 & 3.3 & 5.8 & 9.4 & 10.8 & 14.2 & 12.9\end{array}$

$\begin{array}{lllllll}10 & 7.4 & 13.5 & 19.7 & 18.5 & 23.2 & 24.9\end{array}$

$\begin{array}{llllllll}655550 & 2 & 4.9 & 5.7 & 8.3 & 11.8 & 10.0 & 10.7\end{array}$

$\begin{array}{lllllll}10 & 10.7 & 11.4 & 18.7 & 20.5 & 18.7 & 20.6\end{array}$

$\begin{array}{llllllll}655450 & 2 & 5.7 & 5.7 & 7.0 & 12.7 & 13.1 & 13.6\end{array}$

$\begin{array}{lllllll}10 & 7.5 & 9.8 & 12.5 & 19.6 & 20.1 & 25.0\end{array}$

$\begin{array}{llllllll}654720 & 2 & 3.0 & 5.1 & 8.8 & 12.6 & 13.9 & 11.7\end{array}$

$\begin{array}{llllllll} & \mathbf{1 0} & 7.4 & 10.8 & 14.4 & 21.9 & 20.8 & 19.8 \\ \mathbf{6 5 3 8 7 0} & \mathbf{2} & 1.4 & 2.5 & 5.9 & 9.2 & 11.5 & 12.6\end{array}$

$\begin{array}{lllllll}10 & 4.9 & 5.5 & 11.6 & 15.6 & 19.8 & 22.2\end{array}$

$\begin{array}{llllllll}653760 & 2 & 1.7 & 1.3 & 5.8 & 11.6 & 13.2 & 15.0\end{array}$

$\begin{array}{lllllll}10 & 8.2 & 6.5 & 13.3 & 19.5 & 21.0 & 25.6\end{array}$

$\begin{array}{llllllll}653800 & 2 & 2.3 & 4.8 & 11.2 & 11.2 & 14.1 & 14.1\end{array}$

$\begin{array}{llllllll}10 & 8.7 & 13.8 & 20.2 & 19.4 & 22.1 & 27.1\end{array}$

$\begin{array}{llllllll}653440 & 2 & 2.2 & 2.4 & 5.7 & 11.0 & 14.4 & 21.1\end{array}$

$\begin{array}{lllllll}10 & 3.7 & 4.6 & 10.8 & 17.7 & 23.9 & 34.3\end{array}$

$\begin{array}{llllllll}653380 & 2 & 0.9 & 1.7 & 5.7 & 9.4 & 11.8 & 14.0\end{array}$

$\begin{array}{llllllll} & \mathbf{1 0} & 3.9 & 6.0 & 12.5 & 17.7 & 21.0 & 26.7 \\ \mathbf{6 5 3 3 5 0} & \mathbf{2} & 0.6 & 3.5 & 6.9 & 8.1 & 8.8 & 12.4 \\ & \mathbf{1 0} & 4.3 & 6.8 & 12.7 & 17.2 & 18.0 & 26.9 \\ \mathbf{6 5 5 2 8 0} & \mathbf{2} & 3.0 & 4.2 & 7.4 & 10.1 & 9.7 & 14.7 \\ & \mathbf{1 0} & 7.1 & 6.8 & 11.7 & 17.7 & 25.1 & 23.7\end{array}$


GCM - MRI; Downscaling Method - LARS-WG

SRES A2 (2046 - 2065)

DRWH Reliability (\%)

per capita roof area

Gauge sq. m Jul Aug Sep Oct Nov Dec

$\begin{array}{llllllll}655920 & 2 & 13.4 & 13.3 & 16.6 & 12.8 & 9.7 & 9.7\end{array}$

$\begin{array}{lllllll}10 & 34.3 & 32.7 & 38.6 & 31.3 & 23.5 & 19.7\end{array}$

$\begin{array}{llllllll}655940 & 2 & 10.5 & 5.4 & 5.1 & 9.3 & 9.3 & 7.4\end{array}$

$\begin{array}{lllllll}10 & 22.6 & 17.8 & 18.5 & 24.1 & 23.7 & 18.2\end{array}$

$\begin{array}{llllllll}655990 & 2 & 9.1 & 4.8 & 5.2 & 9.8 & 8.4 & 10.1\end{array}$

$\begin{array}{lllllll}10 & 19.8 & 14.1 & 13.4 & 24.1 & 19.3 & 19.0\end{array}$

$\begin{array}{llllllll}655780 & 2 & 8.9 & 4.5 & 6.7 & 12.8 & 11.8 & 8.4\end{array}$

$\begin{array}{lllllll}10 & 17.6 & 11.1 & 14.7 & 30.2 & 21.3 & 17.2\end{array}$

$\begin{array}{llllllll}655850 & 2 & 11.1 & 7.7 & 9.4 & 17.7 & 10.9 & 8.1\end{array}$

$\begin{array}{lllllll}10 & 20.8 & 18.2 & 20.2 & 34.8 & 22.7 & 16.7\end{array}$

$\begin{array}{llllllll}655480 & 2 & 17.3 & 21.8 & 22.5 & 10.3 & 5.0 & 6.7\end{array}$

$\begin{array}{lllllll}10 & 34.2 & 36.9 & 39.7 & 19.6 & 12.6 & 12.2\end{array}$

$\begin{array}{llllllll}655600 & 2 & 10.8 & 13.1 & 13.1 & 7.5 & 3.8 & 5.1\end{array}$

$\begin{array}{lllllll}10 & 25.6 & 27.4 & 31.9 & 17.5 & 10.6 & 10.6\end{array}$

$\begin{array}{llllllll}655570 & 2 & 7.6 & 8.8 & 10.7 & 9.8 & 8.3 & 6.0\end{array}$

$\begin{array}{lllllll}10 & 21.9 & 20.0 & 26.7 & 24.4 & 22.8 & 12.9\end{array}$

$\begin{array}{llllllll}655630 & 2 & 9.2 & 10.3 & 10.9 & 7.2 & 3.9 & 3.2\end{array}$

$\begin{array}{lllllll}10 & 21.4 & 20.8 & 25.0 & 19.3 & 13.0 & 9.2\end{array}$

$\begin{array}{llllllll}655620 & 2 & 8.6 & 8.1 & 12.1 & 10.2 & 5.0 & 5.5\end{array}$

$\begin{array}{lllllll}10 & 22.9 & 18.5 & 25.3 & 19.0 & 12.5 & 11.1\end{array}$

$\begin{array}{llllllll}655550 & 2 & 11.8 & 15.8 & 15.4 & 8.0 & 3.3 & 5.0\end{array}$

$\begin{array}{lllllll}10 & 22.4 & 28.2 & 30.6 & 17.5 & 10.0 & 10.4\end{array}$

$\begin{array}{llllllll}655450 & 2 & 11.0 & 14.0 & 18.2 & 8.5 & 3.4 & 9.5\end{array}$

$\begin{array}{lllllll}10 & 22.0 & 25.8 & 35.5 & 20.4 & 12.2 & 17.1\end{array}$

$\begin{array}{llllllll}654720 & 2 & 7.7 & 3.6 & 6.7 & 7.9 & 5.6 & 6.2\end{array}$

$\begin{array}{lllllll}10 & 20.3 & 10.6 & 18.1 & 16.6 & 14.3 & 16.4\end{array}$

$\begin{array}{llllllll}653870 & 2 & 9.3 & 5.2 & 7.5 & 6.7 & 3.7 & 4.4\end{array}$

$\begin{array}{lllllll}10 & 20.3 & 13.3 & 20.0 & 14.1 & 10.9 & 13.9\end{array}$

$\begin{array}{llllllll}653760 & 2 & 18.7 & 22.8 & 18.1 & 10.5 & 4.3 & 6.6\end{array}$

$\begin{array}{lllllll}10 & 40.6 & 42.4 & 36.6 & 19.2 & 9.8 & 16.0\end{array}$

$\begin{array}{llllllll}653800 & 2 & 12.4 & 9.7 & 10.8 & 13.3 & 7.4 & 11.1\end{array}$

$\begin{array}{lllllll}10 & 25.7 & 22.5 & 27.9 & 22.3 & 15.0 & 17.6\end{array}$

$\begin{array}{llllllll}653440 & 2 & 13.1 & 11.0 & 14.4 & 11.9 & 5.6 & 6.7\end{array}$

$\begin{array}{lllllll}10 & 28.5 & 21.5 & 27.8 & 18.9 & 11.1 & 10.4\end{array}$

$\begin{array}{llllllll}653380 & 2 & 17.1 & 17.1 & 15.0 & 11.9 & 5.1 & 5.7\end{array}$

$\begin{array}{lllllll}10 & 34.6 & 31.9 & 30.9 & 15.4 & 8.5 & 12.0\end{array}$

$\begin{array}{llllllll}653350 & 2 & 17.7 & 20.9 & 16.8 & 9.8 & 3.5 & 0.6\end{array}$

$\begin{array}{lllllll}10 & 34.6 & 36.9 & 38.7 & 18.0 & 8.1 & 10.3\end{array}$

$\begin{array}{llllllll}655280 & 2 & 19.2 & 25.0 & 18.4 & 12.8 & 3.9 & 3.1\end{array}$

$\begin{array}{lllllll}10 & 32.2 & 39.8 & 25.9 & 29.7 & 15.2 & 7.6\end{array}$


GCM - MRI; Downscaling Method - LARS-WG

SRES A2 (2046 - 2065)

DRWH Reliability (\%)

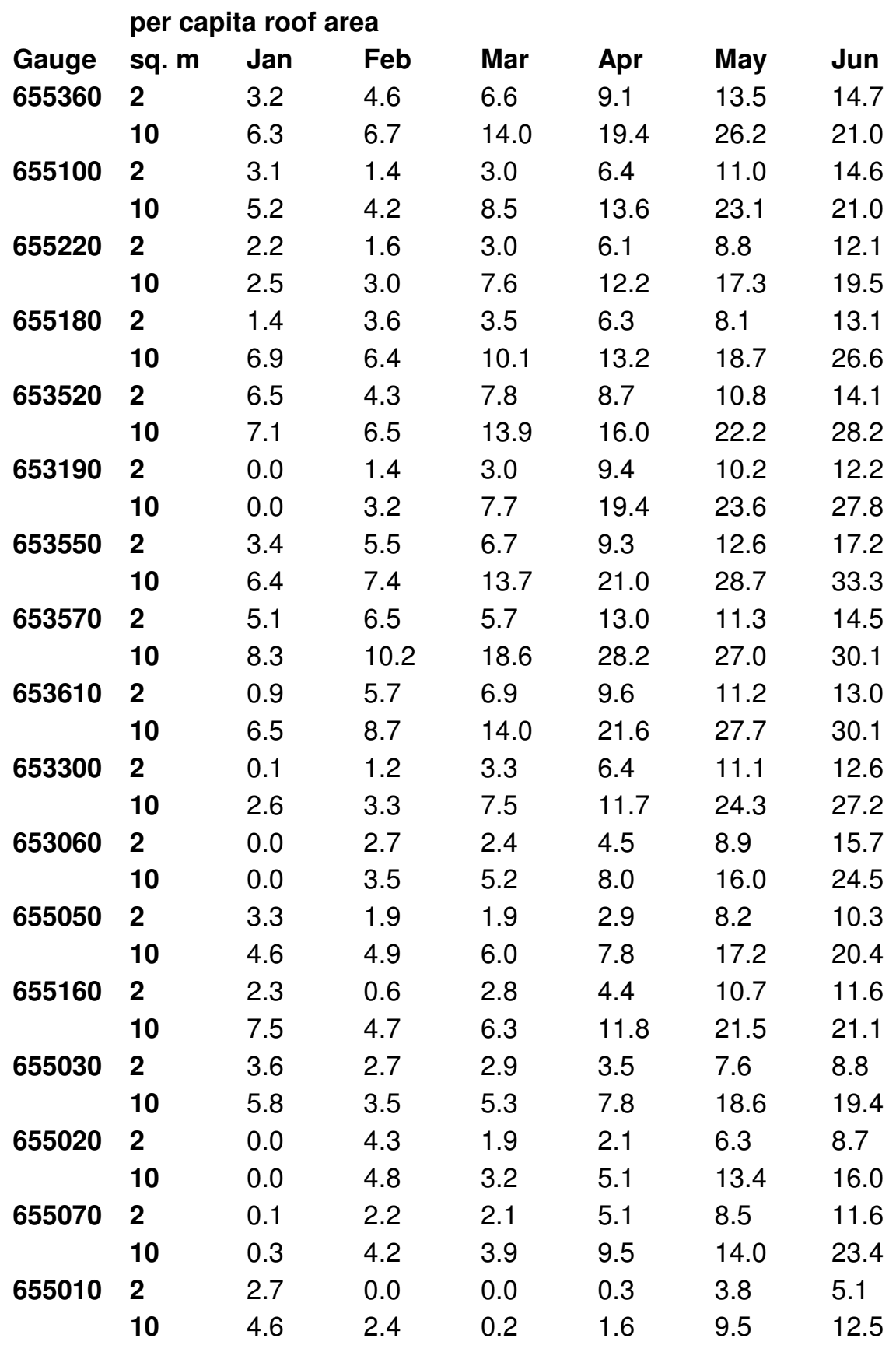


GCM - MRI; Downscaling Method - LARS-WG

SRES A2 (2046 - 2065)

DRWH Reliability (\%)

\begin{tabular}{|c|c|c|c|c|c|c|c|}
\hline \multirow{3}{*}{$\begin{array}{l}\text { Gauge } \\
655360\end{array}$} & \multicolumn{7}{|c|}{ per capita roof area } \\
\hline & sq. $m$ & Jul & Aug & Sep & Oct & Nov & Dec \\
\hline & 2 & 17.7 & 19.6 & 17.9 & 8.3 & 3.0 & 5.6 \\
\hline \multirow{3}{*}{655100} & 10 & 29.5 & 30.7 & 29.2 & 23.1 & 8.3 & 8.5 \\
\hline & 2 & 17.7 & 23.1 & 16.1 & 8.3 & 4.2 & 4.1 \\
\hline & 10 & 30.2 & 35.6 & 29.8 & 18.4 & 7.3 & 9.0 \\
\hline \multirow[t]{2}{*}{655220} & 2 & 15.9 & 17.2 & 18.3 & 8.4 & 4.9 & 3.3 \\
\hline & 10 & 31.6 & 32.5 & 39.5 & 18.3 & 9.3 & 4.9 \\
\hline \multirow[t]{2}{*}{655180} & 2 & 17.2 & 20.1 & 16.8 & 6.5 & 5.8 & 10.6 \\
\hline & 10 & 30.9 & 33.1 & 41.3 & 15.8 & 12.1 & 14.0 \\
\hline \multirow[t]{2}{*}{653520} & 2 & 16.3 & 13.7 & 18.0 & 10.3 & 7.4 & 11.4 \\
\hline & 10 & 30.4 & 27.7 & 39.4 & 17.5 & 10.4 & 13.5 \\
\hline \multirow[t]{2}{*}{653190} & 2 & 18.3 & 22.8 & 22.5 & 10.8 & 5.0 & 1.8 \\
\hline & 10 & 35.1 & 37.0 & 52.3 & 21.9 & 7.3 & 11.3 \\
\hline \multirow[t]{2}{*}{653550} & 2 & 19.2 & 23.0 & 26.1 & 11.8 & 7.6 & 4.6 \\
\hline & 10 & 35.4 & 38.0 & 53.3 & 23.7 & 13.0 & 9.6 \\
\hline \multirow[t]{2}{*}{653570} & 2 & 16.3 & 14.4 & 18.3 & 12.3 & 4.9 & 6.8 \\
\hline & 10 & 32.8 & 27.1 & 43.5 & 24.8 & 10.1 & 10.0 \\
\hline \multirow[t]{2}{*}{653610} & 2 & 18.4 & 18.2 & 22.8 & 11.8 & 8.3 & 3.8 \\
\hline & 10 & 38.1 & 35.2 & 44.7 & 22.9 & 12.6 & 12.9 \\
\hline \multirow[t]{2}{*}{653300} & 2 & 13.5 & 17.7 & 19.4 & 9.5 & 3.4 & 1.3 \\
\hline & 10 & 21.9 & 35.6 & 42.5 & 20.2 & 5.3 & 4.0 \\
\hline \multirow[t]{2}{*}{653060} & 2 & 14.3 & 18.7 & 14.4 & 4.1 & 1.3 & 2.4 \\
\hline & 10 & 21.3 & 35.9 & 38.8 & 12.0 & 3.2 & 2.9 \\
\hline \multirow[t]{2}{*}{655050} & 2 & 14.7 & 22.1 & 14.5 & 8.8 & 7.8 & 4.6 \\
\hline & 10 & 27.7 & 42.2 & 30.8 & 15.7 & 10.4 & 5.7 \\
\hline \multirow[t]{2}{*}{655160} & 2 & 15.6 & 21.6 & 18.0 & 7.5 & 10.8 & 5.5 \\
\hline & 10 & 29.0 & 44.0 & 36.6 & 18.7 & 19.2 & 8.0 \\
\hline \multirow[t]{2}{*}{655030} & 2 & 14.9 & 20.7 & 15.4 & 5.1 & 4.1 & 6.4 \\
\hline & 10 & 28.1 & 41.4 & 29.5 & 13.9 & 9.0 & 8.3 \\
\hline \multirow[t]{2}{*}{655020} & 2 & 12.6 & 17.7 & 14.3 & 5.4 & 7.4 & 3.6 \\
\hline & 10 & 25.8 & 38.7 & 29.2 & 12.4 & 12.7 & 6.4 \\
\hline \multirow[t]{2}{*}{655070} & 2 & 15.7 & 16.1 & 15.2 & 6.2 & 7.7 & 4.6 \\
\hline & 10 & 30.8 & 34.1 & 31.9 & 19.1 & 19.0 & 6.8 \\
\hline \multirow[t]{2}{*}{655010} & 2 & 11.1 & 21.5 & 15.3 & 5.5 & 4.4 & 22.1 \\
\hline & 10 & 23.5 & 33.2 & 22.4 & 7.8 & 6.7 & 27.2 \\
\hline
\end{tabular}


GCM - MRI; Downscaling Method - LARS-WG

SRES B1 (2046 - 2065)

DRWH Reliability (\%)

per capita roof area

$\begin{array}{llllllll}\text { Gauge } & \text { sq. } \mathbf{m} & \text { Jan } & \text { Feb } & \text { Mar } & \text { Apr } & \text { May } & \text { Jun } \\ \mathbf{6 5 5 9 2 0} & \mathbf{2} & 4.8 & 3.2 & 5.4 & 9.5 & 19.2 & 25.2\end{array}$

$\begin{array}{llllllll}655940 & 2 & 3.4 & 2.6 & 4.6 & 9.8 & 15.4 & 20.9\end{array}$

$\begin{array}{lllllll}10 & 8.6 & 5.8 & 8.1 & 17.1 & 27.6 & 38.9\end{array}$

$\begin{array}{llllllll}655990 & 2 & 4.2 & 2.9 & 4.8 & 8.3 & 14.1 & 20.1\end{array}$

$\begin{array}{lllllll}10 & 9.0 & 8.0 & 9.6 & 14.8 & 28.6 & 37.1\end{array}$

$\begin{array}{llllllll}655780 & 2 & 2.3 & 3.3 & 6.5 & 9.8 & 17.6 & 22.7\end{array}$

$\begin{array}{lllllll}10 & 5.3 & 7.0 & 12.5 & 18.9 & 29.1 & 36.4\end{array}$

$\begin{array}{llllllll}655850 & 2 & 4.3 & 5.6 & 8.4 & 9.3 & 16.1 & 21.1\end{array}$

$\begin{array}{lllllll}10 & 8.6 & 10.5 & 14.8 & 18.7 & 29.8 & 36.4\end{array}$

$\begin{array}{llllllll}655480 & 2 & 3.2 & 3.4 & 10.9 & 12.4 & 14.9 & 18.1\end{array}$

$\begin{array}{lllllll}10 & 6.9 & 11.2 & 21.7 & 20.6 & 27.2 & 34.0\end{array}$

$\begin{array}{llllllll}655600 & 2 & 3.2 & 6.5 & 8.9 & 11.8 & 12.7 & 11.1\end{array}$

$\begin{array}{lllllll}10 & 7.0 & 12.0 & 16.0 & 19.5 & 21.0 & 22.9\end{array}$

$\begin{array}{llllllll}655570 & 2 & 4.0 & 7.2 & 11.7 & 14.1 & 14.3 & 14.5\end{array}$

$\begin{array}{lllllll}10 & 9.7 & 14.1 & 22.2 & 25.0 & 26.7 & 30.8\end{array}$

$\begin{array}{llllllll}655630 & 2 & 4.2 & 4.1 & 6.9 & 9.3 & 12.2 & 14.4\end{array}$

$\begin{array}{lllllll}10 & 7.0 & 8.5 & 16.9 & 18.5 & 20.0 & 25.4\end{array}$

$\begin{array}{llllllll}655620 & 2 & 3.0 & 4.5 & 8.6 & 10.4 & 13.0 & 13.7\end{array}$

$\begin{array}{lllllll}10 & 6.4 & 11.2 & 16.8 & 16.9 & 20.3 & 24.8\end{array}$

$\begin{array}{llllllll}655550 & 2 & 4.7 & 4.8 & 7.7 & 12.8 & 11.2 & 11.9\end{array}$

$\begin{array}{lllllll}10 & 8.8 & 10.2 & 16.1 & 20.1 & 17.2 & 20.4\end{array}$

$\begin{array}{llllllll}655450 & 2 & 5.7 & 4.3 & 6.0 & 11.9 & 14.3 & 15.7\end{array}$

$\begin{array}{lllllll}10 & 8.6 & 7.2 & 11.0 & 19.7 & 22.5 & 28.1\end{array}$

$\begin{array}{llllllll}654720 & 2 & 2.9 & 4.3 & 7.6 & 12.7 & 13.8 & 14.6\end{array}$

$\begin{array}{lllllll}10 & 6.6 & 9.2 & 12.6 & 21.2 & 21.0 & 23.4\end{array}$

$\begin{array}{llllllll}653870 & 2 & 1.0 & 1.8 & 4.6 & 8.7 & 11.2 & 14.1\end{array}$

$\begin{array}{lllllll}10 & 4.1 & 4.2 & 9.2 & 14.0 & 19.4 & 23.9\end{array}$

$\begin{array}{llllllll}653760 & 2 & 1.4 & 1.4 & 5.2 & 11.2 & 11.8 & 16.4\end{array}$

$\begin{array}{lllllll}10 & 7.6 & 6.0 & 11.6 & 19.4 & 19.5 & 28.2\end{array}$

$\begin{array}{llllllll}653800 & 2 & 3.5 & 5.2 & 10.4 & 13.0 & 15.5 & 17.0\end{array}$

$\begin{array}{lllllll}10 & 8.5 & 11.0 & 17.7 & 21.5 & 24.3 & 31.1\end{array}$

$\begin{array}{llllllll}653440 & 2 & 1.9 & 2.0 & 5.3 & 12.4 & 16.2 & 23.0\end{array}$

$\begin{array}{lllllll}10 & 3.4 & 3.3 & 9.1 & 19.5 & 26.9 & 36.5\end{array}$

$\begin{array}{llllllll}653380 & 2 & 1.2 & 2.0 & 5.2 & 11.4 & 12.2 & 15.8\end{array}$

$\begin{array}{lllllll}10 & 3.5 & 4.9 & 9.9 & 20.3 & 23.7 & 29.6\end{array}$

$\begin{array}{llllllll}653350 & 2 & 1.8 & 2.8 & 6.5 & 8.8 & 8.8 & 13.7\end{array}$

$\begin{array}{lllllll}10 & 3.8 & 4.9 & 10.9 & 18.5 & 20.3 & 29.0\end{array}$

$\begin{array}{llllllll}655280 & 2 & 1.2 & 2.2 & 6.0 & 8.9 & 8.0 & 12.9\end{array}$

$\begin{array}{lllllll}10 & 5.2 & 4.3 & 9.0 & 16.0 & 21.8 & 27.2\end{array}$ 
GCM - MRI; Downscaling Method - LARS-WG

SRES B1 (2046 - 2065)

DRWH Reliability (\%)

per capita roof area

$\begin{array}{llllllll}\text { Gauge } & \text { sq. } \mathbf{m} & \text { Jul } & \text { Aug } & \text { Sep } & \text { Oct } & \text { Nov } & \text { Dec } \\ \mathbf{6 5 5 9 2 0} & \mathbf{2} & 13.8 & 11.4 & 11.1 & 12.1 & 10.5 & 11.0\end{array}$

$\begin{array}{llllllll}655920 & 2 & 27.7 & 28.4 & 32.8 & 32.3 & 26.6 & 19.5\end{array}$

$\begin{array}{llllllll}655940 & 2 & 9.6 & 4.8 & 3.8 & 7.3 & 9.4 & 7.2\end{array}$

$\begin{array}{lllllll}10 & 19.1 & 16.4 & 15.4 & 19.7 & 27.1 & 16.4\end{array}$

$\begin{array}{llllllll}655990 & 2 & 8.3 & 4.5 & 3.8 & 7.3 & 9.3 & 9.6\end{array}$

$\begin{array}{lllllll}10 & 16.7 & 13.3 & 11.4 & 19.8 & 22.0 & 17.5\end{array}$

$\begin{array}{llllllll}655780 & 2 & 8.1 & 3.9 & 5.5 & 8.8 & 9.1 & 8.1\end{array}$

$\begin{array}{lllllll}10 & 16.3 & 11.0 & 14.3 & 24.3 & 27.2 & 14.7\end{array}$

$\begin{array}{llllllll}655850 & 2 & 11.3 & 7.0 & 8.0 & 13.3 & 10.0 & 7.6\end{array}$

$\begin{array}{lllllll}10 & 22.4 & 17.5 & 19.1 & 29.9 & 27.5 & 14.3\end{array}$

$\begin{array}{llllllll}655480 & 2 & 17.2 & 23.1 & 22.3 & 10.9 & 5.2 & 6.8\end{array}$

$\begin{array}{lllllll}10 & 33.4 & 41.0 & 40.5 & 21.6 & 12.7 & 11.1\end{array}$

$\begin{array}{llllllll}655600 & 2 & 9.8 & 11.6 & 11.1 & 7.3 & 3.2 & 7.2\end{array}$

$\begin{array}{lllllll}10 & 22.9 & 23.9 & 26.2 & 18.9 & 11.3 & 12.1\end{array}$

$\begin{array}{llllllll}655570 & 2 & 7.6 & 7.9 & 10.5 & 9.7 & 7.0 & 7.6\end{array}$

$\begin{array}{lllllll}10 & 21.2 & 18.5 & 23.2 & 25.9 & 22.6 & 15.6\end{array}$

$\begin{array}{llllllll}655630 & 2 & 9.4 & 9.2 & 9.7 & 7.1 & 3.6 & 4.4\end{array}$

$\begin{array}{lllllll}10 & 19.8 & 17.3 & 20.5 & 20.1 & 13.7 & 9.9\end{array}$

$\begin{array}{llllllll}655620 & 2 & 8.4 & 7.1 & 11.1 & 10.1 & 4.7 & 6.9\end{array}$

$\begin{array}{lllllll}10 & 20.9 & 16.0 & 21.7 & 19.9 & 12.6 & 12.4\end{array}$

$\begin{array}{llllllll}655550 & 2 & 11.1 & 13.8 & 14.0 & 8.0 & 3.5 & 6.4\end{array}$

$\begin{array}{lllllll}10 & 20.5 & 23.3 & 26.3 & 17.7 & 9.8 & 12.2\end{array}$

$\begin{array}{llllllll}655450 & 2 & 11.1 & 11.9 & 16.0 & 8.9 & 3.5 & 9.8\end{array}$

$\begin{array}{lllllll}10 & 21.5 & 20.6 & 30.6 & 22.5 & 10.5 & 17.9\end{array}$

$\begin{array}{llllllll}654720 & 2 & 8.4 & 3.8 & 6.3 & 6.8 & 5.5 & 5.3\end{array}$

$\begin{array}{llllllll} & \mathbf{1 0} & 17.1 & 10.2 & 17.2 & 15.1 & 13.5 & 14.0 \\ \mathbf{6 5 3 3 8 7 0} & \mathbf{2} & 8.7 & 4.2 & 6.9 & 5.7 & 3.9 & 3.6 \\ & \mathbf{1 0} & 16.7 & 11.2 & 19.0 & 12.3 & 10.1 & 11.0\end{array}$

$\begin{array}{llllllll}653760 & 2 & 17.5 & 20.7 & 17.8 & 9.9 & 3.9 & 5.4\end{array}$

$\begin{array}{llllllll} & \mathbf{1 0} & 32.7 & 36.8 & 35.8 & 17.7 & 9.1 & 13.4 \\ \mathbf{6 5 3 8 0 0} & \mathbf{2} & 12.5 & 7.8 & 9.3 & 11.4 & 6.8 & 10.8\end{array}$

$\begin{array}{llllllll}10 & 25.9 & 18.7 & 23.0 & 17.9 & 15.4 & 16.8\end{array}$

$\begin{array}{llllllll}653440 & 2 & 12.2 & 8.9 & 13.0 & 9.9 & 4.7 & 5.5\end{array}$

$\begin{array}{lllllll}10 & 27.3 & 18.1 & 23.8 & 14.9 & 10.5 & 8.4\end{array}$

$\begin{array}{llllllll}653380 & 2 & 17.1 & 14.3 & 14.0 & 10.2 & 4.7 & 5.1\end{array}$

$\begin{array}{lllllll}10 & 33.9 & 28.1 & 26.8 & 13.6 & 8.2 & 10.6\end{array}$

$\begin{array}{llllllll}653350 & 2 & 16.8 & 17.5 & 15.3 & 9.3 & 3.5 & 1.1\end{array}$

$\begin{array}{lllllll}10 & 33.9 & 31.7 & 34.3 & 15.9 & 9.1 & 8.6\end{array}$

$\begin{array}{llllllll}655280 & 2 & 18.9 & 22.3 & 20.1 & 10.2 & 3.9 & 2.6\end{array}$

$\begin{array}{lllllll}10 & 32.4 & 35.5 & 41.3 & 32.2 & 12.6 & 12.5\end{array}$


GCM - MRI; Downscaling Method - LARS-WG

SRES B1 (2046 - 2065)

DRWH Reliability (\%)

\begin{tabular}{|c|c|c|c|c|c|c|c|}
\hline \multirow{3}{*}{$\begin{array}{l}\text { Gauge } \\
655360\end{array}$} & \multicolumn{7}{|c|}{ per capita roof area } \\
\hline & sq. $\mathrm{m}$ & Jan & Feb & Mar & Apr & May & Jun \\
\hline & 2 & 2.2 & 2.1 & 4.4 & 7.8 & 12.0 & 14.3 \\
\hline \multirow{3}{*}{655100} & 10 & 5.0 & 3.5 & 9.5 & 13.5 & 23.4 & 28.4 \\
\hline & 2 & 1.8 & 0.6 & 2.2 & 4.7 & 8.4 & 13.1 \\
\hline & 10 & 4.0 & 2.1 & 5.5 & 8.9 & 18.2 & 27.5 \\
\hline \multirow[t]{2}{*}{655220} & 2 & 1.9 & 1.4 & 3.5 & 7.0 & 9.8 & 12.5 \\
\hline & 10 & 2.2 & 2.2 & 8.2 & 13.3 & 20.8 & 23.3 \\
\hline \multirow[t]{2}{*}{655180} & 2 & 1.3 & 0.7 & 2.8 & 5.4 & 7.6 & 14.2 \\
\hline & 10 & 5.8 & 3.1 & 7.2 & 10.6 & 17.6 & 29.0 \\
\hline \multirow[t]{2}{*}{653520} & 2 & 6.9 & 2.0 & 6.1 & 8.2 & 10.4 & 13.5 \\
\hline & 10 & 6.9 & 3.3 & 10.6 & 14.0 & 21.8 & 28.3 \\
\hline \multirow[t]{2}{*}{653190} & 2 & 0.0 & 0.7 & 2.0 & 7.3 & 10.1 & 11.6 \\
\hline & 10 & 0.0 & 1.9 & 5.1 & 13.9 & 23.3 & 29.3 \\
\hline \multirow[t]{2}{*}{653550} & 2 & 3.0 & 2.1 & 4.5 & 7.7 & 12.1 & 18.3 \\
\hline & 10 & 6.0 & 3.7 & 9.8 & 15.6 & 27.0 & 35.6 \\
\hline \multirow[t]{2}{*}{653570} & 2 & 5.8 & 3.3 & 4.2 & 11.5 & 11.2 & 14.5 \\
\hline & 10 & 8.7 & 5.3 & 13.0 & 22.5 & 26.7 & 32.6 \\
\hline \multirow[t]{2}{*}{653610} & 2 & 0.4 & 2.6 & 5.6 & 8.9 & 11.7 & 13.4 \\
\hline & 10 & 6.5 & 4.7 & 11.1 & 18.5 & 27.3 & 33.2 \\
\hline \multirow[t]{2}{*}{653300} & 2 & 0.7 & 0.2 & 1.9 & 5.1 & 11.5 & 13.2 \\
\hline & 10 & 2.9 & 2.0 & 4.7 & 8.8 & 20.3 & 25.6 \\
\hline \multirow[t]{2}{*}{653060} & 2 & 0.0 & 2.0 & 2.2 & 4.1 & 9.0 & 15.6 \\
\hline & 10 & 0.0 & 3.8 & 5.3 & 7.1 & 14.6 & 23.4 \\
\hline \multirow[t]{2}{*}{655050} & 2 & 2.8 & 2.3 & 2.5 & 2.9 & 8.0 & 11.9 \\
\hline & 10 & 4.3 & 4.4 & 7.4 & 6.6 & 17.6 & 24.1 \\
\hline \multirow[t]{2}{*}{655160} & 2 & 2.1 & 0.8 & 3.1 & 3.3 & 10.4 & 13.0 \\
\hline & 10 & 6.2 & 4.4 & 5.8 & 7.8 & 21.6 & 25.0 \\
\hline \multirow[t]{2}{*}{655030} & 2 & 3.0 & 2.5 & 3.0 & 2.9 & 7.7 & 10.0 \\
\hline & 10 & 4.9 & 3.8 & 4.5 & 5.7 & 18.8 & 23.2 \\
\hline \multirow[t]{2}{*}{655020} & 2 & 0.0 & 3.5 & 2.4 & 1.9 & 5.5 & 9.5 \\
\hline & 10 & 0.1 & 4.1 & 3.8 & 3.8 & 12.2 & 19.6 \\
\hline \multirow[t]{2}{*}{655070} & 2 & 0.2 & 3.1 & 3.5 & 4.0 & 8.9 & 15.0 \\
\hline & 10 & 0.4 & 5.9 & 5.3 & 7.7 & 16.8 & 27.4 \\
\hline \multirow[t]{2}{*}{655010} & 2 & 2.5 & 0.2 & 1.2 & 0.2 & 3.8 & 8.7 \\
\hline & 10 & 6.5 & 3.2 & 3.8 & 2.3 & 10.4 & 16.7 \\
\hline
\end{tabular}


GCM - MRI; Downscaling Method - LARS-WG

SRES B1 (2046 - 2065)

DRWH Reliability (\%)

\begin{tabular}{|c|c|c|c|c|c|c|c|}
\hline \multirow{3}{*}{$\begin{array}{l}\text { Gauge } \\
655360\end{array}$} & \multicolumn{7}{|c|}{ per capita roof area } \\
\hline & sq. $\mathrm{m}$ & Jul & Aug & Sep & Oct & Nov & Dec \\
\hline & 2 & 15.9 & 20.8 & 17.4 & 9.4 & 2.3 & 5.2 \\
\hline \multirow{3}{*}{655100} & 10 & 27.9 & 35.3 & 28.1 & 22.9 & 6.1 & 9.2 \\
\hline & 2 & 16.8 & 23.8 & 16.1 & 8.5 & 3.1 & 3.1 \\
\hline & 10 & 28.9 & 38.8 & 28.6 & 17.5 & 5.6 & 9.8 \\
\hline \multirow[t]{2}{*}{655220} & 2 & 17.9 & 18.1 & 18.3 & 9.2 & 3.7 & 4.7 \\
\hline & 10 & 34.0 & 34.6 & 36.4 & 18.6 & 8.1 & 6.1 \\
\hline \multirow[t]{2}{*}{655180} & 2 & 18.2 & 19.7 & 17.1 & 5.0 & 2.9 & 7.4 \\
\hline & 10 & 31.6 & 31.5 & 43.0 & 18.0 & 12.3 & 11.8 \\
\hline \multirow[t]{2}{*}{653520} & 2 & 17.6 & 13.5 & 17.5 & 8.7 & 6.1 & 10.5 \\
\hline & 10 & 29.8 & 26.7 & 40.1 & 18.8 & 10.4 & 12.9 \\
\hline \multirow[t]{2}{*}{653190} & 2 & 19.0 & 21.1 & 24.0 & 9.2 & 4.1 & 1.2 \\
\hline & 10 & 35.5 & 35.0 & 52.7 & 23.6 & 7.3 & 10.3 \\
\hline \multirow[t]{2}{*}{653550} & 2 & 18.9 & 22.3 & 26.4 & 10.2 & 5.4 & 4.0 \\
\hline & 10 & 34.8 & 36.3 & 53.3 & 25.3 & 12.3 & 8.5 \\
\hline \multirow[t]{2}{*}{653570} & 2 & 16.3 & 13.6 & 16.8 & 8.9 & 4.4 & 6.1 \\
\hline & 10 & 31.5 & 25.7 & 43.9 & 25.1 & 10.5 & 9.5 \\
\hline \multirow[t]{2}{*}{653610} & 2 & 18.2 & 17.7 & 21.9 & 9.4 & 5.4 & 2.7 \\
\hline & 10 & 36.6 & 33.5 & 46.2 & 24.1 & 12.3 & 11.2 \\
\hline \multirow[t]{2}{*}{653300} & 2 & 15.2 & 17.2 & 20.9 & 8.2 & 3.6 & 0.6 \\
\hline & 10 & 25.8 & 34.7 & 43.9 & 17.6 & 7.3 & 3.0 \\
\hline \multirow[t]{2}{*}{653060} & 2 & 17.4 & 17.7 & 15.9 & 4.0 & 1.0 & 2.7 \\
\hline & 10 & 26.0 & 34.0 & 41.6 & 11.2 & 2.8 & 2.9 \\
\hline \multirow[t]{2}{*}{655050} & 2 & 16.2 & 21.8 & 15.5 & 10.4 & 7.0 & 4.4 \\
\hline & 10 & 33.3 & 42.3 & 29.4 & 18.1 & 9.3 & 5.4 \\
\hline \multirow[t]{2}{*}{655160} & 2 & 15.8 & 22.0 & 18.1 & 8.4 & 11.1 & 4.4 \\
\hline & 10 & 33.8 & 43.9 & 33.7 & 19.7 & 16.3 & 6.7 \\
\hline \multirow[t]{2}{*}{655030} & 2 & 15.4 & 20.7 & 17.0 & 4.9 & 4.7 & 4.7 \\
\hline & 10 & 33.3 & 41.8 & 27.3 & 13.9 & 7.9 & 6.1 \\
\hline \multirow[t]{2}{*}{655020} & 2 & 14.0 & 18.2 & 14.6 & 6.3 & 9.1 & 3.8 \\
\hline & 10 & 31.0 & 39.5 & 26.0 & 14.0 & 11.7 & 6.0 \\
\hline \multirow[t]{2}{*}{655070} & 2 & 16.9 & 19.5 & 18.1 & 5.9 & 6.0 & 9.5 \\
\hline & 10 & 36.1 & 39.3 & 30.0 & 14.9 & 13.4 & 14.6 \\
\hline \multirow[t]{2}{*}{655010} & 2 & 12.9 & 18.6 & 17.3 & 3.8 & 9.6 & 34.6 \\
\hline & 10 & 25.9 & 27.7 & 27.4 & 10.1 & 13.4 & 34.6 \\
\hline
\end{tabular}

